\title{
Evaluation of Instrumentation and Dynamic Thermal Ratings for Overhead Lines
}

Final Report, October 11, 2013

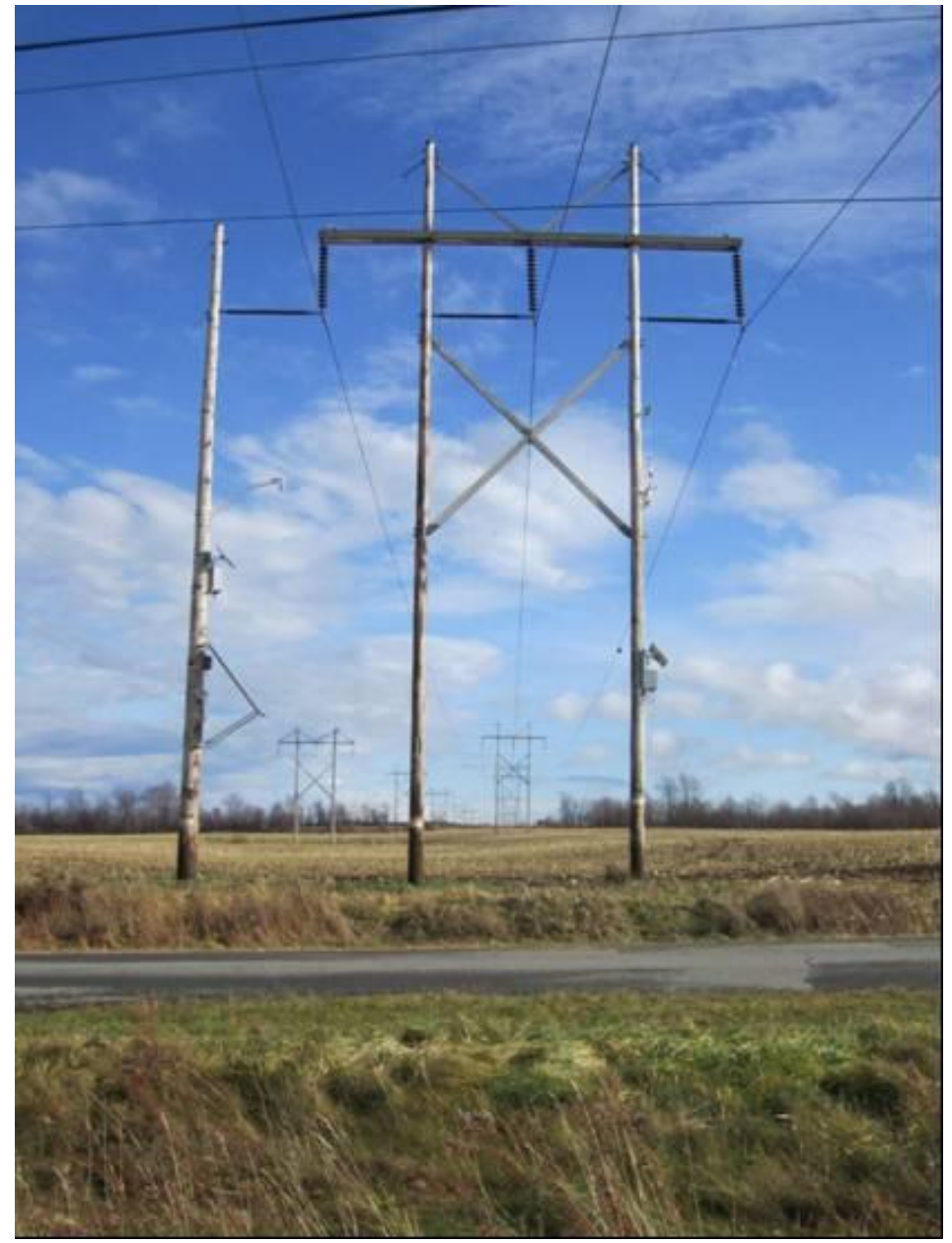

EPRI Project Manager

A. Phillips 


\section{Evaluation of Instrumentation and Dynamic Thermal Ratings for Overhead Lines}

Final Report, October 11, 2013

Work supported by the Department of Energy award number DE-OE0000317 awarded to EPRI.

EPRI Project director: A. Phillips,

Project team members: B. Clairmont, D. Childs, D. Reuger, D. Douglass, J. Bell, D. Birrell 


\section{DISCLAIMER OF WARRANTIES AND LIMITATION OF LIABILITIES}

THIS DOCUMENT WAS PREPARED BY THE ORGANIZATION(S) NAMED BELOW AS AN ACCOUNT OF WORK SPONSORED OR COSPONSORED BY THE ELECTRIC POWER RESEARCH INSTITUTE, INC. (EPRI). NEITHER EPRI, ANY MEMBER OF EPRI, ANY COSPONSOR, THE ORGANIZATION(S) BELOW, NOR ANY PERSON ACTING ON BEHALF OF ANY OF THEM:

(A) MAKES ANY WARRANTY OR REPRESENTATION WHATSOEVER, EXPRESS OR IMPLIED, (I) WITH RESPECT TO THE USE OF ANY INFORMATION, APPARATUS, METHOD, PROCESS, OR SIMILAR ITEM DISCLOSED IN THIS DOCUMENT, INCLUDING MERCHANTABILITY AND FITNESS FOR A PARTICULAR PURPOSE, OR (II) THAT SUCH USE DOES NOT INFRINGE ON OR INTERFERE WITH PRIVATELY OWNED RIGHTS, INCLUDING ANY PARTY'S INTELLECTUAL PROPERTY, OR (III) THAT THIS DOCUMENT IS SUITABLE TO ANY PARTICULAR USER'S CIRCUMSTANCE; OR

(B) ASSUMES RESPONSIBILITY FOR ANY DAMAGES OR OTHER LIABILITY WHATSOEVER (INCLUDING ANY CONSEQUENTIAL DAMAGES, EVEN IF EPRI OR ANY EPRI REPRESENTATIVE HAS BEEN ADVISED OF THE POSSIBILITY OF SUCH DAMAGES) RESULTING FROM YOUR SELECTION OR USE OF THIS DOCUMENT OR ANY INFORMATION, APPARATUS, METHOD, PROCESS, OR SIMILAR ITEM DISCLOSED IN THIS DOCUMENT.

REFERENCE HEREIN TO ANY SPECIFIC COMMERCIAL PRODUCT, PROCESS, OR SERVICE BY ITS TRADE NAME, TRADEMARK, MANUFACTURER, OR OTHERWISE, DOES NOT NECESSARILY CONSTITUTE OR IMPLY ITS ENDORSEMENT, RECOMMENDATION, OR FAVORING BY EPRI.

THE FOLLOWING ORGANIZATION(S), UNDER CONTRACT TO EPRI, PREPARED THIS REPORT:

EPRI

\section{NOTE}

For further information about EPRI, call the EPRI Customer Assistance Center at 800.313.3774 or e-mail askepri@epri.com.

Electric Power Research Institute, EPRI, and TOGETHER...SHAPING THE FUTURE OF ELECTRICITY are registered service marks of the Electric Power Research Institute, Inc. 


\section{ACKNOWLEDGMENTS}

This material is based upon work supported by the Department of Energy under Award Number DE-OE0000317.

This report was prepared by EPRI.

Principal Investigators:
A. Phillips
B. Clairmont
D. Childs
D. Reuger
D. Douglass
J. Bell
D. Birrell

EPRI would like to acknowledge the following individuals from NYPA for their support and contributions to the project:
J. Dering
R. DaSilva
M. Graham
G. Caccavale
L. Hopkins
J. Finnegan and the NYPA Line Crew 


\section{NOTICE}

This report was prepared by EPRI for the New York Power Authority (NYPA), at their request this edition of "Evaluation of Instrumentation and Dynamic Thermal Ratings for Overhead Lines" has been modified from the original document. This was done to protect proprietary information. In this edition the original Chapter 5, Appendix B, and Appendix C have been completely removed. Some elements of the body, figures, and tables have been altered or removed from this edition.

Requests for any removed, altered, or otherwise modified content should be directed to NYPA. 



\section{CONTENTS}

1 INTRODUCTION TO THE DOE/NYPA PROJECT.......................................................1-1

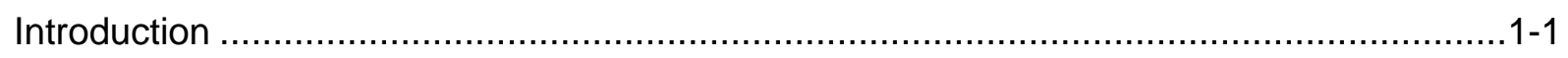

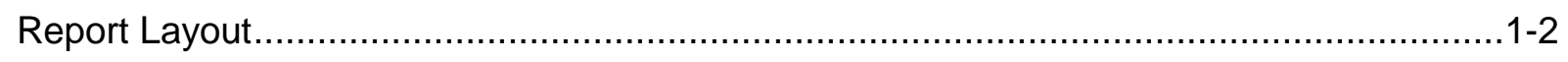

2 OVERVIEW OF THE SCIENCE OF TRANSMISSION LINE RATINGS ...............................2-1

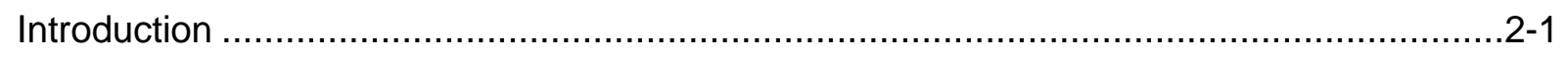

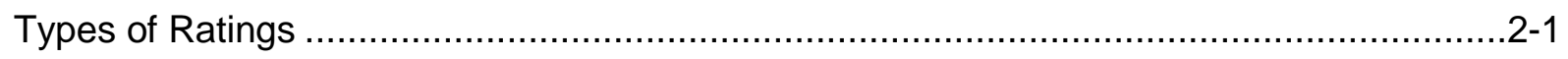

3 INSTRUMENTS AND SOFTWARE FOR DYNAMIC RATING ............................................

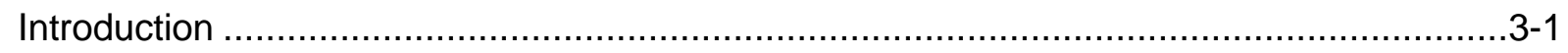

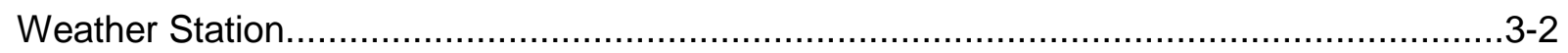

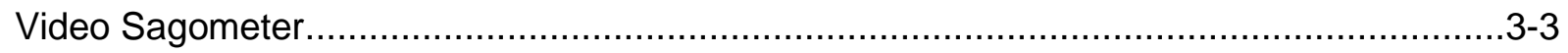

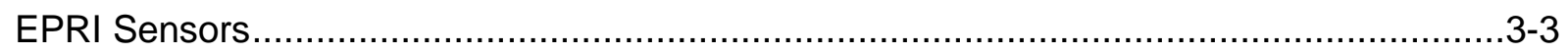

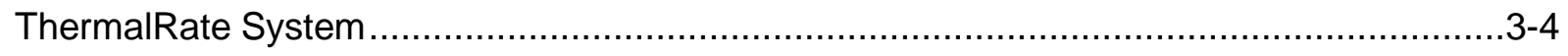

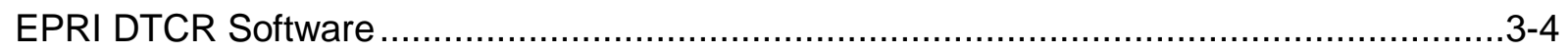

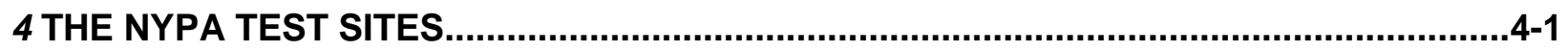

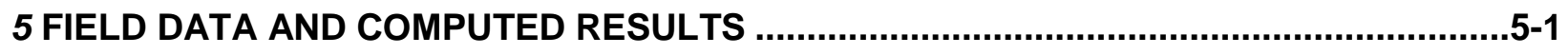

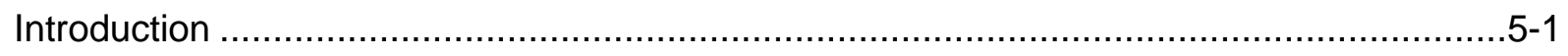

Data Types from the Four Instrument Packages..................................................... $5-1$

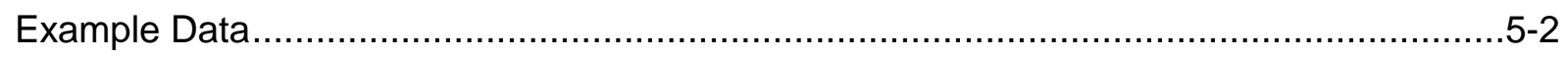

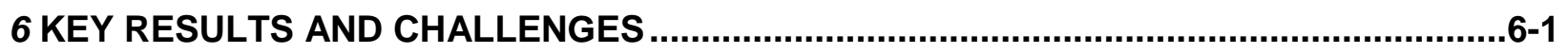

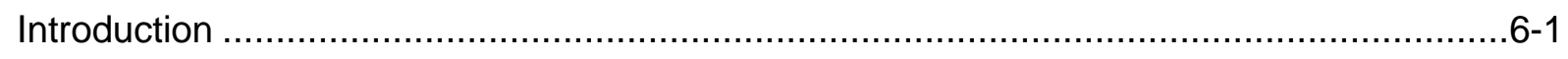

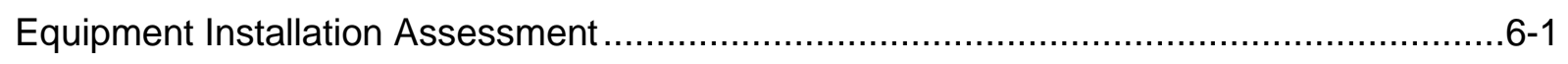

Transmission Corridor - NOAA Weather Correlation ................................................... $6-3$

Advantages of Dynamic Ratings.......................................................................... $6-5$

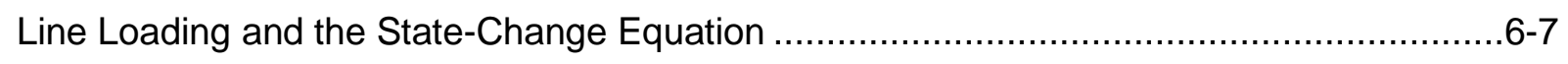

Instrument Performance and Reliability ................................................................. 6 


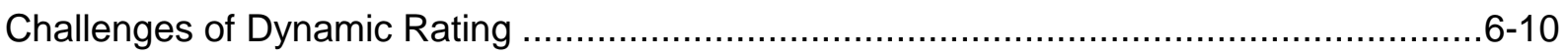

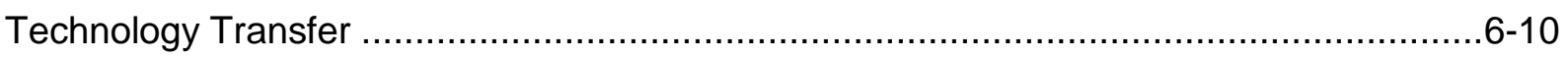

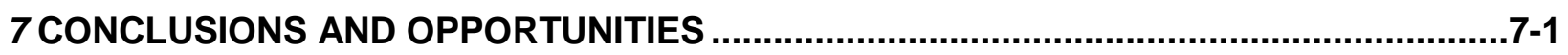

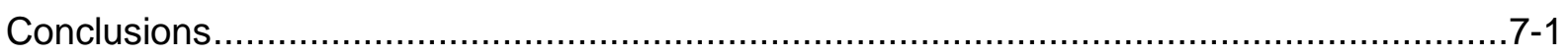

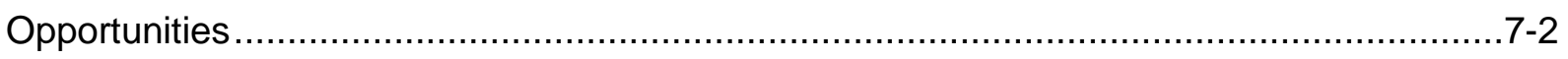

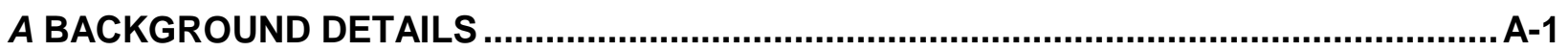

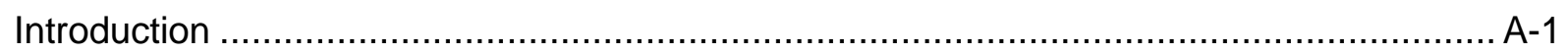

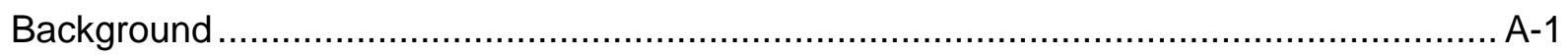

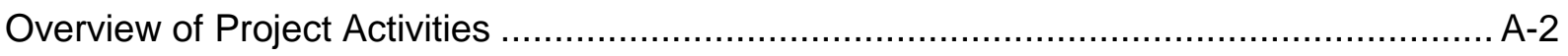

B TRANSMISSION LINE RATINGS - PRINCIPLES AND PRACTICES ............................... B-1

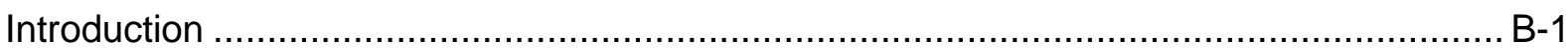

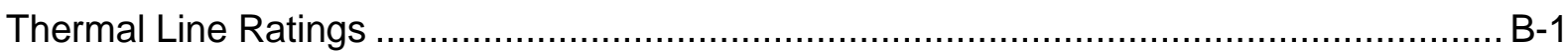

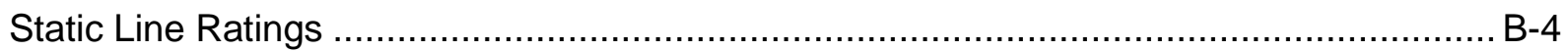

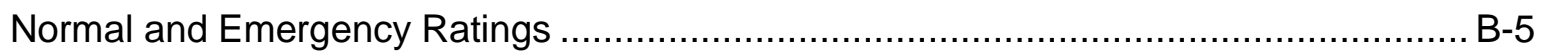

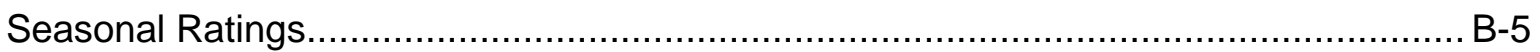

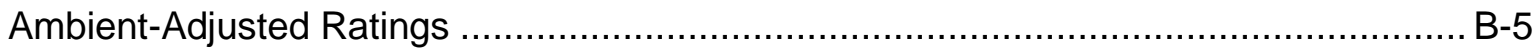

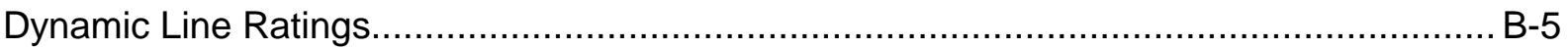

Field Studies of Line Ratings ............................................................................

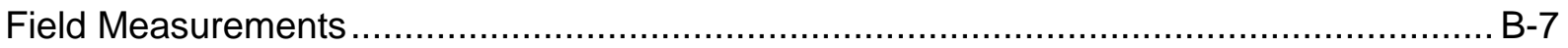

Wind Cooling - Anemometers .......................................................................... B-10

Wind Cooling - ThermalRate Conductor Model ………….................................... B-10

Wind Cooling - Conductor Temperature Monitors …………………….................. B-11

Wind Cooling - Line Sag-Tension Monitors ………………................................... B-12

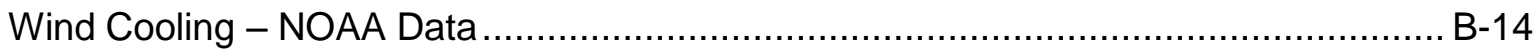

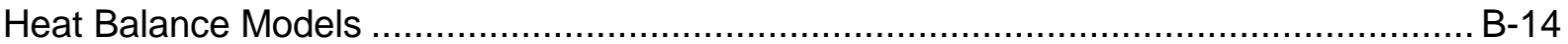

Line Specific vs. System-Wide Ratings ………....................................................... B-15

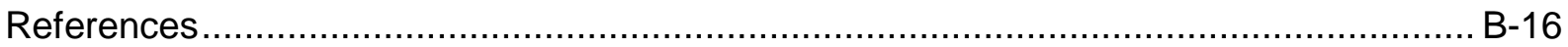

C INSTRUMENTATION, SOFTWARE AND INSTALLATION

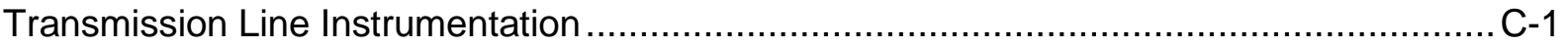

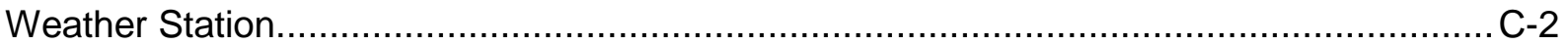

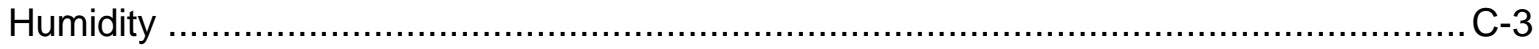

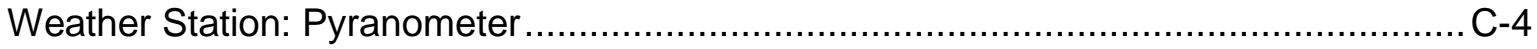




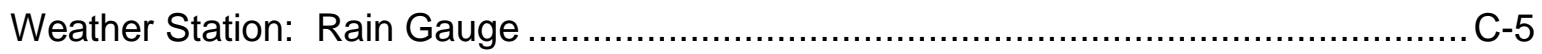

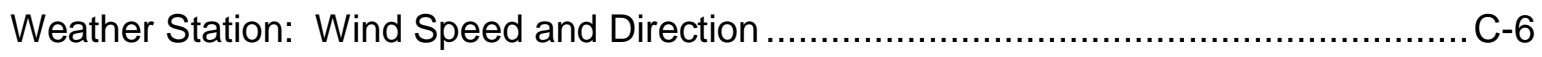

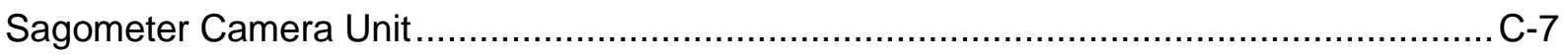

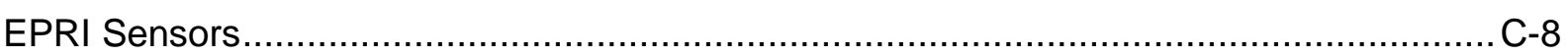

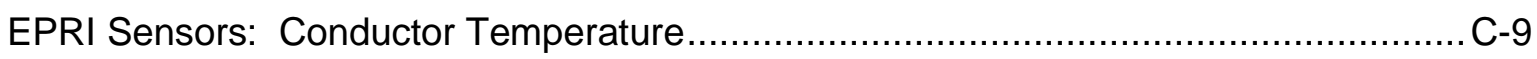

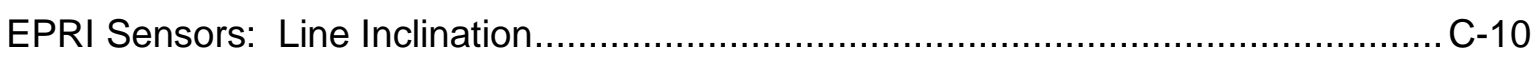

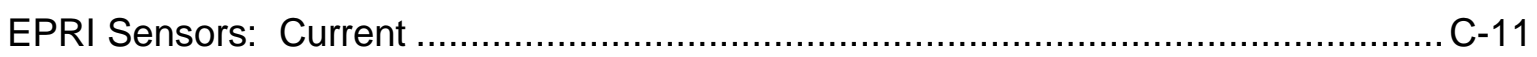

EPRI Sensor Mounting Locations at the Three Sites ................................................ C-12

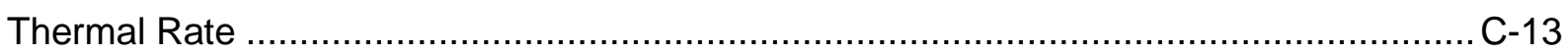

ThermalRate: Effective Perpendicular Wind Speed ……….................................. -13

ThermalRate: Load from SCADA ..........................................................................

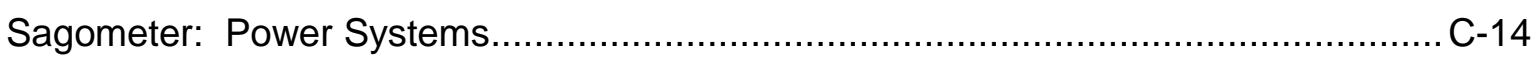

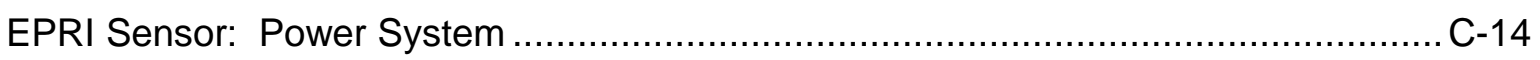

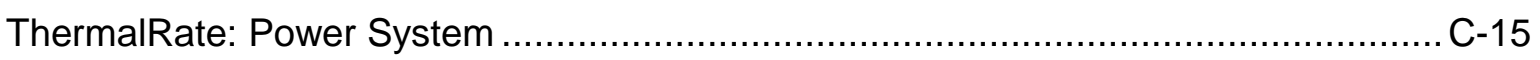

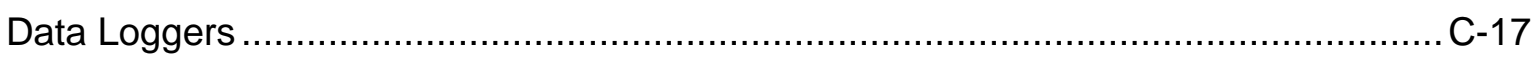

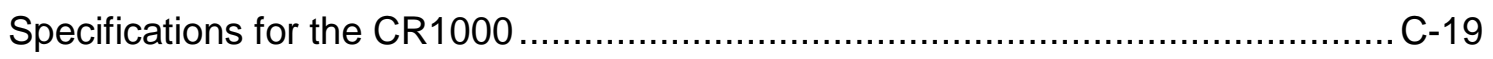

Communications Layout Diagrams ......................................................................

Modems

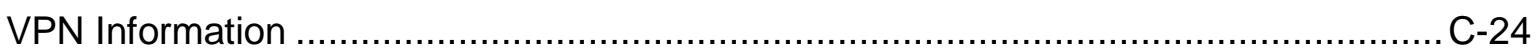

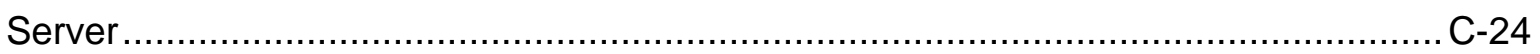

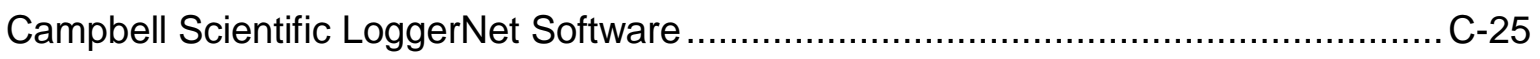

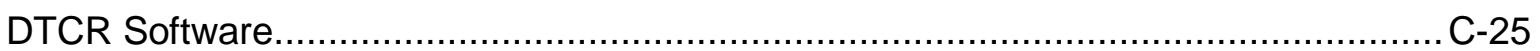

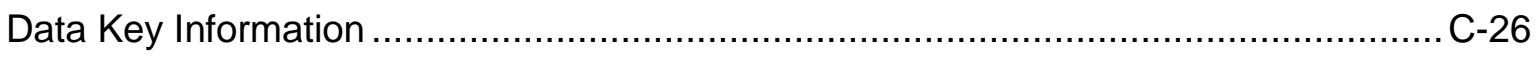

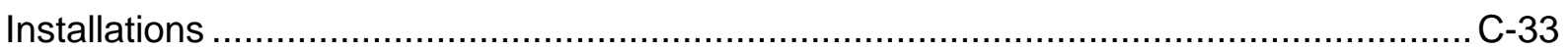

Installation Assessments: Goals and Methods ..................................................... C-33

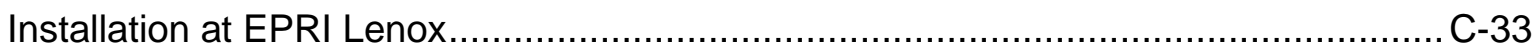

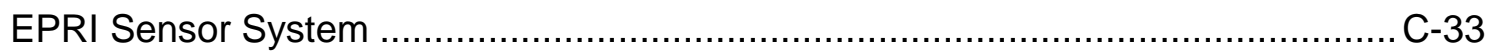

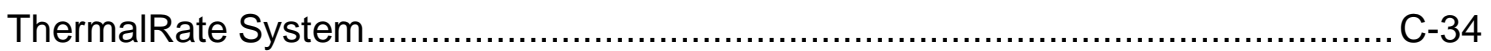

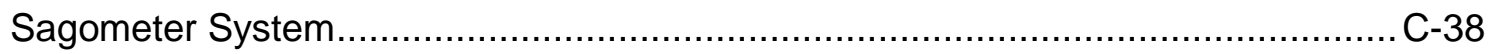

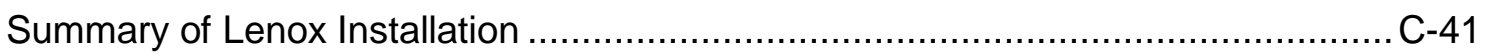

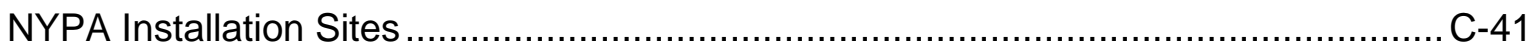

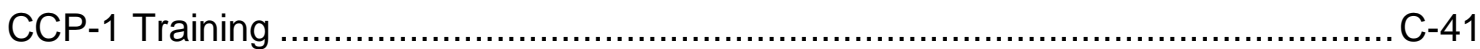

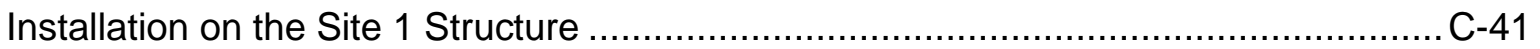

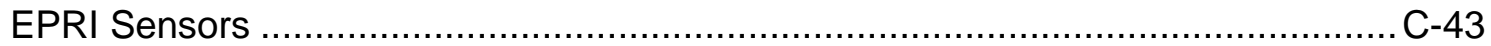

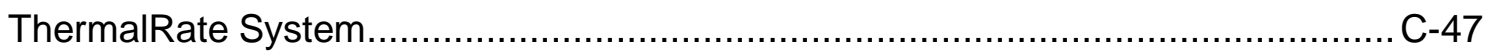


Sagometer System.

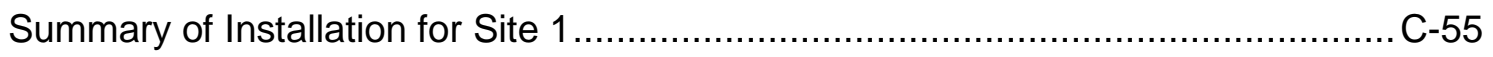

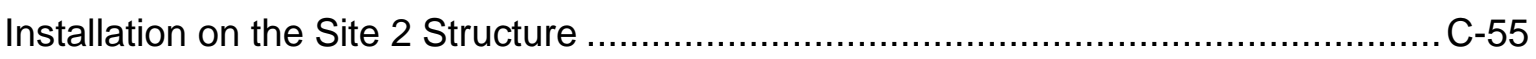

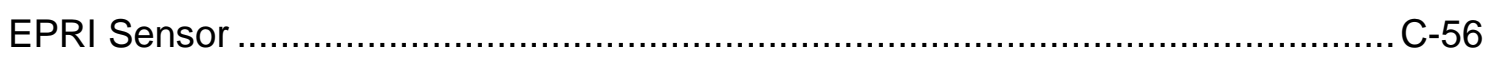

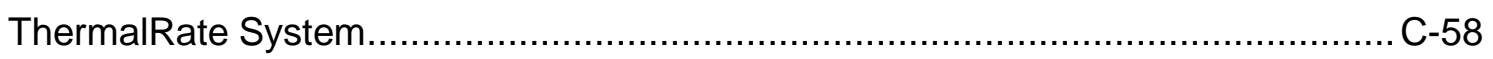

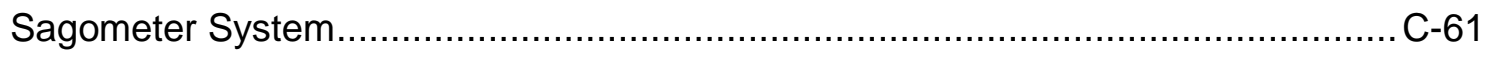

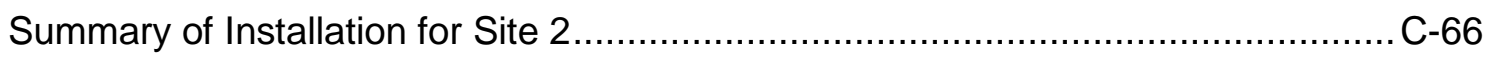

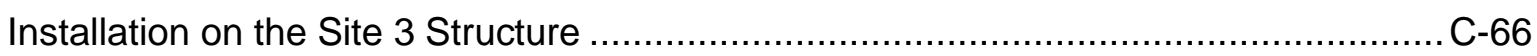

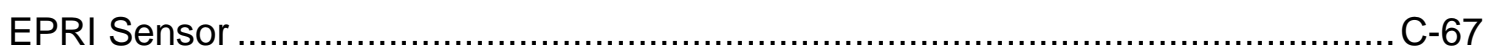

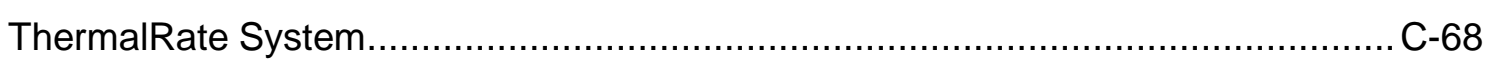

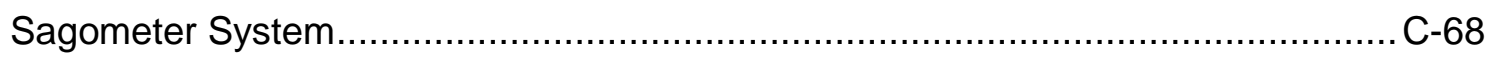

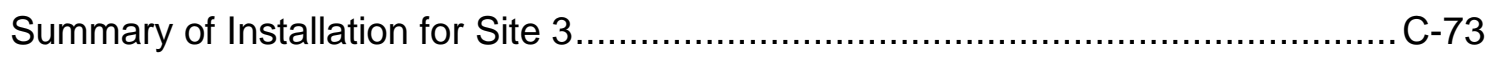

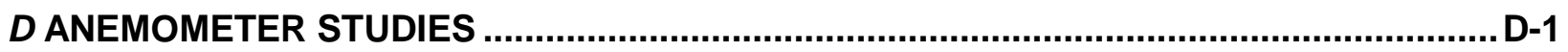

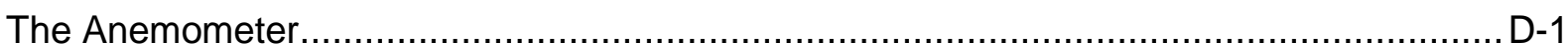

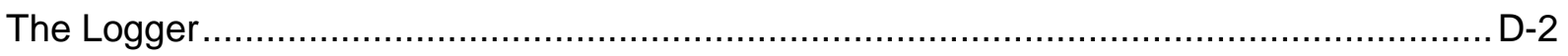

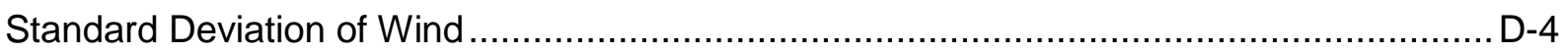

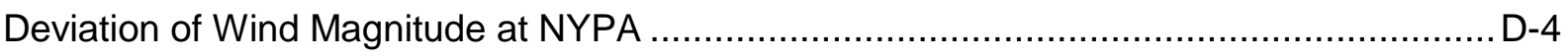

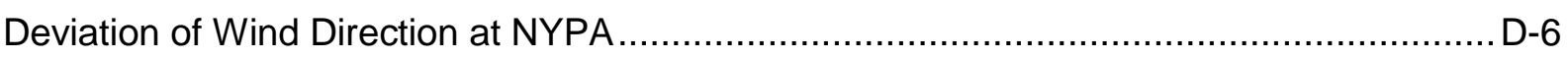

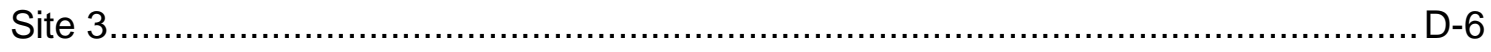

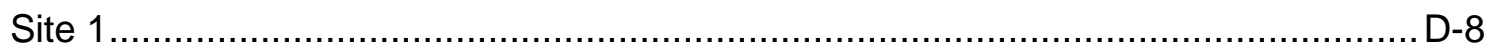

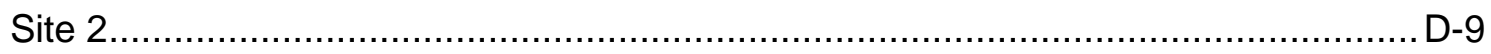

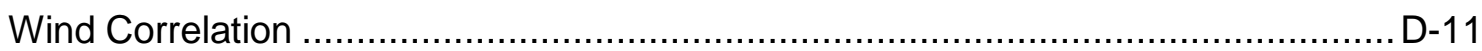

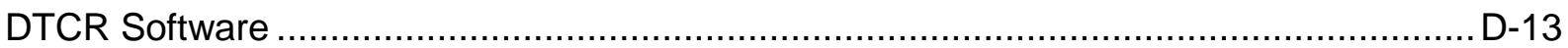

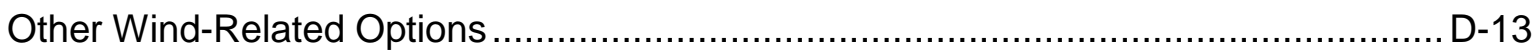

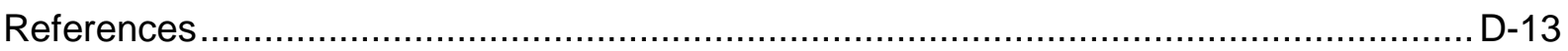

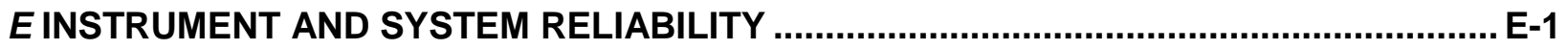

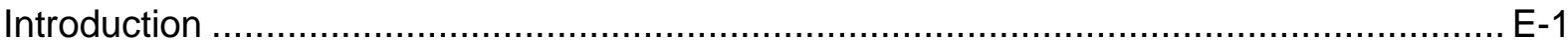

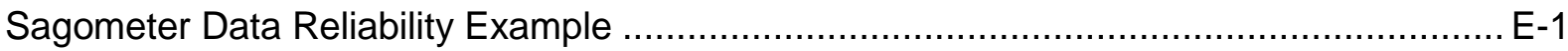

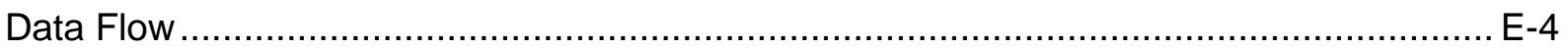

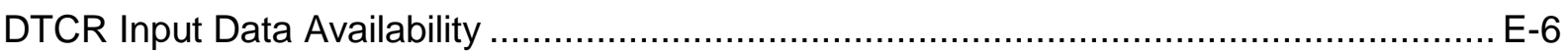

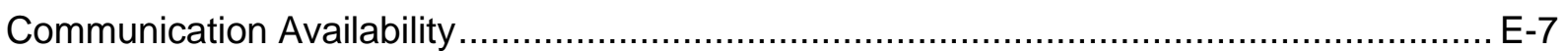

Long Term Raw Rating Data Availability Results ..................................................... E-8

Permanent Loss of Good Data ........................................................................... E-18 
DTCR Server Downtime E-21

Software Down Time

FRATING RESULTS ................................................................................................ F-1

Static Ratings of System Components ..............................................................

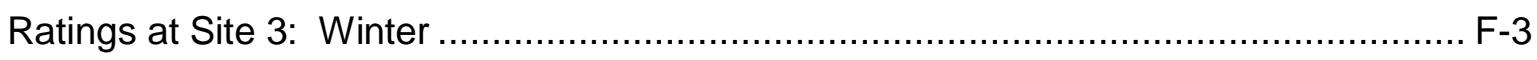

Ratings at Site 3: Summer ......................................................................... F-14

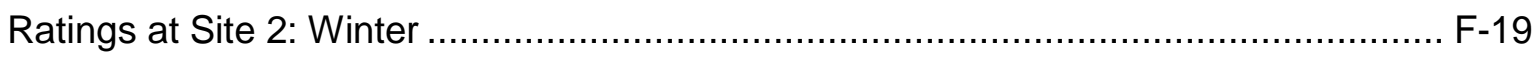

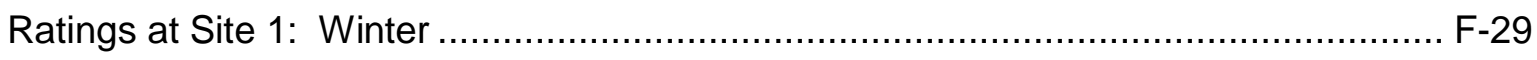

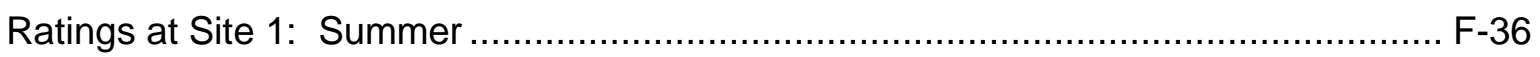

G COMPARISON OF REAL-TIME MONITORING METHODS..............................................

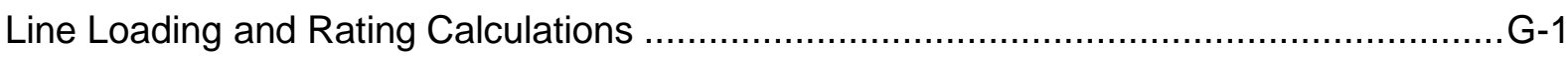

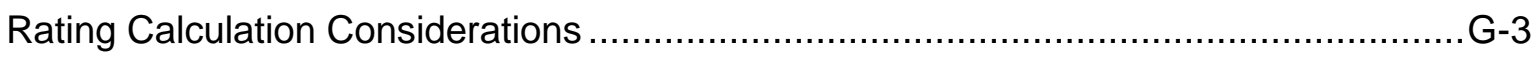

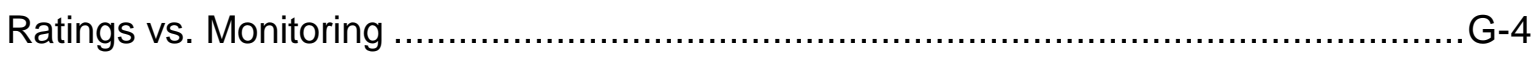

Heat Balance

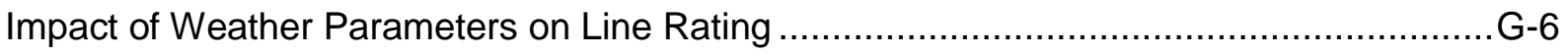

Real-Time Monitoring Methods - Definition and Comparison .....................................

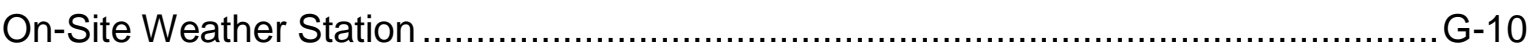

On-Site Weather Station .............................................................................

ThermalRate Conductor Model ..................................................................

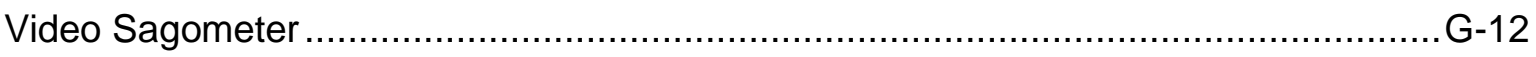

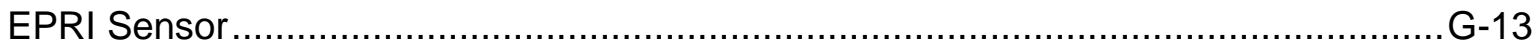

Off-Site Weather Service Data (Backup) .................................................... 15

Substation Wind Measurements ........................................................................ G-15

Summary Comparison of Monitoring Methods ......................................................

Accuracy of Wind Measurements by Monitoring Method............................................

Statistical Comparisons of Effective Wind Speed - Offsite and Onsite Wind Data ........ -19

Comparison of Onsite Weather Station and ThermalRate Wind Speed ............................20

Equivalent Perpendicular Wind Speed for ThermalRate Conductor Model....................20

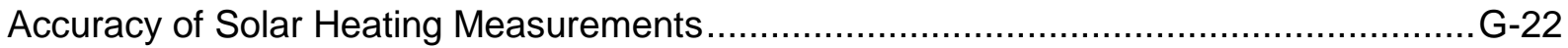

Accuracy of Air Temperature Measurements................................................................24

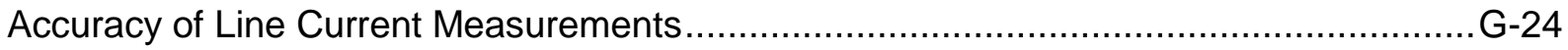

Accuracy of Conductor Temperature Calculations ......................................................

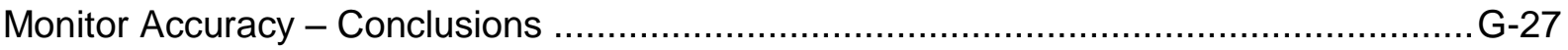




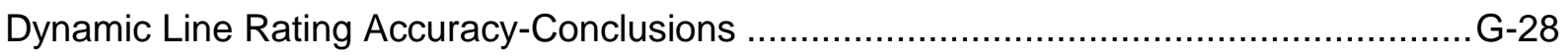

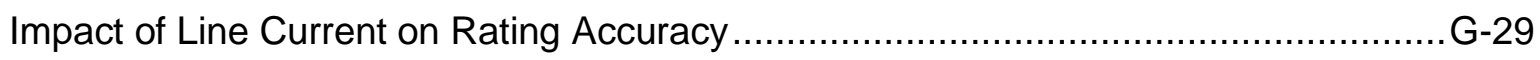

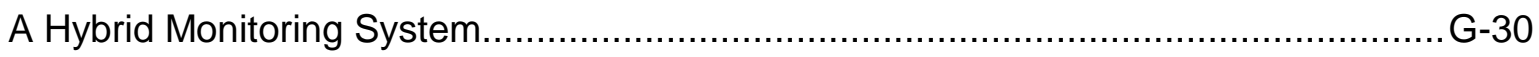

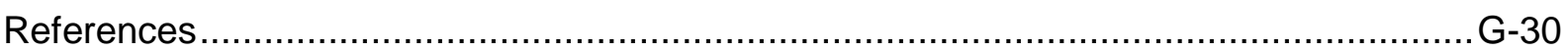

HEVALUATION OF LINE RATINGS: HISTORICAL LINE RATING DATA ...........................

Cumulative Distribution Plots...............................................................................

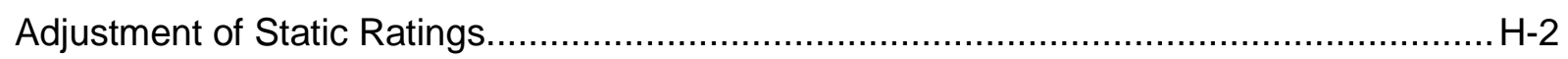

Load and Dynamic Cumulative Distribution Plots for NYPA Lines ................................ H-2

I RATING VS. WIND POWER CORRELATION................................................................ I-1

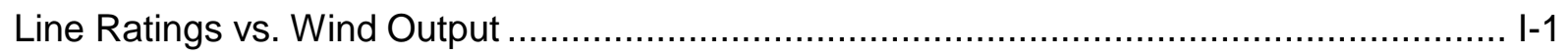

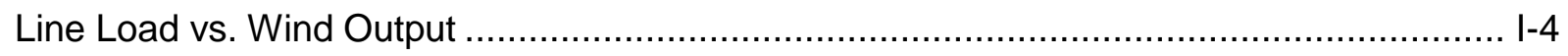

J SUMMARY, CONCLUSIONS AND RECOMMENDATIONS ..................................................

Real-Time Monitoring Methods............................................................................

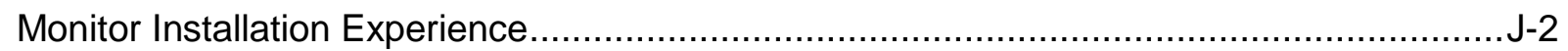

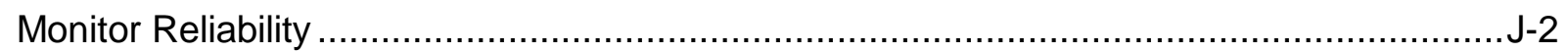

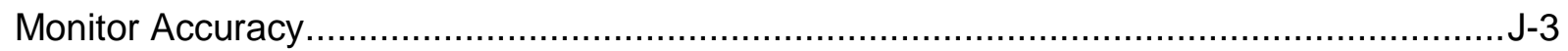

Line Rating Accuracy.......................................................................................

Impact of Line Current on Rating Accuracy .............................................................

A Hybrid Monitoring System................................................................................

Recommendations for Future Work ........................................................................

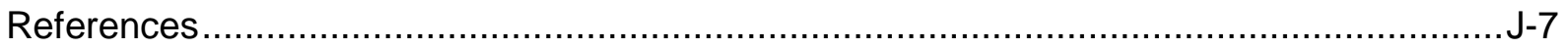

K MONTHLY PLOTS

L DATA QUALITY OF RAW DATA FILES

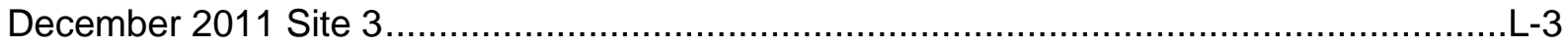

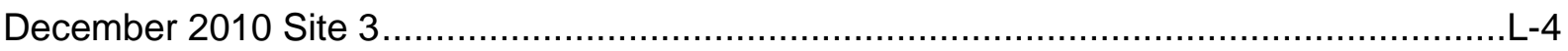

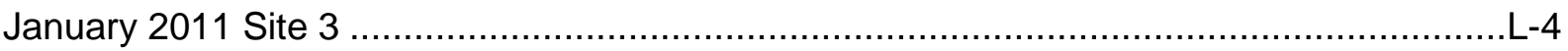

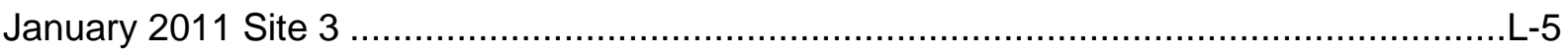

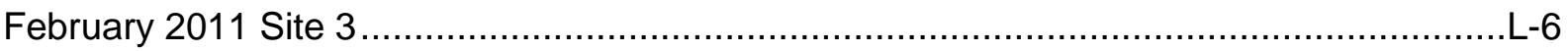

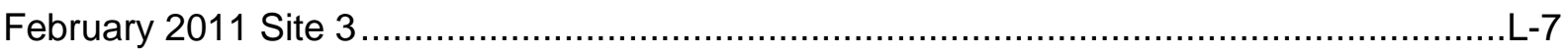

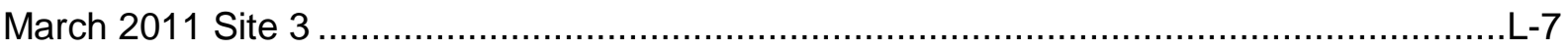

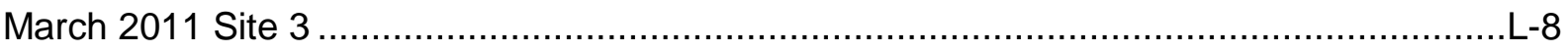




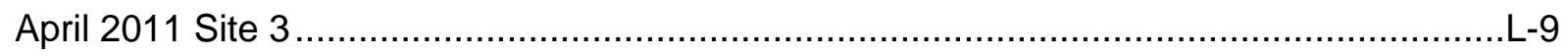

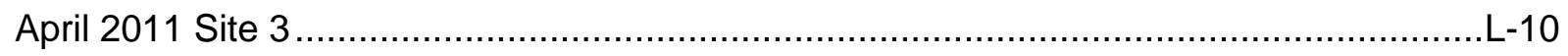

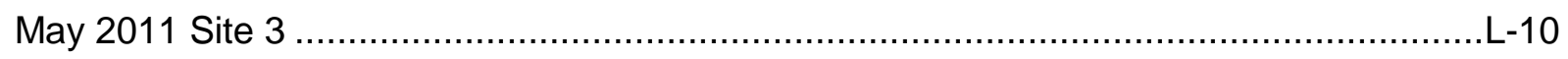

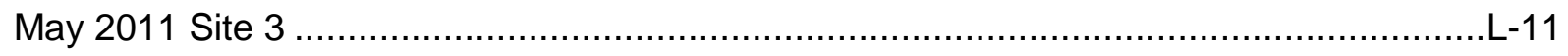

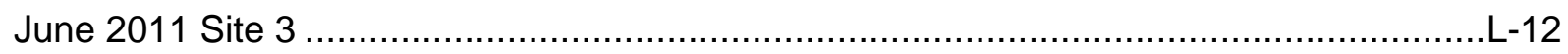

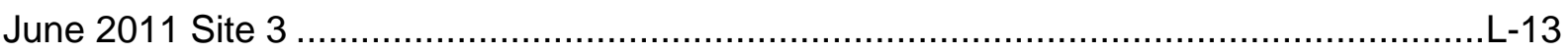

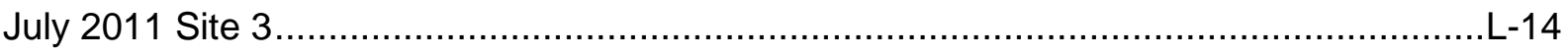

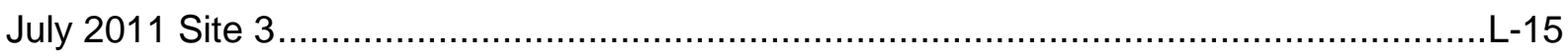

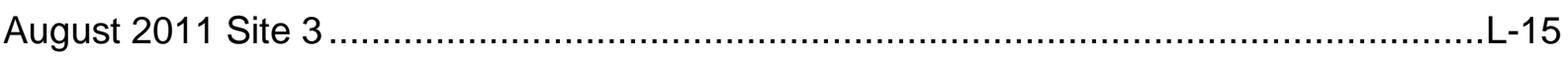

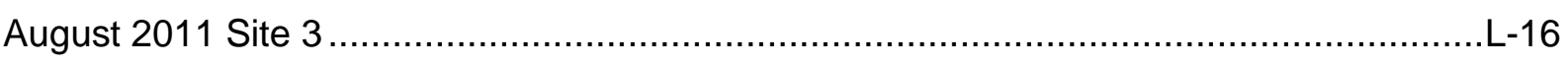

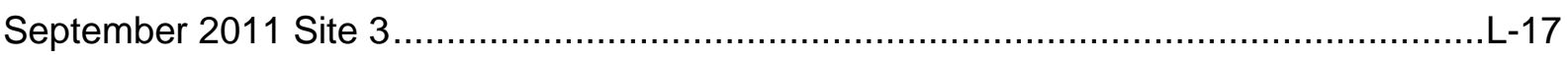

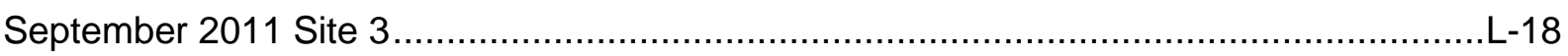

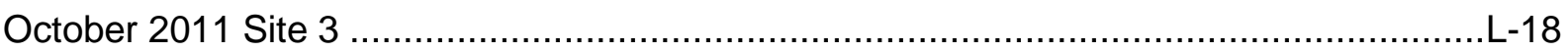

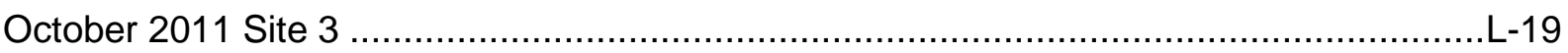

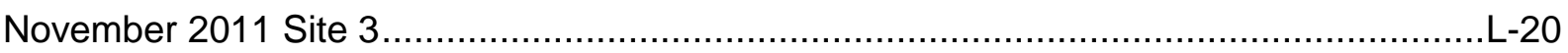

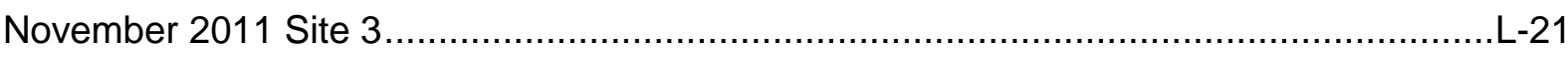

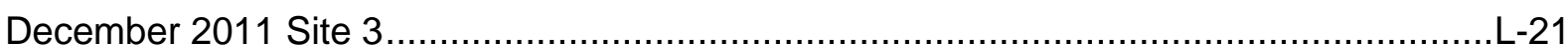

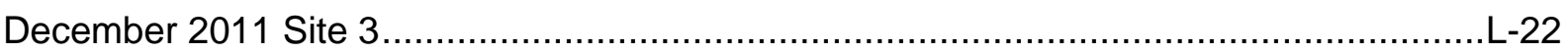

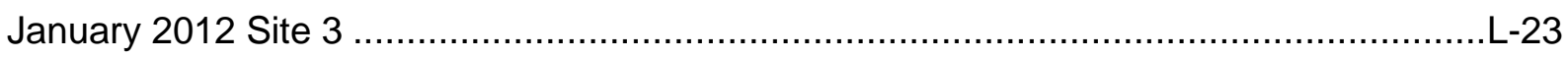

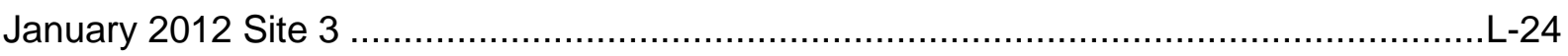

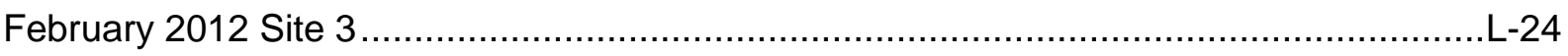

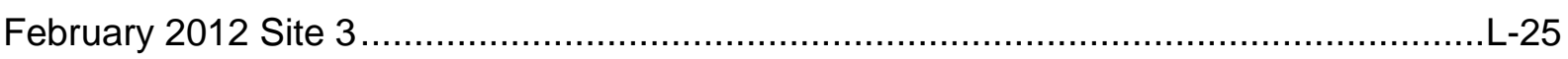

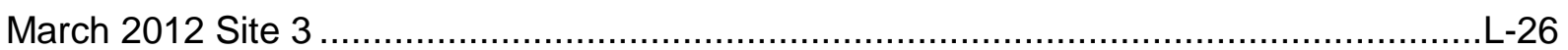

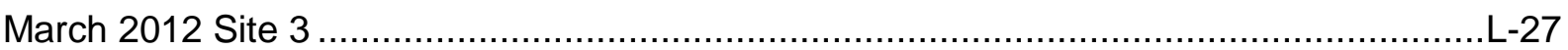

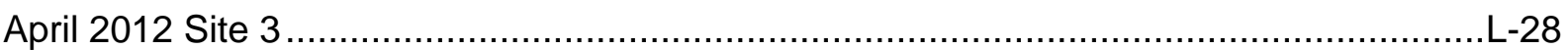

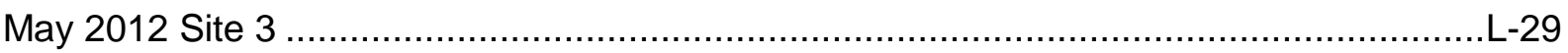

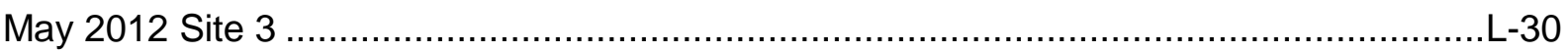

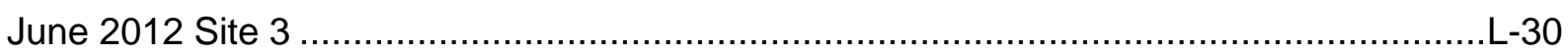

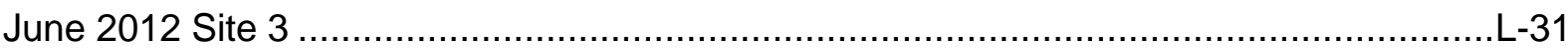

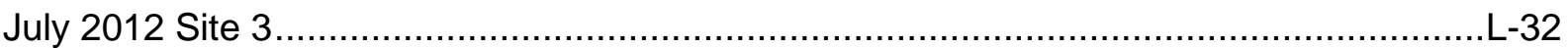

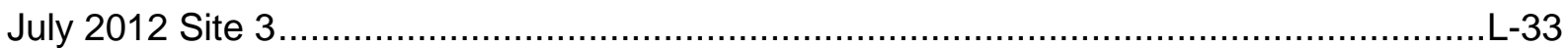

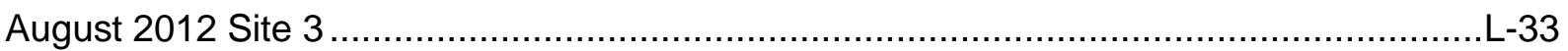

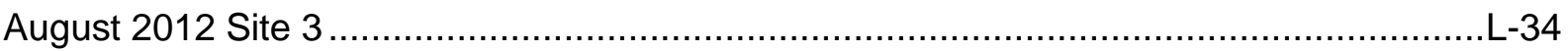

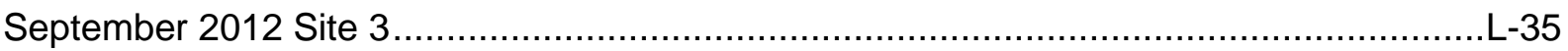

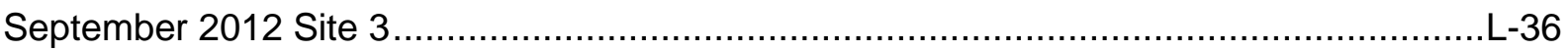




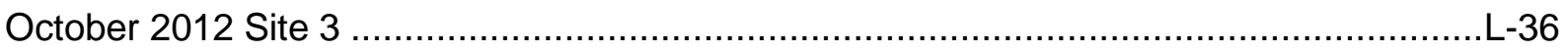

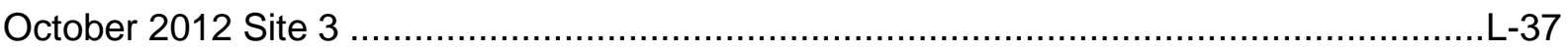

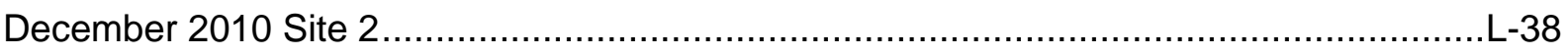

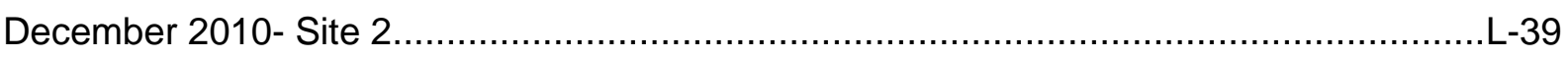

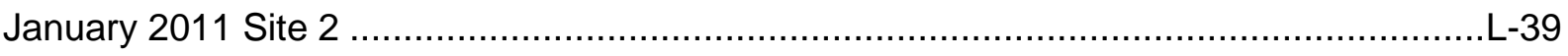

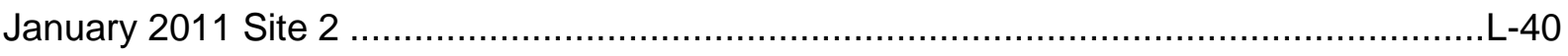

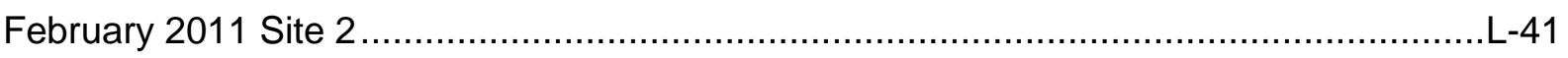

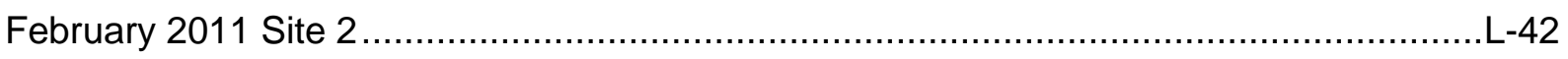

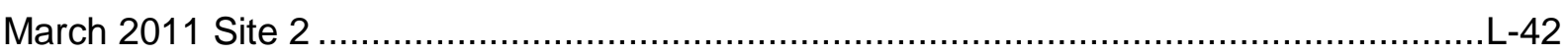

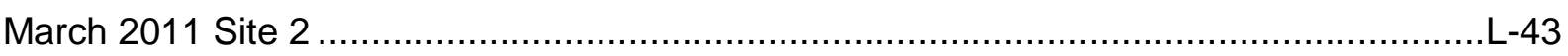

April 2011 Site 2

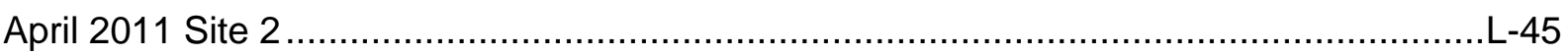

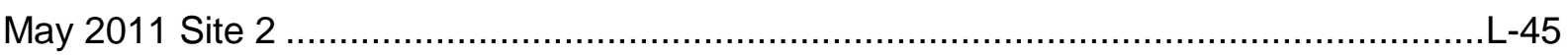

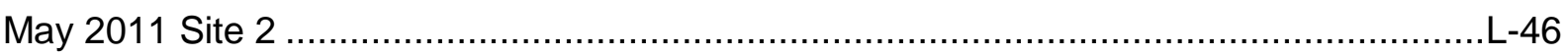

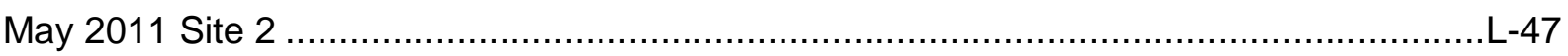

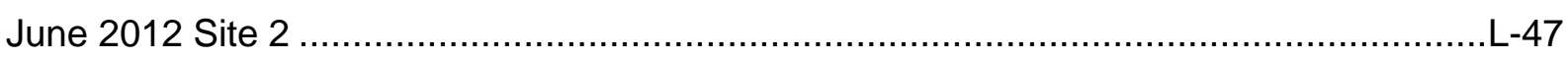

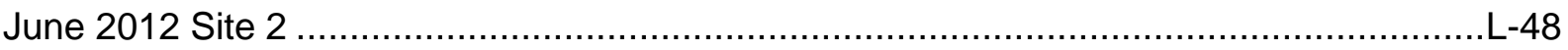

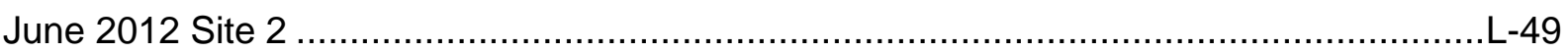

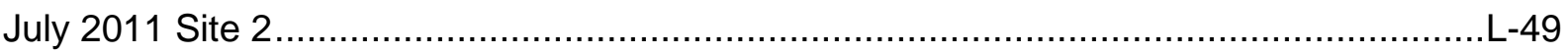

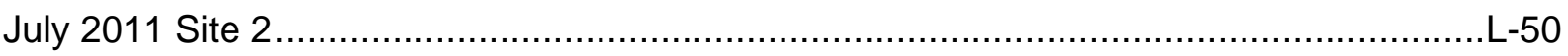

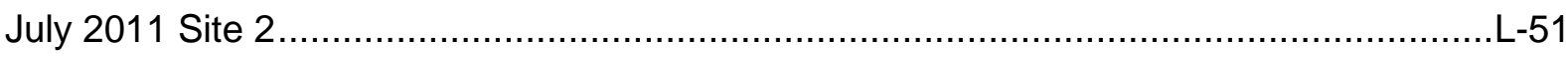

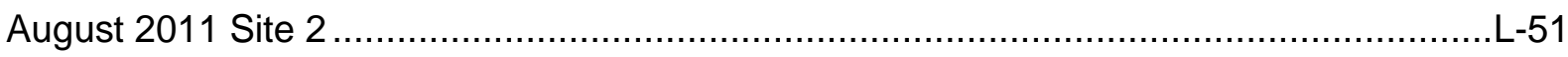

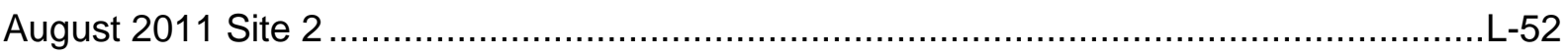

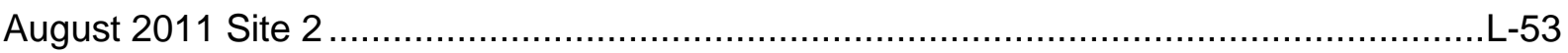

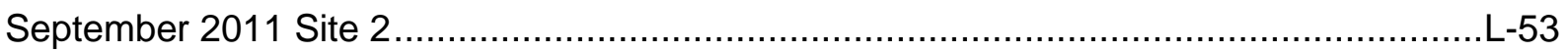

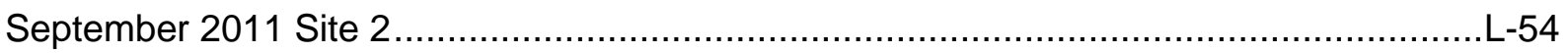

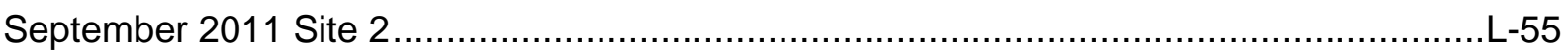

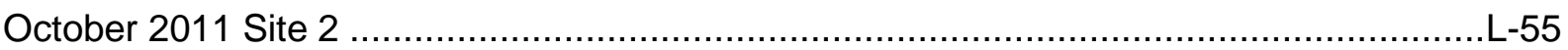

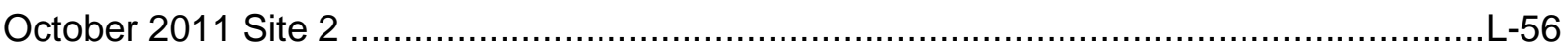

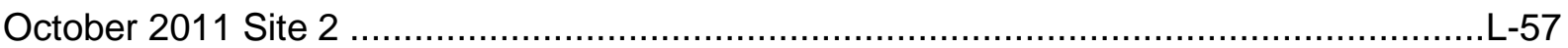

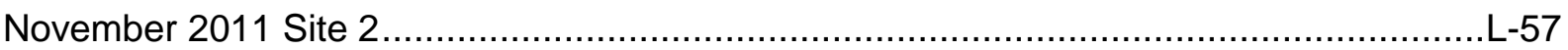

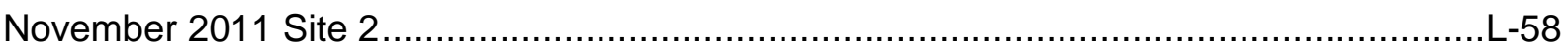

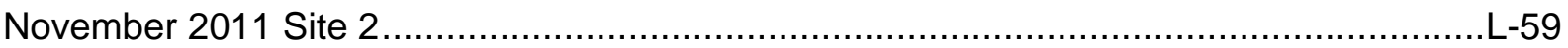

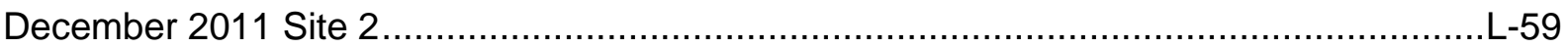

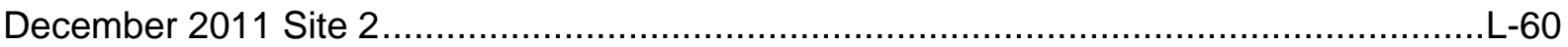




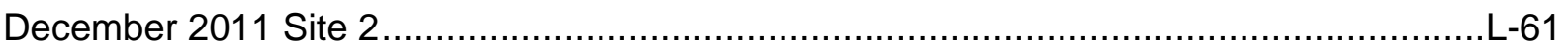

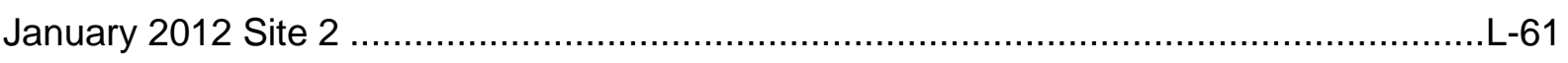

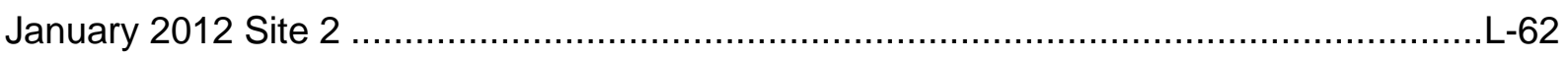

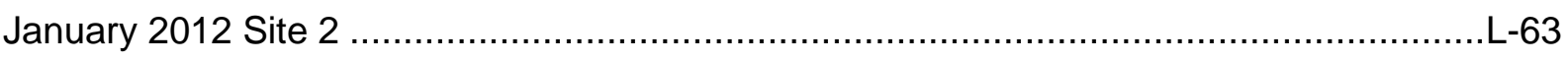

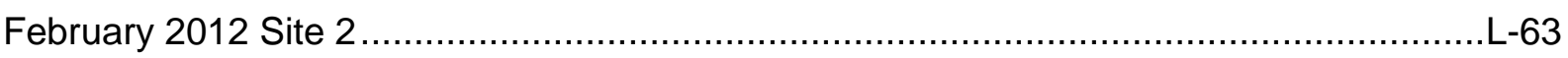

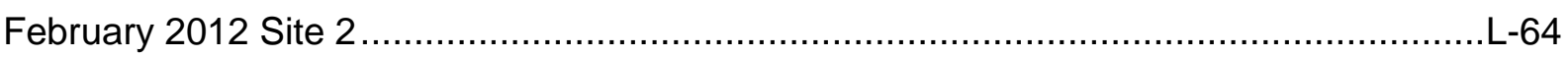

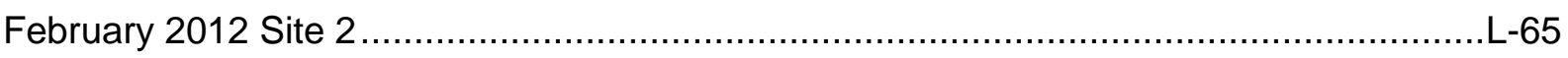

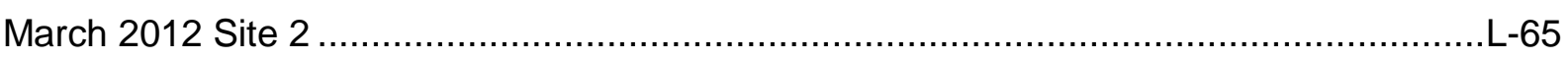

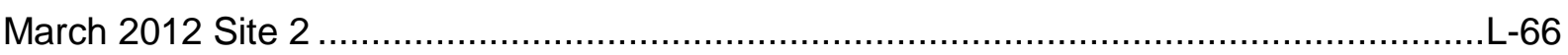

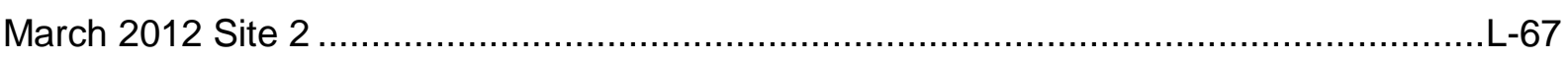

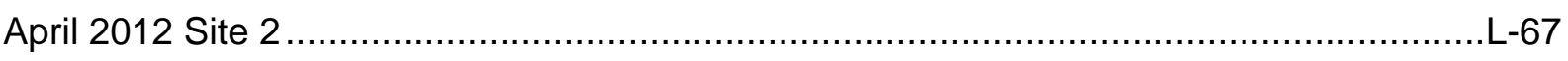

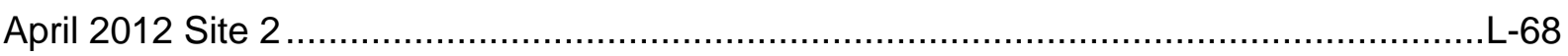

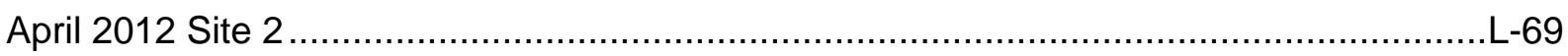

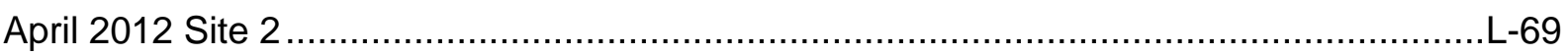

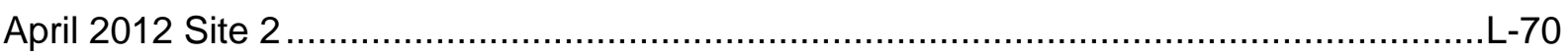

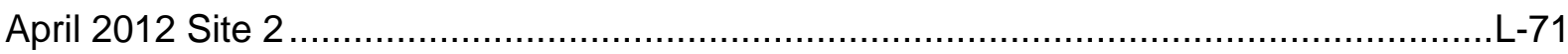

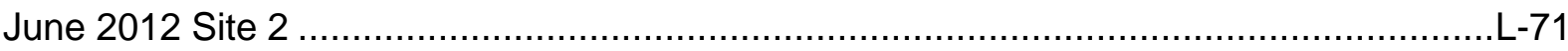

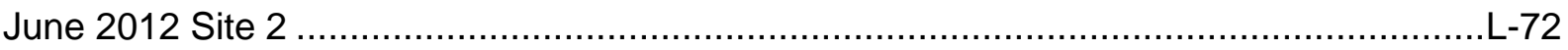

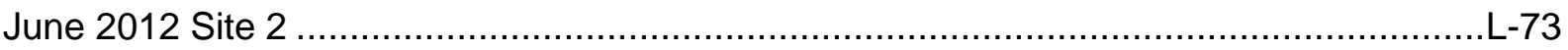

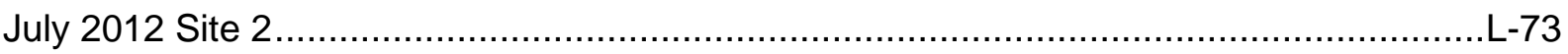

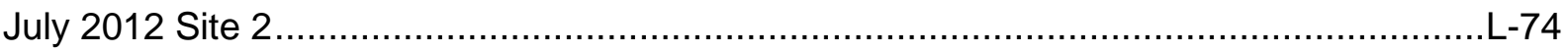

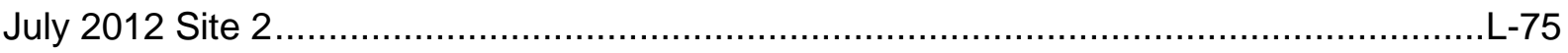

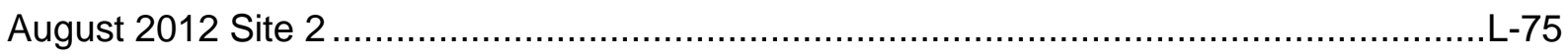

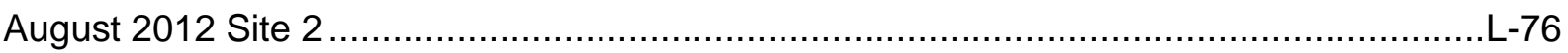

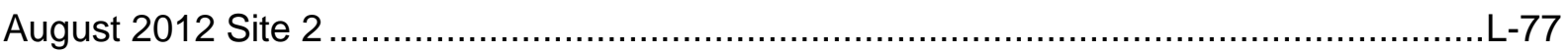

September 2012 Site 2 ..............................................................................

September 2012 Site 2 ..............................................................................

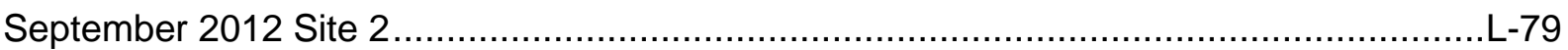

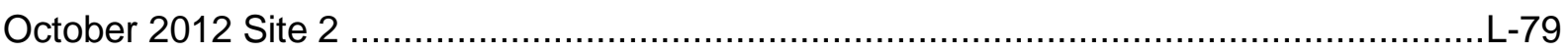

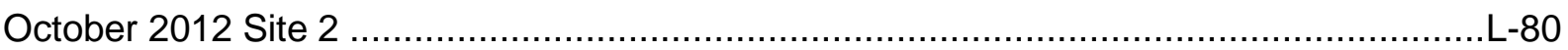

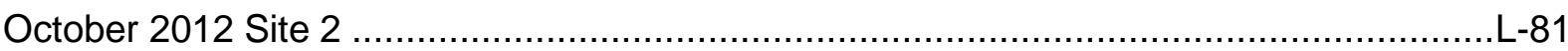

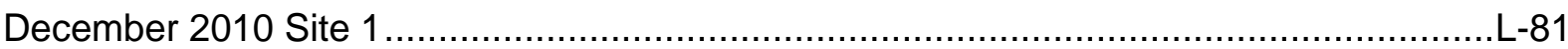

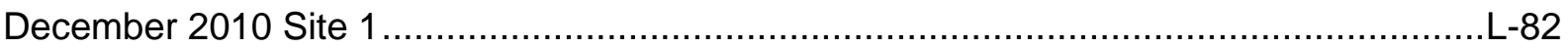

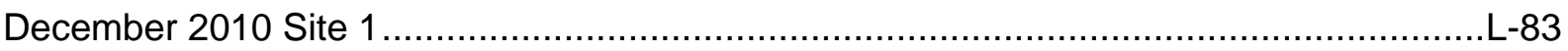

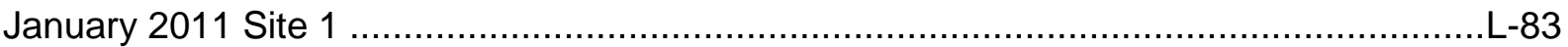




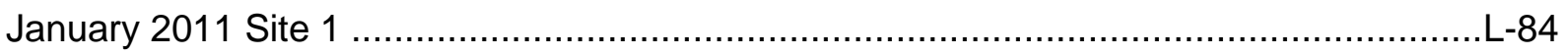

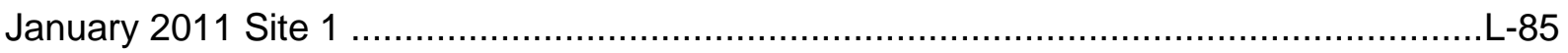

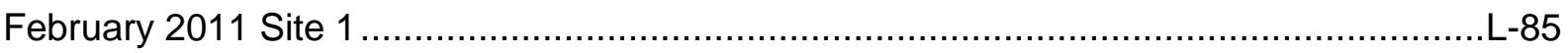

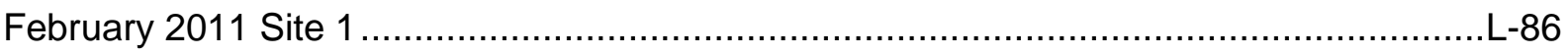

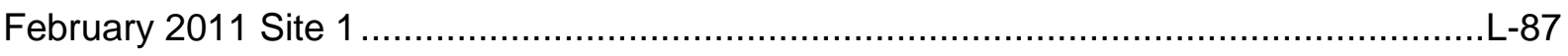

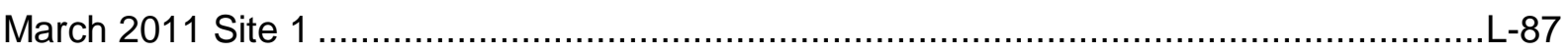

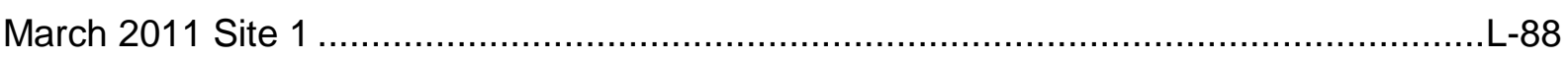

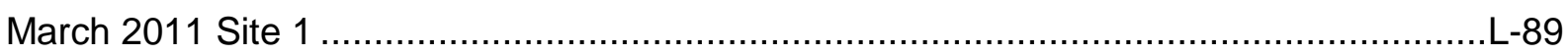

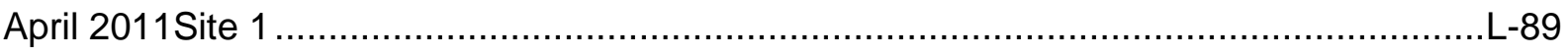

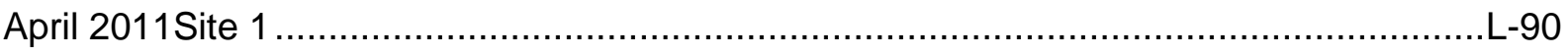

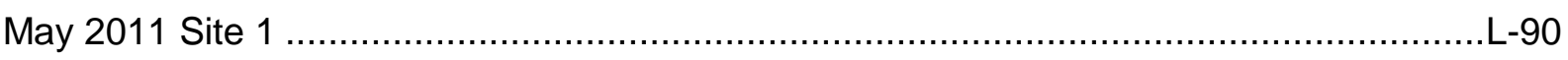

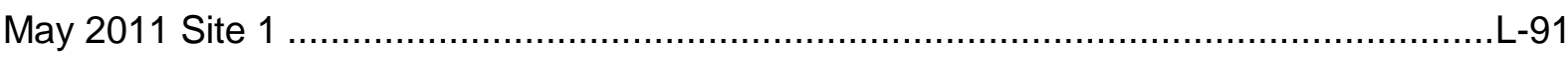

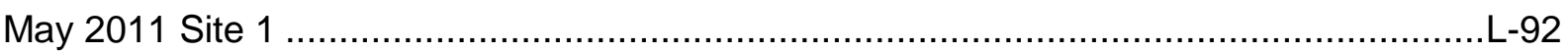

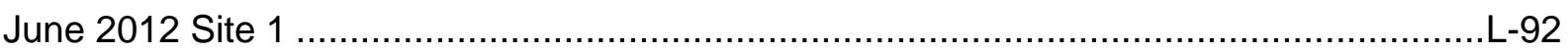

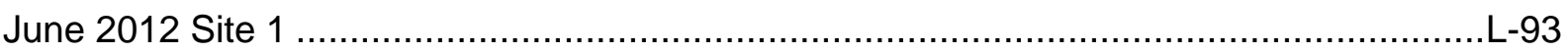

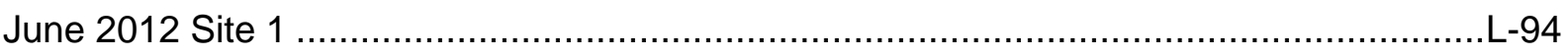

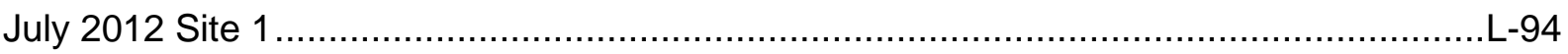

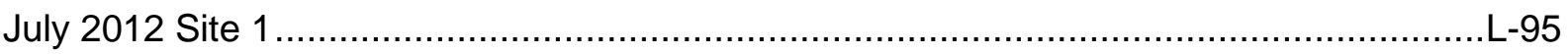

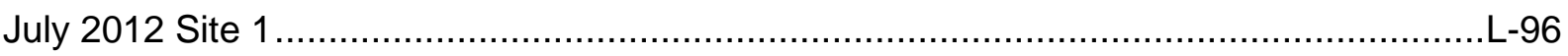

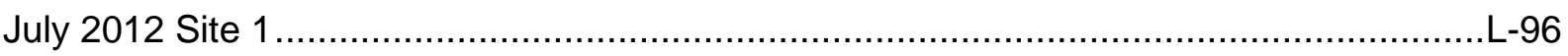

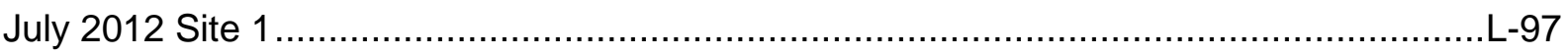

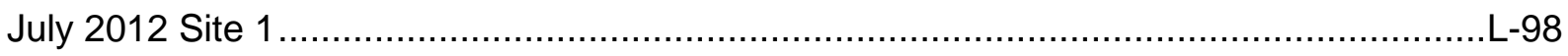

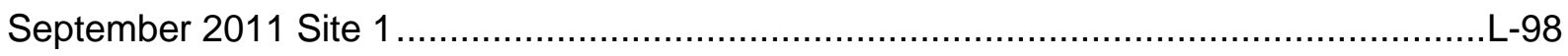

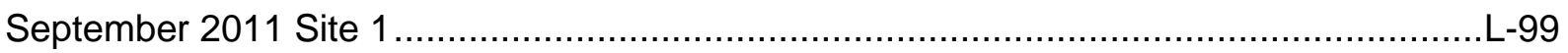

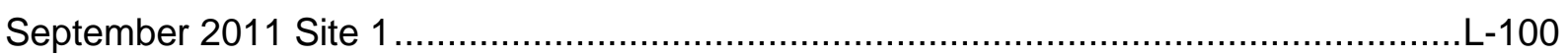

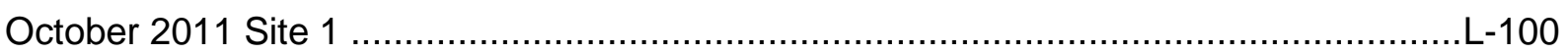

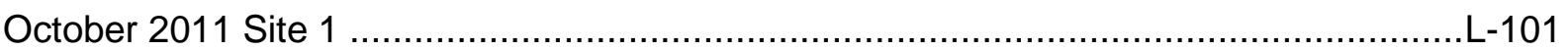

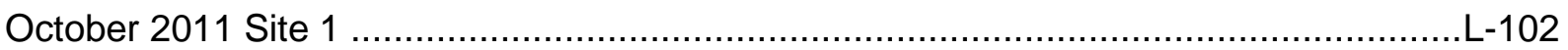

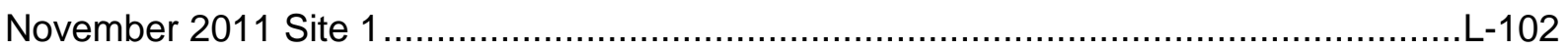

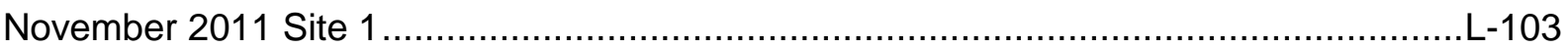

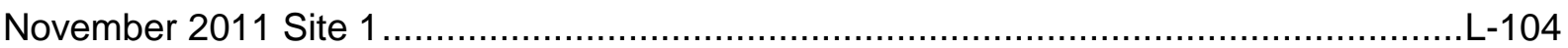

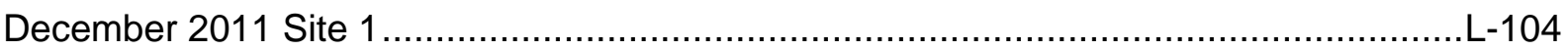

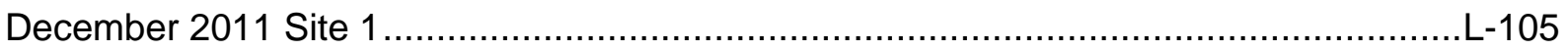

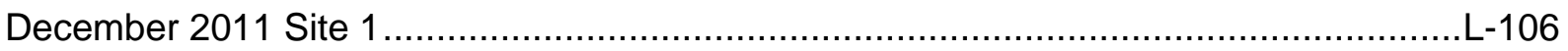

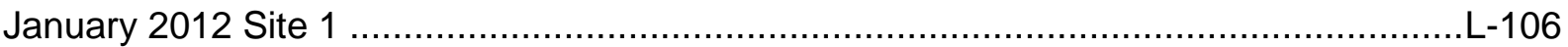




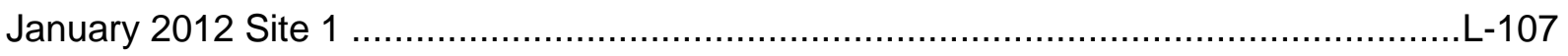

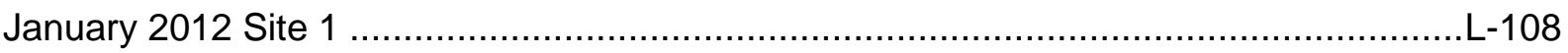

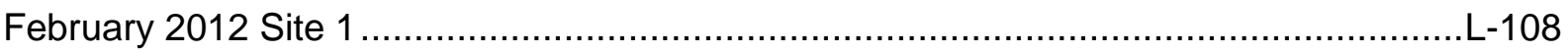

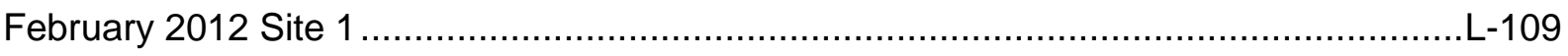

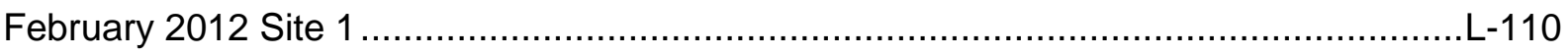

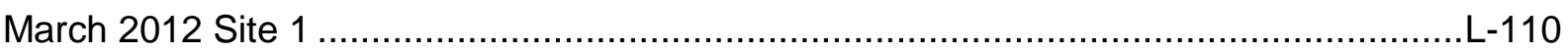

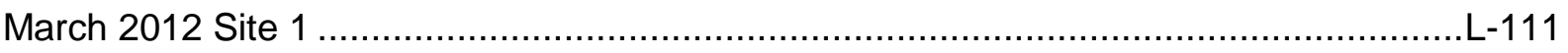

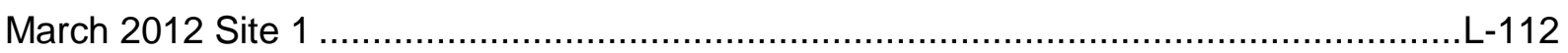

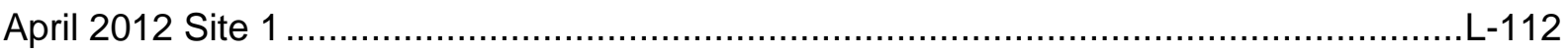

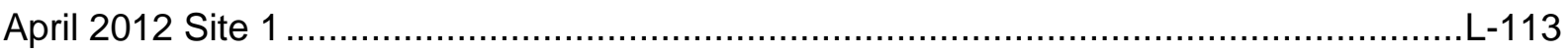

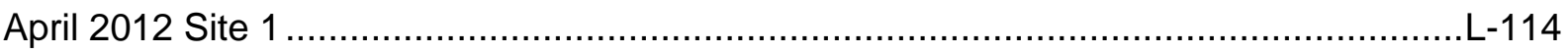

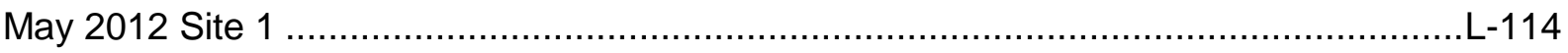

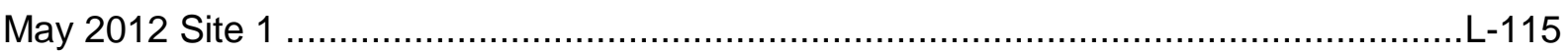

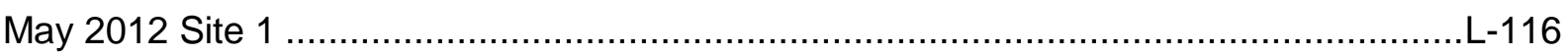

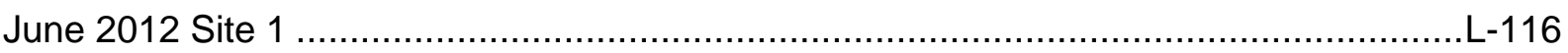

June 2012 Site 1 .................................................................................

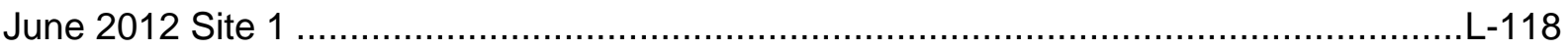

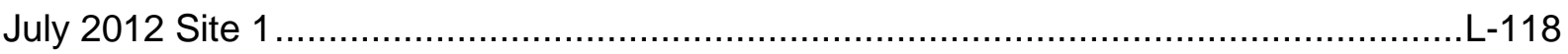

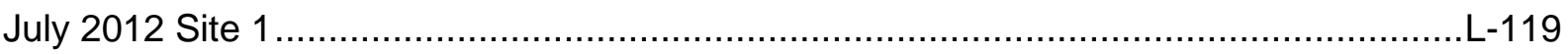

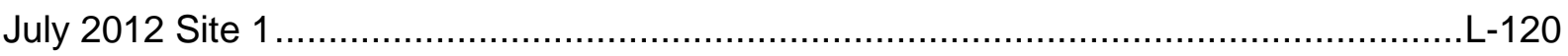

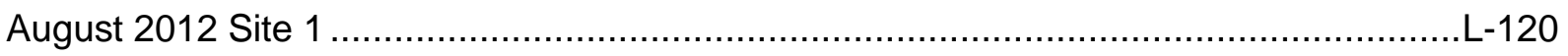

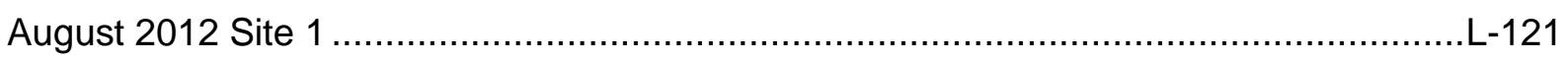

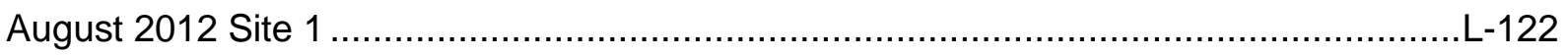

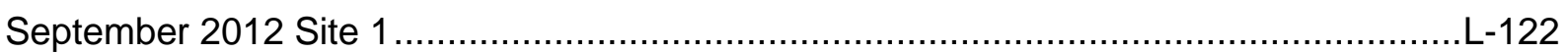

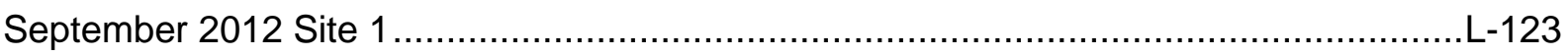

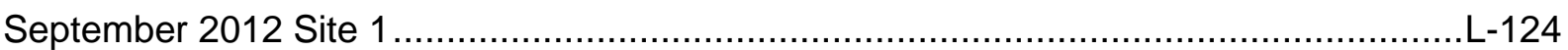

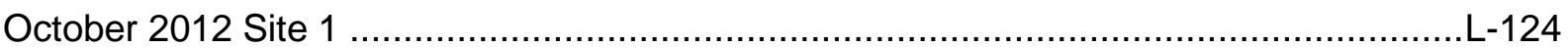

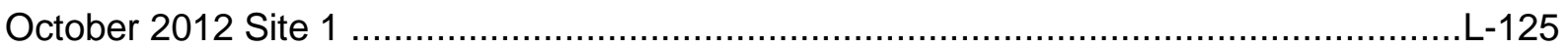

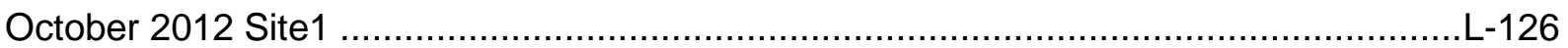




\section{FIGURES}

Figure 1-1 Timeline of the project

Figure 2-1 Example of dynamic ratings, static rating, and load for a 24-hour period for a particular transmission line

Figure 3-1 Weather station sensor array; anemometer, pyranometer, rain gauge,

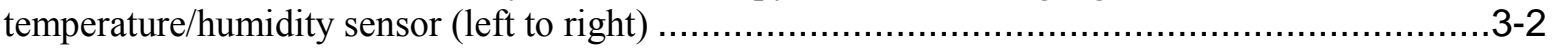

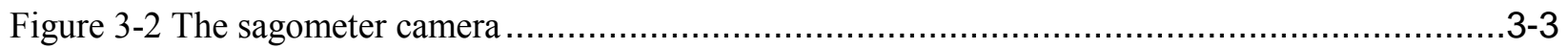

Figure 3-3 EPRI Sensor mounted on a conductor................................................................

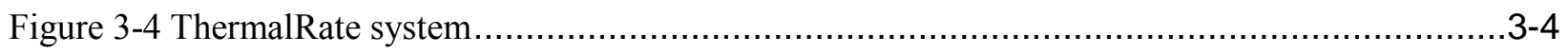

Figure 3-5 Depiction of the DTCR software input and output..........................................

Figure 4-1 Figure 4-1 Photograph of one of the test sites (nearby wind farm in inset).......................4-1

Figure 4-2 Labeled photograph of the structure from Figure $4-1$........................................... $4-2$

Figure 5-1 Plot of the ambient temperature and solar intensity for the month of April 2012 as

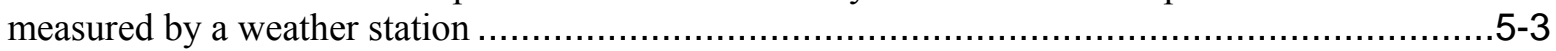

Figure 5-2 Polar plot of wind speed and direction (called a wind rose) for one month as measured by an anemometer.

Figure 5-3 Monthly plot of the conductor ground clearance (directly related to sag) as measured by a Video Sagometer, and the "correlation" factor ....

Figure 5-4 Continuous plot of ratings for one line during the month of April 2012, as derived

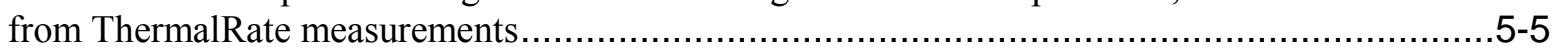

Figure 5-5 Statistical distribution of rating for one line for one month ...................................... $5-5$

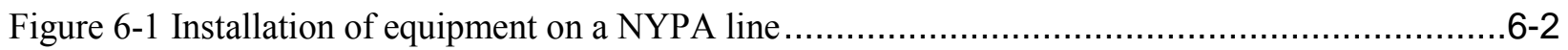

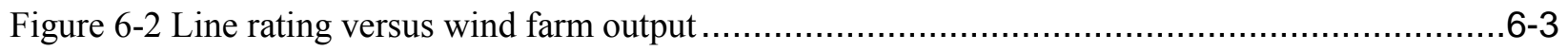

Figure 6-3 Statistical distributions of wind speeds from an anemometer in a transmission line corridor, and as provided by an online weather service

Figure 6-4 Polar plots of wind speed and direction as obtained by an anemometer in the transmission corridor and as obtained from an online NOAA wetaher service

Figure 6-5 Continuous plot of the dynamic ratings for one of the NYPA lines, as determined by

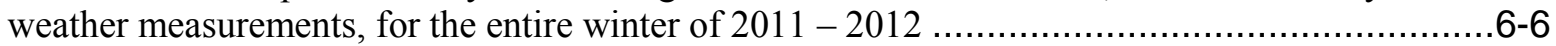

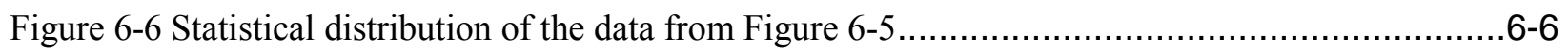

Figure 6-7 Example of a state-change equation for a NYPA line ........................................... $6-8$

Figure 7-1 Flow diagram of how a dynamic rating system could be more fully integrated into

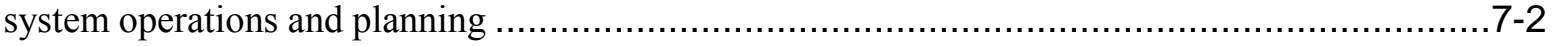

Figure B-1 Depiction of the thermal energy input and output of an overhead line conductor .............. B-2

Figure B-2 Load vs. Rating Probability Distribution example for a transmission line ...................... B-4

Figure B-3 Example of dynamic rating, static rating, and load for a 24-hour period for a particular transmission line..... 
Figure B-4 Impact of changes in air temperature, solar heating, and wind speed and direction on line ratings

Figure B-5 Variation in air temperature between two spans approximately 1.5 miles apart in a typical NYPA transmission line....

Figure B-6 Comparison of simultaneous 10-min average wind speeds (in $\mathrm{ft} / \mathrm{sec}$ ) at two locations about $2 \mathrm{~km}$ apart along a transmission line

Figure B-7 Given the conductor temperature, current, air temperature, and solar heating, the effective perpendicular wind speed can be determined.

Figure B-8 Span-to-Span variation in conductor temperature due to wind speed and direction variations along a line.

Figure B-9 A demonstration of the two-step process for converting tension or sag into an average Sag-Section perpendicular wind speed.

Figure B-10 Comparison of measured and calculated conductor temperature at one of the NYPA lines for the month of July 2012

Figure B-11 Comparison of measured and calculated conductor temperature at a NYPA line for

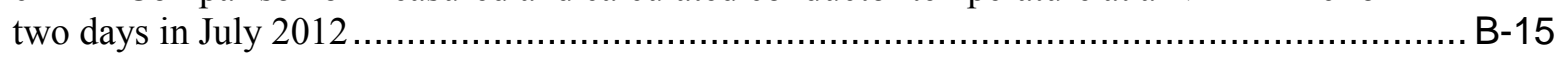

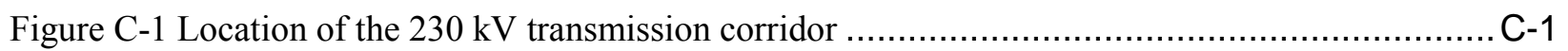

Figure C-2 Geographical Location of the $230 \mathrm{kV}$ Transmission corridor being studied...................... C-2

Figure C-3 Weather Station Array - left to right: anemometer, pyranometer, rain gauge,

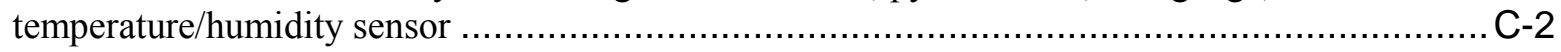

Figure C-4 Wire color-coding of temperature and relative humidity Instrument ........................... C-3

Figure C-5 Temperature sensor removed from housing; temperature and humidity sensors are combined into one unit C-4

Figure C-6 Solar radiation sensor - detailed view (left) and as installed (right) .......................... C-5

Figure C-7 Tipping bucket rain gauge mounted on an aluminum tube ................................... C-6

Figure C-8 Three-axis wind speed and direction sensor Note: The wire junction box should always point south. This keeps the wind direction aligned to a known compass heading. ............

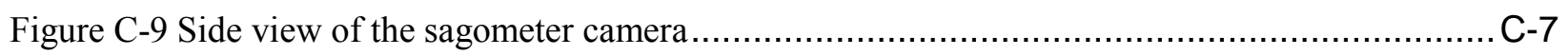

Figure C-10 Camera unit installed showing the front of the camera - The laser target illumination

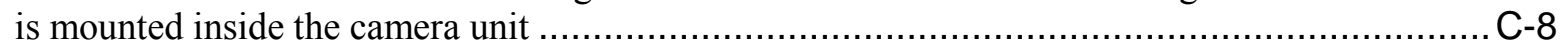

Figure C-11 EPRI Sensor installation and final mounting position on the conductor ........................ C-9

Figure C-12 Comparison of EPRI Sensor performance against thermocouples directly wired to the conductors - range $0-93^{\circ} \mathrm{C}$; six different conductor diameters were utilized ................. C-10

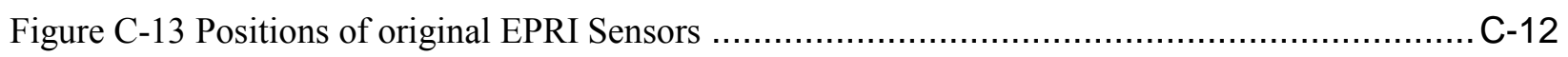

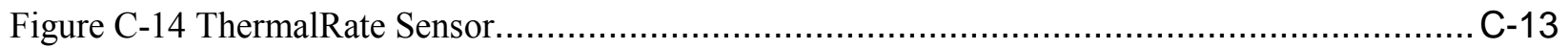

Figure C-15 Radio communication link (MDS TransNet 900) for ThermalRate System .................. C-14

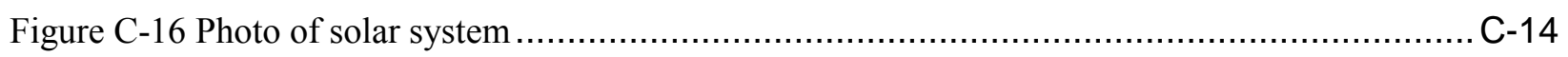

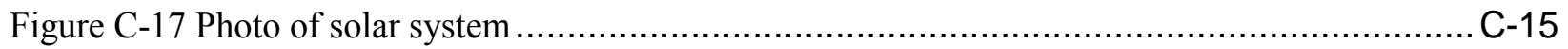

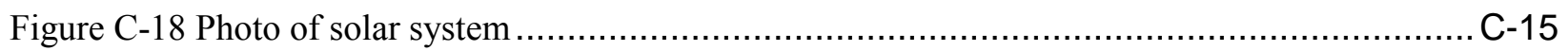

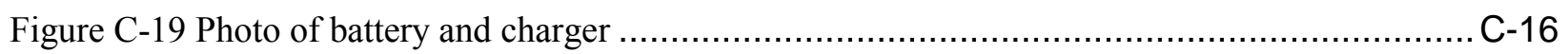

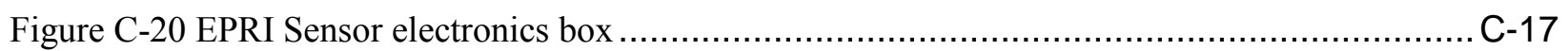

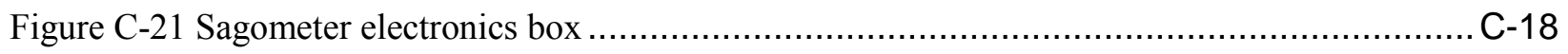


Figure C-22 Isolated view of the Campbell Scientific data logger ......................................... C-19

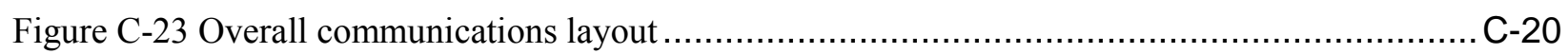

Figure C-24 Detailed communications layout for Video Sagometer ..................................... C-21

Figure C-25 Detailed communications layout for ThermalRate .......................................... C-22

Figure C-26 Detailed communications layout for EPRI Sensor system ......................................23

Figure C-27 Isolated front and rear view of Raven $\mathrm{X}$ - this modem is used by the sagometer............ C-24

Figure C-28 Isolated front and rear view of Raven XT - This modem is used by the EPRI sensor

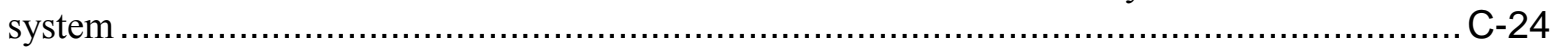

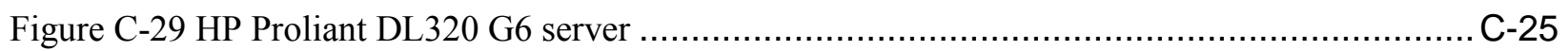

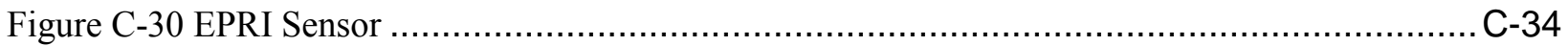

Figure C-31 Bonding tip becomes stuck during the first install attempt .................................. C-34

Figure C-32 ThermalRate sensor being prepared for mounting ......................................... C-35

Figure C-33 ThermalRate sensor installed .................................................................

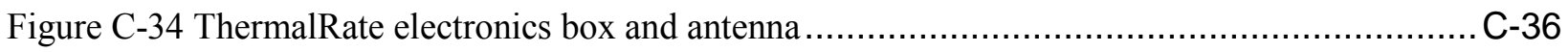

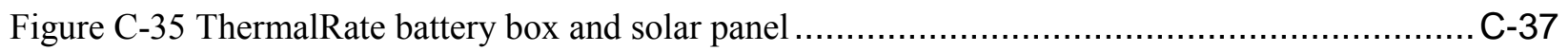

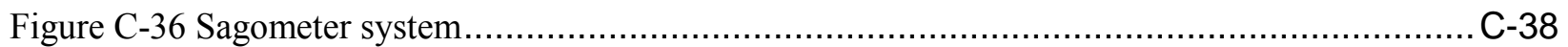

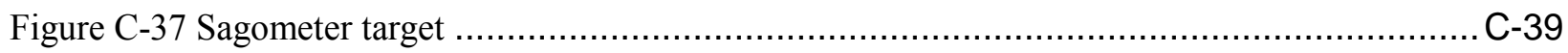

Figure C-38 Near completion of the practice installation at EPRI Lenox ................................. -40

Figure C-39 Locations were marked under the line for the sagometer target and EPRI sensors. ......... C-42

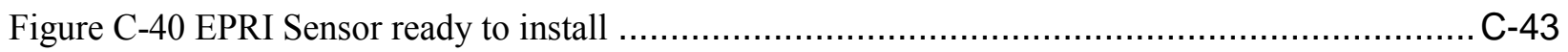

Figure C-41 Lineman tests hot stick prior to installing the EPRI Sensors on the energized $230 \mathrm{kV}$

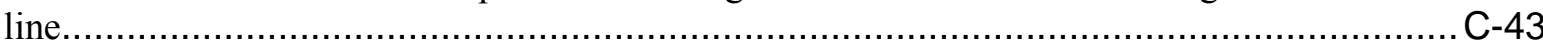

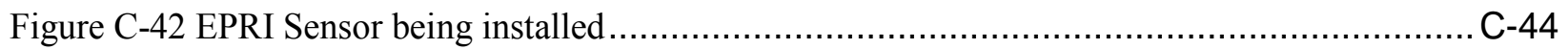

Figure C-43 Remote ZAP Unit used to check communications of the EPRI Sensors in the field ........ C-45

Figure C-44 EPIR Sensor electronics box and solar panel installed .......................................

Figure C-45 ThermalRate sensor being installed.............................................................. C-47

Figure C-46 Solar panel for the ThermalRate system being rigged for installation ....................... C-48

Figure C-47 ThermalRate Battery Box Being Wired ..................................................... C-49

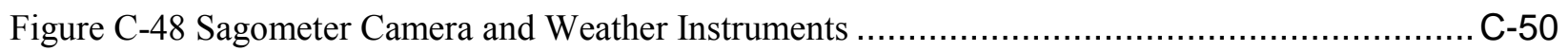

Figure C-49 Sagometer Target Being Installed ............................................................... C-51

Figure C-50 Sagometer Electronic Box and Solar Panel Being Checked After Installation............... C-52

Figure C-51 Site 1 Completed - Looking in an Easterly Direction ....................................... C-53

Figure C-52 Site 1 Completed - Looking in a Westerly Direction........................................... C-54

Figure C-53 EPRI Sensors Ready to Install .......................................................... -56

Figure C-54 EPRI Sensor Electronics Box and Solar Panel Installed ....................................... C-56

Figure C-55 EPRI Sensor Being Installed ..................................................................... C-57

Figure C-56 ThermalRate Sensor Being Readied for Installation ...................................... C-58

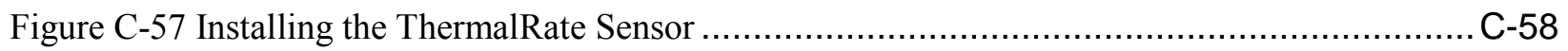

Figure C-58 ThermalRate System Solar Panel and Electrical Box Being Installed ........................ C-59 


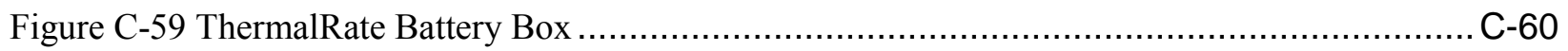

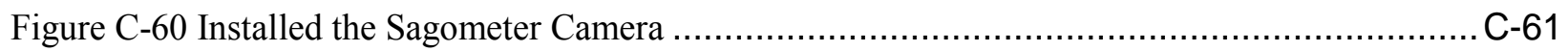

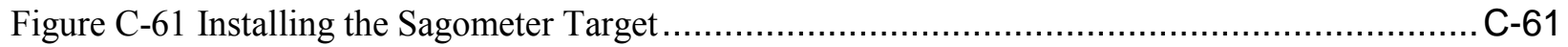

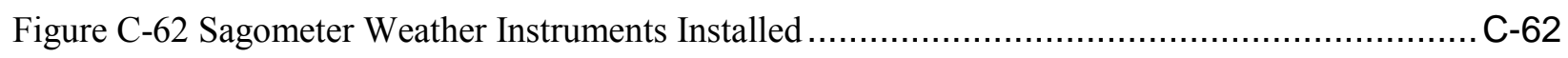

Figure C-63 Sagometer Electronics Box and Solar Panel Installed....................................... C-63

Figure C-64 Install Completed at Site 2 - Looking in a Westerly Direction ................................ C-64

Figure C-65 Install Completed at Site 2 - Looking Back in an Easterly Direction......................... C-65

Figure C-66 EPRI Sensor Electronics Box and Solar Panel ............................................ C-67

Figure C-67 ThermalRate Sensor and Arm Installed ..................................................... -68

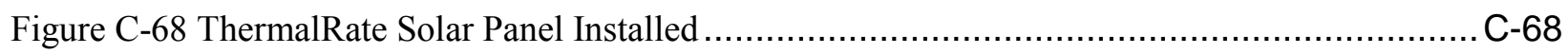

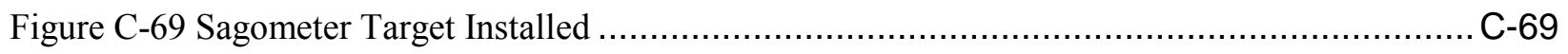

Figure C-70 Sagometer Solar Panel and Electronics Box ........................................................ C-69

Figure C-71 Sagometer Camera, Weather Sensors, Solar Panel and Electronics Box...................... C-70

Figure C-72 Looking Back at Structure Site 3 Before Installation Beings ................................ C-71

Figure C-73 Site 3 Completed - Looking Toward the Substation.......................................... C-71

Figure C-74 Site 3 Completed - Looking Back in the Direction of Willis Substation ...................... C-72

Figure D-1 Deviation of Wind Speed per Rating Cycle .................................................... D-5

Figure D-2 UMass Deviation Data - In this plot the deviation is plotted as a percentage of wind

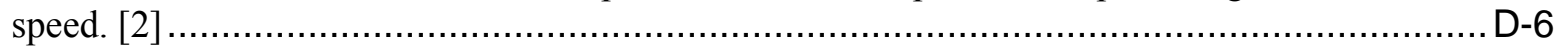

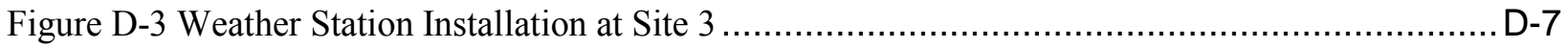

Figure D-4 Standard Deviation of Wind Direction vs. Mean Direction for 10-Minute Periods -

Site 3, One Month of Data (June 2012) .............................................................

Figure D-5 Standard Deviation of Wind Direction vs. Mean Direction for 10-Minute Periods -

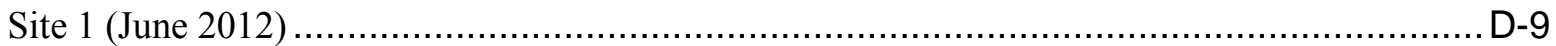

Figure D-6 Standard Deviation of Wind Direction vs. Mean Direction for 10-Minute Periods -

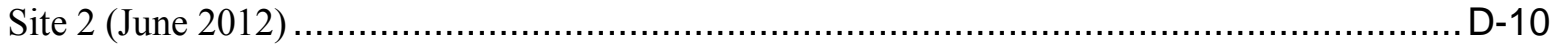

Figure D-7 Aerial Photo East of the Site 2 Showing a More Sheltered Span (Photo from Google

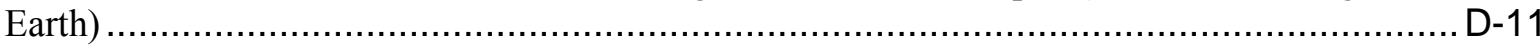

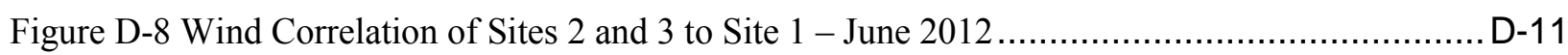

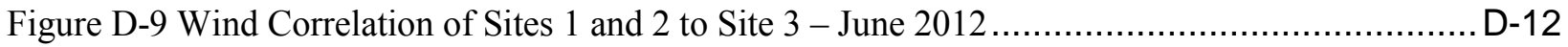

Figure D-10 Wind Correlation of Sites 1 and 3 to Site 2 - June 2012 ..................................... D-12

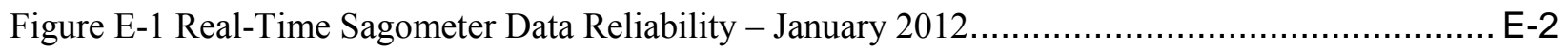

Figure E-2 Sagometer Data Reliability with Communication Delays Removed ............................. E-3

Figure E-3 Flow Chart Depicting Data Flow for All Five Rating Elements Including Airport

Weather Data - This chart is applicable to all three Sites. ............................................. E-5

Figure E-4 The LoggerNet Data Status Monitor Showing Communication Failures in Red and

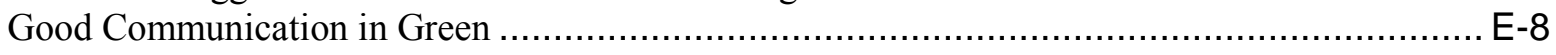

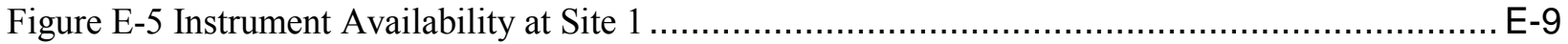

Figure E-6 Real-Time Instrument and Communication Availability at Site 1 ........................... E-10

Figure E-7 Communication Availability Between Data Loggers and DTCR Server at Site 1 ........... E-11 


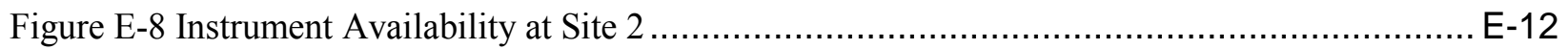

Figure E-9 Real-Time Instrument and Communication Availability at Site 2 ........................... E-13

Figure E-10 Communication Availability Between Data Loggers and DTCR Server at Site 2 .......... E-14

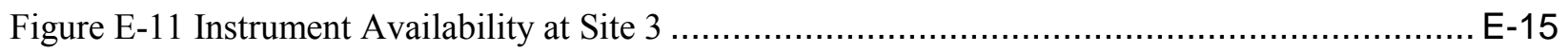

Figure E-12 Real-Time Instrument and Communication Availability at Site 3 .......................... E-16

Figure E-13 Communication Availability Between Data Loggers and DTCR Server at Site 3 ......... E-17

Figure E-14 Data Successfully Archived from Data Loggers at Site 1 ..................................... E-19

Figure E-15 Data Successfully Archived from Data Loggers at Site 2 ................................... E-20

Figure E-16 Data Successfully Archived from Data Loggers at Site 3 ................................... E-20

Figure E-17 Duration of Unscheduled Server Downtime .............................................. E-22

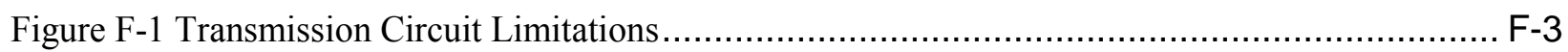

Figure F-2 Overhead Line Ratings Based on the Onsite Weather Station at Site 3 - Winter 2010-

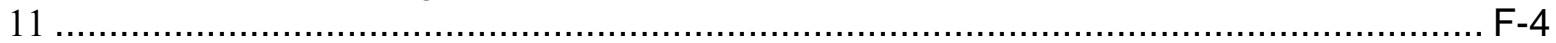

Figure F-3 Overhead line ratings based on ThermalRate at Site 3- Winter 2010-11 ...................... F-5

Figure F-4 Overhead line ratings based on the Sagometer at Site 3 - Winter 2010-11 .................... F-6

Figure F-5 Overhead line ratings based on EPRI Sensors at Site 3 - Winter 2010-11 ..................... F-7

Figure F-6 Overhead line ratings based on Onsite weather station for Site 3 - Summer 2011 ............ F-8

Figure F-7 Overhead line ratings based on ThermalRate device for Site 3 - Summer 2011 ............... F-8

Figure F-8 Overhead line ratings based on the Video Sagometer for Site 3 - Summer 2011 .............. F-9

Figure F-9 Overhead line ratings based on the EPRI Sensors for Site 3 - Summer 2 ...................... F-9

Figure F-10 Overhead line ratings based on the Onsite Weather Station at Site 3- Winter 2011-12 ... F-10

Figure F-11 Overhead line ratings based on ThermalRate at Site 3 - Winter 2011-12 .................. F-11

Figure F-12 Overhead line ratings based on Sagometer at Site 3 - Winter 2011-12 ..................... F-12

Figure F-13 Overhead line ratings based on EPRI Sensors at Site 3 - Winter 2011-12 .................. F-13

Figure F-14 Overhead line ratings based on Offsite Weather Service at Site 3 - Winter 2011-12 ...... F-14

Figure F-15 Overhead line ratings based on the Onsite Weather Station at Site 3 - Summer 2012 ..... F-15

Figure F-16 Overhead line ratings based on ThermalRate at Site 3 - Summer 2012 ..................... F-15

Figure F-17 Overhead line ratings based on the Sagometer at Site 3 - Summer 2012 ................... F-17

Figure F-1 8 Overhead line ratings based on EPRI Sensors at Site 3 - Summer 2012 .................... F-17

Figure F-19 Overhead line ratings based on the Offsite Weather Service at Site 3 - Summer 2012 ..... F-18

Figure F-20 Overhead line ratings based on the Onsite Weather Station at Site 2 - Winter 20102011

Figure F-21 Overhead line ratings based on ThermalRate at Site 2 - Winter 2010-2011 ................ F-20

Figure F-22 Overhead line ratings based on the Video Sagometer at Site 2 - Winter 2010-2011 ........ F-21

Figure F-23 Overhead line ratings based on the EPRI Sensors at Site 2 - Winter 2010-2011 ............ F-21

Figure F-24 Overhead line ratings based on the Onsite Weather Station at Site 2 - Summer 2011 ...... F-22

Figure F-25 Overhead line ratings based on ThermalRate at Site 2 - Summer 2011 ..................... F-22

Figure F-26 Overhead line ratings based on the Sagometer at Site 2 - Summer $2011 \ldots \ldots \ldots \ldots \ldots \ldots \ldots . . . . . . . .23$

Figure F-27 Overhead line ratings based on the EPRI Sensors at Site 2 - Summer $2011 \ldots \ldots \ldots \ldots \ldots . . . . . . .23$ 
Figure F-28 Overhead line ratings based on ThermalRate at Site 2 - Winter 2011-2012 _............... F-24

Figure F-29 Overhead line ratings based on the Sagometer at Site 2- Winter 2011-2012 ............... F-24

Figure F-30 Overhead line ratings based on the EPRI Sensors at Site 2 -Winter 2011-2012 ............ F-25

Figure F-31 Overhead line ratings based on the Off Site Weather Service at Site 2 - Winter 2011-

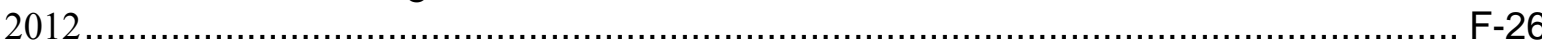

Figure F-32 Overhead line ratings based on the Offsite Weather Service at Site 2 -Summer 2012 .... F-26

Figure F-33 Overhead line ratings based on ThermalRate at Site 2 - Summer 2012 .................... F-27

Figure F-34 Overhead line ratings based on the Sagometer at Site 2 - Summer 2012 ................... F-27

Figure F-35 Overhead line ratings based on EPRI Sensors at Site 2 - Summer 2012 .................... F-28

Figure F-36 Overhead line ratings based on Offsite Weather Service at Site 2 - Summer 2012 ........ F-28

Figure F-37 Overhead line ratings based on the Onsite Weather Station at Site 1- Winter 20102011

Figure F-38 Overhead line ratings based on the ThermalRate at Site 1- Winter 2010-2011 ............. F-30

Figure F-39 Overhead line ratings based on the Sagometer at Site 1- Winter 2010-2011 ............... F-30

Figure F-40 Overhead line ratings based on the EPRI Sensors at Site 1- Winter 2010-2011 ............ F-31

Figure F-41 Overhead line ratings based on the onsite weather station at Site 1 - Summer 2011....... F-31

Figure F-42 Overhead line ratings based on ThermalRate device at Site 1 - Summer 2011 ............ F-32

Figure F-43 Overhead line ratings based on the Sagometer at Site 1 - Summer $2011 \ldots \ldots \ldots \ldots \ldots \ldots . . . . . . . .32$

Figure F-44 Overhead line ratings based on the EPRI Sensors at Site 1 - Summer 2011 ................ F-33

Figure F-45 Overhead line ratings based on the ThermalRate at Site 1- Winter 2010-2011 ............ F-33

Figure F-46 Overhead line ratings based on the Sagometer at Site 1- Winter 2011-2012 ................ F-34

Figure F-47 Overhead line ratings based on EPRI Sensors at Site 1- Winter 2011-2012 ................ F-34

Figure F-48 Overhead line ratings based on Offsite Weather Service at Site 1- Winter 2011-2012 .... F-35

Figure F-49 Overhead line ratings based on the Onsite Weather Station at Site 1- Summer 2012 ...... F-36

Figure F-50 Overhead line ratings based on ThermalRate at Site 1-Summer 2012 ..................... F-36

Figure F-51 Overhead line ratings based on the Sagometer at Site 1 - Summer $2011 \ldots \ldots \ldots \ldots \ldots \ldots \ldots . . . . . . . .37$

Figure F-52 Overhead line ratings based on EPRI Sensors at Site 1- Summer 2012 .................... F-37

Figure F-53 Overhead line ratings based on the Offsite Weather Service at Site 1- Summer 2012 ..... F-38

Figure G-1 Survey Results on Typical Transmission Line Loading..........................................

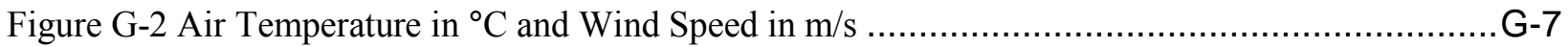

Figure G-3 Line Rating vs. Wind Speed for Different Wind Directions Relative to the Line

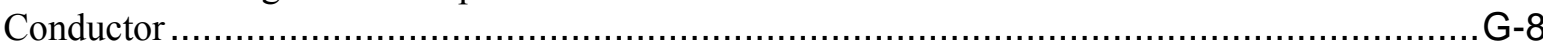

Figure G-4 Impact of Solar Heating on the Rating of a Drake Conductor with $2 \mathrm{f} / \mathrm{s}$ Wind Speed in

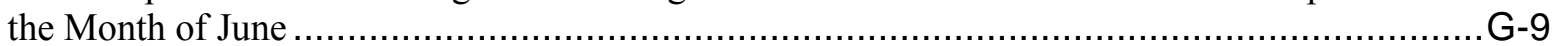

Figure G-5 A ThermalRate Directional Anemometer ..................................................

Figure G-6 The Sagometer Camera and Target Mounted on the Conductor................................. -13

Figure G-7 EPRI Sensor Temperature Monitor.................................................................

Figure G-8 Relationship Between Conductor Temperature and Effective Perpendicular Wind

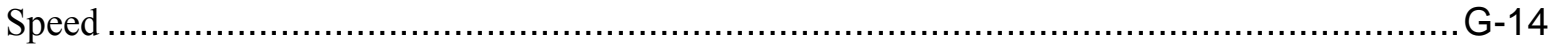

Figure G-9 Instance of Anemometer Stall Evident in NOAA Data....................................... 15 
Figure G-10 Plot of Absolute Wind Speed and Direction Measured by Ultrasonic Anemometer

for the Month of November at Site 2

Figure G-11 Wind Speed and Direction as Provided by NOAA for a Region Encompassing Site 2....G-18

Figure G-12 Forecast Wind Speed and Direction from NOAA National Weather Service..... G-18

Figure G-13 Comparison of Offsite NOAA Interpolated Data and Ultrasonic Anemometer Wind

Speeds at the Onsite Weather Station Located at Site 2

Figure G-14 CDF of Wind Speeds for Site 2 During the Summer of 2011 ................................ 19

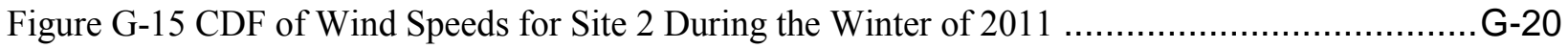

Figure G-16 Effective Perpendicular Wind Speed Estimated by the ThermalRate Conductor

Model Monitoring Method.

Figure G-17 Comparison of EPRI Sensor (Green) to ThermalRate (Black) and Onsite Weather

Station (Red) Models. Ambient Temperature is Shown (blue) as a Reference.....

Figure G-18 Comparison of Solar Temperature Rise as Measured by ThermalRate Conductor

Model to That Calculated from Solar Pyranometer Measurements During a Week in

November of 2011

Figure G-19 Comparison of Solar Temperature Rise as Measured by ThermalRate Conductor

Model to That Calculated from Solar Pyranometer Measurements During a Week in May of 2012 .

Figure G-20 Comparison of Air Temperature Reported by NOAA Airport, the ThermalRate

Conductor Model, and the Onsite Weather Station at Site 2 ....

Figure G-21 Comparison of Line Current Measured by the EPRI Sensor and Reported by NYPA SCADA.

Figure G-22 Comparison of Line Current Measured by EPRI Sensor and NYPA SCADA Data for Site 2 for March 2012.

Figure G-23 Conductor Temperature Above Ambient (Tc - TA) for Video Sagometer, EPRI

Sensor and Onsite Weather Station

Figure G-24 Spot Checks of the Difference in Conductor Temperature Calculations vs. the EPRI

Sensor Conductor Temperature Measurements for a Year-Long Period of the Project ..... G-27

Figure H-1 Winter 2010/11 Ratings Distribution Site 2 Onsite Weather Station ............................. H-4

Figure H-2 Winter 2010/11 Ratings Distribution Site 2 ThermalRate ...................................... H-5

Figure H-3 Winter 2010/11 Ratings Distribution Site 2 Sagometer ...................................... H-6

Figure H-4 Winter 2010/11 Ratings Distribution Site 2 EPRI Sensors ..................................... H-7

Figure H-5 Summer 2011 Ratings Distribution Site 2 Onsite Weather Station ............................... H-8

Figure H-6 Summer 2011 Ratings Distribution Site 2 ThermalRate ......................................... H-9

Figure H-7 Summer 2011 Ratings Distribution Site 2 Sagometer........................................ H-10

Figure H-8 Summer 2011 Ratings Distribution Site 2 EPRI Sensors ........................................ H-11

Figure H-9 Winter 2011/12 Ratings Distribution Site 2 Onsite Weather Station .......................... H-12

Figure H-10 Winter 2011/12 Ratings Distribution Site 2 ThermalRate ................................. H-13

Figure H-11 Winter 2011/12 Ratings Distribution Site 2 Sagometer ...................................... H-14

Figure H-12 Winter 2011/12 Ratings Distribution Site 2 EPRI Sensors .................................. H-15

Figure H-13 Winter 2011/12 Ratings Distribution Site 2 Offsite Weather Service ........................ H-16

Figure H-14 Summer 2012 Ratings Distribution Site 2 Onsite weather station ........................... H-17 
Figure H-15 Summer 2012 Ratings Distribution Site 2 ThermalRate ..................................... 18

Figure H-16 Summer 2012 Ratings Distribution Site 2 Sagometer.................................... H-19

Figure H-17 Summer 2012 Ratings Distribution Site 2 EPRI Sensors .................................... H-20

Figure H-18 Summer 2012 Ratings Distribution Site 2 Offsite Weather Service ........................... H-21

Figure H-19 Winter 2010/11 Ratings Distribution Site 3 Onsite weather station ........................... H-23

Figure H-20 Winter 2010/11 Ratings Distribution Site 3 ThermalRate .................................. H-24

Figure H-21 Winter 2010/11 Ratings Distribution Site 3 Sagometer ...................................... H-25

Figure H-22 Winter 2010/11 Ratings Distribution Site 3 EPRI Sensor ....................................... H-26

Figure H-23 Summer 2011 Ratings Distribution Site 3 Onsite Weather Station ............................ H-27

Figure H-24 Summer 2011 Ratings Distribution Site 3 ThermalRate ................................... H-28

Figure H-25 Summer 2011 Ratings Distribution Site 3 Video Sagometer ................................ H-29

Figure H-26 Summer 2011 Ratings Distribution Site 3 EPRI Sensors ....................................... H-30

Figure H-27 Winter 2011/12 Ratings Distribution Site 3 Onsite weather station ............................ H-31

Figure H-28 Winter 2011/12 Ratings Distribution Site 3 ThermalRate .................................... H-32

Figure H-29 Winter 2011/12 Ratings Distribution Site 3 Sagometer ......................................... H-33

Figure H-30 Winter 2011/12 Ratings Distribution Site 3 EPRI Sensor ....................................H-34

Figure H-31 Winter 2011/12 Ratings Distribution site 3 Offsite Weather Service ......................... H-35

Figure H-32 Summer 2012 Ratings Distribution Site 3 Onsite Weather Station ...............................

Figure H-33 Summer 2012 Ratings Distribution Site 3 ThermalRate .....................................

Figure H-34 Summer 2012 Ratings Distribution Site 3 Sagometer..................................... H-38

Figure H-35 Summer 2012 Ratings Distribution Site 3 EPRI Sensors ......................................H-39

Figure H-36 Summer 2012 Ratings Distribution Site 3 Offsite Weather Service ...........................H-40

Figure H-37 Winter 2010/11 Ratings Distribution Site 1 Onsite Weather Station.......................... H-42

Figure H-38 Winter 2010/11 Ratings Distribution Site 1 ThermalRate .................................... H-43

Figure H-39 Winter 2010/11 Ratings Distribution Site 1 Sagometer ..................................... H-44

Figure H-40 Winter 2010/11 Ratings Distribution Site 1 Sagometer ..................................... H-45

Figure H-41 Summer 2011 Ratings Distribution Site 1 Onsite Weather Station ............................H-46

Figure H-42 Summer 2011 Ratings Distribution Site 1 ThermalRate ...................................... H-47

Figure H-43 Summer 2011 Ratings Distribution Site 1 Sagometer ..................................... H-48

Figure H-44 Summer 2011 Ratings Distribution Site 1 EPRI Sensors ....................................... H-49

Figure H-45 Winter 2011/12 Ratings Distribution Site 1 Onsite Weather Station.......................... H-50

Figure H-46 Winter 2011/12 Ratings Distribution Site 1 ThermalRate ..................................... H-51

Figure H-47 Winter 2011/12 Ratings Distribution Site 1 Sagometer ..................................... H-52

Figure H-48 Winter 2011/12 Ratings Distribution Site 1 EPRI Sensors .....................................

Figure H-49 Winter 2011/12 Ratings Distribution Site 1 Off Site Weather Service ....................... H-54

Figure H-50 Summer 2012 Ratings Distribution Site 1 Onsite Weather Station ............................ H-55

Figure H-51 Summer 2012 Ratings Distribution Site 1 ThermalRate ................................... H-56

Figure H-52 Summer 2011 Ratings Distribution Site 1 Sagometer..................................... H-57

Figure H-53 Summer 2012 Ratings Distribution Site 1 EPRI Sensors .................................... H-58 
Figure H-54 Summer 2012 Ratings Distribution Site 1 Off Site weather service.......................... H-59

Figure I-1 Combined Wind Farm Output vs. Ratings for Site 1 ........................................ I-2

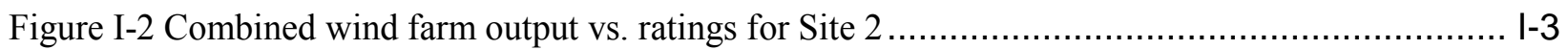

Figure I-3 Ryan Wind Farm output vs. Ratings for Site 3; Correlation Coefficient $=0.29 \ldots \ldots \ldots \ldots \ldots \ldots . . . . . .4$

Figure I-4 Combined Wind Farm Output vs. Load for the Site 1 Line; Correlation Coefficient =

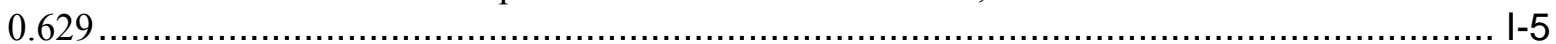

Figure I-5 Ryan Wind Farm Output vs. Load for Sites 2 and 3; Correlation Coefficient $=-0.233 \ldots \ldots \ldots$. I-6

Figure J-1 Statistics of Current Densities Measured on Transmission Lines ......................................5

Figure J-2 Comparison of Diurnal and Real-Time Line Ratings........................................... J-7

K-1 Element: Site 1_WxPlot: Conductor Clearance \& Correlation ....................................... K-2

K-2 Element: Site 1_Wx Plot: Wind Speed \& Direction .................................................... K-2

K-3 Element: Site 1_Wx Plot: Ambient Temperature WX ............................................ K-3

K-4 Element: Site 1_Wx Plot: Load ............................................................................

K-5 Element: Site 1_Wx Plot: Cond. Temp. - EPRI Sensors............................................. K-4

K-6 Element: Site 1_Wx Plot: Wind speed - Airport ....................................................... K-4

K-7 Element: Site 1_Wx Plot: Rating - WX .................................................................

K-8 Element: Site 1_Wx Plot: Wind Rose - Airport ..................................................... K-5

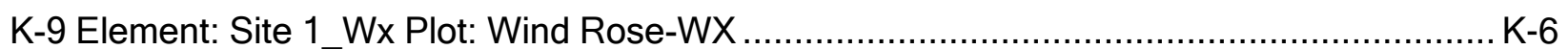

K-10 Element: Site 1_Wx Plot: Cum.Dist. for Wind Speed-WX............................................ K-6

K-11 Element: Site 1_Wx Plot: Data Quality 2 .......................................................... K-7

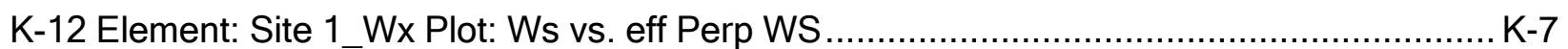

K-13 Element: Site 1_Wx Plot: Rating Cum. Dist............................................................. K-8

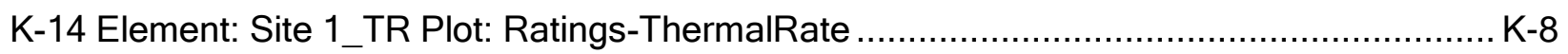

K-15 Element: Site 1_TR Plot: Rating Cum. Dist. ....................................................... K-9

K-16 Element: Site 1_Sag Plot: Ratings-Sagometer..................................................... K-9

K-17 Element: Site 1_Sag Plot: Rating Cum. Dist. .................................................. K-10

K-18 Element: Site 1_Sag Plot: Conductor Temp Compare ........................................ K-10

K-19 Element: Site 1_BS Plot: Ratings-EPRI Sensors ............................................... K-11

K-20 Element: Site 1_BS Plot: Rating Cum. Dist. ........................................................ K-11

K-21 Element: Site 1_AP Plot: Ratings-Airport ....................................................... K-12

K-22 Element: Site 1_AP Plot: Rating Cum. Dist. .......................................................... K-12

K-23 Element: Site 2_Wx Plot: Conductor Clearance \& Correlation ................................. K-13

K-24 Element: Site 2_Wx Plot: Wind Speed \& Direction ............................................... K-13

K-25 Element: Site 2_Wx Plot: Ambient Temperature WX............................................ K-14

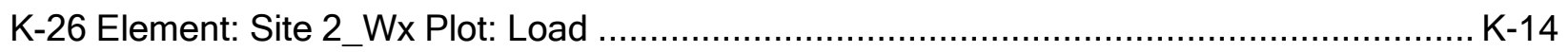

K-27 Element: Site 2_Wx Plot: Cond. Temp. - EPRI Sensors ........................................... K-15

K-28 Element: Site 2_Wx Plot: Wind Speed and Direction - Airport.................................... K-15

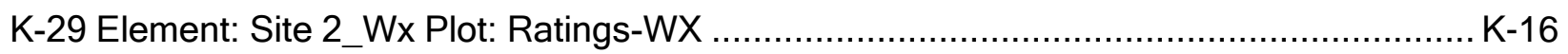

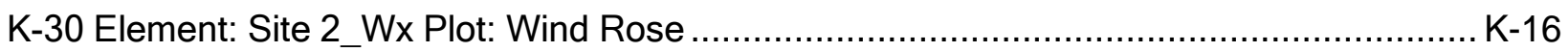


K-31 Element: Site 2_Wx Plot: Data Quality 2 .................................................... K-17

K-32 Element: Site 2_Wx Plot: Ws vs eff Perp WS .................................................. K-17

K-33 Element: Site 2_Wx Plot: Rating Cum. Dist................................................... K-18

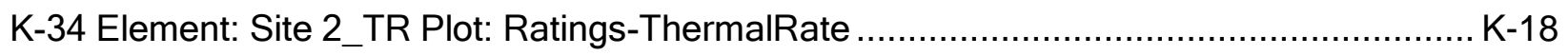

K-35 Element: Site 2_TR Plot: Rating Cum. Dist. .................................................. K-19

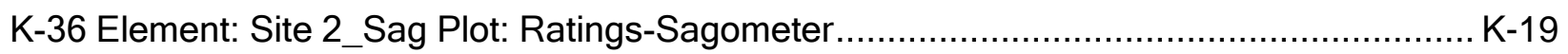

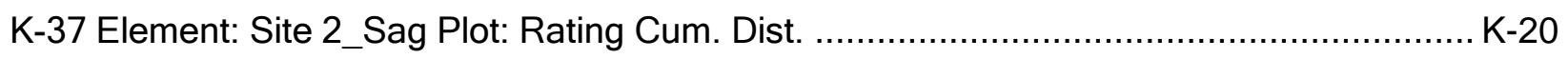

K-38 Element: Site 2_Sag Plot: Conductor Temp Compare ........................................ K-20

K-39 Element: Site 2_BS Plot: Ratings - EPRI Sensors .............................................. K-21

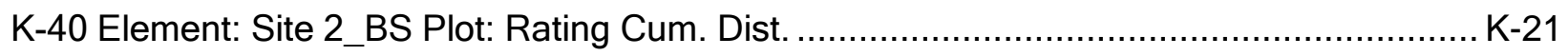

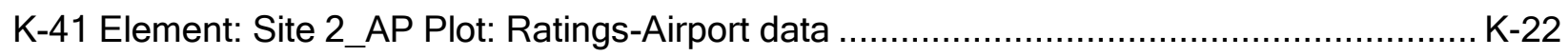

K-42 Element: Site 2_AP Plot: Rating Cum. Dist. ...................................................... K-22

K-43 Element: Site 3_Wx Plot: Conductor Clearance ............................................... K-23

K-44 Element: Site 3_Wx Plot: Wind Speed \& Direction ............................................. K-23

K-45 Element: Site 3_Wx Plot: Ambient Temperature - WX ....................................... K-24

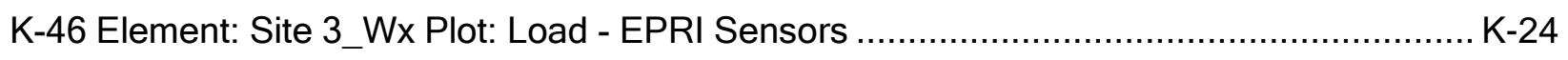

K-47 Element: Site 3_Wx Plot: Cond. Temp. - EPRI Sensors............................................ K-25

K-48 Element: Site 3_Wx Plot: Wind Speed \& Direction-Airport ....................................... K-25

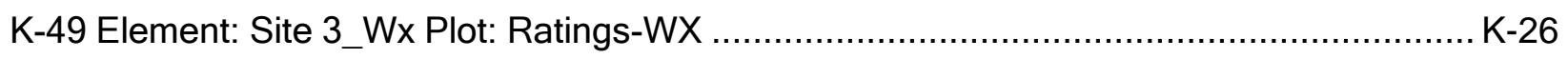

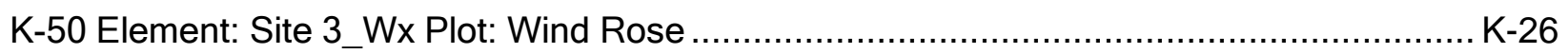

K-51 Element: Site 3_Wx Plot: Wind Rose Airport................................................... K-27

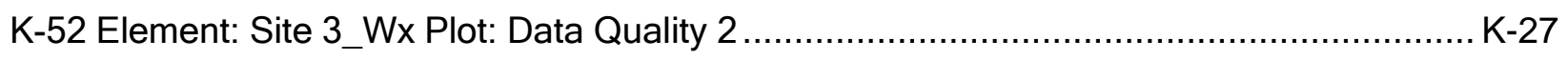

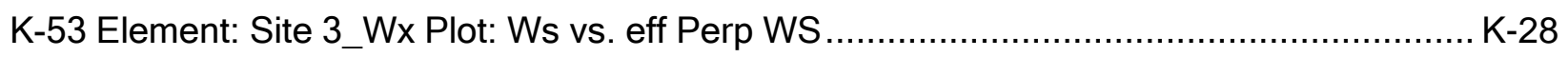

K-54 Element: Site 3_Wx Plot: Rating Cum. Dist.................................................... K-28

K-55 Element: Site 3_TR Plot: Ratings-ThermalRate ............................................ K-29

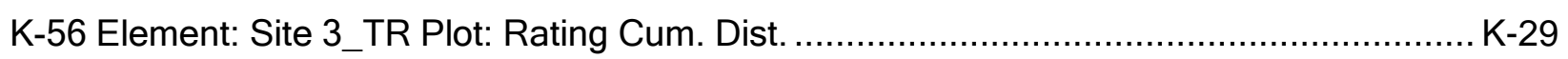

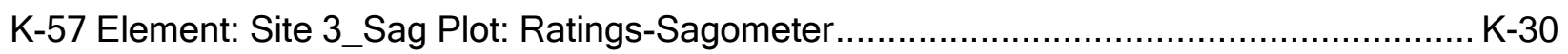

K-58 Element: Site 3_Sag Plot: Rating Cum. Dist. ................................................... K-30

K-59 Element: Site 3_Sag Plot: Conductor Temp Compare ........................................... K-31

K-60 Element: Site 3_BS Plot: Ratings-EPRI Sensors ................................................. K-31

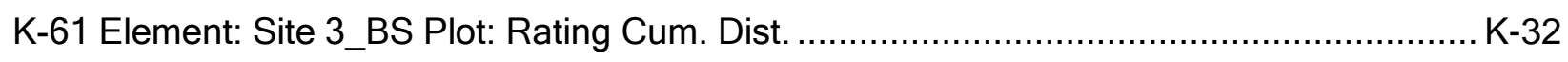

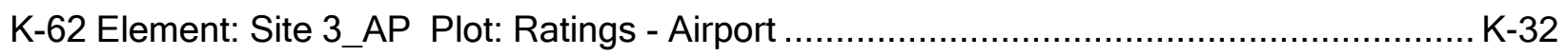

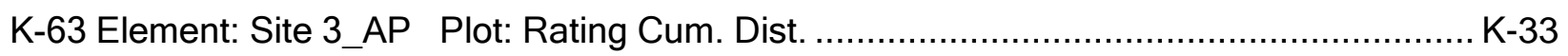

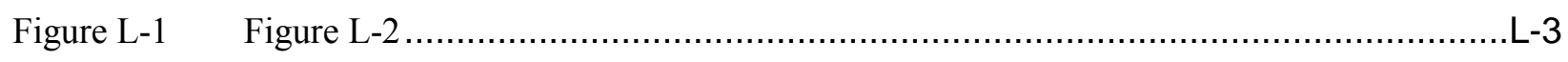

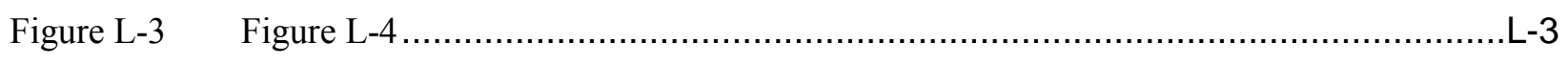

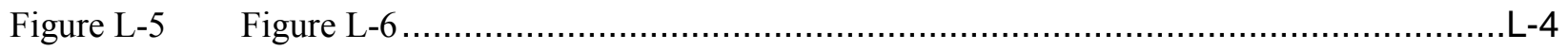

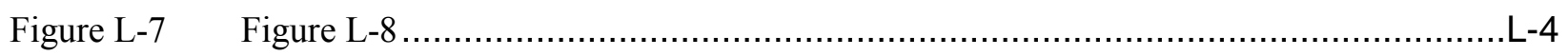

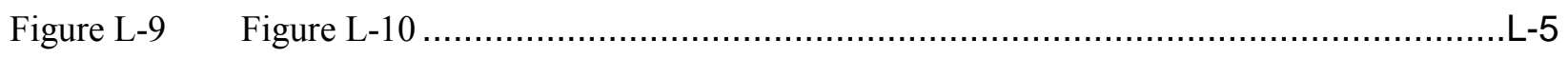

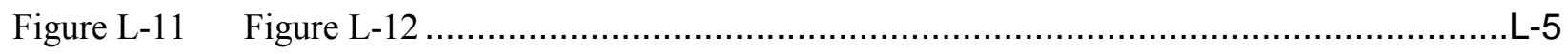


Figure L-13

Figure L-15

Figure L-17

Figure L-19

Figure L-21

Figure L-23

Figure L-25

Figure L-27

Figure L-29

Figure L-31

Figure L-33

Figure L-35

Figure L-37

Figure L-39

Figure L-41

Figure L-43

Figure L-45

Figure L-47

Figure L-49

Figure L-51

Figure L-53

Figure L-55

Figure L-57

Figure L-59

Figure L-61

Figure L-63

Figure L-65

Figure L-67

Figure L-69

Figure L-71

Figure L-73

Figure L-75

Figure L-77

Figure L-79

Figure L-81

Figure L-83

Figure L-85

Figure L-87

Figure L-89
Figure L-14

Figure L-16 L-6

Figure L-1 8 L-7

Figure L-20 L-7

Figure L-22 L-8

Figure L-24 L-8

Figure L-26 L-9

Figure L-28 L-9

Figure L-30

Figure L-32 L-10

Figure L-34

Figure L-36

Figure L-38

Figure L-40

Figure L-42

Figure L-44

Figure L-46

Figure L-48

Figure L-50

Figure L-52

Figure L-54

Figure L-56

Figure L-58

Figure L-60

Figure L-62

Figure L-64

Figure L-66

Figure L-68

Figure L-70

Figure L-72

Figure L-74

Figure L-76

Figure L-78

Figure L-80

Figure L-82

Figure L-84

Figure L-86

Figure L-88

Figure L-90
L-10 L-11 L-11 L-12 L-12 L-13 L-14 L-14 L-15 L-15 L-16 L-16 L-17 L-17 L-18 L-18 


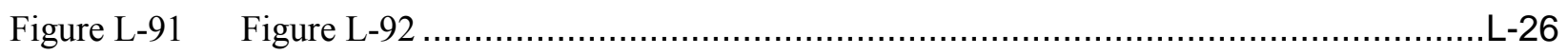

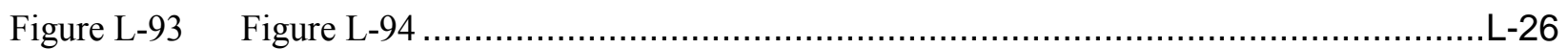

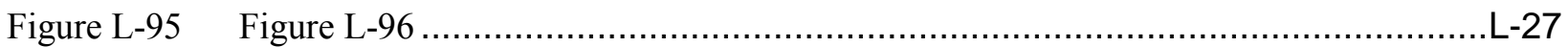

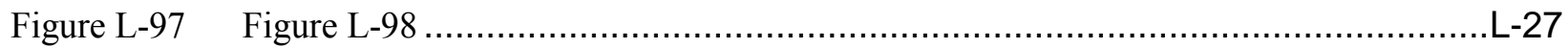

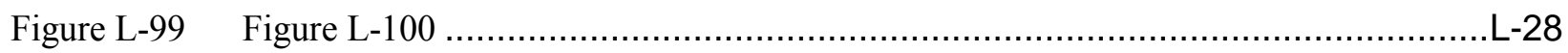

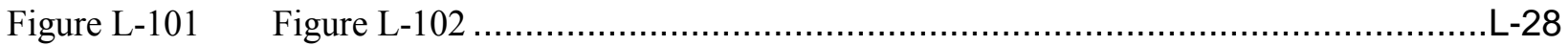

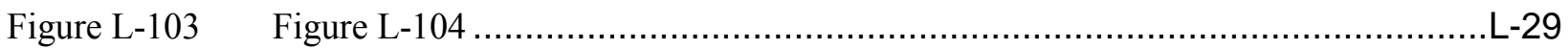

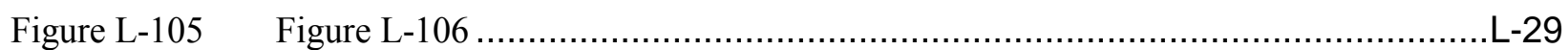

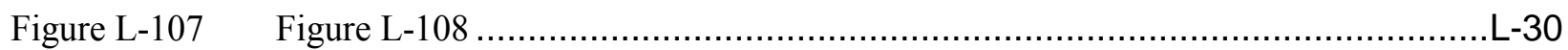

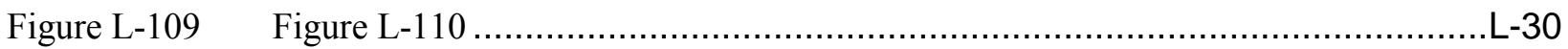

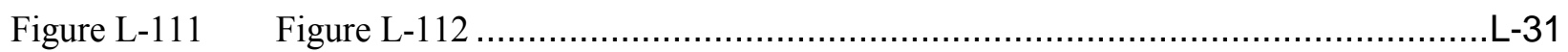

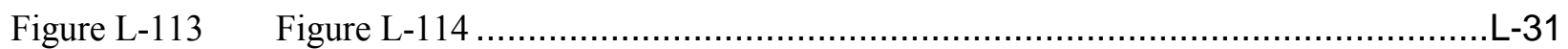

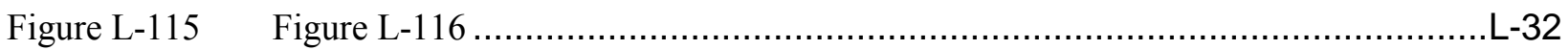

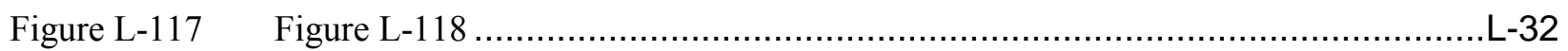

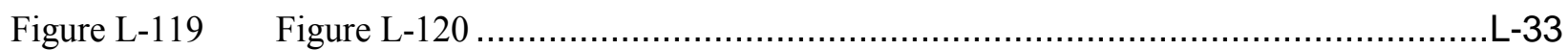

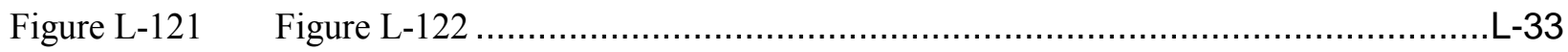

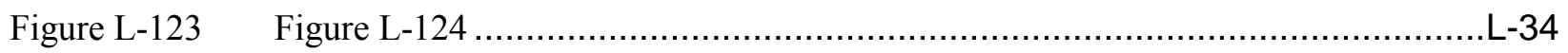

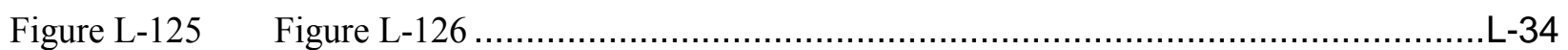

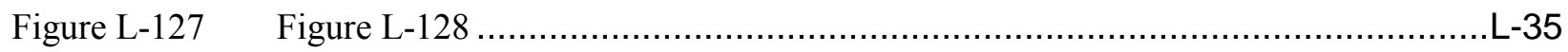

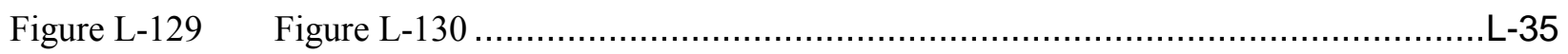

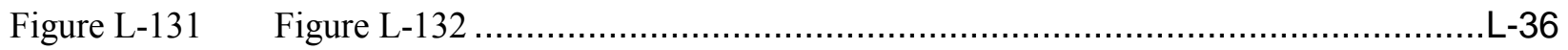

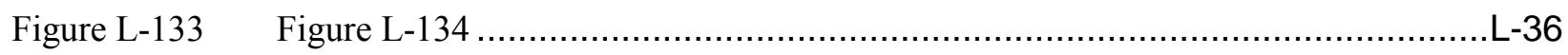

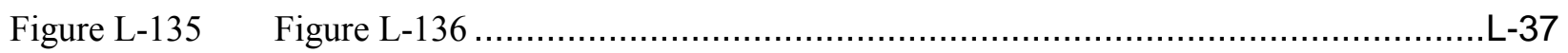

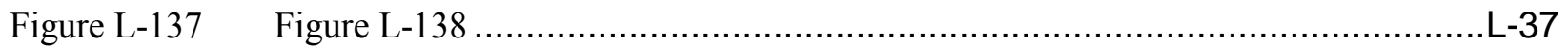

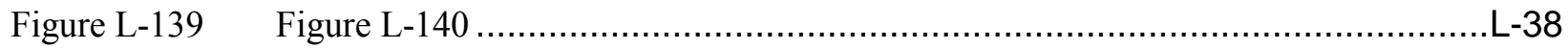

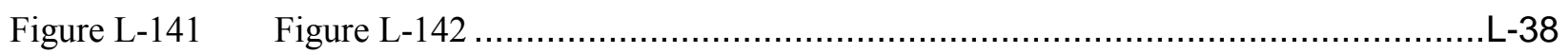

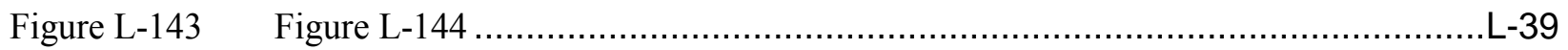

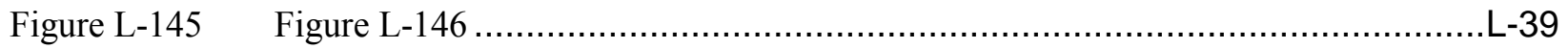

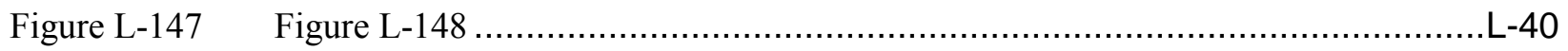

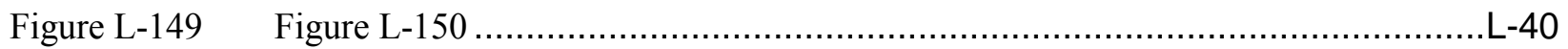

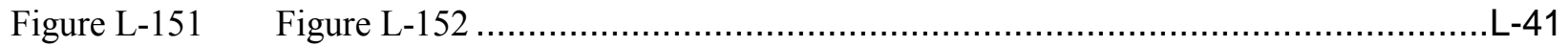

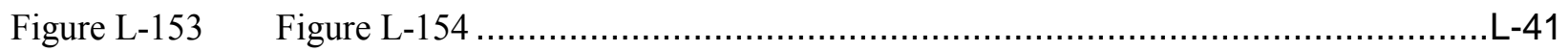

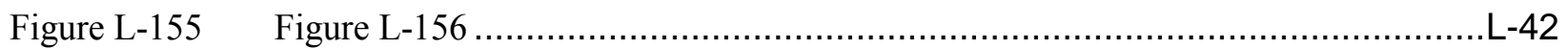

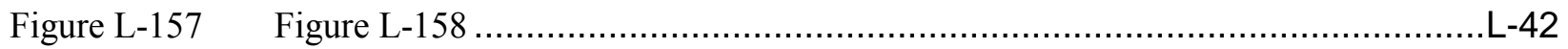

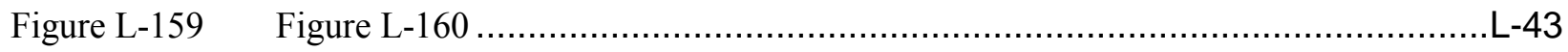

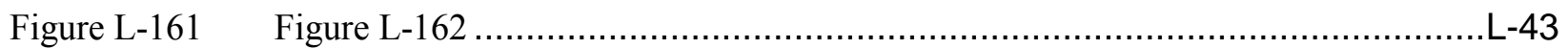

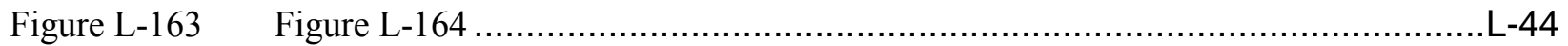

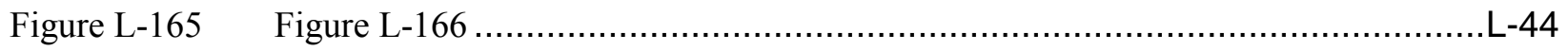

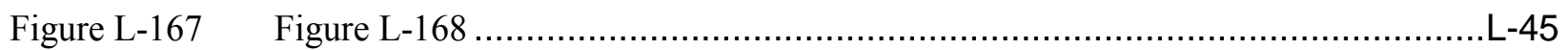




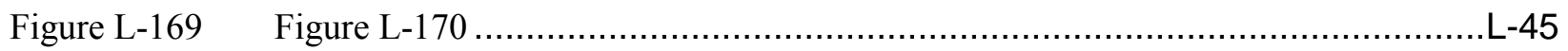

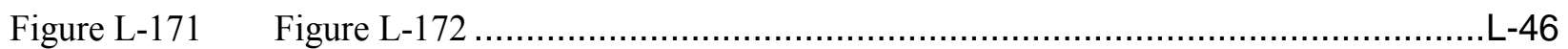

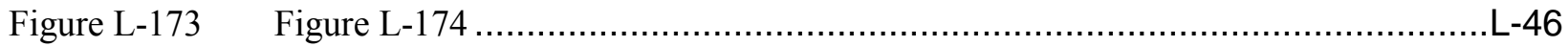

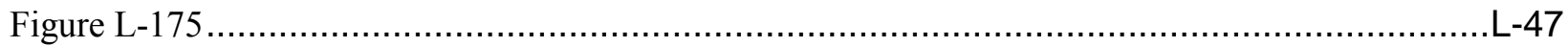

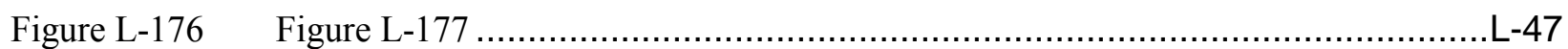

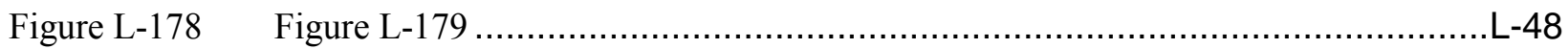

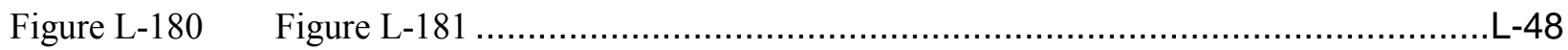

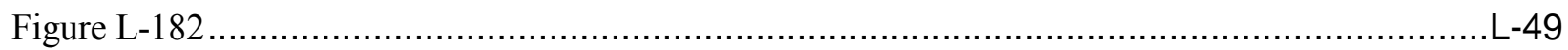

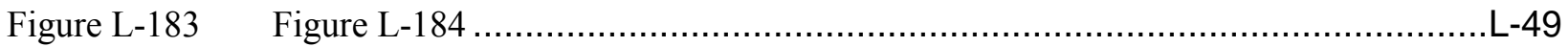

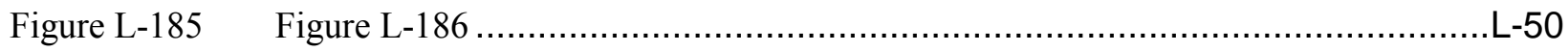

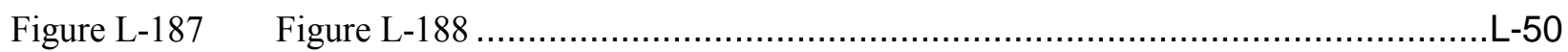

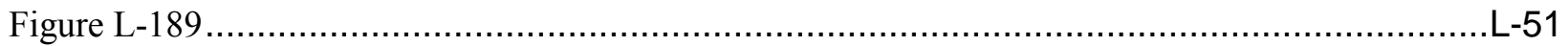

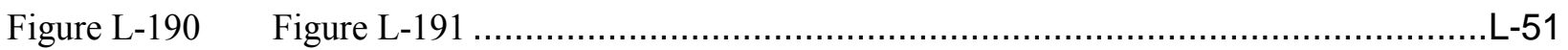

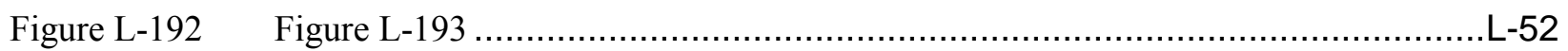

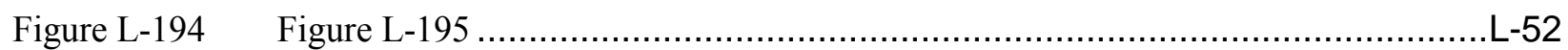

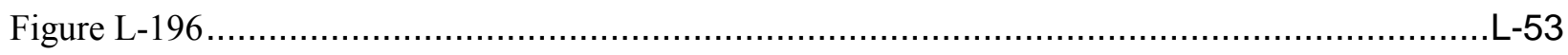

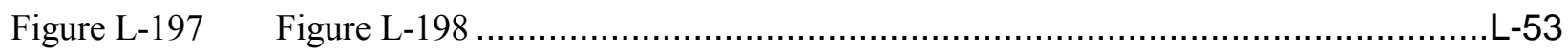

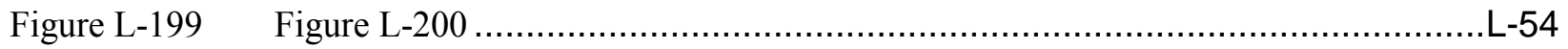

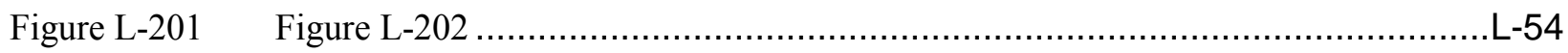

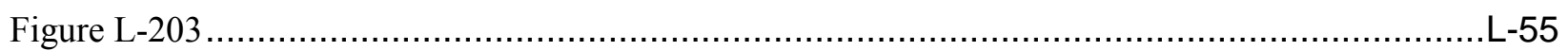

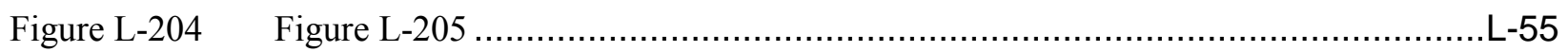

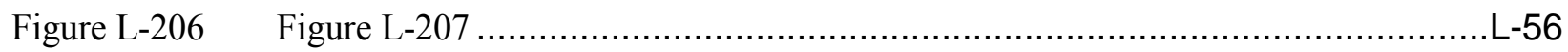

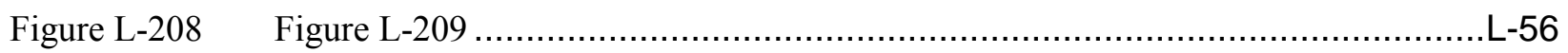

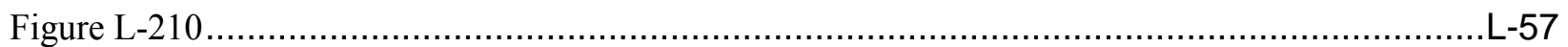

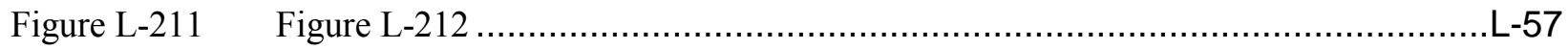

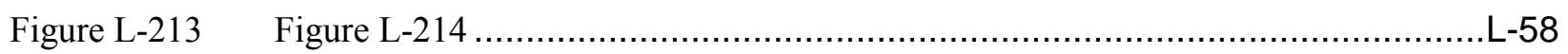

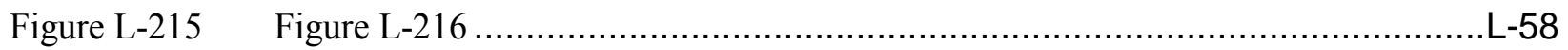

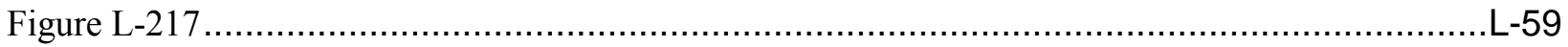

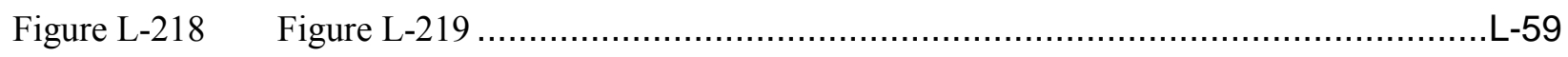

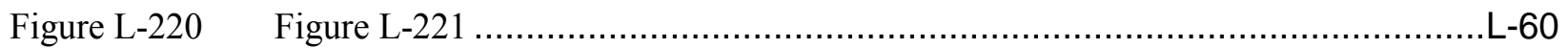

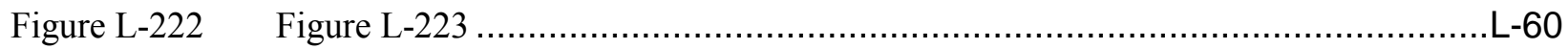

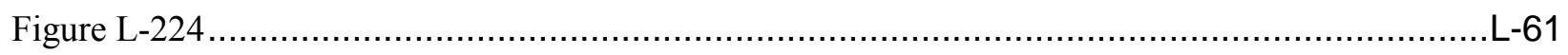

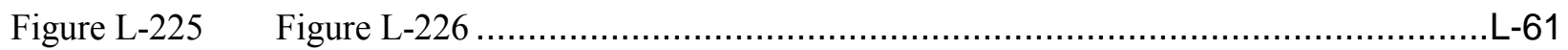

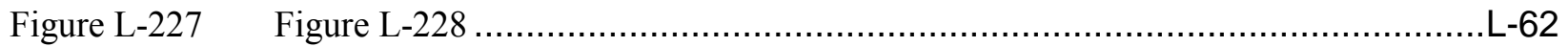

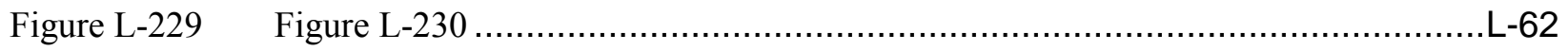

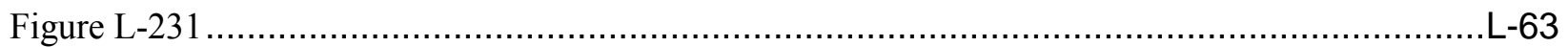

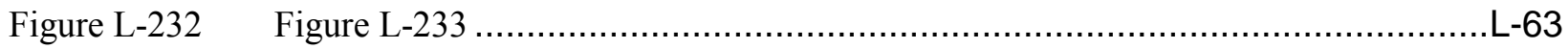

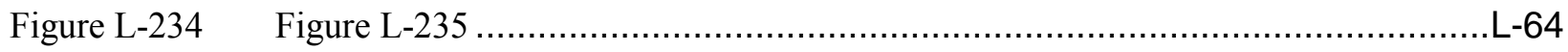

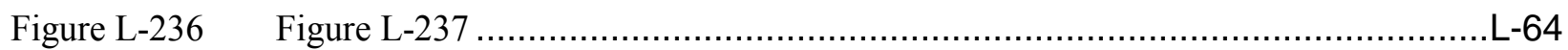


Figure L-238

Figure L-240

Figure L-241

Figure L-242 L-66

Figure L-243

Figure L-244

Figure L-245 L-67

Figure L-246

Figure L-247 L-67

Figure L-248

Figure L-249

Figure L-250

Figure L-251

Figure L-252

Figure L-253

Figure L-254

Figure L-255

Figure L-256

L-69

Figure L-257

Figure L-259

Figure L-258 $L-70$

Figure L-260

Figure L-261

Figure L-262

Figure L-264

Figure L-263

Figure L-266

Figure L-265 L-72

Figure L-267

Figure L-269

Figure L-271

Figure L-268

L-72

Figure L-273

Figure L-270

Figure L-274

Figure L-272

Figure L-276

Figure L-278

Figure L-280

Figure L-281

Figure L-283

Figure L-285

Figure L-287

Figure L-275 $-75$

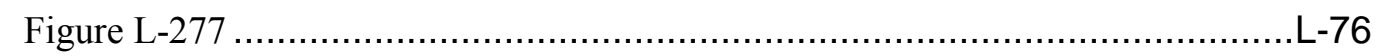

Figure L-288

Figure L-279 L-76

Figure L-290

Figure L-292

Figure L-282 L-77

Figure L-294

Figure L-284

Figure L-286

L-78

Figure L-295

Figure L-297

Figure L-299

Figure L-301

Figure L-302

Figure L-304

Figure L-289

L-78

Figure L-291

L-79

Figure L-293 L-80

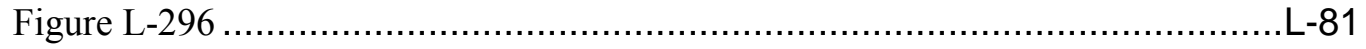

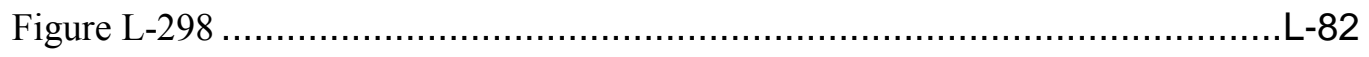

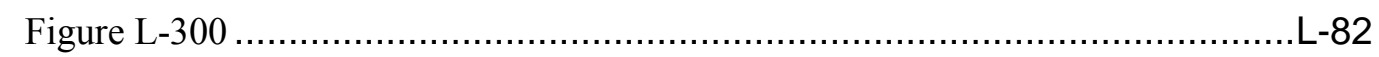

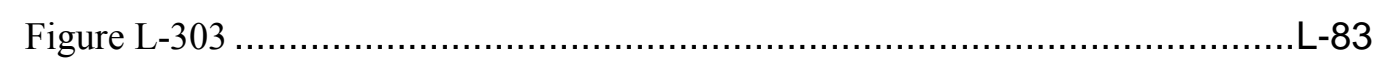

Figure L-305 


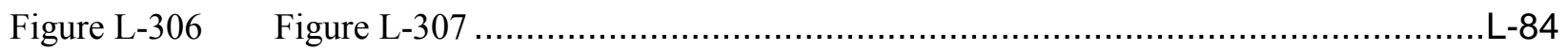

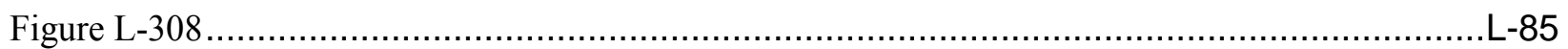

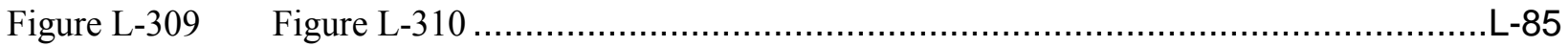

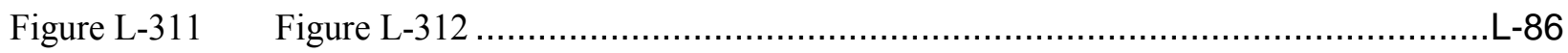

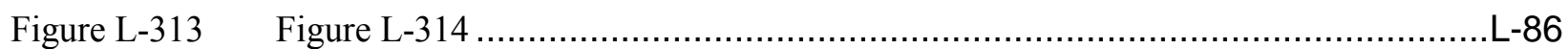

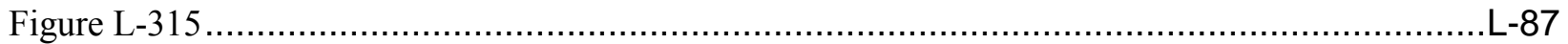

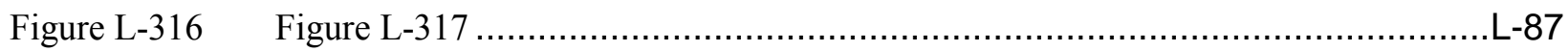

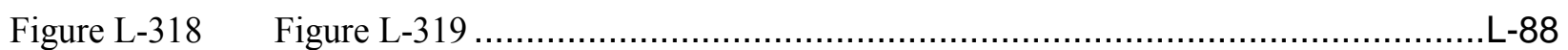

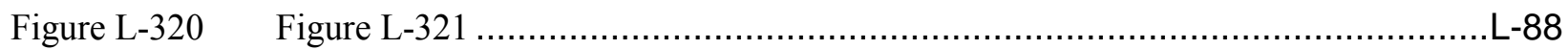

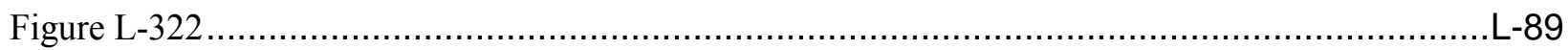

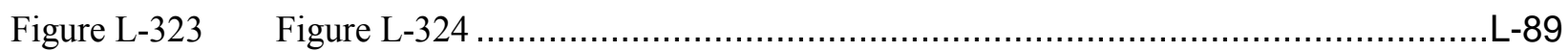

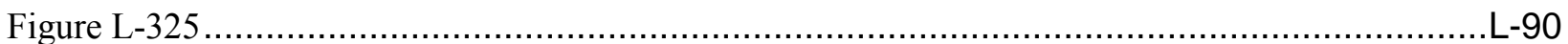

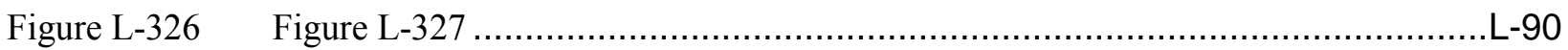

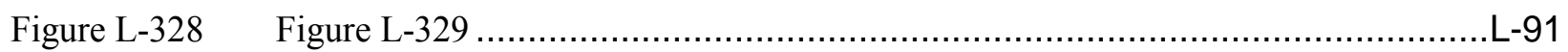

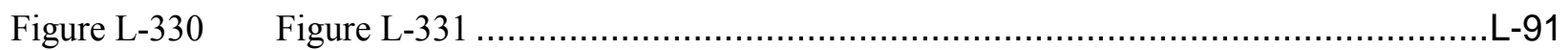

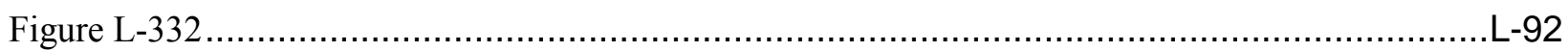

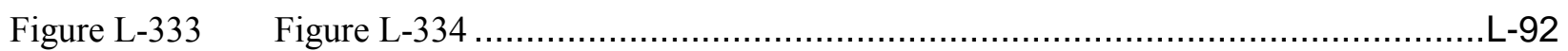

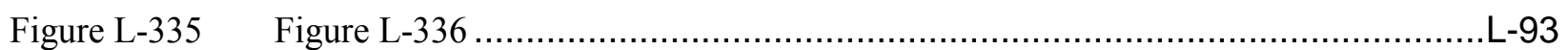

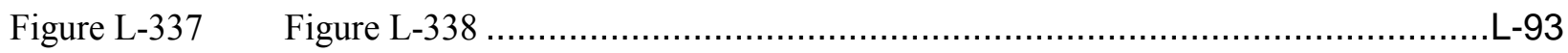

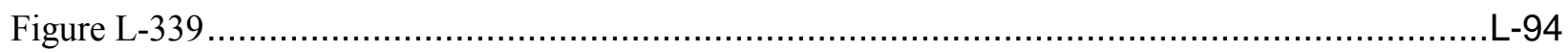

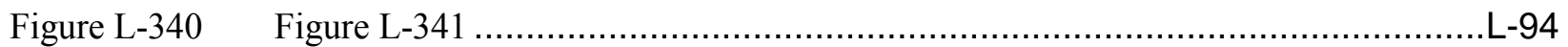

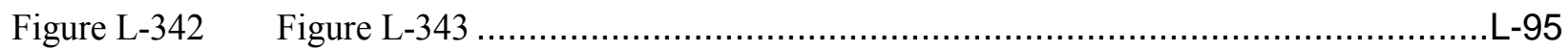

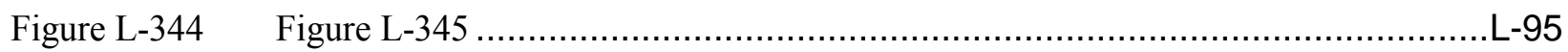

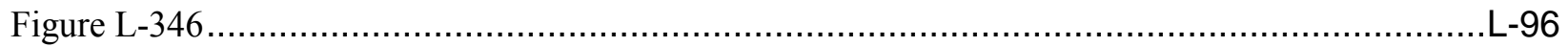

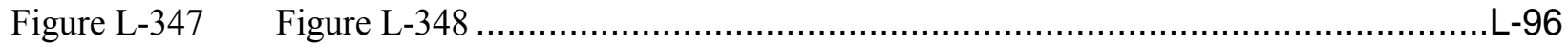

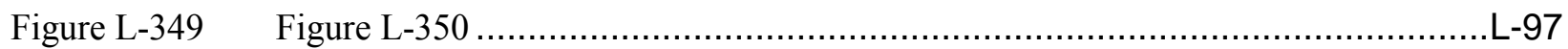

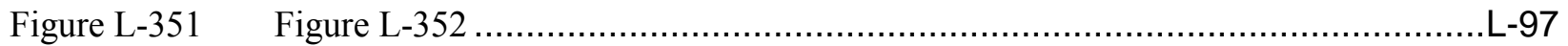

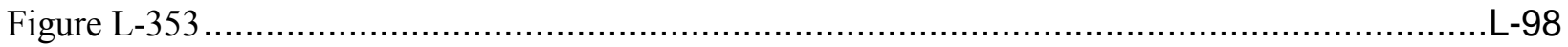

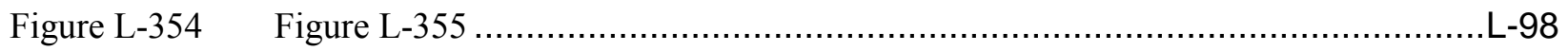

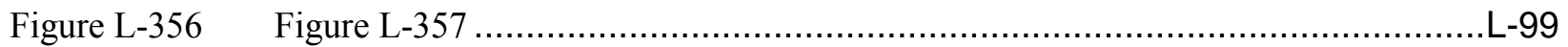

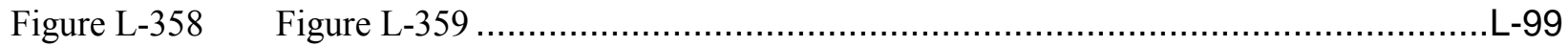

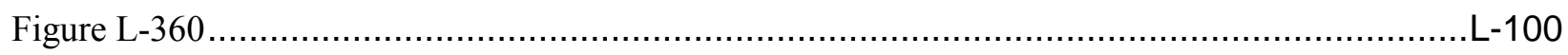

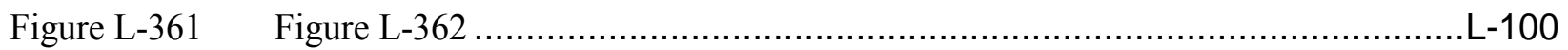

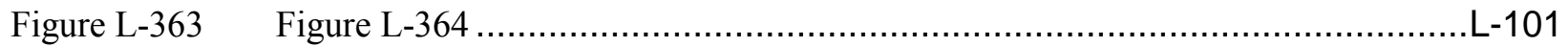

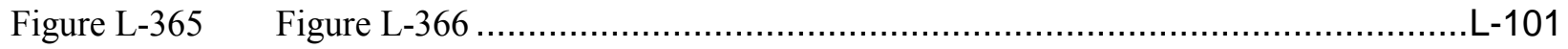

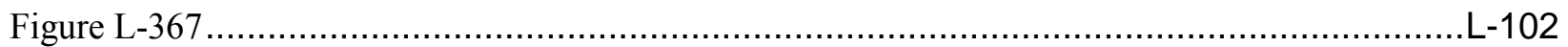

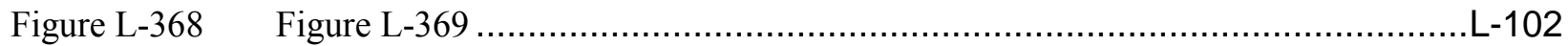

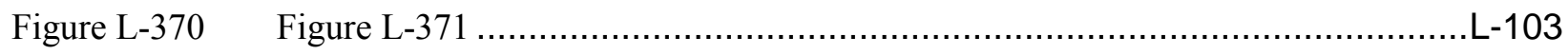

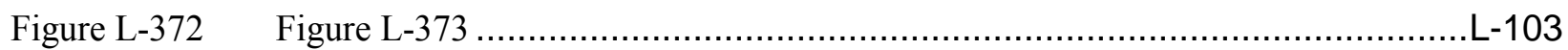


Figure L-374

Figure L-376

Figure L-377

Figure L-378

Figure L-379

Figure L-380

Figure L-381 L-106

Figure L-382

Figure L-383 L-106

Figure L-384

Figure L-385

Figure L-386

Figure L-387 L-107

Figure L-388

Figure L-389

Figure L-390 L-108

Figure L-391

Figure L-392

Figure L-393

Figure L-394 L-109

Figure L-395

Figure L-396

Figure L-397

L-109

Figure L-398

Figure L-399

Figure L-400

Figure L-401

Figure L-402

Figure L-403

Figure L-404 $-110$

Figure L-405

Figure L-406 $-112$

Figure L-407

Figure L-408

Figure L-409

Figure L-410

Figure L-411

Figure L-412

Figure L-413

L-114

Figure L-414

Figure L-415

Figure L-416

Figure L-417

Figure L-418

L-115

Figure L-419

Figure L-421

Figure L-420 L-115

Figure L-423

Figure L-422 L-116

Figure L-424

Figure L-426

Figure L-428

Figure L-430

Figure L-425 L-116

Figure L-431

Figure L-427 L-117

Figure L-433

Figure L-429 L-117

Figure L-435

Figure L-437

Figure L-438

Figure L-440

Figure L-432 L-118 L-119

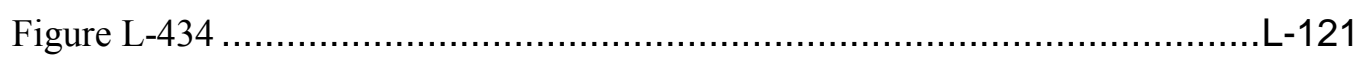

Figure L-436 


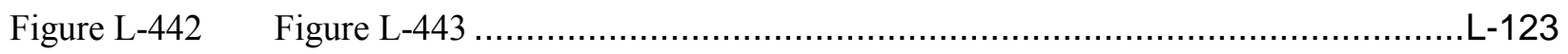

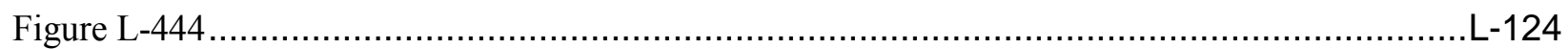

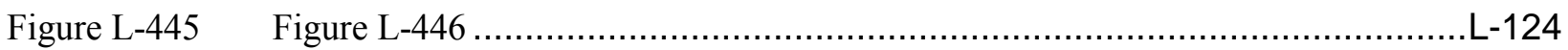

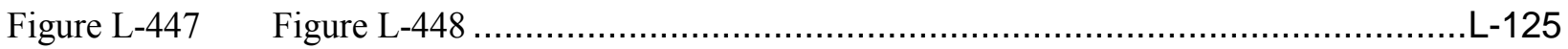

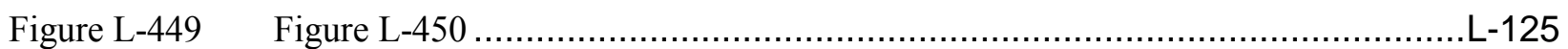

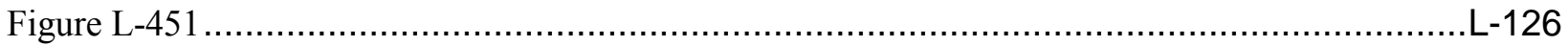




\section{TABLES}

Table 5-1 The main data derived from each of the four instrument types .................................. 5-2

Table 6-1 L-values for Normal, LTE, and STE ratings ................................................... $6-7$

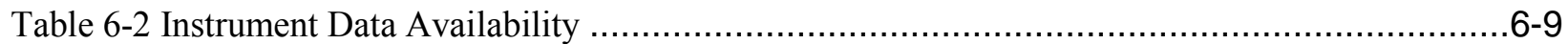

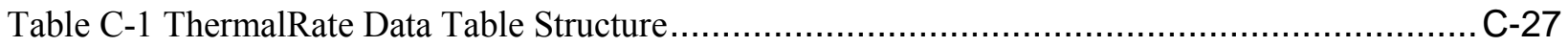

Table C-2 Sagometer Configuration Table Structure ...................................................... C-28

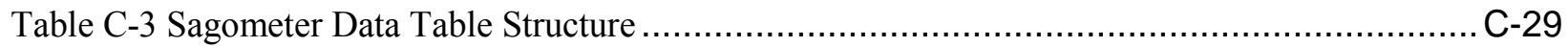

Table C-4 Sagometer Units Table Structure .................................................................... C-31

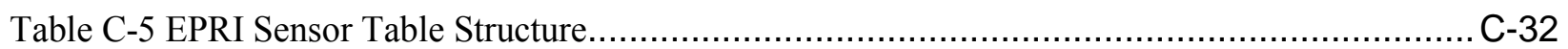

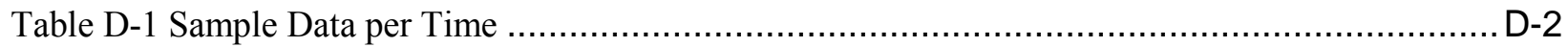

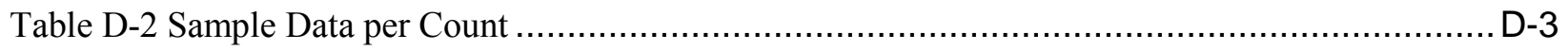

Table E-1 Instrumentation and Communication List by Primary Calculation Element - Some instruments are used in rating calculations in more than one element.................................. E-6

Table E-2 Average Performance of Data Systems at Site 1 from 8/1/11 through 10/31/12 _............. E-11

Table E-3 Average Performance of Data Systems at Site 2 from 8/1/11 through 10/31/12 _............. E-14

Table E-4 Average Performance of Data Systems at Site 3 from 8/1/11 through 10/31/12 .............. E-17

Table E-5 Average Performance of Data Systems at All Sites from 8/1/11 through 10/31/12 .......... E-18

Table F-1 Summary of Static Ratings .................................................................... F-2

Table G-1 Calculation Elements and DTCR Calculation Models ..................................................-1

Table G-2 Comparison of Monitoring Methods ..................................................................

Table H-1 Winter 2010/11 Ratings Distribution Site 2 Onsite Weather Station.............................. H-4

Table H-2 Winter 2010/11 Ratings Distribution Site 2 ThermalRate ......................................... H-5

Table H-3 Winter 2010/11 Ratings Distribution Site 2 Sagometer .......................................... H-6

Table H-4 Winter 2010/11 Ratings Distribution Site 2 EPRI Sensors ........................................

Table H-5 Summer 2011 Ratings Distribution Site 2 Onsite Weather Station ................................ H-8

Table H-6 Summer 2011 Ratings Distribution Site 2 ThermalRate ........................................... H-9

Table H-7 Summer 2011 Ratings Distribution Site 2 Sagometer.......................................... H-10

Table H-8 Summer 2011 Ratings Distribution Site 2 EPRI Sensors ...................................... H-11

Table H-9 Winter 2011/12 Ratings Distribution Site 2 Onsite Weather Station............................ H-12

Table H-10 Winter 2011/12 Ratings Distribution Site 2 ThermalRate ...................................... H-13

Table H-11 Winter 2011/12 Ratings Distribution Site 2 Sagometer.......................................... H-14

Table H-12 Winter 2011/12 Ratings Distribution Site 2 EPRI Sensors ...................................... H-15

Table H-13 Winter 2011/12 Ratings Distribution Site 2 Offsite Weather Service......................... H-16

Table H-14 Summer 2012 Ratings Distribution Site 2 Onsite weather station................................ H-17

Table H-15 Summer 2012 Ratings Distribution Site 2 ThermalRate........................................

Table H-16 Summer 2012 Ratings Distribution Site 2 Sagometer ......................................... H-19 
Table H-17 Summer 2012 Ratings Distribution Site 2 EPRI Sensors....................................... H-20

Table H-18 Summer 2012 Ratings Distribution Site 2 Offsite Weather Service ............................. H-21

Table H-19 Summary Table for Site 2 - L2 Were Used for Purposes of Comparison ...................... H-22

Table H-20 Winter 2010/11 Ratings Distribution Site 3 Onsite weather station ........................... H-23

Table H-21 Winter 2010/11 Ratings Distribution Site 3 ThermalRate .........................................-24

Table H-22 Winter 2010/11 Ratings Distribution Site 3 Sagometer...................................... H-25

Table H-23 Winter 2010/11 Ratings Distribution Site 3 EPRI Sensor......................................... H-26

Table H-24 Summer 2011 Ratings Distribution Site 3 Onsite Weather Station ............................... H-27

Table H-25 Summer 2011 Ratings Distribution Site 3 ThermalRate........................................ H-28

Table H-26 Summer 2011 Ratings Distribution Site 3 Video Sagometer .................................. H-29

Table H-27 Summer 2011 Ratings Distribution Site 3 EPRI Sensors............................................ H-30

Table H-28 Winter 2011/12 Ratings Distribution Site 3 Onsite weather station .......................... H-31

Table H-29 Winter 2011/12 Ratings Distribution Site 3 ThermalRate .................................... H-32

Table H-30 Winter 2011/12 Ratings Distribution Site 3 Sagometer........................................ H-33

Table H-31 Winter 2011/12 Ratings Distribution Site 3 EPRI Sensor........................................ H-34

Table H-32 Winter 2011/12 Ratings Distribution site 3 Offsite Weather Service .......................... H-35

Table H-33 Summer 2012 Ratings Distribution Site 3 Onsite weather station.................................36

Table H-34 Summer 2012 Ratings Distribution Site 3 ThermalRate....................................... H-37

Table H-35 Summer 2012 Ratings Distribution Site 3 Sagometer ........................................ H-38

Table H-36 Summer 2012 Ratings Distribution Site 3 EPRI Sensors........................................... H-39

Table H-37 Summer 2012 Ratings Distribution Site 3 Offsite Weather Service ............................ H-40

Table H-38 Summary Table for Site 2 - L2 Were Used for the Purposes of Comparison .................. H-41

Table H-39 Winter 2010/11 Ratings Distribution Site 1 Onsite Weather Station........................... H-42

Table H-40 Winter 2010/11 Ratings Distribution Site 1 ThermalRate ..................................... H-43

Table H-41 Winter 2010/11 Ratings Distribution Site 1 Sagometer....................................... H-44

Table H-42 Winter 2010/11 Ratings Distribution Site 1 Sagometer....................................... H-45

Table H-43 Summer 2011 Ratings Distribution Site 1 Onsite Weather Station ............................ H-46

Table H-44 Summer 2011 Ratings Distribution Site 1 ThermalRate.........................................47

Table H-45 Summer 2011 Ratings Distribution Site 1 Sagometer ....................................... H-48

Table H-46 Summer 2011 Ratings Distribution Site 1 EPRI Sensors......................................... H-49

Table H-47 Winter 2011/12 Ratings Distribution Site 1 Onsite Weather Station ........................... H-50

Table H-48 Winter 2011/12 Ratings Distribution Site 1 ThermalRate ......................................... H-51

Table H-49 Winter 2011/12 Ratings Distribution Site 1 Sagometer.......................................... H-52

Table H-50 Winter 2011/12 Ratings Distribution Site 1 EPRI Sensors .................................... H-53

Table H-51 Winter 2011/12 Ratings Distribution Site 1 Off Site Weather Service ......................... H-54

Table H-52 Summer 2012 Ratings Distribution Site 1 Onsite Weather Station ............................. H-55

Table H-53 Summer 2012 Ratings Distribution Site 1 ThermalRate........................................... H-56

Table H-54 Summer 2011 Ratings Distribution Site 1 Sagometer ....................................... H-57

Table H-55 Summer 2012 Ratings Distribution Site 1 EPRI Sensors...................................... H-58 
Table H-56 Summer 2012 Ratings Distribution Site 1 Off Site weather service............................ H-59

Table H-57 Summary table for Site 1 - L2 Were Used for Purposes of Comparison ....................... H-60

Table I-1 Site 1 Correlation Coefficients, Wind Output vs. Ratings........................................... I-2

Table I-2 Site 2 Correlation Coefficients, Wind Output vs. Ratings........................................ I-3

Table I-3 Site 3 Correlation Coefficients, Wind Output vs. Ratings.......................................... I-4

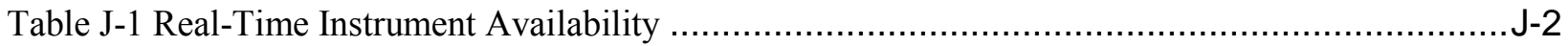

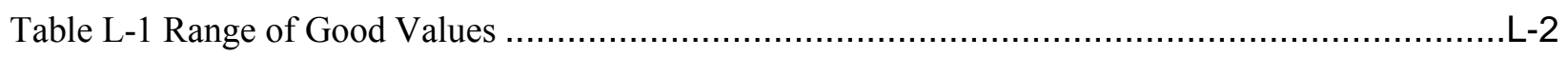





\section{Introduction}

The demand for electric power over transmission circuits is increasing at a faster rate than the construction of new transmission facilities. This trend is pushing the capacity of many transmission circuits beyond operation margins required for contingencies. The power capacity (i.e., the rating) of most overhead transmission lines is prescribed by the so-called "static rating" based on both the conductor configurations and the environmental conditions. Ideally, very conservative worst-case assumptions about environmental conditions are used when developing these static ratings. Due to this conservative approach, significant extra power capacity exists beyond the design margin on most lines most of the time.

As part of its on-going research in this area, EPRI has developed monitors, rating calculation methodologies, and the Dynamic Thermal Circuit Rating (DTCR) software for the purpose of gaining access to the extra power capacity. Prior to undertaking capital intensive activitiessuch as building new lines, reconductoring, raising structure heights, replacing transformers, putting lines underground, etc. - power companies can use these technologies to maximize power throughput of existing assets, defer capital expenditures, and simultaneously increase safe and reliable operation of their assets. This is particularly true for a situation at NYPA where significant bulk power must be transferred a long distance - from where there is significant renewable energy (wind farms in northern New York) to the load center (New York City). It is worth noting that this situation is common in power grids worldwide.

In 2010, a project was initiated through a partnership between the Department of Energy (DOE) and the New York Power Authority (NYPA) to evaluate EPRI's rating technology and instrumentation that can be used to monitor the thermal states of transmission lines and provide the required real-time data for real-time rating calculations.

The project included the installation and maintenance of various instruments at three $230 \mathrm{kV}$ line sites in northern New York. The instruments were monitored, and data collection and rating calculations were performed for about a three year period. The project consisted of ten main tasks listed below (refer to appendices for details)

1. Review Lines and Sites for Study

2. Detailed Design of Instrumentation Mounting, Integration, Power, and Communications

3. Purchase, Configure, Test, and Install Field Instrumentation

4. Maintenance of Field Instrumentation

5. Initial DTCR Setup and Verifications

6. Physical Modeling of Lines and Development of Sag-Temperature Equations

7. DTCR Execution, Training, and Continued Support

8. Training at Operations (Marcy) 
9. Instrumentation, Rating, and Weather Data Analysis

10. Meetings, Reports

A timeline for the project is shown in Figure 1-1

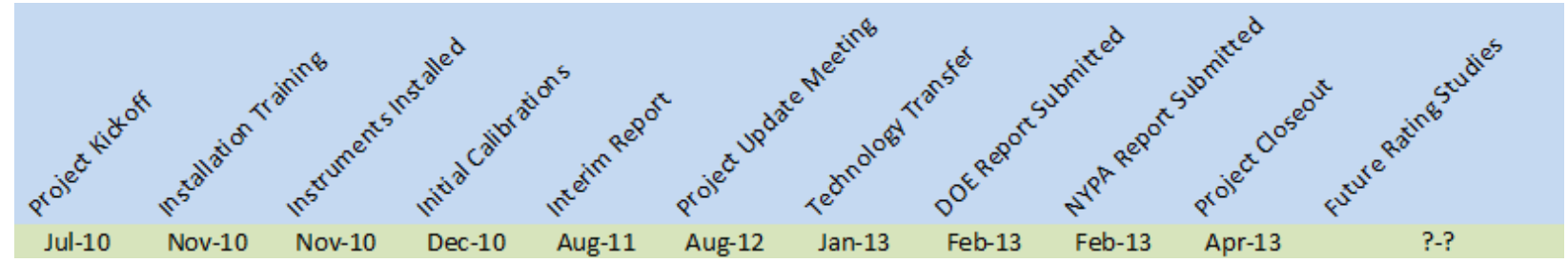

Figure 1-1 Timeline of the project

\section{Report Layout}

The project was very comprehensive, and a thorough documentation of all the details, data, and results required close to 500 pages. The decision was made to arrange the report such that the bulk of the material would be placed in appendices. Twelve appendices were setup for that purpose as listed in the Table-of-Contents. These appendices are referred to frequently in the report herein, and the reader can obtain more detailed information there. In addition, all of the data generated during the project was stored on electronic media and submitted as part of the project. 


\section{OVERVIEW OF THE SCIENCE OF TRANSMISSION LINE RATINGS}

\section{Introduction}

The electric power industry worldwide is experiencing a need to push more power through existing assets. This is a result of the ever growing demand for electric power, and the cost and permitting hurdles of constructing new assets. This is all particularly true for overhead transmission lines, which are the limiting circuit components in most cases, and the most expensive and difficult to replace or upgrade. However, the industry is recognizing that for almost all cases, existing transmission lines have significant extra power capacity, and there is a growing need to take advantage of that extra capacity.

The rating of an overhead transmission line is the specified upper limit of power (amperage) that the conductors are allowed to carry. The amperage must be limited in order to limit the operating temperatures of the conductors. In addition to the amperage, the temperature of a given overhead conductor depends on the ambient conditions (ambient temperature, wind speed, wind direction, solar intensity, and rain rate). The thermal energy input to a conductor comes from the resistive losses and solar input, and the thermal energy dissipation is in the form of heat convection, radiation, cooling by rain, and also in raising the conductor's temperature. The rating of a conductor is calculated from an equation that balances the thermal energy input to the thermal energy output (see details in Appendix B).

\section{Types of Ratings}

For a given transmission line which is designed with a maximum allowed conductor temperature, the rating is determined by the ambient conditions. Ambient conditions which help conductors dissipate heat are favorable to line ratings, and vice versa (see Appendix B). There are several methods used by the power industry to specify the ambient conditions used for rating calculations.

Traditionally, and almost universally still, utilities rate their overhead transmission lines with a static rating. In this case, assumed conservative values are used for ambient conditions. The advantages of using a static rating include the simplicity to calculate, and the fact that the rating is constant. In some cases the static rating is occasionally changed to reflect the differences in ambient conditions between night and day or seasonal variations. The disadvantage of using a static rating is that the actual power capacity of a line is usually significantly greater due to the overly conservative assumptions required. Also, for some periods of time, the actual capacity can be less than the static rating, and the temperature of a conductor may exceed the maximum allowed conductor temperature. 
A concept which is gaining a foothold in the power industry is the use of real-time rating, also referred to as dynamic line rating. In this case, actual real-time field measurements of ambient conditions are made, and the ratings are calculated continuously - typically at ten minute intervals.

The advantage of using dynamic rating is that it provides a better knowledge of the actual line rating, which will be significantly greater than the static rating most of the time. The disadvantage is that the dynamic rating is a varying quantity, and requires instruments for realtime field measurements. In many cases, the thermal inertia of a conductor, due to its heat capacity, can be used to rate a conductor even much higher for a short period of time - referred to as an emergency rating. These concepts are illustrated in Figure 2-2.

\section{Example of Results}

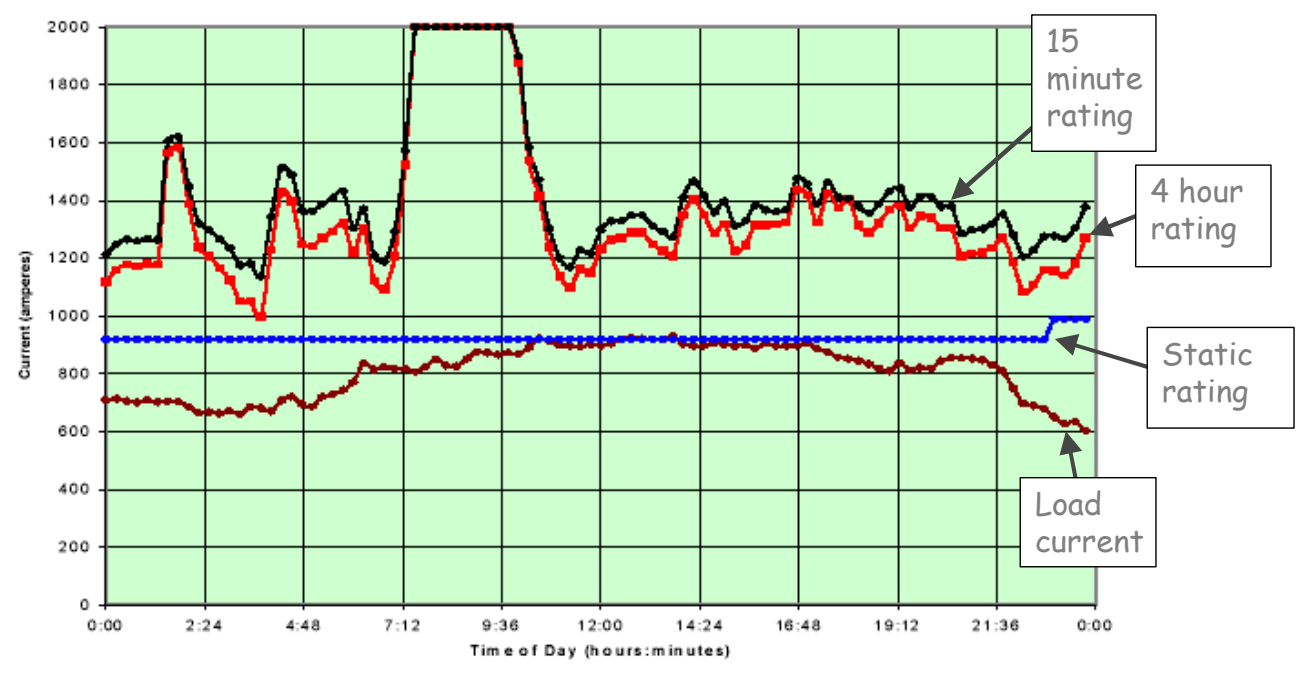

Figure 2-1 Example of dynamic ratings, static rating, and load for a 24-hour period for a particular transmission line

Figure 2-1 shows an example of the ratings for an operating line over a 24-hour period. Plotted are the static rating, the line's load, and the dynamic ratings (4-hour "normal", and 15-minute emergency). The 4-hour rating is the amount of current that a conductor can pass for the next 4 hours without exceeding the predefined maximum allowed operating temperature. Note that during the middle of this particular day the utility was bumping into its static rating when, according to the DTCR rating calculations, there was a significant margin to work with. Also note that a light rain that morning sent the real-time ratings to very high levels.

The focus of the DOE-NYPA project was to install instruments on operating lines in upper New York, and to perform dynamic ratings on those lines for an extended period of time. The results of the project can help NYPA, and other power companies with similar issues, understand the viability of applying real time ratings to their overhead transmission lines. 


\section{INSTRUMENTS AND SOFTWARE FOR DYNAMIC RATING}

\section{Introduction}

There are many different instruments available to the power industry for monitoring transmission lines for real-time rating purposes that are commercially available or under development. Instruments generally come with auxiliary equipment, such as power supplies (e.g. solar panels and batteries), communication devices (e.g. modems and antennae), and supporting electronics and hardware. In every case, the goal of any instrument package is to provide the real-time measurements of ambient conditions needed for real-time rating calculations. The measurements needed include ambient temperature, wind speed; wind direction, solar intensity, and rain rate, as described in Chapter 2 (see Appendix C for details about instruments).

Available instruments come in a wide range of designs which employ various concepts and techniques, but they generally fall into one of four classes:

1. Direct weather measurements

2. Direct conductor temperature measurements

3. Conductor sag measurements

4. Conductor tension measurements

There are pros and cons of each of these classes of instruments. For instance, instruments that make direct weather measurements (e.g. weather stations) are generally cheaper, their components are more reliable, and the data needs little interpretation. However, such instruments measure the ambient conditions only at the site of installation, and may not accurately represent the average conditions along a line. The latter issue can be mitigated by installing multiple units; however, the number of units and their optimal placements need to be determined.

The other three classes of instruments all have one point in common; they all measure conductor temperature. Conductor temperature measurements are used (along with air temperature and solar measurements) to determine an effective wind velocity to be used by the rating calculations. Direct conductor temperature measurements (made with a thermocouple pressed against the conductor) provide the conductor temperature measurements. However, such a measurement only provides data for the point at which it is mounted, and may not truly represent the average conductor temperature along the line. But similar to direct weather measurements, the latter issue can be mitigated by installing multiple units.

Conductor sag or tension measurements can be used to determine the average conductor temperature along an entire line, which is a very clever method of determining the effective wind 
velocity along the line for rating calculations. However, this requires the determination of the state-change equation that relates conductor temperature to conductor sag or tension. The accurate determination of the state-change equation can be very problematic (see Appendix $G$ for details).

Also very important is the fact that using conductor temperature measurements to determine the effective wind velocity can only be performed when the current in the conductor is above a certain threshold - enough to drive the conductor temperature several degrees above ambient temperature. If this is not the case, measurements of conductor temperature directly, or sag or tension measurements, cannot be used for rating calculations. Throughout the entire course of this project, there were virtually no instances when the current was high enough to perform rating calculations with these instruments. The only option in this situation was to rely solely on direct weather measurements.

Due to practical constraints, not all available instruments were tested during the DOE-NYPA project. Four sets of instruments were selected: weather stations, Video Sagometers, EPRI Sensors, and ThermalRate systems. These are briefly described below, and details can be found in Appendices $\mathrm{C}$ and $\mathrm{G}$.

\section{Weather Station}

One weather station (from Campbell Scientific) was installed at each of the three NYPA test sites. Figure 3-1 shows a photograph of a typical installation. The wind was measured with a 3dimensional (3D) ultrasonic anemometer. These devices report the $\mathrm{x}, \mathrm{y}$, and $\mathrm{z}$ components of the wind velocity vector. It has no moving parts, and it has no stall speeds as do propeller or cuptype anemometers. This is a significant advantage because low velocity winds can be very important for rating calculations. Wind cooling is paramount to transmission line ratings, and Appendix D presents details of the wind analysis for the project.

The pyranometer is a sensor that measures the solar power density in terms of watts-per-square meter. The rain gauge measures rain rate, and the sensor to the far right in Figure 3-1 measures ambient temperature and humidity.

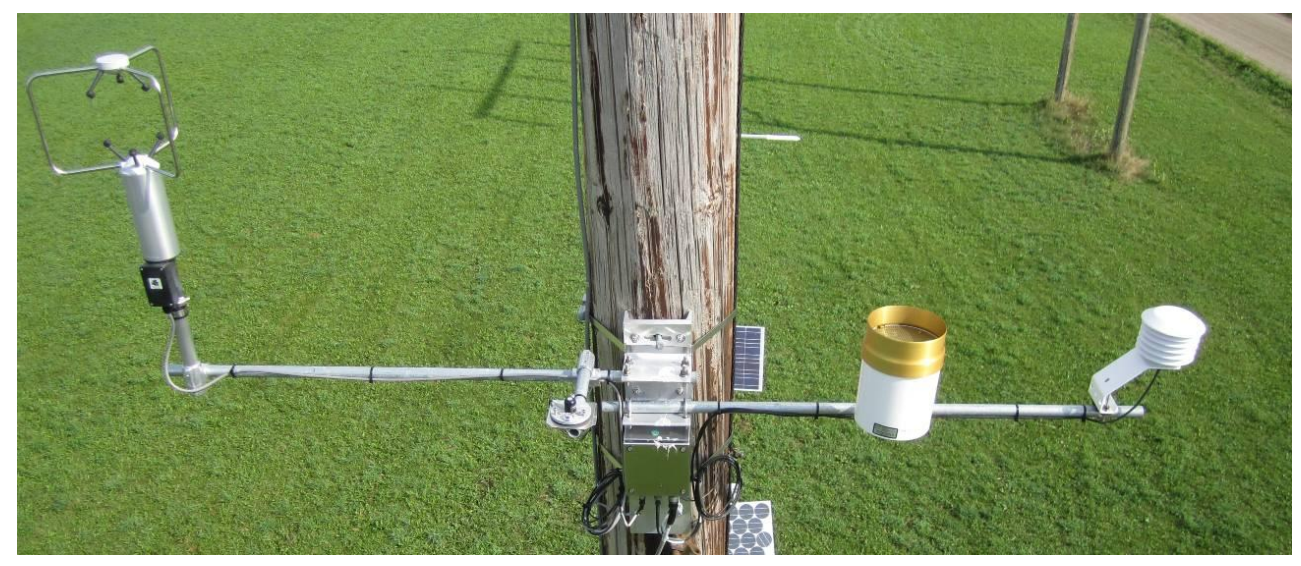

Figure 3-1 Weather station sensor array; anemometer, pyranometer, rain gauge, temperature/humidity sensor (left to right) 


\section{Video Sagometer}

The Video Sagometer (sagometer) consists of a camera mounted to the transmission line structure (see Figure 3-2). Onboard vision-recognition software identifies the line's conductor in the camera's field of view, and determines the sag of the conductor. The sag of a conductor depends on its temperature, and as long as an accurate state-change equation is known, the average conductor temperature can be identified. These devices were initially developed by EPRI, and are now commercialized by Engineering Data Management, International (EDM).

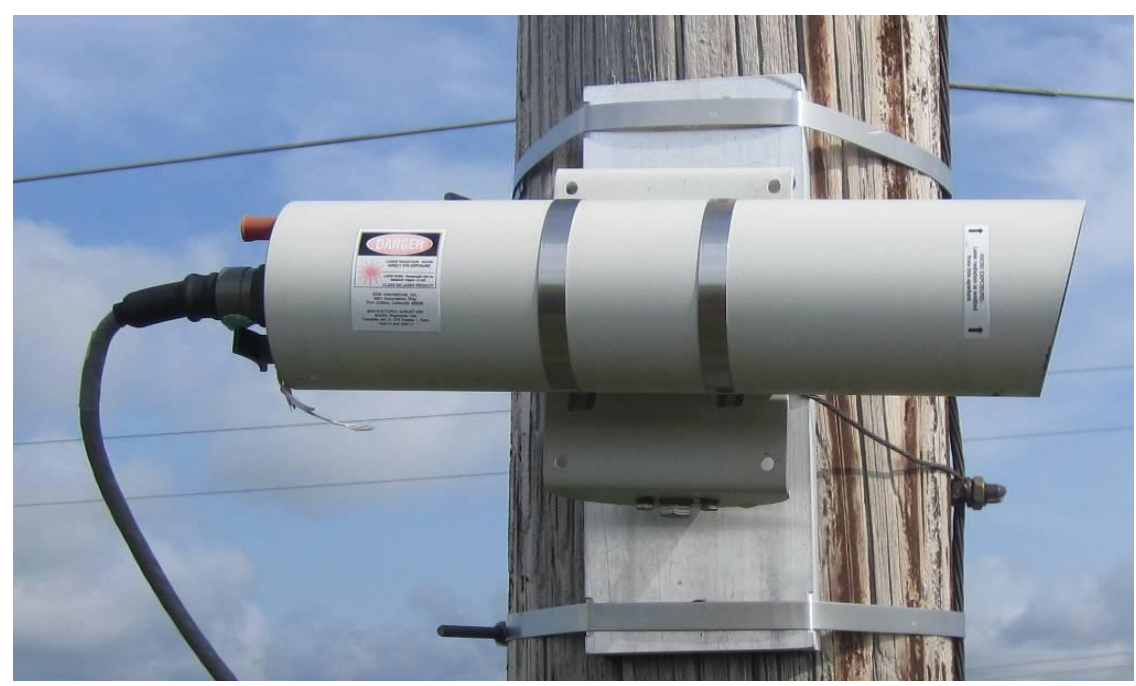

Figure 3-2 The sagometer camera

\section{EPRI Sensors}

EPRI is in the process of developing a device (referred to in this report as the EPRI Sensor) for measuring conductor temperature (at a point), conductor current, conductor vibration, and conductor sag. Unlike the other instruments used in this project, these devices have not been commercialized. For the DOE-NYPA project, the devices were used to measure conductor temperature, and they provided backup current measurements where needed. Figure 3-3 shows a photograph of an installed sensor. 


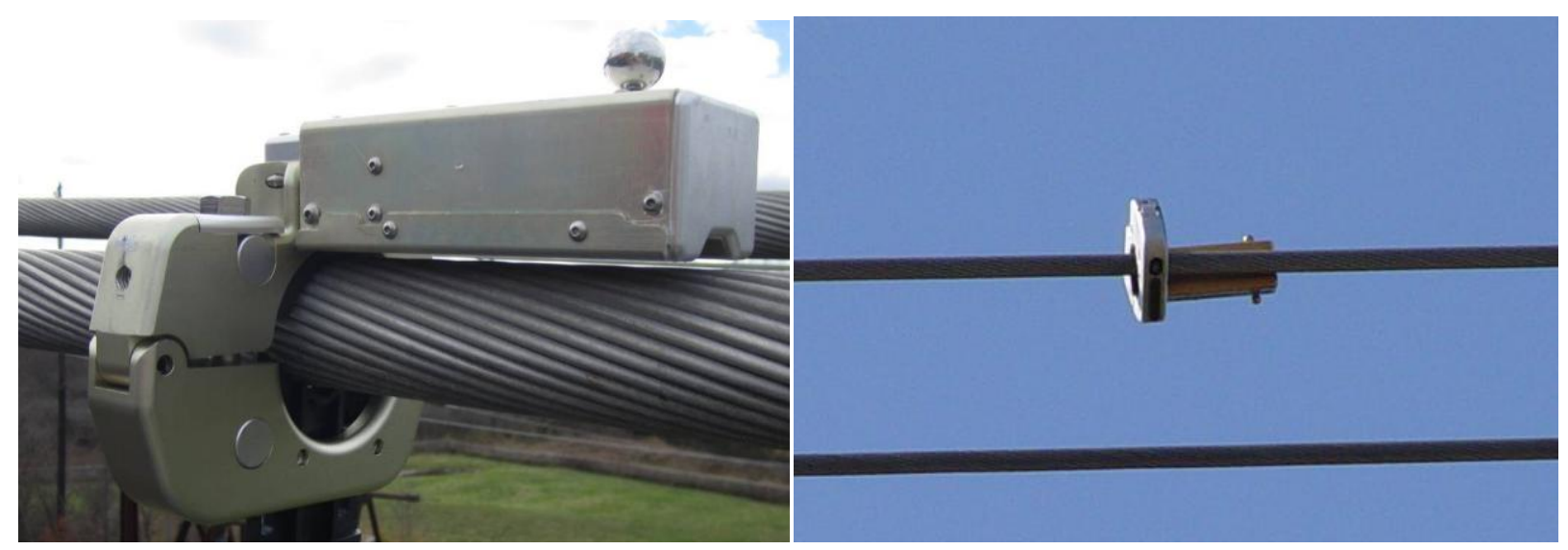

Figure 3-3 EPRI Sensor mounted on a conductor

\section{ThermalRate System}

The ThermalRate system is an instrument by Pike Electric, Inc. It is a unique device that would be classified as a direct weather measuring device. It consists of two rods running parallel to the transmission line conductor (see Figure 3-4). One of the rods is heated with a defined thermal power, and the other rod is not heated. The rods are fabricated to have the same thermal characteristics of the transmission line conductors. By measuring the temperatures of the two rods, enough information can be gleaned to define the ambient conditions for rating calculations.

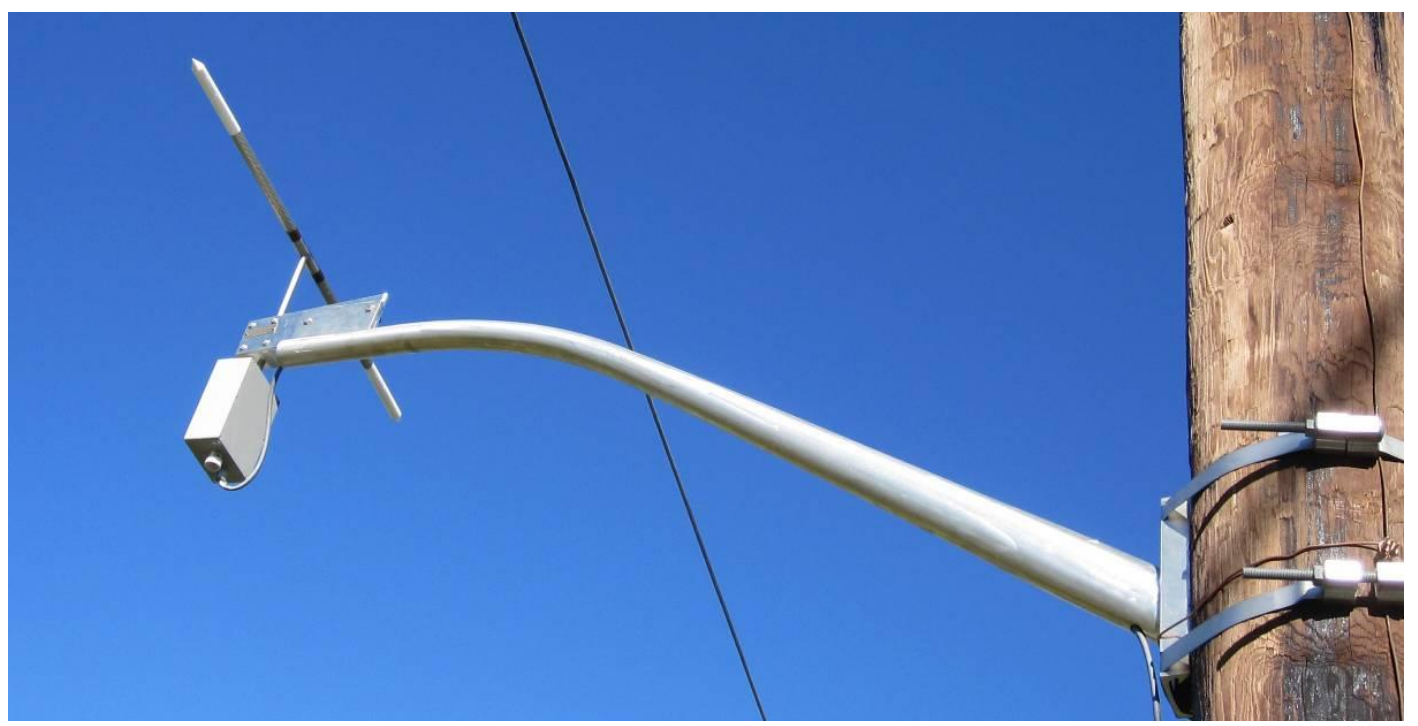

Figure 3-4 ThermalRate system

\section{EPRI DTCR Software}

EPRI's DTCR software is designed to accept real-time (or simulated real-time or forecasted) inputs from a number of field sensors, and computes the normal (and emergency) real-time 
ratings for overhead lines (or other circuit components). The rating calculations, and a host of other relevant data, can be made available to utility personnel (engineers, researchers, operators, planners, etc.), as depicted in Figure 3-5.

For the DOE-NYPA project, rating calculations were performed individually from all the instruments, and comparisons were made (see Appendices F and $\mathrm{G}$ for details).

\section{Field measurements} and load

- Real-time

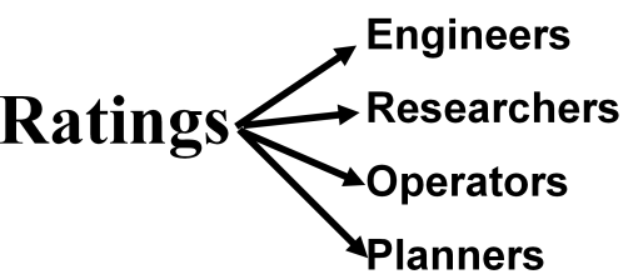

- Simulated real-time

- Forecasted

Figure 3-5 Depiction of the DTCR software input and output 



\section{4 \\ THE NYPA TEST SITES}

The transmission lines identified for study in the DOE-NYPA project were two $230 \mathrm{kV}$ lines in upstate New York that are located very near a significantly large wind farm. Both lines run in approximately an east-west direction where the instruments were installed. Instruments were installed at three sites; one set was installed on a 6.5 mile line, and the other two sets were installed near opposite ends of a 37 mile line.

These particular lines and sites were chosen for three main reasons:

1. They are located adjacent to a large wind farm, and may be called upon to transmit significant wind power to the load center in the south.

2. Because of the open and flat terrain it was thought that these particular lines may have ratings that are well correlated with wind generation, and this hypothesis was to be tested.

3. These particular sites have easy access for installing and maintaining the equipment.

Figure 4-1 shows a photograph of one of the structures on which instruments were installed. It can be seen that the terrain is very flat, and except for some scattered trees, it is very open. From a bucket truck, the wind farm can be seen just beyond the trees in the photograph (see figure inset). Details of all the test sites are presented in Appendix C.

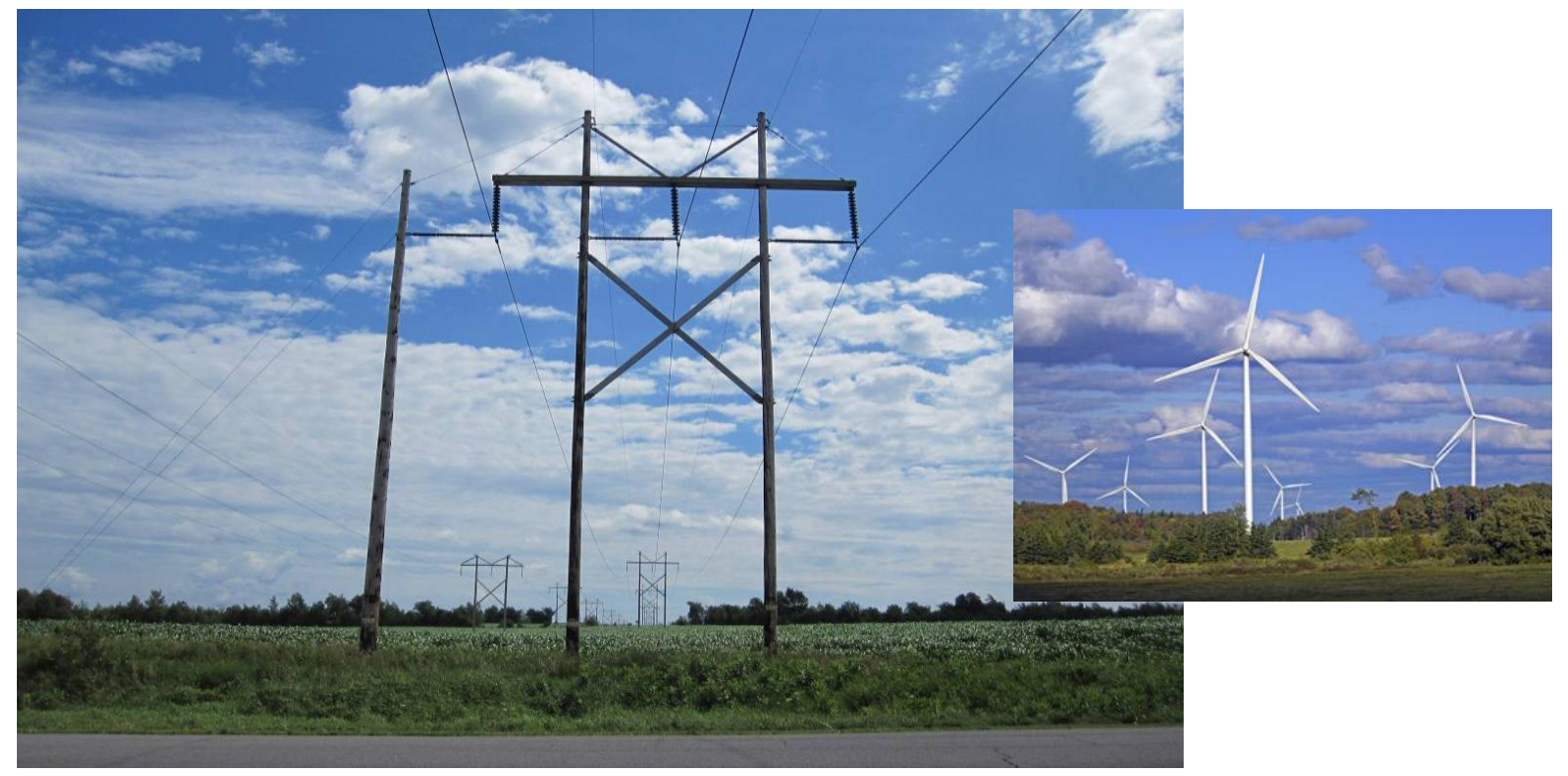

Figure 4-1 Figure 4-1 Photograph of one of the test sites (nearby wind farm in inset) 
Over the course of a several week period in the fall of 2010, the instruments described in Chapter 3 were installed at each of the three sites. A NYPA line crew performed the installations under the direction of EPRI and equipment vendor representatives. Several individuals from DOE, NYPA, and EPRI observed the activities. Figure 4-2 shows a labeled photograph of the structure from Figure 4-1 fully instrumented. Details of each of the installations are presented in Appendix C.

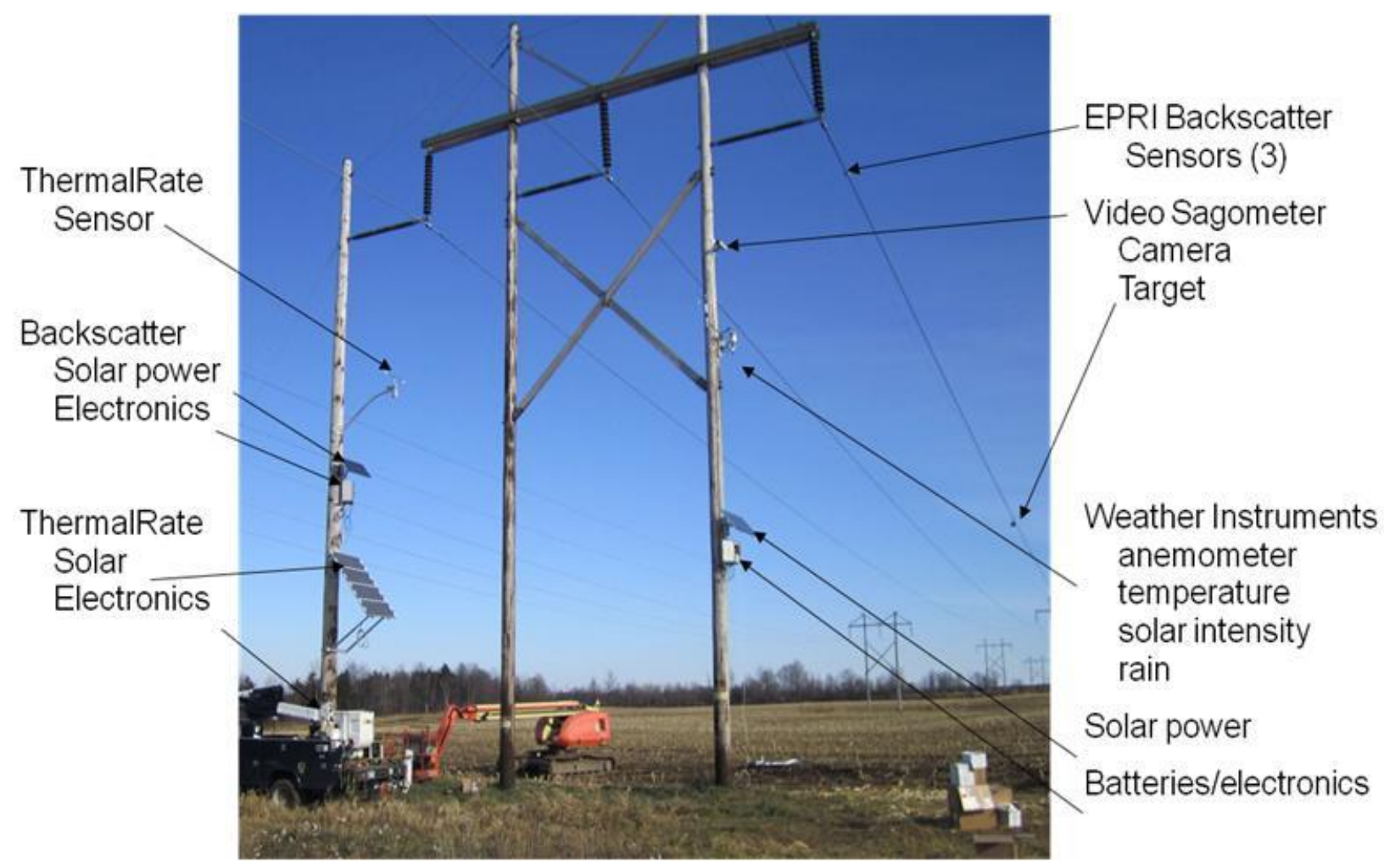

Figure 4-2 Labeled photograph of the structure from Figure 4-1 


\section{5 \\ FIELD DATA AND COMPUTED RESULTS}

\section{Introduction}

There was a considerable amount of data collected and processed during the project. Organizing the data in such a way that it could be assimilated and presented with conclusions drawn was a challenge; Appendices K and L present examples of all this information in an organized manner. Similar data for the entire project was issued only in electronic form because of its large extent.

The data can be grouped into four broad categories:

1. Raw Field Data

2. Calculated Data

3. Statistical Distributions of Data

4. Instrument Performance Statistics

The raw field data are those directly measured in the field by the deployed instruments (such as wind speed from an anemometer). The calculated data are those that have to be calculated using the raw field data as input (such as line rating). In some cases, data that is considered "raw" from some instruments may be considered as "calculated" from other instruments (for instance, measured conductor temperature is "raw" data from the EPRI Sensors, but is "calculated" data from a weather station).

Appendix K shows the raw field data and calculated data in the form of chronological monthly plots, and statistical distributions of the most relevant data. Also, Appendix L shows statistical pie charts of the individual instruments' performances on a monthly basis. As mentioned, the totality of project data was made available through electronic files.

\section{Data Types from the Four Instrument Packages}

As discussed in Chapters 2 and 3, there were four different instrument packages installed at each of three sites. Table 5-1 lists the most relevant raw field data and calculated data derived from each of the four instrument packages. In addition to the four instrument packages, weather data was obtained from a NOAA website, and some comparisons were made. 
Table 5-1 The main data derived from each of the four instrument types

\begin{tabular}{|l|l|l|}
\hline \multicolumn{1}{|c|}{ Instrument Type } & \multicolumn{1}{|c|}{ Raw Field data } & \multicolumn{1}{c|}{ Calculated Data } \\
\hline Weather Station & $\begin{array}{l}\text { Ambient Temperature } \\
\text { Solar Intensity } \\
\text { Wind Speed } \\
\text { Wind Direction } \\
\text { Rain Rate }\end{array}$ & $\begin{array}{l}\text { Effective Perpendicular Wind } \\
\text { Speed } \\
\text { Conductor temperature } \\
\text { Line ratings }\end{array}$ \\
\hline Video Sagometer & Sag & $\begin{array}{l}\text { Effective Perpendicular Wind } \\
\text { Speed } \\
\text { Conductor temperature } \\
\text { Line ratings }\end{array}$ \\
\hline ThermalRate & $\begin{array}{l}\text { Effective Perpendicular Wind ratings } \\
\text { Speed }\end{array}$ & $\begin{array}{l}\text { Line } \\
\text { EPRI Sensors }\end{array}$ \\
\hline $\begin{array}{l}\text { Conductor temperature } \\
\text { Current }\end{array}$ & $\begin{array}{l}\text { Speed } \\
\text { Line ratings }\end{array}$ \\
\hline
\end{tabular}

\section{Example Data}

As discussed above, there was a considerable amount of data collected and processed during the project. Appendices $\mathrm{K}$ and L present comprehensive examples of that data, and all the project data is available via electronic files. Below are some examples of the presented data.

Figure 5-1 shows a plot of the ambient temperature and solar intensity for the month of April 2012 as measured by the weather station at one of the sites. The daily cycles are very evident, and as expected, the solar intensity is greatest at noon, with the highest ambient temperatures occurring shortly afterwards. During a few periods of the month, reduced solar intensity coincided with reduced ambient temperature as would be expected (such as April 22 and 27).

Figure 5-2 shows a polar plot of wind speed and direction (called a wind rose) for one month as measured by an anemometer of one of the weather stations. It can be seen that the prevalent winds were out of the west. Comparisons of such plots with those made from National Oceanic and Atmospheric Administration (NOAA) data demonstrated that it may be very possible to utilize NOAA data for rating purposes for these particular transmission lines. 


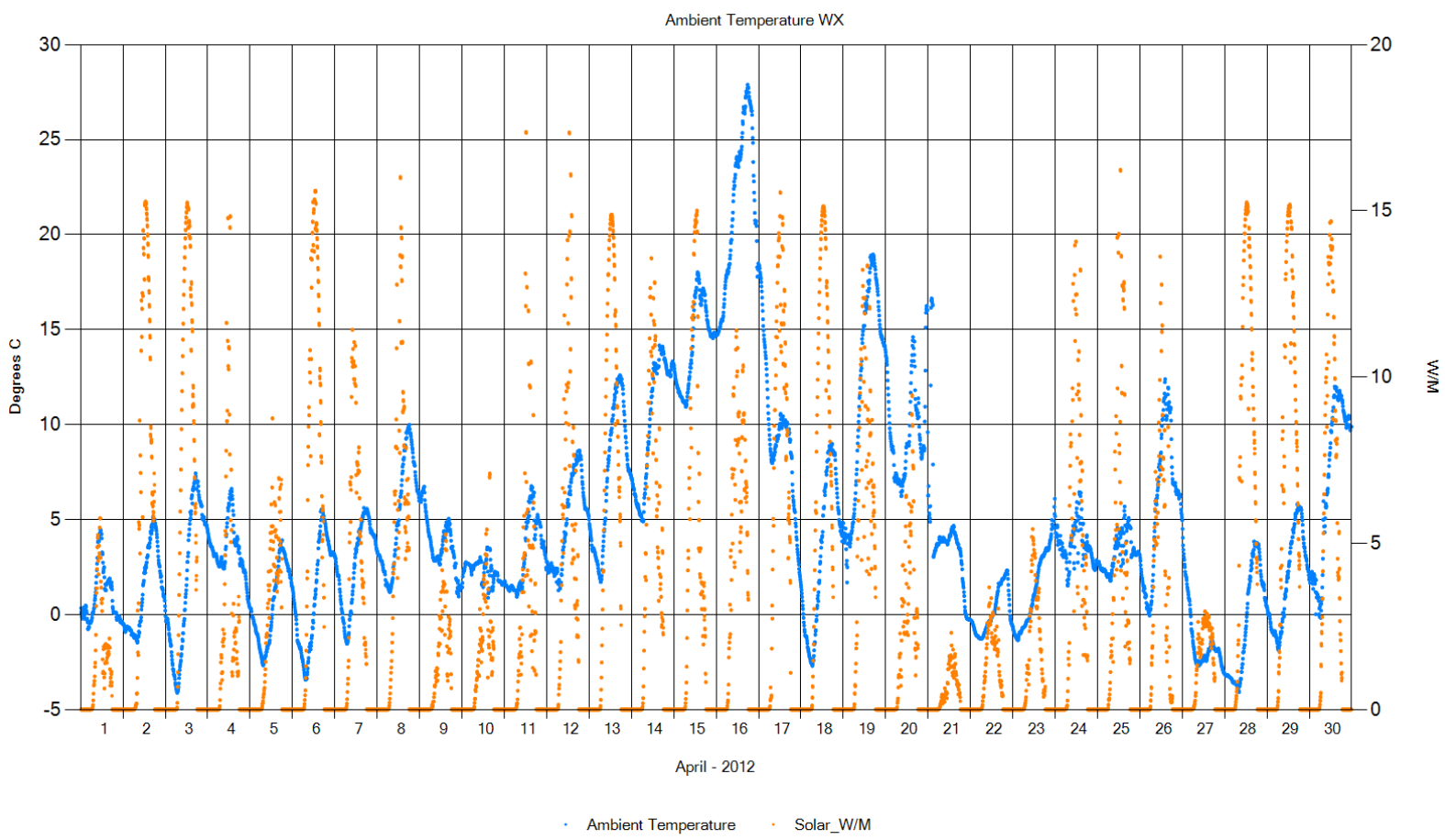

Figure 5-1 Plot of the ambient temperature and solar intensity for the month of April 2012 as measured by a weather station
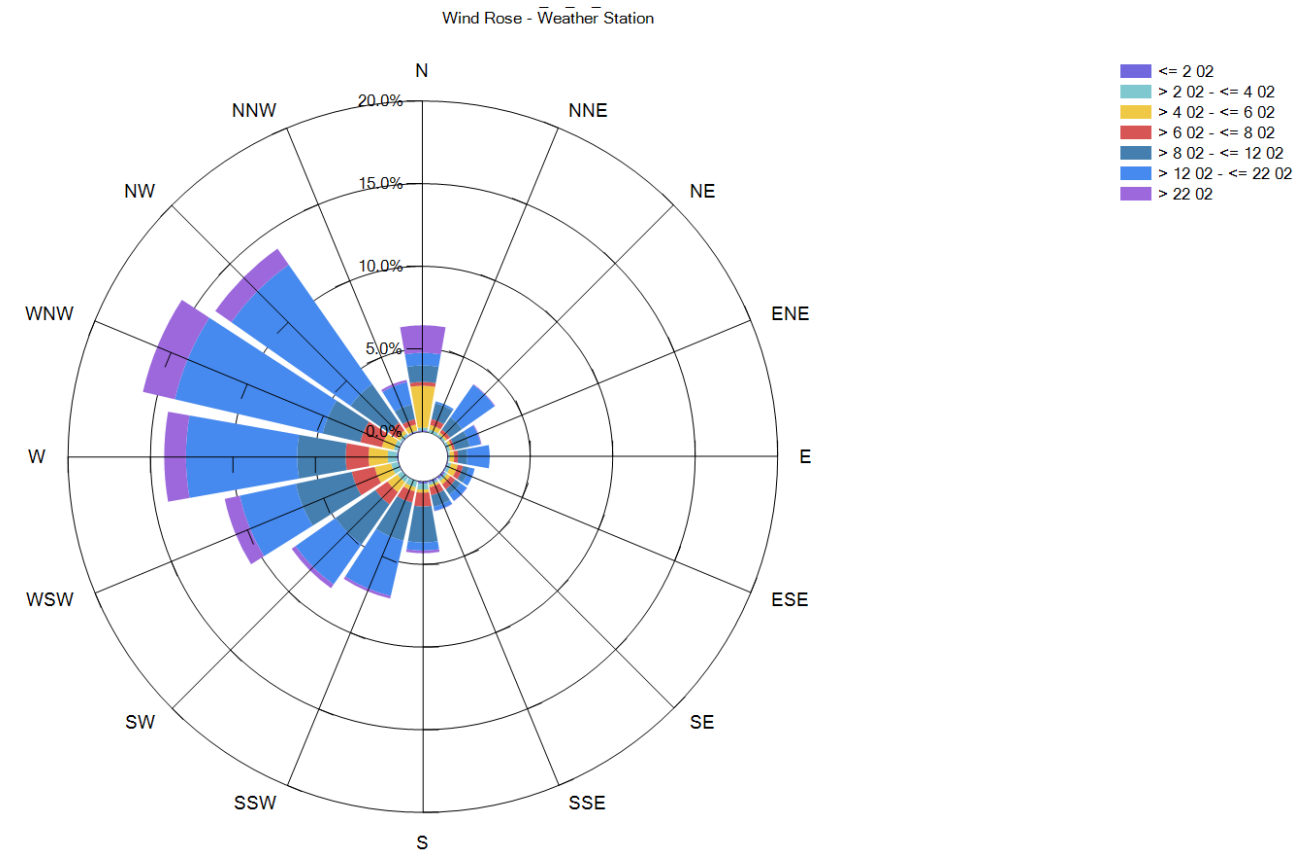

Figure 5-2 Polar plot of wind speed and direction (called a wind rose) for one month as measured by an anemometer

Figure 5-3 shows a monthly plot of the conductor ground clearance (clearance is directly related to sag) as measured by a Video Sagometer, and the "correlation" of the measurements. The daily cyclic nature of the sag (corresponding to conductor temperature) can be seen across the month. 
The correlation of sag measurements is a number between 0 and 1 which indicates the reliability with which the sagometer believes that it has correctly identified the conductor in its camera view. A correlation above 0.5 indicates that the sagometer has correctly identified the conductor, and the sag measurement is believed to be accurate. It can be seen in Figure 5-3 that on occasion the correlation dropped below 0.5 , and during those times it appears that the sag measurement was in error. Such data were used to develop statistics of the sagometer's performance (as shown in Appendix L).

Figure 5-4 shows a continuous plot of ratings for one line during the month of April 2012, as derived from ThermalRate measurements. The normal rating, long term emergency (LTE), short term emergency (STE), and extremely short term emergency (ESTE) are shown (see Appendix B for detailed explanations of ratings). A daily cycle of ratings can be seen, with higher ratings occurring mid-day corresponding to higher winds (this cycle is very typical of ratings generally). Appendix K shows similar plots for all the methods with which ratings were calculated.

Figure 5-5 shows a statistical distribution of rating of one line for one month. The L1, L5, and L50 levels are indicated. L1 (Level 1\%) was that rating that was exceeded 99\% of the time, L5 (Level 5\%) was that rating exceeded 95\% of the time, and L50 (Level 50\%) was that level exceeded $50 \%$ of the time (the median). The median magnitude of normal rating for this line was 2108 amps for the month. The normal rating was over 1355 amps for over $99 \%$ of the month. The present static rating used by NYPA for this line is 1089 amps (see Appendix F for details of NYPA ratings).

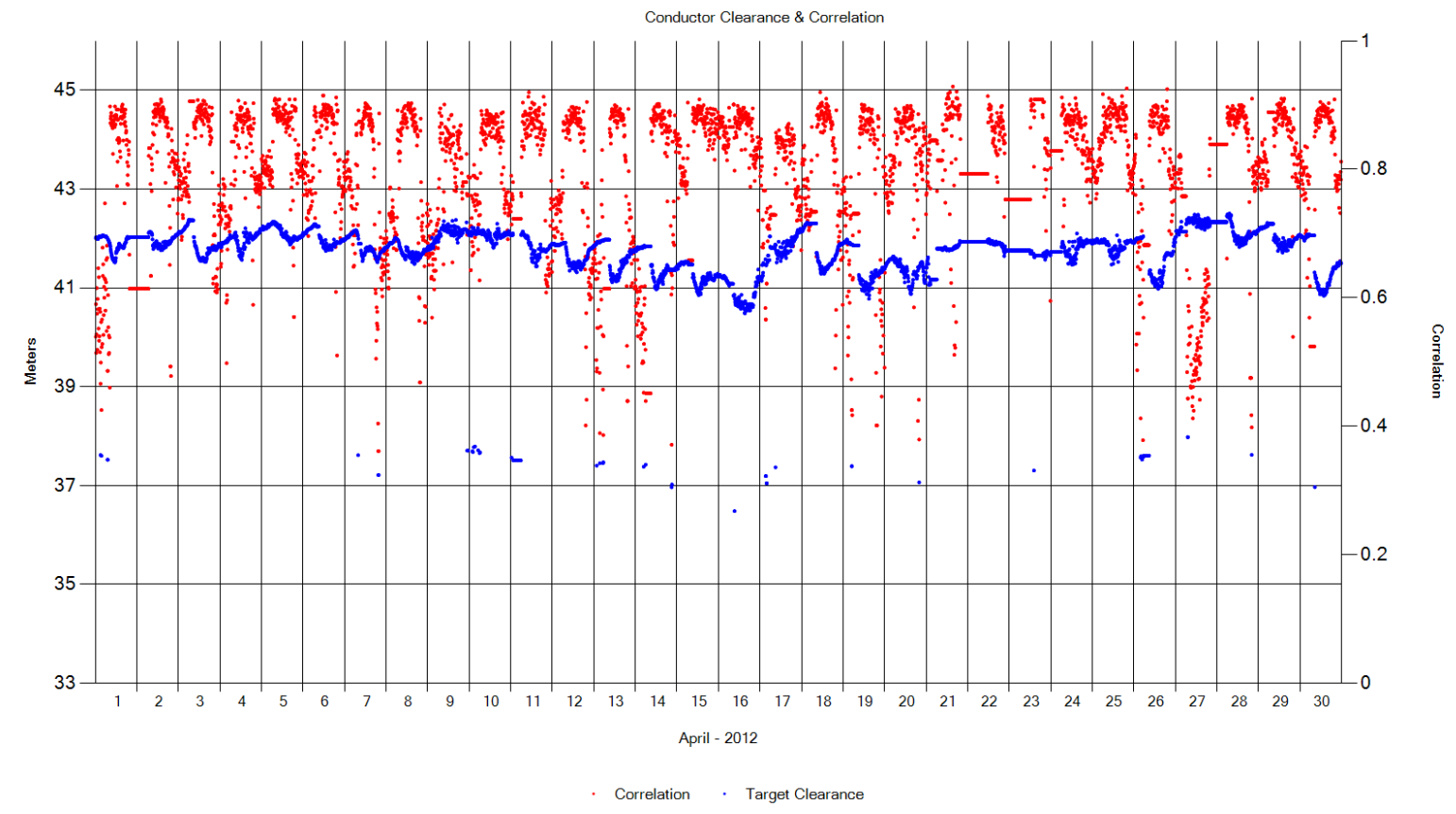

Figure 5-3 Monthly plot of the conductor ground clearance (directly related to sag) as measured by a Video Sagometer, and the "correlation" factor 


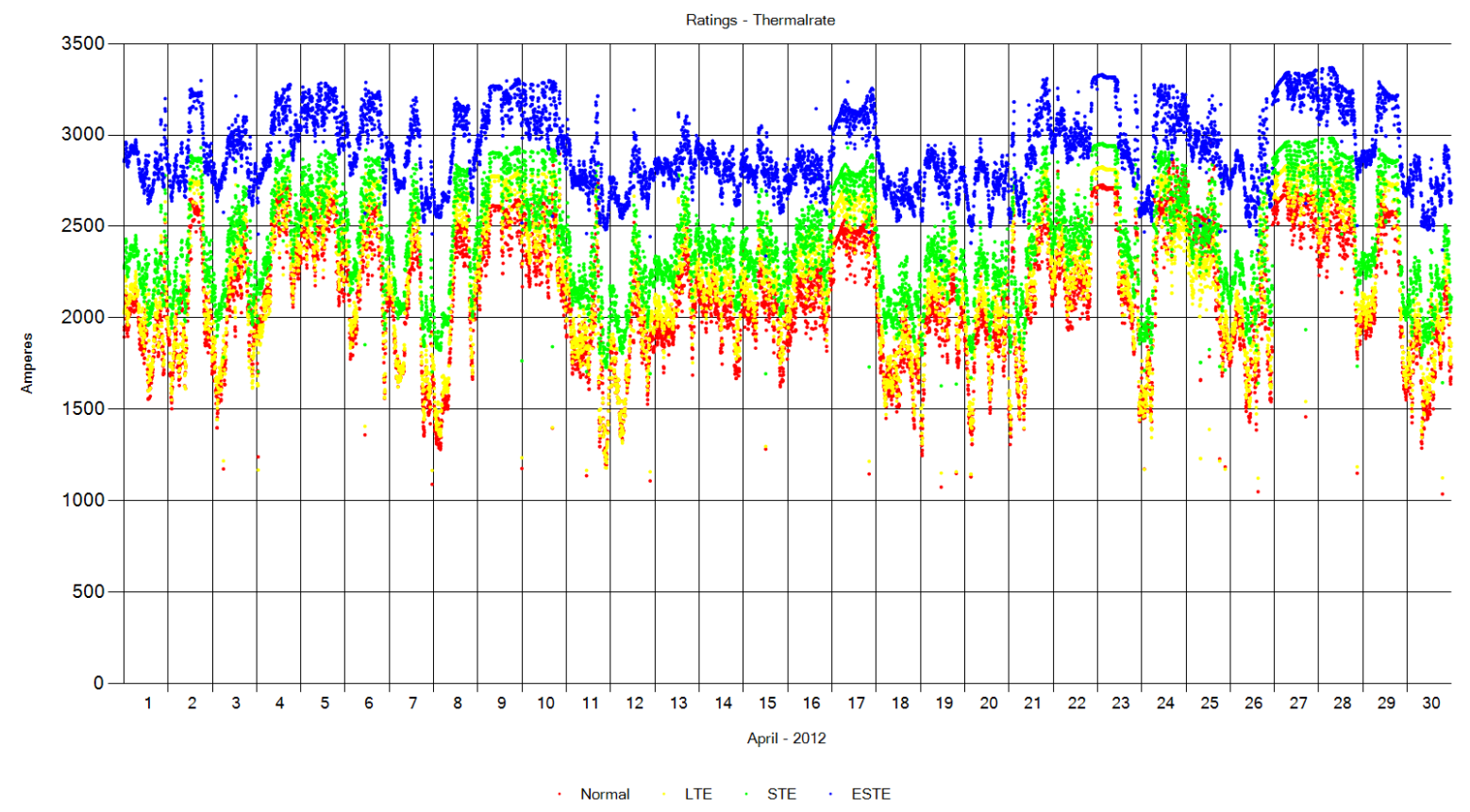

Figure 5-4 Continuous plot of ratings for one line during the month of April 2012, as derived from ThermalRate measurements

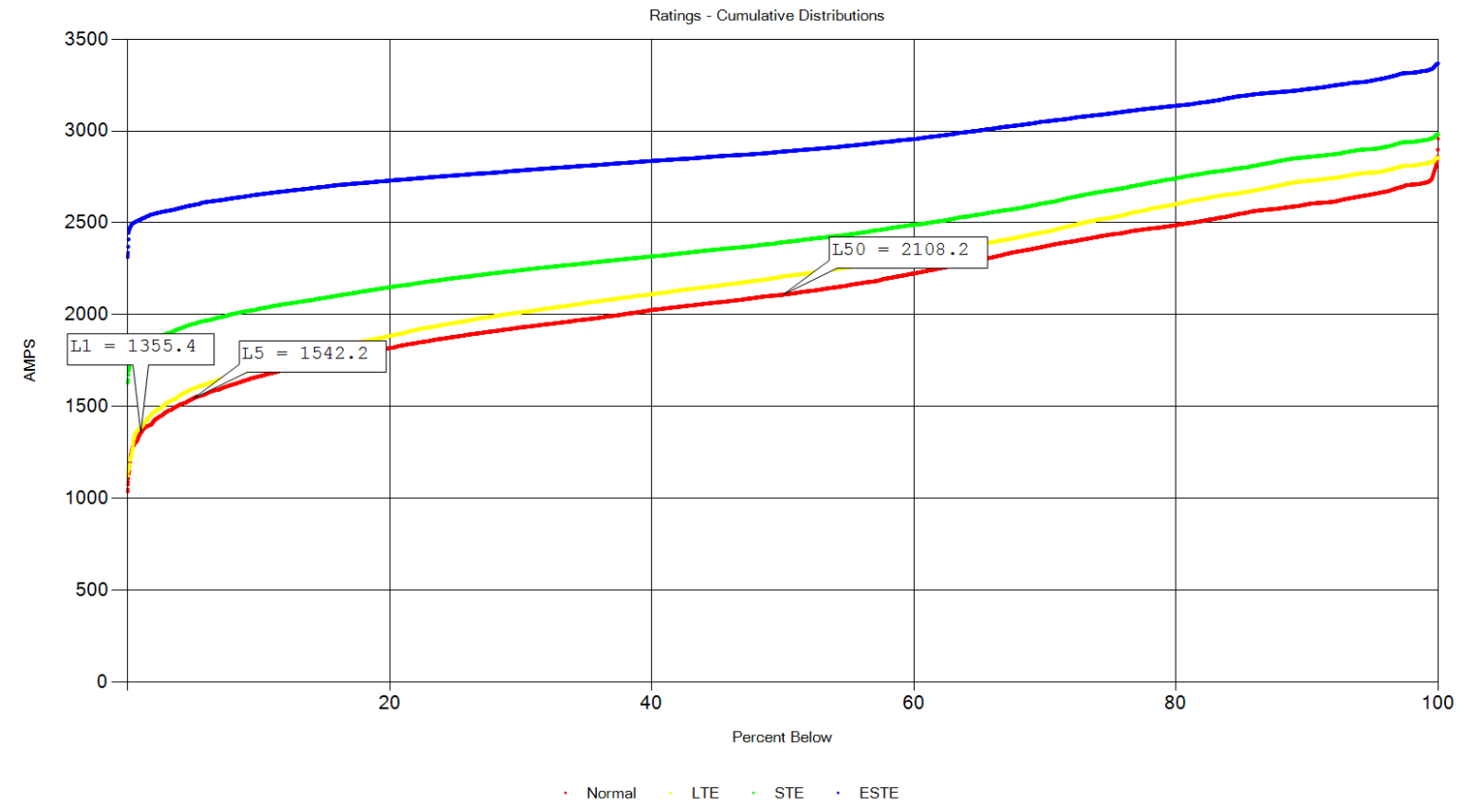

Figure 5-5 Statistical distribution of rating for one line for one month 



\section{KEY RESULTS AND CHALLENGES}

\section{Introduction}

Over the course of this project, many valuable observations were made and results established and confirmed. Most of the details and subtleties are presented in hundreds of pages of appendices. This chapter provides an overview of the key results, and references are made to the appendices where more detail can be found.

\section{Equipment Installation Assessment}

One of the goals of the project was to perform an assessment of the equipment installation process. A significant amount of hardware was installed during the project, including the installation of four sets of instruments, each with multiple components, at three different sites. Line crews do not normally have experience with these specialized instruments, and training had to be provided prior to the field installations. An assessment of the installation process and feasibility was documented based on observations and on discussions with line crew members. The details of the installation process and assessment are provided in Appendix C.

Prior to the field installations on energized NYPA transmission lines, comprehensive training was provided to the NYPA line crew at the EPRI laboratory in Lenox, Massachusetts. At Lenox, the NYPA line crew went through the exercise of installing a complete set of all four instruments on a full-scale test line. The structures of the Lenox test line were similar to those at NYPA, and although not energized, the line crew performed the installation exercise as though the line was energized. Representatives from NYPA, EPRI, and the equipment vendors directed and observed the simulated installations. In addition to gaining an assessment of the installation process, the installation process was itself improved and streamlined and a number of tricks-ofthe-trade were identified.

This exercise served as practice and was a beneficial learning experience for the project team. It provided an understanding of which instruments to install first and what type of spacing worked best. For example, mounting the ThermalRate electronics box under its solar panel reduced the overall footprint for the instruments on the pole and at the same time shielded the electronics box from rain and sunlight. A photo of the Lenox installation can be seen in Figure C-38 of Appendix C.

Following the Lenox simulated installation, the line crew installed full sets of four instruments at the three NYPA test sites. The line crew got better with each subsequent installation, and the process went smoother and faster. Figure 6-1 shows a photo of the NYPA line crew performing equipment installations on one of the lines in upstate New York. 
Prior to the actual field installations, the project team also received training at NYPA's St. Lawrence facility in Massena, NY. NERC requirements were reviewed and CPP-1 (Clearance and Protection Procedure) Operations training was provided for safety requirements.

Some details were learned about improved procedures and tools, however, the overall assessment by the NYPA line crew, and the entire project team, proved that such instruments can readily and successfully be installed by a well-versed line crew. One full day was required to install the four sets of instruments at one site of the project, and it is estimated that it would take a half day to perform an installation of one set of instruments for future real-time rating needs.

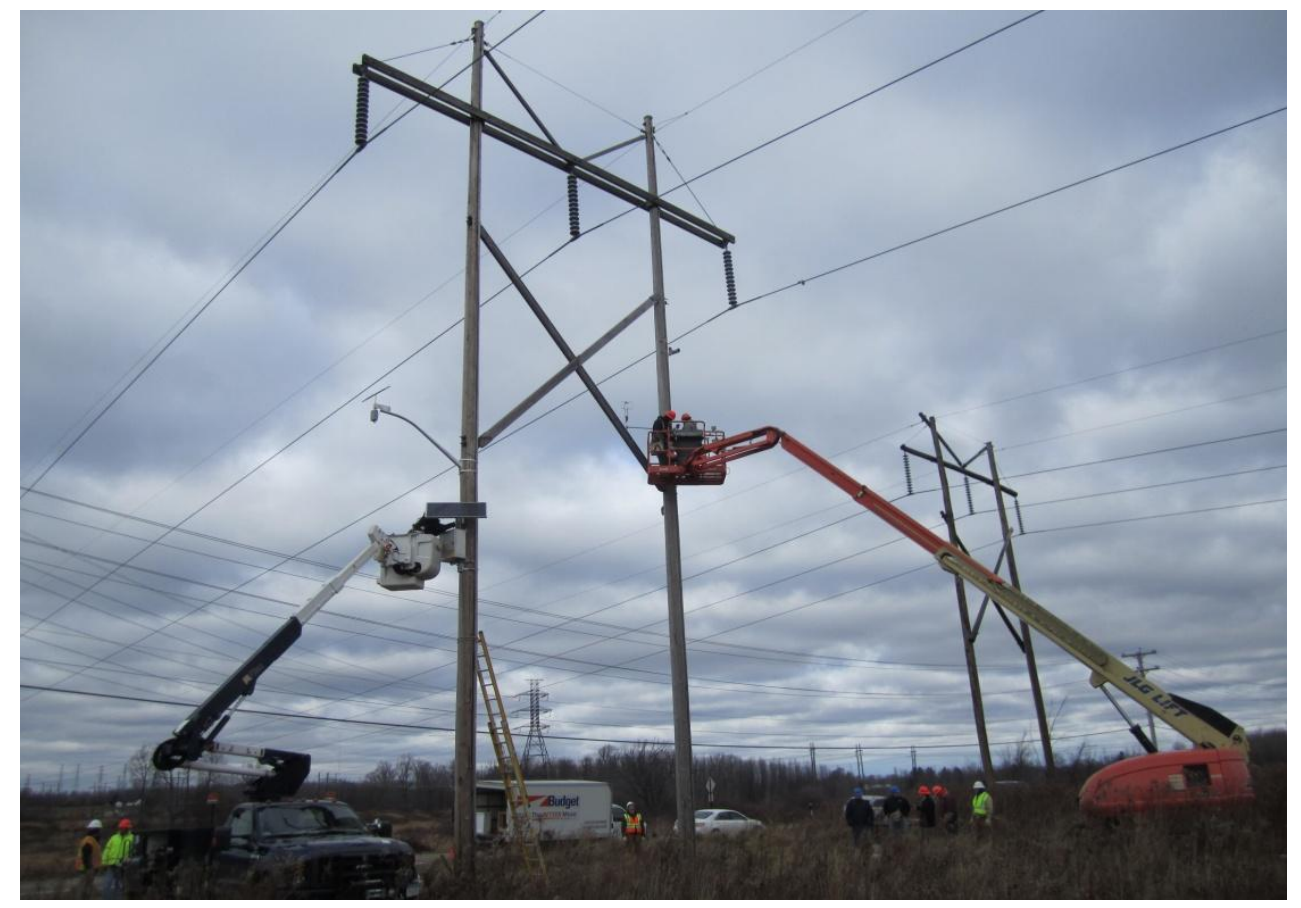

Figure 6-1 Installation of equipment on a NYPA line

One of the goals of the project was to observe any correlation between the potential power output of nearby wind farms and the ratings of the transmission lines. The single most influential weather factor in both rating calculations and wind farm output is wind speed.

A strong positive correlation between ratings and potential wind farm output seems like a logical assumption. However, wind farms are purposely situated in high open terrains where wind speeds are maximized, while transmission lines are built irrespective of local wind speeds, and frequently pass through low lying shielded terrain. Some reports have questioned the correlation between the output of wind farms and line rating.

However, a strong positive correlation was observed at the NYPA sites of this project. In this region, both the wind farms and transmission lines are in flat open terrain, and the wind speeds encountered by the wind farms are very closely correlated with the wind speeds at the lines. The details of this topic are provided in Appendix I.

Figure 6-2 shows an example of the correlation. This figure shows a plot of the ratings for one of the lines versus wind farm output (in terms of amps). The individual data points are widely 
scattered, however, the correlation is evident. Statistically, there is almost a direct proportionality. This supports the view that NYPA's utilization of the wind farm power could be increased if a real-time rating scheme was implemented.

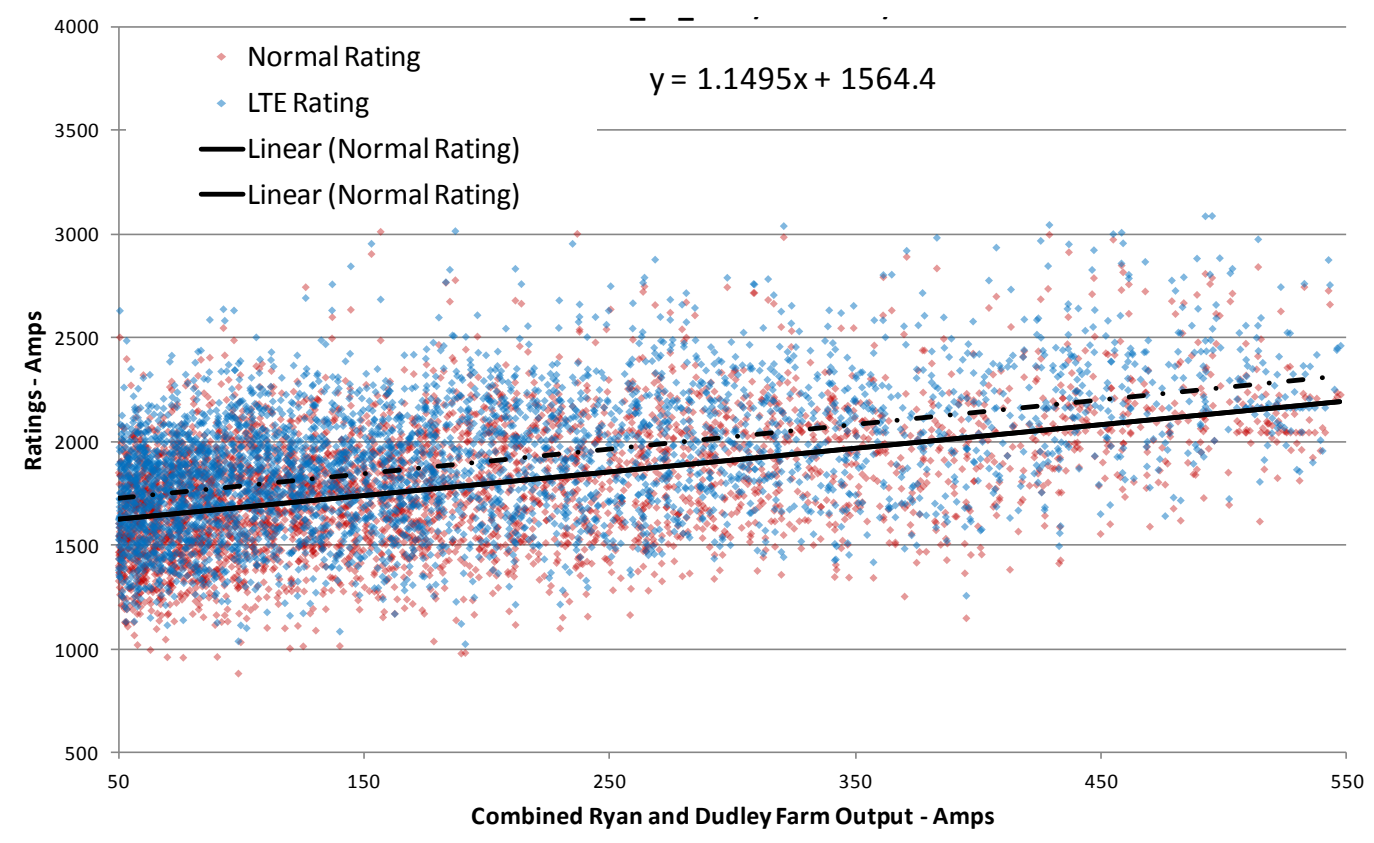

Figure 6-2 Line rating versus wind farm output

\section{Transmission Corridor - NOAA Weather Correlation}

Ultimately, for any given transmission line, ratings depend on the ambient weather conditions. In the DOE-NYPA project, weather was measured from weather stations mounted close to the lines, or inferred by other means. An attractive concept that has been explored by various researchers is the use of weather data provided by online weather services, such as NOAA. The general conclusion regarding this concept is that it is probably not viable. Weather data from such services are generally obtained by measurements at airports, and airports are always located in flat, wide open terrain, and transmission lines, generally, are not. Such weather data may be good for ambient temperature or solar intensity measurements, since these do not vary much over distance or terrain sheltering, but the wind measurements may be very inaccurate for transmission line ratings.

If the concept did work out, it would be very attractive for transmission line rating determinations because it would require no added instrumentation that would need to be purchased, installed, and maintained, and the data would be readily available, and with no communication issues. The idea was considered that, in the region of the project's NYPA lines, being in an area consistently flat and open, perhaps online NOAA data would compare favorably with that data measured in the transmission corridors. It was found that the correlations were, in fact, fairly good. The details of the analysis exploring this concept are presented in Appendix G.

Figure 6-3 shows statistical distributions of wind speeds measured from an anemometer in one of the project's transmission line corridors, and as provided by an online NOAA weather service. 
The weather service data appears "choppy" because of the technique they use to bin their data; however, the correlation with the wind speed measured in the line's corridor is quite reasonable. In fact, at the lower wind speeds, which are generally more important for transmission line ratings, the online data appears to be more conservative (i.e., lower wind speeds), which contradicts the theory that wind speeds are generally higher at NOAA weather sites.

Figure 6-4 shows polar plots (called wind roses) of wind data (magnitude and direction) as obtained over an extended period of time from an anemometer in one of the NYPA transmission corridors and from the NOAA online weather service. A reasonably good correlation is again observed for both wind speed and wind direction. It would appear that the concept of using online weather data for transmission ratings, in some cases, may have merit and should be considered further.

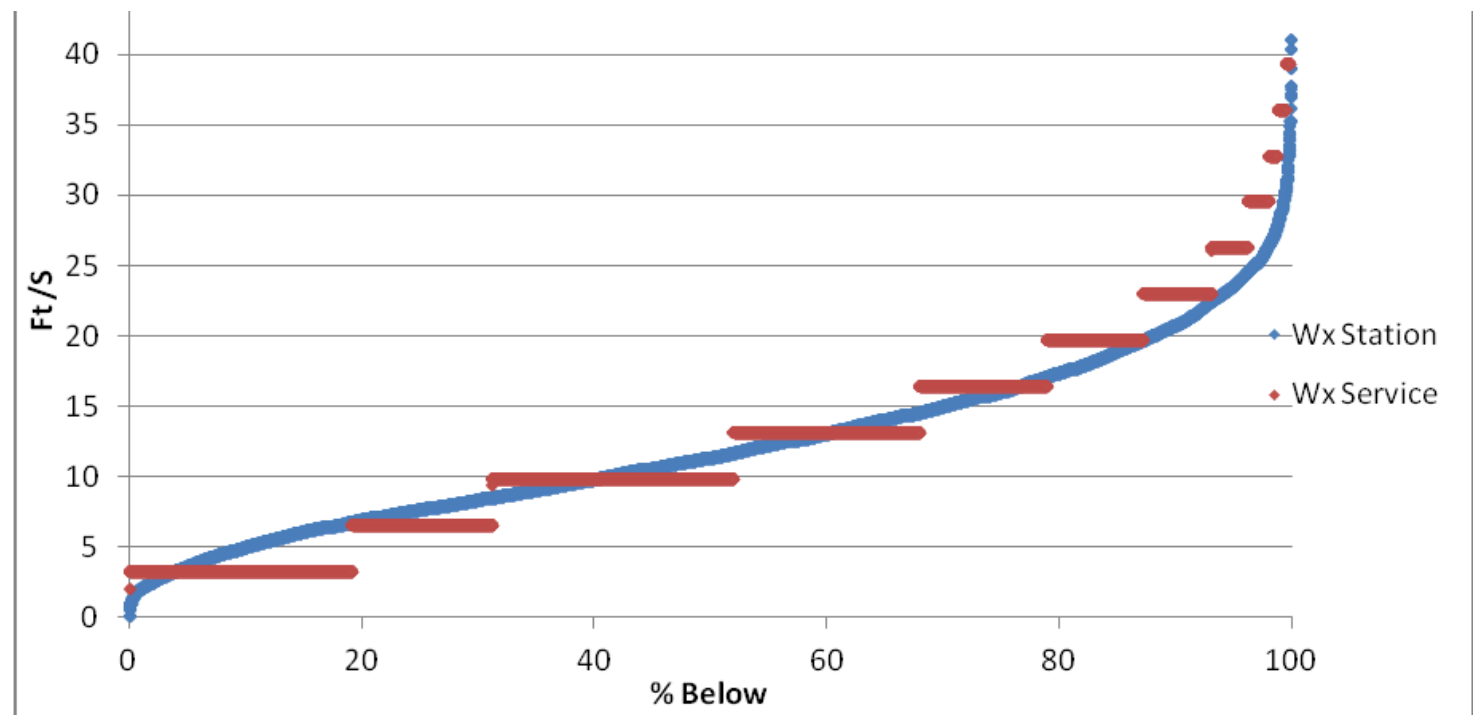

Figure 6-3 Statistical distributions of wind speeds from an anemometer in a transmission line corridor, and as provided by an online weather service 

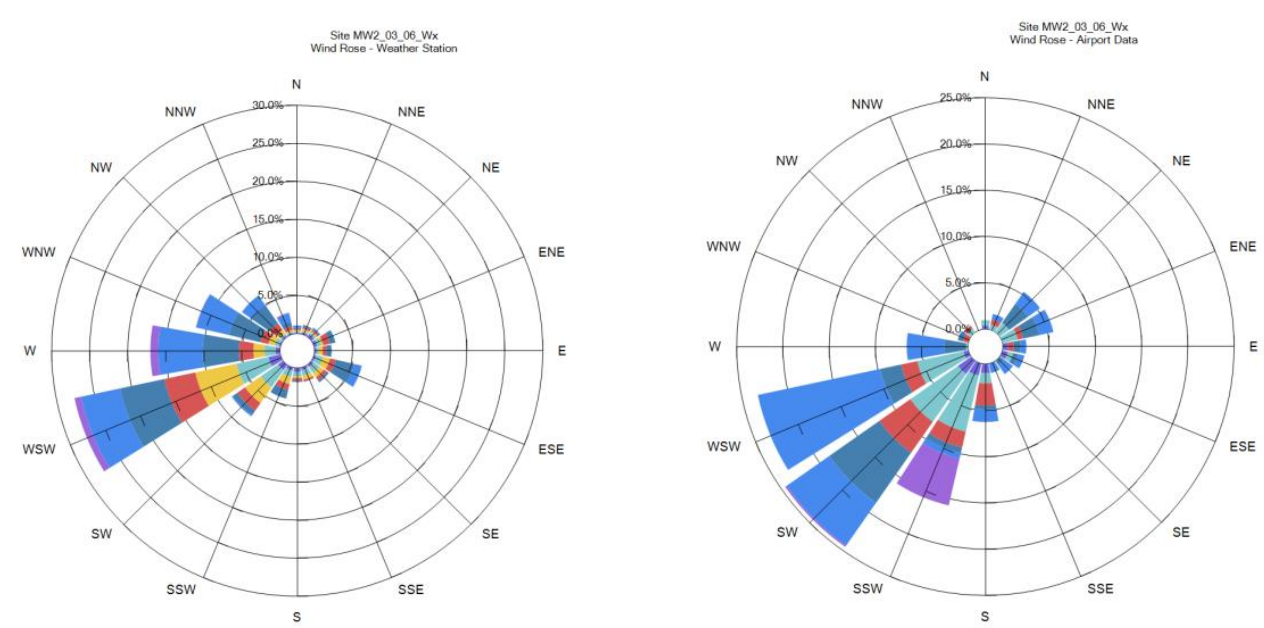

Figure 6-4 Polar plots of wind speed and direction as obtained by an anemometer in the transmission corridor and as obtained from an online NOAA wetaher service

\section{Advantages of Dynamic Ratings}

The advantage of using dynamic ratings is that it can provide a better knowledge of the actual line rating than that provided by static ratings. And in most cases most of the time, dynamic ratings are significantly greater than static ratings. Another very valuable aspect of dynamic rating is the ability to take advantage of the thermal inertia of the conductors to ride through emergency situations when the line would otherwise be assumed to be heading into an overload situation requiring extreme operator intervention (discussed in Chapter 2). A disadvantage of dynamic rating is that it is a varying quantity, and can be a challenge for operators to deal with.

Much of the data and analyses of the project focused on determining dynamic ratings by various means for the NYPA lines, and comparing the results. Much detail is provided in Appendices F and $\mathrm{H}$, and an example is discussed below.

Figure 6-5 shows a continuous plot of the dynamic ratings for one of the NYPA lines, as determined by weather measurements, for the entire winter of $2011-2012$. The Normal, Long Term Emergency (LTE), and Short Term Emergency (STE) ratings are shown, along with the line's load. The Normal static rating for this line during this period was 1331 amps. Sometimes, the dynamic rating actually fell below the static rating (another good piece of information that dynamic rating supplies), but most of the time, by far, the dynamic rating exceeded the static rating. Also, during emergency situations, the dynamic ratings were much higher. The varying nature of dynamic rating is also evident in the figure.

Figure 6-6 shows a statistical distribution of the data from Figure 6-5, and Table 6-1 provides some of the pertinent information in tabular form. It can be seen in Figure 6-6 that the Normal rating for this period ranges from just under 1000 amps up to just over 2500 amps. The LTE was higher by a small amount, and the STE was significantly higher, ranging from about 1500 amps to 3000 amps. Table 6-1 provides the static ratings and the corresponding L-levels of dynamic ratings (see Chapter 5 for definitions of the L-levels). 
From Table 6-1, the L1, L2, and L5 dynamic ratings were all less than the corresponding static ratings, yet for most of the time, by far, the dynamic ratings were higher than the static ratings. For instance, the Normal static rating is 1331 amps, but the median (L50) Normal dynamic rating was 1652 amps - a $24 \%$ increase above the static rating. This extra power capacity is the advantage of using dynamic ratings, and in an increasing number of cases, this increased power capacity advantage outweighs the disadvantages.

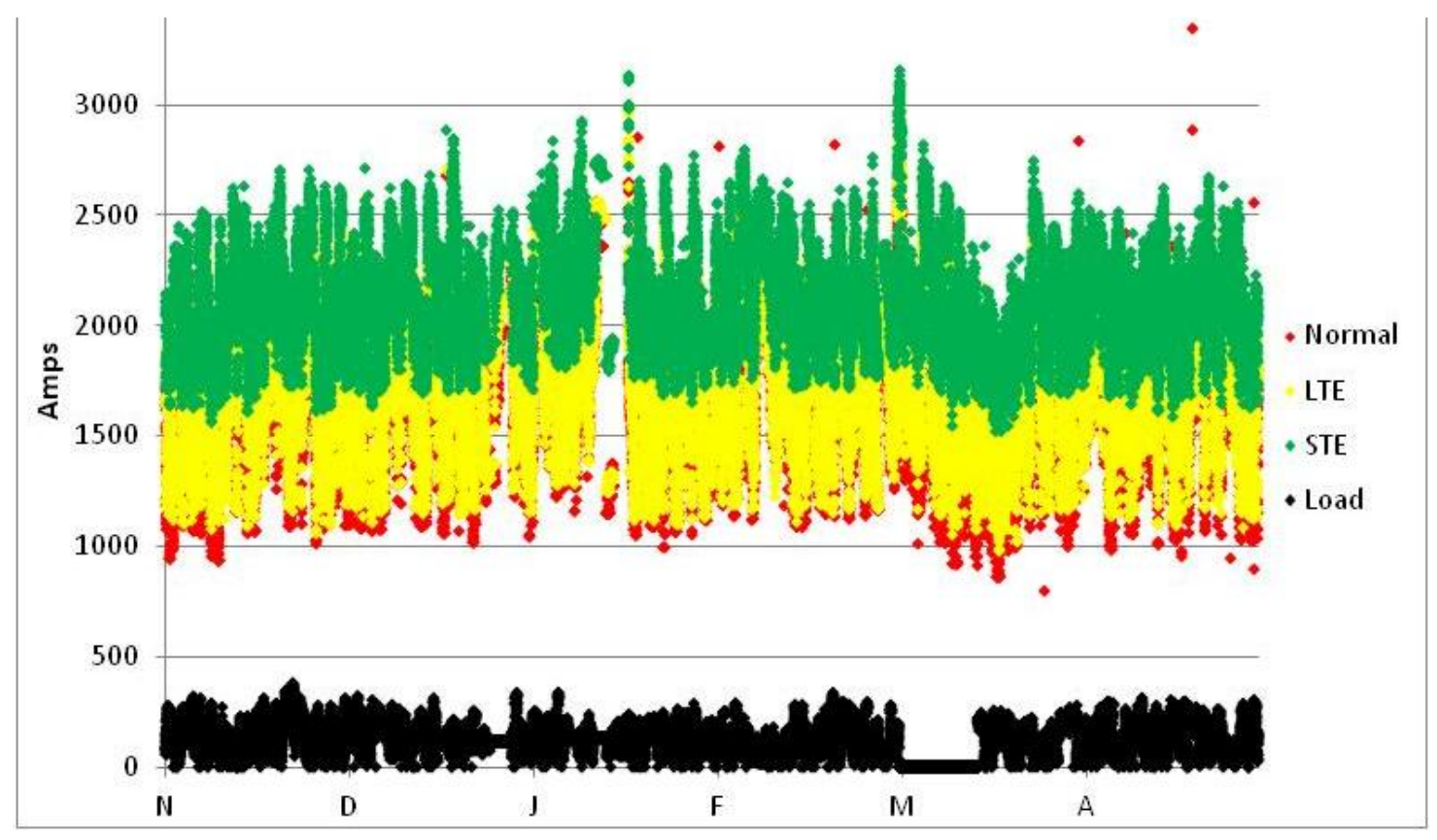

Figure 6-5 Continuous plot of the dynamic ratings for one of the NYPA lines, as determined by weather measurements, for the entire winter of 2011- 2012

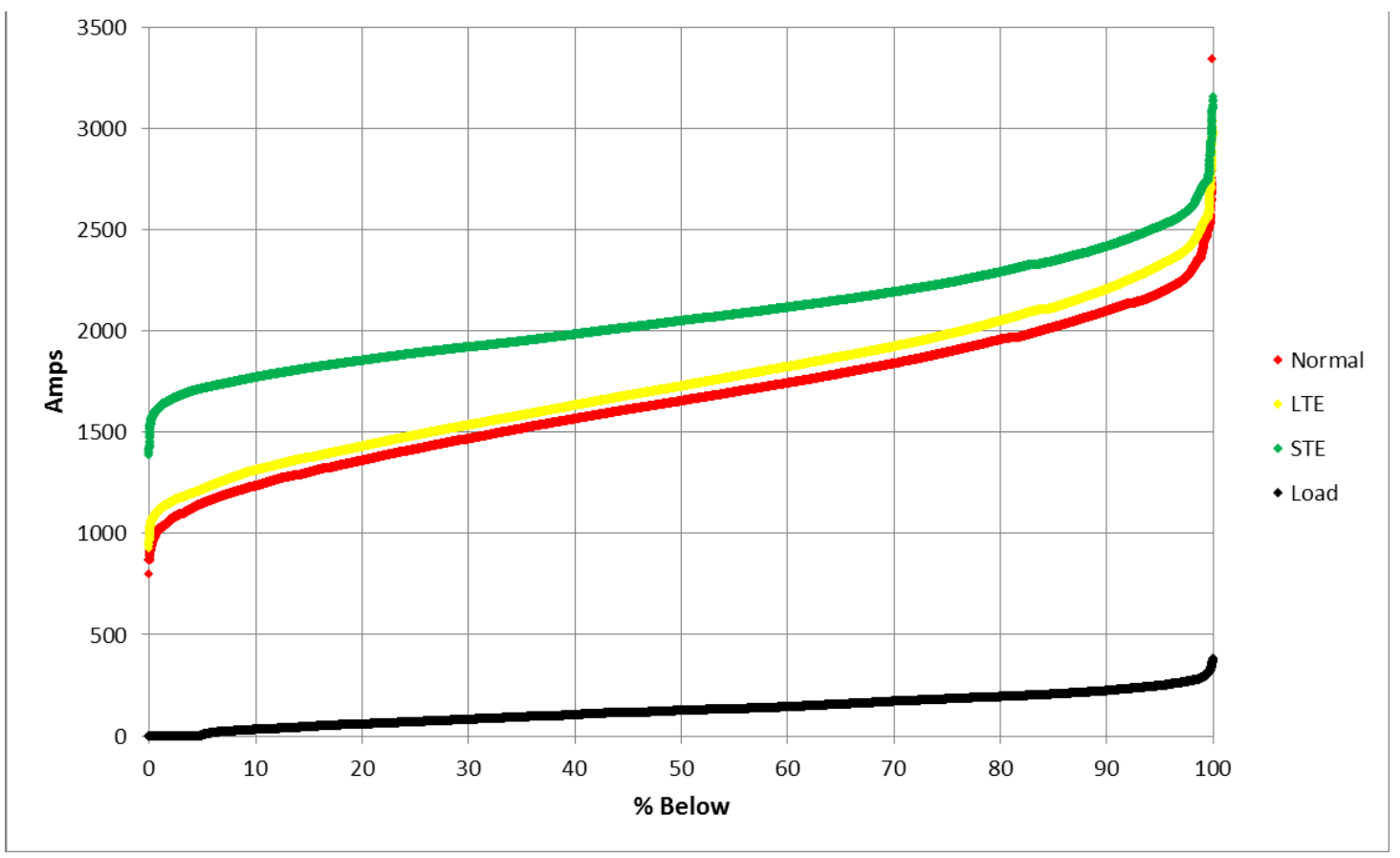

Figure 6-6 Statistical distribution of the data from Figure 6-5 
Table 6-1 L-values for Normal, LTE, and STE ratings

\begin{tabular}{|c|l|l|l|l|l|l|}
\hline & \multicolumn{1}{|c|}{ Static } & \multicolumn{1}{|c|}{ L1 } & \multicolumn{1}{|c|}{ L2 } & \multicolumn{1}{|c|}{ L5 } & \multicolumn{1}{|c|}{ L50 } \\
\hline Normal & 1331 & 1023 & 1066 & 1147 & 1652 & 2185 \\
\hline LTE & 1460 & 1116 & 1152 & 1215 & 1726 & 2321 \\
\hline STE & 1593 & 1622 & 1657 & 1714 & 2048 & 2999 \\
\hline Load & & 0 & 0 & 10 & 120 & 250 \\
\hline
\end{tabular}

\section{Line Loading and the State-Change Equation}

To perform rating calculations, the average temperature of the conductor must be determined. In some cases, such as with weather measurements, the temperature is calculated using the IEEE 738 method (see Appendix B for more details about IEEE 738). However, a weakness of this approach is that the calculations are based on the weather measurements at a single point(s), i.e., the site of the weather station(s), and may not represent the true average weather along the line. For instruments such as the EPRI Sensors, the conductor temperature is measured directly, but only at discreet points along the line.

Sag or tension monitoring instruments determine the average conductor temperature along entire line sections, however, it requires that the mathematical relationship between sag (or tension) and average conductor temperature, $\mathrm{Tc}(\mathrm{sag})$, be determined in the form of a "state-change equation". This requires an accurate as-built physical model of the line, and some up-front data analysis. Figure 6-7 shows an example of a state-change equation (conductor temperature as a function of conductor ground clearance as measured by a sagometer)

However, the state-change equation can fluctuate due to line work, structure movement, varying conductor characteristics, environmental factors, or other reasons. It is important that the statechange equation be monitored and refined as needed. If a state-change equation cannot be accurately determined and maintained, ratings calculated from sag or tension measurements will not be correct. Line rating errors can be very large even if such instrument errors are small. During the course of this project, the state-change equation varied so much that sag or tension measurements would not be of use for rating calculations. 


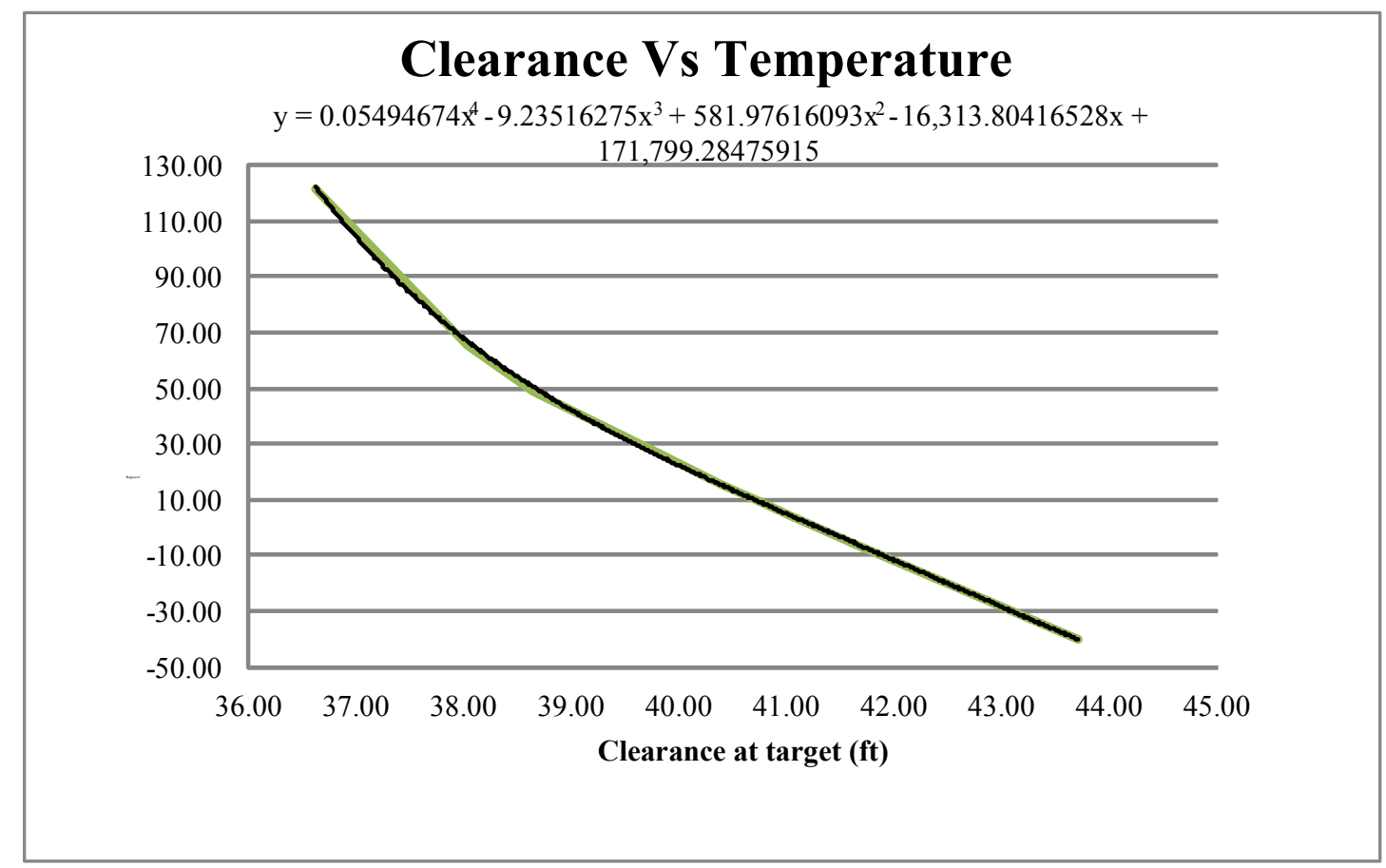

Figure 6-7 Example of a state-change equation for a NYPA line

For line ratings based on sag, tension, or conductor temperature measurements, the measured variables are used to determine an effective perpendicular wind speed (that wind speed when blowing perpendicular to the line gives the same convective cooling as the actual average wind velocity). One of the key contributors to line rating calculation error involves the impact of low current densities on the conversion of sag, tension, or conductor temperature measurements into accurate estimates of effective perpendicular wind speed (refer to Appendices B and D for details).

Wind cooling is the most important environmental variable in determining dynamic line rating, and it is the most difficult one to ascertain. Unlike other variables such as ambient temperature and solar intensity, wind can exhibit large variations in both speed and direction along a line. Determining the values to use when calculating ratings from moment-to-moment can be very difficult and it is the main challenge for any real-time rating scenario.

The heat generated in a conductor depends primarily on the square of the current that passes through it. Thus the heat generated with the line carrying $30 \%$ of its rated load is only $10 \%$ of the heat generated at full rated load, and any conductor temperature rise above ambient temperature - and the resulting change in sag or tension - is quite small. Given normal measurement uncertainties of 1 to $2{ }^{\circ} \mathrm{C}$, the errors inherent in calculating effective perpendicular wind speeds at low current levels can be prohibitively large. As a result, when using sag, tension, or conductor temperature measurements to determine line ratings, the current density in transmission line conductors must be above $0.5 \mathrm{amps} / \mathrm{kcmil}$ (see Appendix D for details). For the two $230 \mathrm{kV}$ lines instrumented in this study, with $795 \mathrm{kcmil}$ Drake conductors, 0.5 amps/kcmil corresponds to 400 amps. The line currents rarely exceeded $350 \mathrm{amps}$, and virtually never reached 400 amps. For the entire duration of the project, sag, tension, or conductor 
temperature measurements could not be used for rating calculations because of the light loading. This is a key observation of the project.

It has generally been observed that the NYPA lines are not unusual in this respect. Because of the need for system reliability, many (if not most), high voltage transmission lines usually operate at current densities less than $0.5 \mathrm{amps} / \mathrm{kcmil}$, and cannot be dynamically rated with devices whose rating accuracy depends on significant heat generation in the conductors.

\section{Instrument Performance and Reliability}

Much work and analysis was performed during the project to assess the quality of instrument performance. Instrument performance was assessed with regards to the percentage of the time that good data was made available in real-time. Data which was "not good" is data that either was there and obviously wrong (out of specified ranges), or missing in real-time (although some data which was missing in real-time, due to problems such as communication breaks, could be retrieved and used later for analysis). Appendices E and L cover these topics in great detail.

Real-time ratings require a stream of input data from sensors in order for real-time ratings to be continuously available. In addition, the DTCR software must be up and running on a server and in communication with the data sources in order to provide real-time rating data. It was found in this project that maintaining these resources with no gaps in coverage on a twenty four hour, seven day a week basis, is challenging. At the present time and state-of-the-science, this challenge makes a compelling case for using multiple redundant means for providing reliable real-time ratings. This statement may be a bit extreme, but that is because real-time rating systems would be expected to be producing good reliable information at all times. Table 6-2 provides a summary of the percent good data available from the various field instruments over the course of the entire project.

Table 6-2

Instrument Data Availability

\begin{tabular}{|c|c|c|}
\hline All Sites; Instrument & \% Available & \% Available Real-time \\
\hline Weather Station Thermometers & 99.4 & 91.6 \\
\hline Weather Station Anemometers & 93.7 & 90.5 \\
\hline Sagometers & 79.3 & 69.4 \\
\hline EPRI Sensors & 71.9 & 70.0 \\
\hline ThermalRate Devices & 85.0 & 75.9 \\
\hline NYPA SCADA RF Link & 75.1 & 69.5 \\
\hline All Logger Communications & NA & 90.3 \\
\hline
\end{tabular}

Progress was made in real-time data reliability over the course of the project, but there is still much room for improvement. Temporary breaks were encountered in all of the links in the chain from the field instruments to the DTCR server. Instrument failures, weather conditions, data logger software issues, cell communication gaps at the site, cell communication gaps at the server, server reliability issues, and DTCR software issues were all contributors to loss of realtime rating information. 
During the entire project, equipment vendors and EPRI were made aware of all hardware and software failures and shortcomings, and many repairs and improvements were made along the way. One of the main benefits that resulted from the DOE-NYPA project was the improvement of all aspects of the real-time rating process.

\section{Challenges of Dynamic Rating}

Many challenges of dynamic rating were known or became known throughout the course of the project. While dynamic rating can offer some truly great benefits to power companies that undertake the challenges, it is not for the "faint of heart".

A detailed analysis needs to be performed to determine if a particular line is a good candidate for benefiting from dynamic ratings, and the proper instruments must be procured, installed, and maintained. Communication links from the field need to be established. At NYPA, some of the instruments needed special analysis and modifications to meet NYPA's security criteria (details are discussed in Appendix J).

One of the biggest challenges recognized during the project is the identification of technical champions of the technology at the utility. The dynamic rating systems tested are not at the state of being plug-and-play, and it requires an engaged technical team to implement and oversee the entire process. This entire report itself is on the order of 500 pages, and with most power companies experiencing a shortage in staffing, being able to assign the proper staff to projects can be a challenge. After a dynamic rating scheme is implemented, it takes a dedicated effort to "stay on top of it" in order to make sure the system is providing good information and to take full advantage of the capabilities.

\section{Technology Transfer}

One goal of the project was to transfer knowledge and technology to NYPA. Several meetings were held to fulfill this goal, including the three meetings in Lenox (2010, 2011, and 2012), and one at White Plains in March 2013. Of particular importance was a meeting at the NYPA Marcy Station near Utica, NY, on January 24-25, 2013, at which the draft Final Report was reviewed in detail, and the fully operational DTCR server was turned over to NYPA along with a live realtime demonstration of the NYPA line ratings with hands-on training for NYPA operations personnel. NYPA retains possession of the server, modems, field instruments, ratings software, and all the project data in electronic form. 


\section{7 \\ CONCLUSIONS AND OPPORTUNITIES}

\section{Conclusions}

The DOE-NYPA project began in mid-2010 and was completed in mid-2013. Over the course of the project, several comprehensive technical meetings were held, status reports were submitted every month, and three drafts of a Final Report were reviewed and revised. The project was successful in having made significant assessments of the technologies involved, and the technologies are undergoing improvements as a result.

Many pros and cons of these technologies were identified and thorough detailed assessments are documented in the appendices, with individual detailed conclusions discussed in Appendix $\mathrm{J}$. The main conclusion is that dynamic rating technologies can be successful in rating transmission lines in real-time, and there does exist extra power capacity beyond the traditional static ratings most of the time - and for the specific lines of this project, that is particularly true during high wind farm output. However, it is clear that some rating technologies tested need improvements before they could reliably go mainstream in the power industry, and it would be a challenge for a utility to implement dynamic ratings without a significant effort upfront and regularly thereafter.

With regards to how NYPA could effectively use the dynamic rating technologies in system engineering, operations, and planning, there are different levels of implementation with different challenges. The DTCR software runs on a standard Windows server, so it is possible to simply have that server residing in an engineering office with a responsible engineer(s) who oversees the results. The advantage of this approach is its simplicity, and the fact that most of the time, by far, the NYPA lines are operating at very low power levels. With the dynamic rating computations being performed in engineering, it saves operations from having to deal with a rating system that is rarely needed. With this approach, operations can communicate with engineering only during the rare events where dynamic ratings may be needed, and engineering can aid operations in interpreting the results. Regardless, engineering has to be involved with the dynamic rating system in order to assure that its equipment is maintained and functional, and the performance of the entire rating system is reliable.

A similar approach is to have the dynamic rating system in the hands of a second or third party that would fill the engineering needs discussed above. For instance, EPRI has a project whereby dynamic rating calculations for some lines are being performed at EPRI, and the dynamic rating results are forwarded to the power company in near real-time.

A more integrated approach would be to integrate the dynamic rating system directly into operations, as illustrated in Figure 7-1. In this scenario, real-time data from either inside SCADA (such as load from the EMS, or other instrument data directly tied to SCADA), or from outside SCADA (such as the data from the instruments tested during the DOE-NYPA project) is imported by the DTCR server. The DTCR server would then export the relevant data back into SCADA where the results could be viewed anywhere that a SCADA interface is made available. 
Also, during this process the server would archive all the data for any post processing that may be desirable, such as for engineering analysis and planning.

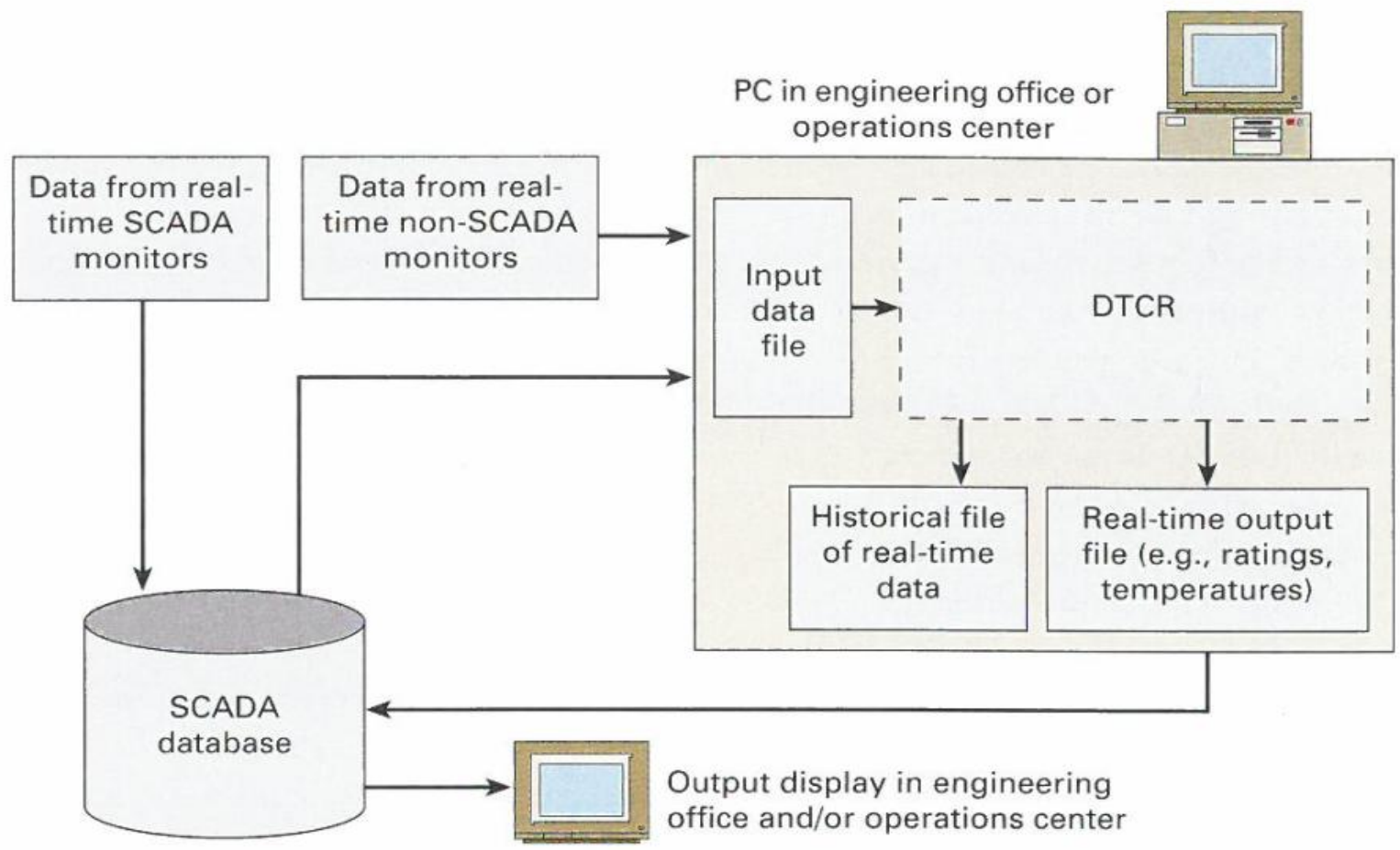

Figure 7-1 Flow diagram of how a dynamic rating system could be more fully integrated into system operations and planning

\section{Opportunities}

As a result of this project, other opportunities for improving transmission line ratings at NYPA have been identified. Primarily, a significant amount of data was collected and archived during the project. This vast collection of data could form the basis of an analytical study to define an improved approach to setting reliable and safe static ratings. This data may also be analyzed to provide a methodology for performing day-ahead forecasted ratings, and possibly up to a week in advance.

For instance, according to CIGRE Technical Brochure 299, the line rating conditions in use by NYISO, which include an effective wind speed of $3 \mathrm{ft} / \mathrm{sec}$ and an air temperature of $35^{\circ} \mathrm{C}$ for summer, need to be justified by field studies of the sort that was done here. The data analyzed here suggest that the NYISO conditions appear to be justified by these measurements. In future upgrades of NERC Reliability Standards FAC-008 and FAC-009, it is conceivable that a statistical analysis of line rating weather conditions may be required in addition to the specification of an industry standard heat balance method and reasonable maximum allowable conductor temperatures. Additional, but somewhat simplified, studies of other NYPA lines in more sheltered areas with lower voltage lines would be very useful. 
The off-site NOAA weather data derived from nearby airport weather stations and accessed over the internet was used as reasonable backup for the other on-site monitoring systems during this project. The emphasis in this project was on the dynamic rating of two specific lines using dedicated real-time monitors deployed along the line. All real-time monitors are located close to the line and within the right-of-way (corridor). The project did not evaluate the possible use of system-wide weather-adjusted ratings based on NOAA or other weather service data, but the possible use of weather service on-line data as a backup to one of the line monitor methods was considered. It may be helpful to pursue similar comparisons at NYPA lines which are in areas having rougher terrain and/or lines heavily shielded by foliage, to determine to what extent online NOAA data could be reliably used for rating determinations.

A related area that NYPA may consider for future study is the consideration of substation terminal equipment ratings. EPRI has begun to develop thermal modeling data for terminal equipment like switches, line traps, and current transformers. The DTCR software is capable of calculating dynamic ratings for terminal equipment and power transformers, as well as for underground cables and overhead lines. In particular, an EPRI project is being organized to model CT ratings. In a significant amount of cases, substation CTs are the limiting factors for line ratings. Similar to overhead lines, CT's are known to have significantly higher power capacities than specified by their nameplate ratings. EPRI is launching a collaborative project to study CT ratings with the goal of defining methodologies to better rate these devices. The project will include laboratory tests of selected samples, including special units fabricated for the project, and forensic analysis. 



\section{BACKGROUND DETAILS}

\section{Introduction}

The demand for electric power over transmission circuits is increasing at a faster rate than the construction of new transmission facilities. This trend is pushing the capacity of many transmission circuits beyond the margins required for contingencies. The power capacity (i.e., the rating) of most overhead transmission lines is prescribed by the so-called "static rating" based on both the conductor configurations and the environmental conditions. Ideally, very conservative worst-case assumptions about environmental conditions are used when developing these "static ratings". Due to this conservative approach, significant extra power capacity exists beyond the design margin on most lines most of the time.

As part of its on-going research in this area, EPRI has developed monitors, rating calculation methodologies, the Dynamic Thermal Circuit Rating (DTCR) software, workshops, and other products for the purpose of gaining access to the extra power capacity. All the related EPRI research results and products are referred to at EPRI as DTCR Technologies. Prior to undertaking capital intensive activities — such as building new lines, reconductoring, raising structure heights, replacing transformers, putting lines underground, etc.- - utilities can use these technologies to maximize power throughput of existing assets, defer capital expenditures, and simultaneously increase safe and reliable operation of their assets.

In 2010, a project was initiated between NYPA and EPRI to evaluate EPRI's Dynamic Thermal Circuit Rating (DTCR) software, along with instrumentation that can be used to monitor the thermal states of transmission lines and provide the required real-time data needed for the DTCR calculations. The main objective of the project was to evaluate the DTCR Technologies, and to demonstrate how they could be used by transmission system engineering, operations, and planning of the New York Power Authority (NYPA).

\section{Background}

There are several types of instrumentation packages commercially available or being developed that can be used to provide the required real-time data for DTCR calculations. It was decided during the formative stages of the project, based on NYPA's initial review and suggestions, that the data input to DTCR would focus on the following four technologies; EPRI's Conductor Temperature and Load Sensors (EPRI Sensors) for measuring conductor temperature and current, Video Sagometers to monitor the conductor position (sag), the ThermalRate systems (TR)to provide calculated conductor temperature and effective perpendicular wind speed, and weather stations (WX) to monitor the relevant weather variables.

Three $230 \mathrm{kV}$ line sites were fully instrumented. Line current measurements were provided to DTCR initially from the EPRI Sensors, but starting in May 2011 these data were also provided from NYPA's SCADA system. 
All the instrumentation and associated equipment was delivered to the EPRI High Voltage Laboratory in Lenox, MA (Lenox). The first set of instruments were fully configured and installed on a test line at Lenox for testing. The hardware and programming for data logging, power supply operation, and communications necessary for the various instruments to work together went through their final design and testing stages. The data link to DTCR was tested and DTCR was tested to verify that it could work with the incoming data stream. NYPA field crew members and engineering staff witnessed and participated in the installation in order to provide training for the final field installations.

The second and third sets of instruments were also delivered to Lenox, and some basic tests were performed to verify operation. The instruments were shipped to the line sites. Each instrumentation vendor provided their own on-site assistance during installation, and EPRI coordinated the activities. NYPA provided bucket trucks and line crews as needed, along with the capability to perform basic span surveys. The NYPA line crew performed the actual installation of all on-site materials, and personnel from EPRI or equipment vendors provided technical direction.

\section{Overview of Project Activities}

Field instrumentation occasionally, (sometimes frequently), needs troubleshooting and/or maintenance. EPRI provided technical support of instrumentation as needed. In some cases, the hardware vendor was directly involved. During on-site maintenance activities NYPA provided bucket trucks and line crew support. There have been several such site visits. As a result of these activities, vendors were able to identify needed improvements for their field instruments, and improvements were made as a side-benefit of the project.

A server was provided for DTCR and other related software. The DTCR software and other associated programs resided at the EPRI High Voltage Laboratory in Lenox, MA where real-time ratings were calculated continuously (every 10 minutes), within equipment limitations, starting in December 2010 through the end of October 2012. This process included the retrieval and archiving of the raw field data. The archived data were also used as input to DTCR in simulation mode to post process a set of historical ratings that included periods missed in real-time calculations due to equipment limitations, including communication interruptions and server downtime. While performing a complete set of DTCR simulations in addition to the real-time calculations was beyond the scope of the original project, these simulations proved extremely valuable in obtaining equipment reliability data, discussed in Appendix E, and for providing a more complete set of historical ratings for risk assessment, presented in Appendix H. The server was shipped to a NYPA location in January of 2013.

To perform rating calculations, the temperature of the conductor must be known. In some cases, such as with a weather station alone, the temperature is calculated. For instruments such as the EPRI Sensors, the temperature is measured directly, but only at discreet points along the line. Sag monitoring instruments determine the average conductor temperature along an entire line section; however, it requires that the mathematical relationship between sag and average conductor temperature, Tc (sag), be determined in the form of a "state change equation". This requires a physical model of the line (using, for instance, SAG10 or PLSCAD), and some upfront data analysis. It is important that this be done correctly, and that the mathematical relationship be monitored and refined if changes occur due to line work, structure maintenance, 
environmental factors, or other reasons. The process of obtaining the state change equations for the three sagometer installations is outlined in Appendix B.

In January 2013 a training program took place to facilitate the transfer of the DTCR server for operation, and integration at NYPA. This training was focused on the use of the software, and on the significance and use of the incoming data and computed results.

Throughout the course of the project, a large amount of data and other information was gathered and processed. The output and operational performance of the different types of instrumentation was documented and analyzed over the project to create an assessment of the field technologies with respect to costs, ease of installation and use, accuracy, maintenance issues, and reliability. The weather and rating data were analyzed to understand the statistical distributions of these data and are presented in this report in chronological form in which ratings were computed, and also as distribution functions for risk assessment. As anticipated, significant extra power capacity was found on the transmission lines during the rating period.

This project was launched in mid-2010 and concluded as scheduled at the end of January 2013. 



\section{TRANSMISSION LINE RATINGS - PRINCIPLES AND PRACTICES}

\section{Introduction}

The current in overhead transmission lines is limited in order to limit undesirable electrical and thermal effects. This project only concerns current limits to limit the temperature of the bare phase conductors and their connectors (i.e., thermal effects). In order to keep the maximum allowable conductor temperature below the line's design value (e.g., $95^{\circ} \mathrm{C}$ ), a maximum line current (or line thermal rating) is typically calculated for suitably conservative weather conditions.

CIGRE Technical Brochure 299[1] suggests that, in the absence of field data, a low wind speed of $2 \mathrm{ft} / \mathrm{sec}$ combined with an air temperature near the seasonal maximum and full solar heating of approximately 1000 watts $/ \mathrm{m}^{2}$ constitutes suitably conservative weather conditions that can be used to calculate static thermal line ratings.

The CIGRE brochure also notes that less conservative weather conditions, which produce higher thermal ratings, may be used if field studies are performed to justify such values or if real-time weather data along the line is available to produce dynamic thermal ratings. The NYISO recommends the use of a $3 \mathrm{ft} / \mathrm{sec}$ wind and a summer air temperature of $35^{\circ} \mathrm{C}$ based on limited field studies.

Section 2 of this report is intended to summarize the principles of static and dynamic thermal line rating calculations and to explain the various practices involved in the use of line and weather monitoring instruments that can be used as the basis of such calculations.

Many types of physical line modification have been implemented to up-rate existing overhead lines by re-tensioning or reconductoring to increase the maximum allowable conductor temperature of the line and produce large rating increases $(20 \%$ to $100 \%)$. However, these methods can be costly, may require extended circuit outages, and encounter regulatory delays. Dynamic rating methods typically yield more modest increases in rating (5\% to $25 \%$ ) but are much lower in cost, easily implemented without extended outages or regulatory delays, and the monitoring instruments are potentially portable for use in other locations.

\section{Thermal Line Ratings}

A maximum allowable conductor temperature is specified for an overhead line's current-carrying phase-conductors in order to limit the aging of the conductor's connectors, the loss of mechanical tensile strength due to annealing of the aluminum (or copper) strands in the conductors, and to maintain minimum electrical clearances at all points along the line. 
Whatever the line current, the temperature of its bare overhead conductors depends on the ambient conditions (air temperature, wind speed, wind direction, solar intensity, and rain rate) along the line. The relationship between current and conductor temperature is calculated by means of a heat balance equation calculated for the outer surface of the conductor, as depicted in Figure B-1 (cooling due to rain is normally ignored, as is corona heating).

\section{Energy Comes = Energy Goes}

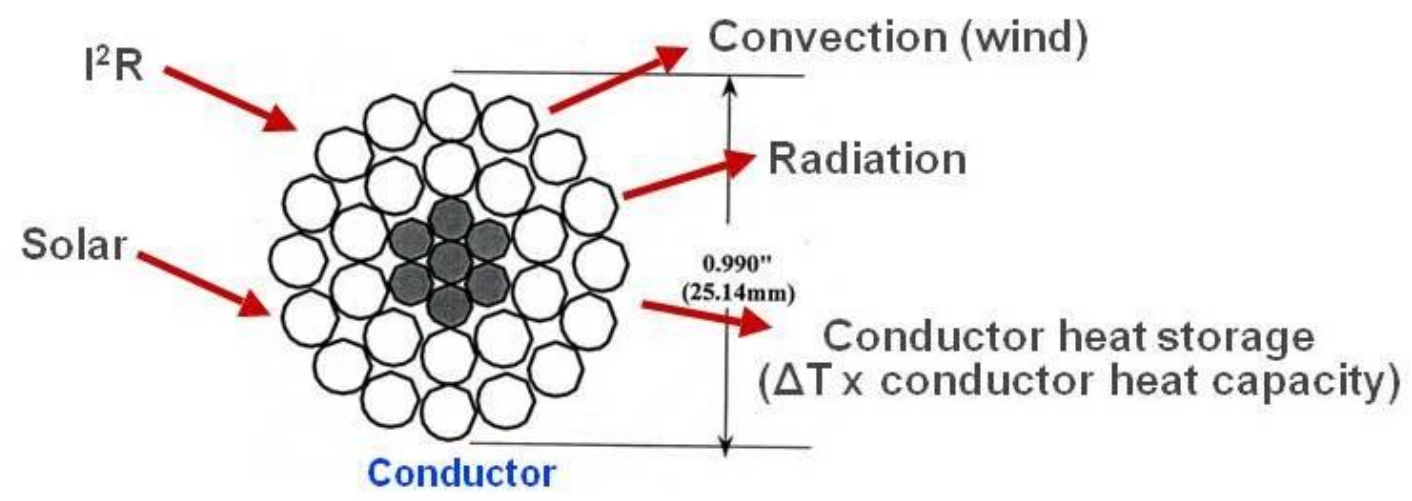

\section{Figure B-1}

Depiction of the thermal energy input and output of an overhead line conductor

The thermal energy input to a conductor comes from Ohmic losses (primarily in the conducting strand layers, not the reinforcing core) and solar heat absorption primarily due to direct solar radiation on the conductor's outer strand layer. For lines in the NYISO, with the power system operating normally, line current may be less than $30 \%$ of line rating and the solar heating can be larger than heat generated by current. At full rated current (100\% rating), however, the Ohmic losses increase with the square of line current and are typically much larger than solar heating.

Heat is lost to the surroundings by means of heat convection, radiation, and through cooling by rain. In most situations the convection heat losses are 3 or 4 times larger than radiation losses.

In the transient state where the line current or weather conditions change significantly within a few minutes, the heat stored in or recovered from the conductor mass per unit length must be included in the heat balance equation.

The thermal rating (in amperes) of an overhead transmission line is calculated by means of a heat balance equation such as shown in Equation B-1, with the conductor temperature set equal to the maximum allowable conductor temperature: 


$$
I^{2} R+Q_{S}=m C \frac{d T}{d t}+Q_{R}+Q_{C}
$$

where:

$$
\begin{aligned}
& \mathrm{I}=\text { current }(\text { amps) } \\
& \mathrm{R}=\text { conductor resistance }(\text { olmms per meter) } \\
& \mathrm{Qs}=\text { solar input (watts per meter) } \\
& \mathrm{m}=\text { conductor mass }(\mathrm{kg} \text { per meter) } \\
& \mathrm{C}=\text { conductor heat capacity }\left(\mathrm{kg}-{ }^{\circ} \mathrm{C} /\right. \text { joule) } \\
& \mathrm{T}=\text { conductor temperature }\left({ }^{\circ} \mathrm{C}\right) \\
& \mathrm{t}=\text { time (seconds) } \\
& \mathrm{Qr}=\text { radiated heat loss (watts } / \text { meter) } \\
& \mathrm{Q} \mathrm{c}=\text { convected heat loss (watts } / \text { meter) }
\end{aligned}
$$

\section{Equation B-1 Heat Balance Equation}

The heat balance equation is described in considerable detail in IEEE 738[2]. In brief:

- The solar energy input, $\mathrm{Q}_{\mathrm{s}}$, depends on the intensity of solar energy, the angle between the conductor axis and the sun, and the absorptivity of the conductor's surface.

- The radiation loss $\left(\mathrm{Q}_{\mathrm{r}}\right)$ depends on the conductor temperature rise above air temperature and the emissivity of the conductor's surface.

- The convection heat loss $\left(\mathrm{Q}_{\mathrm{c}}\right)$ depends on the wind speed and direction, and conductor temperature rise above air temperature.

Over a wide range of conductor temperatures and weather conditions, the convection heat loss rate exceeds the radiation heat loss. With no wind, the natural convection heat loss is twice that of radiation. With crosswinds of 3 to $4 \mathrm{ft} / \mathrm{sec}$, the ratio increases to 4 or more.

There are several different heat balance methods which are used to calculate overhead line thermal ratings. DTCR incorporates two of these - the DYNAMP [3] and the IEEE738 [2] methods. The DYNAMP method is based on an EPRI research study which yielded a report in 1988. The IEEE 738 method is documented in an IEEE Standard 738, the latest version of which was published in 2012; DTCR currently utilizes the previous version published in 2006.

The two methods determine radiation heat loss from the conductor in exactly the same way but they use slightly different formulas for forced and natural convection. The original DYNAMP method, as described in the 1988 report [5], utilizes a different solar heat gain model which includes diffuse as well as direct solar radiation. As implemented in DTCR, for both models, the solar radiation calculation is that of IEEE 738 and the conductor resistance as a function of temperature is the same in the two methods.

In calculating line ratings, the original DYNAMP model almost always produced line ratings which were $3 \%$ to $5 \%$ less than the IEEE 738 model. Most of the difference was due to the different forced convection heat loss terms used in the two methods. By using the IEEE 738 solar model for both methods (as in DTCR), the difference is somewhat less, being $2 \%$ to $4 \%$ lower for DYNAMP. 


\section{Static Line Ratings}

Traditionally, and almost universally, the power flow on overhead transmission lines is limited by the line's static (or "book") ratings. Static ratings are calculated with suitably conservative weather conditions used for the variables in the above heat balance equation. The advantages of using static line ratings are several:

- They are simple to calculate and document, thus the descriptor "book" ratings. Once calculated for a conductor, they can become a permanent part of the utility database.

- They are constant over time so they are completely predictable for operators who must often estimate transmission capacity days in advance.

- They are not line specific, so all lines that use the same conductor at the same maximum conductor temperature have the same rating.

The disadvantages are that the actual power capacity of a line is usually significantly greater than the static rating due to the conservative assumptions used in its calculation, and that those times when the actual capacity is less than the static rating may go undetected. This situation is depicted below in Figure B-2.

\section{Load vs. Rating Distribution}

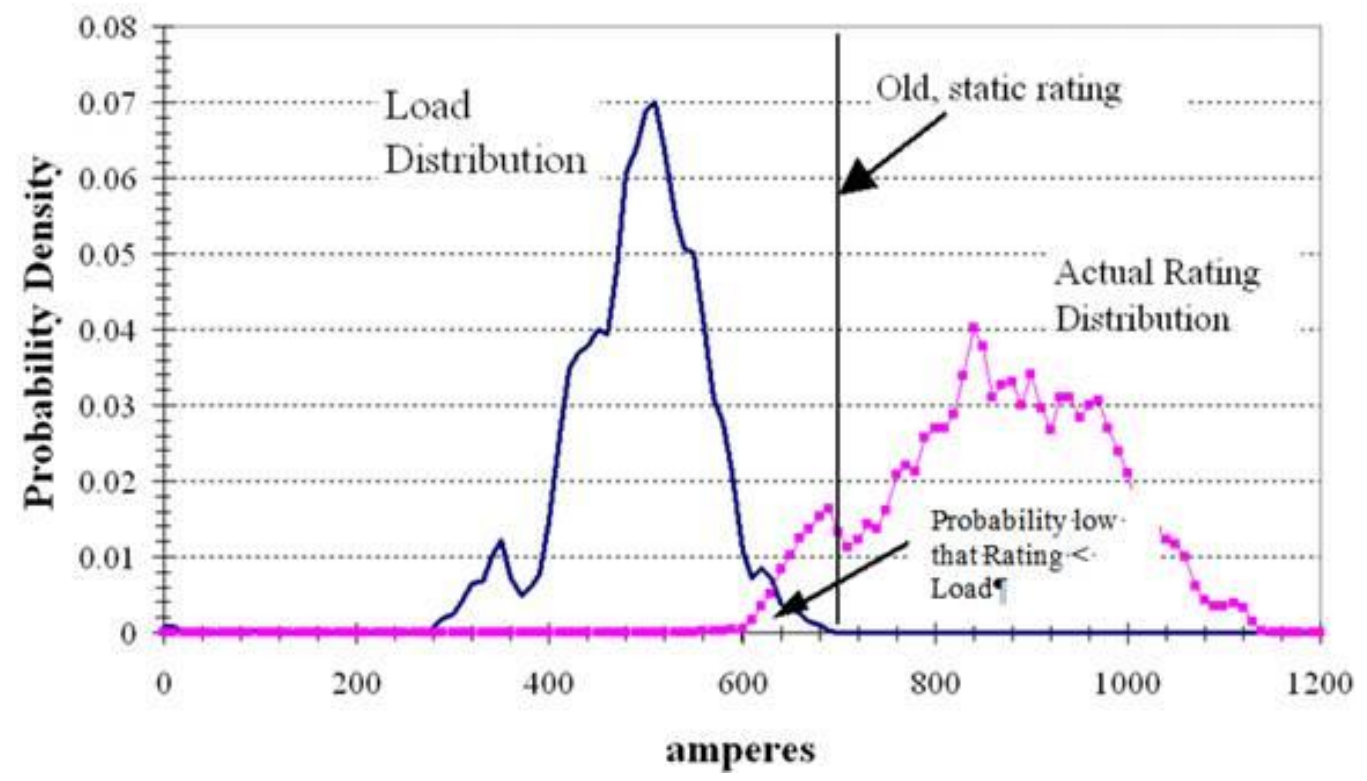

Figure B-2

Load vs. Rating Probability Distribution example for a transmission line

In Figure B-2, the static rating is fixed at about 700A, the load distribution is to the left of the static rating, and goes to zero at the static rating (the operator purposely keeps the load below the static rating). However, the actual line rating distribution is significantly higher than the static rating - with an average of over $20 \%$ greater. Also, there is some small part of the actual rating distribution that falls below the static rating. This is the range of accepted risk in the static rating method. High loads are low probability. Low real-time ratings are also low probability. It is 
extremely unlikely, but not impossible, that these two will occur at the same time. It is an important concept that with the static rating system some element of risk has always been present.

\section{Normal and Emergency Ratings}

Power systems must be reliable. The loss of a bulk transmission line or a major generating station may require high power flows on lower voltage lines that may exceed their normal thermal rating. To accommodate such short term overload events, most power systems calculate both normal and emergency line thermal ratings.

For example, the NYISO suggests the use of normal ratings, and 4-hour (LTE) emergency ratings. The normal rating of Drake is based on a maximum allowable temperature of $95^{\circ} \mathrm{C}$, a temperature at which the annealing of aluminum is negligible even when sustained over long periods of time. The LTE rating of Drake is calculated for a maximum allowable temperature of $115^{\circ} \mathrm{C}$ for a period of time that does not exceed 4 hours. The STE rating of Drake is calculated for a maximum allowable temperature of $125^{\circ} \mathrm{C}$ for a maximum period of time that does not exceed 15 minutes.

\section{Seasonal Ratings}

In non-equatorial regions, there may be a provable difference in maximum air temperature between summer and winter. In New York for example, the air temperature used for summer line ratings is $35^{\circ} \mathrm{C}$ and for winter it is $10^{\circ} \mathrm{C}$. As a result, winter line thermal ratings are about $20 \%$ higher than summer line ratings.

\section{Ambient-Adjusted Ratings}

In many power systems, static ratings are adjusted more frequently than seasonally. In the simplest method, line ratings are adjusted daily to reflect the maximum air temperature predicted for the next 24 hours. Seasonal ratings can be adjusted in this manner. For example, in NY, the summer line rating is calculated for an air temperature of $35^{\circ} \mathrm{C}$. If the maximum air temperature predicted for the next 24 hours is $25^{\circ} \mathrm{C}$, the line rating can be increased by about $10 \%$ for the next 24 hours.

Other power systems have extended the concept of ambient-adjusted ratings to making rating adjustments for day and night, hour-by-hour, or even minute by minute. CIGRE TB 299 notes that making such ambient-adjustments to line ratings may be incorrect since wind speeds are typically correlated with air temperature and solar heating. On-site weather station data suggests that NYPA may be assuming greater risk of exceedance during the winter season using the current system of ambient adjusted ratings.

\section{Dynamic Line Ratings}

A concept which is gaining a foothold in the power industry is the use of real-time rating, referred to as dynamic thermal circuit rating. In this case, actual real-time field measurements are made of the variables needed in the rating equation, and the rating is calculated continuously - typically at ten minute intervals. 
The advantage of using dynamic rating is that it provides a better knowledge of the actual line rating, which is significantly greater than the static rating most of the time, so that higher loads can be carried. The disadvantage is that the dynamic rating is a varying quantity. In many cases, the thermal inertia of a conductor, due to its heat capacity, can be used to rate a conductor even much higher for a short period of time - referred to as an emergency rating. These concepts are illustrated in Figure B-3.

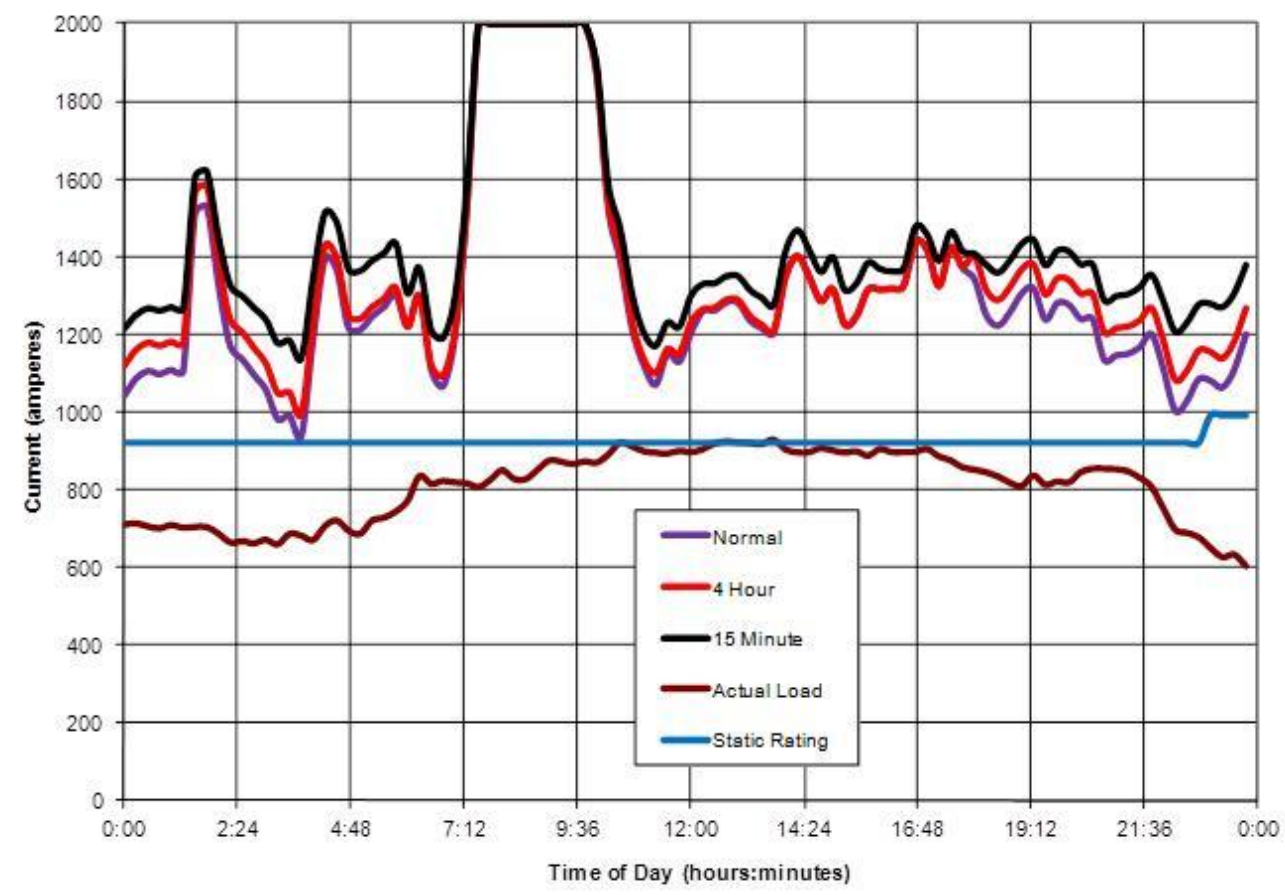

\section{Figure B-3 \\ Example of dynamic rating, static rating, and load for a 24-hour period for a particular transmission line}

Figure B-3 shows an example of the ratings for an operating $161 \mathrm{kV}$ line over a 24 -hour period. Plotted are the static rating, the line's load, and the dynamic ratings (4-hour "normal", and 15minute emergency). The 4-hour rating is the amount of current that a conductor can pass for the next 4 hours without exceeding a predefined maximum allowed operating temperature - if conditions were to remain constant. For all practical purposes, this is equal to the "normal" rating, which is the magnitude of current that can be passed indefinitely, without exceeding a predefined maximum allowed operating temperature - if conditions remained constant.

Note that during the middle of this particular day the utility was bumping into its static rating when, according to the DTCR results, there was a significant margin to work with. Also note that a light rain that morning sent the actual ratings to very high levels.

\section{Field Studies of Line Ratings}

A methodology being developed by EPRI and others is to use the results of dynamic rating calculations as a basis for statistical analyses to optimize a modified approach to static ratings. These rating studies involve gathering a large amount of field data (typically one year of data), executing dynamic rating calculations on the field data, and performing statistical analyses of 
both the field data and the dynamic rating calculation results. This process can provide insight into the associated risk-of-exceedance a utility takes when setting a static-type rating for a given line. Such a risk always accompanies the setting of a static rating (see Figure B-3), but the methodology provides a scientifically based process for making risk-informed decisions.

One of the features of this methodology is the statistical analysis of the DTCR results, as opposed to the statistical analysis of the field data alone. The DTCR computations naturally account for correlations among the field variables that would be missed if the field variables were analyzed separately.

For example, it has been observed that higher winds statistically accompany higher temperatures. This statistical correlation would be missed if the wind speeds and ambient temperatures were analyzed separately, but the DTCR computations automatically account for their correlated impact on the ratings.

\section{Field Measurements}

Referring to Equation B-1 above, it can be seen that for any given transmission line, the rating at any point in time depends on the ambient weather conditions, i.e., air temperature, wind speed, wind direction, and solar intensity. To calculate a line rating with Equation B-1, the line parameters (constants), weather variables, and the maximum permissible conductor operating temperature are used as inputs, and the equation is solved for the current, I (i.e., the rating). In other words, the rating is the current, I, that causes the conductor to reach its maximum allowed temperature.

In the first term of Equation B-1, $\mathrm{I}^{2} \mathrm{R}, \mathrm{R}$ is the conductor resistance (ohms per meter). These resistance values are well known, and are provided in manufacturers' tables. The DTCR software has a built-in database of the values for most conductor types.

The second term in Equation B-1 is the solar input, Qs (watts per meter). The magnitude of Qs depends on the solar intensity at the field site, and is measured by the weather station in real-time with a device called a pyranometer. The magnitude of Qs also depends on line direction and the value of the conductor's absorptivity, which are both of fixed value for a given line.

The third term in Equation B-1 (mC dT/dt) is the heat storage term. The "m" and " $\mathrm{C}$ " are the mass and heat capacity of the conductor, respectively, which are fixed for any given conductor type, and are provided in manufacturers' tables and in the DTCR software conductor database. " $\mathrm{T}$ " is the conductor temperature, and " $\mathrm{t}$ " is time. The derivative, dT/dt, is the rate of change of conductor temperature with time, and accounts for heat storage and the changing conductor temperature when the thermal energy of the conductor is not in a steady state. This term is useful during contingency situations when the conductor can pass more current than its normal rating because the conductor temperature has not yet reached its maximum value due to the heat storage (thermal inertia). In cases where the current (I) is known, Equation B-1 can be solved for conductor temperature $(\mathrm{T})$. When Equation $\mathrm{B}-1$ is used to calculate a rating, $\mathrm{T}$ is assigned the maximum conductor temperature and Equation B-1 is solved for I.

The fourth term in Equation B-1 is $\mathrm{Q}_{\mathrm{r}}$, this is the amount of power, in watts per meter, dissipated to the environment by radiation. This depends on the conductor temperature $(\mathrm{T})$ and the conductor emissivity, which is assigned a constant value. 
The fifth term in Equation B-1 is Qc, the amount of power, again in watts per meter, dissipated to the environment by convection. This term depends on the conductor temperature, $\mathrm{T}$, the ambient temperature, $T_{a}$, and on the wind speed and wind direction relative to the conductor. $T_{a}$ is measured in the field by the weather station's temperature sensor. The wind speed and direction are measured by the weather station's anemometer.

As shown in Figure B-4, changes in wind speed and direction relative to the line have a much larger impact on the line rating than changes in air temperature and solar heating.

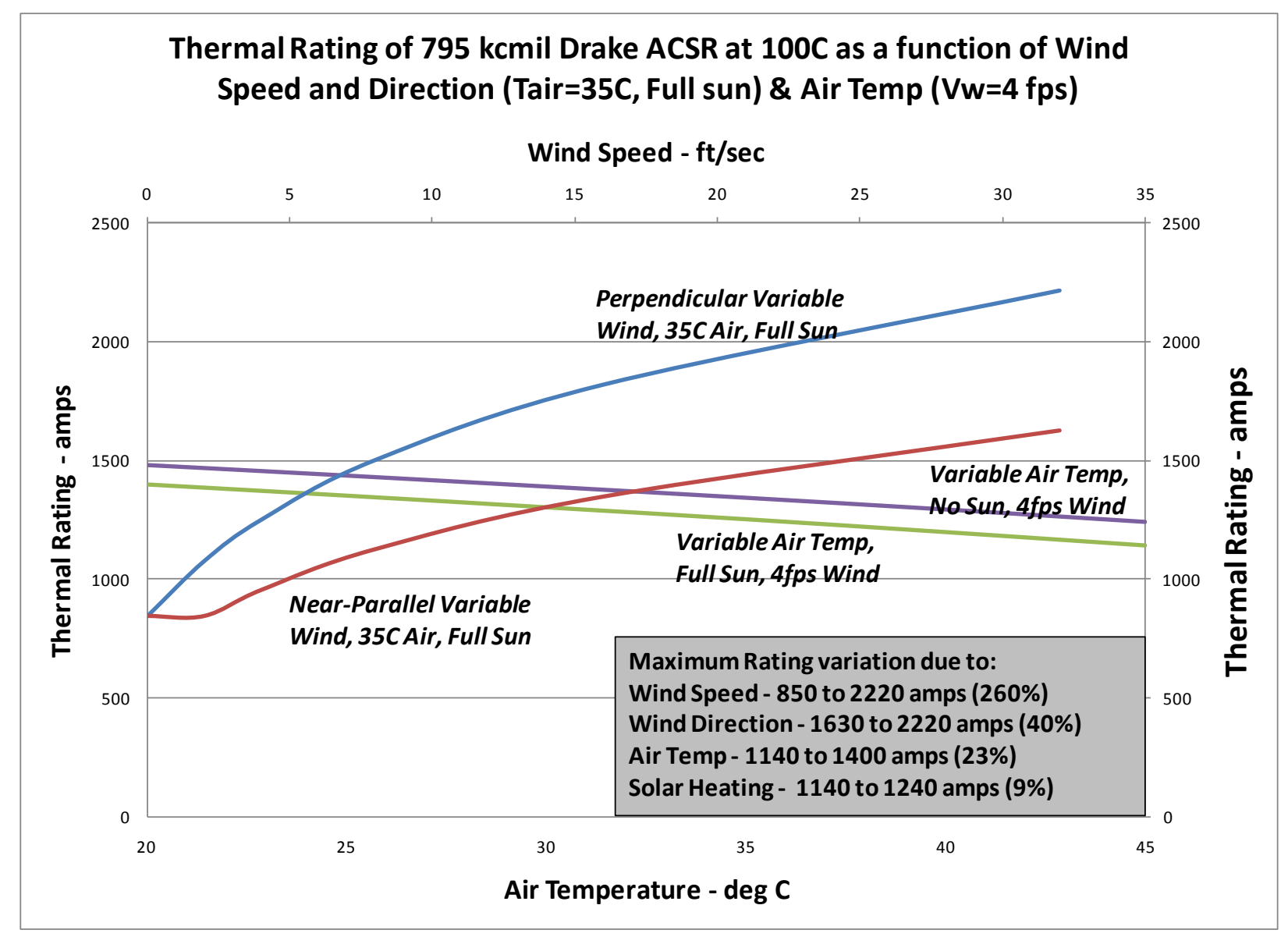

Figure B-4

Impact of changes in air temperature, solar heating, and wind speed and direction on line ratings

Also, solar heating and air temperature are typically similar from span-to-span in most transmission lines. This is shown in Figure B-5, where the air temperature at two locations over a mile apart along a transmission line right-of-way, are shown to be highly correlated over a week of measurements. 


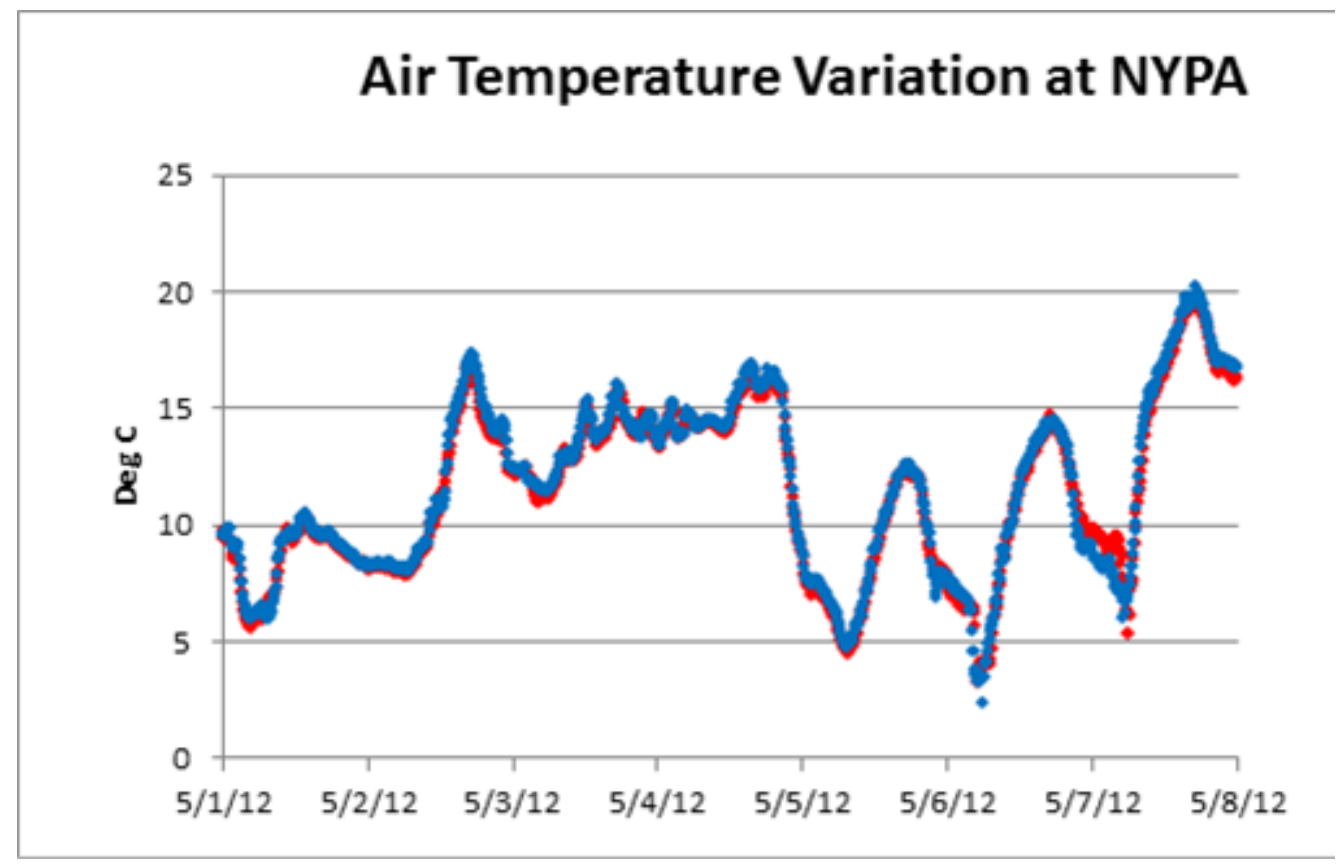

\section{Figure B-5}

Variation in air temperature between two spans approximately 1.5 miles apart in a typical NYPA transmission line

On the other hand, wind speed and wind direction typically vary widely from span to span, especially at low wind speeds that are of primary interest in thermal rating calculations. Figure B-6 is a scatter plot of simultaneous 10-minute average wind speeds at two locations less than 2 miles apart. Notice that the wind speed can be nearly zero at one location and between 2 and 6 $\mathrm{ft} / \mathrm{sec}$ at the other location.

This illustrates the need to measure the wind speed and direction at multiple locations along the line in order to be sure to use the minimum wind cooling for dynamic rating calculations. As explained in this section, this can be done by installing multiple wind anemometers, multiple temperature monitors, or multiple sag-tension monitors. 


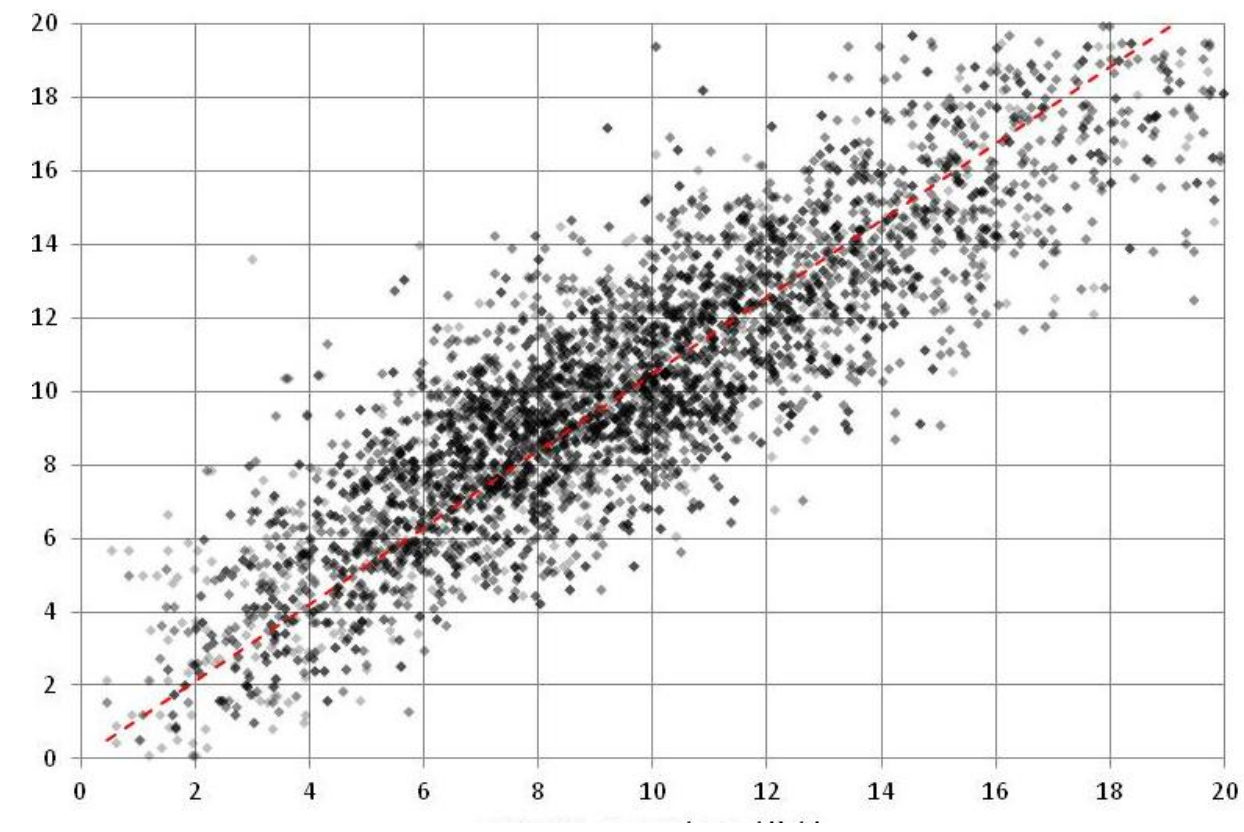

Figure B-6

Comparison of simultaneous 10 -min average wind speeds (in $\mathrm{ft} / \mathrm{sec}$ ) at two locations about $2 \mathrm{~km}$ apart along a transmission line

\section{Wind Cooling - Anemometers}

When using high quality anemometers to determine line rating in real-time, ambient temperature and solar intensity must also be measured in real-time and the heat balance equation solved for conductor rating, $\mathrm{I}$, for an assigned maximum conductor temperature, $\mathrm{T}_{\mathrm{C}}$, at each anemometer location. A sufficient number of anemometers must be located along the line to be sure that sheltered spans are included and the average conductor temperature within the sag-section can be calculated.

A positive feature of using weather stations is that they are relatively inexpensive, are very durable, easy to setup and use, and don't require any special calibrations. They can also be located in sheltered spans to be sure that the maximum conductor temperature (used for annealing calculations) is calculated as well as the average conductor temperature (used for sag clearance calculations).

\section{Wind Cooling - ThermalRate Conductor Model}

The ThermalRate monitoring device consists of a stranded conductor model divided into heated and unheated sections. The rise of the conductor temperature of the unheated section above air temperature measures solar heating. The conductor temperature rise in the heated section can be analyzed to determine the wind speed perpendicular to the nearby transmission line conductor.

In effect, the monitor is a directional anemometer which serves as an alternative to an ultrasonic anemometer. Like a conventional anemometer, the accuracy of the wind speed data that it produces is independent of the actual line current. 


\section{Wind Cooling - Conductor Temperature Monitors}

Another way of determining the local wind cooling is to measure the conductor temperature with a monitor. There are commercially available devices for doing this, and EPRI is in the process of developing an instrument for this purpose, referred to in this report as the EPRI Sensor (EPRI Sensors are used in this NYPA project). The EPRI Sensor measures conductor temperature by a thermocouple pressed against the conductor. From conductor temperature, $\mathrm{T}_{\mathrm{C}}$, an effective perpendicular wind speed can be determined for the location along the line.

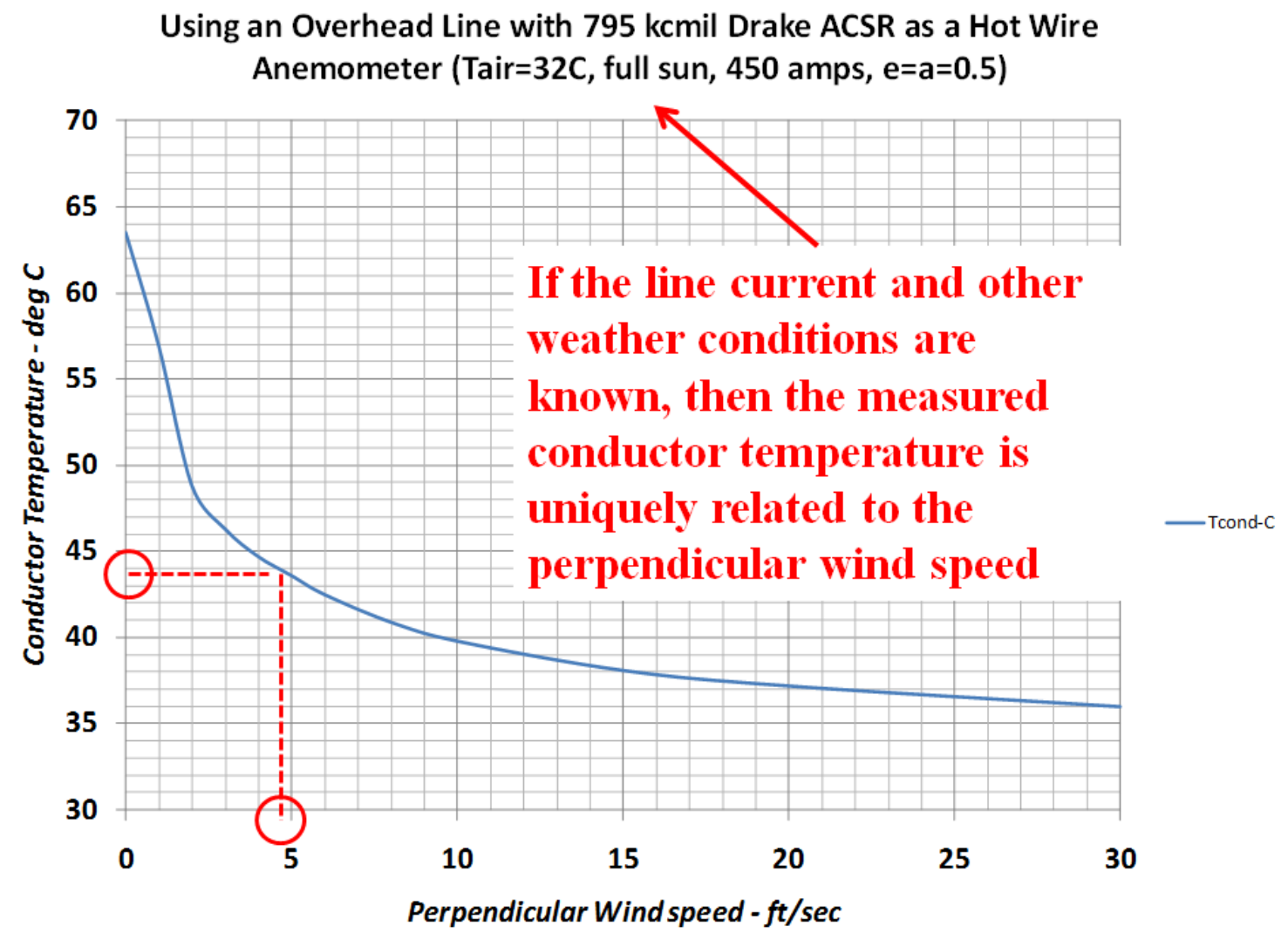

Figure B-7

Given the conductor temperature, current, air temperature, and solar heating, the effective perpendicular wind speed can be determined

Temperature monitors have several advantages over the use of wind anemometers, the largest of which is that they measure the effect of the wind at the surface of the energized conductor rather than some distance away. They also can be designed to measure line current (such as EPRI Sensors) which anemometers cannot. Also, the individual temperature sensors are relatively inexpensive.

Limitations are also significant. The most important limitations involve the possibility of heat sinking and flow disruption due to the presence of the monitor. Another is that the wind speed error can be large when the line current is so low that the conductor temperature is no more than one or two degrees higher than the conductor temperature without any current.

A limitation shared by both anemometers and conductor temperature monitors involves the thermal and mechanical behavior of overhead transmission lines. Most lines are constructed 
with periodic "dead-end" structures which are designed to stop cascade structure failures. Between dead-ends, suspension structures are used where the conductors are supported vertically but are free to move axially. The suspension spans between each pair of dead-ends is called a "sag-section". Because the conductor supports are axially flexible, any variations in tension from span to span, due to variations in load and temperature, are equalized. In this case, the sag in any suspension span depends on the average load and temperature of the line section rather than the load and temperature of that span alone.

At the same time, bare overhead stranded conductors are very poor axial heat conductors and there can be significant differences in the conductor temperature span-to-span due to wind speed and direction variations along the line. This temperature variation typically increases with line current as shown in Figure B-8.

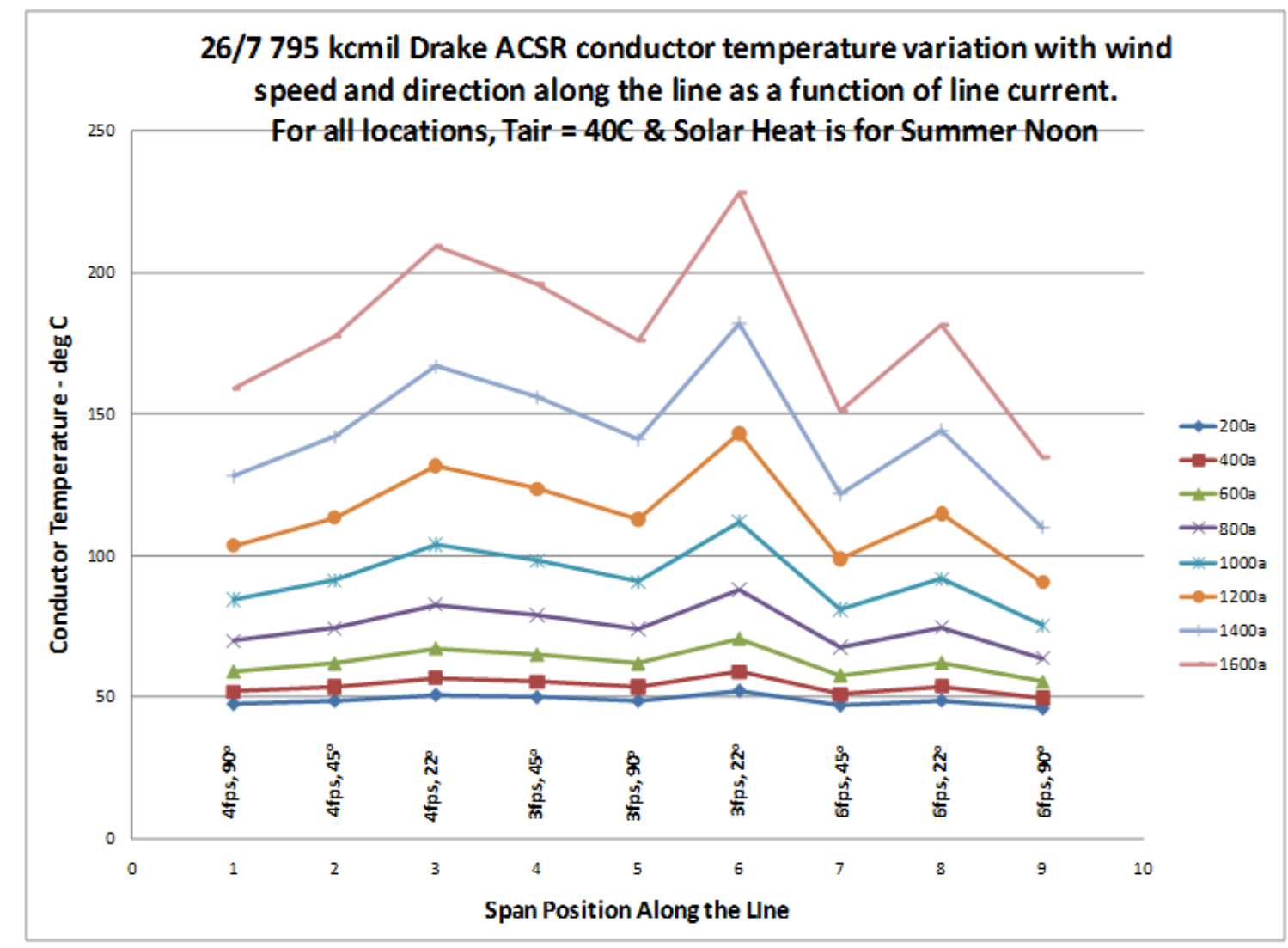

Figure B-8

Span-to-Span variation in conductor temperature due to wind speed and direction variations along a line

\section{Wind Cooling - Line Sag-Tension Monitors}

Instead of using many monitors or anemometers, a different type of conductor monitor, called a sag-tension monitor can be used. These devices do not measure temperature or wind speed and direction, but rather, either the conductor tension or the conductor sag, which reflects the average temperature of the sag-section. Theoretically at least one such monitor can replace multiple temperature or wind monitors, and they measure the actual behavior of the line sag-tension 
change as a function of line current and weather. These sensors cannot measure the maximum conductor temperature in a sheltered area.

As with anemometers and temperature monitors, calculation of the dynamic line rating also requires real-time air temperature, line current, and solar heat intensity, but sag-tension monitors also require the experimental derivation of the state change equation (discussed above) which relates the sag-tension parameter to the average sag-section conductor temperature, which is then related to the effective perpendicular wind speed for the sag-section. This additional step is demonstrated in Figure B-9.

\section{Converting Line Monitor Data into Effective Perp. Wind speed}

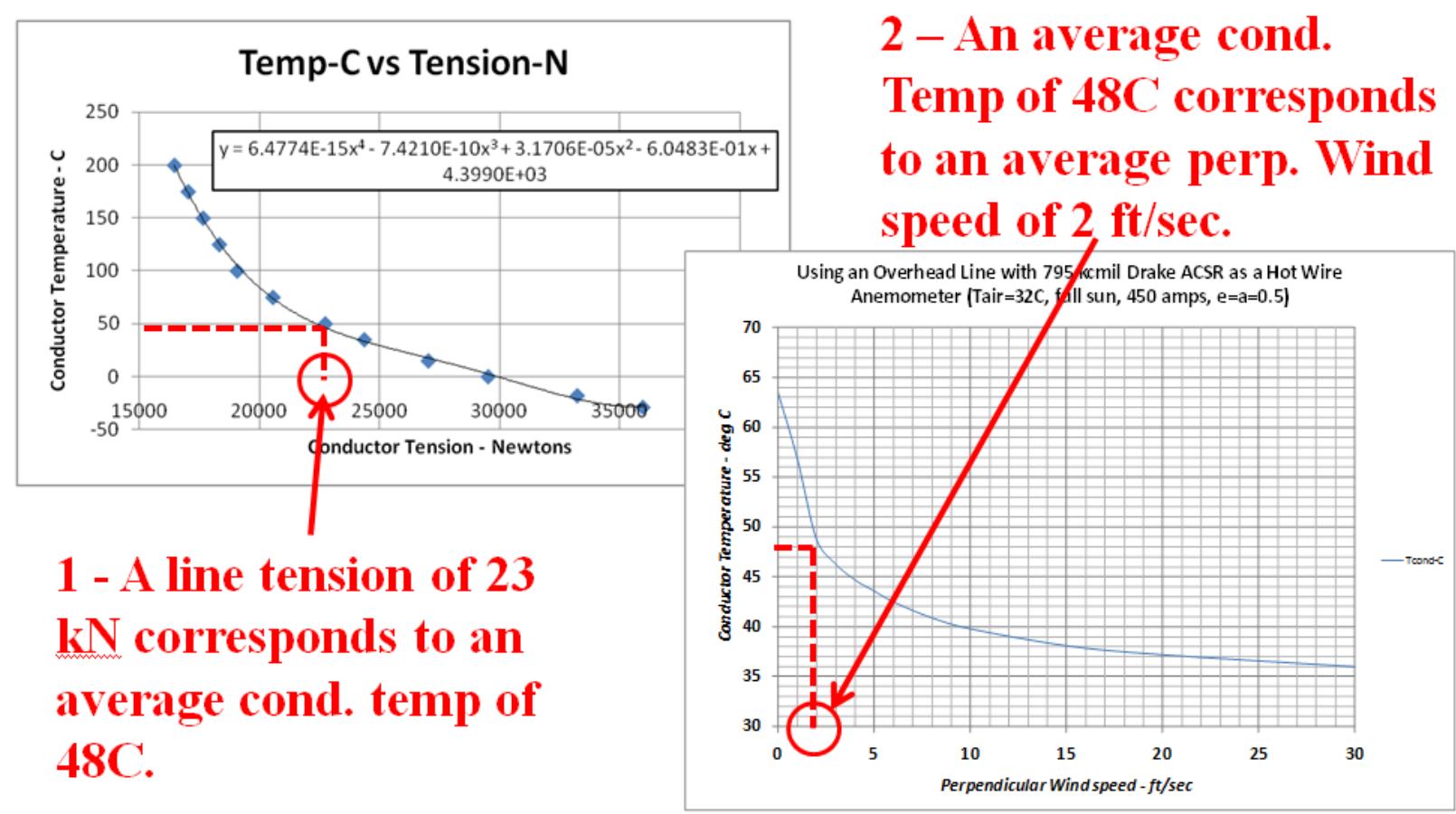

Figure B-9

A demonstration of the two-step process for converting tension or sag into an average Sag-Section perpendicular wind speed

The disadvantage of this type of monitor is that, as is true also of conductor temperature monitors, the line current has to be at least 0.25 to 0.5 amps per Kcmil for the line rating to be reasonably accurate. Also, the process of determining the calibration equation and verifying it can require at least a month of field data and may need to be checked frequently. For instance, for the Video Sagometers installed in this project, the average conductor temperature as a function of sag, i.e., Tc (sag), must be defined (typically as a polynomial) in a process that involves modeling the physical construction of the line with a program such as SAG10 or PLSCAD, and then adjusting the model for as-built conditions by calibrating the model with samples of field data where the current is very low at night. This model must be checked and 
refined occasionally if accuracy is to be maintained as many factors such as the structure movement and conductor stretch cause changes in the sag vs. Tc relationship.

\section{Wind Cooling - NOAA Data}

NOAA data is usually collected at airports rather than along transmission line corridors and the anemometers used are chosen for their ruggedness and high wind speed accuracy. As a result, as noted in CIGRE TB 299, weather service data collected at airports can be used to develop air temperature and solar heating data for lines, but the wind speed measurements are not normally useful due to the distance from transmission line corridor and the phenomenon of anemometer "stall" at speeds of 3 to $5 \mathrm{ft} / \mathrm{sec}$ that is occasionally present in older NOAA equipment.

New NOAA models and services allow their collected data to be interpolated into small grids. An interpolated grid containing a transmission line may yield data that more closely correlates to conditions within the ROW. Further software development and study would be needed to examine this.

In recent years, airport data has been used as the basis for calculating wind speeds and directions which are available from the weather service. Such wind speed models, available "on-line", are an attractive alternative to the use of dedicated monitors in the transmission line right-of-way. The problem with this approach is that the online weather services data may not accurately represent, or even be correlated with, the weather in a line's corridor. It may be that for some sites this approach would work, and for others it will not work. This idea was explored as part of the NYPA project.

\section{Heat Balance Models}

The most commonly used heat balance model is that described in IEEE 738 but there are two other heat balance models that are similar. CIGRE Technical Brochure 207 [3] describes what is commonly referred to as the "CIGRE" model and EPRI has developed a model referred to as the "DynAmp" model [4]. The three heat balance models yield similar results under most weather conditions, but there are some differences that yield thermal rating differences that vary from 2$4 \%$.

In this report, the IEEE 738 model is used as the standard method to which the other methods are compared. The similarity in calculated conductor temperatures given the same weather conditions and line currents are illustrated in the following figures. Estimates of conductor temperature based on ThermalRate measurements are included as well. 


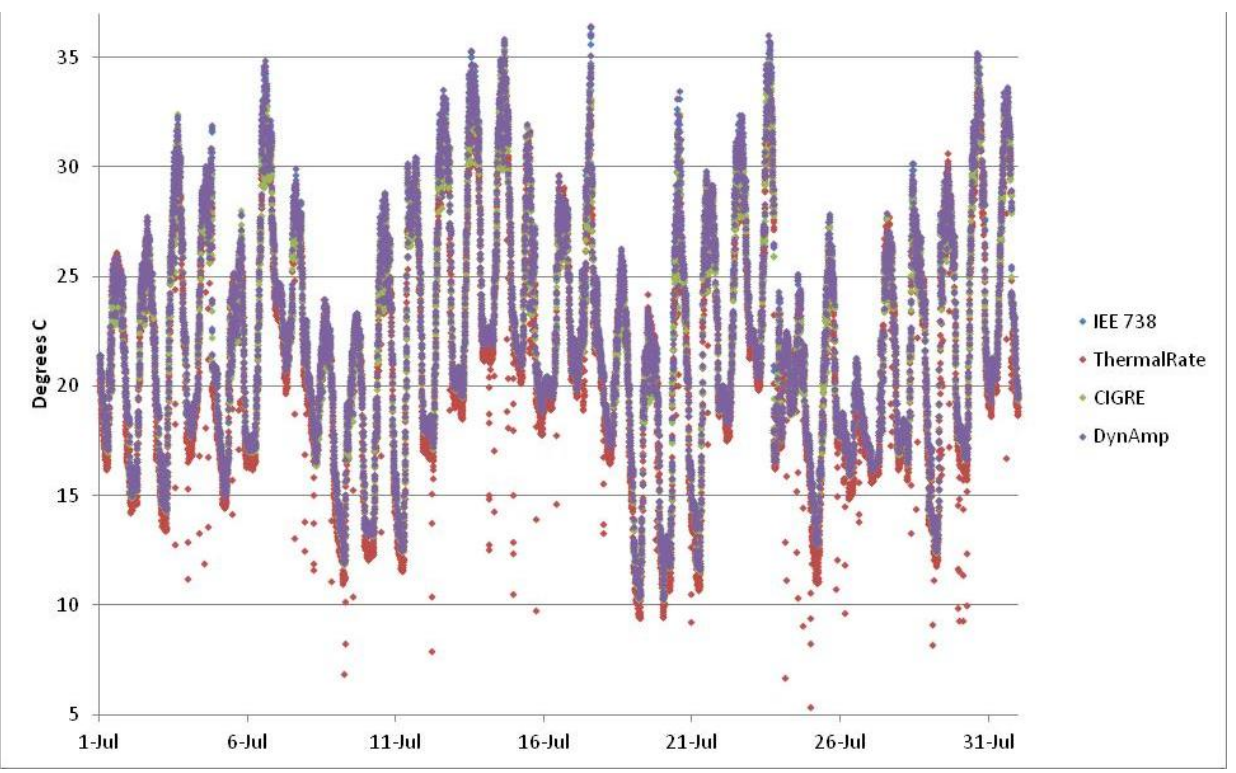

Figure B-10

Comparison of measured and calculated conductor temperature at one of the NYPA lines for the month of July 2012

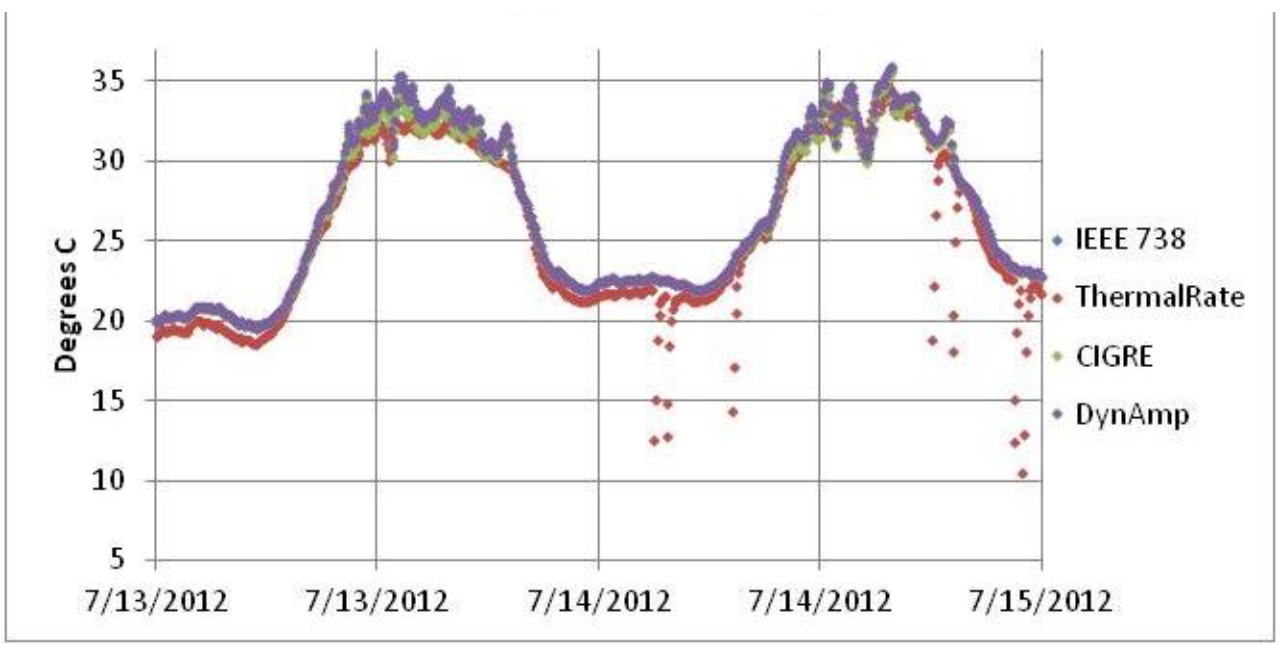

Figure B-11

Comparison of measured and calculated conductor temperature at a NYPA line for two days in July 2012

\section{Line Specific vs. System-Wide Ratings}

Static line ratings are not typically line specific. For example, using the NYISO static rating methods, all lines using $795 \mathrm{Kcmil} 26 / 7$ Drake ACSR, which have sufficient clearance at $125^{\circ} \mathrm{C}$, have the same normal, LTE and STE thermal ratings. This has the advantage of simplicity but it ignores the possibility that the rating of certain lines could be higher, or less commonly, lower. 
Dynamic line ratings, on the other hand, are typically line specific since their calculation usually involves real-time monitors placed along the specific line and it is not obvious that other lines at some distance away or with a different design, have the same dynamic rating.

The accumulation and analysis of multiple weather monitor installations along multiple lines within a given power system may serve as the basis for a revision of system-wide line ratings. This process can be complex and expensive to execute.

\section{References}

CIGRE. 2006. CIGRE Technical Brochure 299. "Guide for Selection of Weather Parameters for Bare Overhead Conductor Ratings.” Working Group B2.12. August, 2006

IEEE. 2012. "Standard for Calculating the Current-Temperature Relationship of Bare Overhead Conductors." PES. IEEE Standard 738-2012.

CIGRE. 2002. CIGRE Technical Brochure 207. "Thermal Behaviour of Overhead Conductors." Working Group 22.12. August, 2002.

Black, W. Z. and W. R. Byrd. 1983. "Real-time Ampacity Model (DynAmp) for Overhead Lines." IEEE Transactions. Vol. PAS-102. No. 7. July. pp. 2289-2293.

EPRI. 1988. EL5707 Research Project 2546-1 “Conductor Temperature Research.” 


\section{INSTRUMENTATION, SOFTWARE AND INSTALLATION}

This chapter contains details on the major hardware and software components used to produce ratings on the NYPA system. Three line sections were instrumented on two NYPA 230kV lines. The sections of line that were instrumented are located in upper New York State and the software and dedicated server were located at the EPRI High Voltage Laboratory in Lenox, Massachusetts.

\section{Transmission Line Instrumentation}

Figure C-1 depicts major components of the transmission system in NY State. The area of interest in this project is the $230 \mathrm{kV}$ corridor located between Massena and Plattsburgh.

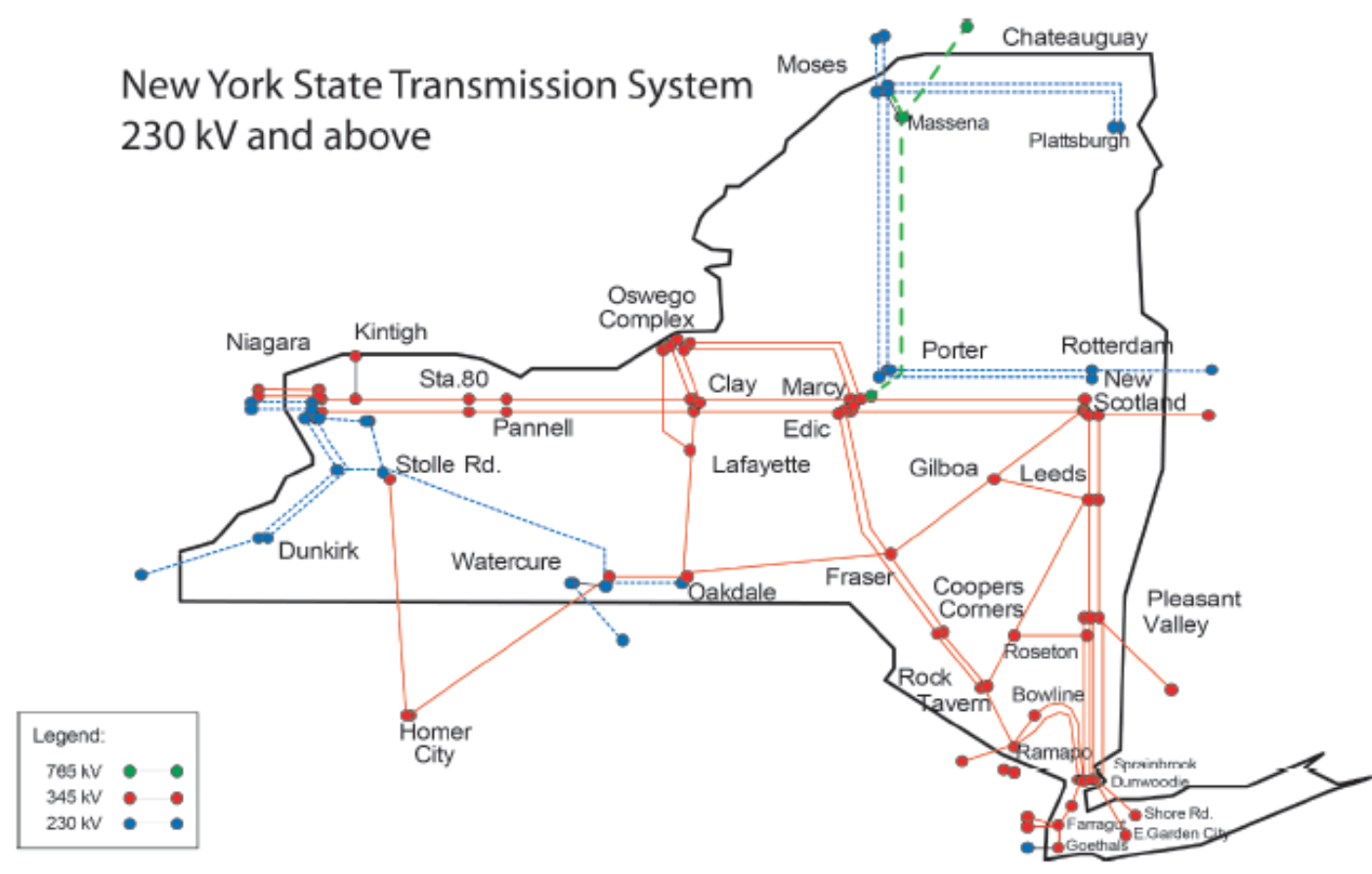

Figure C-1 Location of the $230 \mathrm{kV}$ transmission corridor

Referring to below, Site 3 Site is located near Massena, NY. The other two Sites (Site 2 and Site 1) are near Willis. 


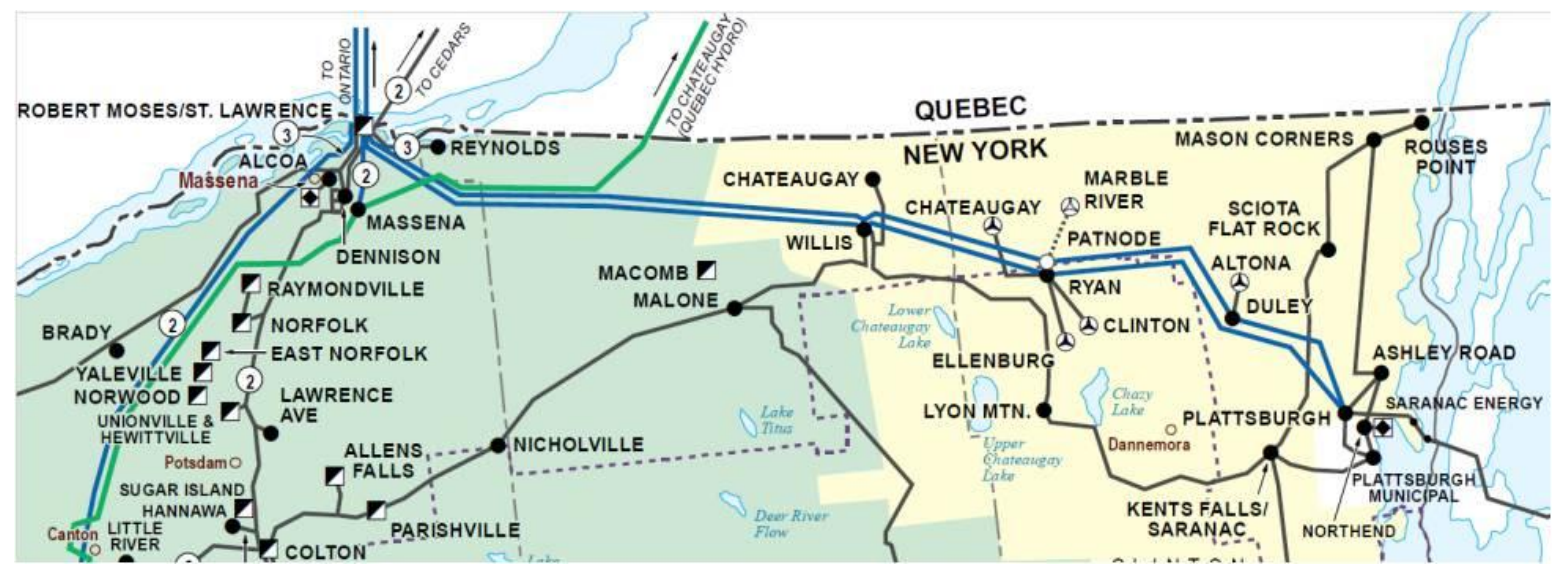

Figure C-2

Geographical Location of the $230 \mathrm{kV}$ Transmission corridor being studied

\section{Weather Station}

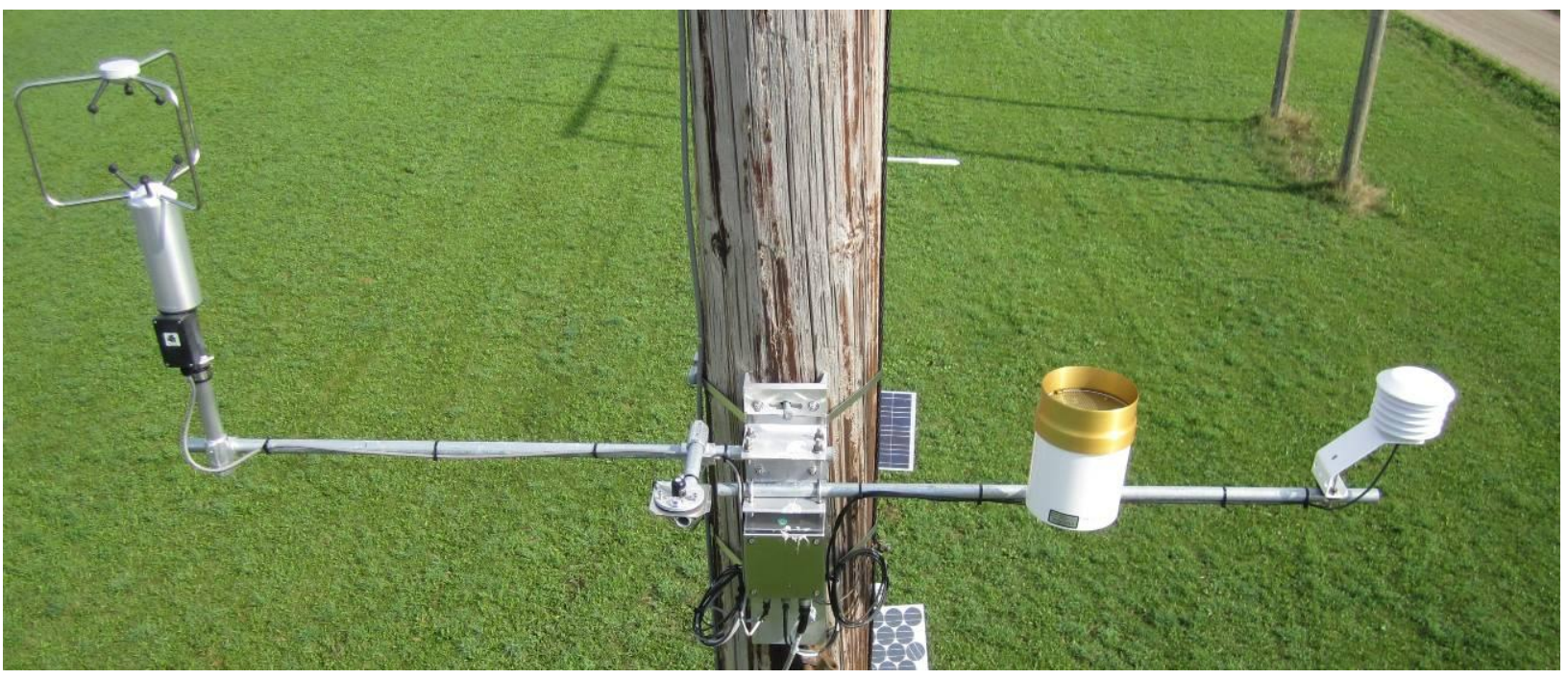

Figure C-3

Weather Station Array - left to right: anemometer, pyranometer, rain gauge, temperature/humidity sensor

Make and model number: Vaisala HMP60 Units: Degree C

Air temperature and relative humidity probes consist of two separate sensors packaged in the same housing. Relative humidity is measured with a capacitive RH sensor, while air temperature is measured by a Platinum Resistance Thermometer (PRT). The most frequently used PRT is the Pt100 - so called because it has a resistance of $100 \Omega$ at $0{ }^{\circ} \mathrm{C}$. At this particular Site a $1000 \Omega$ model is being used.

Solar radiation shields are required for the sensors as otherwise they will be exposed to sunlight and accuracy reduced. A non-absorptive color is used for the housing for the same reason. The HMP60 has stainless steel housing with an ABS plastic over chrome finish. 
The HMP60 probe measures temperature for the range of $-40^{\circ}$ to $60^{\circ} \mathrm{C}$. Within this range its accuracy is $\pm 0.6^{\circ} \mathrm{C}$. Is suitable for long-term, unattended monitoring, and is compatible with all Campbell Scientific data loggers. As this unit is pre-calibrated, replacements can be made with no additional software or logger changes.

PRTs are either wire-wound or metal film resistors. Of these, the latter exhibits the faster response time. A PRT sensor, as a resistor, has a value that can be measured with an Ohmmeter. However, the low resistance of the sensor and its sensitivity $\left(0.385 \Omega /{ }^{\circ} \mathrm{C}\right)$ make accurate measurements difficult when there is lead resistance. To avoid the problem of lead resistance errors two of the wires are used to provide an excitation current and the other two connect a voltmeter over the resistor. This increases the cable length required to cause a $0.5^{\circ} \mathrm{C}$ error to about 100 feet. Santoprene rubber leads are used so degradation will not occur due to exposure to extremes in weather conditions. Corrosion of the wire or connections would also add unwanted resistance. The color coding for these leads is described in Figure C-4.

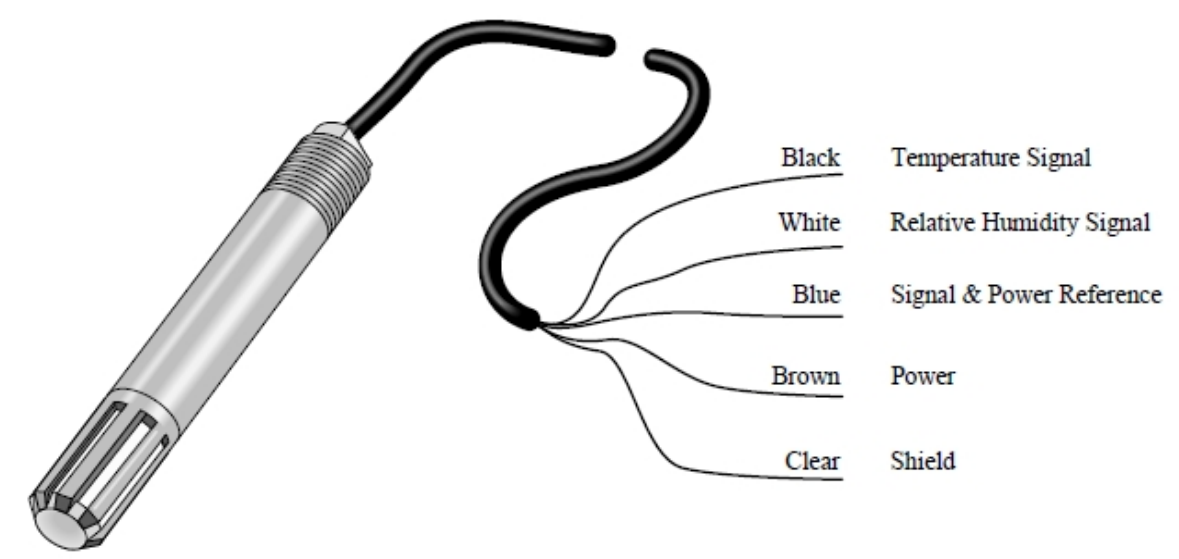

Figure C-4

Wire color-coding of temperature and relative humidity Instrument

\section{Humidity}

Make and model number: Vaisala HMP60 Units: \% Relative

The HMP60 humidity sensor is based on a SHT7x Sensirion chipset. The sensors integrate sensor elements and signal processing in compact form and provide a fully calibrated digital output. This pre-calibration makes replacement units if needed plug-and-play. The accuracy of all humidity sensors varies with ambient temperature. Within a temperature range of $0-40^{\circ} \mathrm{C}$ the sensor has two ranges of accuracy. For a relative humidity below $90 \%$ accuracy is $\pm 3 \%$, above this it is $\pm 5 \%$. 

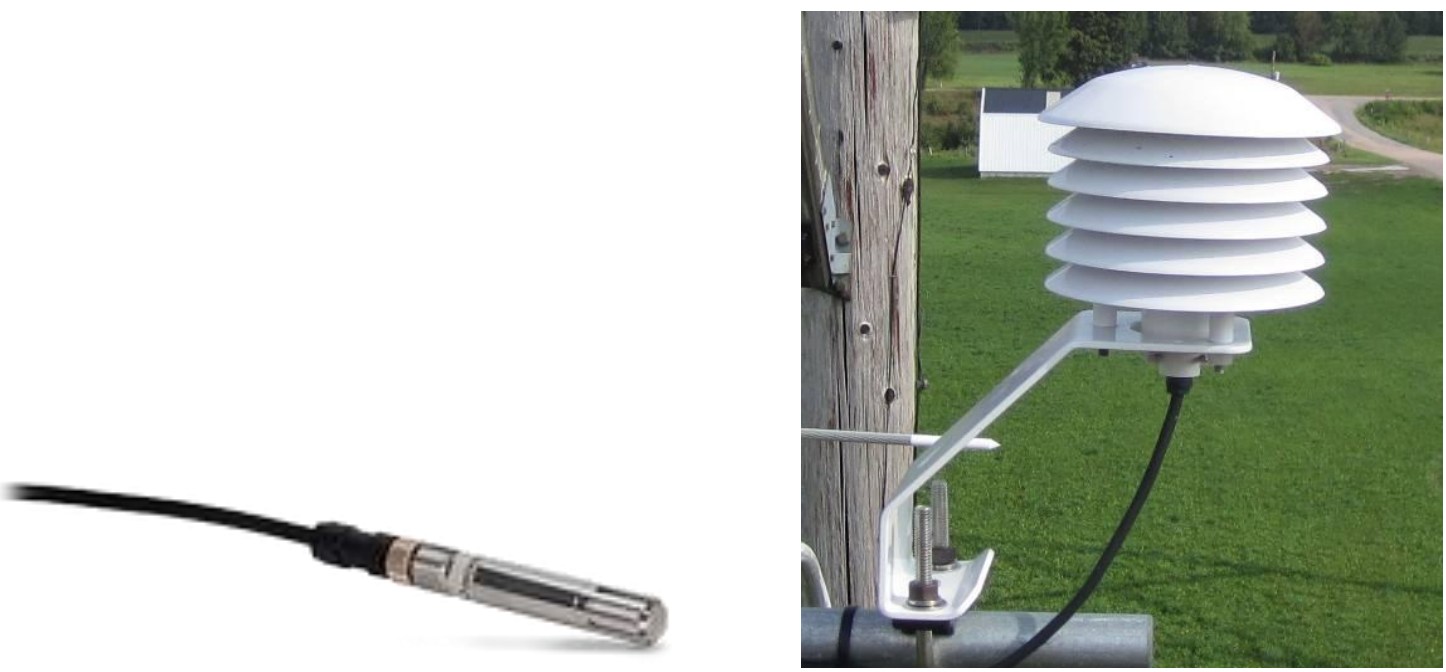

\section{Figure C-5 \\ Temperature sensor removed from housing; temperature and humidity sensors are combined into one unit}

\section{Weather Station: Pyranometer}

Make and Model number: Apogee CS300 Units: Watts/ meter ${ }^{2}$

The CS300 measures total sun and sky solar radiation for solar, agricultural, meteorological, and hydrological applications. Its spectral range of 300 to 1100 nanometers encompasses most of the shortwave radiation that reaches the Earth's surface, visible light being the range from 400 to 800 nanometers. This pyranometer connects directly to the data logger which measures its output in volts, the factory setting is $0.2 \mathrm{mV}$ per $\mathrm{W} / \mathrm{m}^{2}$

The head of the sensor is dome shaped to help prevent water from accumulating on it. To keep other moisture out of the sensor the element is in a fully potted casing. The cables are the same Santoprene rubber as the temperature probe.

The range of the anemometer is 0 to $2000 \mathrm{~W} / \mathrm{m}^{2}$ where full sunlight $\approx 1000 \mathrm{~W} / \mathrm{m}^{2}$. The accuracy is $\pm 5 \%$ for daily total radiation. Of this $<1 \%$ is due to ambient temperature variations. It should be noted that accuracy also depends on the pyranometer being properly levelled at installation and remaining so. Pole tilt will reduce performance though it is not known to what extent, some amount of pole tilt was observed during this project. For increased performance of future installations a self-levelling mount, in place of the one provided with the weather station, could be investigated. 

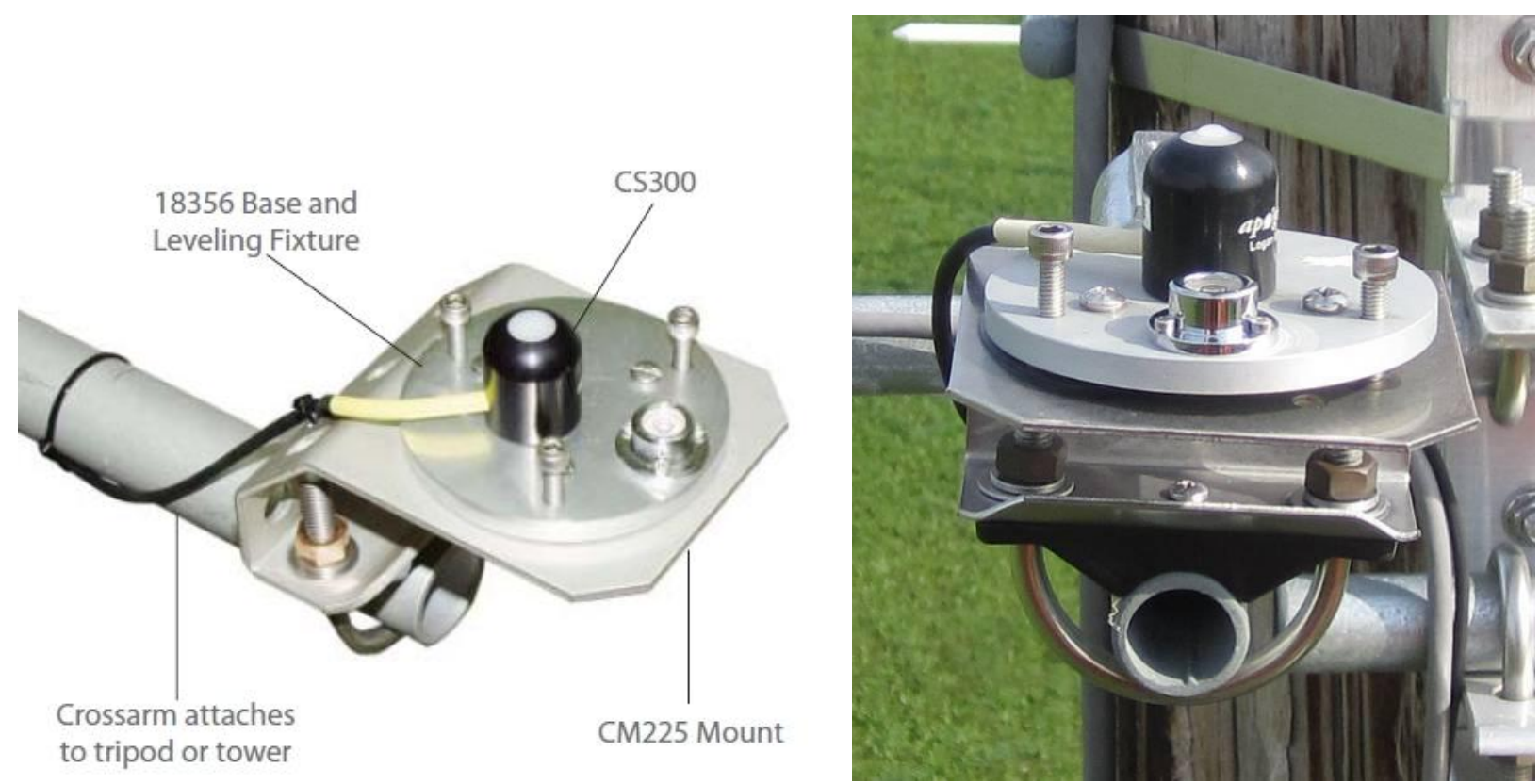

Figure C-6

Solar radiation sensor - detailed view (left) and as installed (right)

\section{Weather Station: Rain Gauge}

\section{Make and Model number: Texas Electronics TE525 Units: Inches per cycle}

The TE525 tipping bucket rain gage has a 6" orifice and measures rainfall in 0.01 " increments. It is compatible with all Campbell Scientific data loggers, and is widely used in environmental monitoring applications.

The tipping bucket rain gauge consists of a funnel that collects and channels the precipitation into a small seesaw-like container. After a pre-set amount of precipitation falls, a lever tips, dumping the collected water and sending an electrical signal.

The advantage of the tipping bucket rain gauge is that the character of the rain (light, medium, or heavy) may be easily obtained. Rainfall character is decided by the total amount of rain that has fallen in a set period and by counting the number of 'clicks' in a 10 minute period. High click counts indicate heavier rainfall. The tipping bucket rain gauge may not be as accurate as a standard rain gauge because the rainfall may stop before the lever has tipped. When the next period of rain begins it may take no more than one or two drops to tip the lever. This is generally not a significant source of error. It takes $.16 \mathrm{oz}$ of water to initiate a tip. Accuracy is $\pm 1 \%$ (up to $1 " / \mathrm{hr})+0,-3 \%$ (1 to $2 \% / \mathrm{hr})+0,-5 \%$ (2 to $3 \% / \mathrm{hr}$ )

Tipping buckets also tend to underestimate the amount of rainfall in mixed precipitation events. The NWS suggests the use of the TE525WS model which has an 8" inlet as well as an adapter that allows it to more accurately handle snow and freezing rain. This may also be useful for predicting icing events on lines. DTCR does not use the rain gauge output in versions 6.1 or earlier. 

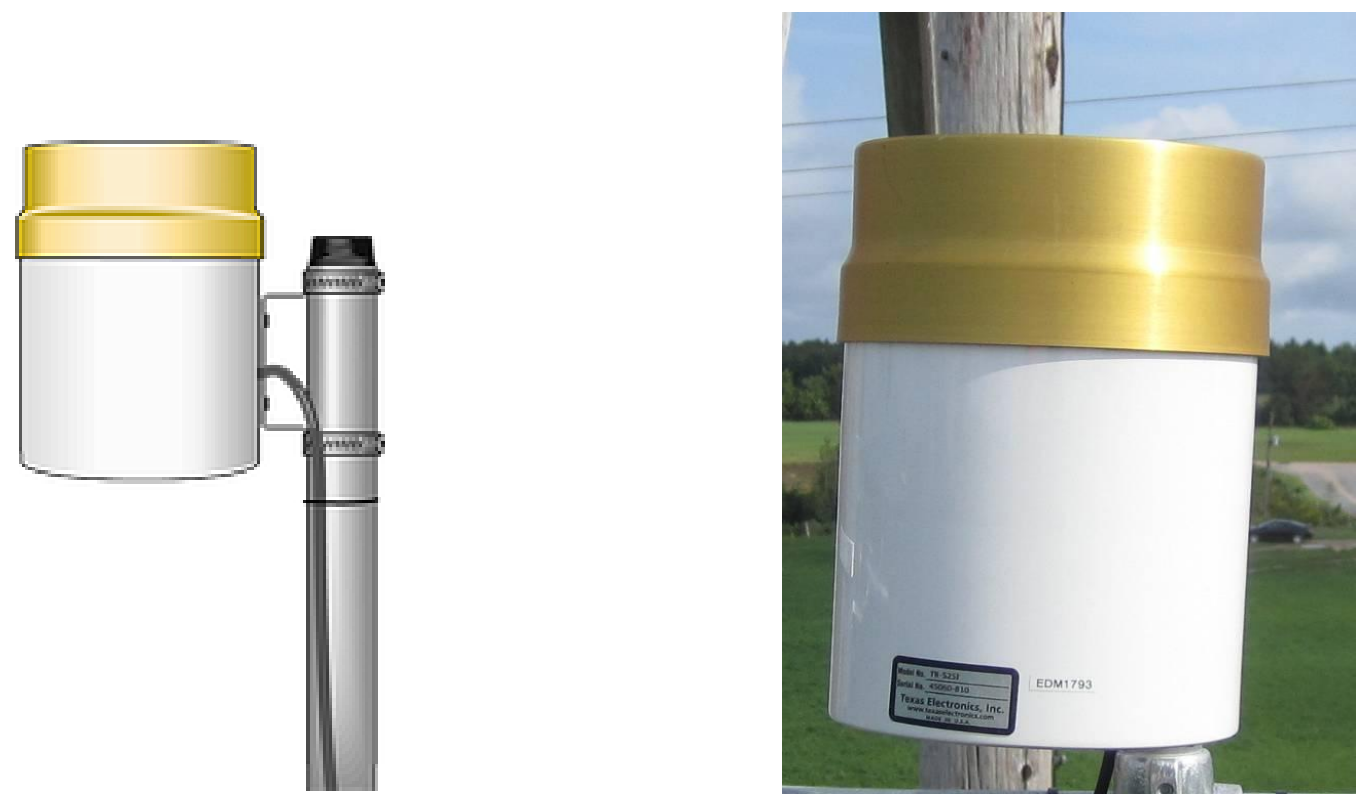

Figure C-7

Tipping bucket rain gauge mounted on an aluminum tube

\section{Weather Station: Wind Speed and Direction}

Make and Model number: RM Young $81000 \quad$ Units: Ft/sec and degrees

The RM Young Model 81000 Ultrasonic Anemometer is a 3-axis, no-moving-parts wind sensor. It is ideal for applications requiring fast response, high resolution and three-dimensional wind measurement.

The sensor features durable corrosion-resistant construction with 3 opposing pairs of ultrasonic transducers supported by stainless steel members. Each 81000 is individually wind-tunnel calibrated. Wind and sonic temperature data is available on four voltage output channels.

Sonic anemometers use ultrasonic sound waves to measure wind velocity. They measure wind speed based on the time of flight of sonic pulses between pairs of transducers. Measurements from pairs of transducers can be combined to yield a measurement of velocity in 2, or 3dimensional flow. The spatial resolution is given by the path length between transducers, which is $15 \mathrm{~cm}$. Sonic anemometers can take measurements with very fine temporal resolution, which makes them well suited for turbulence measurements. From the vendor this resolution is only $1 \mathrm{~Hz}$, which is below the lowest setting of the anemometer $(4 \mathrm{~Hz})$; this can be increased to a maximum of $32 \mathrm{~Hz}$ for the 81000 series. The lack of moving parts makes it appropriate for long term use in exposed automated weather stations where the accuracy and reliability of traditional cup-and-vane anemometer would be adversely affected by debris and wear. The main disadvantage of sonic devices is the distortion of the flow itself by the structure supporting the transducers; this requires a correction based upon wind tunnel measurements.

Due to the complexity and importance of this particular instrument, detailed anemometer information is provided in Appendix D. 

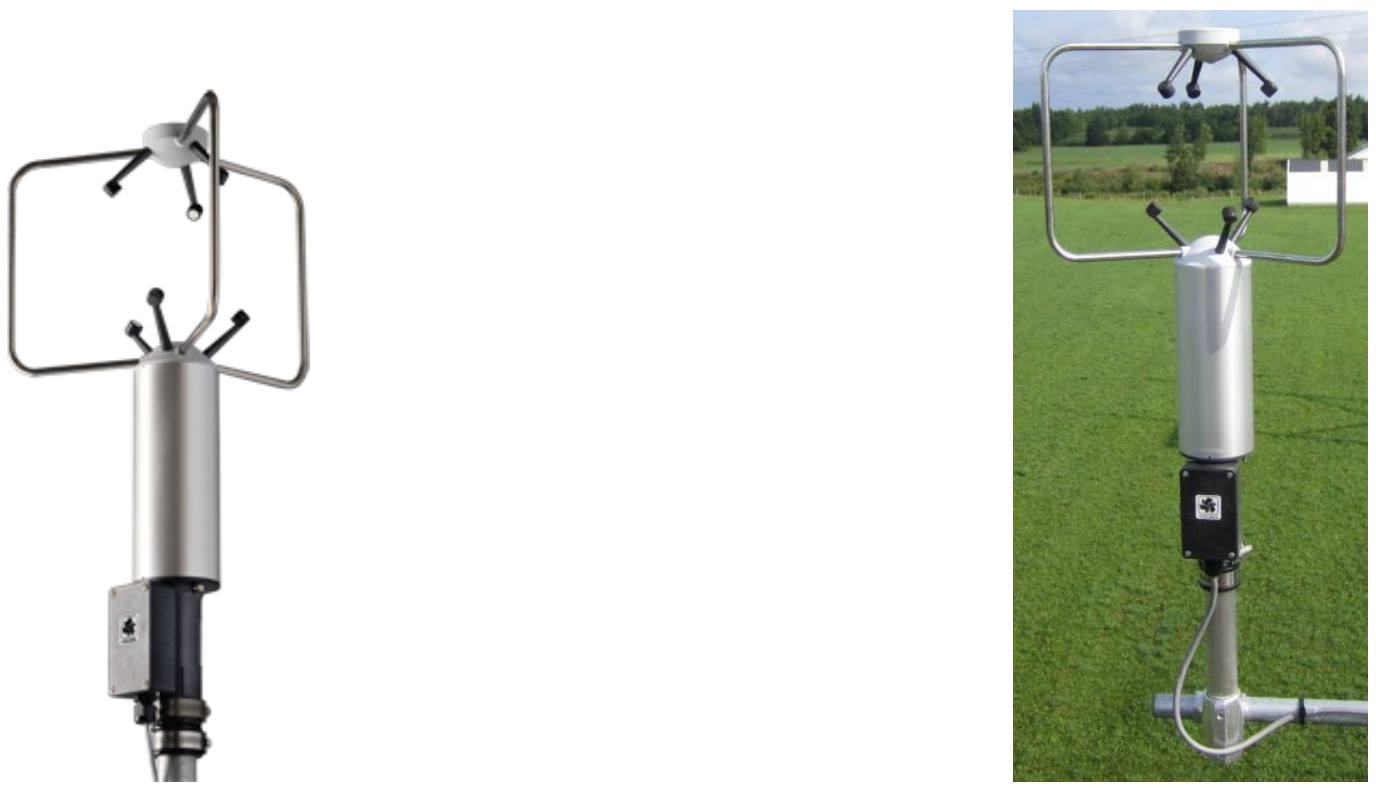

Figure C-8

Three-axis wind speed and direction sensor

Note: The wire junction box should always point south. This keeps the wind direction aligned to a known compass heading.

\section{Sagometer Camera Unit}

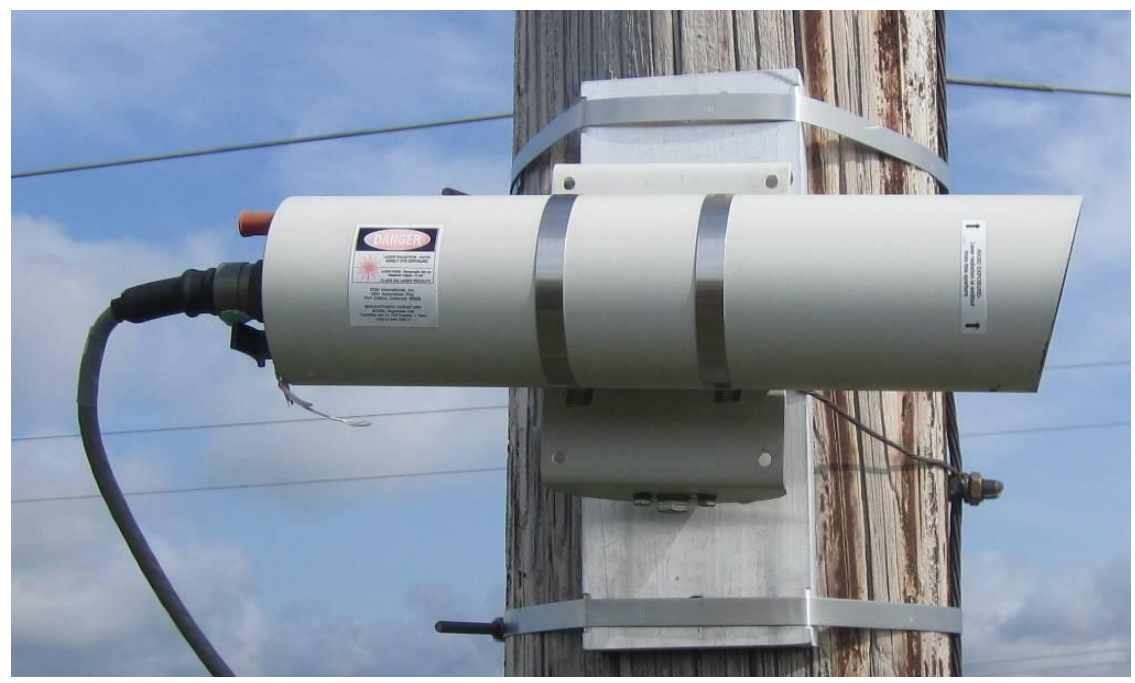

Figure C-9

Side view of the sagometer camera

To measure conductor sag, a camera detects the position of a target that is mounted on the transmission line 150 feet away from the camera lens. The camera has an image of the target stored in memory it compares this image with the one it is currently seeing. Once the pattern of the target is identified the camera locates the target position in the image. It does this by counting pixels; each pixel has been calibrated to equal a certain vertical distance. In this way, the camera can be used to determine the height of the target within its field of view. 
The camera also has tilt sensors, which can help correct for small variations of camera movement that may be a result of solar heating of a steel pole or warping in wood poles. This correction was not used here due to a calibration issue that can cause less data reliability.

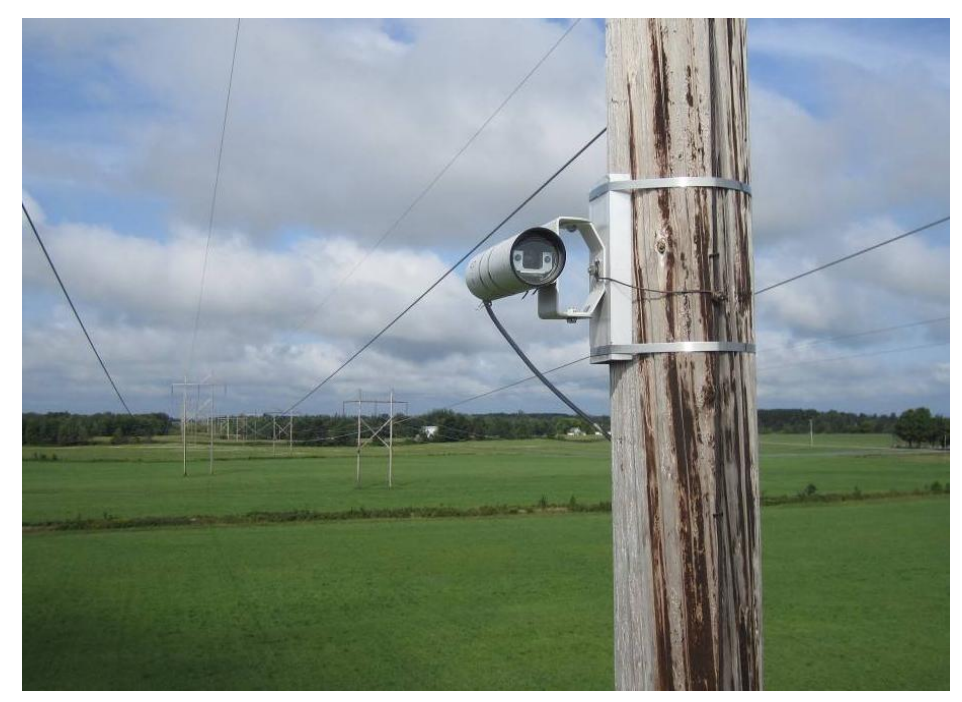

Figure C-10

Camera unit installed showing the front of the camera - The laser target illumination is mounted inside the camera unit

\section{EPRI Sensors}

The EPRI Sensors are designed to attach directly to the conductor and provide various parameter readings as desired within the capability of the sensor unit. The sensor is essentially a data gathering platform with about 25 data channels available on the platform. The sensors were installed by line crews with the conductors energized using a bucket truck and hot sticks Installation and mounted position of a typical EPRI Sensor used for these field tests is shown in Figure C-11.

Each EPRI Sensor was paired directly to its supporting Type I weather station and communicated directly with its dedicated data logger. Although the sensors could gather many parameters, with the proper specific sensing device installed, the six sensors used for the field tests collected only inclination, conductor surface temperature and line current, plus an identification marker, utilizing only three of the twenty-five data channels for the field tests. The EPRI Sensors are powered by harvesting energy from the load current magnetic field surrounding the conductor to which they are attached. 


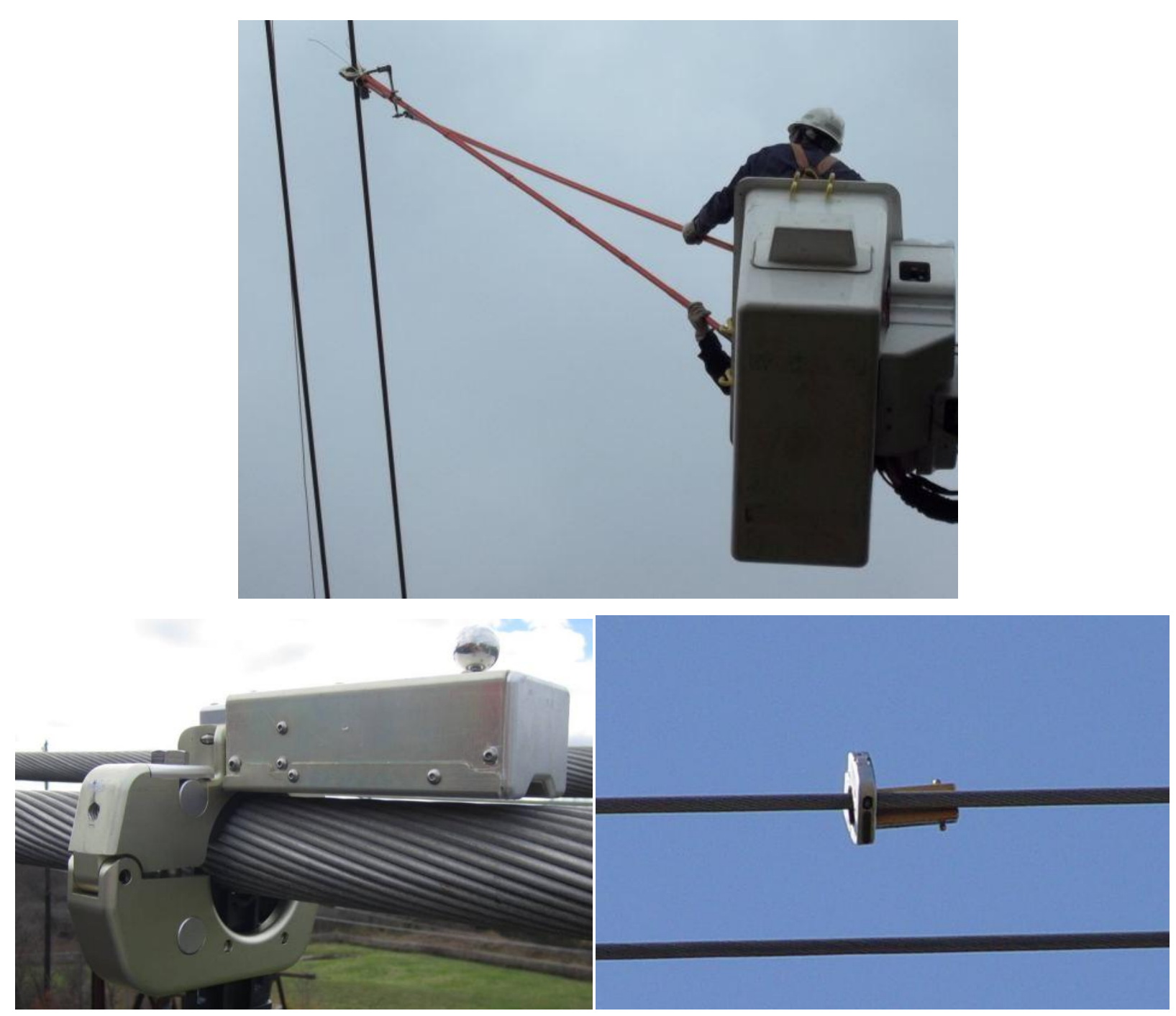

Figure C-11

EPRI Sensor installation and final mounting position on the conductor

\section{EPRI Sensors: Conductor Temperature}

On board thermally isolated thermocouples are used to determine conductor temperature. Significant $R \& D$ has gone into making this device to ensure accurate conductor temperature readings. This is aided by a special mount that allows the sensor tip to shift itself into the stranding seams. The device was originally designed to measure splice temperatures. In modifying it for this application it was not believed critical for the sensor to read below $0^{\circ} \mathrm{C}$ as the conductor temperature is only used when it is driven well above air temperatures. However, the actual conductor temperature can be used to verify other sensor systems, so measures are being taken to increase the functional range in future generations of sensors.

The accuracy of the EPRI Sensor-measured conductor temperature as a function of conductor diameter and thermocouple measured temperature has been evaluated as part of this project. Figure C-12 compares the sensor-measured values against reference wired thermocouples for temperatures of less than $93^{\circ} \mathrm{C}\left(199^{\circ} \mathrm{F}\right)$. For this range the difference between the reference thermocouple and the EPRI Sensor measurements exhibit an absolute mean of $1.5^{\circ} \mathrm{C}\left(2.9^{\circ} \mathrm{F}\right)$ and 
an arithmetic mean of $-0.1^{\circ} \mathrm{C}\left(-0.2^{\circ} \mathrm{F}\right)$ and a standard deviation of $2.0^{\circ} \mathrm{C}\left(3.6^{\circ} \mathrm{F}\right)$. Temperatures of less than $40^{\circ} \mathrm{C}\left(104^{\circ} \mathrm{F}\right)$ exhibit an absolute mean of $0.9^{\circ} \mathrm{C}\left(1.6^{\circ} \mathrm{F}\right)$ and an arithmetic mean of $0.8^{\circ} \mathrm{C}\left(-1.4^{\circ} \mathrm{F}\right)$ and a standard deviation of $0.7^{\circ} \mathrm{C}\left(1.3^{\circ} \mathrm{F}\right)$. For the field tests documented in this report the conductor temperatures never exceeded $40^{\circ} \mathrm{C}$.

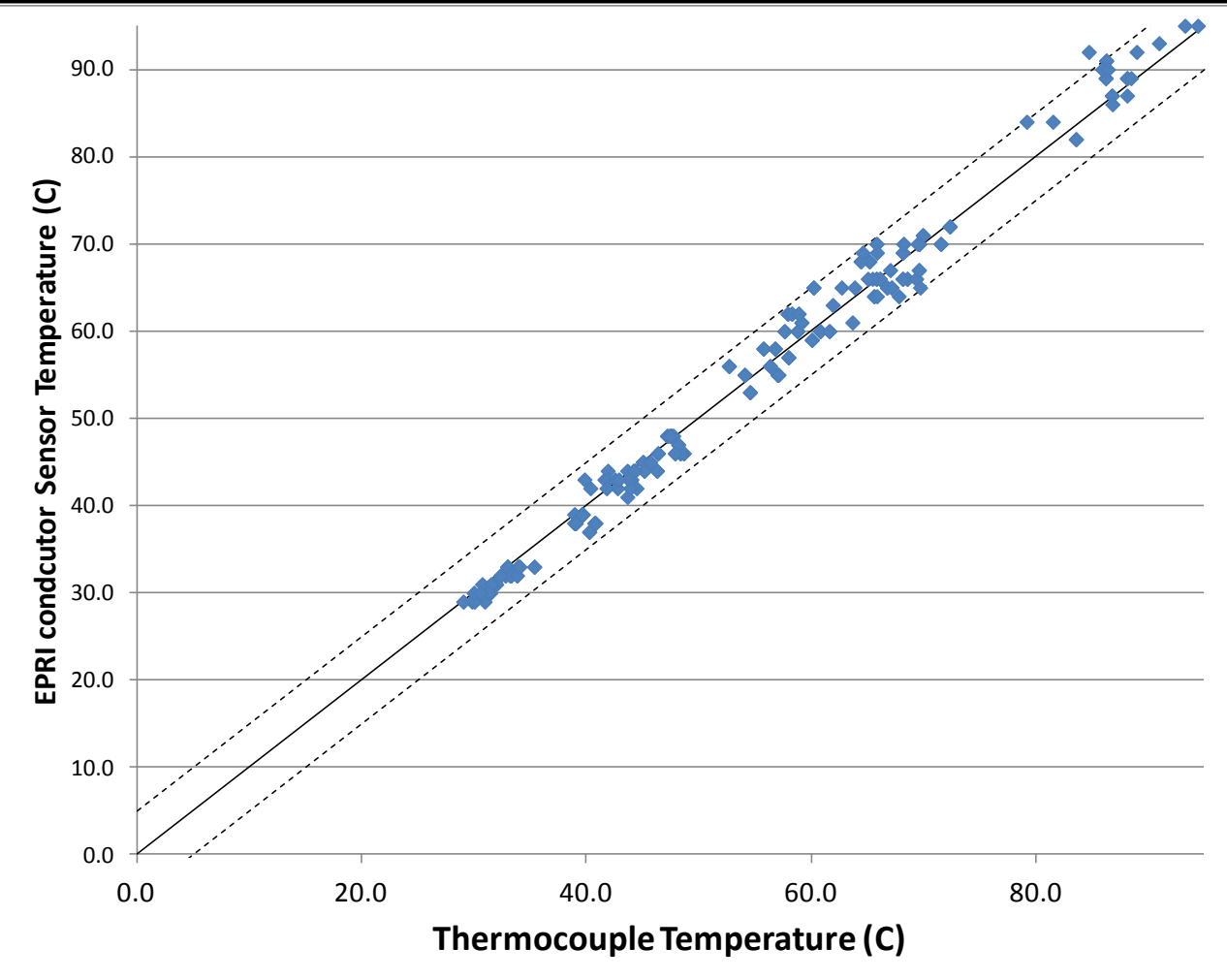

Figure C-12

Comparison of EPRI Sensor performance against thermocouples directly wired to the conductors - range $0-93^{\circ} \mathrm{C}$; six different conductor diameters were utilized

\section{EPRI Sensors: Line Inclination}

The line inclination sensor is experimental at this stage. Fundamentally it is a 3 -axis accelerometer. There is a unique relationship between line inclination and the temperature of the conductor. From line inclination sag can be determined. Once sag has been determined line temperature can be determined. Once line temperature has been determined when the current is high enough, effective perpendicular wind speed can be determined. The advantage of using line inclination is similar to the Sagometer. The entire ruling span section temperature can be measured as opposed to measuring only one point along the line. The disadvantage is that a state equation must be determined for each sensor. The state equation must be periodically adjusted. If the structures are not absolutely stable or if maintenance alters the tension, then additional adjustments must be made accordingly. The inclinometer function was not used to calculate ratings in this project. 


\section{EPRI Sensors: Current}

The EPRI current sensor serves two functions. The sensor is a coil of wire that is in close proximity to the conductor. This coil is magnetically coupled to the conductor. This coupling allows the sensor to charge the on board batteries. It also functions to determine the amount of current flowing down the line. Because the coil is in close proximity to, but not looped around the conductor, it is highly sensitive to small position changes. Consequently the sensor must be calibrated after it is installed, periodic calibration checks were preformed and updated as needed over the course of the project. These calibrations were performed against utility SCADA Load data obtained through the ThermalRate system. 


\section{EPRI Sensor Mounting Locations at the Three Sites}
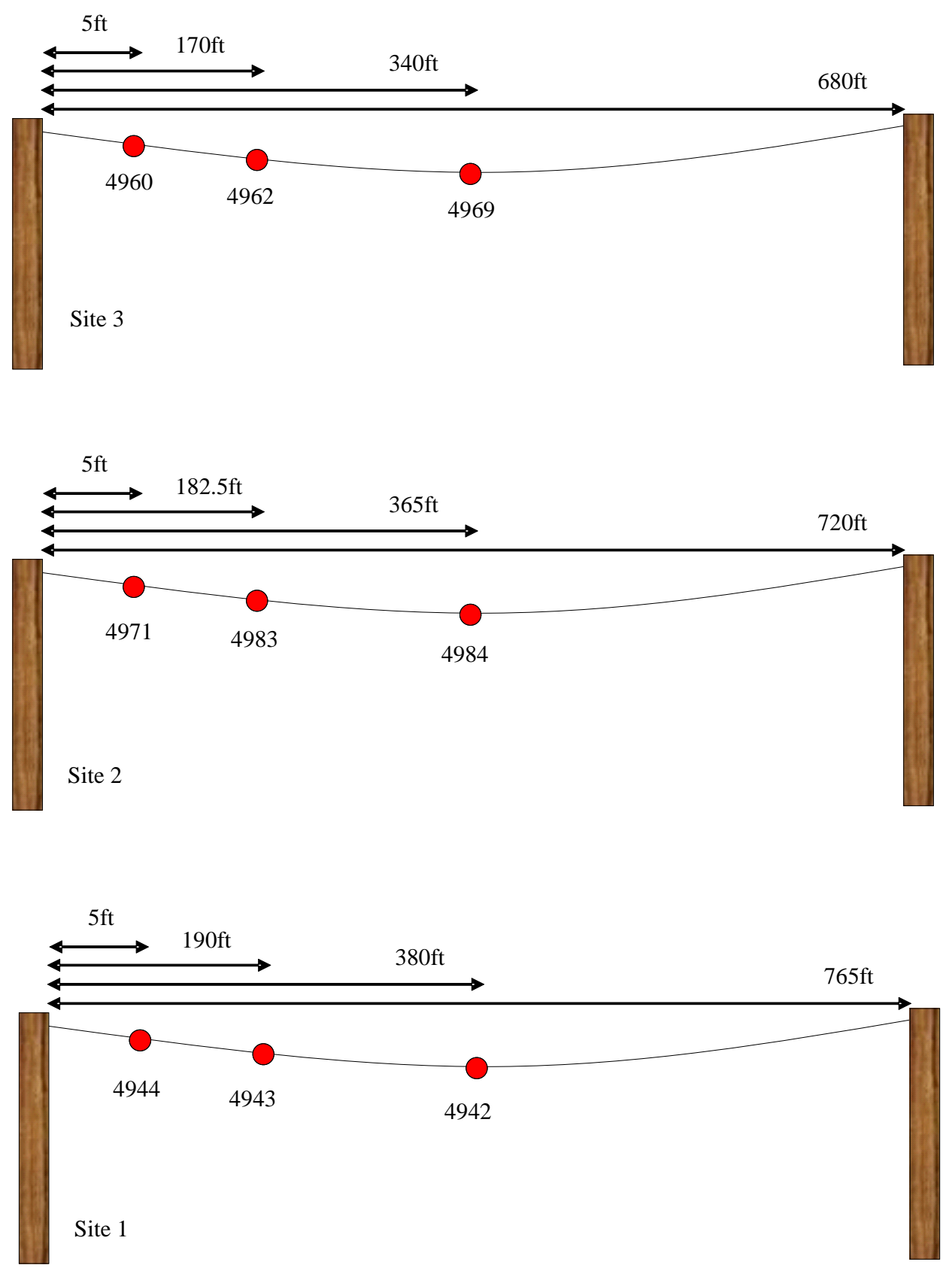

Figure C-13

Positions of original EPRI Sensors

A set of replacement sensors were placed on the MW line in March of 2011, the positions remained the same. 


\section{Thermal Rate}

\section{ThermalRate: Effective Perpendicular Wind Speed}

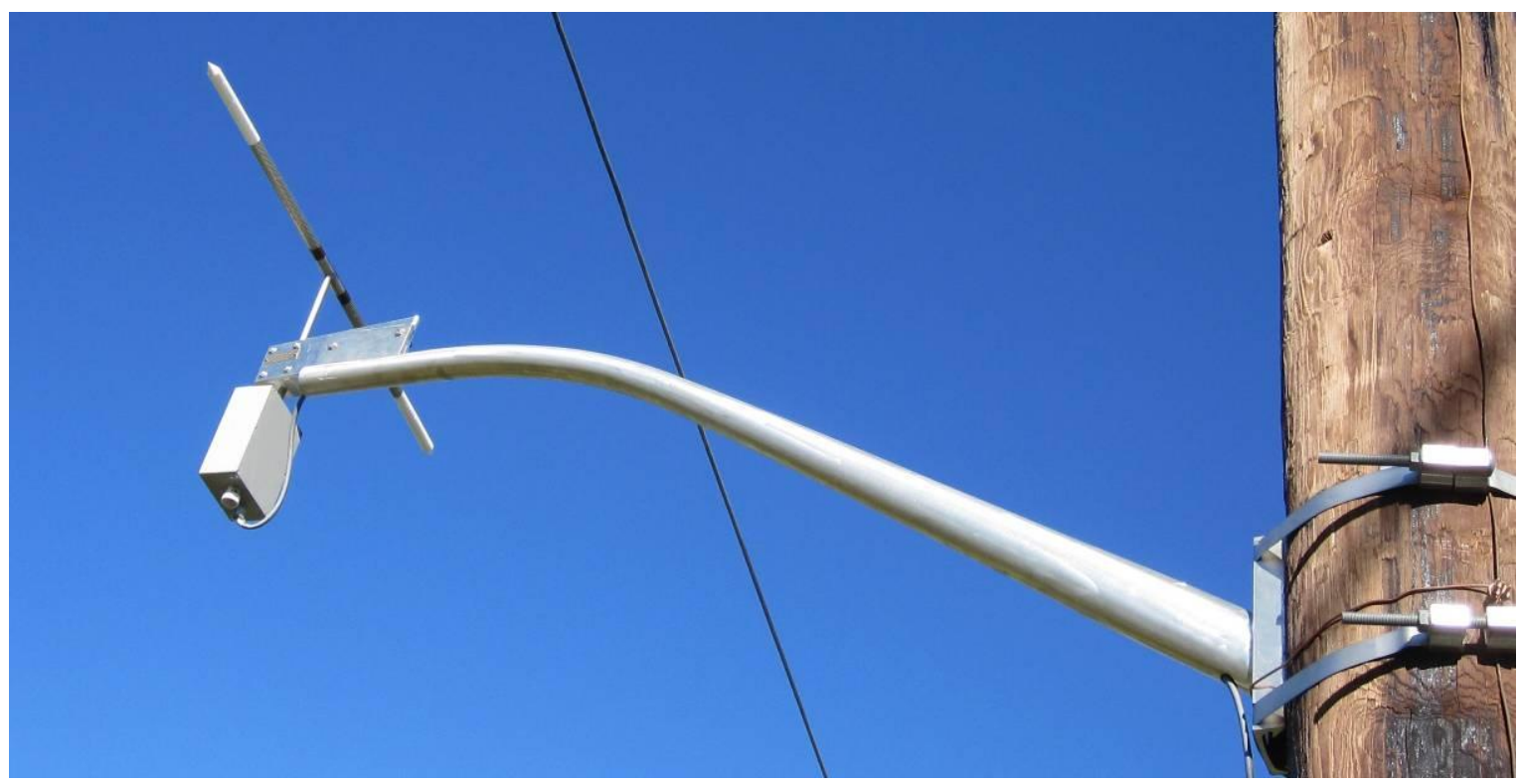

Figure C-14

ThermalRate Sensor

The ThermalRate sensor consists of two parallel replicas of a conductor to mimic how the line behaves thermally in the local weather condition. The replicas have the same material, diameter, and surface as the line conductor and are each approximately one foot in length. Silicone tips are placed over the ends to reduce thermal and wind edge effects. The sensor mounted at the average conductor height and oriented parallel to the line in order to see the same conditions as the line itself. The output is an effective perpendicular wind speed which is reported in feet per second.

\section{ThermalRate: Load from SCADA}

Spread spectrum radio connects the ThermalRate system to the utility SCADA system. This requires that the utility make changes to an RTU so that it can send data to the ThermalRate system. 


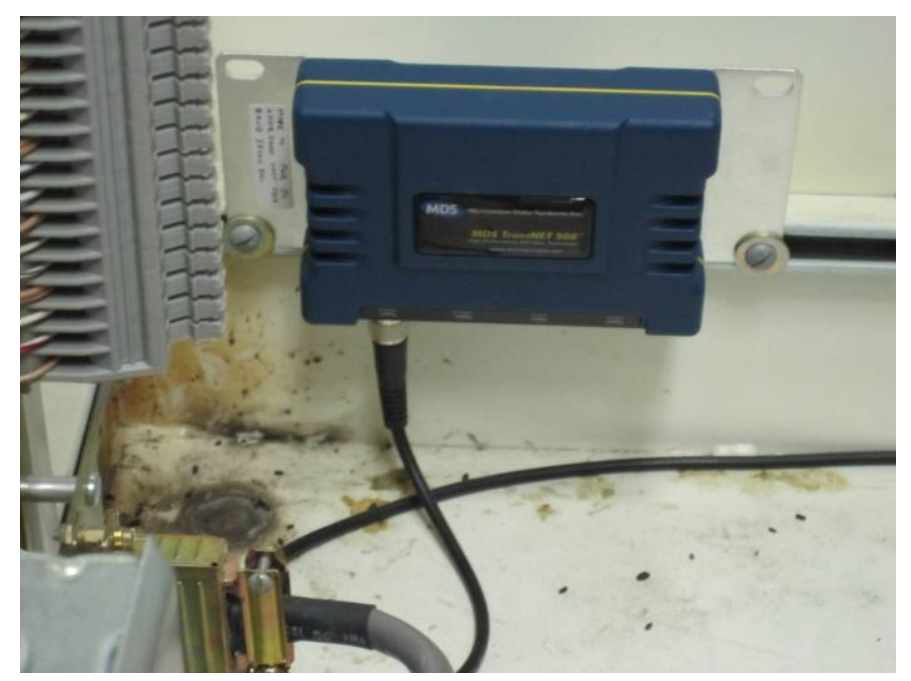

Figure C-15

Radio communication link (MDS TransNet 900) for ThermalRate System

\section{Sagometer: Power Systems}

Solar Cell: Sun Electronics, SUN-ES-C-110, 110W

Battery: Werker, WKDC12-100PUS, 100 Amp-hr @ 20Hr Charger: Stecca, Solsum 8.8F

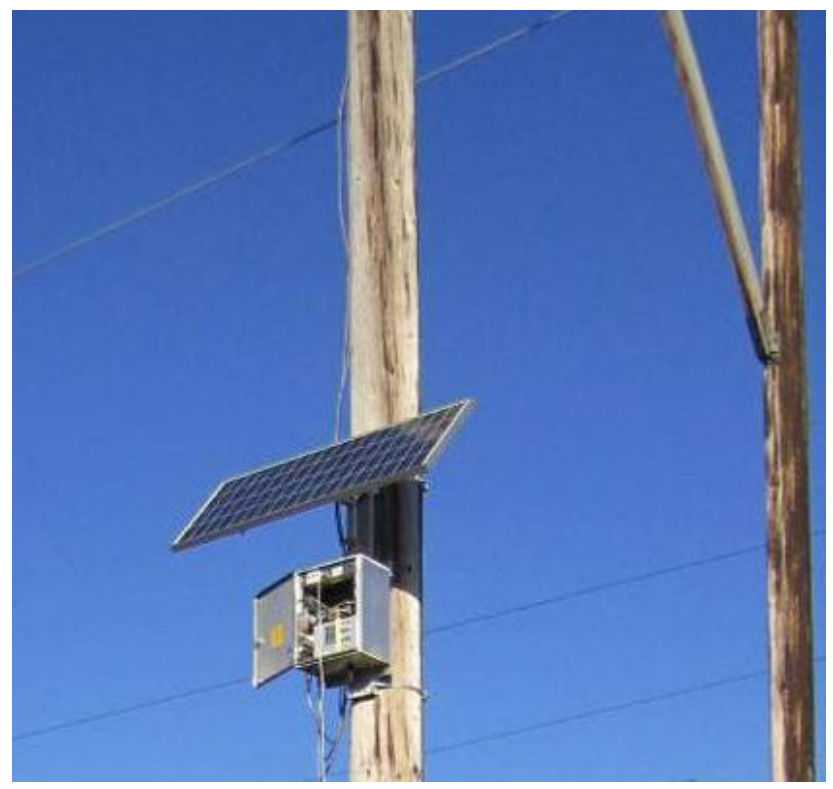

Figure C-16

Photo of solar system

\section{EPRI Sensor: Power System}

Solar Cell: Sharp 80W Solar Panel, Eco Direct P/N: NE-80EJEA

Battery: Power Sonic 12VDC 100 Amp hour battery, Allied Electronics P/N: 621-9000

Charger: Stecca 15 Amp Charger, Eco Direct P/N: PRS 1515 


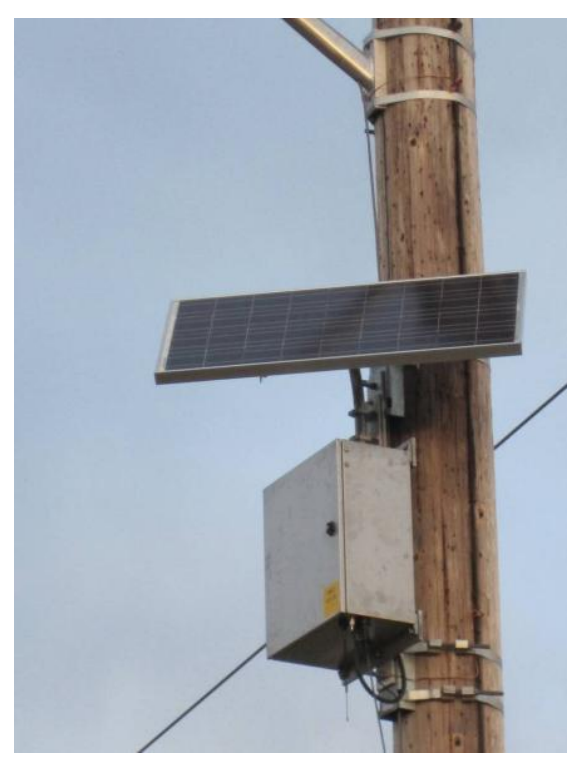

Figure C-17

Photo of solar system

ThermalRate: Power System

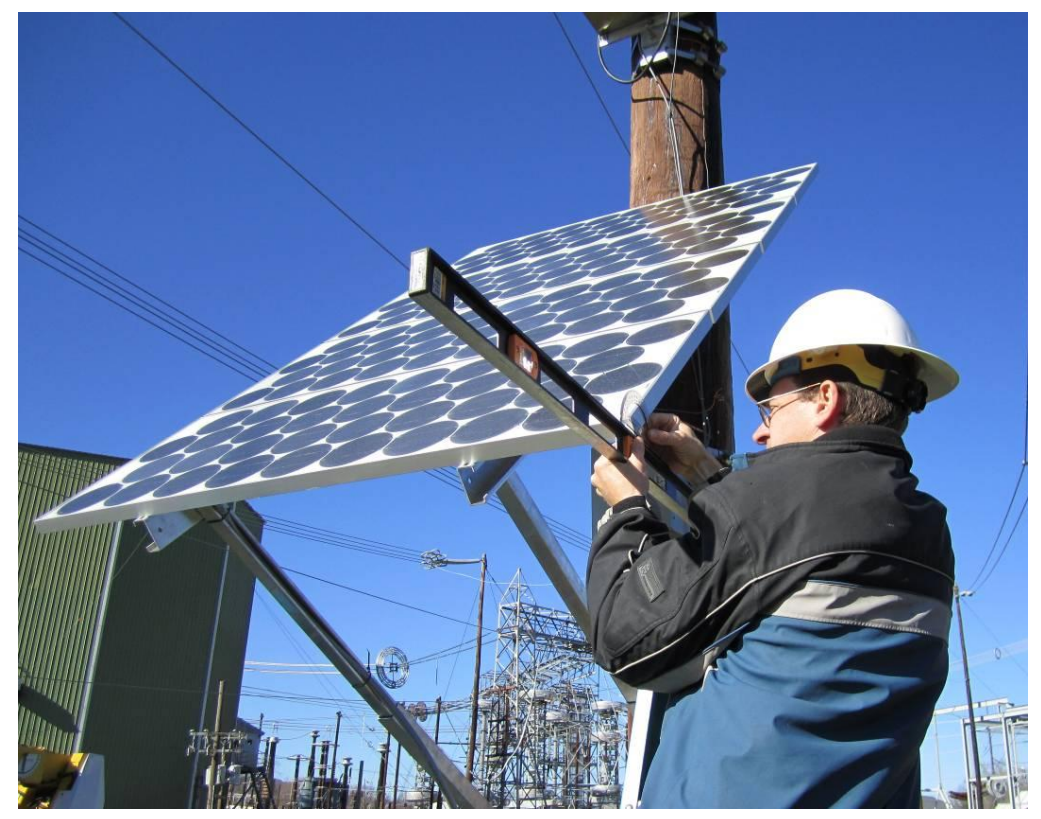

Figure C-18

Photo of solar system 


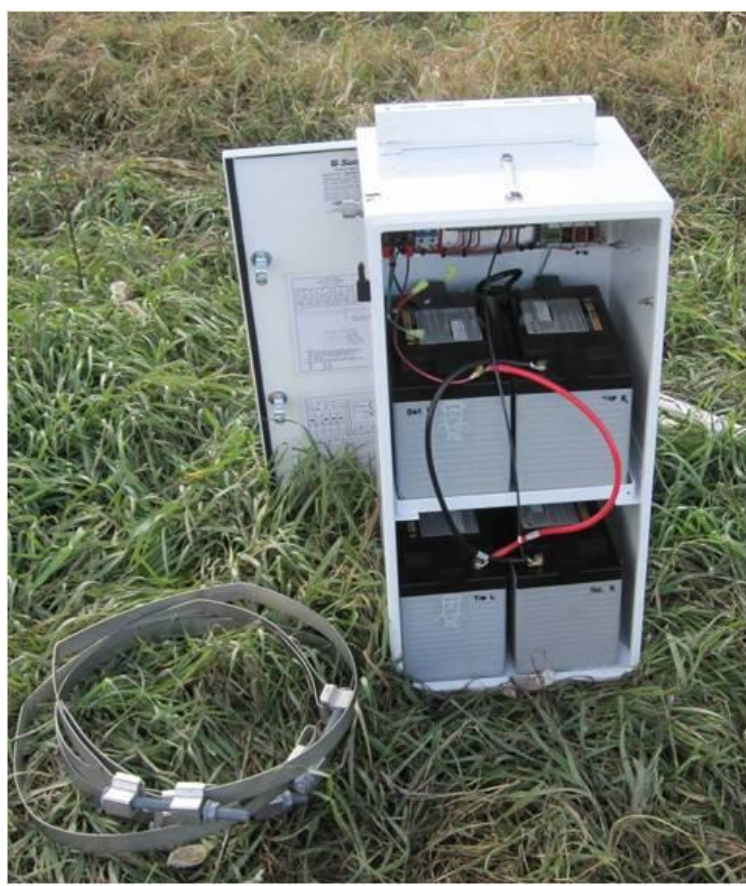

Solar Cell: 360Watts

Battery box: 265A-hrs

ThermalRate draws 17 Watts continuous

Figure C-19

Photo of battery and charger 


\section{Data Loggers}

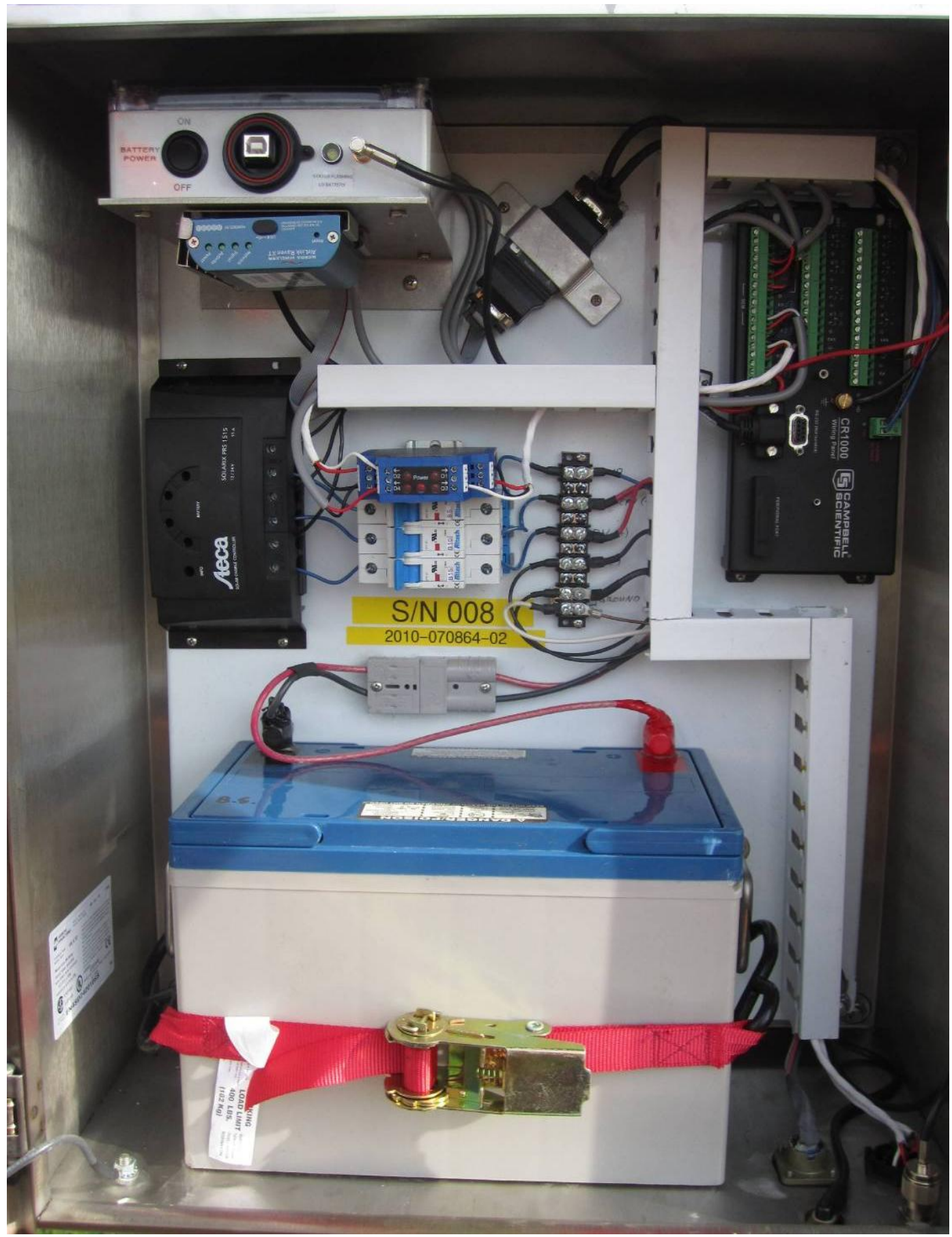

Figure C-20

EPRI Sensor electronics box 


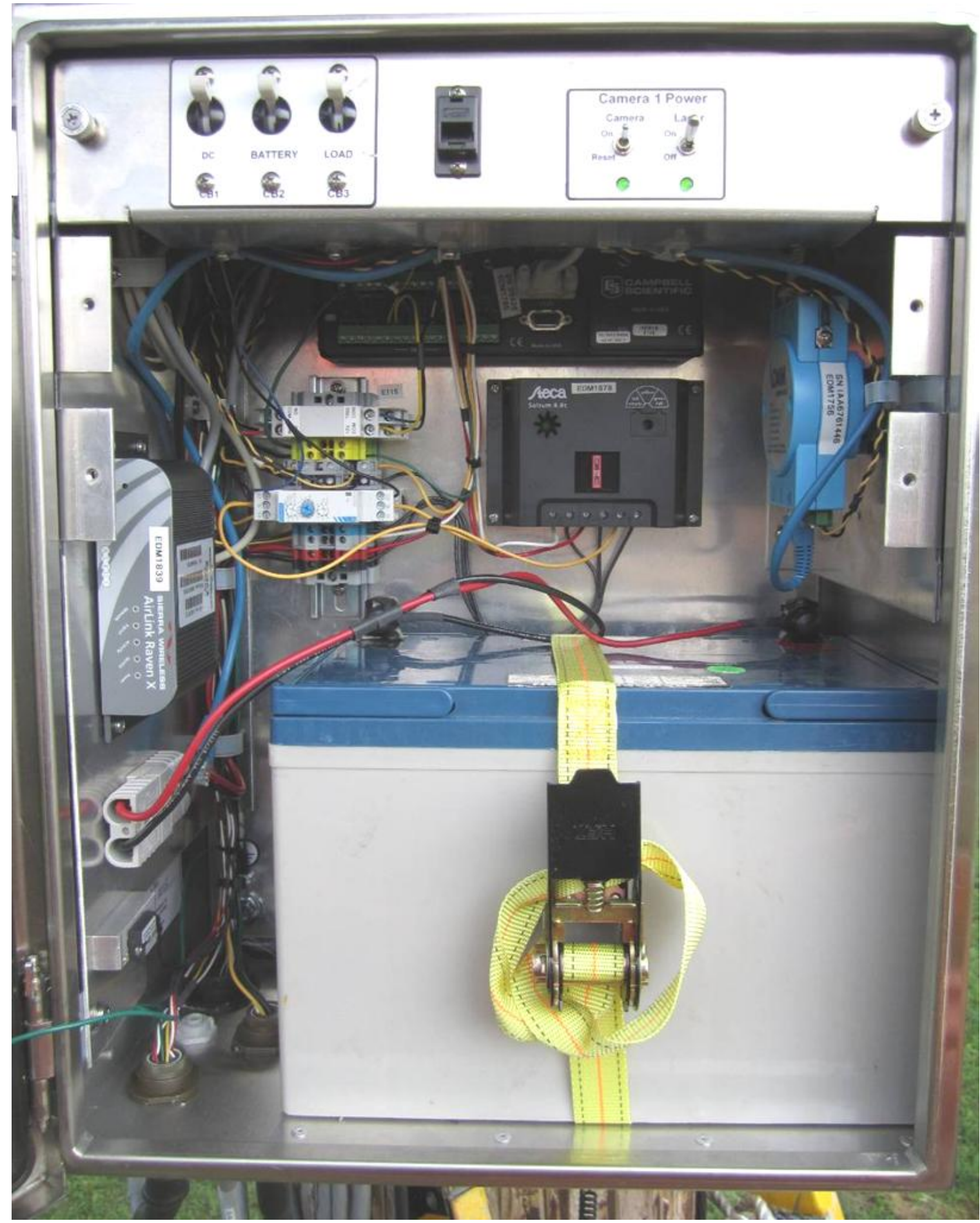

Figure C-21

Sagometer electronics box

All sensors communicate with data loggers. These can be digital or analog signals. The signal can be directly wired into the logger or wireless. The loggers can store the data for a long period of time between downloads. This allows retrieval of data in the event of a server or 
communication failure. However, eventually, if the data is not retrieved the data starts to get overwritten. The loggers used for this project are Campbell Scientific CR1000.

\section{Specifications for the CR1000}

The CR1000 logger stores its program on a 4Mb internal memory chip. This chip allows a program execution rate of up to $100 \mathrm{~Hz}$; they execute from a 16-bit H8S Renesas microcontroller with 32-bit internal CPU architecture. Due to this execution rate sensors that sample faster than $100 \mathrm{~Hz}$ may have to do internal averaging.

Sensor inputs are isolated with gas discharge tubes, additional I/O is allowed by the RS-232 serial ports. Analog data inputs can be processed through an onboard 13-bit A/D converter. If a higher resolution $\mathrm{A} / \mathrm{D}$ conversion is needed, a sensor with onboard conversion would have to be used. Conversely if a device has an 8-bit onboard converter the logger can be used instead thereby increasing sensor resolution.

Communications can be established with remote sensors and control computers through a variety of methods, namely PakBus, Modbus, DNP3, TCP/IP, FTP, and SMTP protocols. This allows a program to be modified remotely via the modem, for example when sensor calibrations need to be uploaded. Any changes to a program increment the version number, so the number of updates that occurred can also be estimated from this number. Some calibrations are pre-processed in DTCR so this does not always require modification of the logger program.

All data input within the logger is stored in a table format with a time stamp and record number. There is a battery backed SRAM memory chip and clock ensuring data, programs, and accurate time are maintained if the CR1000 is disconnected from its main power source. The real-time clock is also temperature compensating, but can be checked remotely from the server for drift. It is best to consider the SRAM memory a circular buffer more than an actual backup. Meaning if data is not retrieved by the server in a timely manner the memory chip will fill up, once full the oldest data points will be discarded. The length of time data can be stored is therefore relative to how often and how much data is being added to the buffer. Available buffer time could be improved in future projects by removing unused columns from data tables that are present in all data files.

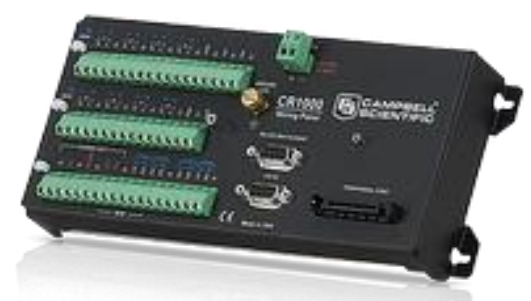

Figure C-22

Isolated view of the Campbell Scientific data logger

\section{Communications Layout Diagrams}

These diagrams were made during the design stage of this project. The purpose of these simple diagrams is to show the data flow along with the mode of communications. 


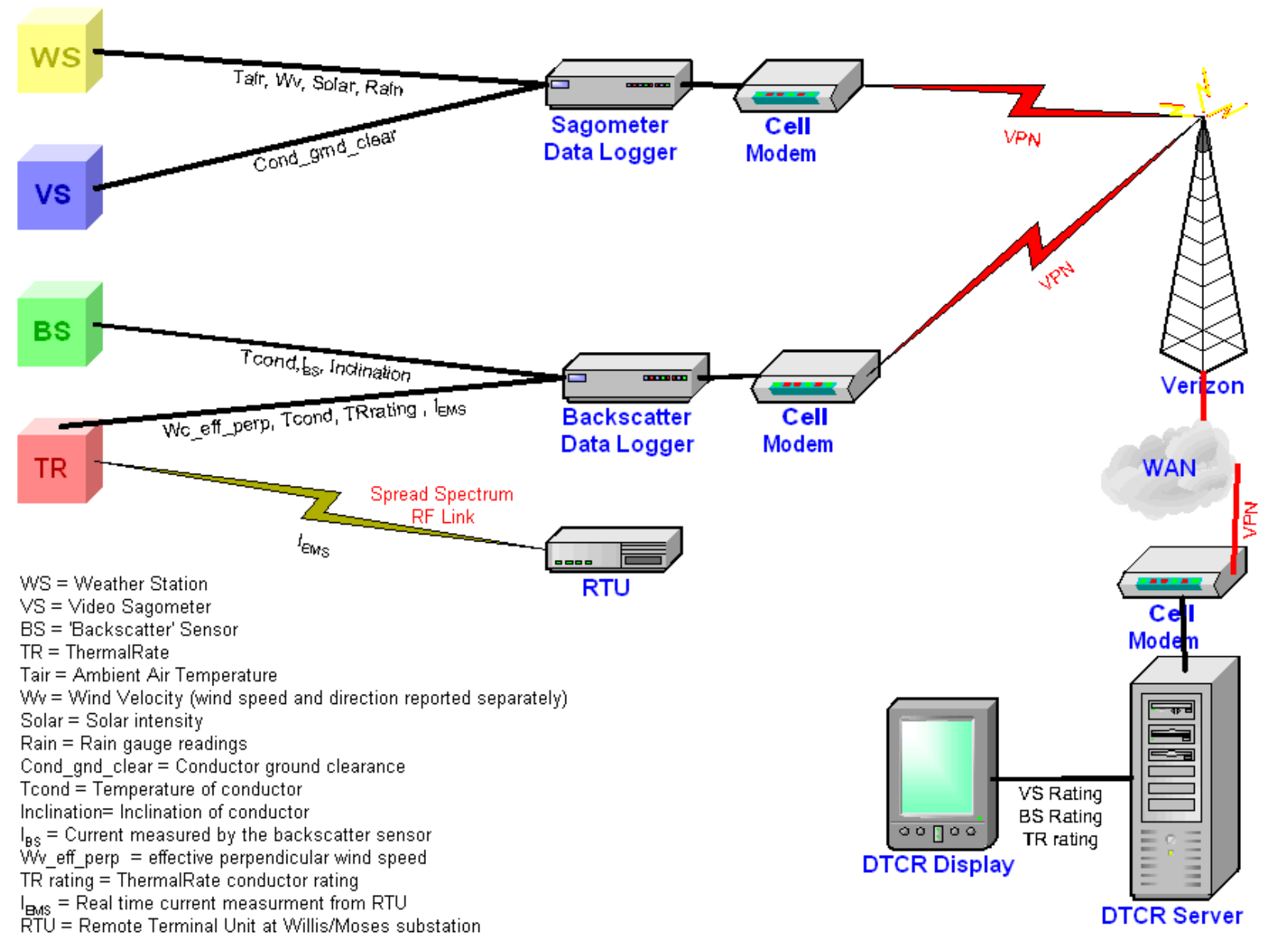

${ }^{*}$ Each device reports health information like battery voltage, internal temperature, signal strength, etc

Figure C-23

Overall communications layout 


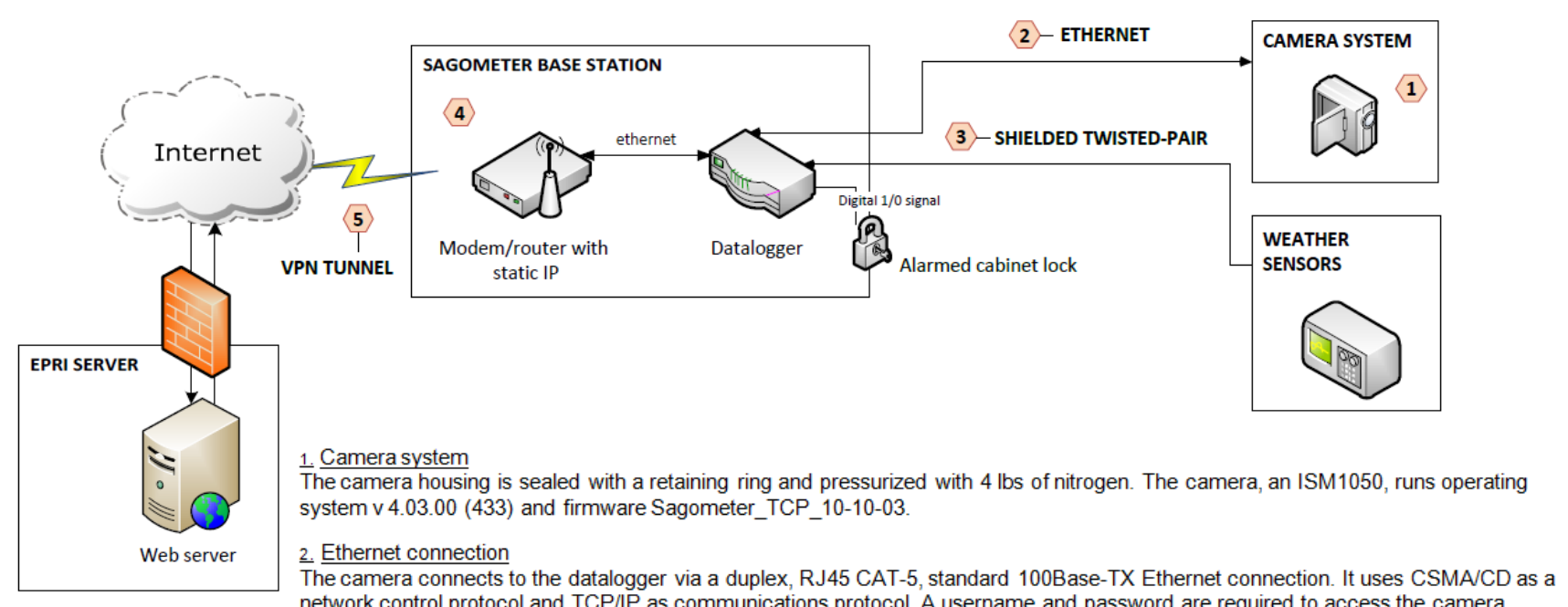
network control protocol and TCP/IP as communications protocol. A username and password are required to access the camera.

3. Shielded twisted-pair

A shielded twisted-pair cable is used for the weather sensor connections. The data transmitted is a combination of analogue signals, where the voltage levels proportional to the measurement value and serial RS-232.

4. Sagometer base station

The base station cabinet is locked and alarmed. The opening of the cabinet triggers an alarm which is immediately routed to the EPRI server through the datalogger. The datalogger is connected to the modem (OS V4221_4.0.6.001 May 4 2010) through a 100Base-TX Ethernet connection using TCP/IP as communications protocol. The datalogger runs CR1000.Std.16.081029 as OS and Sagometer_V42_10-10-05 as firmware.

5. VPN Tunnel

A $1.9 \mathrm{GHz}$ cellular RF encrypted IPSec VPN tunnel through the Internet is used to communicate securely. This is a private network constructed over a shared IP-based backbone that utilizes technologies to ensure privacy of data through a secure path. It uses AES encryption (128 bits) and SHA1 authentication (160 bit digest) algorithms.

Figure C-24

Detailed communications layout for Video Sagometer 


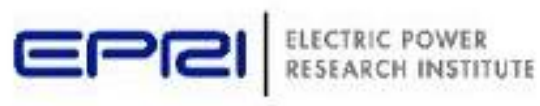

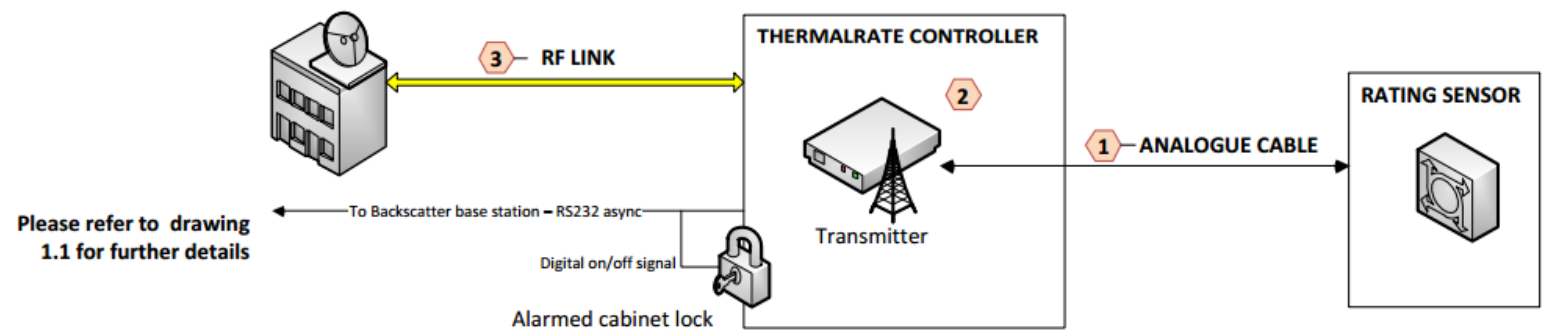

1. Analogue cable

Data from the sensor (Version 2.01) is transmitted to the ThermalRate station through an analogue cable which is also used to power the sensor.

2. ThermalRate ${ }^{\mathrm{TM}}$ controller

The ThermalRate controller calculates the values based on the analogue and RF inputs and sends all the information to the backscatter base station through an asynchronous 5-wire RS232 link. The controller runs software version $2.03 \times 15$.

3. RF Link

The ThermalRate station is connected to the RTU via a $902-928 \mathrm{MHz}$ frequency hopping spread spectrum link. This link is used to obtain the current readings required for the processing of the information.|

Figure C-25

Detailed communications layout for ThermalRate 


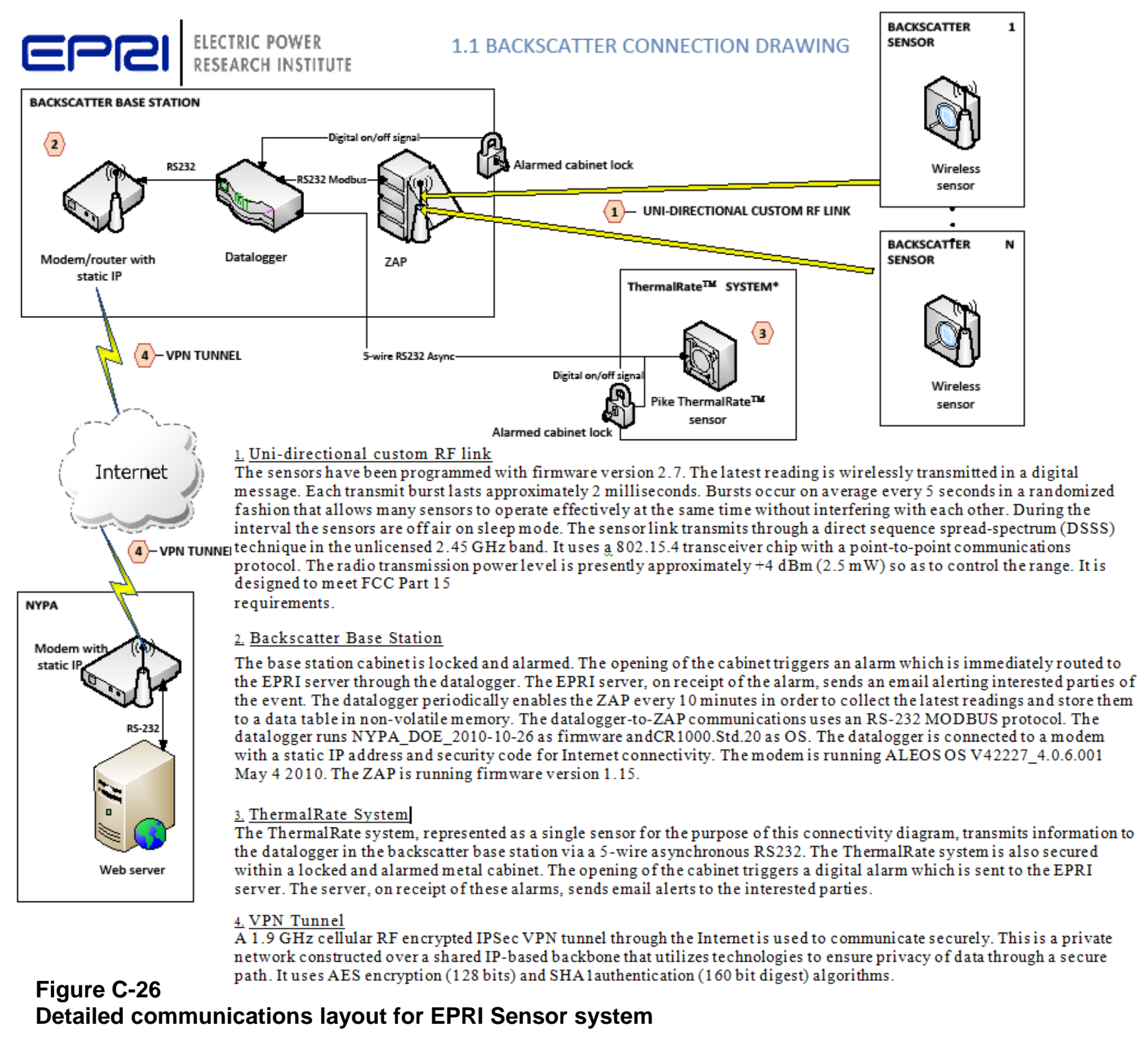

\section{Modems}

Cellular modems are used to move the data from the data loggers to the web. The modems used are Sierra Wireless Raven X and Sierra Wireless Raven XT. The main difference being the XT has a USB interface, the $\mathrm{X}$ model has an Ethernet interface. The Raven $\mathrm{X}$ is a wireless networking device designed to utilize $3 \mathrm{G}$ networks. Its Ethernet port makes the Raven X useful for enterprise applications, while the serial port and embedded machine protocols make it suited for industrial deployments. IPSec VPN provides security for the most sensitive data.

In this case a VPN tunnel is established between the modem and the server downloading the data there is a limit to how many VPN connections a single modem can maintain so the server requires two modems to hold enough secure connections. Verizon is the cellular carrier for this project. The modems are Tri-Band and Quad-Band operable so they will function on most carriers worldwide, the only required change would be the cellular provider. 

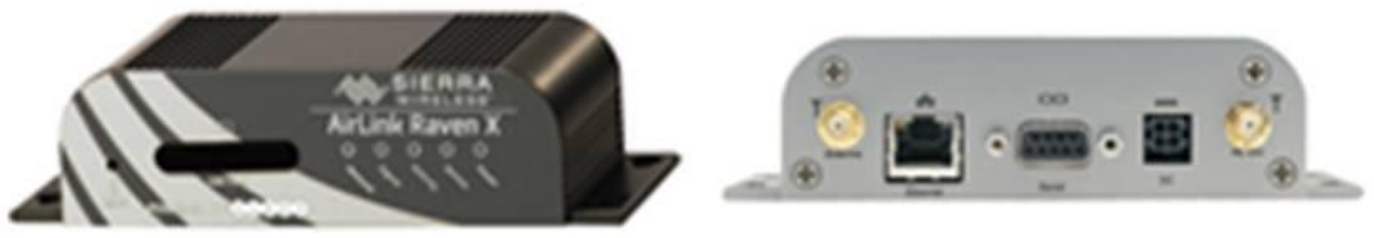

Figure C-27

Isolated front and rear view of Raven $\mathrm{X}$ - this modem is used by the sagometer
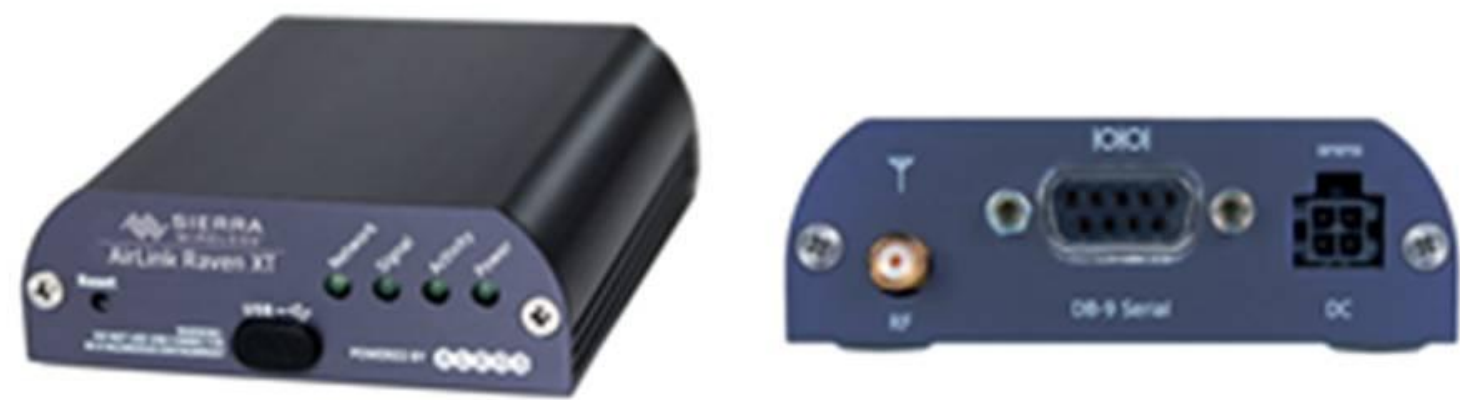

Figure C-28

Isolated front and rear view of Raven XT - This modem is used by the EPRI sensor system

\section{VPN Information}

A virtual private network (VPN) is a mechanism for providing secure, reliable transport over Internet. The VPN uses authentication to deny access to unauthorized users, and encryption to prevent unauthorized users from reading the private network packets. The VPN can be used to send any kind of network traffic securely.

VPNs are frequently used by remote workers or companies with remote offices to share private data and network resources. VPNs may also allow users to bypass regional internet restrictions such as firewalls, and web filtering, by "tunneling" the network connection to a different region.

Technically, the VPN protocol encapsulates network data transfers using a secure cryptographic method between two or more networked devices which are not on the same private network, to keep the data private as it passes through the connecting nodes of a local or wide area network.

\section{Server}

The server is a Hewlett Packard (HP) Proliant DL320 G6 server; it features a quad-core Intel Xenon processor rated at $2.26 \mathrm{GHz}$ with an $8 \mathrm{Mb}$ cache. For RAM it has three $2 \mathrm{~GB}$ HP sticks with a clock speed of $10600 \mathrm{~Hz}$. For permanent storage three of the eight drive bays are being filled with 146 GB $10 \mathrm{krpm}$ drives. To add redundancy to the data storage a raid-3 configuration was chosen. This results in about $300 \mathrm{~GB}$ of effective memory from the set of drives. If large amounts of data were to be post processed, such as running simulations in DTCR, a raid-5 setup should be faster while still providing a backup. When an external backup drive or FTP backup is being employed a raid- 0 configuration would give the fastest simulation runs.

$\mathrm{I} / \mathrm{O}$ is handled through conventional USB, PS2, VGA and Ethernet ports. One of the two Ethernet ports is used to establish an internet connection. This allows access to system updates as 
well as the weather service data provided by NOAA. The second port is used by one of the field data collection modems. A third Ethernet port can be used for remote system management via the Integrated Lights Out software. A use of this technology is the ability to power on the server after an outage remotely.

Two $400 \mathrm{~W}$ power supplied offer redundant power, this allows a dead supply to be replaced without the need for system downtime. The operating system on the server is Windows Server 2003 SP2, the frequency of system updates to the OS has been modified to also reduce the system downtime.

Two wireless modems are connected to the server because 1 modem can only handle 5 VPN connections and the system requires 6 VPN tunnels. The modems are controlled by a Sierra Wireless software package. The modems data is then passed into Campbell Scientifics' LoggerNet software before being processed by EPRI's DTCR 6.0 software.

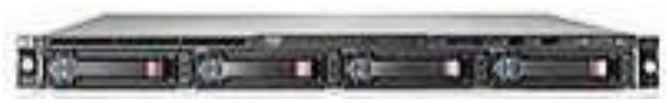

\section{Figure C-29}

HP Proliant DL320 G6 server

\section{Campbell Scientific LoggerNet Software}

LoggerNet is the Campbell Scientific data logger support software package. It supports programming, communication, and data retrieval between data loggers and the server. LoggerNet consists of a server application and several client applications integrated into a single product. It can support connection to a single data logger, but is adept in applications that require telecommunications or scheduled data retrieval used in large data logger networks. The server is loaded with version 4 which is the most recent version of LoggerNet. It features a new tool for designing and configuring PakBus networks, which is the type used for this project. It also contains a more powerful file viewer, an upgrade to RTMC (the real-time viewer), a redesigned toolbar, and other updates to existing clients.

Static IP addresses are assigned to each modem in the system. Future systems may change this to dynamic allocation; this was not an option here due to the VPN link. The data loggers run code that is developed by the venders of each sensor. The code produces data tables, much like an MS Excel spread sheet, and these tables exist on the data logger's memory. The LoggerNet software then connects to the loggers via the wireless modems. LoggerNet compares the data in the server with the data on the logger, after determining what new data is available on the logger it downloads the information to the server. For overhead lines this download interval is typically 5 or 10 minutes. The data tables are saved as files on the server with the file extension of *.DAT. DTCR can directly read these *.DAT files.

\section{DTCR Software}

The DTCR software is designed to accept real-time or simulated real-time inputs from a number of field sensors, and computes the normal (and emergency) ratings for overhead line segments, and for entire circuits. The results can be made available to operators in real-time, or can be used in studies to evaluate a utility's approach to acceptable rating levels in general. 
In 1993, EPRI initiated a project to develop and field test software which would allow the realtime thermal monitoring of transmission circuits. Certain existing thermal models for underground cables, overhead lines, power transformers, and substation equipment such as line traps, circuit breakers, bus, switches, and current transformers, were included in an integrated software model capable of calculating the dynamic thermal rating of transmission circuits which consist of one or more elements. Since that initial project, the software has gone through several fundamental revisions based on a series of field tests and installations at cooperating utilities. The latest version of the software was used during this project.

Dynamic (i.e., "real-time") thermal ratings are calculated based on actual weather conditions (e.g., air temperature, wind speed, etc.) and line monitoring equipment (such as the Video Sagometer) rather than using "worst-case" weather conditions as done for conventional static rating calculations. Also, simulations of dynamic operation based on archived data for an actual line can be useful in the evaluation of present rating methods, and can provide a database for making scientifically justified decisions about uprating methods. Chapter 5 contains details of building the line models used for this NYPA project.

\section{Data Key Information}

The output of the Campbell Scientific software is batch of data files. Included below are a number of data tables that will aid in decoding these files. These tables include the column header in the *.DAT file along with the description of that data. These files can be opened up in MS Excel using comas as the delimiter. Units can be difficult to manage in projects like this so it is important to keep tabs on this parameter. The sagometer device will allow the end user to change the units without any additional data logger reprogramming. 
Table C-1

ThermalRate Data Table Structure

\begin{tabular}{|c|c|c|}
\hline \multicolumn{3}{|c|}{ ThermalRate } \\
\hline Table Column Name & Description & Units \\
\hline ThermalRateString(1) & Debug String & $\mathrm{n} / \mathrm{a}$ \\
\hline ThermalRateString(2) & Date & $\mathrm{n} / \mathrm{a}$ \\
\hline ThermalRateString(3) & Time & $\mathrm{n} / \mathrm{a}$ \\
\hline ThermalRateString(4) & Cold Rod Temperature & ${ }^{\circ} \mathrm{C}$ \\
\hline ThermalRateString(5) & Hot Rod Temperature & ${ }^{\circ} \mathrm{C}$ \\
\hline ThermalRateString(6) & Air Temperature & ${ }^{\circ} \mathrm{C}$ \\
\hline ThermalRateString(7) & Heater Power & Watts \\
\hline ThermalRateString(8) & Normal Rating & Amps \\
\hline ThermalRateString(9) & $1^{\text {st }}$ Emergency Rating & Amps \\
\hline ThermalRateString(10) & $2^{\text {nd }}$ Emergency Rating & Amps \\
\hline ThermalRateString(11) & Conductor Temperature & ${ }^{\circ} \mathrm{C}$ \\
\hline ThermalRateString(12) & Line Current & Amps \\
\hline ThermalRateString(13) & Effective Wind Speed & $\mathrm{ft} / \mathrm{s}$ \\
\hline
\end{tabular}


The Video Sagometer allows the user to change many parameters on the fly. The exact settings for the NYPA Site have been captured here in this data table.

Table C-2

Sagometer Configuration Table Structure

\begin{tabular}{|l|l|l|l|l|}
\hline \multicolumn{5}{|c|}{ User adjustable Setting in Sagometer as of 01/07/2011 } \\
\hline \multicolumn{1}{|c|}{ Name } & Site 1 & \multicolumn{1}{|c|}{ Site 2 } & \multicolumn{1}{c|}{ Site 3 } & \multicolumn{1}{|c|}{ Description } \\
\hline SagCal(1,1) & 32.39 & 36.99 & 37.25 & Vertical Offset \\
\hline SagCal(1,2) & -1.101 & -1.47 & 1.41 & Tilt Offset \\
\hline SagCal(1,3) & 147.058 & 148.56 & 147.07 & Camera to Target Distance \\
\hline SagCal(1,4) & 1.1 & 1.1 & 1.1 & Target Twist Radius \\
\hline SagCal(1,5) & 1.535 & 1.469 & 1.476 & Low Point Constant L1 \\
\hline SagCal(1,6) & 29.702 & 30.458 & 25.51 & Low Point Constant L2 \\
\hline SagCal(1,7) & 49673 & 47125 & 42883 & Tension Constant T1 \\
\hline SagCal(1,8) & 53.02 & 52.76 & 50.44 & Tension Constant T2 \\
\hline SagCal(2,1) & 0 & 0 & 0 & Vertical Offset \\
\hline SagCal(2,2) & 0 & 0 & 0 & Tilt Offset \\
\hline SagCal(2,3) & 150 & 150 & 150 & Camera to Target Distance \\
\hline SagCal(2,4) & 0.75 & 0.75 & 0.75 & Target Twist Radius \\
\hline SagCal(2,5) & 0 & 0 & 0 & Low Point Constant L1 \\
\hline SagCal(2,6) & 0 & 0 & 0 & Low Point Constant L2 \\
\hline SagCal(2,7) & 0 & 0 & 0 & Tension Constant T1 \\
\hline SagCal(2,8) & 0 & 0 & 0 & Tension Constant T2 \\
\hline
\end{tabular}


Table C-3

Sagometer Data Table Structure

\begin{tabular}{|c|c|c|}
\hline \multicolumn{3}{|c|}{ Sagometer } \\
\hline Variable Name & Description & Units \\
\hline Sag_Avg $(1,1)$ & Conductor clearance at target & $\mathrm{ft}$ \\
\hline Sag_Avg $(1,2)$ & $\begin{array}{l}\text { Sag_Avg }(1,1) \text { corrected for twisting (rotating) of target } \\
\text { from vertical }\end{array}$ & $\mathrm{ft}$ \\
\hline Sag_Avg $(1,3)$ & Sag_Avg $(1,2)$ corrected for change in camera tilt & $\mathrm{ft}$ \\
\hline Sag_Avg $(1,4)$ & $\begin{array}{l}\text { Conductor clearance at low-point on catenary, calculated } \\
\text { from Sag_Avg }(1,4) \text { and low point parameters }\end{array}$ & $\mathrm{ft}$ \\
\hline Sag_Avg $(1,5)$ & Conductor Tension & $\mathrm{ft}$ \\
\hline Sag_Avg $(1,6)$ & $\begin{array}{l}\text { Horizontal position of target } \\
\text { Negative values = left of center } \\
\text { Positive values = right of center }\end{array}$ & $\mathrm{ft}$ \\
\hline Sag_Avg $(1,7)$ & $\begin{array}{l}\text { Twist (rotation) of target from vertical, caused by twisting } \\
\text { of conductor }\end{array}$ & degrees \\
\hline Sag_Avg $(1,8)$ & Correlation/Score & $\mathrm{n} / \mathrm{a}$ \\
\hline Tilt_Avg(1) & Camera Tilt & degrees \\
\hline Tilt_StdDev(1) & Camera Tilt, Standard Deviation & degrees \\
\hline Sag_Avg(2,1) & Conductor clearance at target & $\mathrm{ft}$ \\
\hline Sag_Avg(2,2) & $\begin{array}{l}\text { Sag_Avg }(2,1) \text { corrected for twisting (rotating) of target } \\
\text { from vertical }\end{array}$ & $\mathrm{ft}$ \\
\hline Sag_Avg(2,3) & Sag_Avg $(2,2)$ corrected for change in camera tilt & $\mathrm{ft}$ \\
\hline Sag_Avg $(2,4)$ & $\begin{array}{l}\text { Conductor clearance at low-point on catenary, calculated } \\
\text { from Sag_Avg }(2,4) \text { and low point parameters }\end{array}$ & $\mathrm{ft}$ \\
\hline Sag_Avg(2,5) & Conductor Tension & lbs \\
\hline Sag_Avg $(2,6)$ & $\begin{array}{l}\text { Horizontal position of target } \\
\text { Negative values = left of center } \\
\text { Positive values = right of center }\end{array}$ & $\mathrm{ft}$ \\
\hline Sag_Avg $(2,7)$ & $\begin{array}{l}\text { Twist (rotation) of target from vertical, caused by twisting } \\
\text { of conductor }\end{array}$ & degrees \\
\hline Sag_Avg $(2,8)$ & Correlation/Score & $\mathrm{n} / \mathrm{a}$ \\
\hline Tilt_Avg(2) & Camera Tilt & degrees \\
\hline Tilt_StdDev(2) & Camera Tilt, Standard Deviation & degrees \\
\hline AmbTemp_Avg & Ambient Temperature, Avg & C \\
\hline Rad_Avg & Solar Radiation, Avg & $\mathrm{W} / \mathrm{m}^{2}$ \\
\hline
\end{tabular}

C-29 


\begin{tabular}{|c|c|c|}
\hline Rain_Tot & Rain, Totalized & in \\
\hline Humid_Avg & Relative Humidity, Avg & $\%$ \\
\hline WSMax(1) & Wind Speed, Max, Anemometer \#1 & \multirow[t]{2}{*}{$\mathrm{ft} / \mathrm{sec}$} \\
\hline WSAvg(1) & Wind Speed, Avg, Anemometer \#1 & \\
\hline WDAvg(1) & Wind Direction, Avg, Anemometer \#1 & \multirow[t]{2}{*}{ degrees } \\
\hline WDStdDev(1) & Wind Direction Standard Deviation, Anemometer \#1 & \\
\hline WS3D_Avg & Wind Speed, 3D (includes vertical), Avg & $\mathrm{ft} / \mathrm{sec}$ \\
\hline WDV_Avg & Wind Direction, Vertical Only, Avg & degrees \\
\hline SOSAvg(1) & Speed of Sound, Anemometer \#1 & $\mathrm{m} / \mathrm{s}$ \\
\hline WSMax(2) & Wind Speed, Max, Anemometer \#2 & \multirow[t]{2}{*}{$\mathrm{ft} / \mathrm{sec}$} \\
\hline WSAvg(2) & Wind Speed, Avg, Anemometer \#2 & \\
\hline WDAvg(2) & Wind Direction, Avg, Anemometer \#2 & \multirow[t]{2}{*}{ degrees } \\
\hline WDStdDev(2) & Wind Direction Standard Deviation, Anemometer \#2 & \\
\hline SOSAvg(2) & Speed of Sound, Anemometer \#2 & $\mathrm{m} / \mathrm{s}$ \\
\hline BattVolt & Battery Voltage, Avg & volts \\
\hline IntTemp & Internal Temperature, Avg & $\mathrm{C}$ \\
\hline
\end{tabular}


The following data table shows what units are available for on the fly adjustment within the Video Sagometer.

Table C-4

Sagometer Units Table Structure

\begin{tabular}{|c|c|c|}
\hline \multicolumn{3}{|c|}{ Sagometer user adjustable settings } \\
\hline Variable Name & Description & Units \\
\hline SagCal $(1,1)$ & Vertical Offset & $\mathrm{ft}$ or $\mathrm{m}$ \\
\hline SagCal $(1,2)$ & Tilt Offset & Degrees \\
\hline SagCal $(1,3)$ & Camera to Target Distance & ft or $m$ \\
\hline SagCal $(1,4)$ & Target Twist Radius & ft or $m$ \\
\hline SagCal $(1,5)$ & Low Point Constant L1 & none \\
\hline SagCal $(1,6)$ & Low Point Constant L2 & none \\
\hline SagCal $(1,7)$ & Tension Constant T1 & none \\
\hline SagCal $(1,8)$ & Tension Constant T2 & none \\
\hline SagCal $(2,1)$ & Vertical Offset & $\mathrm{ft}$ or $\mathrm{m}$ \\
\hline SagCal(2,2) & Tilt Offset & Degrees \\
\hline SagCal $(2,3)$ & Camera to Target Distance & $\mathrm{ft}$ or $\mathrm{m}$ \\
\hline $\operatorname{SagCal}(2,4)$ & Target Twist Radius & $\mathrm{ft}$ or $\mathrm{m}$ \\
\hline SagCal(2,5) & Low Point Constant L1 & none \\
\hline $\operatorname{SagCal}(2,6)$ & Low Point Constant L2 & none \\
\hline SagCal $(2,7)$ & Tension Constant T1 & none \\
\hline SagCal $(2,8)$ & Tension Constant T2 & none \\
\hline Units(1) & Length/Distance Units & $0-\mathrm{ft}$ \\
\hline Units(2) & Tension Units & $0-\mathrm{lbs}$ \\
\hline Units(3) & Temperature Units & $0-{ }^{\circ} \mathrm{F}$ \\
\hline Units(4) & Wind Speed Units & $0-\mathrm{ft} / \mathrm{sec}$ \\
\hline Units(5) & Precipitation Units & $0-$ in \\
\hline ModemPower(1) & Periodic interval when modem is turned ON & Min \\
\hline ModemPower(2) & Duration modem remains ON after being turned ON & Min \\
\hline RollWindow & length of rolling average window & Min \\
\hline
\end{tabular}


Table C-5

EPRI Sensor Table Structure

EPRI Sensors

\begin{tabular}{|c|c|c|}
\hline column heading & description & units \\
\hline TIMESTAMP & timestamp when data was recorded & date hour:min:sec \\
\hline RECORD & record number of data & integer \\
\hline ETempSensorReading $(1,1)$ & Id number of first sensor & integer \\
\hline ETempSensorReading $(1,2)$ & latest temp reading & Celsius \\
\hline ETempSensorReading $(1,3)$ & latest current reading & amps \\
\hline ETempSensorReading $(1,4)$ & peak temp reading & Celsius \\
\hline ETempSensorReading $(1,5)$ & current reading at peak temp & amps \\
\hline ETempSensorReading $(1,6)$ & battery voltage & volts \\
\hline ETempSensorReading $(1,7)$ & Max $\mathrm{X}$ acceleration & ADC counts \\
\hline ETempSensorReading $(1,8)$ & Min $X$ acceleration & ADC counts \\
\hline ETempSensorReading $(1,9)$ & Max $\mathrm{Y}$ acceleration & ADC counts \\
\hline ETempSensorReading $(1,10)$ & Min $Y$ acceleration & ADC counts \\
\hline ETempSensorReading $(1,11)$ & Max $Z$ acceleration & ADC counts \\
\hline ETempSensorReading $(1,12)$ & Line inclination & $1 / 100$ ths of a degree \\
\hline ETempSensorReading $(1,13)$ & $\mathrm{X}$ acceleration & 1/1000th of a G \\
\hline ETempSensorReading $(1,14)$ & Y acceleration & 1/1000th of a G \\
\hline ETempSensorReading $(1,15)$ & $\mathrm{Z}$ acceleration & 1/1000th of a G \\
\hline ETempSensorReading $(1,16)$ & Stale Inclination Count & integer \\
\hline ETempSensorReading $(1,17)$ & Min $\mathrm{Z}$ acceleration & ADC counts \\
\hline ETempSensorReading $(1,18)$ & Average absolute deviation $\mathrm{X}$ acceleration & integer \\
\hline ETempSensorReading $(1,19)$ & Average absolute deviation $\mathrm{Y}$ acceleration & integer \\
\hline ETempSensorReading $(1,20)$ & Average absolute deviation $\mathrm{Z}$ acceleration & integer \\
\hline ETempSensorReading $(1,21)$ & latest on board temp & ADC counts \\
\hline ETempSensorReading $(1,22)$ & firmware version & 8 bit number \\
\hline ETempSensorReading $(1,23)$ & latest on board temp & Celsius \\
\hline ETempSensorReading $(1,24)$ & RSSI value & $\mathrm{dB}$ \\
\hline ETempSensorReading $(1,25)$ & missed reading cycle count & integer \\
\hline ETempSensorReading $(1,26)$ & AAD threshold & integer \\
\hline ETempSensorReading $(1,27)$ & Inclination threshold & integer \\
\hline
\end{tabular}




\section{Installations}

The first installation was a practice exercise performed at EPRI's High-Voltage Laboratory in Lenox, MA under controlled conditions. This provided the project team and line crew an opportunity to familiarize themselves with the instruments and installation methods. The next three installations were at actual field Sites on NYPA's $230 \mathrm{kV}$ transmission system. NYPA refers to these Sites as Site 1, Site 2 and Site 3

\section{Installation Assessments: Goals and Methods}

The purpose of assessing the installations was to collect and document information for the benefit of industry personnel and utilities tasked with implementing these instruments in the future. Side-by-side field trials and evaluations of three transmission line sensor systems that monitor an overhead transmission line's sag, temperature and local ambient weather conditions were initiated in November 2010.

The assessments look at the following: work methods and tools required for installation, ease of installation, start-up and operability requirements, time to install the instruments and follow-up maintenance.

The four installations are described separately in the sections below. They are organized in the order in which they were performed starting with the trial at EPRI Lenox followed by the NYPA Sites; designated Site 1, Site 2and Site 3.

\section{Installation at EPRI Lenox}

The EPRI High Voltage Laboratory is located at 115 East New Lenox Road, in Lenox MA. One of the objectives of the practice installation was to provide the project team a chance to familiarize themselves with the instruments and systems so best practices for field assembly could be determined. This was done by installing the devices with live line tools on a deenergized line at the EPRI's High Voltage laboratory. Installation was performed on a wooden H-frame structure similar to those used in NYPA's system. The test install also helped determine a more efficient sequence of installation. Some of these changes will be visible when comparing the photographs of the test line with those from the field installation. One example being the placement of data logger cabinets was changed to being directly under the solar panels as opposed to being mounted beneath their lower brackets.

As all the devices used were not likely to be common to work crews, it was beneficial to go through the actual wiring and connections of the instruments to the junction boxes. This ensured the proper tooling would be present during the live line installs, this also afforded time to troubleshoot any possible communications.

\section{EPRI Sensor System}

The EPRI Sensor system included sensors that attach directly to the transmission line conductor, electronics that communicate with the sensors and solar panels to power the system.

An EPRI Sensor is shown in the figure below. Before installing, the clamping jaw was preset for the conductor size. Presetting the jaw ensures a good fit to the conductor and more accurate thermocouple placement. The clamping jaw is made of a composite material and is adjusted 
with two screws as seen in the figure below. This composite material helps reduce the ability of the sensor to act as a heat sink thereby ensuring the conductor near the sensor truly represents the line section. The jaws and sensor body were also designed in such a way as to prevent audible and visible corona at typical line voltages.

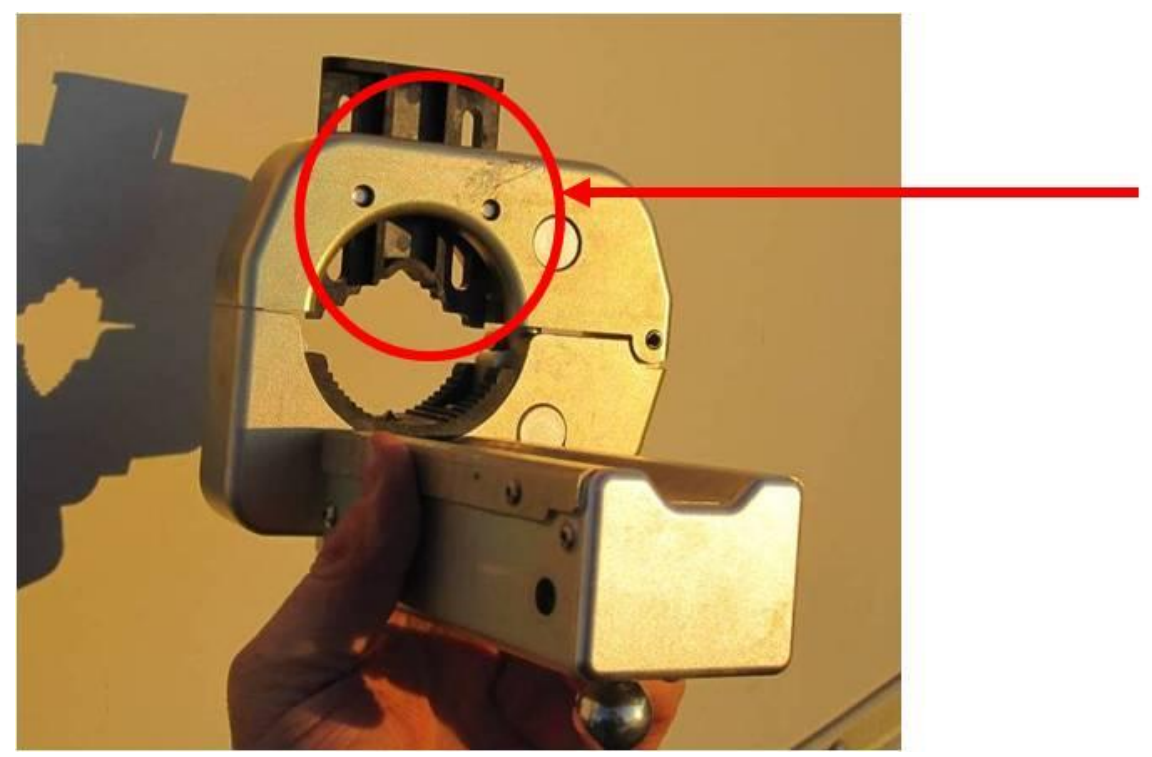

Adjustable clamping jaw

\section{Figure C-30}

EPRI Sensor

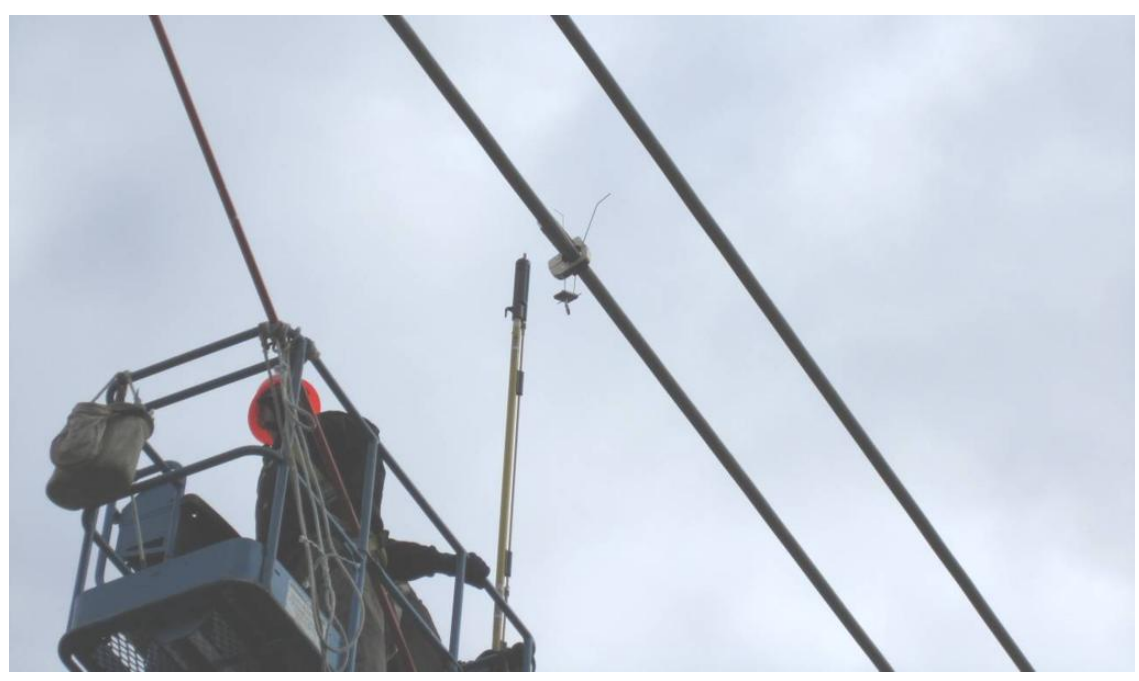

\section{Figure C-31}

Bonding tip becomes stuck during the first install attempt

As shown above in Figure C-31, the linemen become familiar with the methods and tools required for attaching the EPRI Sensor to the conductor. During the first attempt, the bonding leads became stuck under the clamping mechanism. With a little practice the lineman became comfortable with installing the sensors.

\section{ThermalRate System}


The ThermalRate sensor was attached to an aluminum arm mounted to one of the wooden $\mathrm{H}$ Frame poles. The figure below shows the line crew pulling the communications wire through the sensor's mounting arm.

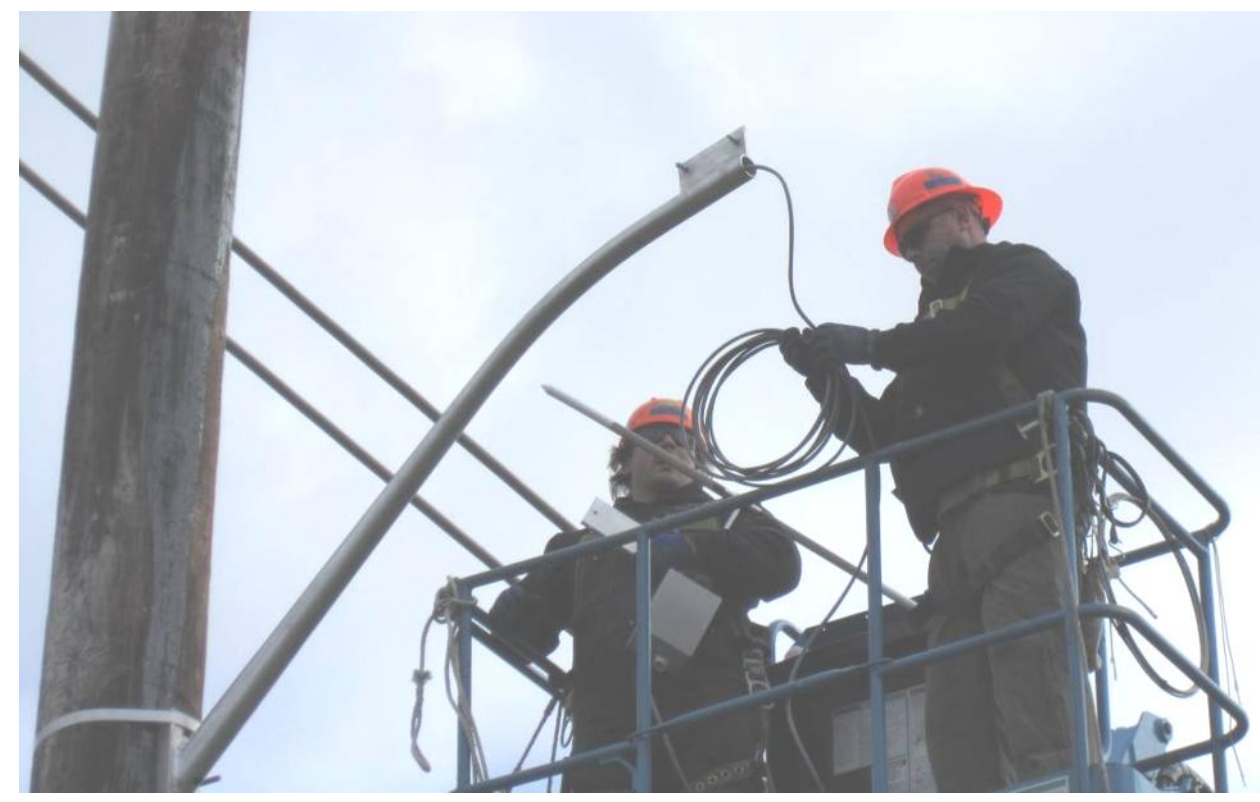

Figure C-32

ThermalRate sensor being prepared for mounting

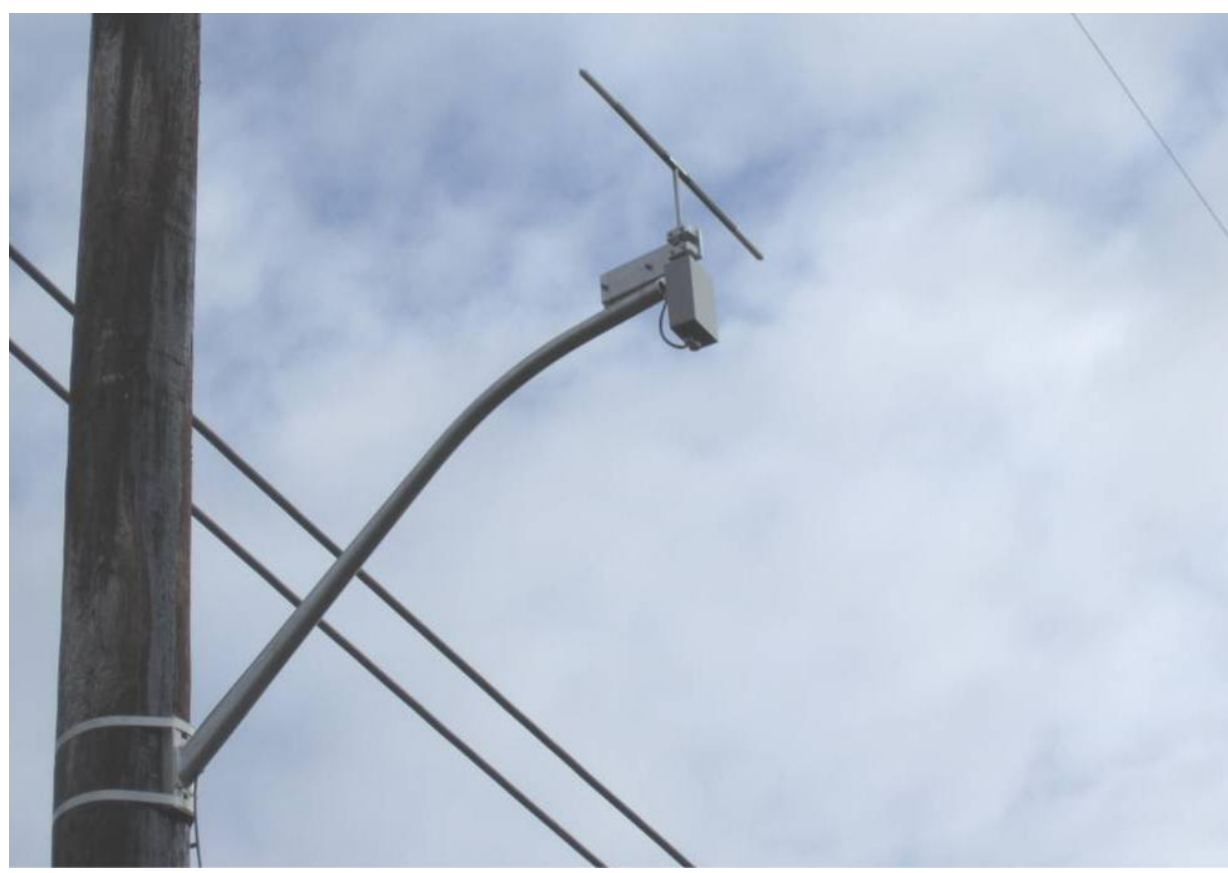

Figure C-33

ThermalRate sensor installed 


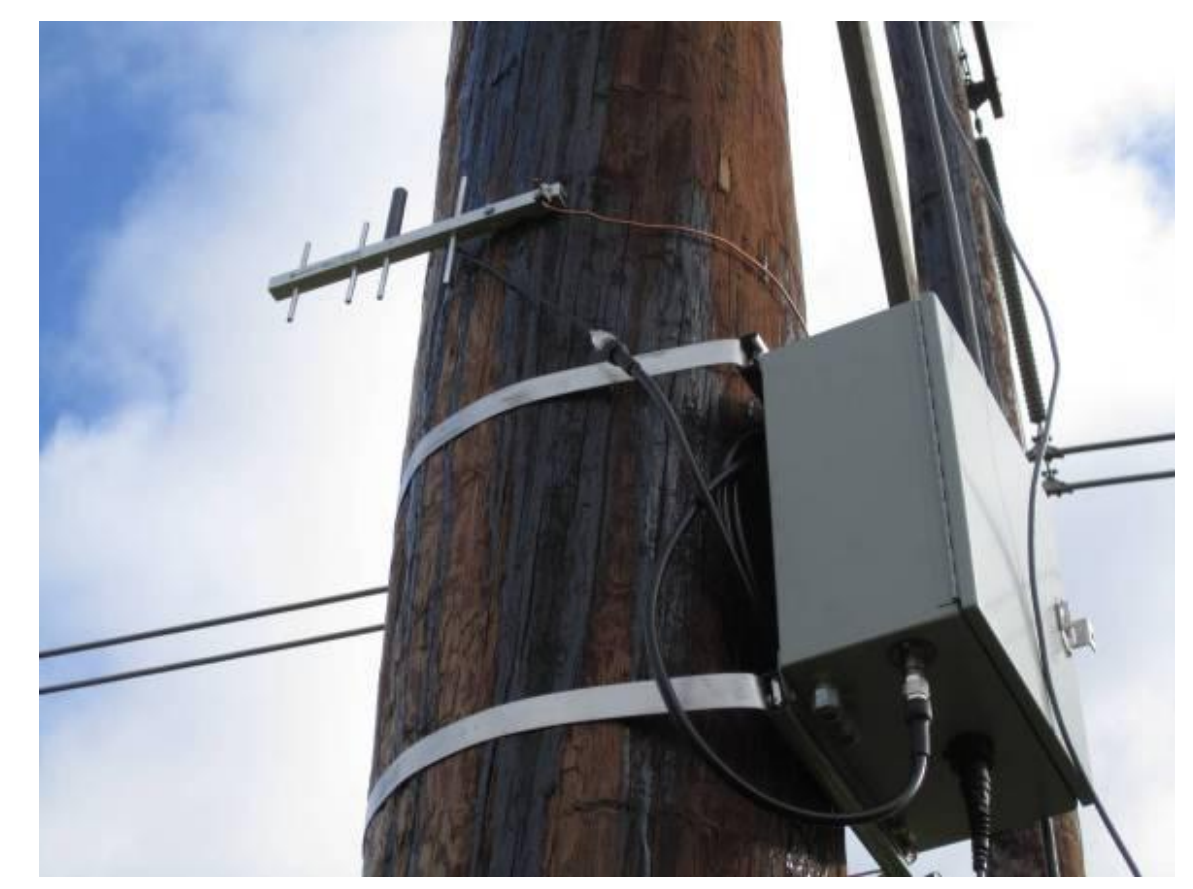

Figure C-34

ThermalRate electronics box and antenna 


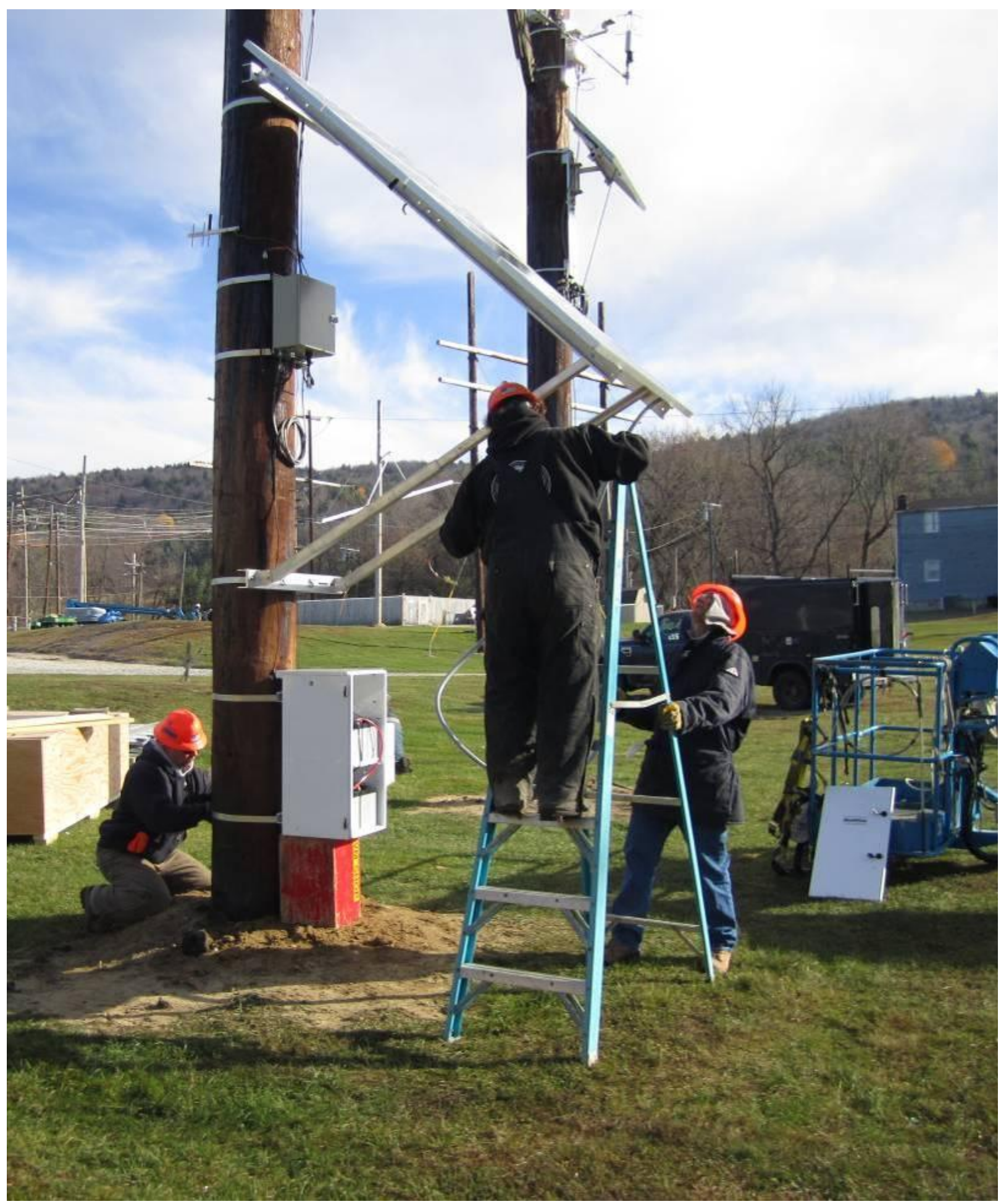

Figure C-35

ThermalRate battery box and solar panel 


\section{Sagometer System}

The sagometer system was mounted to the wooded pole adjacent to that of the ThermalRate. The sagometer camera, weather instruments, solar panel and electronics box are shown in the Figure below.

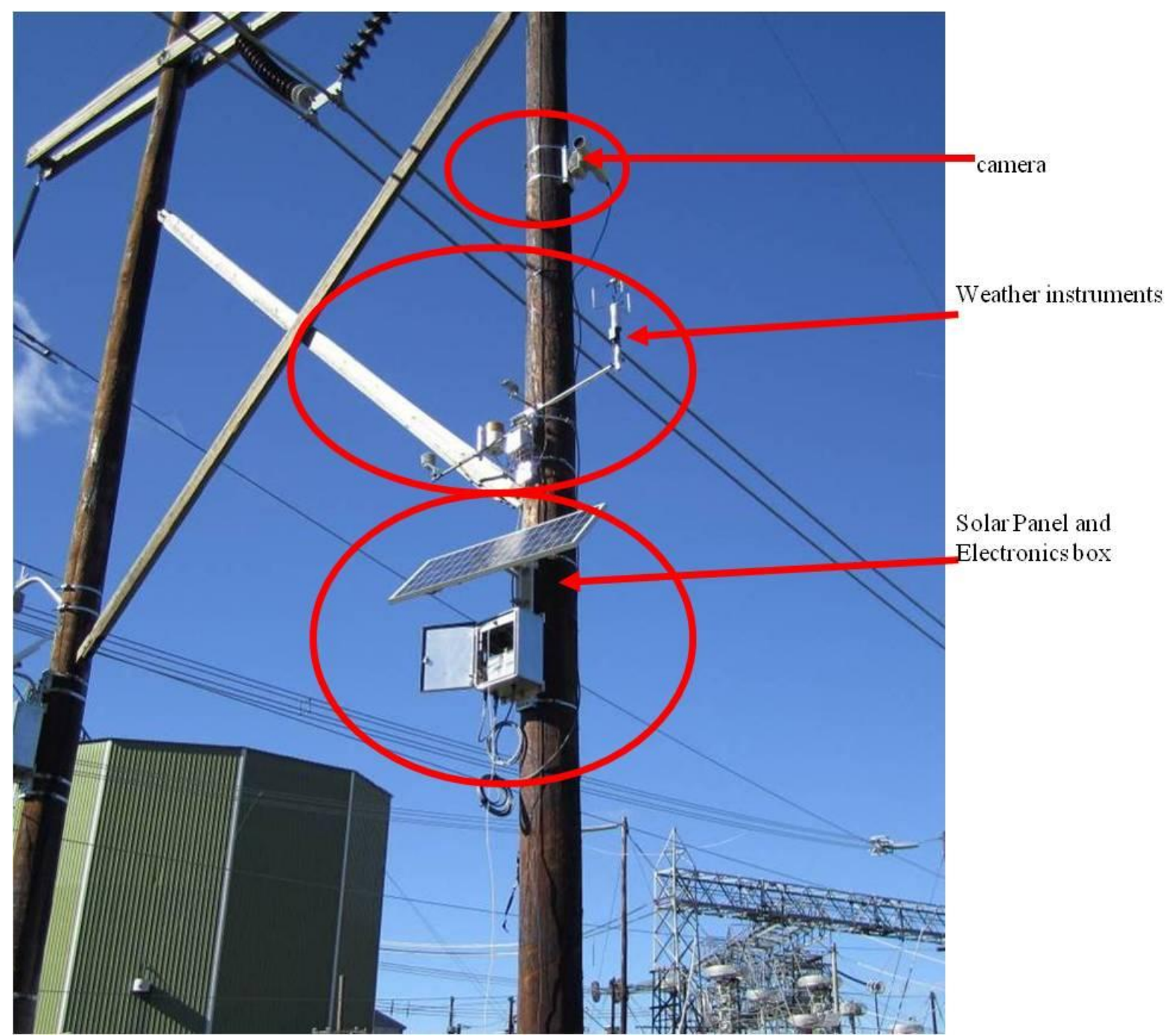

Figure C-36

Sagometer system

The sagometer target is shown in the following figure. The target mounting clamp was made to accommodate a Drake 795 ACSR conductor and so did not fit correctly on the test line which was a larger diameter conductor. The target was mounted on a smaller section of conductor which was then secured to the test line. 


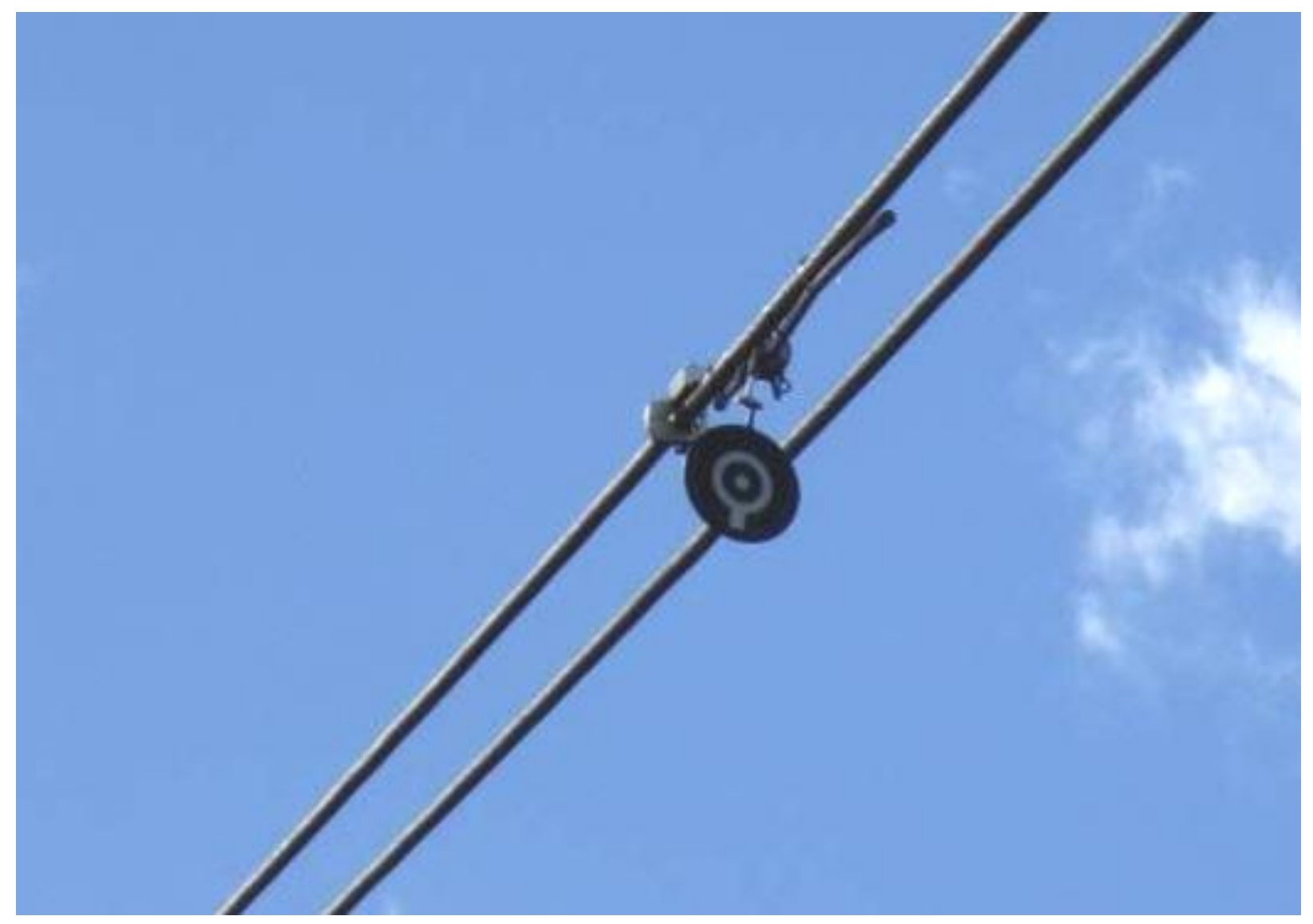

Figure C-37

Sagometer target 


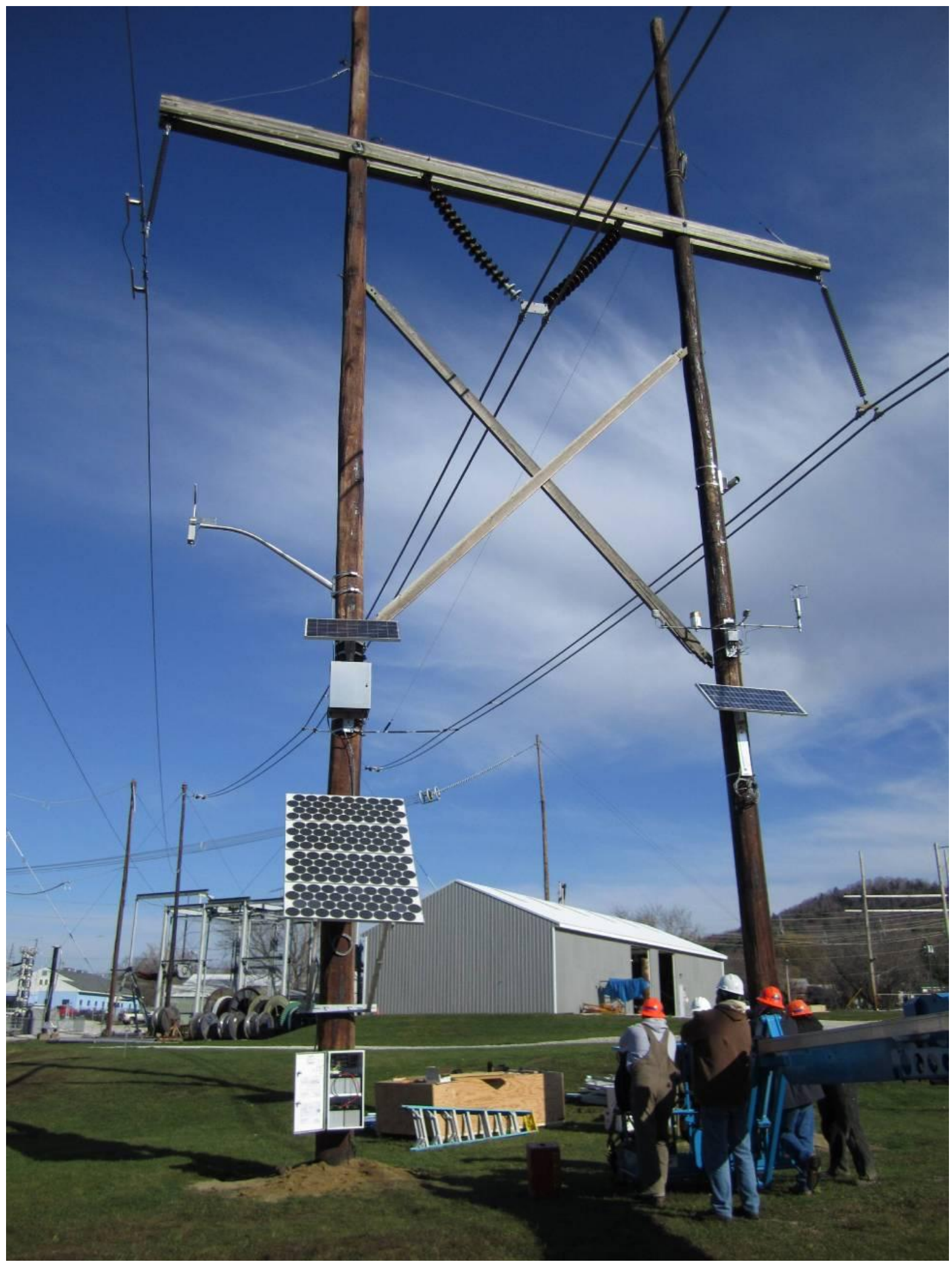

Figure C-38 Near completion of the practice installation at EPRI Lenox 


\section{Summary of Lenox Installation}

The Lenox installation was performed over three days from November $1^{\text {st }}$ thru $3^{\text {rd }} 2010$.

This served as practice and was a beneficial learning experience for the project team. It provided an understanding of which instruments to install first, what order and what type of spacing worked best. For example, mounting the ThermalRate electronics box under its solar panel reduces the overall footprint for the instruments on the pole and at the same time shielded the electronics box from rain and sun light.

The exact heights and spacing of all the instruments on the wood poles were somewhat open for adjustment at the start of the install. After completion, the relative positioning of the instruments were better identified and then noted for the field installs.

The line crew practiced installing the EPRI Sensors on a de-energized test line. The line crew gained confidence with the methods and tools required for securing the sensors after trying a couple of times. One lesson learned was to be careful not to tighten the sensor clamp down onto the bonding leads, this happen during the first attempt to install a sensor as shown.

The method for measuring and installing the pole bands that secured the instruments was practiced several times. This helped saved time during the field installs.

After the systems were installed, time was spent working out communication issues between the data logger modems and server modems. As the systems were relatively close to the receiver modems, they could be manually checked; this allowed the team to troubleshoot in an efficient manner.

The large solar panel and batteries for the ThermalRate system were the heaviest components therefore the solar panel required some rigging to lift. The rigging method worked out during the EPRI Lenox install for lifting and adjusting the solar panel was used later at the actual field Sites. This certainly saved time during the field installation.

The line crew was able to assess the types of tools were necessary for the installation. Having the right tools on hand saved time during the actual installations.

\section{NYPA Installation Sites}

\section{CCP-1 Training}

The project team received training at NYPA's St. Lawrence facility in Massena, NY. NERC requirements were reviewed and CPP-1 (Clearance and Protection Procedure) Operations training was provided at the LEM Building. The Clearance and Protection Procedure establishes the administrative program and controls which provide a safe working environment for all personnel while working at NYPA's Sites.

\section{Installation on the Site 1 Structure}

This site was nearest the Willis Substation. The relevant structure is at an elevation of approximately 1100 feet and this section of line is $81^{\circ}$ clockwise from north, based on information extracted from the plan and profile drawings. 
The conductor is $795 \mathrm{Kcmil}$ Drake ACSR. The wood structure at this site is classified as type "BB". The system voltage on this line is $230 \mathrm{kV}$.

As can be seen in the photo below, this section of line is mostly open level terrain with minimal sheltering from trees and other objects. Therefore, ruling span assumptions should be quite accurate. This site is in close proximity to a wind farm which suggested the line segment is located in a windy area as well as increased loading during high winds. This provides ideal conditions for dynamic ratings as the ratings should increase linearly with generation capability and load.

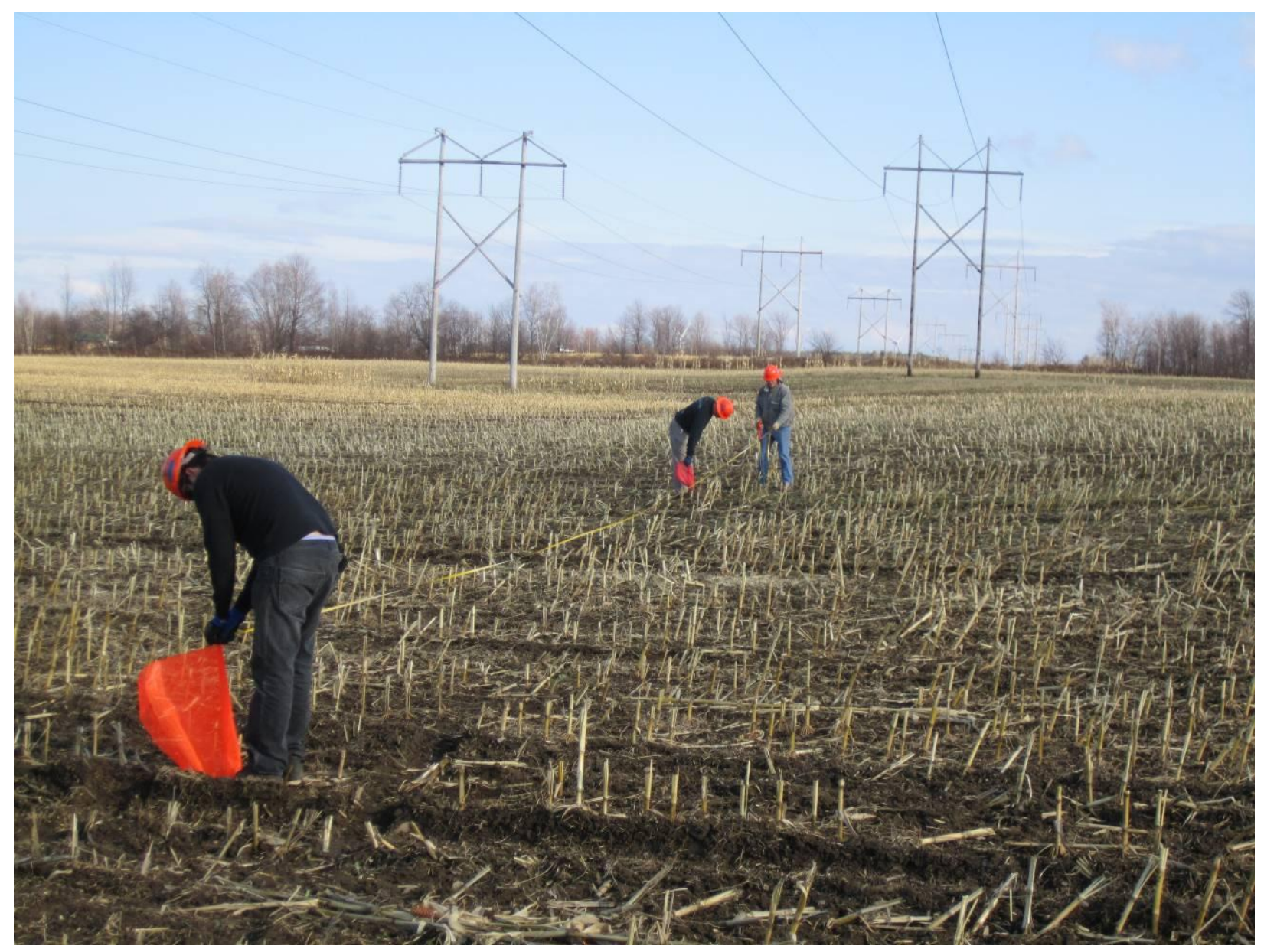

Figure C-39

Locations were marked under the line for the sagometer target and EPRI sensors. 


\section{EPRI Sensors}

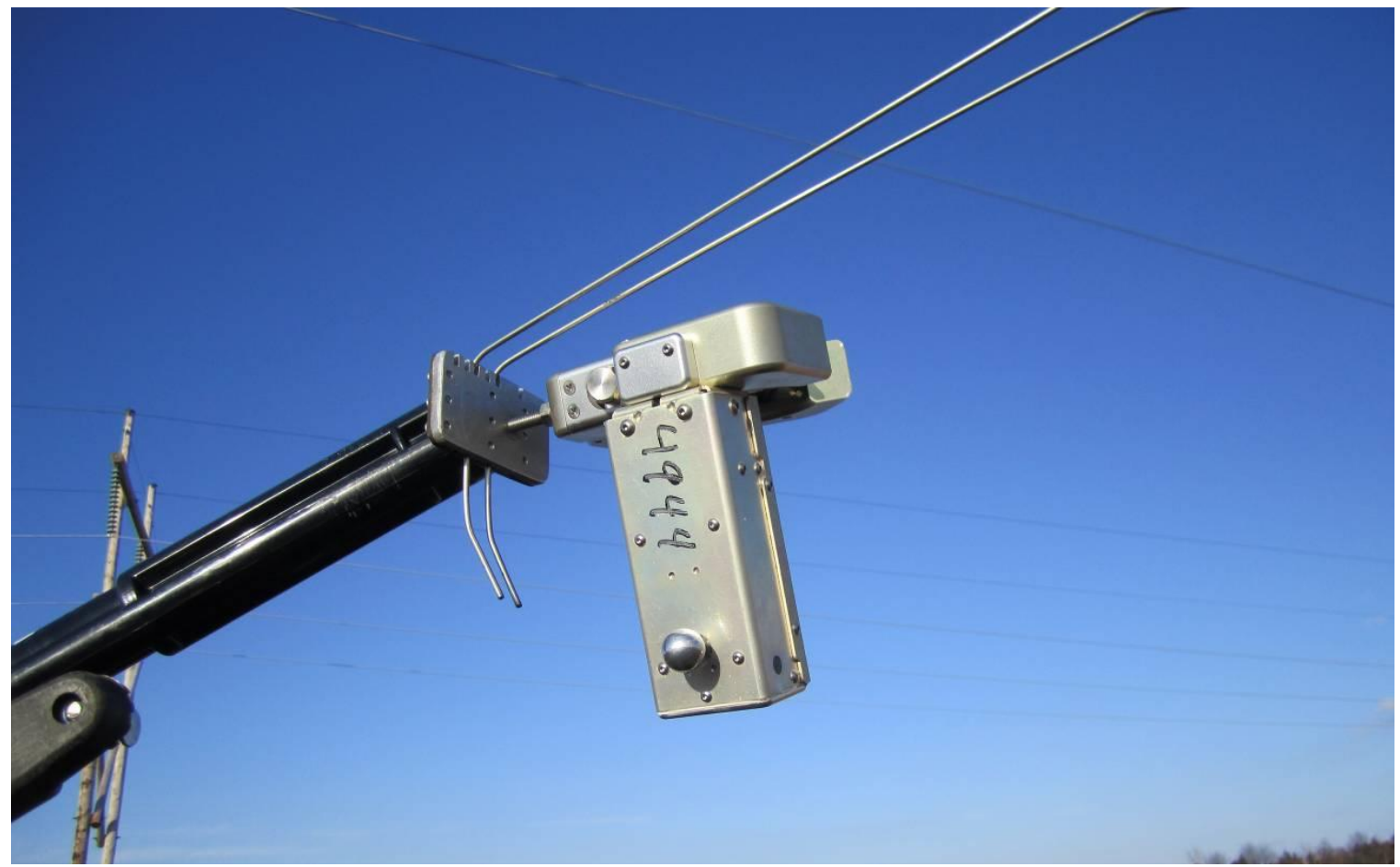

Figure C-40

EPRI Sensor ready to install

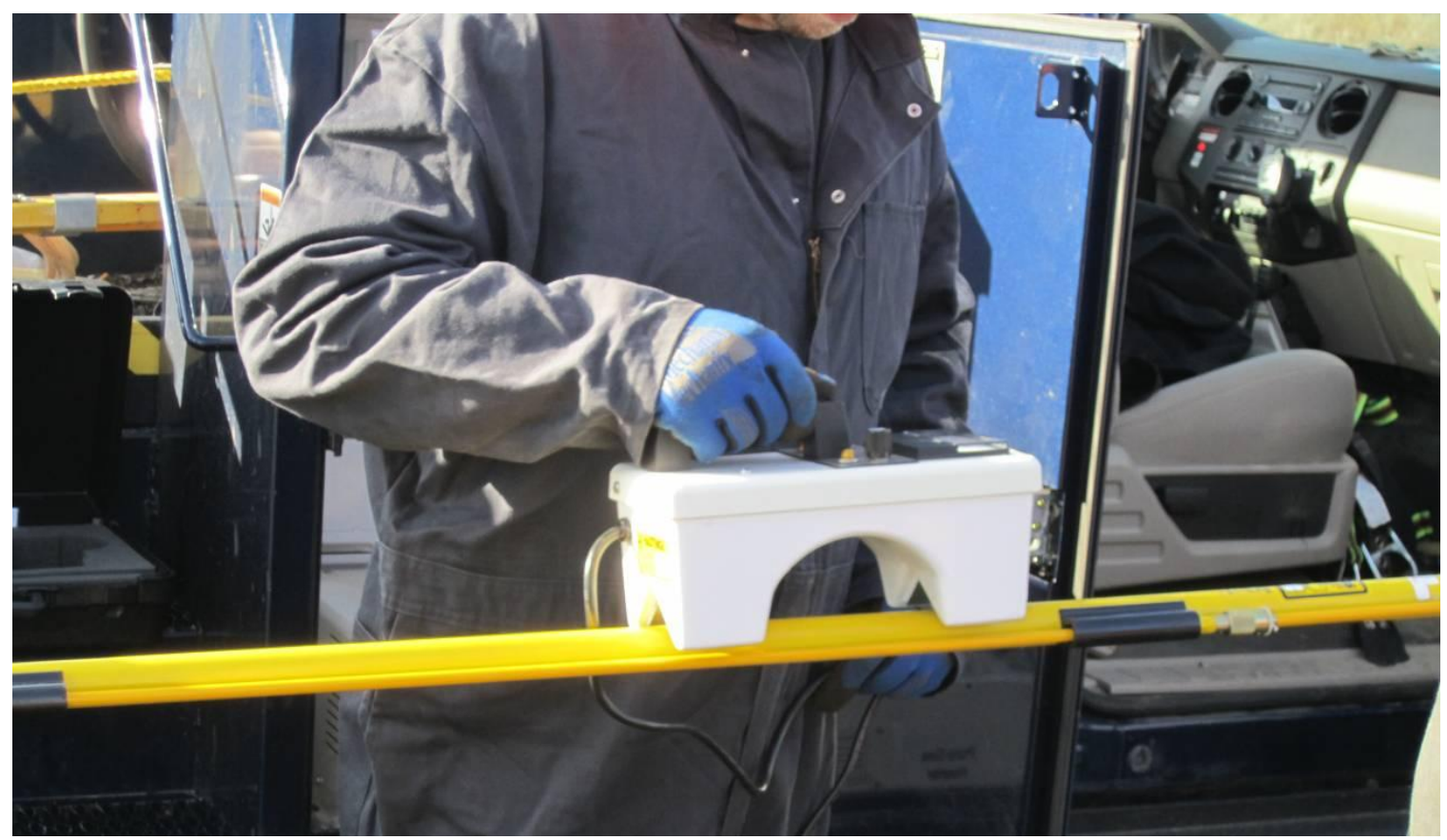

Figure C-41

Lineman tests hot stick prior to installing the EPRI Sensors on the energized $230 \mathrm{kV}$ line 


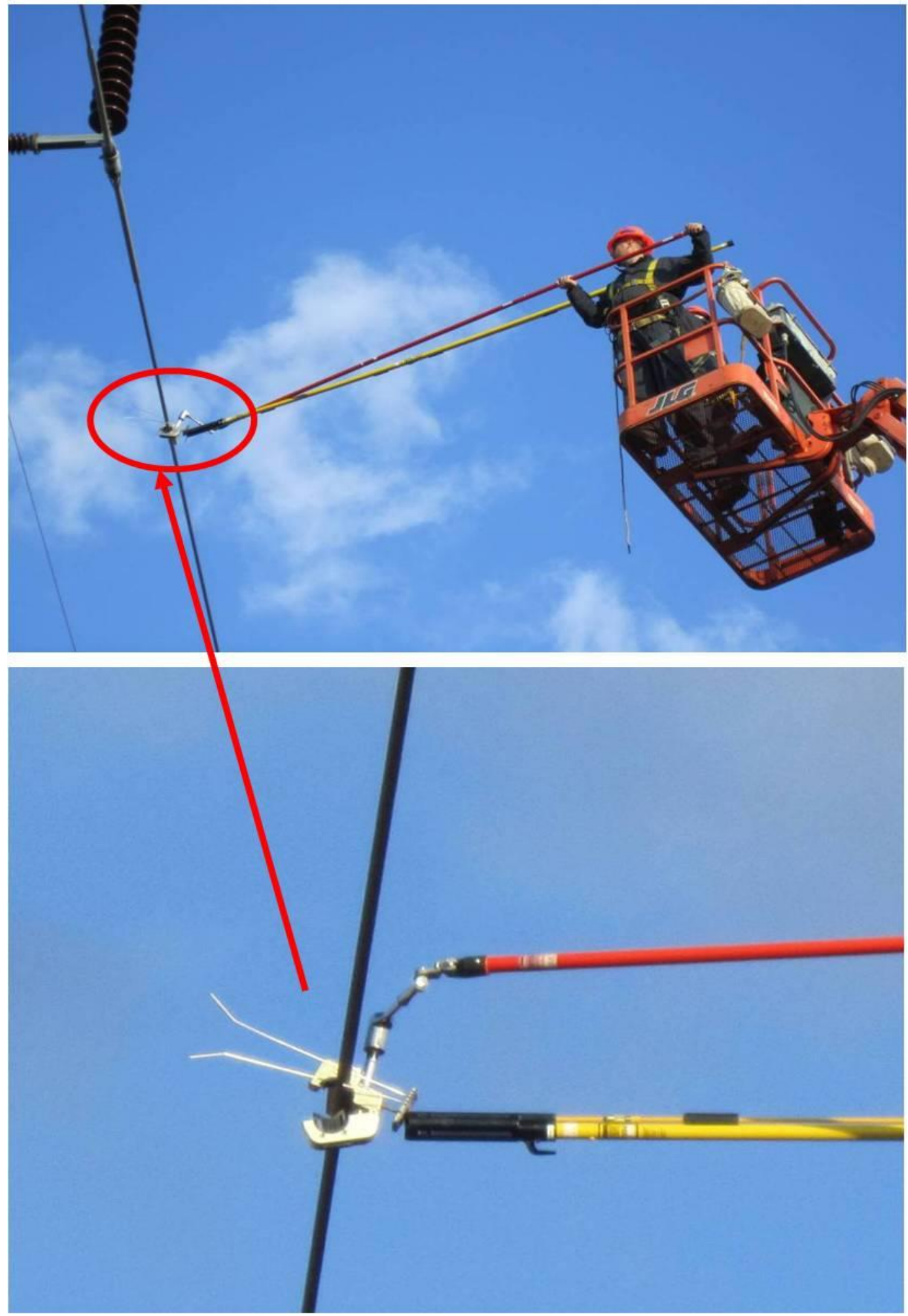

Figure C-42

EPRI Sensor being installed 


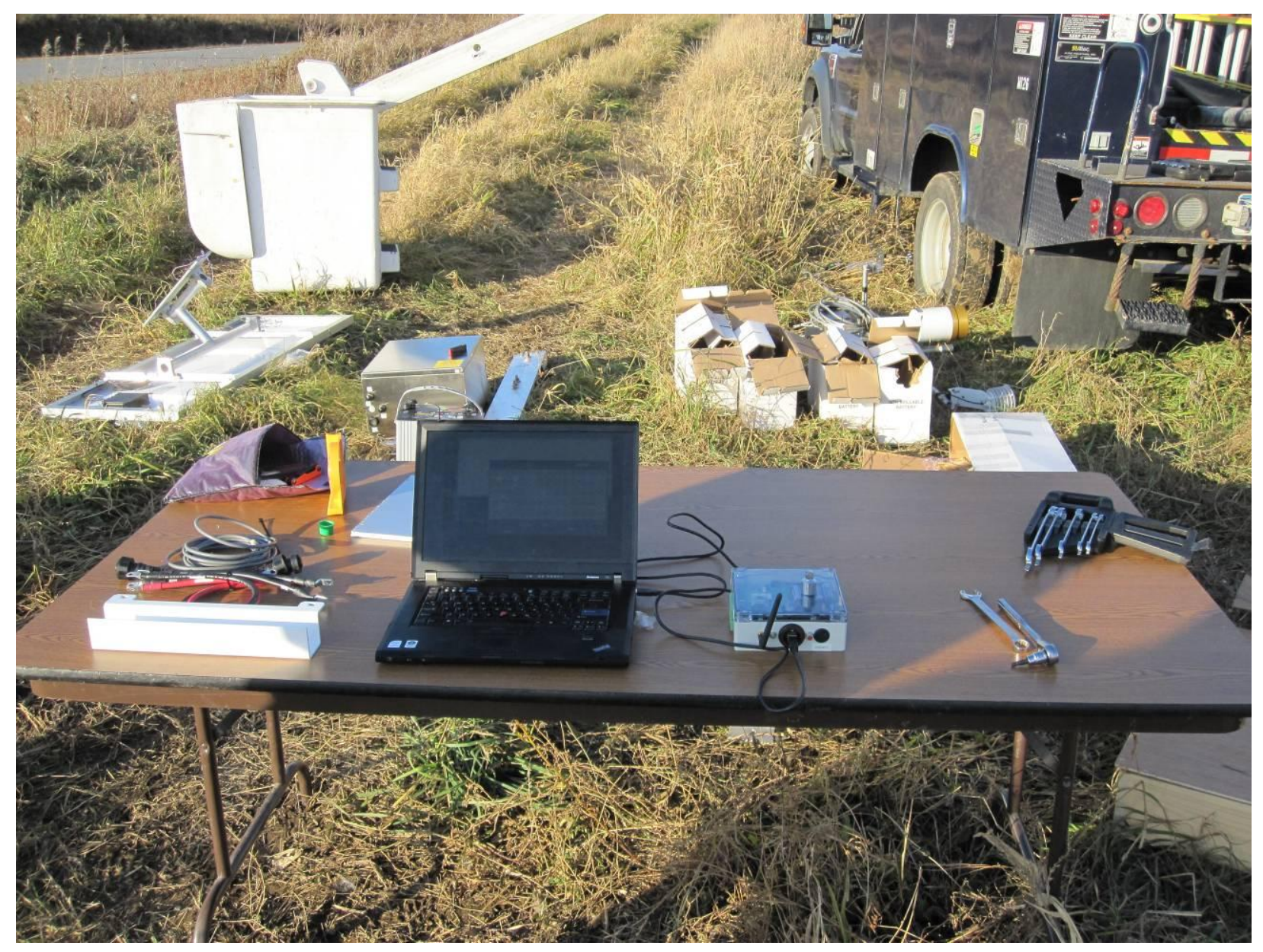

Figure C-43

Remote ZAP Unit used to check communications of the EPRI Sensors in the field 


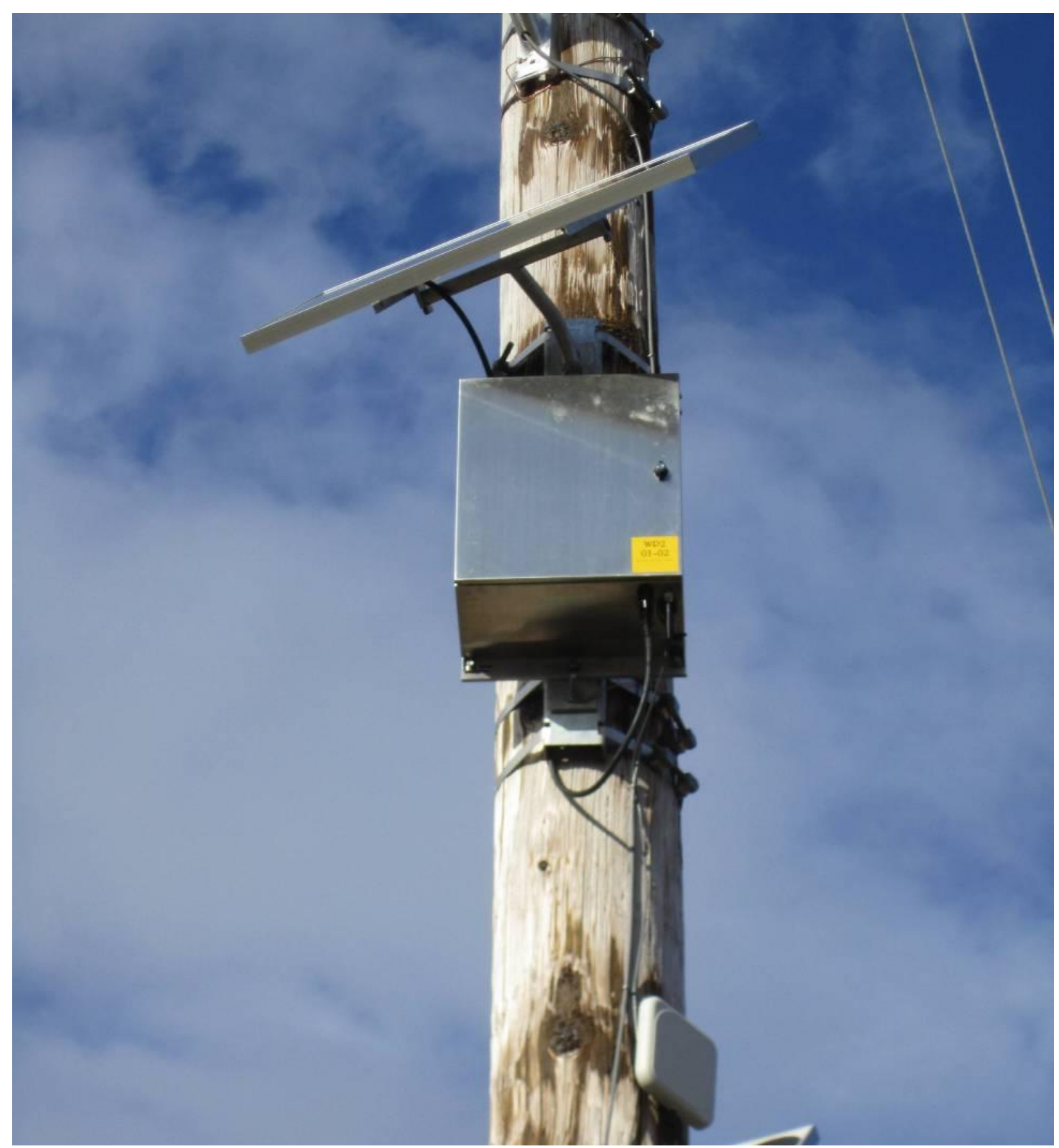

Figure C-44

EPIR Sensor electronics box and solar panel installed 
ThermalRate System

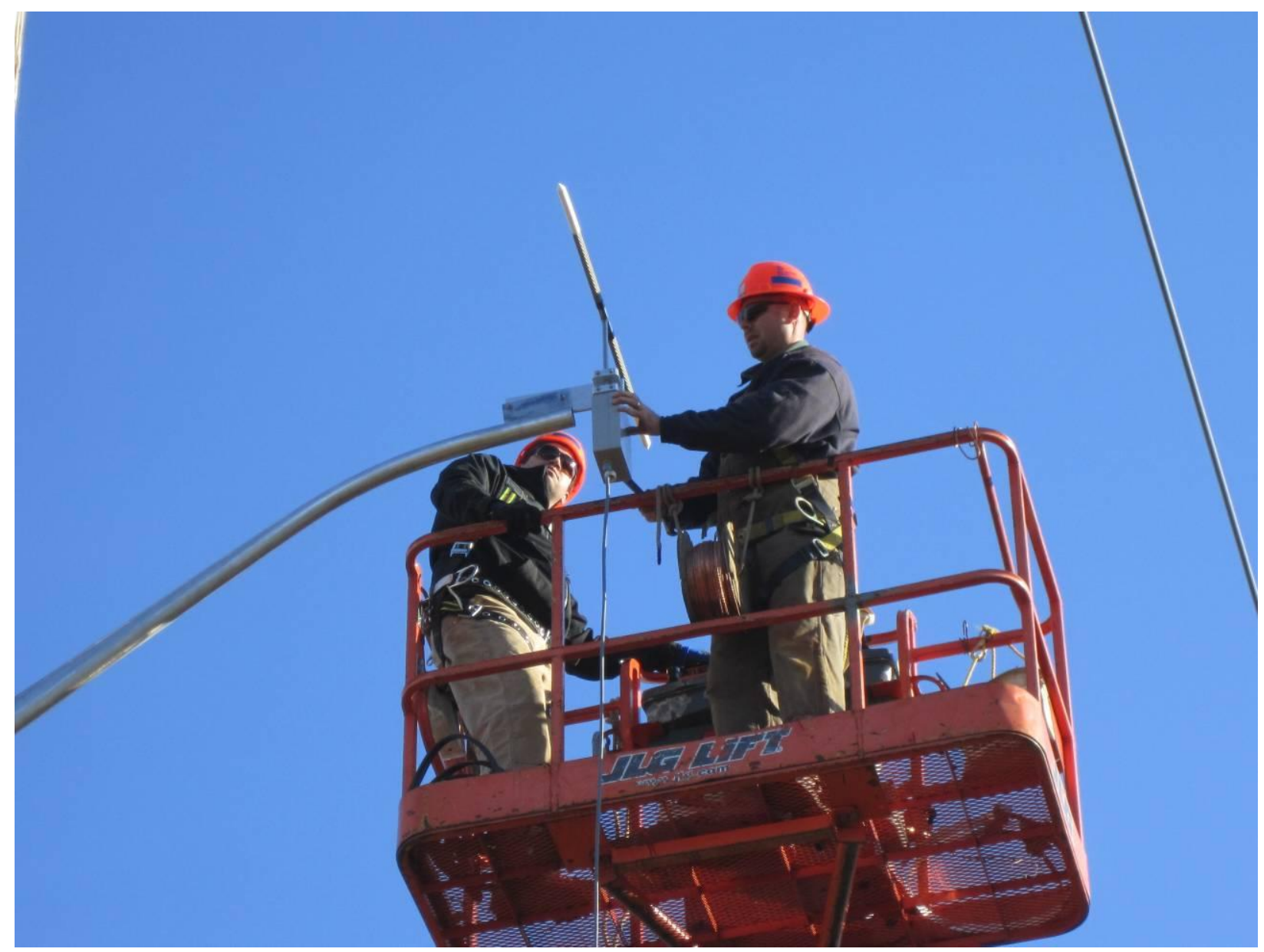

Figure C-45

ThermalRate sensor being installed 


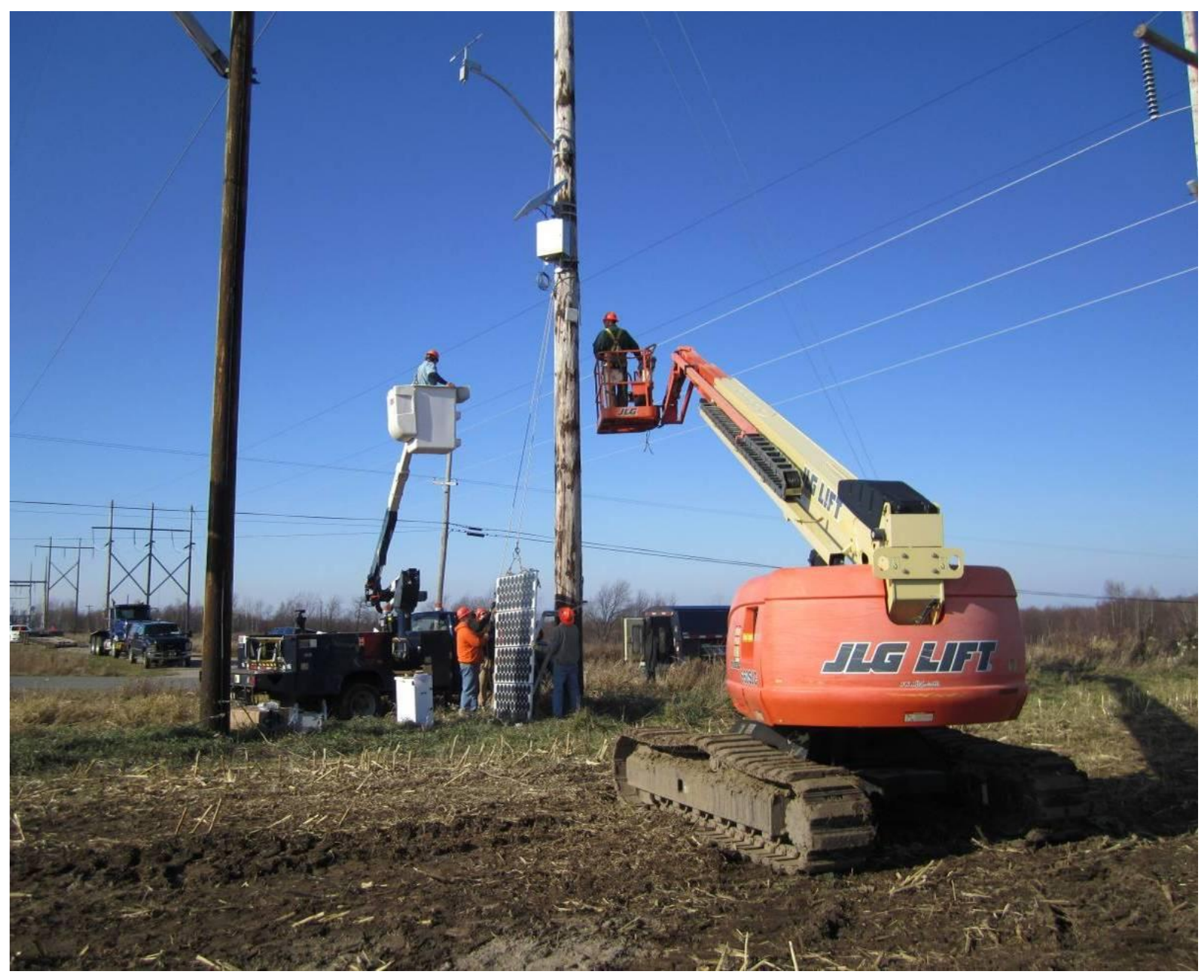

Figure C-46

Solar panel for the ThermalRate system being rigged for installation 


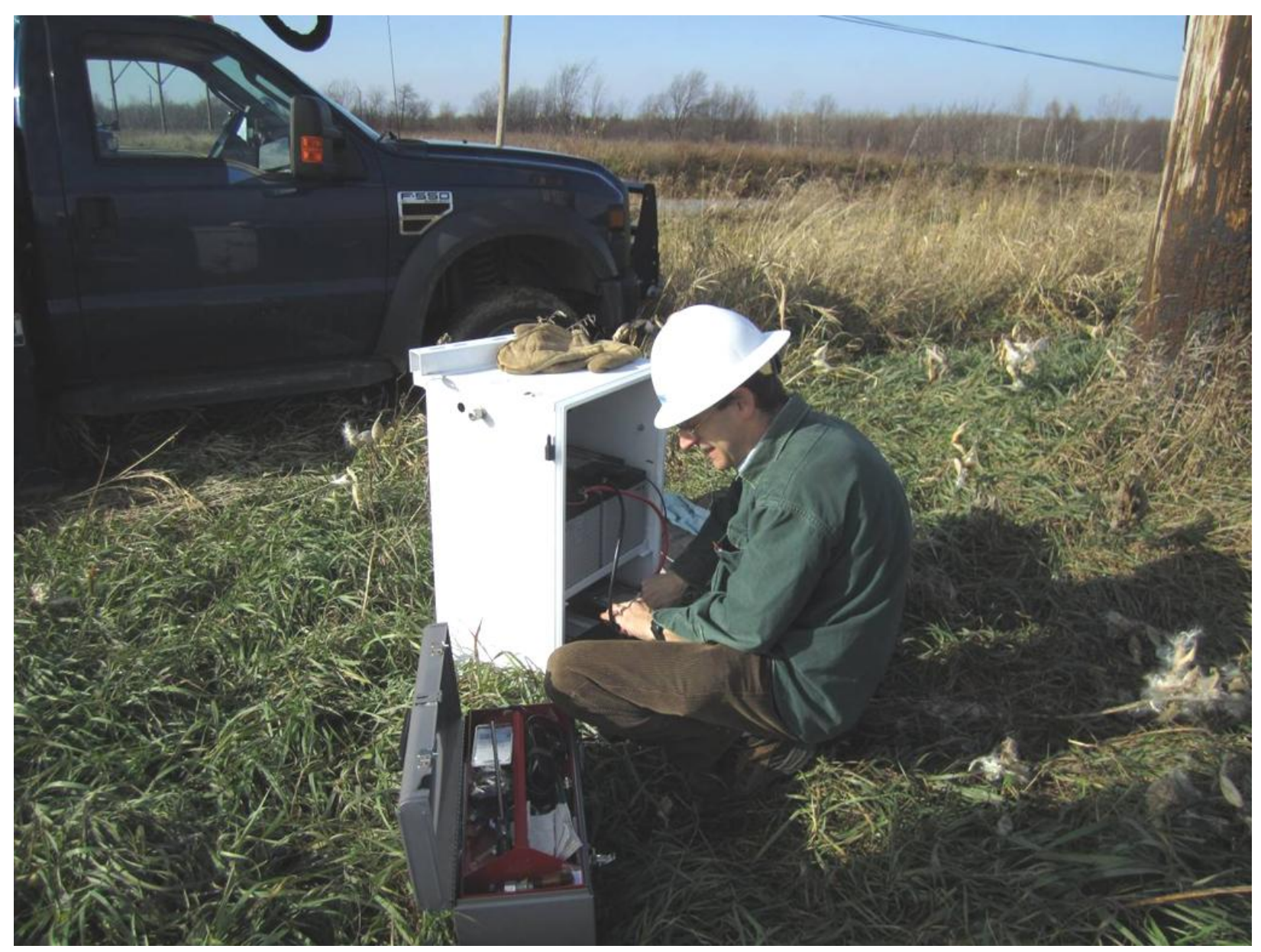

Figure $\mathrm{C}-47$

ThermalRate Battery Box Being Wired

Sagometer System 


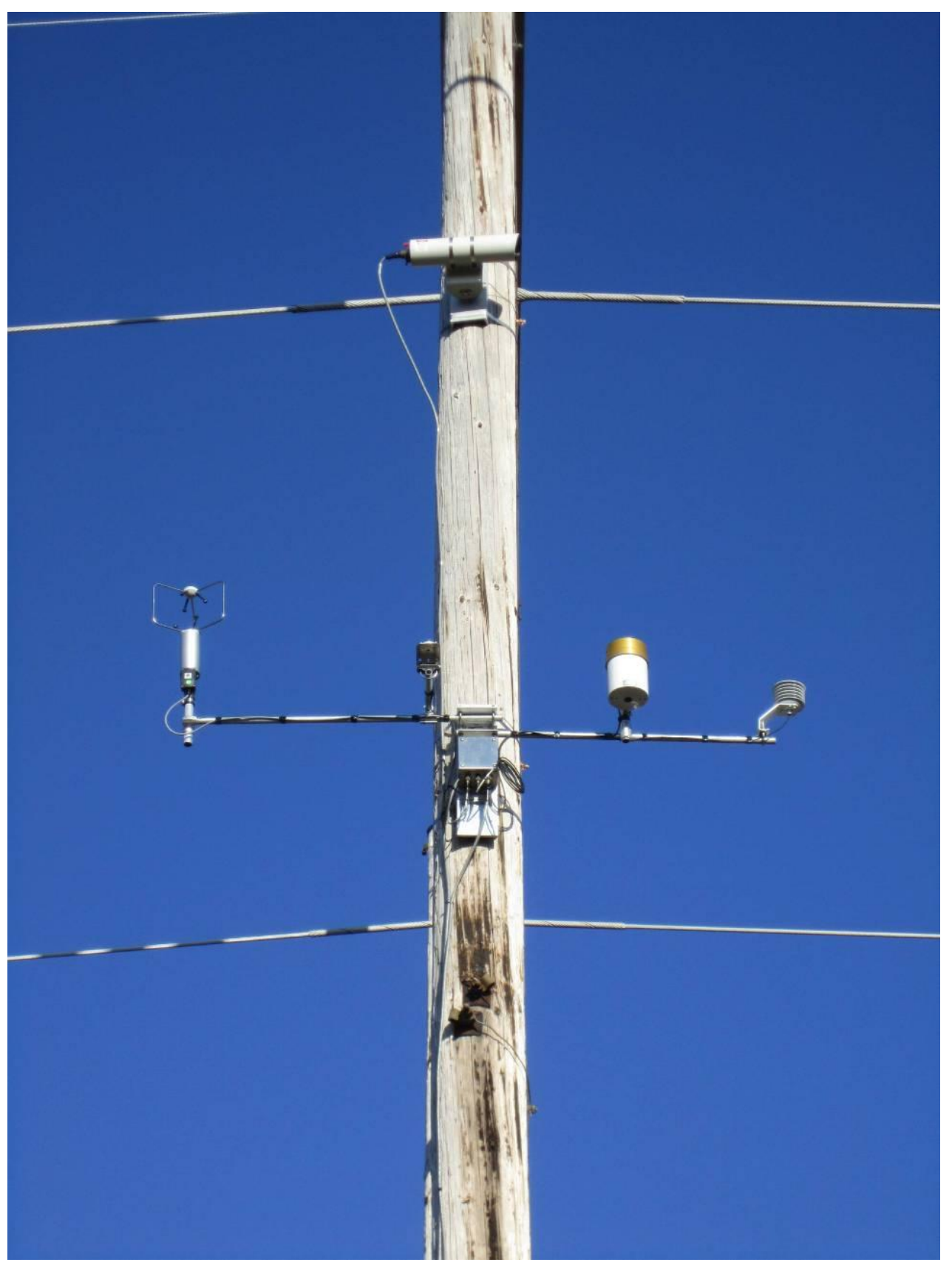

Figure C-48

Sagometer Camera and Weather Instruments

The conductor attachment point at structure Site 1 is 51 feet above the ground. The conductor attachment point at structure Site 1 is 52 feet above the ground. The height of the conductor at mid-span during the survey on 11/16/2010 was 34 feet. The height of the anemometer on structure Site 1 is 35 feet. 


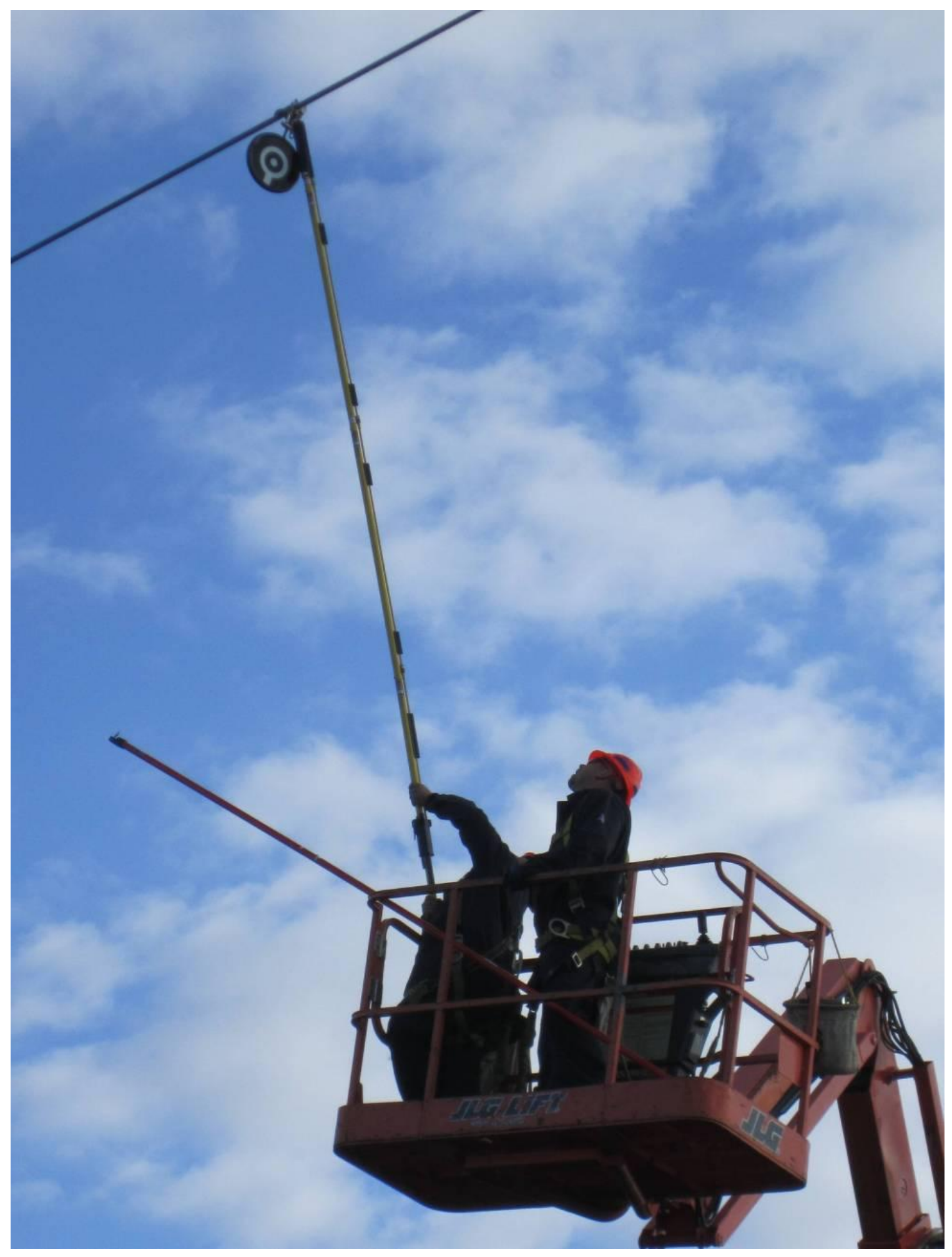

Figure C-49

Sagometer Target Being Installed 


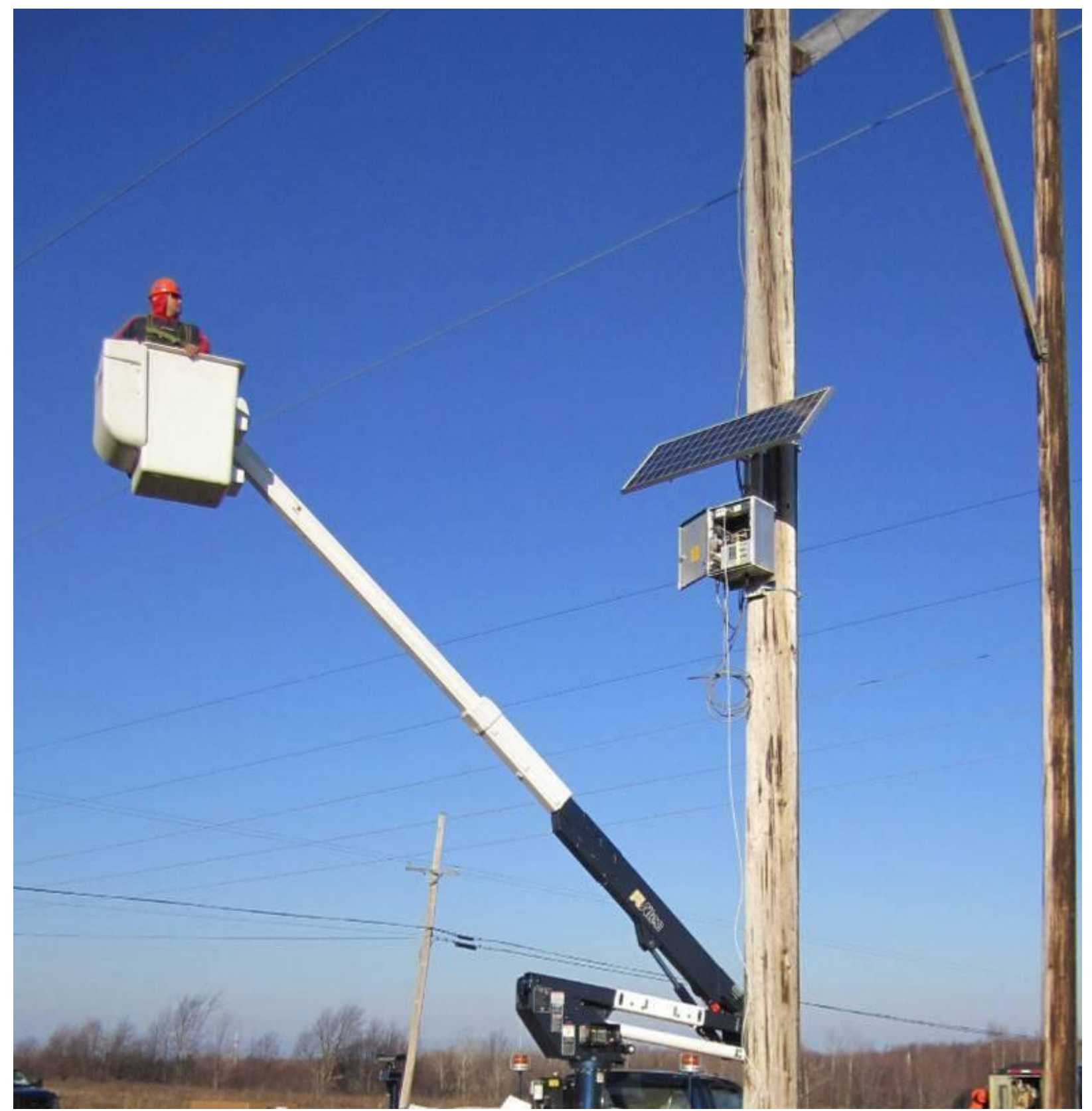

Figure C-50

Sagometer Electronic Box and Solar Panel Being Checked After Installation 


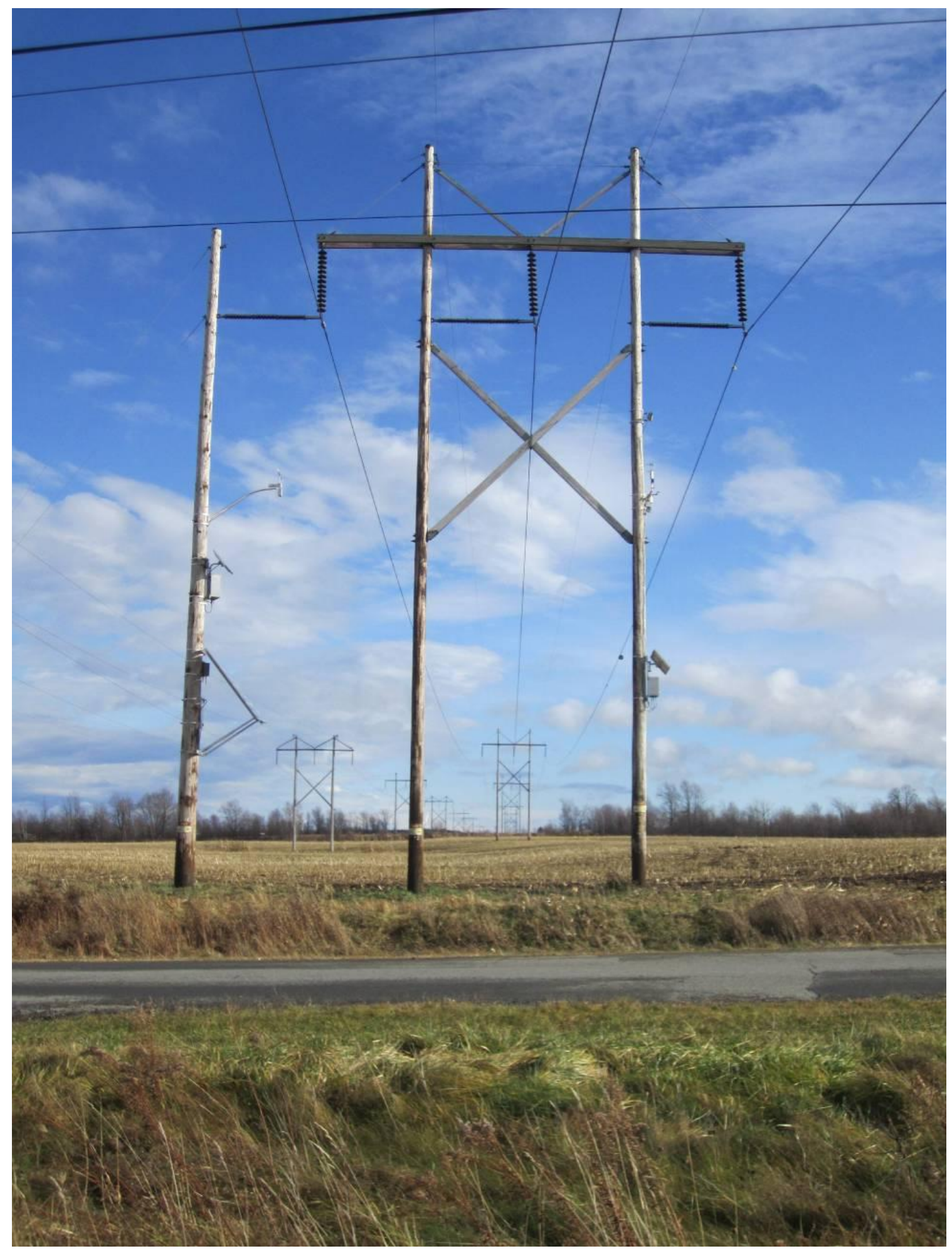

Figure C-51

Site 1 Completed - Looking in an Easterly Direction 


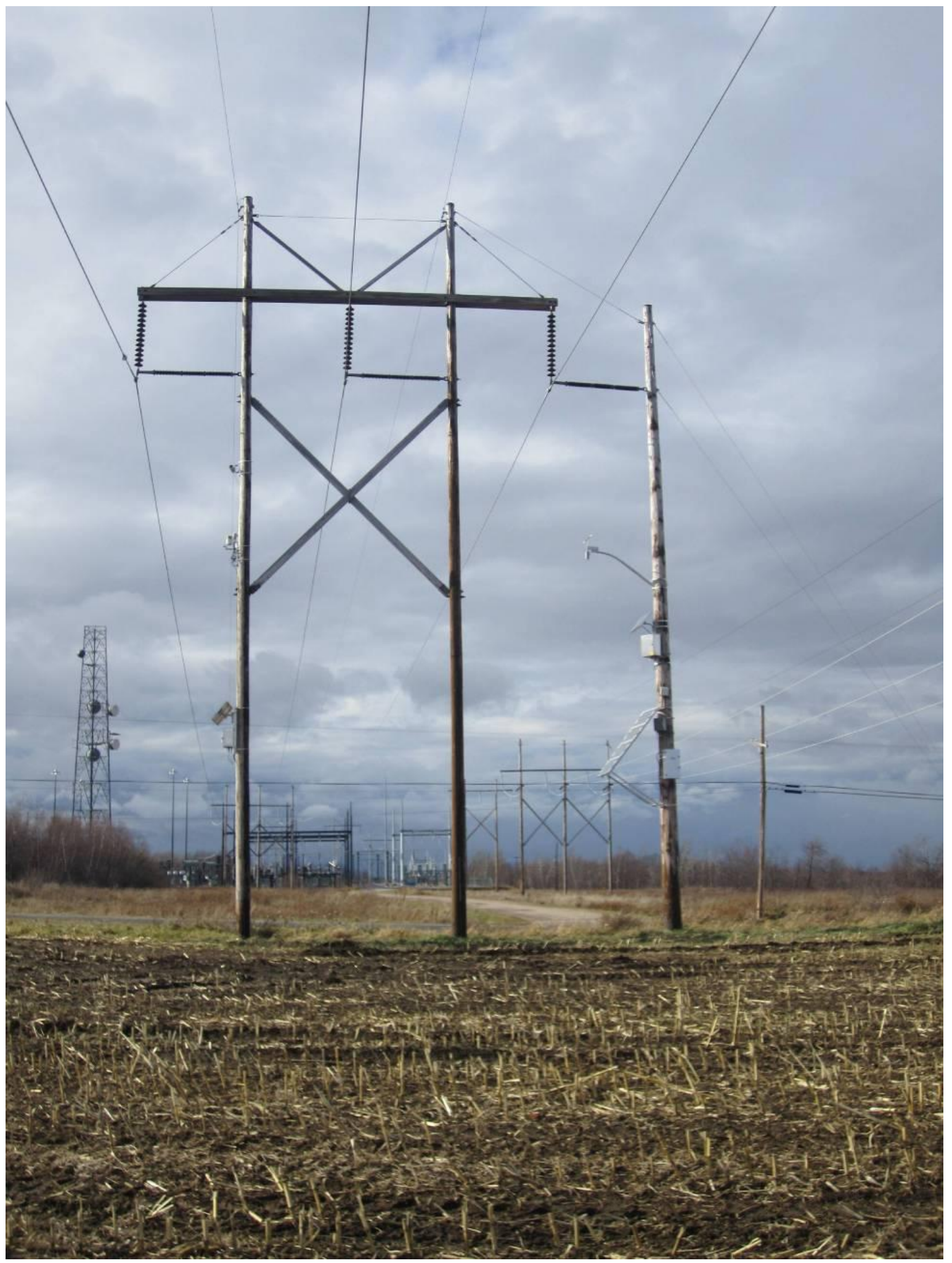

Figure C-52

Site 1 Completed - Looking in a Westerly Direction 


\section{Summary of Installation for Site 1}

The installation at Site 1 was performed from November $15^{\text {th }}$ thru $16^{\text {th }} 2010$. It took time on site to explain what was required for the survey of the first line. This was expected to some extent. However, it may work better to have a question and answer session prior to the first field install. This is something that could be added to test installs such as the Lenox one.

The line crew noted that during installation of the EPRI Sensors a wrench handle attached to the end of the hot stick fell off a few of times. The hot stick being used had a composite universal attachment end. When applying torque, this end can flex and loosen. It was noted by the line crew that a metal universal attachment ends work better in this type of application. This would not however relieve the dropping socket problem, only the dropping of the wrench. It will be beneficial for future projects to develop a workaround for this problem as well as have metal hot stick ends available.

A connector wire was broken off in the ThermalRate electronics box. The electronics box was taken down and swapped out with another one. The broken connector was repaired the next day. It may be beneficial to have an assortment of spare parts available for these types of situation; in such remote locations any setback can cause very long delays.

The ThermalRate system was not able to communicate with the RTU at Willis substation due to ongoing issues that could not be resolved during the field install. The main objective of this connection was to obtain the line current in real-time. During the months this issue took place the calculated real-time current from the EPRI Sensors was used.

There were a few initial communications issues between the sagometer and remote server. These were mostly associated with modem settings. These issues were corrected on site.

The best method for installing the EPRI Sensors and sagometer target on the line was to start with the sensors farthest away and work back towards the structure.

The solar panel support arms for the ThermalRate system needed to be retightened. It was thought that the wind may loosen the lockdown screws.

\section{Installation on the Site 2 Structure}

This site was located a few miles from the Willis Substation. This structure is at an elevation of approximately 1000 feet and this section of line is $86^{\circ}$ clockwise from north, based on information extracted from the plan and profile drawings. The conductor is $795 \mathrm{Kcmil}$ Drake ACSR. The wooden structure at this site is classified as type "AA".

As can be seen in the photo above, this section of line is mostly open level terrain with minimal sheltering from trees and other objects. Therefore, ruling span assumptions should be quite accurate. This Site is also in close proximity to a wind farm which suggests the line segment is located in a windy area. 


\section{EPRI Sensor}

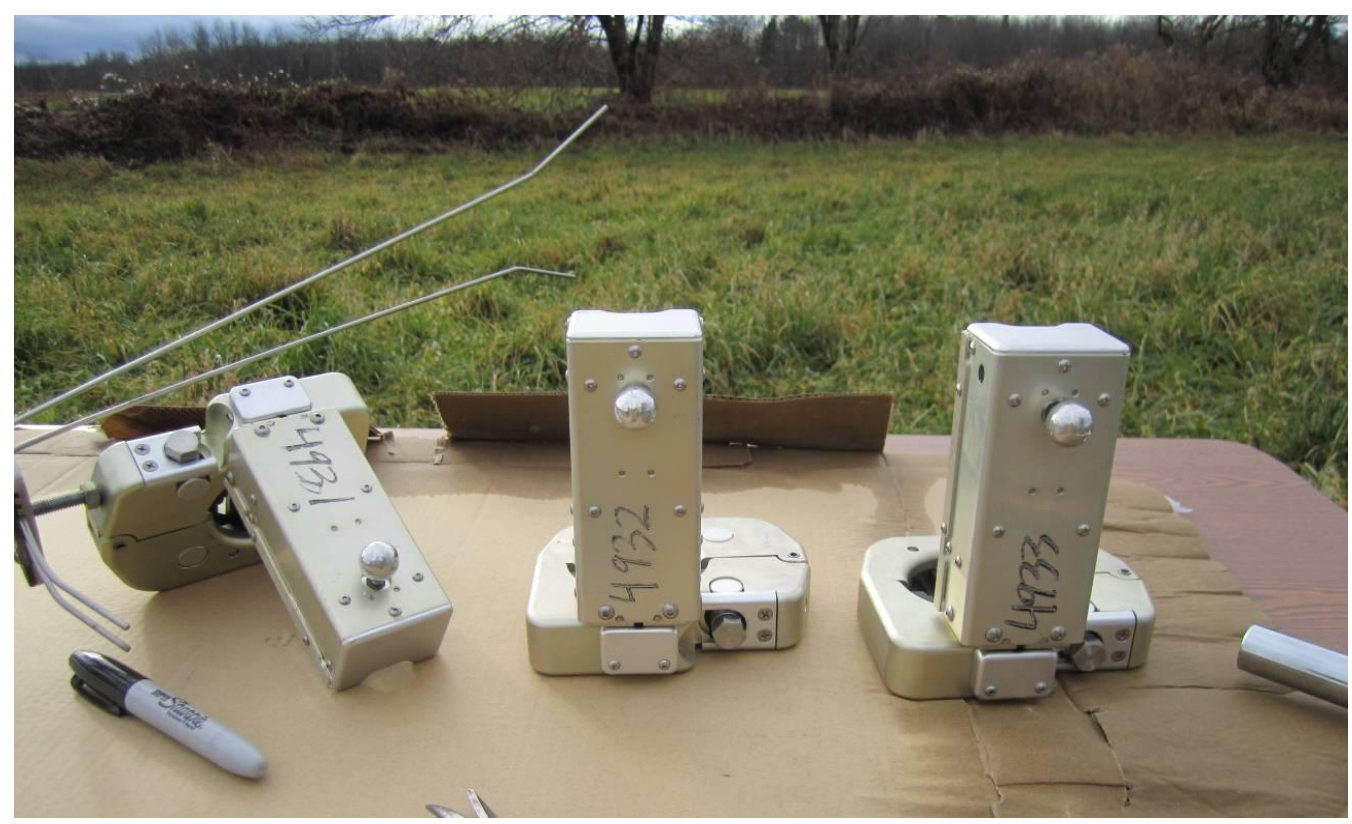

Figure C-53

EPRI Sensors Ready to Install

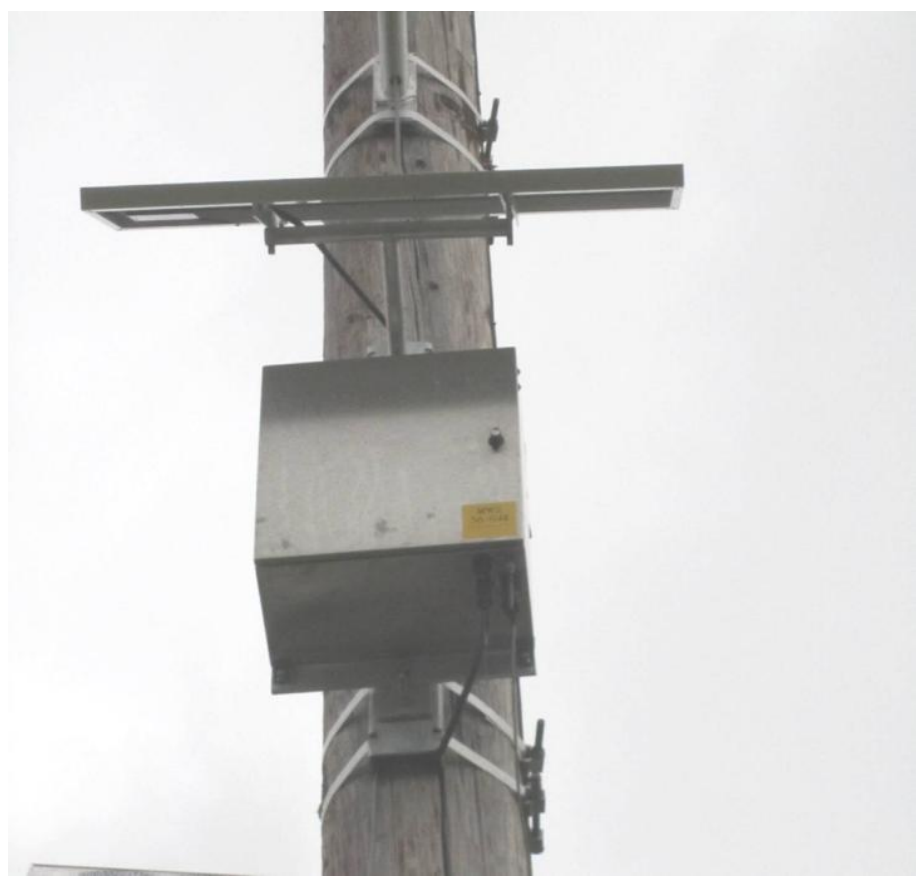

Figure C-54

EPRI Sensor Electronics Box and Solar Panel Installed 


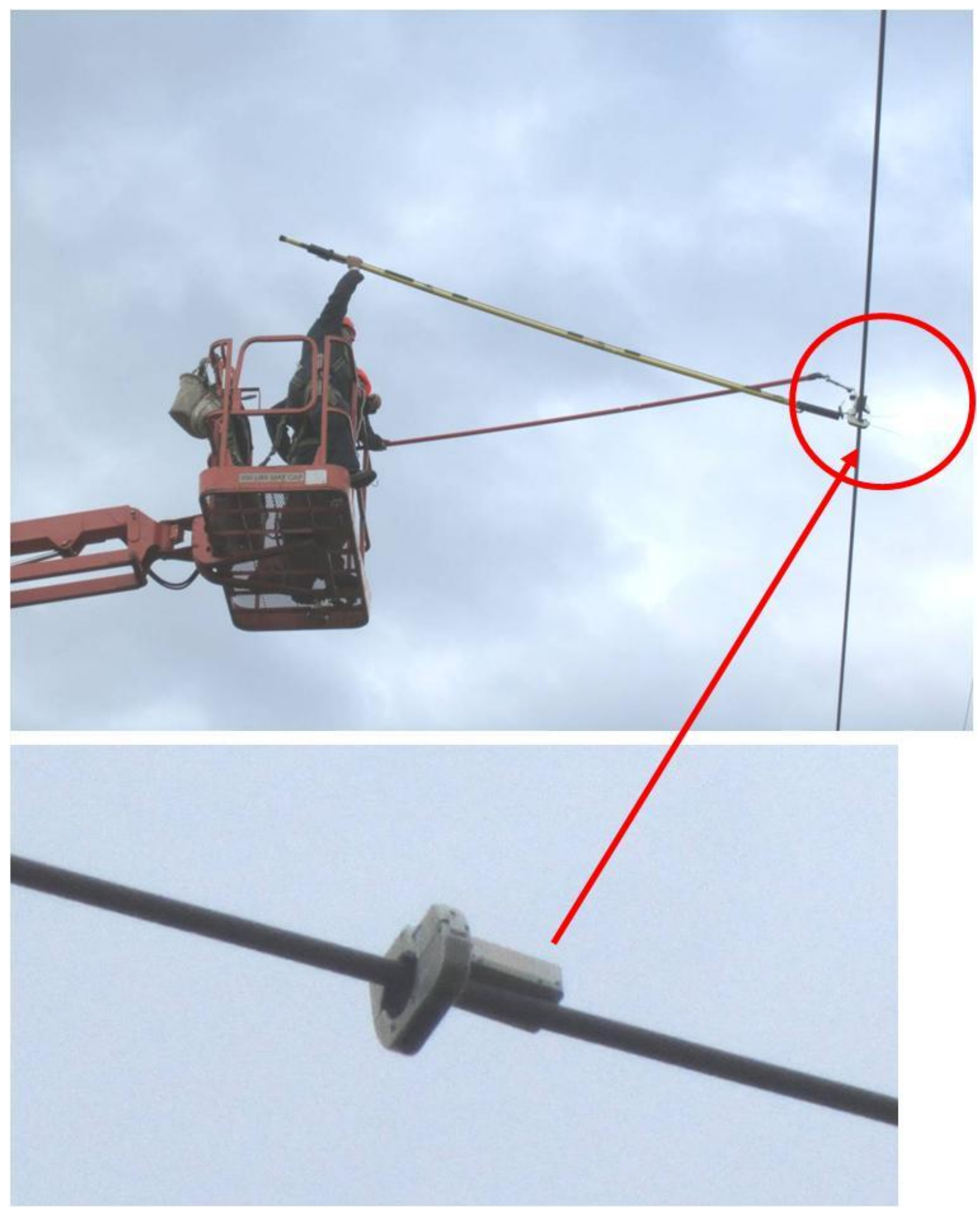

Figure C-55

EPRI Sensor Being Installed 
ThermalRate System

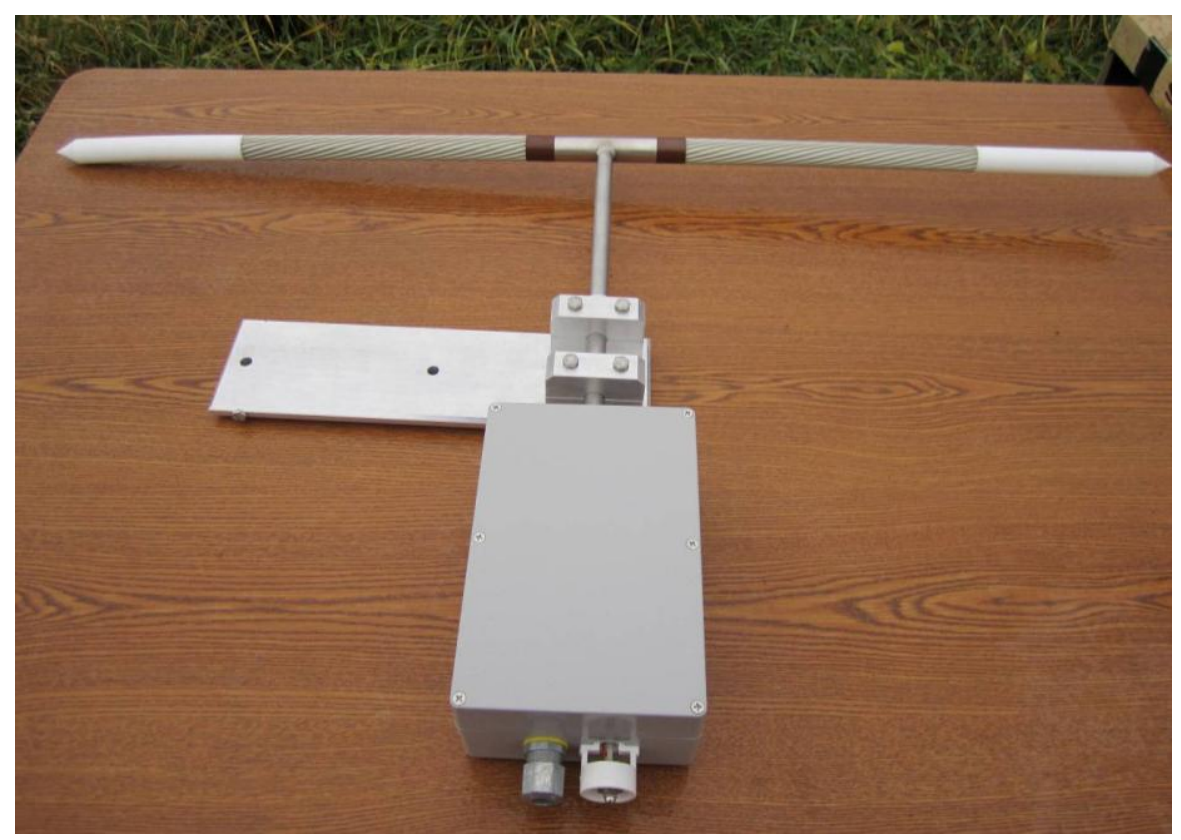

Figure C-56

ThermalRate Sensor Being Readied for Installation

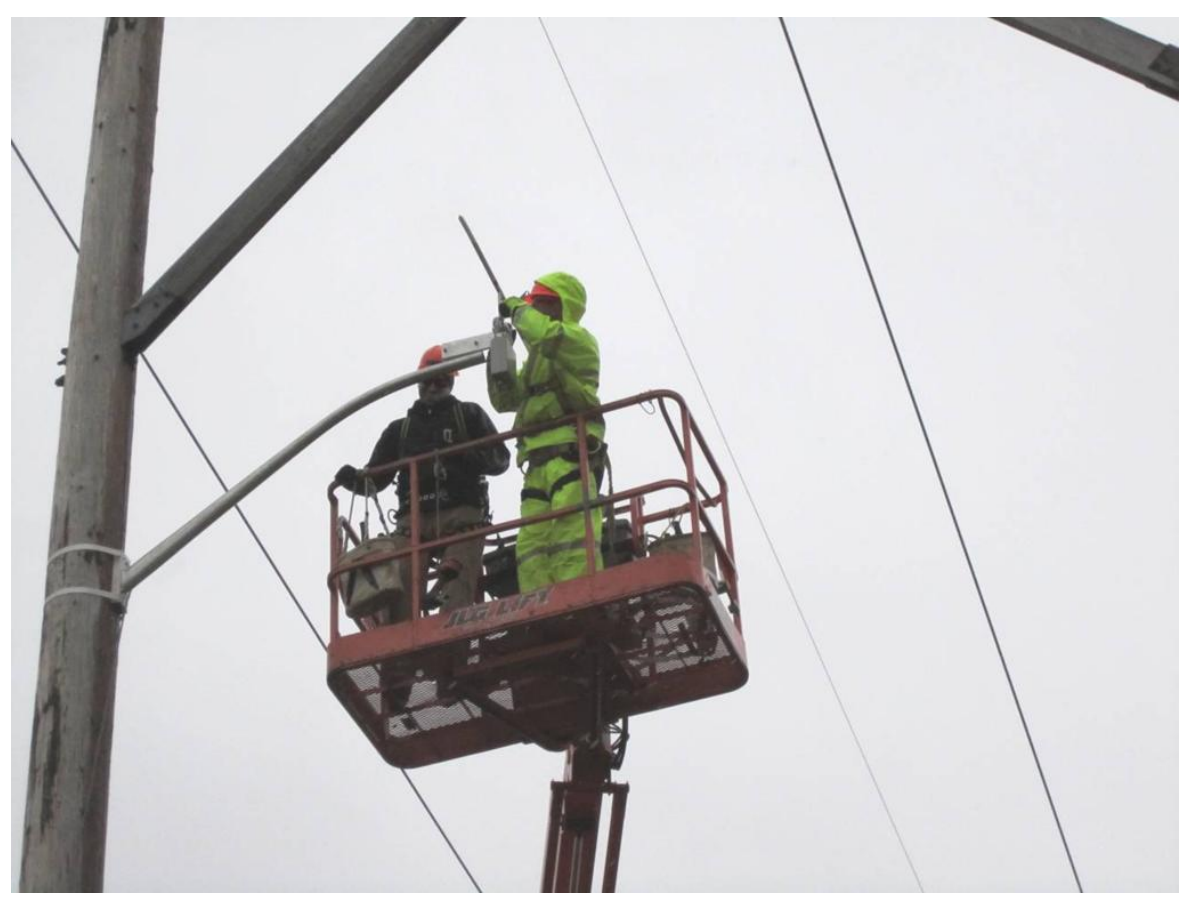

Figure C-57

Installing the ThermalRate Sensor 

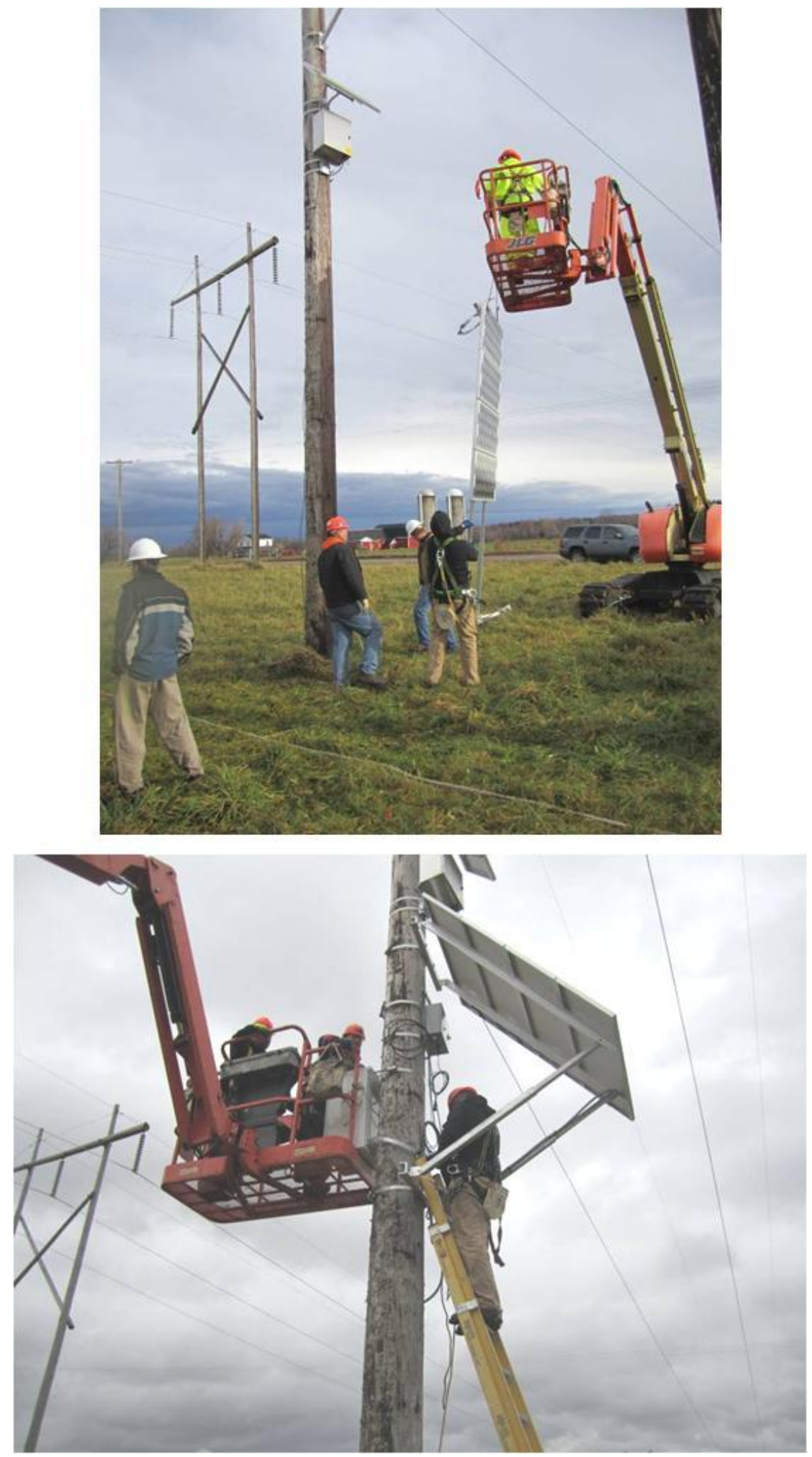

Figure C-58

ThermalRate System Solar Panel and Electrical Box Being Installed 


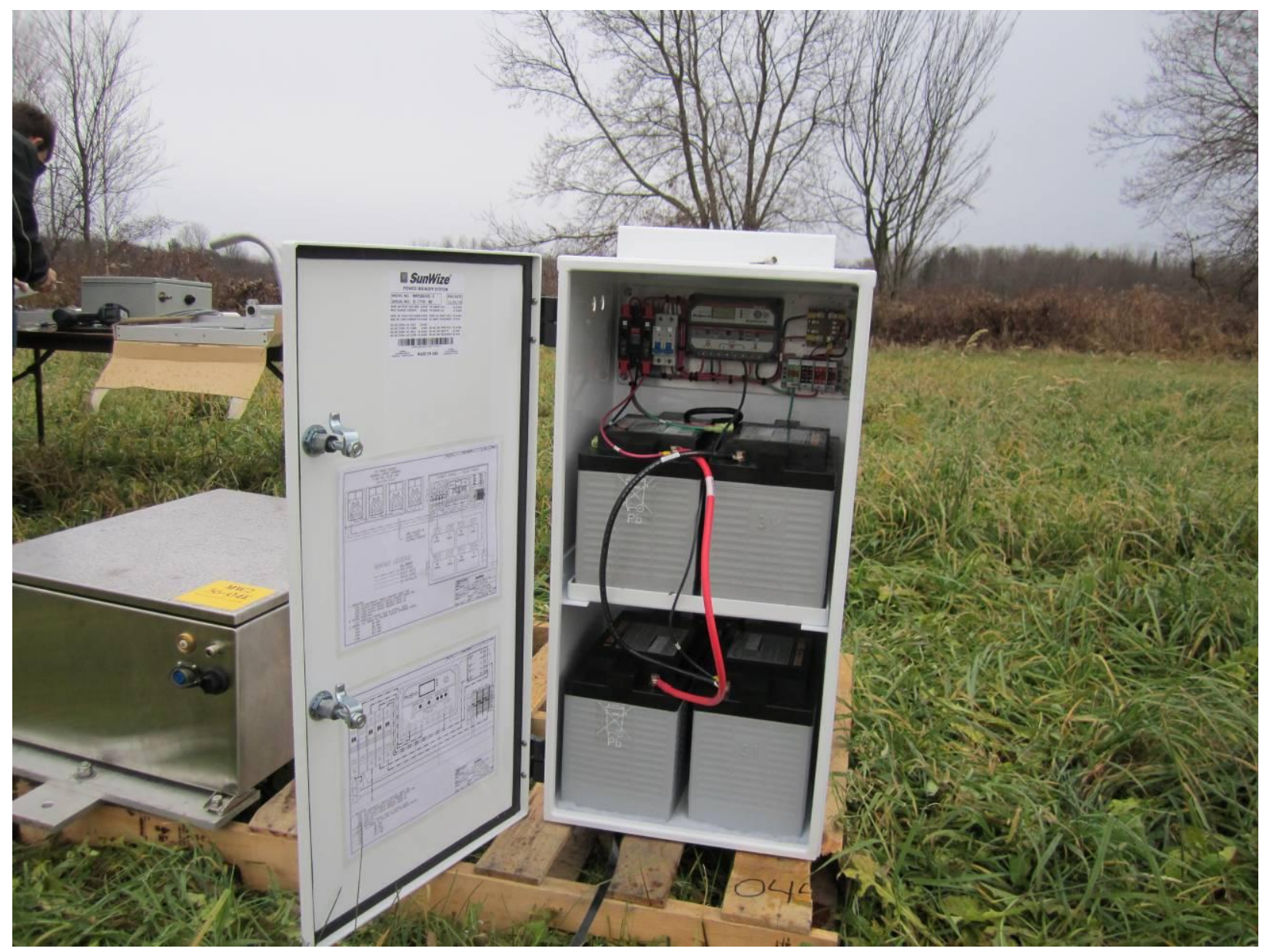

Figure C-59

ThermalRate Battery Box 


\section{Sagometer System}

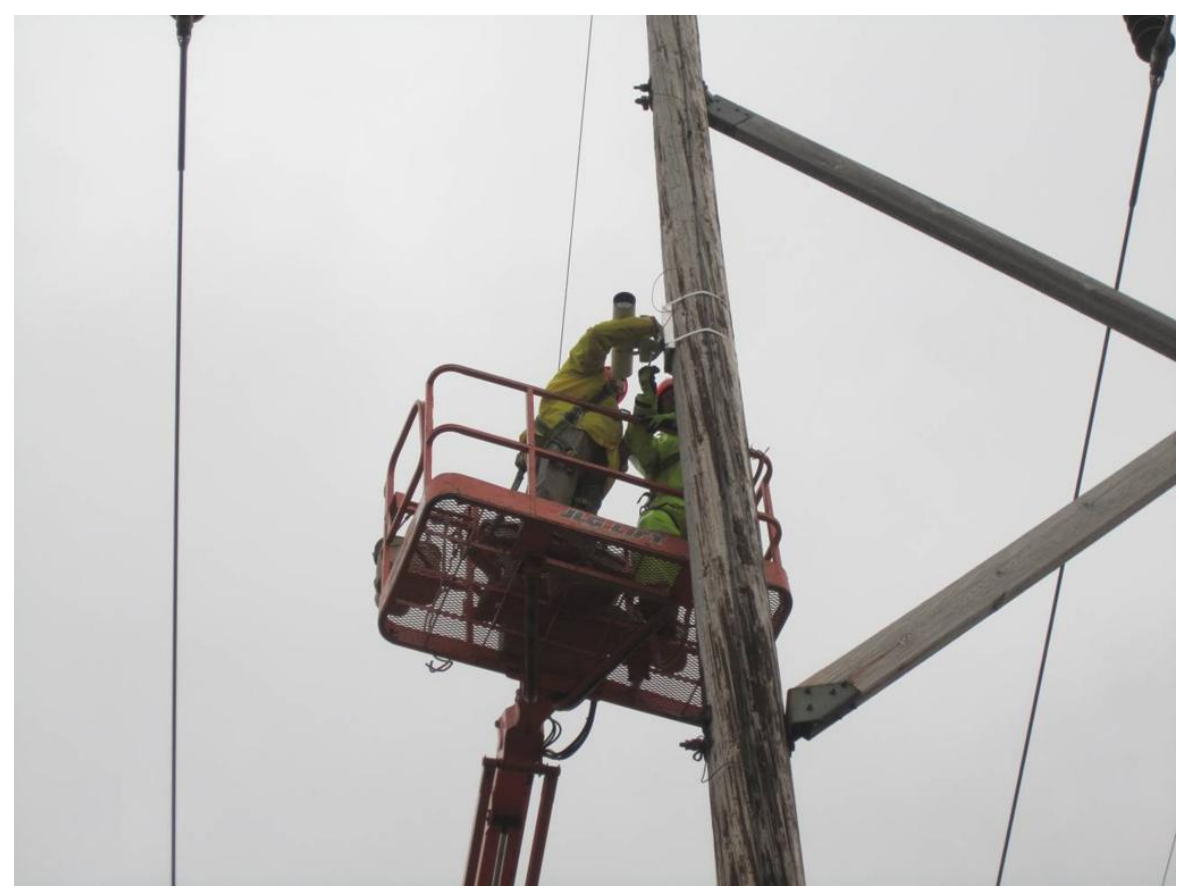

Figure C-60

Installed the Sagometer Camera

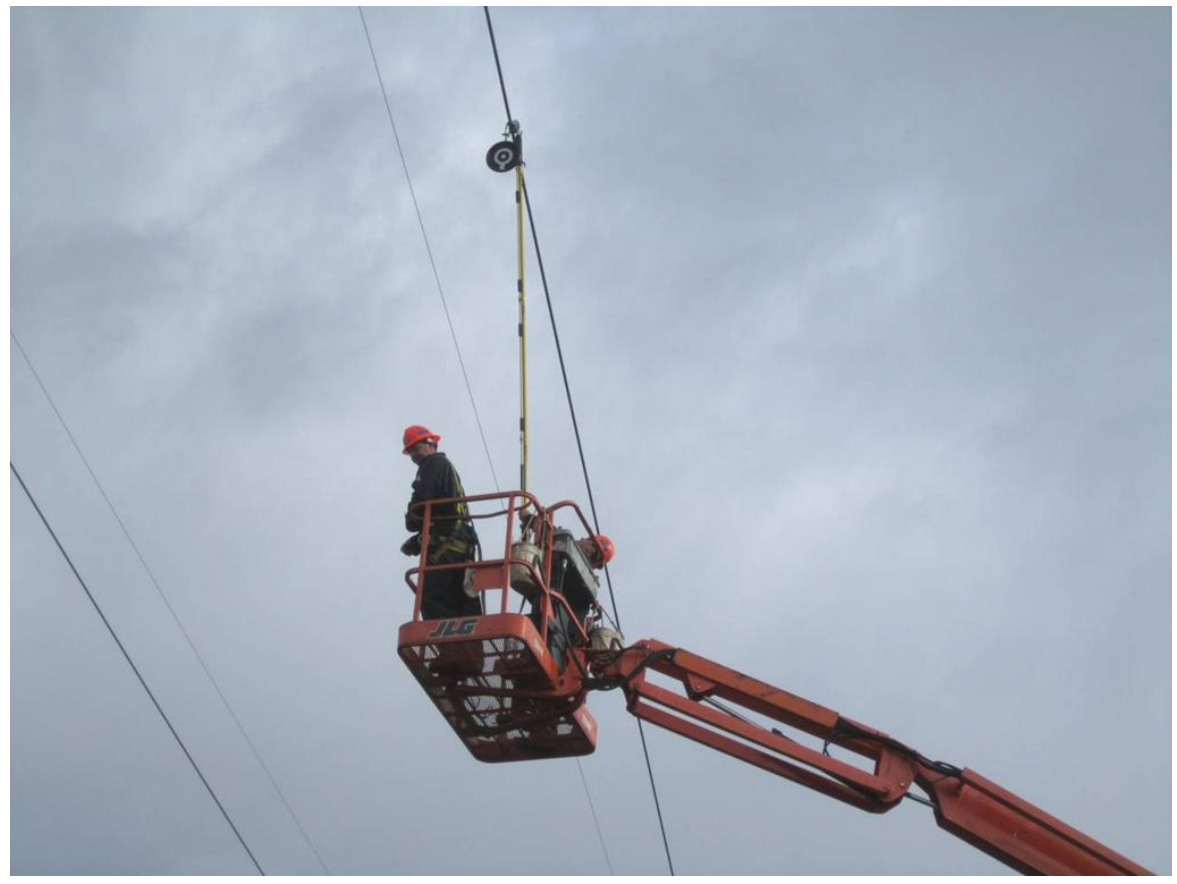

Figure C-61

Installing the Sagometer Target 


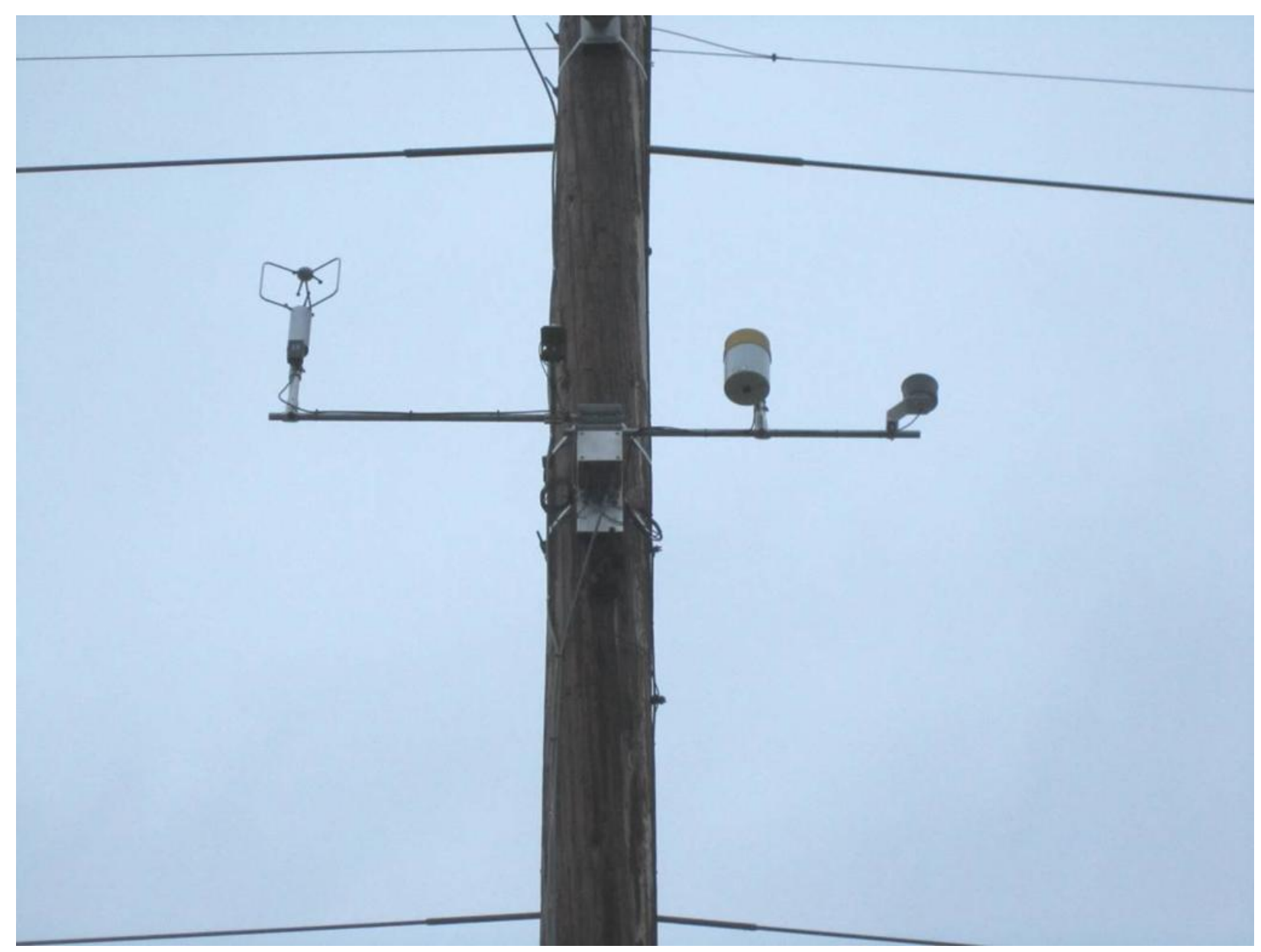

Figure C-62

Sagometer Weather Instruments Installed

The conductor attachment point is $\mathbf{5 7}$ feet above the ground.

The conductor attachment point is 42 feet above the ground.

The height of the conductor at mid-span during the survey on 11/17/2010 was 33 feet.

The height of the anemometer is 38 feet. 


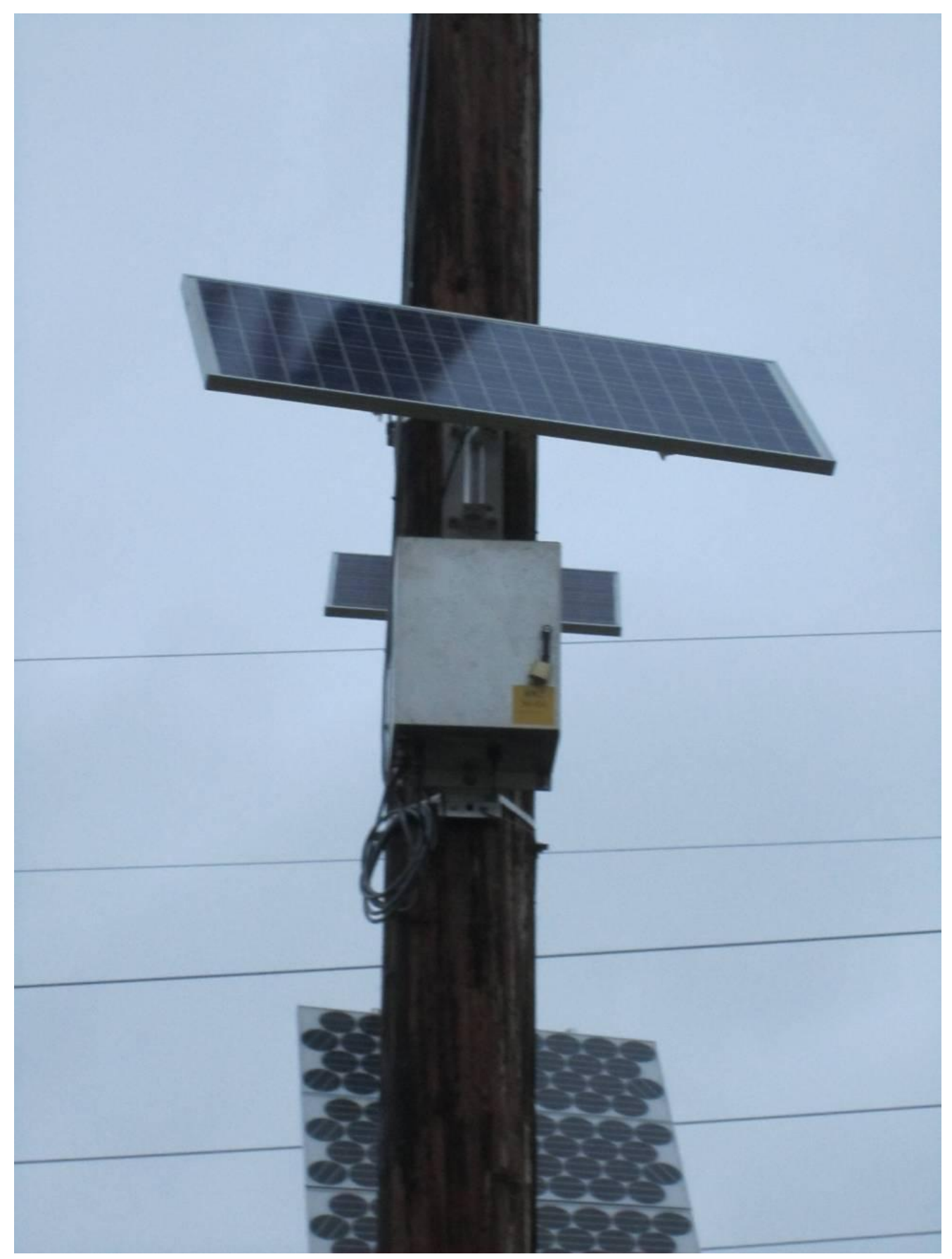

Figure C-63

Sagometer Electronics Box and Solar Panel Installed 


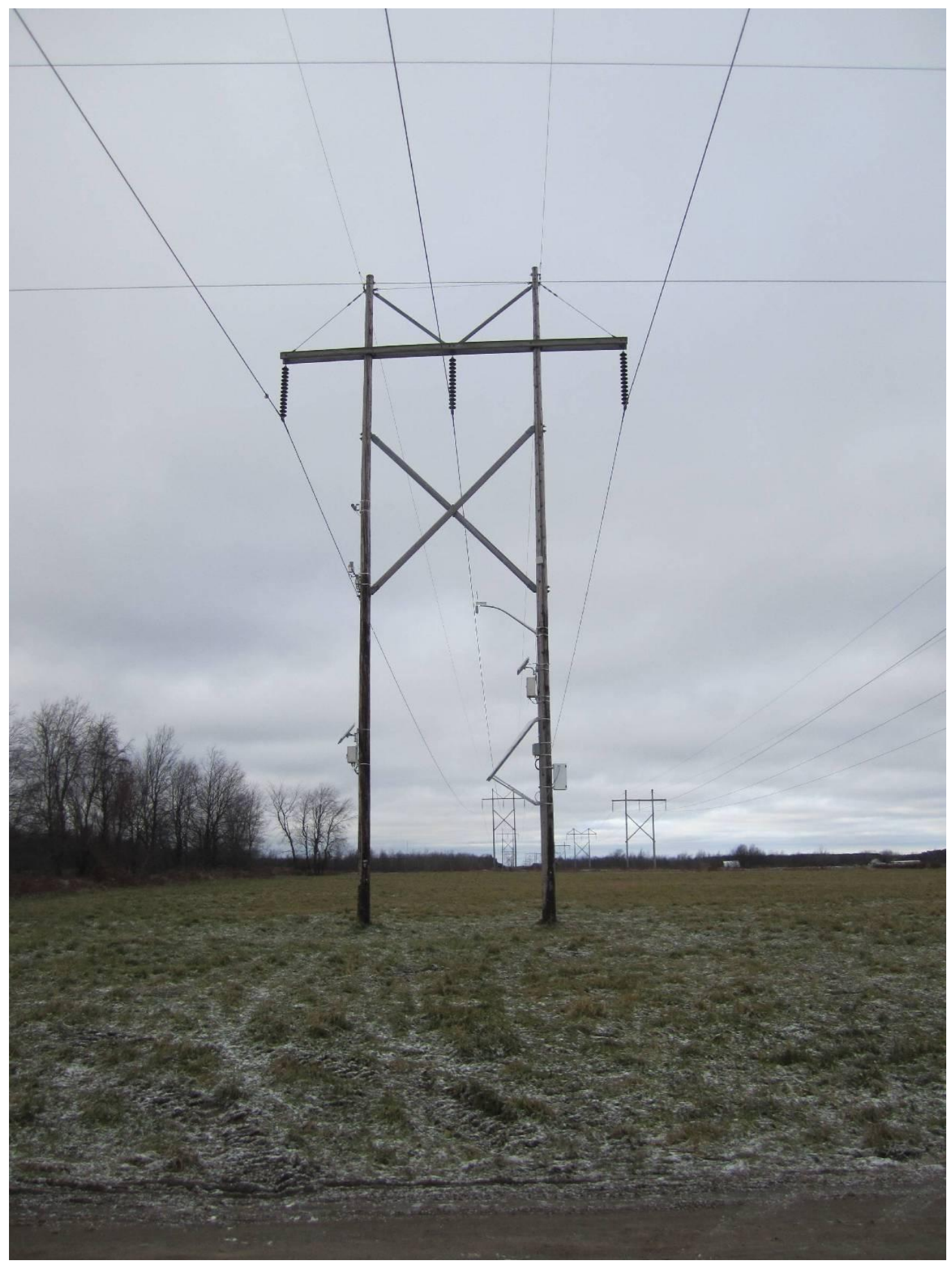

Figure C-64 Install Completed at Site 2 - Looking in a Westerly Direction 


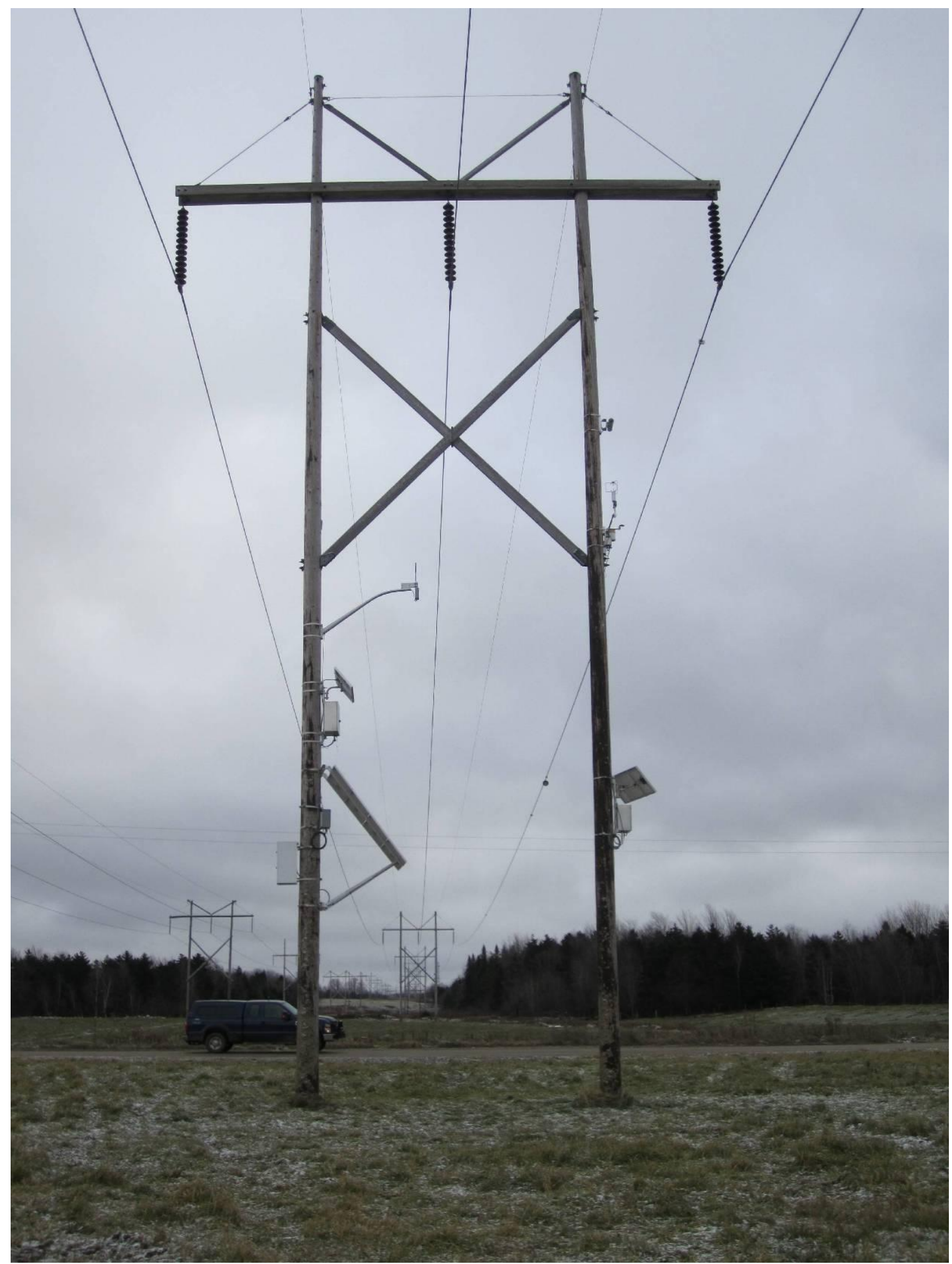

Figure C-65

Install Completed at Site 2 - Looking Back in an Easterly Direction 


\section{Summary of Installation for Site 2}

The installation at Site 2 was completed on November $17^{\text {th }} 2010$. The site installation was completed in just one day. Heavy rain in the morning caused a shutdown of work for about 45 minutes.

EPRI Sensors were installed relatively quickly at this site. The line crew was more familiar with the installation method and it only took a few minutes at each conductor location to install the sensor. The electronic boxes and other instruments were installed relatively quickly at this site as well. The Sagometer target was moving around somewhat due to windy conditions during the calibration process. Excessive movement of the target can affect calibration and one should be aware of this effect. The communications check with all three systems went smoothly. No major issues were noted.

\section{Installation on the Site 3 Structure}

This site was located near Moses Substation. This structure is at an elevation of approximately 200 feet and this section of line is $121^{\circ}$ clockwise from north, based on information extracted from the plan and profile drawings. The conductor is $795 \mathrm{Kcmil}$ Drake ACSR. The wooden structure at this Site is classified as type "AA".

As can be seen in the photos below, this section of line is mostly open level terrain with minimal sheltering from trees and other objects. Therefore, ruling span assumptions should again be quite accurate. 


\section{EPRI Sensor}
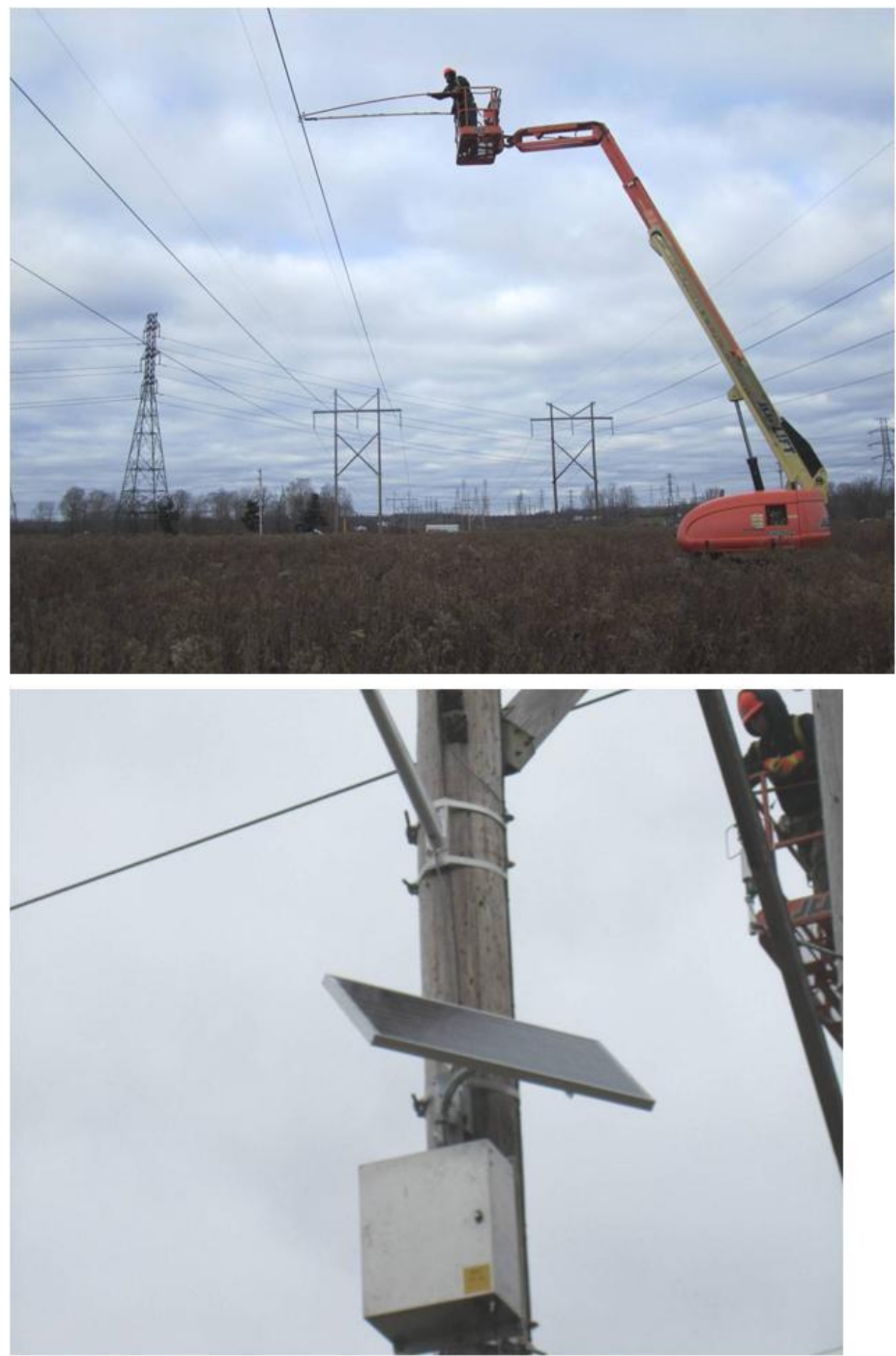

Figure C-66

EPRI Sensor Electronics Box and Solar Panel 
ThermalRate System

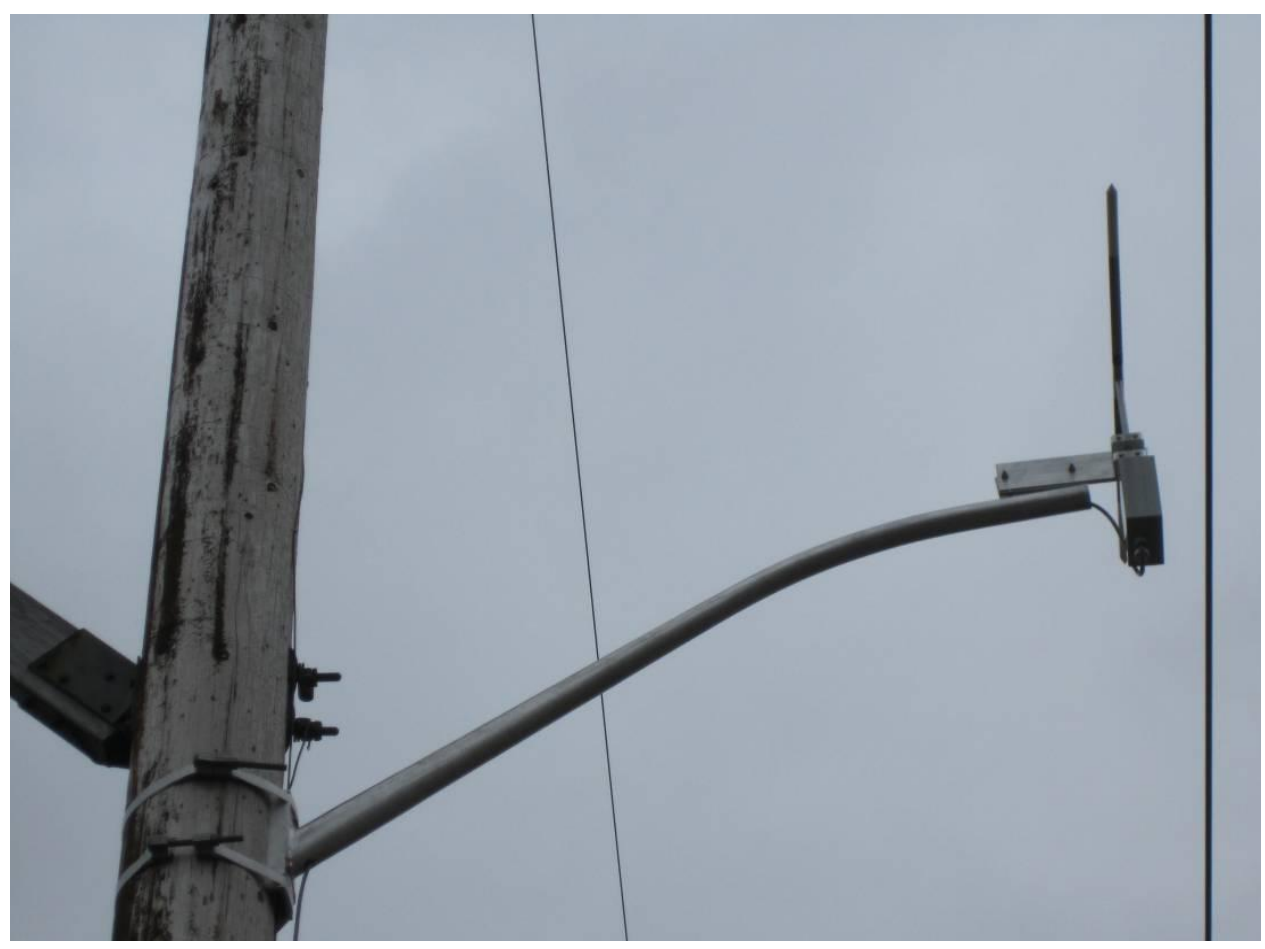

Figure C-67

ThermalRate Sensor and Arm Installed

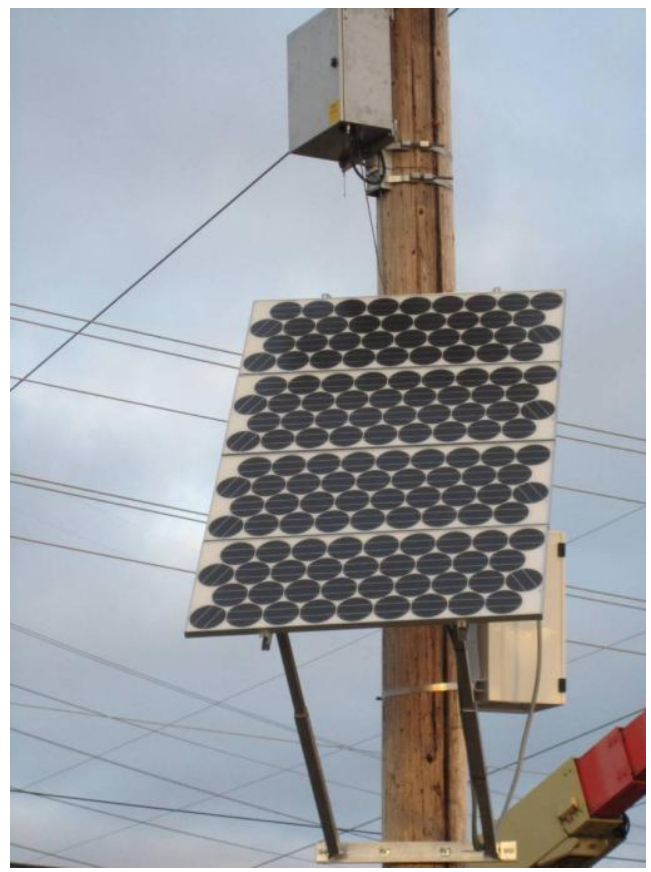

Figure C-68

ThermalRate Solar Panel Installed

Sagometer System 


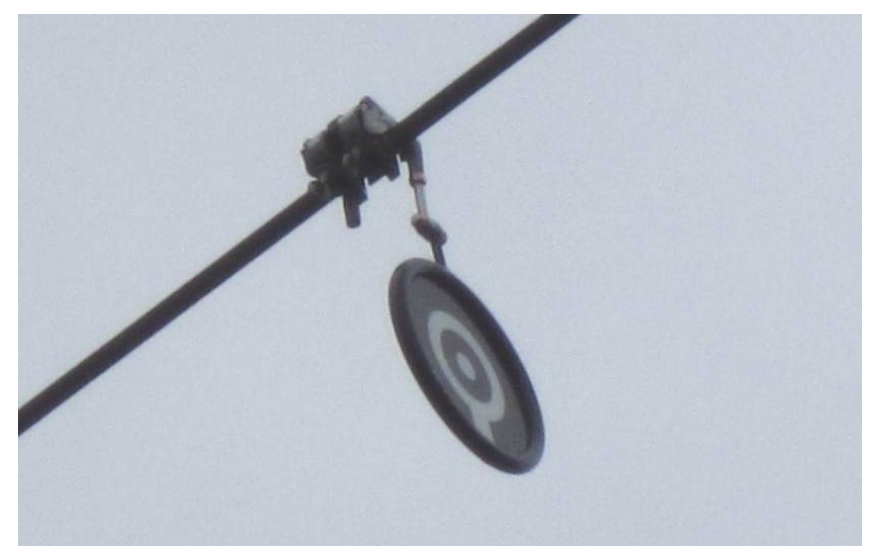

Figure C-69

Sagometer Target Installed

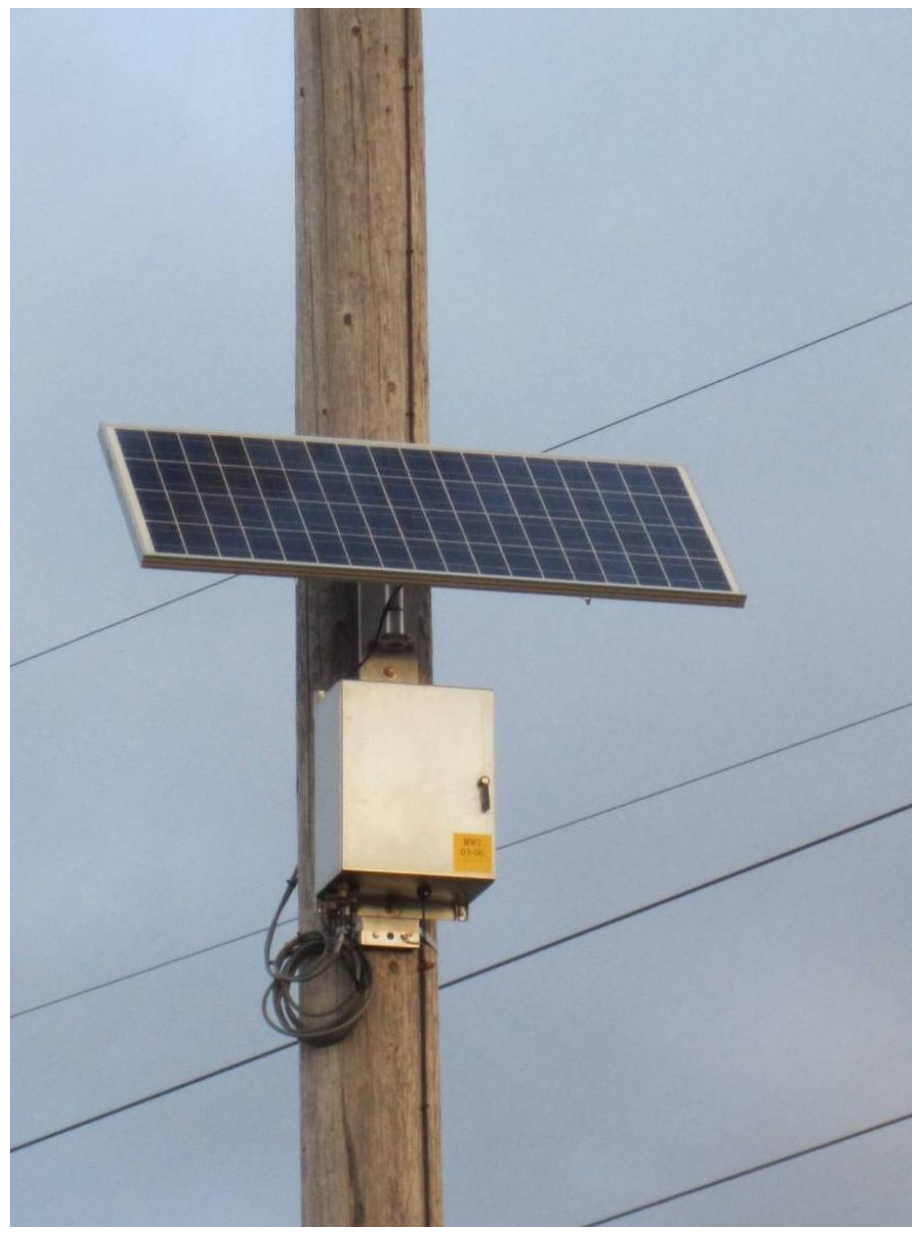

Figure C-70

Sagometer Solar Panel and Electronics Box 


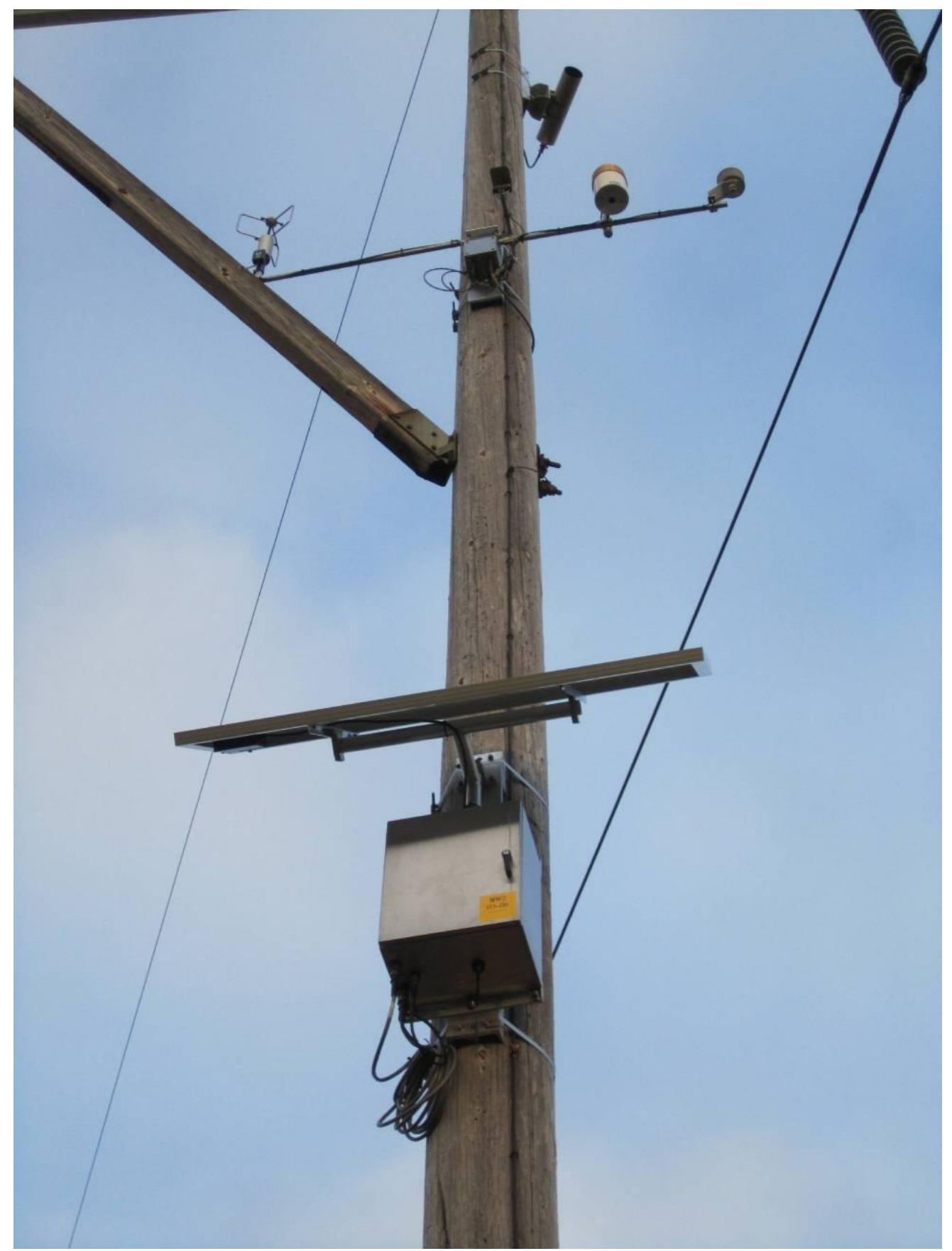

Figure C-71

Sagometer Camera, Weather Sensors, Solar Panel and Electronics Box

The conductor attachment point at structure Site 3 is 52 feet above the ground. The conductor attachment point at structure Site 3 is 48 feet above the ground. The height of the conductor at mid-span during the survey on 11/18/2010 was 36 feet. The height of the anemometer on structure Site 3 is 36 feet. 


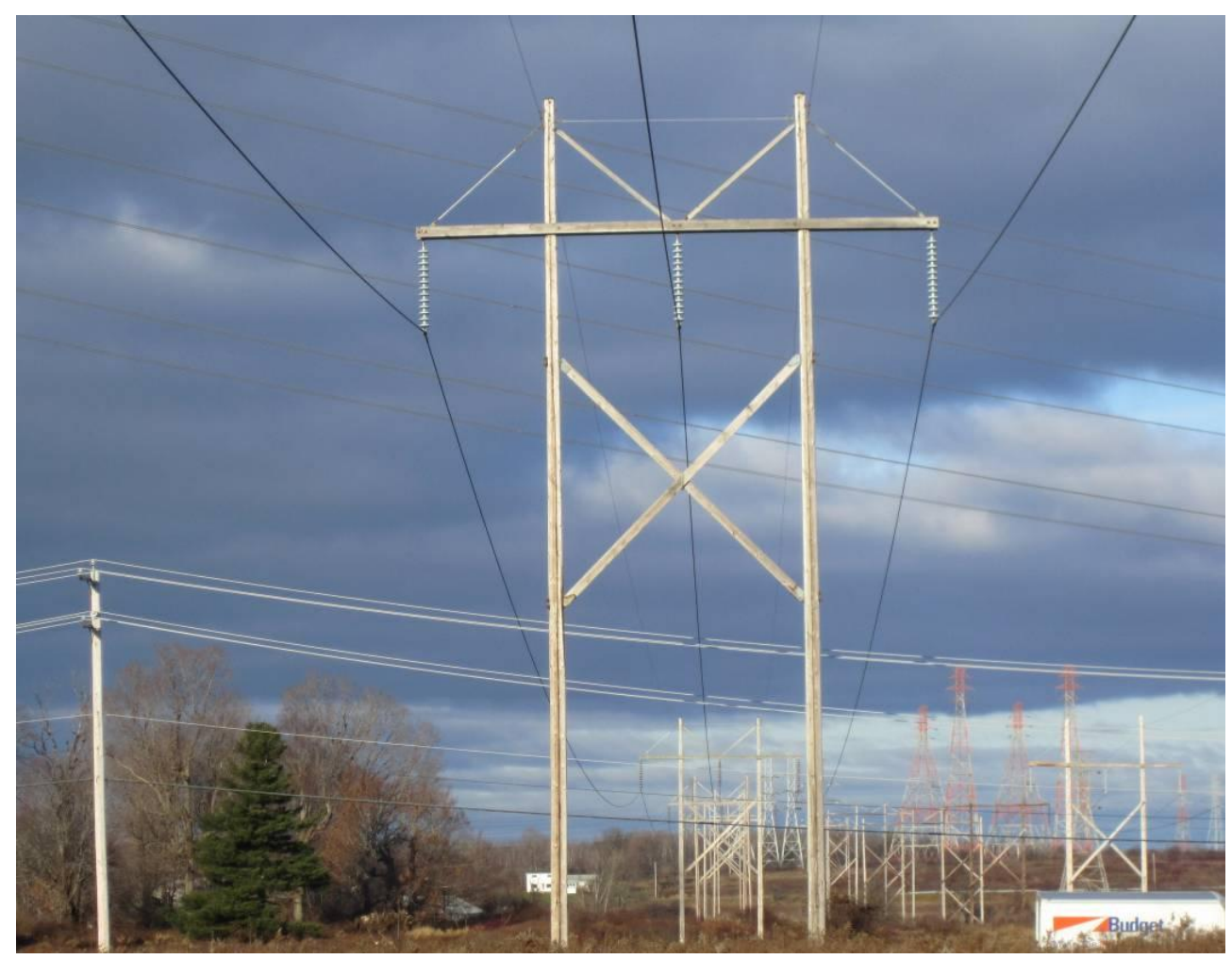

Figure C-72

Looking Back at Structure Site 3 Before Installation Beings

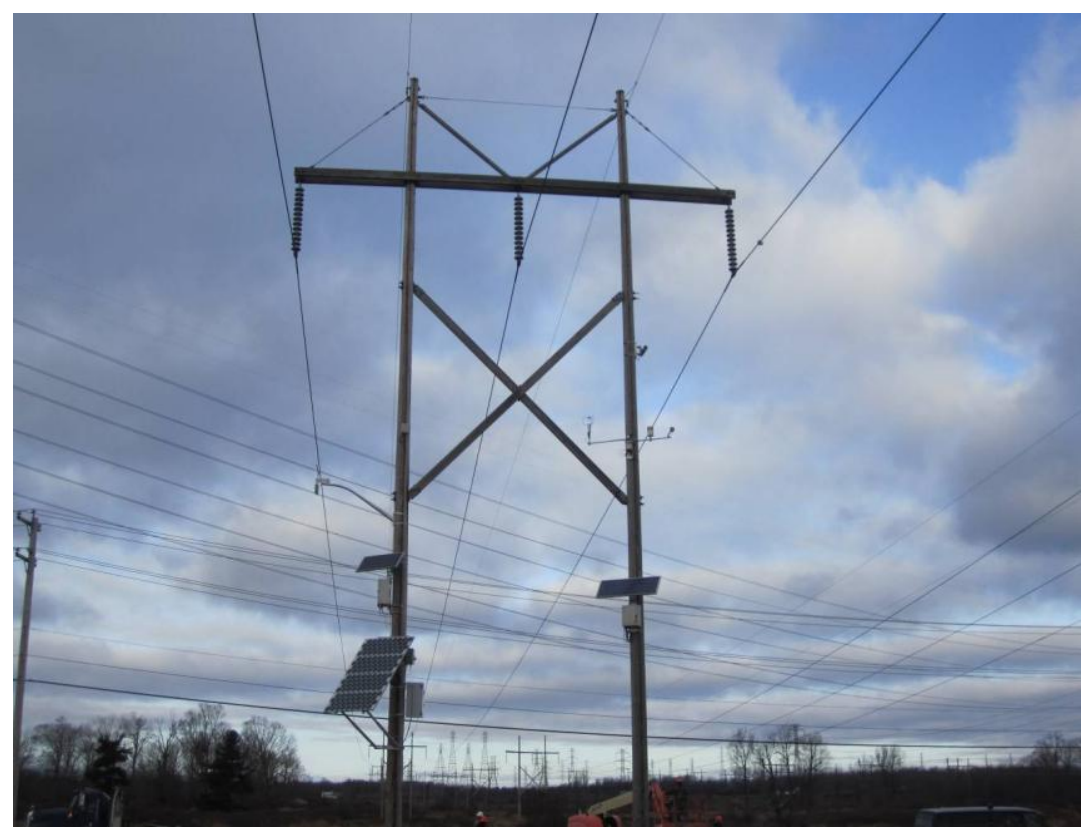

Figure C-73

Site 3 Completed - Looking Toward the Substation 


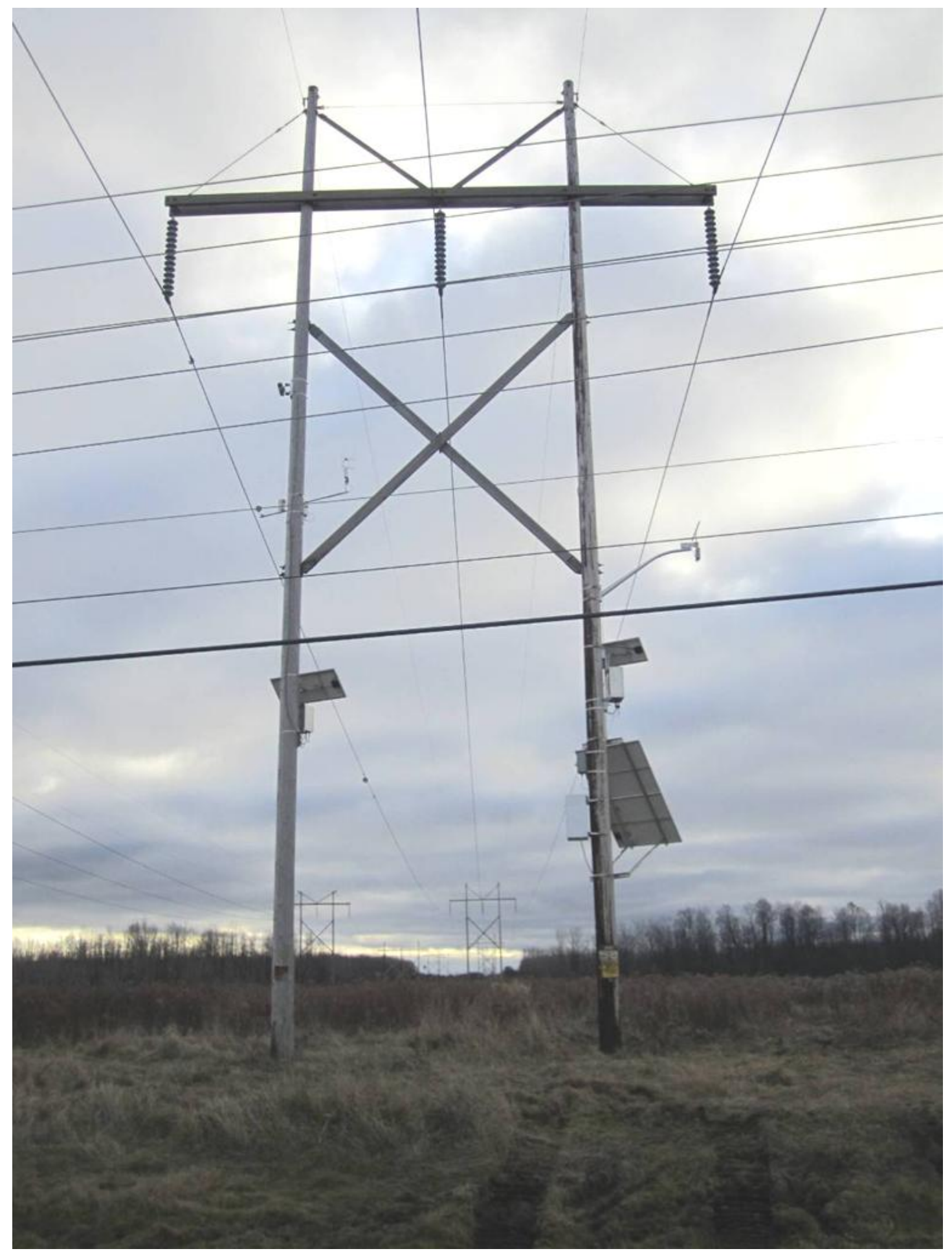

Figure C-74

Site 3 Completed - Looking Back in the Direction of Willis Substation

It is unclear why at this Site the ThermalRate boxes were placed behind the solar panel as opposed to directly underneath as was decided on in previous installation notes. 
It is unclear why at this Site the ThermalRate boxes were placed behind the solar panel as opposed to directly underneath as was decided on in previous installation notes.

\section{Summary of Installation for Site 3}

The installation at Site 3 was completed on November $18^{\text {th }} 2010$. This was the fourth installation of the project. Again the installation of this Site was completed in one day. The team had worked out an efficient method for installing the instruments. Below is a chronological order of the install at this Site:

- $8: 15 \mathrm{am}$ - team arrived

- $8: 30$ am - unloaded truck and instruments prepared

- 9:15 am - lifts and line crew arrive

- 10:00 am - hot sticks checked and tested

- 10:15 am - first EPRI Sensor installed

- 11:00 am - other two EPRI Sensors and target installed

- $12: 15-1: 15 \mathrm{pm}-$ lunch

- 1:15 - 3:15 pm - Video Sagometer and control box installed on north pole; EPRI Sensor control box and ThermalRate sensor and control box installed on south pole

- 3:25 pm Thermal Rate solar panel and battery box installed

- There was a software communication issue between the sagometer camera and data logger during setup and calibration. A software switch enabled to shut power off to the camera during normal field operation was set "on". This condition made the camera appear to be down. The problem was corrected by bypassing the camera "off" setting while adjustments were being made during calibration and then reset to power off at a specific interval later. As the camera is in a sealed case the unit had to be re-pressurized before operation.

- The line crew dropped the tool socket once while installing the first EPRI Sensor. 



\section{$D$ \\ ANEMOMETER STUDIES}

Wind speed and direction are the variables that have the greatest effect on real-time ratings. For the IEEE 738 weather based model in DTCR, the wind speed and direction data are converted to effective perpendicular wind speed from which the heat transfer is derived for use in the rating calculations. However, wind data used by DTCR in this project are not simple analog measurements. Before the wind data are ever logged and communicated to the DTCR server a complex series of calculations surround determining the wind speed and direction. A summary of these calculations and methodology follows.

\section{The Anemometer}

At the NYPA Sites, RM Young 81000 3-D anemometers configured by EDM are used. This type of anemometer has an adjustable response rate from 4 to $32 \mathrm{~Hz}$. The default system setting allows the device to sample four data points per second, perform a scalar average and transmit that to the logger. The EDM configuration has the anemometer neglecting three of these data points and simply transmitting the last data point each cycle, which creates an output at $1 \mathrm{~Hz}$.

The NYPA anemometer model uses Doppler technology to determine wind speed and direction using no moving parts. There is no aerodynamic stall for this technology as there is in mechanical anemometers but the device has a minimum reported wind velocity limit that for historical reasons is programmed as a "stall speed" of $0.2 \mathrm{~m} / \mathrm{s}(0.65 \mathrm{ft} / \mathrm{s})$. If the wind speed is below $0.2 \mathrm{~m} / \mathrm{s}$ the device reports zero. The stall speed limit can be overridden, down to a speed of $.01 \mathrm{~m} / \mathrm{s}$. The NYPA anemometers report zero wind speed when the wind is below stall speed. When setting DTCR error checking limits for the NYPA anemometers, zero is a valid wind speed. Some anemometers report the stall speed when the wind speed is below the limit. For these anemometers zero represents bad data so these are important details to note for future rating projects.

At certain speeds and directions, the support struts can cause wind deflections noticeable in the results. The manufacturer performs wind tunnel tests to determine the wake effect for these support struts. The 81000 has a mode titled "Wake Correction" which was designed and tested to compensate for the wake effect. For this NYPA project, the correction mode is active. The wake correction algorithm is specific to the machine, not the installation so the body of the anemometer is compensated for but not the pole or other nearby structures.

The outputs of the anemometer to the data logger are wind speed, 2D wind direction, wind elevation and local speed of sound. The sonic temperature is also calculated internally by the anemometer from measurements of the real-time local speed of sound. If the sonic temperature were also logged, then it could be compared to the ambient temperature and used for error checking. If the variation is more than $+/-2^{\circ} \mathrm{C}$ (per the anemometer manual) then there may be something interfering with the receivers and the values may not be as accurate. The most common cause for this is rain or snow, though dust particles or heavy fogs may also cause issues. 
Enabling this error check could reduce data availability, but increase data quality. It is not being performed by EDM for this project.

\section{The Logger}

The 1-second interval data is sent to the CR1000 data logger. The logger collects these points for 10 minutes and creates a 10-minute average of a total 600 points. The calculations are performed by Campbell Scientific subroutine called "WindVector()".

WindVector() uses wind speed measurements of zero when processing scalar wind speed but measurements at zero wind speed are not used for direction calculations.

The anemometer has a minimum sample rate of $4 \mathrm{~Hz}$, though its output to the CR1000 logger is $1 \mathrm{~Hz}$. There are a variety of averaging options that can be used for these samples. The configuration by EDM bypasses these methods and reports to the logger the last value only. A one second data burst such as in the table below would be sent to the CR1000 as a speed of 1.7 at a direction of $110^{\circ}$.

Table D-1

Sample Data per Time

\begin{tabular}{|l|l|l|}
\hline \multicolumn{1}{|c|}{ Time (seconds) } & \multicolumn{1}{|c|}{ Wind Speed $\mathbf{S}_{\mathbf{n}}(\mathbf{f t} / \mathbf{s})$} & \multicolumn{1}{|c|}{$\begin{array}{c}\text { Wind Direction } \boldsymbol{\theta}_{\mathbf{n}} \text { (degrees } \\
\mathbf{C W} \text { from North) }\end{array}$} \\
\hline 0.25 & 2.0 & 120 \\
\hline 0.50 & 2.2 & 140 \\
\hline 0.75 & 1.8 & 100 \\
\hline 1.0 & 1.7 & 110 \\
\hline
\end{tabular}

The logger then converts the 600 points in a 10 minute cycle to a single speed and direction. The table below contains only five samples but is used here to demonstrate averaging methods: 
Table D-2

Sample Data per Count

\begin{tabular}{|c|c|c|}
\hline $\mathbf{n}$ & Wind Speed $\mathbf{S}_{\mathbf{n}}(\mathbf{f t} / \mathbf{s})$ & $\begin{array}{c}\text { Wind Direction } \boldsymbol{\theta}_{\mathbf{n}} \text { (degrees } \mathbf{C W} \\
\text { from } \mathbf{\text { orth }}\end{array}$ \\
\hline 1 & 8 & 300 \\
\hline 2 & 3 & 10 \\
\hline 3 & 6 & 325 \\
\hline 4 & 4 & 350 \\
\hline 5 & 12 & 335 \\
\hline
\end{tabular}

Let $\mathrm{N}$ be the total number of points being averaged, in this case five. The 10-minute wind speed is calculated as a scalar as follows:

$$
\begin{gathered}
S=\sum_{1}^{N} s_{n} / N \\
S=(8+3+6+4+12) / 5=6.6 \mathrm{ft} / \mathrm{s}
\end{gathered}
$$

\section{Equation D-1}

If the wind direction $\theta$ is computed as an arithmetic average of the scalar values the result would be $264^{\circ}$ but wind is a vector and the scalar average is not considered meaningful. In addition to a scalar average, average wind direction can be calculated relative to either 0 or 180 degrees with the following method; which weights the value of each vector with the magnitude of wind $\mathrm{S}_{\mathrm{n}}$ : The weighted result for the example data set is:

$$
\theta=\arctan \left[\frac{\sum_{1}^{N} s_{n} \sin \left(\theta_{n}\right) / N}{\sum_{1}^{N} s_{n} \cos \left(\theta_{n}\right) / N}\right]=\arctan \frac{-3.12295}{5.336852}=-30^{\circ}=330^{\circ}
$$

\section{Equation D-2}

However in this project the direction is calculated by EDM by the preferred method used by NOAA neglecting $S_{n}$ and instead using unit vectors. The unit vector result for the example data set is:

$$
\theta=\arctan \left[\frac{\sum_{1}^{N} \sin \left(\theta_{n}\right) / N}{\sum_{1}^{N} \cos \left(\theta_{n}\right) / N}\right]=\arctan \frac{0.144648}{0.473927}=-24^{\circ}=336^{\circ}
$$

\section{Equation D-3}

The above is close to the weighted average. The results of the two methods are likely to converge over larger data sets. 
In summary 600 data pairs of speed and direction sent from the anemometer to the logger over a 10 minute period (represented in this example by the five pairs of data) are reduced to a single pair of data representing a wind vector (in this case $6.6 \mathrm{ft} / \mathrm{s}$ at $336^{\circ}$ ). These are the data that are permanently logged and sent to DTCR for rating calculations.

\section{Standard Deviation of Wind}

The standard deviation of both the wind speed and direction gives an indication of the degree of turbulence. Turbulence increases with the presence of local obstacles upstream and to a lesser extent downstream of the measurement point. The minimum amount of turbulence is also a function of wind speed. At low wind speeds the range of possible deviations can approach $0^{\circ}$ as well as $90^{\circ}$, while the maximum deviation at high wind speeds is lower, the minimum deviation can no longer approach $0^{\circ}$; this is shown in Figure D-1. While deviation data are not used by DTCR in rating calculations the standard deviations are calculated by the data logger. These data were analyzed to get insight into anemometer installation practices, as well as differences in rating models, namely ThermalRate and IEEE-738 onsite weather based ratings.

The standard deviation of wind speed magnitude is calculated assuming these data are scalars in a normal distribution. For our five-sample data set:

$$
\sigma_{s n}=3.58 f p s
$$

\section{Equation D-4}

The standard deviation of wind direction is calculated in the data logger as follows:

Where:

$$
\begin{aligned}
& \sigma=\arcsin (\mathrm{E})\left(1+0.1547 \mathrm{E}^{3}\right)^{\circ} \\
& \mathrm{E}=\sqrt{1-\left[\left(\sum_{1}^{N} \sin \left(\theta_{n}\right) / N\right)^{2}+\left(\sum_{1}^{N} \cos \left(\theta_{n}\right) / N\right)^{2}\right]}=0.3967
\end{aligned}
$$

\section{Equation D-5}

For the five-sample example:

$$
\sigma=24.8^{\circ}
$$

\section{Deviation of Wind Magnitude at NYPA}

Deviations in wind speed magnitude were examined for all Sites. The Site 3 data shown below is typical of the other Sites. Figure D-1 is a plot of standard deviation of wind speed magnitude in the horizontal plane vs. the wind speed magnitude calculated for the 10 minute interval for the month of June 2012: 


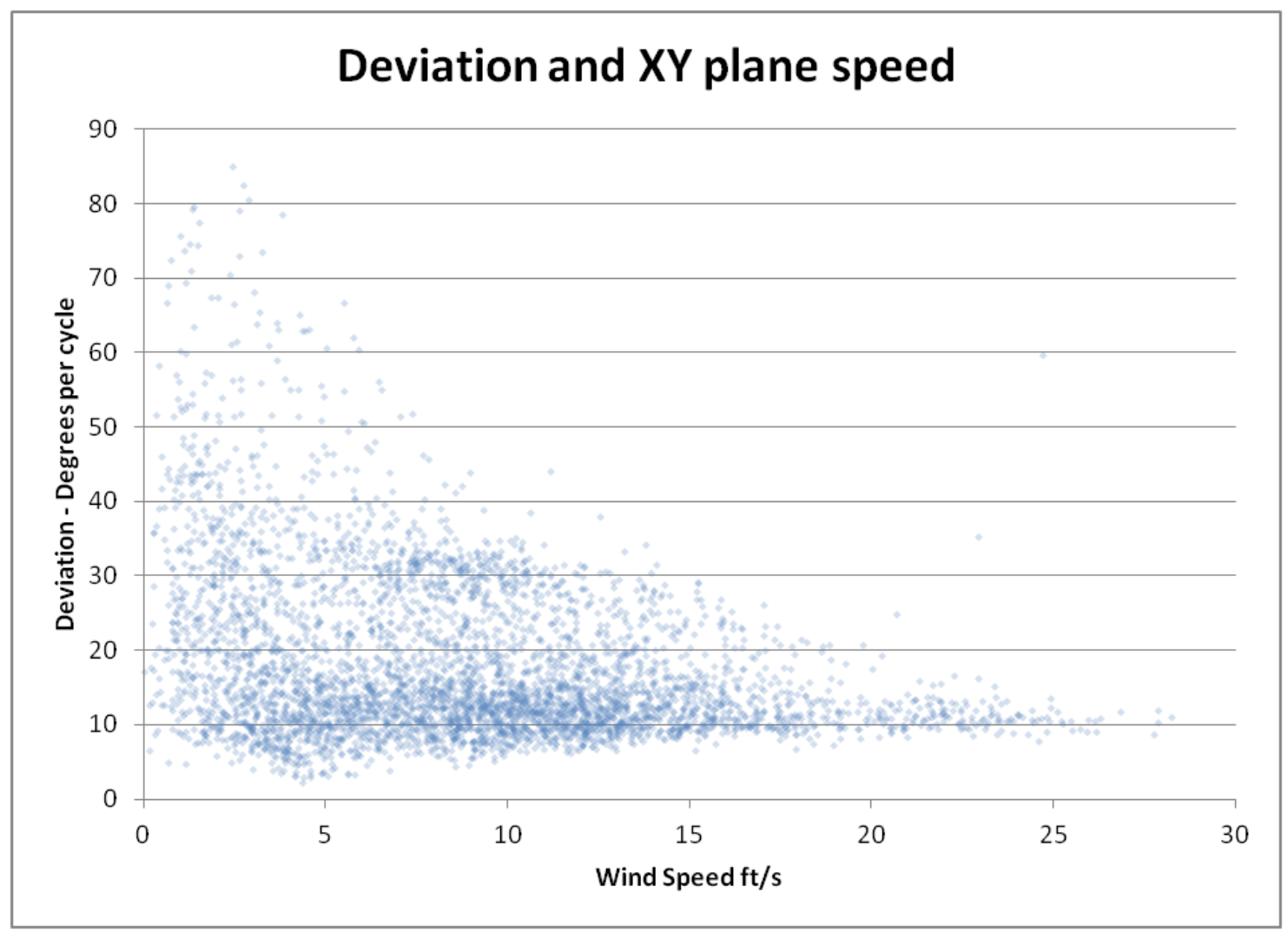

\section{Figure D-1}

Deviation of Wind Speed per Rating Cycle

In comparing our results to a recent report from for Wind Energy Center prepared at UMass Amherst, the lift in deviation at higher wind speeds is to be expected [1]. This same study found an average deviation of $19 \%$, very comparable to the results here with an average deviation of $18 \%$. This is evidence that the installation and sampling methods used for wind speed at NYPA are consistent with other findings. 


\section{Turbulence Intensities}

MMR Turbulence Intensity, 49m - Jan 2012 through Mar 2012

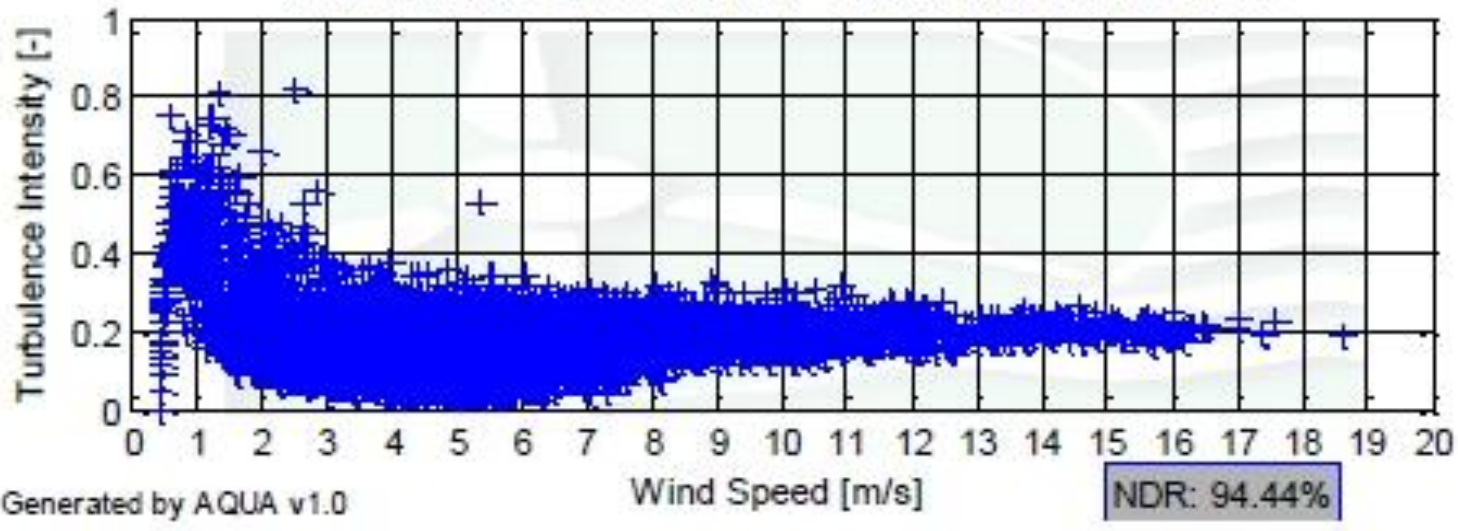

Figure D-2

UMass Deviation Data - In this plot the deviation is plotted as a percentage of wind speed.

[2]

\section{Deviation of Wind Direction at NYPA}

Standard deviation of wind direction at the NYPA was investigated and revealed some effects of installation and environmental details on the wind data.

\section{Site 3}

Site 3 is in open terrain with no trees near the anemometer. The Site is unique among the three Sites due to the way the anemometer is positioned with respect to the structure. As seen in Figure D-3Error! Reference source not found., at this Site the station is perpendicular to the line. This position gives the pyranometer a favorable angle; this may come at the cost of a less favorable angle for the anemometer. At the other two installations the weather station bracket is parallel with the line. 


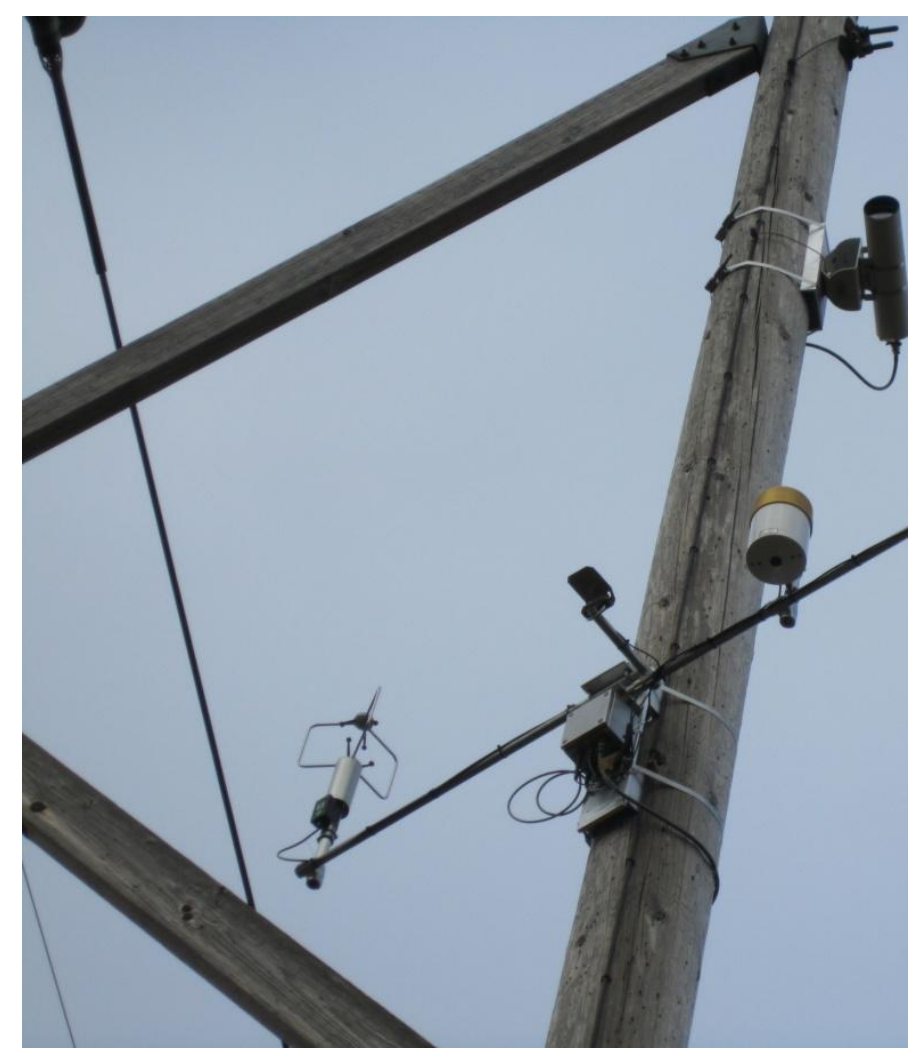

\section{Figure D-3}

Weather Station Installation at Site 3

Figure D-4 is a plot of the standard deviation of wind direction over the 10 minute sampling period versus the corresponding calculated average wind angle for the 10 minute period. These data are for June 2012.

There are noticeable "lobes" in the data where standard deviations are higher than average. These lobes are associated with structure features. Winds at $\sim 80$ degrees have the most prominent lobe. Winds from this direction pass the near pole, and weather station to reach the anemometer; this causes a disturbance resulting in turbulent flow and the resulting higher standard deviations. 


\section{Deviation vs Direction}

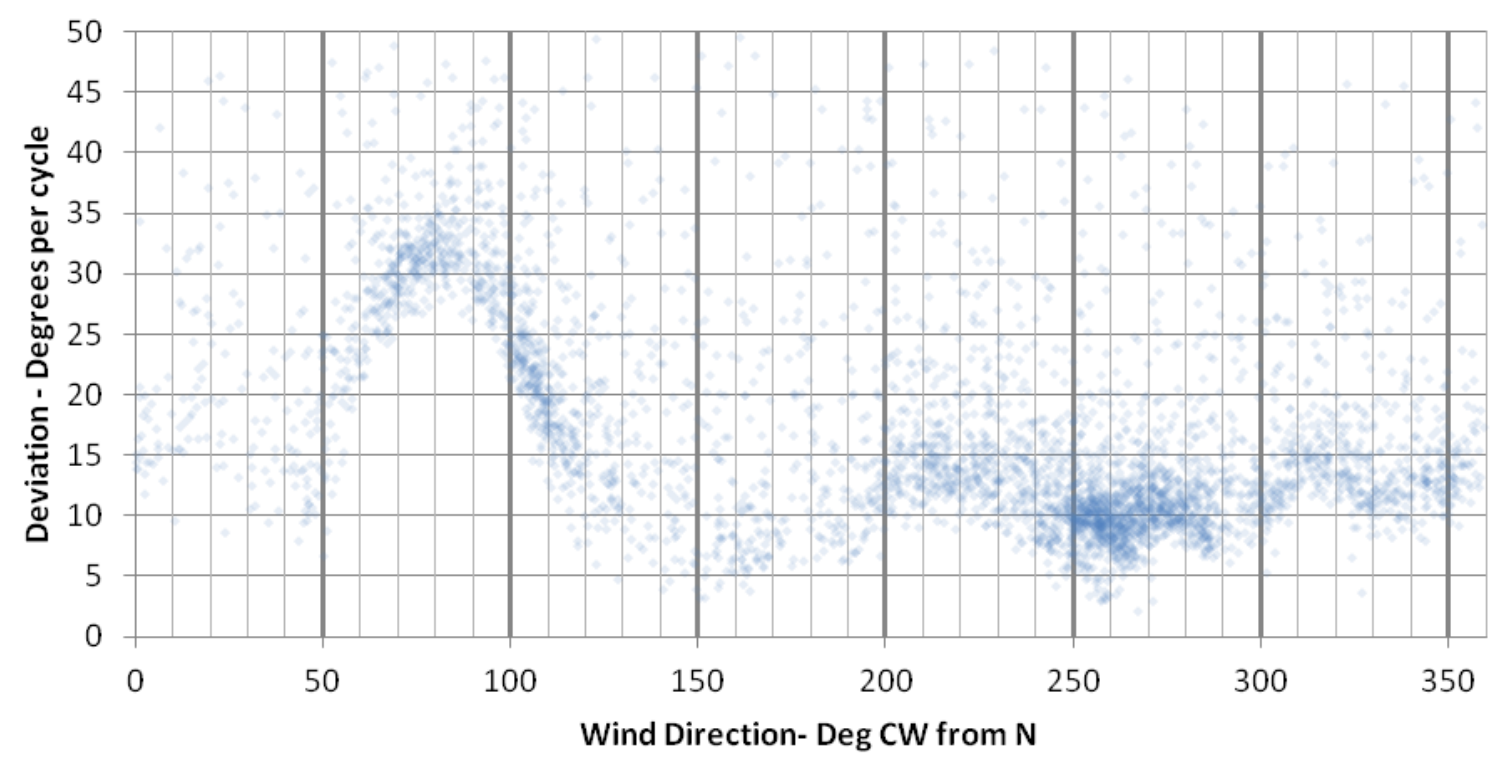

Figure D-4

Standard Deviation of Wind Direction vs. Mean Direction for 10-Minute Periods - Site 3, One Month of Data (June 2012)

Winds at $\sim 215$ degrees pass the far pole to reach the anemometer; the turbulence associated by the far pole is unlikely to be different from the near pole but is attenuated by the distance from the anemometer when compared to the near pole. Thus the flow tends more toward laminar and the standard deviations are lower.

Winds at $\sim 315$ are directed toward a diagonal brace downstream of the anemometer; this causes a slight disturbance, similar to the "stagnation streamline" effect as defined Bernoulli's equations of fluid dynamics. In short these equations show that air approaching an obstacle will either come to rest, or if it has sufficient velocity it will turbulently bend about the object. The denser and faster the airflow, the further from the obstacle this effect can be seen.

Winds at $\sim 135$ pass the same diagonal brace to reach the anemometer, though there is not a pronounced lobe of scattering at this angle, note the relative "thinness" of data from the direction of the brace, this may be due to shielding or local weather conditions. The nearby dominant wind direction can also be seen by comparison to the weather service data.

\section{Site 1}

Site 1 is also in open terrain with no trees near the anemometer. The Site has the anemometer positioned parallel with the line, again giving the pyranometer a favorable angle. At the other two installations, the weather station bracket is facing southerly. Figure D-5 is the deviation plot for wind direction at this site. 


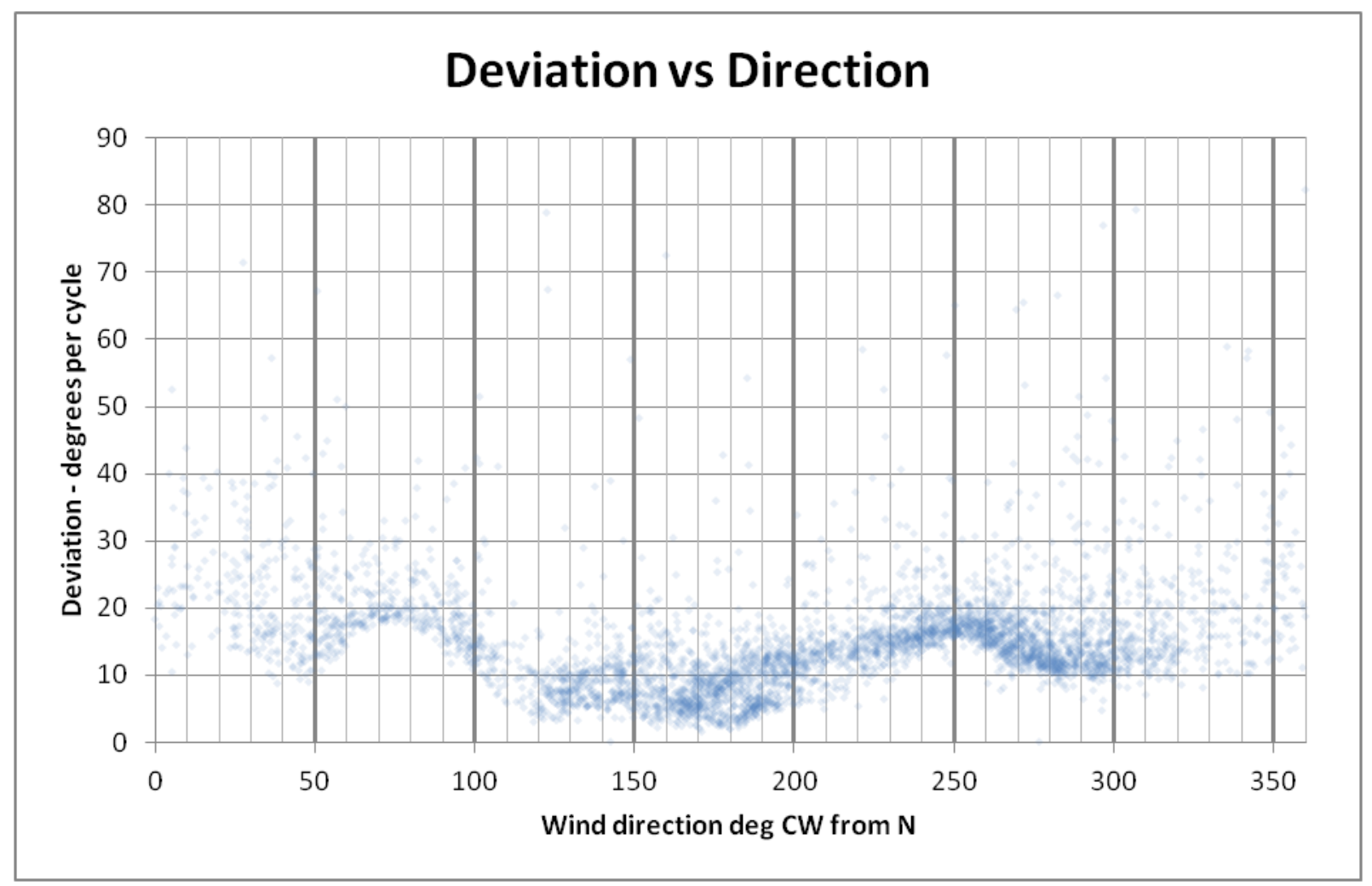

Figure D-5

Standard Deviation of Wind Direction vs. Mean Direction for 10-Minute Periods - Site 1 (June 2012)

Winds at $\sim 75$ degrees pass weather station and near pole; this causes turbulence and a pronounced lobe but, less so, than at Site 3 due to the increased distance from the far pole and bracing, as well as the bracket placing the anemometer non-linear with dominants winds and sources of disturbance.

Winds at $\sim 255$ degrees are passing into the pole; this causes the less pronounced upstream turbulence from the stagnation streamline.

Note that this anemometer installation has less wind interference from the structure in general than Site 3. When the weather station is mounted parallel with the line no effects from the far pole or cross bracing are prominent in the data. Observe also that at this Site the anemometer is located on the windward side of the pole so prevailing westerly winds are less affected by the pole than the less frequent easterly winds.

\section{Site 2}

Site 2 is in less open terrain with some trees near the anemometer. The anemometer is positioned parallel to the line. The layout here is nearly identical to Site 1varying in line direction by only 5 degrees so the wind direction deviation at this site has characteristics similar to Site 1 with additional influence from trees. Figure D-6 is the deviation plot for wind direction at this site. 


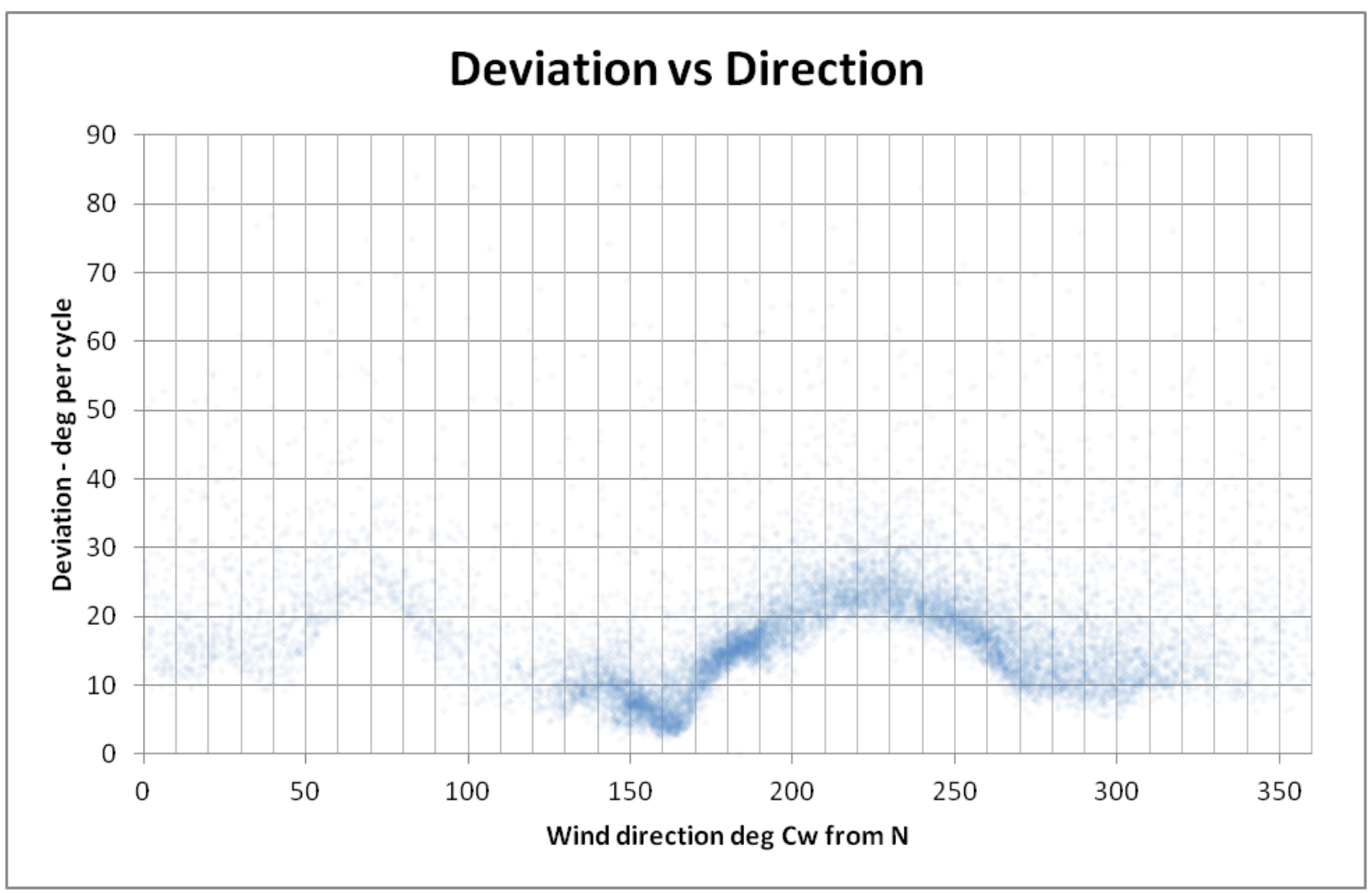

Figure D-6

Standard Deviation of Wind Direction vs. Mean Direction for 10-Minute Periods - Site 2 (June 2012)

The winds from $\sim 80$ degrees are obstructed by the pole and weather station; and the lobe is similar to the Site 1 as expected.

A large lobe is also present centered at $\sim 220$ degrees. A lobe is expected at about 260 degrees due to upstream stagnation from the pole but when compared to the Site 1 plot the lobe subtends a wider angle down to $180^{\circ}$. The turbulence associated with this lobe is likely caused by a combination of the pole and a sheltering line of trees parallel to the line in the vicinity of the anemometer. All winds from the southwest may be affected by the tree line. Winds from the west include stagnation due to the pole. These two effects produce a wide angle of turbulent wind flow.

Two structures to the east of Site 2 is shown in figure D-7, here the line enters a sheltered area that is forested both to the North and South of the right of way. A more turbulent wind state is likely to be dominant there but more importantly the wind speeds are likely to be lower and the conductor temperature higher that on the span at Site 2. An anemometer mounted in this span would likely produce lower line ratings on average. This emphasizes the point that turbulent winds do not mean that the data are poor quality. If the conditions measured are representative of the span or span section then the degree of turbulence is also representative. The value of examining the deviation data is the ability to, in some cases, see influences that are NOT representative. This is easier at open sites such as those in this project. 


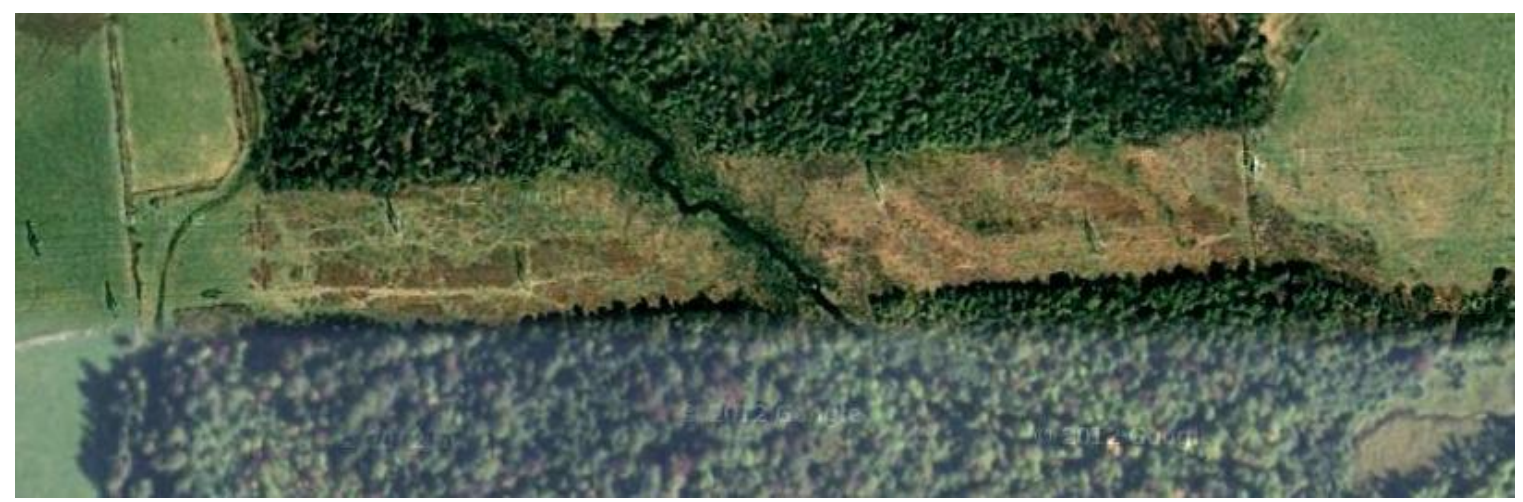

Figure D-7

Aerial Photo East of the Site 2 Showing a More Sheltered Span (Photo from Google Earth)

\section{Wind Correlation}

It is desirable to place weather stations at locations that accurately represent critical sections of the line. Distance to the anemometer is one obvious factor on whether a particular section of line is well represented. Also to be considered are terrain features and sheltering vegetation. The wind data for the three Sites were compared for correlation to see how consistent wind conditions were over the area of this project. Figure D-8 through Figure D-10 are the results of the comparisons.

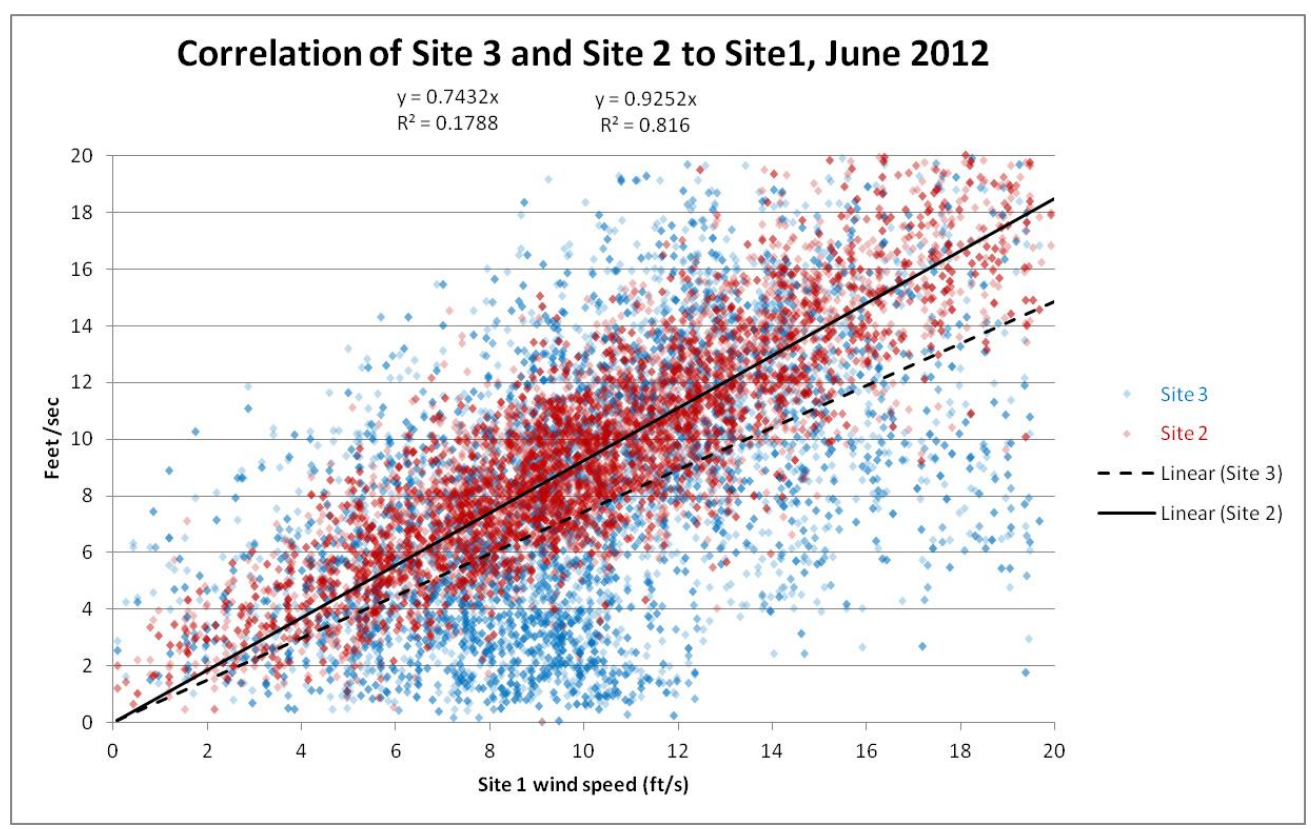

Figure D-8

Wind Correlation of Sites 2 and 3 to Site 1 - June 2012 


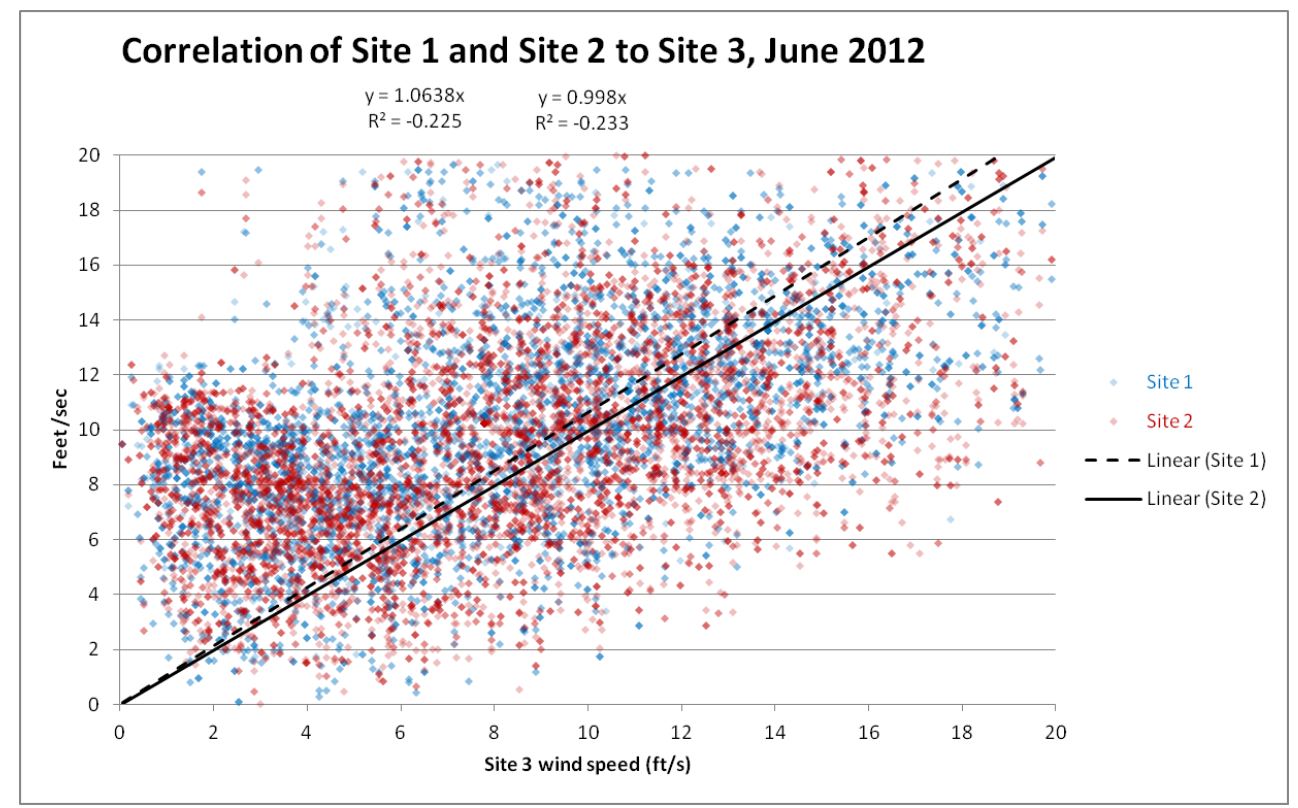

Figure D-9

Wind Correlation of Sites 1 and 2 to Site 3 - June 2012

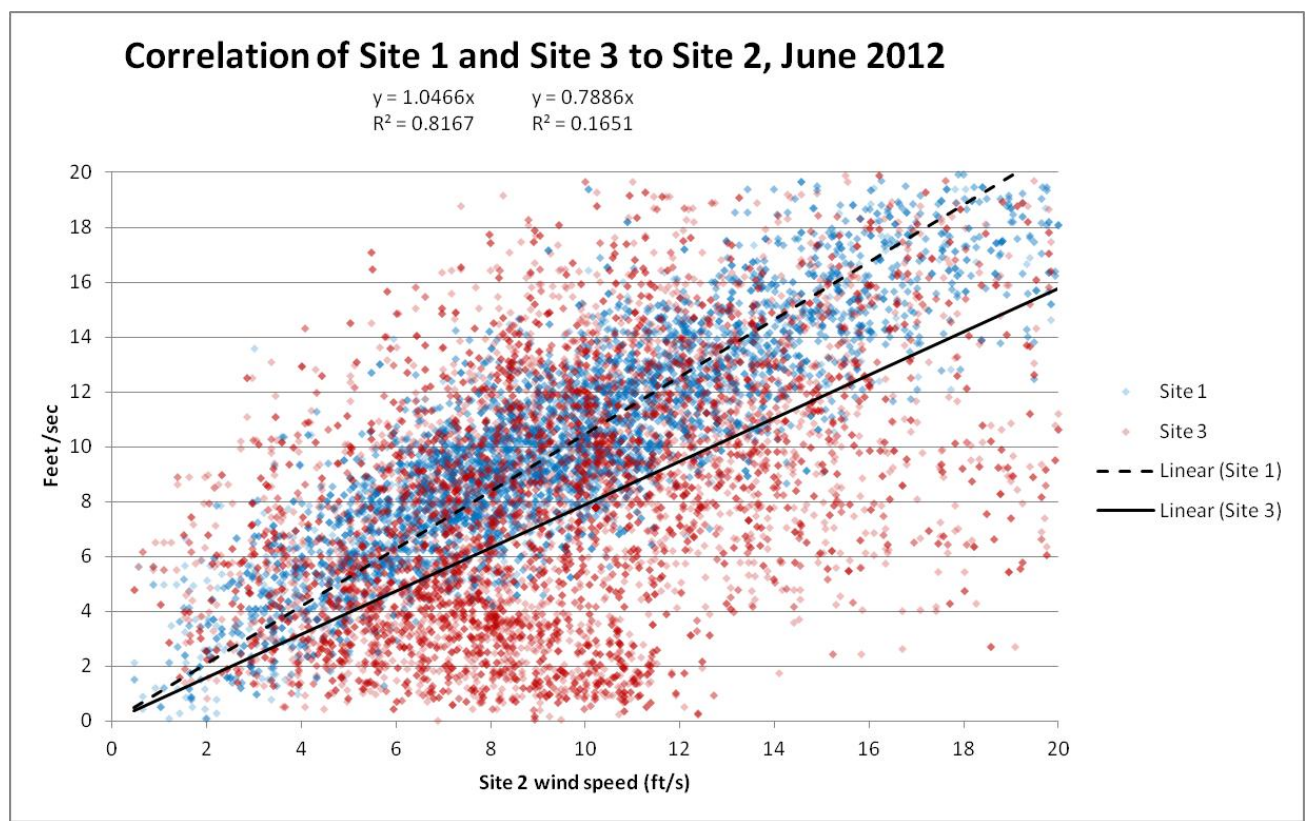

\section{Figure D-10}

Wind Correlation of Sites 1 and 3 to Site 2 - June 2012

All three sites are in relatively open and flat terrain. The data depicted in these plots confirm that the proximity of Site 1 and Site 2 makes them strongly correlated (.82 with 1.0 being perfect correlation). The correlation of the two eastern sites to Site 3 was poor $(\sim 0.2)$ indicating that wind data from the other Sites would be a poor substitute for the local anemometer. 


\section{DTCR Software}

Every 10 minutes the logger is polled via a cell modem connection by the DTCR server. When DTCR reads the wind speed and direction it is checked against a user defined range. The range for good input on wind speed is 0 to $200 \mathrm{ft} / \mathrm{s}$. The range for a good wind direction is -360 to $360^{\circ}$; if text data is received it is flagged as bad data. If wind speed data is missing or flagged "bad" at this stage the last good value is presented for a period of 30 minutes, then "NAN" (Not a Number) is reported. For wind direction a period of 60 minutes is allowed before "NAN" is reported.

A variety of options are presented within DTCR for how to handle bad input. However, a maximum of 24 hours is imposed on all rules. After that point "NAN" will be reported regardless of the rule selected. The option chosen determines the level of risk assumed when bad data is present. The full list of options is detailed in the DTCR user manual.

Note the limits here are not necessarily those used to determine bad data in data quality plots.

\section{Other Wind-Related Options}

When standard deviation is included in wind data the standard deviation calculation could be modified, using a method of sub-intervals. Quite simply, a standard deviation is calculated over a few subsets of the data then those deviations are combined. Averaging sub-intervals minimizes the effects of meander under light wind conditions, and it provides more complete information for periods of transition. The method used in the data logger may more accurately account for how a dust mote may move over the average course of time, but is less precise at explaining the cooling potential of wind. The Campbell Scientific manual for the logger suggests a 4:1 ratio of subintervals, the manual further mentions this is the method preferred by agencies such as the EPA.[2]

Three-dimensional wind speed data are available from many anemometers including the ones used in this project. For this project only the 2-D information was used, neglecting the cooling potential of wind in the z-axis.

The sample rate of the anemometer itself could be utilized using for example an $8 \mathrm{~Hz}$ rate. This internal high speed sampling may help reduce errors caused by turbulent winds as no other noise filtering method is currently being employed. If this option is chosen, the internal averaging method within the anemometer needs to be considered. The method that best matches the one used within the logger should be chosen. The methods used by the logger discussed in this chapter are the dominant factor and therefore changes to the anemometer sampling method would have only secondary effects on rating results.

\section{References}

1. Preeti, Verma, James Manwell, and Anthony Ellis. "Wind Data Report." Massachusetts Clean Energy Center. (2012): Print.

2. Cr1000 measurement and control system. (Rev. 7th Ed). Logan, Utah: Campbell Scientific Inc. (2011) www.campbellsci.com 



\section{INSTRUMENT AND SYSTEM RELIABILITY}

\section{Introduction}

All five element models that were built in DTCR to compute real-time ratings at the three NYPA sites require a stream of input data from sensors at the monitoring sites or from other remote sources in order for real-time ratings to be continuously available. In addition DTCR must be up and running on a server and in communication with the data sources to provide rating data. It was found in this project that maintaining these resources with no gaps in coverage on a twenty four hour seven day a week basis, while a worthy goal, is challenging. At the present time and state of the science, this challenge makes a compelling case for using multiple simultaneous rating calculations and/or data sources.

Progress was made in real-time data reliability over the course of the project but there is still much room for improvement. Losses were encountered in all of the links in the chain from the field instrument to the DTCR server. Instrument failures, weather conditions, data logger software issues, cell communication gaps at the site, cell communication gaps at the server, server reliability issues and DTCR software issues were all contributors to loss of real-time rating information.

Defining reliability is not a completely straightforward endeavor. Useful if less precise ratings can be calculated from data that is less recent. If one sensor reading is lost but the next is collected on time the rating calculation is affected much less than if the data stream is interrupted for longer periods. Sensors at the sites were logging data at either five minute or ten minute intervals. Ten minute intervals are more than adequate to provide precise rating calculations. It is likely that in most situations that do not include rapid weather changes ratings are not seriously compromised until raw data lapses greater than an hour are incurred. It is a more serious reliability problem if DTCR rating calculation results cannot be accessed due to software or server problems since in that case any data that exist are not available for operation decisions.

\section{Sagometer Data Reliability Example}

Reliability data were calculated on a monthly basis for the sagometer elements and kept in the monthly plots for reference by project engineers. Figure E-1 is a data quality pie chart for the sagometer element at Site 1 from the real-time DTCR output for the month of January 2012: 


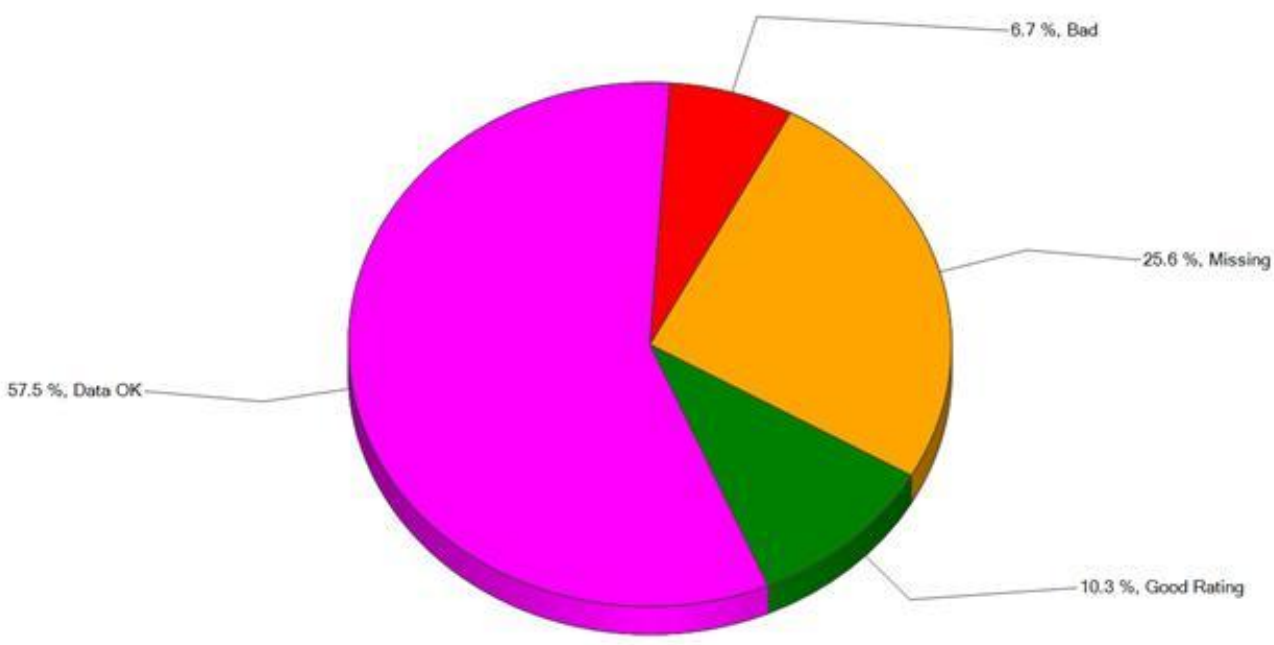

Figure E-1

Real-Time Sagometer Data Reliability - January 2012

Figure E-1 was constructed from the real-time DTCR output file (010312_NYPA_input_file-012012.RTD) and indicates not only the reliability of the instruments and communications but also of the weather conditions. The pink and green sections indicate data is present and all systems are operating as designed. For these two categories, data is available every 10 minutes for rating calculations. The green section is good data that was taken during a period load current was above 350A. A current of 350 Amps is approximately the lowest current for which rating calculations can be made using the sag based DTCR model. Although there was $10.3 \%$ of the time data met the load criteria for rating calculations, the majority of that time ratings could not be preformed. The load is an indicator the conductor temperature has risen at least $10^{\circ} \mathrm{C}$ above the air temperature. If wind or rain prevents this from happening, then ratings can still not be performed. The red category is marked as bad and is data that is out of range or flagged as unreliable. For the sagometer element this category applies mainly to measurements taken by the sagometer camera when weather conditions produced optical interference. The correlation coefficient is calculated for each optical measurement. When the correlation function from EDM was below 0.65 the data was flagged as unreliable. While this affects availability it is an expected occurrence and not due to a system malfunction. The missing data indicates that the data was not logged for the 10 minute interval but it is unknown in this graph whether the lapse was followed by a good measurement in the following 10 minutes or whether there was a longer blackout period when no data were available. If these data were missing for over an hour then by the criteria discussed above ratings were compromised. This issue is addressed in Appendix D. 
Figure E-2 is a similar plot for the same equipment over the same time period but constructed from the output file of a simulation performed at a later date (new-01-2012.RTD):

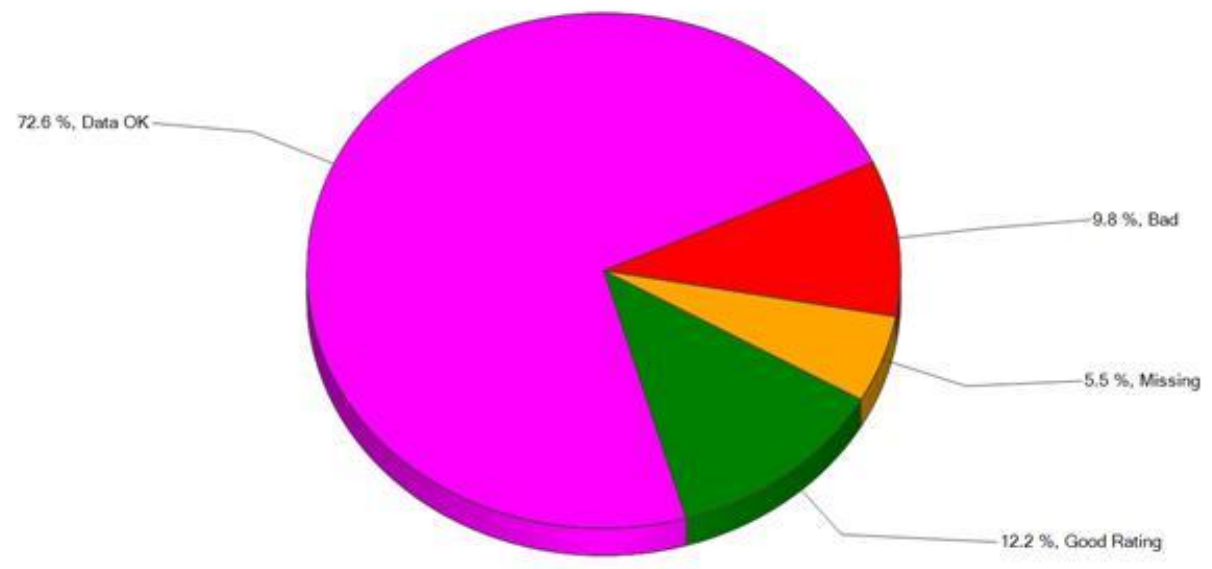

\section{Figure E-2}

Sagometer Data Reliability with Communication Delays Removed

Figure E-2 shows better performance than Figure E-1 and the reason has been isolated to communication interruptions between the data logger at the site and the DTCR server in Lenox MA. DTCR did not have real-time data for some periods even though the measurements had been made and logged at the site and therefore this data is flagged as missing. However, when communications were restored then any unsent data stored in the logger buffer were sent to the DTCR server. DTCR uses all of the available data, including the data that was unavailable realtime when the same month is run in simulation mode. Data that were overwritten in the logger due to limited buffer size and long communication delays were permanently lost. This data loss is smaller and addressed separately.

The difference in the missing data percentage between real-time and simulation output is an indication of communication reliability but not a direct measurement. DTCR allows a data coast in which it fills in missing data with the last good reading. The duration of allowable coasts is a user set parameter in DTCR (see Chapter 5 for details). The maximum coast period that applies to the data stream (clearance at target) used to construct Figure E-1 and Figure E-2 was set to 4 hours in the DTCR software. Therefore, the difference in missing data between the real-time and simulation output indicates the percentage of time that communication outages lasted more than 4 hours. As discussed above shorter communication outages have less effect on rating accuracy than longer outages. Outages of 4 hours can have a small but significant effect on rating accuracy so though the sagometer clearance at target data was not the parameter chosen to evaluate communication reliability described below but the methodology used is the same.

There is value in considering the data quality of Figure E-2 as a stand-alone indication of data quality for the purpose of performing line studies. If a primary or secondary objective exists to 
evaluate risks associated with static ratings based on measured data then Figure E-2 is a good indicator since the ratings can be calculated in simulations at any time after the data are collected and are then available for statistical evaluation. The simulation reliability for the Video Sagometer depicted in Figure E-2 is a monthly plot and this data for all three sites for the length of the project is in Appendix A which contains all of the monthly plots for the entire project.

For this particular month data quality improved from $76 \%$ to $95 \%$ availability when the communication failures longer than 4 hours were removed from the causal list. This month was particularly poor for cell communications at this site. Early in the program there were startup issues with much of the equipment. By late 2011 and 2012 most of these issues were resolved and cell communication failure became the dominant cause of missing data for real-time rating calculations over instrument failure, this is the case for all elements except the sites with failed EPRI Sensors and the airport data which uses no cell communications. However, cell communication also improved with time reflecting both improvements in the project equipment and the cell provider performance.

\section{Data Flow}

The example above is a snapshot of the reliability of one element at one site for one month of the project. Analysis of DTCR simulation output, real-time DTCR Output and the LoggerNet raw data files were all used to capture similar reliability information over the course of the project for the four elements that use data gathered and transmitted from the three NYPA Sites. The nature of the sensors and data-links are different for the five elements and the reliability also differed. Complicating the reliability picture is the fact that some equipment and data links were shared between elements.

Figure E-3 is a chart of data flow from the individual sensors to the DTCR rating server. Only sensors that were used in DTCR rating calculations are depicted. For example the weather station rain gauge data is ignored by DTCR so it cannot affect rating reliability. The EPRI Sensor load data is not ignored even though not used for most of the project it was used for the first several months before the NYPA SCADA data was made available through a spread spectrum radio link. The reliability of load is of particular importance since load is a global parameter in DTCR with only one source per circuit for all elements. If the single source load information is not available then all elements cease to produce ratings. This single point of failure due to dependency on load data with no backup source option in the software will be addressed in future versions of DTCR software.

Figure E-3 is valid for all three sites. Note that there is no NYPA SCADA load information communicated at Site 3. The data collected at Site 2 are used for both sites: 


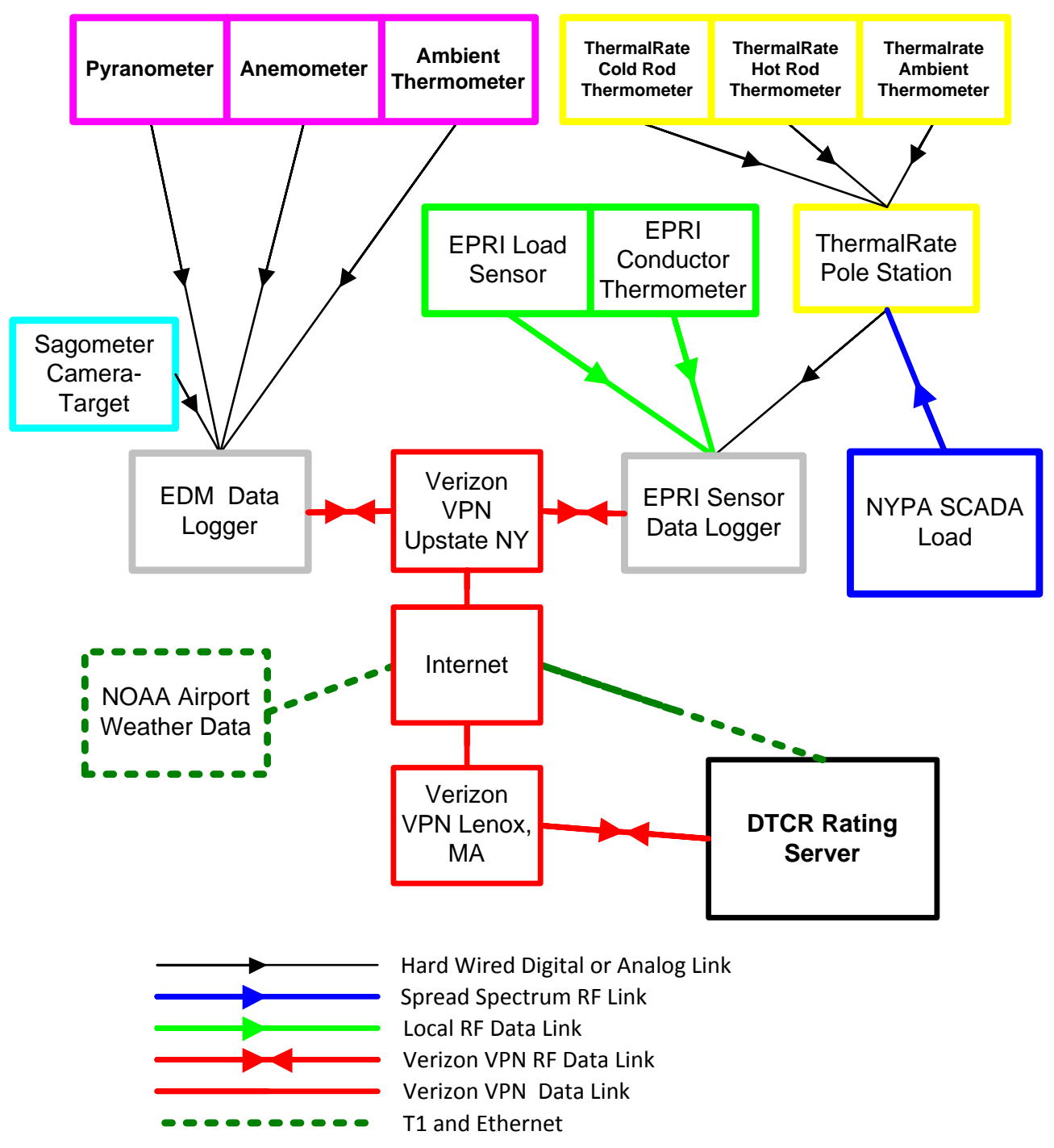

Figure E-3

Flow Chart Depicting Data Flow for All Five Rating Elements Including Airport Weather Data - This chart is applicable to all three Sites.

Figure E-3 can be used to help map the locations where the data generation and communication process may fail. In most cases, the failure mapping cannot be isolated to a single process or instrument since the loss cannot be measured in all parts of the flow. For instance, when an EPRI Sensor does not report data it could be due to the sensor or to the local RF link. Therefore, both of those losses are attributed to the instrument. 
Table E-1 summarizes the instruments and communication parameters by DTCR rating element.

Table E-1

Instrumentation and Communication List by Primary Calculation Element - Some instruments are used in rating calculations in more than one element.

\begin{tabular}{|c|c|c|c|c|c|}
\hline Element & Sensor/Source & $\begin{array}{c}\text { Maximum } \\
\text { Coast } \\
\text { time (hr) }\end{array}$ & $\begin{array}{l}\text { Logger } \\
\text { used }\end{array}$ & $\begin{array}{l}\text { Data link to } \\
\text { logger }\end{array}$ & $\begin{array}{l}\text { Data Link to } \\
\text { DTCR }\end{array}$ \\
\hline Global (All) & NYPA SCADA Load & 0.5 & EPRI & Spread Spectrum & Cell-internet-cell \\
\hline \multirow{4}{*}{$\begin{array}{c}\text { Weather } \\
\text { Based (WX) }\end{array}$} & Pyranometer & 1.0 & \multirow{6}{*}{ EDM } & \multirow{6}{*}{ Hard Wire } & \multirow{6}{*}{ Cell-internet-cell } \\
\hline & WX Ambient Temp & 3.0 & & & \\
\hline & Anemometer Speed & 0.5 & & & \\
\hline & $\begin{array}{c}\text { Anemometer Wind } \\
\text { Direction }\end{array}$ & 1.0 & & & \\
\hline \multirow{2}{*}{$\begin{array}{l}\text { Sagometer } \\
\text { (SAG) }\end{array}$} & Camera clearance & 4.0 & & & \\
\hline & Camera correlation & 0.2 & & & \\
\hline \multirow[b]{2}{*}{$\begin{array}{l}\text { EPRI Sensor } \\
\text { (BS) }\end{array}$} & Conductor Temp & 0.2 & \multirow[t]{2}{*}{ EPRI } & \multirow[t]{2}{*}{ Local RF } & \multirow[t]{2}{*}{ Cell-internet-cell } \\
\hline & EPRI Conductor Load & 0.2 & & & \\
\hline \multirow{3}{*}{$\begin{array}{l}\text { Thermal-Rate } \\
\text { (TR) }\end{array}$} & Cold Rod Temp & 24 & \multirow{3}{*}{ EPRI } & \multirow{3}{*}{ Hard Wire } & \multirow{3}{*}{ Cell-internet-cell } \\
\hline & Hot Rod Temp & 24 & & & \\
\hline & TR Ambient Temp & 24 & & & \\
\hline Airport (AP) & Amb T, WS, WD & 24 & none & NA & internet \\
\hline
\end{tabular}

\section{DTCR Input Data Availability}

Methods similar to those described above were used on all data sets to quantify data availability and to place bounds on the possible source of the losses. In the following sections, data availability from the weather station, Sagometer, EPRI Sensor and ThermalRate device are presented in two categories:

1. Instrument availability

2. Real-time instrument and communication availability

Instrument availability is a measure of the percentage of time valid data is logged in the data logger at the site and communicated either real-time or at a later time to the DTCR server. Referring to Figure E-3 all processes including and prior to the logger are evaluated when determining this value. LoggerNet raw data files stored at the DTCR server are used for this analysis and no DTCR coast errors are introduced

Real-time availability is the percentage of time instrument AND the communications to the DTCR server are both available at the same time. Coasting is included in this measurement and increases the availability by allowing short lapses in communications. The maximum coast 
times for individual data streams are listed in Table E-1. Communication can be unavailable for the shortest coast time associated with an element before DTCR rating calculations from that element are interrupted. For example, the shortest coast time for the WX element is 0.5 hours so real-time availability for WX is the percentage of time both the instrument data and communications are available within the preceding 30 minutes.

\section{Communication Availability}

The communication availability is derived by subtracting the difference in DTCR simulated availability and DTCR real-time availability from $100 \%$. Coasting is included in this value. The effect of coasting is minimized by basing the difference not on all of the parameters but on a single parameter from each logger with good reliability and a short maximum coast time.

There are only two data loggers, EDM and EPRI, at each Site and the communication availability value captures the effects of losses from each of these loggers to the server. The availability of only one data parameter for each logger is used to derive these numbers:

$\begin{array}{ll}\text { EDM logger; } & \text { Wind speed (maximum } 0.5 \text { hour coast) } \\ \text { EPRI logger } & \text { NYPA SCADA Load (maximum } 0.2 \text { hour coast) }\end{array}$

The two parameters do not have the same coast time which does bias performance in favor of the EDM logger, and this is consistent with the results. However, there were no other parameters that had both adequate instrument performance and a 0.5 hour coast time on the EPRI logger so NYPA SCADA Load was chosen for this study.

The communication availability includes losses from both cell communication at the upstate NY end and cell communication at the DTCR server in Lenox MA. Internet data transfer failures are also in this path as seen in Figure E-3 but are likely an insignificant percentage.

After some startup issues the cell communication at the DTCR server in Lenox, MA was very good. The antennas and antenna placement were factors in the first month but this was resolved early in 2011. The DTCR real-time file structure changed to its final form in July of 2011 and the analysis method used required the final file structure so communication is evaluated in this report from $8 / 11$ to the end of the project. No significant changes to the cell communication equipment at the DTCR server or at the sites during this period.

From November 2011 through October of 2012 a qualitative assessment was made of the cell performance at the DTCR server and indicates that the upstate NY cell communication produced most of the real-time data losses while the Lenox cell communications were a minor loss factor. This assessment is based on a status monitor for logger communications that flags real-time data loss from the six upstate data sources and displays it at the server for the preceding 24 hours in graphical form. This monitor is depicted in Figure E-4. 


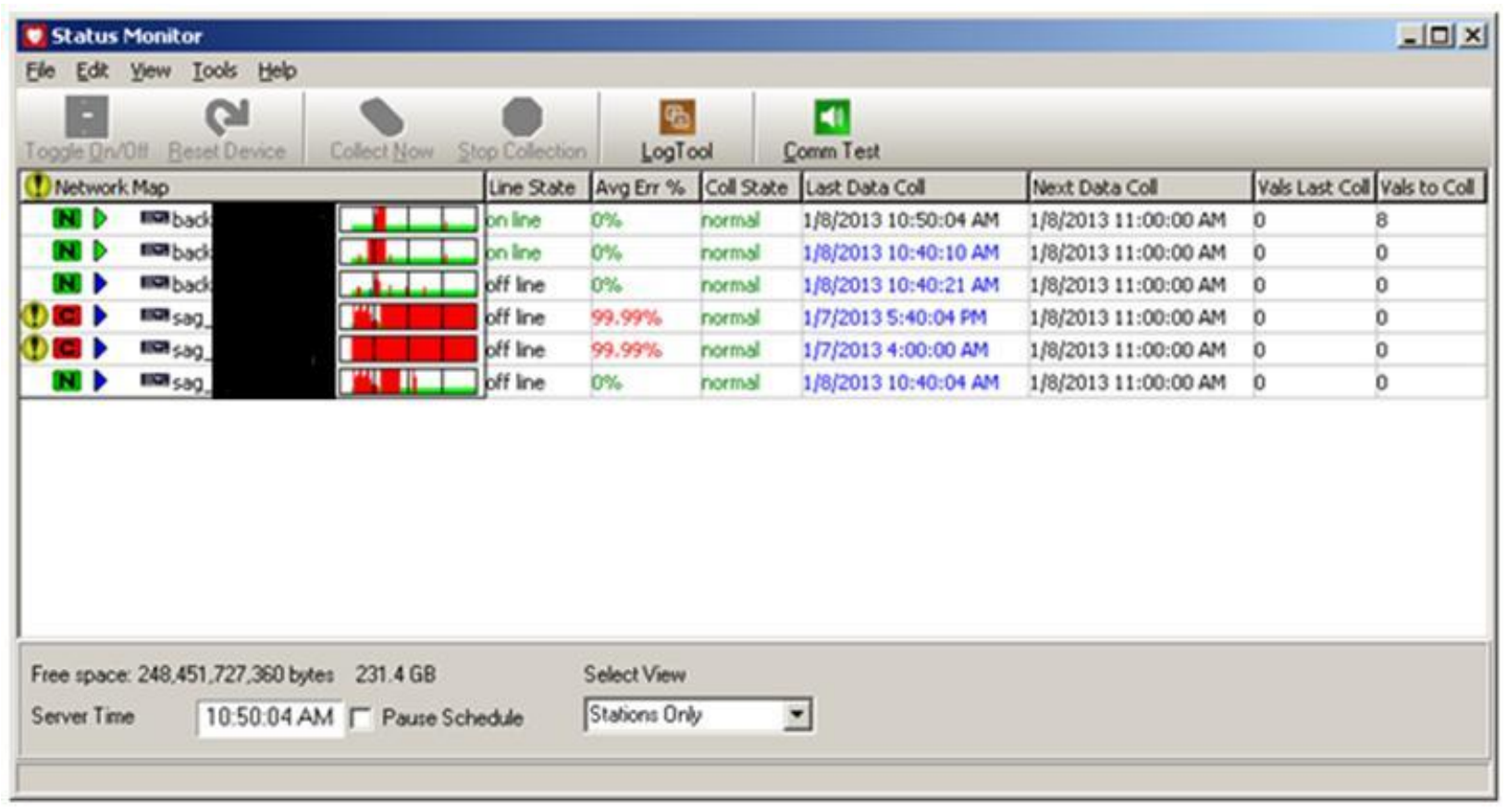

Figure E-4

The LoggerNet Data Status Monitor Showing Communication Failures in Red and Good Communication in Green

Project engineers frequently examined this data when working with the DTCR server and it was extremely rare that all six reporting data loggers were down simultaneously which would be the case if problems existed with the local cell connection at the server end. The screen shot in Figure E-4 was taken in January 2013 after data collection ceased but the communication links were still active. This snapshot into communication performance shows that communication was down at two sites for the EDM logger for nearly a day. The fact that four loggers are reporting normally indicates that the problem is not at the server end.

The status monitor data are overwritten in the LoggerNet software and are therefore not available for post processing so this assessment cannot be quantitatively confirmed. Generally, however, with moderate confidence the communication losses depicted in the following charts can be attributed to cell communications at the individual sites. Since nearly all communication lapses were self repairing, needing no Site visits before communications were restored, it is suspected that the Site modems are not the primary cause of communication problems.

\section{Long Term Raw Rating Data Availability Results}

The instrument availability, real-time availability and communication availability for all three NYPA sites are depicted in chart form in the Figures E-5 through E-13 and Tables E-2 through E-4 list the average data availability over the course of the project for the respective sites. Since all sites had similar instrumentation, overall availability figures were also compiled by type of instrument. These values are depicted in Figure E-5.

Note that EPRI Sensor instrument availability was calculated using three EPRI Sensors at each site. If any of the three instruments had valid readings in the LoggerNet files for a given time stamp then the instrument was considered available. A method has been developed to allow an 
array of sensors to be used simultaneously that was not available during the NYPA project, but resulted from this experience.

In contrast the instrument and communication availability for EPRI Sensors was based on the single instrument that was assigned to rating calculations in DTCR. Thus for the EPRI Sensor, real-time availability of valid EPRI Sensor data was reduced by more than just communication availability. There were no other redundant instruments at the three sites.

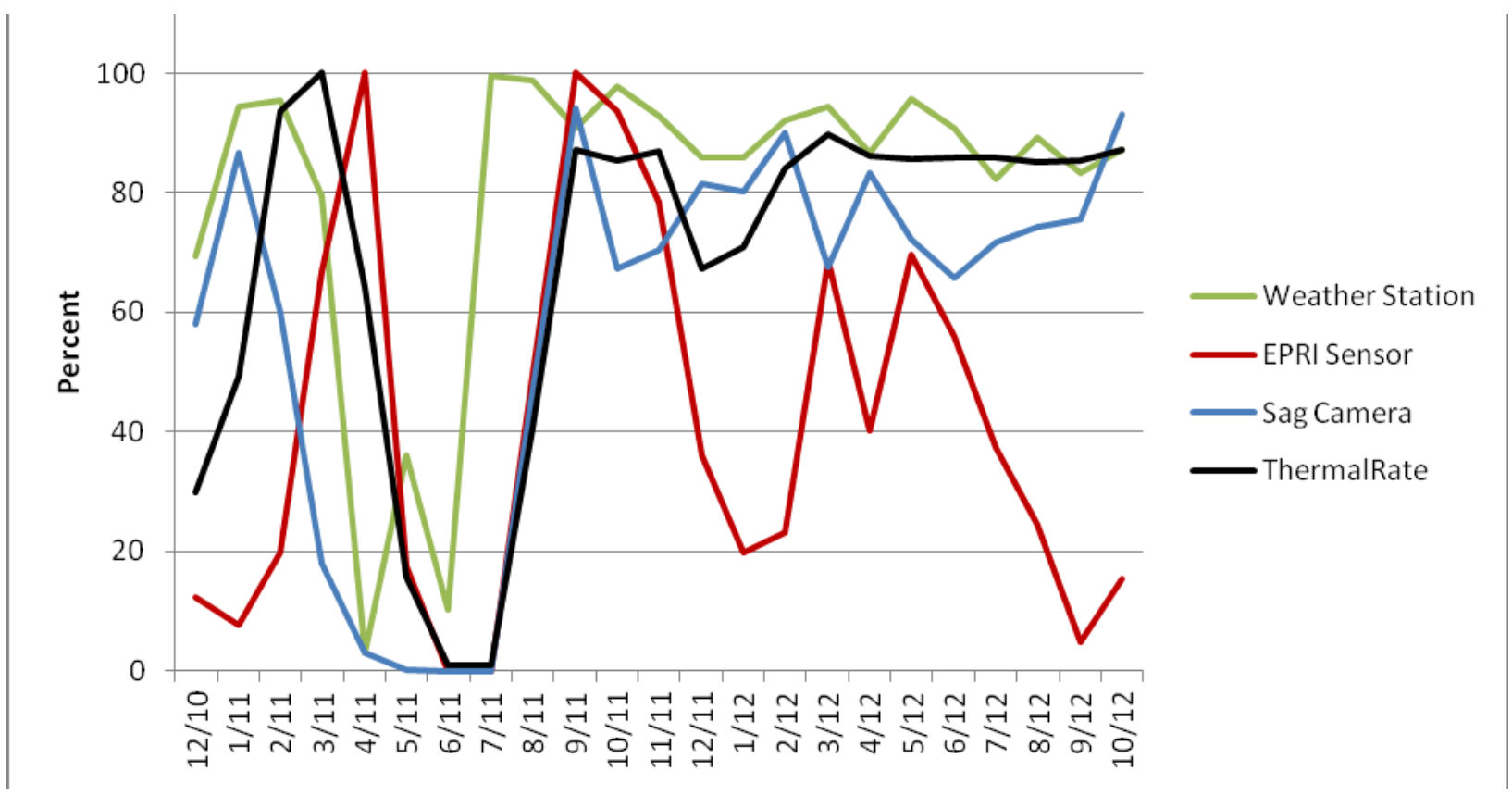

Figure E-5

Instrument Availability at Site 1

All instrument reliability improved over the first half of the project with the exception of the EPRI Sensors. EPRI Sensors were replaced at the other two Sites on March 27, 2011 but not at this site and moisture ingress continued to degrade performance to the end of the project.

A breaker problem on the EDM solar panel occurred and affected reliability on all Weather Station instruments and the Sagometer Camera which use the EDM Logger. The affected period was between April 4, 2011 and May 6, 2011. In the same general time period a communication problem existed between the EPRI Sensors and the logger. This was resolved by August 1, 2011. Refer to the maintenance logs in Appendix C for more details.

The Sagometer cameras suffered periodic communication lapses to the logger at all sites. This required a manual reset of the camera which could be done remotely but resulted in periods of lost data before EDM became aware of the problem and performed the reset.

From August 2011 on most of these startup issues were resolved and the DTCR program was running in a configuration that saw only minor changes. Figure E-6 below shows the availability to DTCR at the period from this time to the end of the project. 


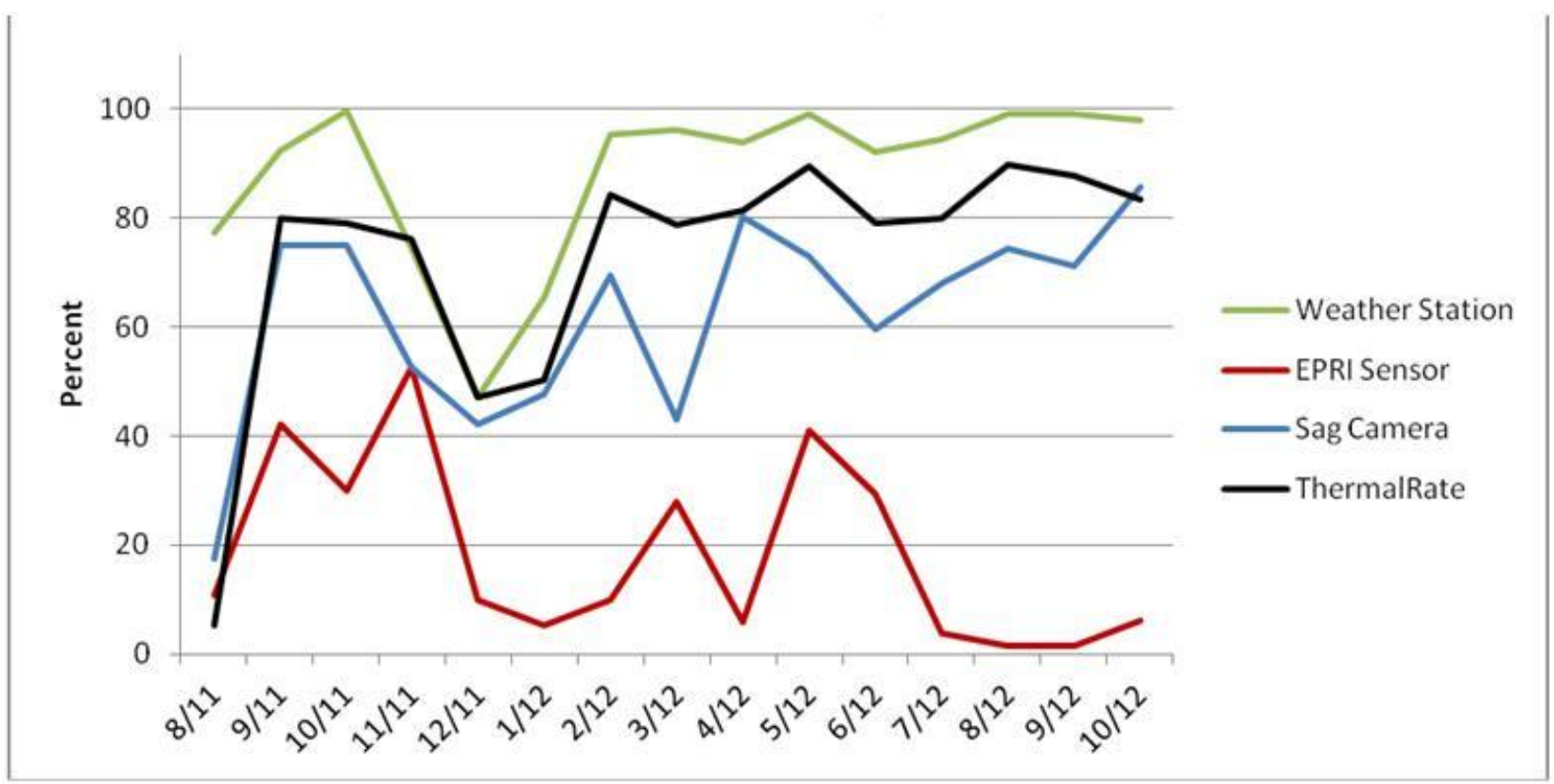

Figure E-6

Real-Time Instrument and Communication Availability at Site 1

When communication losses from the two data loggers to the DTCR server are added, availability drops for all instruments.

Note that only the EPRI Sensor used for calculations is included in Figure E-5 while E-6 is the availability when the criteria of a minimum of one sensor of three reporting. This produced a significant decrease in performance. There is also a decrease in the EPRI Sensor performance due to a large number of coasts that were between 0.5 hours and 1.0 hours. The ratings are not seriously affected by these coasts. Multiple modifications to the EPRI Sensors have been made as a result of this project.

The weather station and ThermalRate availability were the best over the second half of the project at this site. 


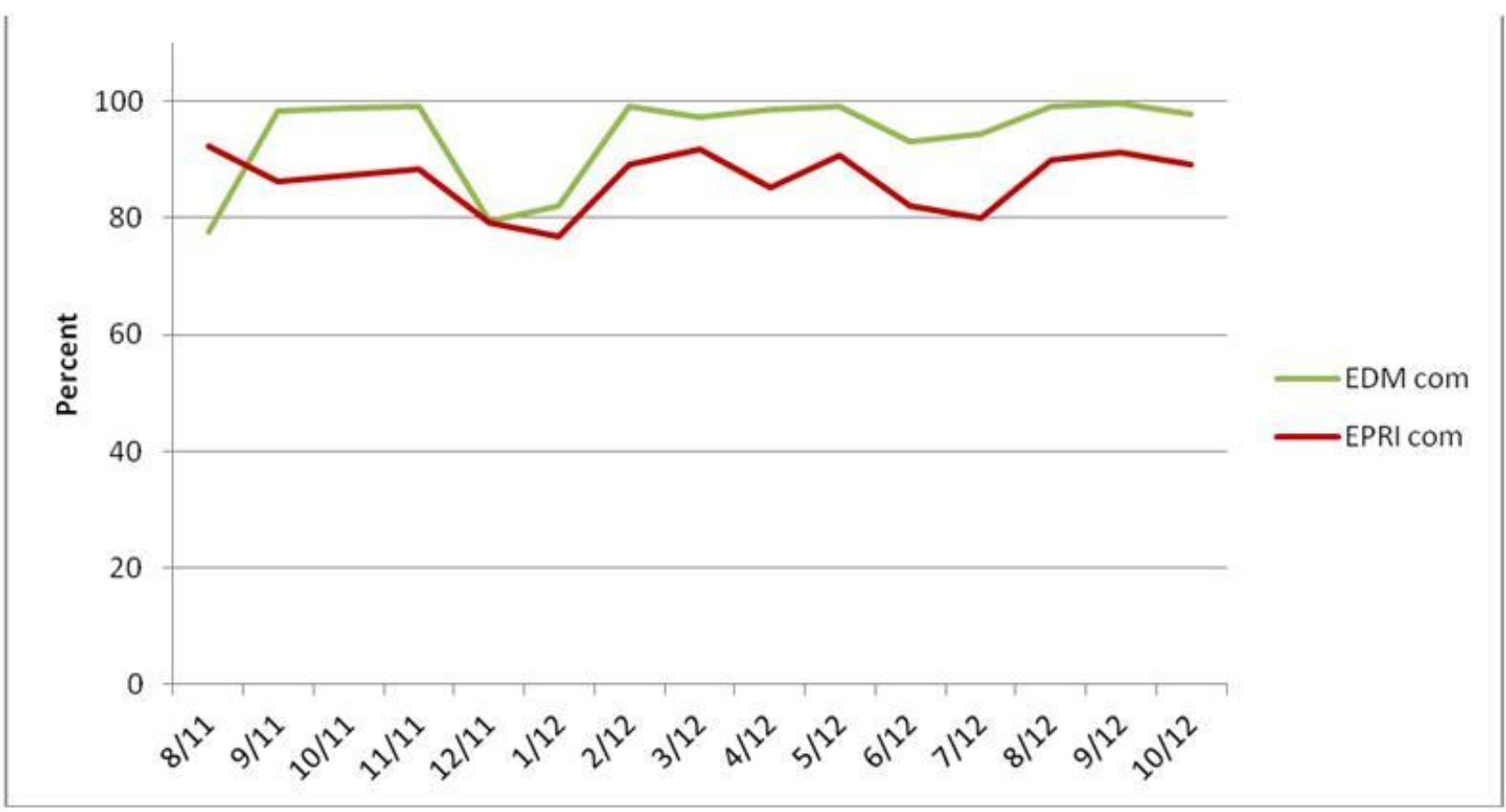

Figure E-7

Communication Availability Between Data Loggers and DTCR Server at Site 1

Communications remained relatively consistent at this site throughout the project. Note that the EDM performance is based on communications every 30 minutes and the EPRI Logger every 10 minutes so these two plots are based on different availability criteria.

Table E-2 summarizes the reliability of the data systems at Site 1 for the final 14 months of monitoring.

Table E-2

Average Performance of Data Systems at Site 1 from 8/1/11 through 10/31/12

\begin{tabular}{|c|c|c|}
\hline Site $\mathbf{1}$ Instrument & \% Available & \% Available Real-time \\
\hline Weather Station Thermometer & 99.4 & 89.0 \\
\hline Weather Station Anemometer & 90.8 & 88.2 \\
\hline Sagometer Camera & 75.6 & 57.3 \\
\hline EPRI Sensor & 47.8 & 16.5 \\
\hline ThermalRate Device & 80.9 & 67.6 \\
\hline NYPA SCADA RF Link & 79.8 & 68.9 \\
\hline EDM Logger communications & NA & 94.3 \\
\hline EPRI Logger Communications & NA & 86.7 \\
\hline
\end{tabular}




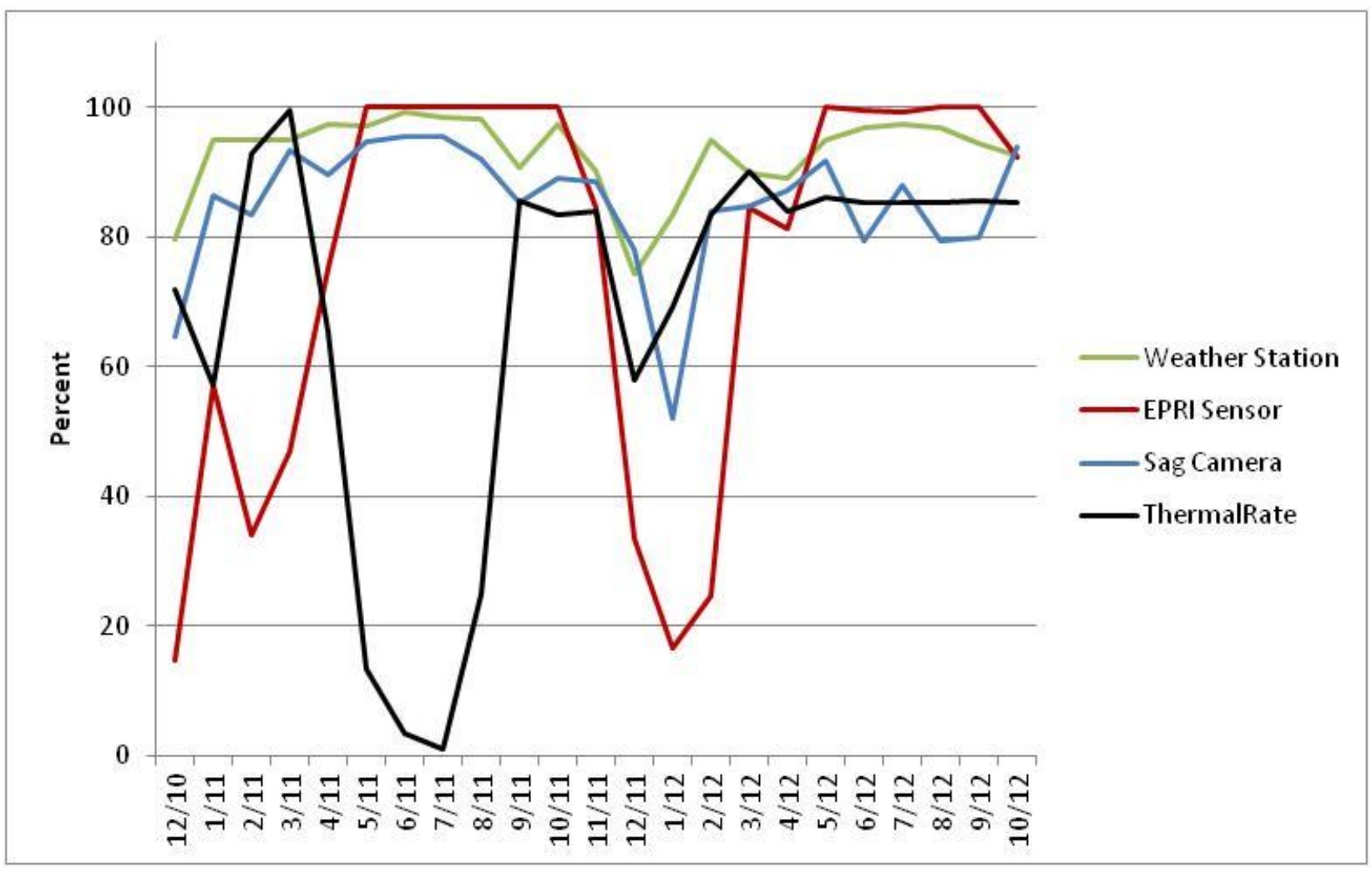

Figure E-8

Instrument Availability at Site 2

EPRI Sensors were replaced at the end of March 2011at this site and performance increased significantly. Although not reflected in this data there is some decreased availability near the end of the project from the EPRI Sensors, this may be due to the aforementioned suspected moisture ingress. Further detail can be seen in the data quality plots in Appendix D. The EPRI Sensors also show an unavailable state when the conductor temperature is below $2 \operatorname{deg} \mathrm{C}$, this is reflected in Figure E-8 noticeably in the winter months.

ThermalRate performance suffered between May 2011 and July 2012 due to a polling issue from the EMS RTU. Polling was set to every 2 seconds, which overloaded the ThermalRate processor. The polling interval was increased to 30 seconds and performance increased accordingly.

The sagometer camera at this site required manual resets similar to Site 1 . 


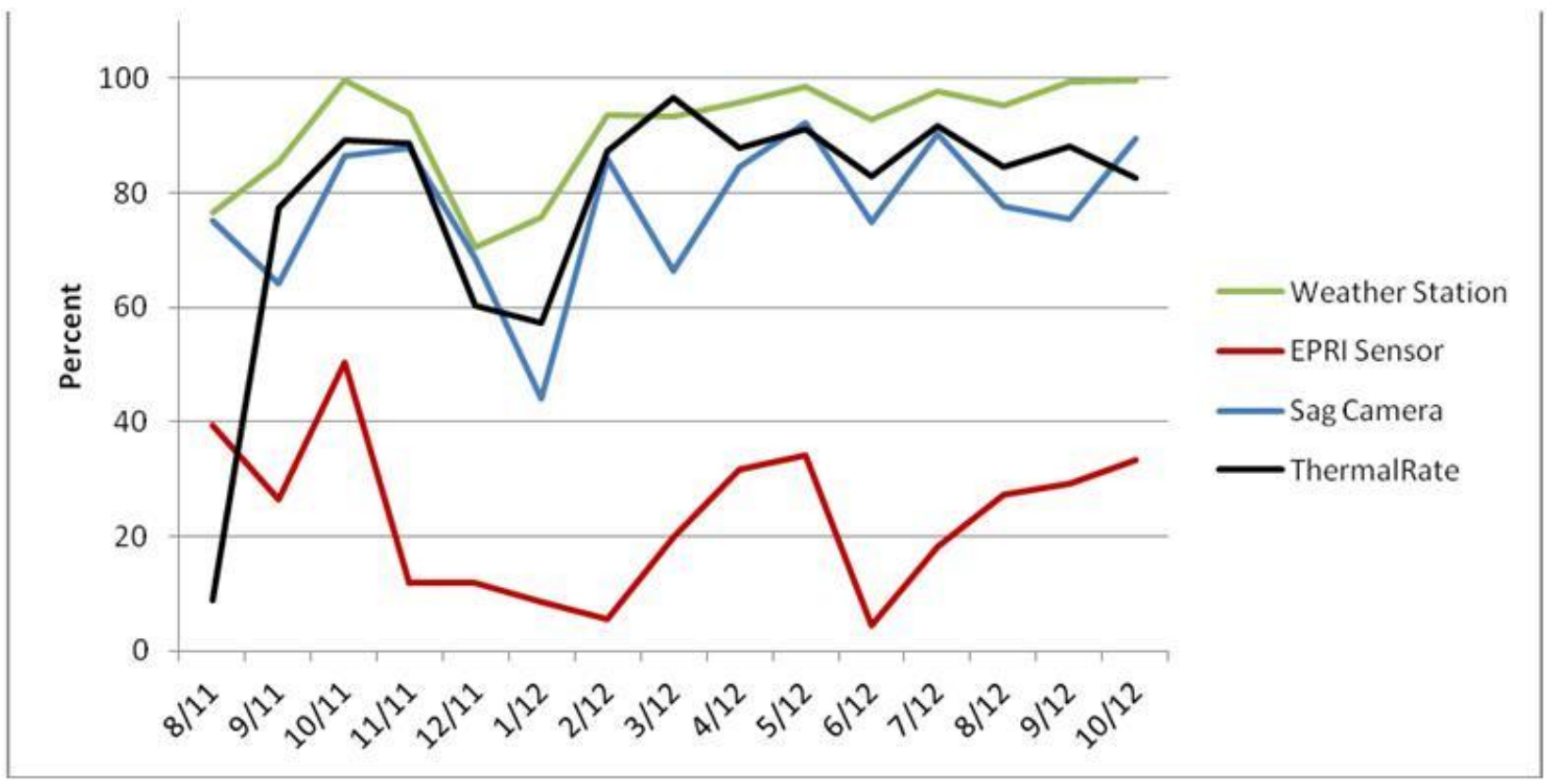

Figure E-9

Real-Time Instrument and Communication Availability at Site 2

When communication losses from the two data loggers to the DTCR server are added availability drops for all instruments.

There is again a decrease in the EPRI Sensor performance due to a large number of coasts that were between 0.5 hours and 1.0 hours. The ratings are not seriously affected by these coasts.

The weather station and ThermalRate availability were the best over the second half of the project at this site. 


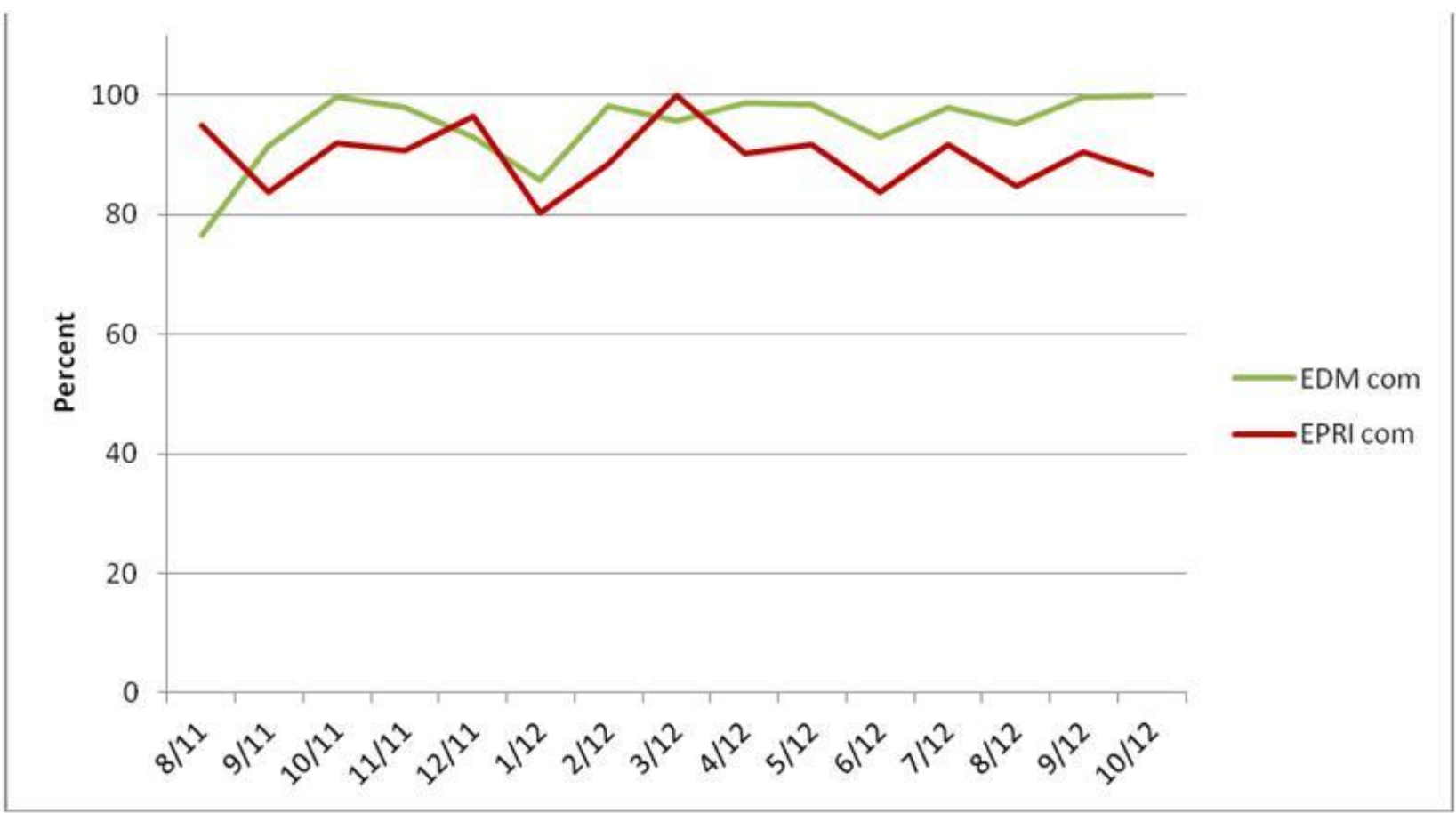

Figure E-10

Communication Availability Between Data Loggers and DTCR Server at Site 2

Table E-3

Average Performance of Data Systems at Site 2 from 8/1/11 through 10/31/12 summarizes the reliability of the data systems at Site 2 for the final 14 months of monitoring.

Table E-3

Average Performance of Data Systems at Site 2 from 8/1/11 through 10/31/12

\begin{tabular}{|c|c|c|}
\hline Site 2 Instrument & \% Available & \% Available Real-time \\
\hline Weather Station Thermometer & 99.4 & 92.7 \\
\hline Weather Station Anemometer & 92.6 & 91.2 \\
\hline Sagometer Camera & 83.6 & 77.6 \\
\hline EPRI Sensor & 81.1 & 23.6 \\
\hline ThermalRate Device & 78.4 & 78.3 \\
\hline NYPA SCADA RF Link & 75.5 & 70.1 \\
\hline EDM Logger communications & NA & 94.8 \\
\hline EPRI Logger Communications & NA & 89.8 \\
\hline
\end{tabular}




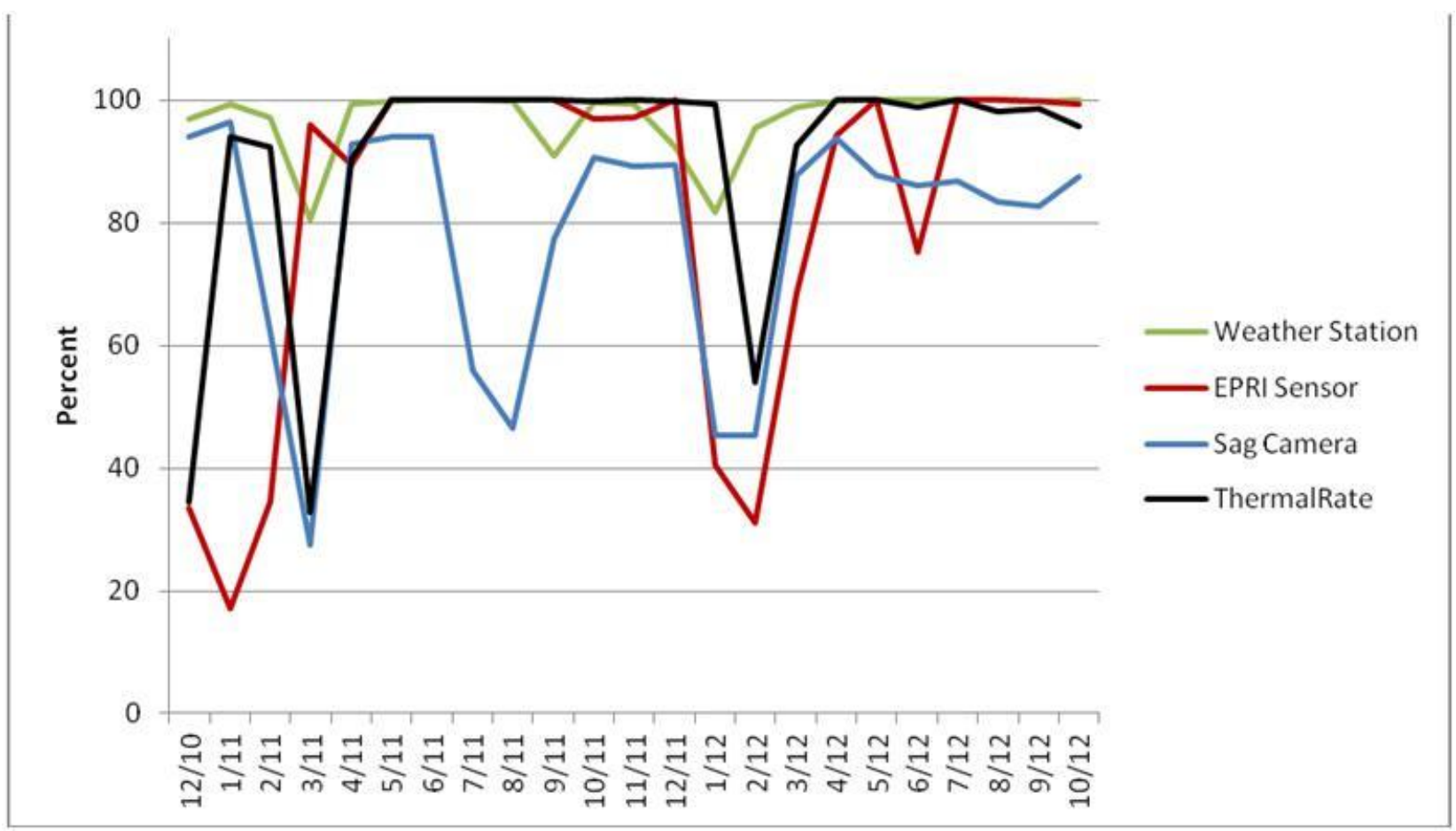

Figure E-11

Instrument Availability at Site 3

EPRI Sensors were replaced at the end of March 2011at this site and performance increased significantly. Unlike Site 2 the DTCR accessed EPRI Sensor continued to report accurate data through the end of the project so the availability depicted here is a good representation of performance.

ThermalRate performance was not affected by polling issues at this site since there is no RTU at Site 3. There was an unresolved ThermalRate data issue in February of 2012 that did affect ratings. Troubleshooting from the server end was unsuccessful but the problem resolved itself, the cause was unknown.

The Sagometer camera at this site required manual resets similar to Site 1. 


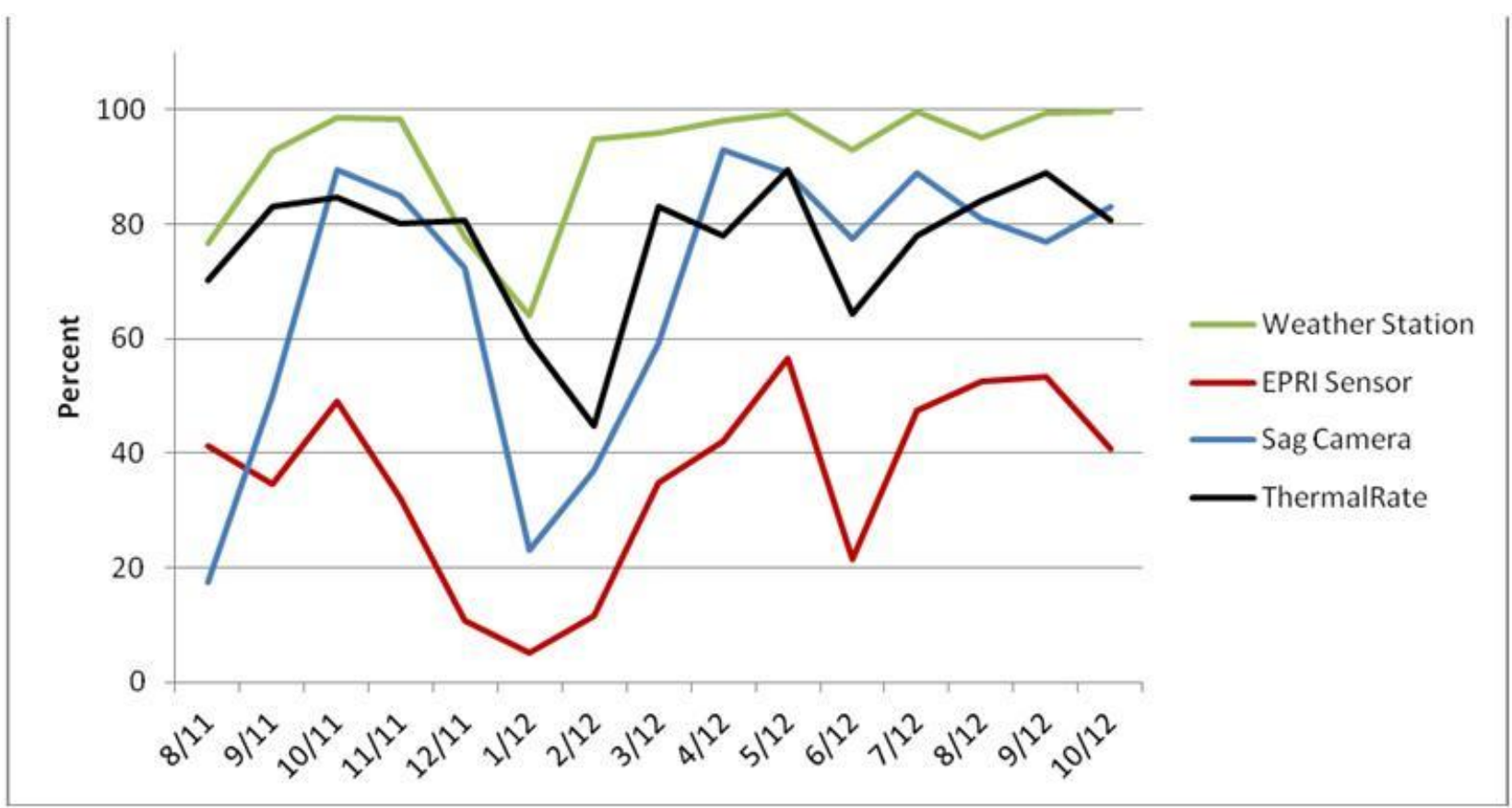

Figure E-12

Real-Time Instrument and Communication Availability at Site 3

When communication losses from the two data loggers to the DTCR server are added availability drops for all instruments.

There is again a decrease in the EPRI Sensor performance due to a large number of coasts that were between 0.5 hours and 1.0 hours. The ratings are not seriously affected by these coasts.

The weather station and ThermalRate availability were the best over the second half of the project at this site.

A DTCR server outage contributed to the poor performance in January of 2012. 


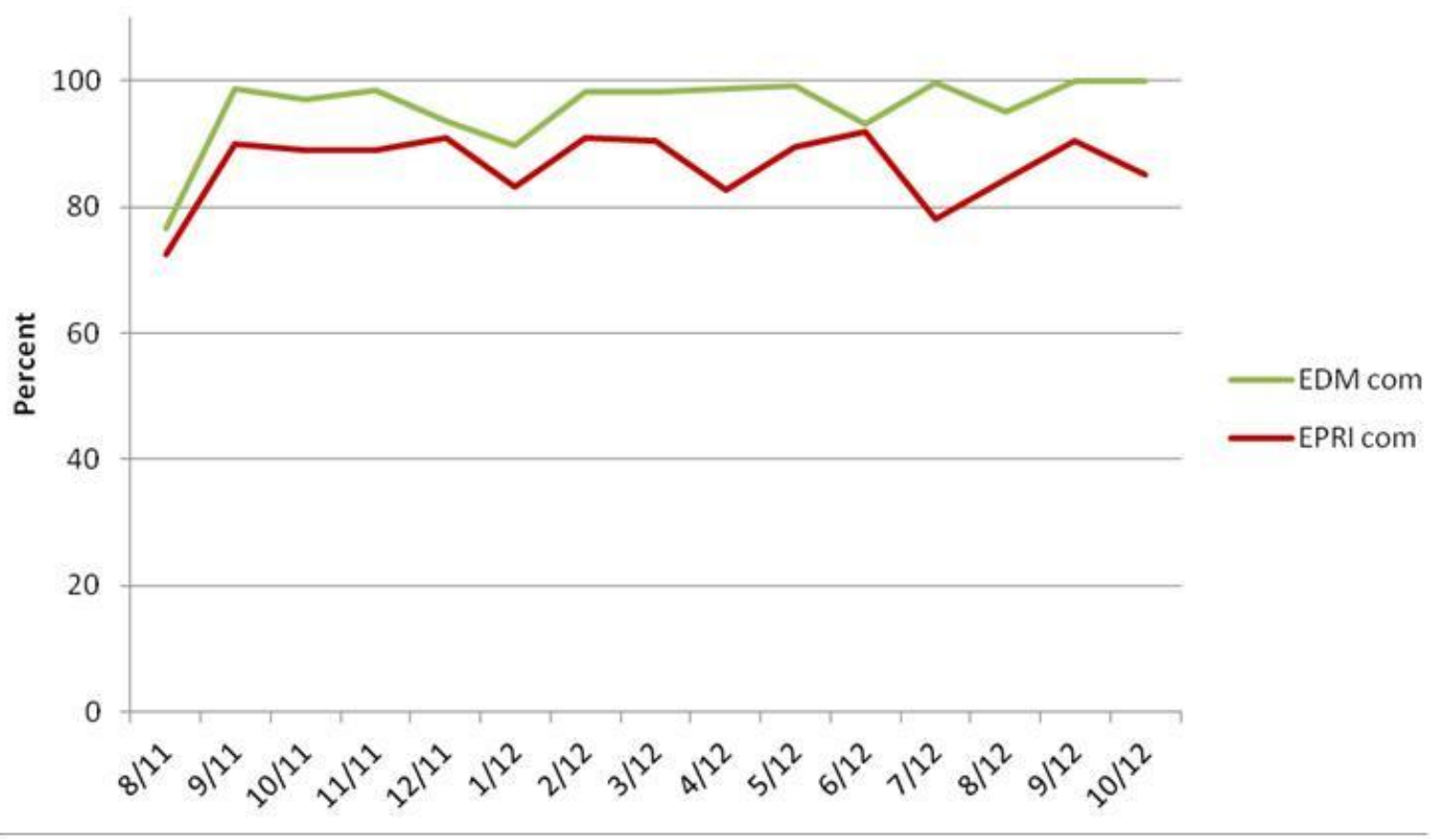

Figure E-13

Communication Availability Between Data Loggers and DTCR Server at Site 3

Table E-4 summarizes the reliability of the data systems at Site 2 for the final 14 months of monitoring.

Table E-4

Average Performance of Data Systems at Site 3 from 8/1/11 through 10/31/12

\begin{tabular}{|c|c|c|}
\hline Site $\mathbf{3}$ Instrument & \% Available & \% Available Real-time \\
\hline Weather Station Thermometer & 98.9 & 93.0 \\
\hline Weather Station Anemometer & 97.4 & 92.2 \\
\hline Sagometer Camera & 78.1 & 68.2 \\
\hline EPRI Sensor & 81.5 & 35.6 \\
\hline ThermalRate Device & 90.5 & 76.7 \\
\hline NYPA SCADA RF Link & none installed & none installed \\
\hline EDM Logger communications & NA & 95.8 \\
\hline EPRI Logger Communications & NA & 86.6 \\
\hline
\end{tabular}


Table E-5 summarizes the reliability of the data systems at all sites for the final 14 months of monitoring.

Table E-5

Average Performance of Data Systems at All Sites from 8/1/11 through 10/31/12.

\begin{tabular}{|c|c|c|}
\hline All Sites; Instrument & \% Available & \% Available Real-time \\
\hline Weather Station Thermometer & $\mathbf{9 9 . 4}$ & 91.6 \\
\hline Weather Station Anemometer & $\mathbf{9 3 . 7}$ & 90.5 \\
\hline Sagometer Camera & $\mathbf{7 9 . 3}$ & 69.4 \\
\hline EPRI Sensor & $\mathbf{7 1 . 9}$ & 25.9 \\
\hline ThermalRate Device & $\mathbf{8 5 . 0}$ & 75.9 \\
\hline NYPA SCADA RF Link & $\mathbf{7 5 . 1}$ & 69.5 \\
\hline All Logger Communications & NA & 90.3 \\
\hline
\end{tabular}

Table E-5 captures combined data availability from all sites and instruments. The weather station proved to be the most reliable instrument group in the project followed by ThermalRate, Video Sagometer and EPRI Sensors. Of these systems, the EPRI Sensors are the only prototype systems in the study. All other instrumentation is commercially available.

\section{Permanent Loss of Good Data}

Some of the communication failures resulted not only in the loss of real-time rating information but in permanent raw data loss. These losses have the added consequence of impinging on the data set available for DTCR simulations.

If communications are lost between the logger and the DTCR server then the logger will store the data until the communications are restored. The data are transmitted at the next available opportunity. However, data are constantly overwritten in the logger due to limited buffer size. As new data is transmitted from the instruments to the logger and recorded, the oldest data are overwritten. If communications interruptions are prolonged, then untransmitted data may be overwritten in the logger before a successful transmission to the DTCR server occurs. This data is permanently lost since the data archive exists on the DTCR server and the first opportunity to back up the data is the from the server archive. Missing time stamps in the archived raw data from a logger are evidence of such an occurrence.

Fortunately, this was not a common occurrence but prolonged interruptions did occur. Figures E-14 through E-16 depicts this data loss over the course of the project for the EDM and EPRI loggers at all three Sites. 


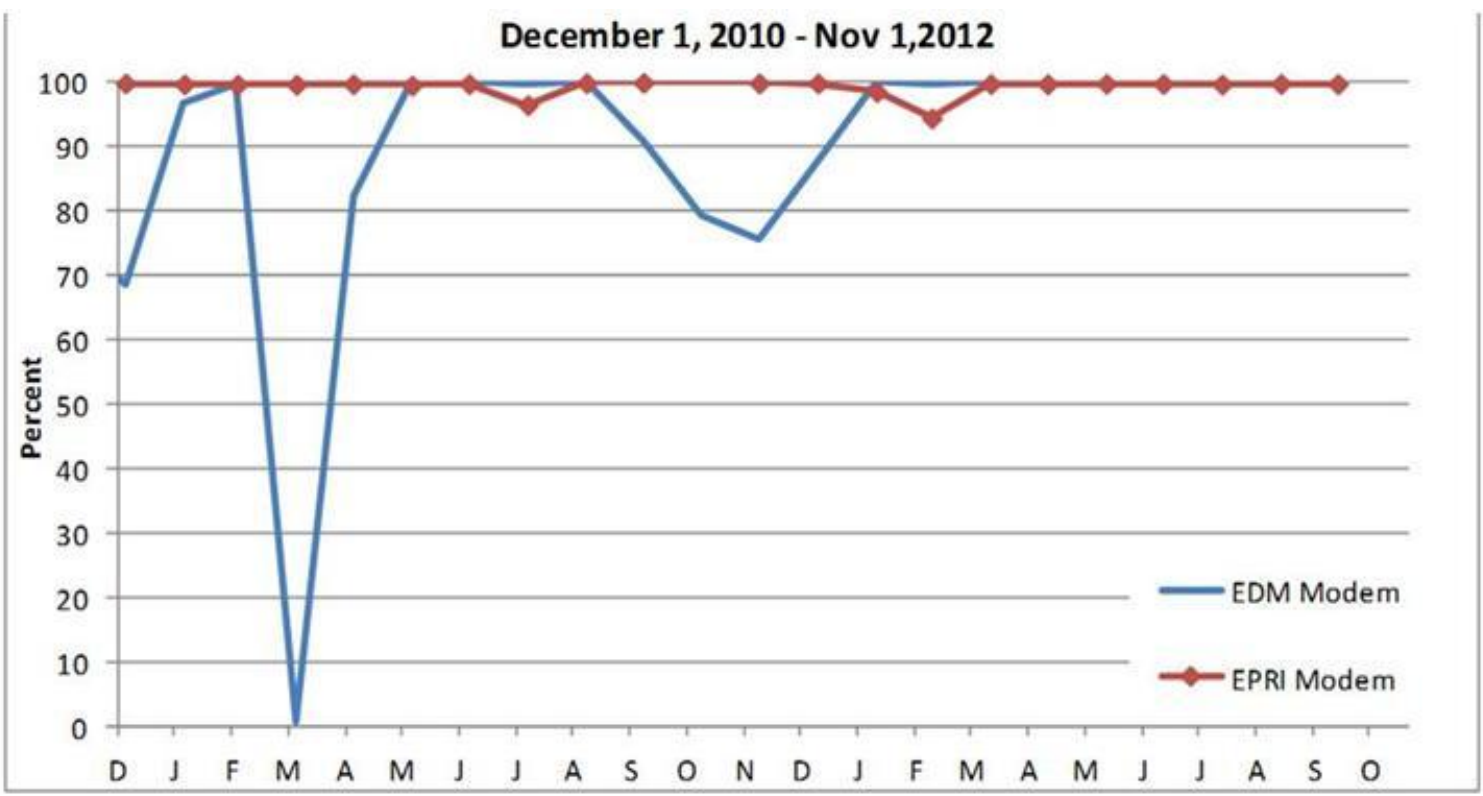

Figure E-14

Data Successfully Archived from Data Loggers at Site 1

Note that the EPRI modem logger combination had fewer long-term communication interruptions than the EDM pair at this site. An interview with a NYPA lineman in August 2012 during a maintenance visit revealed the anecdotal information that cell communications varied over short distances in the area and even when facing different directions. The site is near the Canadian border and occasional inappropriate roaming messages also occur on cell phones. The EPRI personnel visiting the site had similar experiences over two days in the area. It is unknown if and how much these issues affect the site modems or if they could affect two modems within 20 feet of each other on the same structure differently. However a possible explanation for prolonged outages (such as the two shown in Figure E-14 is that the site modem locks onto the Canadian cell provider signal for these periods and data transfer is interrupted until the Verizon signal becomes the stronger of the two. 


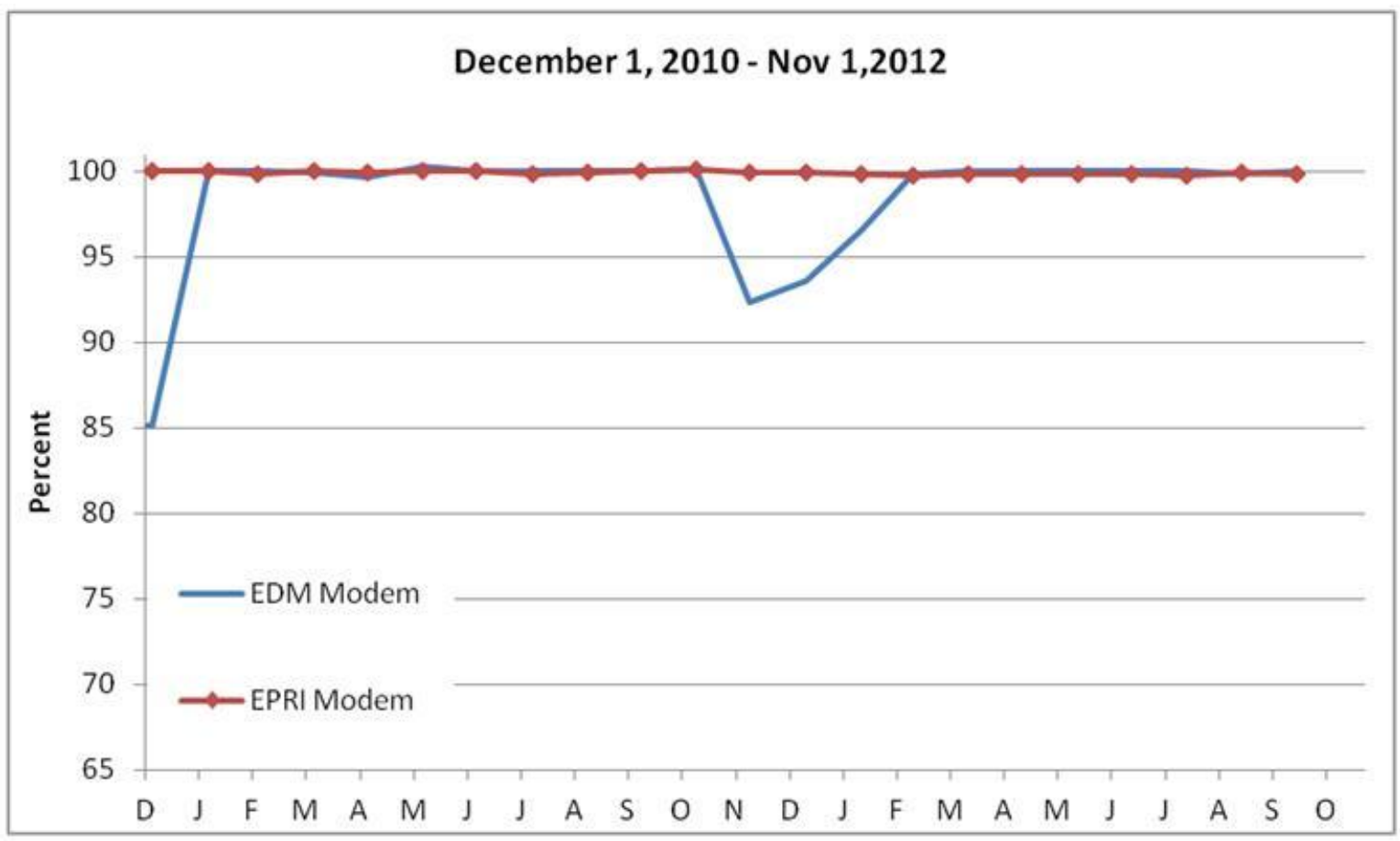

Figure E-15

Data Successfully Archived from Data Loggers at Site 2

Note that the EPRI Modem logger combination also performed better than the EDM pair at site 2 and that both pairs performed marginally better here than at Site 1though the two sites are within 2 miles of each other.

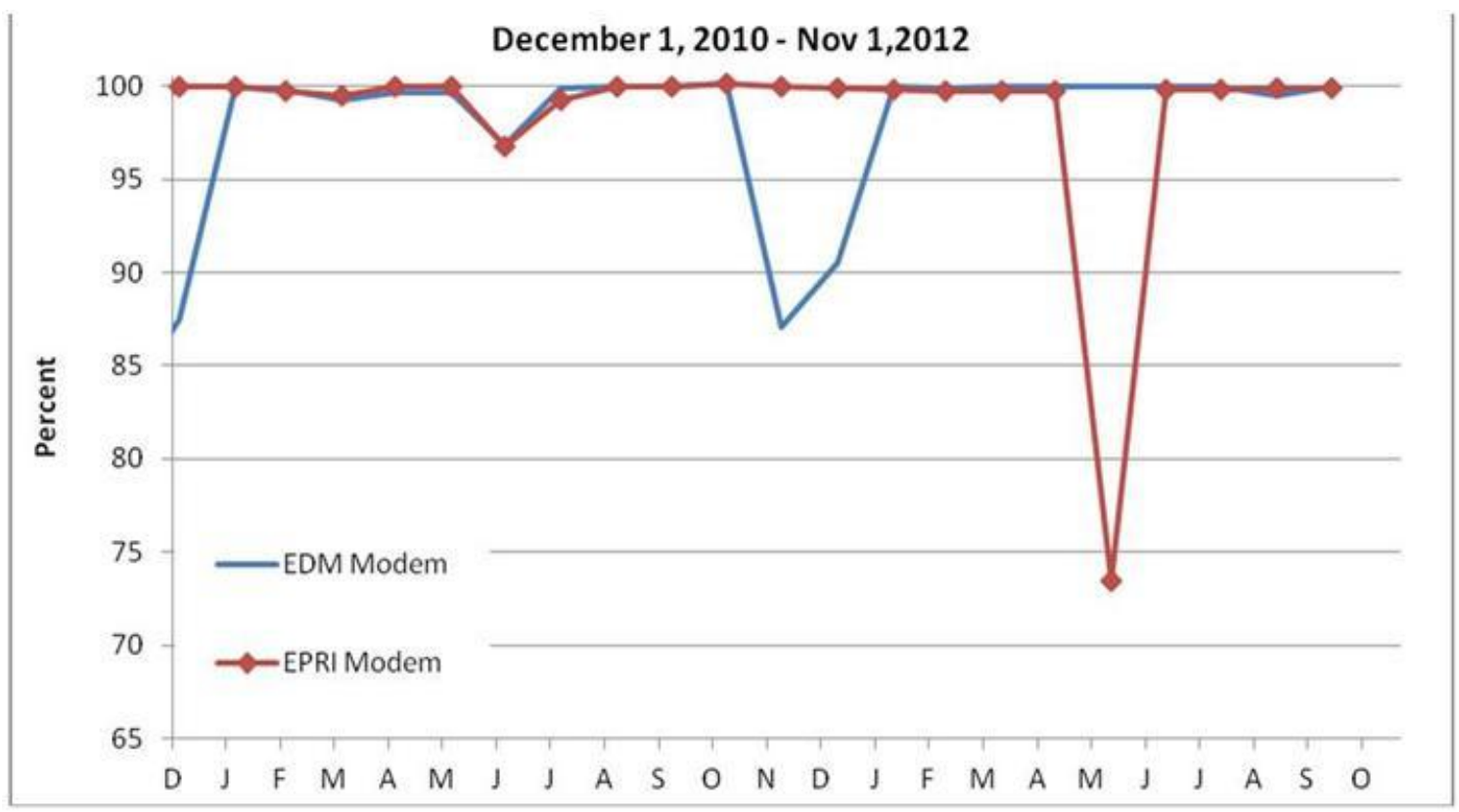

Figure E-16

Data Successfully Archived from Data Loggers at Site 3 
Note that the EPRI Modem logger combination performed worse than the EDM pair at Site 3 in contrast to the other two sites. This was primarily due to an extended incident of non communication in May 2012. Note that the EPRI logger at this site does not log NYPA load data. At the other two sites a loss of data from the EPRI logger prevents rating calculations for all elements because SCADA load is a required global parameter. Additionally, the loss of load data due to communications failure from the EPRI modem at Site 2 will also prevent ratings calculations at Site 3 regardless of its connection status. This dependence can be removed in post processing for periods where EPRI Sensor load data is available.

\section{DTCR Server Downtime}

Server reliability applies directly to real-time availability of all rating calculation elements. Experience in this project was good with the HP server which is described in detail in Appendix C. It has redundant power supplies to allow for maintenance on one power supply without shutting the server down. This redundancy does not protect against facility wide power outages. The server in Lenox was subjected to power outages as well as other interruptions. Since this server was not continuously monitored there was usually a delay before the server was restarted

Figure E-18 shows a point for each occurrence of unexpected downtime, this is found by looking in the server management logs and looking for event codes that show an unexpected power failure or service termination, then looking for the event code for a power up and finding the time in between. The plot goes back no further than March 2011 as this was the oldest logs available.

The Lenox facility is a test lab and during some high voltage switching operations the server was affected. Improvements were made to the power quality in 2011 and a dedicated UPS was added in December 2011. The dedicated UPS improved server performance in 2012. The latest event in Figure E-17 was from a prolonged outage caused by Hurricane Sandy. In this event the UPS was unable to sustain operations. 


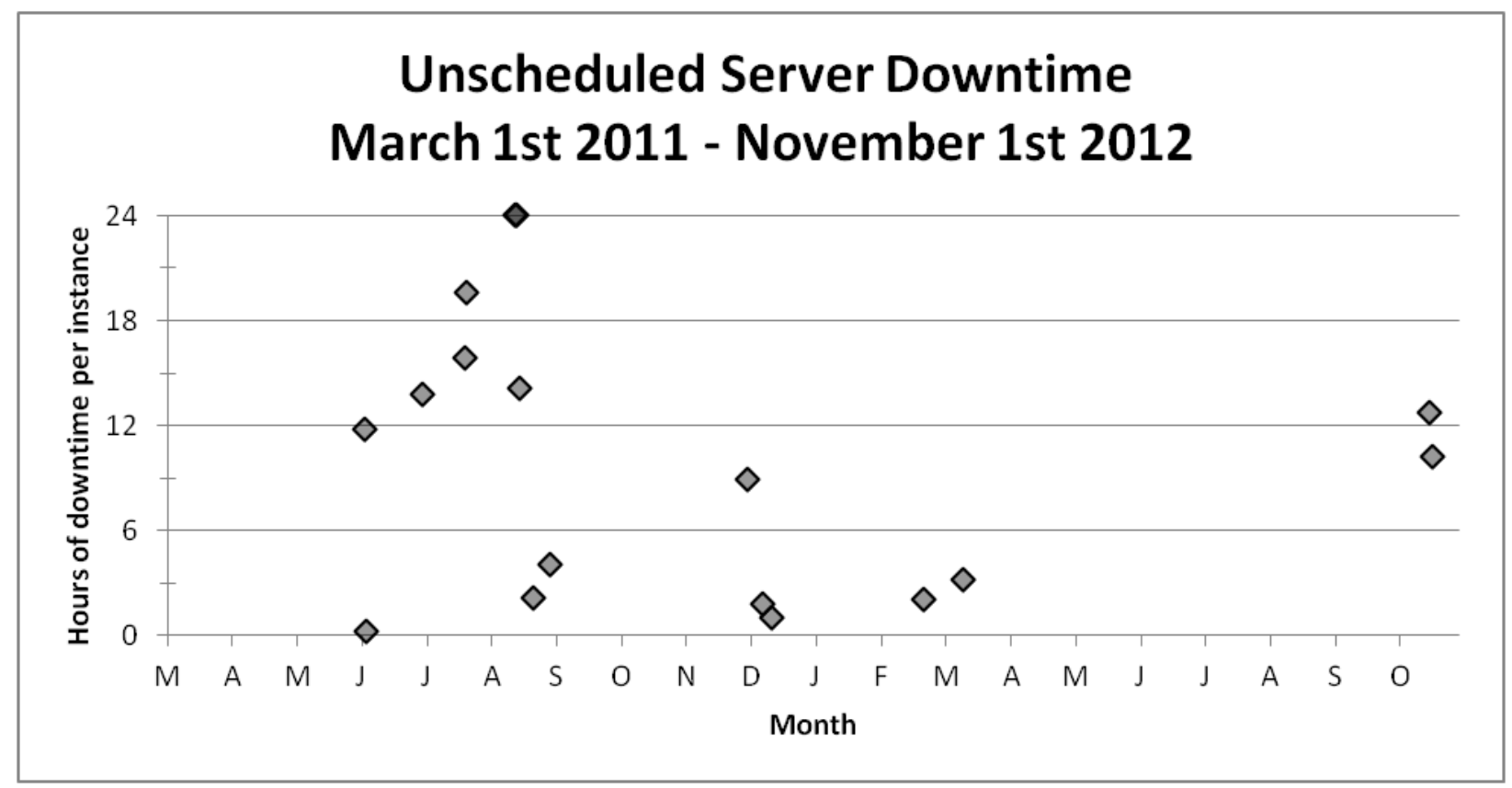

Figure E-17

Duration of Unscheduled Server Downtime

\section{Software Down Time}

Scheduled software downtime is roughly estimated from the operator notes to have been in the order $0.4 \%$; about half of this was due to planned server downtime. Typical operations included updating the DTCR input file (element models), updating the server operating system, and revising LoggerNet data locations.

Some downtime was due to a memory leak in the DTCR GUI. After a few weeks of operation this leak typically locks the GUI up but rating calculations continue normally. On one occasion on $6 / 13 / 12$ the server crashed due to overloaded memory. When the GUI is closed in DTCR rating calculations are not halted. Closing the GUI cleans the memory. Therefore DTCR version 6.0 can be run continuously by closing and reopening the GUI every few days. After the leak was confirmed in June 2012 the practice became to close the GUI when not actively monitoring the server. 


\section{RATING RESULTS}

This chapter presents line rating data as a function of time for summer and winter seasons. Variation in ratings over summer and winter periods will be discussed. The seasonal start and stop dates have been determined by the NYISO as summer (June 1 to November 1) and winter (November 1 to June 1).

Wind speeds are often above and air temperature below the weather conditions used by the NYISO. Therefore, the ratings of the instrumented lines are often higher than the static NYISO line ratings. Appendix K (and the associated data files located on an FTP site documented in Appendix K) contain all time based rating data. This chapter will show typical rating data and is not intended to be all inclusive. All rating data was made available to NYPA in the digital release that accompanies Appendix K.

Static ratings of components other than the overhead lines are also discussed. This chapter section identifies some system components that may become limiting factors when dynamic ratings are introduced. For instance the connectors coming from the substation to the overhead line have a lower static thermal limit than the lines themselves. In some cases the current transformers are the limit of the line. While transformers can be dynamically rated in DTCR there may be some components in the circuit that cannot. If the static or dynamic circuit rating needs to be increased, replacing the connectors and other low cost terminal equipment is an easy way to accomplish it.

Reviewing time series rating data helps gain a better understanding of the fluctuation in dynamic line ratings. The sometimes rapid fluctuations are the result of changes in wind speed and direction combined with the relatively small thermal time constant of bare overhead conductors (5 to 15 minutes). This short time constant makes it difficult to utilize all of the true capacity when dynamically rating over head lines but substantial increases in power flow can be realized on the NYPA lines even when the higher fluctuations are ignored. Subsequent chapters will discuss the risk levels associated with increasing static ratings.

The time series graphs depicted in this chapter will show some "holes" in the data. This is due to a number of rating reliability factors which are reviewed in Appendix E.

In general the dynamic ratings are significantly higher than the static ratings. Statistical evaluation of ratings can be used to evaluate the additional capacity. Statistical studies of the ratings shown here are discussed in Appendix $\mathrm{H}$.

\section{Static Ratings of System Components}

While this report focuses on improving on the static ratings for the overhead line sections a listing of other limiting components on the line is made here as a resource for a top level evaluation of the next limiting factors when dynamic overhead ratings are used. 
Table F-1 below summarizes the static ratings for the overall circuit and for the line section with which the sensors are associated.

Table F-1

Summary of Static Ratings

\begin{tabular}{|c|c|c|}
\hline \multicolumn{3}{|c|}{$\begin{array}{c}795 \text { ACSR } 26 / 7 \\
\text { Drake Conductor Site } 3 \text { and Site } 2\end{array}$} \\
\hline Time Period & Summer (Amps) & Winter (Amps) \\
\hline Normal (24 hrs) & 1087 & 1331 \\
\hline Long Term Emergency (4hrs) & 1256 & 1460 \\
\hline Short Term Emergency (15min) & 1410 & 1593 \\
\hline \multicolumn{3}{|c|}{795 AAC connectors } \\
\hline Time Period & Summer (Amps) & Winter (Amps) \\
\hline Normal (24 hrs) & 876 & 1121 \\
\hline Long Term Emergency (4hrs) & 968 & 1188 \\
\hline Short Term Emergency (15min) & 1104 & 1299 \\
\hline \multicolumn{3}{|c|}{ Site 1795 ACSR 26/7 } \\
\hline Time Period & Summer (Amps) & Winter (Amps) \\
\hline Normal (24 hrs) & 1089 & 1331 \\
\hline Long Term Emergency (4hrs) & 1256 & 1460 \\
\hline Short Term Emergency (15min) & 1410 & 1593 \\
\hline \multicolumn{3}{|c|}{ Site 1795 ACSR $26 / 7$ connectors and Current Transformers } \\
\hline Time Period & Summer (Amps) & Winter (Amps) \\
\hline Normal (24 hrs) & 996 & 1200 \\
\hline Long Term Emergency (4hrs) & 1152 & 1200 \\
\hline Short Term Emergency (15min) & 1263 & 1428 \\
\hline
\end{tabular}

Note that in many circuits, the dynamic overhead line rating exceeds the static rating of many other system components. If the limiting circuit element is relatively inexpensive, it should be replaced in order to realize the higher dynamic circuit rating. Of course, if the thermal rating of the transmission circuit does not limit power flow (e.g., it is limited by voltage drop), then there is no reason to make such modifications or to implement dynamic rating methods. 
If power flow on the circuit is limited by the thermal rating, and a power transformer or line switch has a lower rating than the line, then both the line and the terminal equipment could be dynamically rated with DTCR.

Proprietary information about thermally limited components on the specific circuits in this study could not be included but Figure F-Error! Reference source not found. 1 includes data from a atabase of many transmission lines from many utilities. The overhead conductor static thermal limit is usually the limiting circuit element but when dynamic ratings are used and high winds are present, other components quickly come into play. Statistically CT's are the next load limiting equipment after the overhead line.

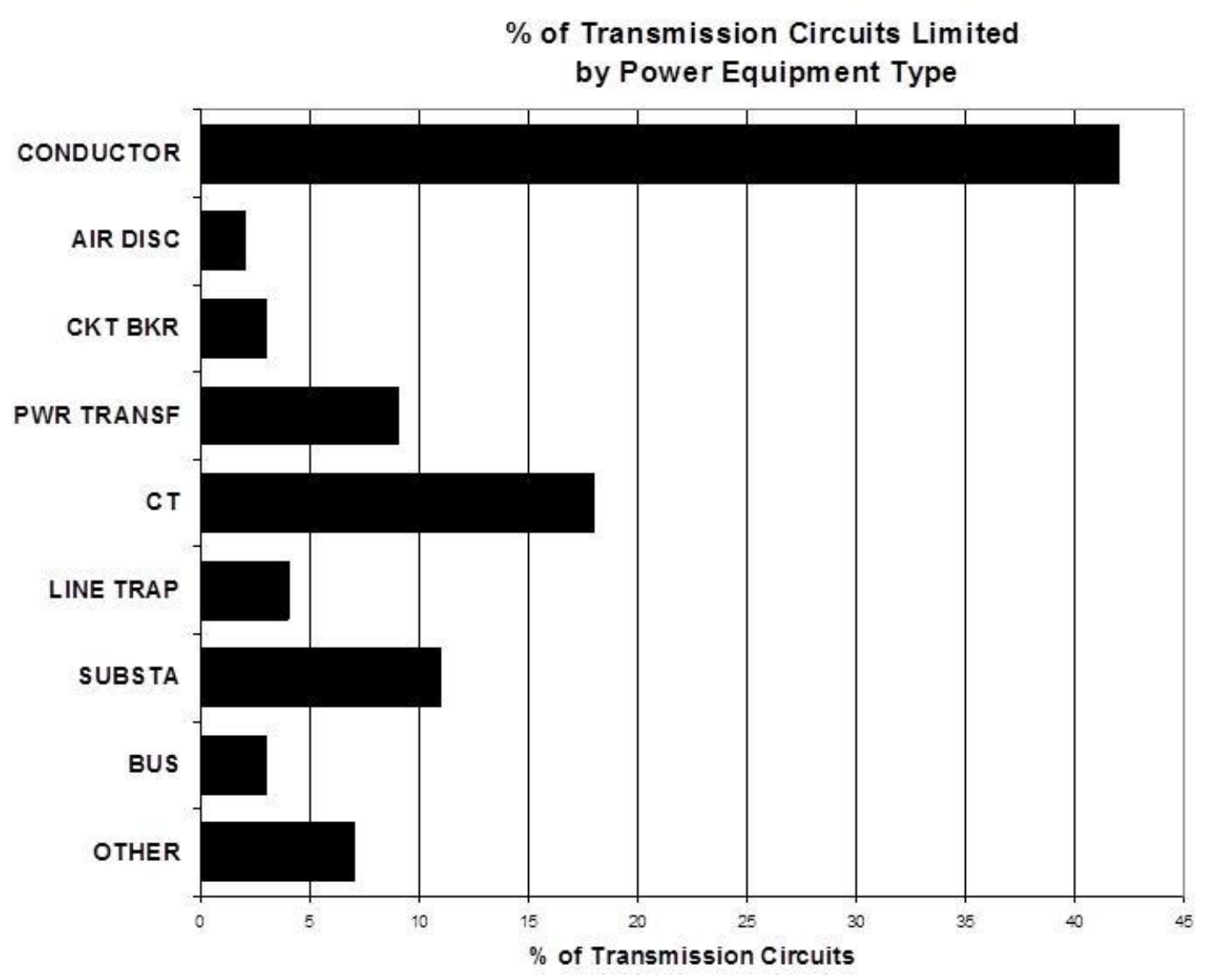

Figure F-1

Transmission Circuit Limitations

\section{Ratings at Site 3: Winter}

Figure F-Error! Reference source not found. 2 is a plot of dynamic ratings based on the onsite eather station and the IEEE-738 standard. The flat spot in the ratings around March $24^{\text {th }}$ was due to a failure of the weather instruments. Weather conditions indicate this may have been due to ice and snow buildup. It was clearly not a communication issue as the weather instruments and sagometer share a modem and sag data was being retrieved during this time. This condition persisted about three days. 
The flat spot in the ratings during the middle of December was due to prolonged communication failure with the EDM modem. This can be verified because sag data was also lost during this time. It was not due to a prolonged server outage as data from both the EPRI Sensors and ThermalRate device were received from the second data logger at the Site. For this flat spot to be visible in a simulation the communications had to be down so long that data was lost off the circular buffer. The flat spot in the load data during this time is thought to be accurate. There was a gradual taper to zero and temperature data was still being received from the sensor during the entire period.

For these months the average rating was 1678 Amps, which shows that a majority of the time the increased power flow provided by weather based dynamic rating would be over $25 \%$. The average downtime in simulation mode was less than 5\% which shows a high degree of reliability for the onsite weather station system.

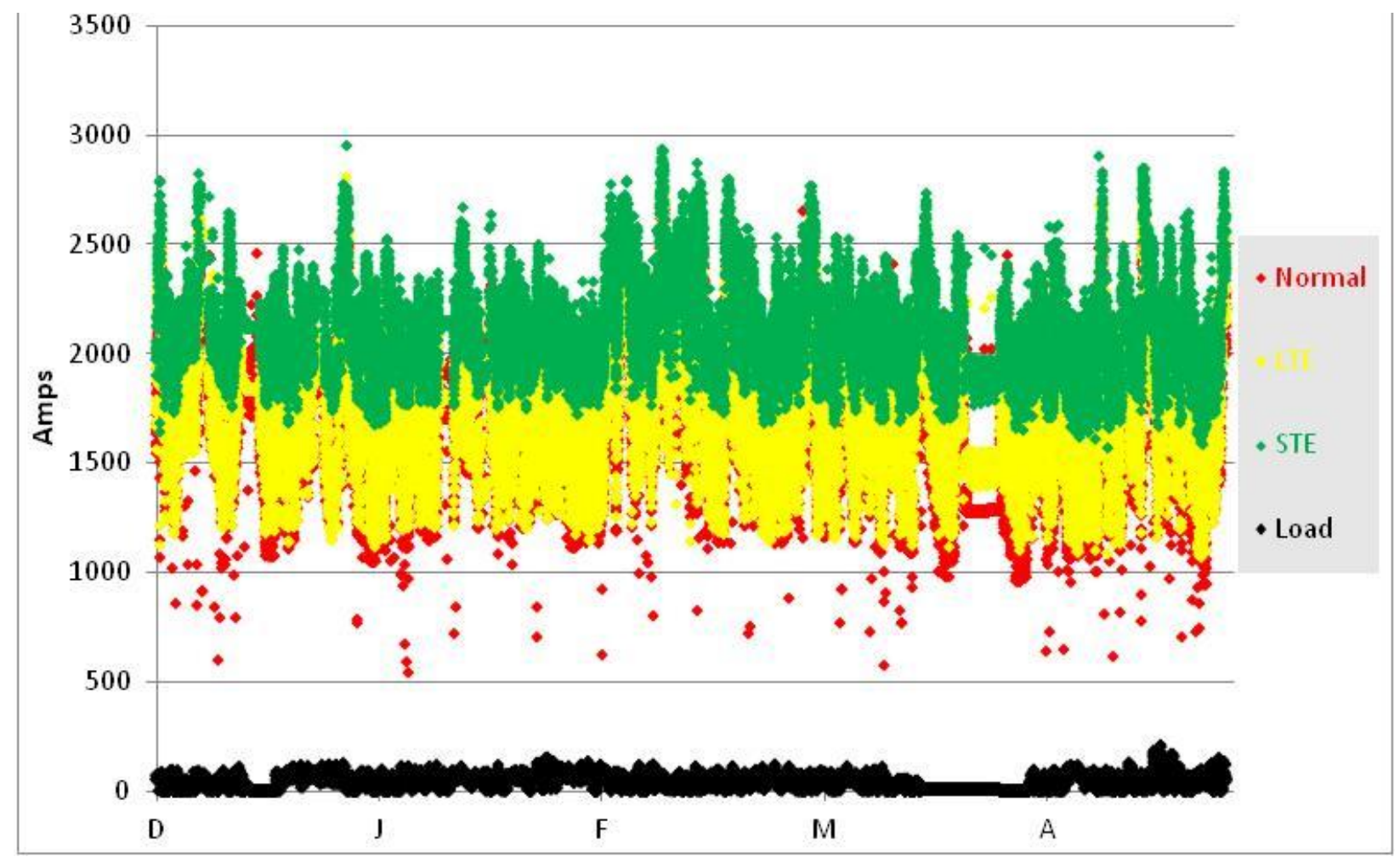

Figure F-2

Overhead Line Ratings Based on the Onsite Weather Station at Site 3 - Winter 2010-11

Load is omitted in the following plots to allow a more detailed view; it will be displayed on the Weather Station plot for each section. Below are the rating calculations for the same interval but using the ThermalRate sensor and the IEEE-738 rating model. The average rating of the line with this model is closer to 2000 Amps. ThermalRate produced the highest dynamic ratings of the five rating elements. Though less conservative than the onsite Weather Station results the result has some evidence of support in detailed analysis. Evidence supporting accuracy of ThermalRate ratings are discussed in Appendix G. 
The gap during December was caused by a run in period of communications debugging. Once communications were established data was then clearly retrieved without an incident noticeable on this scale.

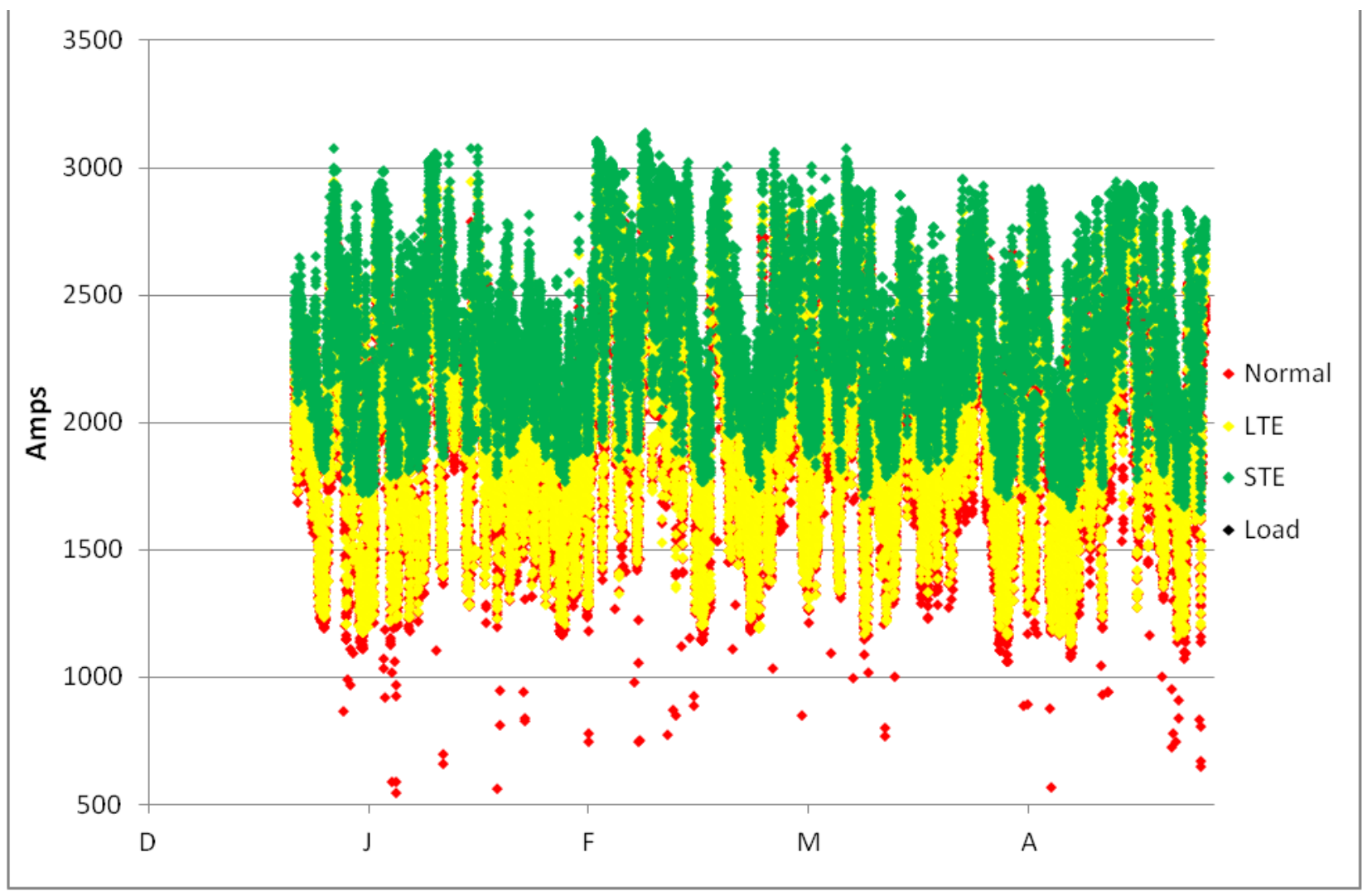

Figure F-3

Overhead line ratings based on ThermalRate at Site 3- Winter 2010-11 
Figure F-4 below is a plot for the same time scale and location, it uses data from the Sagometer based DTCR sag/tension model. The communications error visible in the weather model is again visible in December and mid January here because they share a common communication path (see Appendix $\mathrm{G}$ for details). There are also intermittent errors at the end of December, and these are due to camera errors. The camera also reported missing data throughout the majority of March.

When the conductor temperature is not driven high above ambient, the load sag/tension DTCR models revert to a weather based rating. The load threshold for the ambient-conductor temperature difference to allow Sag models to take effect is 350A. This occurs less than 5\% of the time so over a large time period ratings follow those of the weather model.

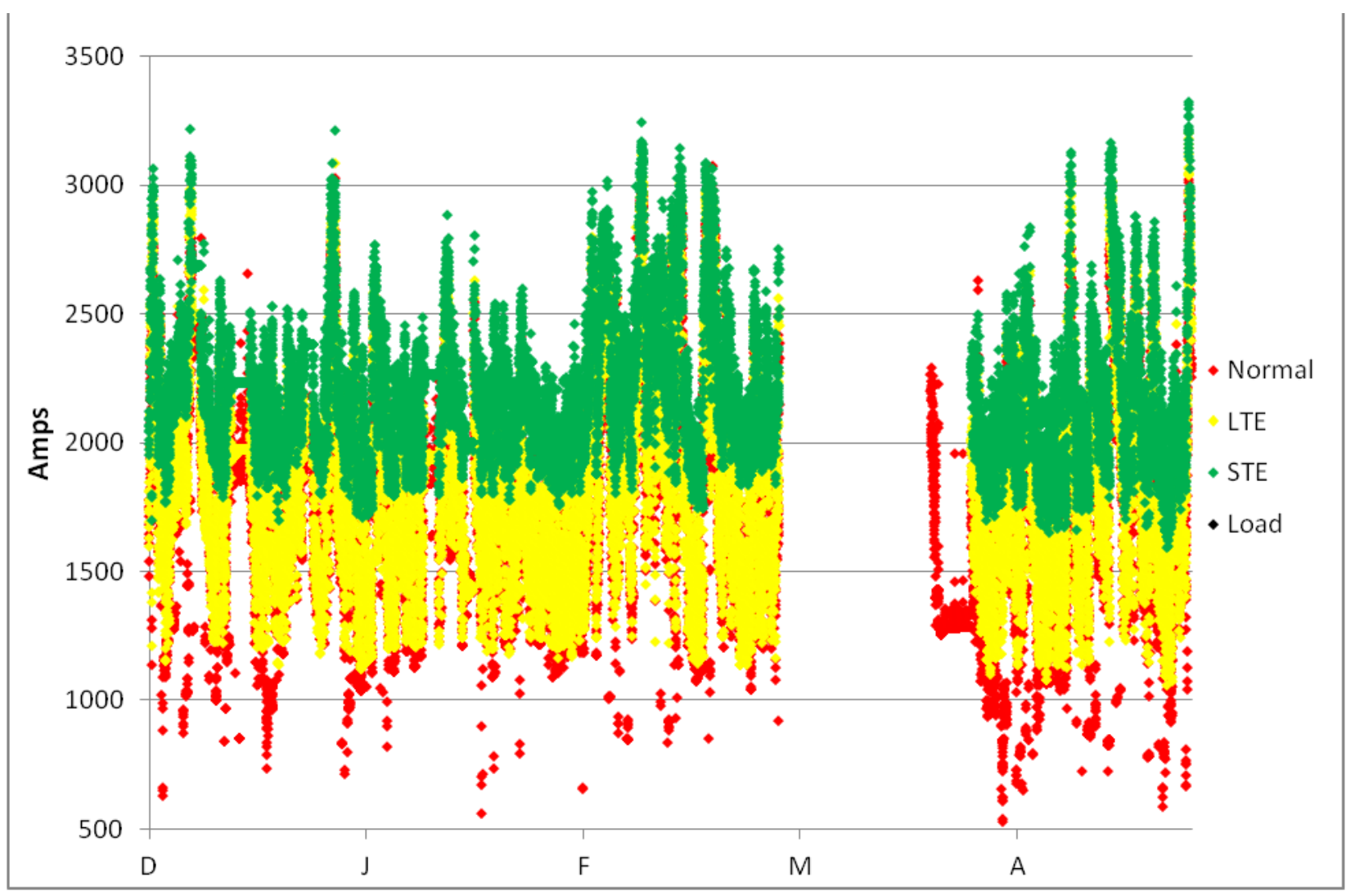

Figure F-4

Overhead line ratings based on the Sagometer at Site 3 - Winter 2010-11

Some of the technologies had ongoing problems that took time to resolve. In the case of the Sagometer the camera would unexpectedly shut down. This problem persisted for a considerable time before it was finally resolved. Once the problem was better understood, EPRI would actively check for it on a weekly basis. When the camera when down EPRI would contact EDM and they would reset it. The camera would come back on line at that point.

Figure F-5 below is the plot of the rating data as based on EPRI Sensor readings for the winter of 2010 into 2011. Just as in the Sagometer case the calculation reverts to a weather model when the line is lightly loaded (below $350 \mathrm{~A}$ ) so the holes present in the weather data are again viewed here. 


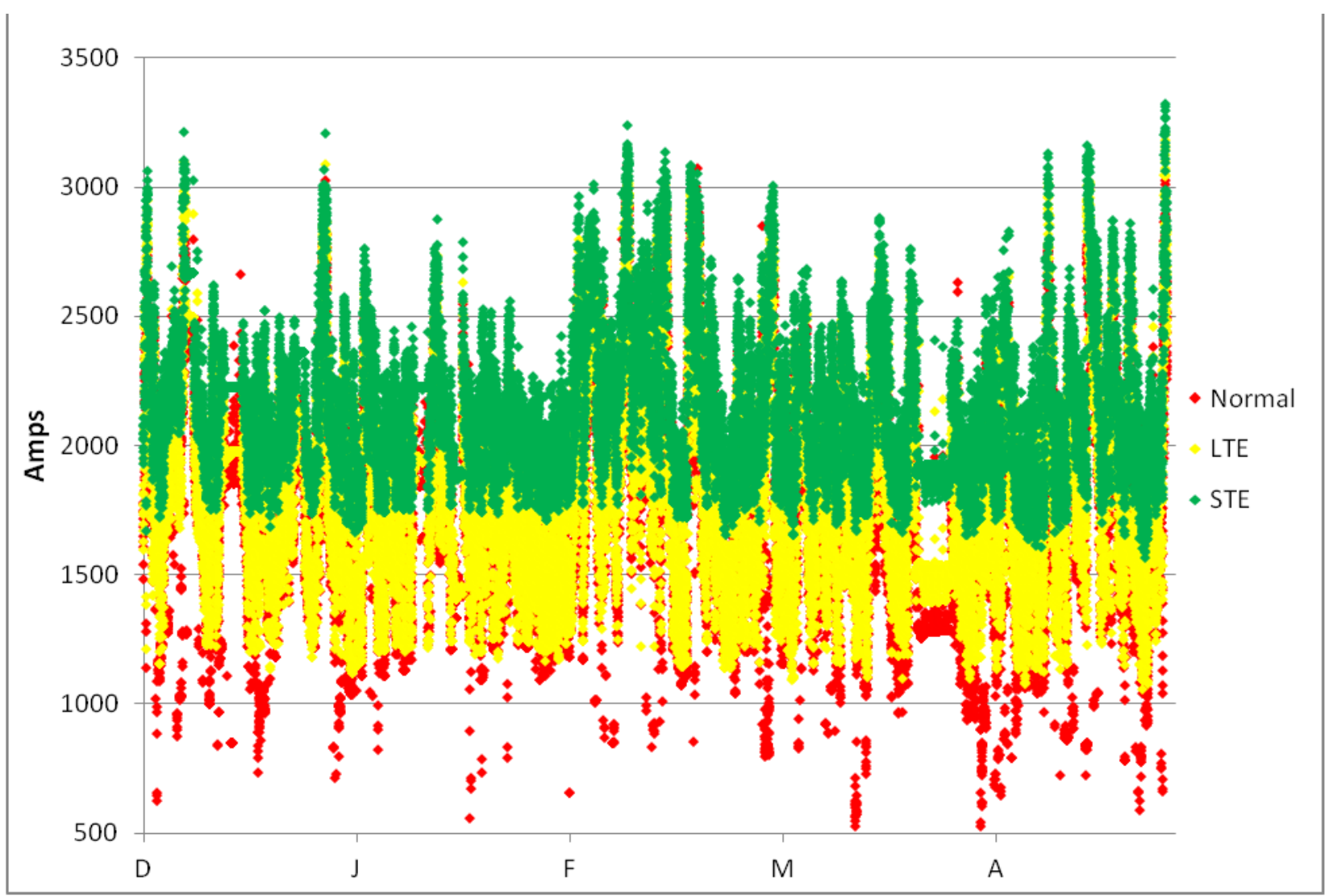

Figure F-5

Overhead line ratings based on EPRI Sensors at Site 3 - Winter 2010-11

Plots of the offsite weather service (airport) results are not available for this time period. There is no data archived for DTCR simulations, there is limited data shown in the section containing monthly real-time data. 


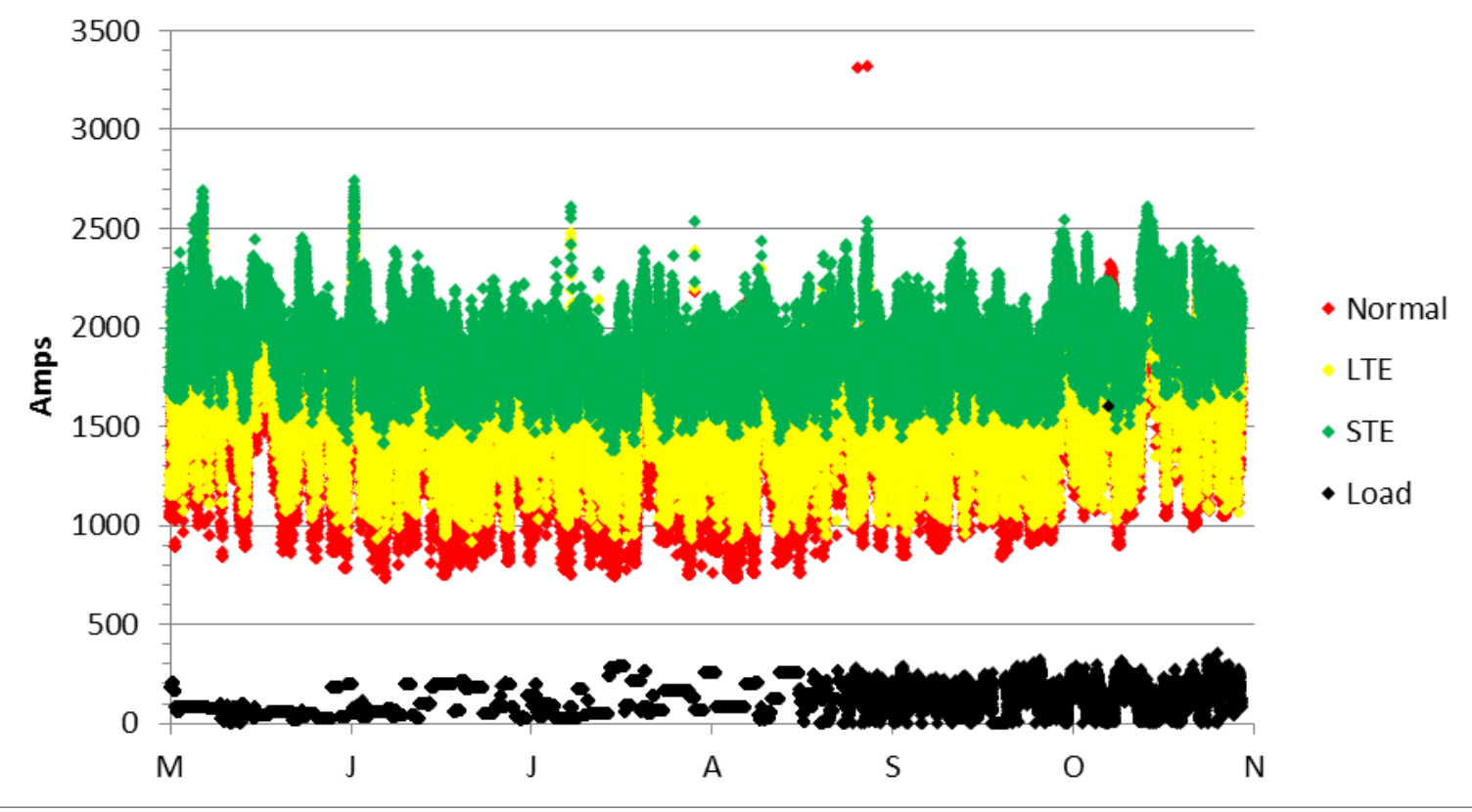

Figure F-6

Overhead line ratings based on Onsite weather station for Site 3 - Summer 2011

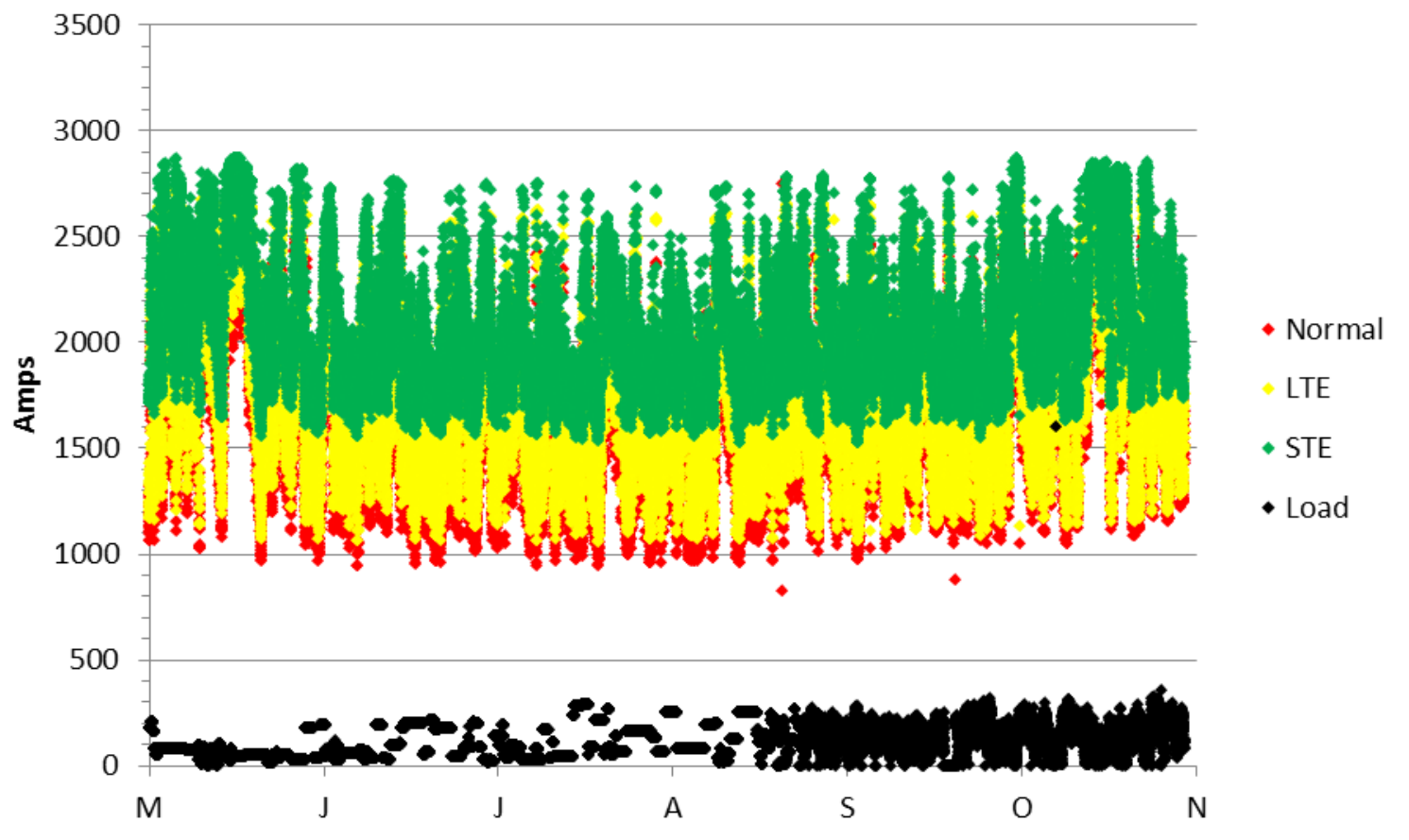

Figure F-7

Overhead line ratings based on ThermalRate device for Site 3 - Summer 2011 


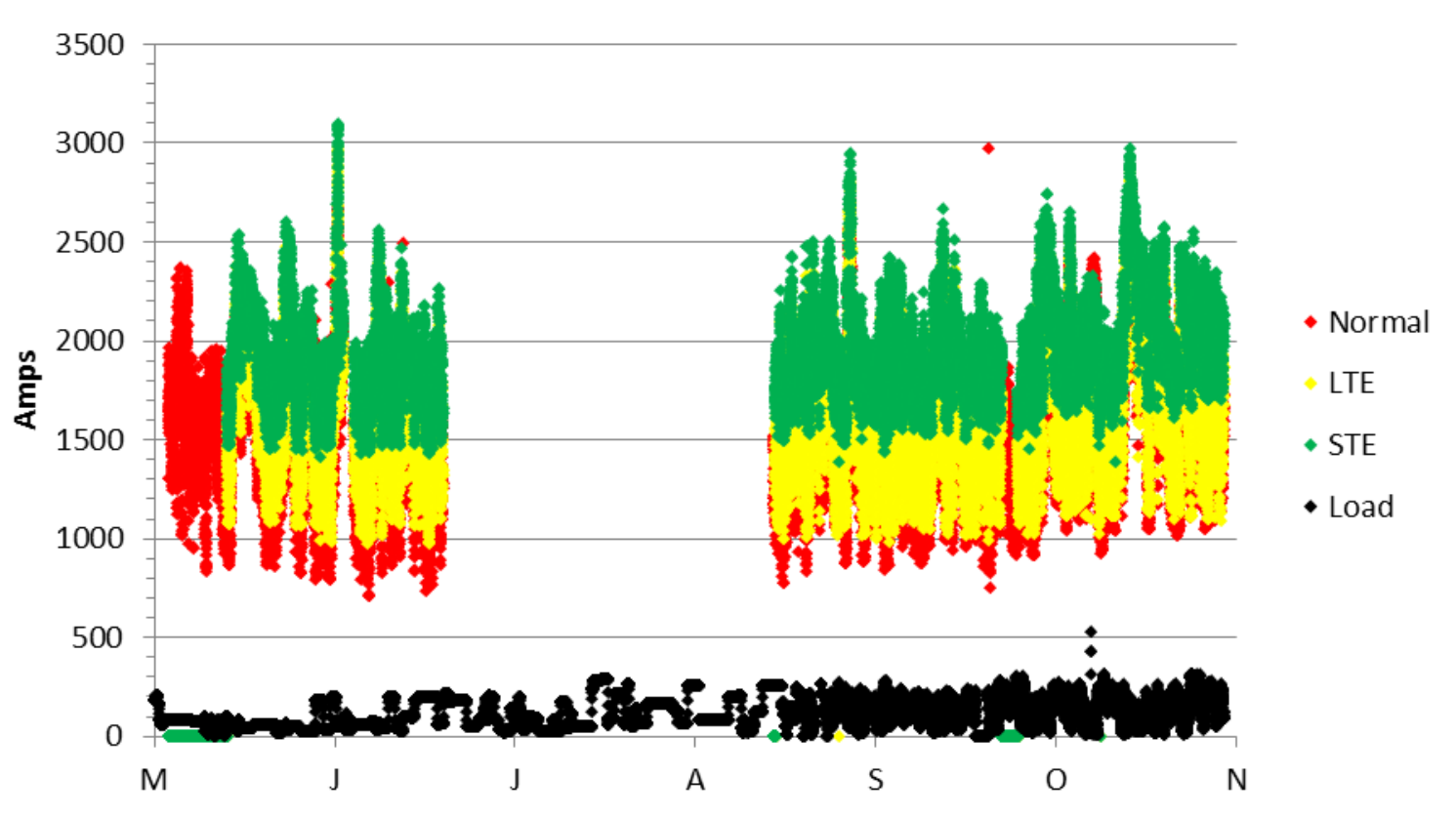

Figure F-8

Overhead line ratings based on the Video Sagometer for Site 3 - Summer 2011

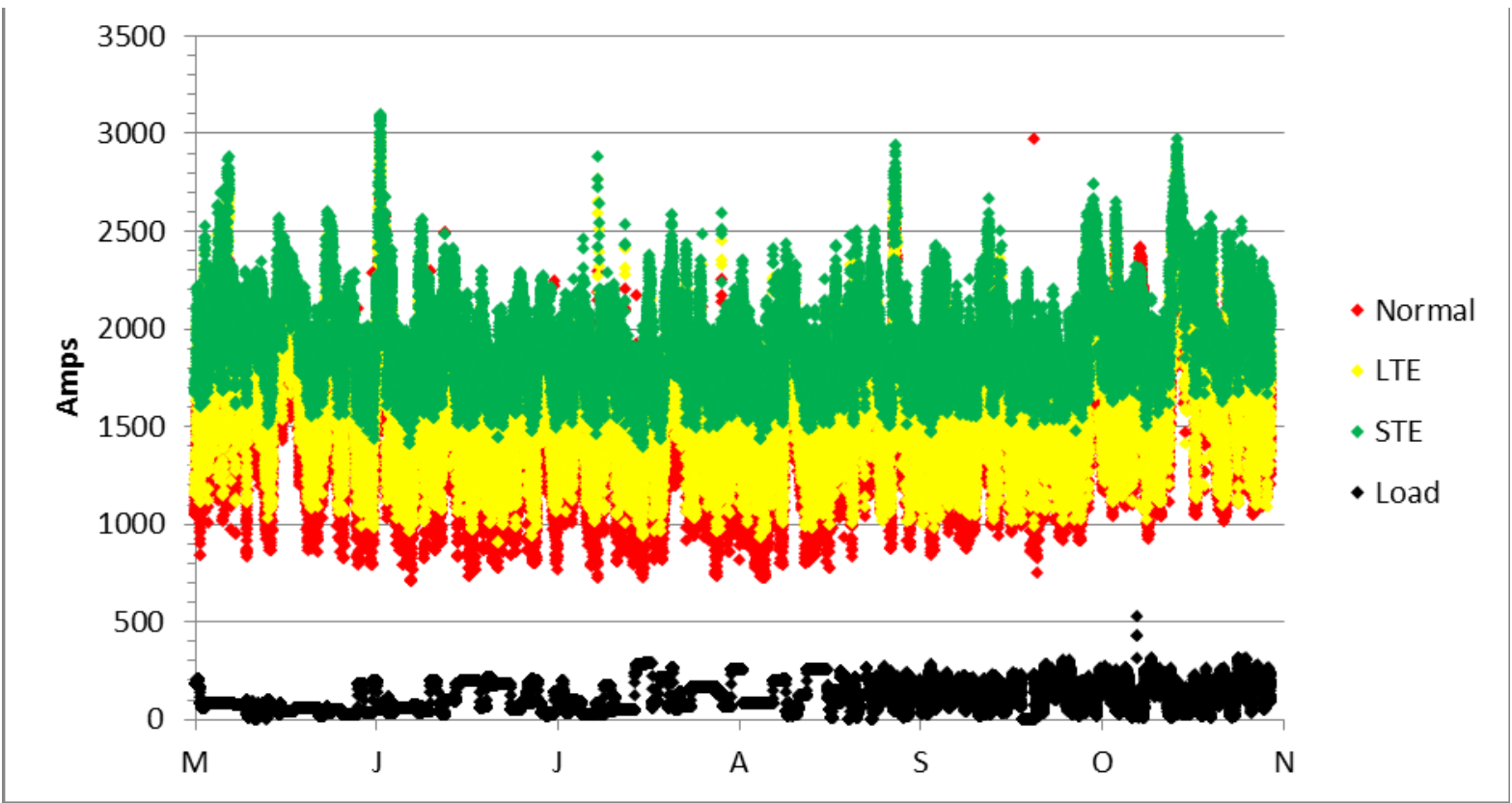

Figure F-9

Overhead line ratings based on the EPRI Sensors for Site 3 -Summer 2 
For the winter of 2011-2012 the onsite weather station average rating was about 20 Amps lower than the previous year, but again giving large gains over the static rating. This slightly lower average is along with variations in weather patterns also influenced by the presence of the November data. It is shown here that the first half of November had some of the lower ratings of the winter season which is to be expected.

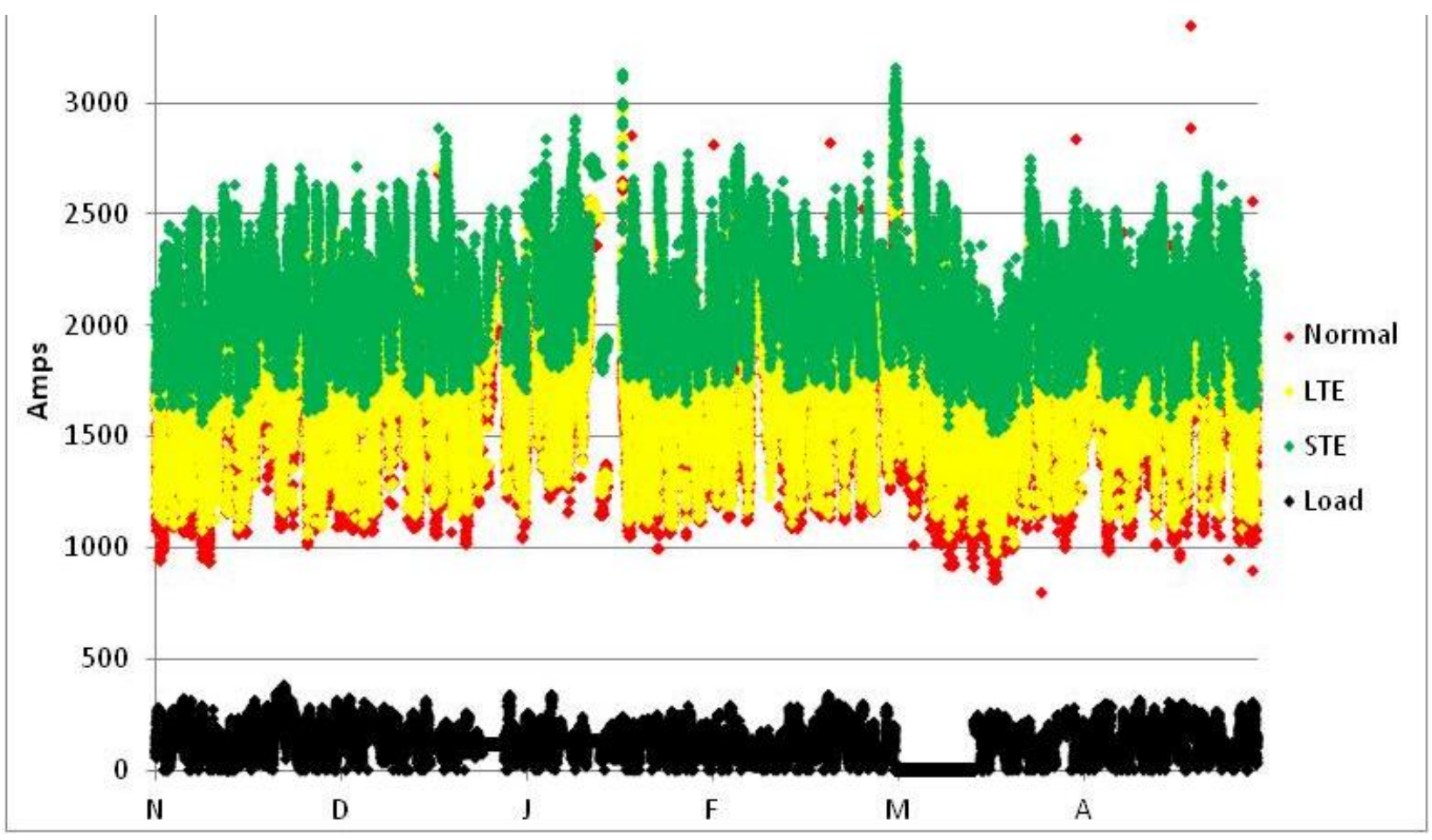

Figure F-10

Overhead line ratings based on the Onsite Weather Station at Site 3-

Winter 2011-12

All of these technologies require careful and frequent monitoring. Catching the problem early helps restore the system back to working order more quickly. Venders typically were able to solve the problems remotely and quickly.

The following is a plot of ratings for the same period from the ThermalRate device. The gap in the data during February is due to an instrument failure. The device was reporting the expected effective wind speeds but was unable to determine the air temperature for comparison. A simulation using an alternate source of data (from the weather station) could be to fill this gap since the hot and cold rod data is available. The advantages of building a hierarchy of data sources for use within DTCR software has been noted due to this and similar rating lapses as a result of this project. 


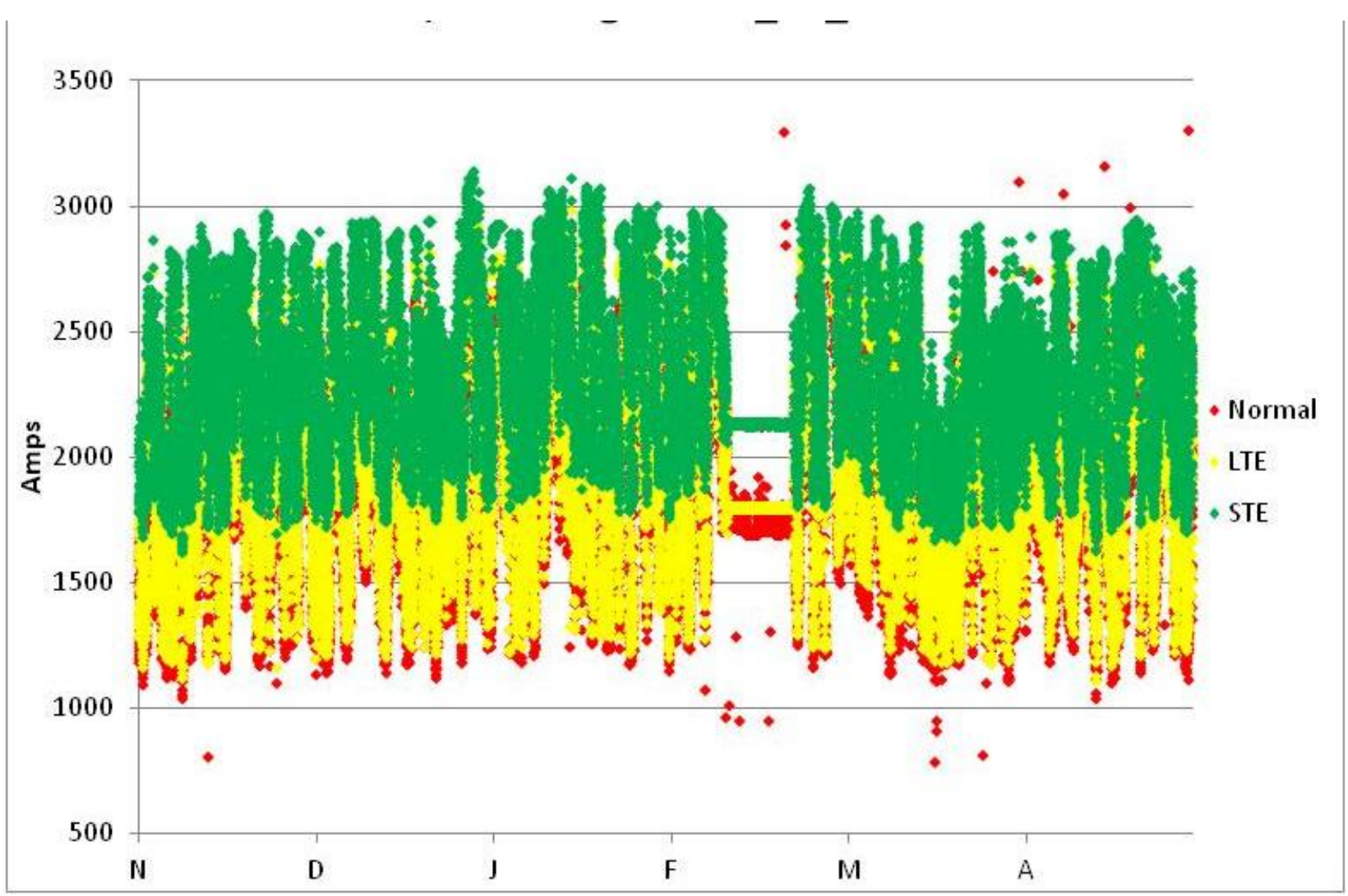

Figure F-11

Overhead line ratings based on ThermalRate at Site 3 - Winter 2011-12

Sometimes venders needed to go to the Site to make repairs. It is recommended that when buying devices like these that some agreement is made between the utility and the vender about onsite repairs if the system cannot be fixed remotely. 
The following is a plot of ratings for the same period but for the Sagometer device. Due to the weather model reversion previously mentioned the hole in the January data is also present here. The large gap present in the February data is due to a system failure, the camera was not reporting any data from the end of January to mid February.

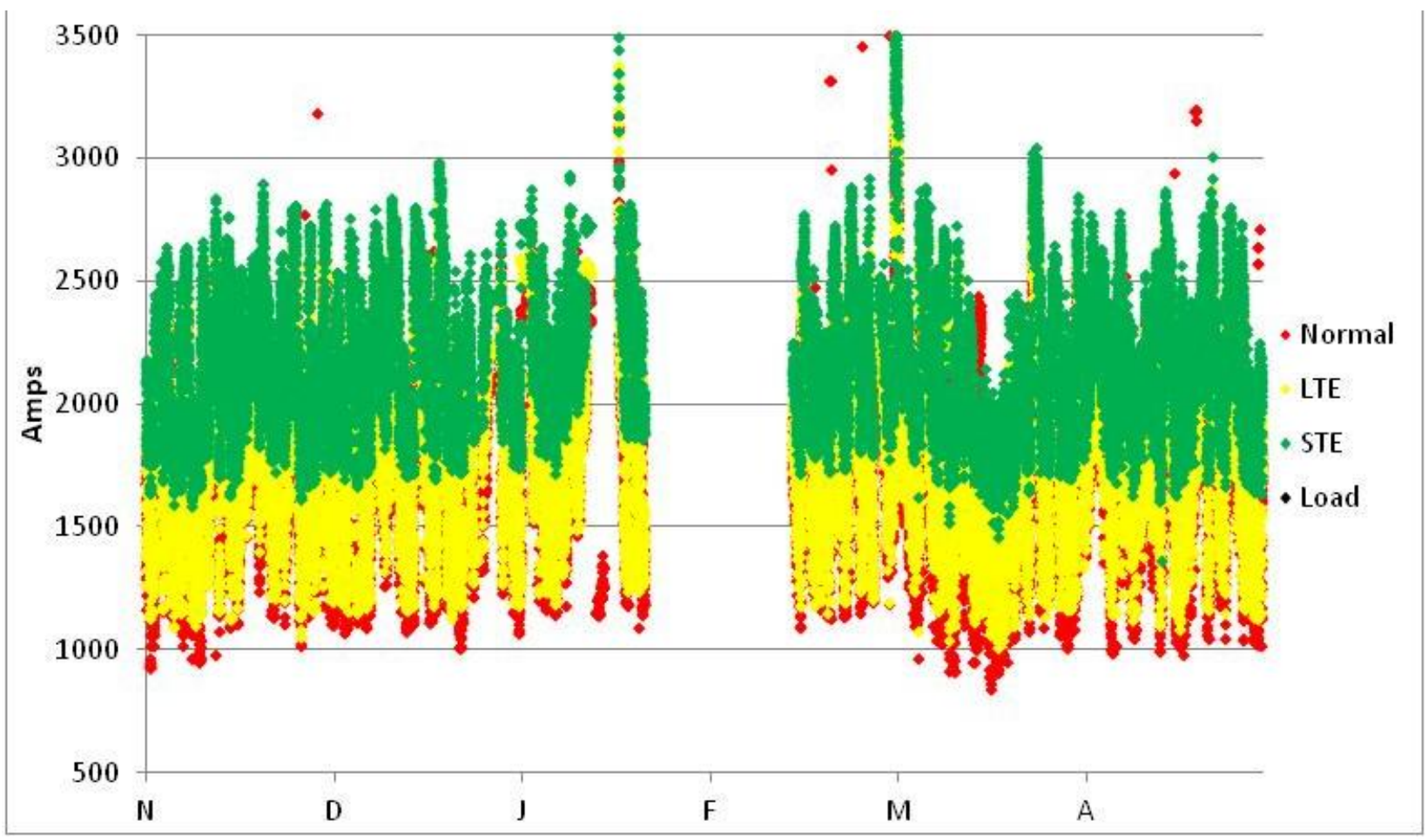

Figure F-12

Overhead line ratings based on Sagometer at Site 3 - Winter 2011-12

The responsibility to keep a dynamic rating system up and running is considerable. It is recommended that a minimum of weekly checks be done on the systems. These checks will take about 1 hour. In the beginning of the project these check were less frequent and often resulted in larger gaps in the data. 
The following is a plot of ratings for the same period but for the EPRI Sensor element. Again we see the EPRI Sensors following the weather model due to the low loads on the line.

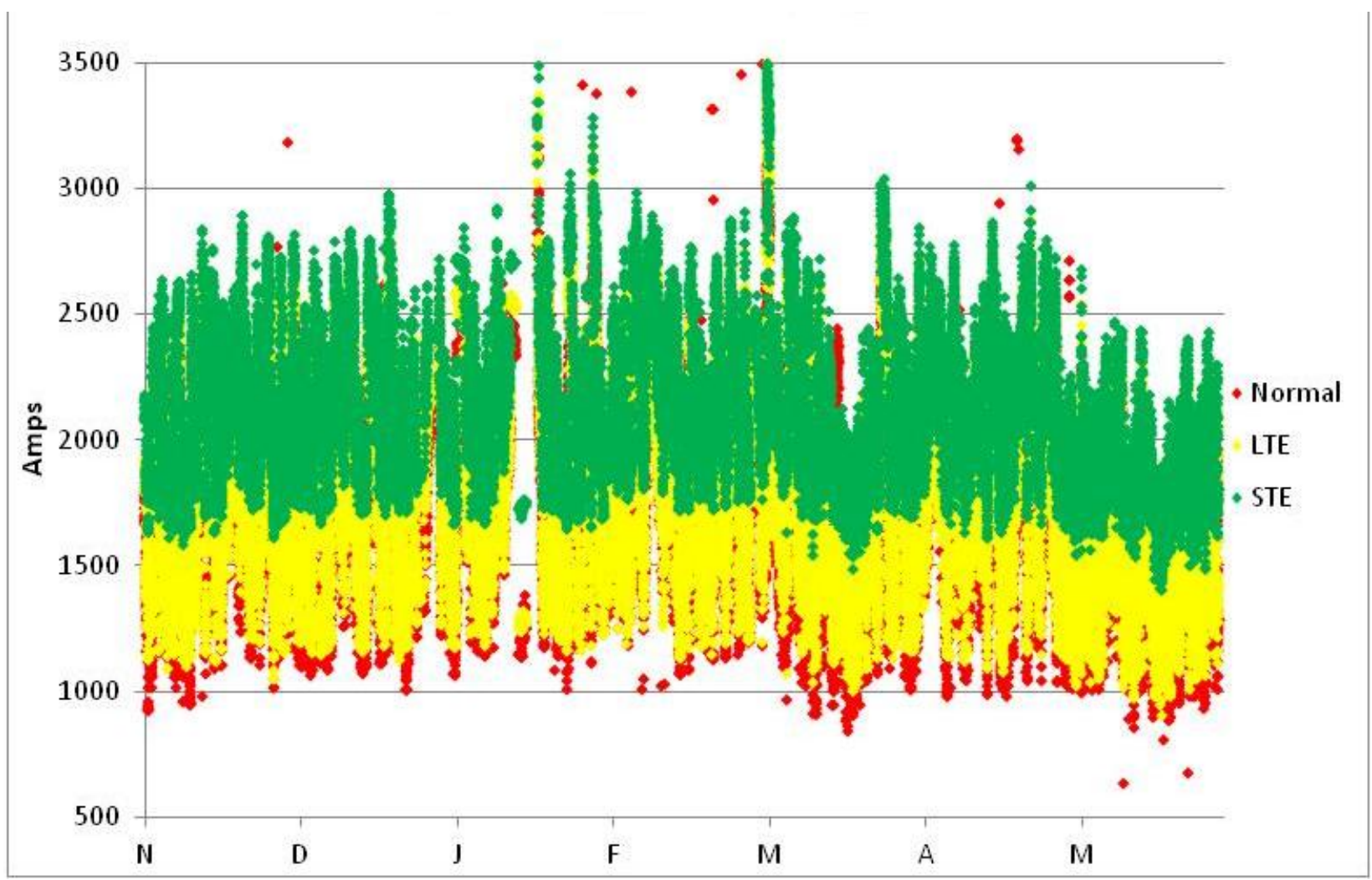

Figure F-13

Overhead line ratings based on EPRI Sensors at Site 3 - Winter 2011-12 
The following is a plot of ratings for the same data range as above but for the NOAA off site weather service data. The data appears more "spotty" due to the update interval of the NOAA data stream. Data points are updated hourly so there are 6 overlapping data points with identical data.

The holes in the data were both caused by server downtime. The server downtime was shorter than the data gap in February so there must have been a secondary issue that caused the delay in data retrieval. Unlike the field sensors there is no buffer for weather service data. If it is not immediately retrieved it is lost. Therefore any downtime of the server, the hard-line internet connection, or the NOAA server will cause holes in this data.

The data is slightly more conservative for Normal rating minimums in comparison to the onsite weather station. This does not however indicate the ratings are always more conservative, in fact the offsite weather service data on average yields equal or slightly higher ratings.

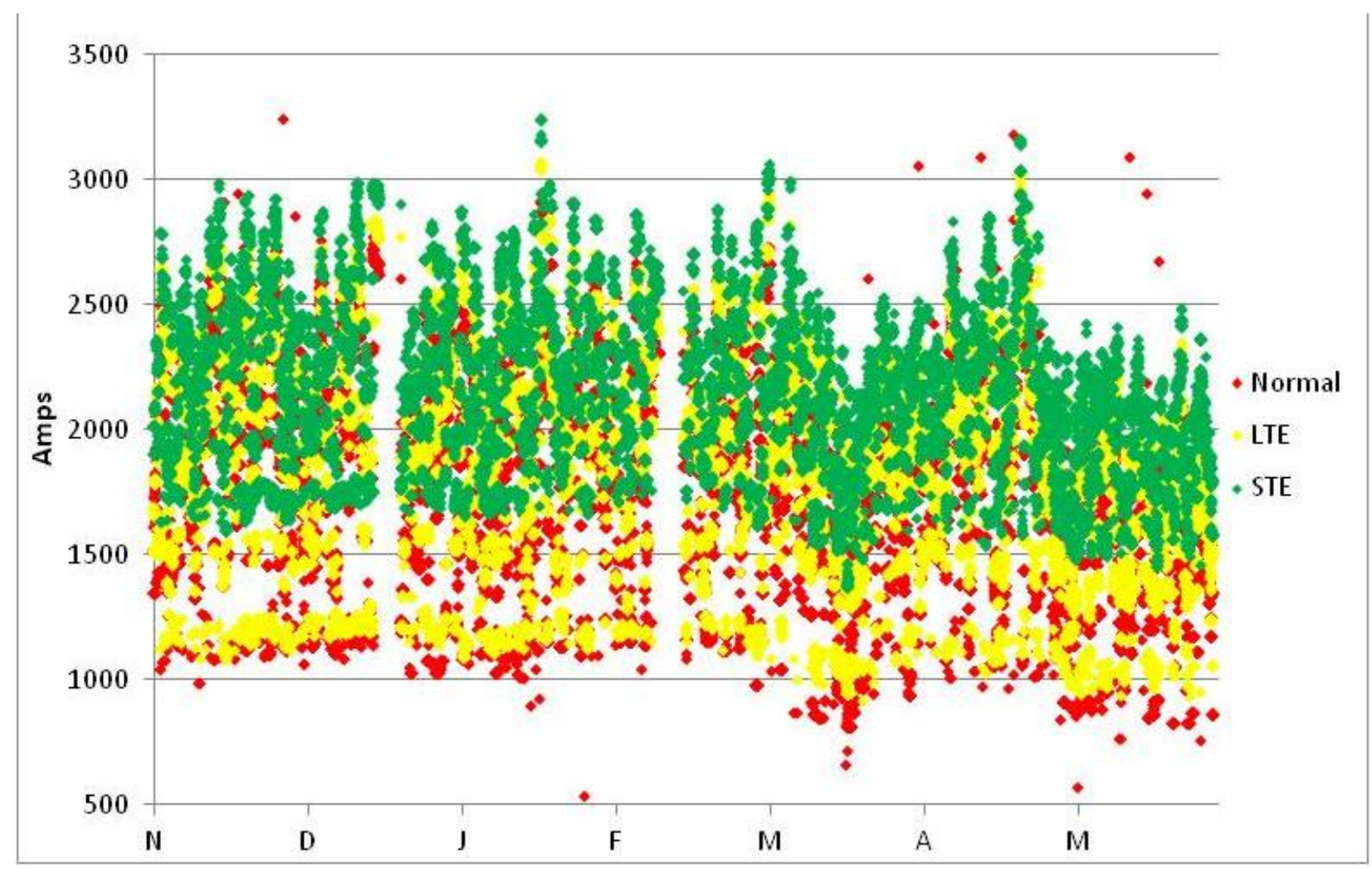

Figure F-14

Overhead line ratings based on Offsite Weather Service at Site 3 - Winter 2011-12

\section{Ratings at Site 3: Summer}

Similar data are shown in this section for the summer period.

Figure F-15 depicts ratings from the onsite weather station. The hole in the data at the end of July is due to a DTCR error and will be present in all plots spanning that date. 


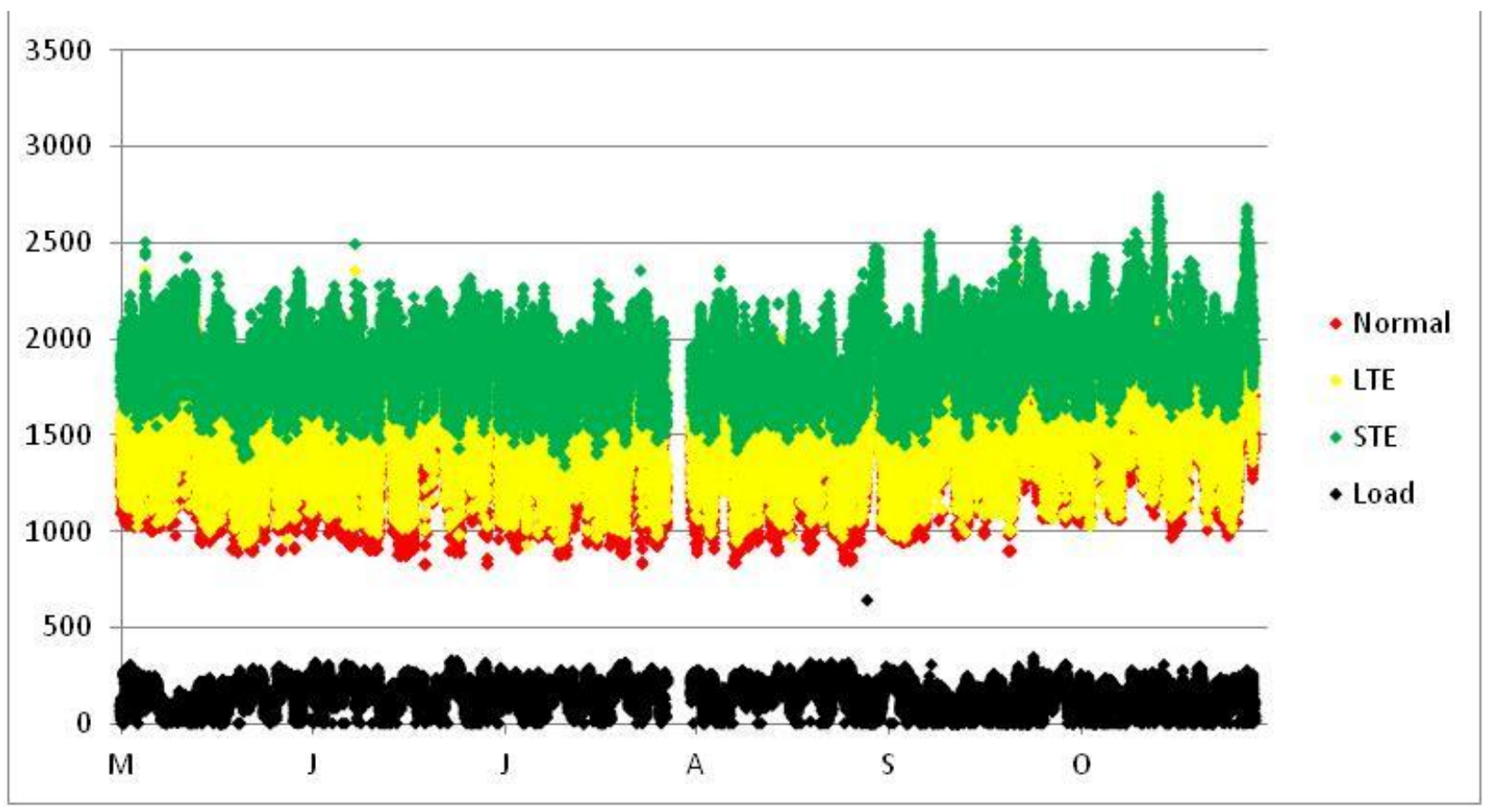

Figure F-15

Overhead line ratings based on the Onsite Weather Station at Site 3 - Summer 2012

DTCR will be undergoing significant changes in the next few years. It will be called Thermal Rating Workstation (TRW). EPRI plans to improve reliability of the software by the addition of backup data channels. This will allow other sources of data to be substituted should the primary source become unavailable.

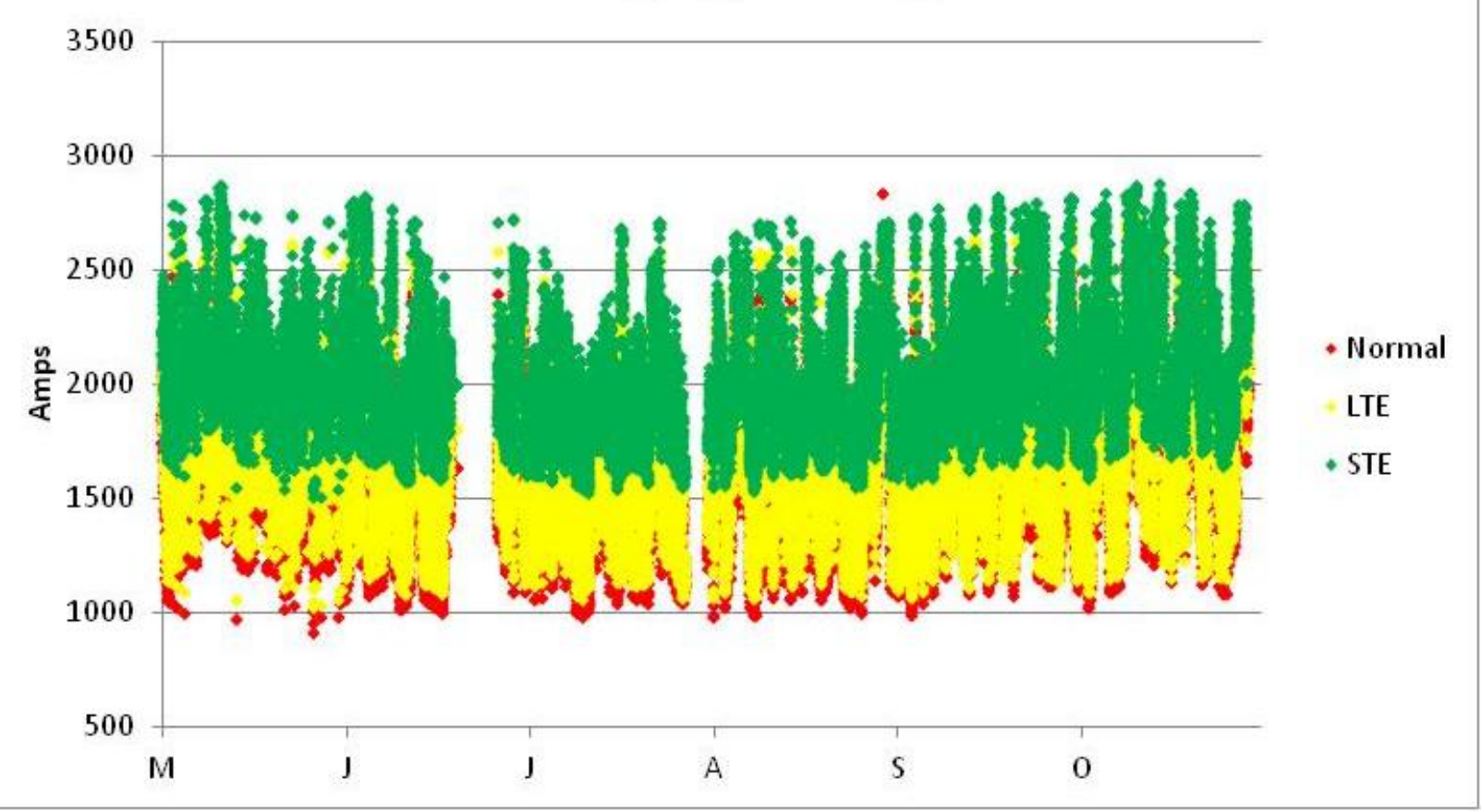

Figure F-16

Overhead line ratings based on ThermalRate at Site 3 - Summer 2012 
Time series plots emphasize the dynamic nature of overhead ratings. As discussed in other chapters ambient adjusted ratings would not have as much variation in ratings, however, ambient adjusted ratings often result in ratings that increase when thermal capacity as determined by dynamic rating is decreasing. Later chapters will show cumulative distribution plots of these same data sets. Cumulative distribution plots (shown in the next chapter) in many ways are more useful than time series plots because they help define risk levels. 


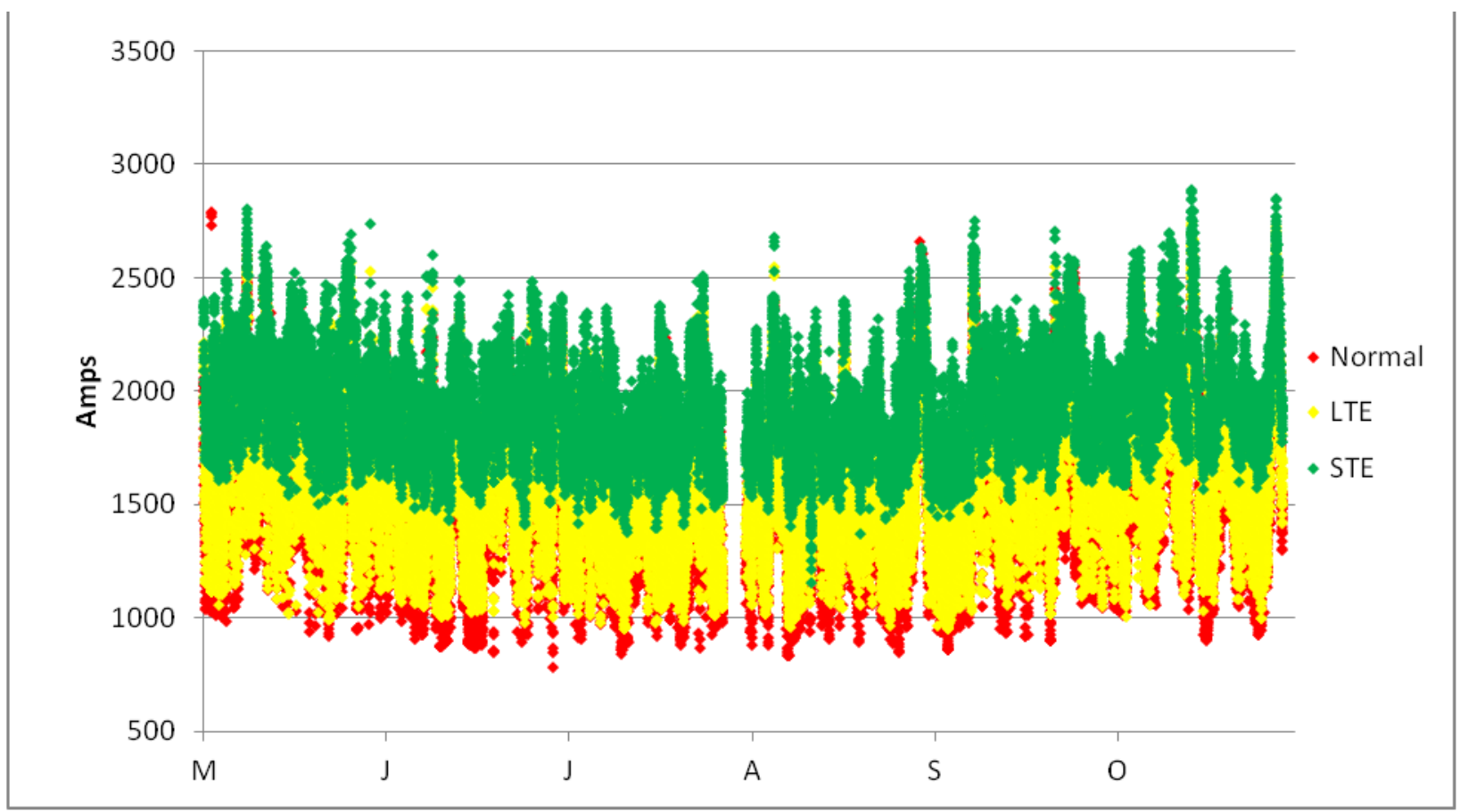

Figure F-17

Overhead line ratings based on the Sagometer at Site 3 - Summer 2012

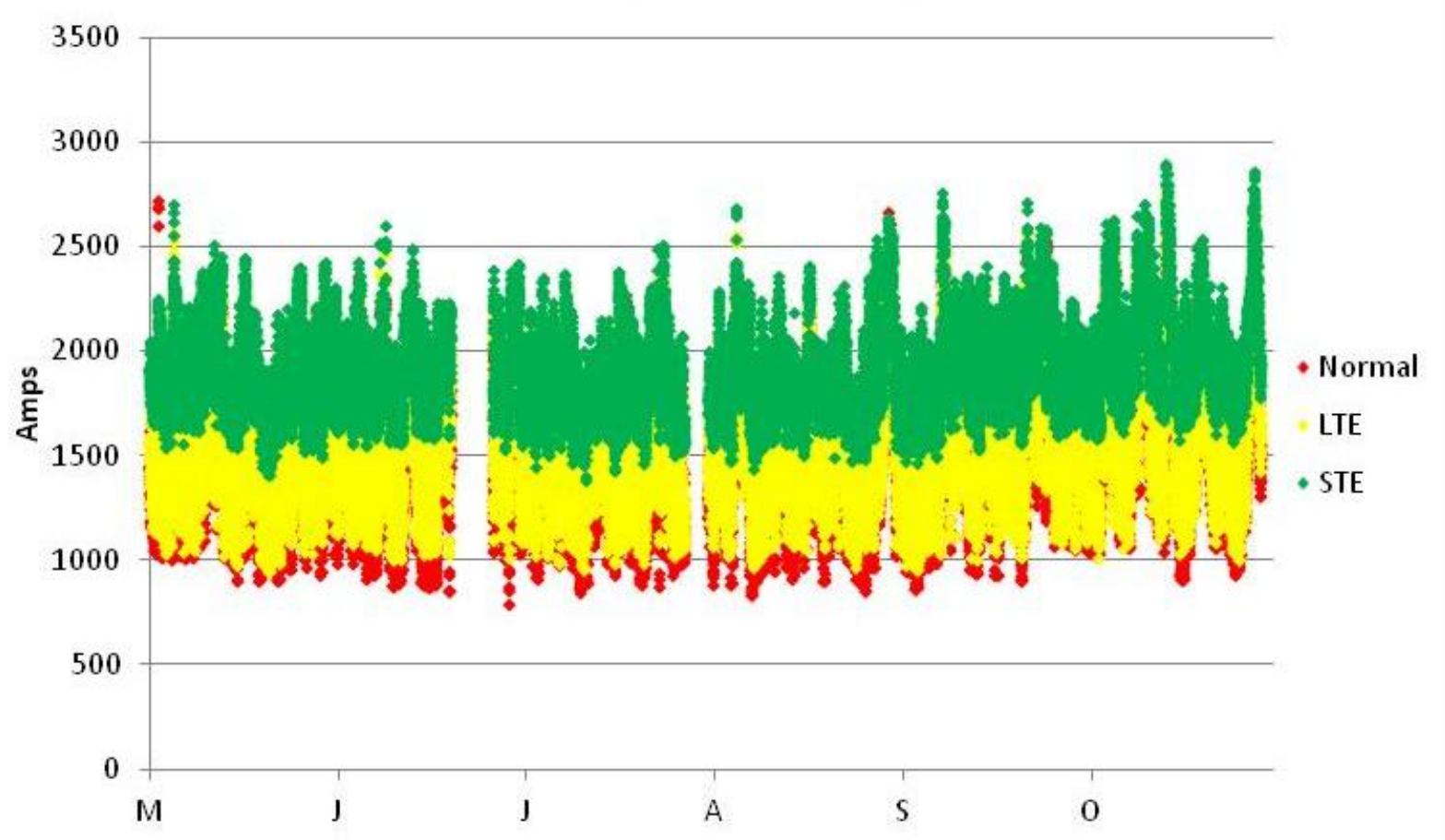

Figure F-18

Overhead line ratings based on EPRI Sensors at Site 3 - Summer 2012 
The gap in the data in Figure F-19 at the end of August was due to a software issue that interrupted the archiving of NOAA weather data. This line uses a different NOAA data file than the Site 2 and Site 1 Sites so this hole is unique to Site 3.

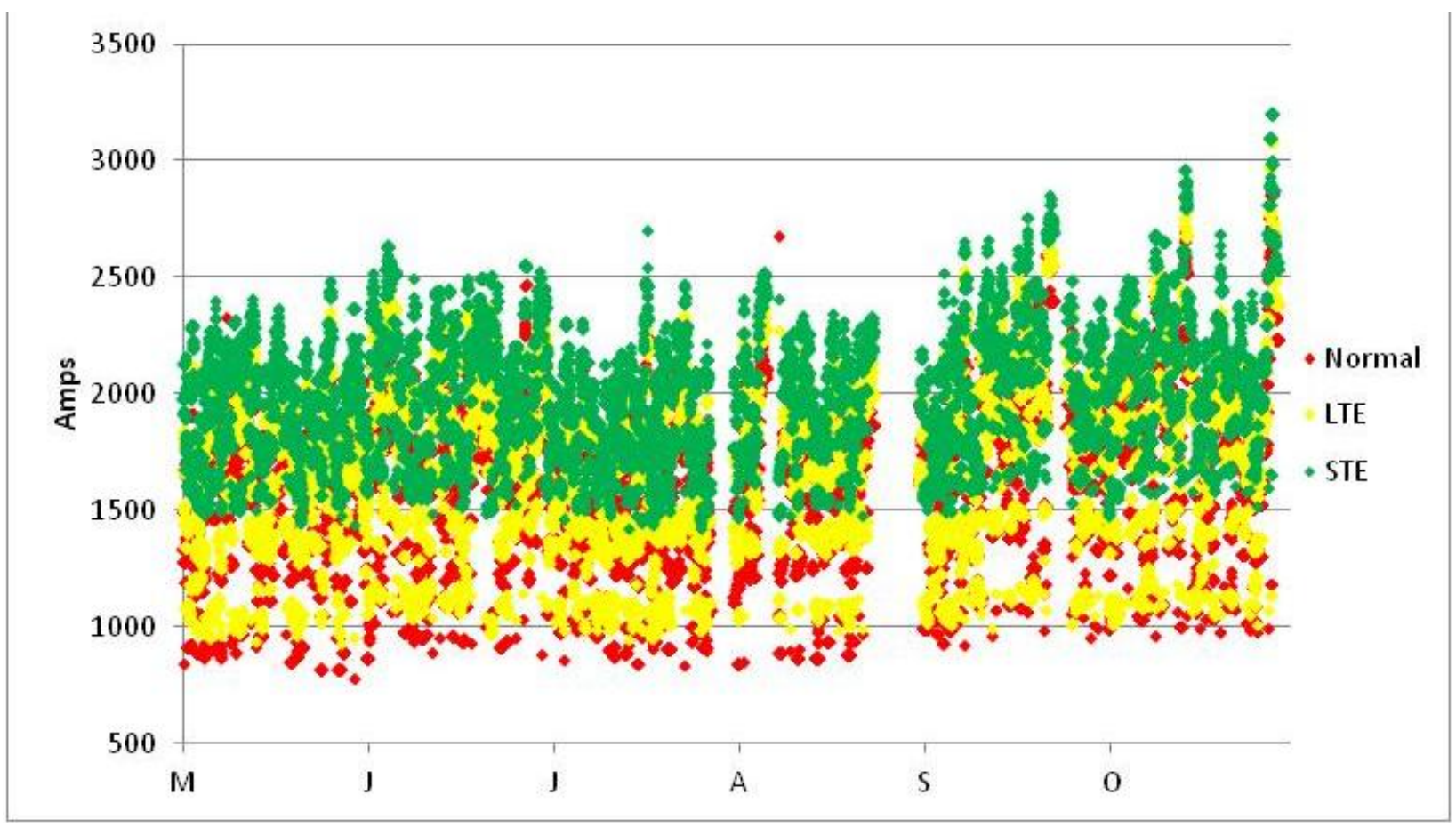

Figure F-19

Overhead line ratings based on the Offsite Weather Service at Site 3 - Summer 2012 


\section{Ratings at Site 2: Winter}

The figure below contains the rating and load data for Site 2 for the winter spanning 2010 and 2011 from the weather station data. The flat spot in the data on December $21^{\text {st }}$ is due to a failure of the anemometer to report data. Other weather instruments remained functional at this time. The Sagometer camera also failed during this time.

One possible reason for this would be a heavy snowfall or icing event. This hypothesis is also supported by the fact this error is seen in the Site 1data for this period as well, due to their proximity the weather patterns are likely to be shared.

Outside of this brief period the weather station functioned with a high degree of reliability.

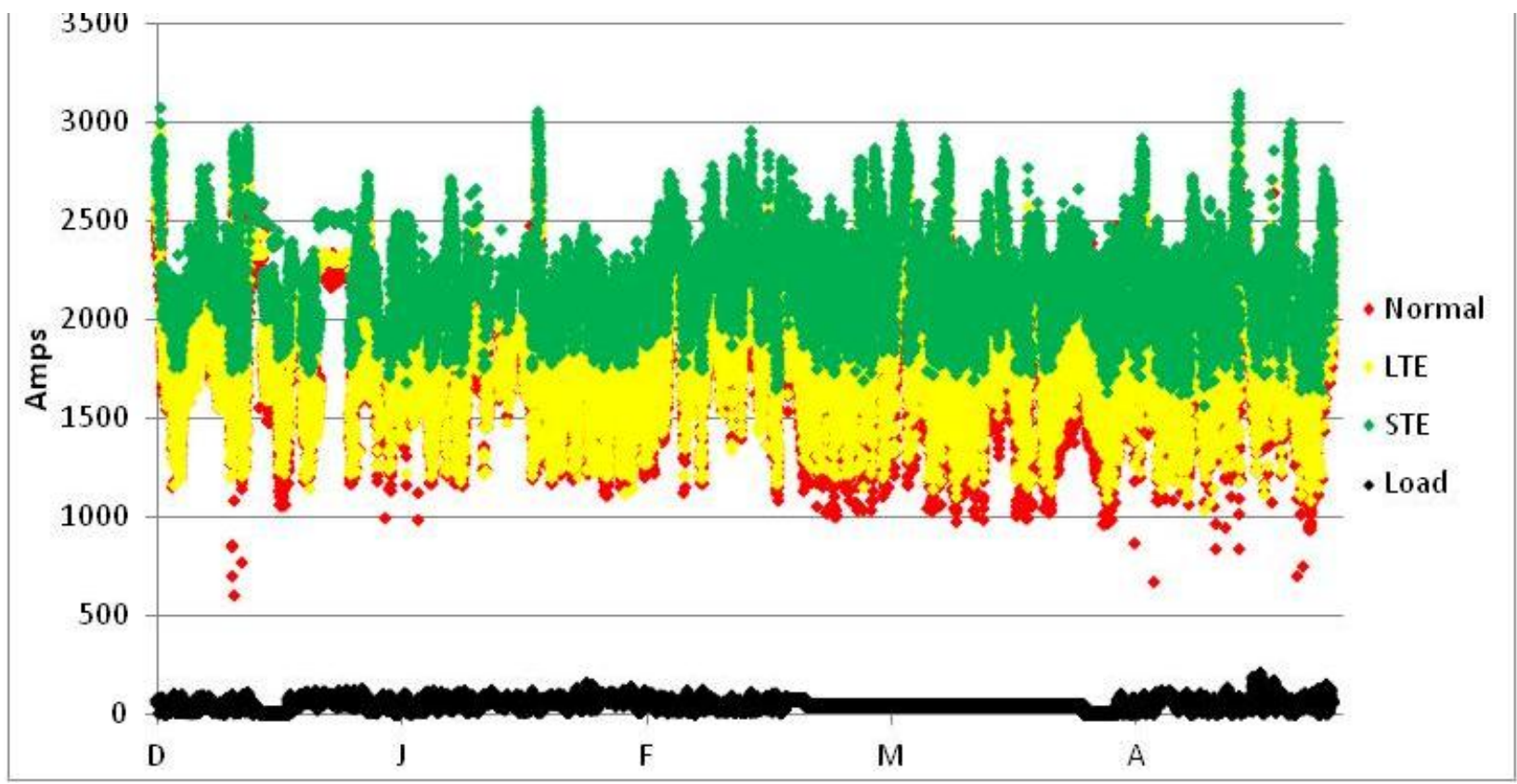

Figure F-20

Overhead line ratings based on the Onsite Weather Station at Site 2 -

Winter 2010-2011

The reader may notice that onsite weather station data tends to be the most complete. This is analyzed in more detail in the reliability chapter. 
The figure below contains the data from the ThermalRate device during the same time period. The spotty nature of the data near the end of the winter was due to attempts to integrate the SCADA data.

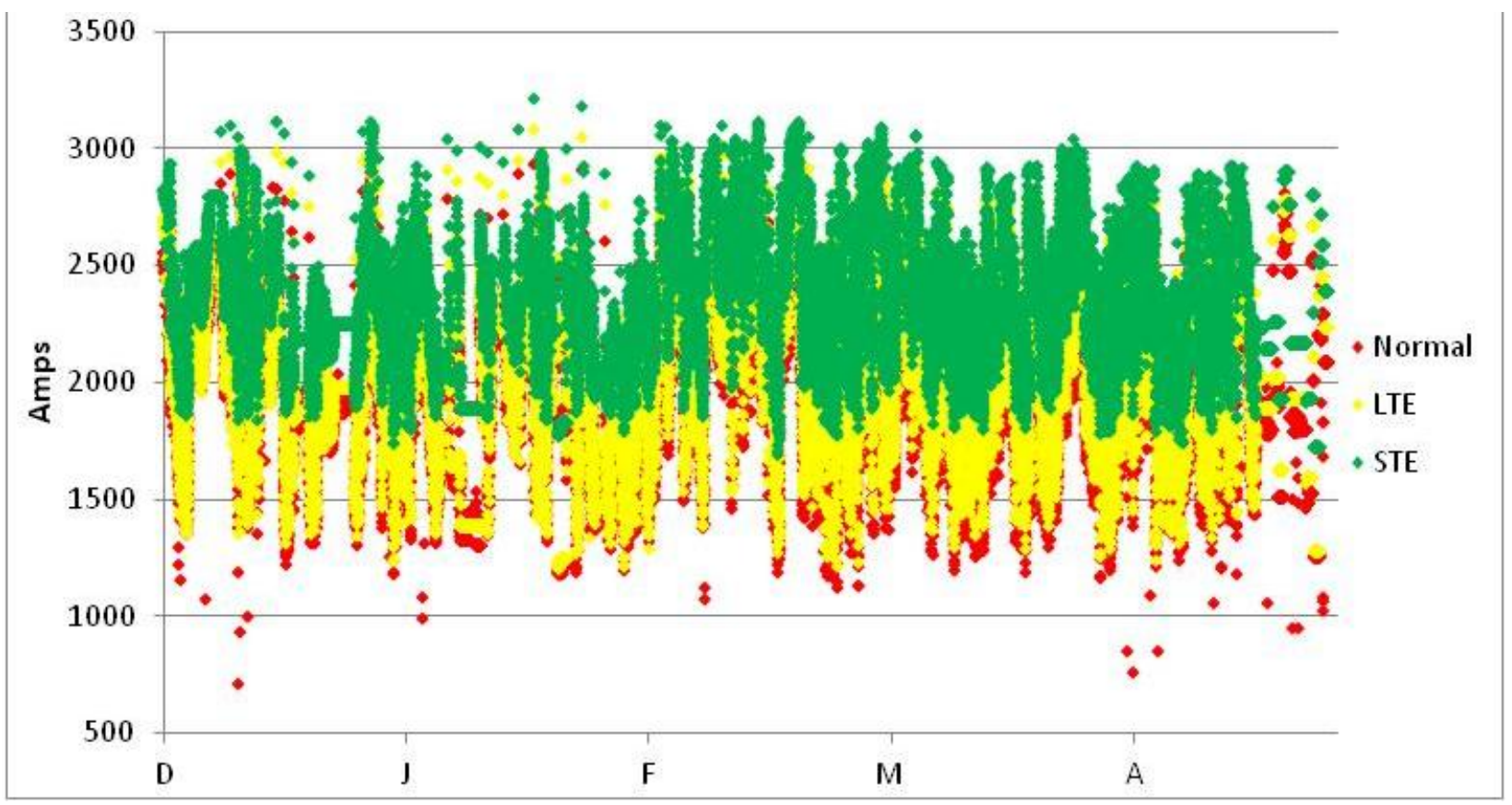

Figure F-21

Overhead line ratings based on ThermalRate at Site 2 - Winter 2010-2011 


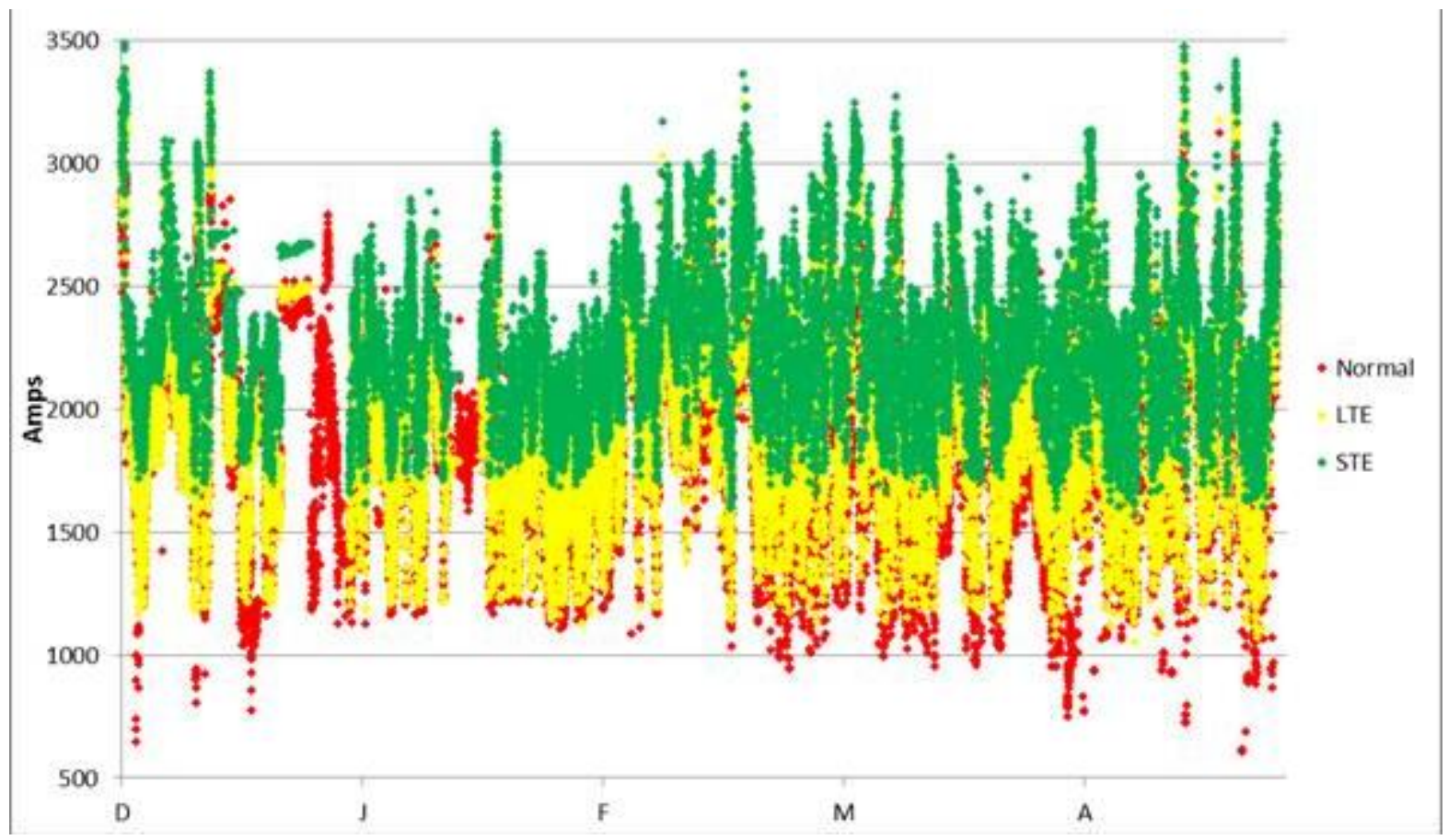

Figure F-22

Overhead line ratings based on the Video Sagometer at Site 2 - Winter 2010-2011

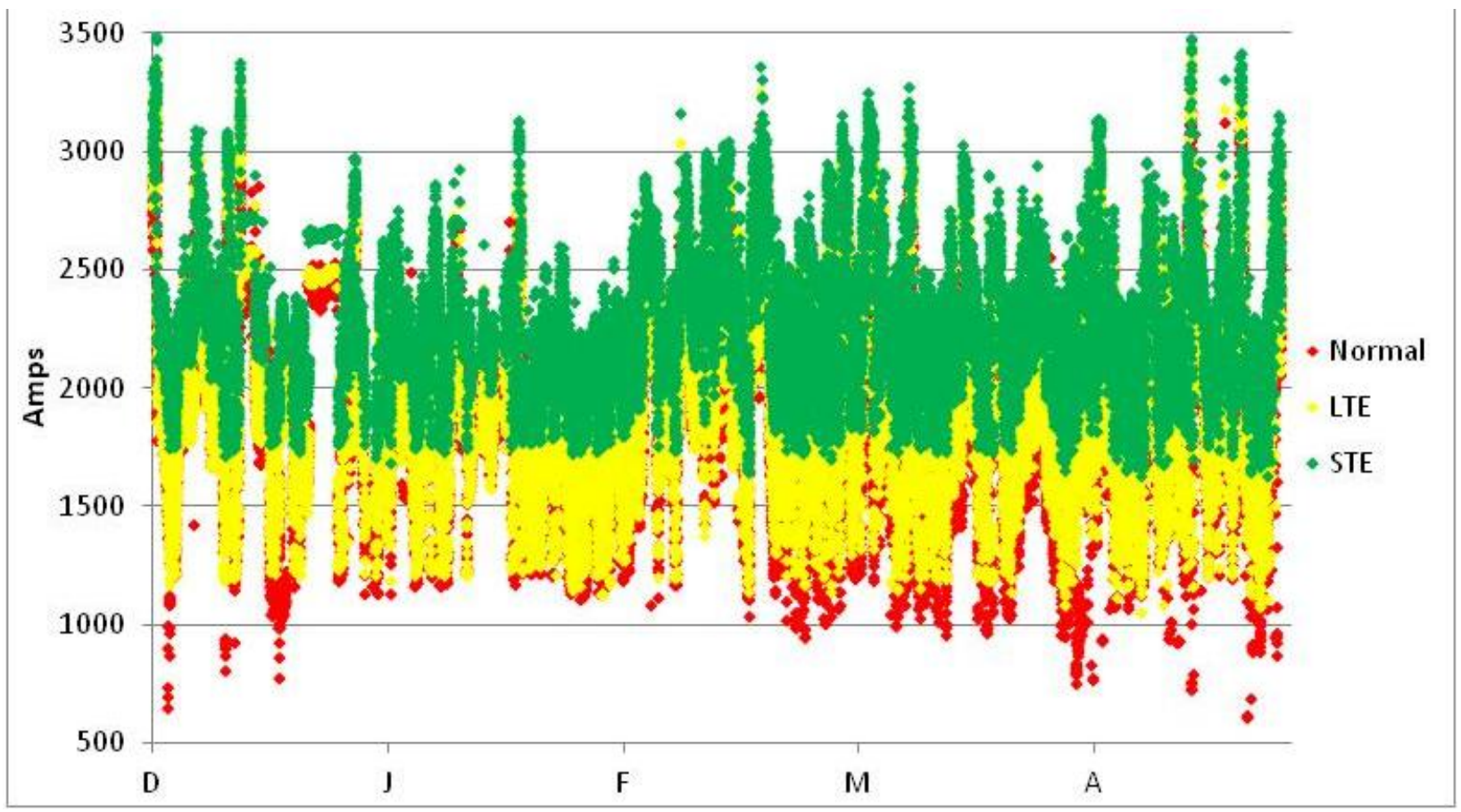

Figure F-23

Overhead line ratings based on the EPRI Sensors at Site 2 - Winter 2010-2011 


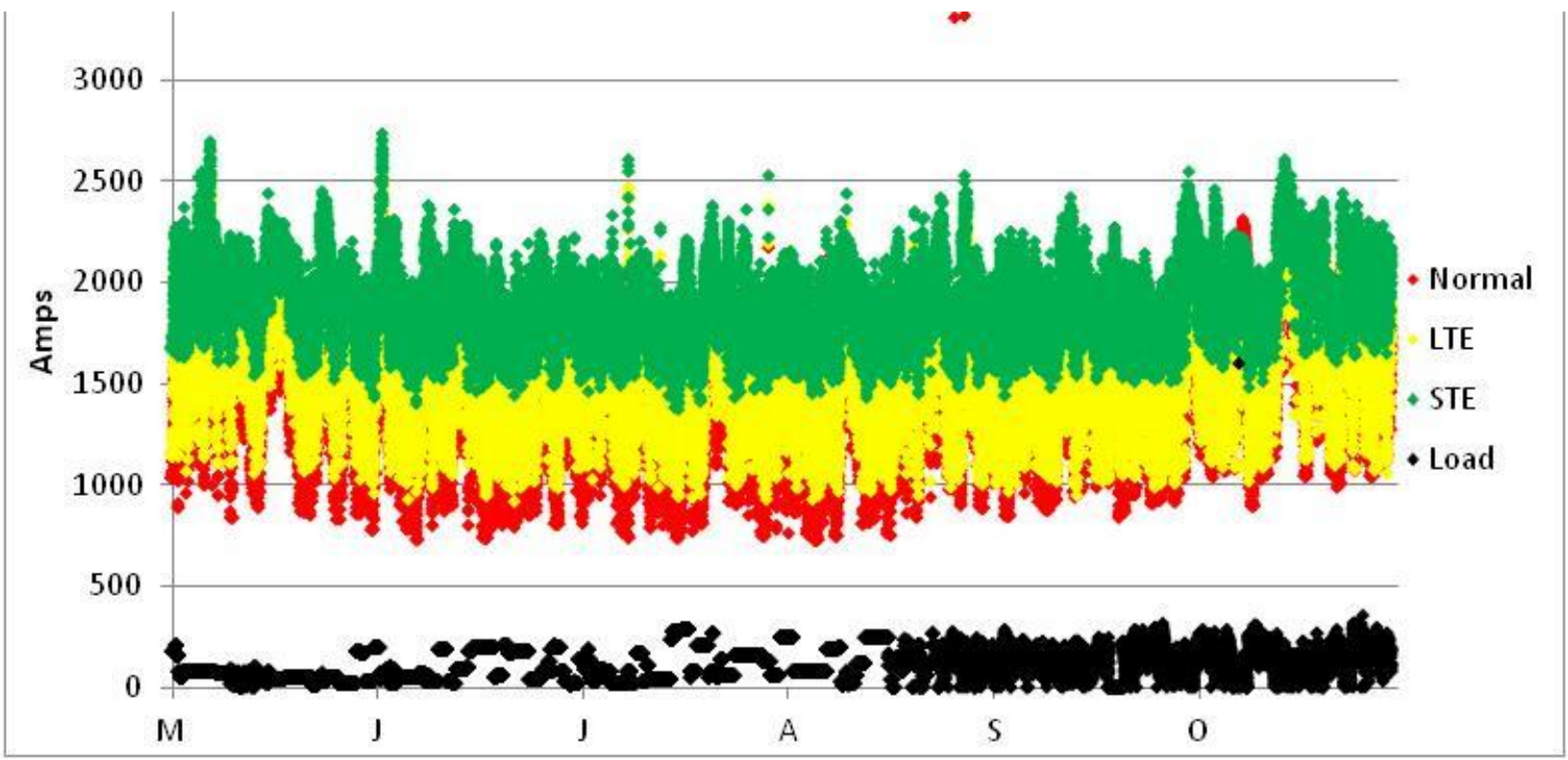

Figure F-24

Overhead line ratings based on the Onsite Weather Station at Site 2 - Summer 2011

Load data is available from two locations, the SCADA system and the EPRI Sensors. Some startup problems made it difficult to get load data from the SCADA system. This has been discussed in more detail in other chapters. Spread spectrum radio link connects the utility SCADA data to the ThermalRate device. This data is then transmitted to the EPRI Sensor data logger. From there the data is downloaded to the server where it can be processed. It is necessary not to overload the ThermalRate device with SCADA data. Data transfer should be less frequent than every 10 seconds. It is also important to note that the load data is needed for all elements of the DTCR calculations.

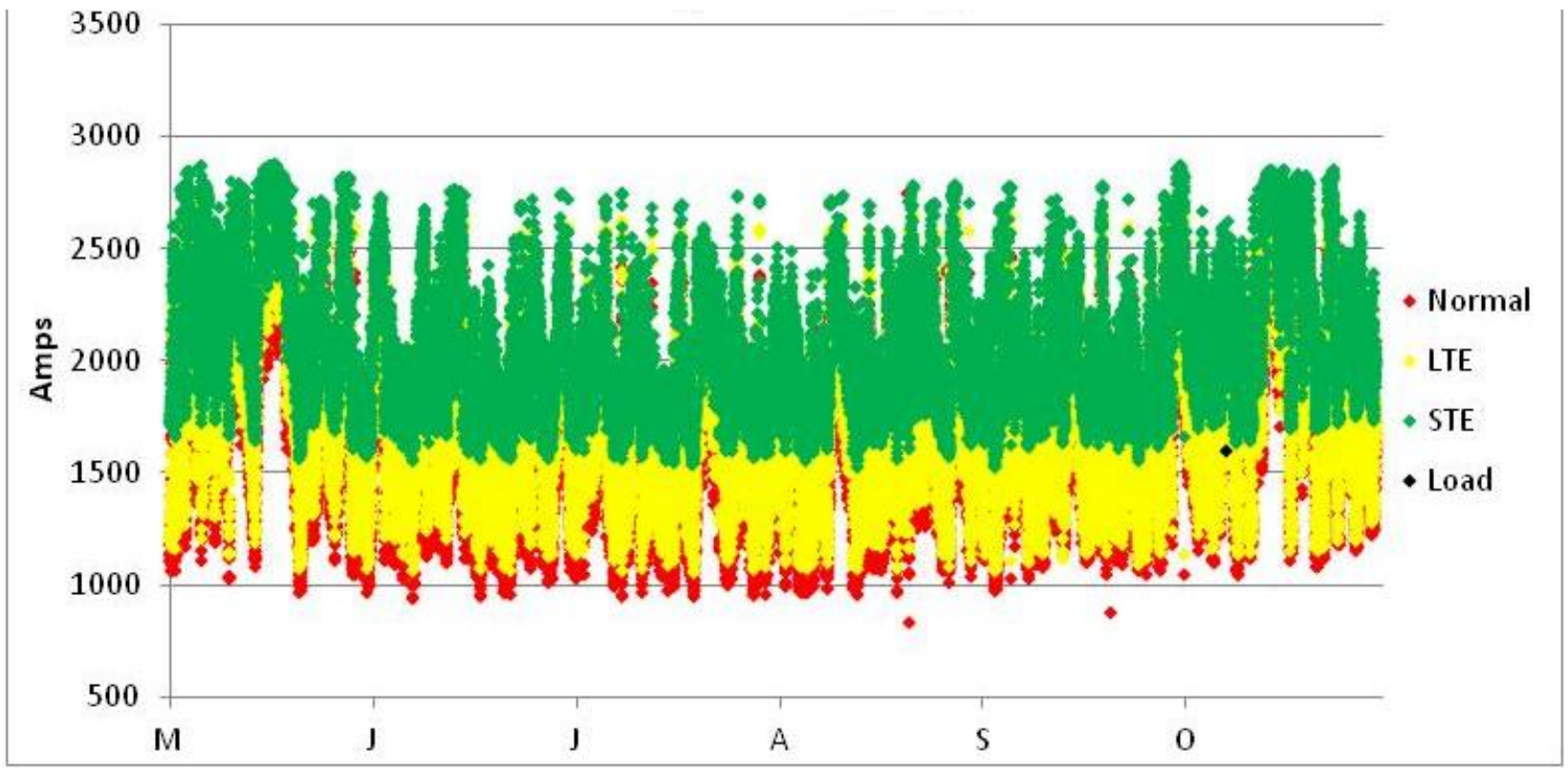

Figure F-25

Overhead line ratings based on ThermalRate at Site 2 - Summer 2011 


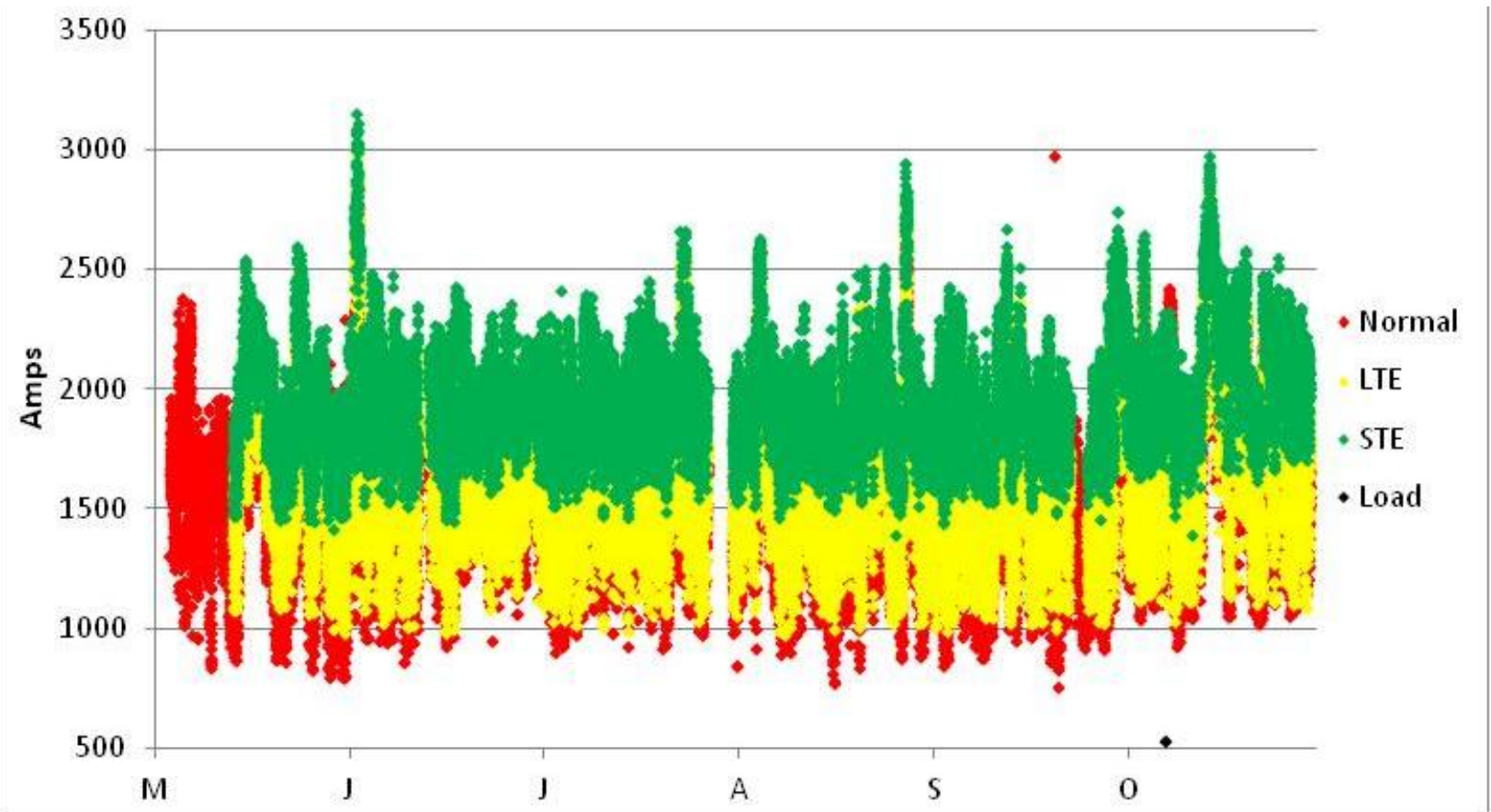

Figure F-26

Overhead line ratings based on the Sagometer at Site 2 - Summer 2011

Many plots in this section are here so the reader can view the changes from season to season. Few additional comments are required.

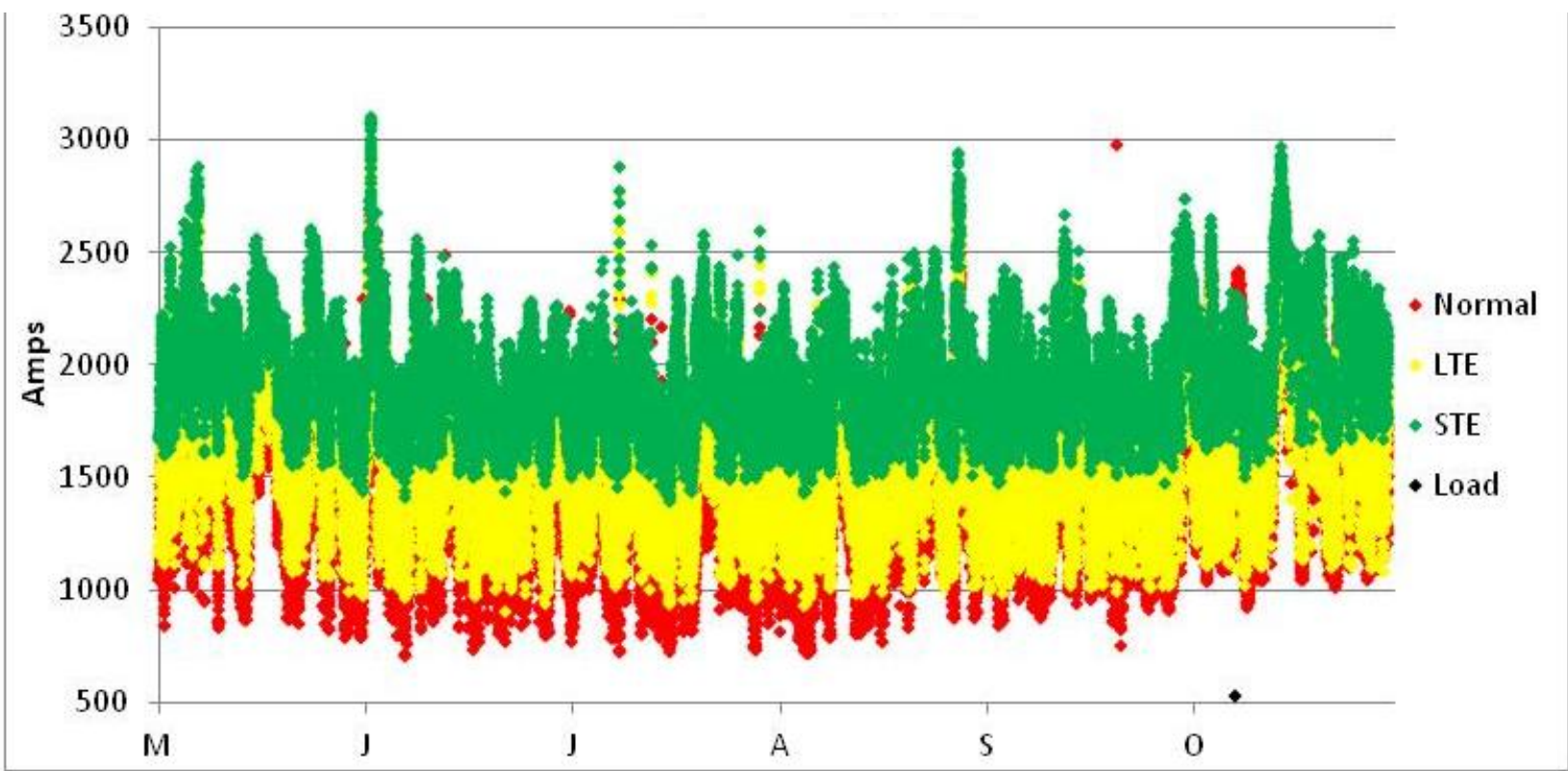

Figure F-27

Overhead line ratings based on the EPRI Sensors at Site 2 - Summer 2011 


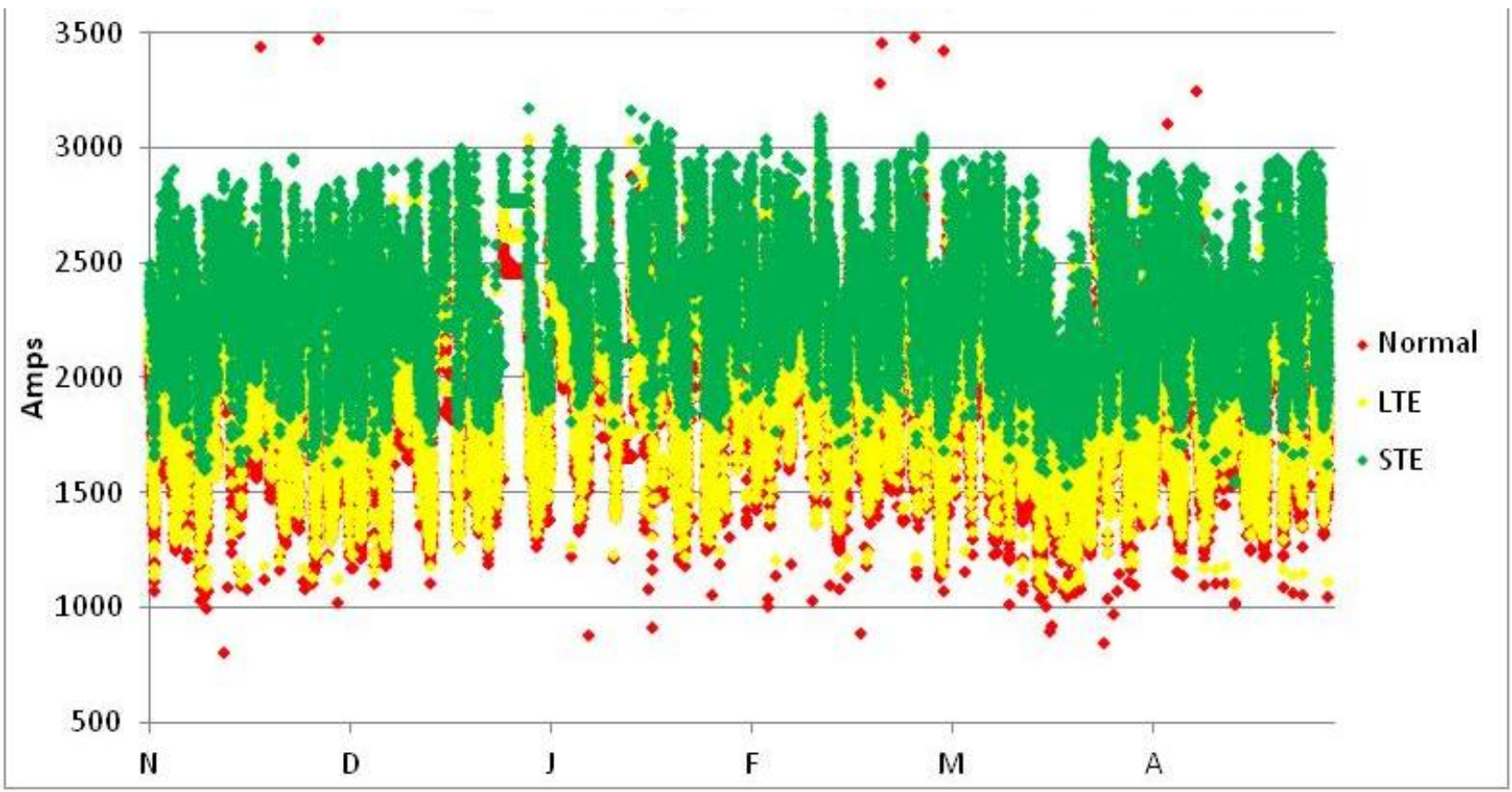

Figure F-28

Overhead line ratings based on ThermalRate at Site 2 - Winter 2011-2012

In Figure F-29, the hole in the data for January was due to a failing of the Sagometer camera.

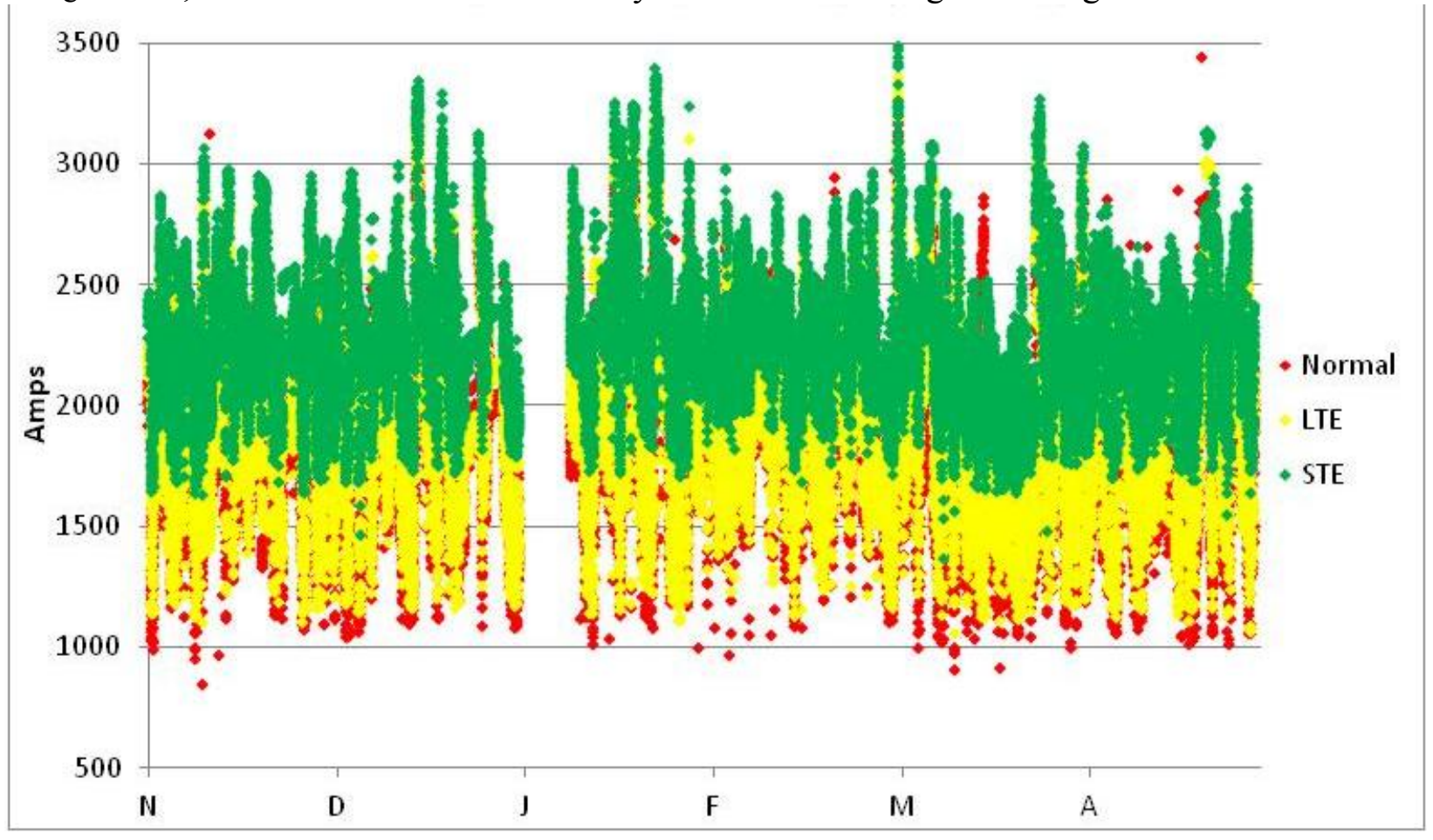

Figure F-29

Overhead line ratings based on the Sagometer at Site 2- Winter 2011-2012 


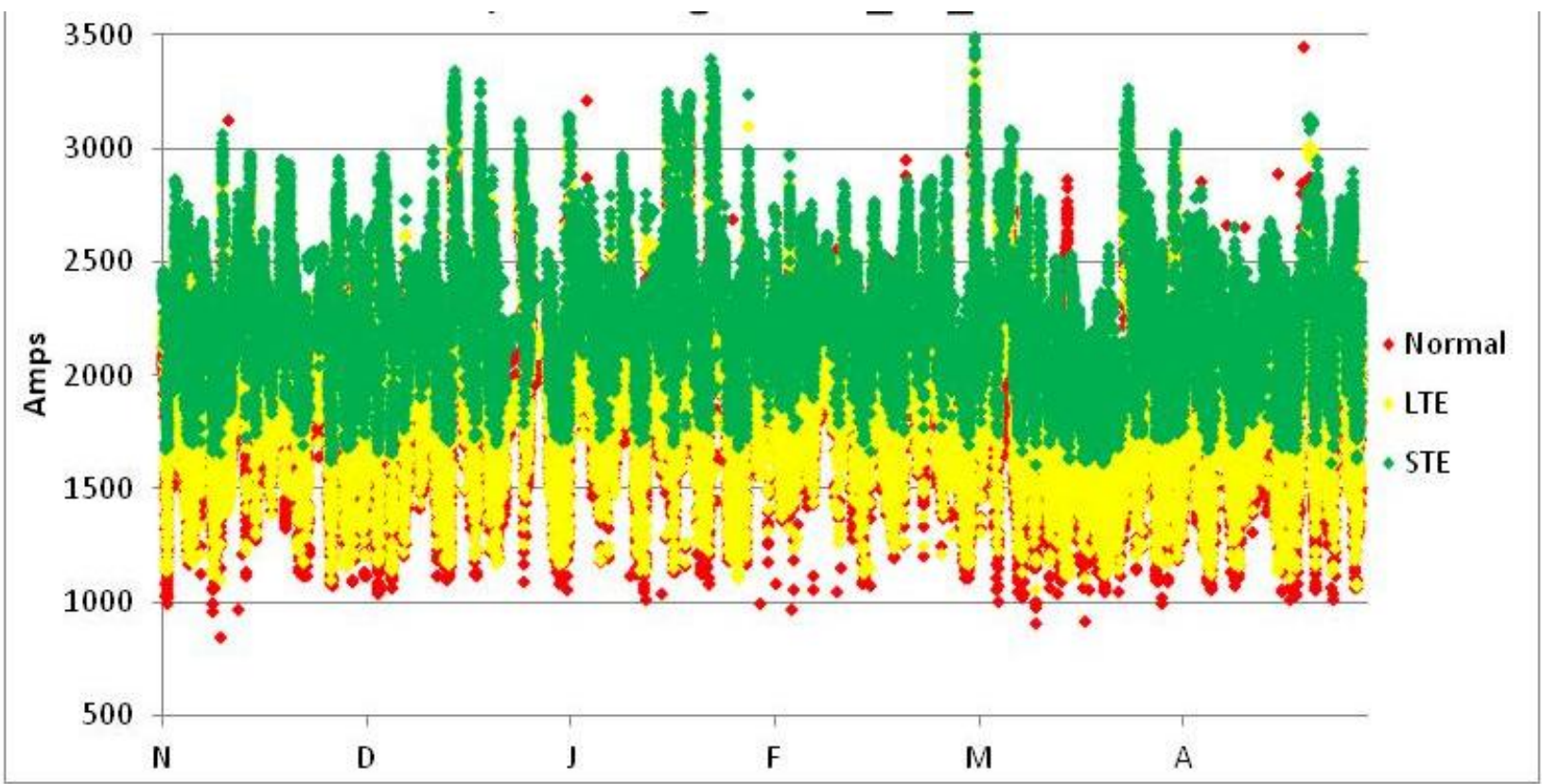

Figure F-30

Overhead line ratings based on the EPRI Sensors at Site 2 -Winter 2011-2012 
The hole in the December data below represents downtime of the DTCR server over the course of a weekend.

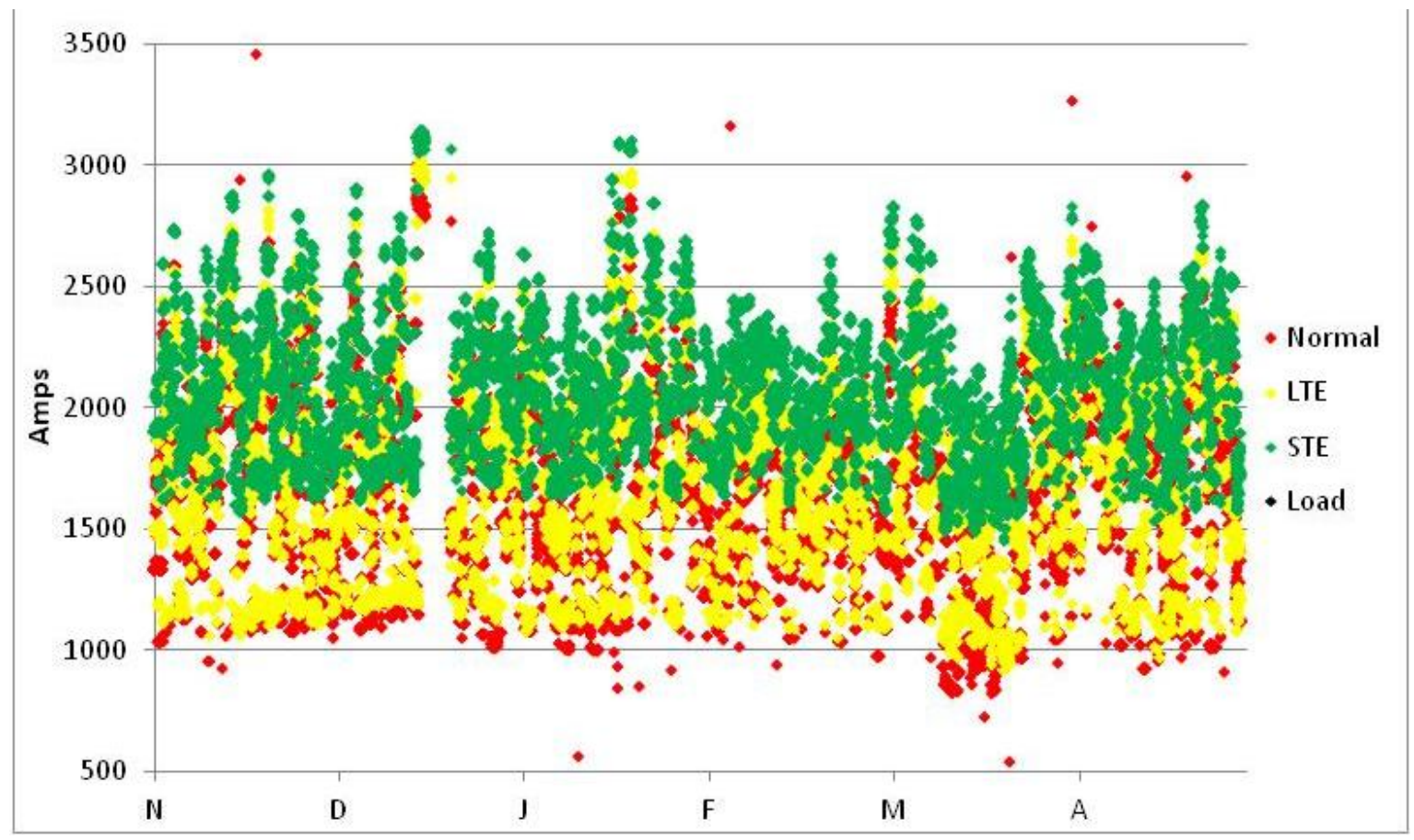

Figure F-31

Overhead line ratings based on the Off Site Weather Service at Site 2 - Winter 2011-2012

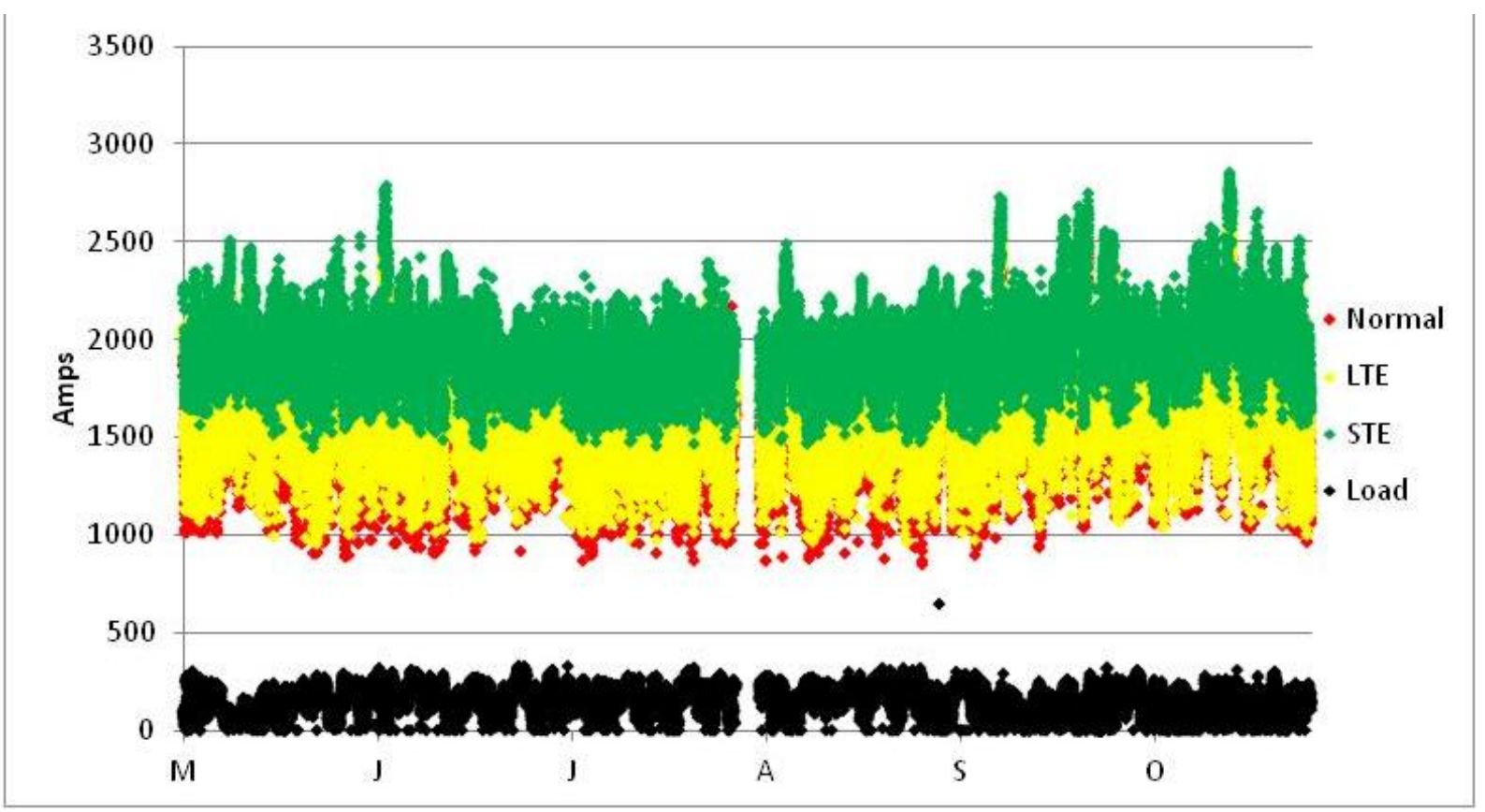

Figure F-32

Overhead line ratings based on the Offsite Weather Service at Site 2 -Summer 2012 


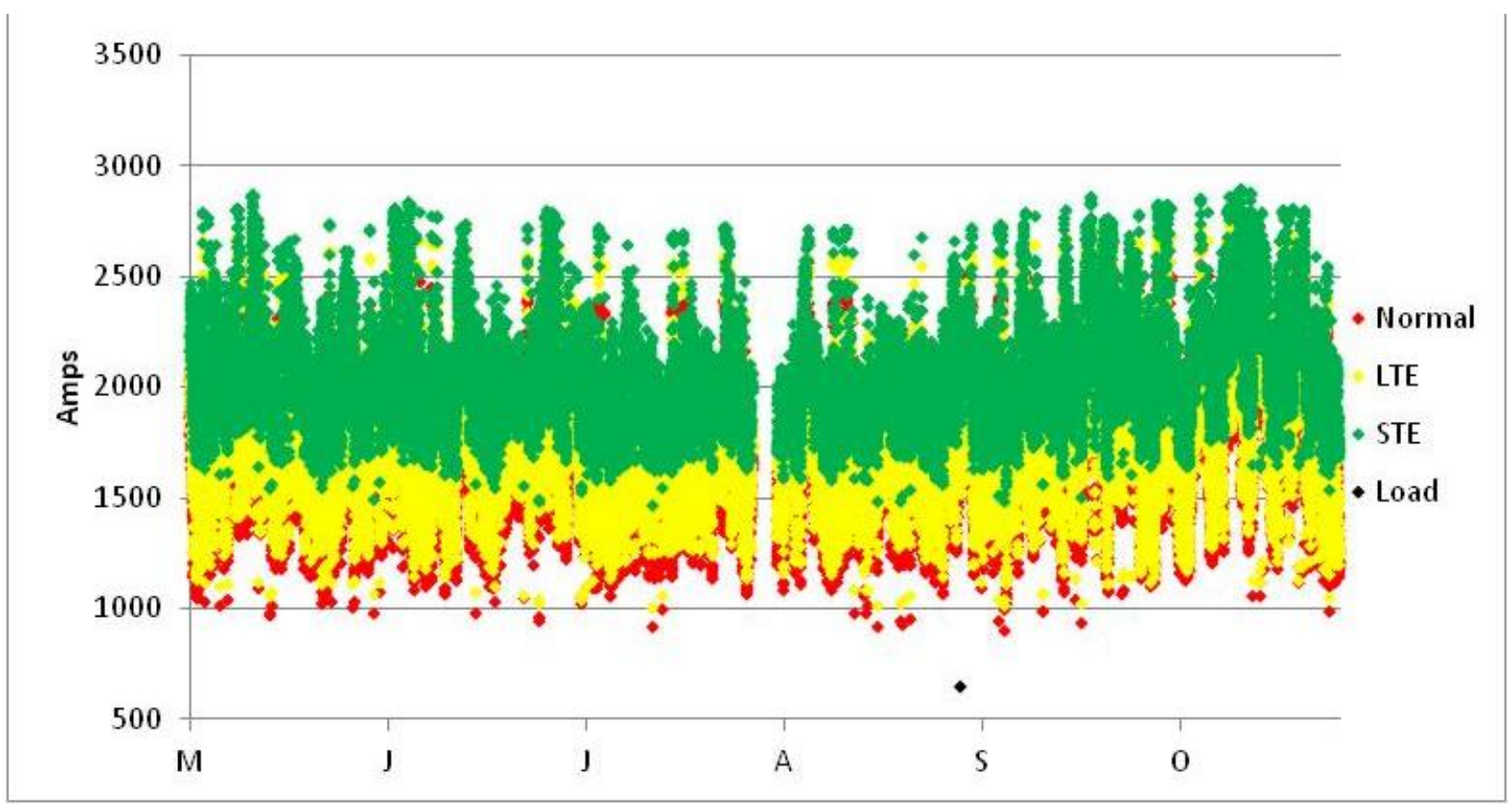

Figure F-33

Overhead line ratings based on ThermalRate at Site 2 - Summer 2012

The hole in the month of September in Figure F-34 was caused by issues with the Sag Camera.

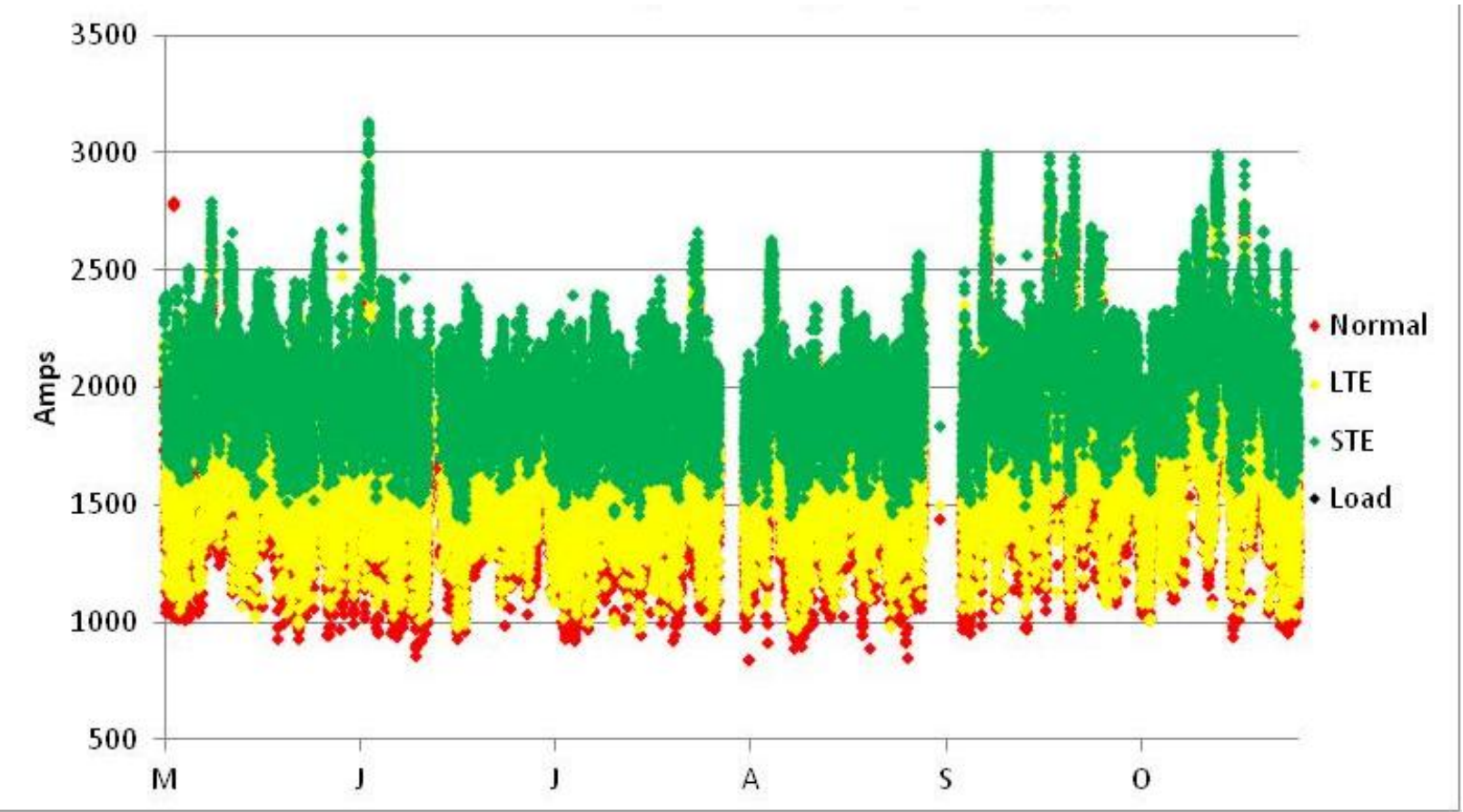

Figure F-34

Overhead line ratings based on the Sagometer at Site 2 - Summer 2012 


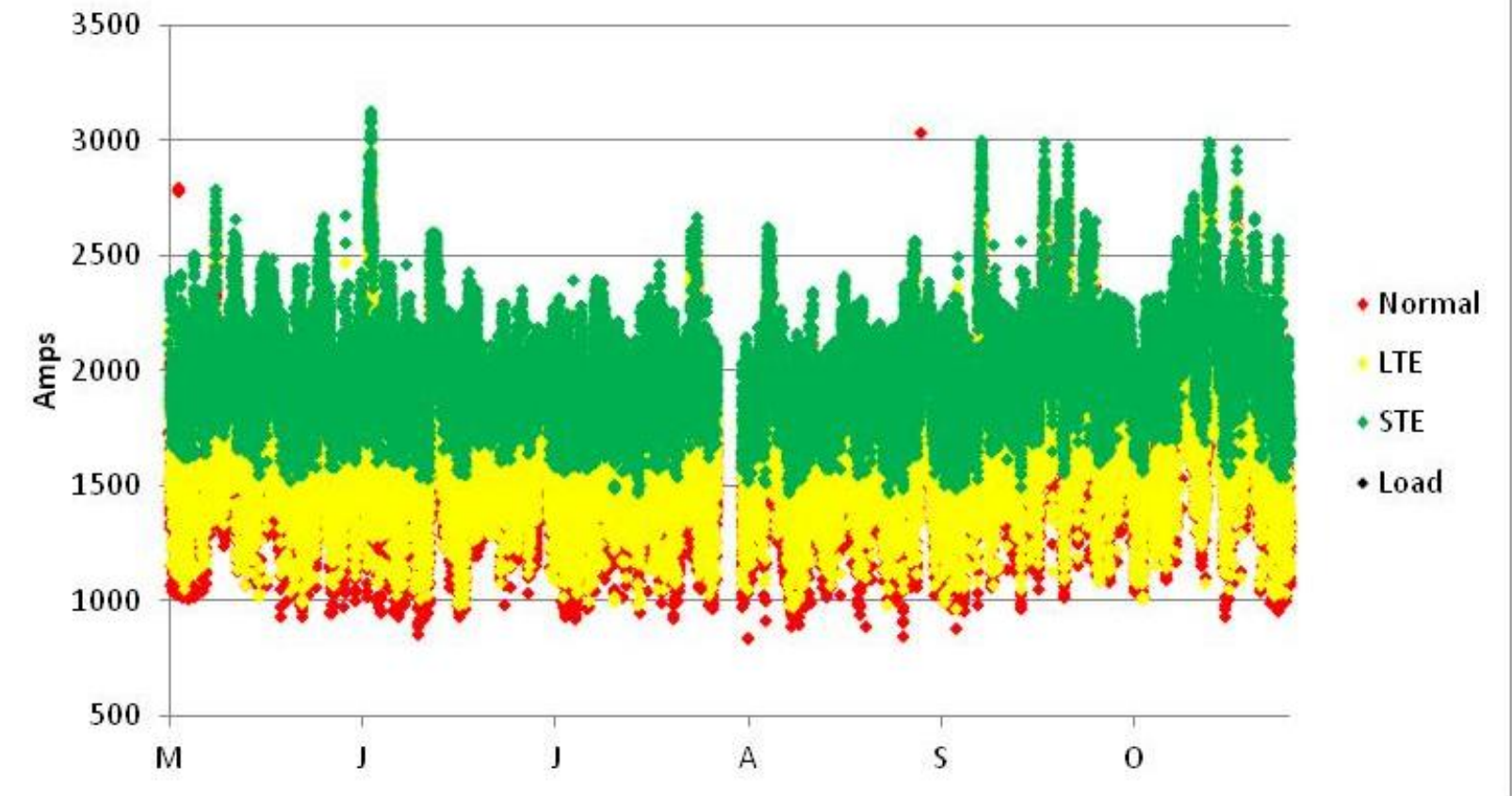

Figure F-35

Overhead line ratings based on EPRI Sensors at Site 2 - Summer 2012

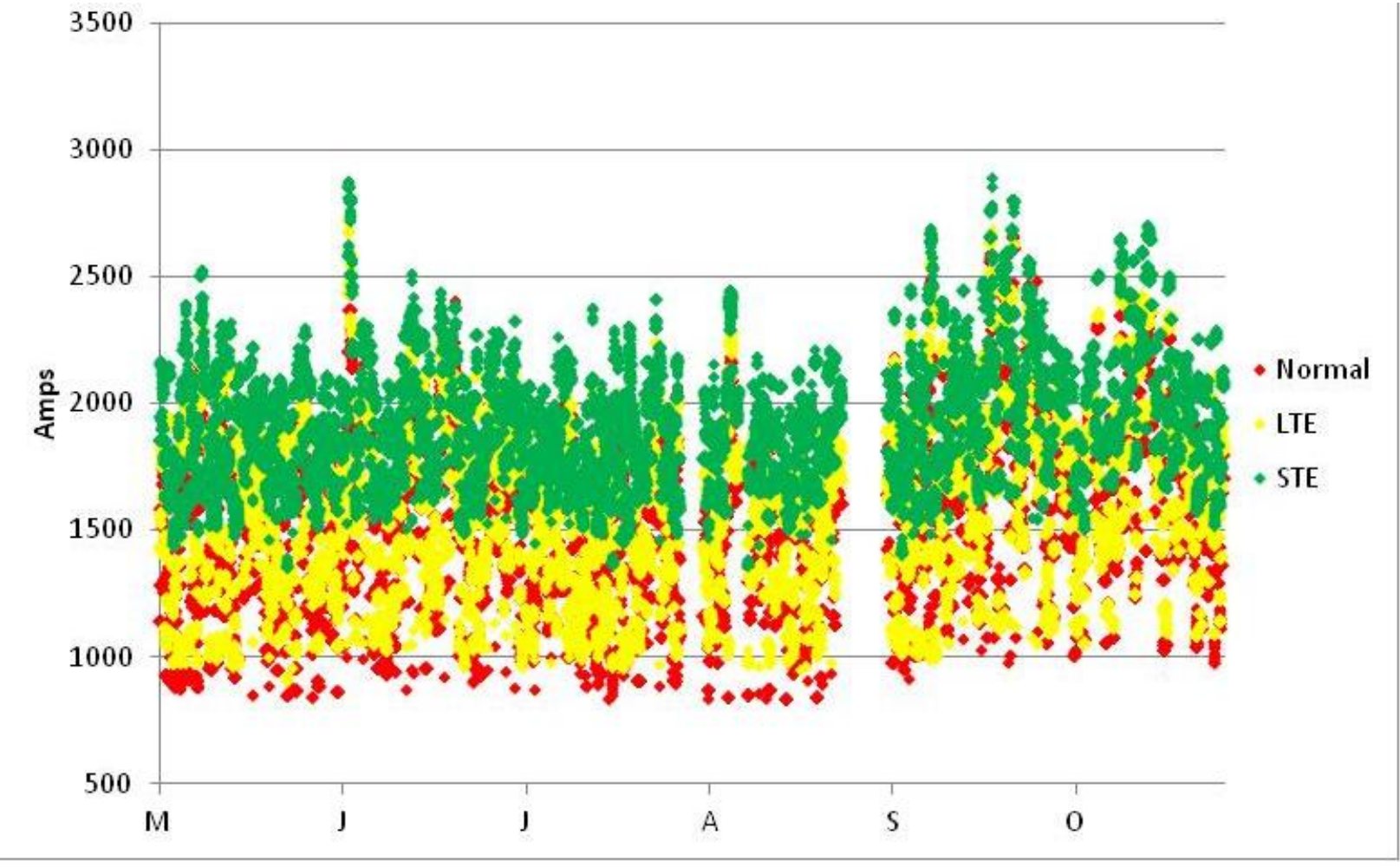

Figure F-36

Overhead line ratings based on Offsite Weather Service at Site 2 - Summer 2012 


\section{Ratings at Site 1: Winter}

The hole in the April data below was due to issues with the EDM base station and communications. As the Sagometer and EPRI Sensor share this weather information the hole will be present there as well.

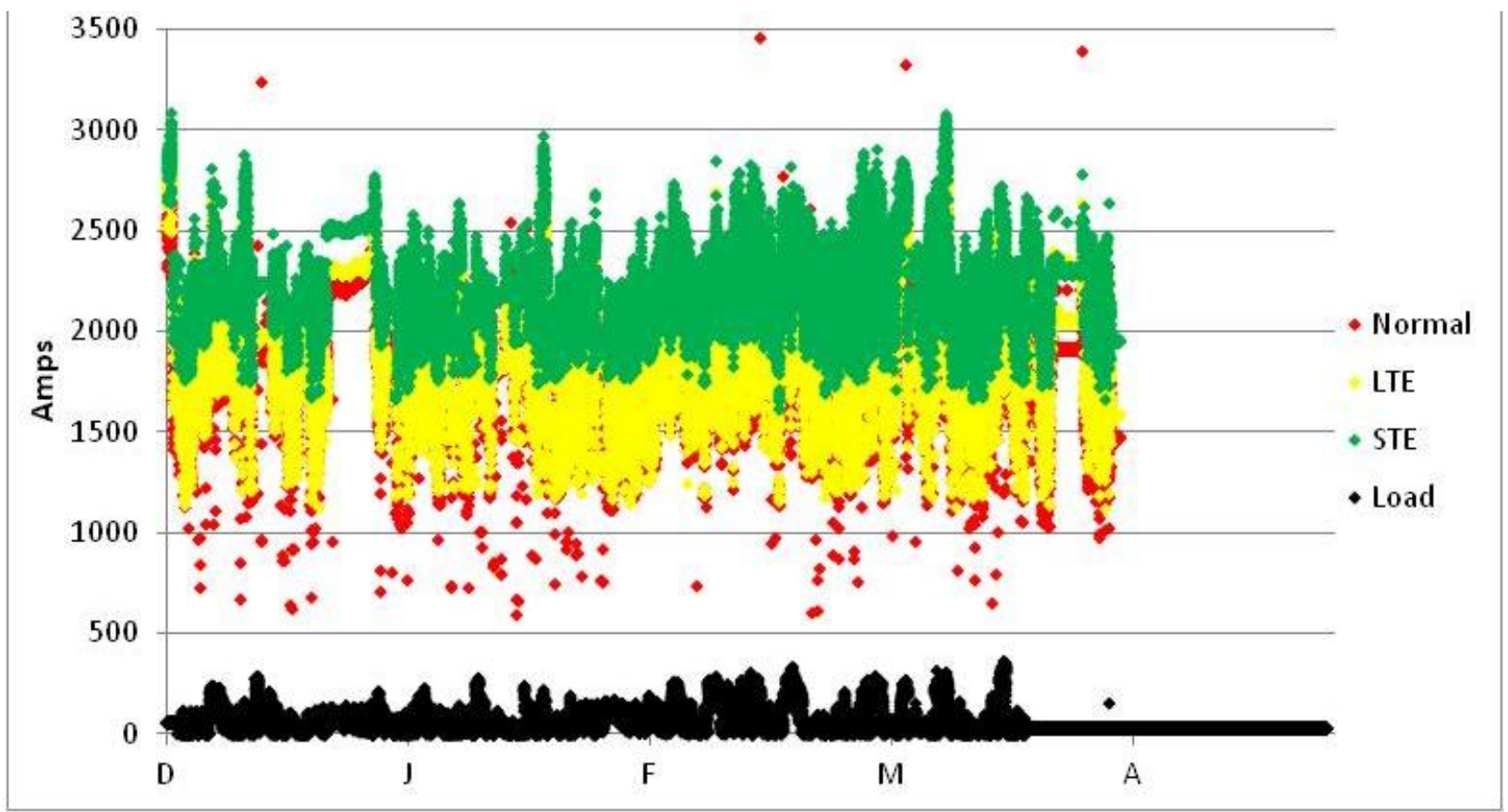

Figure F-37

Overhead line ratings based on the Onsite Weather Station at Site 1-

Winter 2010-2011 


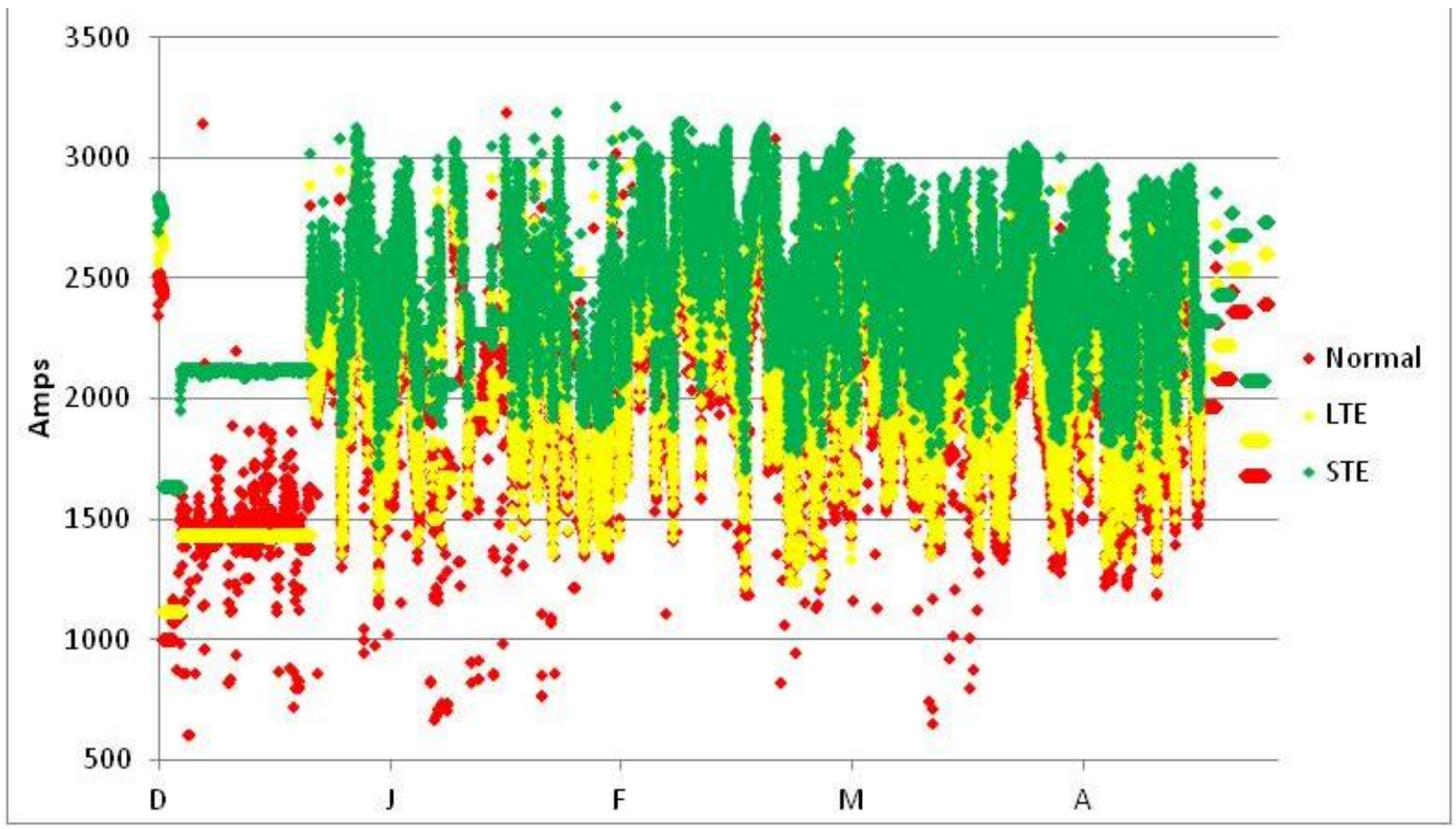

Figure F-38

Overhead line ratings based on the ThermalRate at Site 1- Winter 2010-2011

In the figure below, the hole in the March data is due to a missing sag data from the camera

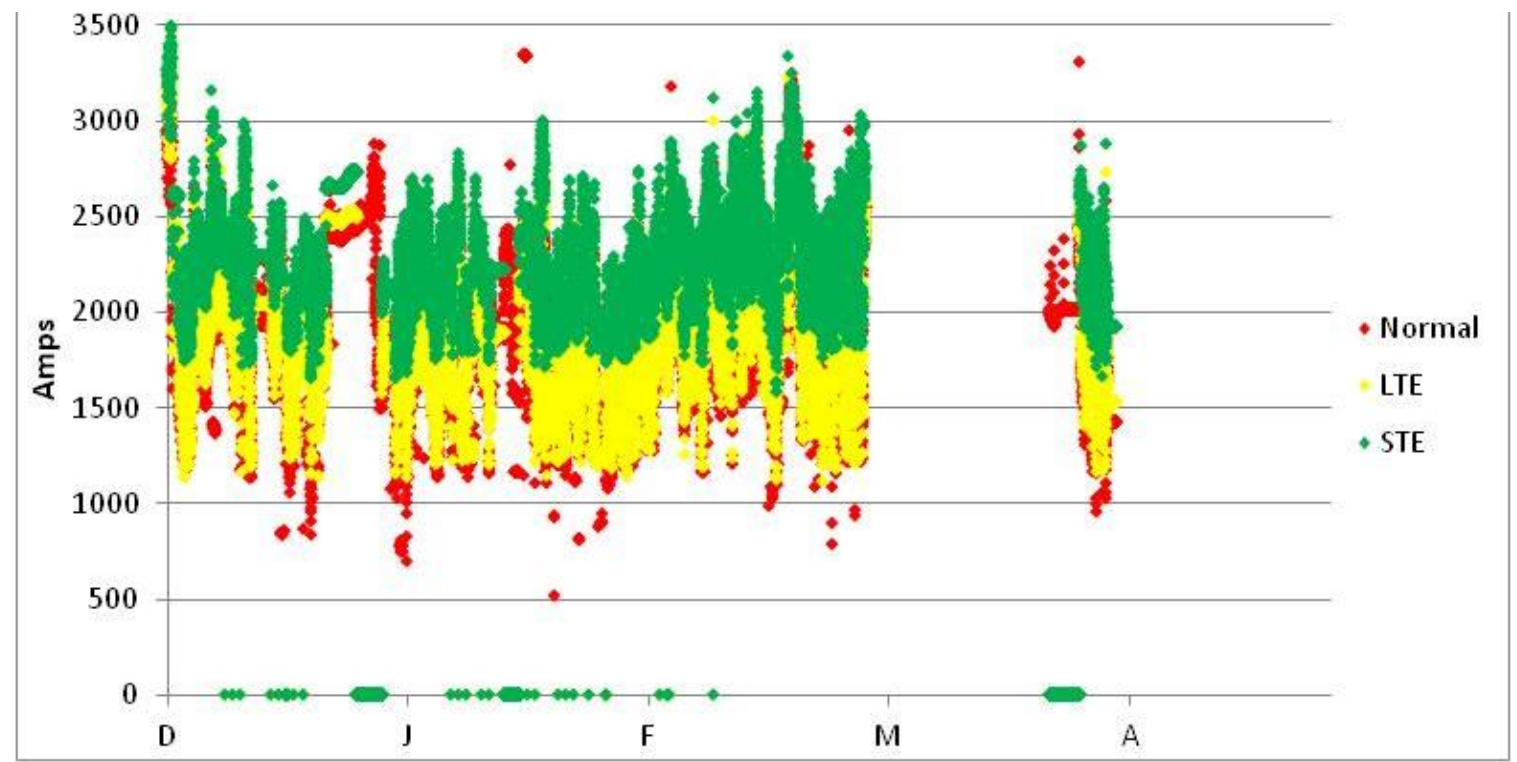

Figure F-39

Overhead line ratings based on the Sagometer at Site 1- Winter 2010-2011 


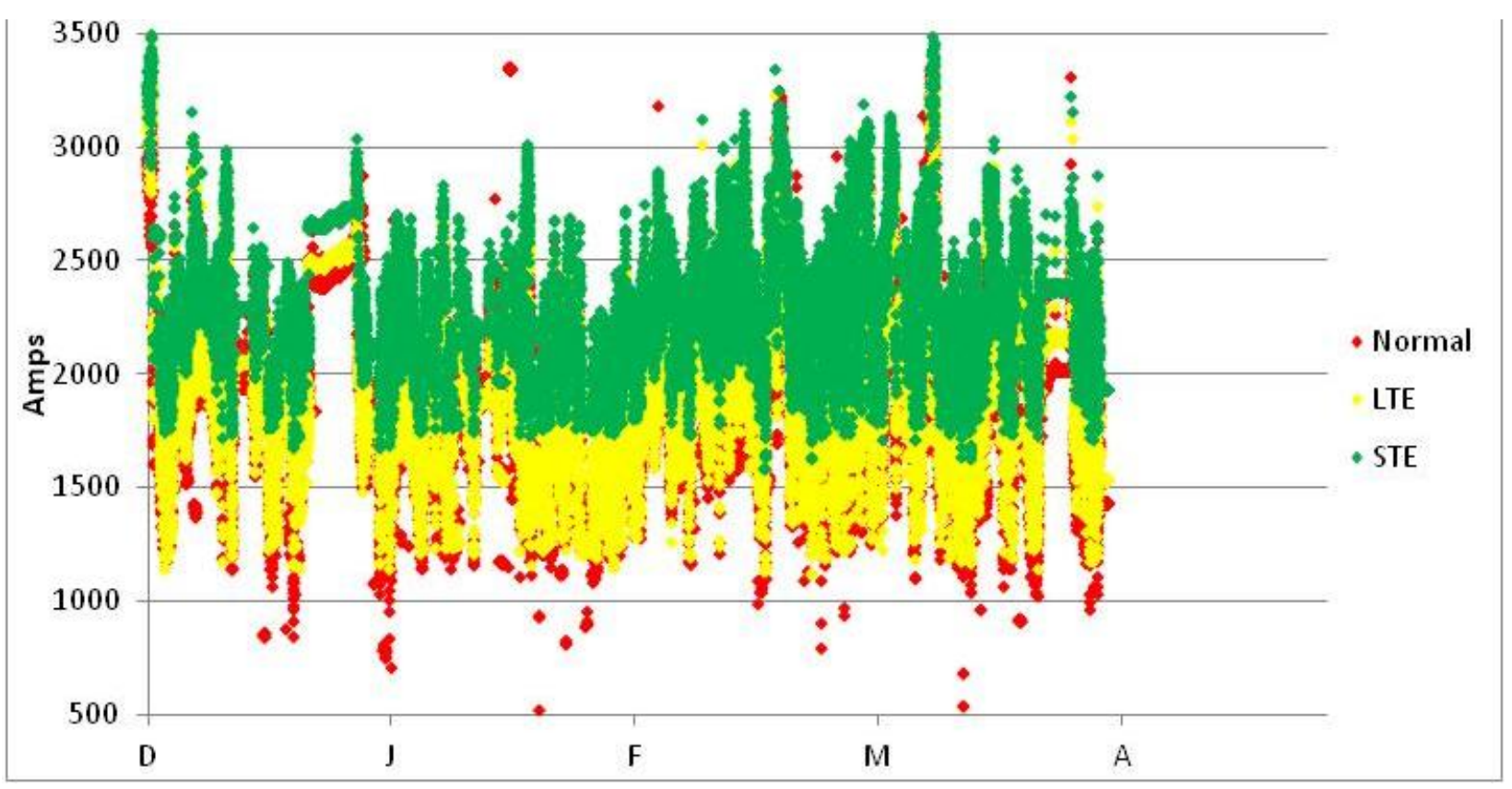

Figure F-40

Overhead line ratings based on the EPRI Sensors at Site 1- Winter 2010-2011

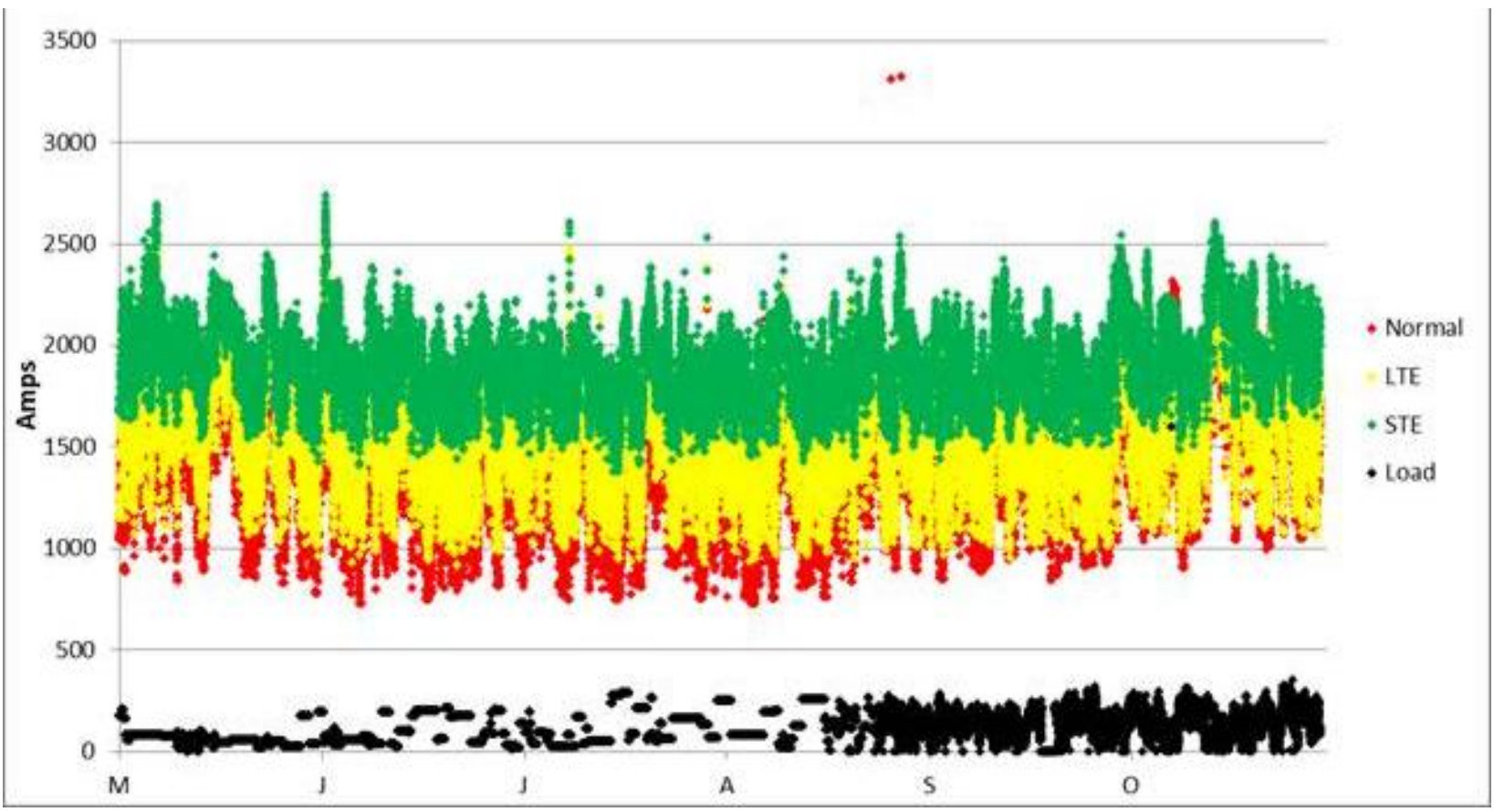

Figure F-41

Overhead line ratings based on the onsite weather station at Site 1 - Summer 2011 


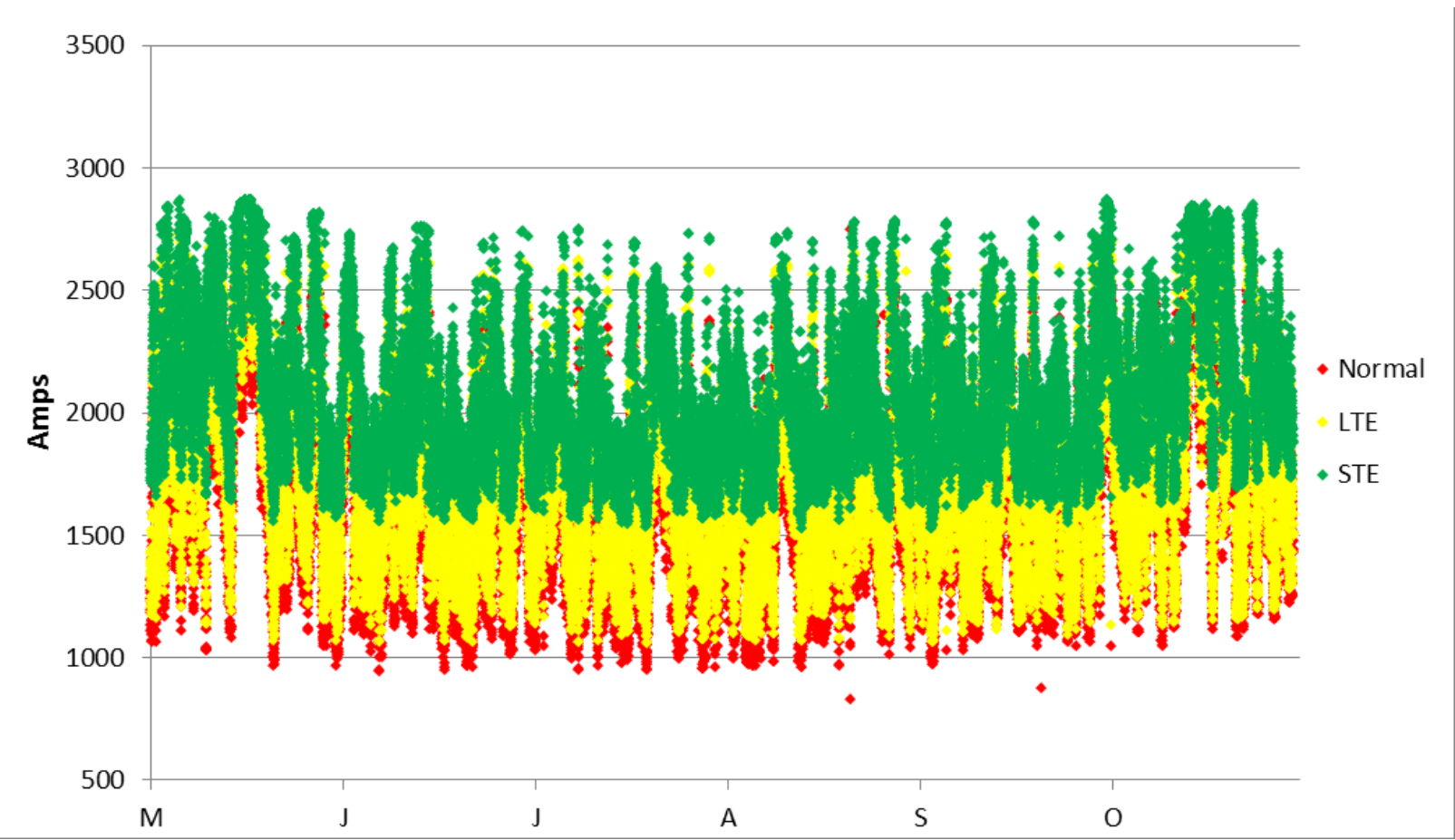

Figure F-42

Overhead line ratings based on ThermalRate device at Site 1 - Summer 2011

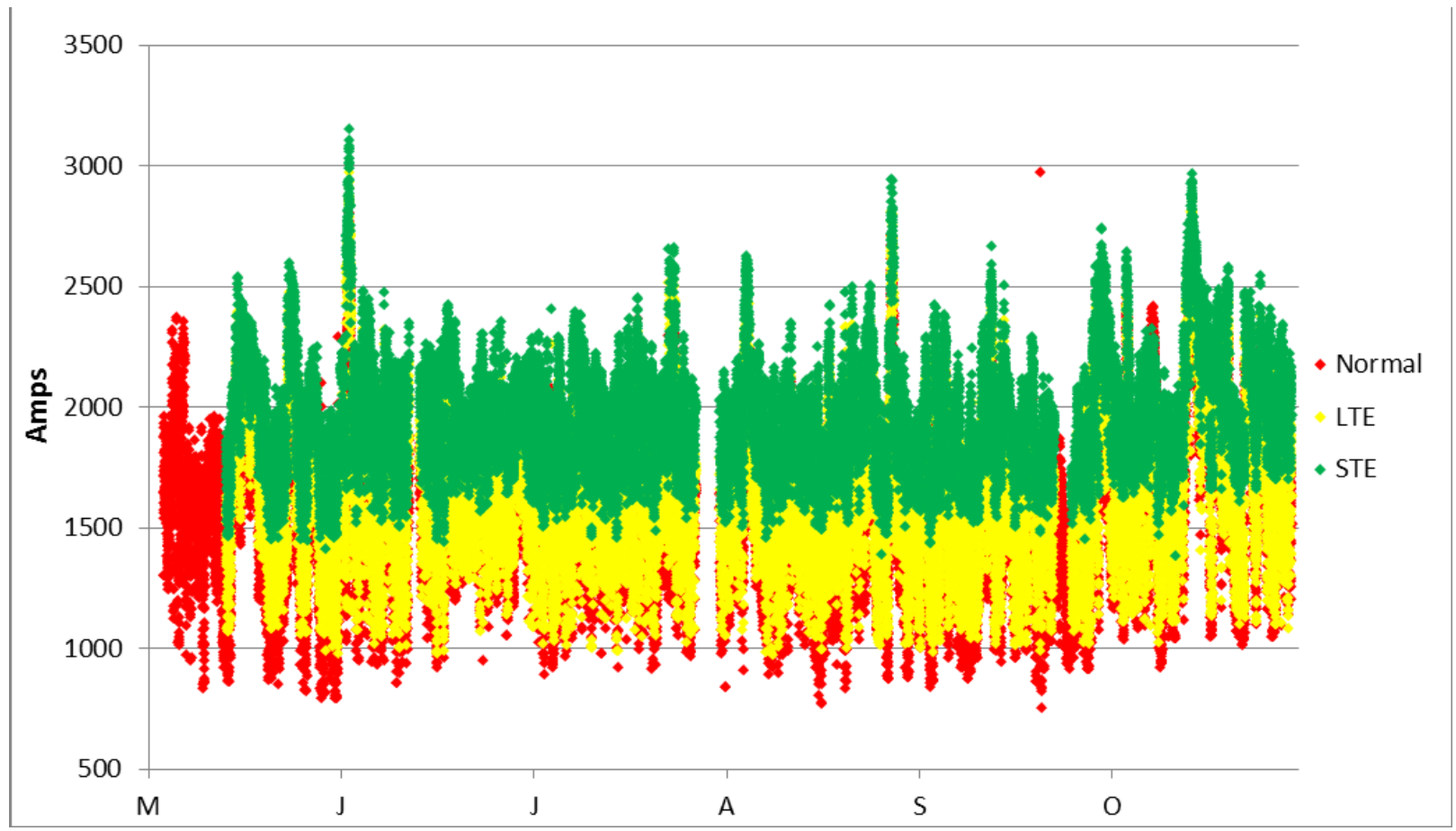

Figure F-43

Overhead line ratings based on the Sagometer at Site 1 - Summer 2011 


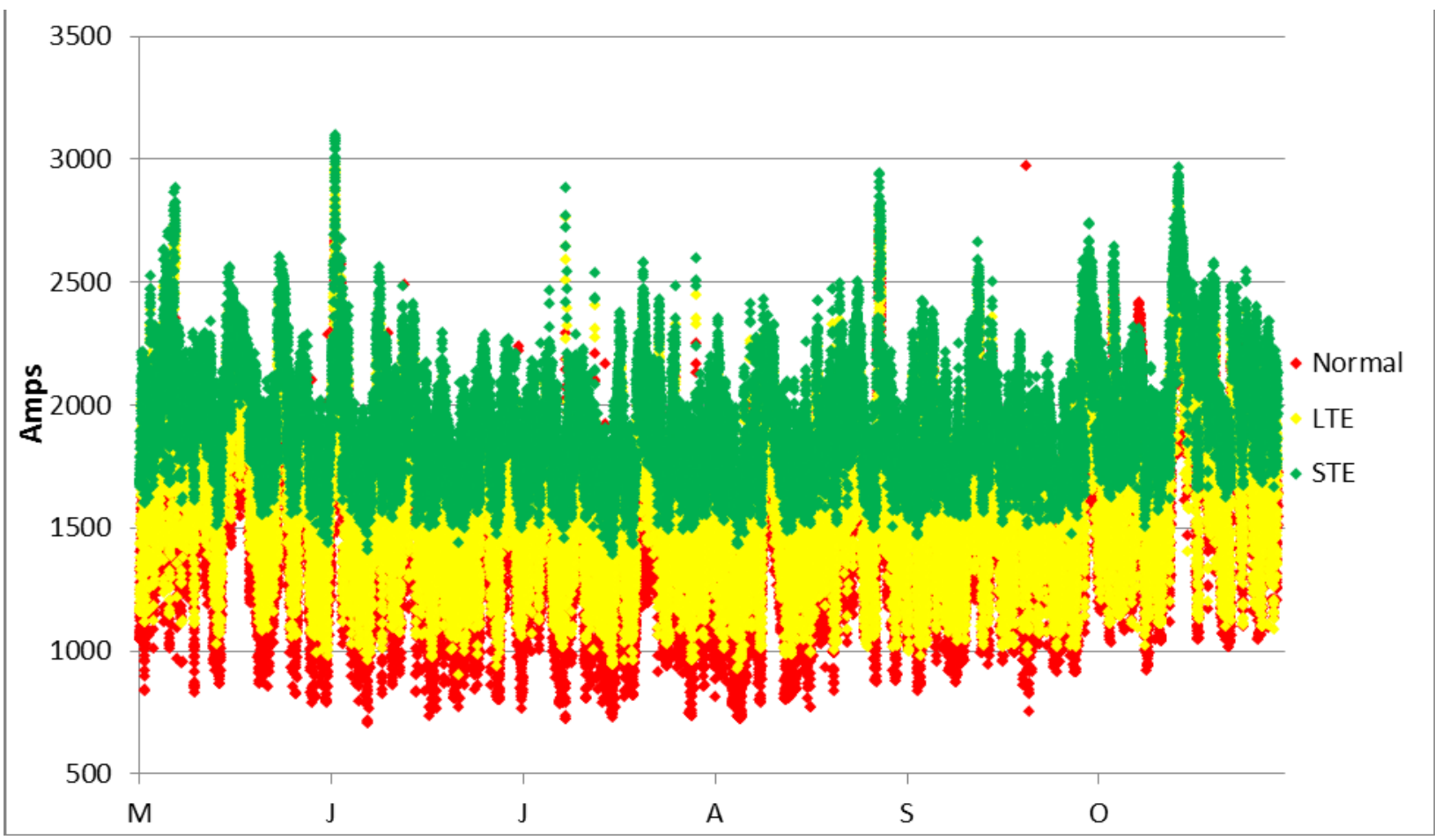

Figure F-44

Overhead line ratings based on the EPRI Sensors at Site 1 - Summer 2011

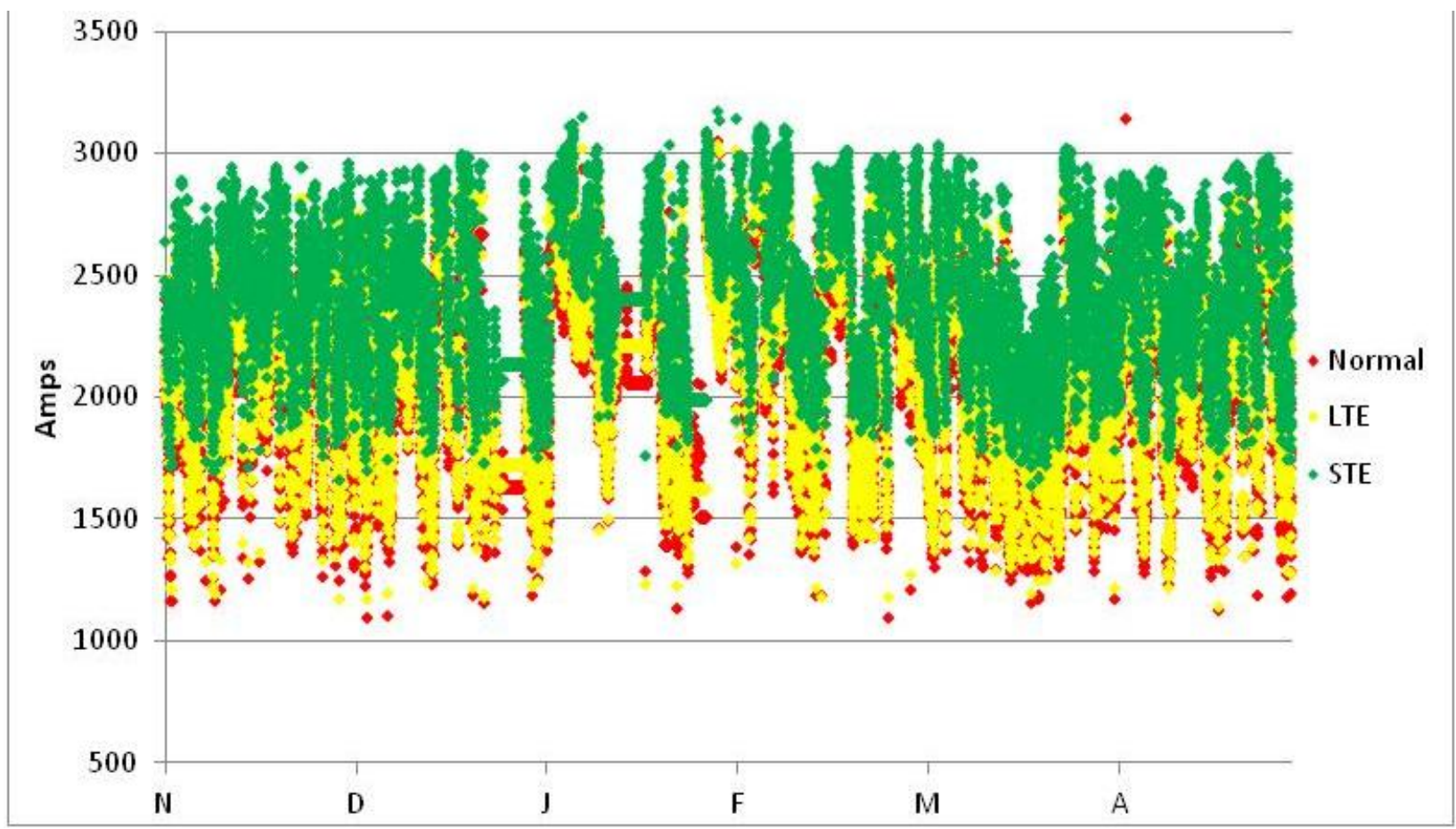

Figure F-45

Overhead line ratings based on the ThermalRate at Site 1- Winter 2010-2011 


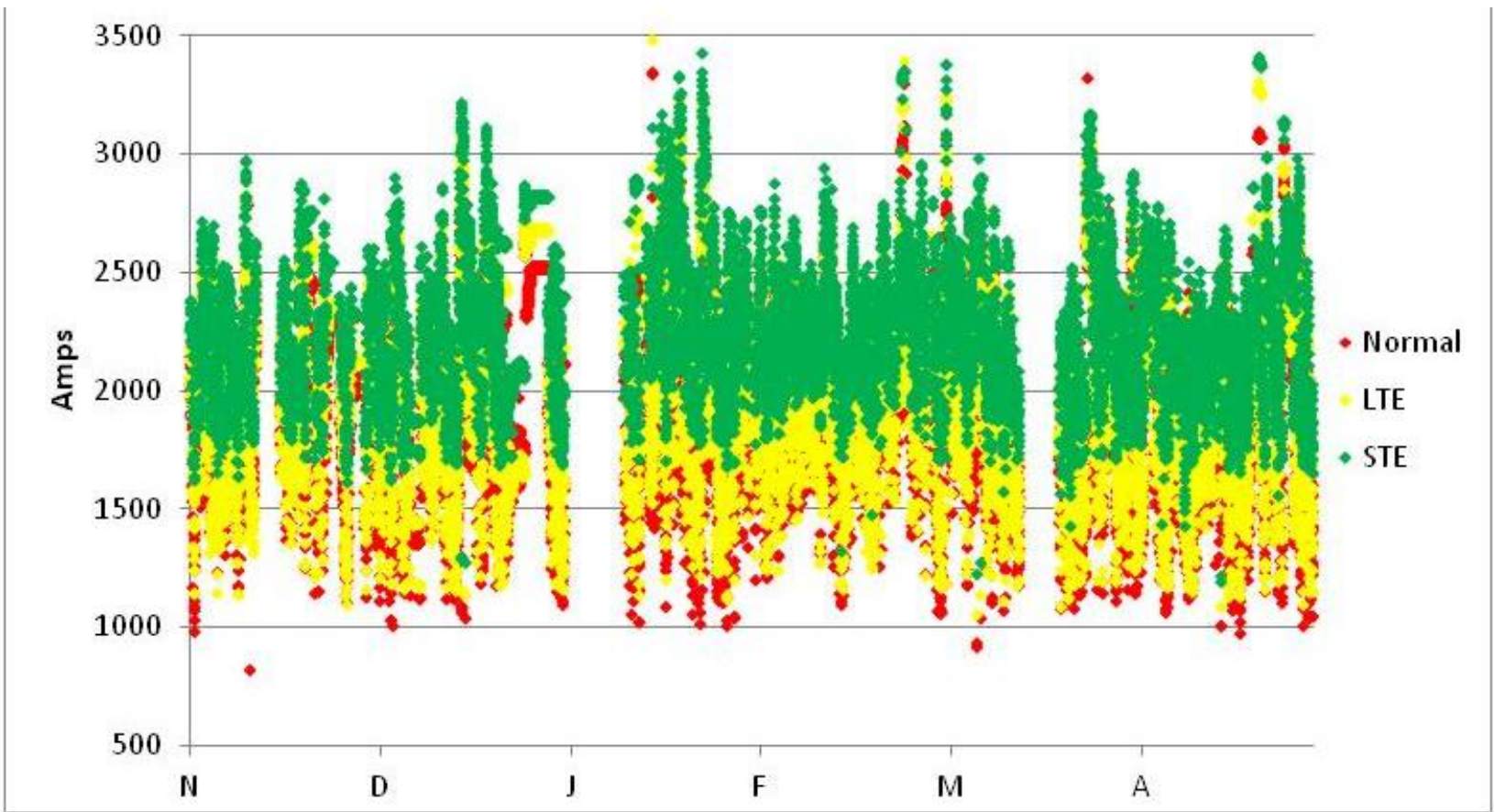

Figure F-46

Overhead line ratings based on the Sagometer at Site 1- Winter 2011-2012

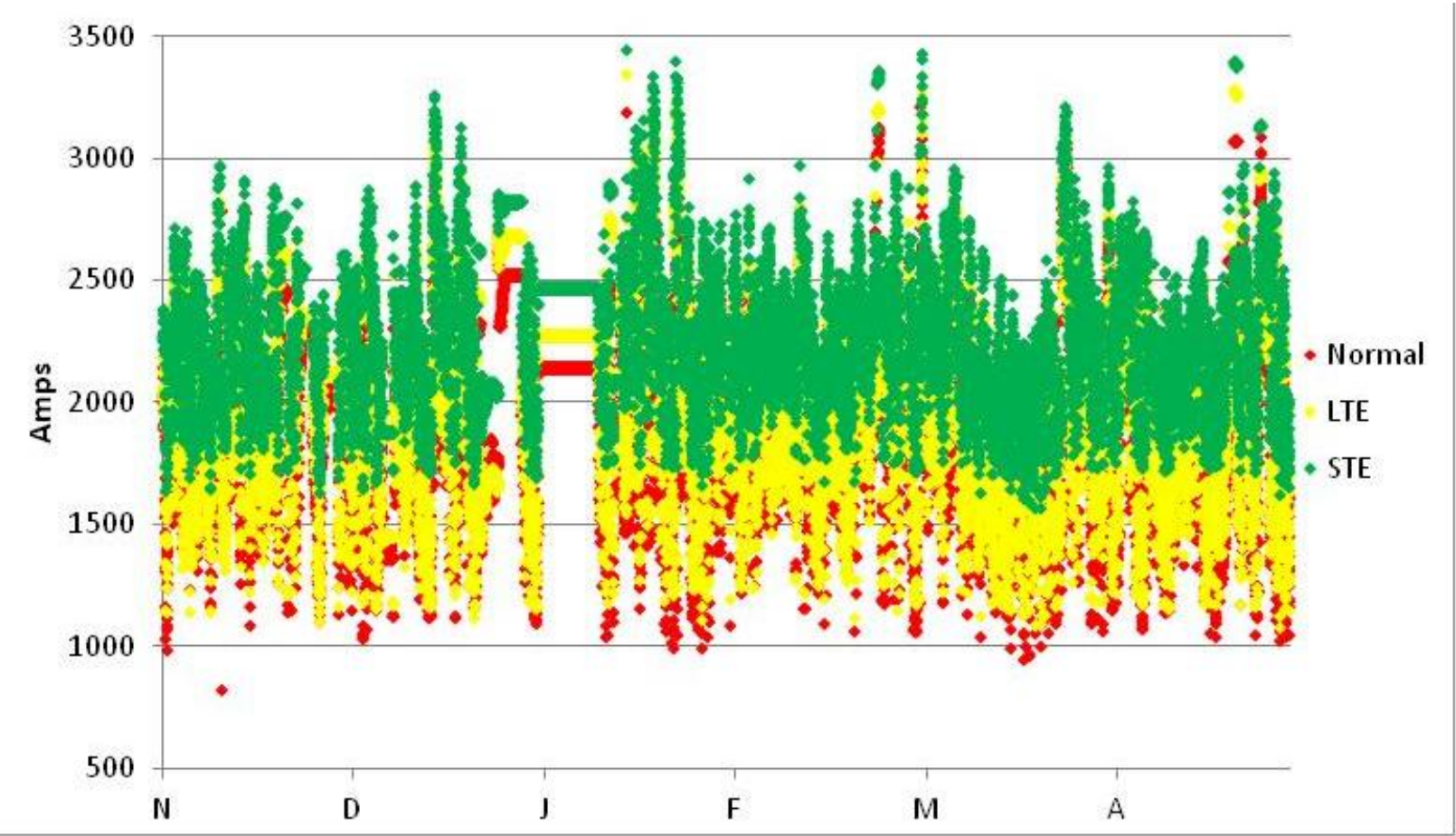

Figure F-47

Overhead line ratings based on EPRI Sensors at Site 1- Winter 2011-2012 


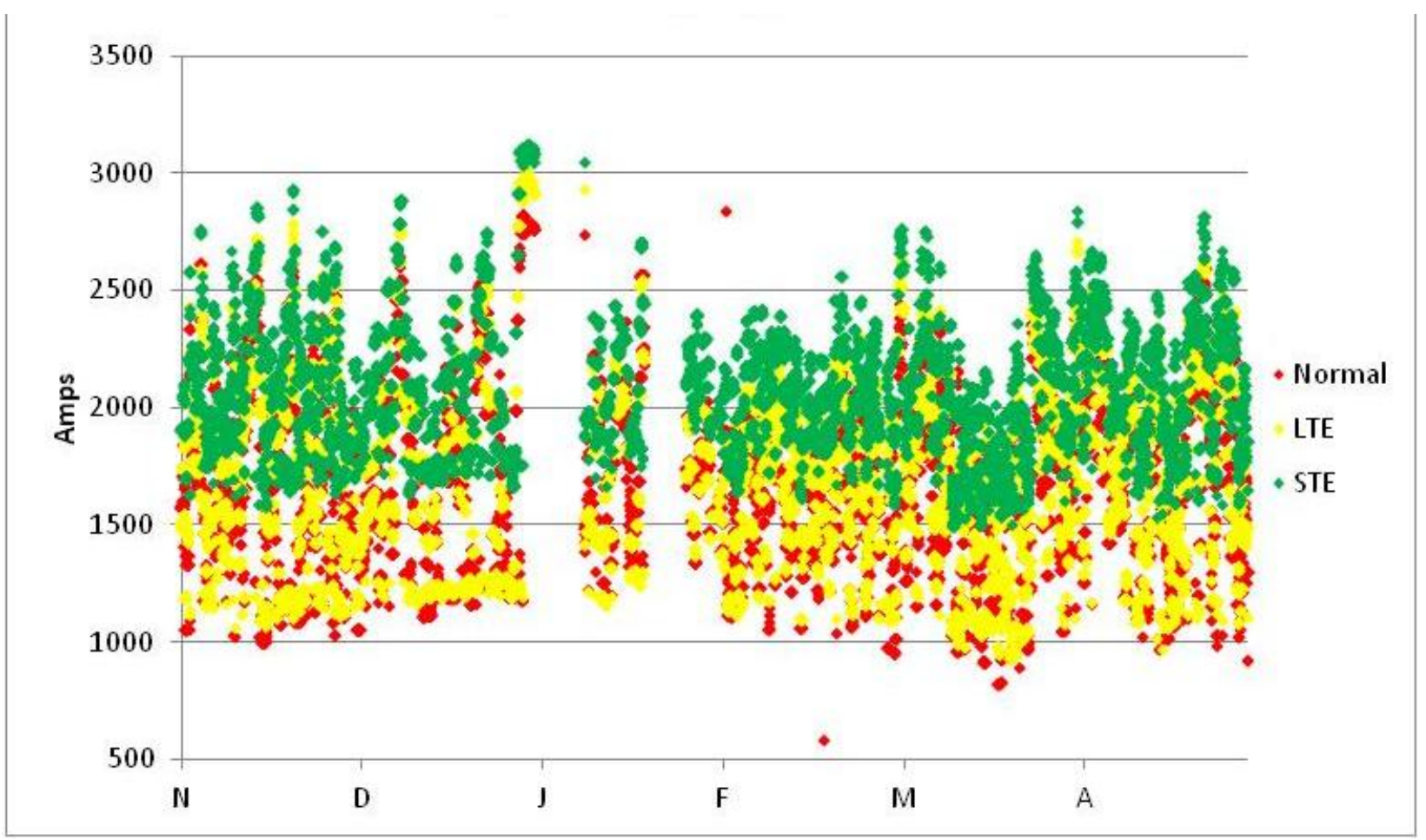

Figure F-48

Overhead line ratings based on Offsite Weather Service at Site 1- Winter 2011-2012 


\section{Ratings at Site 1: Summer}

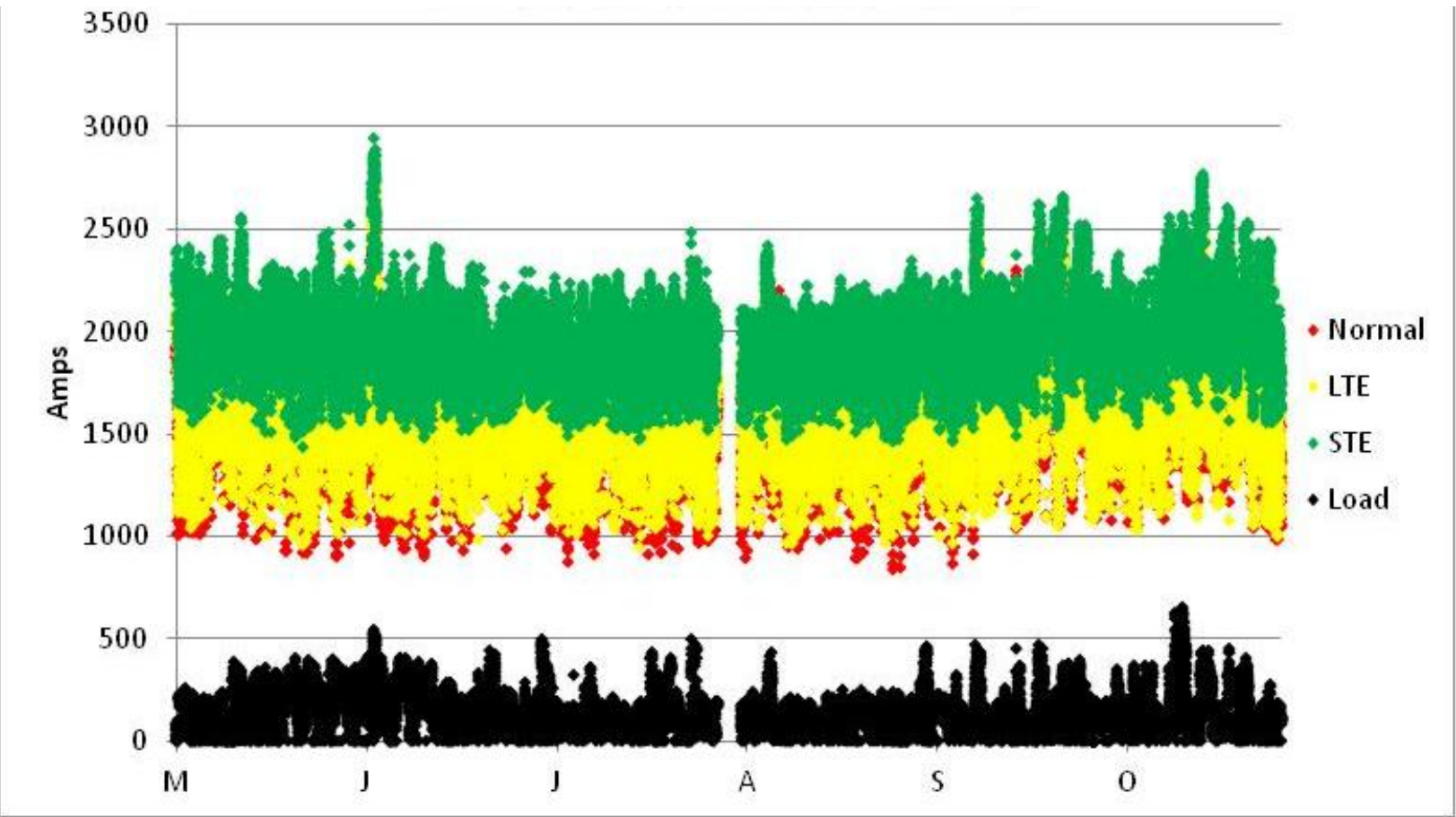

Figure F-49

Overhead line ratings based on the Onsite Weather Station at Site 1- Summer 2012

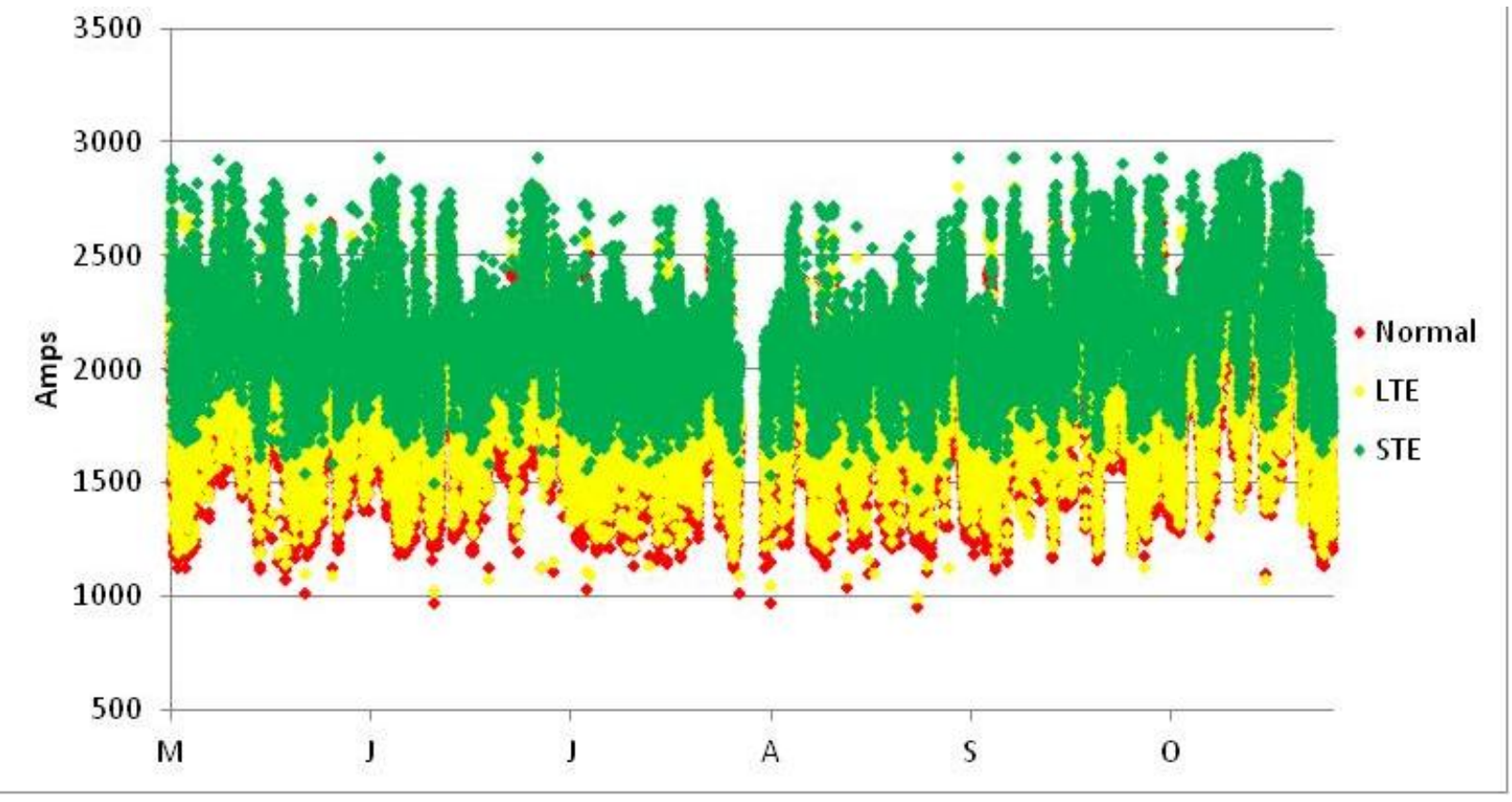

Figure F-50

Overhead line ratings based on ThermalRate at Site 1- Summer 2012 


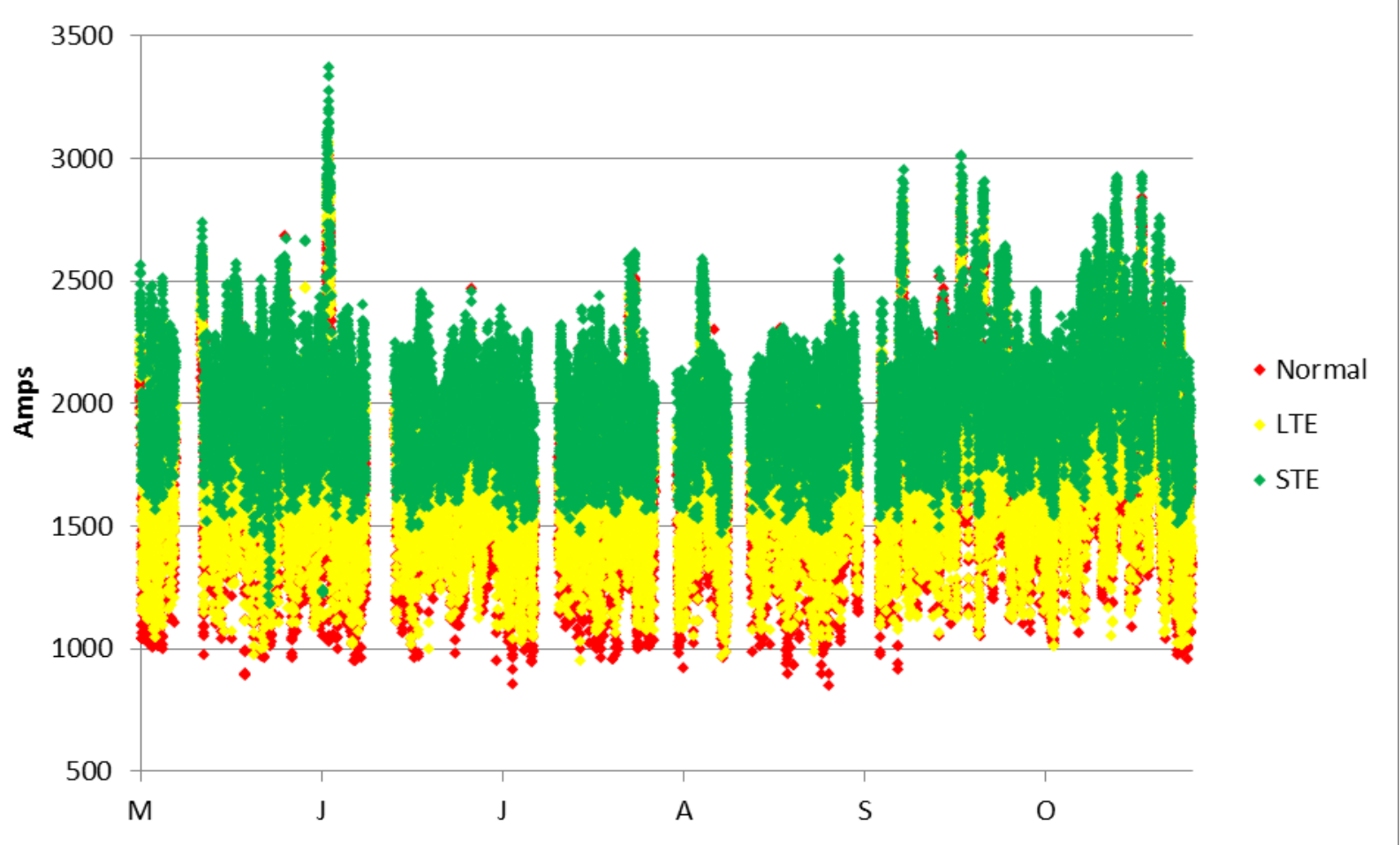

Figure F-51 Overhead line ratings based on the Sagometer at Site 1 - Summer 2011

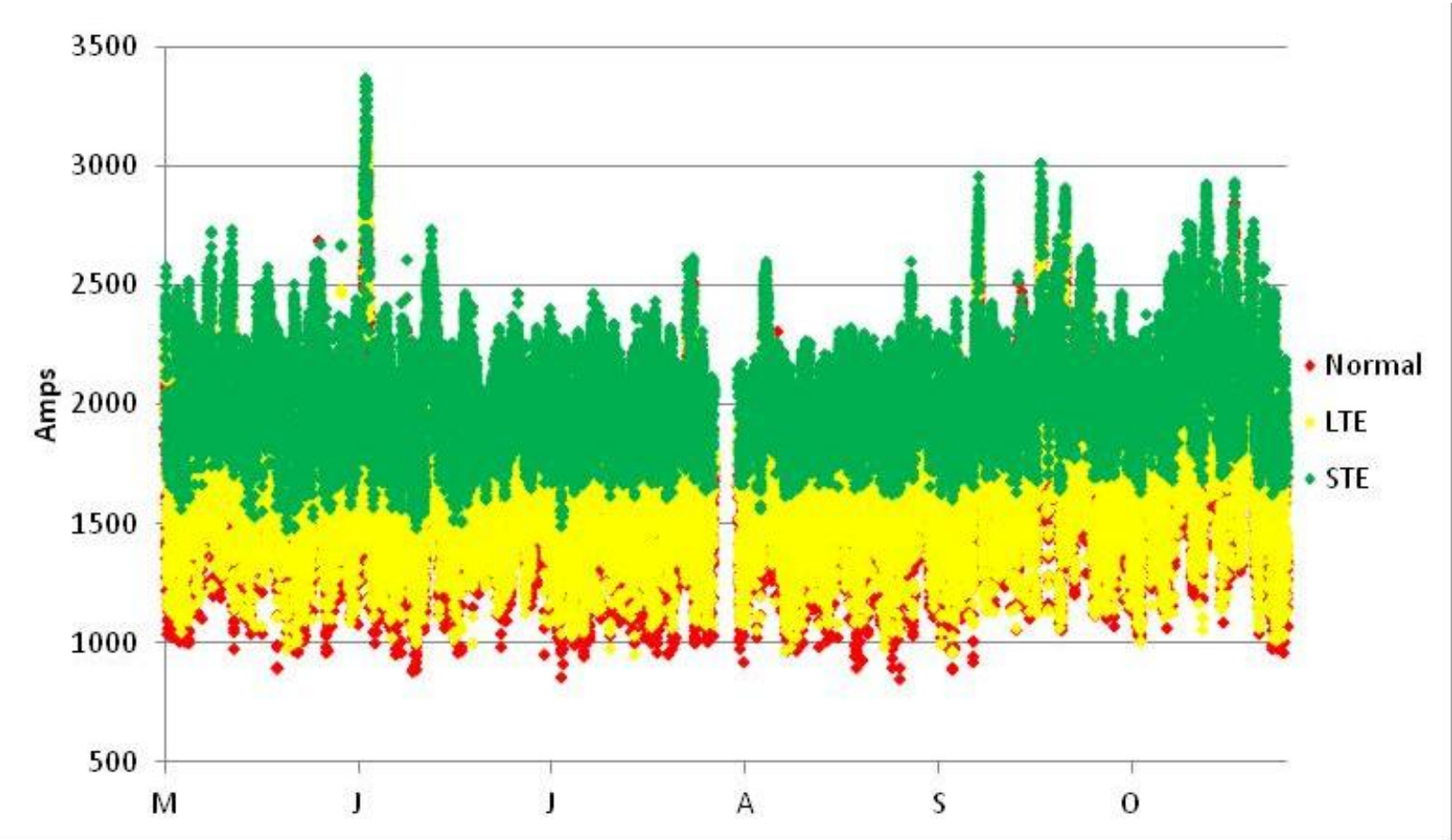

Figure F-52

Overhead line ratings based on EPRI Sensors at Site 1- Summer 2012 


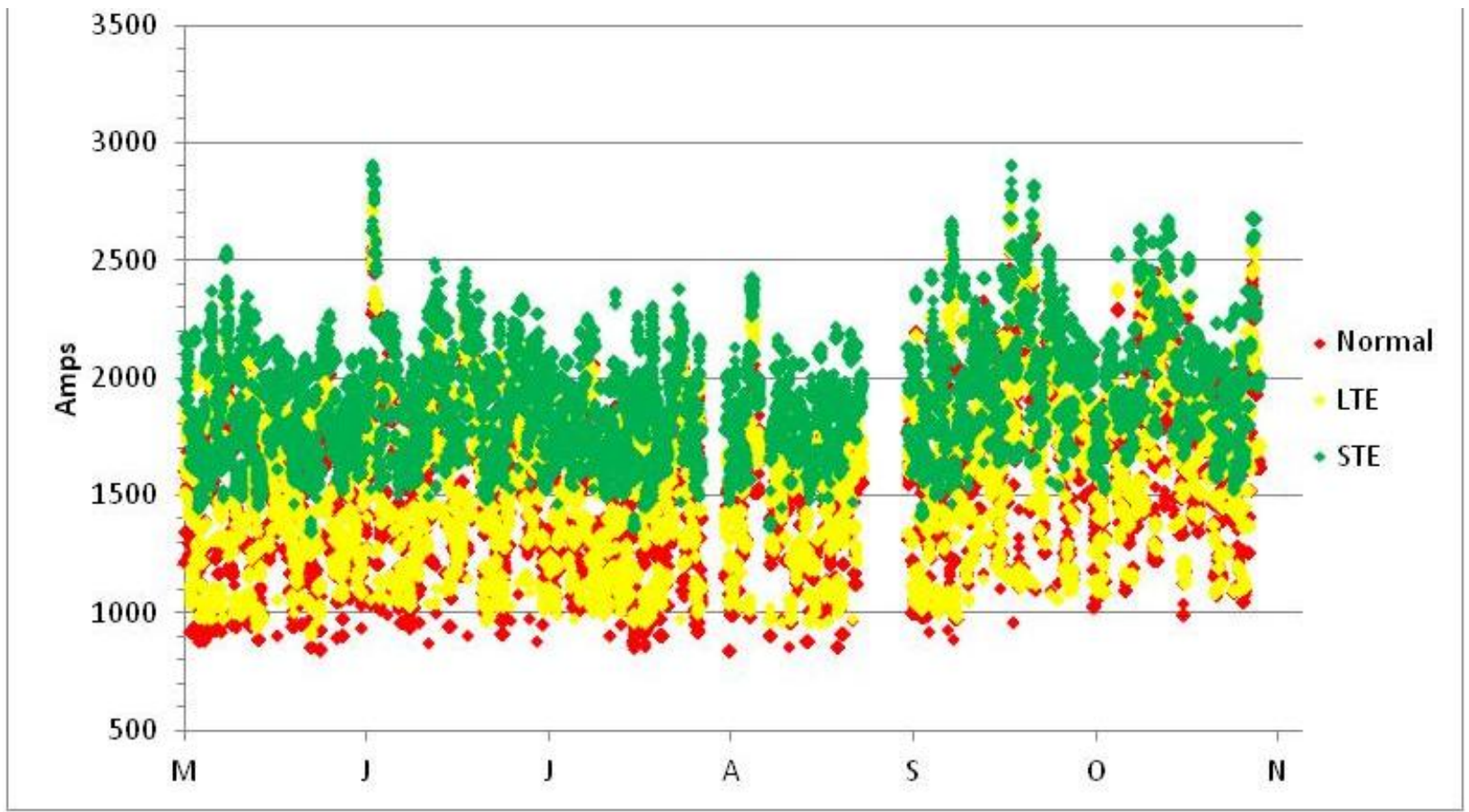

Figure F-53

Overhead line ratings based on the Offsite Weather Service at Site 1- Summer 2012 


\section{COMPARISON OF REAL-TIME MONITORING METHODS}

In this chapter a technical comparison is made between the five rating elements used in this project for dynamic line ratings. Table G-1 summarizes the five elements and the rating model used in DTCR:

Table G-1

Calculation Elements and DTCR Calculation Models

\begin{tabular}{|c|c|c|}
\hline Element & $\begin{array}{c}\text { Rating Calculation method } \\
\text { used in DTCR }\end{array}$ & $\begin{array}{c}\text { Backup Rating Method } \\
\text { (For low line currents) }\end{array}$ \\
\hline Onsite Weather Station (WX) & IEEE 738 & none \\
\hline Video Sagometer (SAG) & SAG & DynAmp \\
\hline EPRI Sensor (BS) & SAG & DynAmp \\
\hline ThermalRate (TR) & IEEE 738 \\
\hline Offsite Weather Service (AP) & IEEE 738 & None \\
\hline
\end{tabular}

There is no exact method for calculating real-time dynamic line ratings that can be used as a standard but the data from nearly two years of monitoring using five different methods, each with its own raw data set, can be analyzed and compared to gain insight into the effectiveness of the different elements for producing useful real-time ratings. For some elements there was very little data to do a proper evaluation. This in itself is a result that can be used in decision making on what strategies to employ in future dynamic line rating projects.

\section{Line Loading and Rating Calculations}

The temperature of the bare overhead conductors in an overhead transmission line depends on the line current and the ambient weather parameters in the line corridor. At any point in time the line current the air temperature and the level of solar heating vary little from span to span, however, the wind speed and direction can vary significantly from span to span.

When the line current is high, the variation in wind speed and direction along the line can cause the conductor temperature to vary sharply along the line. In most lines tension equalization at structure supports prevents equally sharp variations in tension along each line section.

Therefore, the sag (and thus the electrical clearance of the line's energized conductors to ground and other conductors) varies with the average line section temperature rather than with the local conductor temperature.

When the line current is low, the variation in wind speed and direction along the line does not produce a large conductor temperature variation, either within a span or from span to span. 
When the line current in the NYPA lines studied in this project are at $30 \%$ of the line's normal static rating the conductor temperature is typically no more than $5^{\circ} \mathrm{C}$ above air temperature and that rise is largely due to solar heating.

Power flow of less than $30 \%$ of the normal rating is typical rather than an exception on high voltage $(69-230 \mathrm{kV})$ transmission lines. The only time the line current approaches the thermal rating of such lines is after an N-1 or N-2 contingency. An exception to this can occur for radial transmission lines which may connect a physically isolated generating source such as a wind farm to the system.

Two of the technologies evaluated here; sagometer and EPRI Sensor need a substantial conductor temperature rise in order to produce accurate ratings. At "low" line currents, below $0.44 \mathrm{~A} / \mathrm{Kcmil}$ or $350 \mathrm{~A}$ ( $32 \%$ of NYISO normal summer rating), these methods are not useful for ratings calculations. The two $230 \mathrm{kV}$ lines instrumented in this study were not typically loaded to this level. Line currents only rarely exceeded the minimum 350A load required for these elements to generate ratings based on the sag/tension model in DTCR and therefore, these elements reverted to an alternate method (DynAmp) to produce ratings nearly all of the time. Since sag/tension based methods have the advantage of averaging the conductor temperature over many spans the inability to properly evaluate these technologies in this program was disappointing. However, this is actually a significant finding in the project. There is evidence from other sources that the lines instrumented at NYPA are not lightly loaded but rather "typically" loaded. An EPRI LiDAR document on related technologies relates the following information:

"The amount of power being generated must balance the amount of power being consumed almost instantaneously in order to maintain system electrical stability, large amounts of thermal capacity must be available to absorb fluctuations in current flow both during normal operation and during a system emergency. Common operating levels for lines within an electrical system are typically $15-40 \%$ of rated thermal line capacity.

While this may not be true for all lines it is the general case as illustrated by a survey of utility project participants. Project participants were canvassed to provide current densities during the LiDAR field surveys they have performed to date. The response provided $222 \mathrm{LiDAR}$ field survey current densities and is summarized on Figure 2.5 \{note; figure G-1 in this report\}. Approximately $65 \%$ of the current densities are less than or equal to $0.25 \mathrm{~A} / \mathrm{kcmil}$ and another $30 \%$ were less than or equal to $0.50 \mathrm{~A} / \mathrm{kcmil}$ with only about $5 \%$ exceeding $0.50 \mathrm{~A} / \mathrm{kcmil}$. This distribution in current densities during a LiDAR field survey is consistent with the normal operation of an electrical system". [1]

Figure G-1 below is a reproduction of Figure 2.5 referred to in the quote above and shows the results of the LiDAR survey: 


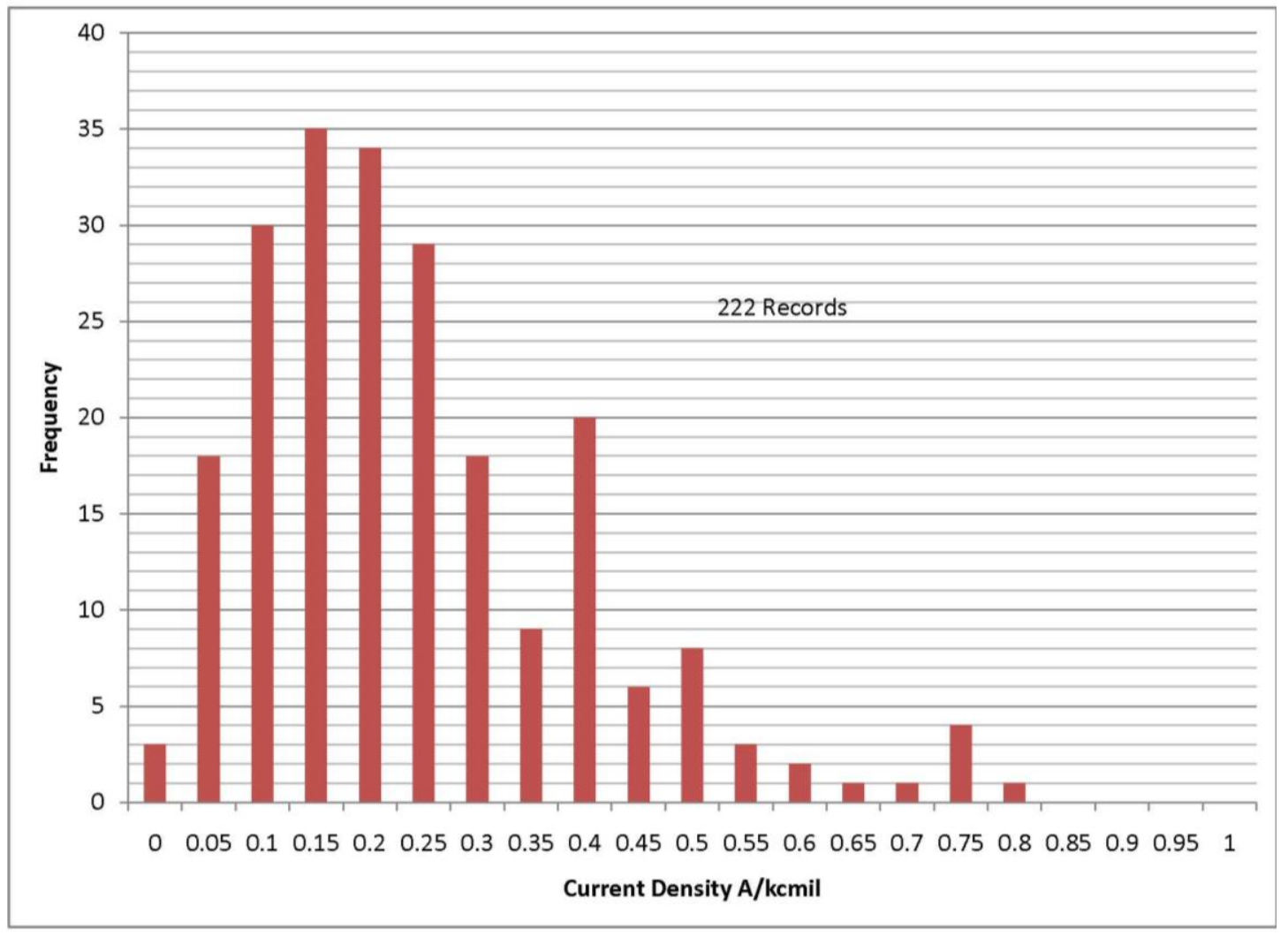

\section{Figure G-1}

\section{Survey Results on Typical Transmission Line Loading}

According this survey, 350Amps $(0.44 \mathrm{~A} / \mathrm{Kcmil})$ on the NYPA lines is in the upper end of the typical operating range and therefore $350 \mathrm{~A}$ should only be occasionally exceeded. This indicates that sag/tension based calculations will only rarely produce valid ratings on typical lines, which is consistent with the findings of this project.

Even though sag/tension based ratings were almost never performed by DTCR in this project due to low currents, DTCR would utilize sag based ratings in a contingency situation if one had arisen and any advantages of these methods would then be realized when they are most needed. However, the need for an alternative rating calculation that is accurate at low loads is indicated for typical lines. A comprehensive evaluation of sag based technologies may require a controlled study in which the line is carefully selected or where loading can be manipulated. In this chapter limited data during high load periods will be presented.

\section{Rating Calculation Considerations}

In evaluating the accuracy of all of the dynamic rating methods, some of the most important factors include the following:

- Current Density (the line current divided by the conductor cross section area )

- The existence or absence of wind sheltering by terrain and foliage 
- Whether the "Maximum Allowable Conductor Temperature" of the line is set to assure adequate electrical clearance or to limit the deterioration of the conductor system.

- The length of line sections and the overall length of the line.

The sag of the phase conductors in a particular span is a function of that span length, the line section ruling span, and the average temperature of the conductor in the mechanically isolated line section between strain structures. If real-time monitors measure the conductor temperature or weather conditions at a single location within a span, then multiple monitors may be required to estimate the sag behavior of the line.

If it is raining then the rain acts as a significant cooling agent that drives the rating so high that other parts of the system become the limiting factor. Consequently, precipitation is not factored into the ratings. Though not used for ratings, a rain gauge can prove useful for diagnosing discrepancies between weather and sag/tension models, as the latter models have instruments that will account for the cooling effects. The ThermalRate device for example does not distinguish between cooling caused by wind or precipitation.

Anemometers (including the directional anemometer of the ThermalRate device) measure wind cooling independently of power flow level on the line. Temperature and sag-tension monitors measure wind cooling indirectly and the accuracy of the calculated wind cooling for these devices is dependent on the power flow on the line among other factors.

\section{Ratings vs. Monitoring}

The system operator has little interest in the conductor temperature but is always interested in the line rating since the rating can be compared to the line current which is displayed to the operator in real-time. Thus any comparison of real-time monitoring methods comes down to how well the real-time monitor data can be converted into a useful real-time dynamic line rating.

\section{Heat Balance}

As described in Appendix B, the calculation of dynamic line ratings involves the solution of the following "heat-balance" equation:

$$
q_{c}+q_{r}=q_{s}+I^{2} \cdot R\left(T_{C}\right)
$$

\section{Equation G-1}

Where $q_{c}$ is the convection heat loss $(\mathrm{W} / \mathrm{m}), q_{r}$ is the radiation heat loss $(\mathrm{W} / \mathrm{m})$, both of which are a function of conductor temperature rise over air temperature $\left(T_{C}-T_{A}\right), T_{\mathrm{C}}$ is the average temperature of the conducting conductor strands $\left({ }^{\circ} \mathrm{C}\right), q_{s}$ is the heat gain from solar radiation $(\mathrm{W} / \mathrm{m}), I$ is the RMS current (A), and $R$ is the temperature-dependent resistance of the conductor $(\Omega / \mathrm{m})$.

This equation can be solved for the thermal rating of the line by specifying the conductor temperature equal to the Maximum Allowable Conductor Temperature: 


$$
I=\sqrt{\frac{q_{c}+q_{r}-q_{s}}{R\left(T_{C}\right)}}
$$

\section{Equation G-2}

With reference to the specific equation components described in IEEE 738, it is possible to develop a simplified heat balance equation for a specific conductor based on the linearization of the heat loss terms:

$$
K_{c} \cdot V_{\text {wind }}^{0.6} \cdot\left(T_{C}-T_{A}\right)+K_{r} \cdot\left(T_{C}-T_{A}\right)+m \cdot C_{p} \cdot \frac{d T_{C}}{d t}=q_{s}+I^{2} \cdot R\left(T_{C}\right)
$$

\section{Equation G-3}

Where $C_{p}$ is the heat capacity of the conductor $\left(\mathrm{W} /\left(\mathrm{kg} \mathrm{C}^{\circ}\right)\right), m$ is the mass of the conductor $(\mathrm{kg} / \mathrm{m}), K_{\mathrm{r}}$ is a constant unique to the conductor as described in IEEE738. $K_{\mathrm{C}}$ is also a conductor dependent constant.

$V_{\text {wind }}$ is the equivalent perpendicular wind speed. $V_{\text {wind }}$ is equal to wind speed when the wind is perpendicular to the conductor but is less than the actual wind speed when the wind angle is other than perpendicular. The ratio of $V_{\text {wind }} /$ windspeed is a minimum of $\sim 0.2$ when the wind is parallel to the line. The conversion of raw wind speed to $V_{\text {wind }}$ involves complex convective heat transfer theory and empirical data and is one of the key focuses of research and of the IEEE738 methodology.

Equation G-3 can be used to calculate the conductor temperature if it is not measured. This is the method used to track Tc by both on site and off site weather station elements. ThermalRate uses this method but bypasses many of the calculations. Instead of calculating the cooling effects of the wind using heat transfer theory, and the wind speed and angle of wind to the line, the cooling effects are measured directly.

ThermalRate calculates $V_{\text {wind }}$ by measuring the actual heat transfer from the hot rod side of the instrument and subtracting the solar gain. The internal power input into the hot rod is not due to line load but is an accurately known value that simulates a high loading so the heat transfer in turn can be accurately calculated. In this way the equations used to determine $V_{\text {wind }}$ as a function of wind speed and angle are bypassed completely. The effectiveness of this methodology for NYPA ratings is addressed in a later section.

Rearranging G-3 gives G-4; once $V_{\text {wind }}$ is determined by any of the methods and line current is measured, equation 4 allows the calculation of conductor temperature over time ("tracking conductor temperature") by integrating the following version:

$$
\frac{d T C}{d t}=\frac{\left[q_{s}+I^{2} \cdot R\left(T_{C}\right)-K_{c} \cdot V \operatorname{wind}^{0.6} \cdot\left(T_{C}-T_{A}\right)-K_{r} \cdot\left(T_{C}-T_{A}\right)\right]}{m \cdot C_{p}}
$$

\section{Equation G-4}

Given conductor temperature, the air temperature, the resistance, the solar heat input to the conductor and the line current, the equation can be used to calculate the conductor temperature as a function of the weather parameters: 


$$
V_{\text {WIND }}=\left[\frac{q_{s}+I^{2} \cdot R\left(T_{C}\right)-\operatorname{Krad} \cdot\left(T_{C}-T_{A}\right)-m \cdot C_{p} \cdot \frac{d T_{C}}{d t}}{\operatorname{Kconv} \cdot\left(T_{C}-T_{A}\right)}\right]^{1.67}
$$

\section{Equation G-5}

When using a temperature monitor such as the EPRI Sensor, the $V_{\text {wind }}$ can be calculated with Equation G-5. Subsequently, the line rating can be calculated using

For sag/tension monitors such as the Video Sagometer, the sag/tension must be converted into a conductor temperature (average over a line section). For the Video Sagometer this requires a state change equation that converts the target height to conductor temperature for use in Equation G-5. In this way $V_{\text {wind }}$ becomes the average wind speed over the line section rather than just a value at a point along the line.

The methodology used to calculate the state equations for the three Video Sagometers is outlined in the removed Appendix B. Adjustments were made to the state equations during the project. The state equations for sag/tension monitors are only as stable as the structures that the line is mounted on. Plastic deformation of the conductor (stretch) also changes the state equation. On the NYPA lines the state equations appeared to become less accurate with time. Evidence of state equation drift is addressed later in this chapter.

\section{Impact of Weather Parameters on Line Rating}

The most critical parameter in Equations 1 through 5 is the convective heat loss variable. Consequently, wind speed and direction is the most important ambient weather condition that is required for a rating calculation. Unfortunately wind is also the most unpredictable and volatile weather parameter.

Figure G-2 shows the effect of a changing wind speed versus air temperature. This graph will demonstrate the impact of various parameters. 


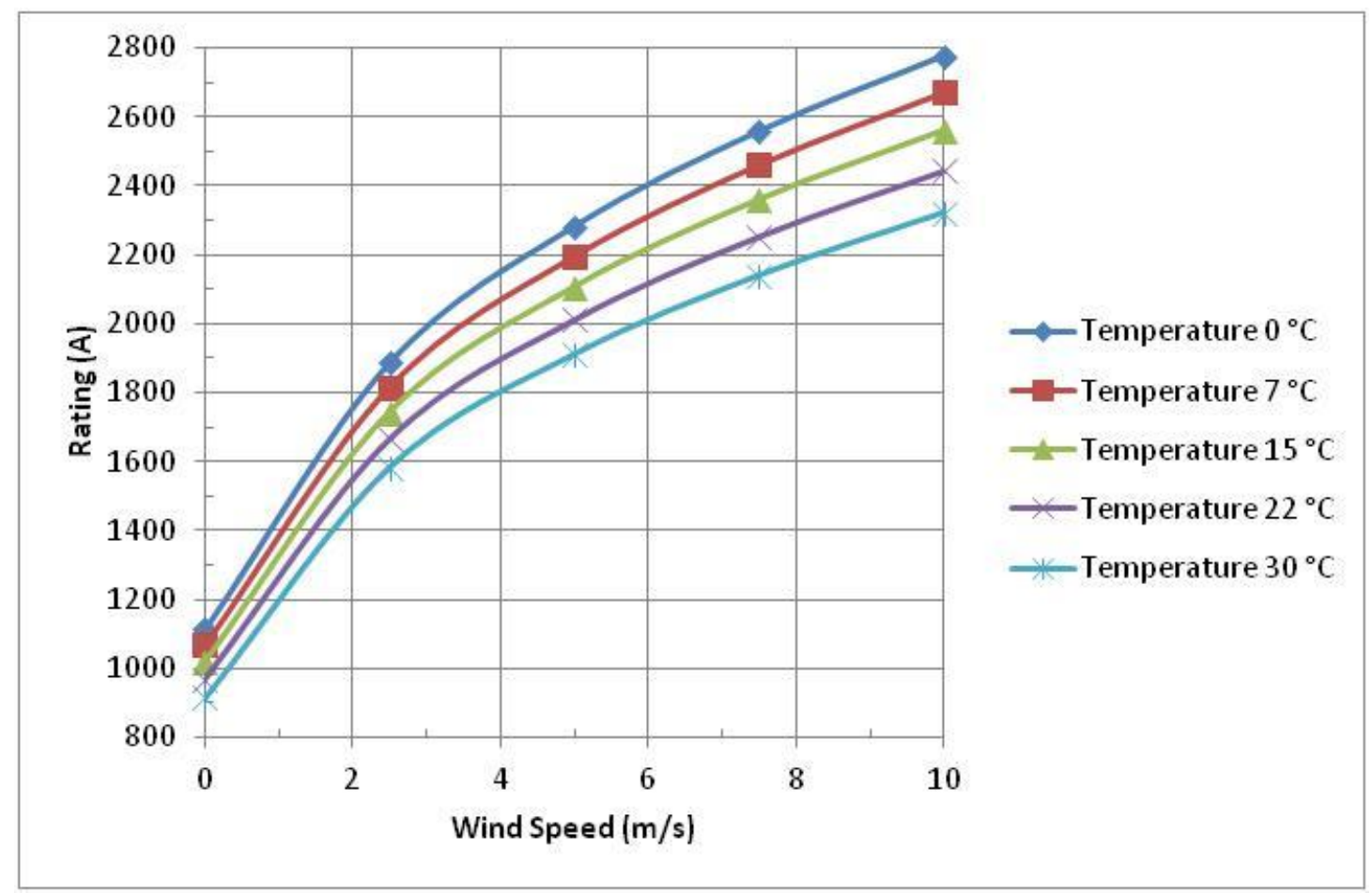

\section{Figure G-2}

\section{Air Temperature in ${ }^{\circ} \mathrm{C}$ and Wind Speed in $\mathrm{m} / \mathrm{s}$}

There is an approximate 200 Amps difference in rating with a $30^{\circ} \mathrm{C}$ difference in ambient air temperature. There is about 1660 amps difference in rating going from $0 \mathrm{~m} / \mathrm{s}$ to $10 \mathrm{~m} / \mathrm{s}$ wind speed with a wind direction that is perpendicular to the line. Not shown in the graph, even if the wind was parallel to the line the difference in rating would be just a little less than 700 amps. Clearly wind speed is the most important parameter in overhead line rating. The graph above also emphasizes the importance of low wind speeds. There is a large difference in rating between 0 and $2 \mathrm{~m} / \mathrm{s}$ wind speeds.

Sheltering from a row of trees could dramatically reduce the wind speed and consequently reduce the rating. Open geography like much of upper New York State makes it easy to place the anemometer. It is likely that winds near the line will be the same as winds a mile or two away when there are no trees or significant hills to disturb the flow of air. This provides a good environment for comparing technologies that use spot measurements to those that use distributed measurements.

Figure G-3 shows the effect of wind speed and wind direction. 


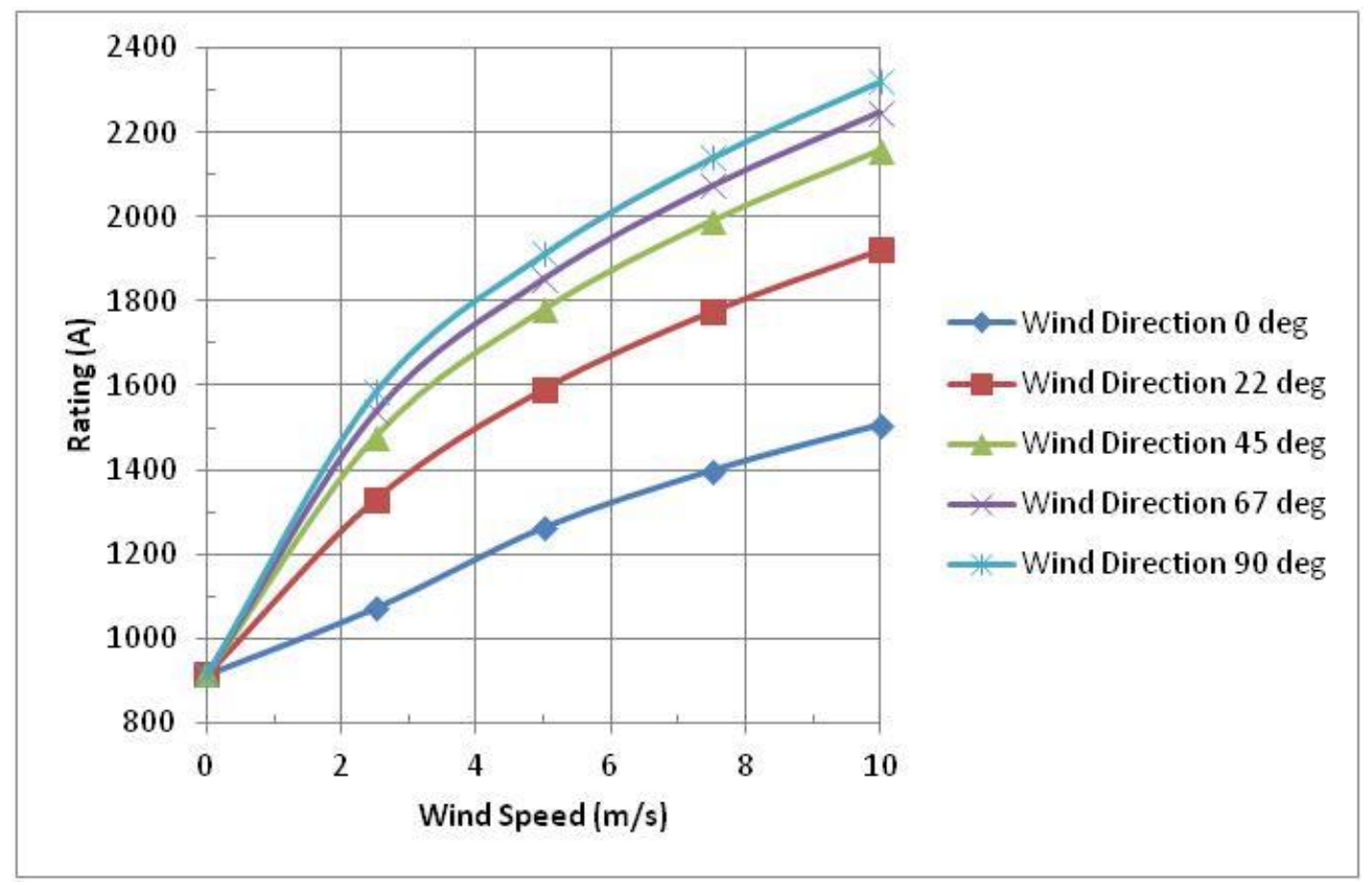

\section{Figure G-3}

Line Rating vs. Wind Speed for Different Wind Directions Relative to the Line Conductor

Wind direction plays a more important role in rating when compared to temperature. At 2.5 $\mathrm{m} / \mathrm{sec}$ the difference between 0 and 90 degrees wind angle in ratings is about 511 amps. At 10 $\mathrm{m} / \mathrm{sec}$ the difference between 0 and 90 degrees is about 815 amps. In contrast at $2.5 \mathrm{~m} / \mathrm{s}$ the difference in ratings between $0^{\circ} \mathrm{C}$ and $30^{\circ} \mathrm{C}$ is only $250 \mathrm{Amps}$ and at $10 \mathrm{~m} / \mathrm{s}$ the difference is 500 Amps.

It should be noted that a weather station must be situated in a location that is exposed to the same wind that the overhead line in exposed to if dynamic ratings are to be accurate and useful. If the line direction were to change and the wind were to remain constant, the rating of the line would change as a function of line direction. As Figure G-3 shows, this change is significant. 


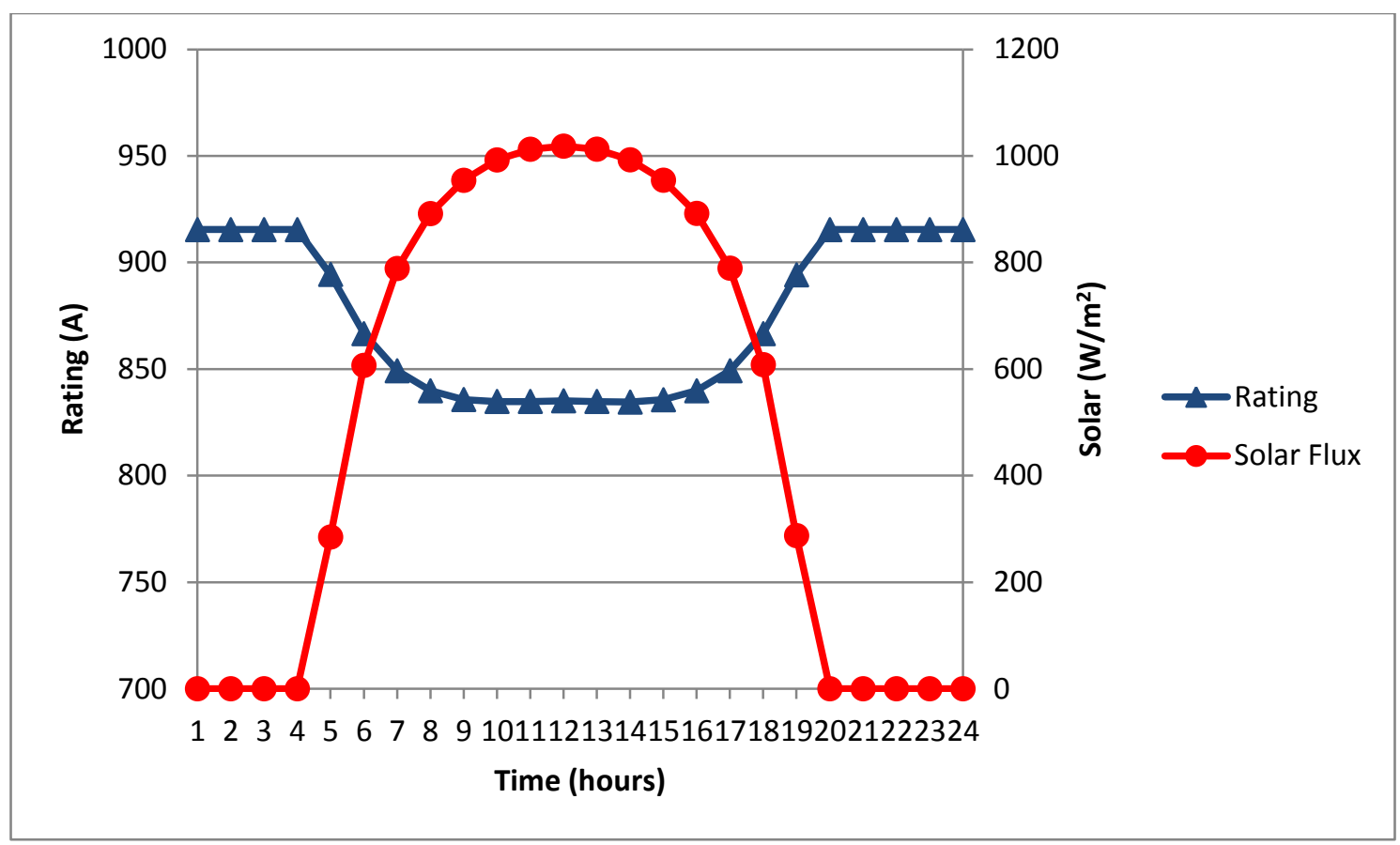

Figure G-4

Impact of Solar Heating on the Rating of a Drake Conductor with $2 \mathrm{f} / \mathrm{s}$ Wind Speed in the Month of June

Figure G-4 shows the effect of solar heating. Much like temperature the impact of the solar input is not as significant as wind. For the particular situation above, the difference between full sun and no sun is about 80 amps. As shown previously, wind speed and direction can make a rating difference of hundreds of amps.

\section{Real-Time Monitoring Methods - Definition and Comparison}

The graphs above show that it is critical that each rating device provide accurate and representative wind information. When using an anemometer it is necessary for DTCR to model the effect of wind blowing over the conductor so that it can accurately calculate the convective heat lost parameter. The ThermalRate device determines this by actually measuring this parameter and then calculating the equivalent perpendicular wind speed, so its location criteria are the same as the anemometer.

Air temperature is easy to measure and it is consistent over a large area. It is not significantly impacted by subtle terrain changes. Conductor load information can be obtained from the utility's SCADA system, the EPRI Sensor also has the ability to estimate the current flowing through the line. There are often some challenges getting the utility system to talk to other systems like the ones used on this project so the EPRI Sensor can fill this data gap if required.

There are a number of devices on the market that can measure solar flux. Solar flux, much like temperature is consistent over a large geographic area. Solar heat gain can also be determined by measuring the temperature of a similar conductor that does not have current flowing in it as the ThermalRate device does. 
Given the criticality of the wind, this section of the report will primarily focus how each device reports forced convection.

Three of the models used in this report are weather based. These models rely on a point measurement in space. These models are not affected by the amount of current flowing in the line. They will always provide a rating. Two of the models are conductor based. These models do require significant current flowing in the line before they can provide accurate ratings.

\section{On-Site Weather Station}

\section{On-Site Weather Station}

Generally, measurements of air temperature and solar heating at locations near the line are accurate enough to be used for thermal calculations at multiple spans. Wind speed and direction, however, must be measured along the line.

The simplest measurement of wind speed and direction is by means of an anemometer in the line right-of-way near the conductor. Traditionally, cup-type and even research-grade propeller-type anemometers are inaccurate at low wind speeds, especially if they were not frequently serviced to re-lubricate the bearings. With the advent of ultrasonic anemometers such as those used here at NYPA and described in Appendices C and D, many of these difficulties disappeared.

It is easy to do a sanity check on anemometers used for ratings by simply looking at other weather stations in the area. However, equations to convert the data are required so that the results reflect the way the line corridor responds to the wind. Because of this, this report uses the weather (wind based) model as a base line of comparison. It should be kept in mind that using this method for the control does not make it more "right" than other methods.

It is important to note that not all weather stations are created equal. When utilities are looking at doing line rating it is important to use high quality anemometers. Propeller and cup types do not respond to low wind speeds. These anemometers also require maintenance. Experience with these devices has shown that dusty environments can result in failed bearings, leading to loss of rating data, in as little a year time. Also, stall speeds will result in significantly reduced ratings. Ultrasonic devices have a number of advantages; these devices do not have the stall speed issues, they have no moving parts. Some of these devices could be more prone to high EMF issues than others. No such issues were observed at NYPA. Overall results between the weather station and the NOAA data are in good agreement, suggesting that accuracy of the individual sensors was not an issue. Detailed information on anemometer characteristics and output for this study are presented in Appendix D.

\section{ThermalRate Conductor Model}

ThermalRate physically simulates the heat transfer of the overhead conductor using a heated and an unheated conductor section mounted near the line. This in concept has a number of advantages described earlier in this chapter. It is difficult to check the ThermalRate directly against other weather stations since the output parameters are not the same but in this project the ThermalRate predicted conductor temperature could be checked against the EPRI Sensor measured conductor temperature. In this study checks revealed that the ThermalRate device 
predicted conductor temperature as measured directly with the EPRI Sensors more consistently and accurately than any other calculation element.

The ThermalRate device is a special purpose weather station with built-in electronics to calculate real-time line ratings. It can be installed at any point within the line's ROW though it should be at the height of the middle third of the conductor span and not too far from the energized conductor. Since it does not mount on the energized conductor, and since there is no need to "calibrate" the device, no line outage is required.

Figure G-5 shows ThermalRate directional anemometer mounted adjacent to a transmission line. The accuracy of this device in measuring the effective perpendicular wind speed at the energized conductors of the transmission line, require that it be mounted closer to the line and at approximately the average of the lowest of the energized conductors.

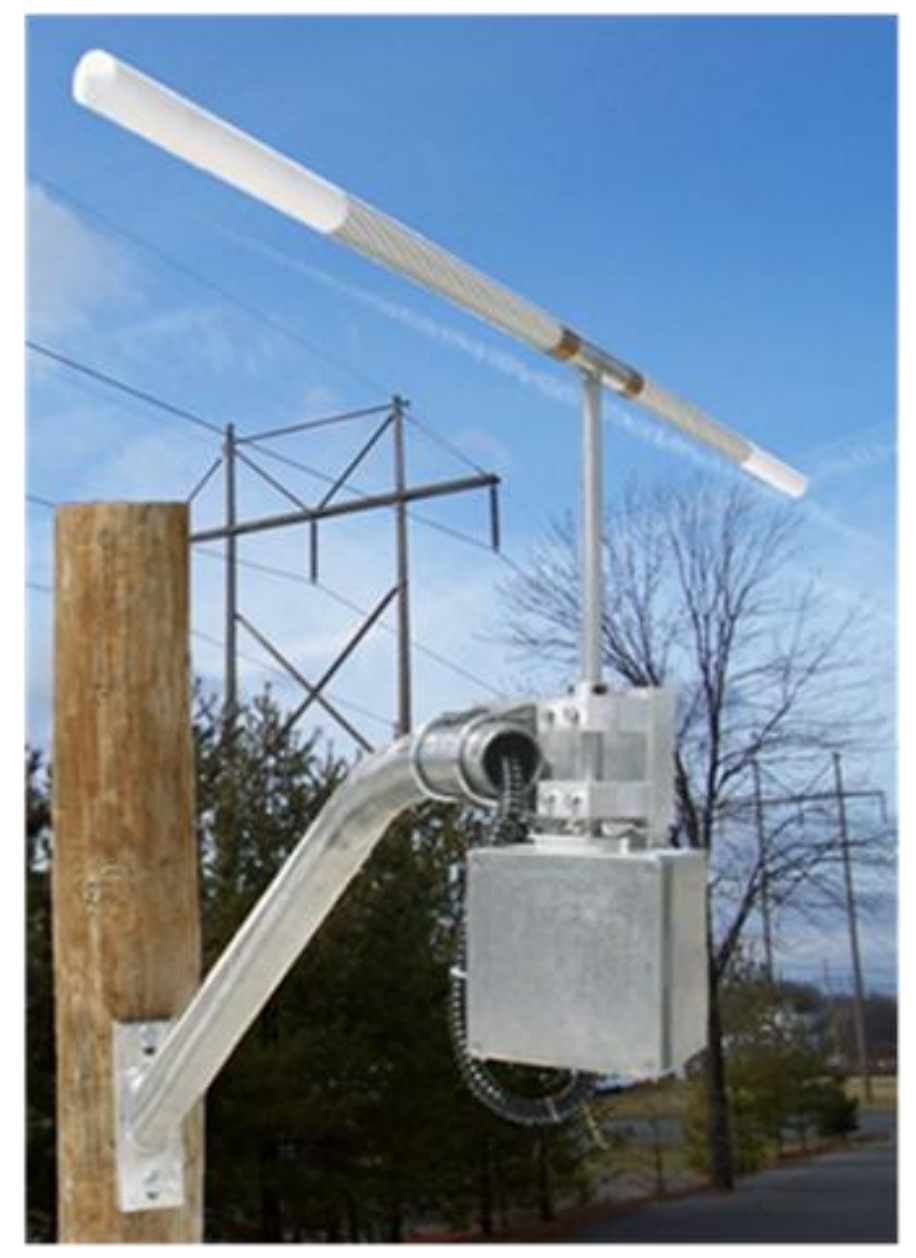

Figure G-5

A ThermalRate Directional Anemometer

The ThermalRate device consists of two machined aluminum rods that are thermally separated, have the same outside diameter and stranding pattern as the line conductor, and are oriented in the direction of the adjacent span. One half of the device is heated only by the sun and air, while the other half is heated also by an internal core heater. The temperature of each half is monitored by high precision thermocouples. 
The unheated half of the device is at the solar temperature of the line conductors, that is, the temperature of the conductors with no current flowing in them. It is typically about $5^{\circ} \mathrm{C}$ above air temperature on a sunny day.

The other half of the device is at the temperature of the energized conductors with simulated high load times the known Ohmic heat per unit length equal to that of the core heater watts. By comparing the temperatures of the two halves, the conductor temperature rise above solar temperature under this simulated load is known and $V_{\text {wind }}$ for the line section can be calculated.

The device had a few issues during the project but overall it proved to be reliable. One issue with this device is the power consumption. This device required large solar panels to provide the constant power to the hot rod. If AC power could be used this would dramatically reduce the foot print of this device. It may also be possible to better optimize the solar array and battery pack depending on reliability requirements. Reducing the solar array and battery packs would reduce the wind load on the structure.

Limitations on accuracy involve how close the device can be installed to the energized conductor that it is modeling and the extent to which the ThermalRate device emissivity and absorptivity are the same as the line conductors. The device provides $V_{\text {wind }}$ as a point measurement for the line near the installation. Like a weather station it can be located in the most sheltered area if conductor temperature rather than clearance is the primary thermal constraint.

\section{Video Sagometer}

The pole mounted camera and conductor mounted target system logged clearance and correlation data regardless of current levels but requires significant current in the line to produce ratings. The camera unit had more reliability issues than would be expected for a commercially available device. It seems that the power supply was slightly undersized, which contributed to some of the failures. There were a number of communications hang-ups between the camera and data logger that required a manual reset of the camera (performed remotely by cell phone). After some time many of these issues were addressed making the system more reliable. Given the lightly loaded lines at these Sites this device was not useful for rating most of the time.

The Sagometer was developed by EPRI but is now commercially available. There occasionally have been difficulties in maintenance mostly related to communications links. The system is put together by EDM in conjunction with the standard Fisher Scientific instrumentation package and the high quality on site weather station (including ultrasonic anemometer). It is fair to say that the Sagometer is a mature instrument (3rd version). The sagometer can be installed without taking an outage but it may need an outage in order to ensure proper calibration. 


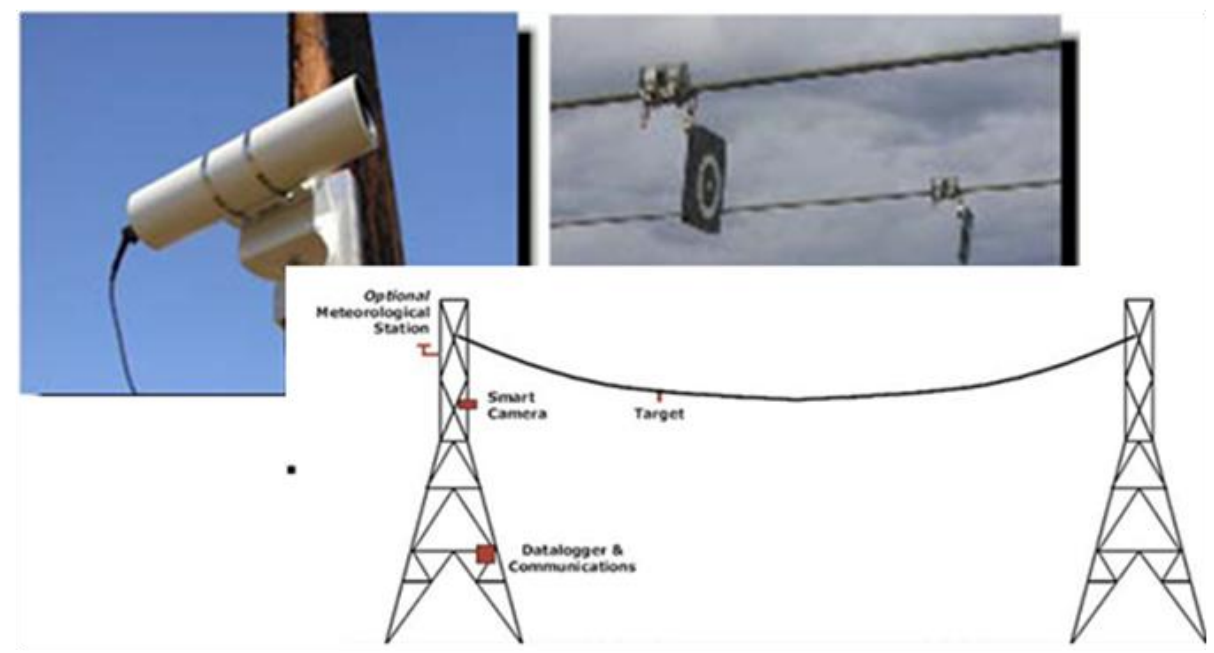

\section{Figure G-6}

The Sagometer Camera and Target Mounted on the Conductor

Both the Video Sagometer and tension monitors require the state change equation procedure outlined in Appendix B to determine the relationship between line sag/tension and the average conductor temperature in the sag-section where they are mounted.

\section{EPRI Sensor}

The EPRI Sensor for the purpose of rating was of limited value much like the Sagometer, due to the loading conditions. However, it is extremely useful for providing line loading information. It also provided a direct measurement to compare with the calculated conductor temperature from other technologies. These devices are prototypes; consequently, reliability expectations are lower than they would be for a commercial device. These devices became less reliable as time went on and field degradation is being addressed in future versions. EPRI Sensors are in the development stage where constant updates and improvements are being implemented. Many upgrades are a direct result of this project. Short comings of this device are temperature range, communications distance, and reliability. The latter two issues have already been worked on and notable improvements have been made.

The EPRI Sensors measure the temperature, line current and the span inclination of the energized conductor on which it is mounted. Only the first two parameters were used in this project. The DTCR software converts the measured conductor temperature into an effective perpendicular wind speed which can be used to calculate the dynamic rating of the line by using the SAG equation option and using a simple state equation of $\mathrm{T}=1 \times \mathrm{x}$ T measured. This is accomplished in DTCR by including an input 1 as the $x^{\wedge} 1$ multiplier and setting all other multipliers to 0 in the $4^{\text {th }}$ order polynomial input dialog box (see Chapter 5) 


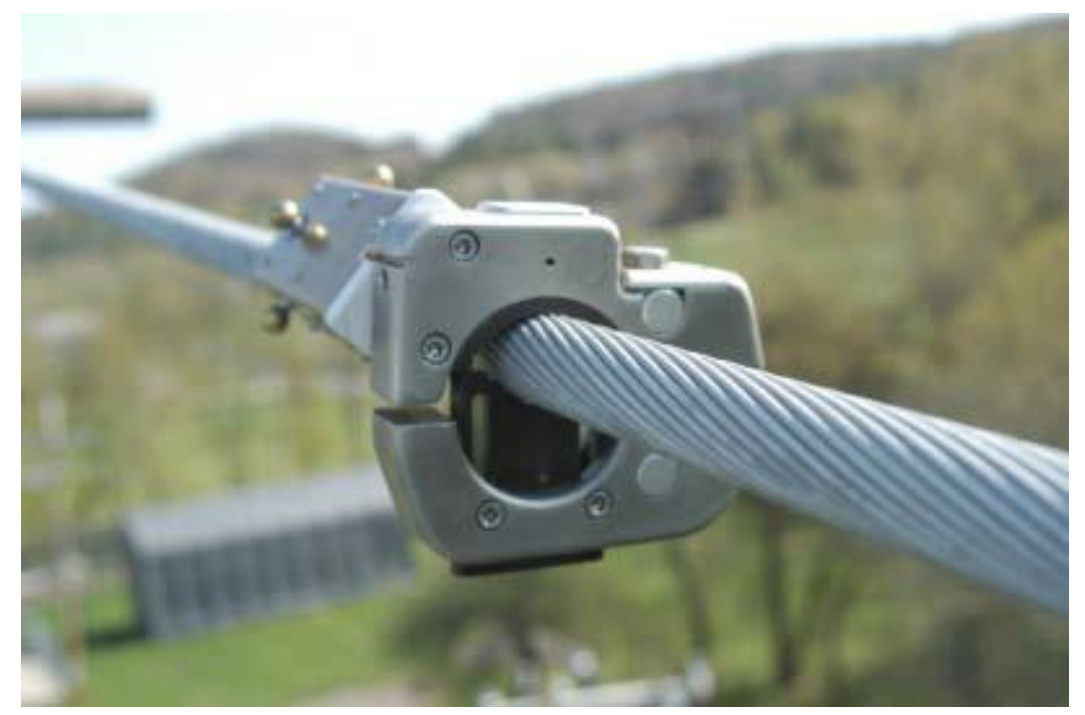

Figure G-7

EPRI Sensor Temperature Monitor

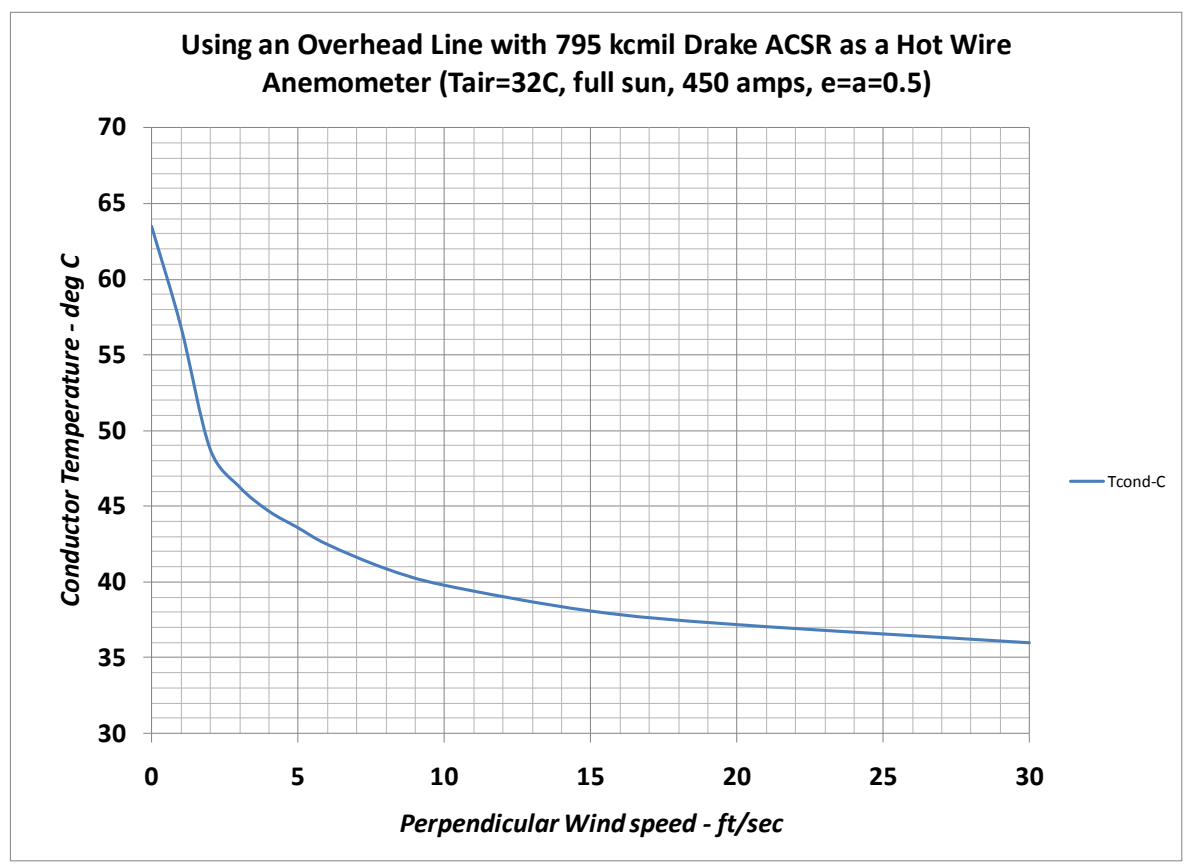

Figure G-8

Relationship Between Conductor Temperature and Effective Perpendicular Wind Speed

Thermal rating is calculated for a hypothetical typical 1 meter length of conductor where the air temperature, the solar heating, and the wind speed and direction relative to the conductor are known. The errors in calculating convection and radiation heat loss are very small in comparison to the errors that can result from using incorrect weather data, particularly wind speed and direction.

The use of real-time monitors does not guarantee accurate line ratings unless the monitors yield an accurate estimate of wind speed and direction across the conductor. The accuracy can be estimated by comparing the calculated conductor temperature from a specific device to that 
directly measured by the thermocouples in the various EPRI Sensors. In the case of this study where there the variations in conductor temperature with respect to line position are minimal, the EPRI Sensor output could be used to assess accuracy of the other elements and even to calibrate the state equation of the Video Sagometer.

\section{Off-Site Weather Service Data (Backup)}

Second party weather stations use equipment that is out of the control of the utility. Older cuptype anemometers stall at wind speeds higher than ultrasonic anemometers. Current commercially available cup-type anemometers report stall speeds of about $0.4 \mathrm{~m} / \mathrm{s}$ whereas the ultrasonic anemometer used on site could have been set as low as $0.01 \mathrm{~m} / \mathrm{s}$. This stall speed can create an issue if there are low winds. Meaning at lower wind speeds, the ratings provided may be overly conservative. The instances of zero wind speed in Figure G-9 are an indication that some stall speed issues could be confused lack of data. The NOAA service reports zero wind speed when the system is in an error state. The weather service wind measurements were only updated hourly, yielding a poorer quality estimate of wind speed and direction for nearby transmission line rights-of-way than the onsite weather station in real-time. Figure G-9 shows both downsides of the weather service data. On 7/19/12 the service was not available so a wind speed of zero was reported. The weather service could closely match or surpass the onsite weather station for availability with an attentive operator. On 7/27/12 the low wind speeds were "rounded down" due to the step-like nature of the data and therefore a more conservative rating would be calculated. Both on and off site weather data indicate the wind speed was below the static rating assumptions for multiple periods during this brief sample, this was not a rare occurrence.

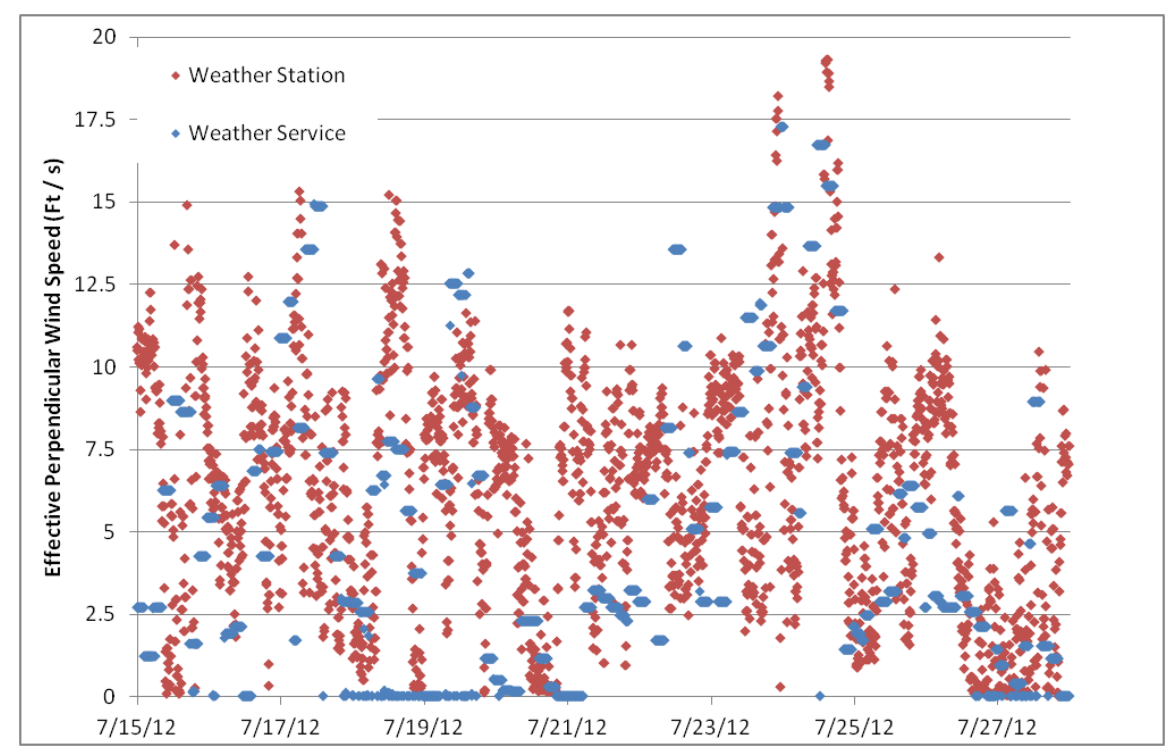

Figure G-9

Instance of Anemometer Stall Evident in NOAA Data

\section{Substation Wind Measurements}

High accuracy ultrasonic anemometers placed in a transmission line substation, can produce much better estimates of wind speed in the transmission line right-of-way than airport 
measurements do, however, sheltering by trees and terrain in the ROW can only be estimated and the line spans furthest from the substation location may experience different wind speeds and directions from those measured. Placement in the substation simplifies maintenance, reduces vandalism, and makes communication of real-time data easier.

Offsite weather data has several drawbacks but is potentially useful as a backup to the other monitoring methods. Location is perhaps the largest of them; the location is not necessarily comparable to the location of the transmission line. Studies or modeling would have to be done to determine the effect of local terrain on critical spans. For the lines studied here, the offsite data typically was more conservative than the onsite data. Meaning although it was not as accurate as the ROW data, using it would not (on average) increase the operator's risk factor. The data used was only updated once per hour, it would be preferable to use a service that updates more frequently if STE ratings were to be accurately determined.

Also, the data vendor can change the format of the data without notification. This did happen during this project. Temperature data from the internet sources should be more accurate than wind data. Solar information should be good as well. Wind is the challenge. It is conceivable that with some studies, airport data could be used as a backup to a weather station on the line. In other words, to improve real-time rating reliability, airport data at this site could temporarily be substituted for weather station data. It may be desirable to add some level of conservatism to the backup data. Any time data from a weather service is going to be used with no other instrumentation for the purpose of line ratings caution would be advised. It will be necessary to show strong correlation between the reported wind and the wind along the line.

\section{Summary Comparison of Monitoring Methods}

Some of the key aspects of the four different monitoring methods studied in this project are summarized in Table G-2.

Table G-2

Comparison of Monitoring Methods

\begin{tabular}{|c|c|c|c|c|}
\hline Monitor Method & $\begin{array}{c}\text { Min L } \\
\text { (A/Kcmil) }\end{array}$ & $\begin{array}{l}\text { Local or } \\
\text { Line-section }\end{array}$ & Quantities Measured & Power Source \\
\hline Onsite Weather & 0 & Local & Wind, Ta, Qs & solar \\
\hline ThermalRate Model & 0 & Local & Model Temp, Ta, Ts* & solar \\
\hline EPRI Temp sensor & 0.5 & Multi-Local & Line Temp, current & battery \\
\hline Video Sagometer & 0.5 & Line Section & Target Clearance & Solar \\
\hline Off Site (NOAA) & 0 & Remote & Wind, Ta, Qs & NA \\
\hline
\end{tabular}

The ThermalRate model measures the solar temperature, Ts, of the conductor model rather than the solar heat intensity, Qs.

Note that:

- The weather based and ThermalRate conductor models methods work well even if the line current is zero. 
- The Video Sagometer does not work well at low line currents but it measures average quantities over the entire line section in which it installed and it gives a direct sag clearance measurement at high current levels. As result, if conductor clearance is the design constraint on the line then it is possible to use fewer Video Sagometer monitors than temperature/wind monitors in rating long lines.

- If conductor temperature, rather than clearance, is the design constraint then the three local technologies installed at the most sheltered location(s) provide ratings based on the hottest conductor section and averaging of conductor temperature (using a Video Sagometer) is actually a disadvantage in this case.

\section{Accuracy of Wind Measurements by Monitoring Method}

The measurement of wind speed with the ultrasonic anemometer shows no evidence of a stall speed below which the instrument reports zero wind speeds. The plot shown in figure G-10 shows a time series of the wind speed and wind direction as reported by the onsite weather station at Site 2. Each data point is a 10-minute average of 1 -second data values. This process has been discussed in detail in Appendix D.

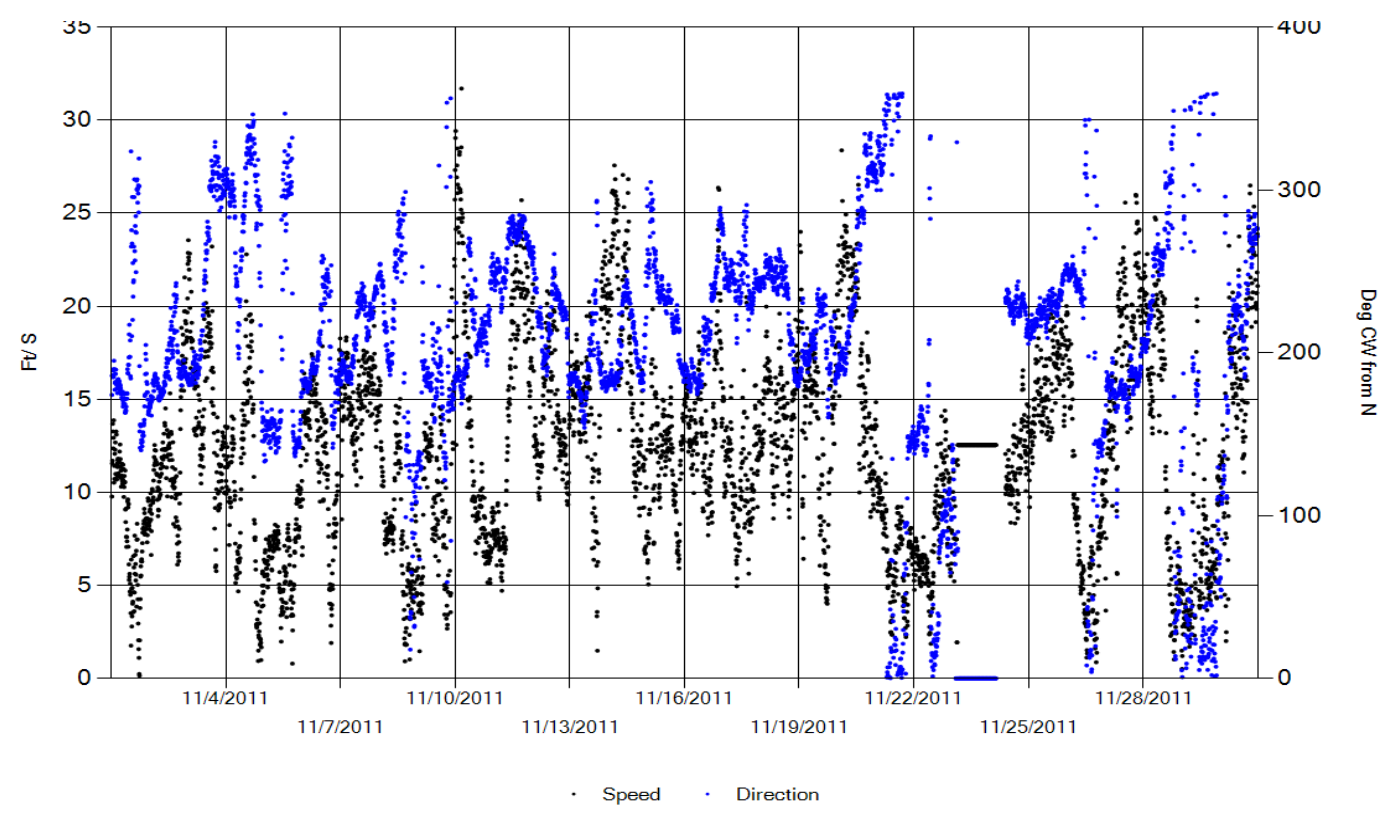

Figure G-10

Plot of Absolute Wind Speed and Direction Measured by Ultrasonic Anemometer for the Month of November at Site 2 


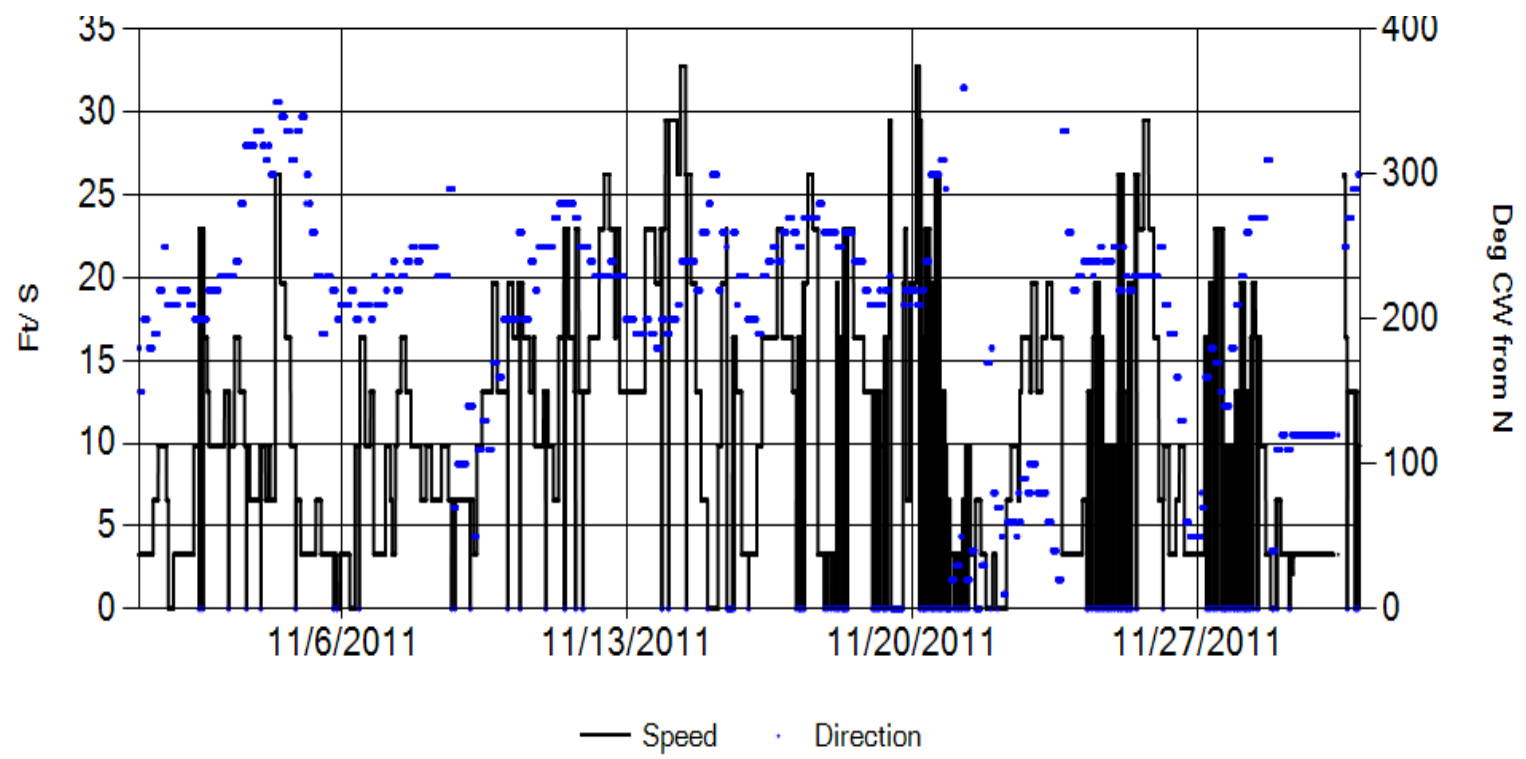

Figure G-11

Wind Speed and Direction as Provided by NOAA for a Region Encompassing Site 2

Figure G-11Error! Reference source not found. shows wind speed and direction based on data aken from the offsite weather service. It is apparent that the wind data quality and precision is much less than that of an onsite ultrasonic anemometer, however, the offsite estimates covers a wide area and includes forecasts of wind speed and direction. These forecasts can be used as inputs in the rating software to improve both onsite and offsite weather models. A sample of the software inputs is shown below in Figure G-12.

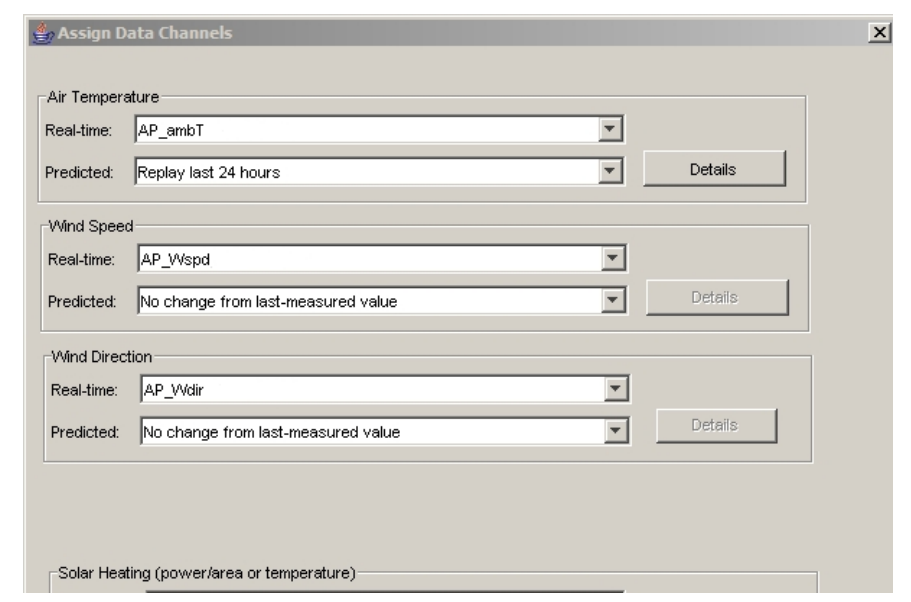

Figure G-12

Forecast Wind Speed and Direction from NOAA National Weather Service

NOAA derives the offsite wind data through a complex process of interpolating multi-station data. Though the mathematics is powerful, the quality of airport wind data can still occasionally be poor. Due to the nature of the region, the interpolated data is largely determined by airport data in this region of New York.

Another limitation to this particular offsite weather data is that it is updated only once an hour. Since the typical thermal time constant of bare overhead conductors is in the range of $\sim 15$ 
minutes, an hour interval is most useful as a backup data source if the primary monitoring method is out of service. This difference in update interval is clearly seen in Figure G-13

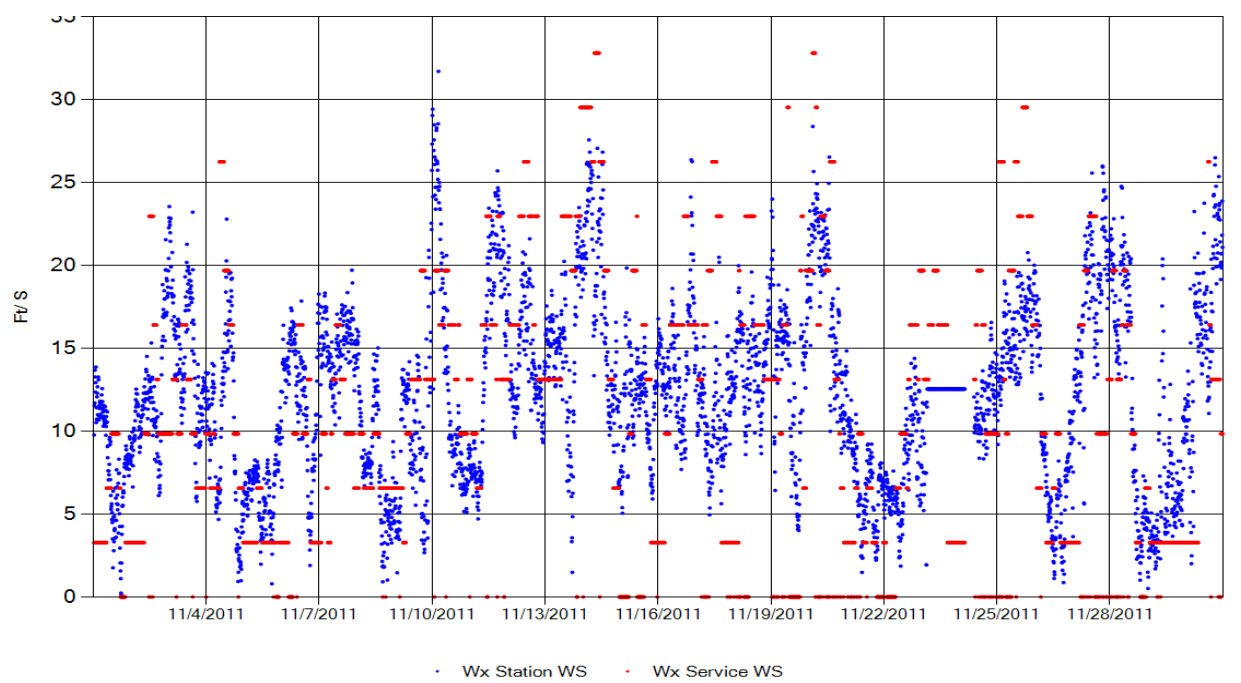

Figure G-13

Comparison of Offsite NOAA Interpolated Data and Ultrasonic Anemometer Wind Speeds at the Onsite Weather Station Located at Site 2

\section{Statistical Comparisons of Effective Wind Speed - Offsite and Onsite Wind Data}

The data precision limitation of offsite wind data is illustrated in Figure G-13and Figure G-15, which compares the cumulative distributions of wind speed for summer and winter.

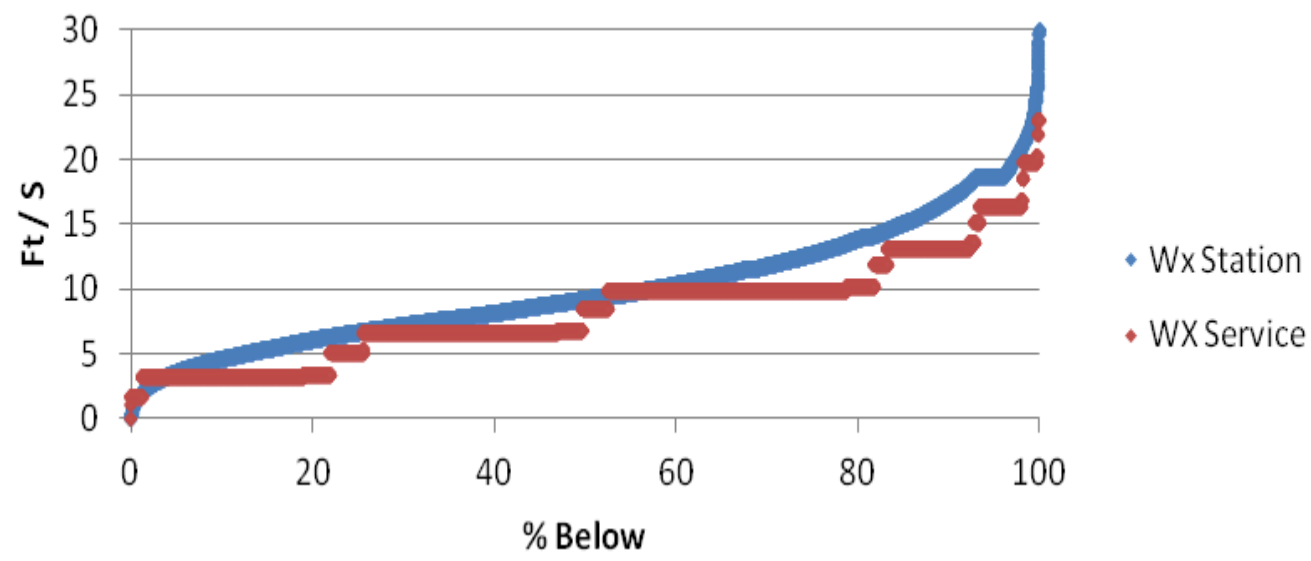

Figure G-14

CDF of Wind Speeds for Site 2 During the Summer of 2011

The CDF plots show that the speed data distribution is similar but that the measurement interval of the offsite NOAA data is much larger. This makes little difference in the evaluation of highspeed winds but can be a serious limitation in calculating line ratings where the difference in line rating for a $3 \mathrm{ft} / \mathrm{sec}$ and $5 \mathrm{ft} / \mathrm{sec}$ crosswind can be as much as $10-15 \%$. 
As shown in both figures over a large enough sample the data correlates much more closely than it does on a per measurement basis. This underlines the potential to utilize this data for ratings calculations, though with the current state of the science, it may not be the best option for realtime ratings. To that effect, further study is needed.

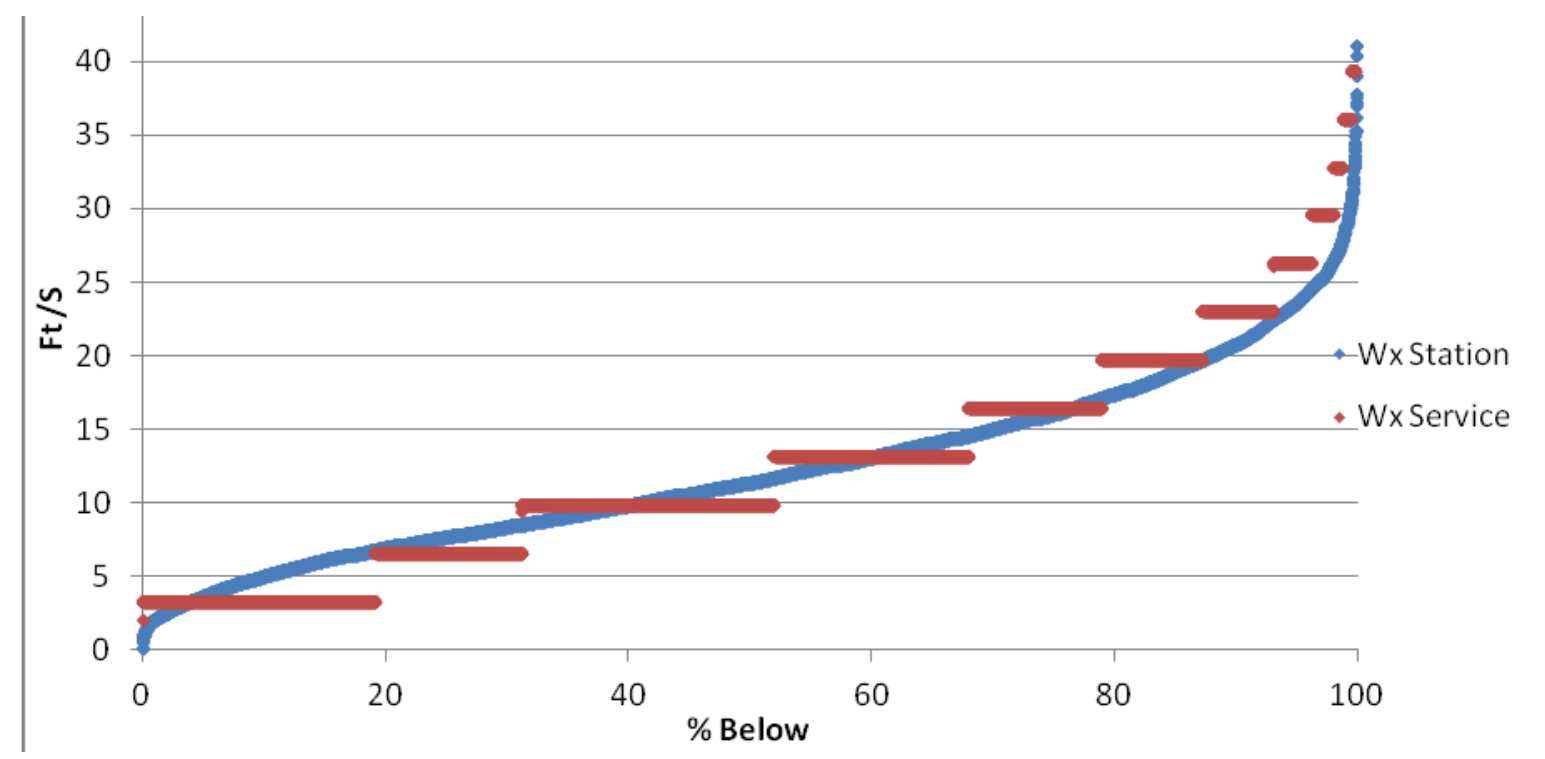

Figure G-15

CDF of Wind Speeds for Site 2 During the Winter of 2011

\section{Comparison of Onsite Weather Station and ThermalRate Wind Speed}

\section{Equivalent Perpendicular Wind Speed for ThermalRate Conductor Model}

The ThermalRate monitoring system compares the temperature of the heated conductor model with a known heat input to the unheated conductor model. The monitoring system uses the conductor heat balance equation shown here as the basis for estimating an effective perpendicular wind speed $\left(V_{\text {wind }}\right)$.

$$
K_{c} \cdot V_{\text {wind }}^{0.6} \cdot\left(T_{C}-T_{S}\right)+K_{r} \cdot\left(T_{C}-T_{S}\right)=\text { HeatInput }
$$

\section{Equation G-6}




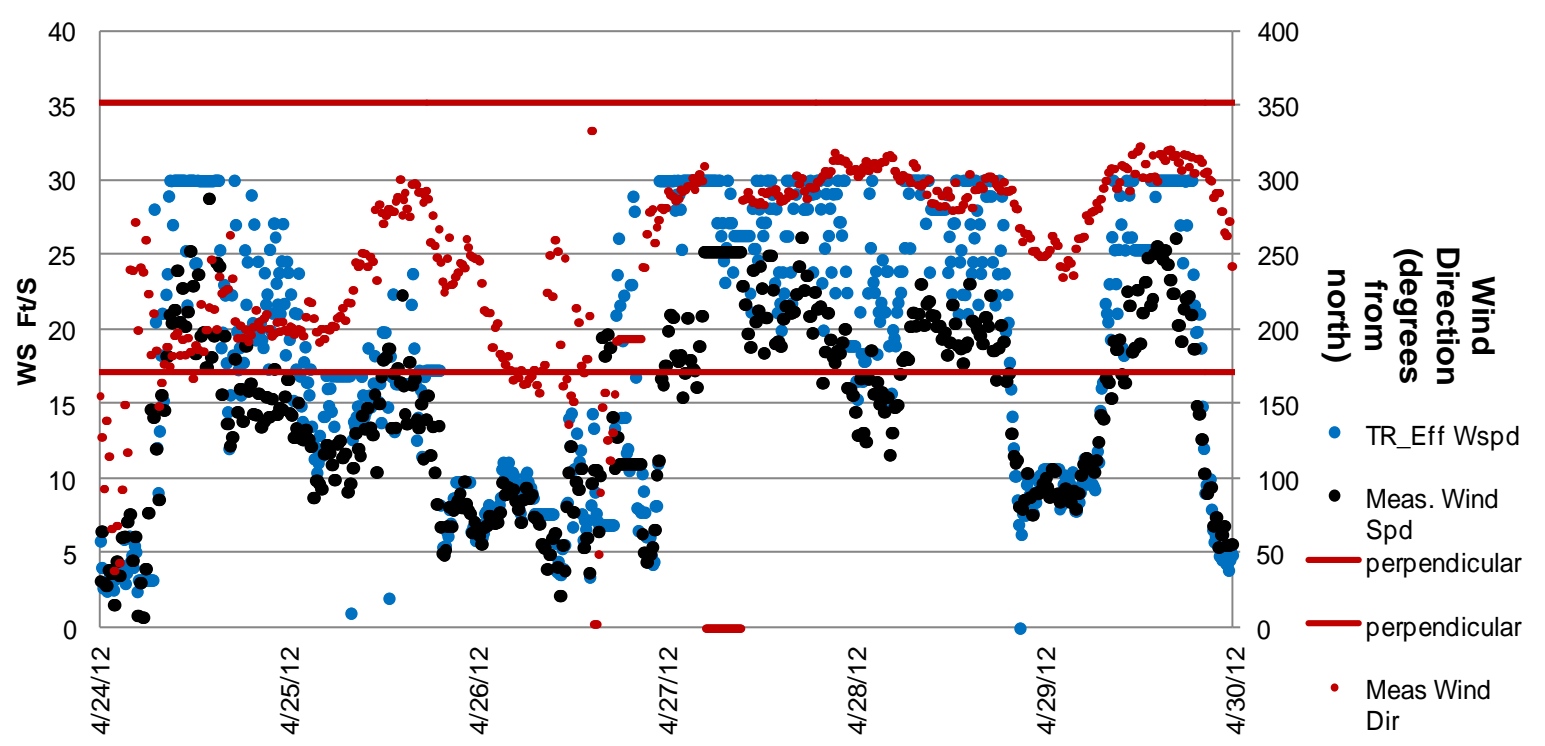

Figure G-16

Effective Perpendicular Wind Speed Estimated by the ThermalRate Conductor Model Monitoring Method

The ThermalRate $V_{\text {wind }}$ results are a monthly plot in Appendix K. The ThermalRate $V_{\text {wind }}$ results are compared here to on site weather station data. Figure G-16 is a typical comparison for a week long period in April 2012. Recall that $V_{\text {wind }}$ should vary from being identical with absolute wind speed when the wind is perpendicular and about 0.2 times absolute wind speed when the wind is parallel to the conductor. In theory $V_{\text {wind }}$ should never be higher than absolute wind speed.

Figure G-16 shows that effective perpendicular wind speeds calculated by ThermalRate were often higher than absolute wind speed. Clearly this was not an anticipated result. Many hours of EPRI and manufacturer engineering time were spent in trying to find an error in the data including running additional wind tunnel tests on the ThermalRate device. No errors were identified.

The ThermalRate device includes stranded replicas of the Drake conductor used on the lines. IEEE-738 calculations model conductors as round smooth cylinders. The ThermalRate manufacturer believes that the stranding increases the heat transfer and that the ThermalRate device takes stranding into account while the present theoretical methods including IEEE-738 do not. A possible effect of stranding is to increase turbulence in the boundary layer of air near the conductor. Turbulence in this location increases heat transfer dramatically because heat transfer is dominated by mixing rather than heat conduction through laminar layers of air (which is a poor heat conductor). This increase in heat transfer shows up as higher wind velocity.

One piece of evidence supporting the manufacturer's claim is that turbulence increases with speed so the effects should be greater at greater wind speed. This is the case in Figure G-16.

Figure G-17 shows 11 days of conductor temperature data for ThermalRate (calculated), the onsite weather station model (calculated) and the EPRI Sensor (measured). The primary heat input into the conductor was solar for this period and a diurnal period is the resulting pattern. 


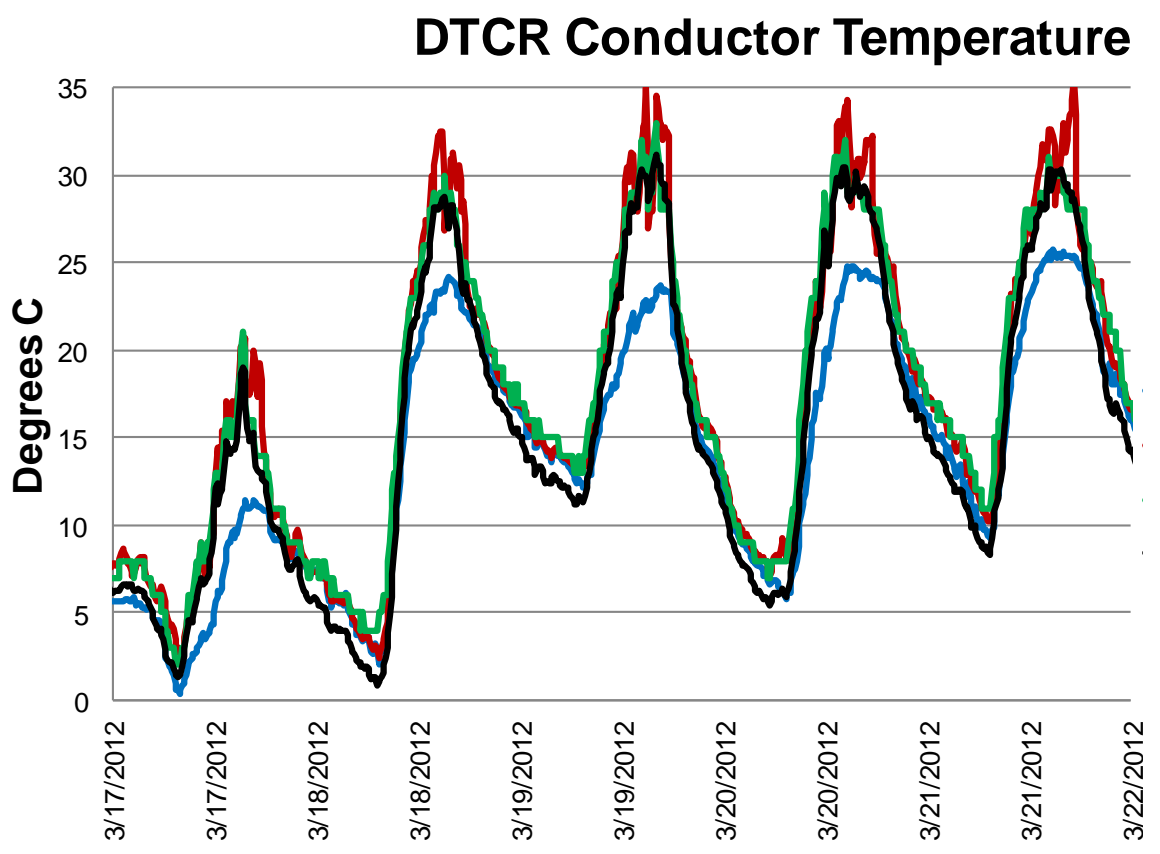

Figure G-17

Comparison of EPRI Sensor (Green) to ThermalRate (Black) and Onsite Weather Station (Red) Models. Ambient Temperature is Shown (blue) as a Reference

For this period the ThermalRate device followed EPRI Sensor measured values well. The ThermalRate measured ambient temperature was slightly lower so a small bias to low temperature shows up at night (low temperatures) on its predicted conductor temperature. The weather station clearly predicts less heat transfer than measured by the EPRI Sensor or than predicted by ThermalRate. This graph is an indication that the ThermalRate $V_{\text {wind }}$ predicts the correct heat transfer even though the results appear physically impossible when considered as a wind vector. Higher line load should accentuate the differences and provide stronger evidence.

A clear conclusion is that ThermalRate is less conservative in predicting heat transfer than the onsite weather station and IEEE-738 methodology. This is consistent with the higher ratings produced by ThermalRate as shown in Appendices E and G.

\section{Accuracy of Solar Heating Measurements}

Since the monitored NYPA lines carried relatively low currents throughout most of the project, the accuracy of effective perpendicular wind speed calculations with the Video Sagometer and the EPRI Sensor, is very sensitive to errors in the solar heat input to the conductor.

For example, a $795 \mathrm{Kcmil}$ Drake conductor carrying 350 amperes, with an air temperature of $25 \mathrm{C}$ and a measured conductor temperature of $35 \mathrm{C}$, the calculated perpendicular effective wind speed is $4.5 \mathrm{ft} / \mathrm{sec}$ if the conductor is receiving the summer maximum solar heating and it is 0.6 $\mathrm{ft} / \mathrm{sec}$ if there is no solar heating. Therefore, the accurate measurement of solar heat input is critical to accuracy with the Video Sagometer and the EPRI Sensor methods. 


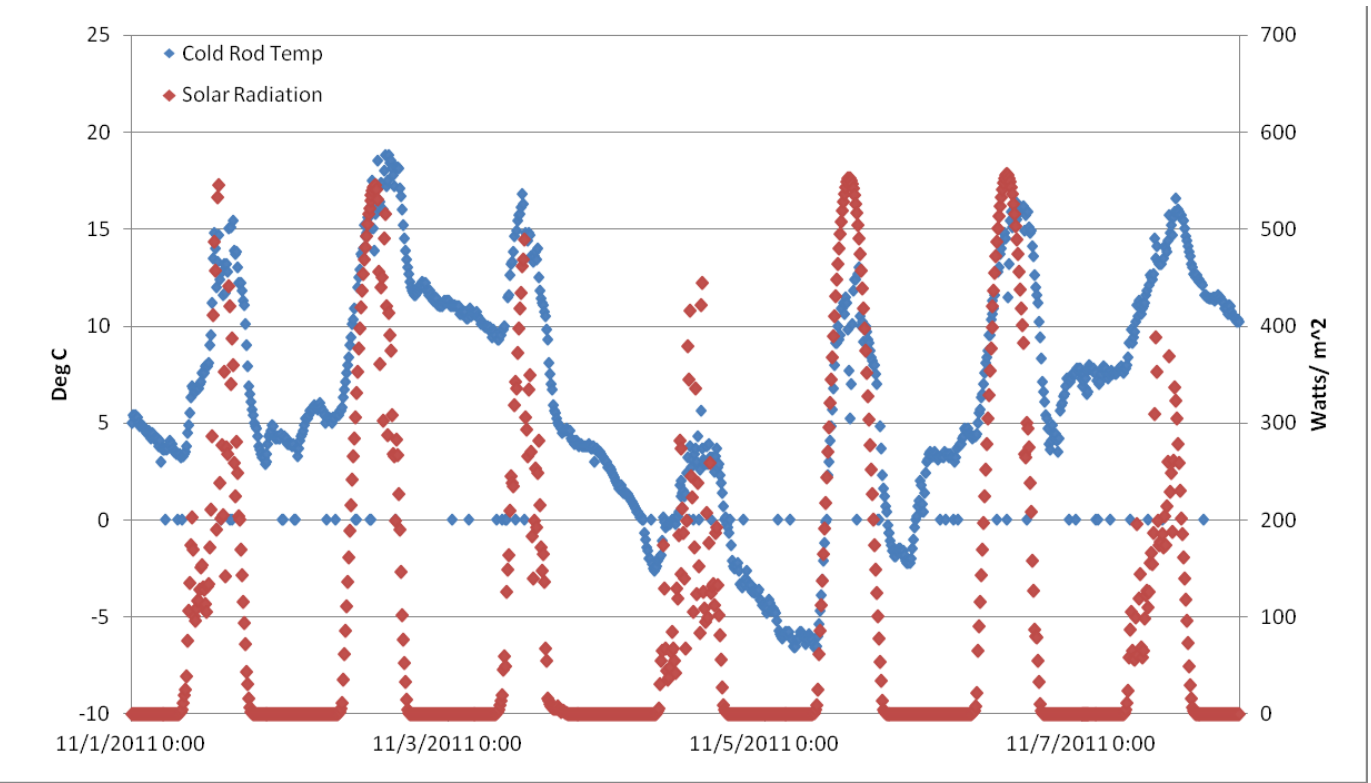

Figure G-18

Comparison of Solar Temperature Rise as Measured by ThermalRate Conductor Model to That Calculated from Solar Pyranometer Measurements During a Week in November of 2011

This graph shows the ThermalRate cold rod temperature and the weather station pyranometer. These two different methods can be used in conjunction with DTCR to determine the effect of solar heating.

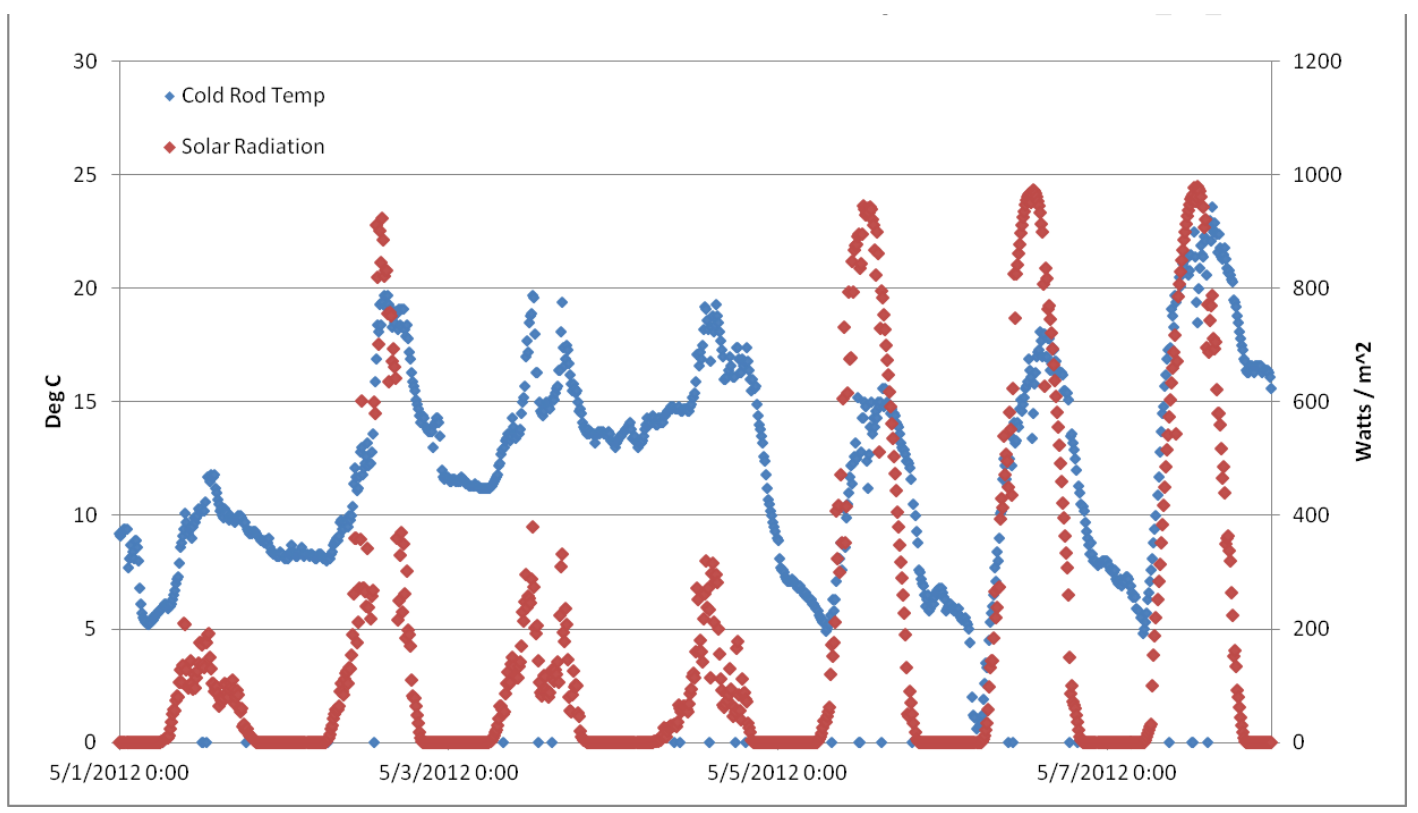

Figure G-19

Comparison of Solar Temperature Rise as Measured by ThermalRate Conductor Model to That Calculated from Solar Pyranometer Measurements During a Week in May of 2012

This graph shows the ThermalRate cold rod temperature and the weather station pyranometer. Each of these techniques has strengths and weaknesses. Cold rod temperature method requires 
that the sensor mimic the surface of the conductor. Emissivity and absorptivity for example must be well matched with the line. The pyranometer must have a frequency response that is appropriate for the heating of the conductor. The equations must then appropriately model the response of the conductor.

\section{Accuracy of Air Temperature Measurements}

Air temperature is generally similar over fair distances unless there are large changes in elevation. There were three sources of air temperature in this project: the ThermalRate conductor model measures air temperature, the onsite weather station has a high quality air temperature sensor; and the offsite weather service estimates air temperature. Figure G-20 illustrates the good agreement between the three estimates for the month of November, 2011. It also shows that the offsite weather service does not provide as fine an interval in both magnitude and time as the other two monitors.

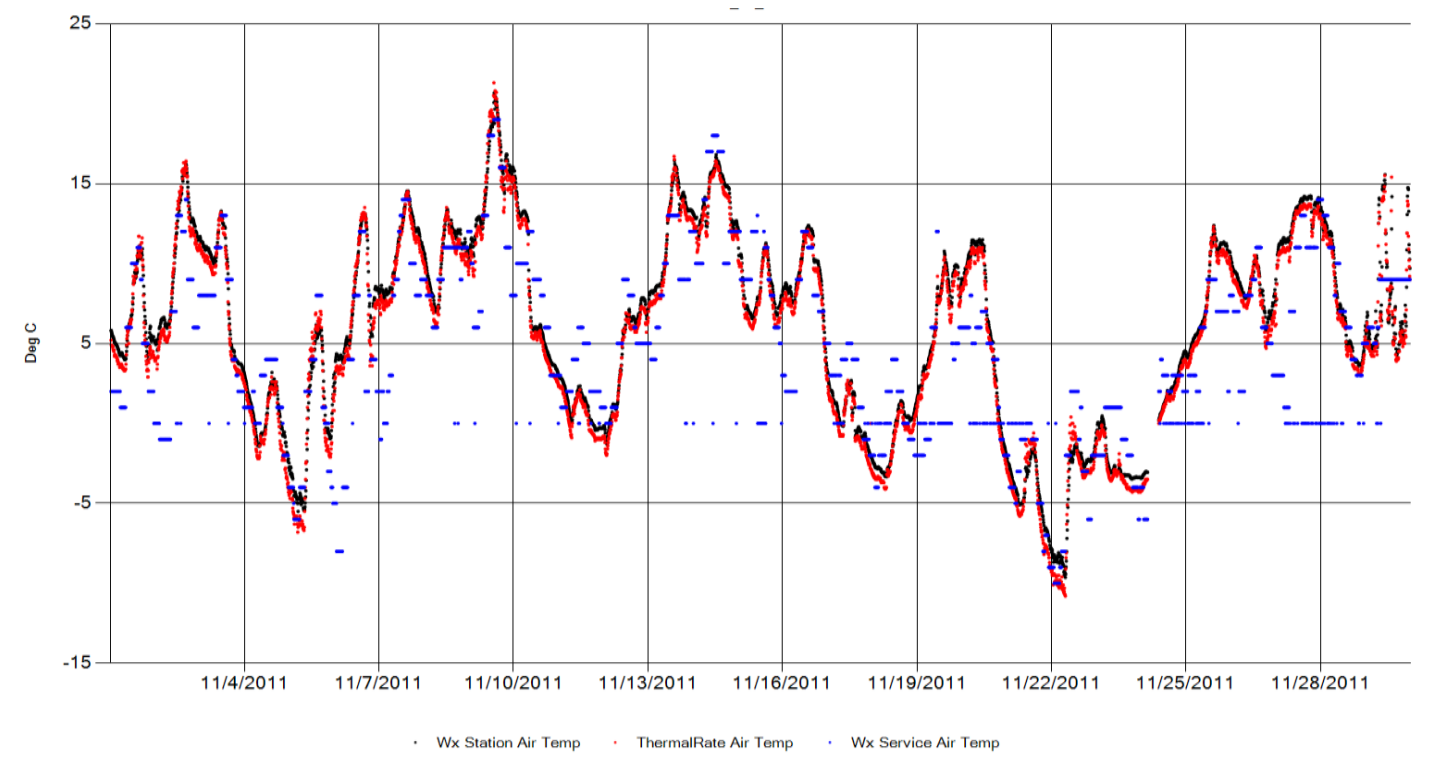

Figure G-20

Comparison of Air Temperature Reported by NOAA Airport, the ThermalRate Conductor Model, and the Onsite Weather Station at Site 2

\section{Accuracy of Line Current Measurements}

It is good to see that line current as measured by the EPRI Sensor method and that provided by NYPA SCADA data agree so well. 


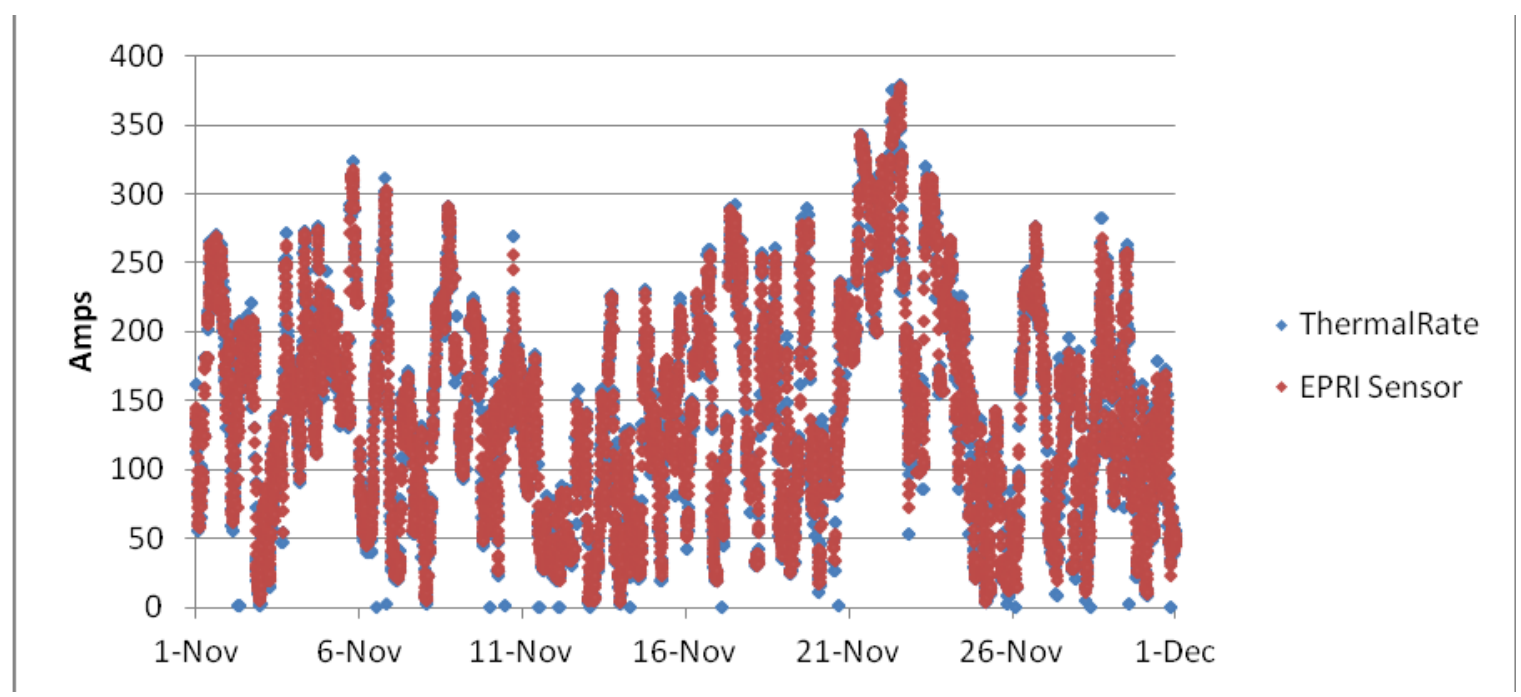

Figure G-21

Comparison of Line Current Measured by the EPRI Sensor and Reported by NYPA SCADA

In Figure G-22 the line current from SCADA is compared to the line current measured by the calibrated EPRI Sensor at Site 2. Clearly, the EPRI Sensor is less accurate at line currents below 100 amps.

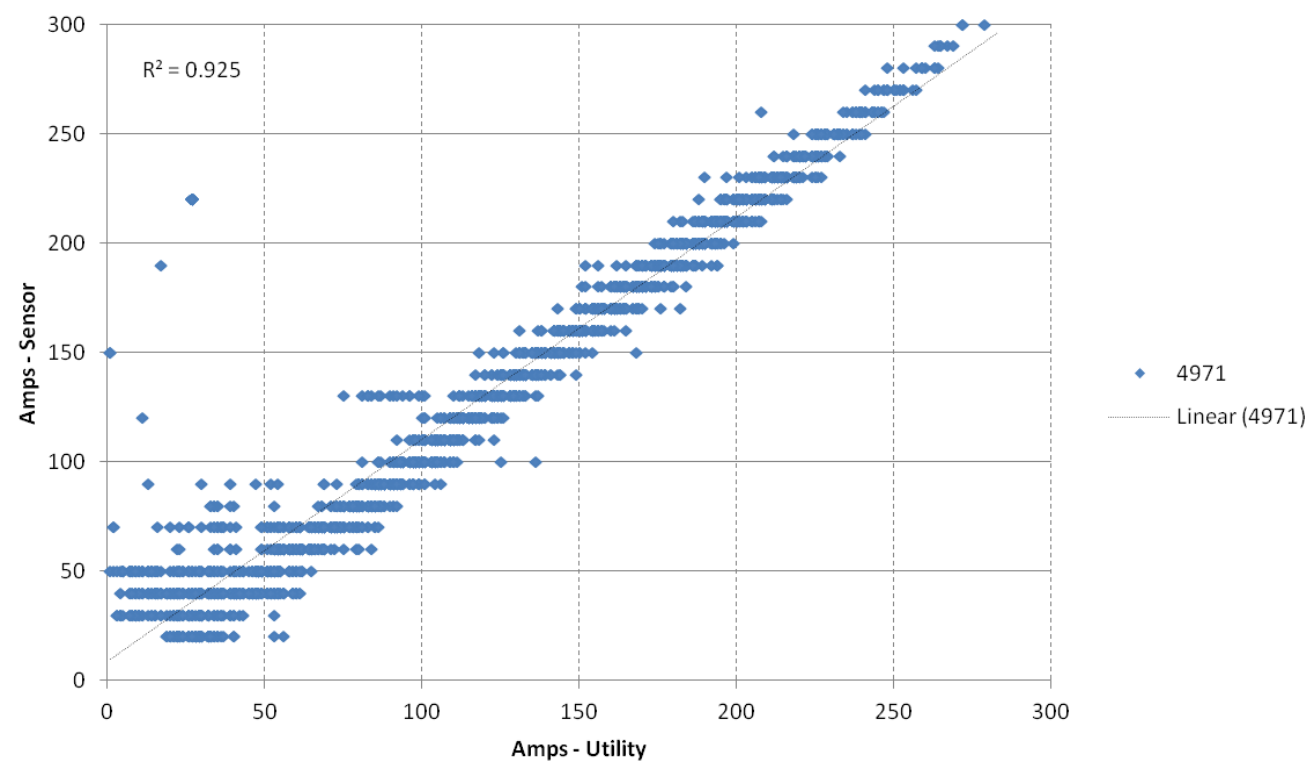

Figure G-22

Comparison of Line Current Measured by EPRI Sensor and NYPA SCADA Data for Site 2 for March 2012

\section{Accuracy of Conductor Temperature Calculations}

Conductor Temperature $T c$ is a calculated value for all methods except the EPRI Sensors which measure the temperature directly. The EPRI Sensor measurements are point measurements on the conductor. The Video Sagometer Tc measurements are distributed over the line section. At 
Site 2 there is very little elevation change or sheltering for the line section in question so there should be only a small difference in point and distributed measurements.

Conductor temperature $T c$ varies from ambient only when there is solar gain or when load is high so a period of high load is desirable for comparing technologies. Figure G-23 is a plot of conductor temperature above ambient during a rare period when the load rose above $350 \mathrm{~A}$ $(0.44 \mathrm{~A} / \mathrm{Kcmil})$ on line containing Site 1 . The EPRI Sensor measurements in red represent the only measured value. The striations on this measurement are due digitizing. The line segments are sloped due to the fact that the ambient temperature $T_{A}$ is changing. The winds were high during this period approximately $20 \mathrm{fps}$ so $T c-T_{A}$ is less than 5 degrees for most of the period in spite of the high load.

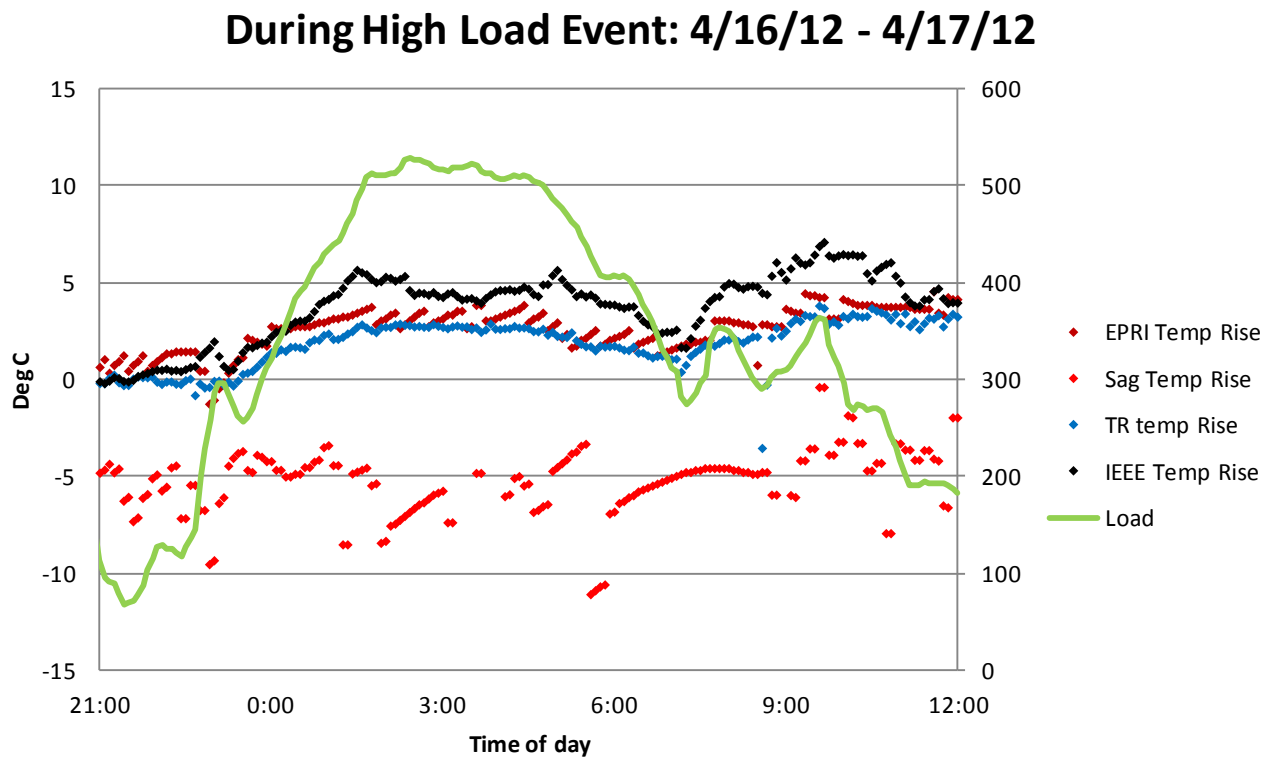

\section{Figure G-23 \\ Conductor Temperature Above Ambient (Tc - TA) for Video Sagometer, EPRI Sensor and Onsite Weather Station}

ThermalRate $T c$ agrees with the measured value within instrument precision. The onsite weather model is also very close and slightly more conservative which is consistent with earlier findings above. This is an indication that the ThermalRate high $V_{\text {wind }}$ values at high absolute wind speeds may produce an accurate representation of the actual heat transfer. Figure G-23 provides evidence here that the ThermalRate device predicts accurate conductor temperatures in winds above $10 \mathrm{fps}$ even though in this range it consistently gives higher values of $V_{\text {wind }}$ than the other elements.

The Video Sagometer Tc was lower than $T_{A}$ suggesting that the instrument was not as accurate for this period. The SAG measurement also did not trend with the other curves. The Sagometer state equation did not appear to be/stay well calibrated. The state equation only holds when the catenary curve stays within the range it matches the calculated fourth order polynomial.

The state equations for the Video Sagometers produced inconsistent conductor temperatures in this project. Figure G-23 is a plot of spot checks of conductor temperatures for the Sagometer, EPRI Sensor and onsite weather station over the course of a year. The state equation was not changed during this period. The plot is not of absolute temperature but temperature differences 
between the models. Of particular interest are differences between a model and the EPRI Sensor which is a direct measurement. (IEEE738 is the On-Site Weather station (WX) in this graph).

Sagometer vs. EPRI sensors vs. IEEE 738

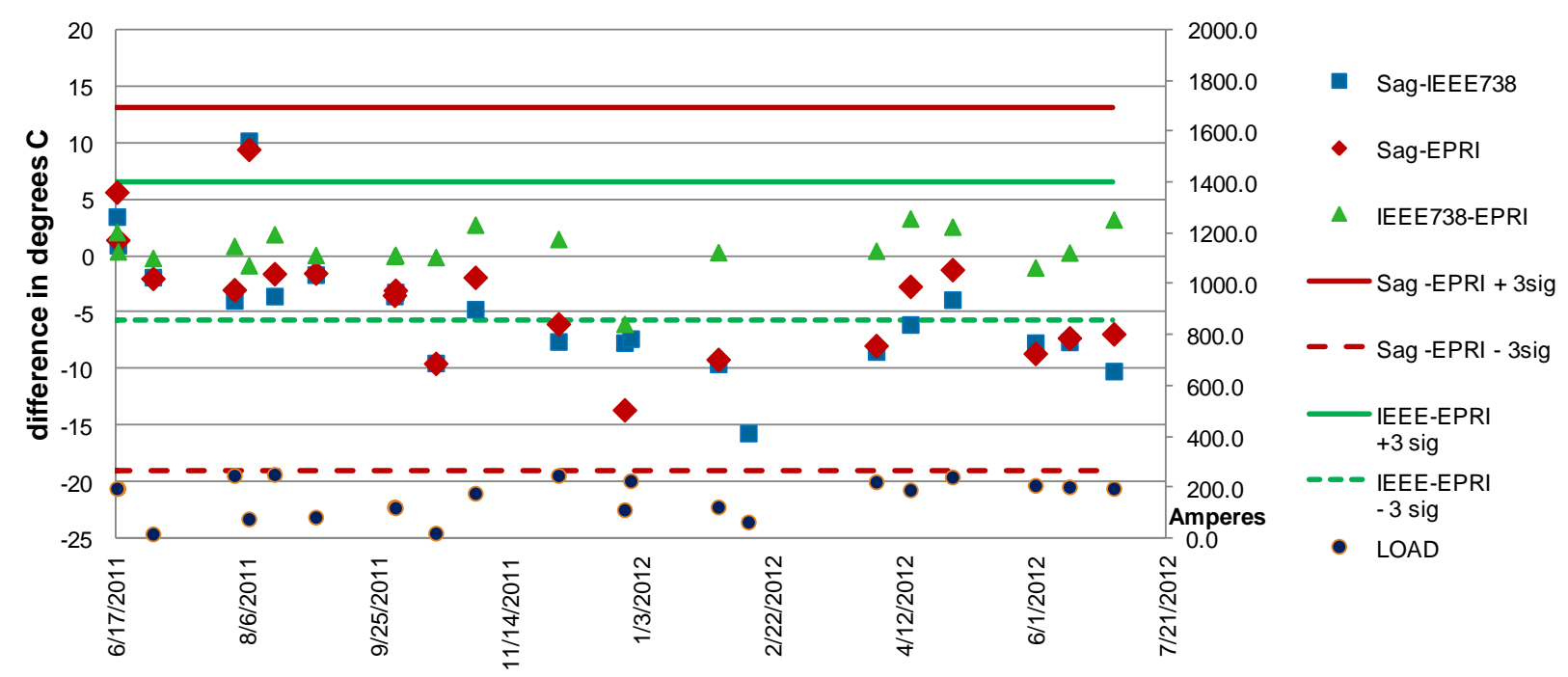

\section{Figure G-24}

Spot Checks of the Difference in Conductor Temperature Calculations vs. the EPRI Sensor Conductor Temperature Measurements for a Year-Long Period of the Project

Figure G-24 includes plus and minus 3 standard deviation lines which gives an idea of the spread of the data. The WX data is in good agreement with the EPRI Sensor both in the spread of the data and in absolute terms. The Sagometer temperatures not only vary statistically but seasonally with low values through the winter months. The cause of this variation in the sag values is not known. The poles are wood and the area is subject to deep winter frost. Structure movement is suspected.

It should be noted here that this data represents one of the longest periods of loading above 350A for the entire project. However, Sagometer values were still not used for ratings by DTCR. This is because there is a second criteria that $T_{C}-T_{A}>10^{\circ} \mathrm{C}$ before the clearance values are utilized for rating calculations. This criterion was not met during this period because of high winds. There are no periods in the entire project for any Site where both criteria were met.

\section{Monitor Accuracy - Conclusions}

The EPRI Sensor's temperature monitors, the anemometer wind speed, etc. are reasonably accurate. Similarly, the weather monitoring devices which measure air temperature and solar heating are also very accurate. The Video Sagometer clearance was the least accurate instrument.

Similarly, the heat balance algorithms (e.g., IEEE-738) used to calculate the relationship of conductor temperature and conductor current given weather conditions are also quite accurate and slightly conservative. We confirmed that the conductor temperature at a point along the overhead line can be tracked with accuracy adequate for rating calculations with three of the four technologies, if the line's recent current history is known and the real-time weather conditions 
are measured near the conductor whose temperature is to be determined. The exception was the Video Sagometer which showed variation in the relationship between measured sag and conductor temperature over the course of the project.

The ThermalRate and EPRI Sensor systems showed extremely good agreement. Inaccuracies in determining conductor temperature from weather station measurements probably result from inaccuracies in the IEEE-738 model for determining convective cooling, particularly from low and/or parallel winds. Inaccuracies in determining conductor temperature from sagometer measurements result from inaccuracies and instabilities (variation in time) in the state-change equation.

We have also shown that the conductor temperature in a nearby span of the same line may be quite different from the conductor temperature which is measured or calculated for the local span. This implies the need for multiple monitoring locations along any dynamically rated overhead line in order to assure that the dynamic rating is conservative.

Location of the monitor within an individual span can affect the measurement of temperature. Conductor temperature monitors should be located at the low point of conductor sag and/or in sheltered areas to be sure they reflect the lowest wind cooling.

It is also shown from the field investigation that the conductor temperature can vary rapidly with changes in wind cooling which yields large prediction errors when the prediction period exceeds one hour. Also, any chronological error in conductor temperature increases with line current and during periods of low wind cooling.

\section{Dynamic Line Rating Accuracy-Conclusions}

Wind cooling is the most important environmental variable in determining dynamic line rating, and it is the most difficult one to ascertain. Unlike other variables such as ambient temperature and solar intensity, wind can exhibit large variations in both speed and direction at any location along the line and between spans in the line. To determine what values to use when calculating ratings from moment-to-moment can be very difficult, and is the main challenge for any realtime rating scenario.

Wind speed measurements were directly made (whether the load was high or low) by the weather station anemometers, nearby NOAA stations, and indirectly by the ThermalRate conductor modeling devices. Overall, these measurements compared favorably. There was also reasonable agreement for the limited periods where the line load was high enough to estimate wind speed from the EPRI Sensors.

To the system operator, the primary interest concerns the line rating accuracy, since the operator assures safe operation of the power system by keeping the line current below the appropriate line rating or allowing it to exceed the rating for limited periods of time. The primary concern of the system operator centers on the accuracy of line rating predictions. It is impossible to make operational decisions such as load shedding or re-dispatch of generation to keep the line current below the rating, if the rating either increases or decreases rapidly. Also, it is impossible to set contract flow limits a day or more in advance, if the ratings used in determining the contract flows change. 
Line rating errors can be very large even if instrument errors are tiny. One of the key contributors to line rating calculation error involves the impact of low current densities on the conversion of sag clearance and conductor temperature measurements into accurate estimates of line section average wind speed.

\section{Impact of Line Current on Rating Accuracy}

As described in Appendix B, the line rating calculation involves the use of a heat balance equation with weather data inputs (wind speed, wind direction, air temperature, and solar radiation). The wind speed and direction parameters can be replaced by an "effective wind speed" which equals that wind speed, blowing perpendicular to the conductor axis, which produces the same convective cooling. Each of the monitoring methods must provide air temperature, solar heat and convective cooling data to allow a line rating calculation.

The onsite weather stations method measures all the necessary weather data parameters to allow a real-time line rating calculation. The ThermalRate conductor model method measures air temperature and solar heating with the unheated portion of the conductor model and calculates an "effective wind speed" based on the conductor temperature of the internally heated part of the conductor model. In neither method is the line rating accuracy affected by the actual line current flowing on the line.

In calculating line ratings based on the Video Sagometer monitoring method and the EPRI Sensor conductor temperature monitoring method, the measured parameters are conductor target clearance and conductor temperature, respectively. Clearance and conductor temperature must be converted to effective wind speed before the line rating can be calculated.

The heat generated in the line's conductor depends primarily on the square of the current that passes through it. Thus the heat generated with the line carrying 30\% of its rated load is only $10 \%$ of the heat generated at full rated load and any temperature rise above ambient and change in sag clearance is quite small. Given normal measurement uncertainties of 1 to $2^{\circ} \mathrm{C}$, the errors inherent in calculating effective wind speeds at low current levels are prohibitively large. When the sometimes large errors involved in converting sag clearance to average conductor temperature in a line section are considered, the Video Sagometer monitoring method is only reasonably accurate when the current density in the $795 \mathrm{Kcmil}$ Drake conductor is equal to 0.5 amps/Kcmil or more, (i.e., greater than 400 Amps ).

In a case where the lines are lightly loaded, as was the case at NYPA most of the time, the ratings cannot be accurately estimated from the Video Sagometer or from the EPRI conductor sensor monitoring methods. For the two $230 \mathrm{kV}$ lines instrumented in this study, the line currents rarely exceeded 350A ( $0.44 \mathrm{amps} / \mathrm{Kcmil})$ so that these monitoring methods were not useful.

The Video Sagometer has an advantage for sag limited lines because it effectively averages the conductor temperature over many spans and the average temperature determines clearance values. The same device has a disadvantage in thermally limited lines because the averaging shields the ability to see the local conductor hot sections in sheltered areas. Because many lines are sag limited the inability to properly evaluate these technologies in this program was disappointing. However this is actually a significant finding in the project. Evidence has been presented that the lines instrumented at NYPA are not unusual and that such devices typically go for long periods without providing rating information. This leads to the conclusion that, because 
of the need for system reliability, many high voltage lines operate at current densities less than $0.5 \mathrm{amps} / \mathrm{Kcmil}$ and cannot be monitored accurately with devices whose rating accuracy depend on significant heat generation in the conductors.

\section{A Hybrid Monitoring System}

The Video Sagometer should become more accurate as the line current increases and may even be more accurate for ratings based on clearance limits than the onsite weather stations or the ThermalRate conductor model during high current events such as after an N-1 or N-2 contingency if the state equations are kept in calibration. In fact, it can be argued that there is a significant advantage to the direct measurement of sag-tension during those infrequent times when the line current is close to the line rating.

To the extent that this is true, it appears that a hybrid system that involves two monitoring methods such as the ThermalRate Conductor model and the Video Sagometer could be the most effective and accurate method of determining dynamic line ratings under the full range of weather and loading conditions.

\section{References}

Evaluation of Emerging Line Inspection Technologies, Results of 2011 Field Tests, 1026639 Technical Update, November 2012 


\section{$\boldsymbol{H}$ \\ EVALUATION OF LINE RATINGS: HISTORICAL LINE RATING DATA}

The two NYPA Lines in this study have had real-time ratings calculated by DTCR using multiple methods (elements) for nearly 2 years. The rating data available real-time can be used for operating decisions but past data remains valuable after all real-time operation decisions have been made. These data can be used for evaluation of the current carrying capacity that the line possessed over the course of those two years, regardless of how much of the capacity was utilized. In this project the historical data indicate that most of the time line capacity exceeds the static ratings by a wide margin. This will be shown quantitatively in this chapter. These data are an indication of the incentive to continue real-time rating operations and can also be used as an aid to assess the risks associated with the existing static rating and the risks associated with changes to the static ratings. A complete risk assessment is beyond the scope of this project but the data are presented here in a useful form for future analysis and consideration.

As discussed in Appendix E, due to communication delays the archived raw measured data used to make the rating calculations is higher quality than the real-time rating data. A more complete evaluation of the rating history of the lines can be made from DTCR simulations using the archived raw data as input. The historical rating data used in this chapter are from DTCR simulations.

\section{Cumulative Distribution Plots}

A cumulative distribution plot is a graphical representation of the cumulative distribution function (CDF). The CDF is a one to one function that gives the proportion of values in a set that are less than or equal to any individual value. This function also gives the probability a random value from the data set will be less than or equal to any individual value.

Take for example the following set of wind speeds in $\mathrm{ft} / \mathrm{sec} ;\{1,2,3,4\}$ The distribution function would give $50 \%$ for $2 \mathrm{fps}$ because if chosen at random you have a $50 \%$ chance of getting a number less than or equal to 2 . The function would give $100 \%$ for $4 \mathrm{fps}$ because when chosen at random you will always get a number less than or equal to 4 . The terminology used to describe the percentage is the "L-number." In the above example L-50 would be 2, and L-100 would be 4.

The L-number is useful in risk assessment. If the line is operated at a constant current for a prolonged period of time the L-number associated with that load is the percentage of time that the actual capacity of the line is less than the load. Suppose a data set existed for a line that represented the full range of environmental conditions. Suppose also that the CDF for the rating data set gives L-1 at 1000 Amps. It is therefore likely in future operations that the line will be operated above its capacity about $1 \%$ of the time if the line is operated at a constant 1000 Amps. 
Transmission lines are not typically operated at or near the rating, (dynamic or static), nor are they typically operated at constant load. The CDF of line actual loading can therefore be just as important as the CDF of capacity. Figure H-1 is a CDF plot showing both ratings and load for the time period of $11 / 1 / 2010$ to $5 / 1 / 2012$ for Site 2 . Comparison of low dynamic rating Lnumbers with high load L numbers on this plot is a top level indication of the loading margin. For this transmission line a low risk is indicated because in this data set the load L-100 is less than the rating L-0. This indicates that the highest loads did not exceed the lowest dynamic rating over this period of historical operation. However the data in this history may not be representative of future loads and dynamic ratings. For example in this case a larger load data set that includes contingency situations that forced very high loads would provide a more appropriate CDF for assessing risks. An investigation into how typical the weather was over the measurement period of the rating data set might also indicate that the ratings were either optimistic or conservative when considering future operations.

In the situation when the load exceeds L-0 for dynamic normal rating. Risk may still be low because the conditions that produced both extreme dynamic rating and load L-numbers are unlikely to coincide. If the conditions creating the low dynamic rating extremes are short lived then the thermal capacity of the conductor may also eliminate any consequences if there were a coincidence of high load and low steady state (normal) capacity. The thermal capacity is the basis of the higher Emergency ratings. Therefore comparing L-numbers is useful in the assessment of risk but is not a direct measurement of risk.

\section{Adjustment of Static Ratings}

If assessing the risks associated with increasing the static ratings is undertaken then the actual load data need not be included. In this case the lowest dynamic ratings alone are the key factors in the decisions on how high the static ratings might be raised. If any correlation between high loads and the dynamic rating exist then this can be factored into the risk assessment. For instance, if high loads are due to wind generation then a positive correlation of load and dynamic rating may be present. The stronger the correlation the lower the risks associated with a higher static rating.

\section{Load and Dynamic Cumulative Distribution Plots for NYPA Lines}

Winter is defined as the period of time from November 1st to April 30th; conversely, summer is defined as the time from May 1st to October 31st. The plots shown in this chapter are good for assessing ratings over a period of time. Please note the sites represented here were not chosen for the purpose of determining the limiting section of overhead conductor. It is possible that there are sections of line that are sheltered from the wind by local geological factors such as a valley or hills. Trees also shelter a line from the wind. Ideally, a monitoring site used to determine the rating of the line would be chosen in the most wind sheltered area of the corridor. If not explicitly explained most comparisons focus on the normal ratings and to a lesser degree on the LTE ratings. The STE ratings tend to be more volatile because they are more strongly affected by the initial conditions of the temperature of the line.

It should also be noted that consistent data availability is a strong factor in comparing CDF plots. For example if during one month the Video Sagometer camera was out, meaning no ratings we calculated, then the loss of that data will affect the seasonal CDF. A summer season with missing 
data for an entire month, such as the sagometer for July 2011 at Site 3, is likely to give the impression of a higher seasonal rating than is actually present. Some of the inconsistencies between EPRI Sensors and the Video Sagometer, which predominately should be nearly identical due to the DTCR software reverting to the weather model as previously described, are not due to the difference in technologies. It is actually in the difference of times when the models were not calculable. To this end, the onsite weather model, by virtue of having the most complete data set, should be the most accurate representation of that models variation by season and year.

Note also that even though the monthly rating plots of offsite weather data are coarse in nature, the seasonal CDF plots contain enough data to eliminate this effect. This indicates this "steplike" nature in offsite weather data can be effectively averaged out over a long enough period; in this case a 6 month rating season. 


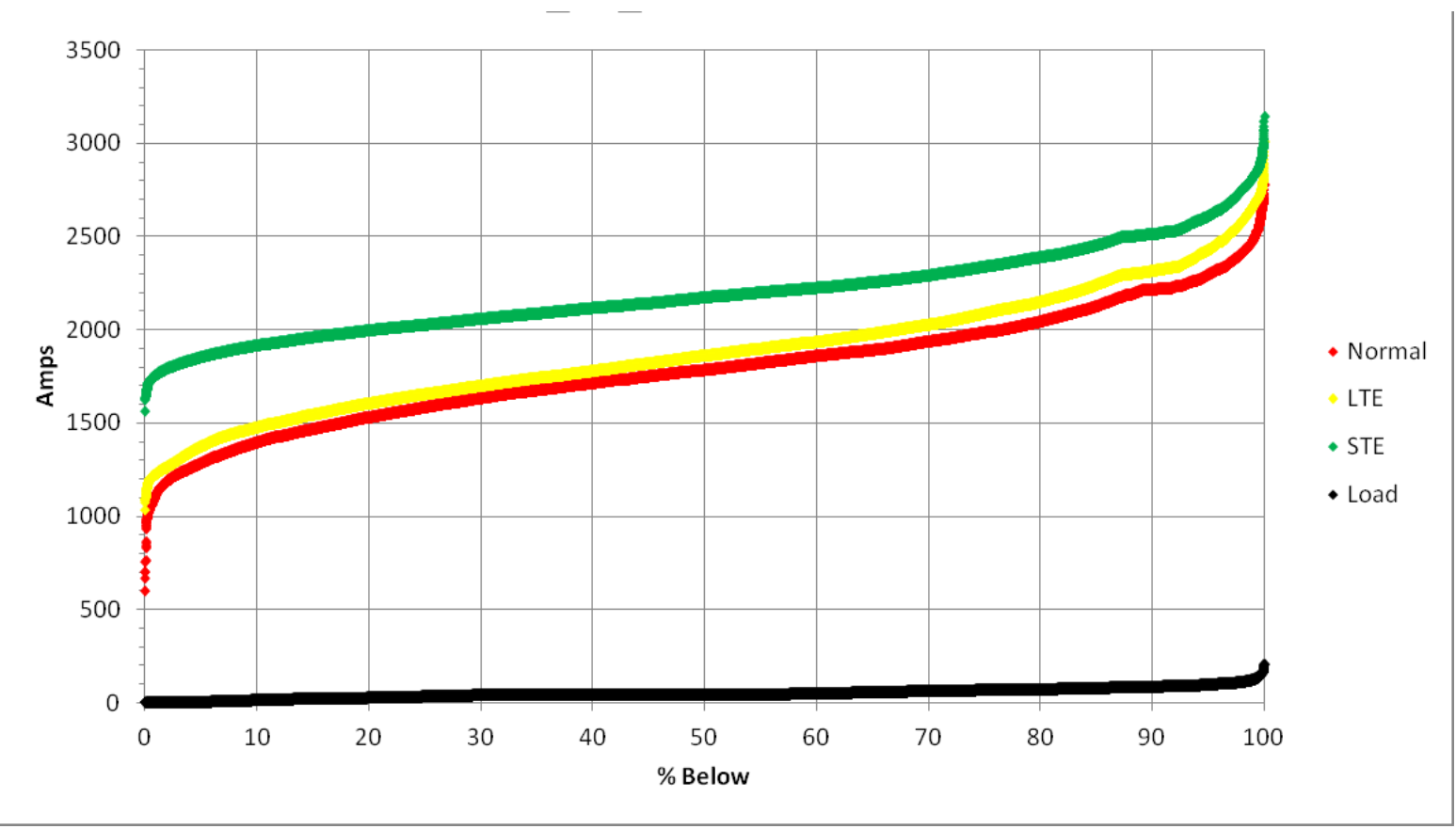

Figure $\mathrm{H}-1$

Winter 2010/11 Ratings Distribution Site 2 Onsite Weather Station

Table H-1

Winter 2010/11 Ratings Distribution Site 2 Onsite Weather Station

\begin{tabular}{|c|l|l|l|l|l|l|}
\hline & \multicolumn{1}{|c|}{ Static } & \multicolumn{1}{|c|}{ L1 } & \multicolumn{1}{|c|}{ L2 } & \multicolumn{1}{|c|}{ L5 } & \multicolumn{1}{|c|}{ L50 } \\
\hline Normal & 1331 & 1122 & 1192 & 1285 & 1784 & 2296 \\
\hline LTE & 1460 & 1228 & 1266 & 1374 & 1860 & 2486 \\
\hline STE & 1593 & 1760 & 1794 & 1853 & 2173 & 2609 \\
\hline Load & & 0 & 0 & 5 & 40 & 96 \\
\hline
\end{tabular}

The Site 2 winter 2010/11 local weather station data can be compared to the Site 2 winter 2010/11 ThermalRate data. It can be seen in the tables that there is approximately 100 amps difference between the two. The weather station based rating is the more conservative of the two. 


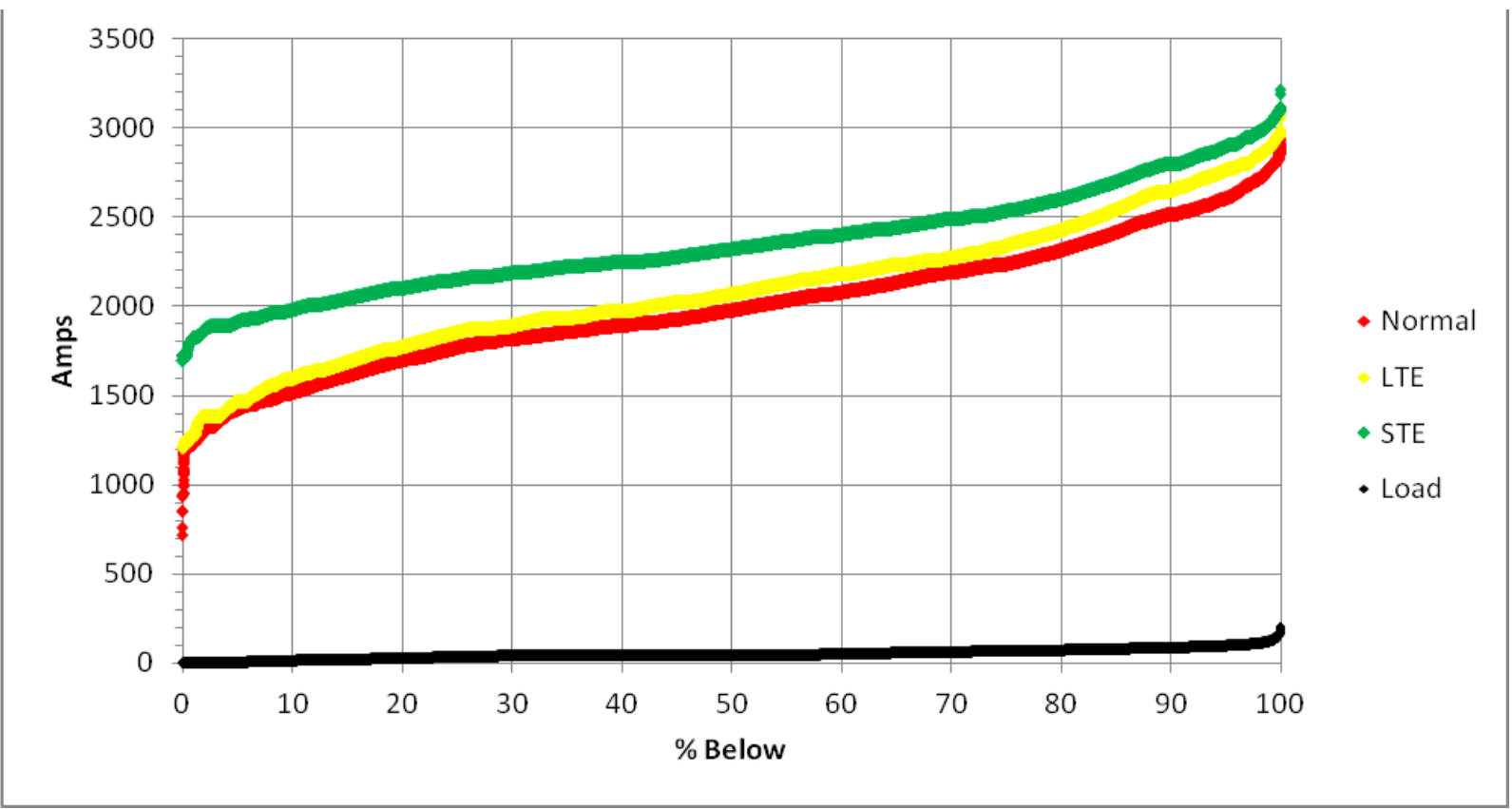

Figure $\mathrm{H}-2$

Winter 2010/11 Ratings Distribution Site 2 ThermalRate

Table H-2

Winter 2010/11 Ratings Distribution Site 2 ThermalRate

\begin{tabular}{|c|l|l|l|l|l|l|}
\hline & \multicolumn{1}{|c|}{ Static } & \multicolumn{1}{|c|}{ L1 } & \multicolumn{1}{|c|}{ L2 } & \multicolumn{1}{|c|}{ L5 } & \multicolumn{1}{|c|}{ L50 } & \multicolumn{1}{|l|}{} \\
\hline Normal & 1331 & 1245 & 1300 & 1421 & 1975 & 2605 \\
\hline LTE & 1460 & 1281 & 1384 & 1468 & 2077 & 2762 \\
\hline STE & 1593 & 1824 & 1863 & 1992 & 2319 & 2894 \\
\hline Load & & 0 & 0 & 5 & 40 & 96 \\
\hline
\end{tabular}

The Site 2 winter 2010/11 ThermalRate data can be compared to the Site 2 winter 2011/12 thermal rate data. It can be seen in the tables that there is approximately 15 amps difference between the two. This difference is small suggesting consistency from year to year. 


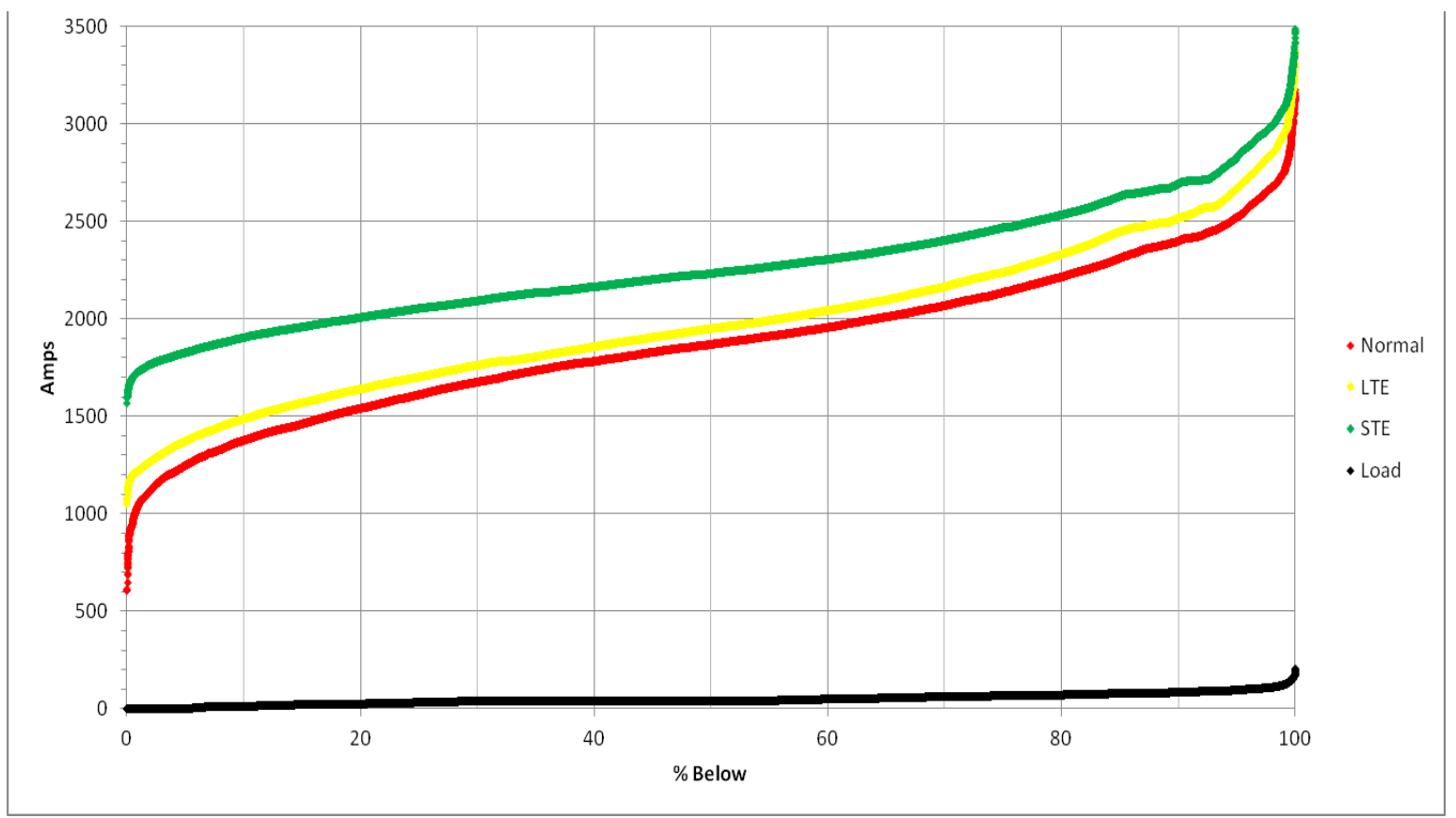

Figure H-3

Winter 2010/11 Ratings Distribution Site 2 Sagometer

Table H-3

Winter 2010/11 Ratings Distribution Site 2 Sagometer

\begin{tabular}{|l|l|l|l|l|l|l|}
\hline & Static & L1 & L2 & L5 & L50 & L95 \\
\hline Normal & 1331 & 1049 & 1122 & 1248 & 1870 & 2545 \\
\hline LTE & 1460 & 1222 & 1270 & 1373 & 1950 & 2673 \\
\hline STE & 1593 & 1731 & 1766 & 1826 & 2231 & 2835 \\
\hline Load & & 0 & 0 & 5 & 40 & 96 \\
\hline
\end{tabular}

The Site 2 winter 2010/11sagometer data can be compared to the Site 2 winter 2010/11 EPRI Sensor data. The values and shape of these curves will be the same because most of the time DTCR is forced to fall back on the weather station model. If the line had more load, it would be possible to properly evaluate the technology. It is likely there would be differences. Some of these differences would be explained by state change equations and point measurements versus ruling span measurements. 


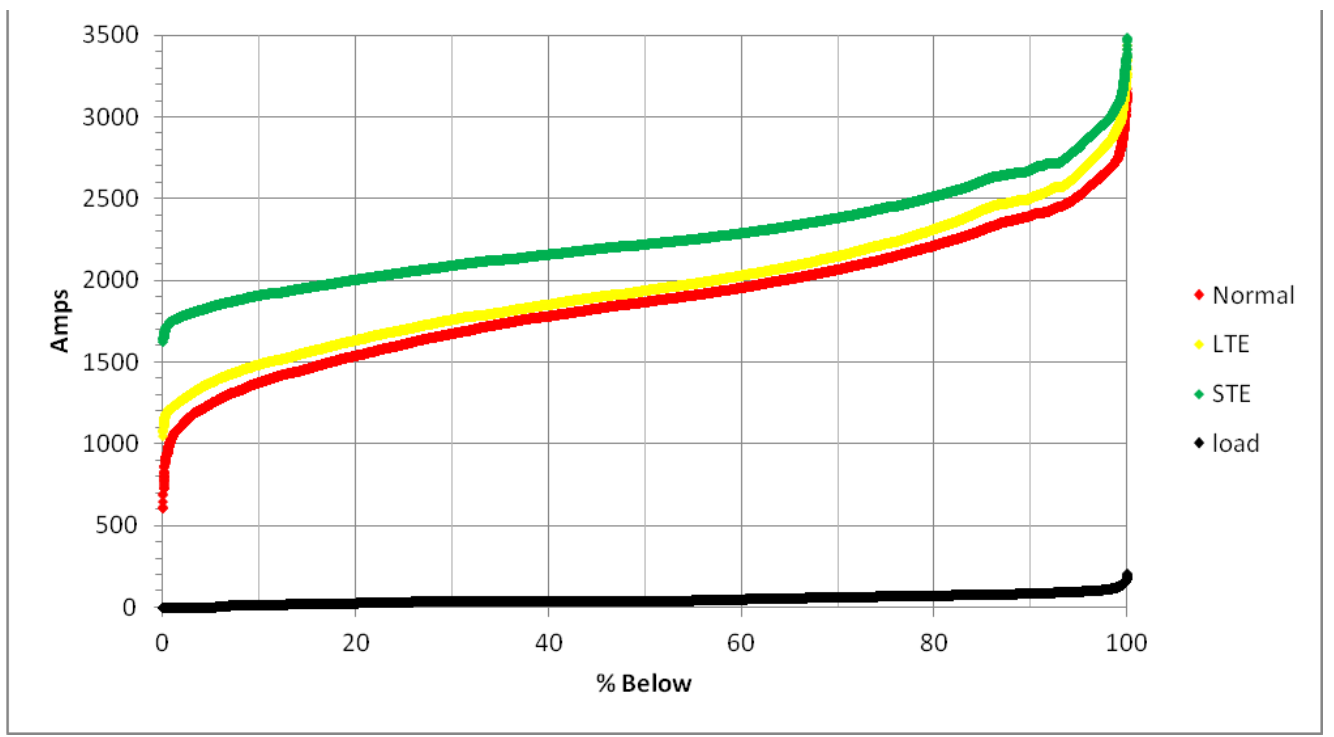

Figure H-4

Winter 2010/11 Ratings Distribution Site 2 EPRI Sensors

Table H-4

Winter 2010/11 Ratings Distribution Site 2 EPRI Sensors

\begin{tabular}{|c|l|l|l|l|l|l|}
\hline & \multicolumn{1}{|c|}{ Static } & \multicolumn{1}{|c|}{ L1 } & \multicolumn{1}{|c|}{ L2 } & \multicolumn{1}{|c|}{ L5 } & \multicolumn{1}{|c|}{ L50 } \\
\hline Normal & 1331 & 1049 & 1122 & 1248 & 1868 & 2520 \\
\hline LTE & 1460 & 1224 & 1271 & 1376 & 1939 & 2659 \\
\hline STE & 1593 & 1754 & 1781 & 1840 & 2221 & 2815 \\
\hline Load & & 0 & 0 & 5 & 40 & 96 \\
\hline
\end{tabular}

The Site 2 winter 2010/11sagometer data can be compared to the Site 2 winter 2010/11 EPRI Sensor data. When comparing the L2 levels it can be seen in the tables that the Normal and the LTE ratings are about the same, however, there is some difference in the STE level. DTCR is forced to use the weather model when the line is lightly loaded. Less than 5 percent of the time the load was high enough for DTCR to fully utilize the Sagometer end EPRI Sensors. Thermal inertia plays little to no roll in LTE or Normal rating calculations. However, it does play a significant part in the STE calculation. Because of the importance of thermal inertia, a temperature difference in the measured or calculated conductor temperature will show up in the STE rating calculation, as it does here. 


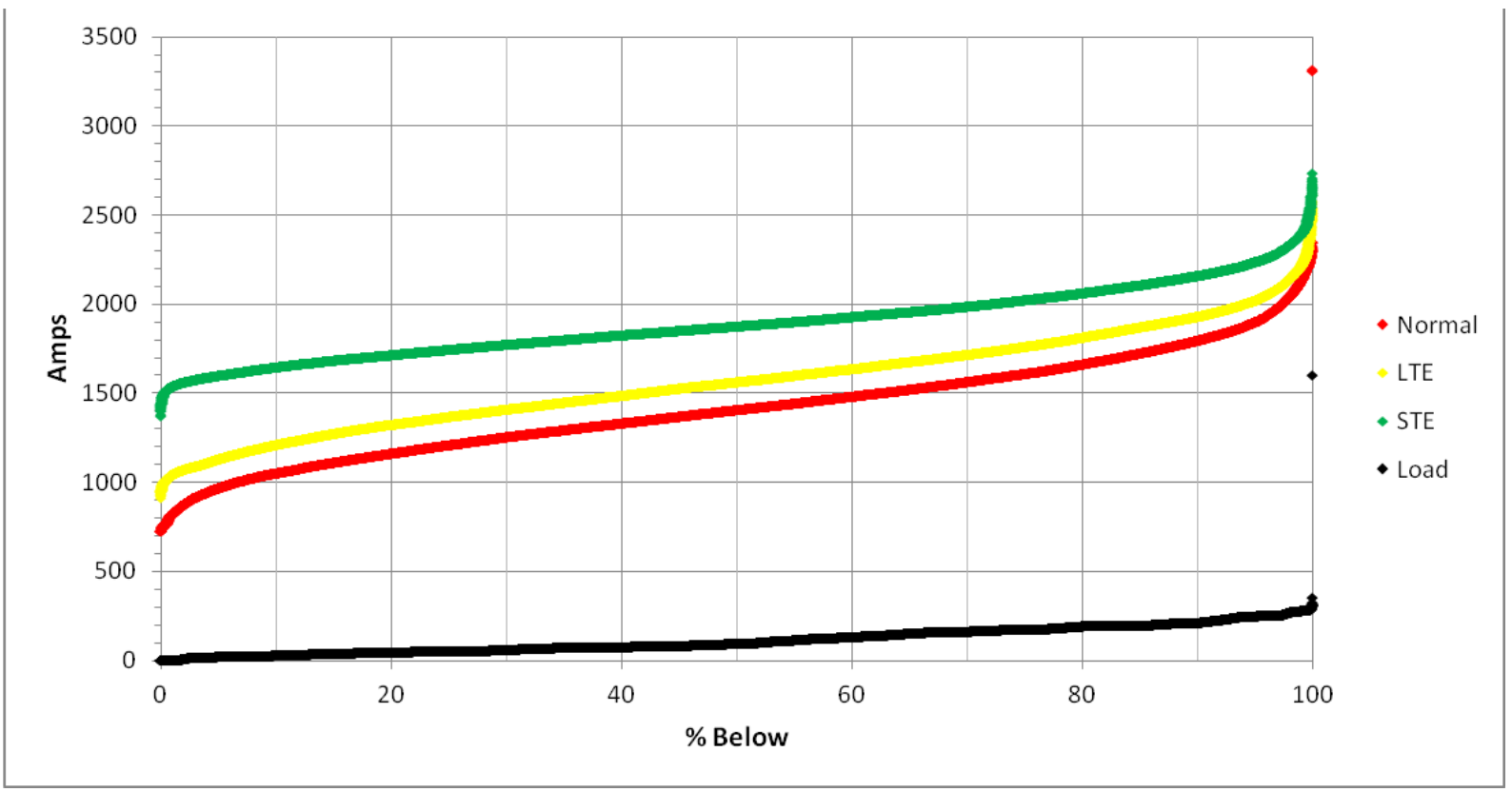

Figure H-5

Summer 2011 Ratings Distribution Site 2 Onsite Weather Station

Table H-5

Summer 2011 Ratings Distribution Site 2 Onsite Weather Station

\begin{tabular}{|c|l|l|l|l|l|l|}
\hline & \multicolumn{1}{|c|}{ Static } & \multicolumn{1}{|c|}{ L1 } & \multicolumn{1}{|c|}{ L2 } & \multicolumn{1}{|c|}{ L5 } & \multicolumn{1}{|c|}{ L95 } \\
\hline Normal & 1089 & 818 & 875 & 967 & 1410 & 1901 \\
\hline LTE & 1256 & 1042 & 1072 & 1130 & 1566 & 2022 \\
\hline STE & 1410 & 1538 & 1561 & 1599 & 1878 & 2238 \\
\hline Load & & 0 & 10 & 25 & 95 & 250 \\
\hline
\end{tabular}

The Site 2 summer 2011 weather station data can be compared to the Site 2 summer 2011 ThermalRate sensor data. It can be seen in the tables that there is approximately 200amps difference between the two. The weather station based rating is the more conservative of the two. 


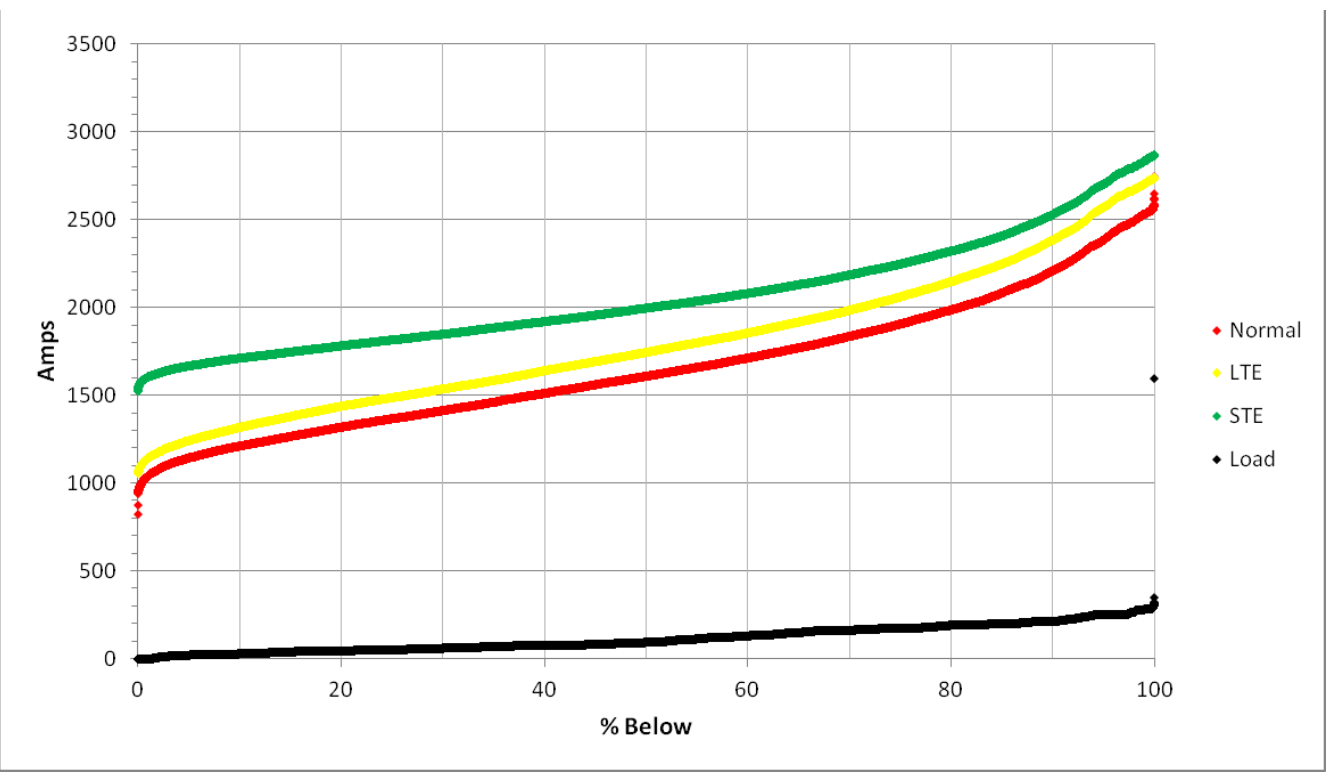

Figure H-6

Summer 2011 Ratings Distribution Site 2 ThermalRate

Table H-6

Summer 2011 Ratings Distribution Site 2 ThermalRate

\begin{tabular}{|c|l|l|l|l|l|l|}
\hline & \multicolumn{1}{|c|}{ Static } & \multicolumn{1}{|c|}{ L1 } & \multicolumn{1}{|c|}{ L2 } & \multicolumn{1}{|c|}{ L5 } & \multicolumn{1}{|c|}{ L50 } & \multicolumn{1}{|l|}{ L95 } \\
\hline Normal & 1089 & 1047 & 1079 & 1147 & 1611 & 2388 \\
\hline LTE & 1256 & 1147 & 1177 & 1244 & 1747 & 2572 \\
\hline STE & 1410 & 1608 & 1628 & 1670 & 1999 & 2706 \\
\hline Load & & 0 & 10 & 25 & 95 & 250 \\
\hline
\end{tabular}

The Site 2 summer 2011 ThermalRate data can be compared to the Site 2 summer 2012 ThermalRate sensor data. It can be seen in the tables that there is approximately 100amps difference between the two. This higher rating is a result of increased wind speeds during the month of September the average wind speed was $1.2 \mathrm{ft} / \mathrm{s}$ faster in 2012. 


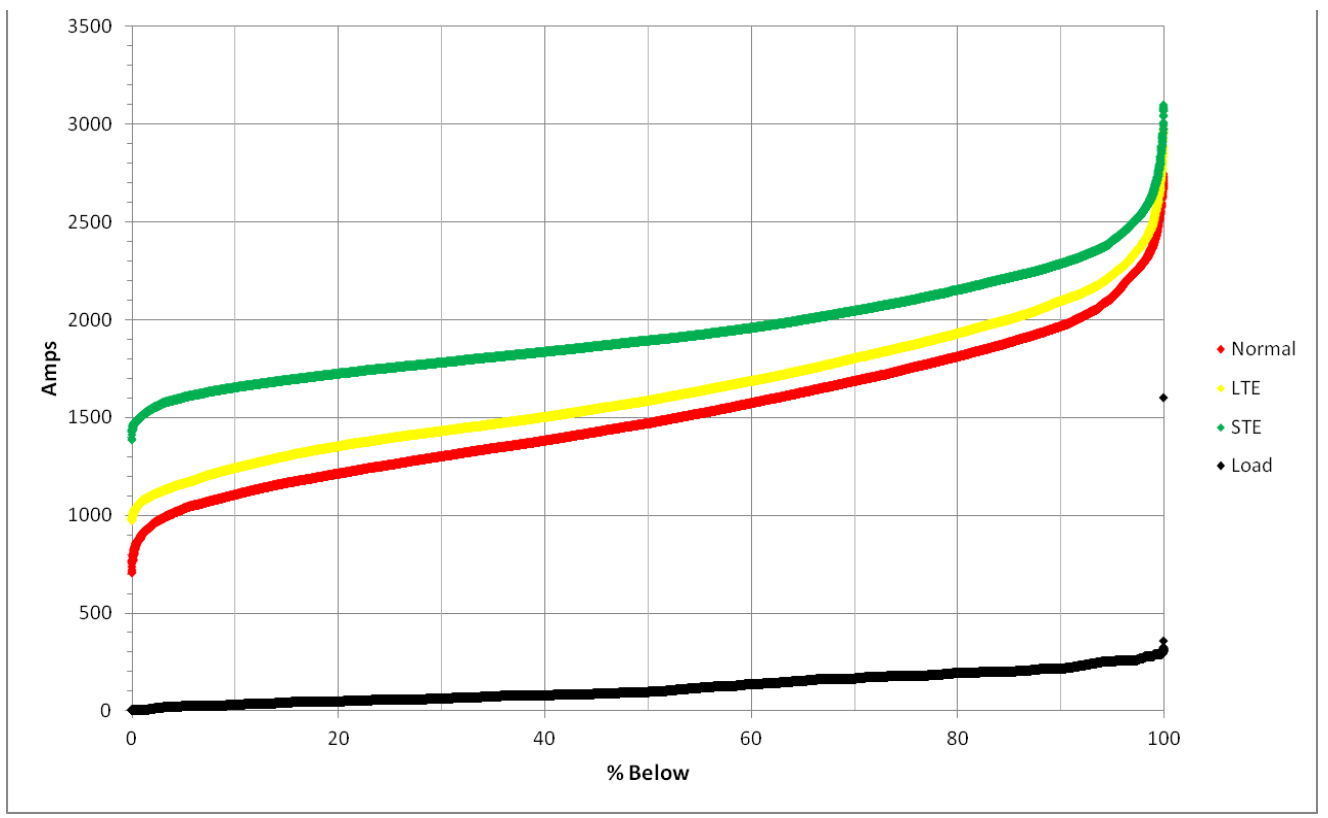

Figure H-7

Summer 2011 Ratings Distribution Site 2 Sagometer

Table H-7

Summer 2011 Ratings Distribution Site 2 Sagometer

\begin{tabular}{|c|l|l|l|l|l|l|}
\hline & \multicolumn{1}{|c|}{ Static } & \multicolumn{1}{|c|}{ L1 } & \multicolumn{1}{|c|}{ L2 } & \multicolumn{1}{|c|}{ L5 } & \multicolumn{1}{|c|}{ L95 } \\
\hline Normal & 1089 & 898 & 950 & 1031 & 1471 & 2117 \\
\hline LTE & 1256 & 1070 & 1101 & 1159 & 1584 & 2225 \\
\hline STE & 1410 & 1507 & 1545 & 1601 & 1891 & 2400 \\
\hline Load & & 0 & 10 & 25 & 95 & 250 \\
\hline
\end{tabular}

Summer L95 loads are larger than winter L95 loads. This fact results in more periods of time when the Sagometer can be used for ratings rather than simply reverting to weather based calculations. Consequently, when comparing Site 2 summer 2011 Sagometer data with Site 2 summer 2011 EPRI Sensor data, a more significant difference can be viewed. One of the issues with devices that measure the average temperature over the ruling span section is the state change equation can be a moving target. This can be a result of heating and cooling cycles of the structure holding the line. A small change in position of the structure can result in a large difference in calculated conductor temperature. 


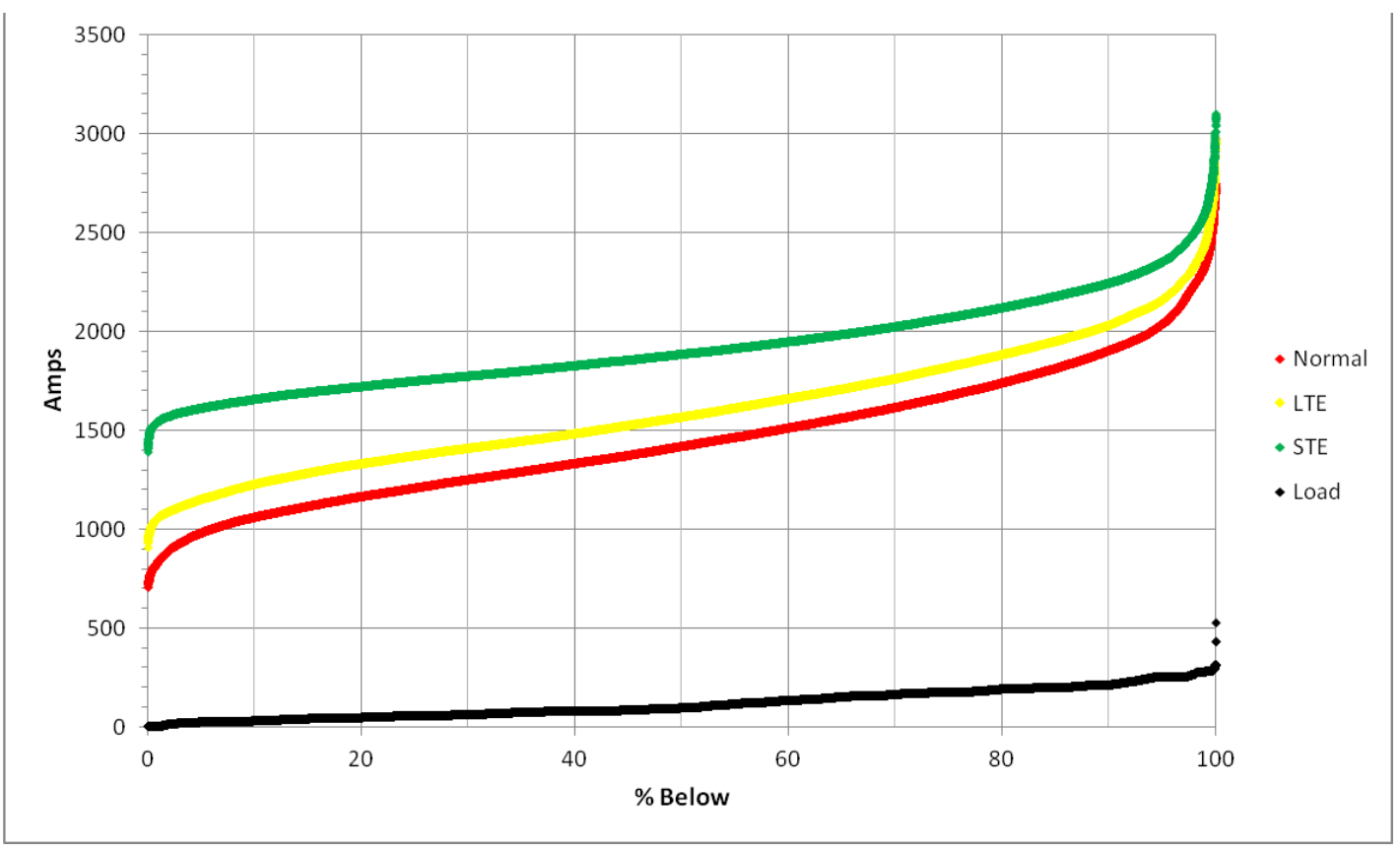

Figure H-8

Summer 2011 Ratings Distribution Site 2 EPRI Sensors

Table H-8

Summer 2011 Ratings Distribution Site 2 EPRI Sensors

\begin{tabular}{|c|l|l|l|l|l|l|}
\hline & \multicolumn{1}{|c|}{ Static } & \multicolumn{1}{|c|}{ L1 } & \multicolumn{1}{|c|}{ L2 } & \multicolumn{1}{|c|}{ L50 } & \multicolumn{1}{|l|}{ L95 } \\
\hline Normal & 1089 & 838 & 894 & 981 & 1418 & 2036 \\
\hline LTE & 1256 & 1060 & 1087 & 1150 & 1566 & 2158 \\
\hline STE & 1410 & 1544 & 1570 & 1610 & 1883 & 2349 \\
\hline Load & & 0 & 10 & 25 & 95 & 250 \\
\hline
\end{tabular}

One advantage to the EPRI Sensor is that it directly measures conductor temperature.

Consequently the state change equation is 1 . The disadvantage is the sensor is a single point measurement. It would take a number of sensors along that line section to start to approach the distributed measurements that you get with a load cell or sag measuring device. The conductor temperature then gets converted into an equivalent perpendicular wind speed. Then the line rating calculation is made. So it is critical that the conductor temperature be accurate! Small differences would result in changes like we see between Site 2 summer 2011 Sagometer data and Site 2 summer 2011 EPRI Sensor data. 


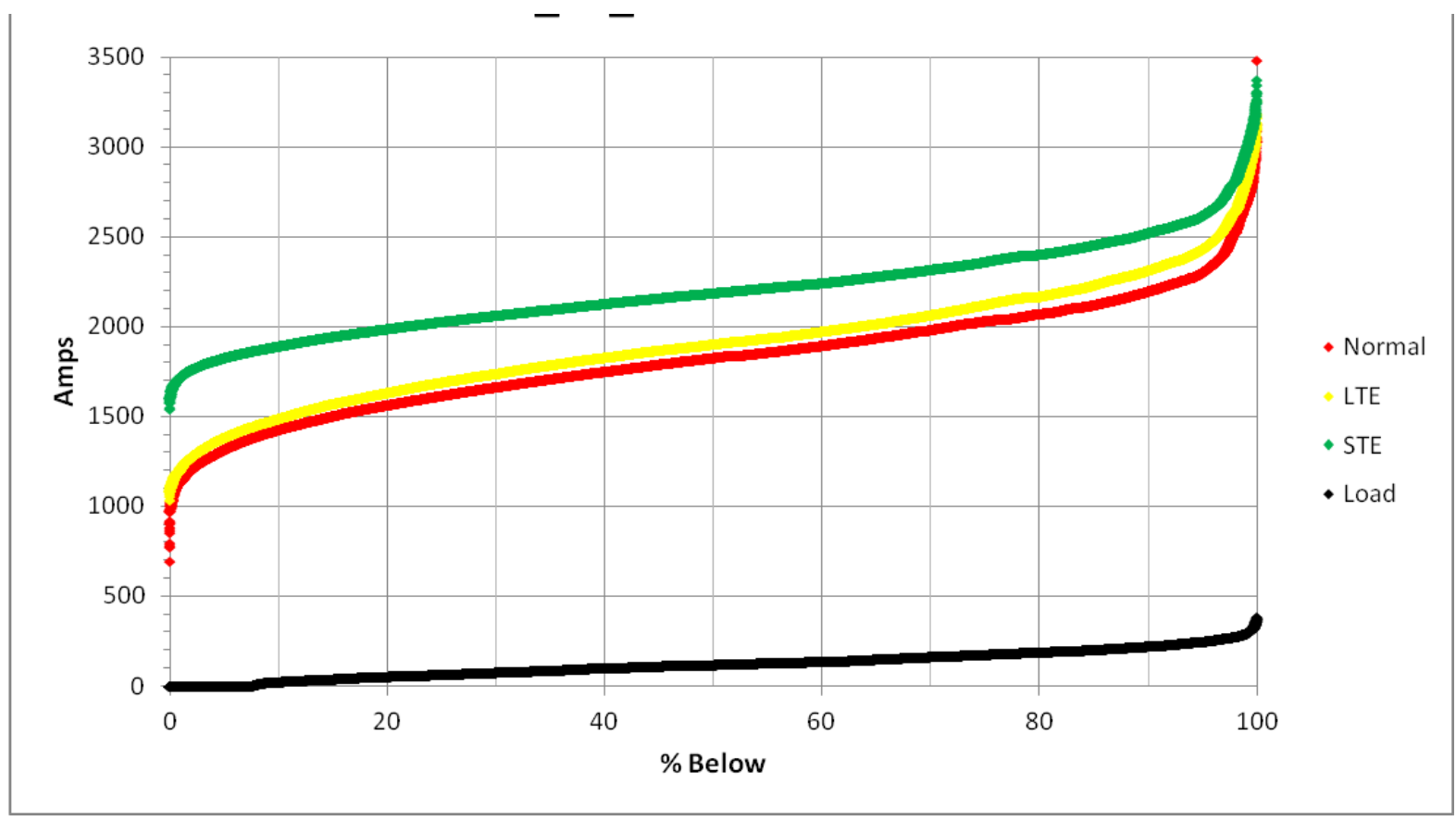

Figure $\mathrm{H}-9$

Winter 2011/12 Ratings Distribution Site 2 Onsite Weather Station

Table H-9

Winter 2011/12 Ratings Distribution Site 2 Onsite Weather Station

\begin{tabular}{|c|l|l|l|l|l|l|}
\hline & \multicolumn{1}{|c|}{ Static } & \multicolumn{1}{|c|}{ L1 } & \multicolumn{1}{|c|}{ L2 } & \multicolumn{1}{|c|}{ L5 } & \multicolumn{1}{|c|}{ L50 } \\
\hline Normal & 1331 & 1138 & 1204 & 1314 & 1826 & 2303 \\
\hline LTE & 1460 & 1212 & 1276 & 1380 & 1902 & 2432 \\
\hline STE & 1593 & 1720 & 1760 & 1826 & 2186 & 2619 \\
\hline Load & & 0 & 0 & 5 & 120 & 250 \\
\hline
\end{tabular}

The Site 2 winter 2011/12 Onsite weather station data can be compared to the Site 2 winter 2010/11 Onsite weather station sensor data. There is less that 10 amps difference between the two seasons at the L2 level. This would suggest that there may be some consistency in these ratings from season to season. 


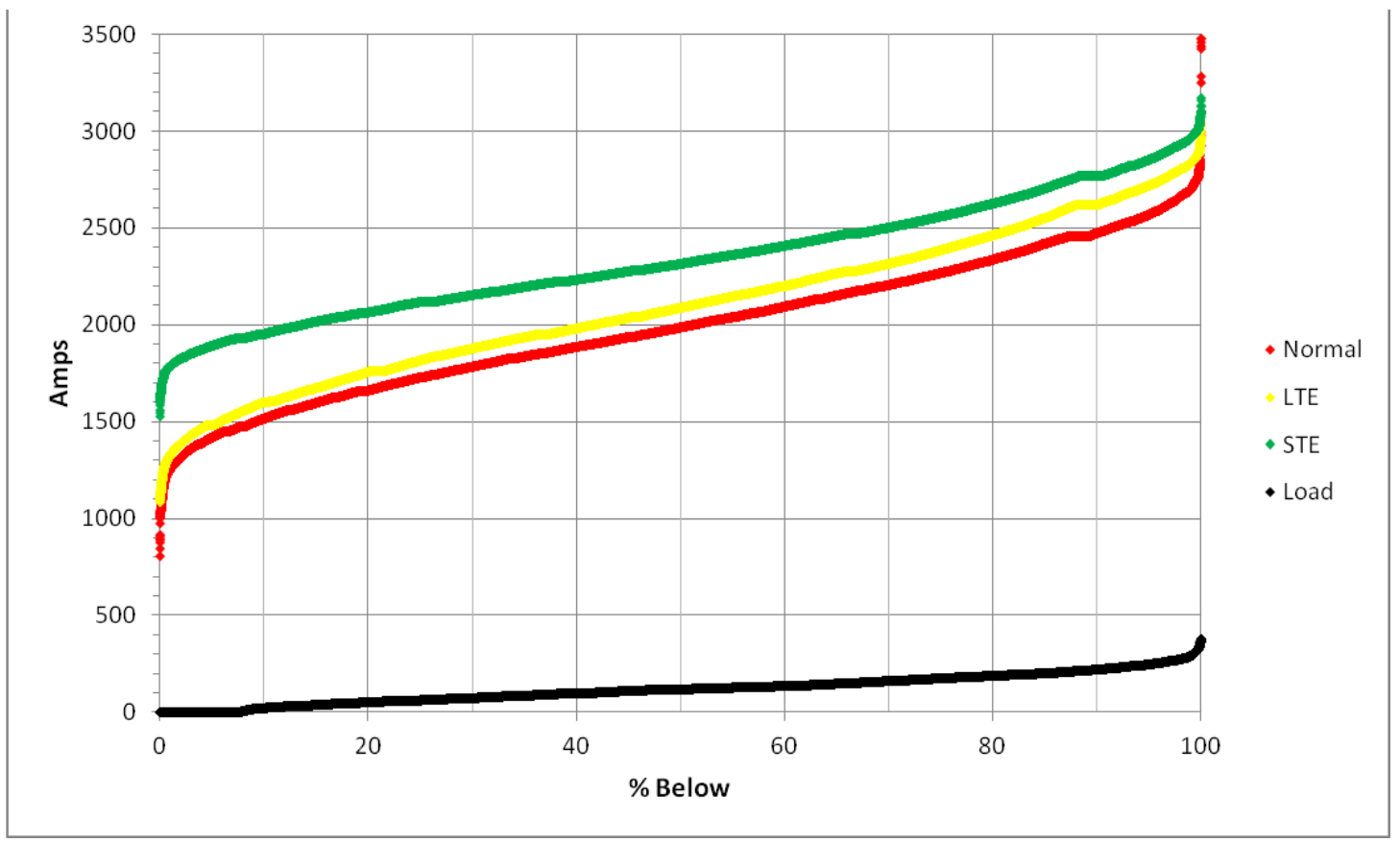

Figure $\mathrm{H}-10$

Winter 2011/12 Ratings Distribution Site 2 ThermalRate

Table H-10

Winter 2011/12 Ratings Distribution Site 2 ThermalRate

\begin{tabular}{|c|l|l|l|l|l|l|}
\hline & \multicolumn{1}{|c|}{ Static } & \multicolumn{1}{|c|}{ L1 } & \multicolumn{1}{|c|}{ L2 } & \multicolumn{1}{|c|}{ L5 } & \multicolumn{1}{|c|}{ L50 } \\
\hline Normal & 1331 & 1258 & 1315 & 1417 & 1986 & 2569 \\
\hline LTE & 1460 & 1328 & 1385 & 1472 & 2087 & 2719 \\
\hline STE & 1593 & 1786 & 1824 & 1892 & 2313 & 2852 \\
\hline Load & & 0 & 0 & 5 & 120 & 250 \\
\hline
\end{tabular}

The Site 2 winter 2011/12 ThermalRate can be compared to the Site 2 winter 2010/11 ThermalRate sensor data. It can be seen in the tables that there is less than 10 amps difference between the two for the normal and LTE ratings. This would indicate that the ratings may be consistent from season to season. 


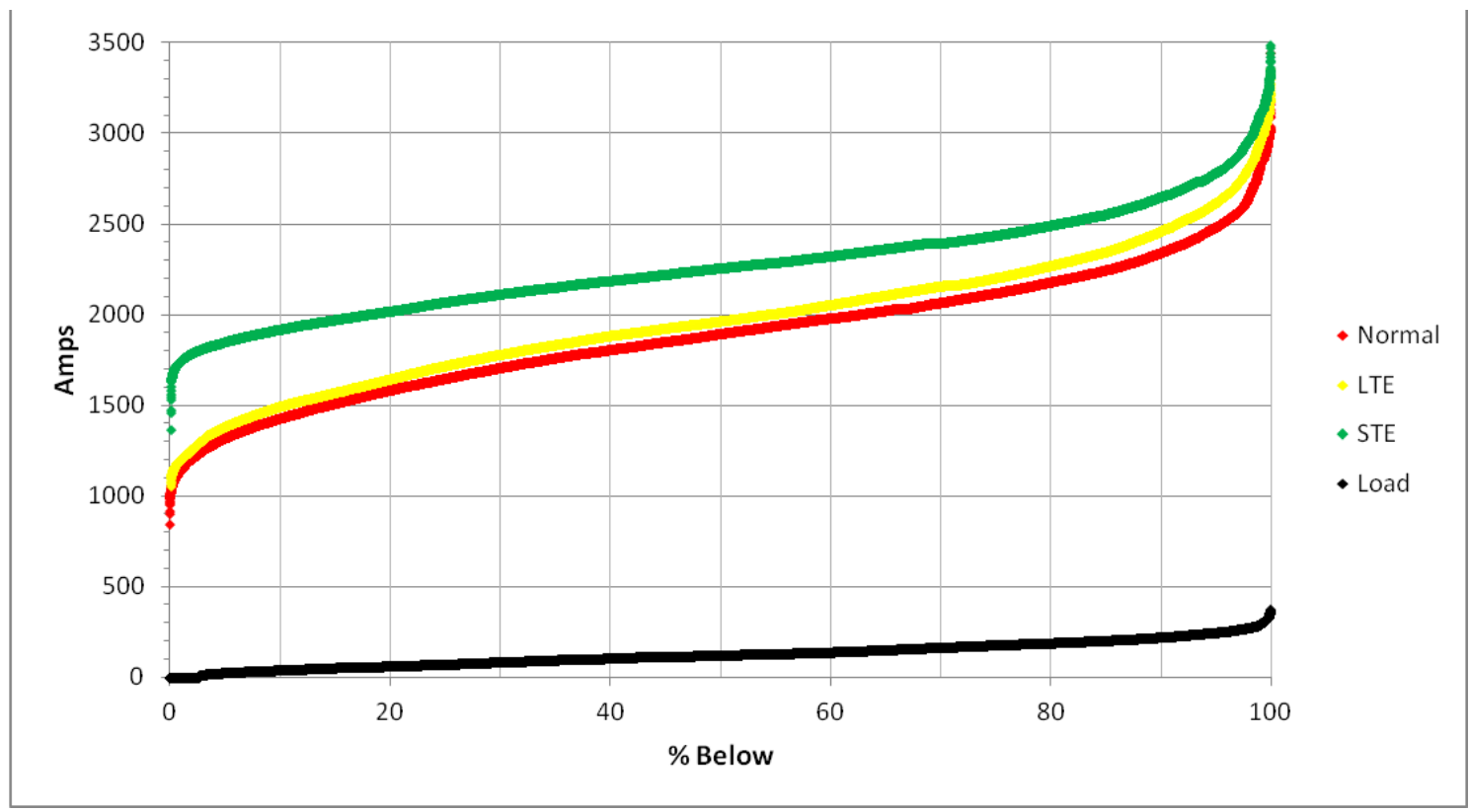

Figure $\mathrm{H}-11$

Winter 2011/12 Ratings Distribution Site 2 Sagometer

Table H-11

Winter 2011/12 Ratings Distribution Site 2 Sagometer

\begin{tabular}{|c|l|l|l|l|l|l|}
\hline & \multicolumn{1}{|c|}{ Static } & \multicolumn{1}{|c|}{ L1 } & \multicolumn{1}{|c|}{ L2 } & \multicolumn{1}{|c|}{ L5 } & \multicolumn{1}{|c|}{ L50 } \\
\hline Normal & 1331 & 1140 & 1205 & 1318 & 1895 & 2484 \\
\hline LTE & 1460 & 1200 & 1260 & 1382 & 1964 & 2621 \\
\hline STE & 1593 & 1744 & 1788 & 1849 & 2257 & 2785 \\
\hline Load & & 0 & 0 & 5 & 120 & 250 \\
\hline
\end{tabular}

The Site 2 winter 2011/12 sagometer data can be compared to the Site 2 winter 2011/12 EPRI Sensor data. The values and shape of these curves will be the same because most of the time DTCR is forced to fall back on the weather station model. If the line had more load, it would be possible to properly evaluate the technology. It is likely there would be differences. Some of these differences would be explained by state change equations and point measurements versus ruling span measurements. 


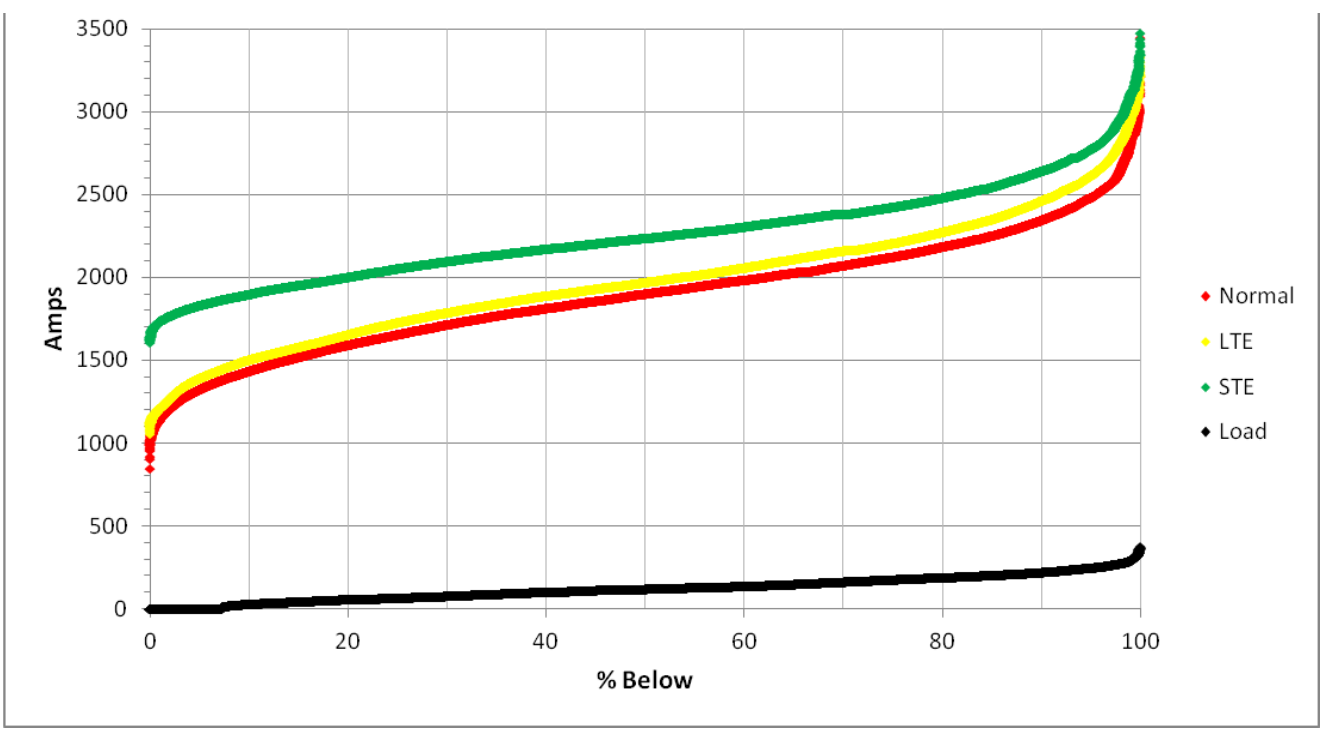

Figure $\mathrm{H}-12$

Winter 2011/12 Ratings Distribution Site 2 EPRI Sensors

Table H-12

Winter 2011/12 Ratings Distribution Site 2 EPRI Sensors

\begin{tabular}{|c|l|l|l|l|l|l|}
\hline & \multicolumn{1}{|c|}{ Static } & \multicolumn{1}{|c|}{ L1 } & \multicolumn{1}{|c|}{ L2 } & \multicolumn{1}{|c|}{ L5 } & \multicolumn{1}{|c|}{ L50 } & \multicolumn{1}{|c|}{ L95 } \\
\hline Normal & 1331 & 1140 & 1205 & 1322 & 1899 & 2484 \\
\hline LTE & 1460 & 1206 & 1266 & 1389 & 1968 & 2615 \\
\hline STE & 1593 & 1734 & 1765 & 1830 & 2235 & 2774 \\
\hline Load & & 0 & 0 & 5 & 120 & 250 \\
\hline
\end{tabular}

The Site 2 winter 2011/12 sagometer data can be compared to the Site 2 winter 2011/12 EPRI Sensor data. When comparing the L2 levels it can be seen in the tables that the Normal and the LTE ratings are about the same, however, there is some difference in the STE level. DTCR is forced to use the weather model when the line is lightly loaded. Less than 5 percent of the time the load was high enough for DTCR to fully utilize the Sagometer end EPRI Sensors. Thermal inertia plays no role in the Normal rating calculations and only a small role in the LTE rating calculations. However, it does play a significant part in the STE calculation. Because of the importance of thermal inertia, a temperature difference in the measured or calculated conductor temperature will show up in the STE rating calculation, as it does here. 


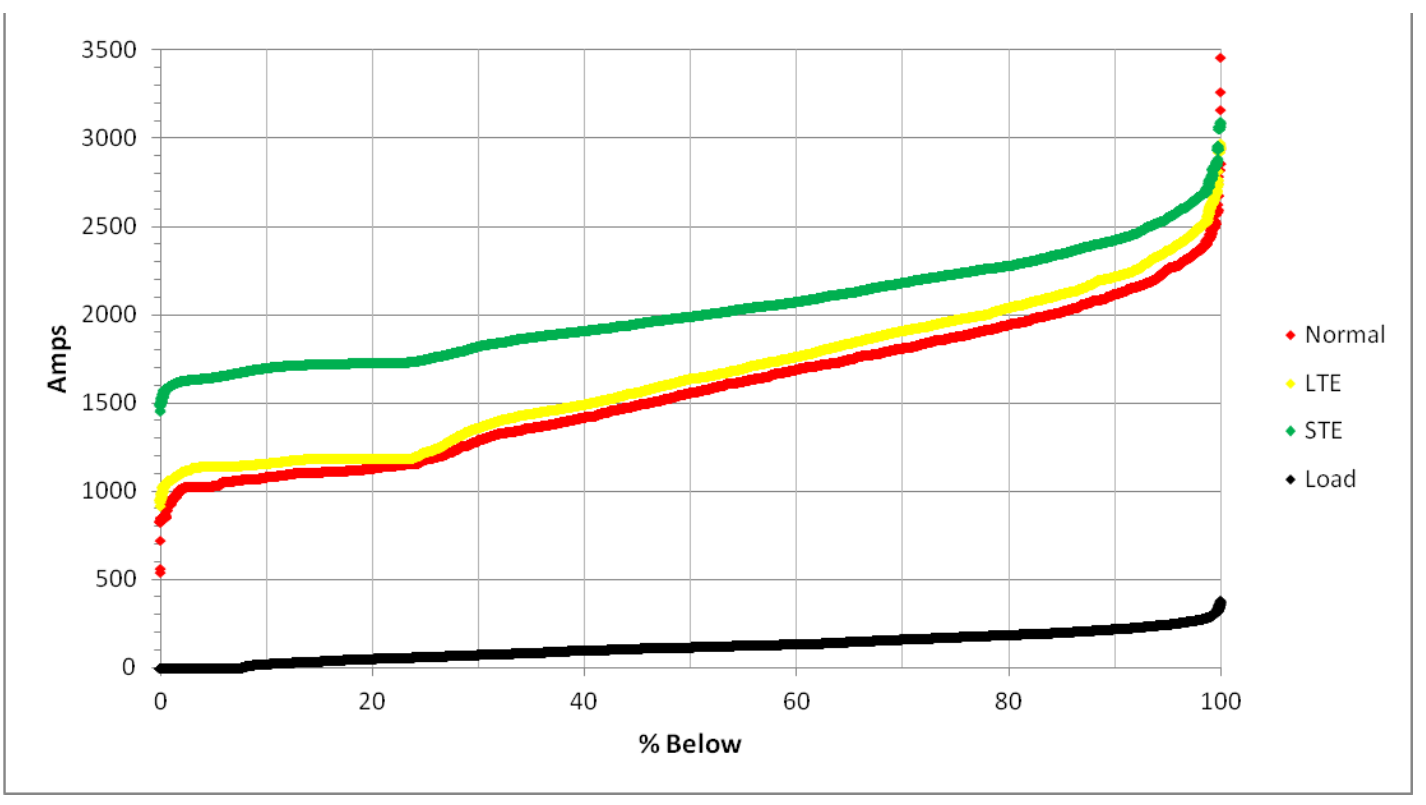

Figure $\mathrm{H}-13$

Winter 2011/12 Ratings Distribution Site 2 Offsite Weather Service

Table $\mathrm{H}-13$

Winter 2011/12 Ratings Distribution Site 2 Offsite Weather Service

\begin{tabular}{|c|l|l|l|l|l|l|}
\hline & \multicolumn{1}{|c|}{ Static } & \multicolumn{1}{|c|}{ L1 } & \multicolumn{1}{|c|}{ L2 } & \multicolumn{1}{|c|}{ L5 } & \multicolumn{1}{|c|}{ L50 } & \\
\hline Normal & 1331 & 937 & 1014 & 1033 & 1560 & 2259 \\
\hline LTE & 1460 & 1063 & 1104 & 1140 & 1639 & 2366 \\
\hline STE & 1593 & 1598 & 1625 & 1646 & 1991 & 2557 \\
\hline Load & & 0 & 0 & 5 & 120 & 250 \\
\hline
\end{tabular}

The Site 2 winter 2011/12 off site weather service data can be compared to the Site 2 winter 2011/12 Onsite weather station data. It can be seen in the tables that there is approximately 200amps difference between the two. The offsite weather service based rating is the more conservative of the two. 


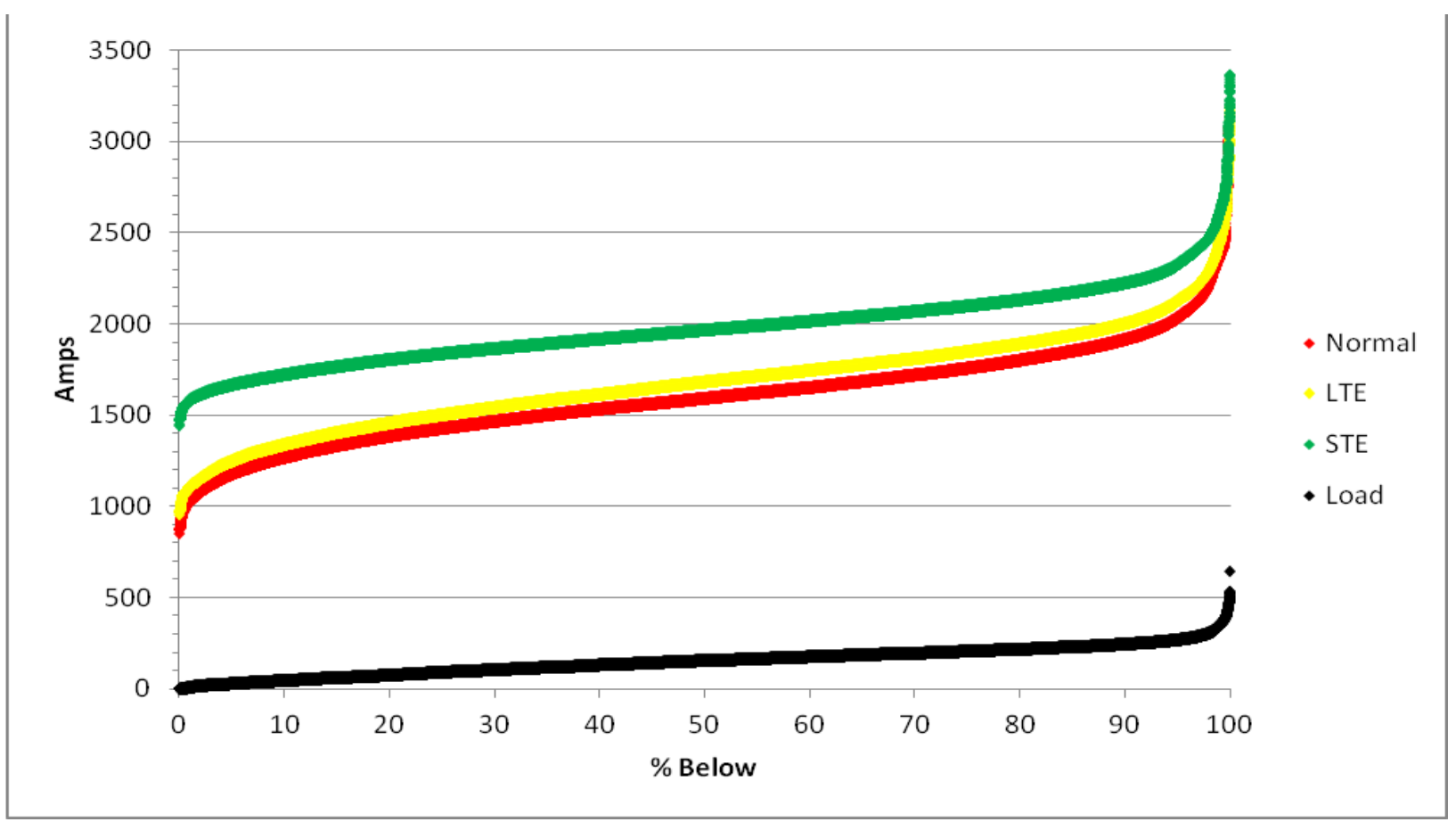

Figure $\mathrm{H}-14$

Summer 2012 Ratings Distribution Site 2 Onsite weather station

Table H-14

Summer 2012 Ratings Distribution Site 2 Onsite weather station

\begin{tabular}{|c|l|l|l|l|l|l|}
\hline & \multicolumn{1}{|c|}{ Static } & \multicolumn{1}{|c|}{ L1 } & \multicolumn{1}{|c|}{ L2 } & \multicolumn{1}{|c|}{ L5 } & \multicolumn{1}{|c|}{ L50 } & \multicolumn{1}{c|}{ L95 } \\
\hline Normal & 1089 & 982 & 1055 & 1165 & 1587 & 2016 \\
\hline LTE & 1256 & 1071 & 1130 & 1235 & 1677 & 2105 \\
\hline STE & 1410 & 1552 & 1598 & 1657 & 1962 & 2306 \\
\hline Load & & 0 & 10 & 25 & 150 & 260 \\
\hline
\end{tabular}

The Site 2 summer 2012 onsite weather station data can be compared to the Site 2 summer 2012 ThermalRate data. It can be seen in the tables that there is approximately 100 amps difference between the two. The onsite weather station based rating is the more conservative of the two. 


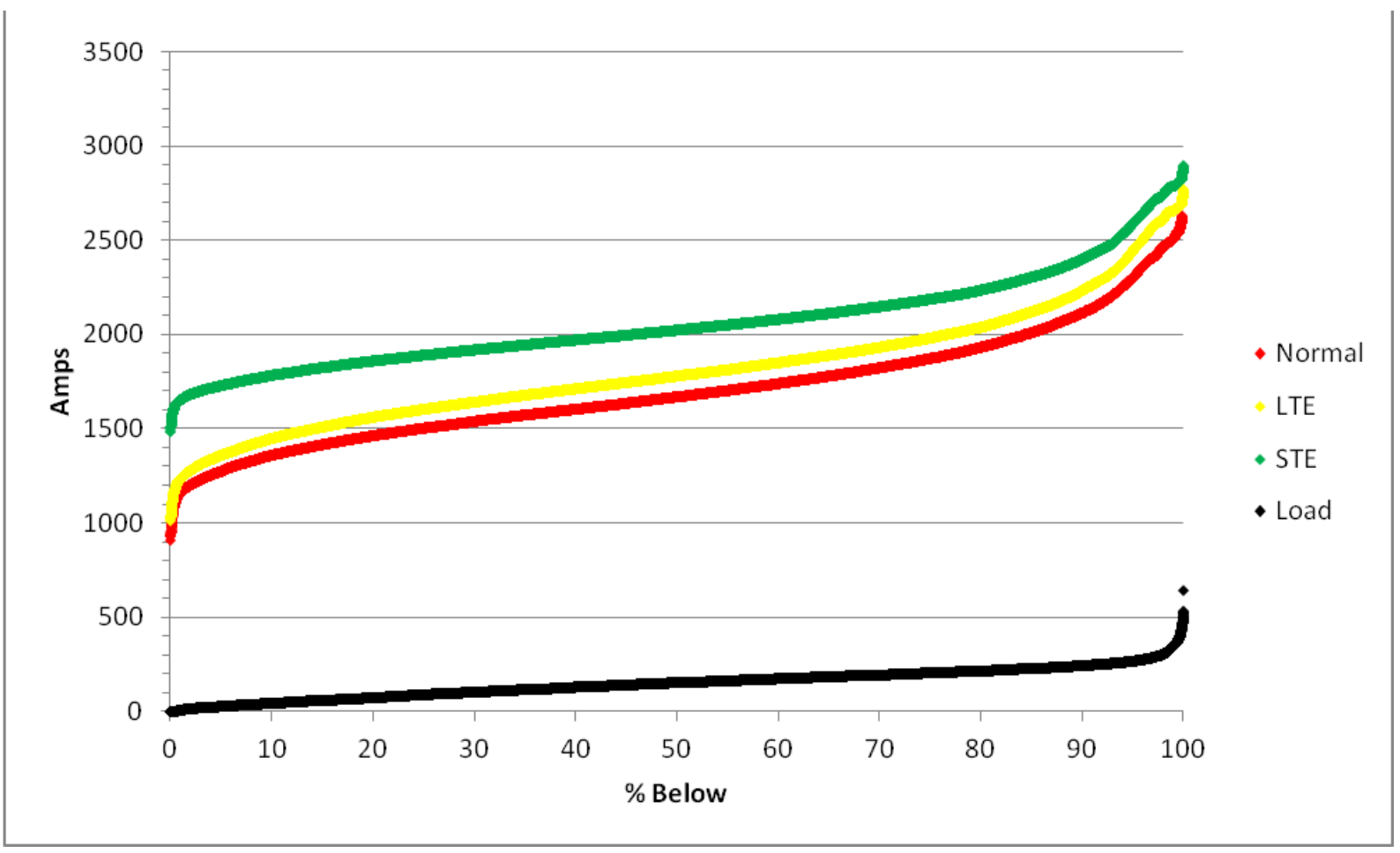

Figure $\mathrm{H}-15$

Summer 2012 Ratings Distribution Site 2 ThermalRate

Table H-15

Summer 2012 Ratings Distribution Site 2 ThermalRate

\begin{tabular}{|l|l|l|l|l|l|l|}
\hline & Static & L1 & L2 & L5 & L50 & L95 \\
\hline Normal & 1089 & 1117 & 1185 & 1264 & 1665 & 2279 \\
\hline LTE & 1256 & 1191 & 1259 & 1346 & 1775 & 2412 \\
\hline STE & 1410 & 1625 & 1669 & 1721 & 2019 & 2557 \\
\hline Load & & 0 & 10 & 25 & 150 & 260 \\
\hline
\end{tabular}

The Site 2 summer 2012 ThermalRate data can be compared to the Site 2 summer 2012 EPRI Sensor data. It can be seen in the tables that there is approximately 100 amps difference between the two. The EPRI Sensor based rating is the more conservative of the two. This is because the EPRI Sensors are based primarily on weather data as result of low loads. 


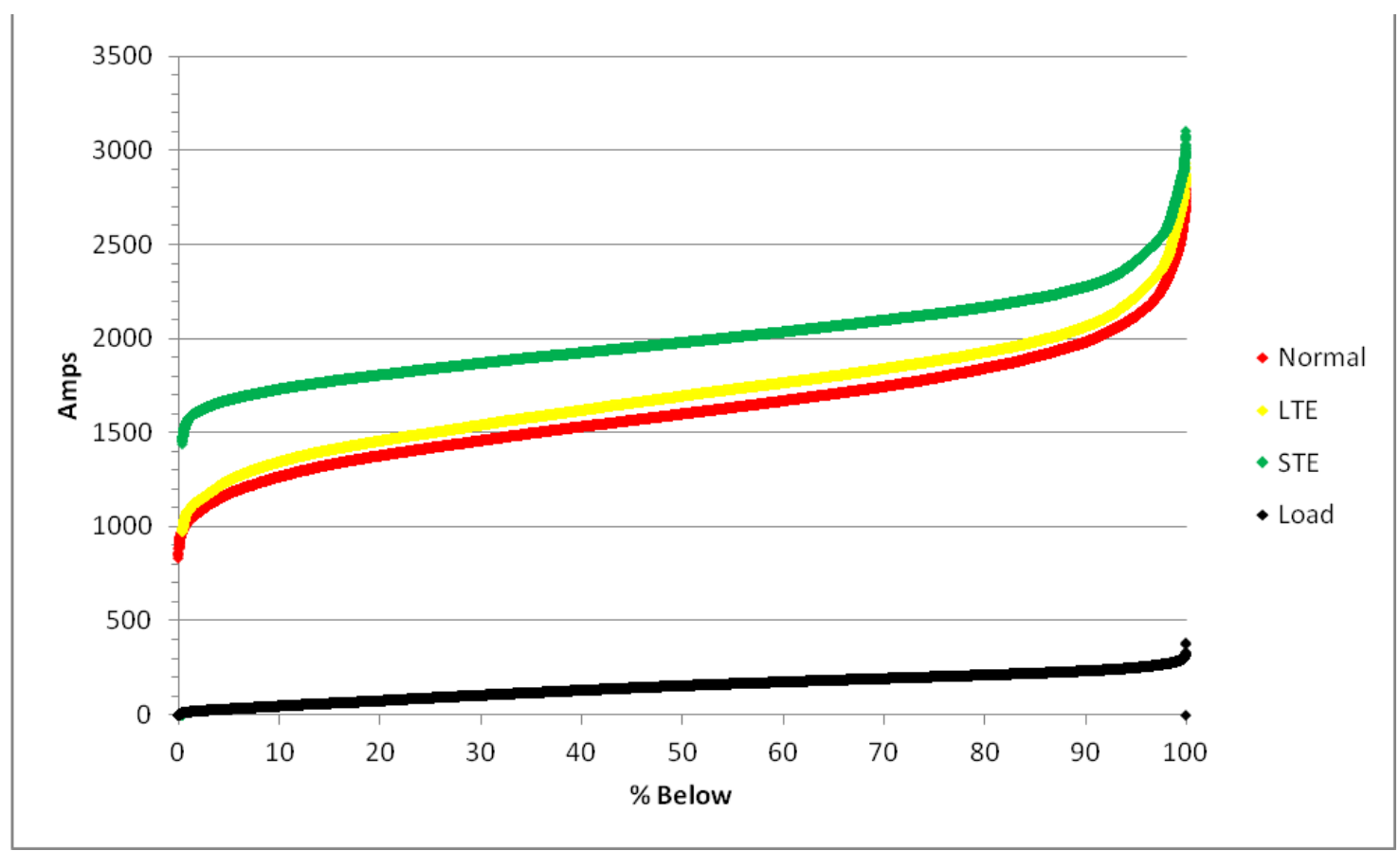

Figure H-16

Summer 2012 Ratings Distribution Site 2 Sagometer

Table H-16

Summer 2012 Ratings Distribution Site 2 Sagometer

\begin{tabular}{|c|l|l|l|l|l|l|}
\hline & \multicolumn{1}{|c|}{ Static } & \multicolumn{1}{|c|}{ L1 } & \multicolumn{1}{|c|}{ L2 } & \multicolumn{1}{|c|}{ L50 } & \multicolumn{1}{|c|}{ L95 } \\
\hline Normal & 1089 & 1033 & 1076 & 1175 & 1600 & 2120 \\
\hline LTE & 1256 & 1086 & 1139 & 1246 & 1695 & 2225 \\
\hline STE & 1410 & 1571 & 1614 & 1674 & 1980 & 2413 \\
\hline Load & & 0 & 10 & 25 & 150 & 260 \\
\hline
\end{tabular}




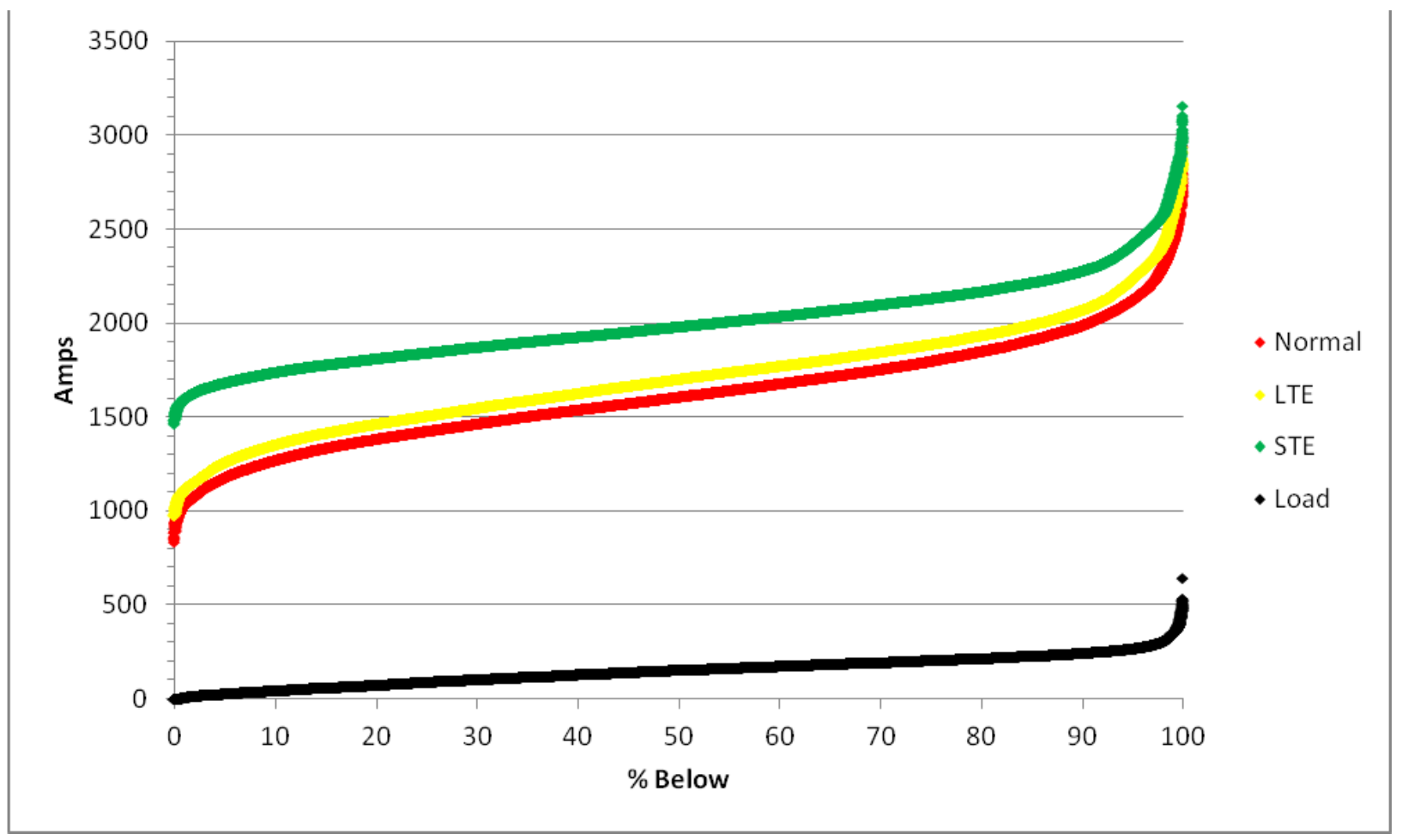

Figure $\mathrm{H}-17$

Summer 2012 Ratings Distribution Site 2 EPRI Sensors

Table H-17

Summer 2012 Ratings Distribution Site 2 EPRI Sensors

\begin{tabular}{|c|l|l|l|l|l|l|}
\hline & \multicolumn{1}{|c|}{ Static } & \multicolumn{1}{|c|}{ L1 } & \multicolumn{1}{|c|}{ L2 } & \multicolumn{1}{|c|}{ L5 } & \multicolumn{1}{|c|}{ L95 } \\
\hline Normal & 1089 & 993 & 1057 & 1165 & 1602 & 2105 \\
\hline LTE & 1256 & 1078 & 1137 & 1245 & 1697 & 2209 \\
\hline STE & 1410 & 1569 & 1614 & 1675 & 1977 & 2395 \\
\hline Load & & 0 & 10 & 25 & 150 & 260 \\
\hline
\end{tabular}

The Site 2 summer 2012 EPRI temperature sensor data can be compared to the Site 2 summer 2012 off site weather station data. It can be seen in the tables that there is approximately 100 amps difference between the two. The offsite weather service based rating is the more conservative of the two. 


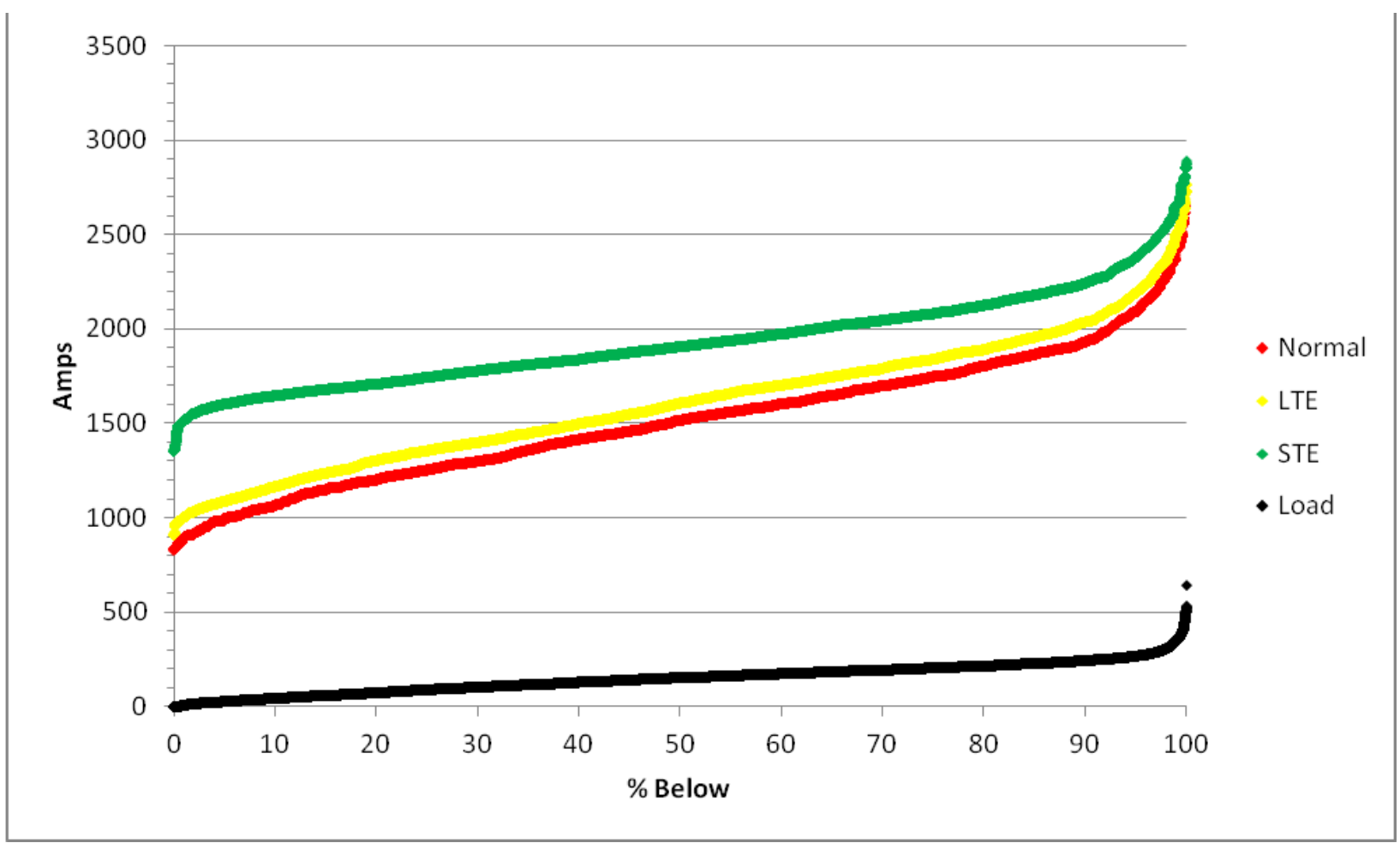

Figure $\mathrm{H}-18$

Summer 2012 Ratings Distribution Site 2 Offsite Weather Service

Table H-18

Summer 2012 Ratings Distribution Site 2 Offsite Weather Service

\begin{tabular}{|c|l|l|l|l|l|l|}
\hline & \multicolumn{1}{|c|}{ Static } & \multicolumn{1}{|c|}{ L1 } & \multicolumn{1}{c|}{ L2 } & \multicolumn{1}{c|}{ L5 } & \multicolumn{1}{c|}{ L50 } & \multicolumn{1}{|c|}{ L95 } \\
\hline Normal & 1089 & 866 & 906 & 979 & 1510 & 2081 \\
\hline LTE & 1256 & 981 & 1021 & 1078 & 1600 & 2166 \\
\hline STE & 1410 & 1489 & 1533 & 1595 & 1902 & 2355 \\
\hline Load & & 0 & 10 & 25 & 150 & 260 \\
\hline
\end{tabular}

The Site 2 summer 2012 off site weather service data can be compared to the Site 2 summer 2012 onsite weather station data. It can be seen in the tables that there is approximately 100amps difference between the two. The offsite weather service based rating is the more conservative of the two. 
Table H-19

Summary Table for Site 2 - L2 Were Used for Purposes of Comparison

\begin{tabular}{|l|l|l|l|}
\hline \multicolumn{1}{|c|}{ Description } & \multicolumn{1}{c|}{$\begin{array}{c}\text { L2 } \\
\text { Norm }\end{array}$} & \multicolumn{1}{|c|}{ L2 LTE } & \multicolumn{1}{|c|}{ L2 STE } \\
\hline Site 2 Onsite Weather Station Winter 2010/11 & 1192 & 1266 & 1794 \\
\hline Site 2 ThermalRate Winter 2010/11 & 1300 & 1384 & 1863 \\
\hline Site 2 Sagometer Winter 2010/11 & 1122 & 1270 & 1766 \\
\hline Site 2 EPRI Temperature Sensor Winter 2010/11 & 1122 & 1271 & 1781 \\
\hline Site 2 Onsite Weather Station Summer 2011 & 875 & 1072 & 1561 \\
\hline Site 2 ThermalRate Summer 2011 & 1079 & 1177 & 1628 \\
\hline Site 2 Sagometer Summer 2011 & 950 & 1101 & 1545 \\
\hline Site 2 EPRI Temperature Sensor Summer 2011 & 894 & 1087 & 1570 \\
\hline Site 2 Onsite Weather Station Winter 2011/12 & 1204 & 1276 & 1760 \\
\hline Site 2 ThermalRate Winter 2011/12 & 1315 & 1385 & 1824 \\
\hline Site 2 Sagometer Winter 2011/12 & 1205 & 1260 & 1788 \\
\hline Site 2 EPRI Temperature Sensor Winter 2011/12 & 1205 & 1266 & 1765 \\
\hline Site 2 Off Site Weather Service Winter 2011/12 & 1014 & 1104 & 1625 \\
\hline Site 2 Onsite Weather Station Summer 2012 & 1055 & 1130 & 1598 \\
\hline Site 2 ThermalRate Summer 2012 & 1185 & 1259 & 1669 \\
\hline MW236 04 Sagometer Summer 2012 & 1175 & 1246 & 1674 \\
\hline Site 2 EPRI Temperature Sensor Summer 2012 & 1057 & 1137 & 1614 \\
\hline Site 2 Off Site Weather Service Summer 2012 & 906 & 1021 & 1533 \\
\hline
\end{tabular}




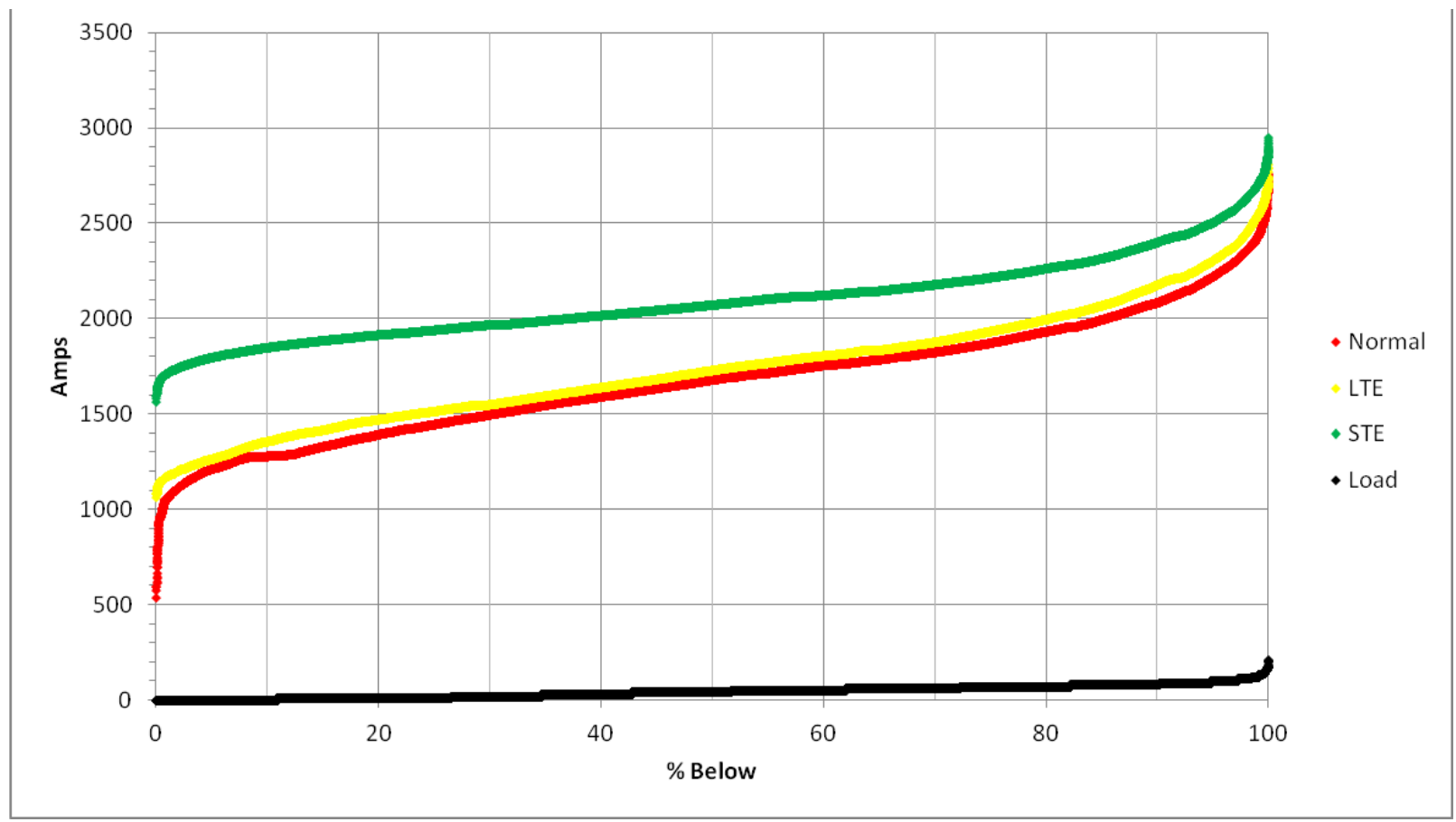

Figure H-19

Winter 2010/11 Ratings Distribution Site 3 Onsite weather station

Table H-20

Winter 2010/11 Ratings Distribution Site 3 Onsite weather station

\begin{tabular}{|c|l|l|l|l|l|l|}
\hline & \multicolumn{1}{|c|}{ Static } & \multicolumn{1}{|c|}{ L1 } & \multicolumn{1}{|c|}{ L2 } & \multicolumn{1}{c|}{ L50 } & \multicolumn{1}{|c|}{ L95 } \\
\hline Normal & 1331 & 1061 & 1116 & 1210 & 1678 & 2222 \\
\hline LTE & 1460 & 1174 & 1204 & 1264 & 1729 & 2305 \\
\hline STE & 1593 & 1715 & 1743 & 1796 & 2072 & 2504 \\
\hline Load & & 0 & 0 & 0 & 40 & 100 \\
\hline
\end{tabular}

The Site 3 winter 2010/11 local weather station data can be compared to the Site 3 winter 2010/11 ThermalRate data. It can be seen in the tables that there is approximately 100 amps difference between the two. The weather station based rating is the more conservative of the two. 


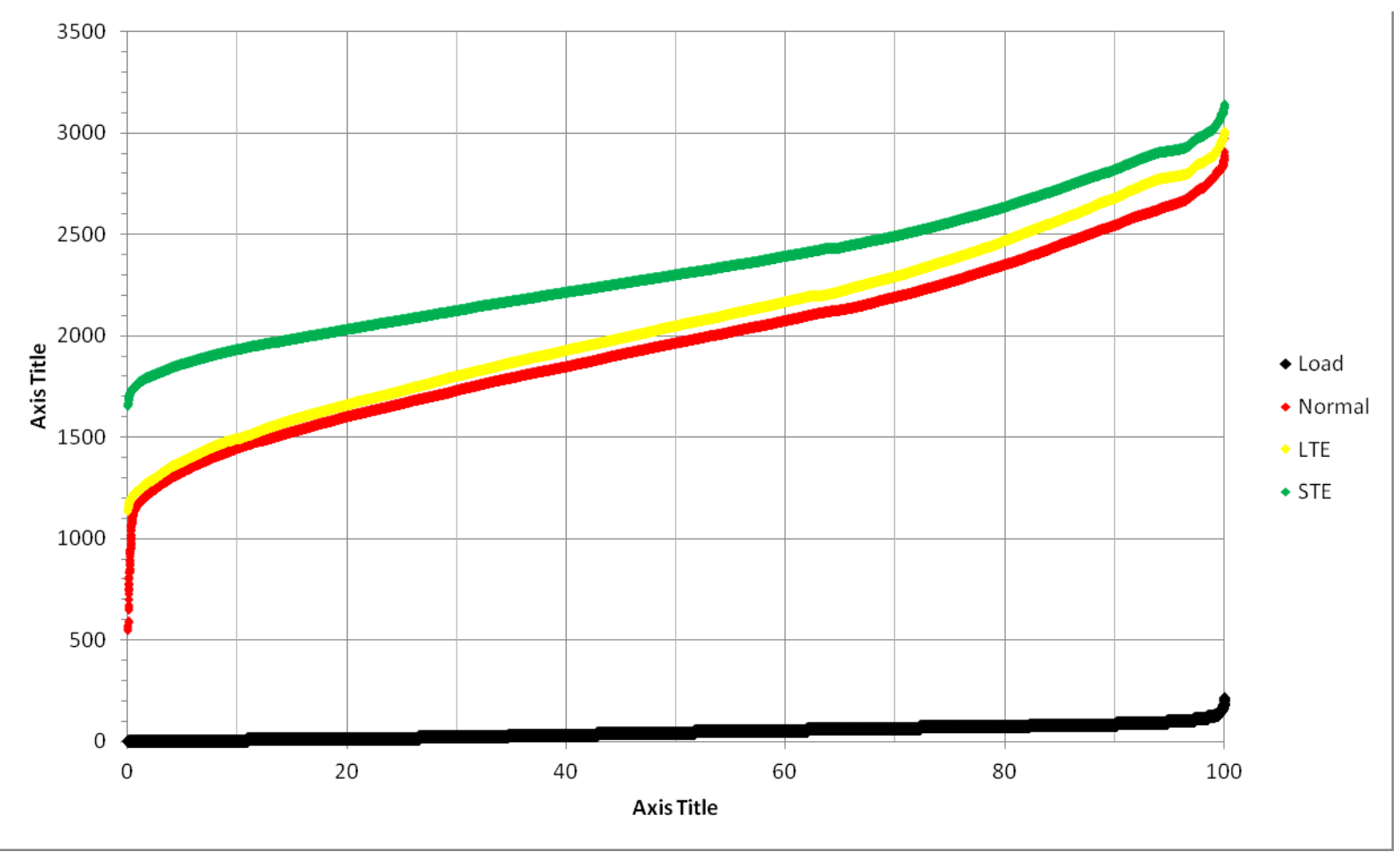

Figure $\mathrm{H}-20$

Winter 2010/11 Ratings Distribution Site 3 ThermalRate

Table H-21

Winter 2010/11 Ratings Distribution Site 3 ThermalRate

\begin{tabular}{|c|l|l|l|l|l|l|}
\hline & \multicolumn{1}{|c|}{ Static } & \multicolumn{1}{|c|}{ L1 } & \multicolumn{1}{|c|}{ L2 } & \multicolumn{1}{|c|}{ L50 } & \multicolumn{1}{|c|}{ L95 } \\
\hline Normal & 1331 & 1175 & 1226 & 1331 & 1964 & 2641 \\
\hline LTE & 1460 & 1239 & 1282 & 1381 & 2047 & 2782 \\
\hline STE & 1593 & 1769 & 1798 & 1861 & 2300 & 2912 \\
\hline Load & & 0 & 0 & 0 & 40 & 100 \\
\hline
\end{tabular}

The Site 3 winter 2010/11 ThermalRate data can be compared to the Site 3 winter 2011/12 ThermalRate data. It can be seen in the tables that there is approximately 20 amps difference between the two. This difference is small suggesting consistency from year to year. 


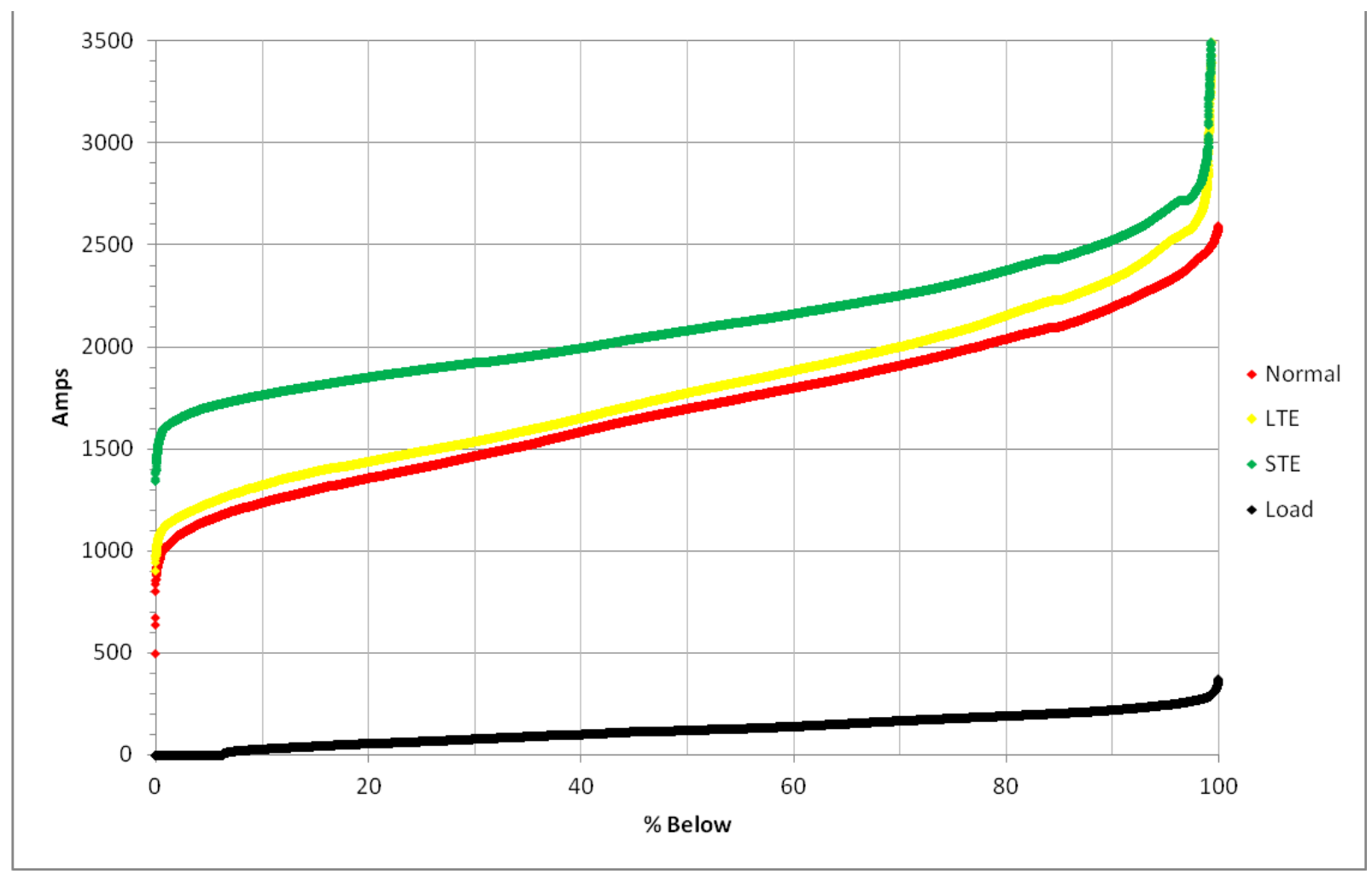

Figure $\mathrm{H}-21$

Winter 2010/11 Ratings Distribution Site 3 Sagometer

Table H-22

Winter 2010/11 Ratings Distribution Site 3 Sagometer

\begin{tabular}{|c|l|l|l|l|l|l|}
\hline & \multicolumn{1}{|c|}{ Static } & \multicolumn{1}{|c|}{ L1 } & \multicolumn{1}{|c|}{ L2 } & \multicolumn{1}{|c|}{ L5 } & \multicolumn{1}{|c|}{ L50 } \\
\hline Normal & 1331 & 1021 & 1071 & 1153 & 1702 & 2335 \\
\hline LTE & 1460 & 1127 & 1161 & 1234 & 1776 & 2504 \\
\hline STE & 1593 & 1610 & 1645 & 1707 & 2802 & 2670 \\
\hline Load & & 0 & 0 & 0 & 40 & 100 \\
\hline
\end{tabular}




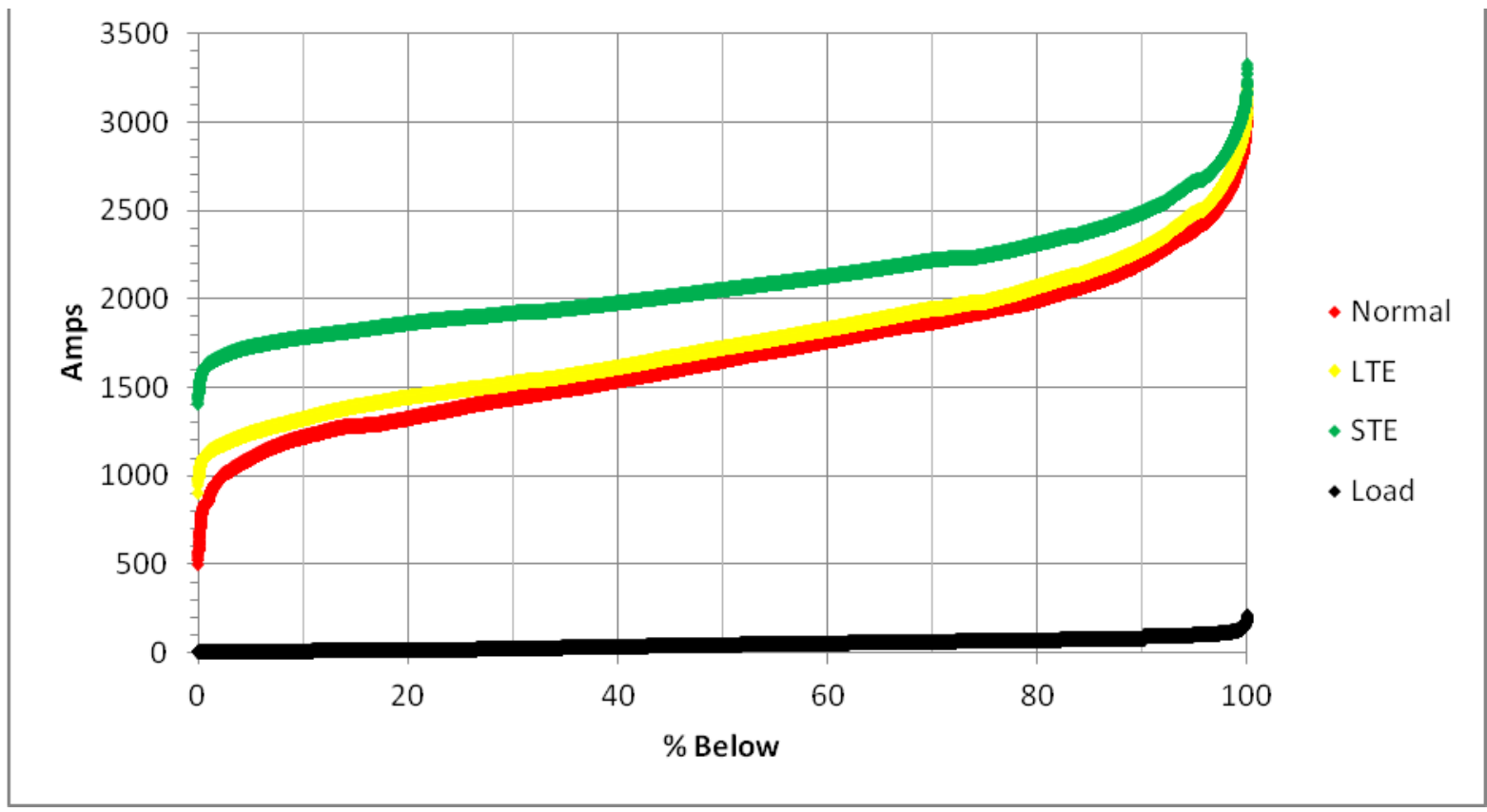

Figure $\mathrm{H}-22$

Winter 2010/11 Ratings Distribution Site 3 EPRI Sensor

Table H-23

Winter 2010/11 Ratings Distribution Site 3 EPRI Sensor

\begin{tabular}{|c|l|l|l|l|l|l|}
\hline & \multicolumn{1}{|c|}{ Static } & \multicolumn{1}{|c|}{ L1 } & \multicolumn{1}{|c|}{ L2 } & \multicolumn{1}{|c|}{ L5 } & \multicolumn{1}{|c|}{ L95 } \\
\hline Normal & 1331 & 869 & 979 & 1095 & 1647 & 2395 \\
\hline LTE & 1460 & 1133 & 1167 & 1242 & 1721 & 2487 \\
\hline STE & 1593 & 1633 & 1666 & 1727 & 2051 & 2665 \\
\hline Load & & 0 & 0 & 0 & 40 & 100 \\
\hline
\end{tabular}

The Site 3 winter 2010/11sagometer data can be compared to the Site 3 winter 2010/11 EPRI Sensor data. When comparing the L2 levels it can be seen in the tables that the Normal and the LTE ratings are about the same, however, there is some difference in the STE level. DTCR is forced to use the weather model when the line is lightly loaded. Less than 5 percent of the time the load was high enough for DTCR to fully utilize the Sagometer end EPRI Sensors. Thermal inertia plays little to no roll in LTE or Normal rating calculations. However, it does play a significant part in the STE calculation. Because of the importance of thermal inertia, a temperature difference in the measured or calculated conductor temperature will show up in the STE rating calculation, as it does here. 


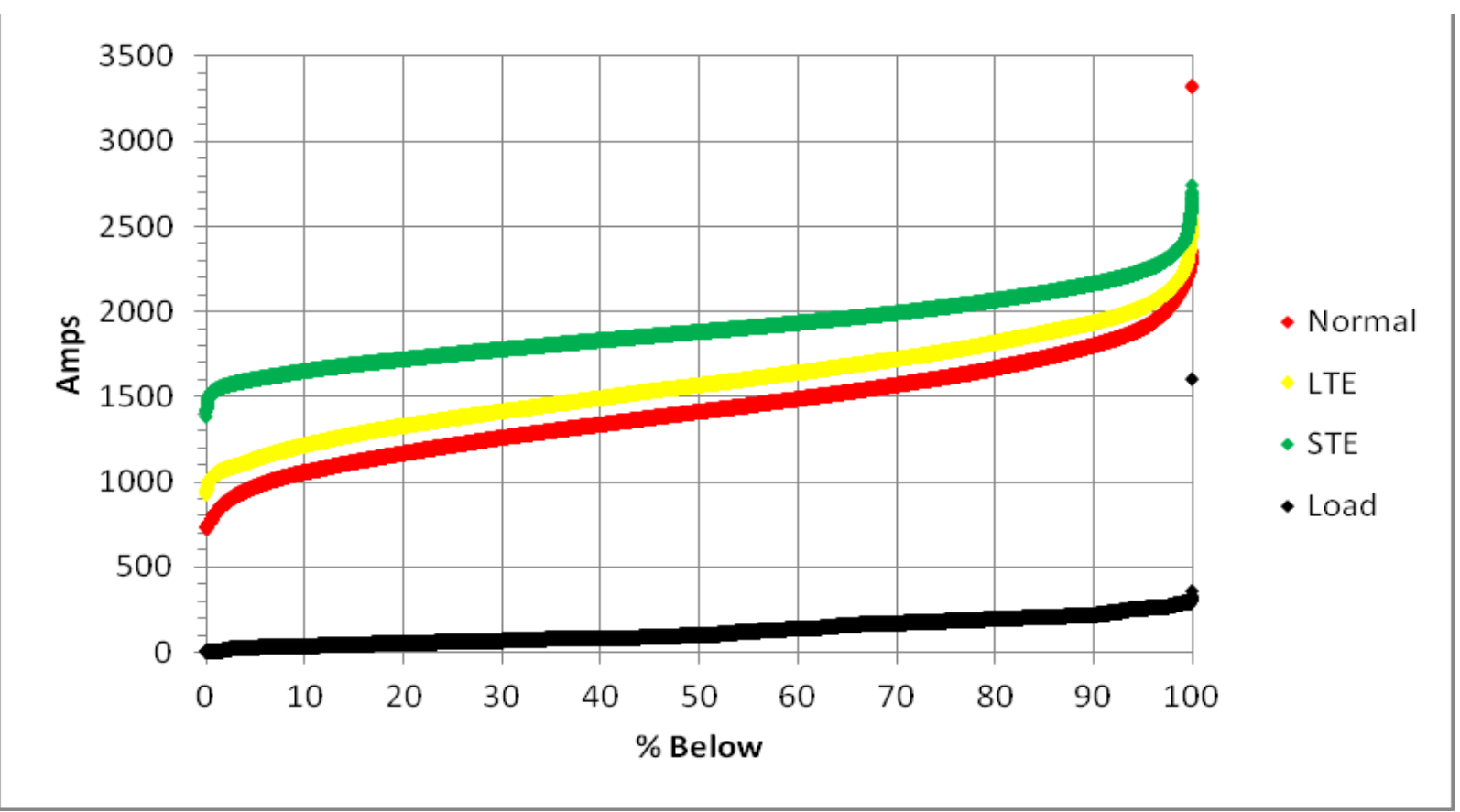

Figure $\mathrm{H}-23$

Summer 2011 Ratings Distribution Site 3 Onsite Weather Station

Table H-24

Summer 2011 Ratings Distribution Site 3 Onsite Weather Station

\begin{tabular}{|c|l|l|l|l|l|l|}
\hline & \multicolumn{1}{|c|}{ Static } & \multicolumn{1}{|c|}{ L1 } & \multicolumn{1}{|c|}{ L2 } & \multicolumn{1}{|c|}{ L50 } & \multicolumn{1}{|c|}{ L95 } \\
\hline Normal & 1089 & 817 & 875 & 967 & 1407 & 1900 \\
\hline LTE & 1256 & 1042 & 1072 & 1130 & 1563 & 2021 \\
\hline STE & 1410 & 1536 & 1561 & 1600 & 1875 & 2237 \\
\hline Load & & 0 & 10 & 25 & 100 & 250 \\
\hline
\end{tabular}

The Site 3 summer 2011 weather station data can be compared to the Site 3 summer 2011 ThermalRate sensor data. It can be seen in the tables that there is approximately 200amps difference between the two. The weather station based rating is the more conservative of the two. 


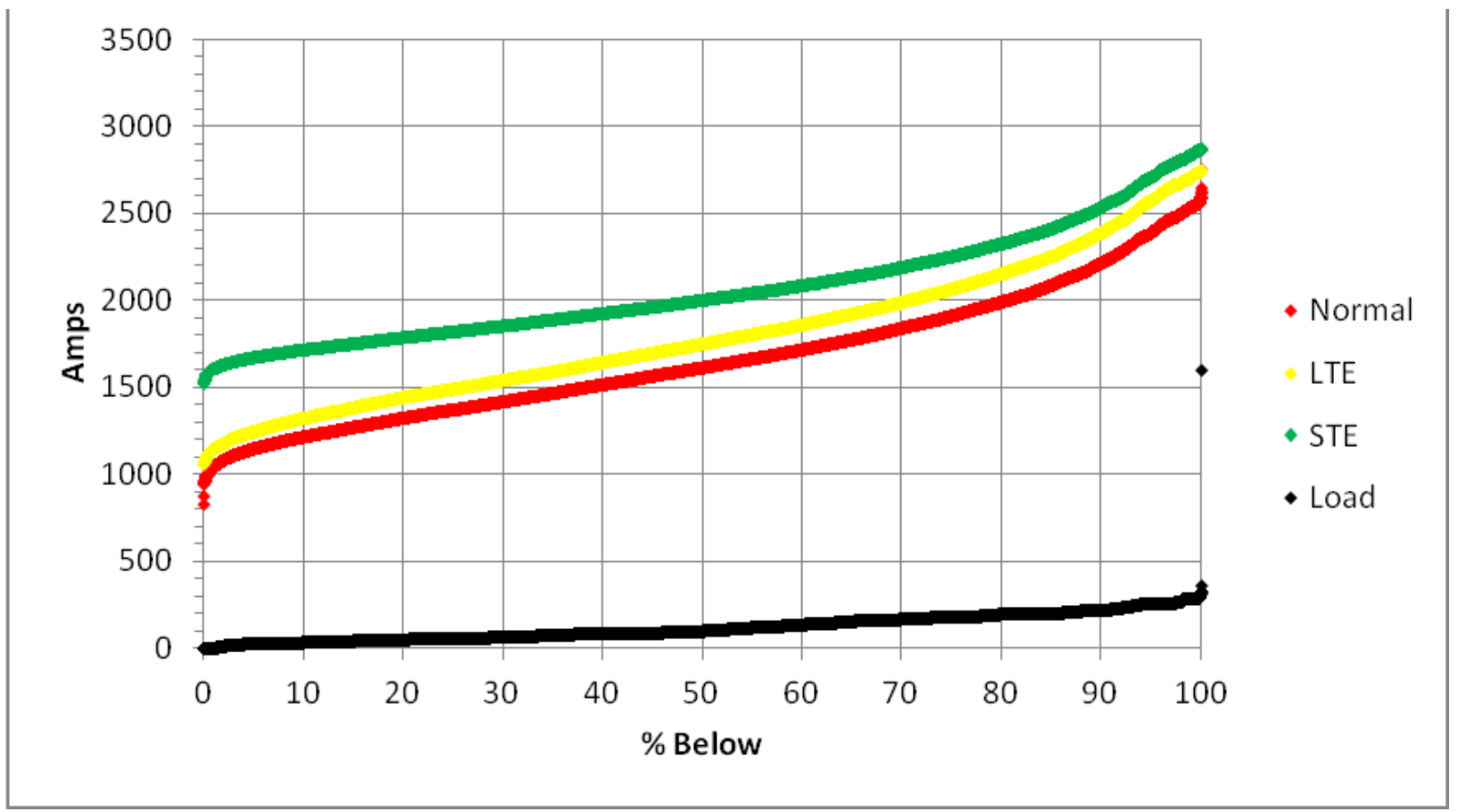

Figure H-24

Summer 2011 Ratings Distribution Site 3 ThermalRate

Table H-25

Summer 2011 Ratings Distribution Site 3 ThermalRate

\begin{tabular}{|c|l|l|l|l|l|l|}
\hline & \multicolumn{1}{|c|}{ Static } & \multicolumn{1}{|c|}{ L1 } & \multicolumn{1}{|c|}{ L2 } & \multicolumn{1}{c|}{ L5 } & \multicolumn{1}{|c|}{ L95 } \\
\hline Normal & 1089 & 1042 & 1078 & 1145 & 1611 & 2388 \\
\hline LTE & 1256 & 1145 & 1176 & 1243 & 1747 & 2572 \\
\hline STE & 1410 & 1606 & 1627 & 1669 & 1999 & 2706 \\
\hline Load & & 0 & 10 & 25 & 100 & 250 \\
\hline
\end{tabular}

The Site 3 summer 2011 ThermalRate data can be compared to the Site 3 summer 2012 ThermalRate sensor data. It can be seen in the tables that there is approximately 30 amps difference between the two normal ratings. This higher rating is a result of a slightly increased wind speed September of 2012. 


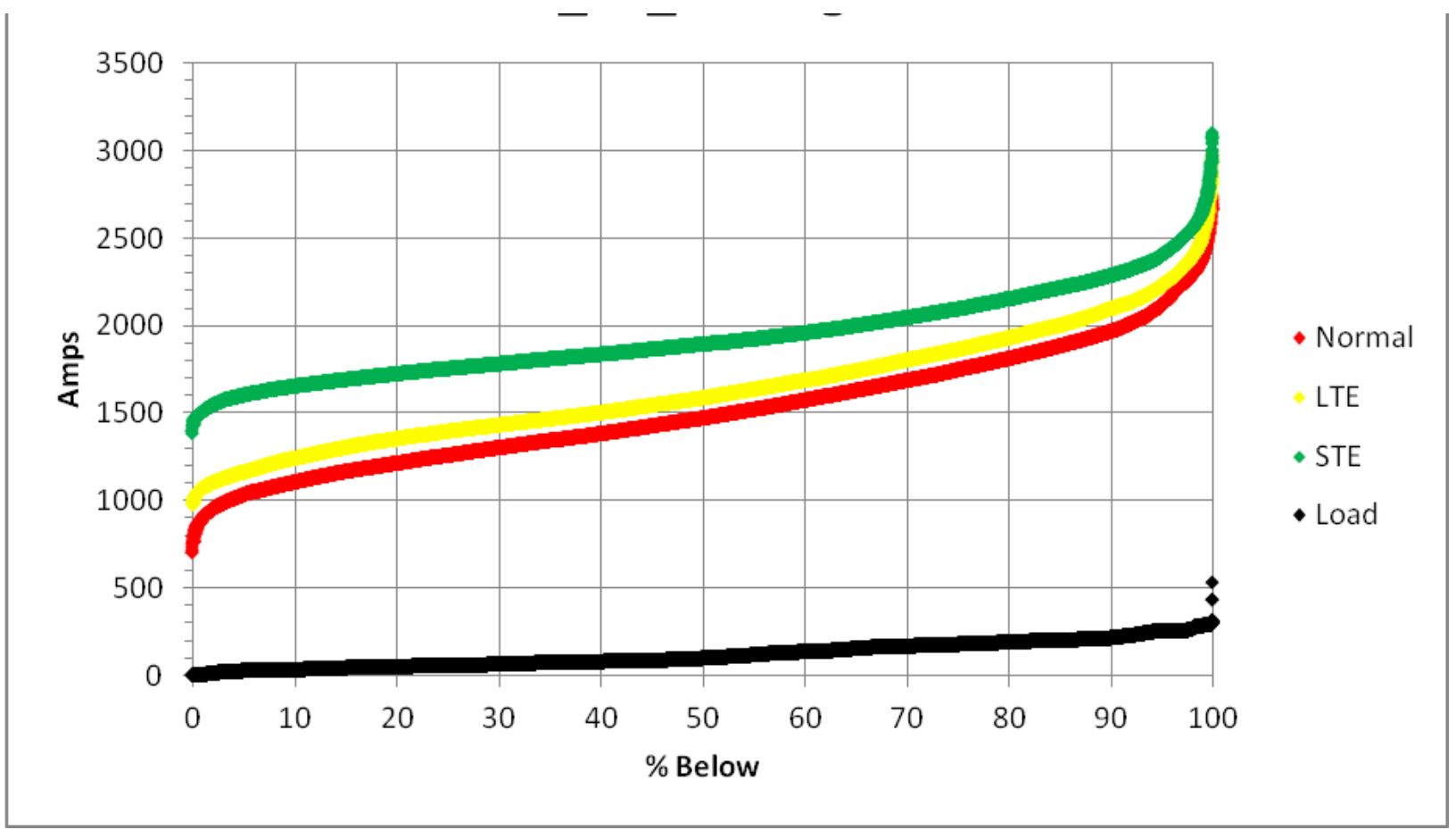

Figure $\mathrm{H}-25$

Summer 2011 Ratings Distribution Site 3 Video Sagometer

Table H-26

Summer 2011 Ratings Distribution Site 3 Video Sagometer

\begin{tabular}{|c|l|l|l|l|l|l|}
\hline & \multicolumn{1}{|c|}{ Static } & \multicolumn{1}{|c|}{ L1 } & \multicolumn{1}{|c|}{ L2 } & \multicolumn{1}{|c|}{ L5 } & \multicolumn{1}{|c|}{ L95 } \\
\hline Normal & 1089 & 898 & 949 & 1031 & 1468 & 2117 \\
\hline LTE & 1256 & 1070 & 1100 & 1159 & 1584 & 2226 \\
\hline STE & 1410 & 1506 & 1543 & 1601 & 1892 & 2403 \\
\hline Load & & 0 & 10 & 25 & 100 & 250 \\
\hline
\end{tabular}




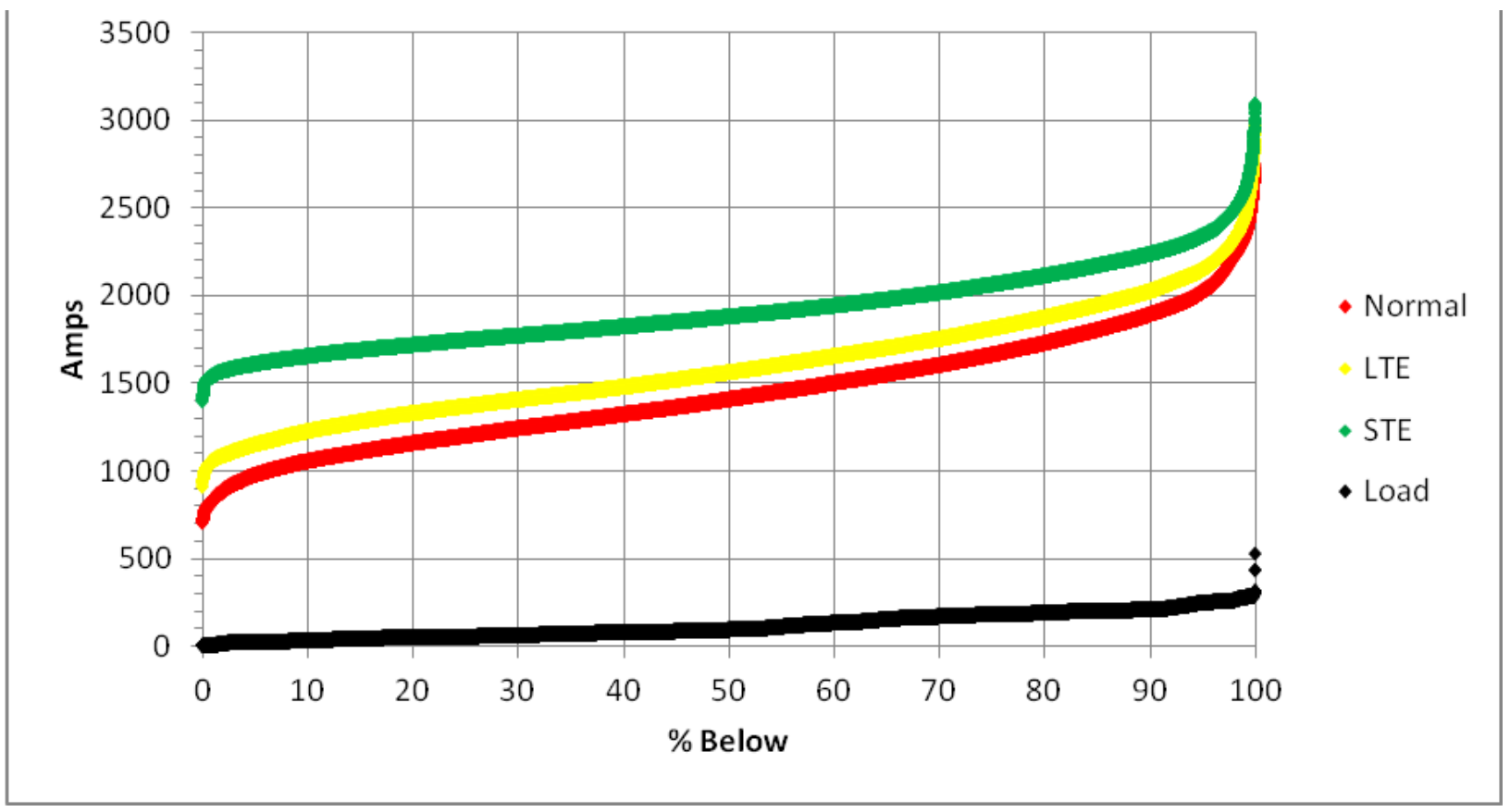

Figure H-26

Summer 2011 Ratings Distribution Site 3 EPRI Sensors

Table H-27

Summer 2011 Ratings Distribution Site 3 EPRI Sensors

\begin{tabular}{|c|l|l|l|l|l|l|}
\hline & \multicolumn{1}{|c|}{ Static } & \multicolumn{1}{|c|}{ L1 } & \multicolumn{1}{c|}{ L2 } & \multicolumn{1}{c|}{ L5 } & \multicolumn{1}{|c|}{ L50 } & \multicolumn{1}{|c|}{} \\
\hline Normal & 1089 & 835 & 889 & 976 & 1407 & 2023 \\
\hline LTE & 1256 & 1057 & 1086 & 1150 & 1559 & 2149 \\
\hline STE & 1410 & 1542 & 1569 & 1608 & 1878 & 2341 \\
\hline Load & & 0 & 10 & 25 & 100 & 250 \\
\hline
\end{tabular}

One advantage to the EPRI Sensor is that it directly measures conductor temperature.

Consequently the state change equation is 1 . The disadvantage is the sensor is a single point measurement. It would take a number of sensors along that line section to start to approach the distributed measurements that you get with a load cell or sag measuring device. The conductor temperature then gets converted into an equivalent perpendicular wind speed. Then the line rating calculation is made. So it is critical that the conductor temperature be accurate! Small differences would result in changes like we see between Site 3 summer 2011 sagometer data and Site 3 summer 2011 EPRI Sensor data 


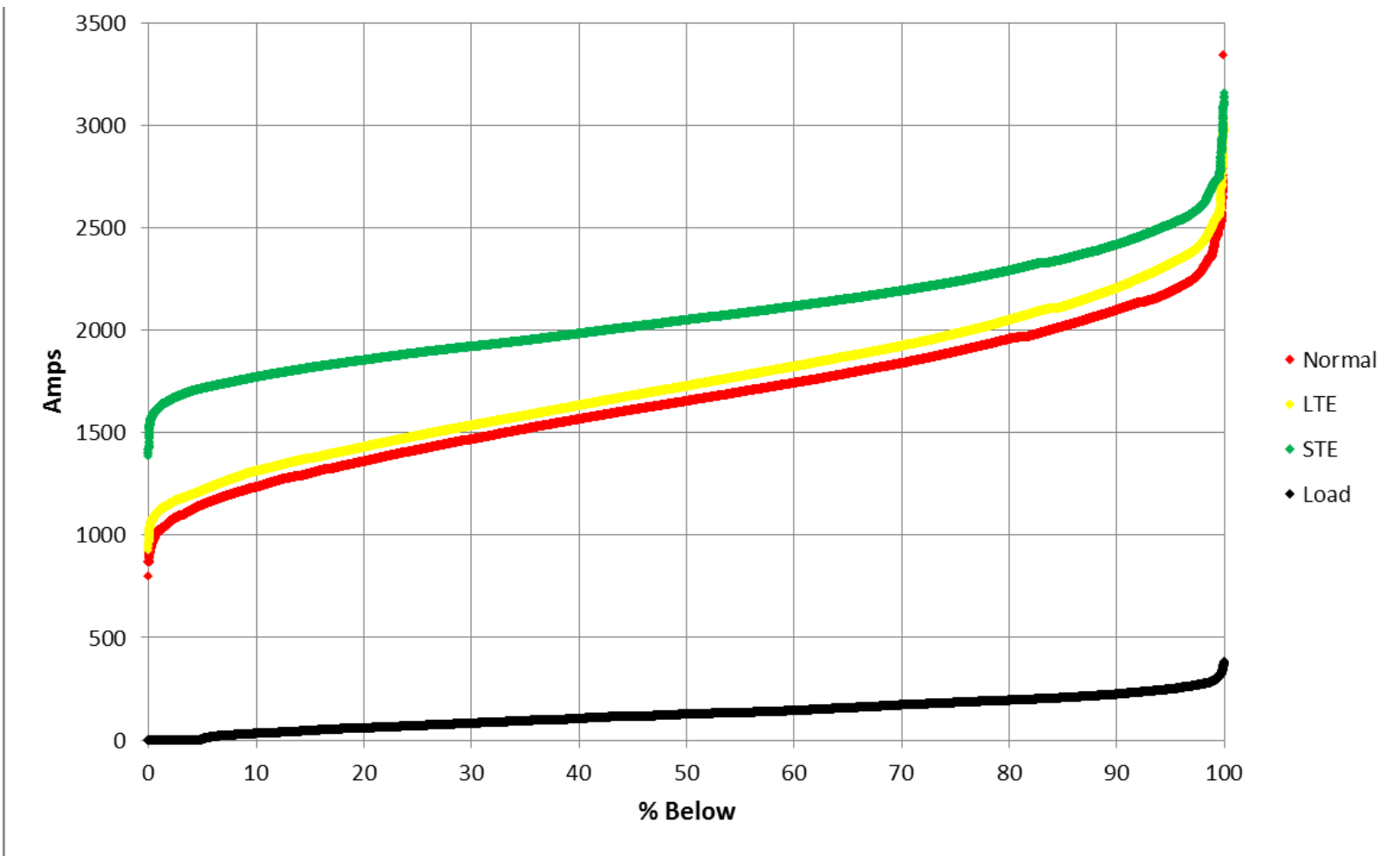

Figure $\mathrm{H}-27$

Winter 2011/12 Ratings Distribution Site 3 Onsite weather station

Table H-28

Winter 2011/12 Ratings Distribution Site 3 Onsite weather station

\begin{tabular}{|c|l|l|l|l|l|l|}
\hline & \multicolumn{1}{|c|}{ Static } & \multicolumn{1}{|c|}{ L1 } & \multicolumn{1}{|c|}{ L2 } & \multicolumn{1}{|c|}{ L5 } & \multicolumn{1}{|c|}{ L50 } \\
\hline Normal & 1331 & 1023 & 1066 & 1147 & 1652 & 2185 \\
\hline LTE & 1460 & 1116 & 1152 & 1215 & 1726 & 2321 \\
\hline STE & 1593 & 1622 & 1657 & 1714 & 2048 & 2999 \\
\hline Load & & 0 & 0 & 10 & 120 & 250 \\
\hline
\end{tabular}

The Site 3 winter 2011/12 Onsite weather station data can be compared to the Site 3 winter 2010/11 Onsite weather station sensor data. There is about 50 amps difference between the two seasons at the L2 level. This is a larger difference than seen at the other end of this line. 


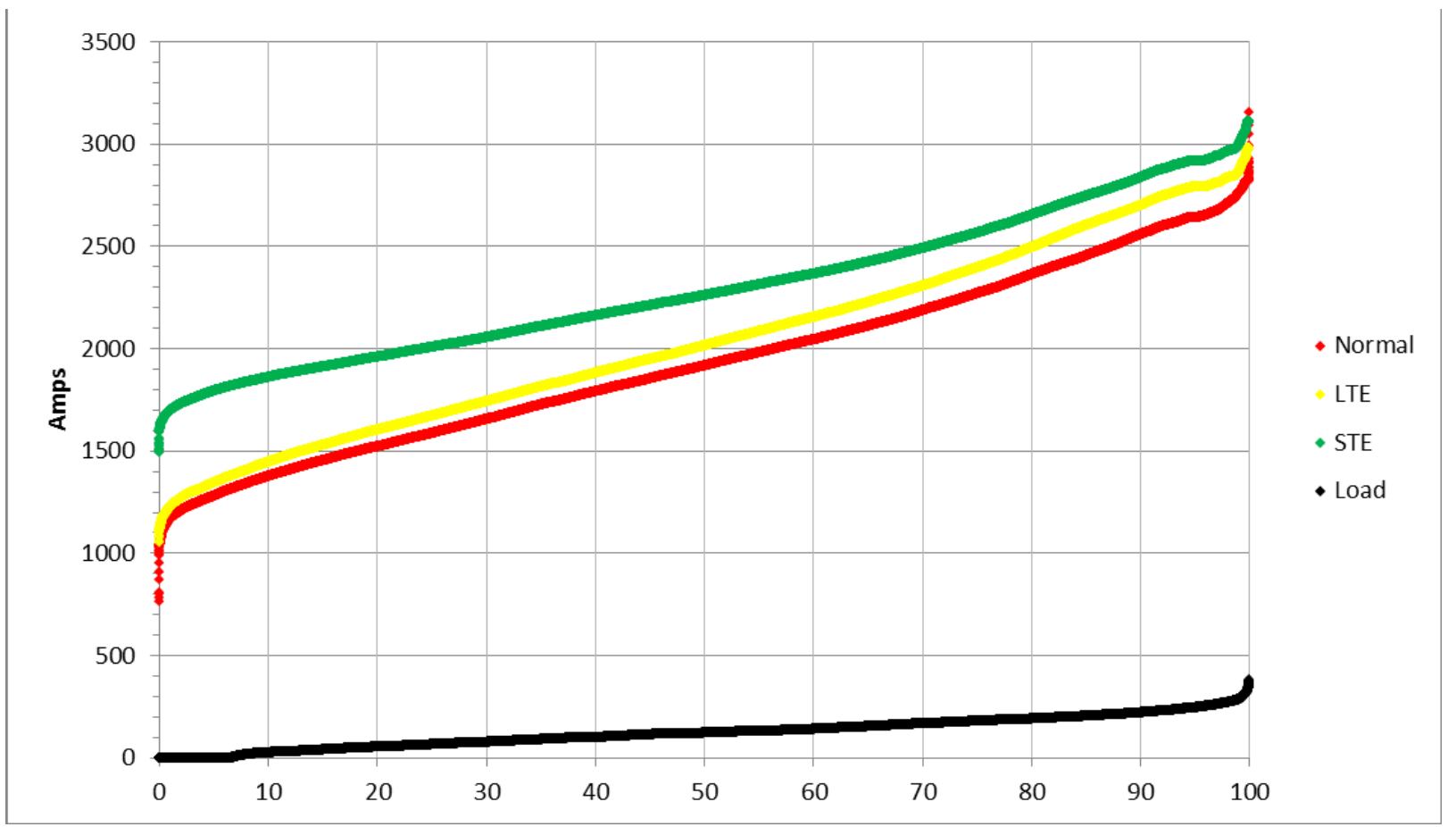

Figure H-28

Winter 2011/12 Ratings Distribution Site 3 ThermalRate

Table H-29

Winter 2011/12 Ratings Distribution Site 3 ThermalRate

\begin{tabular}{|c|l|l|l|l|l|l|}
\hline & \multicolumn{1}{|c|}{ Static } & \multicolumn{1}{|c|}{ L1 } & \multicolumn{1}{|c|}{ L2 } & \multicolumn{1}{|c|}{ L5 } & \multicolumn{1}{|c|}{ L95 } \\
\hline Normal & 1331 & 1170 & 1208 & 1279 & 1916 & 2642 \\
\hline LTE & 1460 & 1227 & 1270 & 1344 & 2014 & 2788 \\
\hline STE & 1593 & 1696 & 1731 & 1796 & 2560 & 2917 \\
\hline Load & & 0 & 0 & 10 & 120 & 250 \\
\hline
\end{tabular}

The Site 3 winter 2011/12 ThermalRate can be compared to the Site 3 winter 2010/11 ThermalRate sensor data. It can be seen in the tables that there is less than 20 amps difference between the two for the normal and LTE ratings. This would indicate that the ratings may be consistent from season to season. 


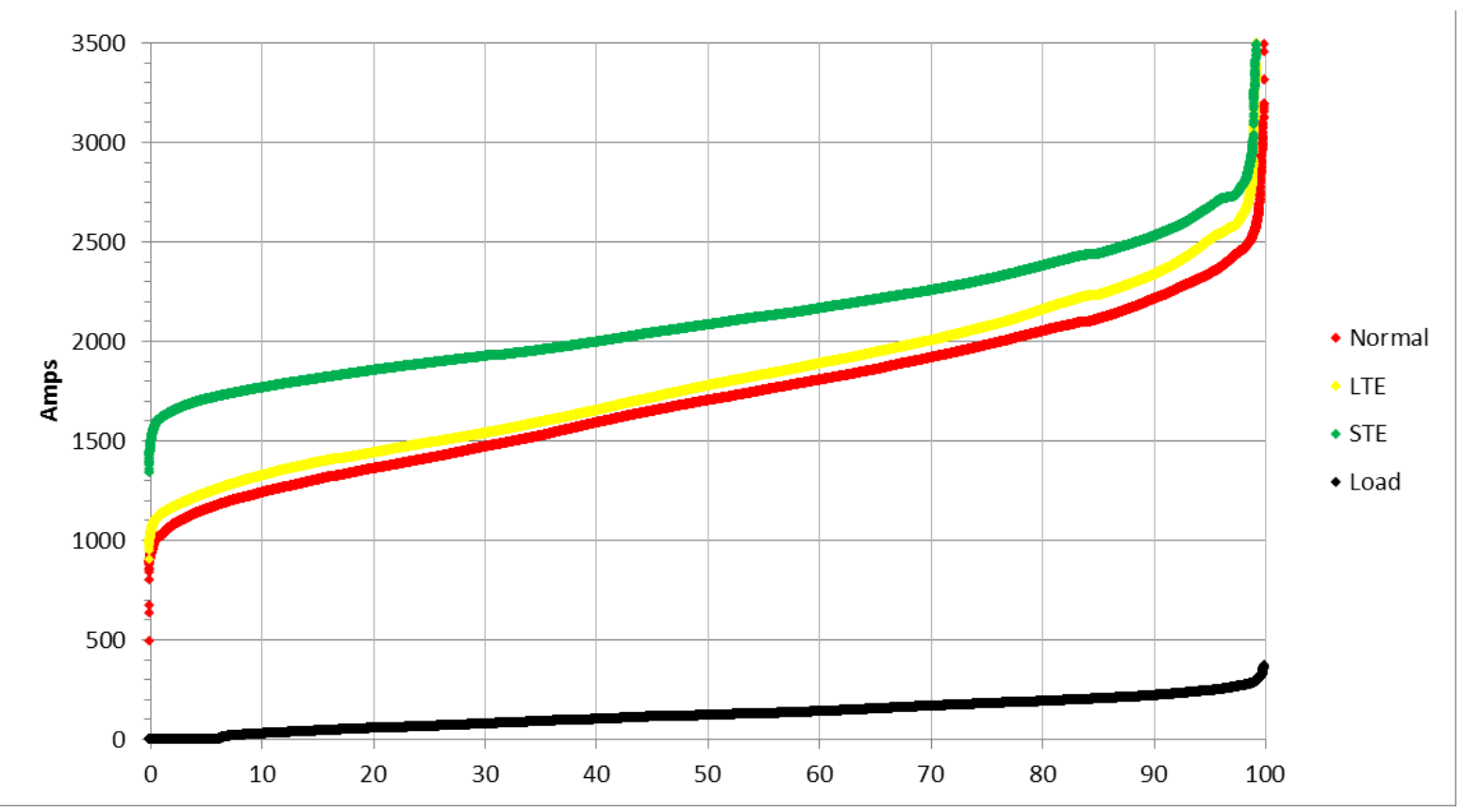

Figure H-29

Winter 2011/12 Ratings Distribution Site 3 Sagometer

Table H-30

Winter 2011/12 Ratings Distribution Site 3 Sagometer

\begin{tabular}{|c|l|l|l|l|l|l|}
\hline & \multicolumn{1}{|c|}{ Static } & \multicolumn{1}{|c|}{ L1 } & \multicolumn{1}{|c|}{ L2 } & \multicolumn{1}{|c|}{ L5 } & \multicolumn{1}{|c|}{ L95 } \\
\hline Normal & 1331 & 1021 & 1070 & 1153 & 1699 & 2318 \\
\hline LTE & 1460 & 1128 & 1161 & 1233 & 1772 & 2478 \\
\hline STE & 1593 & 1611 & 1645 & 1707 & 2080 & 2648 \\
\hline Load & & 0 & 0 & 10 & 120 & 250 \\
\hline
\end{tabular}

The Site 3 winter 2011/12 sagometer data can be compared to the Site 3 winter 2011/12 EPRI Sensor data. The values and shape of these curves will be the same because most of the time DTCR is forced to fall back on the weather station model. If the line had more load, it would be possible to properly evaluate the technology. It is likely there would be differences. Some of these differences would be explained by state change equations and point measurements versus ruling span measurements. 


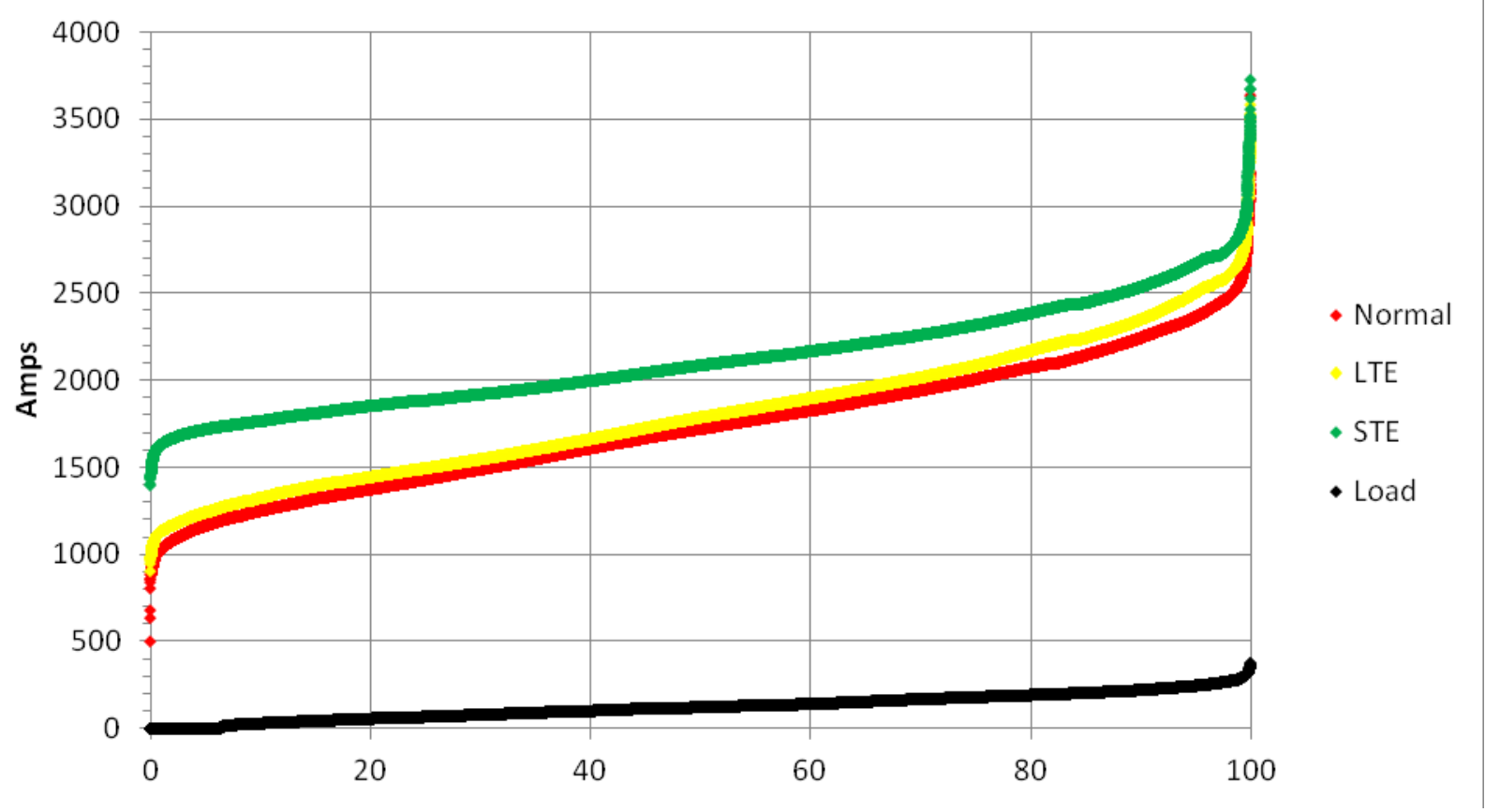

Figure $\mathrm{H}-30$

Winter 2011/12 Ratings Distribution Site 3 EPRI Sensor

Table H-31

Winter 2011/12 Ratings Distribution Site 3 EPRI Sensor

\begin{tabular}{|c|l|l|l|l|l|l|}
\hline & \multicolumn{1}{|c|}{ Static } & \multicolumn{1}{|c|}{ L1 } & \multicolumn{1}{c|}{ L2 } & \multicolumn{1}{c|}{ L50 } & \multicolumn{1}{|c|}{ L95 } \\
\hline Normal & 1331 & 1027 & 1078 & 1163 & 1720 & 2363 \\
\hline LTE & 1460 & 1133 & 1167 & 1241 & 1786 & 2503 \\
\hline STE & 1593 & 1633 & 1666 & 1718 & 2085 & 2669 \\
\hline Load & & 0 & 0 & 10 & 120 & 250 \\
\hline
\end{tabular}

The Site 3 winter 2011/12 Sagometer data can be compared to the Site 3 winter 2011/12 EPRI Sensor data. When comparing the L2 levels it can be seen in the tables that the Normal and the LTE ratings are about the same, however, there is some difference in the STE level. DTCR is forced to use the weather model when the line is lightly loaded. Less than 5 percent of the time the load was high enough for DTCR to fully utilize the Sagometer end EPRI Sensors. Thermal inertia plays no role in the Normal rating calculations and only a small role in the LTE rating calculations. However, it does play a significant part in the STE calculation. Because of the importance of thermal inertia, a temperature difference in the measured or calculated conductor temperature will show up in the STE rating calculation, as it does here. 


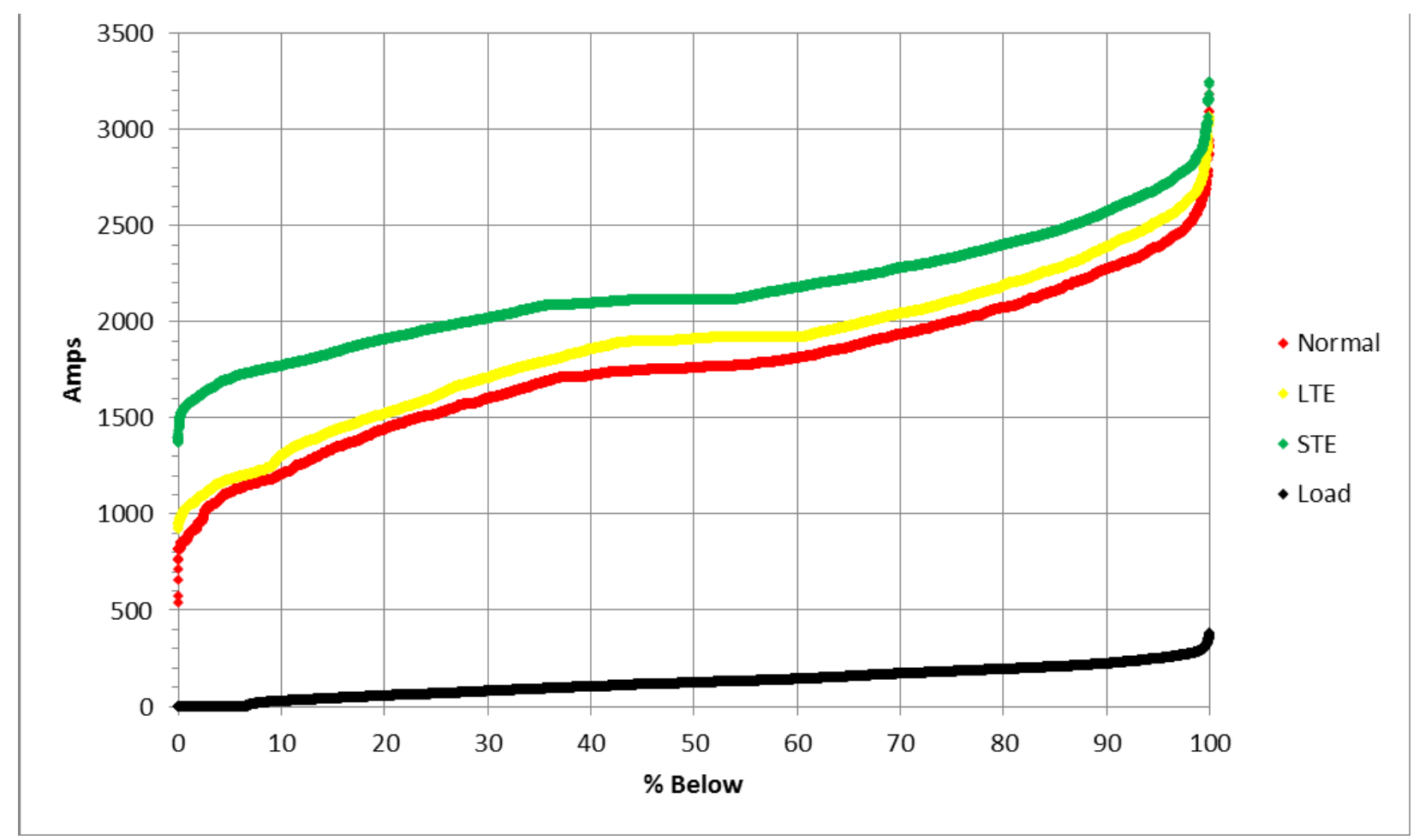

Figure H-31

Winter 2011/12 Ratings Distribution site 3 Offsite Weather Service

Table H-32

Winter 2011/12 Ratings Distribution site 3 Offsite Weather Service

\begin{tabular}{|c|l|l|l|l|l|l|}
\hline & \multicolumn{1}{|c|}{ Static } & \multicolumn{1}{|c|}{ L1 } & \multicolumn{1}{|c|}{ L2 } & \multicolumn{1}{|c|}{ L50 } & \multicolumn{1}{|c|}{ L95 } \\
\hline Normal & 1331 & 885 & 953 & 1107 & 1758 & 2385 \\
\hline LTE & 1460 & 1041 & 1083 & 1178 & 1908 & 2517 \\
\hline STE & 1593 & 1570 & 1610 & 1698 & 2110 & 2689 \\
\hline Load & & 0 & 0 & 10 & 120 & 250 \\
\hline
\end{tabular}

The Site 3 Winter 2011/12 Offsite weather service data can be compared to Site 3 winter 2011/12 Onsite weather station data. Typically weather data taken at an air port results in ratings that are higher than ratings based on calculation that are based off of a weather station in the ROW. However, given the layout of the geography and the geometry of the line, it turns out that the National Weather Service data results in more conservative ratings. A difference of approximately 100 amps can be observed. 


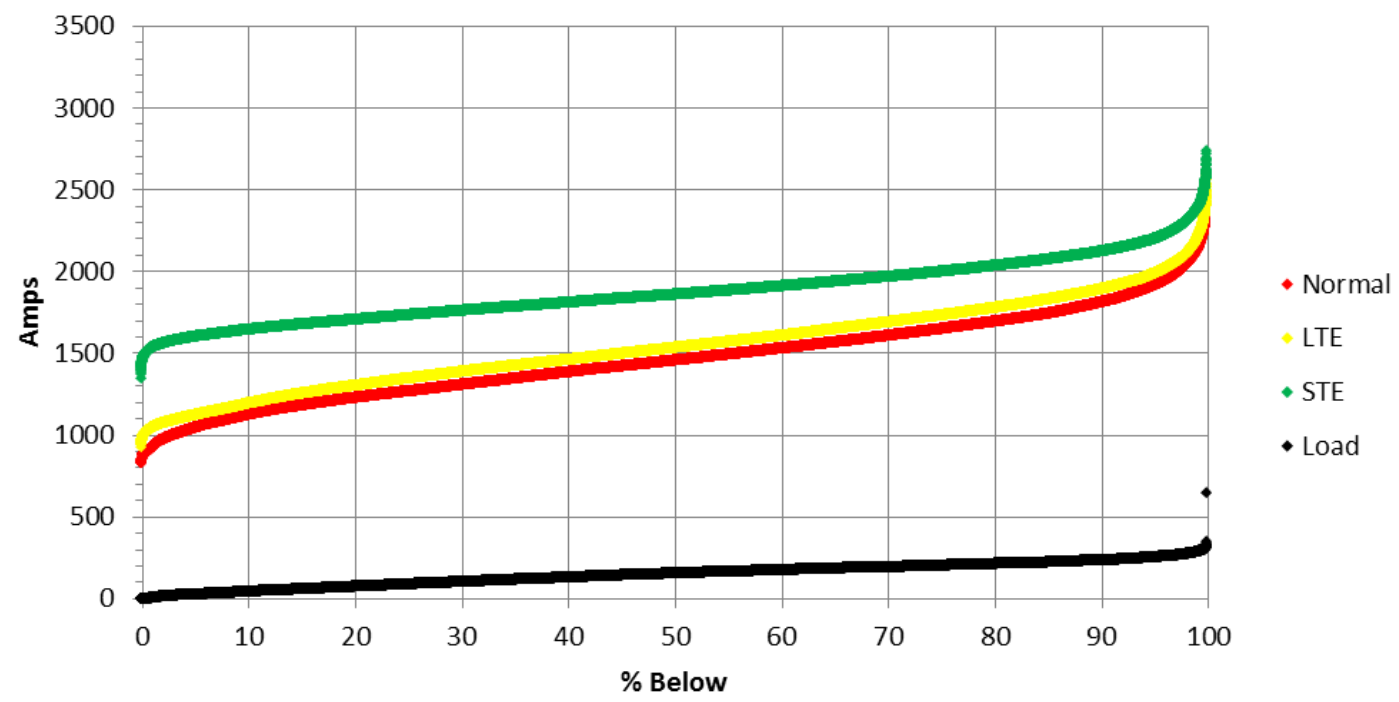

Figure H-32

Summer 2012 Ratings Distribution Site 3 Onsite Weather Station

Table H-33

Summer 2012 Ratings Distribution Site 3 Onsite weather station

\begin{tabular}{|c|l|l|l|l|l|l|}
\hline & \multicolumn{1}{|c|}{ Static } & \multicolumn{1}{|c|}{ L1 } & \multicolumn{1}{c|}{ L2 } & \multicolumn{1}{c|}{ L5 } & \multicolumn{1}{c|}{ L95 } \\
\hline Normal & 1089 & 925 & 971 & 1045 & 1456 & 1917 \\
\hline LTE & 1256 & 1048 & 1074 & 1122 & 1534 & 1988 \\
\hline STE & 1410 & 1534 & 1560 & 1602 & 1858 & 2199 \\
\hline Load & & 0 & 20 & 30 & 160 & 250 \\
\hline
\end{tabular}




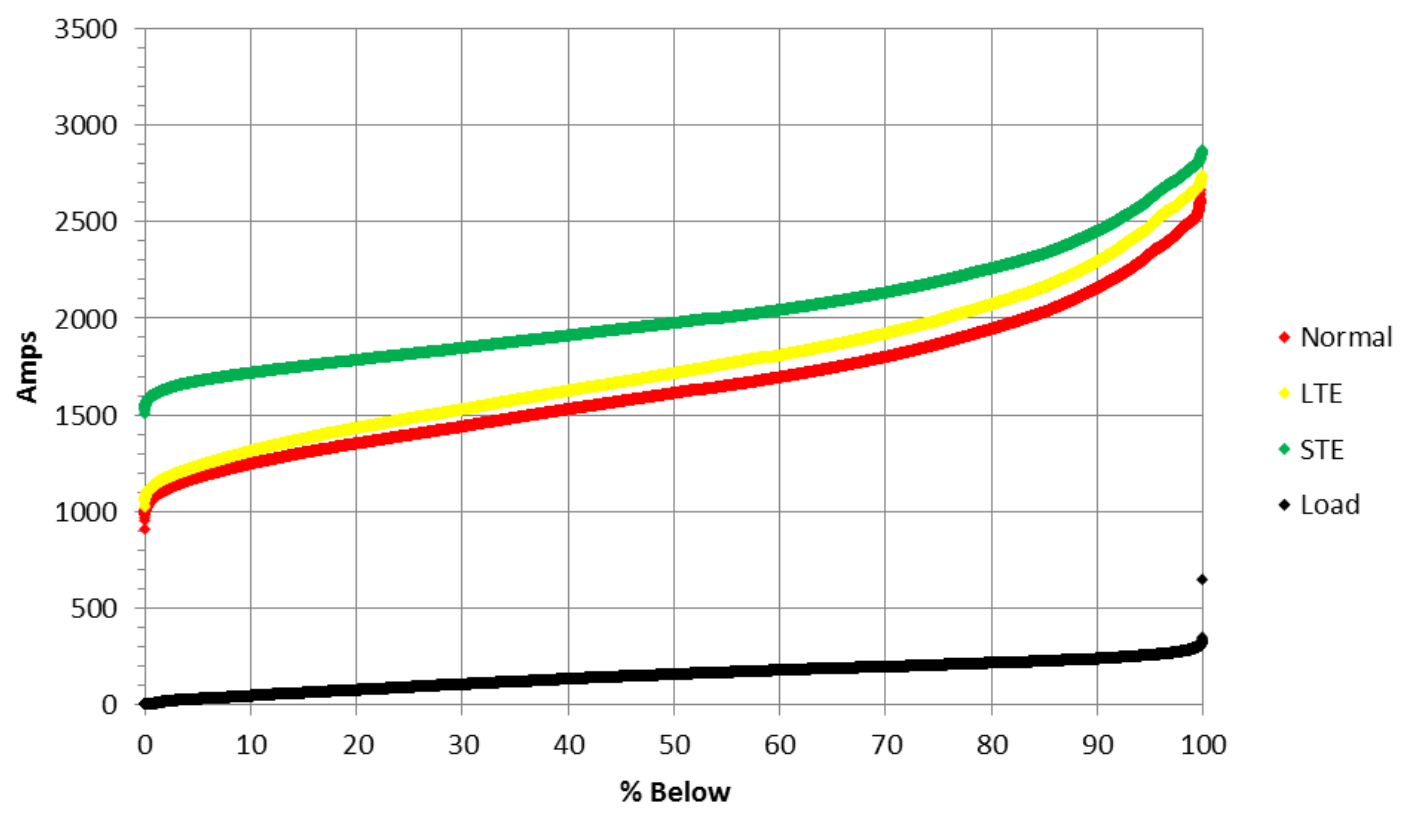

Figure H-33

Summer 2012 Ratings Distribution Site 3 ThermalRate

Table H-34

Summer 2012 Ratings Distribution Site 3 ThermalRate

\begin{tabular}{|c|l|l|l|l|l|l|}
\hline & \multicolumn{1}{|c|}{ Static } & \multicolumn{1}{|c|}{ L1 } & \multicolumn{1}{|c|}{ L2 } & \multicolumn{1}{|c|}{ L50 } & \multicolumn{1}{|c|}{ L95 } \\
\hline Normal & 1089 & 1080 & 1109 & 1173 & 1610 & 2329 \\
\hline LTE & 1256 & 1138 & 1170 & 1231 & 1713 & 2473 \\
\hline STE & 1410 & 1605 & 1630 & 1671 & 1973 & 2613 \\
\hline Load & & 0 & 20 & 30 & 160 & 250 \\
\hline
\end{tabular}

The Site 3 summer 2012 ThermalRate data can be compared to the Site 3 summer 2012 EPRI Sensor data. It can be seen in the tables that there is approximately 100amps difference between the two. The EPRI Sensor based rating is the more conservative of the two. This is because the EPRI Sensors are based primarily on weather data as result of low loads.

The Site 3 summer 2012 Onsite weather station data can be compared to the Site 3 summer 2012 ThermalRate data. It can be seen in the tables that there is approximately 100 amps difference between the two. The Onsite weather station based rating is the more conservative of the two. 


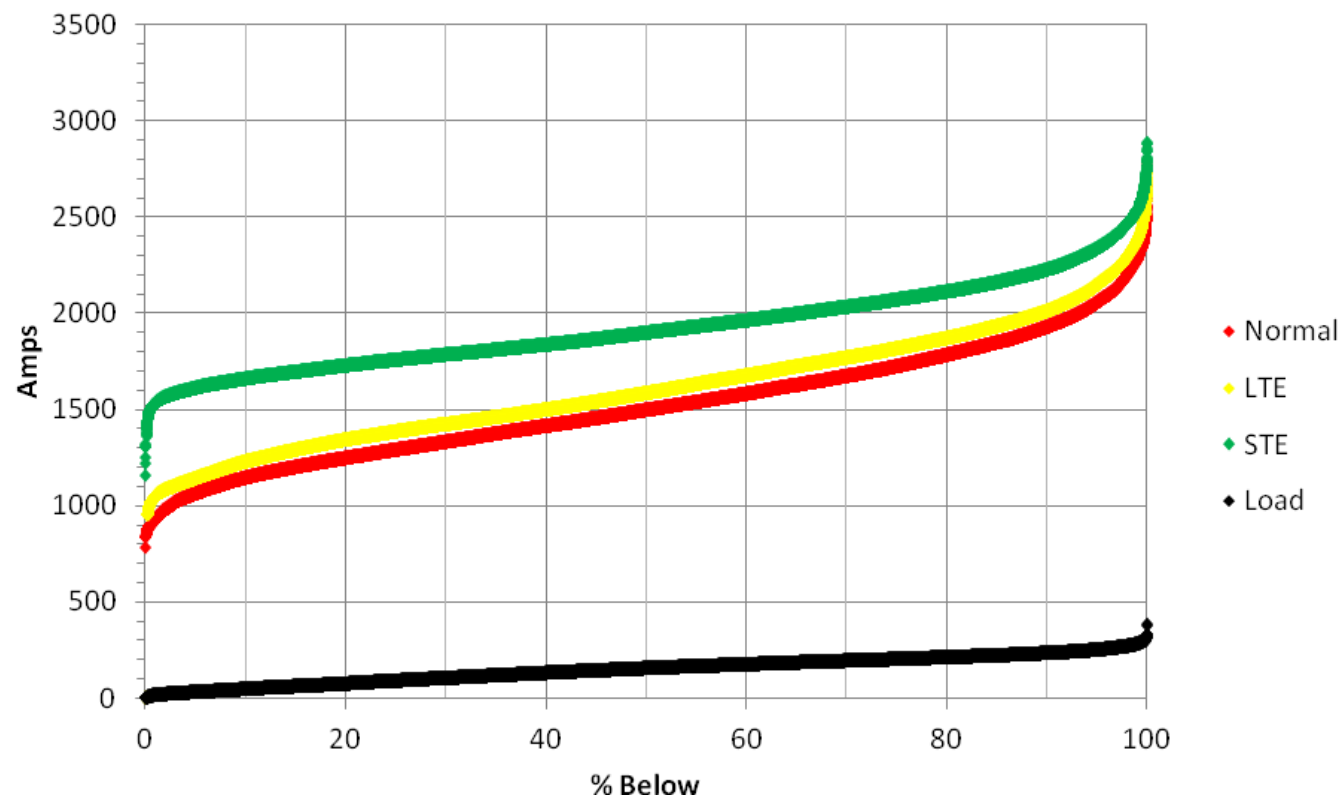

Figure H-34

Summer 2012 Ratings Distribution Site 3 Sagometer

Table H-35

Summer 2012 Ratings Distribution Site 3 Sagometer

\begin{tabular}{|c|l|l|l|l|l|l|}
\hline & \multicolumn{1}{|c|}{ Static } & \multicolumn{1}{|c|}{ L1 } & \multicolumn{1}{|c|}{ L2 } & \multicolumn{1}{c|}{ L5 } & \multicolumn{1}{|c|}{ L95 } \\
\hline Normal & 1089 & 936 & 983 & 1064 & 1497 & 2058 \\
\hline LTE & 1256 & 1050 & 1084 & 1142 & 1585 & 2150 \\
\hline STE & 1410 & 1534 & 1568 & 1612 & 1599 & 2339 \\
\hline Load & & 0 & 20 & 30 & 160 & 250 \\
\hline
\end{tabular}




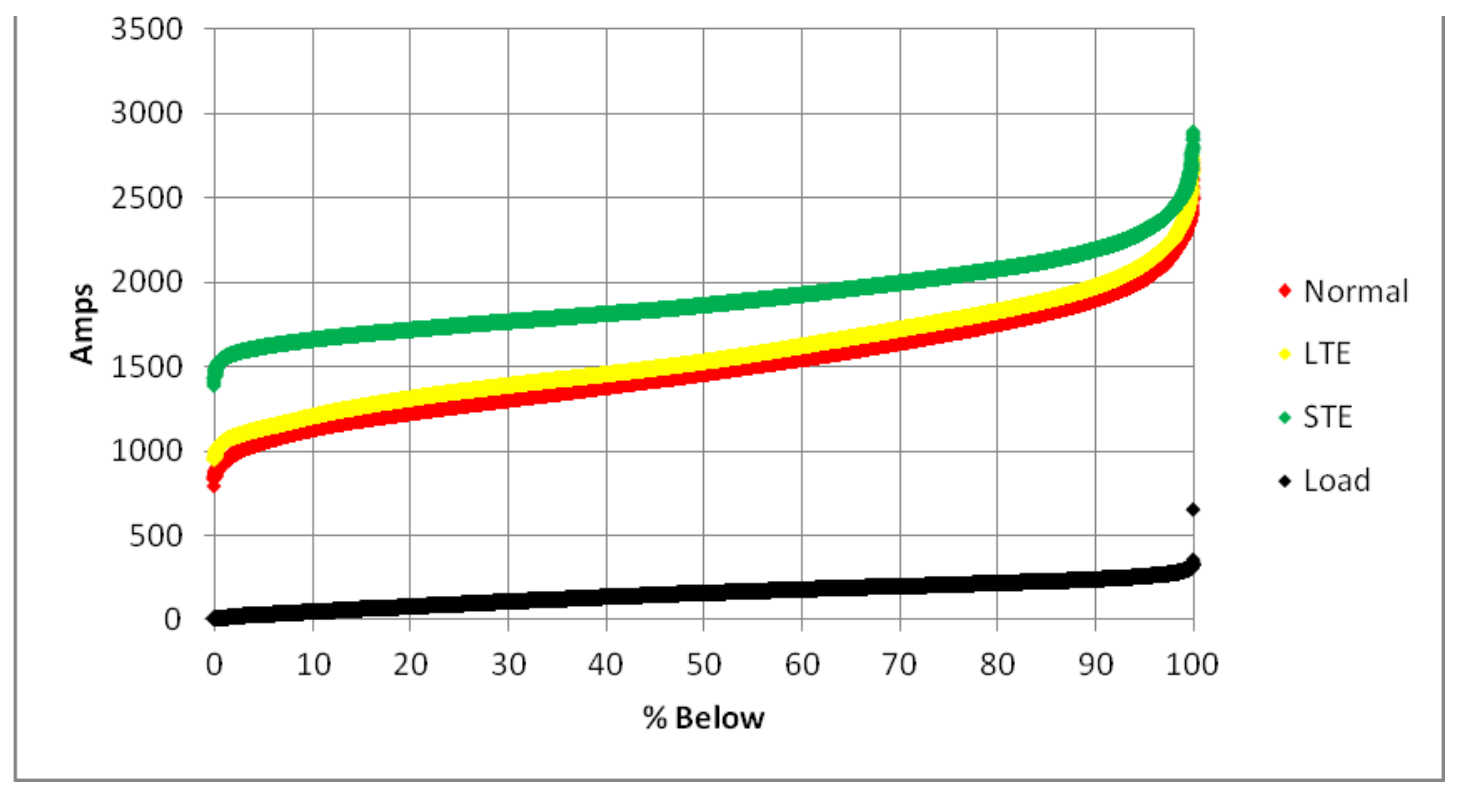

Figure $\mathrm{H}-35$

Summer 2012 Ratings Distribution Site 3 EPRI Sensors

Table H-36

Summer 2012 Ratings Distribution Site 3 EPRI Sensors

\begin{tabular}{|c|l|l|l|l|l|l|}
\hline & \multicolumn{1}{|c|}{ Static } & \multicolumn{1}{|c|}{ L1 } & \multicolumn{1}{|c|}{ L2 } & \multicolumn{1}{|c|}{ L5 } & \multicolumn{1}{|c|}{ L95 } \\
\hline Normal & 1089 & 929 & 977 & 1048 & 1454 & 2015 \\
\hline LTE & 1256 & 1050 & 1081 & 1131 & 1525 & 2102 \\
\hline STE & 1410 & 1545 & 1572 & 1613 & 1857 & 2300 \\
\hline Load & & 0 & 20 & 30 & 160 & 250 \\
\hline
\end{tabular}

The Site 3 summer 2012 EPRI temperature sensor data can be compared to the Site 3 summer 2012 off site weather station data. It can be seen in the tables that there is approximately 100 amps difference between the two. The offsite weather service based rating is the more conservative of the two. 


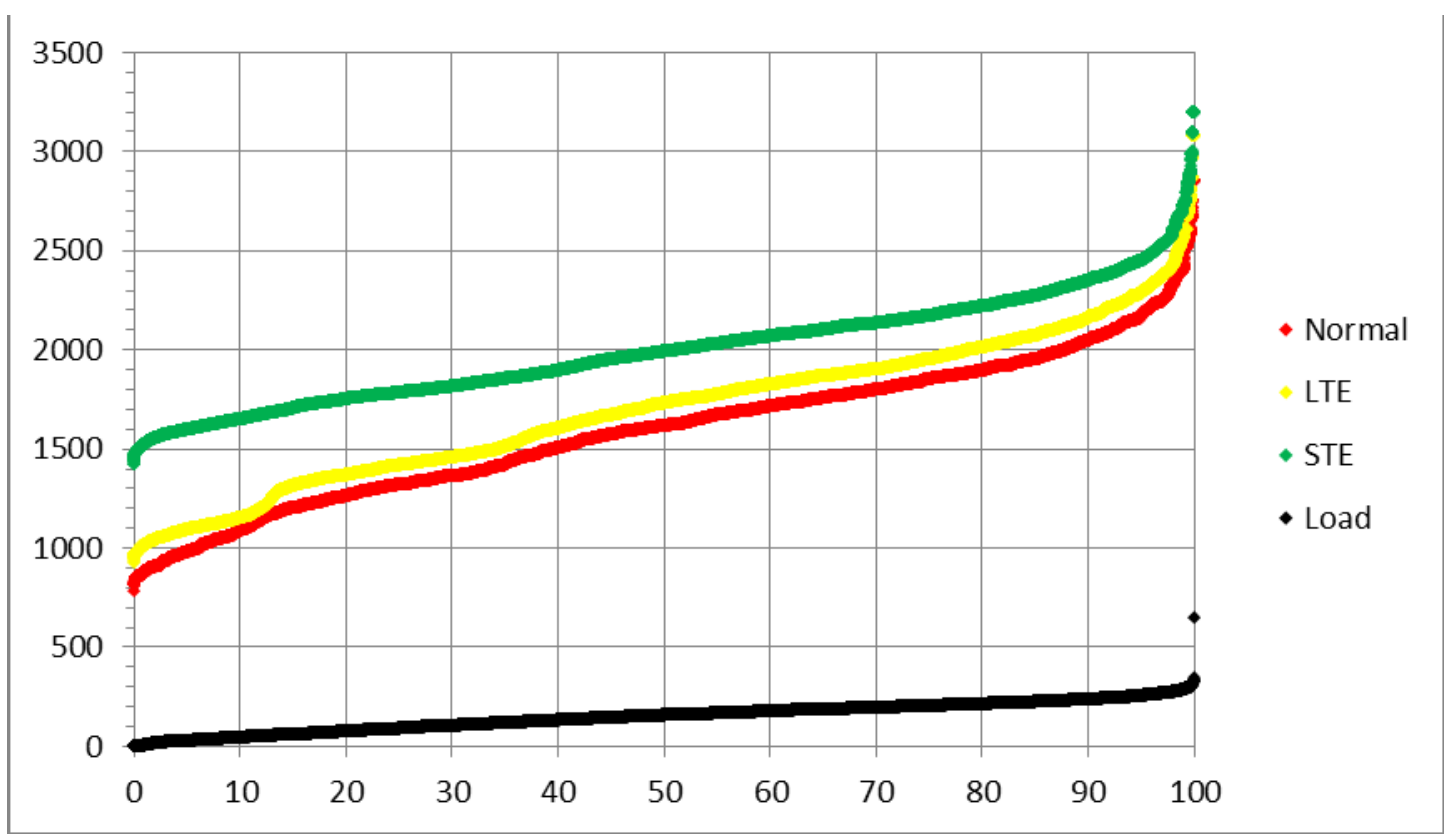

Figure H-36

Summer 2012 Ratings Distribution Site 3 Offsite Weather Service

Table H-37

Summer 2012 Ratings Distribution Site 3 Offsite Weather Service

\begin{tabular}{|c|l|l|l|l|l|l|}
\hline & \multicolumn{1}{|c|}{ Static } & \multicolumn{1}{|c|}{ L1 } & \multicolumn{1}{c|}{ L2 } & \multicolumn{1}{c|}{ L5 } & \multicolumn{1}{c|}{ L50 } & \multicolumn{1}{|c|}{ L95 } \\
\hline Normal & 1089 & 880 & 907 & 977 & 1615 & 2167 \\
\hline LTE & 1256 & 1013 & 1042 & 1091 & 1731 & 2288 \\
\hline STE & 1410 & 1522 & 1554 & 1597 & 1990 & 2450 \\
\hline Load & & 0 & 20 & 30 & 160 & 250 \\
\hline
\end{tabular}

The Site 3 summer 2012 off site weather service data can be compared to the Site 3 summer 2012 Onsite weather station data. It can be seen in the tables that there is approximately 100 amps difference between the two. The offsite weather service based rating is the more conservative of the two. 
Table H-38

Summary Table for Site 2 - L2 Were Used for the Purposes of Comparison

\begin{tabular}{|l|l|l|l|}
\hline \multicolumn{1}{|c|}{ Description } & \multicolumn{1}{|c|}{$\begin{array}{c}\text { L2 } \\
\text { Norm }\end{array}$} & L2 LTE & \multicolumn{1}{|c|}{ L2 STE } \\
\hline Site 3 Onsite Weather Station Winter 2010/111 & 1116 & 1204 & 1743 \\
\hline Site 3 ThermalRate Winter 2010/11 & 1226 & 1282 & 1798 \\
\hline Site 3 Sagometer Winter 2010/11 & 1153 & 1234 & 1707 \\
\hline Site 3 EPRI Temperature Sensor Winter 2010/11 & 979 & 1167 & 1666 \\
\hline Site 3 Onsite Weather Station Summer 2011 & 875 & 1072 & 1561 \\
\hline Site 3 ThermalRate Summer 2011 & 1078 & 1176 & 1627 \\
\hline MW203 06 Sagometer Summer 2011 & 1031 & 1159 & 1601 \\
\hline Site 3 EPRI Temperature Sensor Summer 2011 & 889 & 1086 & 1569 \\
\hline Site 3 Onsite Weather Station Winter 2011/12 & 1066 & 1152 & 1651 \\
\hline MW203 06 ThermalRate Winter 2011/12 & 1208 & 1270 & 1731 \\
\hline Site 3 Sagometer Winter 2011/12 & 1070 & 1161 & 1645 \\
\hline Site 3 EPRI Temperature Sensor Winter 2011/12 & 1078 & 1167 & 1666 \\
\hline Site 3 Off Site Weather Service Winter 2011/12 & 953 & 1083 & 1610 \\
\hline Site 3 Onsite Weather Station Summer 2012 & 971 & 1074 & 1560 \\
\hline Site 3 ThermalRate Summer 2012 & 1109 & 1170 & 1630 \\
\hline MW203 06 Sagometer Summer 2012 & 1153 & 1233 & 1707 \\
\hline Site 3 EPRI Temperature Sensor Summer 2012 & 977 & 1081 & 1572 \\
\hline Site 3 Off Site Weather Service Summer 2012 & 907 & 1042 & 1554 \\
\hline
\end{tabular}




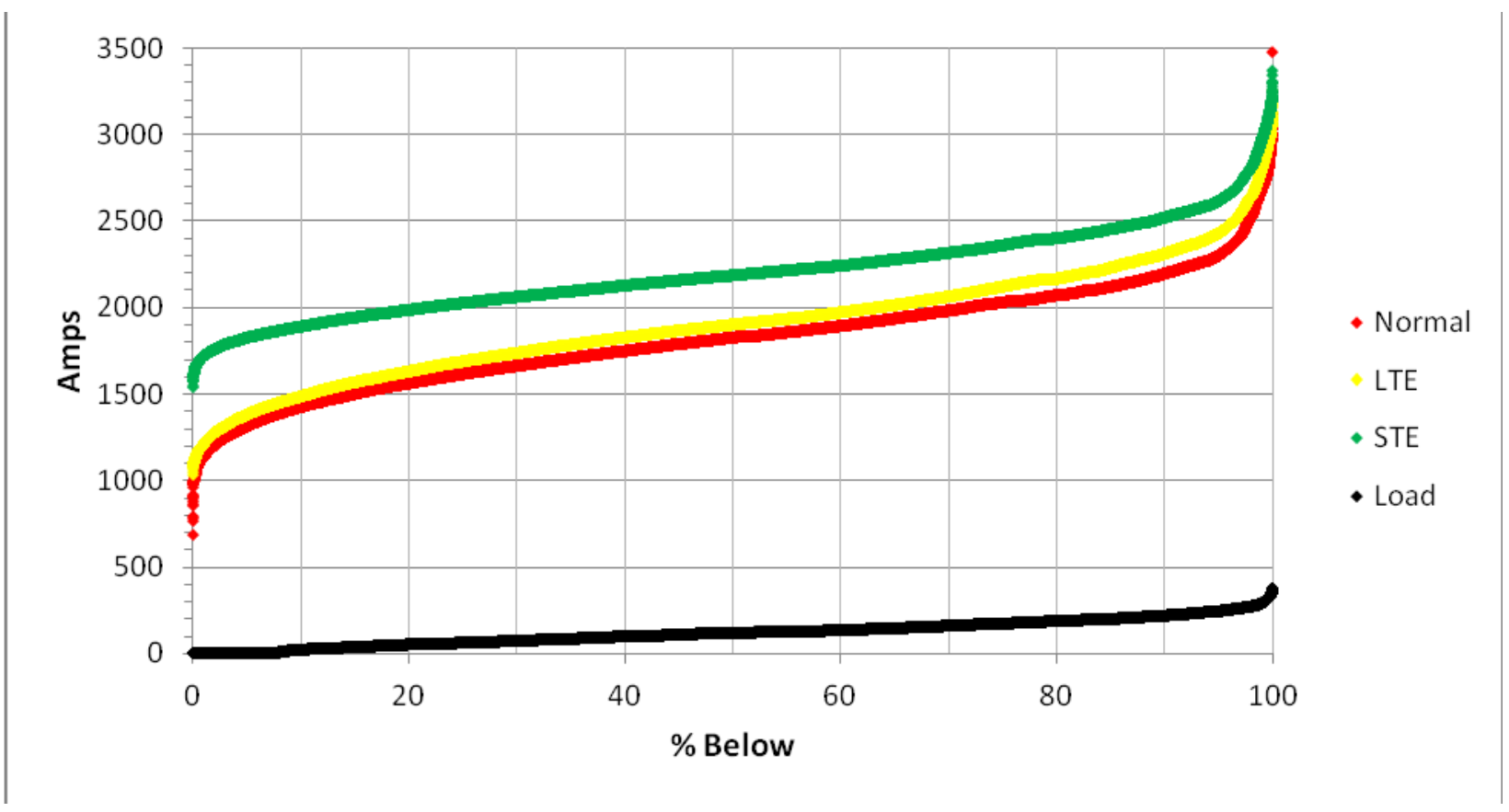

Figure $\mathrm{H}-37$

Winter 2010/11 Ratings Distribution Site 1 Onsite Weather Station

Table H-39

Winter 2010/11 Ratings Distribution Site 1 Onsite Weather Station

\begin{tabular}{|c|l|l|l|l|l|l|}
\hline & \multicolumn{1}{|c|}{ Static } & \multicolumn{1}{|c|}{ L1 } & \multicolumn{1}{c|}{ L2 } & \multicolumn{1}{c|}{ L5 } & \multicolumn{1}{|c|}{ L95 } \\
\hline Normal & 1331 & 1138 & 1204 & 1314 & 1826 & 2301 \\
\hline LTE & 1460 & 1211 & 1276 & 1380 & 1902 & 2430 \\
\hline STE & 1593 & 1720 & 1760 & 1826 & 2186 & 2617 \\
\hline Load & & 0 & 0 & 10 & 120 & 250 \\
\hline
\end{tabular}

The Site 1 winter 2010/11 local weather station data can be compared to the Site 1 winter 2010/11 ThermalRate data. It can be seen in the tables that there is approximately 100 amps difference between the two. The weather station based rating is the more conservative of the two. 


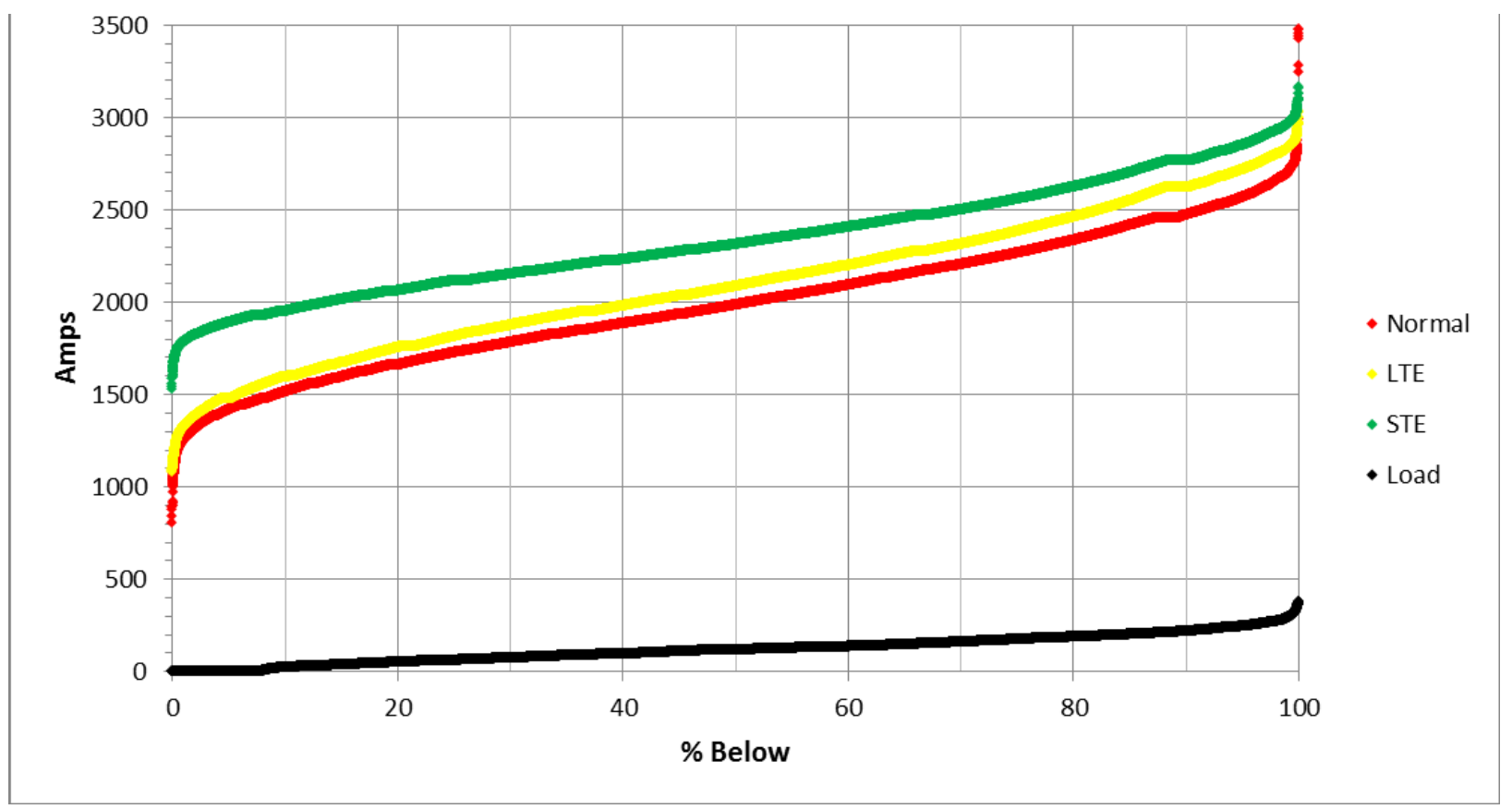

Figure H-38

Winter 2010/11 Ratings Distribution Site 1 ThermalRate

Table H-40

Winter 2010/11 Ratings Distribution Site 1 ThermalRate

\begin{tabular}{|c|l|l|l|l|l|l|}
\hline & \multicolumn{1}{|c|}{ Static } & \multicolumn{1}{|c|}{ L1 } & \multicolumn{1}{|c|}{ L2 } & \multicolumn{1}{|c|}{ L50 } & \multicolumn{1}{|c|}{ L95 } \\
\hline Normal & 1331 & 1258 & 1315 & 1417 & 1986 & 2568 \\
\hline LTE & 1460 & 1327 & 1385 & 1482 & 2087 & 2718 \\
\hline STE & 1593 & 1786 & 1824 & 1892 & 2313 & 2850 \\
\hline Load & & 0 & 0 & 10 & 120 & 250 \\
\hline
\end{tabular}

The Site 1 winter 2010/11 ThermalRate data can be compared to the Site 1 winter 2011/12 ThermalRate data. It can be seen in the tables that there is approximately 70 amps difference between the two. This difference is greater than expected. 


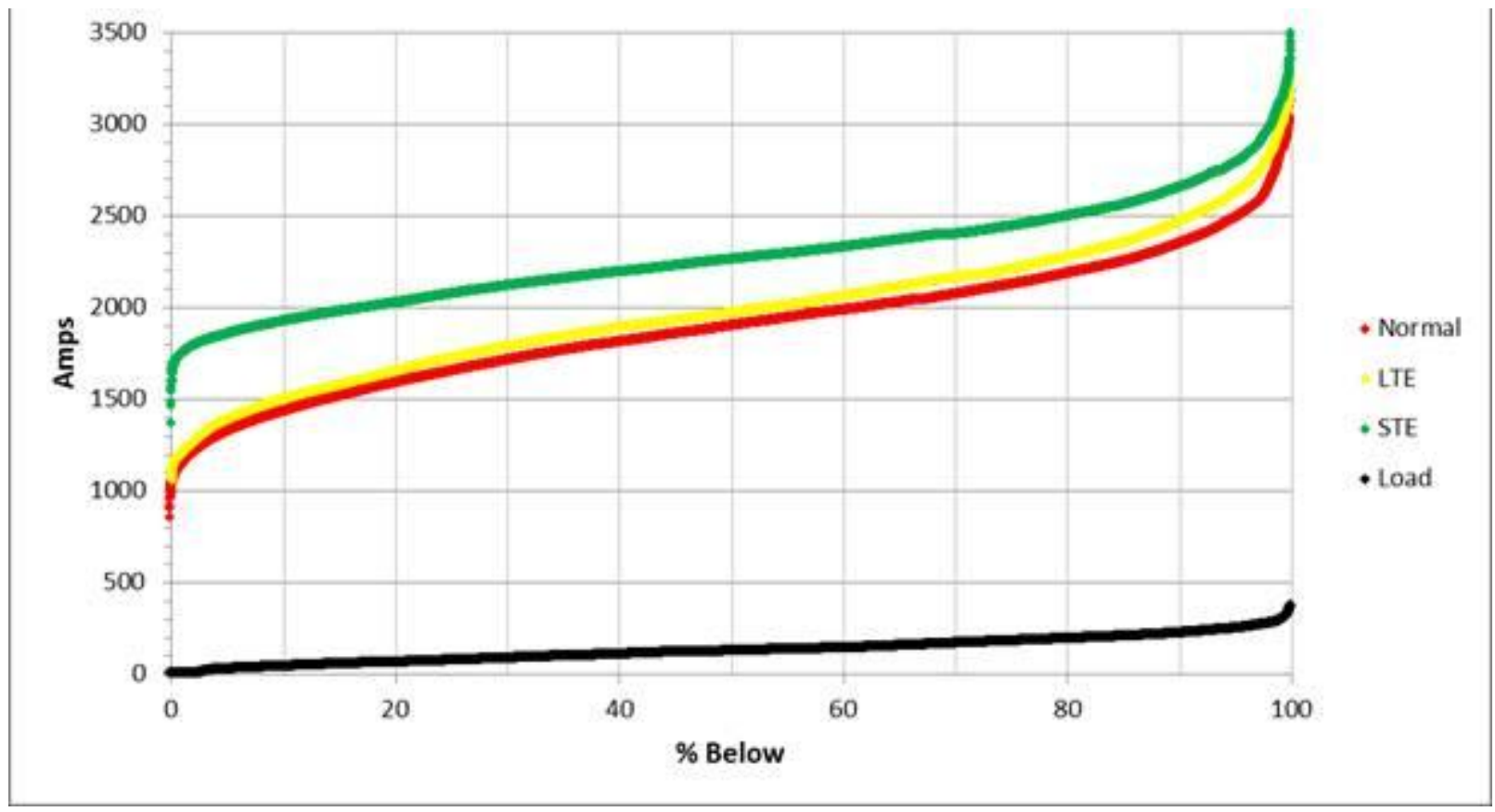

Figure H-39

Winter 2010/11 Ratings Distribution Site 1 Sagometer

Table H-41

Winter 2010/11 Ratings Distribution Site 1 Sagometer

\begin{tabular}{|c|l|l|l|l|l|l|}
\hline & \multicolumn{1}{|c|}{ Static } & \multicolumn{1}{|c|}{ L1 } & \multicolumn{1}{|c|}{ L2 } & \multicolumn{1}{|c|}{ L5 } & \multicolumn{1}{|c|}{ L50 } & \multicolumn{1}{|c|}{ L95 } \\
\hline Normal & 1331 & 1140 & 1203 & 1318 & 1895 & 2483 \\
\hline LTE & 1460 & 1200 & 1255 & 1381 & 1964 & 2618 \\
\hline STE & 1593 & 1744 & 1786 & 1850 & 2888 & 2782 \\
\hline Load & & 0 & 0 & 10 & 120 & 250 \\
\hline
\end{tabular}

The Site 1 winter 2010/11 Sagometer data can be compared to the Site 1 winter 2010/11 EPRI Sensor data. The values and shape of these curves will be the same because most of the time DTCR is forced to fall back on the weather station model. If the line had more load, it would be possible to properly evaluate the technology. It is likely there would be differences. Some of these differences would be explained by state change equations and point measurements versus ruling span measurements. 


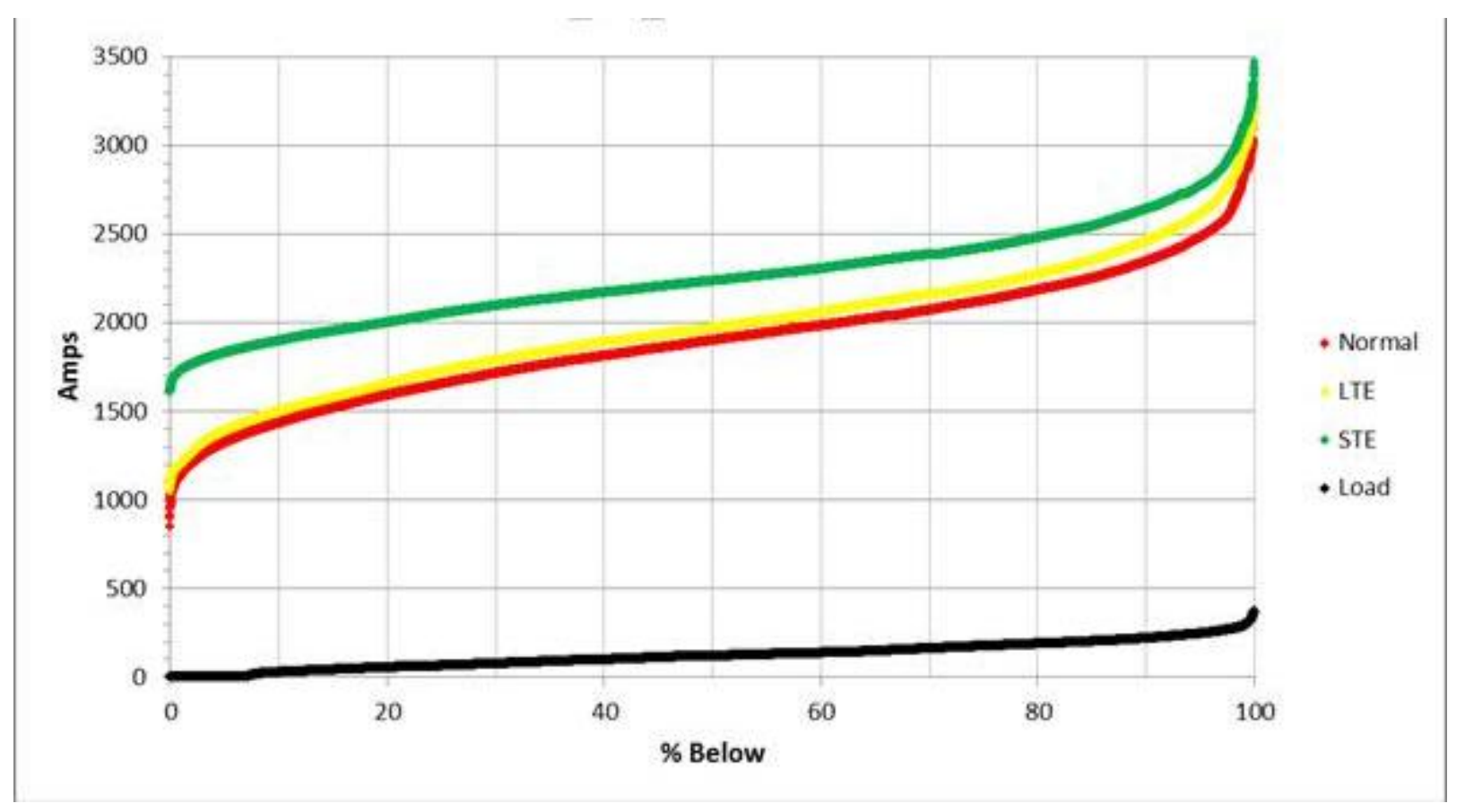

Figure $\mathrm{H}-40$

Winter 2010/11 Ratings Distribution Site 1 Sagometer

Table H-42

Winter 2010/11 Ratings Distribution Site 1 Sagometer

\begin{tabular}{|c|l|l|l|l|l|l|}
\hline & \multicolumn{1}{|c|}{ Static } & \multicolumn{1}{|c|}{ L1 } & \multicolumn{1}{|c|}{ L2 } & \multicolumn{1}{|c|}{ L5 } & \multicolumn{1}{|c|}{ L50 } & \multicolumn{1}{|c|}{ L95 } \\
\hline Normal & 1331 & 1142 & 1205 & 1322 & 1895 & 2481 \\
\hline LTE & 1460 & 1206 & 1266 & 1389 & 1964 & 2616 \\
\hline STE & 1593 & 1733 & 1765 & 1830 & 2888 & 2772 \\
\hline Load & & 0 & 0 & 10 & 120 & 250 \\
\hline
\end{tabular}

The Site 1 winter 2010/11 sagometer data can be compared to the Site 1 winter 2010/11 EPRI Sensor data. When comparing the L2 levels it can be seen in the tables that the Normal and the LTE ratings are about the same, however, there is some difference in the STE level. DTCR is forced to use the weather model when the line is lightly loaded. Less than 5 percent of the time the load was high enough for DTCR to fully utilize the Sagometer end EPRI Sensors. Thermal inertia plays little to no roll in LTE or Normal rating calculations. However, it does play a significant part in the STE calculation. Because of the importance of thermal inertia, a temperature difference in the measured or calculated conductor temperature will show up in the STE rating calculation, as it does here. 


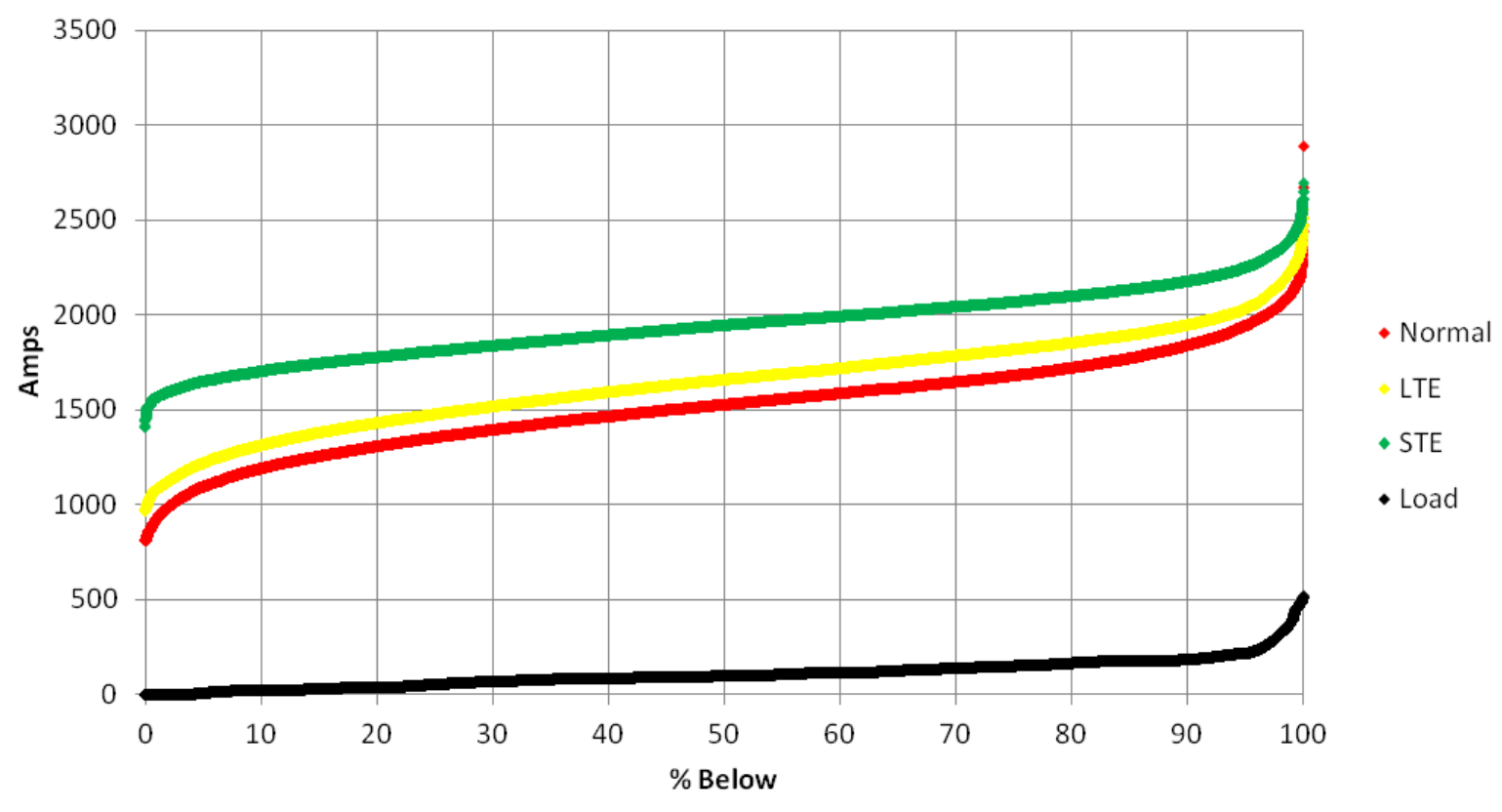

Figure $\mathrm{H}-41$

Summer 2011 Ratings Distribution Site 1 Onsite Weather Station

Table H-43

Summer 2011 Ratings Distribution Site 1 Onsite Weather Station

\begin{tabular}{|c|l|l|l|l|l|l|}
\hline & \multicolumn{1}{|c|}{ Static } & \multicolumn{1}{|c|}{ L1 } & \multicolumn{1}{|c|}{ L2 } & \multicolumn{1}{|c|}{ L5 } & \multicolumn{1}{|c|}{ L95 } \\
\hline Normal & 1089 & 929 & 988 & 1096 & 1533 & 1947 \\
\hline LTE & 1256 & 1078 & 1123 & 1220 & 1665 & 2038 \\
\hline STE & 1410 & 1567 & 1594 & 1651 & 1951 & 2255 \\
\hline Load & & 0 & 5 & 10 & 100 & 250 \\
\hline
\end{tabular}

The ratings here are lower than anticipated, and in comparison to other sites and years. This is partly due to less favorable weather, but also a result of a considerable portion of the season in which the EDM onsite weather station had been transmitting air temperature data that were clearly incorrect. This affects the quality of the Video Sagometer and EPRI Sensor ratings as well. 


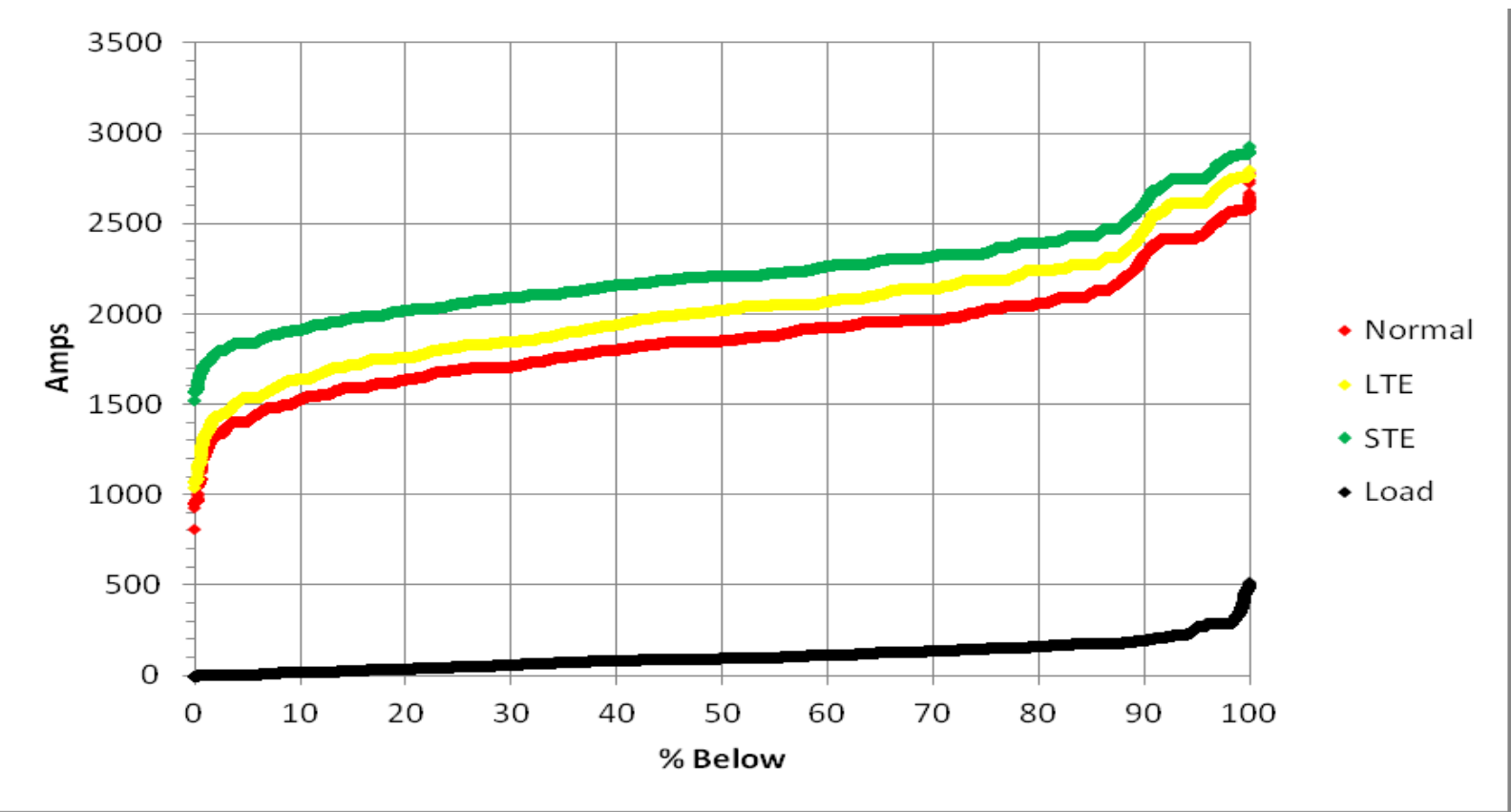

Figure $\mathrm{H}-42$

Summer 2011 Ratings Distribution Site 1 ThermalRate

Table H-44

Summer 2011 Ratings Distribution Site 1 ThermalRate

\begin{tabular}{|c|l|l|l|l|l|l|}
\hline & \multicolumn{1}{|c|}{ Static } & \multicolumn{1}{|c|}{ L1 } & \multicolumn{1}{|c|}{ L2 } & \multicolumn{1}{|c|}{ L5 } & \multicolumn{1}{|c|}{ L95 } \\
\hline Normal & 1089 & 1235 & 1332 & 1406 & 1855 & 1861 \\
\hline LTE & 1256 & 1335 & 1435 & 1541 & 2024 & 1988 \\
\hline STE & 1410 & 1724 & 1784 & 1842 & 2215 & 2210 \\
\hline Load & & 0 & 5 & 10 & 100 & 250 \\
\hline
\end{tabular}




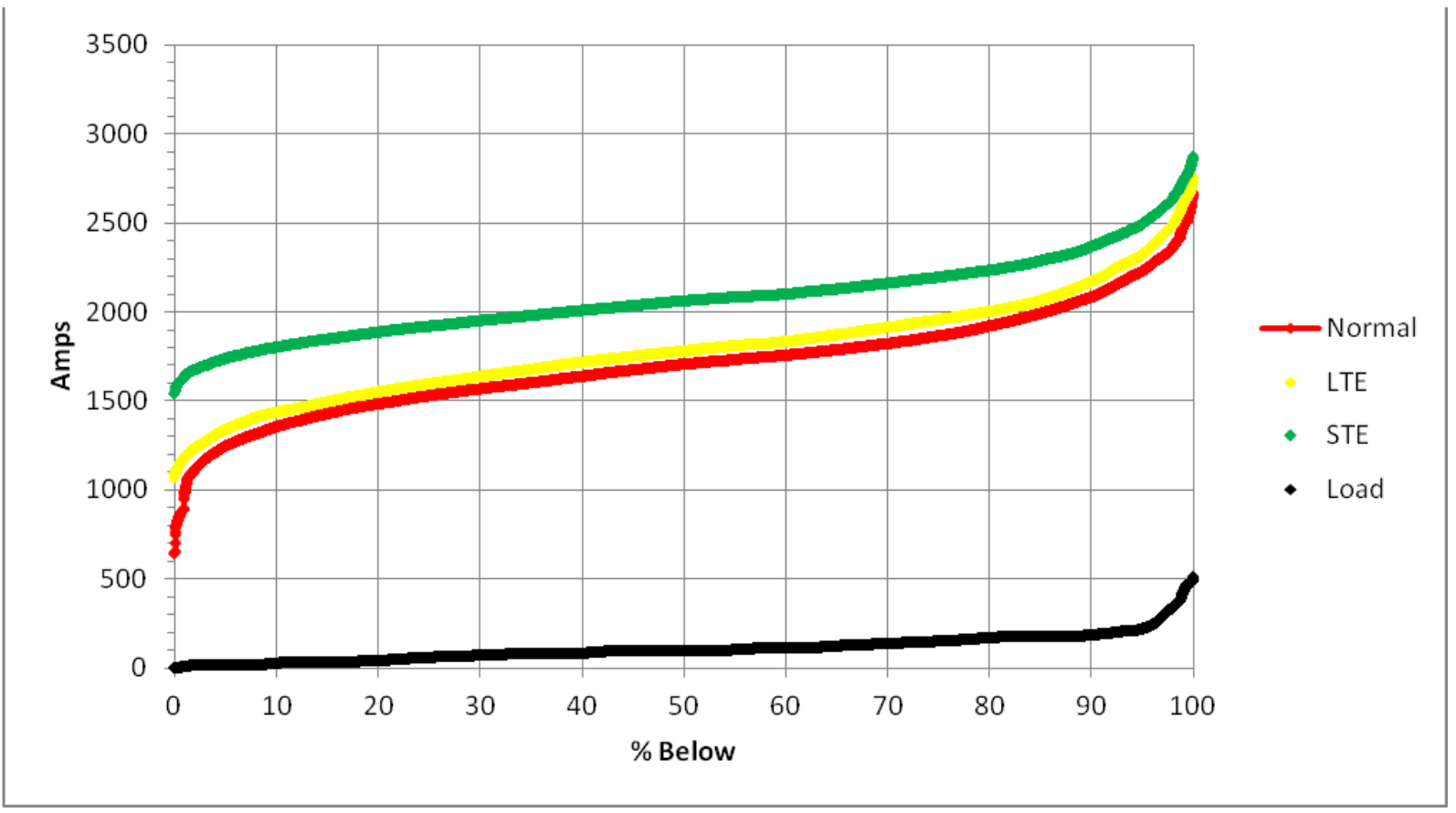

Figure H-43

Summer 2011 Ratings Distribution Site 1 Sagometer

Table H-45

Summer 2011 Ratings Distribution Site 1 Sagometer

\begin{tabular}{|c|l|l|l|l|l|l|}
\hline & \multicolumn{1}{|c|}{ Static } & \multicolumn{1}{|c|}{ L1 } & \multicolumn{1}{|c|}{ L2 } & \multicolumn{1}{|c|}{ L5 } & \multicolumn{1}{|c|}{ L50 } \\
\hline Normal & 1089 & 988 & 1116 & 1247 & 1708 & 2229 \\
\hline LTE & 1256 & 1185 & 1236 & 1341 & 1781 & 2323 \\
\hline STE & 1410 & 1641 & 1678 & 1740 & 2063 & 2495 \\
\hline Load & & 0 & 5 & 10 & 100 & 250 \\
\hline
\end{tabular}




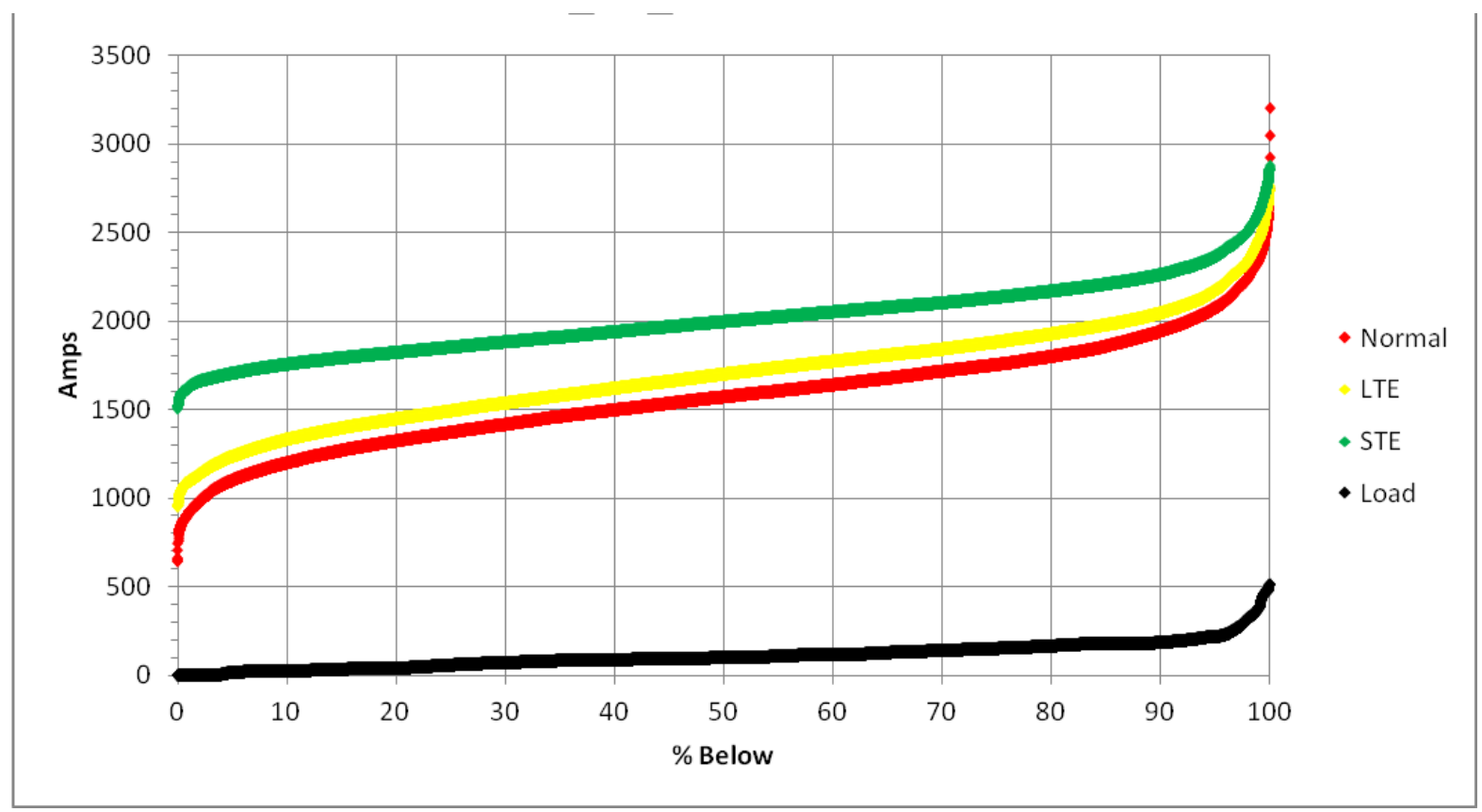

Figure H-44

Summer 2011 Ratings Distribution Site 1 EPRI Sensors

Table H-46

Summer 2011 Ratings Distribution Site 1 EPRI Sensors

\begin{tabular}{|c|l|l|l|l|l|l|}
\hline & \multicolumn{1}{|c|}{ Static } & \multicolumn{1}{|c|}{ L1 } & \multicolumn{1}{|c|}{ L2 } & \multicolumn{1}{|c|}{ L5 } & \multicolumn{1}{|c|}{ L95 } \\
\hline Normal & 1089 & 916 & 983 & 1100 & 1570 & 2073 \\
\hline LTE & 1256 & 1093 & 1135 & 1234 & 1699 & 2172 \\
\hline STE & 1410 & 1626 & 1659 & 1704 & 1995 & 2361 \\
\hline Load & & 0 & 5 & 10 & 100 & 250 \\
\hline
\end{tabular}




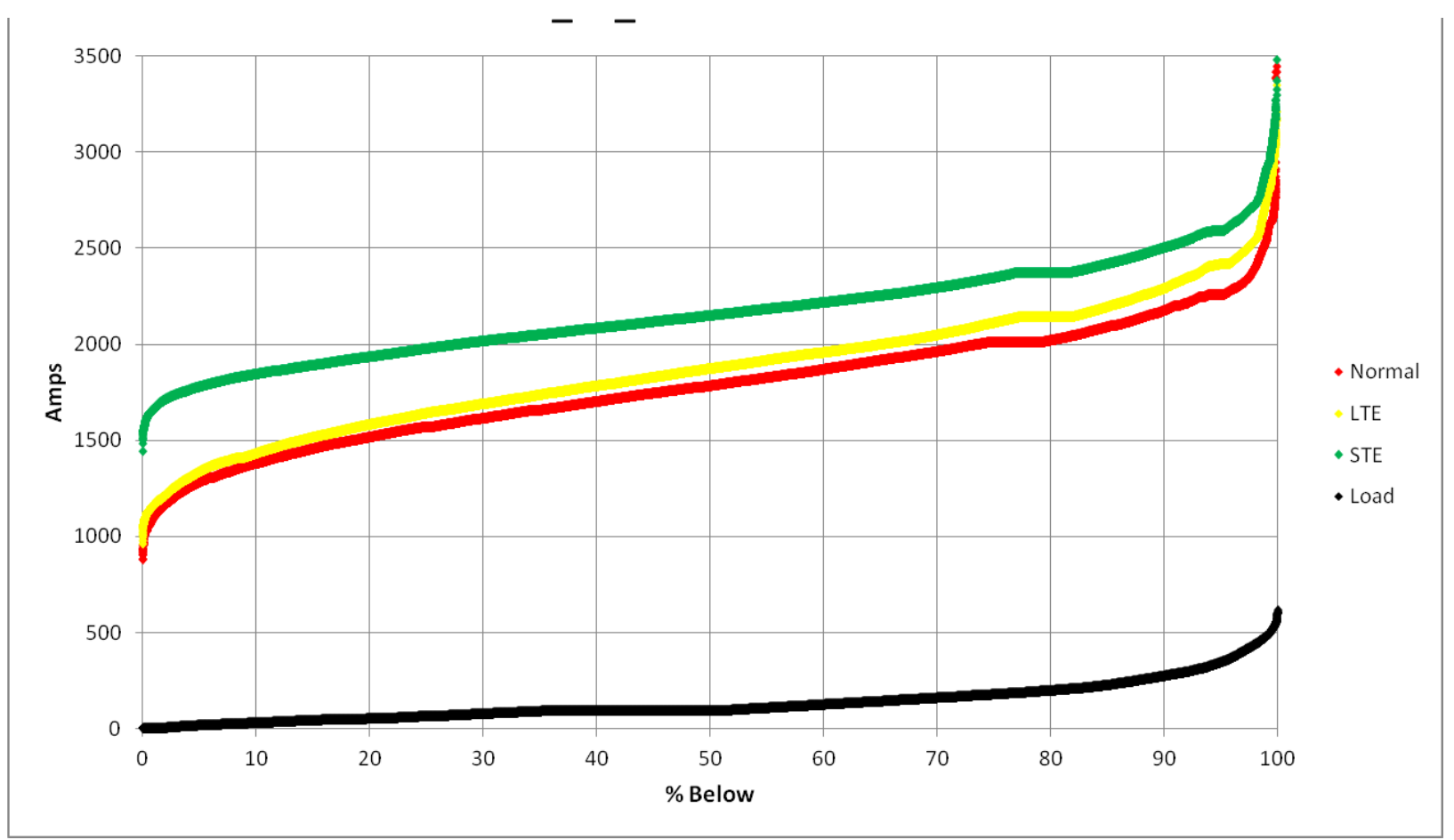

Figure H-45

Winter 2011/12 Ratings Distribution Site 1 Onsite Weather Station

Table H-47

Winter 2011/12 Ratings Distribution Site 1 Onsite Weather Station

\begin{tabular}{|c|l|l|l|l|l|l|}
\hline & \multicolumn{1}{|c|}{ Static } & \multicolumn{1}{|c|}{ L1 } & \multicolumn{1}{|c|}{ L2 } & \multicolumn{1}{|c|}{ L5 } & \multicolumn{1}{|c|}{ L95 } \\
\hline Normal & 1331 & 1101 & 1166 & 1283 & 1783 & 2256 \\
\hline LTE & 1460 & 1166 & 1215 & 1336 & 1872 & 2416 \\
\hline STE & 1593 & 1664 & 1713 & 1779 & 2149 & 2588 \\
\hline Load & & 0 & 5 & 15 & 90 & 345 \\
\hline
\end{tabular}

The Site 1 winter 2011/12 onsite weather station data can be compared to the Site 1 winter 2010/11 onsite weather station sensor data. There is less that 40 amps difference between the two seasons at the L2 level. This would suggest that there may be some consistency in these ratings from season to season; however, the correlation is not as good as other sites. 


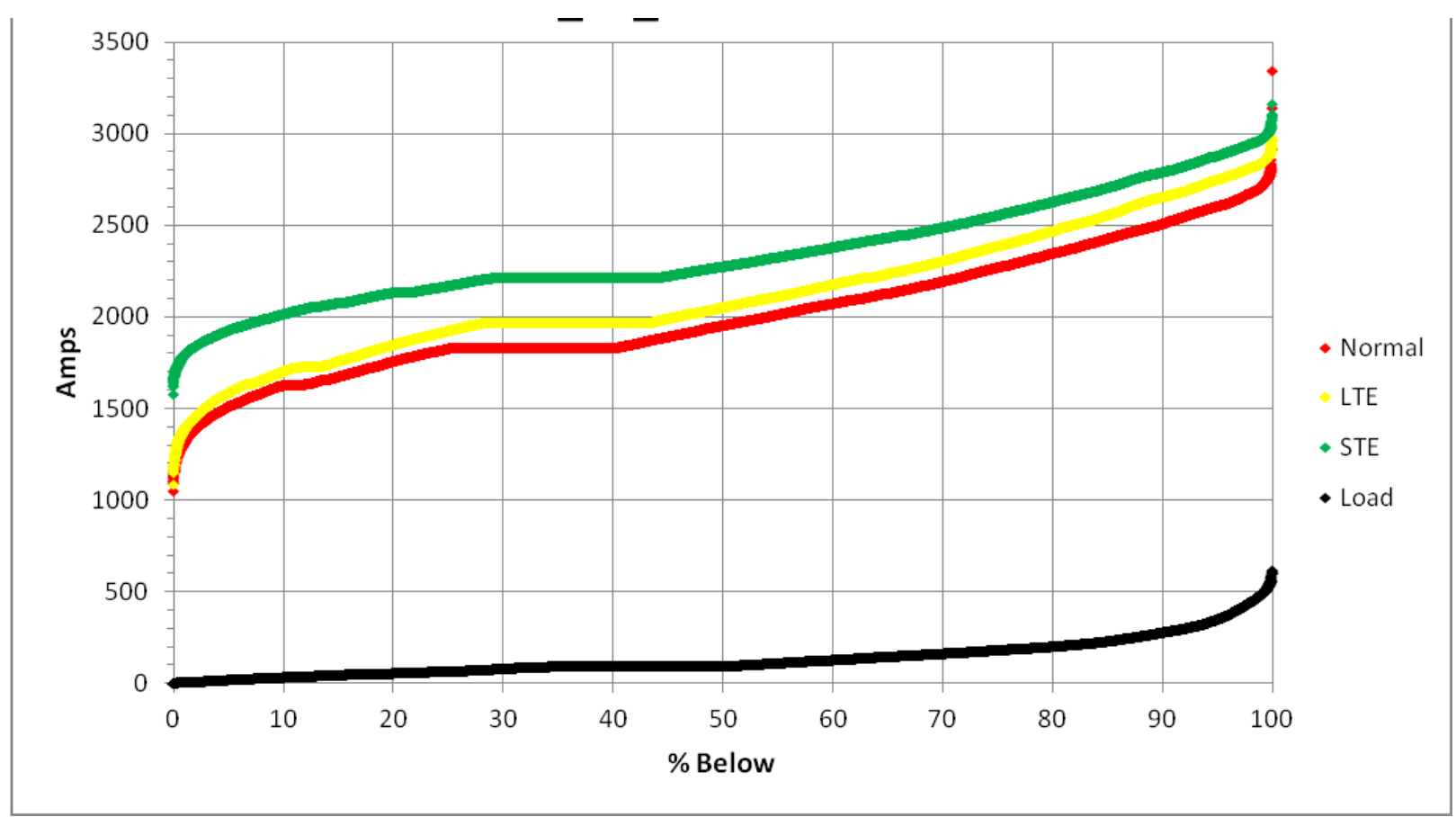

Figure H-46

Winter 2011/12 Ratings Distribution Site 1 ThermalRate

Table H-48

Winter 2011/12 Ratings Distribution Site 1 ThermalRate

\begin{tabular}{|c|l|l|l|l|l|l|}
\hline & \multicolumn{1}{|c|}{ Static } & \multicolumn{1}{|c|}{ L1 } & \multicolumn{1}{|c|}{ L2 } & \multicolumn{1}{|c|}{ L50 } & \multicolumn{1}{|c|}{ L95 } \\
\hline Normal & 1331 & 1309 & 1384 & 1507 & 1950 & 2601 \\
\hline LTE & 1460 & 1385 & 1454 & 1582 & 2049 & 2745 \\
\hline STE & 1593 & 1789 & 1837 & 1924 & 2272 & 2875 \\
\hline Load & & 0 & 5 & 15 & 90 & 345 \\
\hline
\end{tabular}

The Site 1 winter 2011/12 ThermalRate can be compared to the Site 1 winter 2010/11 ThermalRate sensor data. It can be seen in the tables that there is less than 70 amps difference between the two for the normal and LTE ratings. This is higher than expected and could be explored. 


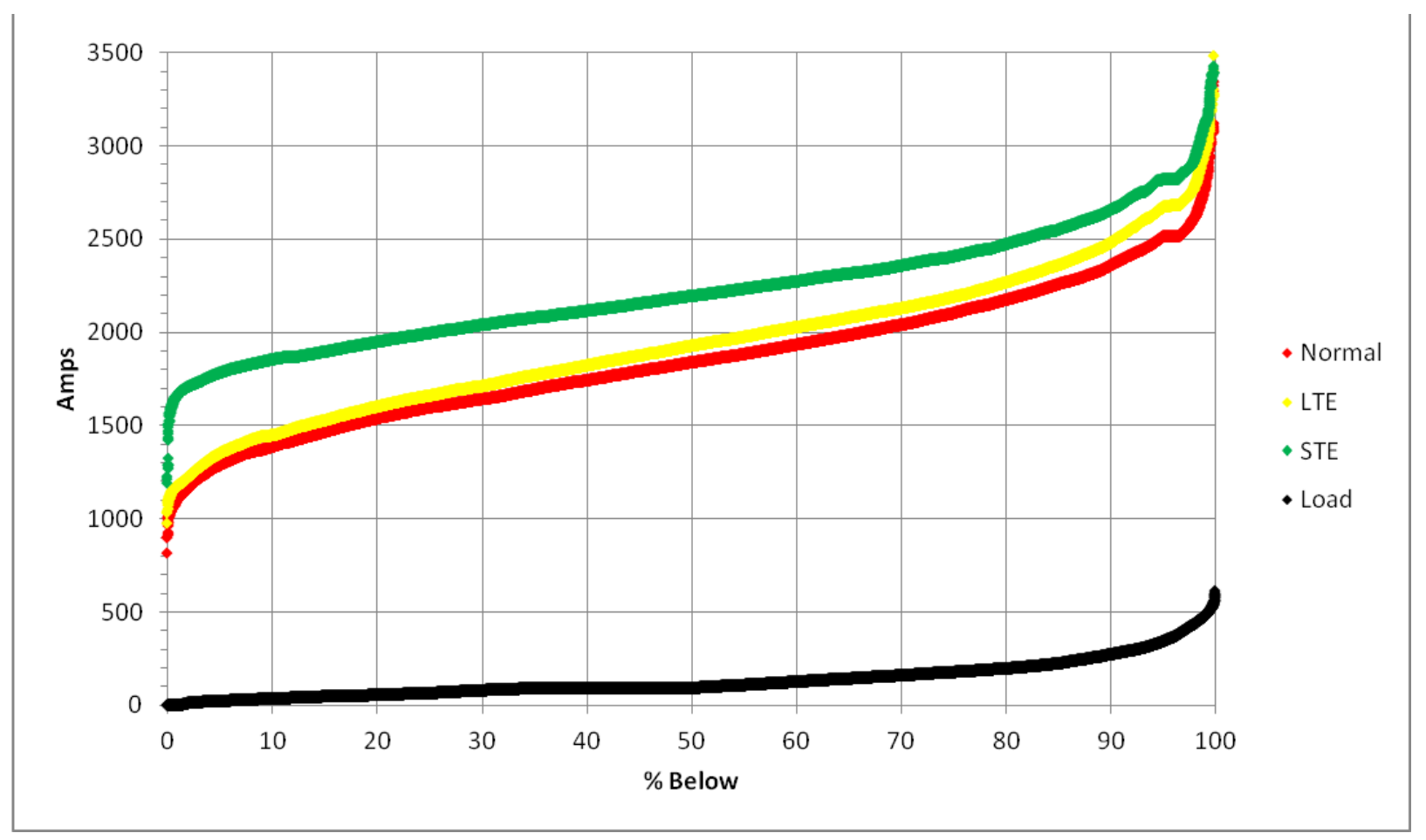

Figure $\mathrm{H}-47$

Winter 2011/12 Ratings Distribution Site 1 Sagometer

Table H-49

Winter 2011/12 Ratings Distribution Site 1 Sagometer

\begin{tabular}{|c|l|l|l|l|l|l|}
\hline & \multicolumn{1}{|c|}{ Static } & \multicolumn{1}{|c|}{ L1 } & \multicolumn{1}{|c|}{ L2 } & \multicolumn{1}{|c|}{ L5 } & \multicolumn{1}{|c|}{ L95 } \\
\hline Normal & 1331 & 1105 & 1161 & 1286 & 1908 & 2513 \\
\hline LTE & 1460 & 1176 & 1224 & 1350 & 2005 & 2673 \\
\hline STE & 1593 & 1665 & 1707 & 1782 & 2255 & 2819 \\
\hline Load & & 0 & 5 & 15 & 90 & 345 \\
\hline
\end{tabular}

The Site 1 winter 2011/12 sagometer data can be compared to the Site 1 winter 2011/12 EPRI Sensor data. The values and shape of these curves will be the same because most of the time DTCR is forced to fall back on the weather station model. If the line had more load, it would be possible to properly evaluate the technology. It is likely there would be differences. Some of these differences would be explained by state change equations and point measurements versus ruling span measurements. 


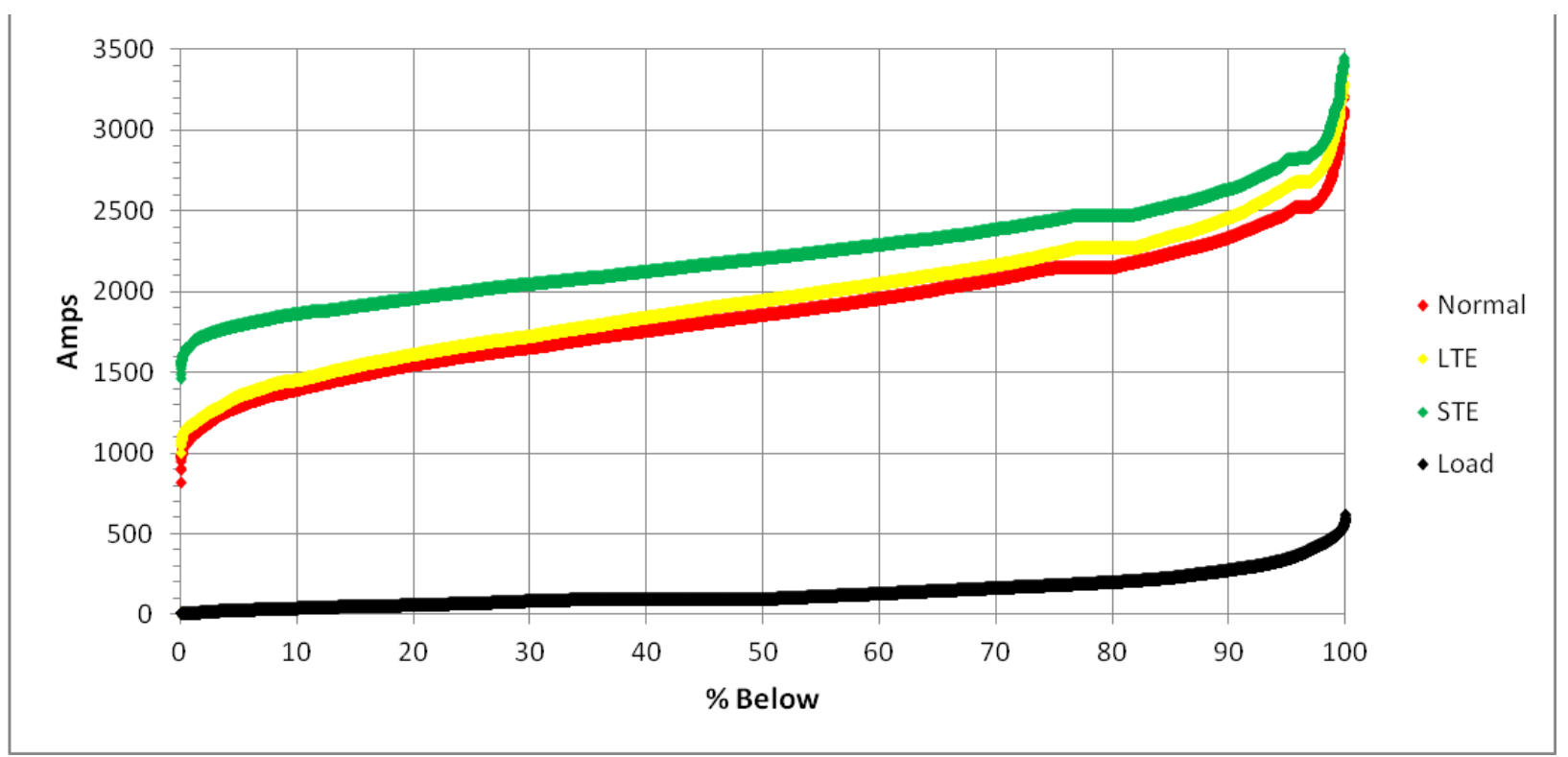

Figure H-48

Winter 2011/12 Ratings Distribution Site 1 EPRI Sensors

Table H-50

Winter 2011/12 Ratings Distribution Site 1 EPRI Sensors

\begin{tabular}{|c|l|l|l|l|l|l|}
\hline & \multicolumn{1}{|c|}{ Static } & \multicolumn{1}{|c|}{ L1 } & \multicolumn{1}{|c|}{ L2 } & \multicolumn{1}{|c|}{ L5 } & \multicolumn{1}{|c|}{ L95 } \\
\hline Normal & 1331 & 1105 & 1167 & 1290 & 1868 & 2658 \\
\hline LTE & 1460 & 1175 & 1229 & 1355 & 1958 & 2663 \\
\hline STE & 1593 & 1675 & 1725 & 1790 & 2215 & 2818 \\
\hline Load & & 0 & 5 & 15 & 90 & 345 \\
\hline
\end{tabular}

The Site 1 winter 2011/12 sagometer data can be compared to the Site 1 winter 2011/12 EPRI Sensor data. When comparing the L2 levels it can be seen in the tables that the Normal and the LTE ratings are about the same, however, there is some difference in the STE level. DTCR is forced to use the weather model when the line is lightly loaded. Less than 5 percent of the time the load was high enough for DTCR to fully utilize the Sagometer end EPRI Sensors. Thermal inertia plays no role in the Normal rating calculations and only a small role in the LTE rating calculations. However, it does play a significant part in the STE calculation. Because of the importance of thermal inertia, a temperature difference in the measured or calculated conductor temperature will show up in the STE rating calculation, as it does here. 


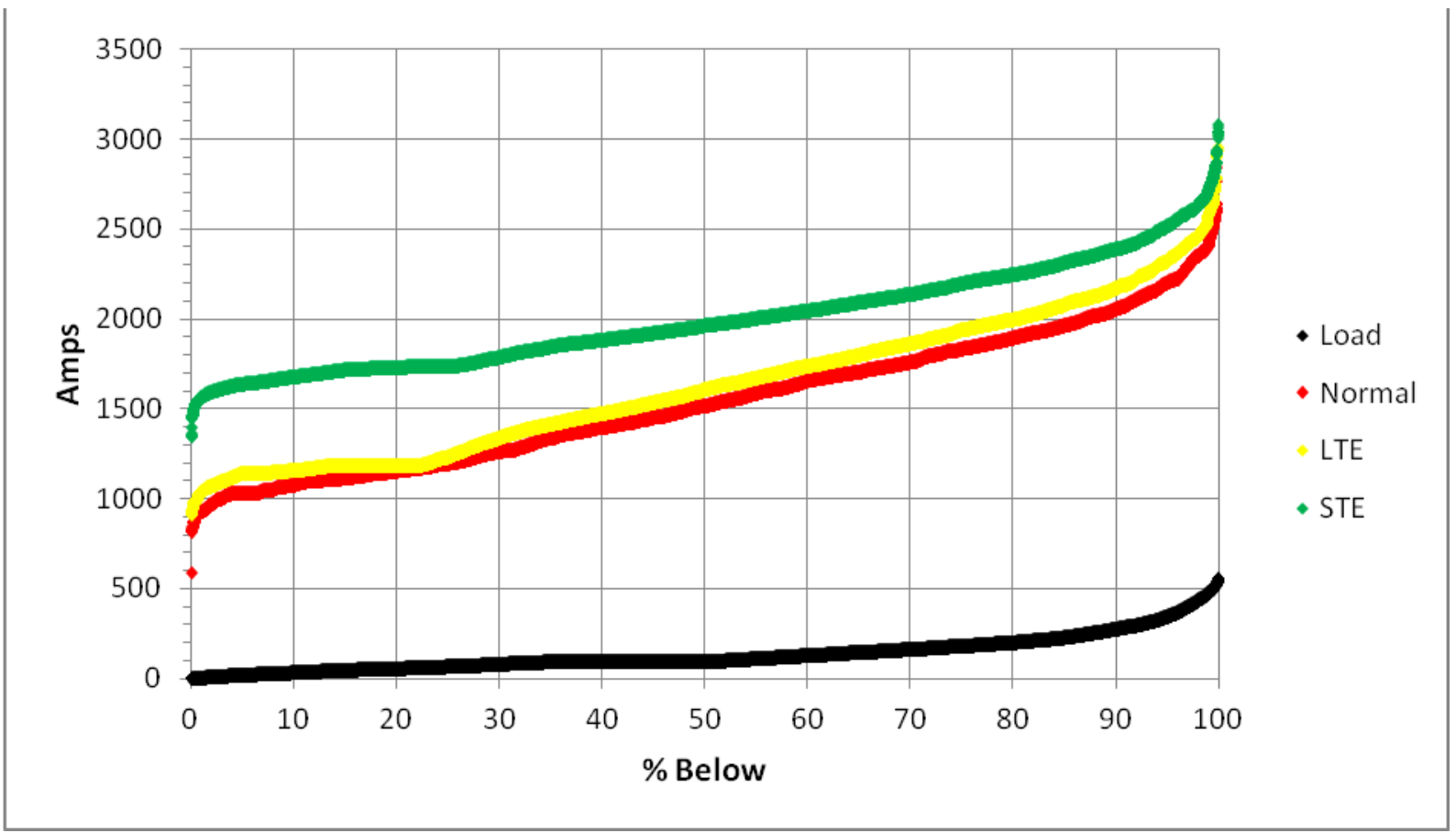

Figure $\mathrm{H}-49$

Winter 2011/12 Ratings Distribution Site 1 Off Site Weather Service

Table H-51

Winter 2011/12 Ratings Distribution Site 1 Off Site Weather Service

\begin{tabular}{|c|l|l|l|l|l|l|}
\hline & \multicolumn{1}{|c|}{ Static } & \multicolumn{1}{|c|}{ L1 } & \multicolumn{1}{|c|}{ L2 } & \multicolumn{1}{|c|}{ L5 } & \multicolumn{1}{|c|}{ L95 } \\
\hline Normal & 1331 & 926 & 972 & 1027 & 1515 & 2200 \\
\hline LTE & 1460 & 1037 & 1069 & 1138 & 1605 & 2321 \\
\hline STE & 1593 & 1562 & 1592 & 1635 & 1961 & 2517 \\
\hline Load & & 0 & 5 & 15 & 90 & 345 \\
\hline
\end{tabular}

The Site 1 winter 2011/12 off site weather service data can be compared to the Site 1 winter $2011 / 12$ onsite weather station data. It can be seen in the tables that there is approximately 200amps difference between the two. The offsite weather service based rating is the more conservative of the two. 


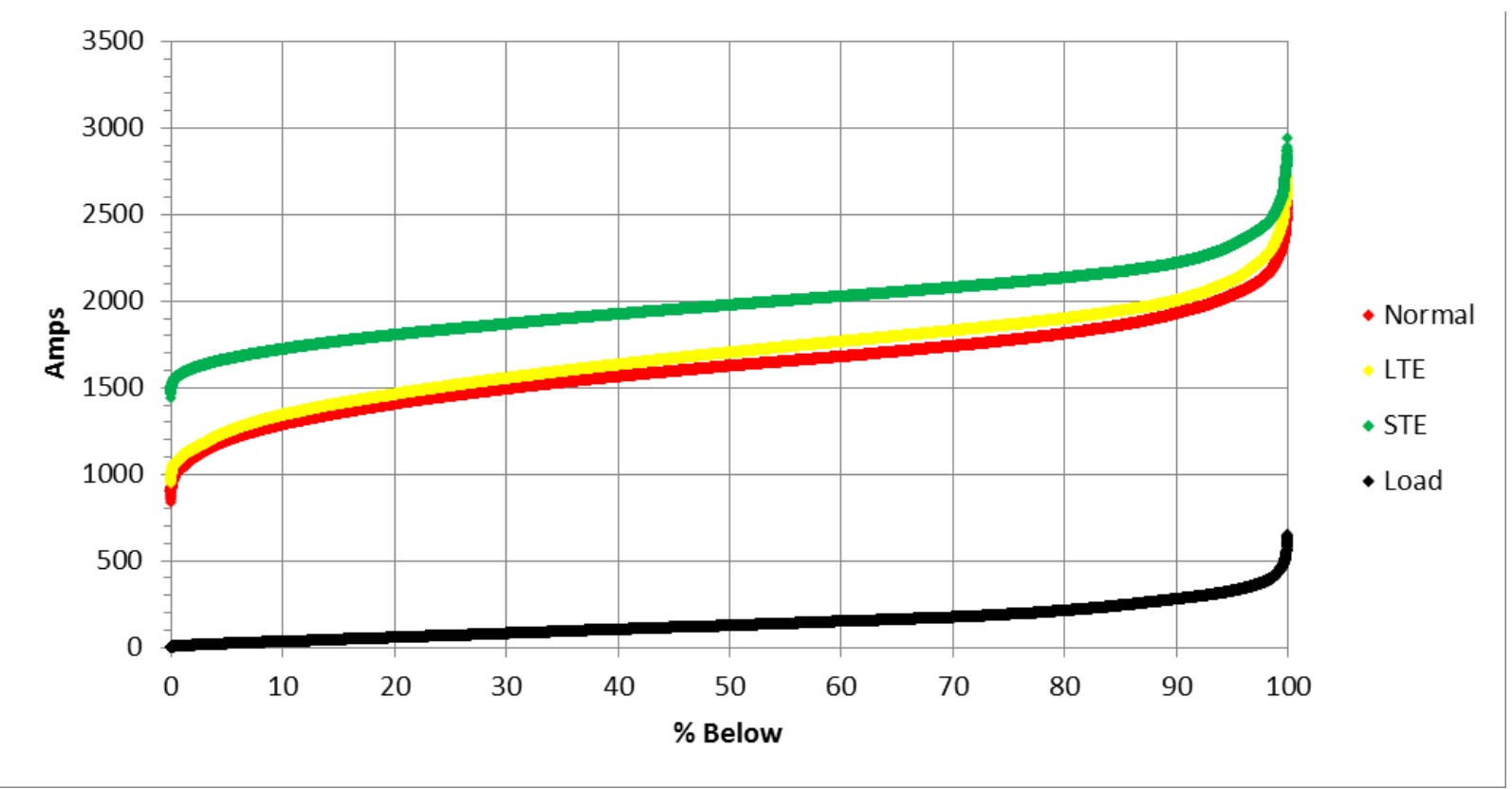

Figure H-50

Summer 2012 Ratings Distribution Site 1 Onsite Weather Station

Table H-52

Summer 2012 Ratings Distribution Site 1 Onsite Weather Station

\begin{tabular}{|c|l|l|l|l|l|l|}
\hline & \multicolumn{1}{|c|}{ Static } & \multicolumn{1}{|c|}{ L1 } & \multicolumn{1}{|c|}{ L2 } & \multicolumn{1}{|c|}{ L50 } & \multicolumn{1}{|c|}{ L95 } \\
\hline Normal & 1089 & 1038 & 1090 & 1191 & 1622 & 2031 \\
\hline LTE & 1256 & 1099 & 1141 & 1241 & 1699 & 2115 \\
\hline STE & 1410 & 1578 & 1607 & 1661 & 1973 & 2319 \\
\hline Load & & 0 & 10 & 20 & 130 & 330 \\
\hline
\end{tabular}

The Site 1 summer 2012 Onsite weather station data can be compared to the Site 1 summer 2012 ThermalRate data. It can be seen in the tables that there is approximately 200 amps difference between the two. The Onsite weather station based rating is the more conservative of the two. 


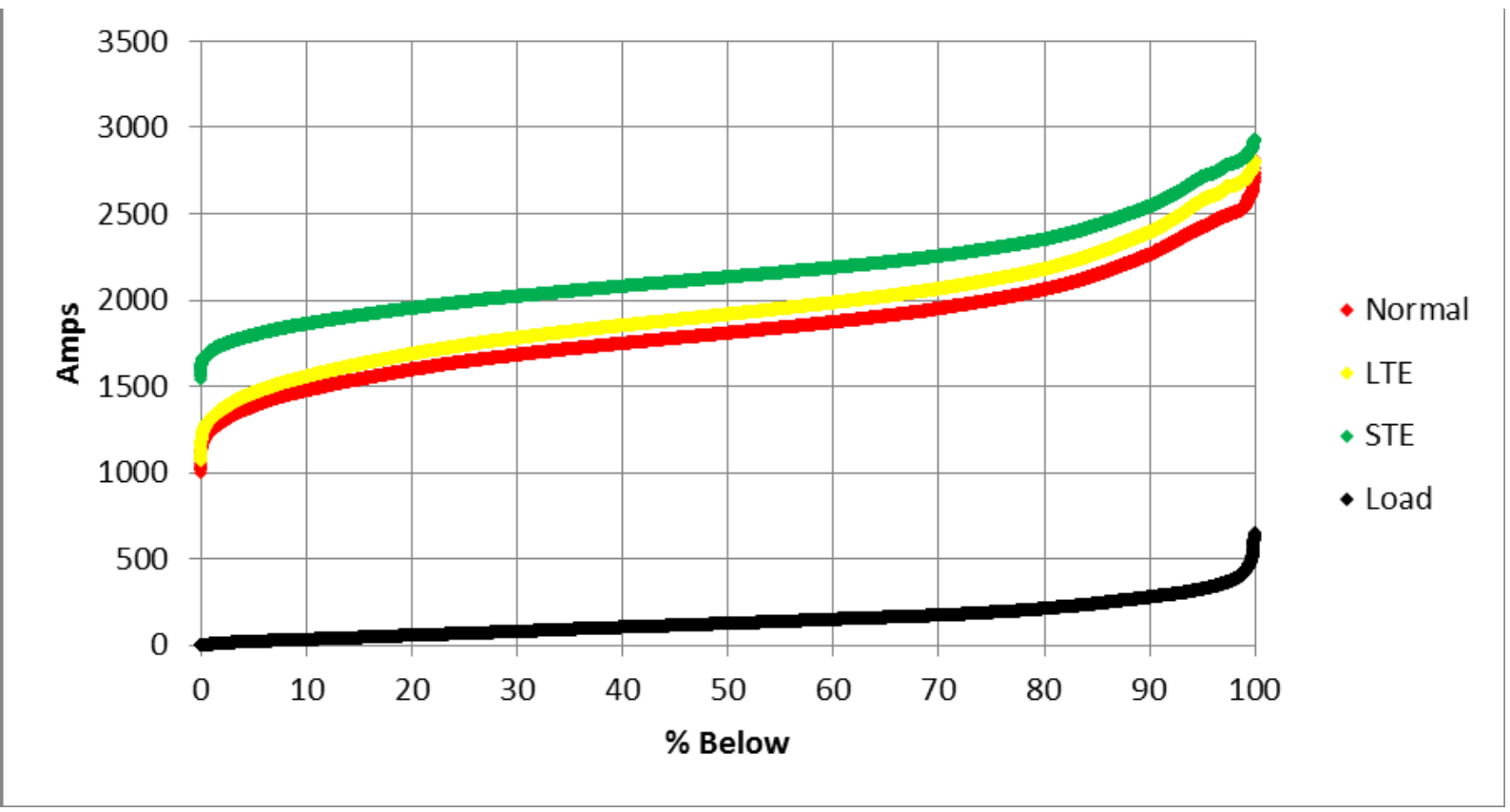

Figure $\mathrm{H}-51$

Summer 2012 Ratings Distribution Site 1 ThermalRate

Table H-53

Summer 2012 Ratings Distribution Site 1 ThermalRate

\begin{tabular}{|c|l|l|l|l|l|l|}
\hline & \multicolumn{1}{|c|}{ Static } & \multicolumn{1}{|c|}{ L1 } & \multicolumn{1}{|c|}{ L2 } & \multicolumn{1}{|c|}{ L5 } & \multicolumn{1}{|c|}{ L95 } \\
\hline Normal & 1089 & 1204 & 1270 & 1368 & 1802 & 2403 \\
\hline LTE & 1256 & 1273 & 1339 & 1447 & 1910 & 2556 \\
\hline STE & 1410 & 1673 & 1720 & 1784 & 2127 & 2691 \\
\hline Load & & 5 & 10 & 20 & 130 & 330 \\
\hline
\end{tabular}

The Site 1 summer 2012 ThermalRate data can be compared to the Site 1 Summer 2012 EPRI Sensor data. It can be seen in the tables that there is approximately $200 \mathrm{amps}$ difference between the two. The EPRI Sensor based rating is the more conservative of the two. This is because the EPRI Sensors are based primarily on weather data as result of low loads. 


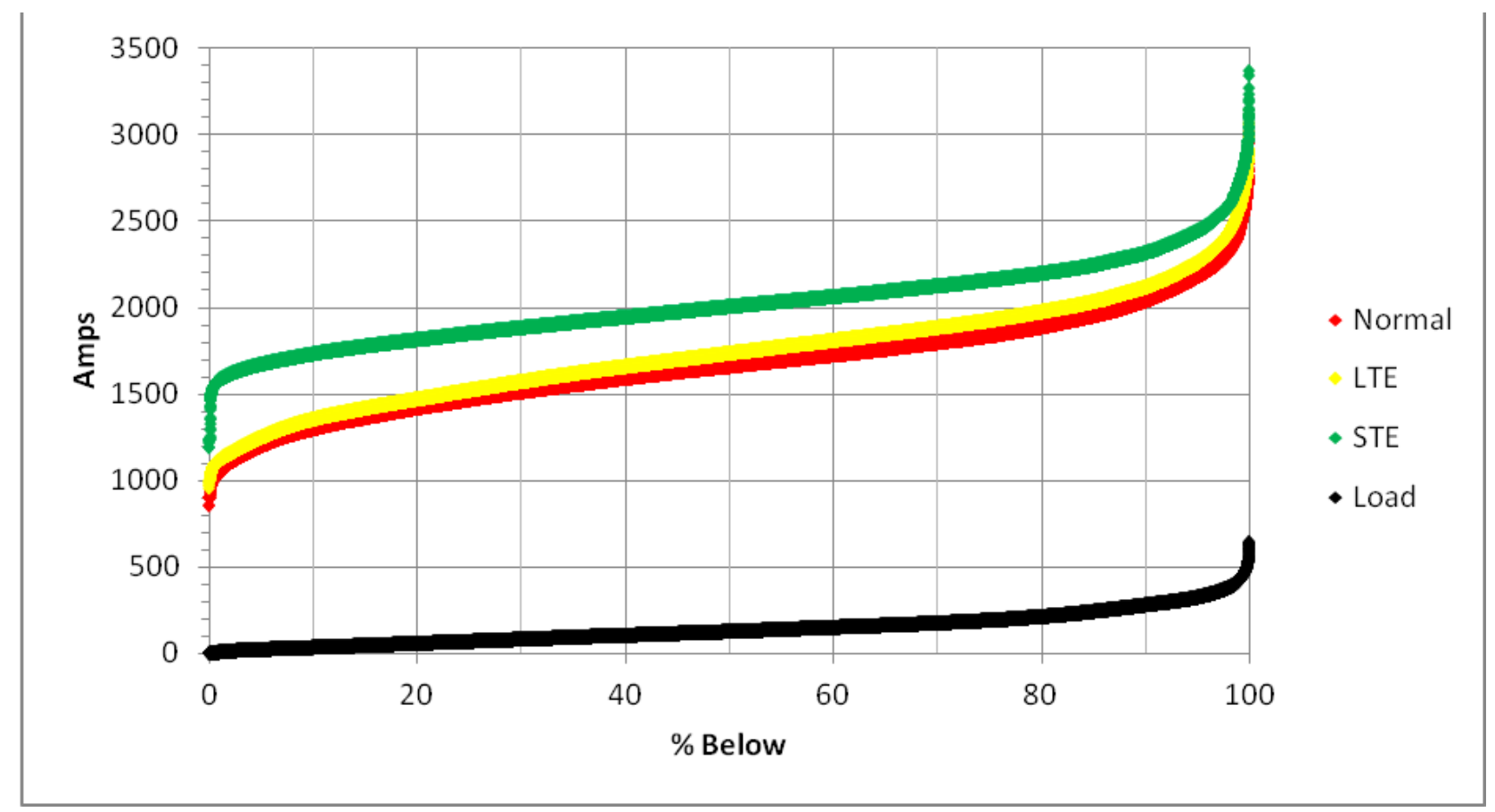

Figure H-52

Summer 2011 Ratings Distribution Site 1 Sagometer

Table H-54

Summer 2011 Ratings Distribution Site 1 Sagometer

\begin{tabular}{|c|l|l|l|l|l|l|}
\hline & \multicolumn{1}{|c|}{ Static } & \multicolumn{1}{|c|}{ L1 } & \multicolumn{1}{|c|}{ L2 } & \multicolumn{1}{|c|}{ L5 } & \multicolumn{1}{|c|}{ L95 } \\
\hline Normal & 1089 & 1048 & 1102 & 1197 & 1656 & 2179 \\
\hline LTE & 1256 & 1116 & 1154 & 1247 & 1733 & 2258 \\
\hline STE & 1410 & 1580 & 1613 & 1669 & 2003 & 2443 \\
\hline Load & & 5 & 10 & 20 & 130 & 330 \\
\hline
\end{tabular}




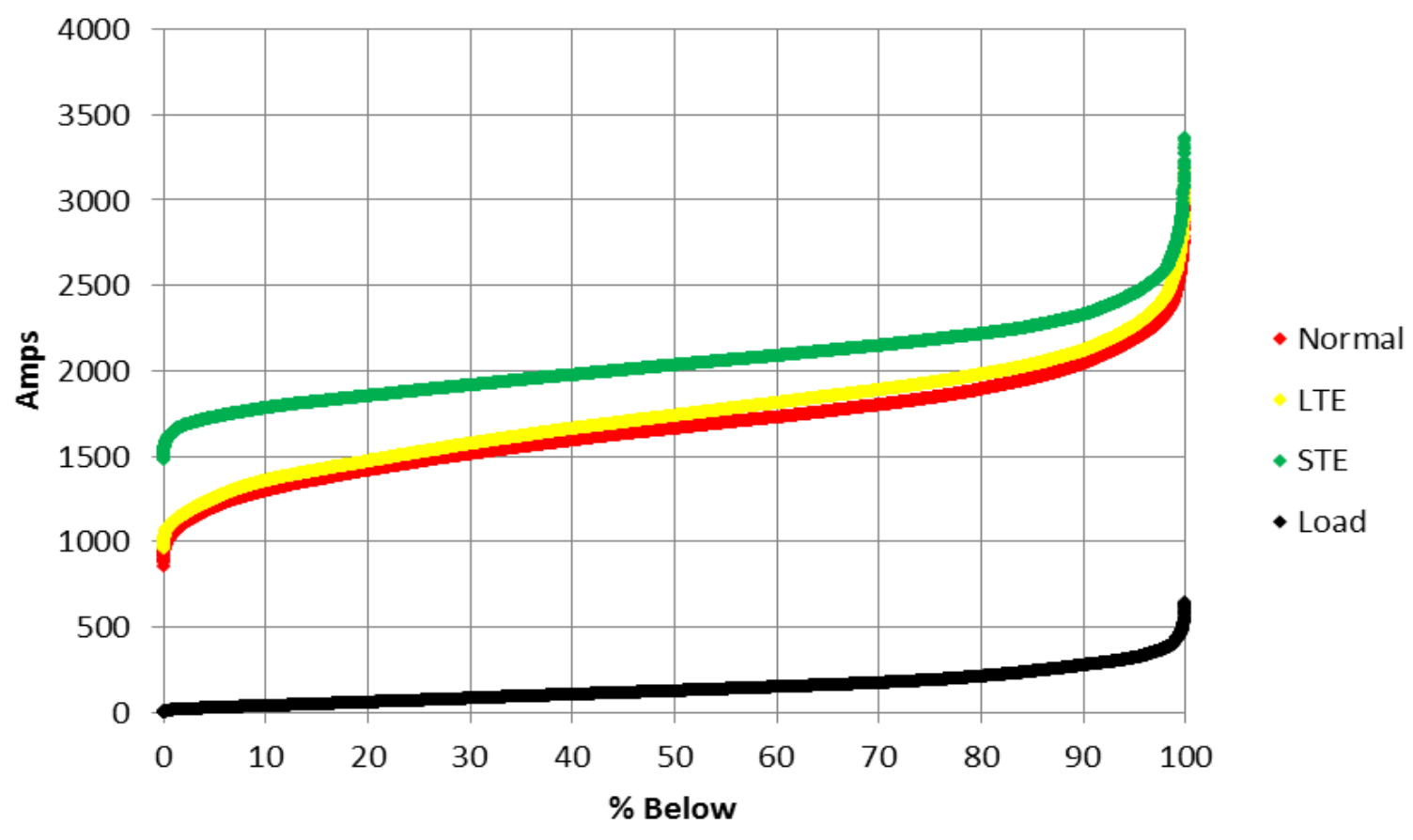

Figure H-53

Summer 2012 Ratings Distribution Site 1 EPRI Sensors

Table H-55

Summer 2012 Ratings Distribution Site 1 EPRI Sensors

\begin{tabular}{|c|l|l|l|l|l|l|}
\hline & \multicolumn{1}{|c|}{ Static } & \multicolumn{1}{|c|}{ L1 } & \multicolumn{1}{|c|}{ L2 } & \multicolumn{1}{|c|}{ L50 } & \multicolumn{1}{|c|}{ L95 } \\
\hline Normal & 1089 & 1011 & 1082 & 1189 & 1656 & 2166 \\
\hline LTE & 1256 & 1085 & 1140 & 1238 & 1732 & 2241 \\
\hline STE & 1410 & 1611 & 1660 & 1720 & 2028 & 2437 \\
\hline Load & & 5 & 10 & 20 & 130 & 330 \\
\hline
\end{tabular}

The Site 1 summer 2012 EPRI temperature sensor data can be compared to the Site 1 summer 2012 off site weather station data. It can be seen in the tables that there is approximately 150 amps difference between the two. The offsite weather service based rating is the more conservative of the two. 


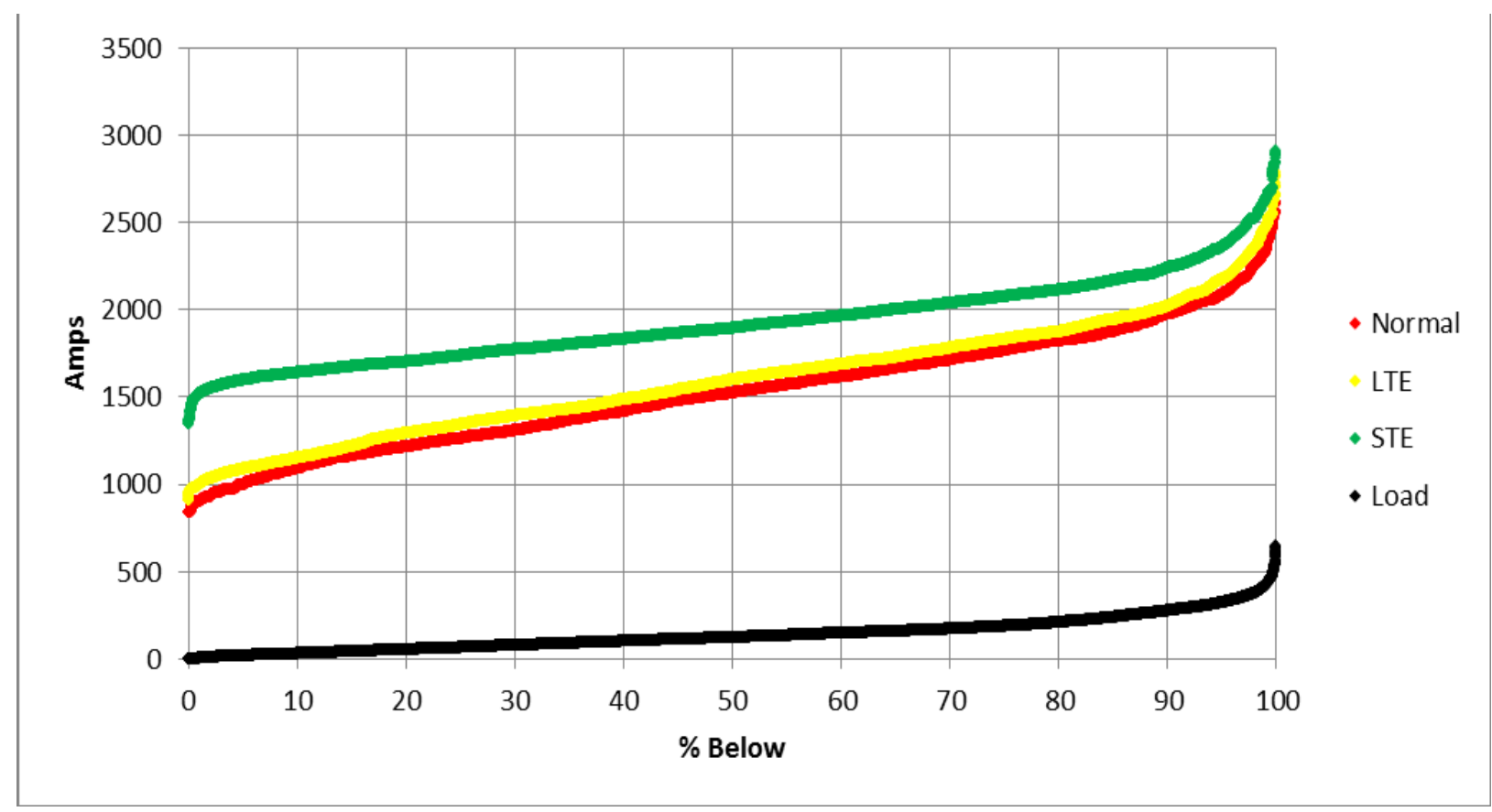

Figure H-54

Summer 2012 Ratings Distribution Site 1 Off Site weather service

Table H-56

Summer 2012 Ratings Distribution Site 1 Off Site weather service

\begin{tabular}{|c|l|l|l|l|l|l|}
\hline & \multicolumn{1}{|c|}{ Static } & \multicolumn{1}{|c|}{ L1 } & \multicolumn{1}{c|}{ L2 } & \multicolumn{1}{c|}{ L50 } & \multicolumn{1}{|c|}{ L95 } \\
\hline Normal & 1089 & 886 & 920 & 988 & 1517 & 2067 \\
\hline LTE & 1256 & 979 & 1021 & 1080 & 1593 & 2159 \\
\hline STE & 1410 & 1488 & 1536 & 1590 & 1890 & 2346 \\
\hline Load & & 5 & 10 & 20 & 130 & 330 \\
\hline
\end{tabular}

The Site 1 summer 2012 off site weather service data can be compared to the Site 1 summer 2012 onsite weather station data. It can be seen in the tables that there is less than 200 amps difference between the two. The offsite weather service based rating is the more conservative of the two. 
Table H-57

Summary table for Site 1 - L2 Were Used for Purposes of Comparison

\begin{tabular}{|l|l|l|l|}
\hline Site 1 Onsite Weather Station Winter 2010/11 & 1204 & 1276 & 1760 \\
\hline Site 1 ThermalRate Winter 2010/11 & 1315 & 1383 & 1824 \\
\hline Site 1 Sagometer Winter 2010/11 & 1203 & 1255 & 1786 \\
\hline Site 1 EPRI Temperature Sensor Winter 2010/11 & 1205 & 1266 & 1765 \\
\hline Site 1 Onsite Weather Station Summer 2011 & 988 & 1123 & 1594 \\
\hline Site 1 ThermalRate Summer 2011 & 1332 & 1435 & 1784 \\
\hline Site 1 Sagometer Summer 2011 & 1247 & 1341 & 1740 \\
\hline Site 1 EPRI Temperature Sensor Summer 2011 & 983 & 1135 & 1689 \\
\hline Site 1 Onsite Weather Station Winter 2011/12 & 1166 & 1215 & 1713 \\
\hline Site 1 ThermalRate Winter 2011/12 & 1384 & 1454 & 1837 \\
\hline Site 1 Sagometer Winter 2011/12 & 1161 & 1224 & 1707 \\
\hline Site 1 EPRI Temperature Sensor Winter 2011/12 & 1167 & 1229 & 1725 \\
\hline Site 1 Off Site Weather Service Winter 2011/12 & 972 & 1069 & 1592 \\
\hline Site 1 Onsite Weather Station Summer 2012 & 1090 & 1141 & 1607 \\
\hline Site 1 ThermalRate Summer 2012 & 1270 & 1339 & 1720 \\
\hline Site 1 Sagometer Summer 2012 & 1197 & 1247 & 1669 \\
\hline Site 1 EPRI Temperature Sensor Summer 2012 & 1082 & 1140 & 1660 \\
\hline Site 1 Off Site Weather Service Summer 2012 & 920 & 1021 & 1536 \\
\hline
\end{tabular}




\section{RATING VS. WIND POWER CORRELATION}

Wind Power Generation in upstate New York is tied to the NYPA $230 \mathrm{kV}$ system. The single most influential weather factor in both rating calculations and wind farm output is wind speed. A strong positive correlation between ratings and wind output (load) is a logical assumption. However in ratings other parameters such as solar gain, temperatures etc. are also important. Wind direction is highly influential. Line orientation is fixed so cooling is a function of both wind angle and speed. In contrast, wind generation is direction independent so the actual correlations from empirical data are of interest, and are included here.

There are two wind farms in the vicinity of the study lines. Ryan Farm is located near Ryan Substation at the end of the Site 1 line, and Dudley Wind Farm located on a line that is not rated in this project. Ryan Wind Farm has a greater impact on loads on the two lines rated in this project than Dudley Farm due to the latter farm's smaller size and the relative locations on the system. The output of both farms though should have an impact on power flow and therefore line loading. Therefore, in addition to correlations between wind output and dynamic rating, correlations between wind output and load on the two lines are examined here.

\section{Line Ratings vs. Wind Output}

Comparisons of ratings for the two transmission lines studied in the rating project with combined Ryan Farm and Dudley Farm output are depicted here in scatter plots. Correlation coefficients between the combined wind farm output and line ratings are also given.

Figure I-1 is a time-synchronized plot of dynamic line rating vs. wind farm output for Site 1. Rating data were from DTCR simulations using historical weather data from the onsite weather station. Wind farm output was supplied by NYPA in Megawatts. A power factor of $96 \%$ was assumed in the conversion from Megawatts to load on the $230 \mathrm{kV}$ system (based on Site 2 and 3 line Megawatts and MVAr information also supplied by NYPA). 


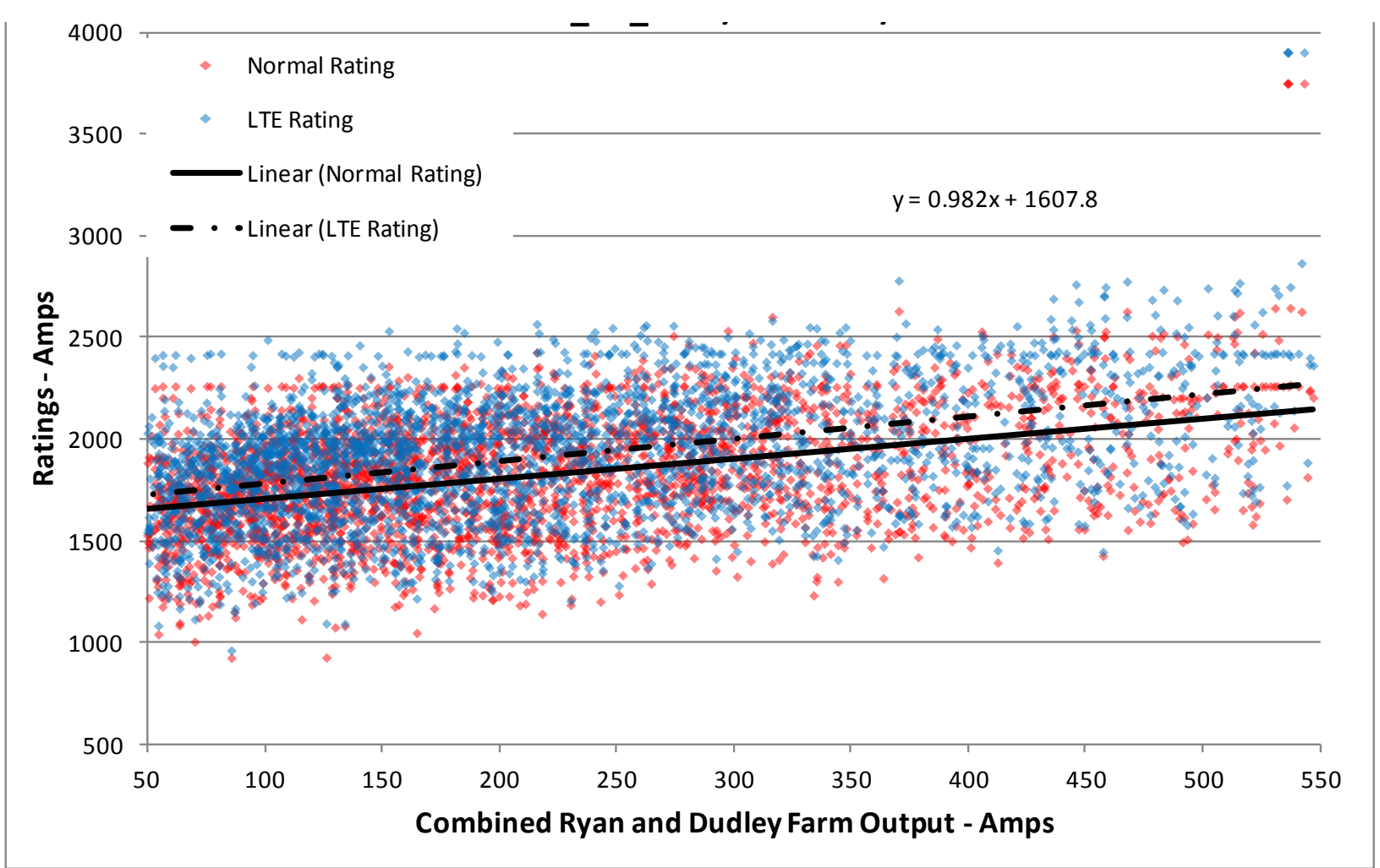

Figure l-1

Combined Wind Farm Output vs. Ratings for Site 1

\section{Table I-1}

Site 1 Correlation Coefficients, Wind Output vs. Ratings

\begin{tabular}{|c|c|}
0.462 & wind output and normal rating \\
\hline 0.462 & wind output and LTE \\
\hline
\end{tabular}

Though there is a moderate correlation coefficient of 0.462 (perfect correlation $=1.0$ ) between the two data sets, the minimum and maximum data retain a very consistent slope with the overall trend throughout the combined wind farm output range. The scatter may be partially due to wind angle with the maximum rating values for a given output corresponding to winds that are near perpendicular to the line and minimum values corresponding to winds parallel to the line. Wind patterns that vary from the farm to the monitoring site, precipitation and ambient temperature will also add to the scatter.

Note the slope of the trend line in Figure I-1 is $+0.98 \mathrm{~A}_{\text {rating }} / \mathrm{A}_{\text {output }}$. This indicates there is very close to a one to one relationship between wind output and rating. Because on average, the rating trend increases at the same rate as the system wind farm output, the Site 1 overhead line capacity is not likely to be a limiting factor to wind farm output if dynamic line ratings based on the WX element are used. This is the case even in the most conservative situation that all wind farm load on the system is carried by one line. This is not a general finding; if the wind farms were twice as big then the line rating would only go up half as fast as wind output. This is simply a fortunate coincidence for this transmission system. 
Similar results were found at Site 2. Figure I-2 is a plot of dynamic line rating vs. combined wind farm output load for Site 2. The Correlation Coefficients of wind output to Normal and Long Term Emergency ratings for this site are shown in Table I-2.

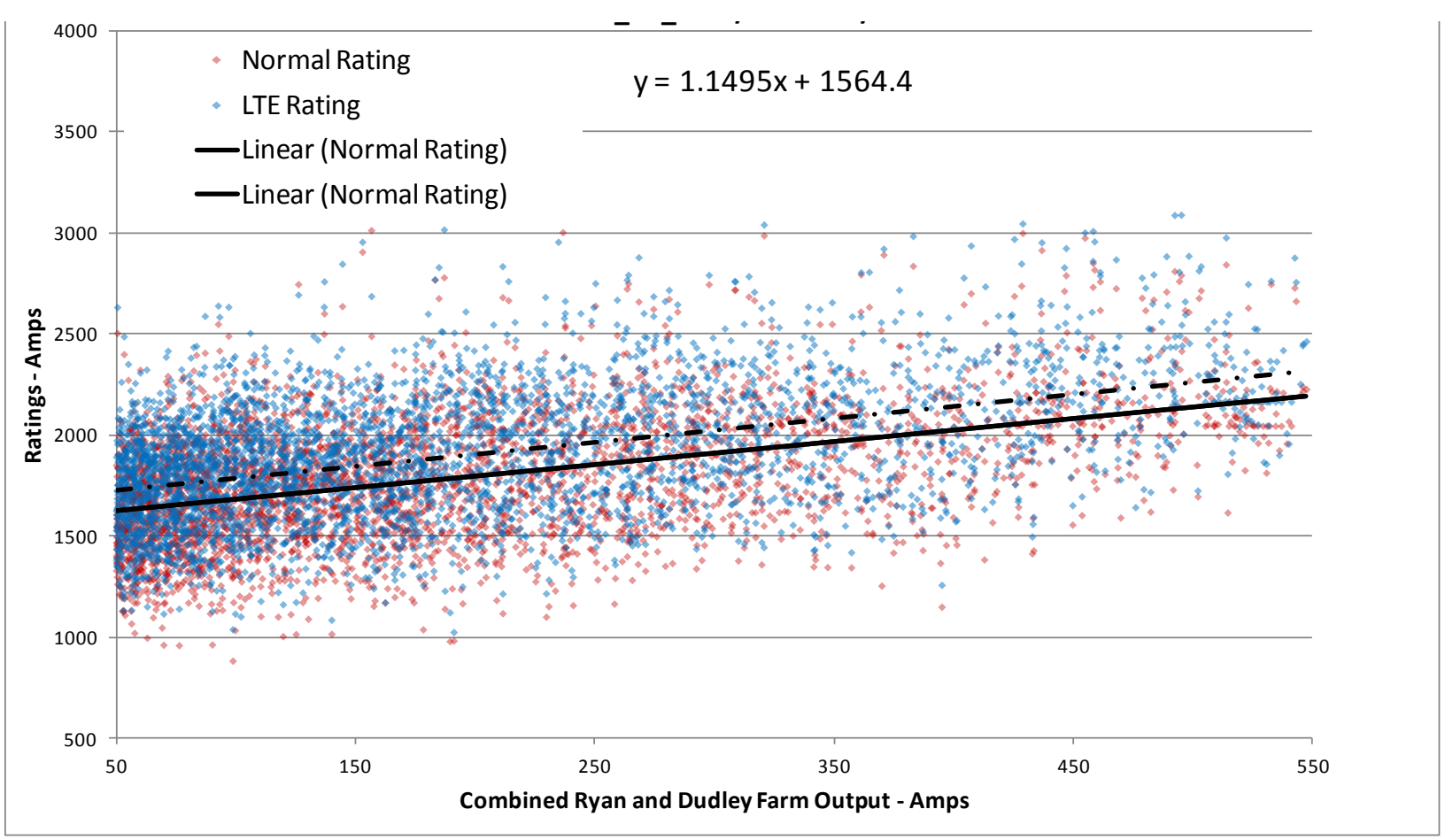

Figure I-2

Combined wind farm output vs. ratings for Site 2

\section{Table I-2}

Site 2 Correlation Coefficients, Wind Output vs. Ratings

\begin{tabular}{|c|c|}
0.451 & wind output and normal rating \\
\hline 0.455 & wind output and LTE \\
\hline
\end{tabular}

A linear relation between actual wind farm output and the dynamic rating with a correlation of 0.45 is indicated at this site also. These results are similar to Site 1which is not surprising since Site 2 is located very close to Site land the Ryan Wind Farm.

Note the slope of the trend line in Figure I-2 is $1.15 \mathrm{~A}_{\text {rating }} / \mathrm{A}_{\text {output }}$. The rating trend increases $15 \%$ faster than wind farm output at this site as compared to a one to one relationship at Site 1. This may be due to a more favorable line orientation.

Figure I-3 is a plot of dynamic line rating vs. combined wind farm output load for Site 3. The correlation coefficients of wind output to normal and LTE ratings for this site are shown in Table I-3 


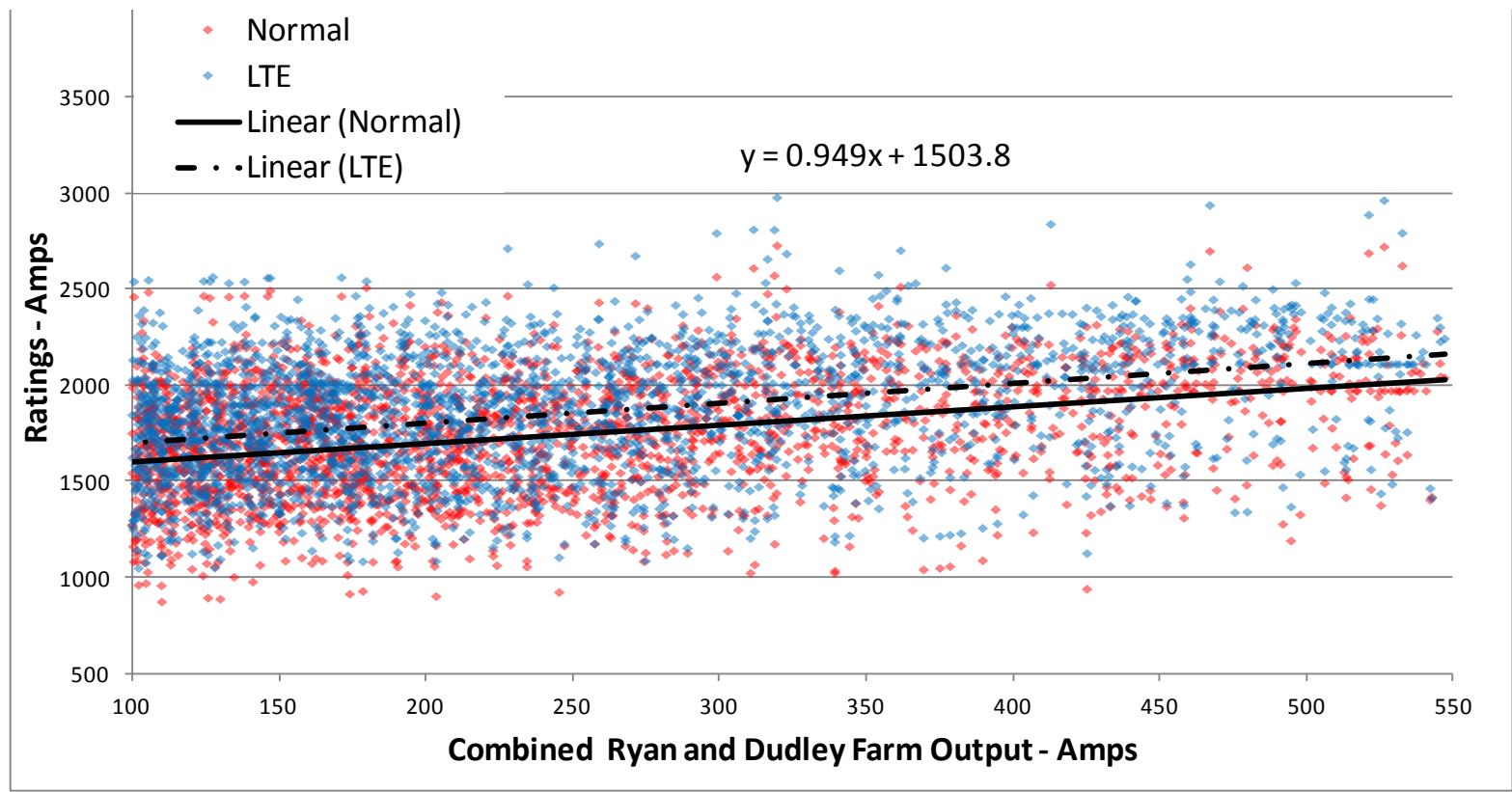

Figure I-3

Ryan Wind Farm output vs. Ratings for Site 3; Correlation Coefficient $=0.29$

Table I-3

Site 3 Correlation Coefficients, Wind Output vs. Ratings

\begin{tabular}{|c|c|}
\hline 0.373 & wind output and normal rating \\
\hline 0.396 & wind output and LTE \\
\hline
\end{tabular}

A correlation between combined wind farm output and the dynamic rating is indicated at Site 3 similar to Site 2 but there is more scatter and the correlation coefficient is slightly weaker than at the other 2 Sites. This may be due to the location of Site 3. This site is approximately 40 miles farther west than the other two sites. The wind farm sites are located east of both of the rated lines and all of the instrumented sites. Therefore, Site 3 is the most remote site in relation to the wind resource. The weaker correlation is consistent with wind patterns differing more over greater distances. The slope of the trend-line at this site is similar to Site 1.

\section{Line Load vs. Wind Output}

Power flow on a given line is a function of many factors including the relative locations of the lines in relation to the generation and power usage. It should be noted that the influence of both wind farms is seen in the power flow even though the correlation may be positive for one farm and negative for the other. The wind resource at these two farms is likely highly correlated but the wind farm locations on the system mean that output can have very different effects on power flow on any one line as is seen in this case. In this study the two lines had significant but different correlations between line load and wind farm output. 


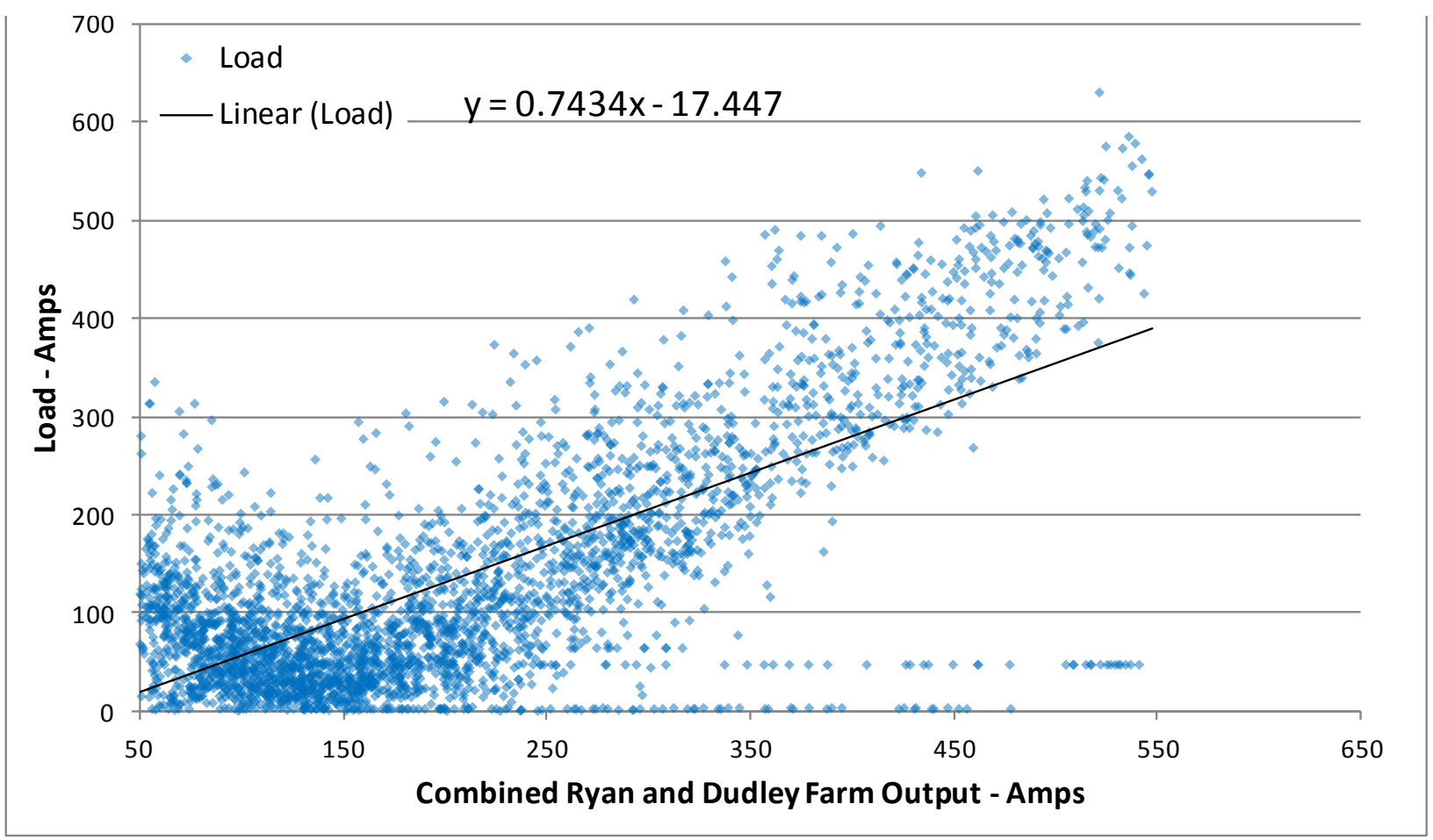

Figure I-4

Combined Wind Farm Output vs. Load for the Site 1 Line; Correlation Coefficient $=\mathbf{0 . 6 2 9}$

A linear curve fit to the data indicate that load increases with wind power output on Site 1 but at a slower rate than wind farm output. Many factors influence power flow and Figure I-4 indicates that the influence of the Ryan Wind Farm on Site 1 load is attenuated by these other factors. The load to output relationship trends to approximately $0.74 \mathrm{~A}_{\text {load }} / \mathrm{A}_{\text {output. }}$ Recall from Figure I-1 that the ratings at Site 1 increased at $0.988 \mathrm{~A}_{\text {rating }} / \mathrm{A}_{\text {output. }}$

The load on Site 1 increases on average less than the Wind Power output at and therefore the ratings on average increase as fast as, or faster than wind power output. A closer look though, indicates that the overall trend is highly affected by the data during low wind power output. When the data above 250A of wind power output is considered, the relation approaches 1 $\mathrm{A}_{\text {load }} / \mathrm{A}_{\text {output. }}$ In either case, this result supports the conclusion that if dynamic line ratings are used, wind farm output is not likely to be limited by the Site 1 line capacity in the existing system configuration and the dispatch conditions encountered during this project.

Figure I-5 shows the load vs. combined wind farm output on the Site 2 and 3 lines: 


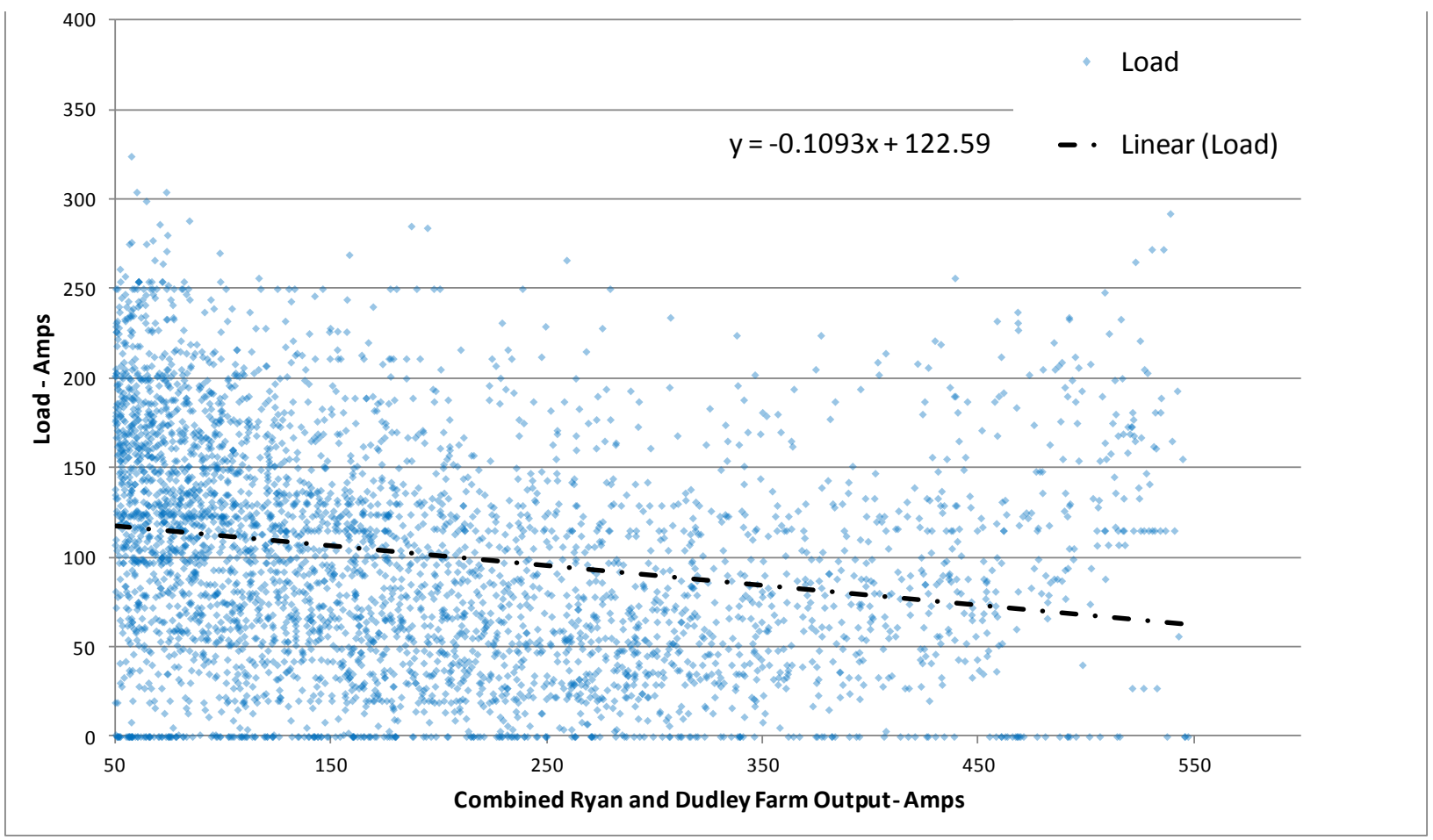

Figure I-5

Ryan Wind Farm Output vs. Load for Sites 2 and 3; Correlation Coefficient $=\mathbf{- 0 . 2 3 3}$

There is a weak negative correlation between loadings on Sites 2 and 3 and combined wind farm output. This indicates that wind farm output may supplant load normally flowing over the Site 2 and 3 lines in the typical dispatch situations encountered during this study. In this case wind power output would obviously not be limited by overhead capacity on the Site 2 and 3 lines. 


\section{SUMMARY, CONCLUSIONS AND RECOMMENDATIONS}

Peak normal and post contingency emergency power flows in existing transmission systems such as NYPA's, are increasing faster than new transmission capacity is being added. This is particularly true for certain older high voltage overhead lines where peak power flows approach or threaten to exceed the line's thermal ratings during N-1 system emergencies or as a result of peak power generation from newly added wind generation farms.

A relatively inexpensive and flexible method of increasing transmission line thermal capacity involves the application of real-time monitoring and dynamic line ratings, where the thermal rating is based on actual weather conditions along a specific transmission line rather than using conservative assumptions of high air temperature and low wind speed. The main objective of this project was to evaluate real-time DTCR Technologies, and to demonstrate how they could be used by transmission system engineering, operations, and planning of the New York Power Authority (NYPA).

The emphasis in this project has been on the dynamic rating of two specific lines using dedicated real-time monitors deployed along the line. All real-time monitors are located close to the line and within the right-of-way (corridor). The project did not evaluate the possible use of systemwide weather-adjusted ratings based on NOAA or other weather service data, but the possible use of weather service on-line data as a backup to one of the line monitor methods was considered.

\section{Real-Time Monitoring Methods}

EPRI's Dynamic Thermal Circuit Rating software (DTCR) can track the line conductor temperature and calculate dynamic line ratings for multiple locations along the line. At each monitoring location, the key weather parameters are wind speed and wind direction, but the air temperature and solar heating must also be known if the line rating is to be calculated accurately. In this project, four types of real-time monitoring devices were used to measure wind at the monitoring locations. They are:

- On-site weather stations

- ThermalRate devices (by Pike)

- Video Sagometers (by EDM)

- EPRI Sensors

- Off-site weather service data (backup) 
All of these monitoring methods produce data that can be entered in real-time to DTCR software, and in combination with the line current, air temperature, and solar heating, can be used to calculate line ratings. The weather stations provided air temperature and solar heat intensity to DTCR for all but the ThermalRate system, which used its own solar and air temperature monitor.

The measurements of conductor temperature, and the sagometer measurements, are converted by DTCR into effective wind speeds before calculating the line rating.

\section{Monitor Installation Experience}

The NYPA line crew was provided training on the installation of all the field instruments at the EPRI high voltage laboratory in Lenox, Massachusetts. Subsequently, the line crew installed the equipment in the field at three sites on operating lines. The line crew was interviewed about their experiences and provided insight into any problems or issues encountered. Overall, the line crew was pleased with the installation process. There were a few minor bumps in the first set of instruments installed, but by the time they installed the third set, all issues were resolved, and the instruments were all installed with relative ease.

The one issue that did take an extended period of time to resolve was to provide a communication link between the instruments and an RTU in a nearby substation. This relatively small task took several months to resolve because of the required logistical processes. It was learned that for future applications, these processes need to be considered and addressed upfront.

\section{Monitor Reliability}

All the rating instruments tested during the course of the project had some reliability issues more than what would be hoped for or expected. The instrument vendors responded quickly, and in most cases the issues were resolved. Data from the instruments were available throughout the projects as follows:

Table J-1

Real-Time Instrument Availability

\begin{tabular}{|c|c|c|}
\hline All Sites; Instrument & \% Available & \% Available Real-time \\
\hline Weather Station Thermometer & 99.4 & 91.6 \\
\hline Weather Station Anemometer & 93.7 & 90.5 \\
\hline Sagometer Camera & 79.3 & 69.4 \\
\hline EPRI Sensor & $71.9^{*}$ & 69.5 \\
\hline ThermalRate Device & 85.0 & 75.9 \\
\hline NYPA SCADA RF Link & 75.1 & 69.5 \\
\hline All Logger Communications & NA & 90.3 \\
\hline
\end{tabular}

* Available and conductor temperature above 1 degree

One of the more useful outcomes of this project is that all the vendors learned more about the performance of their products, and in every case the vendors have made improvements to their 
products. The performance issues encountered during this project are expected to be significantly reduced in future applications. In addition, limitations and requirements for the DTCR rating software were identified, and the software too is undergoing improvements as a result.

\section{Monitor Accuracy}

The errors produced by the real-time monitoring devices were small. Similarly, other weather monitoring devices which measure air temperature and solar heating are also accurate. These field studies confirm that this was true for this project.

Similarly, the heat balance algorithms (e.g., IEEE 738) used to calculate the relationship of conductor temperature and conductor current given weather conditions were also shown to be accurate and slightly conservative. Slight inaccuracies in determining conductor temperature from weather station measurements probably results from inaccuracies in the IEEE 738 model for determining convective cooling, particularly from low and/or parallel winds.

Evidence was presented that the ThermalRate device was less conservative but as accurate as and possibly more accurate than IEEE 738 when calculating this relationship.

The EPRI sensors measured the local conductor temperature directly and so this method was as accurate as the instrument output. Because of the open and flat terrain in this project there is reasonable confidence that the local temperature was representative of the conductor temperature over the distance between strain structures. This allowed the EPRI sensors to be used as comparison to evaluate other technologies whether they produced point or span section results.

Data were presented showing that the Video Sagometer in this project did not have a stable relationship between line sag and conductor temperature. The open and flat nature of the terrain suggests that this instability is probably not due to variations in conductor temperature along the span section. The instability is possibly due to structure movement which will affect the state change equation relating target clearance to conductor temperature. The Video Sagometer was the least accurate of the four onsite methods in predicting conductor temperature.

It was confirmed that the conductor temperature at a point along the overhead line can be tracked with considerable accuracy if the line's recent current history is known and the real-time weather conditions are measured near the conductor whose temperature is to be determined.

It is known that in general the conductor temperature in a nearby span of the same line may be quite different from the conductor temperature which is measured or calculated for the local span. This implies the need for multiple monitoring locations along any dynamically rated overhead line in order to assure that the dynamic rating is conservative. Local conductor temperature monitors should be located in the most sheltered location (or near the low point of conductor sag if the line is in flat open terrain) to be sure they reflect the lowest wind cooling.

It is also shown from the field investigation that the conductor temperature can vary rapidly with changes in wind cooling, which yields large prediction errors when the prediction period exceeds one hour. Also, any chronological error in conductor temperature increases with line current and with periods of low wind cooling. 


\section{Line Rating Accuracy}

Wind cooling is the most important environmental variable in determining dynamic line rating, and it is the most difficult one to ascertain. Unlike other variables such as ambient temperature and solar intensity, wind can exhibit large variations in both speed and direction at any location along the line and between spans in the line. To determine what values to use when calculating ratings from moment-to-moment can be very difficult, and is the main challenge for any realtime rating scenario.

Wind speed measurements were directly made (whether the load was high or low) by the weather station anemometers and nearby NOAA stations, and indirectly by the ThermalRate devices. Overall, these measurements compared favorably. There was also reasonable agreement for the limited periods where the line load was high enough to estimate wind speed from the Video Sagometers and the EPRI Sensors.

To the system operator, the primary interest concerns the line rating accuracy since the operator assures safe operation of the power system by keeping the line current below the appropriate line rating, or allowing it to exceed the rating for limited periods of time. It is impossible to make operational decisions, such as load shedding or re-dispatch of generation, to keep the line current below the rating if the rating either increases or decreases rapidly. Also, it is impossible to set contract flow limits a day or more in advance if the ratings used in determining the contract flows change.

Line rating errors can be very large, even if instrument errors are tiny, if the measurements are applied incorrectly. For example, sag, tension, and conductor temperature measurements should not be used when the line current is low.

\section{Impact of Line Current on Rating Accuracy}

As described in Appendix B, the line rating calculation involves the use of a heat balance equation with weather data inputs (wind speed, wind direction, air temperature, and solar radiation). The wind speed and direction parameters can be replaced by an "effective wind speed" which equals that wind speed, blowing perpendicular to the conductor axis, which produces the same convective cooling. Each of the monitoring methods must provide air temperature, solar heat, and convective cooling data to allow a line rating calculation.

The on-site weather stations measure all the necessary weather data parameters to allow a realtime line rating calculation. The ThermalRate device measures air temperature and solar heating with the unheated portion of its conductor model and calculates an "effective wind speed" based on the conductor temperature of the internally heated part of the its conductor model. In neither method is the line rating accuracy affected by the actual line current flowing on the line.

In calculating line ratings based on the Video Sagometer monitoring method and the EPRI Sensor conductor temperature monitoring method, the measured parameters are conductor target clearance and conductor temperature, respectively. Clearance and conductor temperature must be converted to effective wind speed before the line rating can be calculated.

The heat generated in the line's conductor depends primarily on the square of the current that passes through it. Thus, the heat generated with the line carrying $30 \%$ of its rated load is only $10 \%$ of the heat generated at full rated load, and any temperature rise above ambient, and change 
in sag, is quite small. Given normal measurement uncertainties of 1 to $2{ }^{\circ} \mathrm{C}$, the errors inherent in calculating effective wind speeds at low current levels are prohibitively large. When the sometimes large errors involved in converting sag clearance to average conductor temperature in a line section are considered, the Video Sagometer monitoring method is only reasonably accurate when the current density in the $795 \mathrm{Kcmil}$ Drake conductors is equal to $0.5 \mathrm{amps} / \mathrm{Kcmil}$ or more.

In cases where a line is more lightly loaded, (as was the case at NYPA most of the time), the ratings cannot be accurately estimated from the Video Sagometer or the EPRI conductor sensor monitoring methods. For the two $230 \mathrm{kV}$ lines instrumented in this study, the line currents rarely exceeded 350A (0.45 amps/Kcmil), so these monitoring methods were not useful.

Since sag-based methods have the advantage of averaging the conductor temperature over many spans, the inability to properly evaluate these technologies in this project was unfortunate. However, this is actually a significant finding in the project. There is evidence from other sources that the lines instrumented at NYPA are not unusual. A recent EPRI Technical Update [1] regarding the use of LiDAR with in-service lines includes a statistical estimate of line currents on over 200 high voltage transmission lines (see Figure J-1).

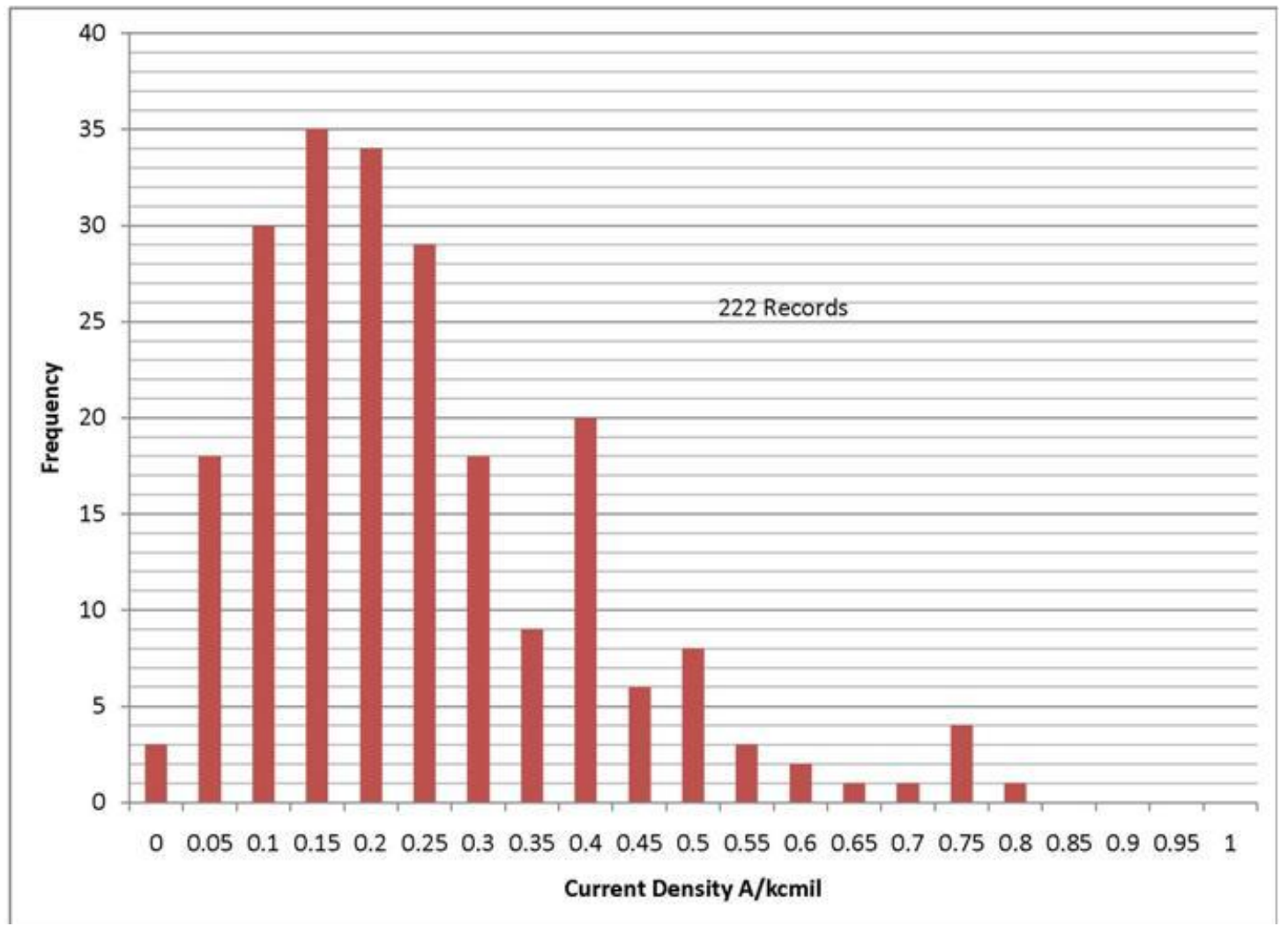

Figure J-1

Statistics of Current Densities Measured on Transmission Lines

Clearly, because of the need for system reliability, many high voltage lines operate at current densities less than $0.5 \mathrm{amps} / \mathrm{Kcmil}$ and cannot be monitored accurately with devices whose rating accuracy depend on significant heat generation in the conductors. 


\section{A Hybrid Monitoring System}

On the other hand, the Video Sagometer should become more accurate as the line current increases and may even be more accurate than on-site weather stations or the ThermalRate system during high current events such as after an N-1 or N-2 contingency. In fact, it can be argued that there is a significant advantage to the direct measurement of sag-tension during those infrequent times when the line current is close to the line rating.

To the extent that this is true, it appears that a hybrid system that involves two monitoring methods such as the weather station and the Video Sagometer would be the most effective and accurate method of determining dynamic line ratings under the full range of weather and loading conditions. However, sag or tension monitoring for rating purposes require the identification of an accurate state-change equation, which can be challenging itself (see Appendix $G$ and the removed Appendix B).

\section{Recommendations for Future Work}

The off-site NOAA weather data derived from nearby airport weather stations and accessed over the internet was used as reasonable backup for the other on-site monitoring systems. It may be helpful to pursue similar comparisons at NYPA lines which are in areas having rougher terrain and/or lines heavily shielded by foliage.

According to CIGRE Technical Brochure 299[2], the line rating conditions in use by NYISO, which include an effective wind speed of $3 \mathrm{ft} / \mathrm{sec}$ and an air temperature of $35^{\circ} \mathrm{C}$ for summer, need to be justified by field studies of the sort that was done here. The data analyzed here suggest that the NYISO conditions appear to be justified by these measurements. In future upgrades of NERC Reliability Standards FAC-008 and FAC-009, it is conceivable that a statistical analysis of line rating weather conditions may be required in addition to the specification of an industry standard heat balance method and reasonable maximum allowable conductor temperatures. Additional, but somewhat simplified, studies of other NYPA lines in more sheltered areas with lower voltage lines would be very useful.

A related area that NYPA may consider for future study is the consideration of substation terminal equipment ratings. EPRI has begun to develop thermal modeling data for terminal equipment like switches, line traps, and current transformers. The DTCR software is capable of calculating dynamic ratings for terminal equipment and power transformers, as well as for underground cables and overhead lines. In particular, an EPRI project is being organized to model CT ratings. In a significant amount of cases, substation CTs are the limiting factors for line ratings. Similar to overhead lines, CT's are known to have significantly higher power capacities than specified by their nameplate ratings. EPRI is launching a collaborative project to study CT ratings with the goal of defining methodologies to better rate these devices. The project will include laboratory tests of selected samples (including special units fabricated for the project) and forensic analysis.

To build on the significant data collection and analysis performed in this study, it is suggested that an additional study be undertaken to evaluate the use of simplified approaches to dynamic line ratings. In particular, EPRI is engaging some utilizes about novel methods for predictive ratings that may apply well to NYPA lines. 
One such approach which is presently be studied at two other member companies is the use of diurnal-cycle line ratings, which combines the statistical analysis of wind cooling as a function of time during the daily solar cycle with real-time air temperature. The resulting line ratings are far more predictable than dynamic ratings based on real-time wind monitoring and simpler to implement in operations.

As an example, Figure J-2 shows a two day period in a previous study where the higher more volatile dynamic rating and the lower but less volatile diurnal rating are compared to the line's static rating. Air temperature and wind speed are also shown.

The advantage to this approach is that, once the diurnal wind along the line has been statistically analyzed, it only requires real-time air temperature to be implemented. This also makes it possible to predict diurnal ratings on the basis of predicted air temperature which is usually available for days in advance.

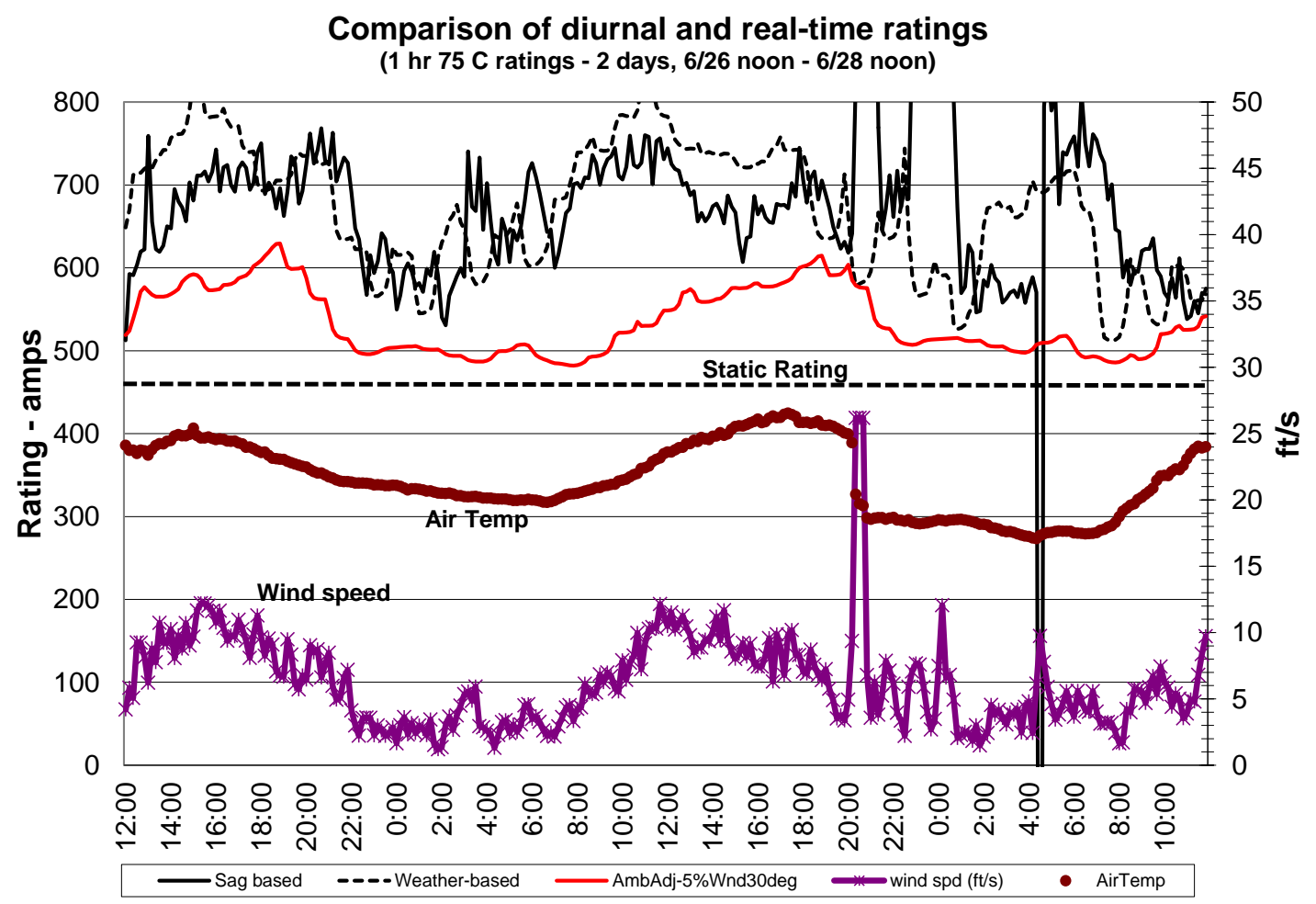

Figure J-2

Comparison of Diurnal and Real-Time Line Ratings

\section{References}

1. Evaluation of Emerging Line Inspection Technologies Results of 2011 Field Tests, Technical Update 1026639, November 2012

2. CIGRE Technical Brochure 299 "Guide for Selection of Weather Parameters for Bare Overhead Conductor Ratings.” Working Group B2.12. August, 2006 



\section{K \\ MONTHLY PLOTS}

Throughout the Project monthly plots were made of key data and rating results for the two lines. These plots were used by project engineers to identify trends, instrumentation problems, communication problems and key project result. The plot set evolved over the course of the project to better visualize and keep track of the performance of the lines and the systems.

This appendix contains sample plots from April 2012. The plot set was the latest used at the end of the project. The plotted data was all from DTCR software output (*.RTD files) and the graphing software was the Data Analysis Package (DAP) included with DTCR version 6.0. The plots were exported in RTD format, imported into Microsoft Word where they were sized and formatted in a usable form.

Binders exist at the EPRI facility in Lenox that contain real-time plots throughout the project. The plots here are not duplicates of those plots. Instead the latest DAP document collection was combined with the latest simulations to provide the most complete data set possible. .Appendix E (and the removed chapter 5) has a detailed explanation of DTCR simulation output and how it differed from real-time DTCR output. A short description of the difference is that real-time communication efficiency between the Site and the DTCR server was less than $100 \%$. Most of the data affected was delayed rather than lost. Simulations utilized this delayed data to give a more complete rating picture.

All monthly Plots are available to qualified users in digital form. Plot formats are PDF and Word .docx files. In addition the DTCR output files in .RTD form are also included. With the RTD files the user can use DTCR version 6.0 DAP application to view the project output data in user defined plot format, as well as modify the plots presented here. The location of the posted digital data is as follows: 


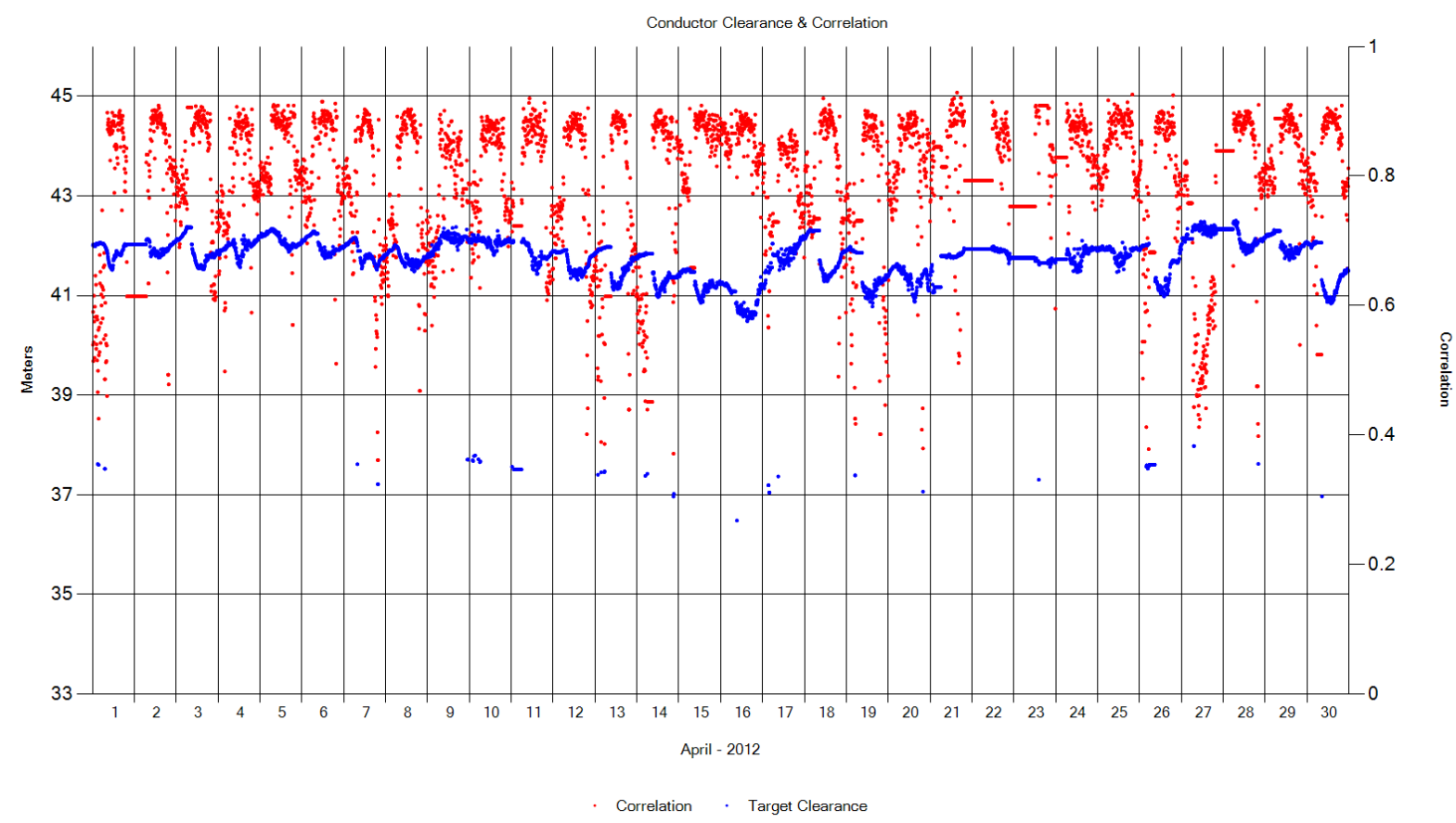

K-1 Element: Site 1_WxPlot: Conductor Clearance \& Correlation

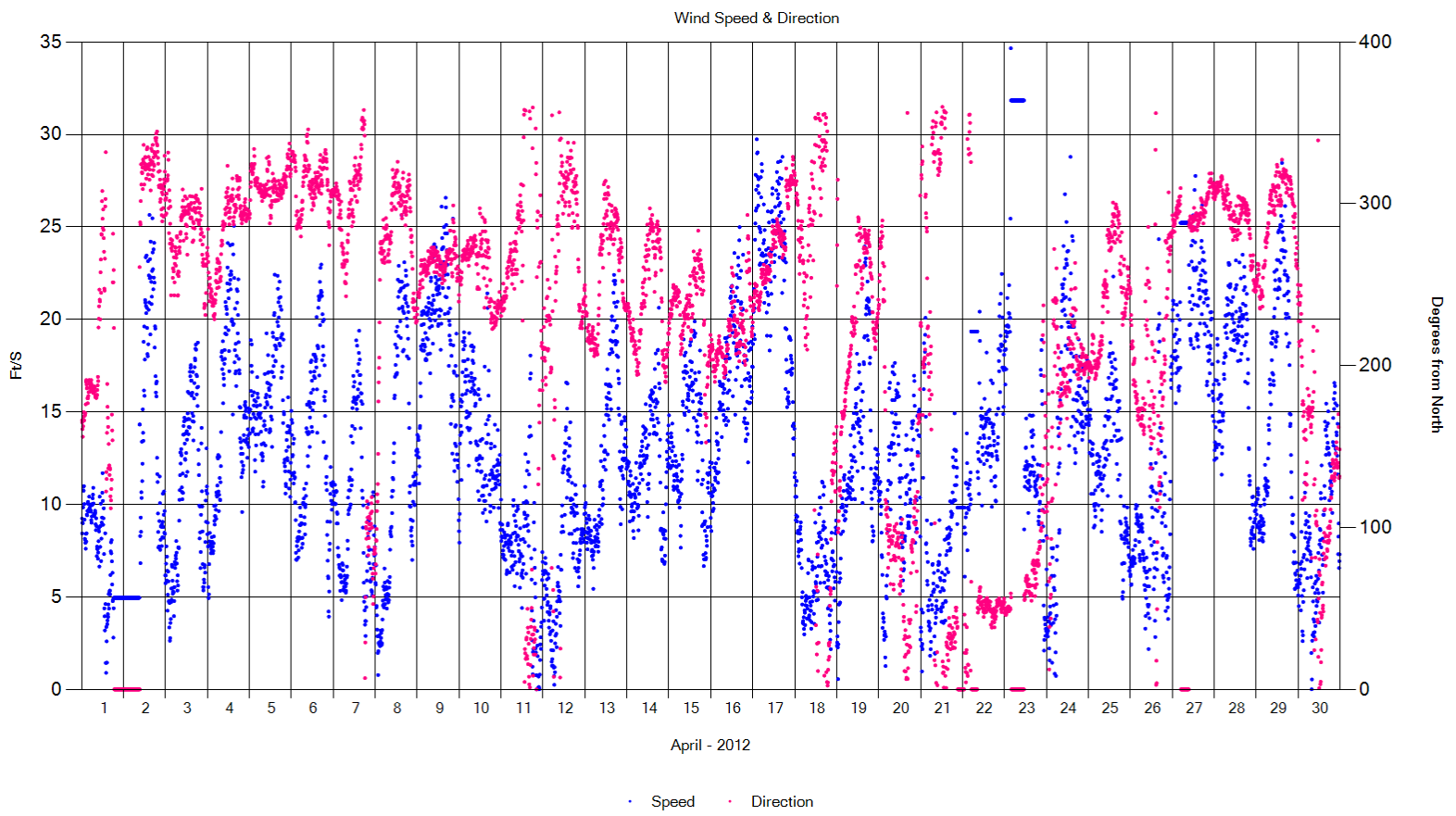

K-2 Element: Site 1_Wx Plot: Wind Speed \& Direction 


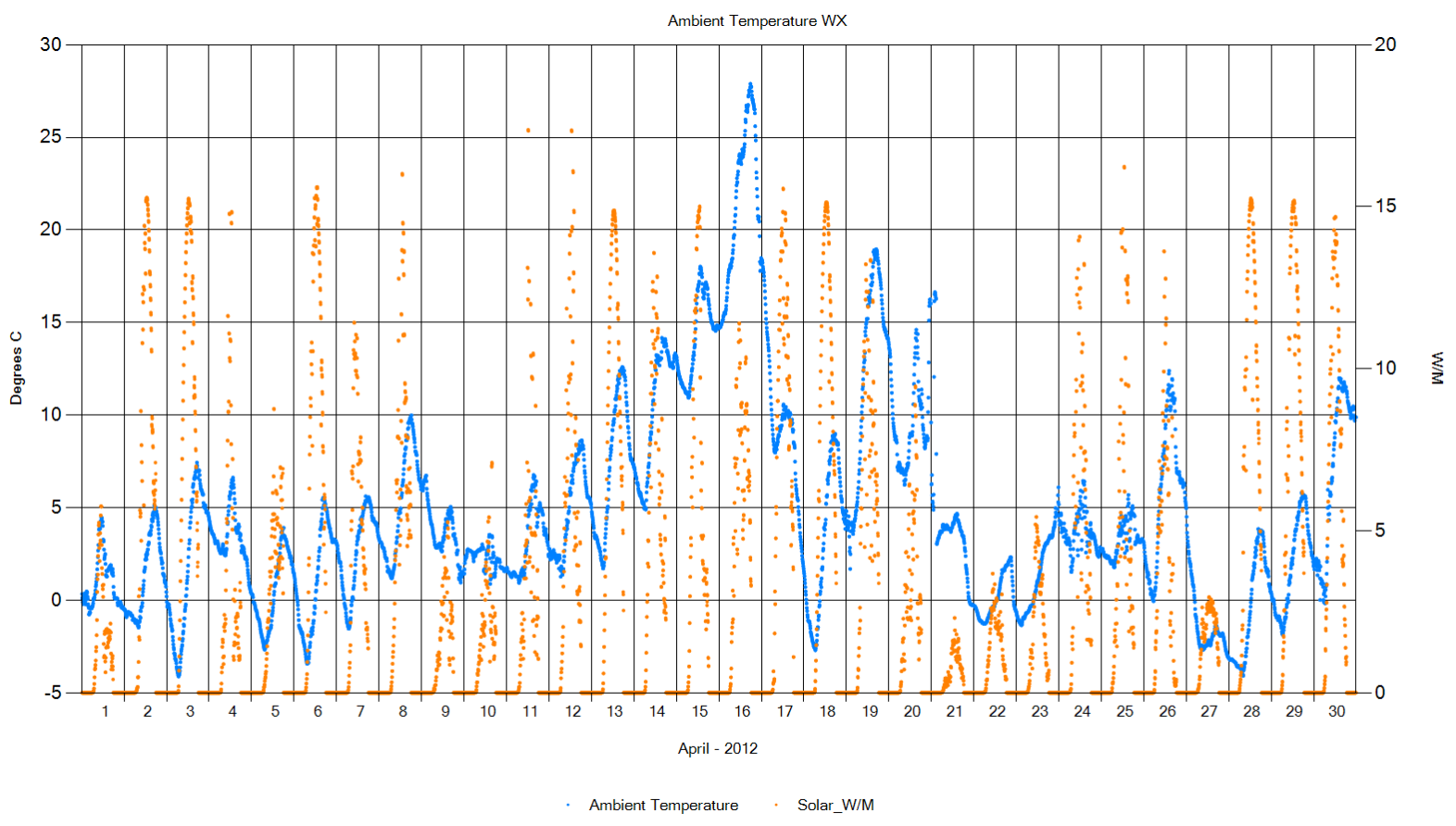

K-3 Element: Site 1_Wx Plot: Ambient Temperature WX

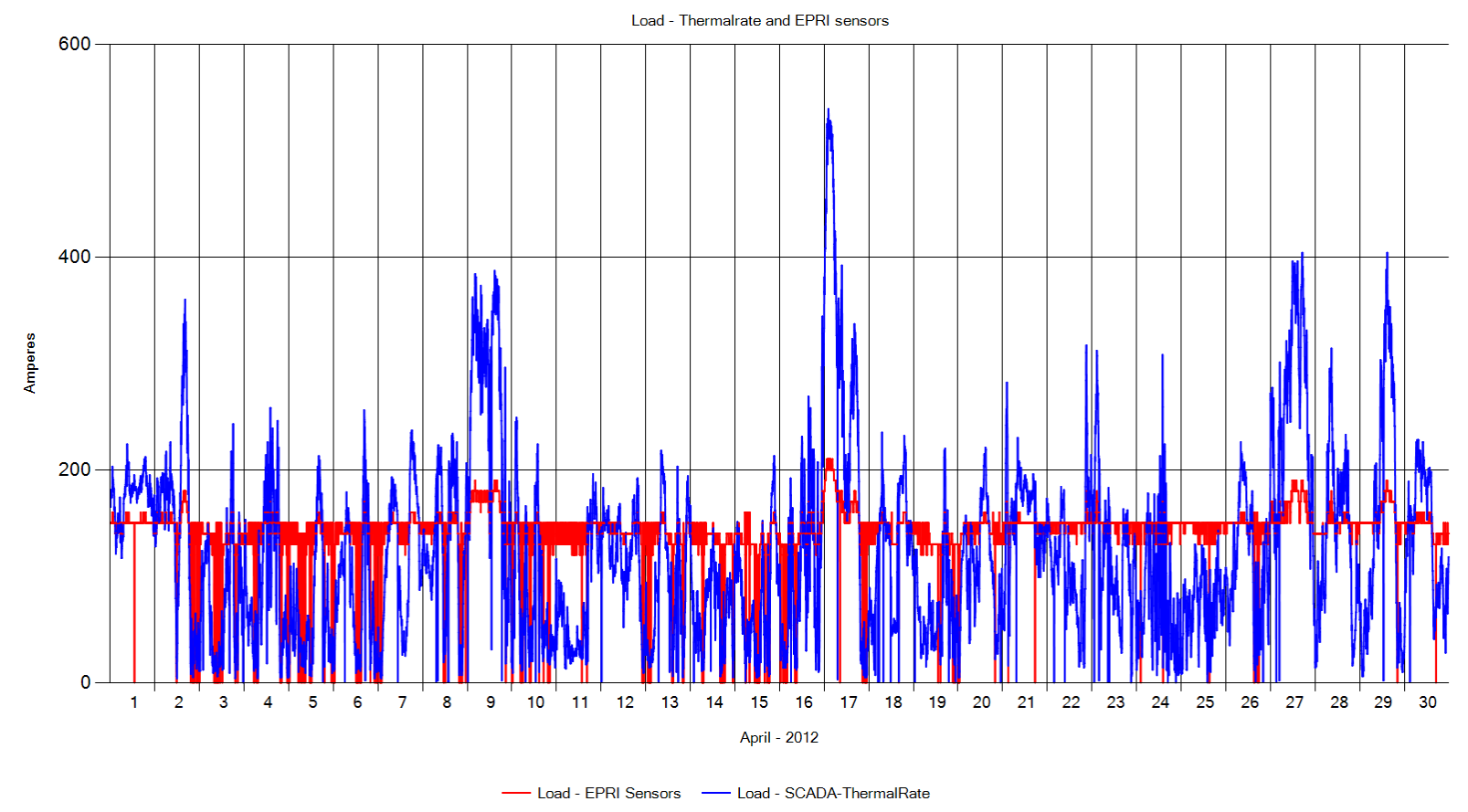

K-4 Element: Site 1_Wx Plot: Load 


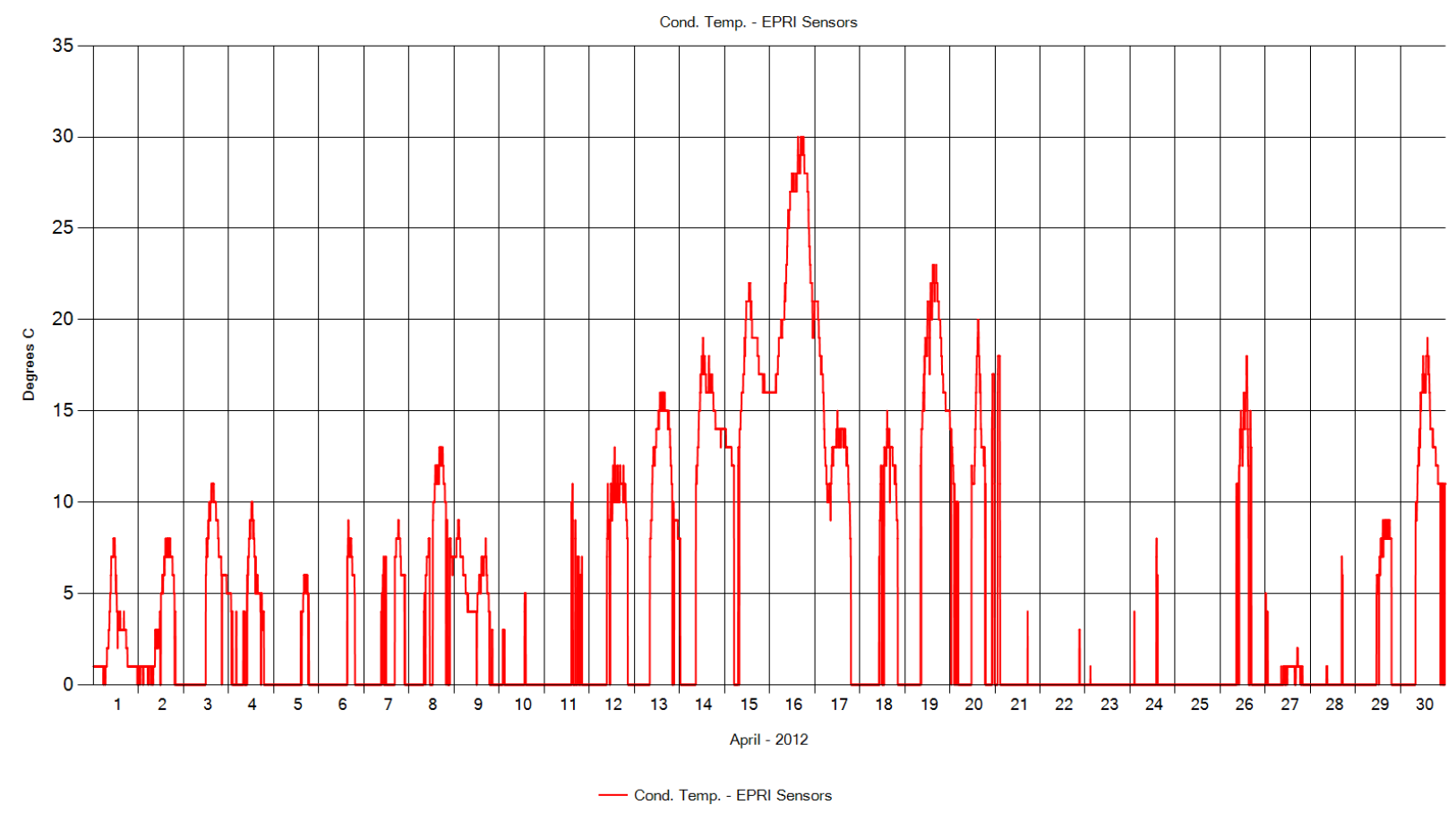

K-5 Element: Site 1_Wx Plot: Cond. Temp. - EPRI Sensors

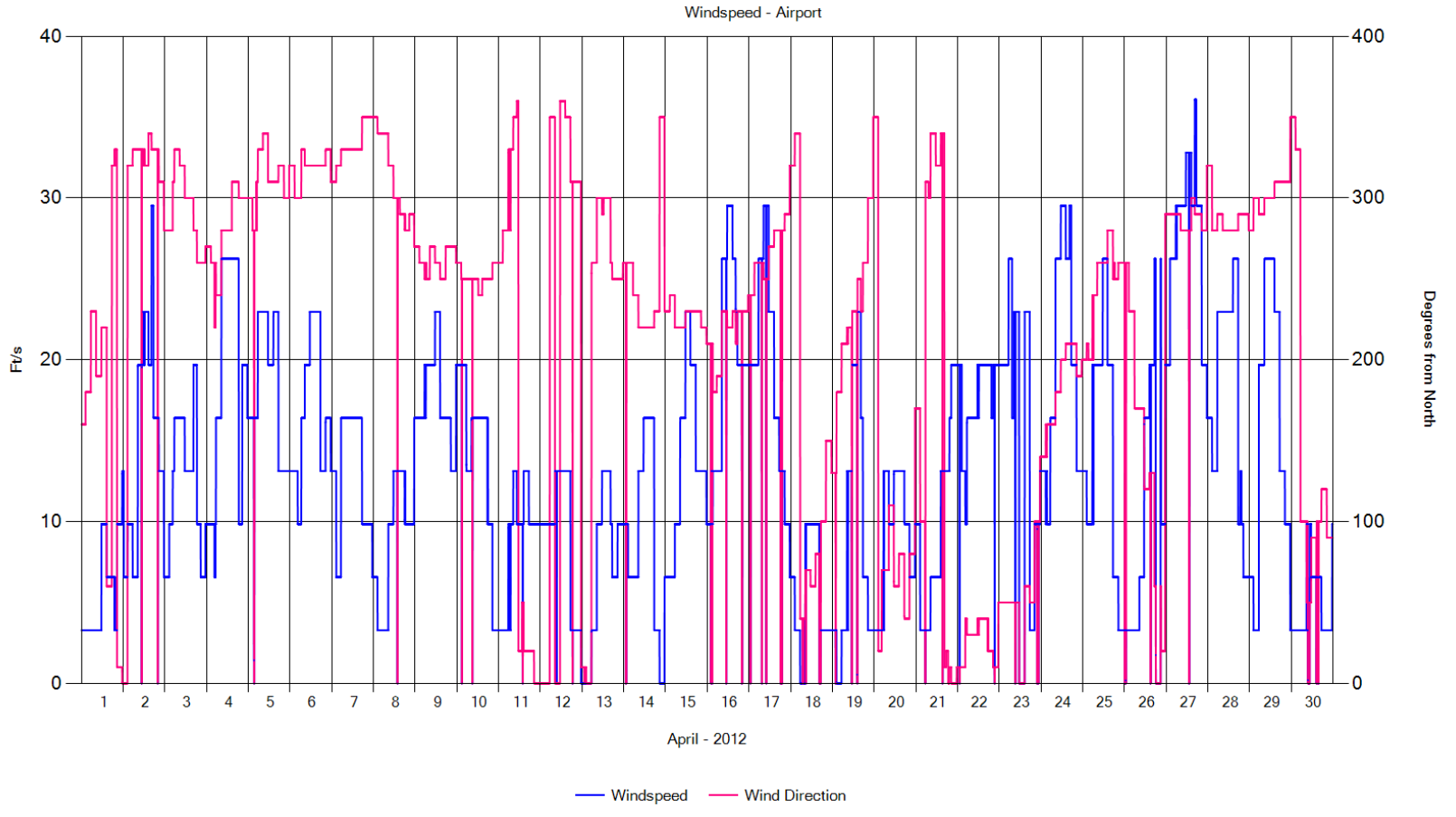

K-6 Element: Site 1_Wx Plot: Wind speed - Airport 


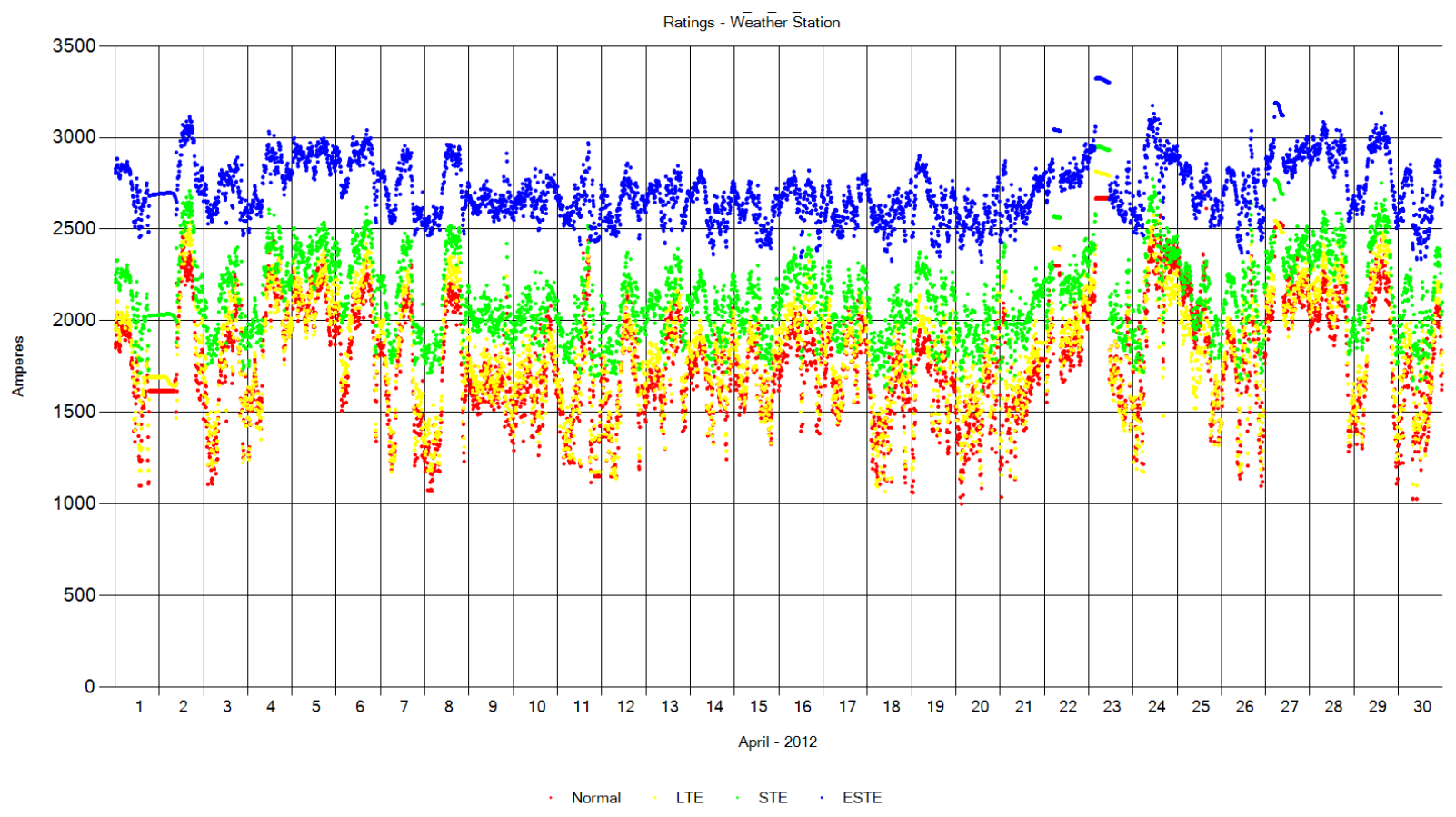

K-7 Element: Site 1_Wx Plot: Rating - WX

Wind Rose - Airport
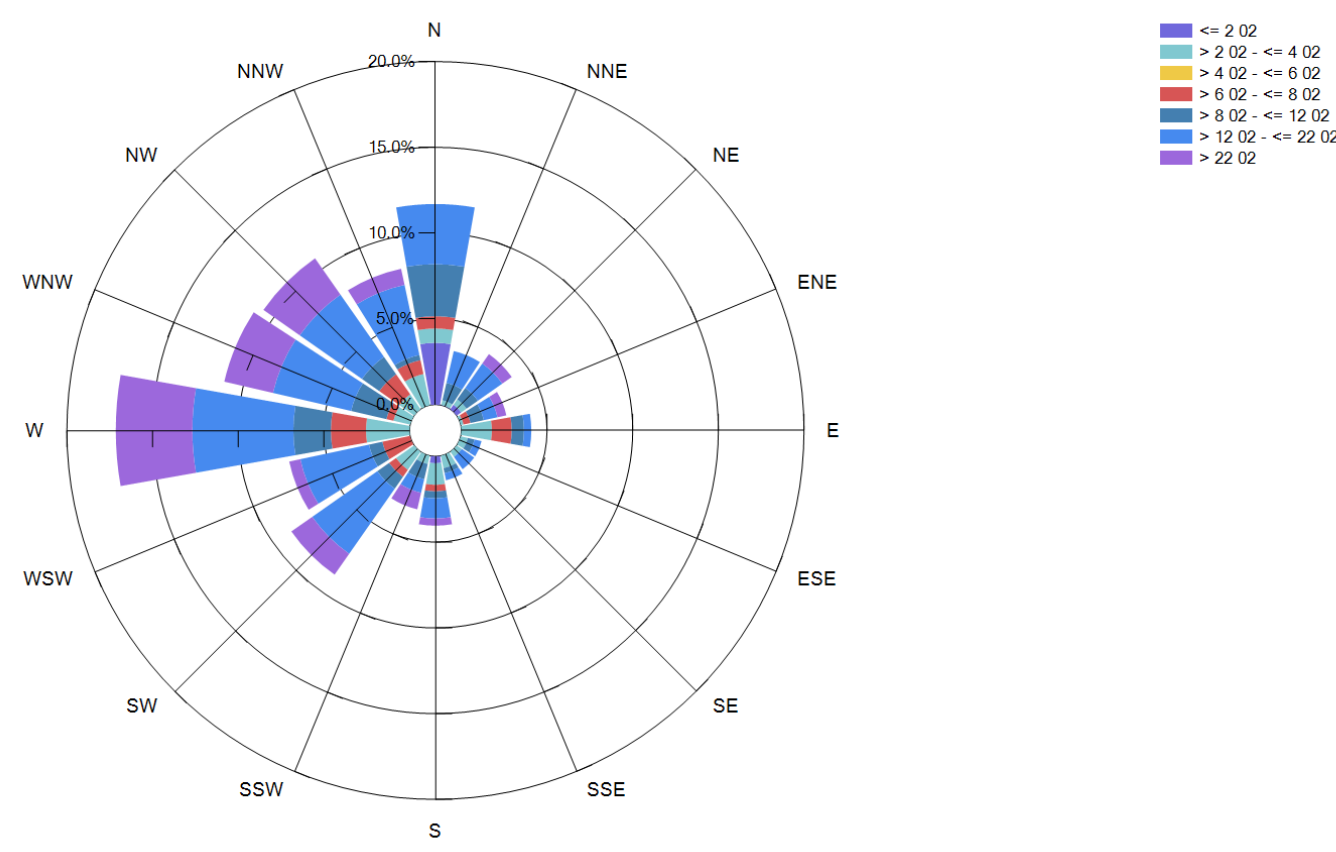

K-8 Element: Site 1_Wx Plot: Wind Rose - Airport 

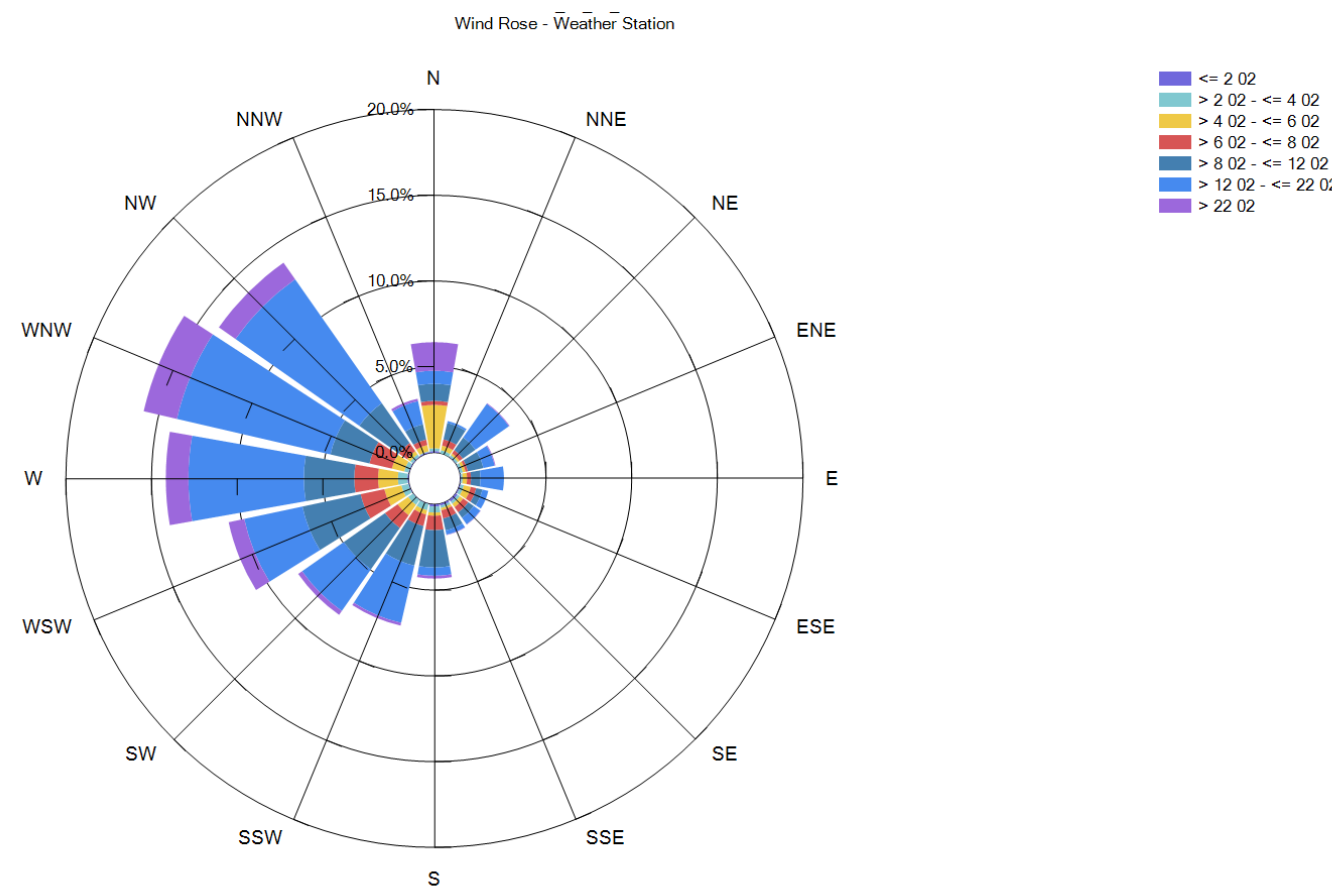

$>1202-<=22$

\section{K-9 Element: Site 1_Wx Plot: Wind Rose-WX}

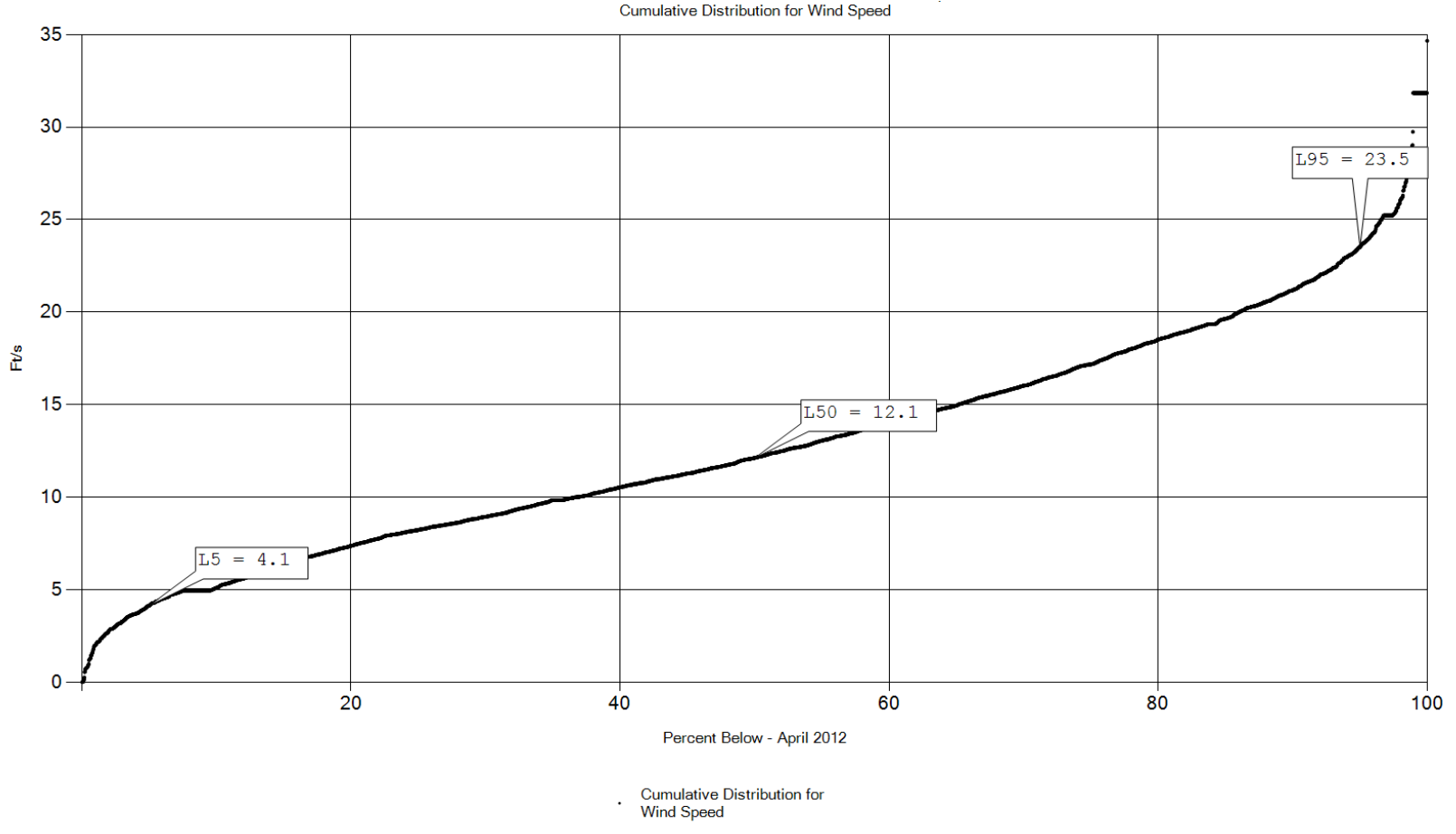

K-10 Element: Site 1_Wx Plot: Cum.Dist. for Wind Speed-WX 


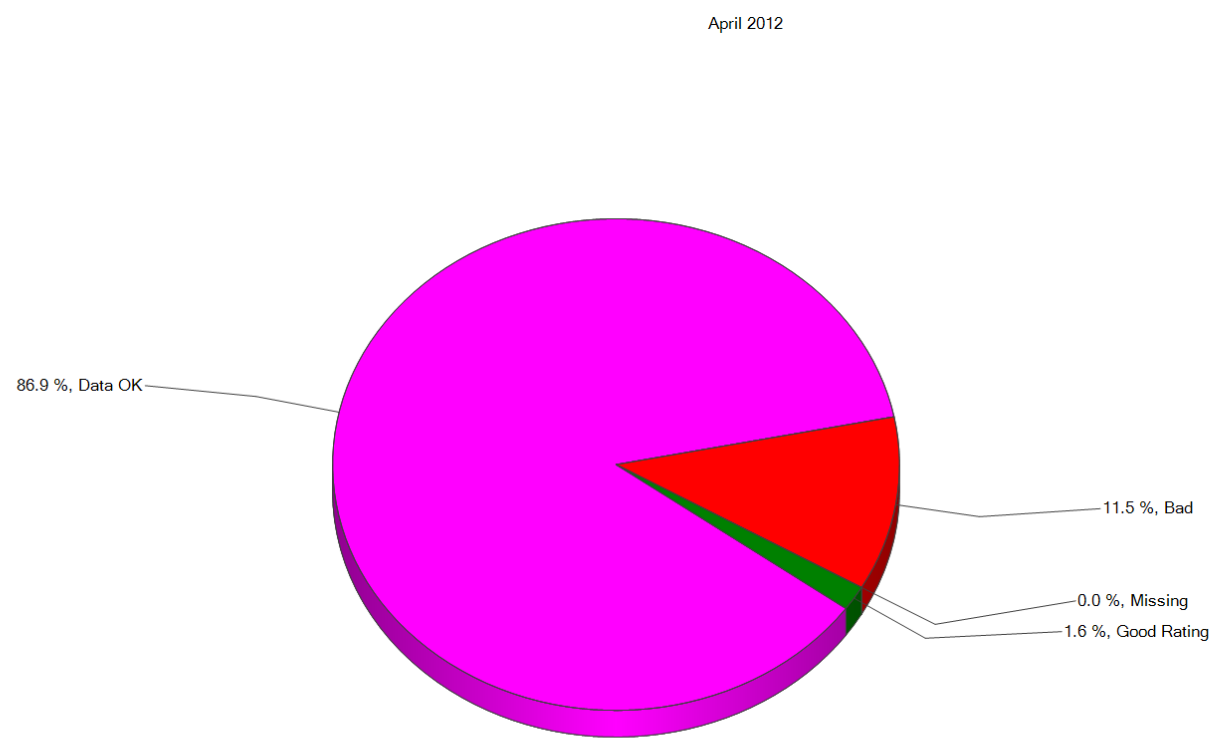

\section{K-11 Element: Site 1_Wx Plot: Data Quality 2}

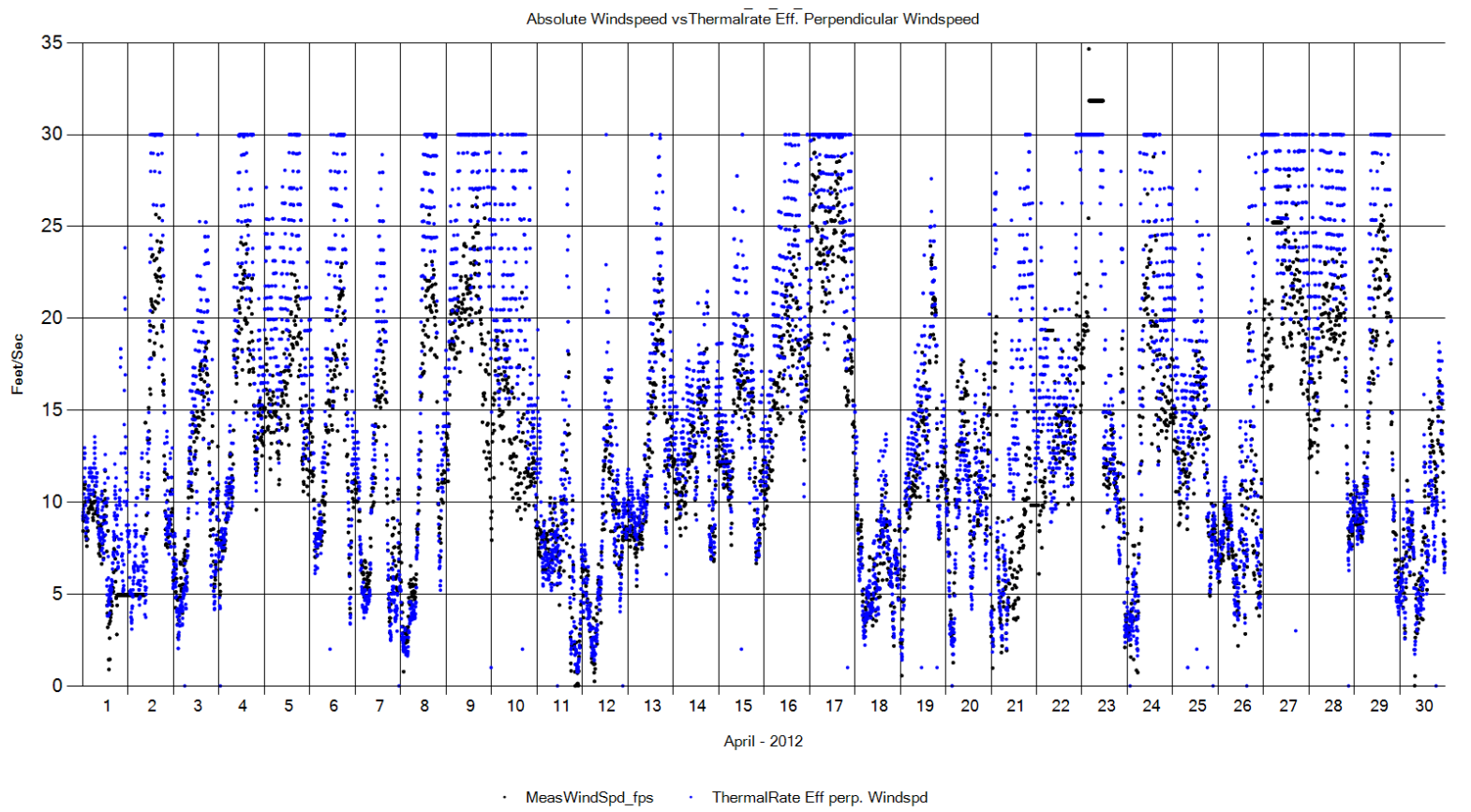

K-12 Element: Site 1_Wx Plot: Ws vs. eff Perp WS 


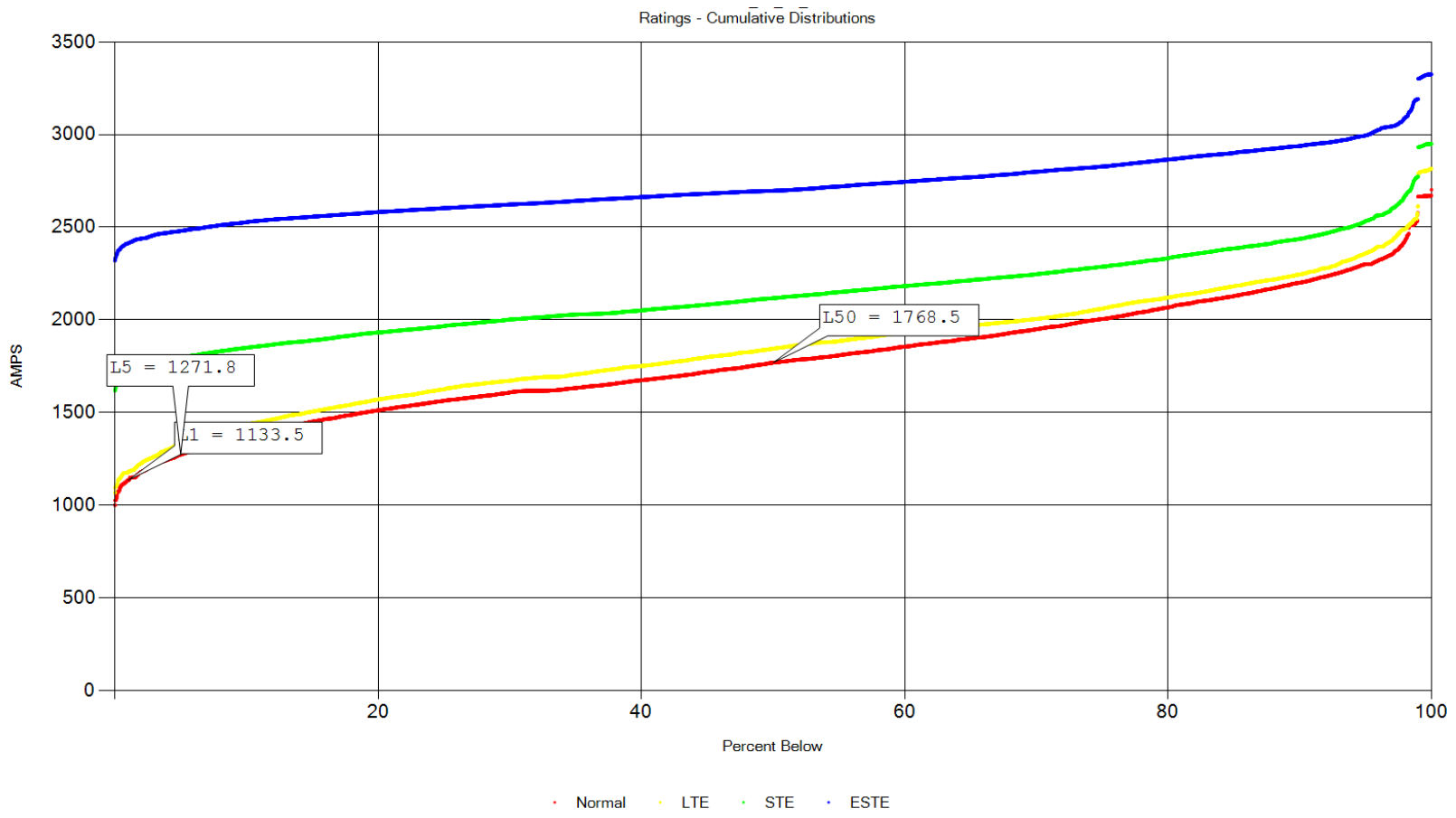

K-13 Element: Site 1_Wx Plot: Rating Cum. Dist.

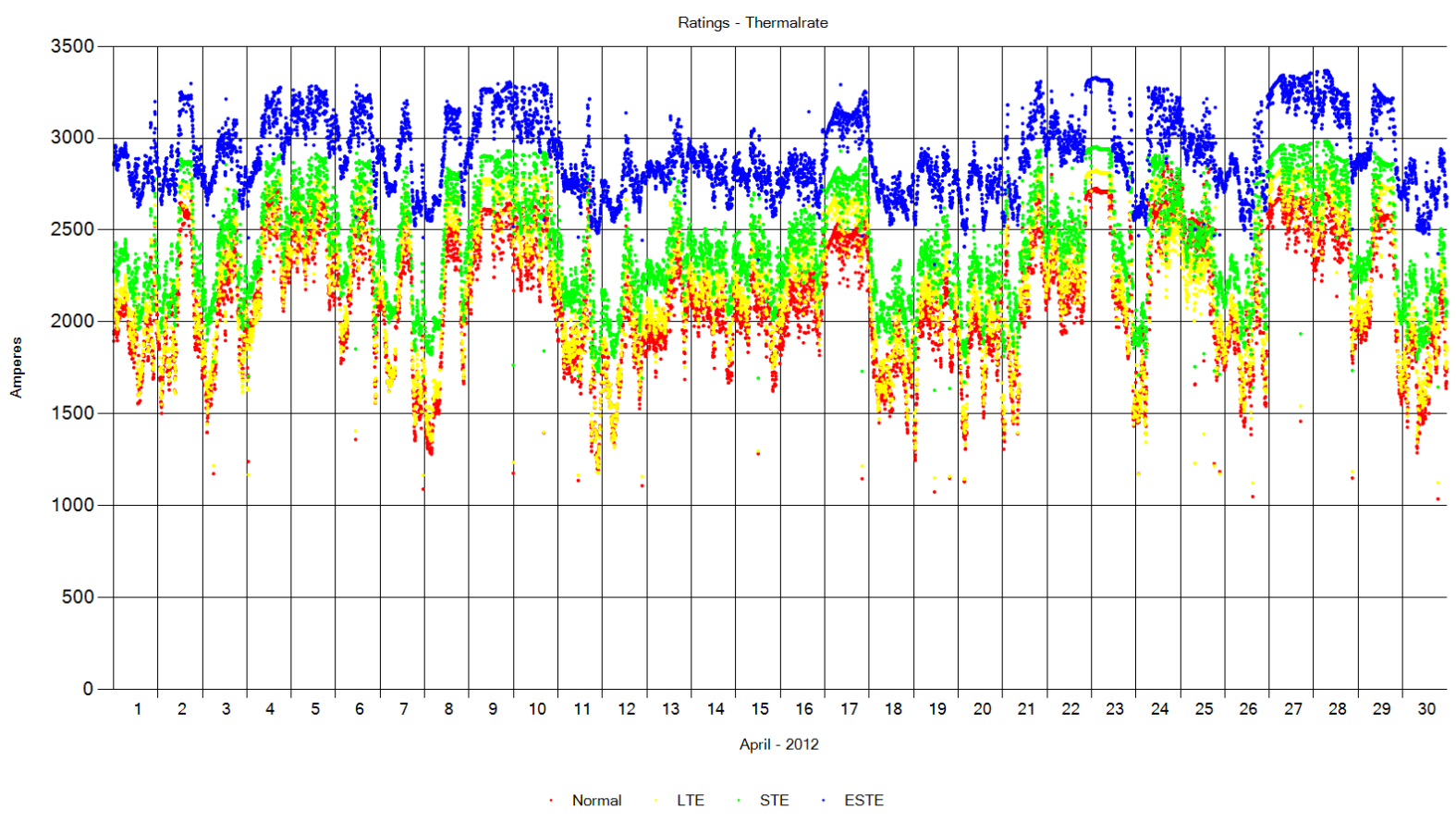

K-14 Element: Site 1_TR Plot: Ratings-ThermalRate 


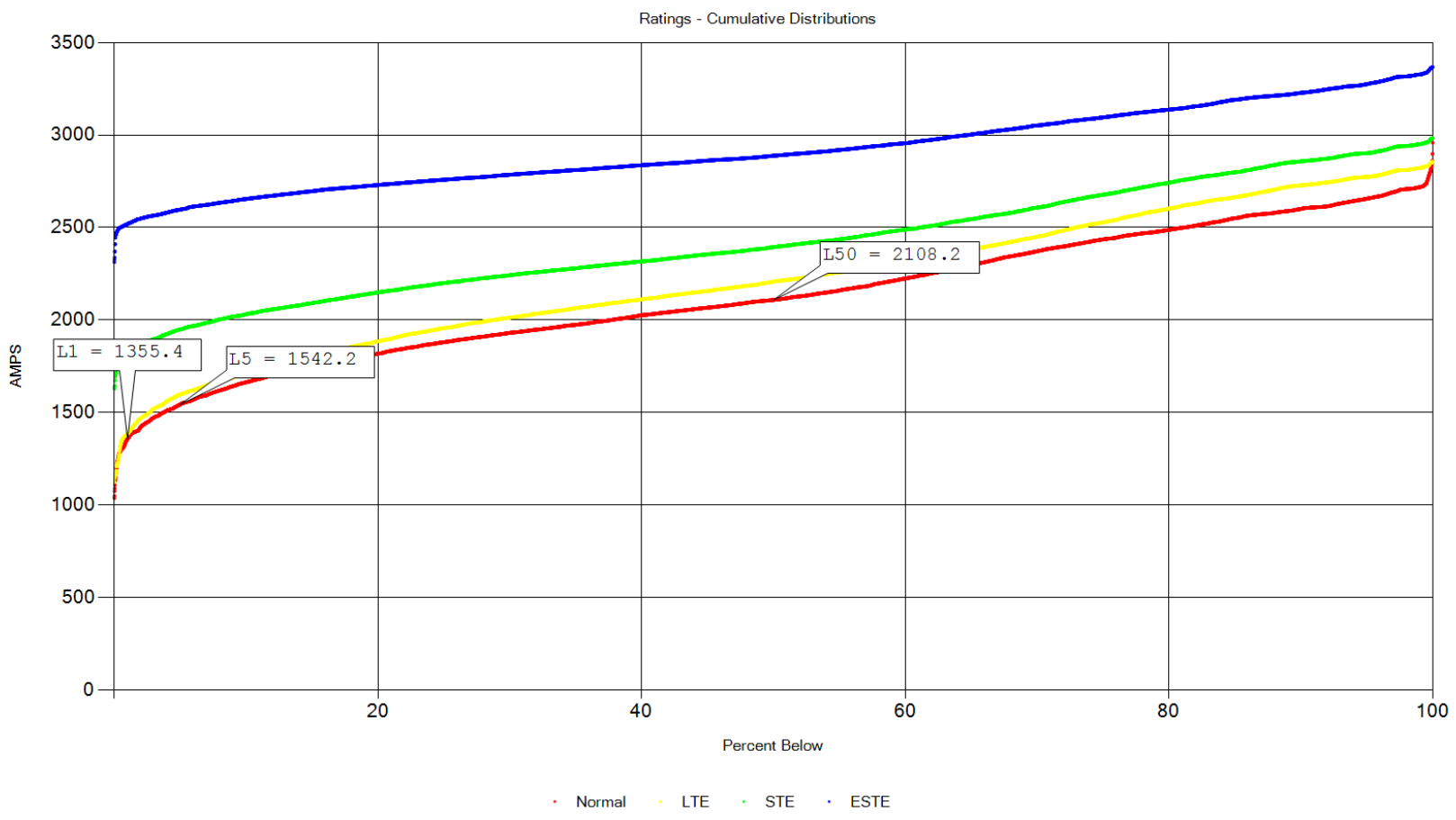

K-15 Element: Site 1_TR Plot: Rating Cum. Dist.

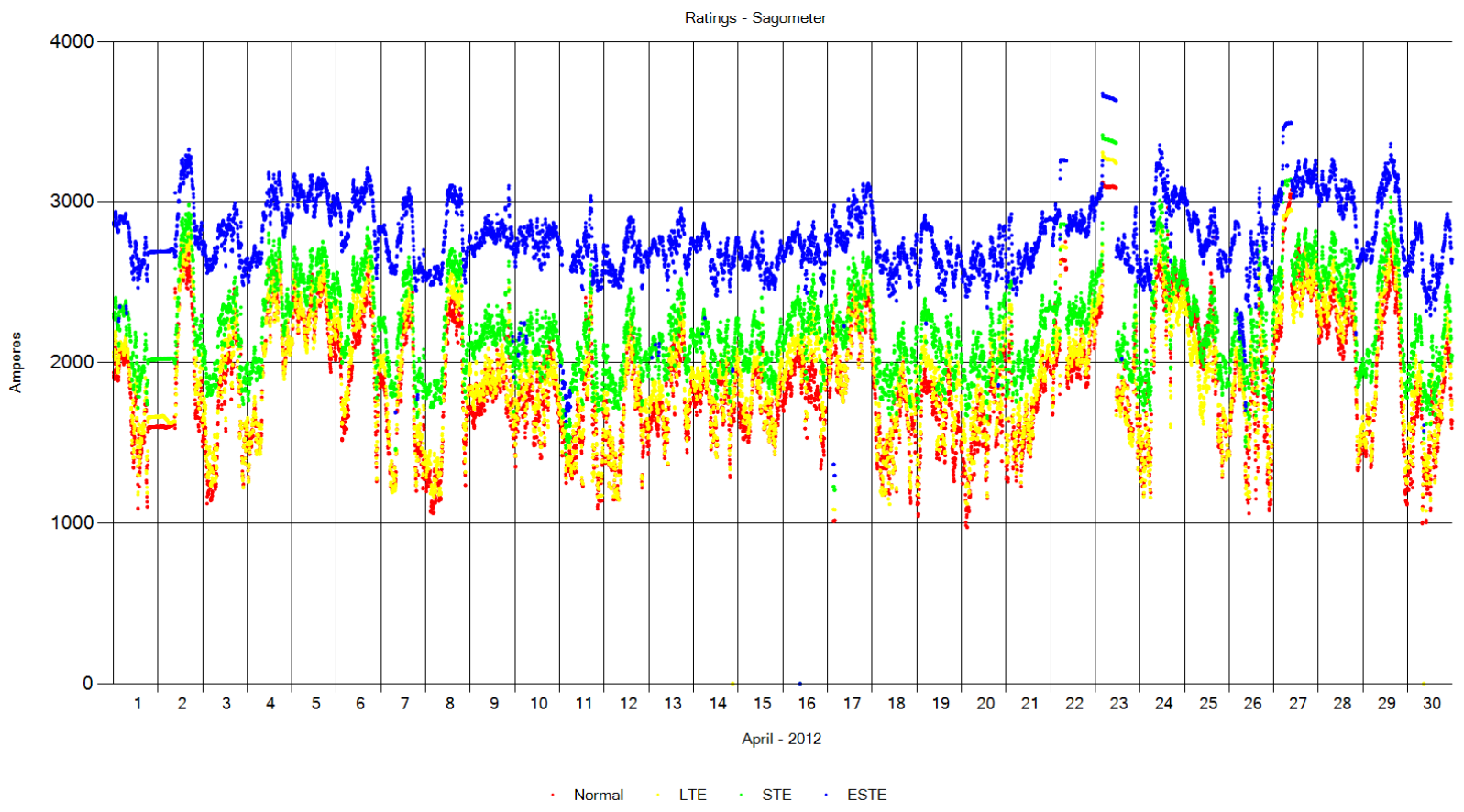

K-16 Element: Site 1_Sag Plot: Ratings-Sagometer 


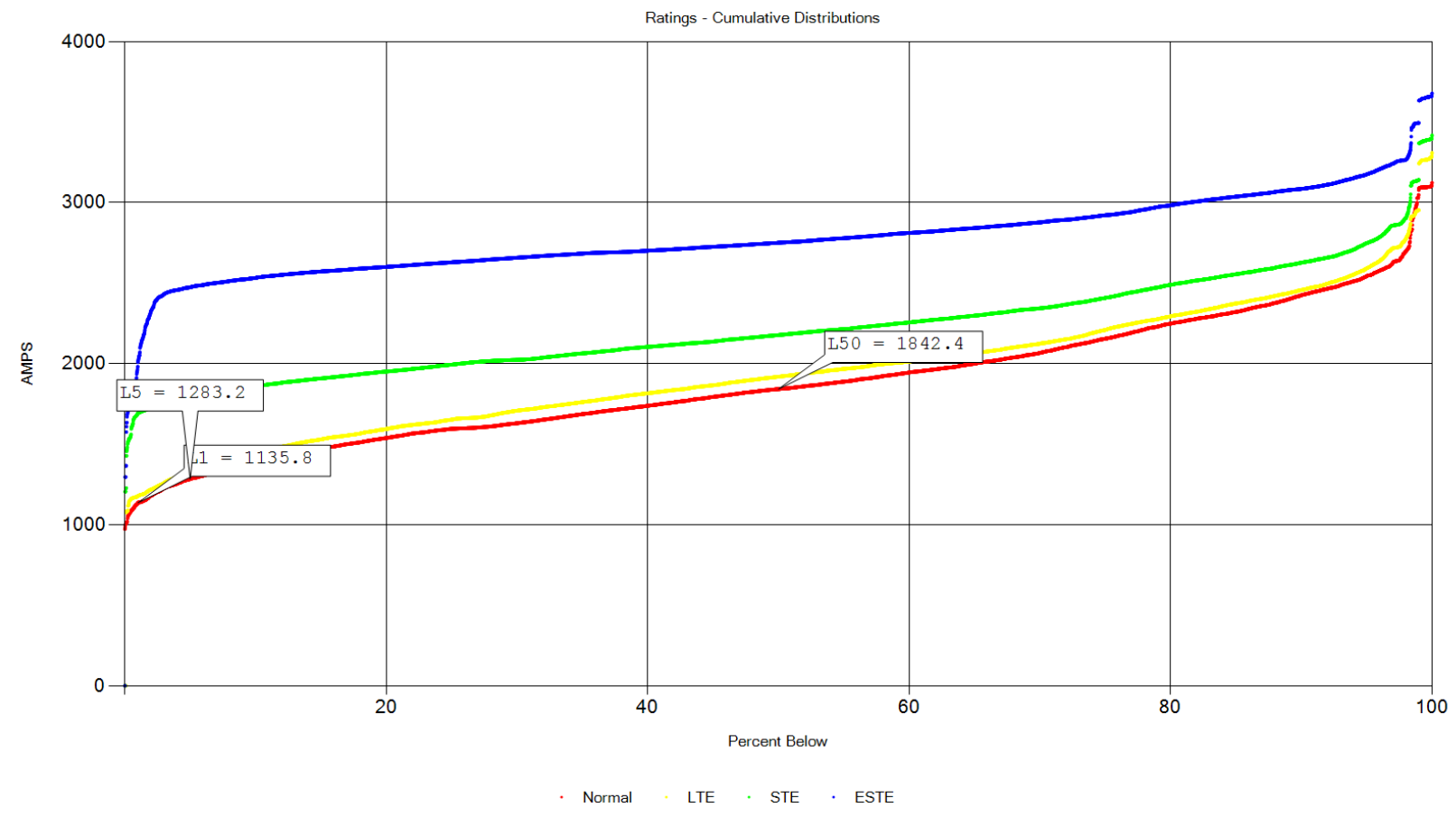

K-17 Element: Site 1_Sag Plot: Rating Cum. Dist.

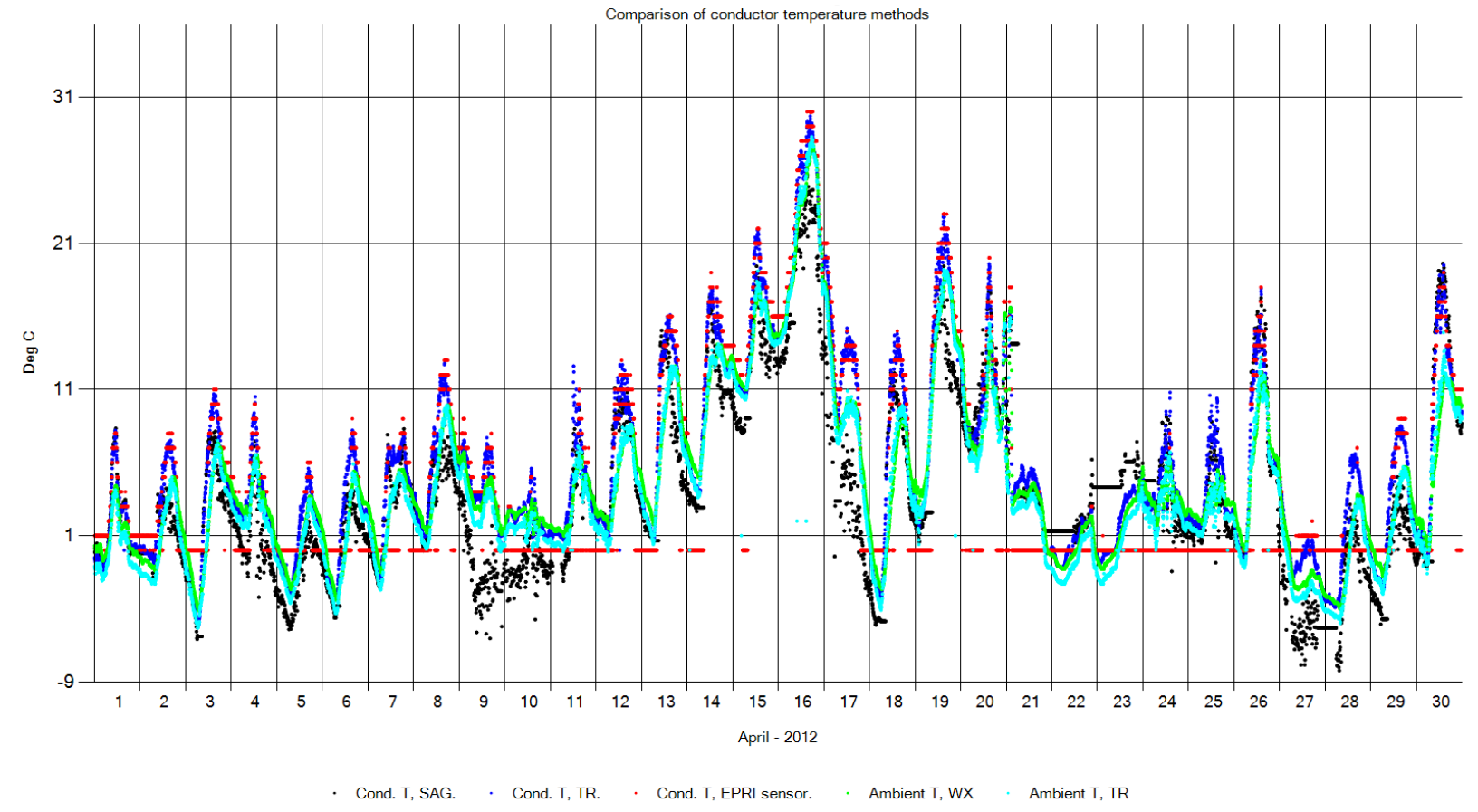

K-18 Element: Site 1_Sag Plot: Conductor Temp Compare

K-10 


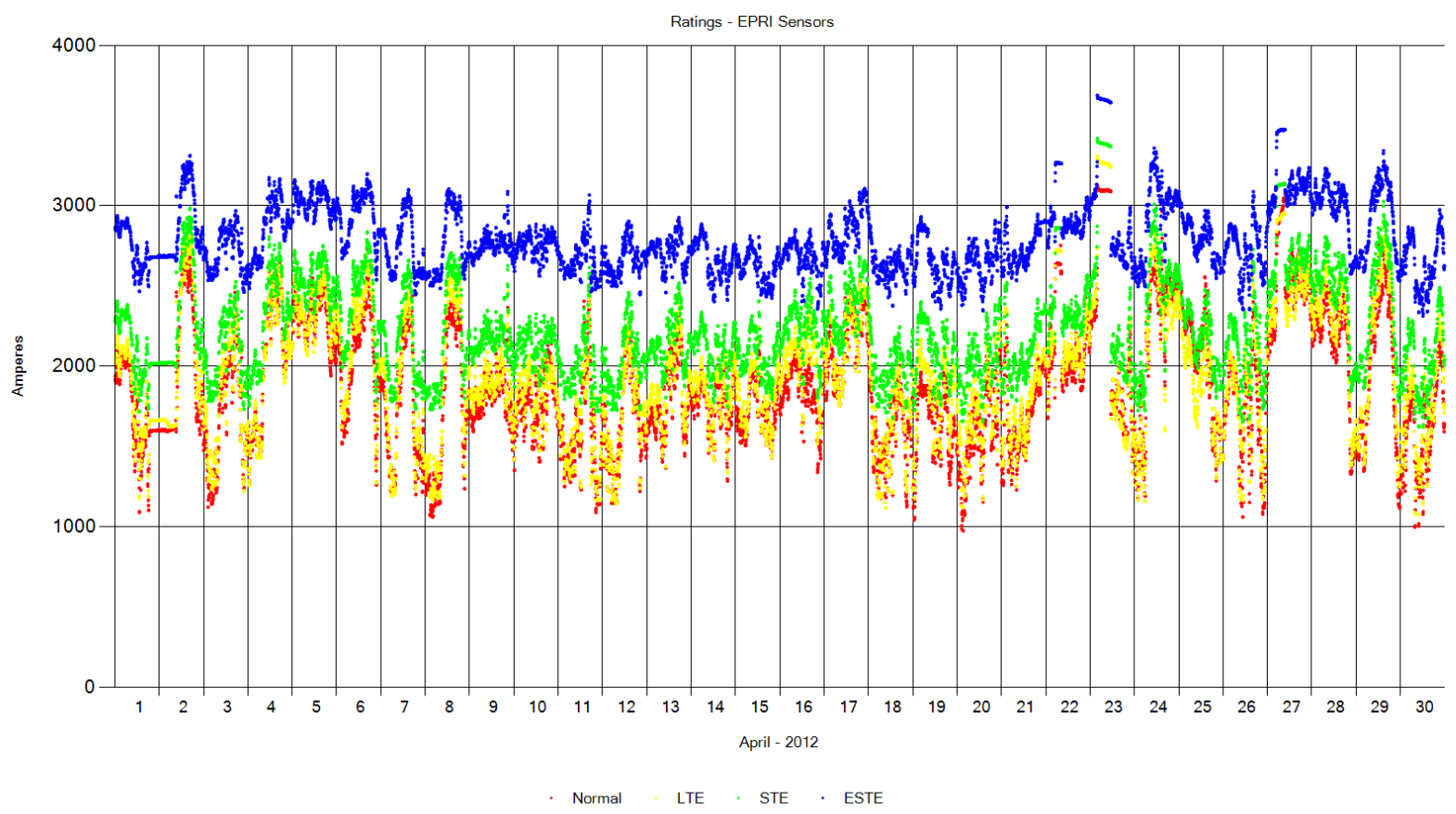

K-19 Element: Site 1_BS Plot: Ratings-EPRI Sensors

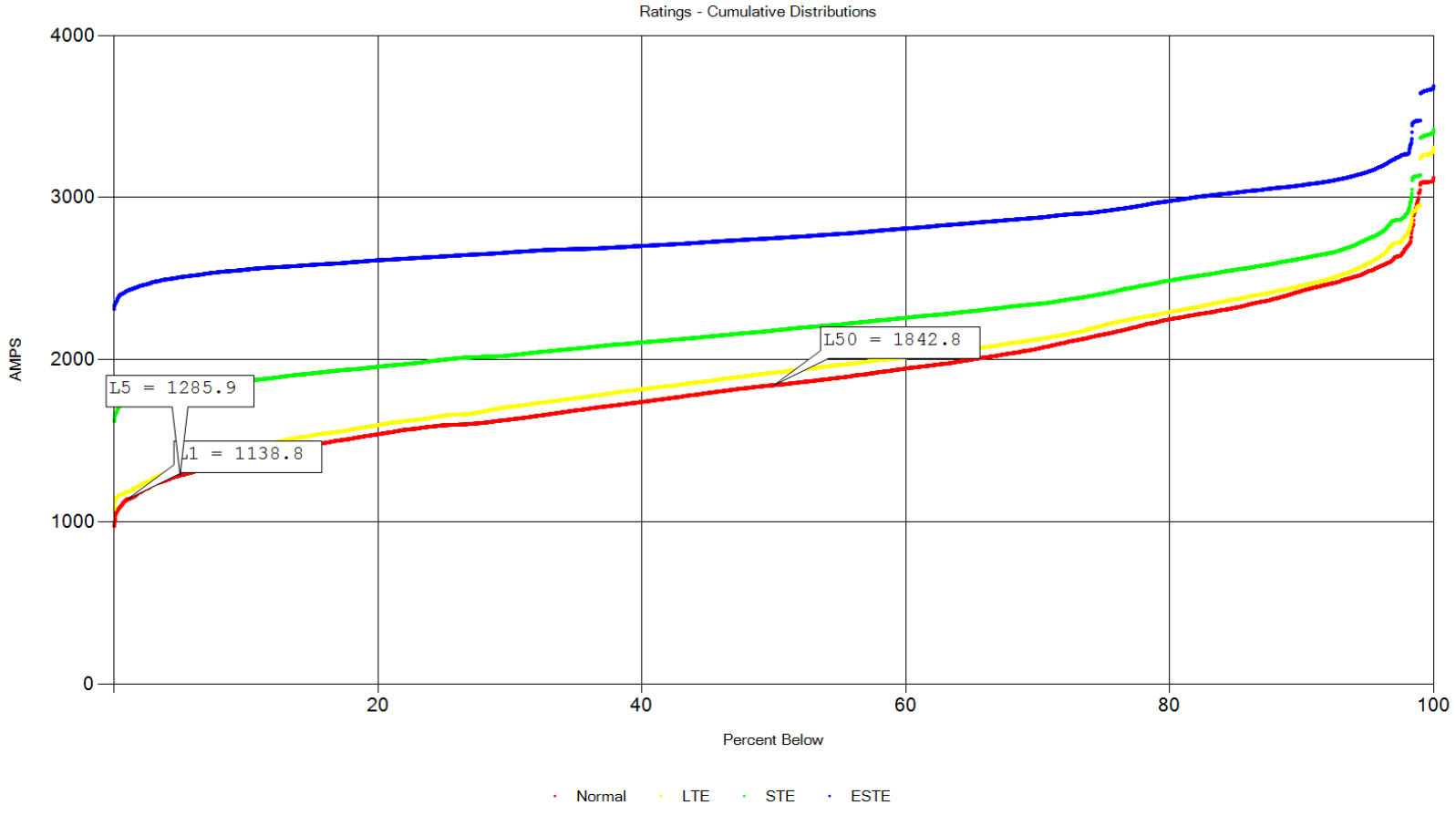

K-20 Element: Site 1_BS Plot: Rating Cum. Dist. 


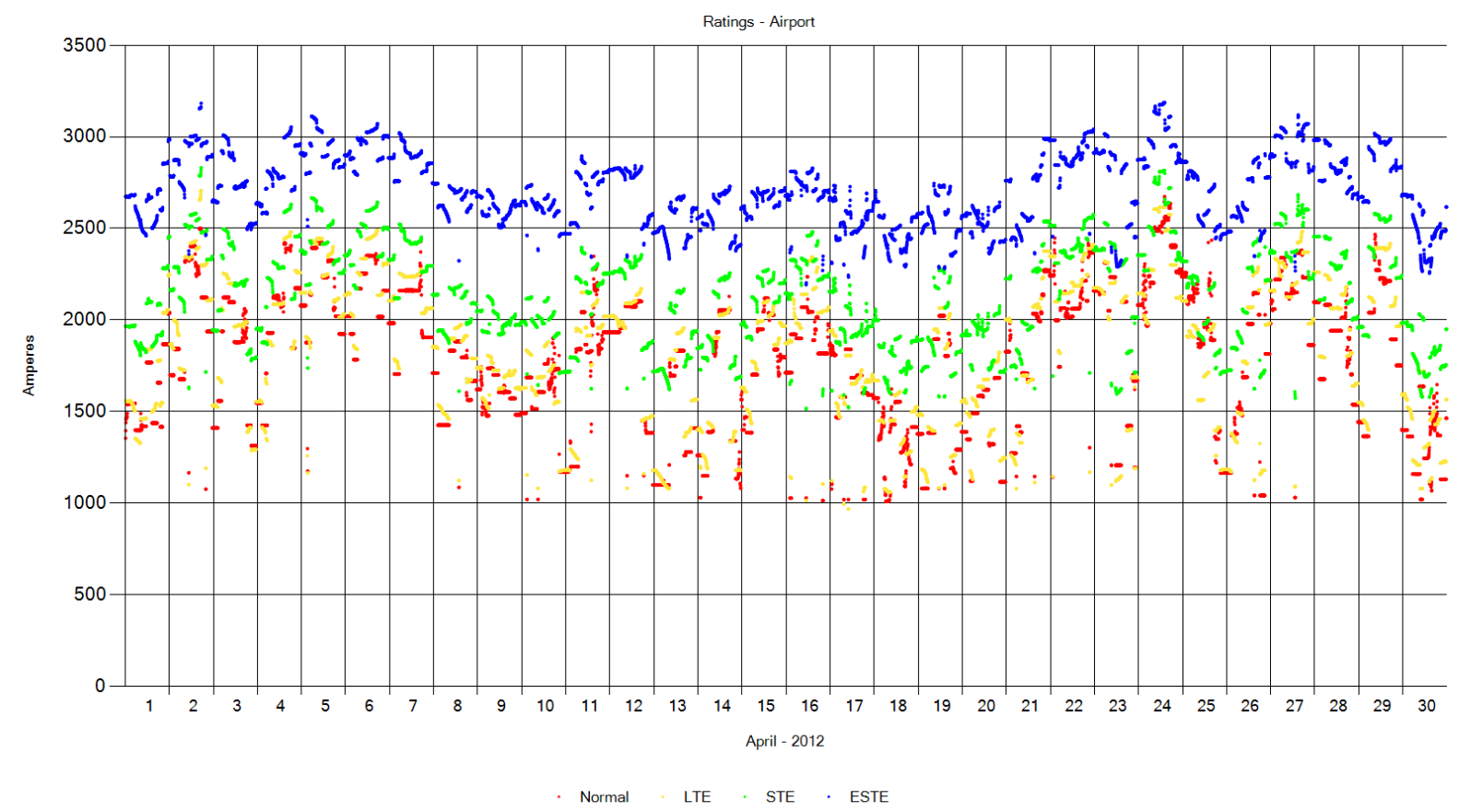

K-21 Element: Site 1_AP Plot: Ratings-Airport

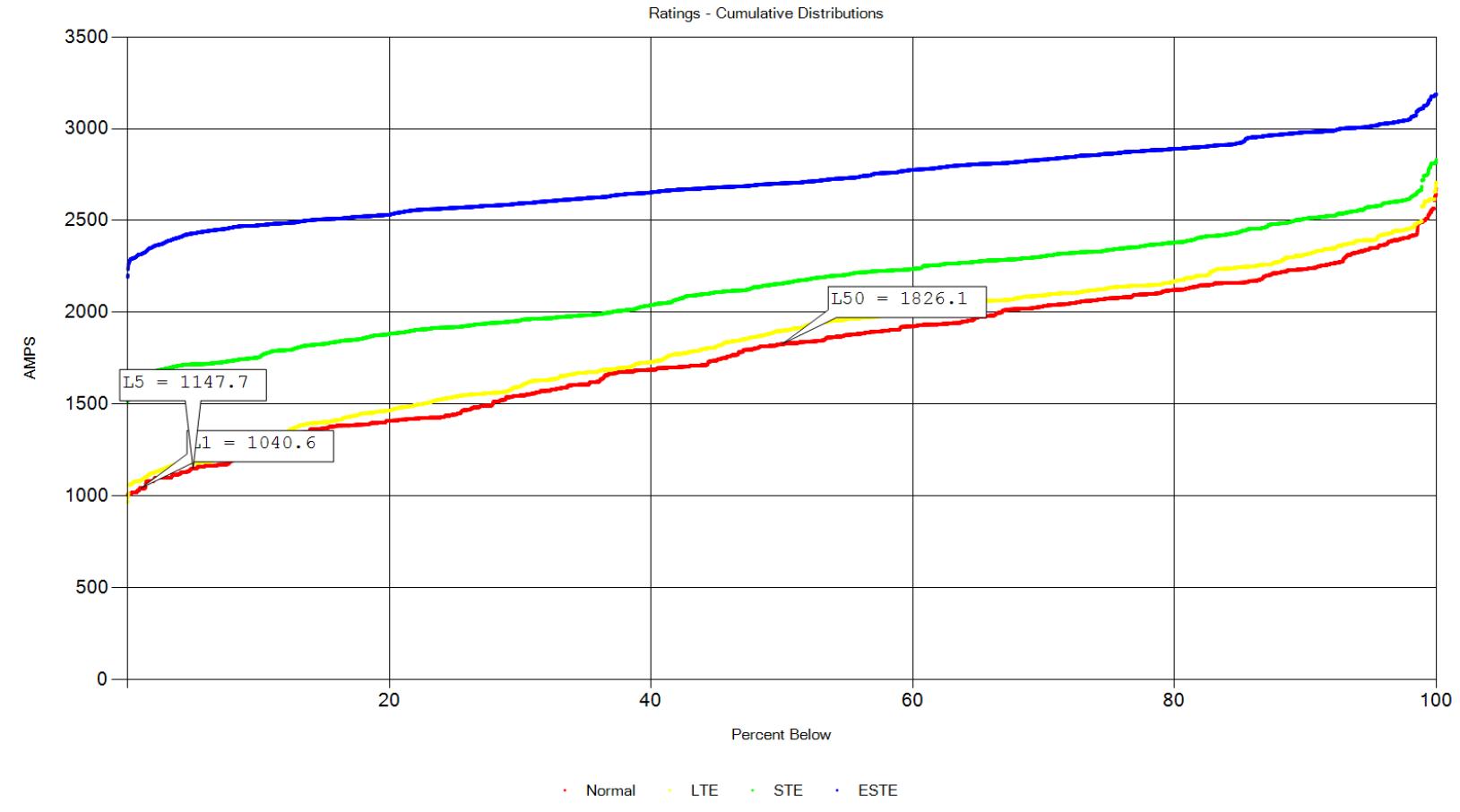

K-22 Element: Site 1_AP Plot: Rating Cum. Dist. 


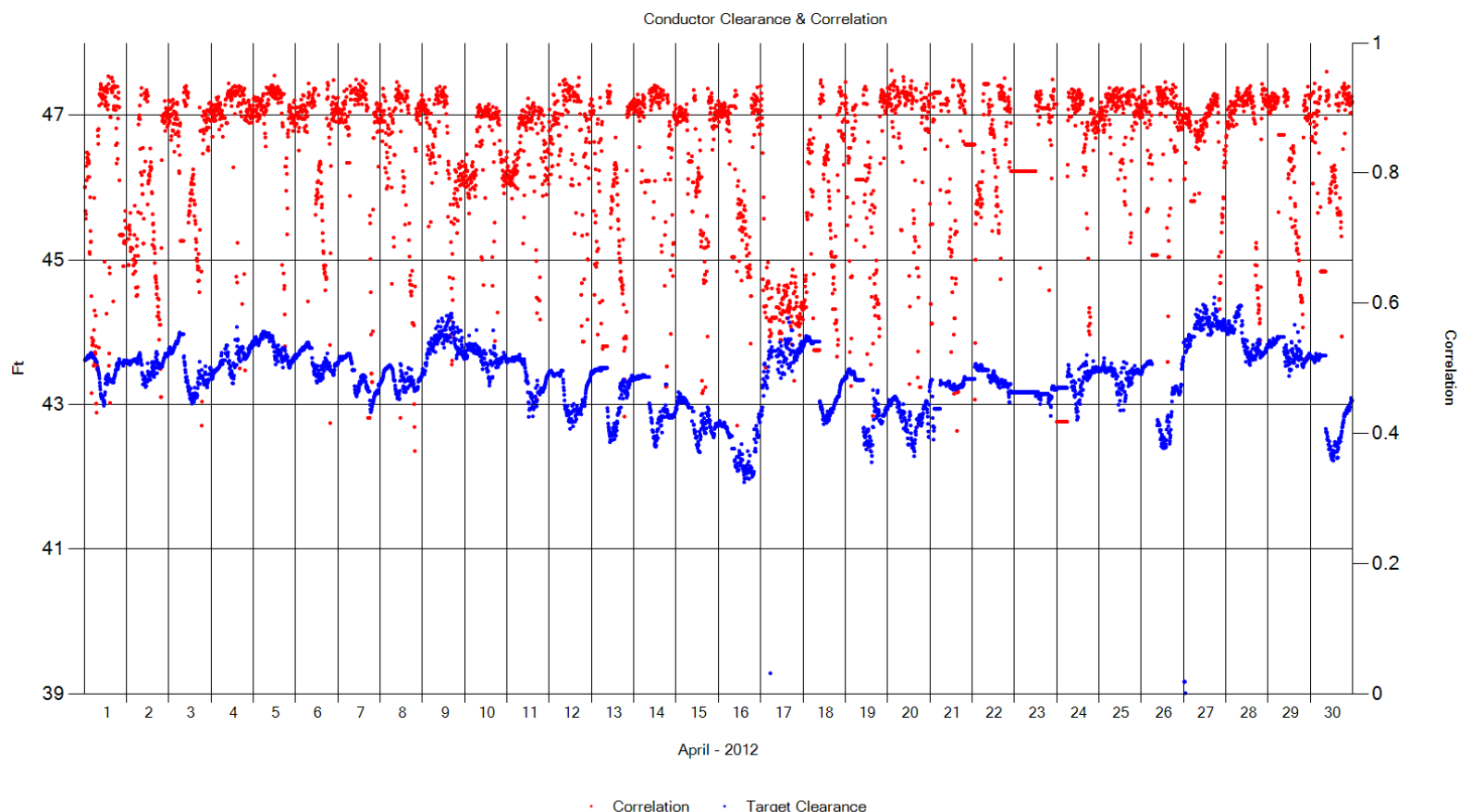

K-23 Element: Site 2_Wx Plot: Conductor Clearance \& Correlation

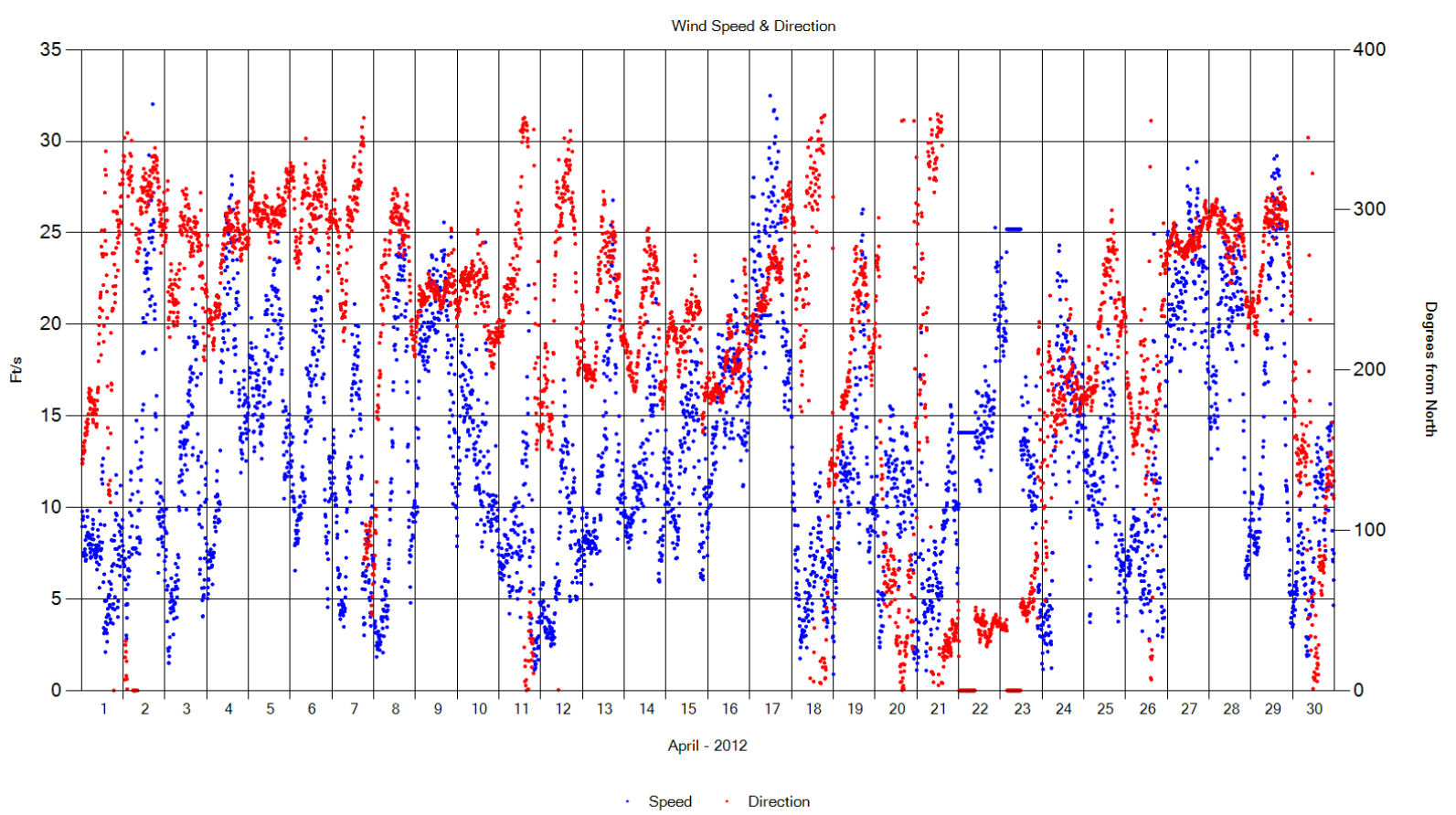

K-24 Element: Site 2_Wx Plot: Wind Speed \& Direction 


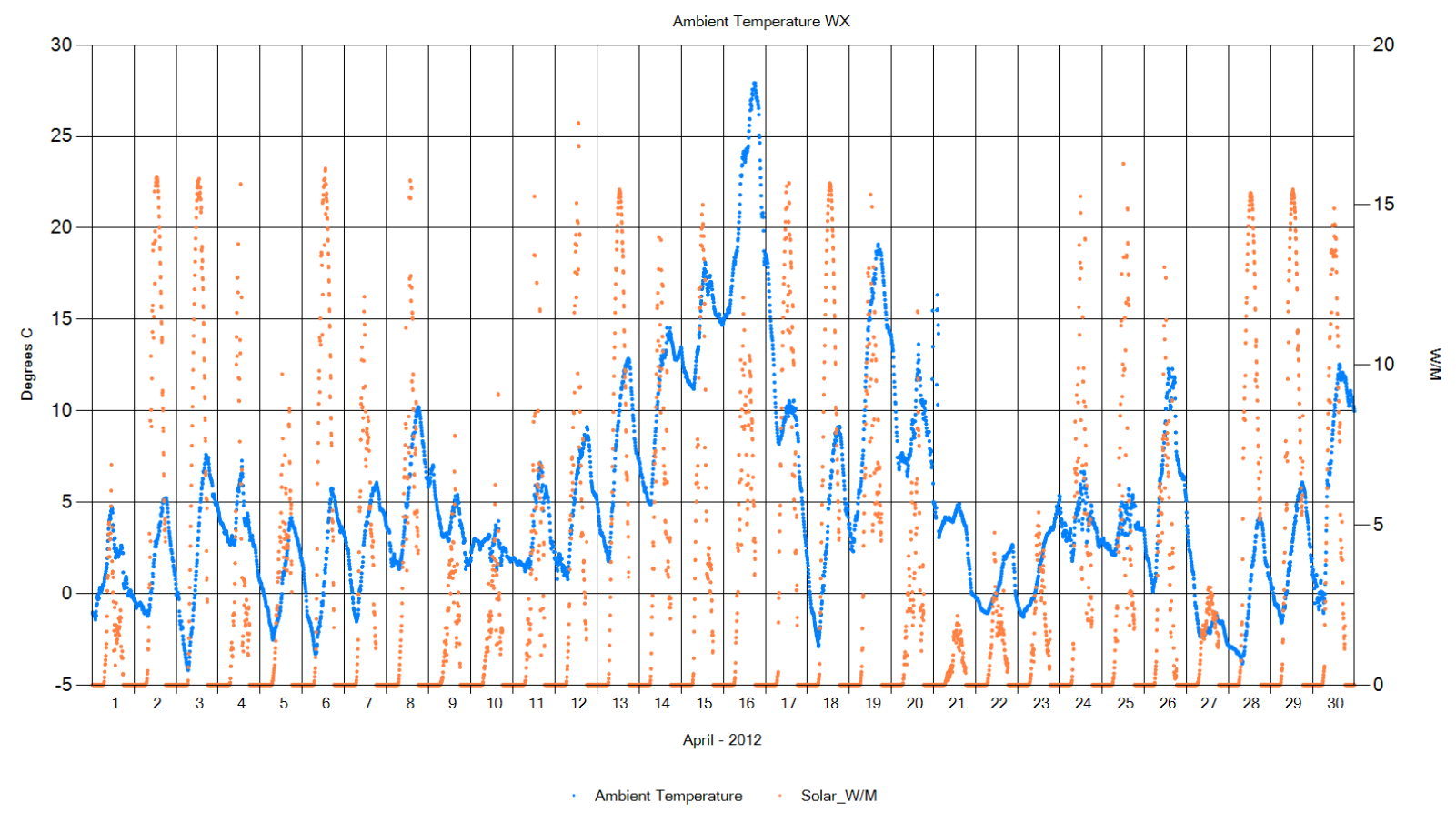

K-25 Element: Site 2_Wx Plot: Ambient Temperature WX

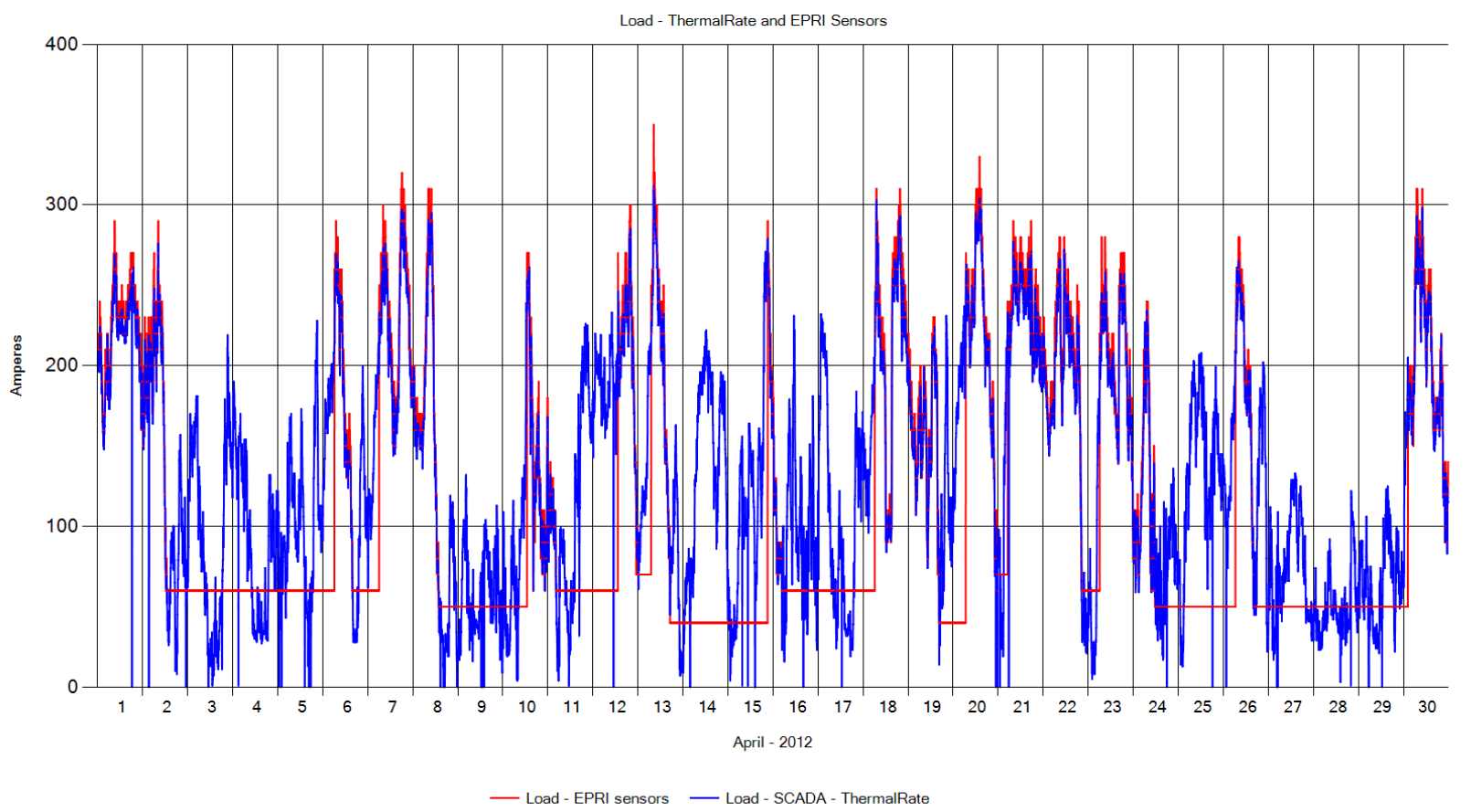

K-26 Element: Site 2_Wx Plot: Load 


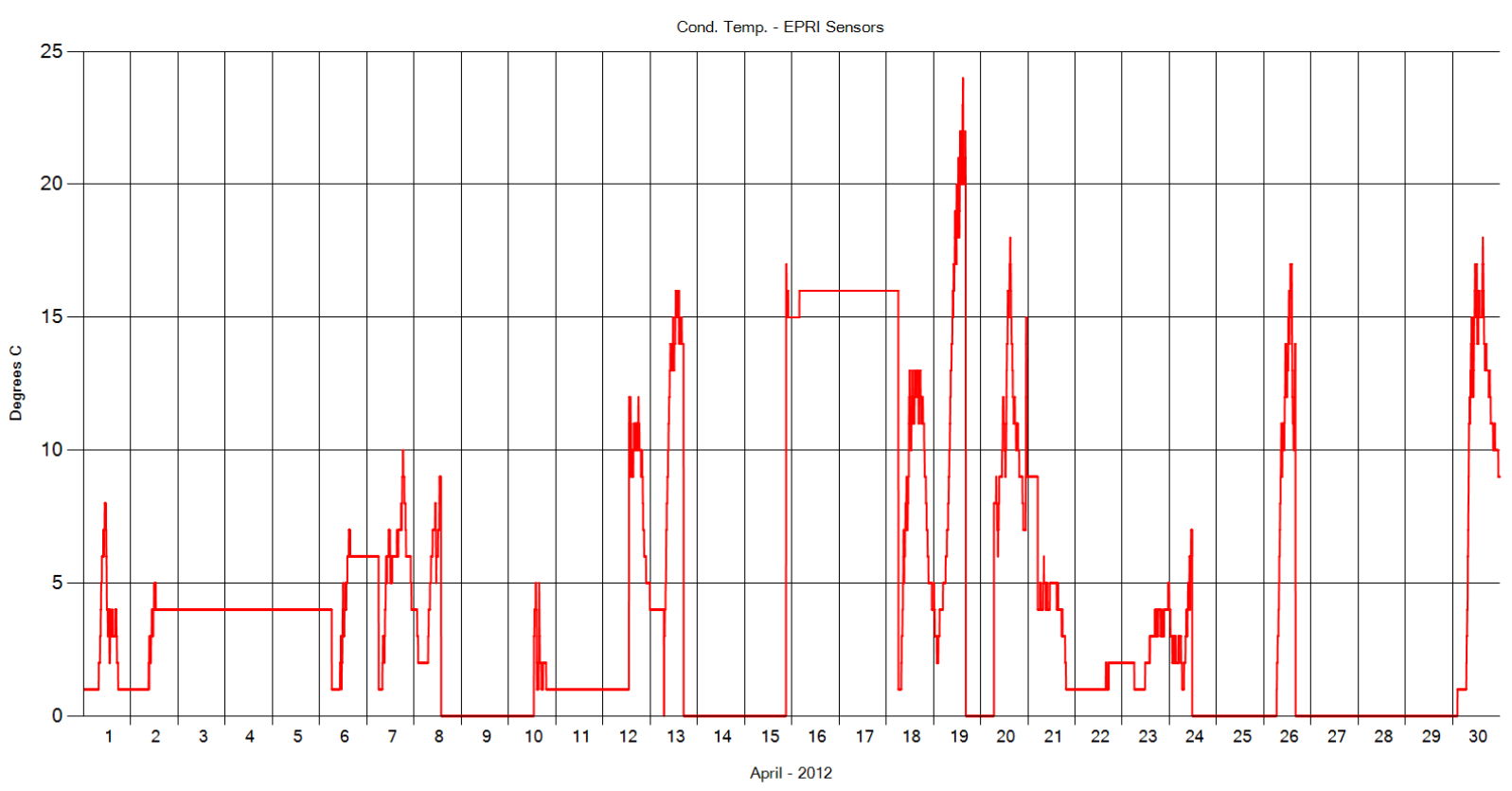

— Cond. Temp. - EPRI Sensors

K-27 Element: Site 2_Wx Plot: Cond. Temp. - EPRI Sensors

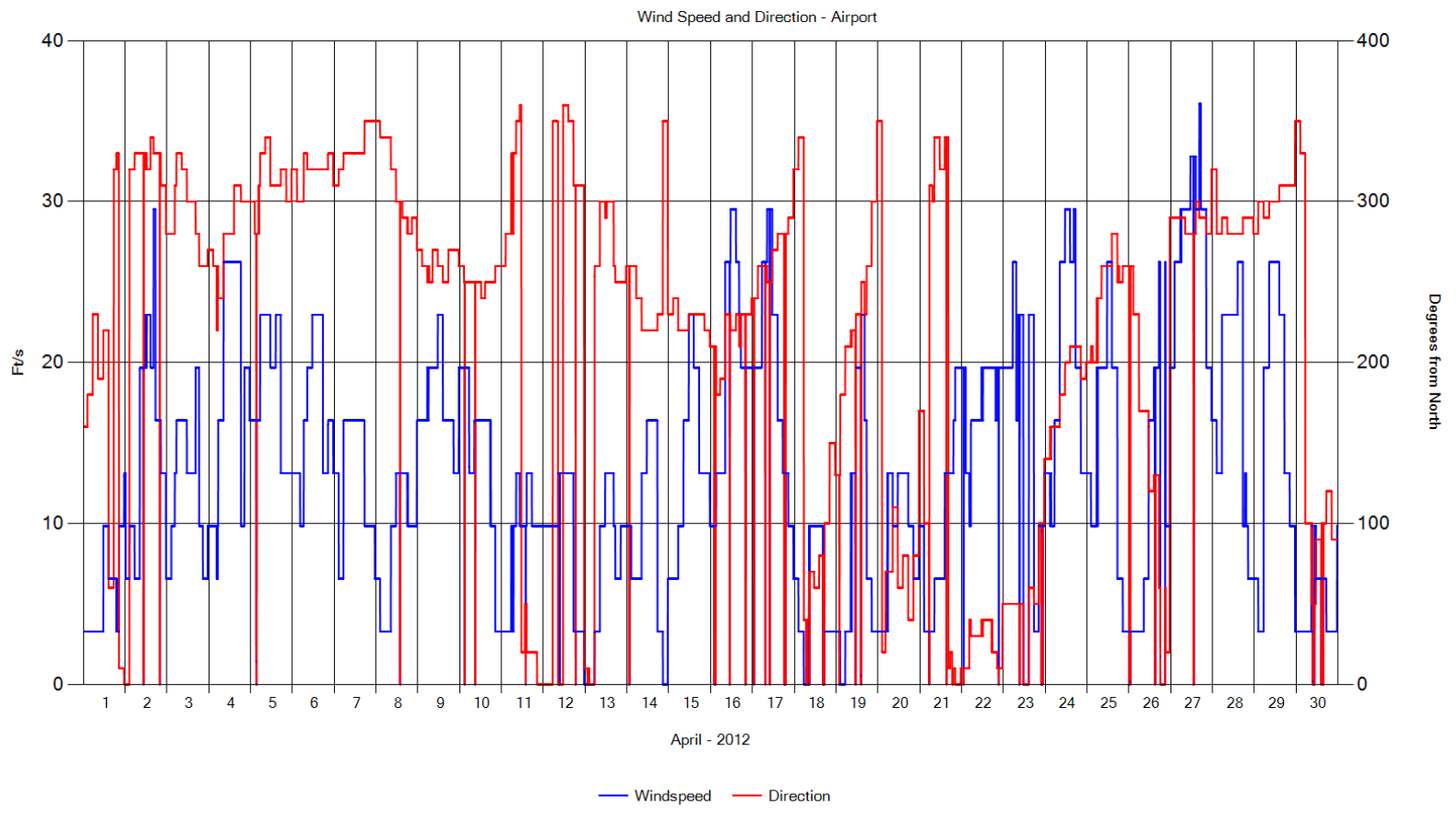

K-28 Element: Site 2_Wx Plot: Wind Speed and Direction - Airport 


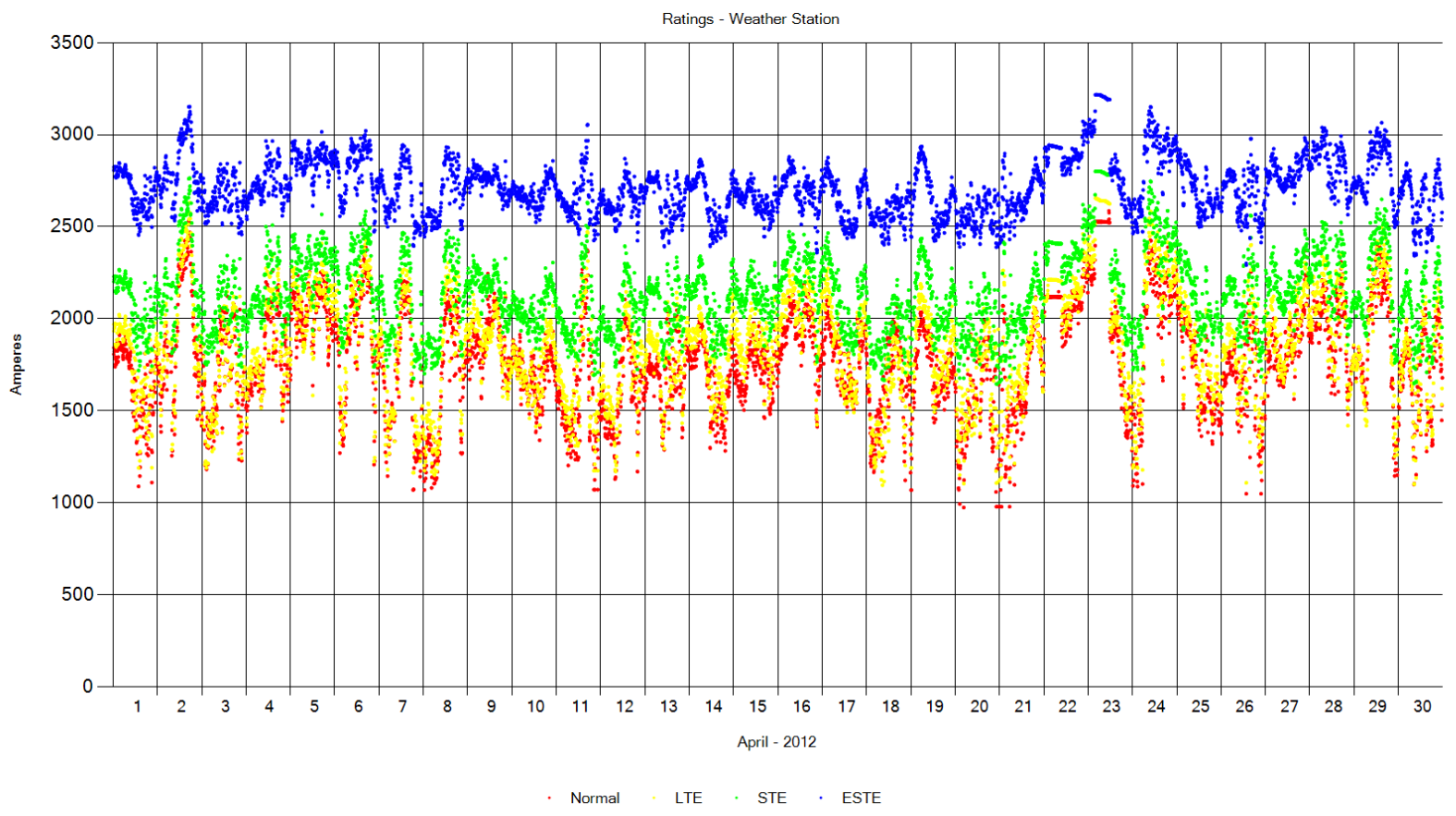

K-29 Element: Site 2_Wx Plot: Ratings-WX
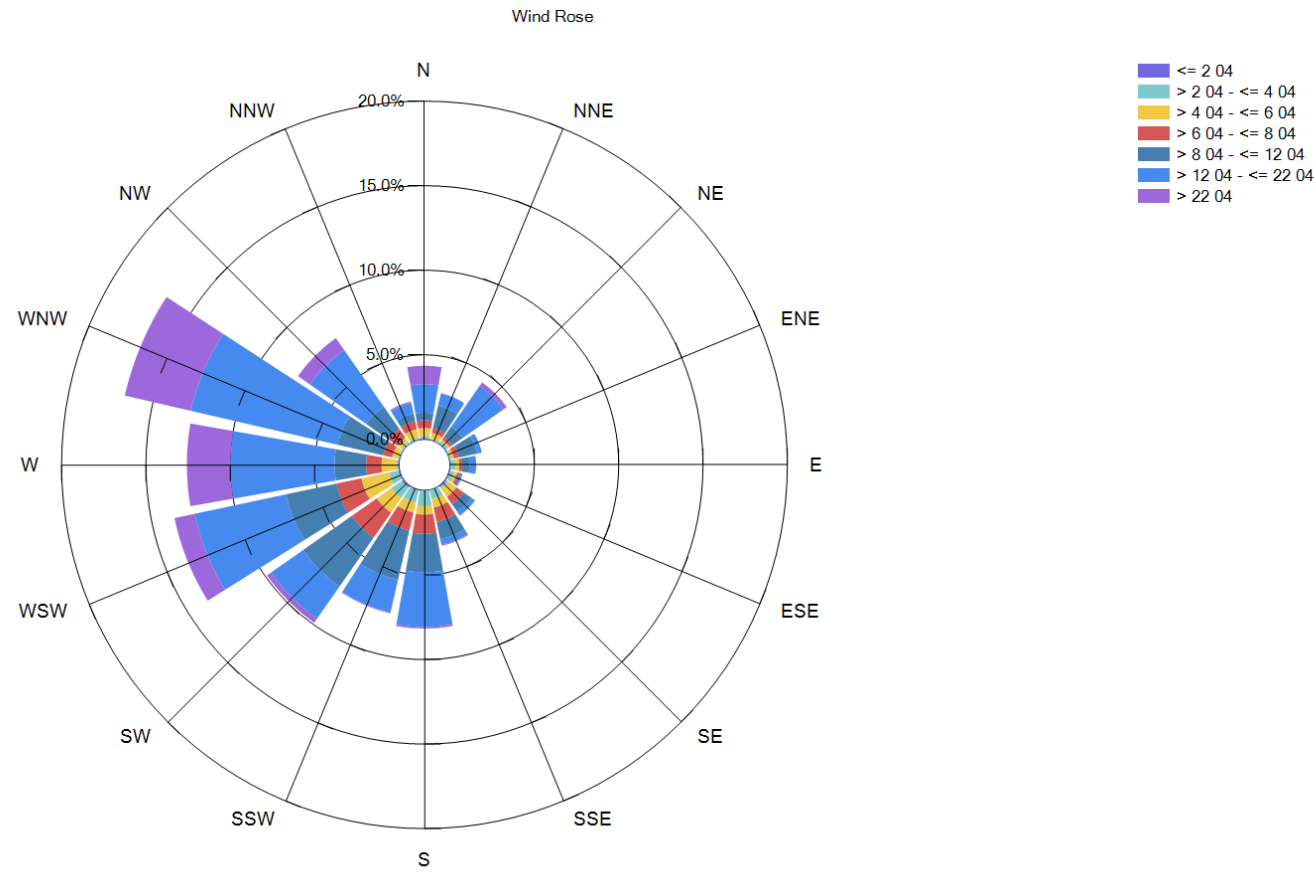

K-30 Element: Site 2_Wx Plot: Wind Rose 


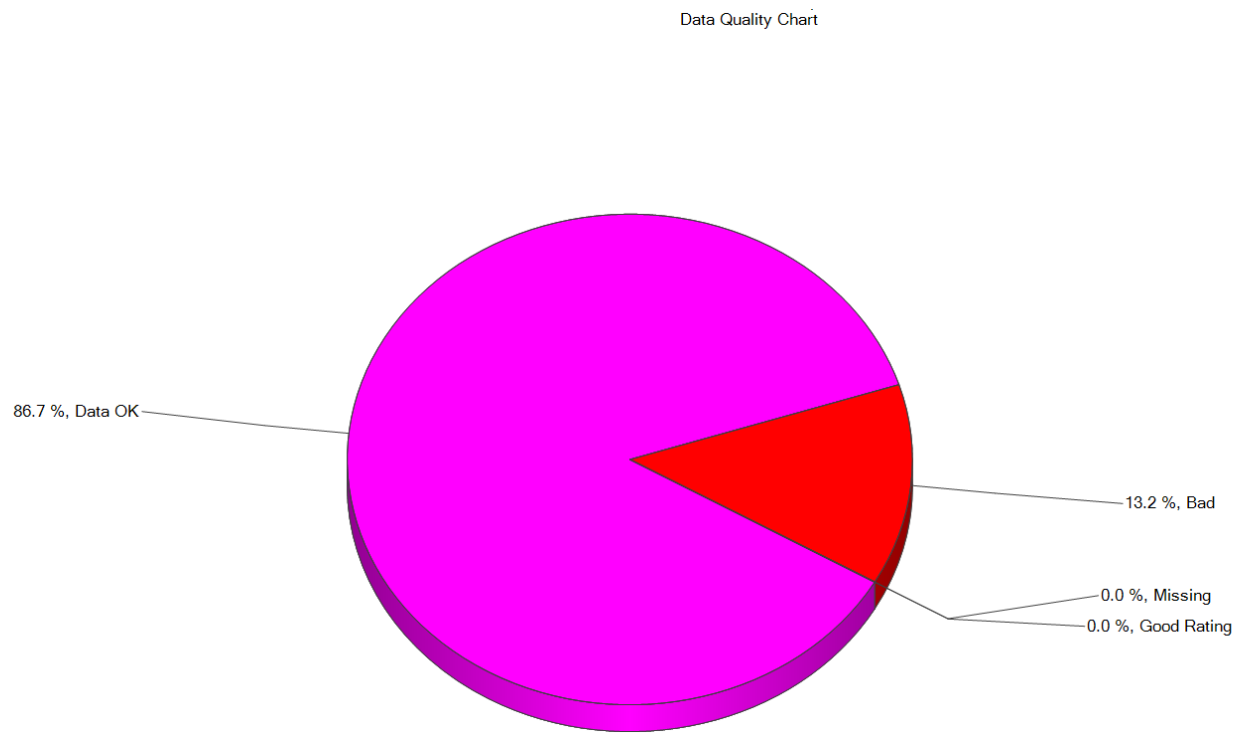

K-31 Element: Site 2_Wx Plot: Data Quality 2

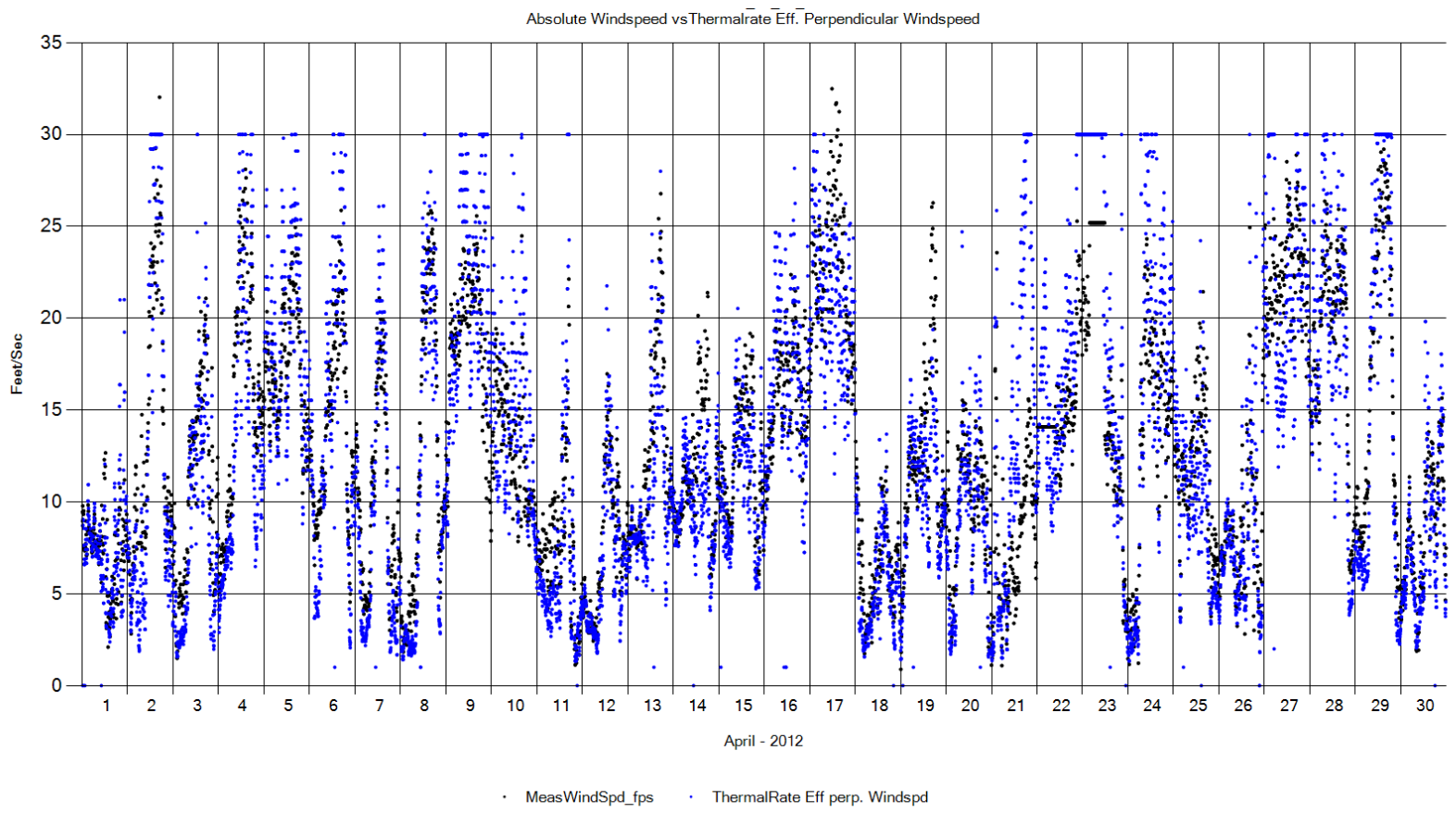

K-32 Element: Site 2_Wx Plot: Ws vs eff Perp WS 


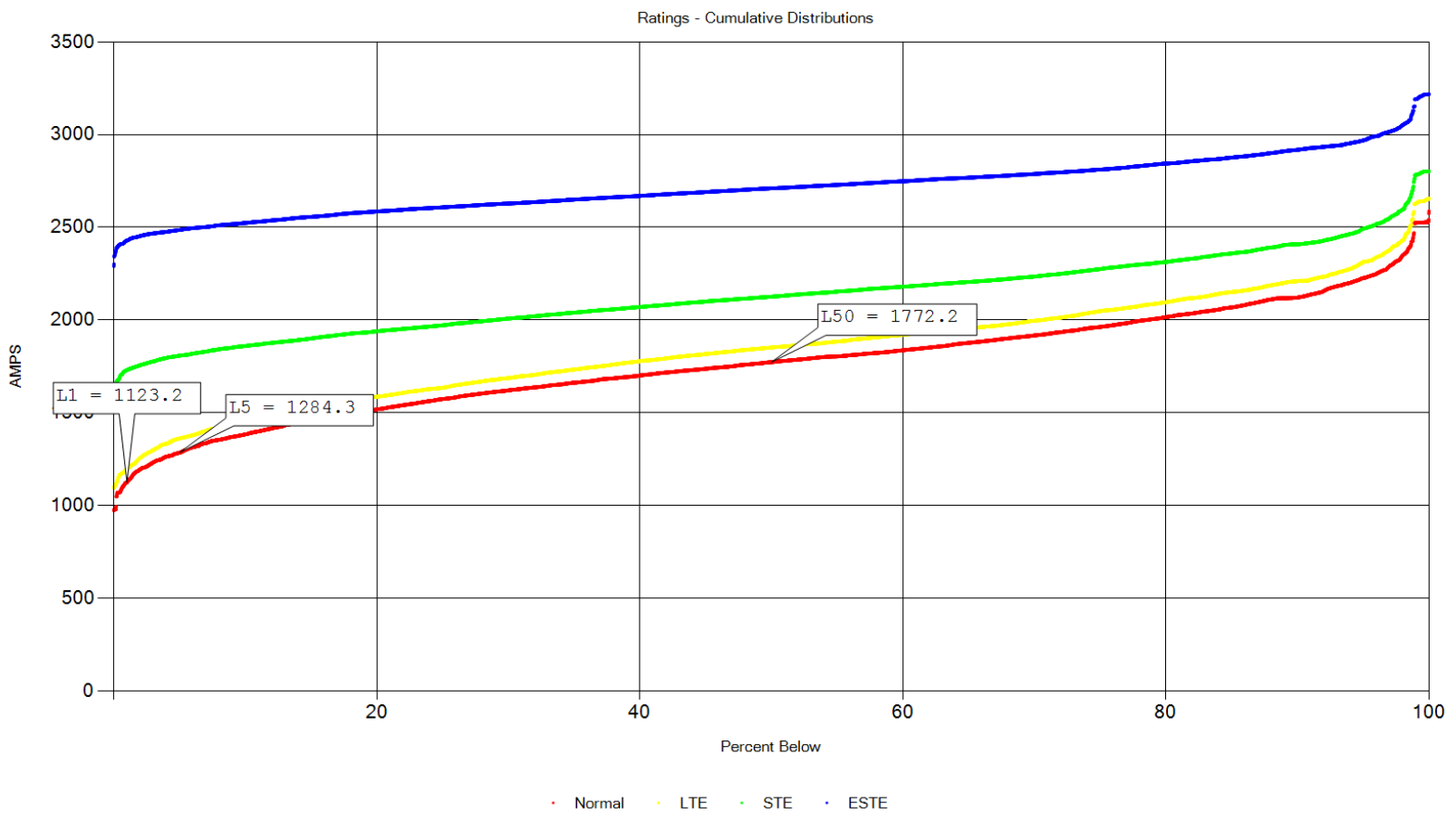

K-33 Element: Site 2_Wx Plot: Rating Cum. Dist.

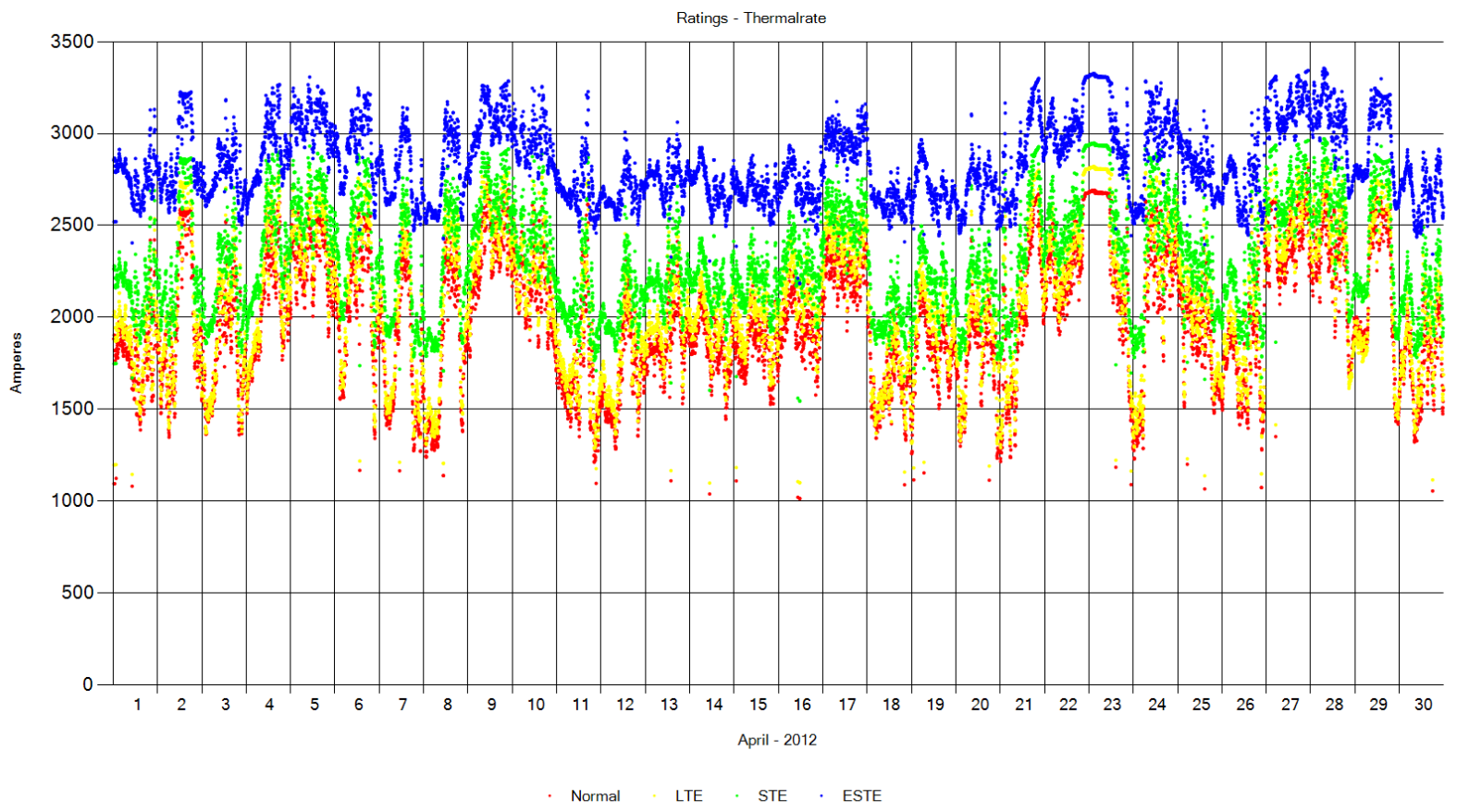

K-34 Element: Site 2_TR Plot: Ratings-ThermalRate

K-18 


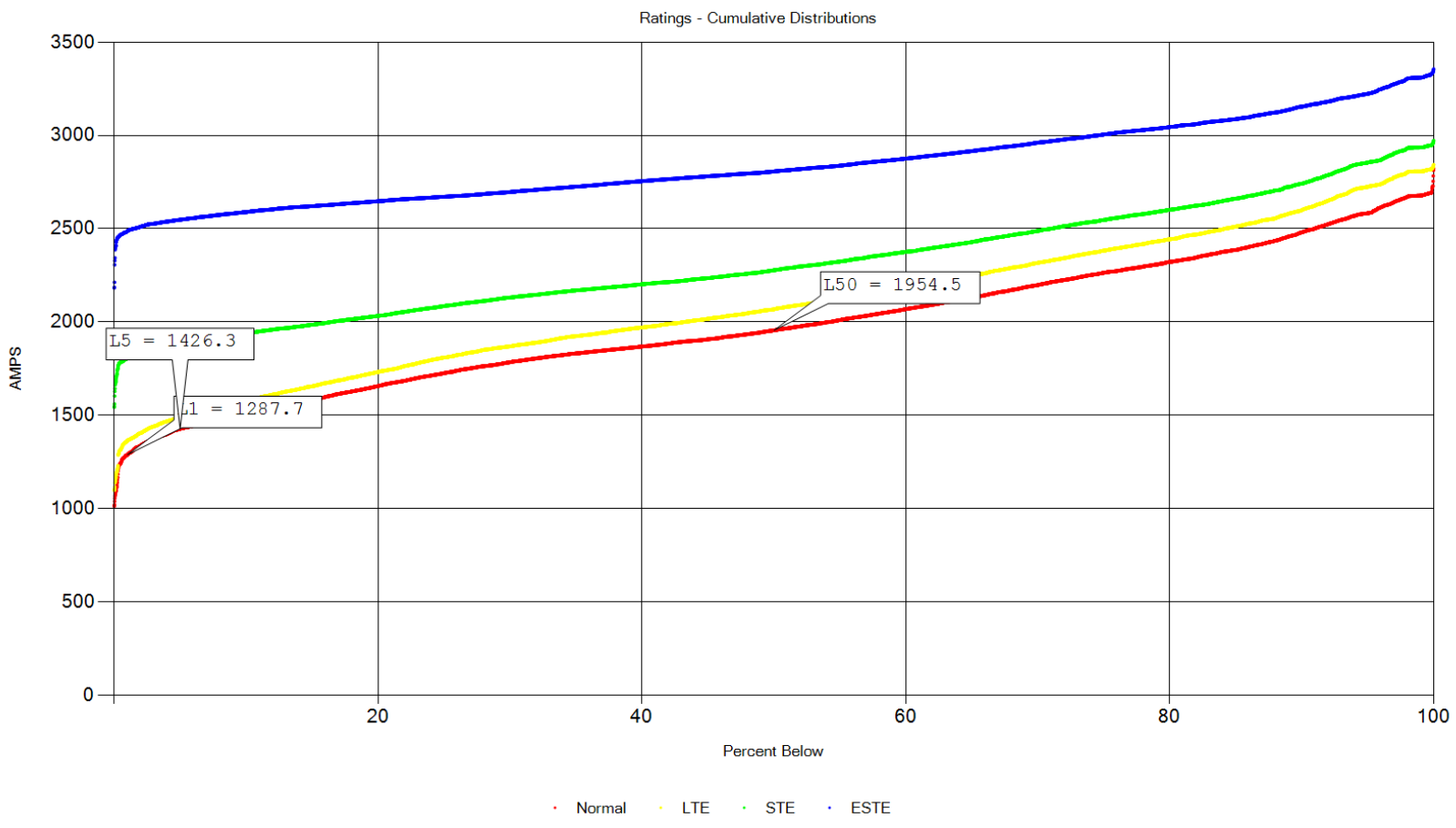

K-35 Element: Site 2_TR Plot: Rating Cum. Dist.

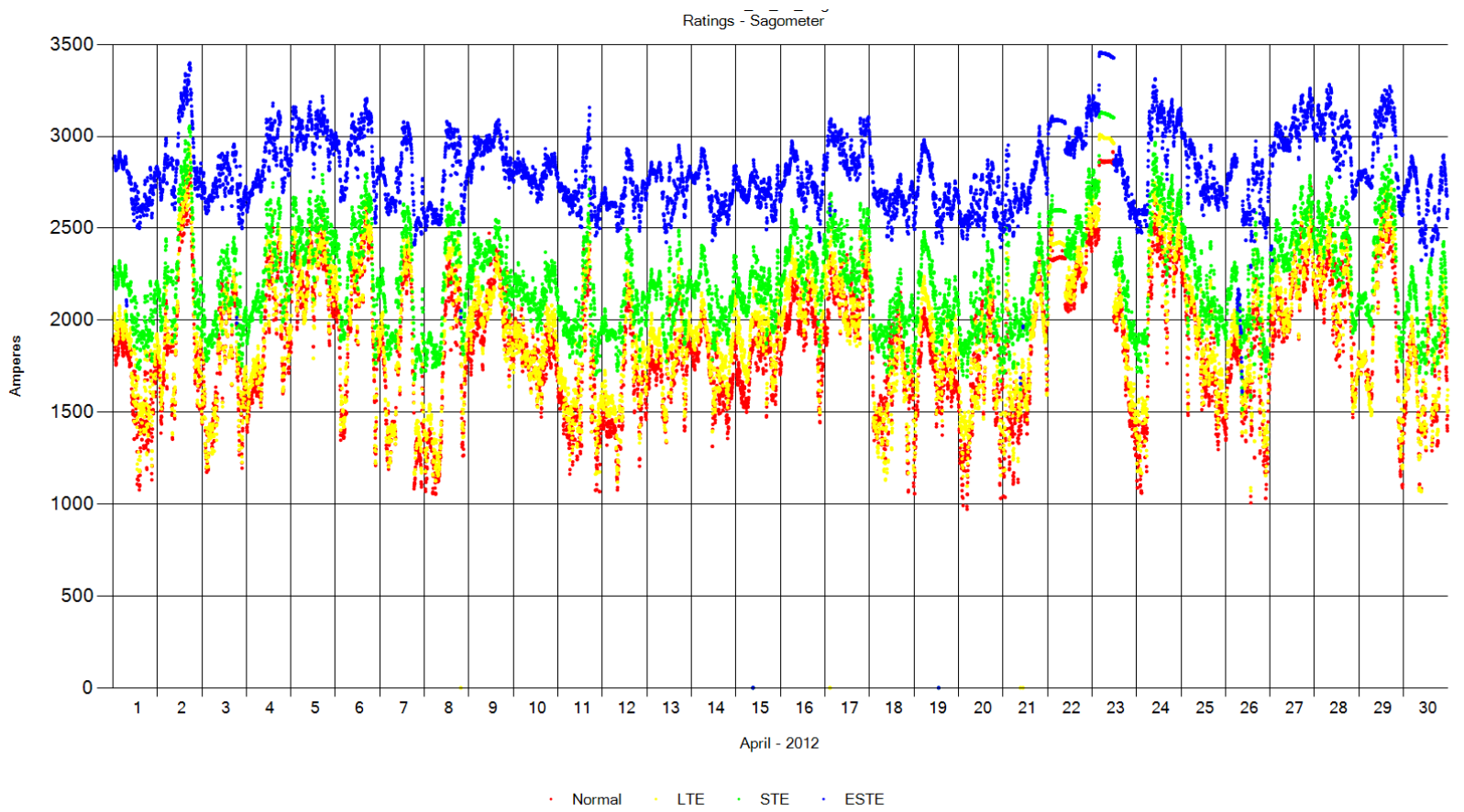

K-36 Element: Site 2_Sag Plot: Ratings-Sagometer 


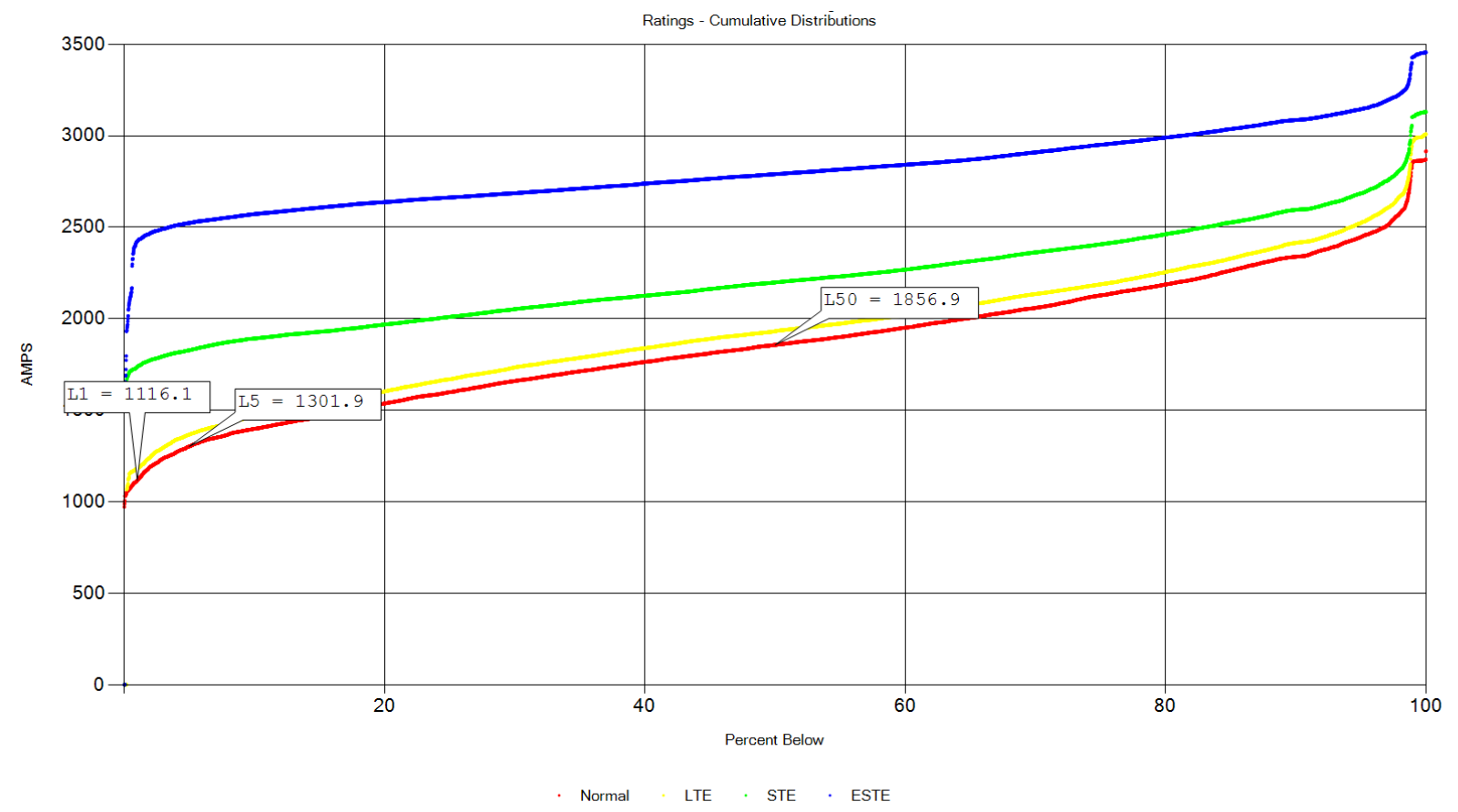

K-37 Element: Site 2_Sag Plot: Rating Cum. Dist.

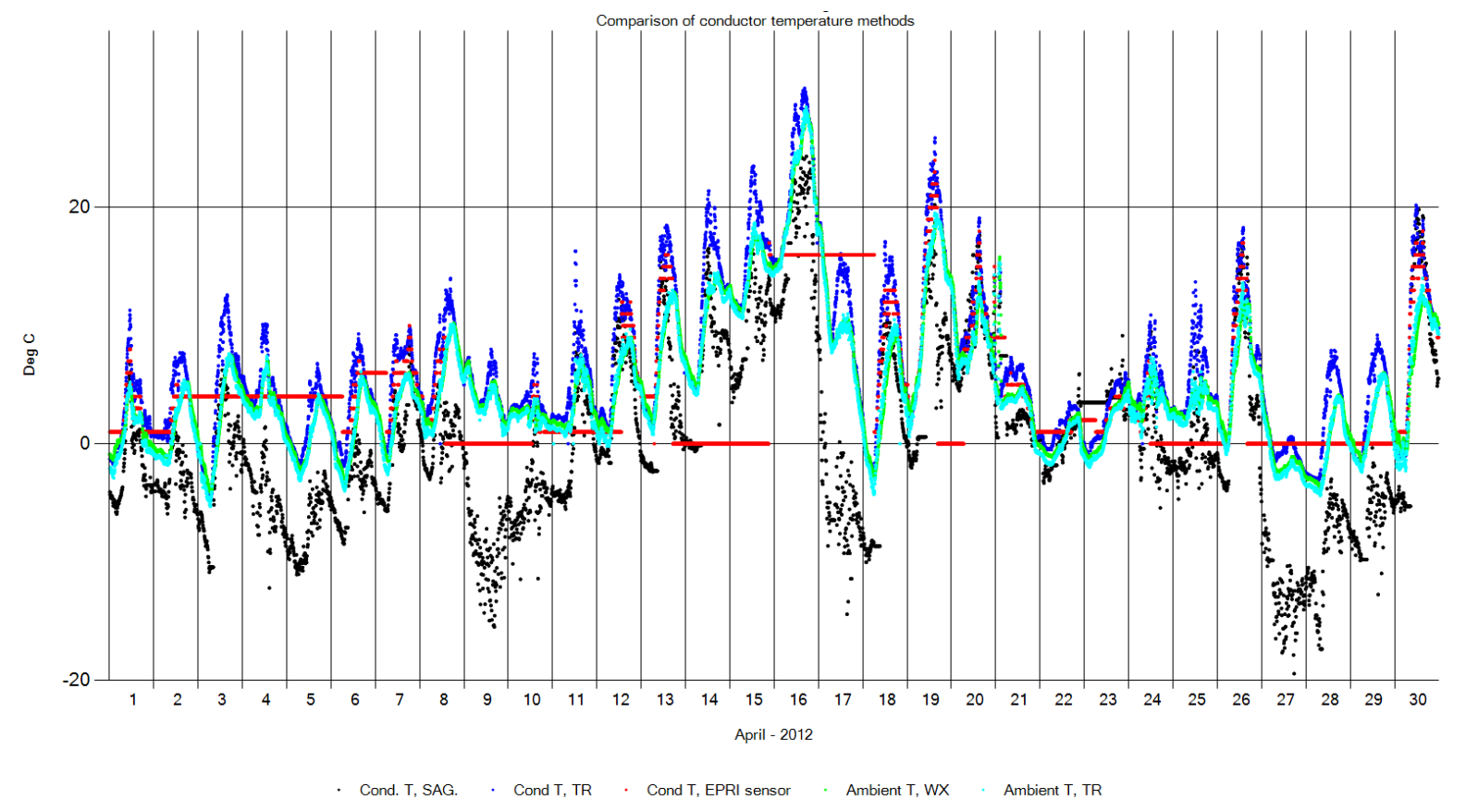

K-38 Element: Site 2_Sag Plot: Conductor Temp Compare 


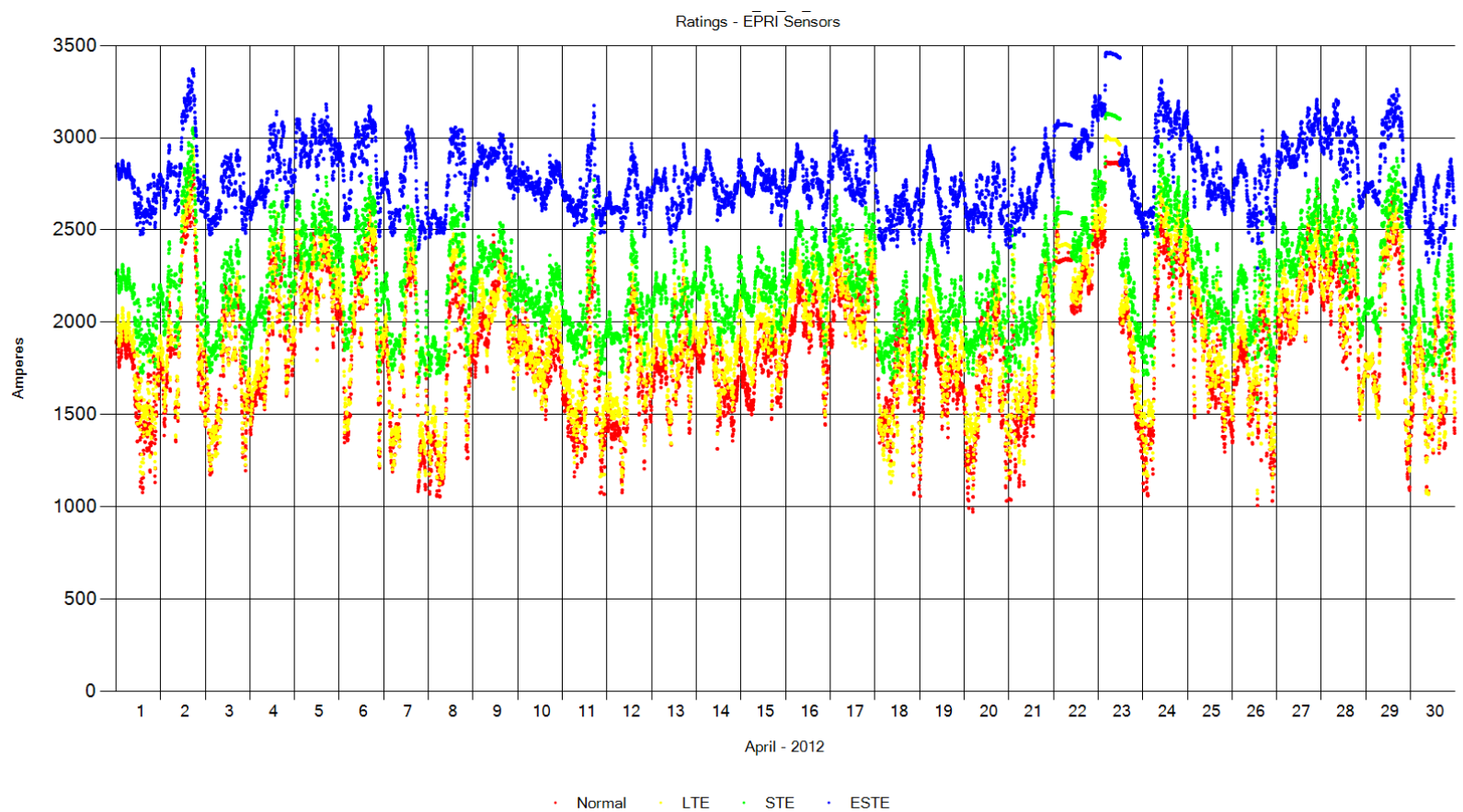

K-39 Element: Site 2_BS Plot: Ratings - EPRI Sensors

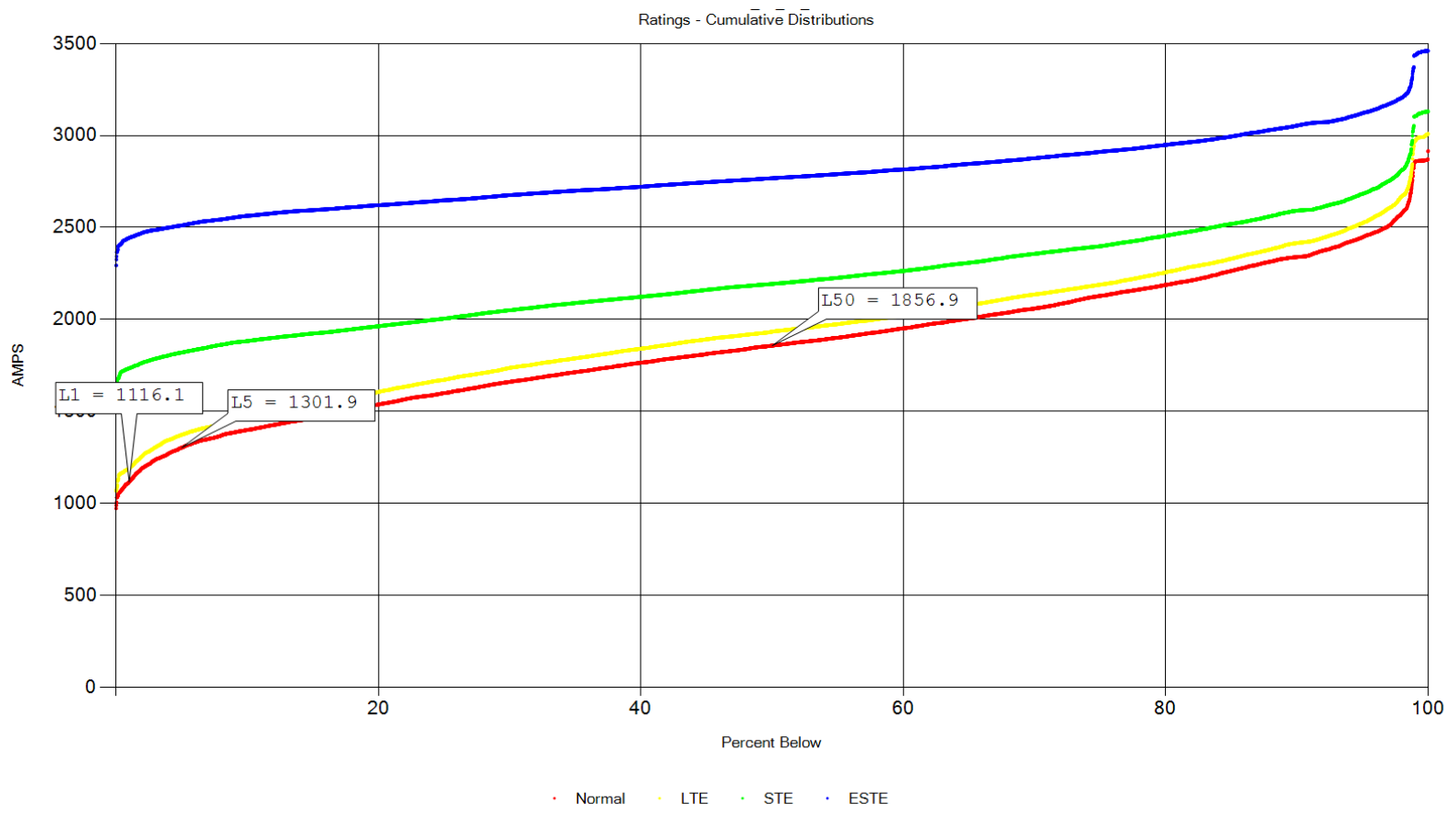

K-40 Element: Site 2_BS Plot: Rating Cum. Dist. 


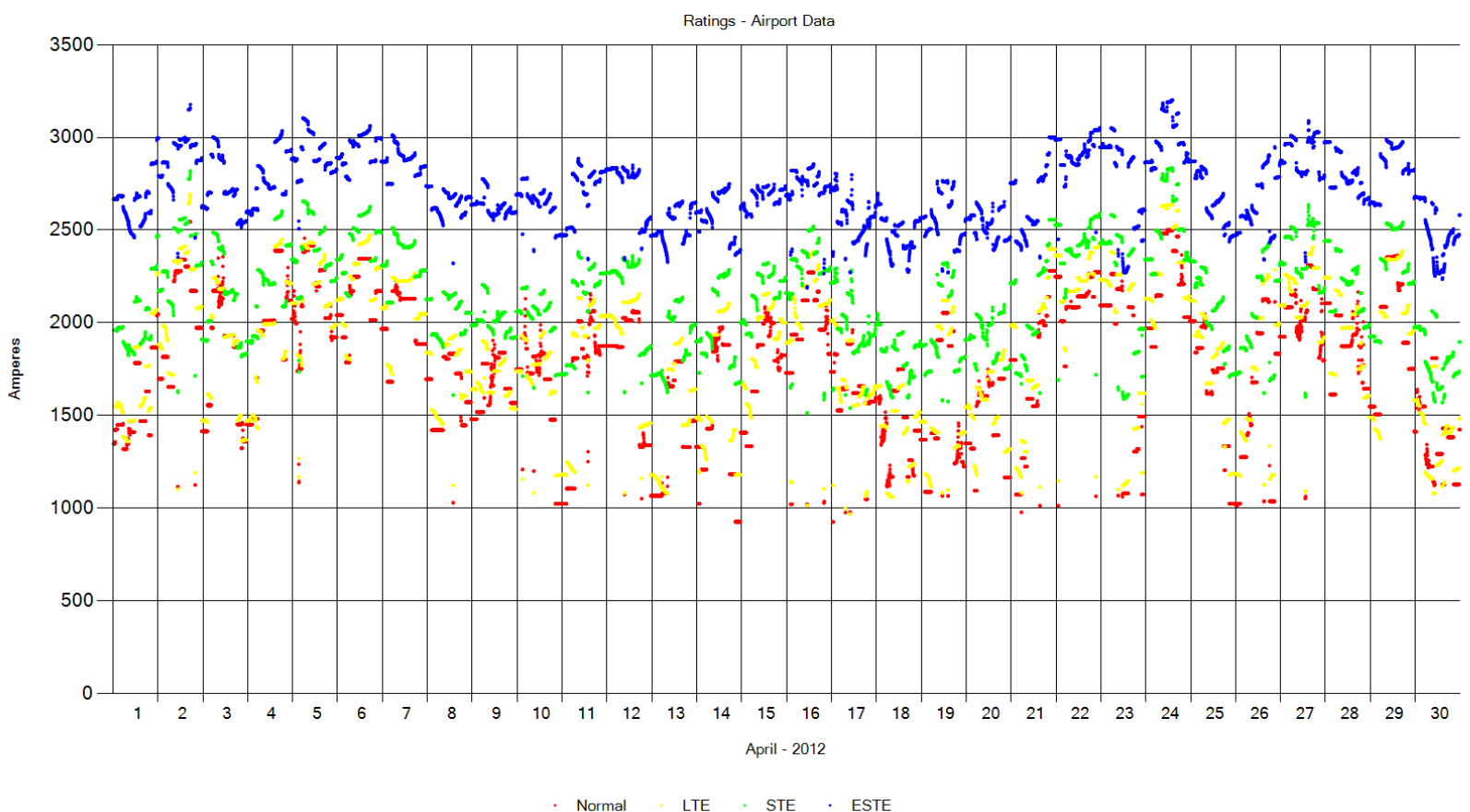

\section{K-41 Element: Site 2_AP Plot: Ratings-Airport data}

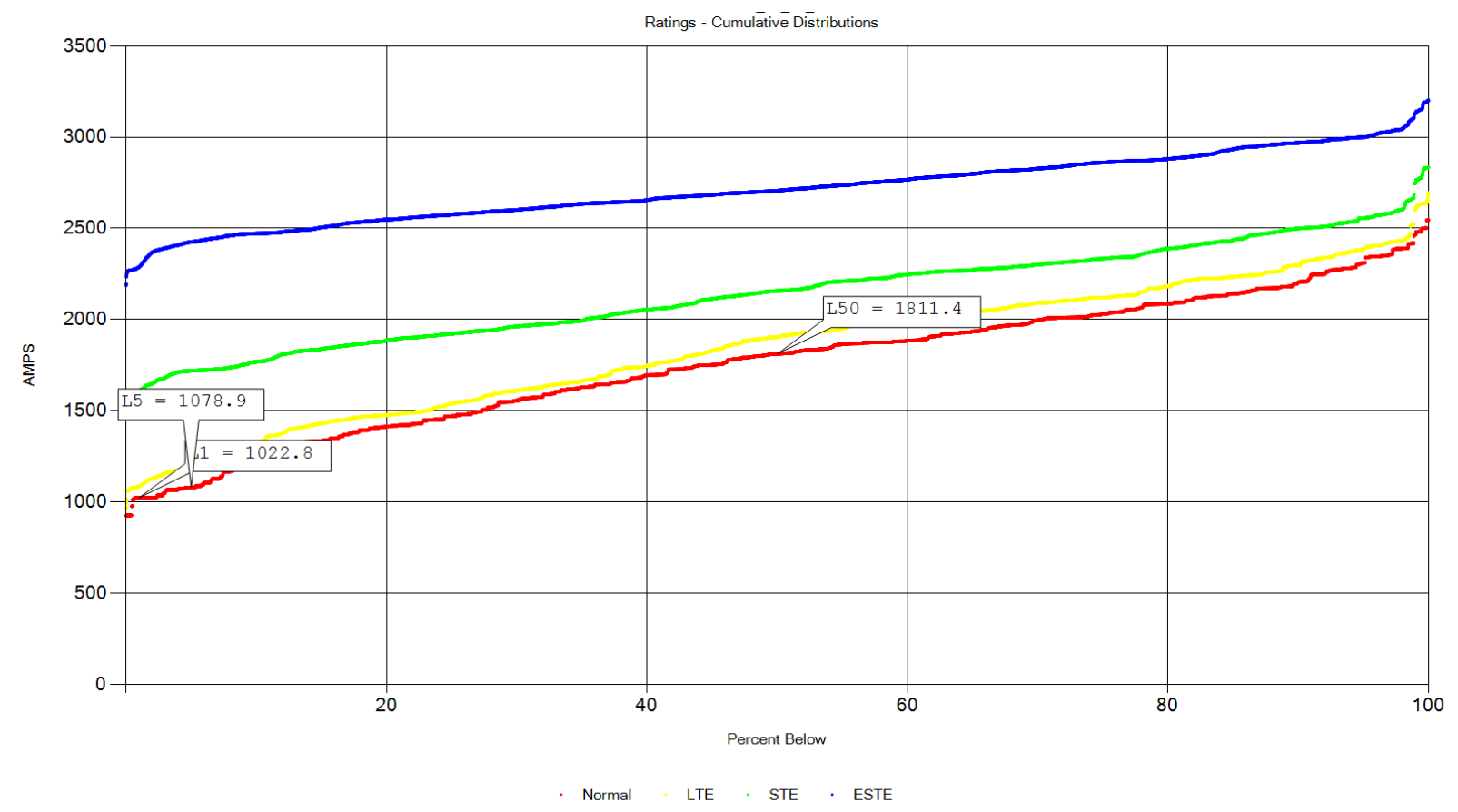

K-42 Element: Site 2_AP Plot: Rating Cum. Dist. 


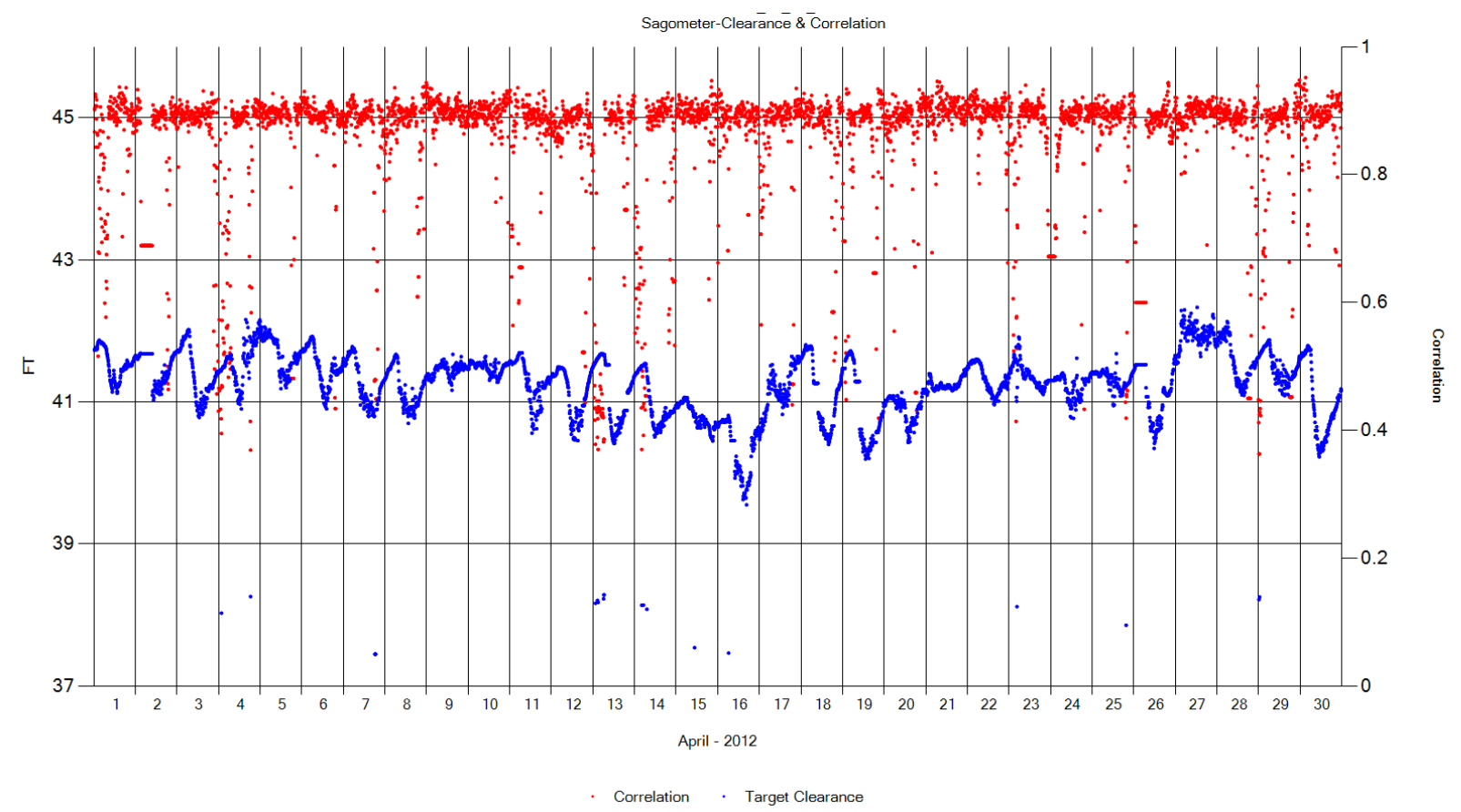

K-43 Element: Site 3_Wx Plot: Conductor Clearance

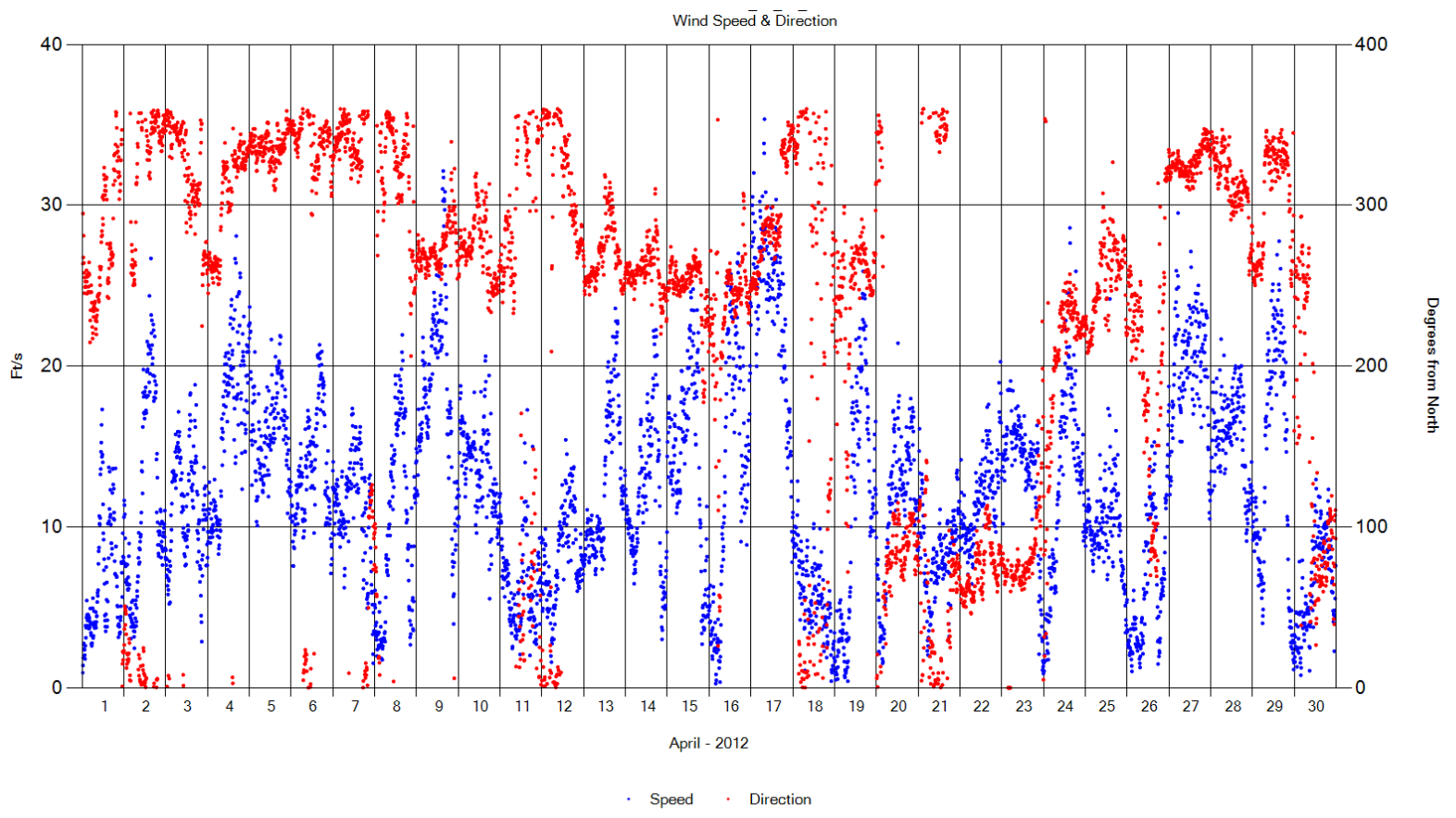

K-44 Element: Site 3_Wx Plot: Wind Speed \& Direction 


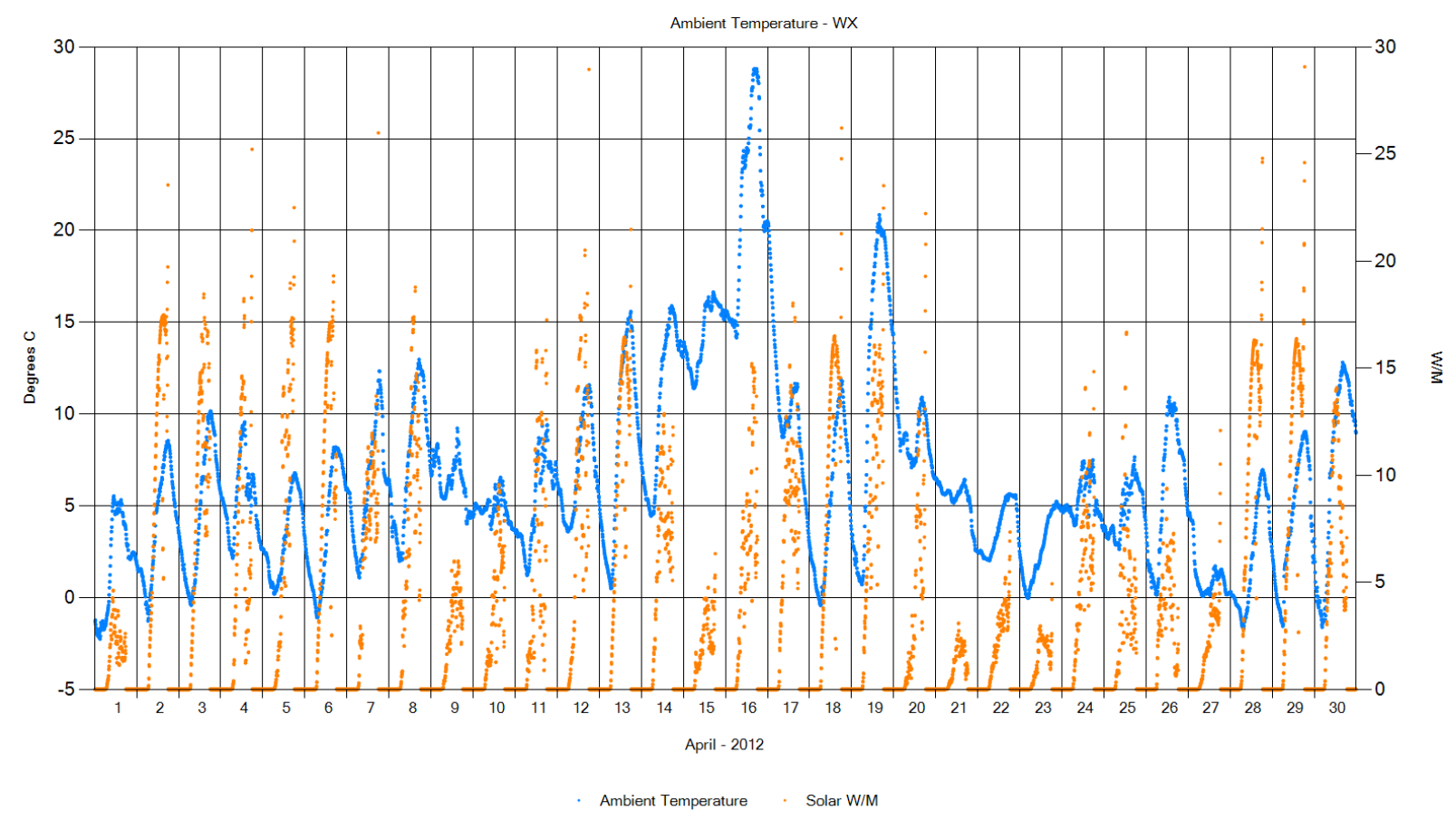

K-45 Element: Site 3_Wx Plot: Ambient Temperature - WX

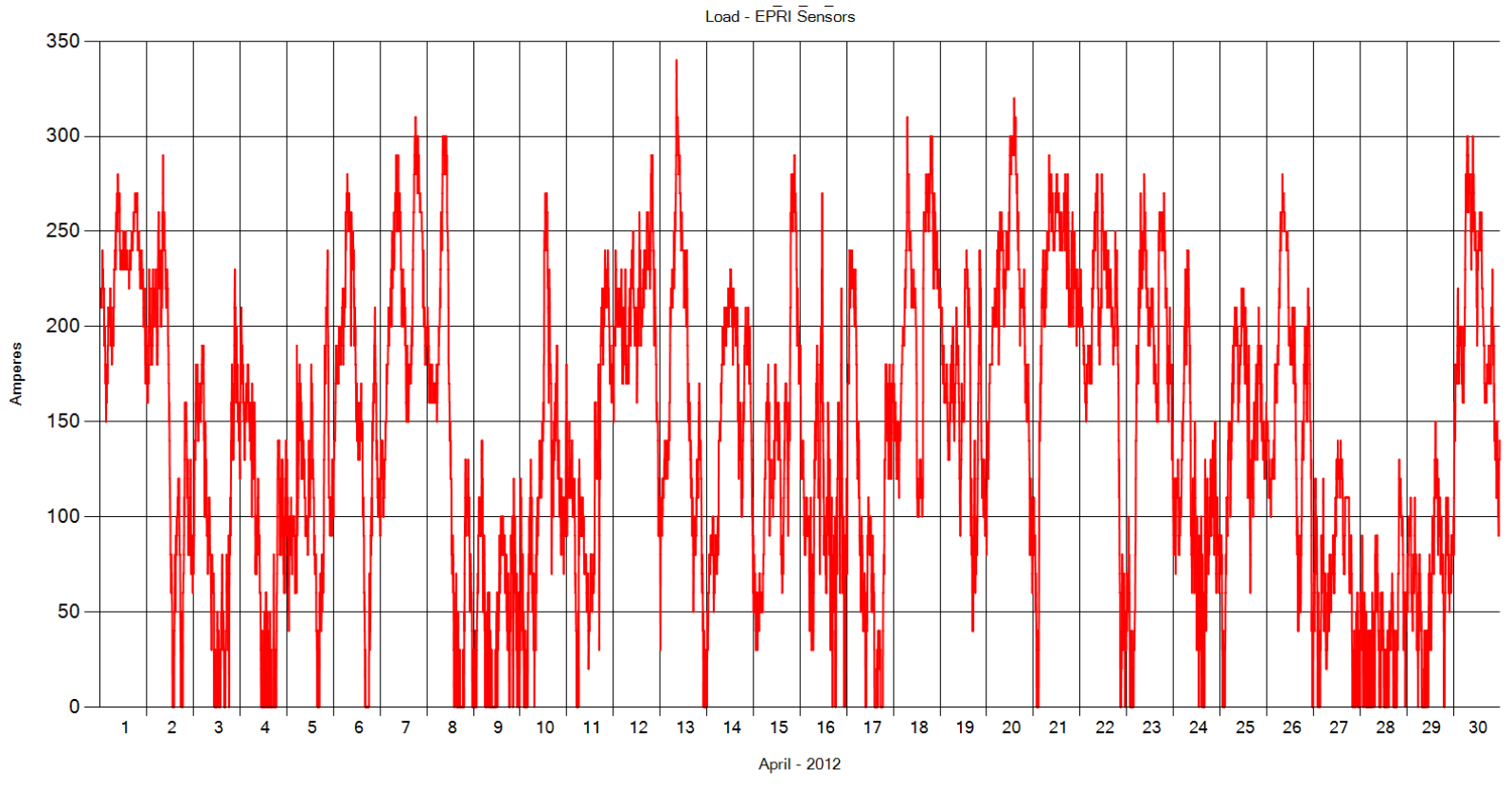

— Load - EPRI Sensors

K-46 Element: Site 3_Wx Plot: Load - EPRI Sensors 


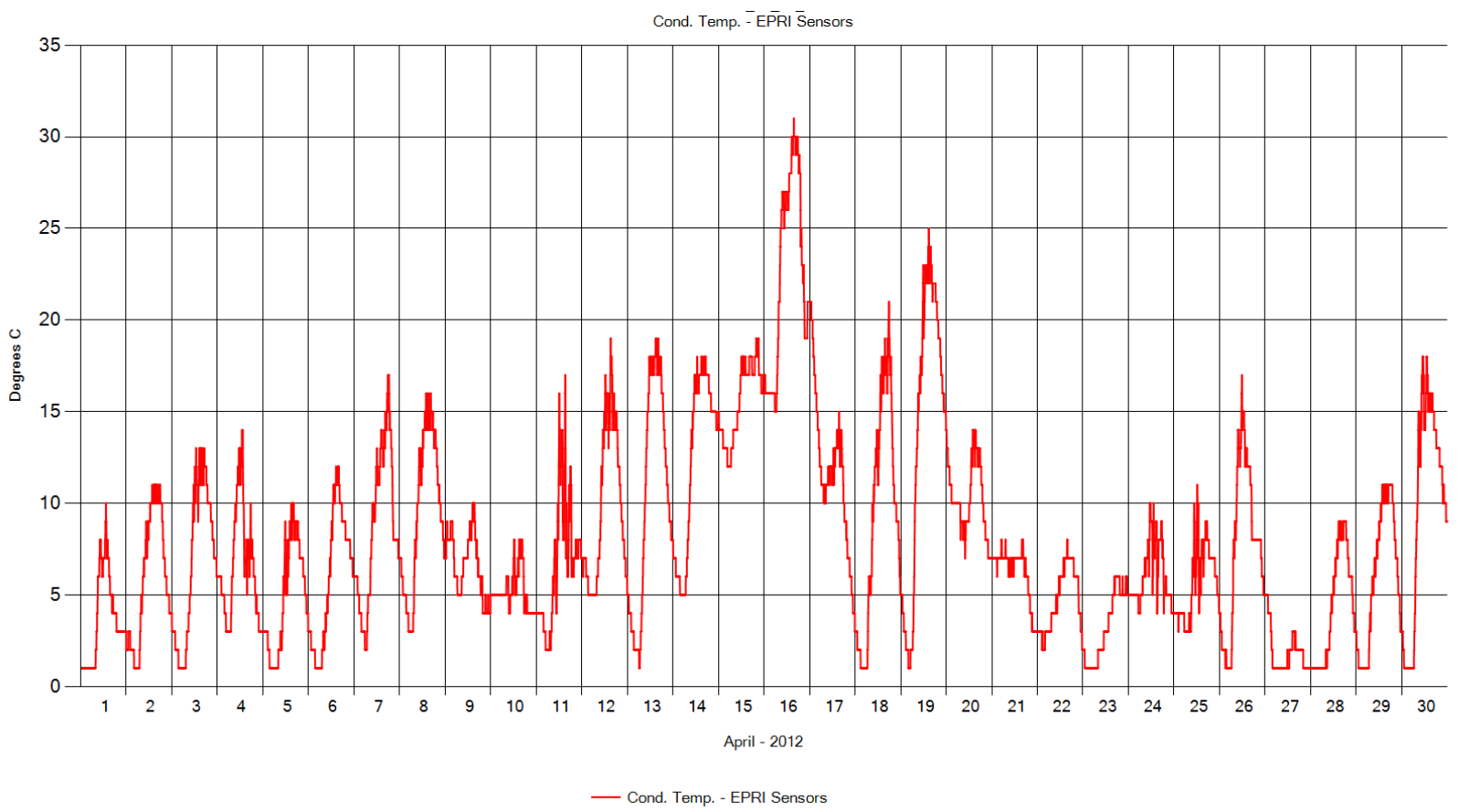

K-47 Element: Site 3_Wx Plot: Cond. Temp. - EPRI Sensors

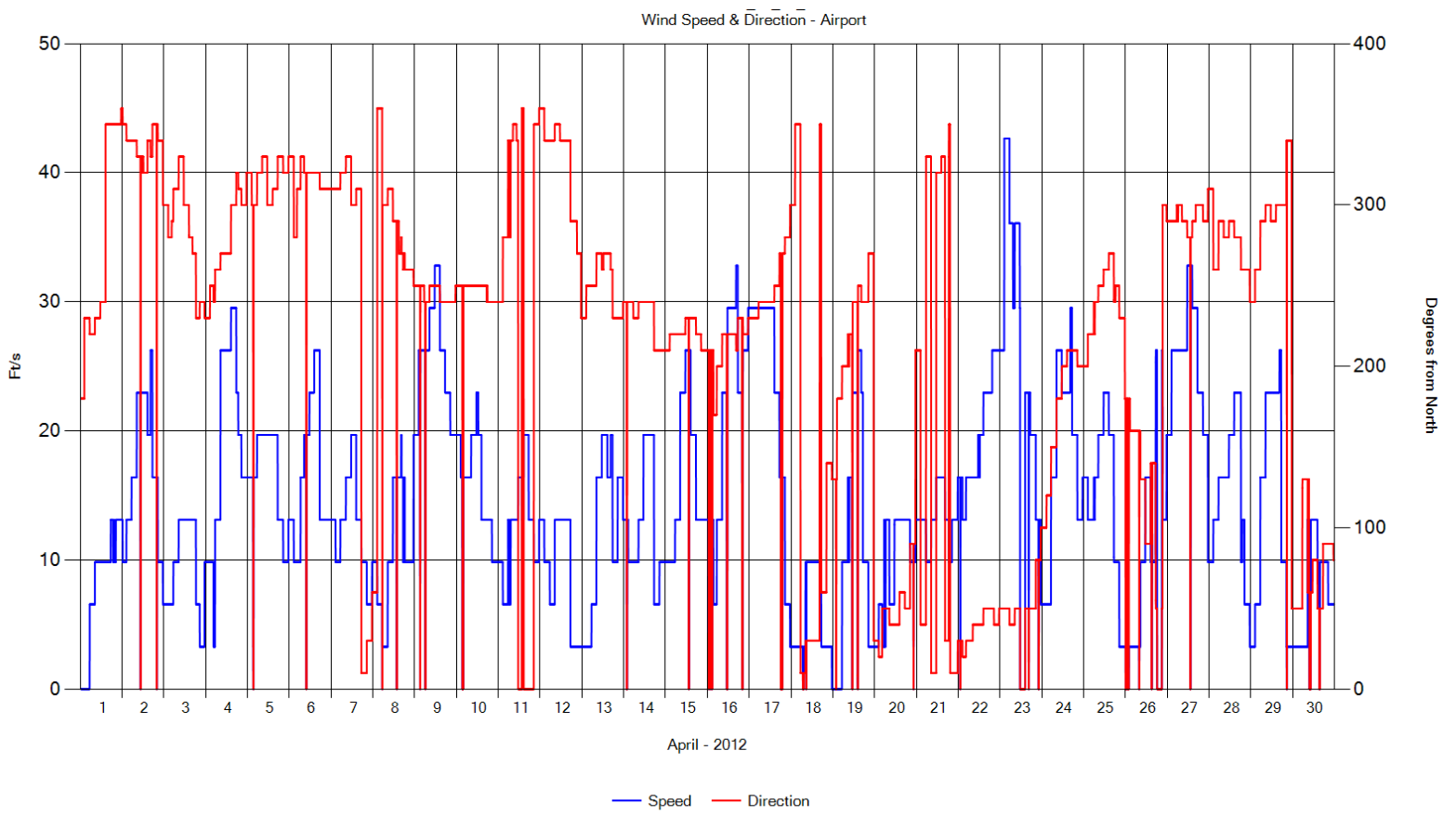

K-48 Element: Site 3_Wx Plot: Wind Speed \& Direction-Airport 


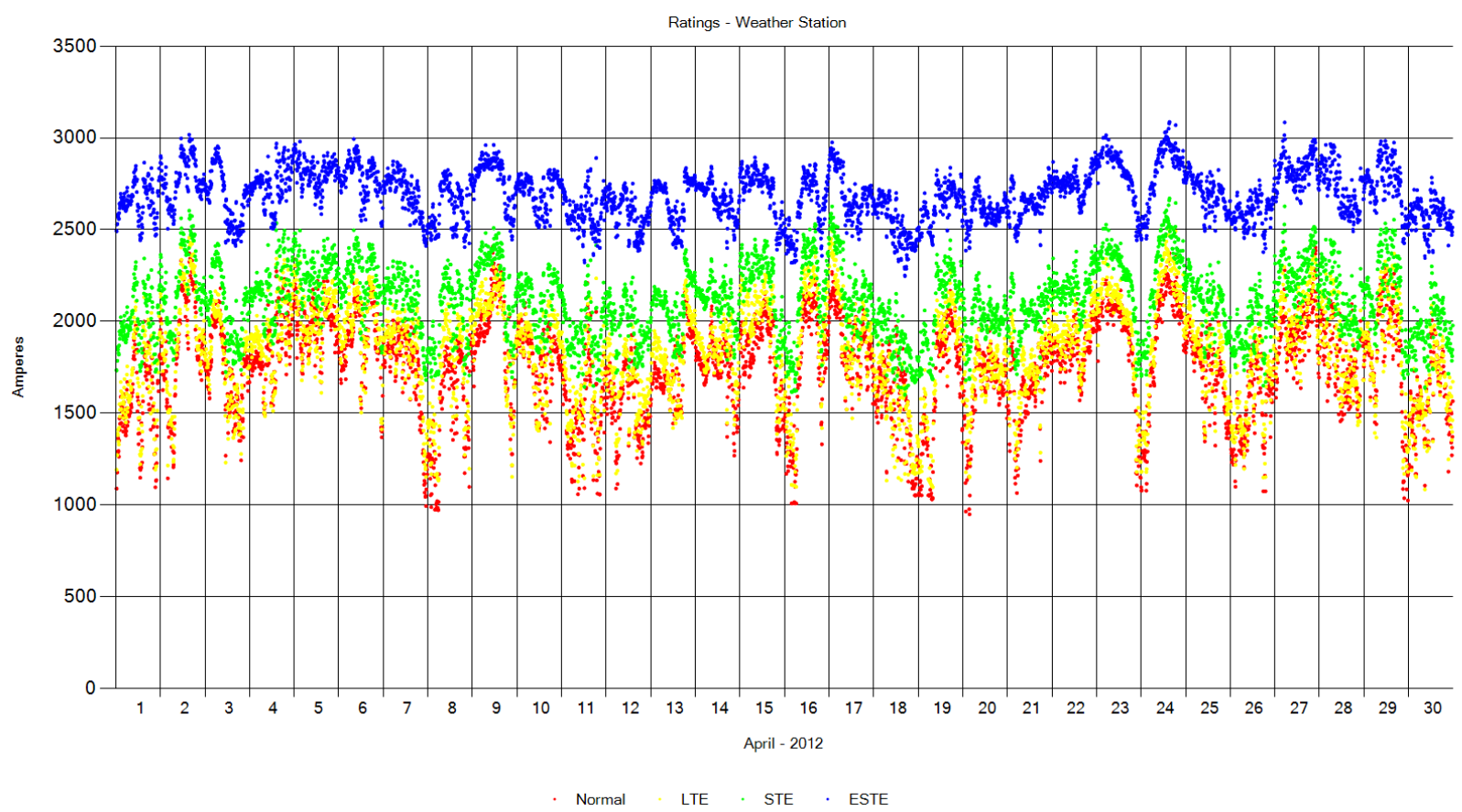

K-49 Element: Site 3_Wx Plot: Ratings-WX

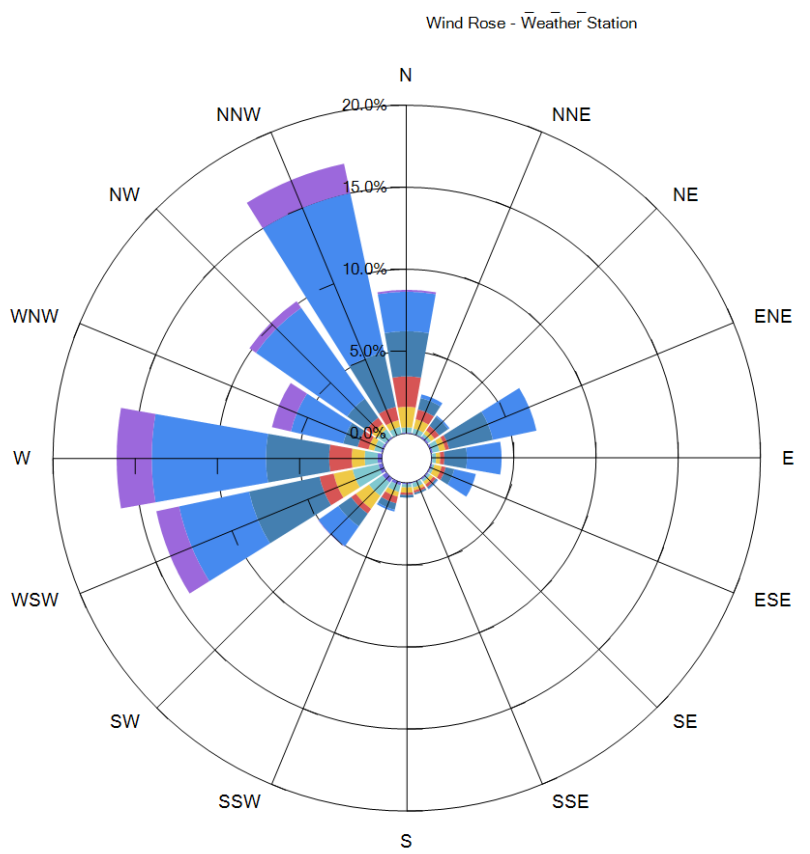

$<=206$

$>606-<=806$

$>806-<=1206$
$>1206-<=2206$

$>2206$ 


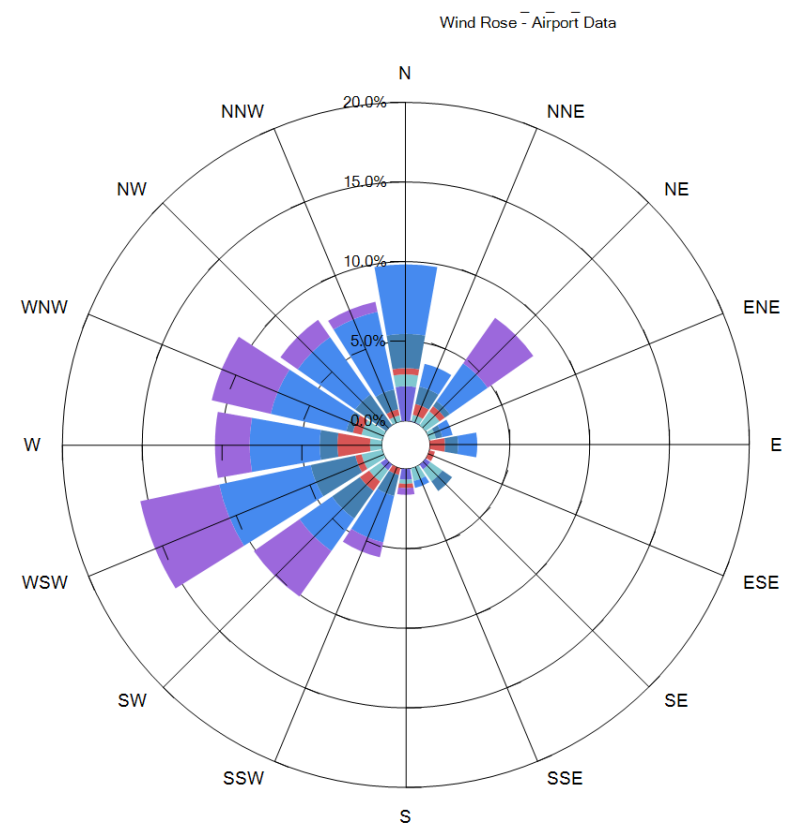

$<=206$
$>206-<=406$
$>406-<=606$

$>406-<=606$

$>606-<=806$
$>806-<=1206$

$>>806-<=1206$
$>1206-<=2206$

$>2206$

K-51 Element: Site 3_Wx Plot: Wind Rose Airport

Dāa Quality Chart

Good Rating - 0

Bad -602

Bad - 602
Missing - 2

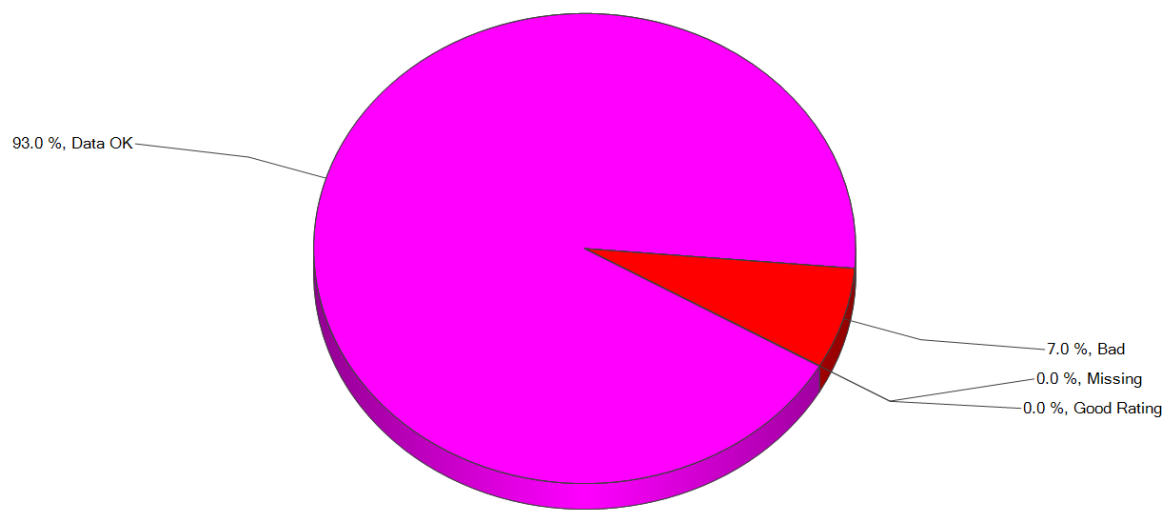

K-52 Element: Site 3_Wx Plot: Data Quality 2 


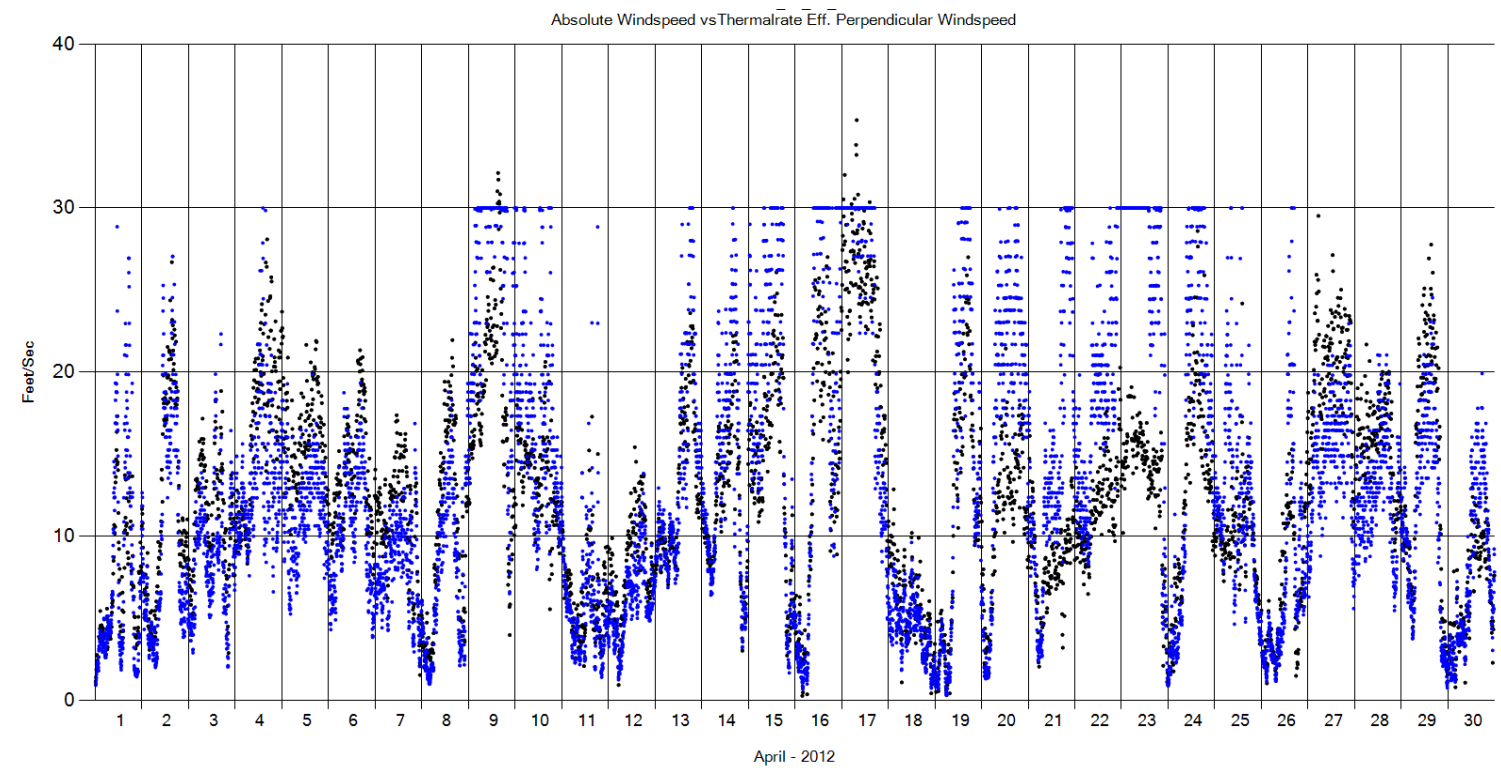

- MeasWindSpd_fps . ThermalRate Eff perp. Windspd

\section{K-53 Element: Site 3_Wx Plot: Ws vs. eff Perp WS}

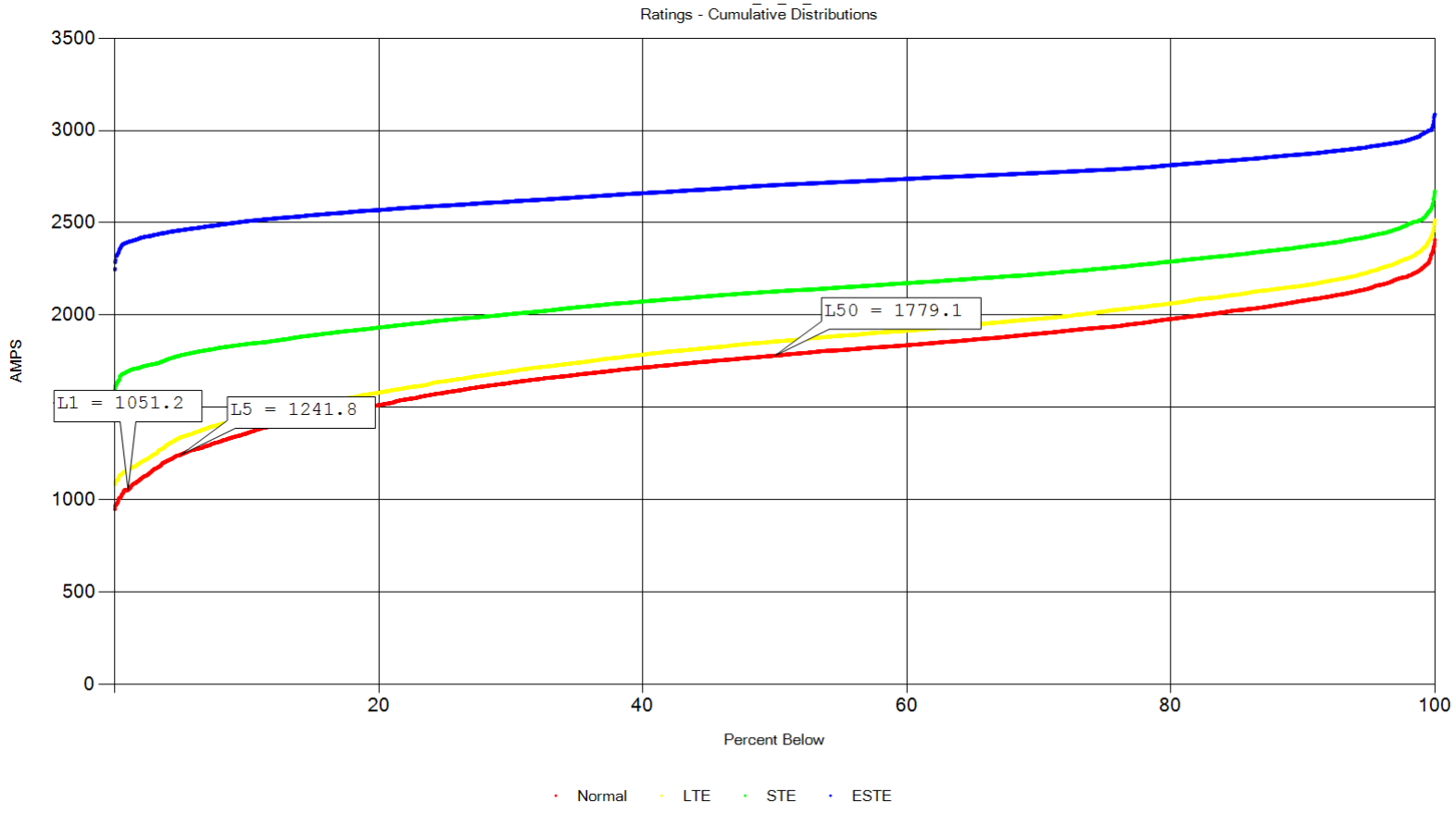

K-54 Element: Site 3_Wx Plot: Rating Cum. Dist. 


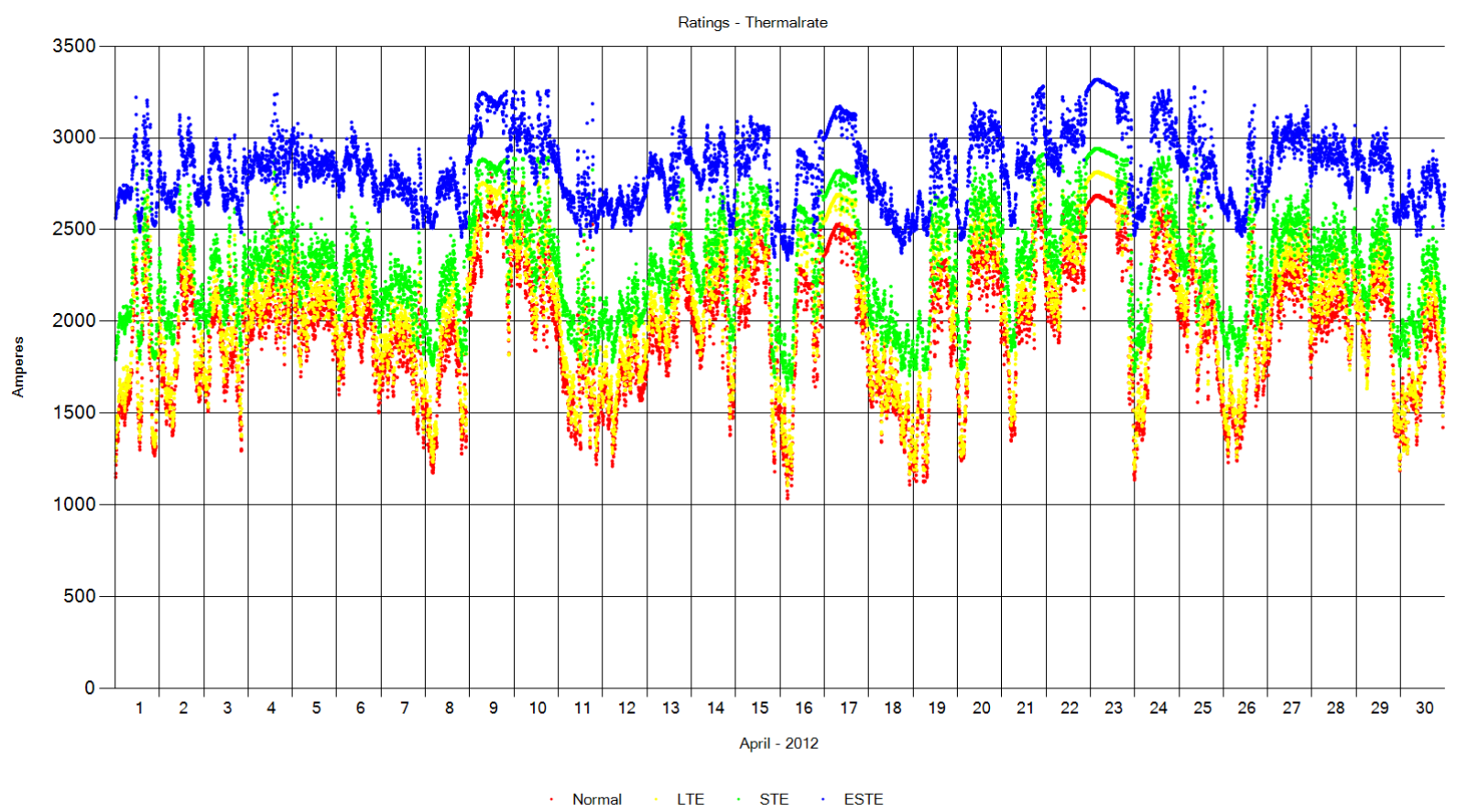

K-55 Element: Site 3_TR Plot: Ratings-ThermalRate

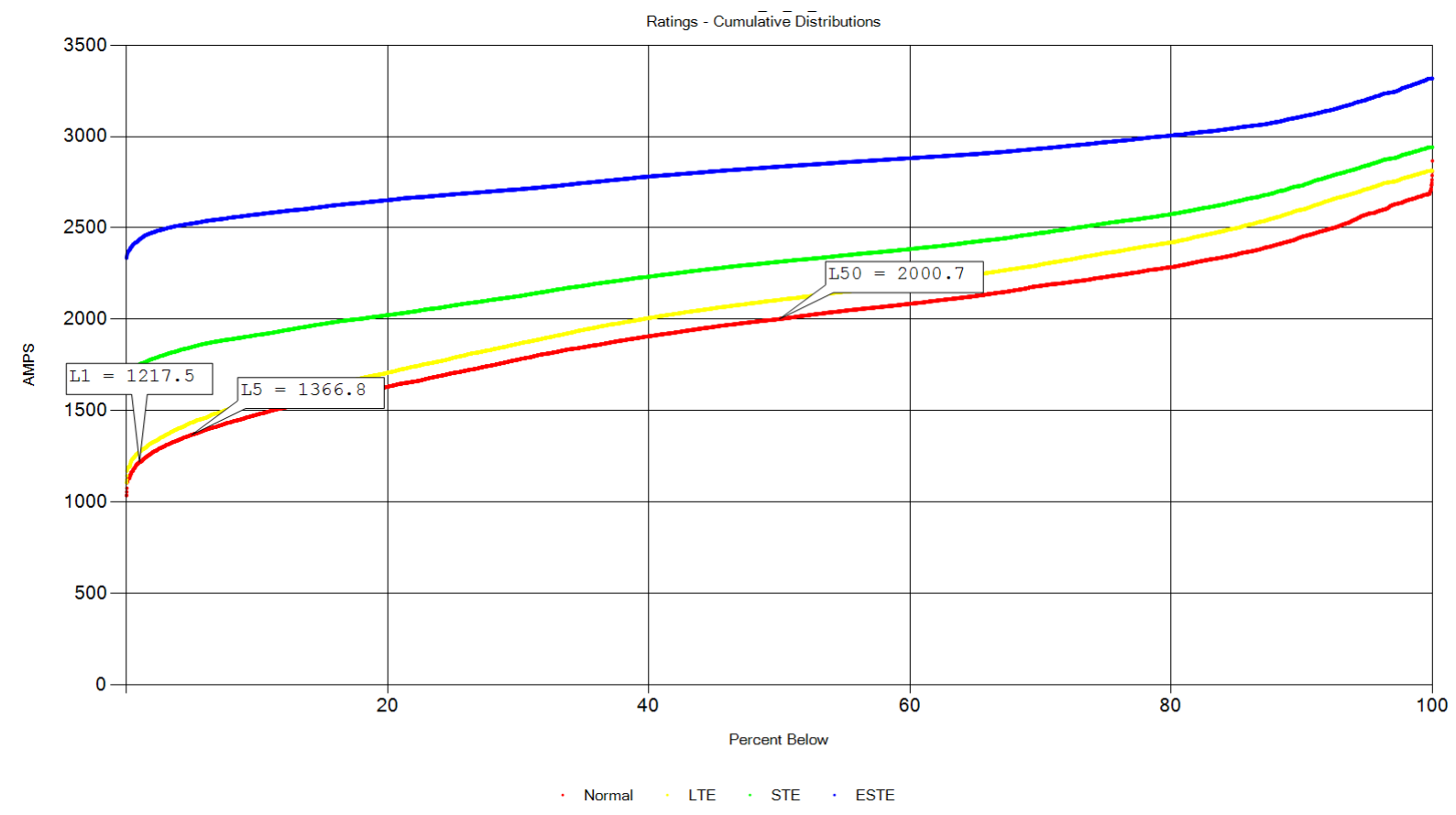

K-56 Element: Site 3_TR Plot: Rating Cum. Dist. 


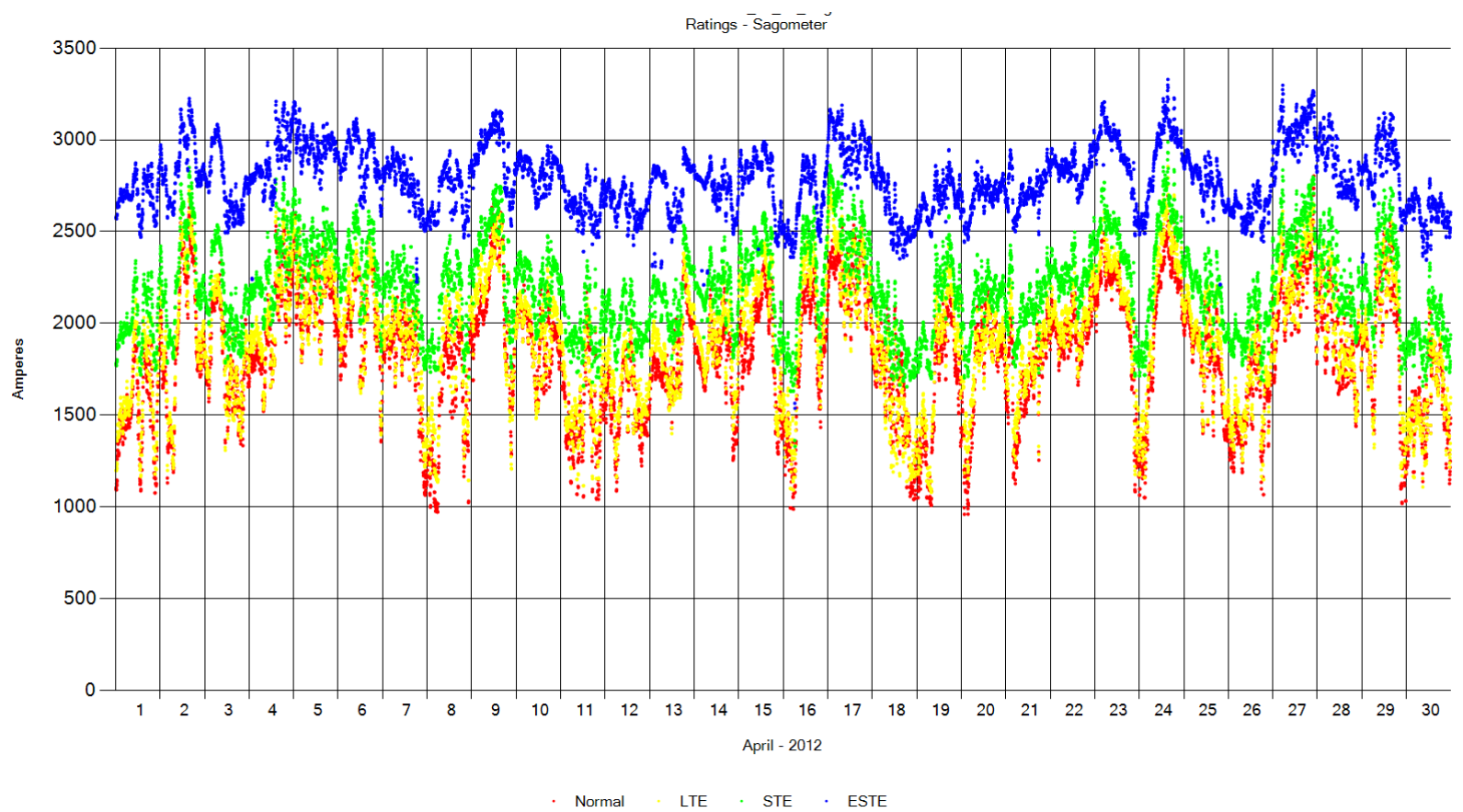

K-57 Element: Site 3_Sag Plot: Ratings-Sagometer

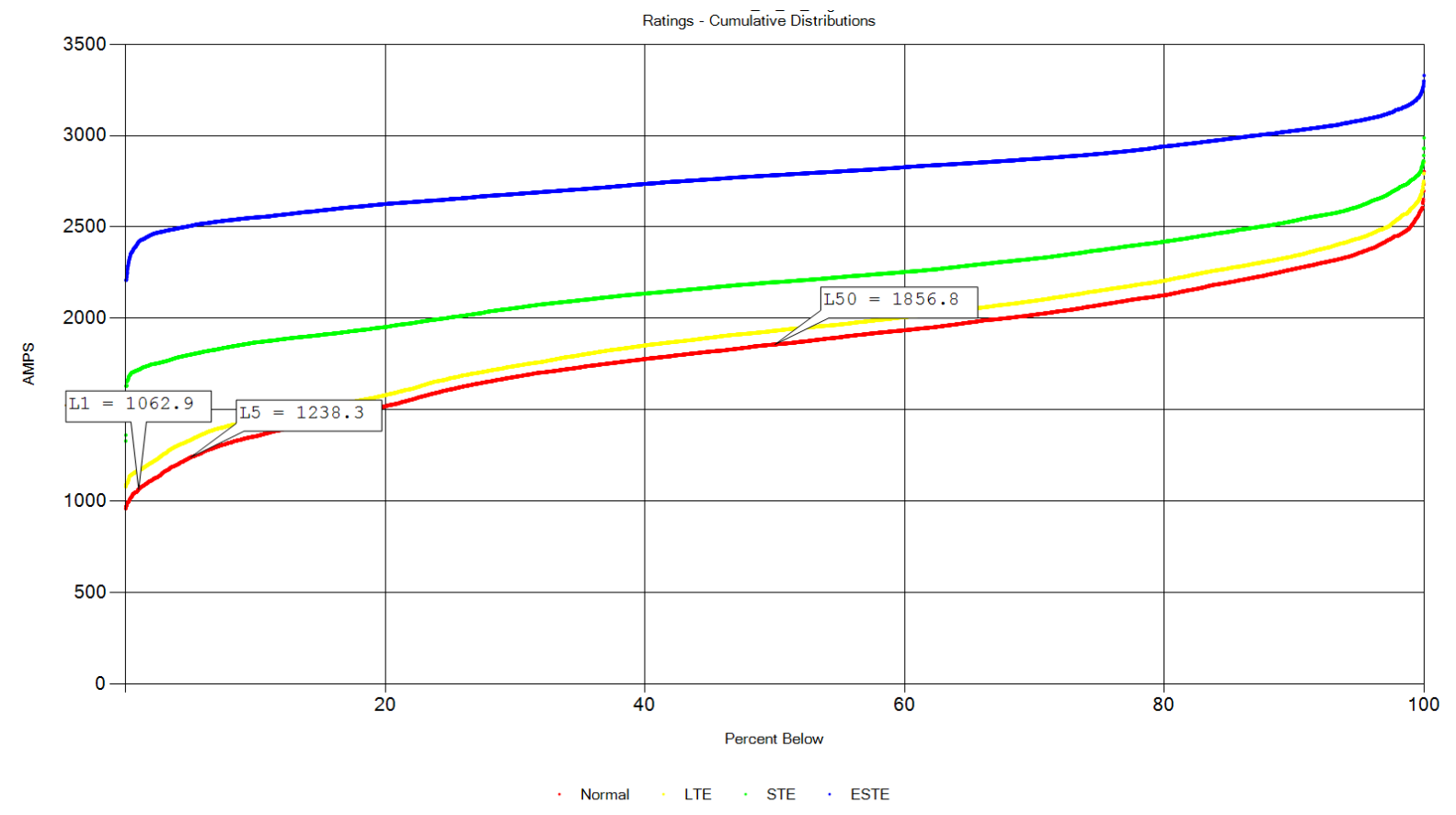

K-58 Element: Site 3_Sag Plot: Rating Cum. Dist.

K-30 


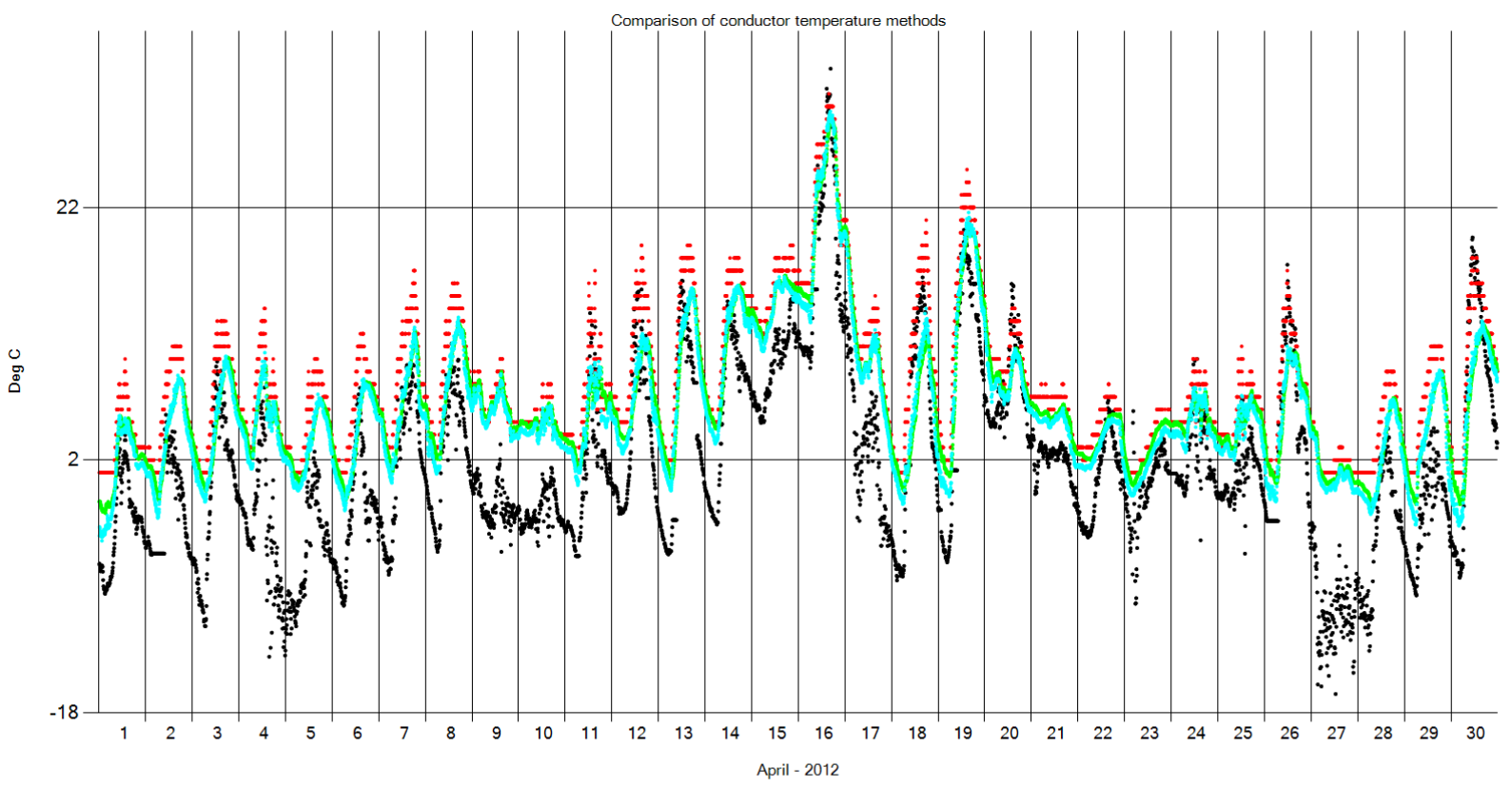

- Cond. T, SAG. - Cond T, Epri sensor - Ambient T, WX - Ambient T, TR

K-59 Element: Site 3_Sag Plot: Conductor Temp Compare

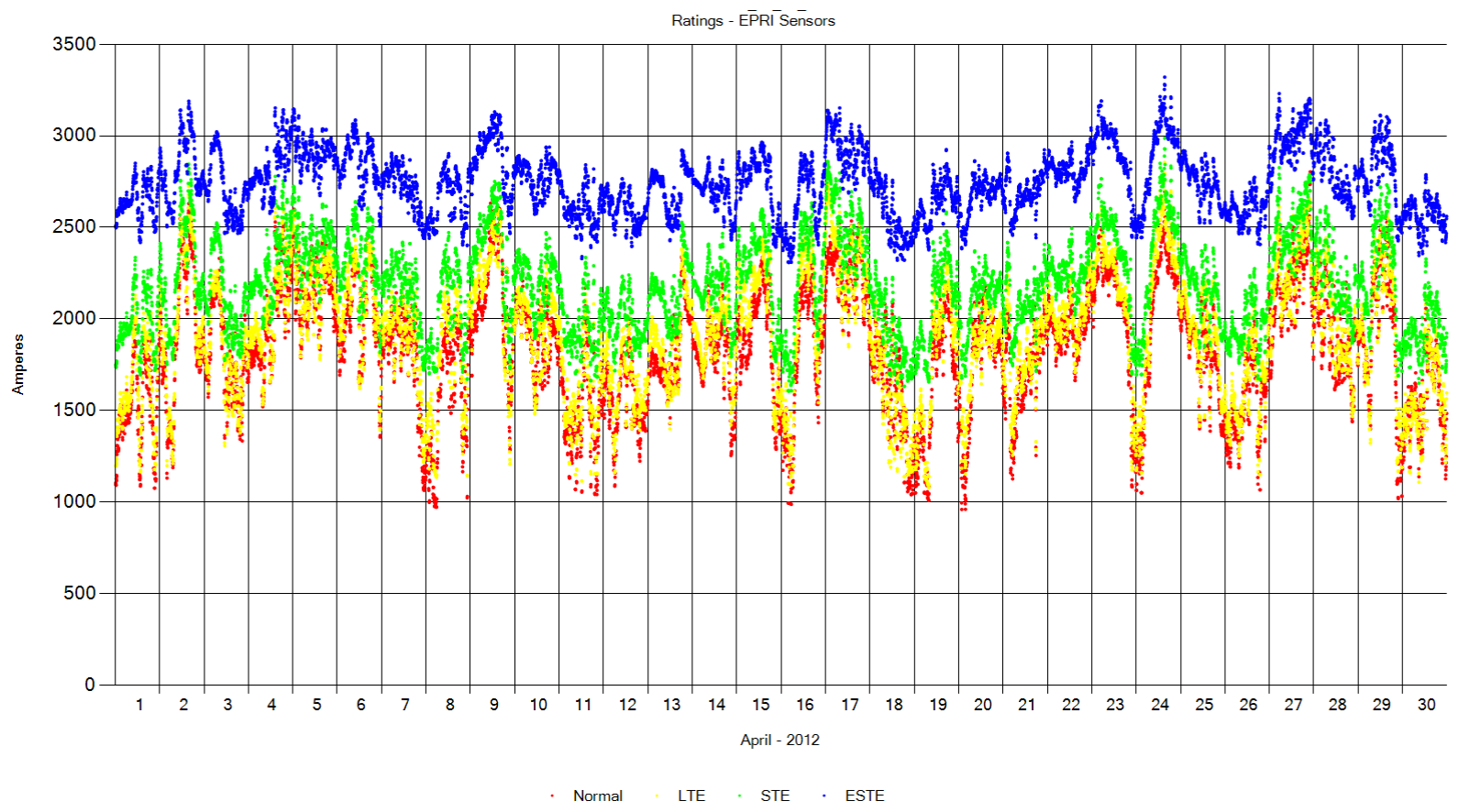

K-60 Element: Site 3_BS Plot: Ratings-EPRI Sensors 


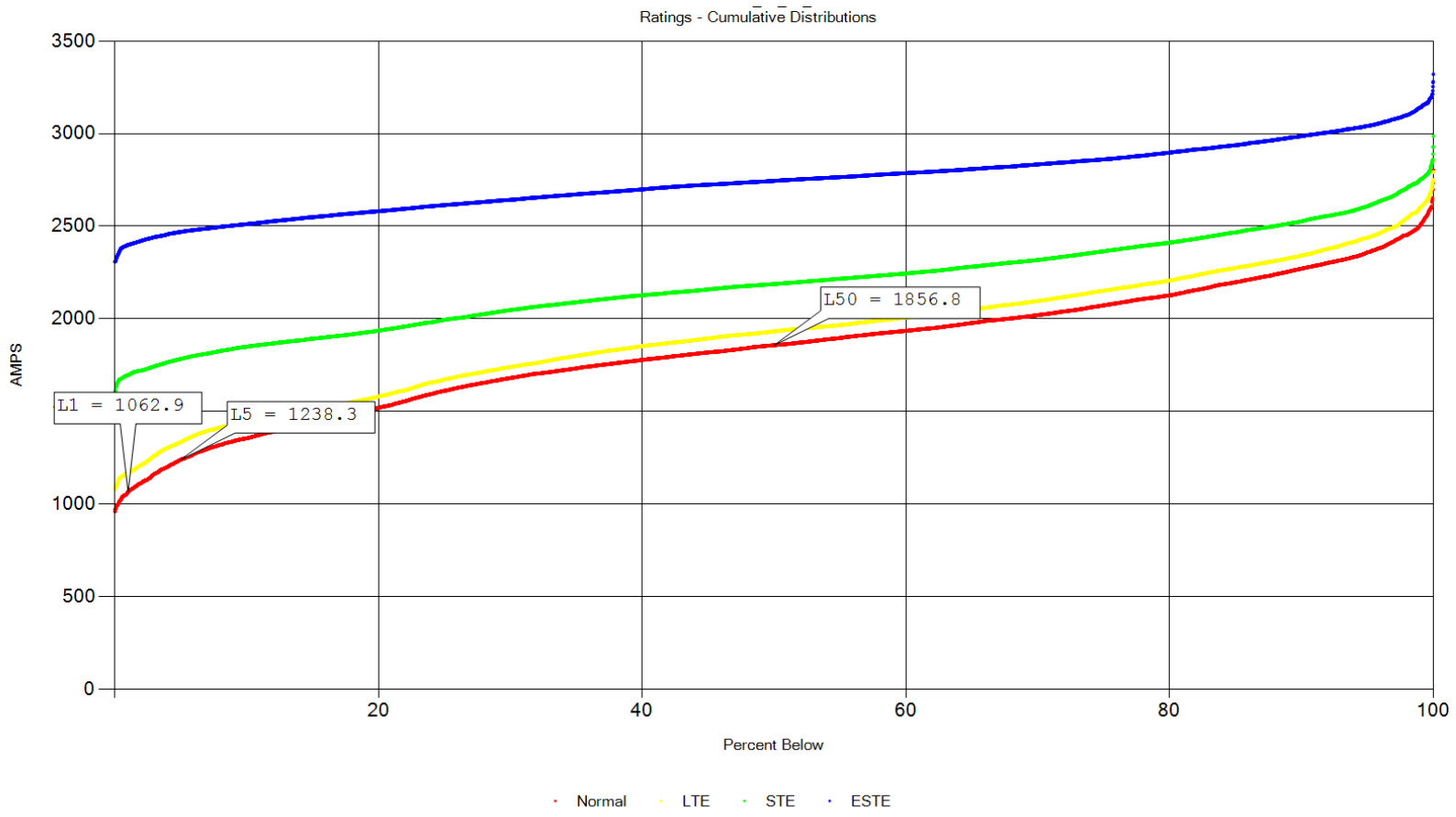

K-61 Element: Site 3_BS Plot: Rating Cum. Dist.

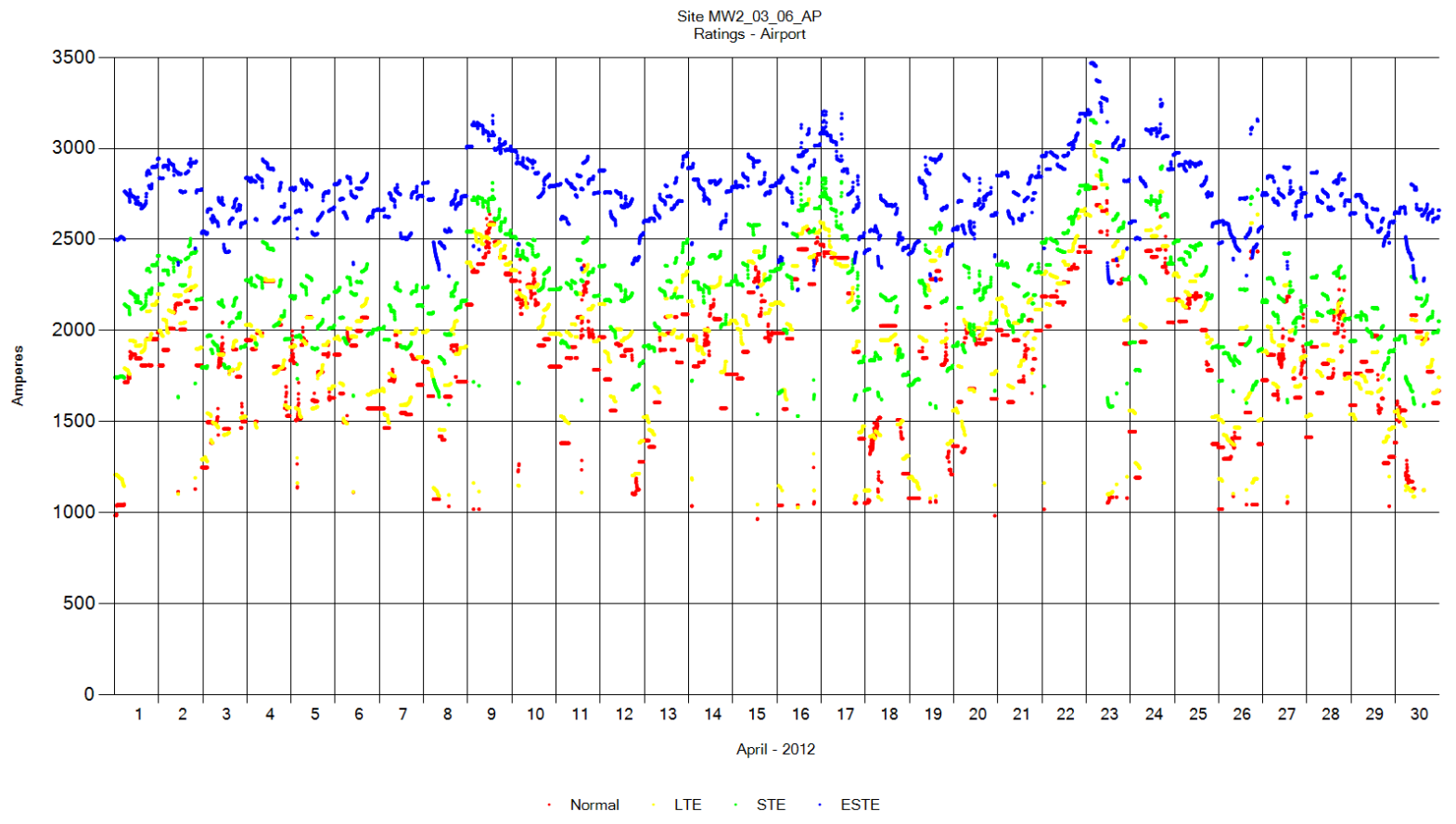

K-62 Element: Site 3_AP Plot: Ratings - Airport 


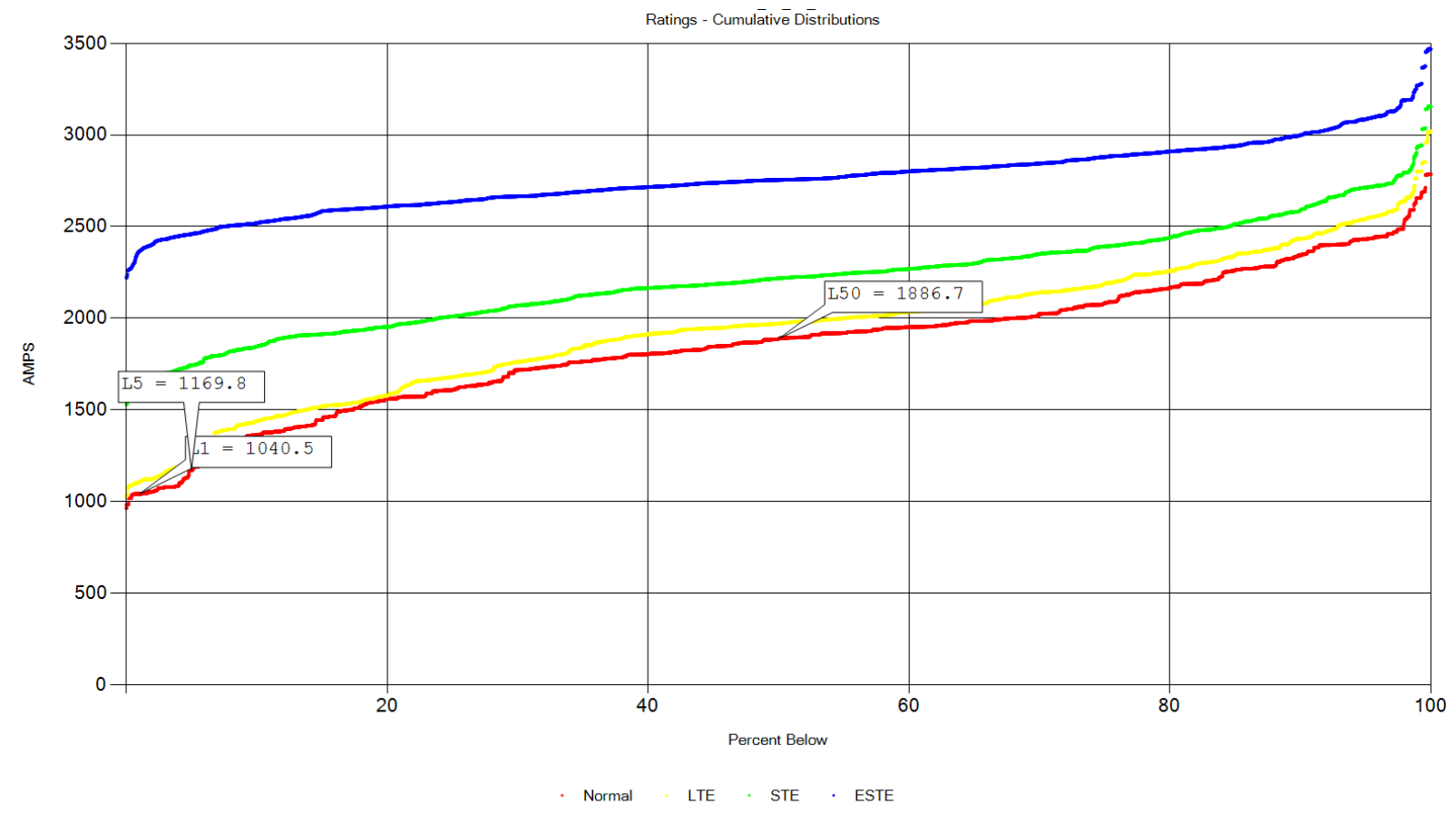

K-63 Element: Site 3_AP Plot: Rating Cum. Dist. 



\section{DATA QUALITY OF RAW DATA FILES}

As a primary focus of the study was to evaluate the reliability of various rating instruments the following data is being presented. It is a collection of data quality plots for many of the devices across the entire duration of the project. This analysis was performed on the data in the format that it is collected in from the field data logger before it is passed through any ratings software. Therefore this data set well represents the performance capabilities if there were no software and communications issues. Due to the nature of the field loggers' internal buffer a prolonged communication error of many days may still manifest itself in these figures. Analysis was done on additional elements, only those most directly involved in ratings calculations are presented.

"Bad" data is data that is outside the acceptable range, for each sensor a different range has been determined; this may not be the same as the ranges used in the rating calculations software.

"Missing" data is data that has been either flagged by the logger as missing with a specific code, or when there is a noticeable gap in the data files.

"Coasting" data is data in which the same value persists for more than one hour. This value can be neither bad nor a value that indicates missing data, for example "9999." At this point the entire initial hour is also counted towards the duration of the coast. It is common for sensors to repeat their last measured value under certain error conditions. As this repeated value is not in the bad data category then ratings calculation software would not detect the fault and continue running. Less accurate rating calculations may result from sensors with large amounts of data coasting. Coasting was not calculated for the solar radiation sensor as the condition of night (no solar radiation for many hours) would register as a coast.

"Good" data is any data that is not contained within any of the above categories.

The range of good value is shown in the table below. Note that the Sagometer height shown is from Site 3 and these limits may vary by site and over time as the natural conductor position shifts. These limits were chosen not only due to the expected parameters of these line sections but also the idiosyncrasies of the individual sensors. For example, the ThermalRate device cannot report a wind speed higher than $30 \mathrm{ft} / \mathrm{s}$ by design, so that is the appropriate limit for it; while the limits on the ultrasonic anemometer is much higher. 
Table L-1

Range of Good Values

\begin{tabular}{|l|c|c|l|}
\hline \multicolumn{1}{|c|}{ Sensor } & Lower Limit & Upper Limit & \multicolumn{1}{|c|}{ Units } \\
\hline $\begin{array}{l}\text { Sagometer } \\
\text { At Target }\end{array}$ & 35 & 45 & Feet \\
\hline Air Temp & -40 & 50 & Deg C \\
\hline Wind Speed & 0 & 98 & $\mathrm{Ft} / \mathrm{s}$ \\
\hline Solar Radiation & 0 & 1500 & $\mathrm{~W} / \mathrm{m}^{2}$ \\
\hline $\begin{array}{l}\text { EPRI Sensor } \\
\text { Temperature }\end{array}$ & 2 & 150 & $\mathrm{Deg} \mathrm{C}$ \\
\hline $\begin{array}{l}\text { ThermalRate } \\
\text { Load }\end{array}$ & 1 & 1700 & $\mathrm{Amps}$ \\
\hline $\begin{array}{l}\text { ThermalRate } \\
\text { Wind Speed }\end{array}$ & 0 & 30 & $\mathrm{Ft} / \mathrm{s}$ \\
\hline
\end{tabular}




\section{December 2011 Site 3}

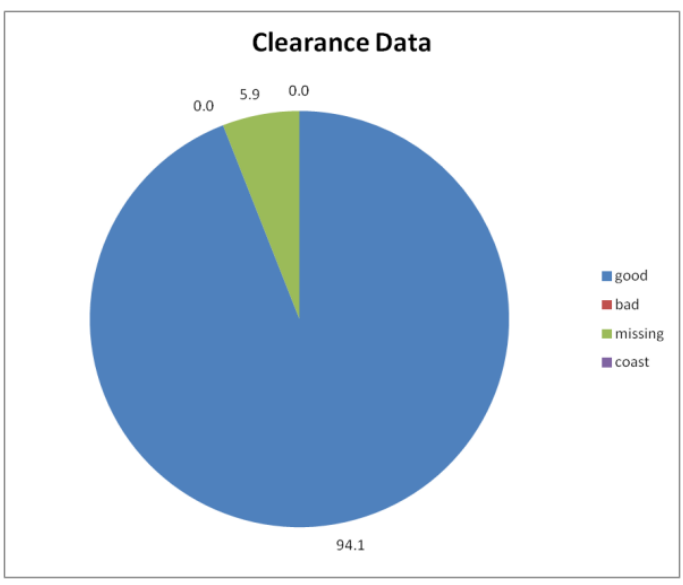

Figure L-1

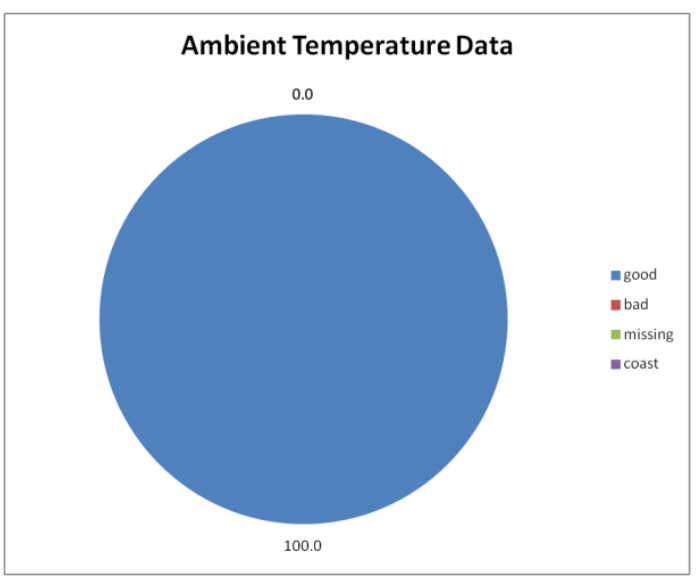

Figure L-3

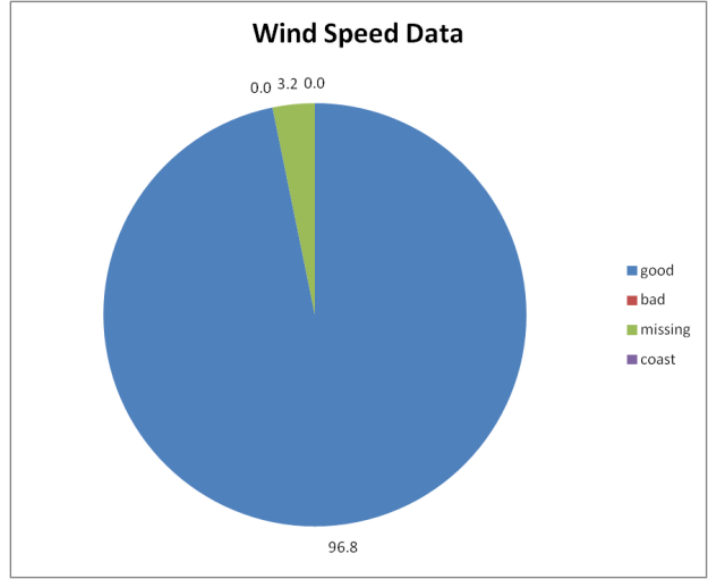

Figure L-2

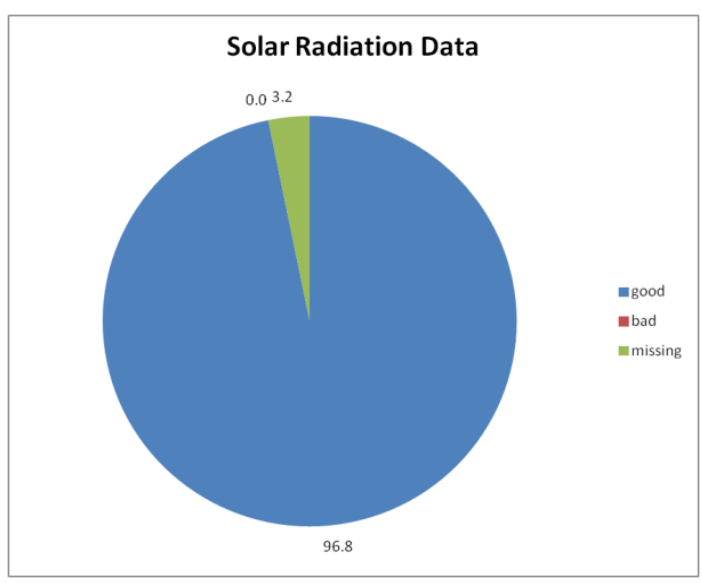

Figure L-4 


\section{December 2010 Site 3}

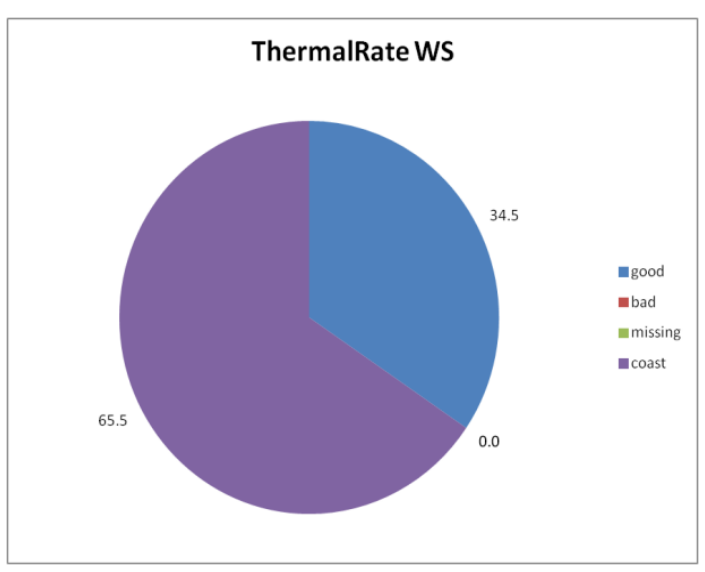

Figure L-5

\section{January 2011 Site 3}

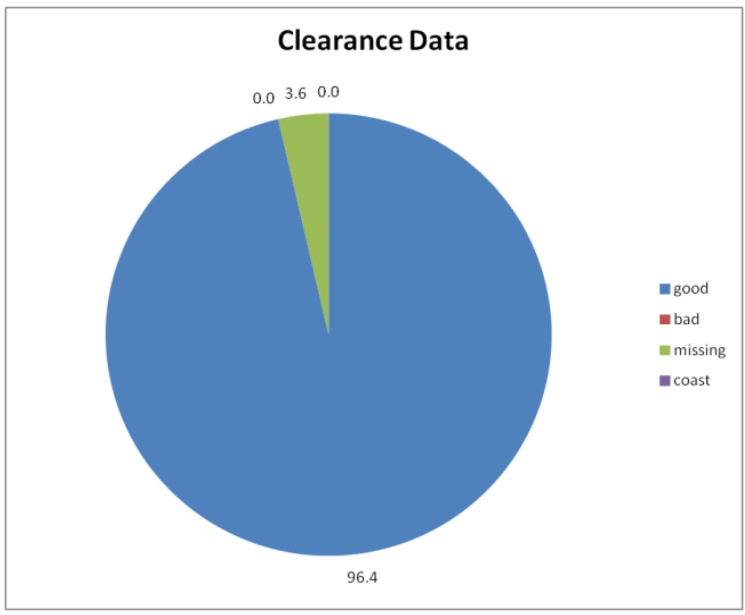

Figure L-7

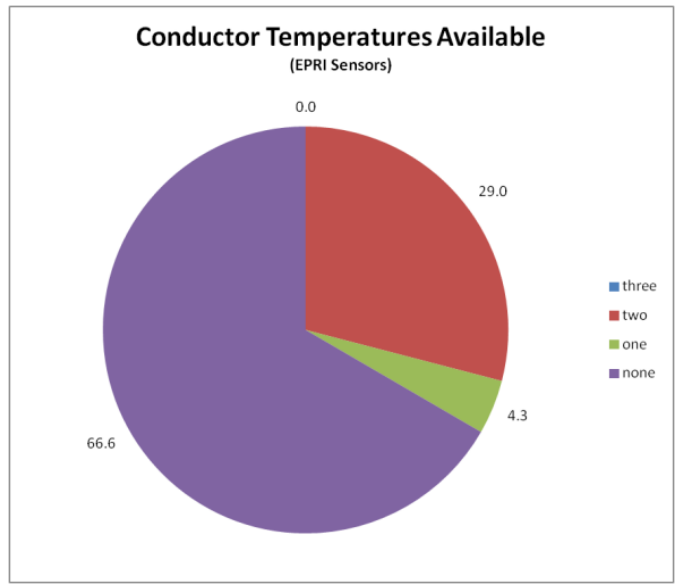

Figure L-6

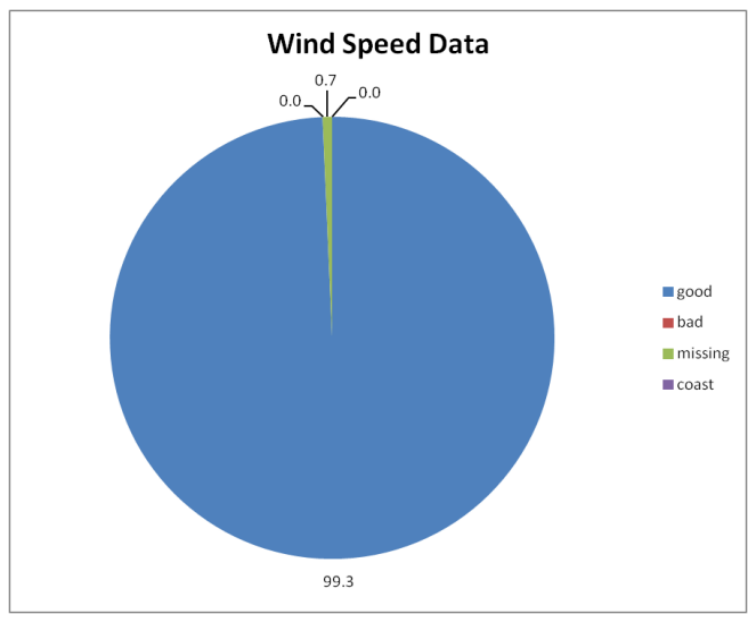

Figure L-8 


\section{January 2011 Site 3}

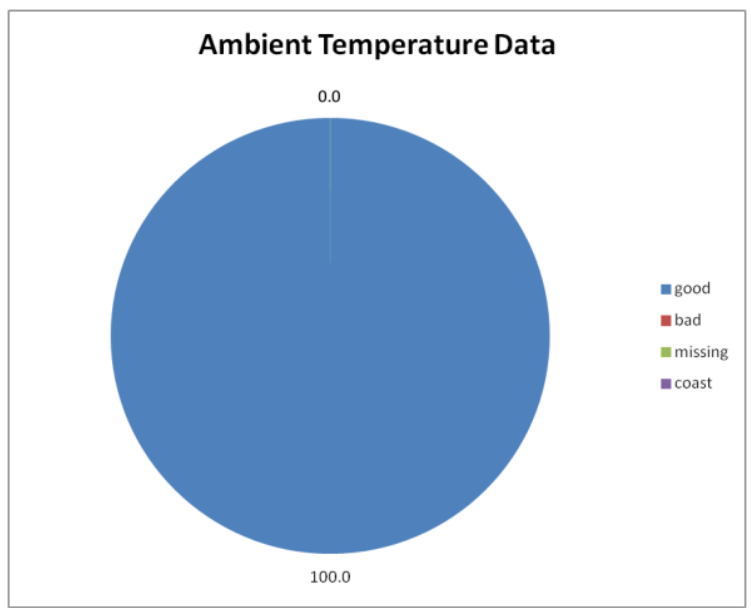

Figure L-9

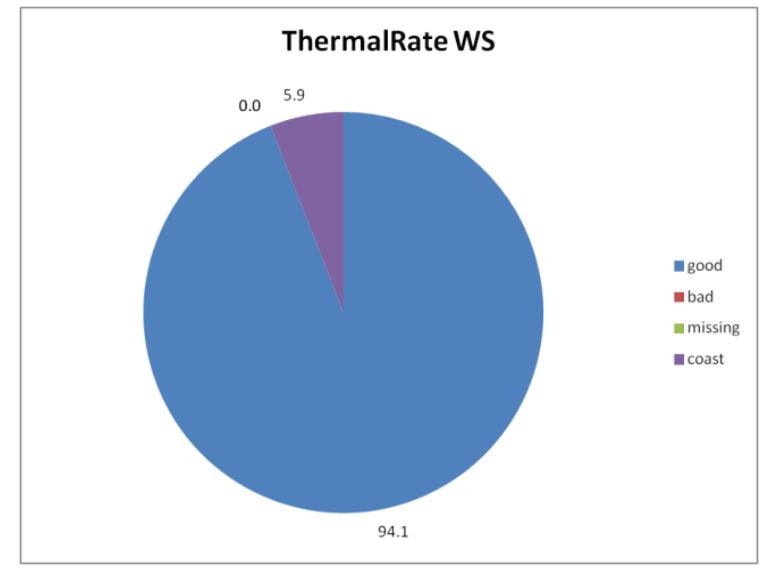

Figure L-11

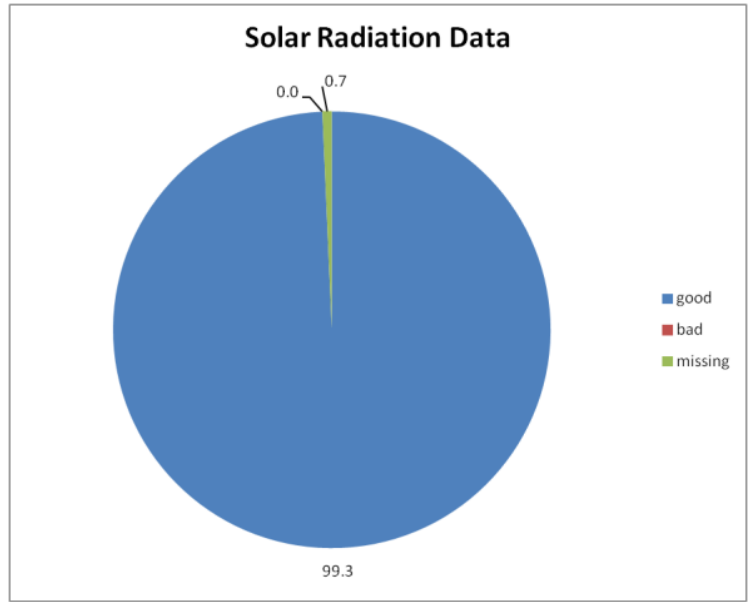

Figure L-10

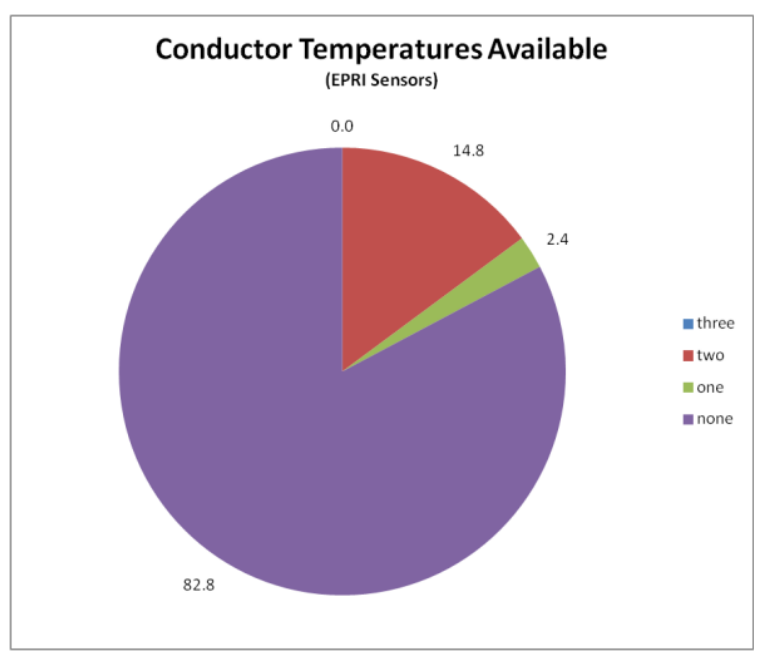

Figure L-12 


\section{February 2011 Site 3}

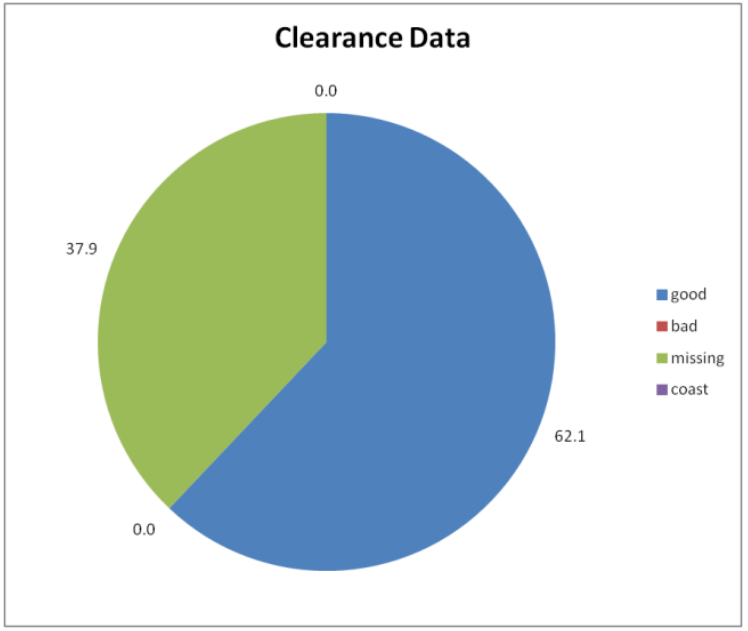

Figure L-13

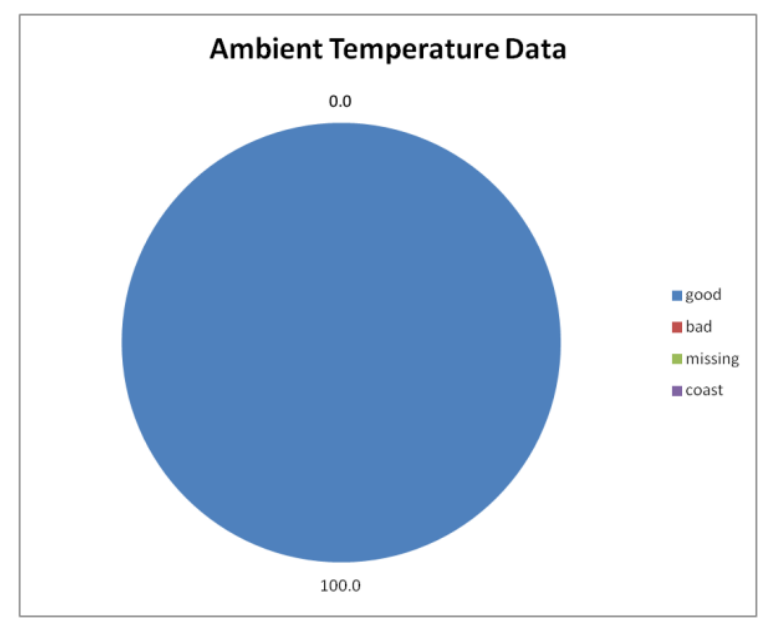

Figure L-15

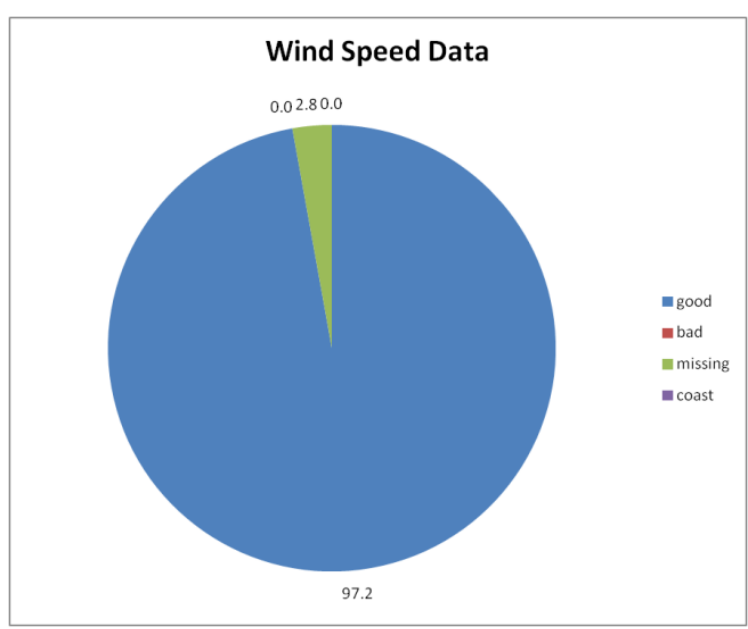

Figure L-14

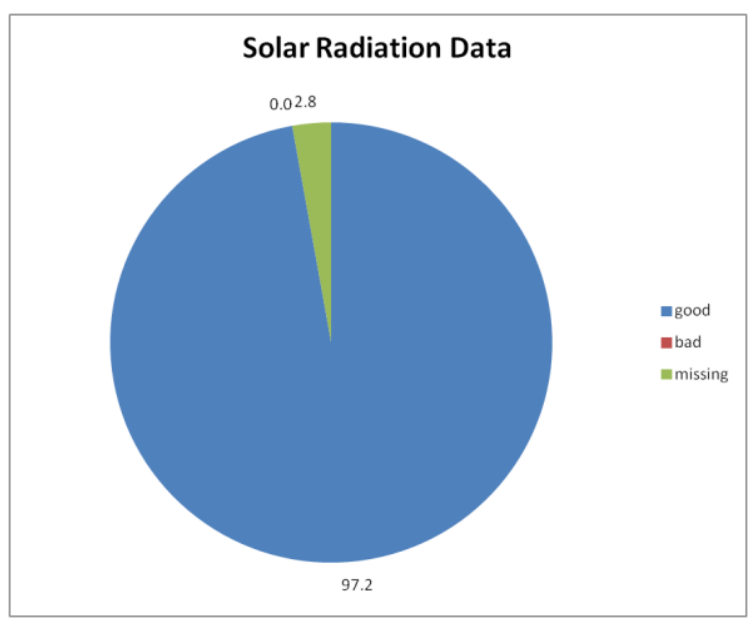

Figure L-16 
February 2011 Site 3

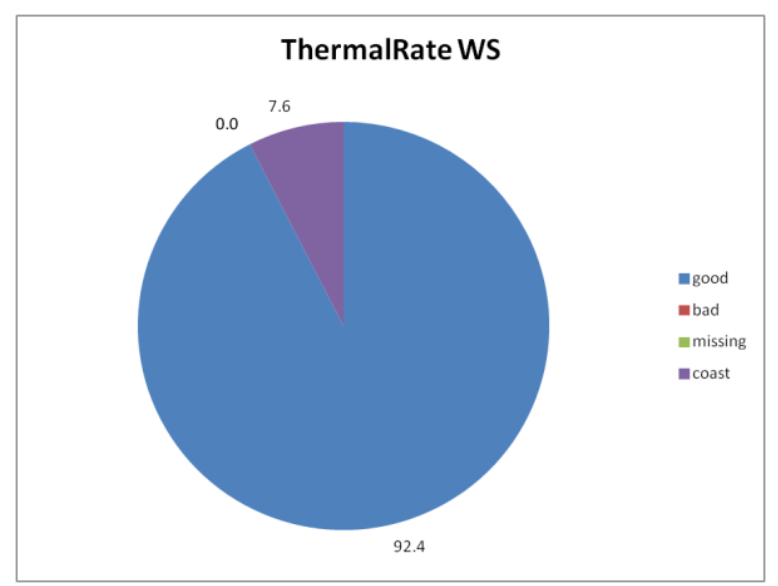

Figure L-17

\section{March 2011 Site 3}

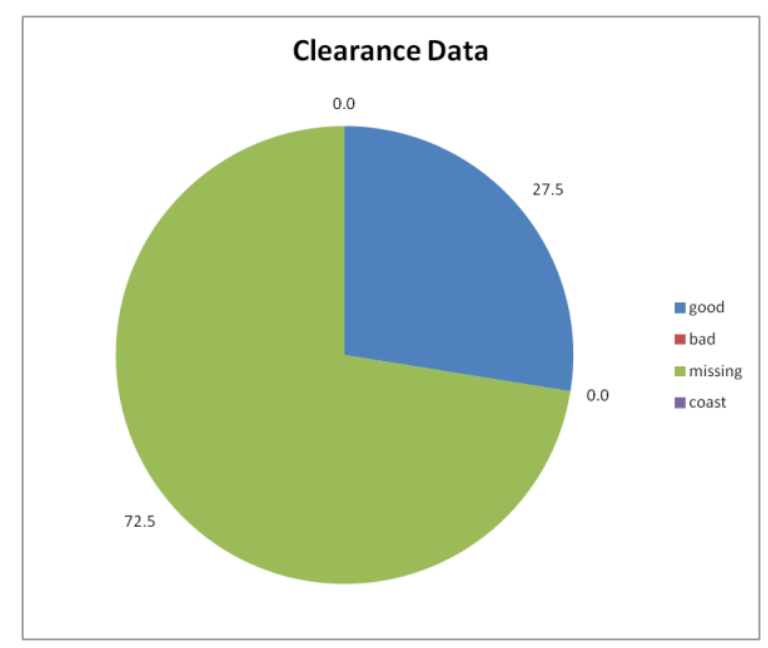

Figure L-19

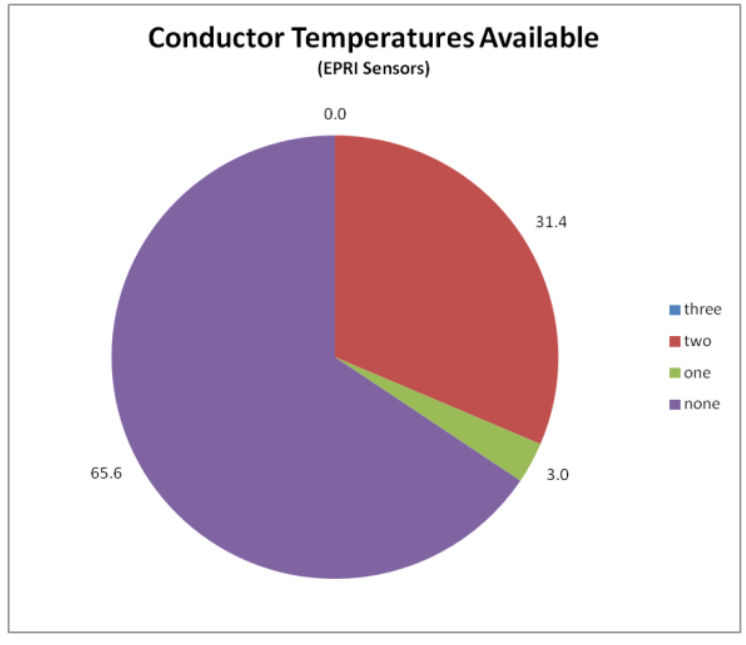

Figure L-18

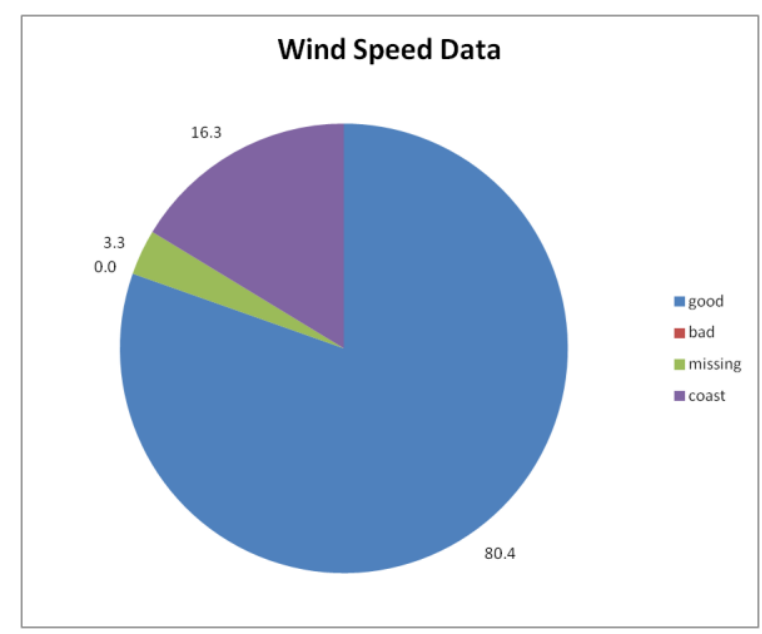

Figure L-20 
March 2011 Site 3

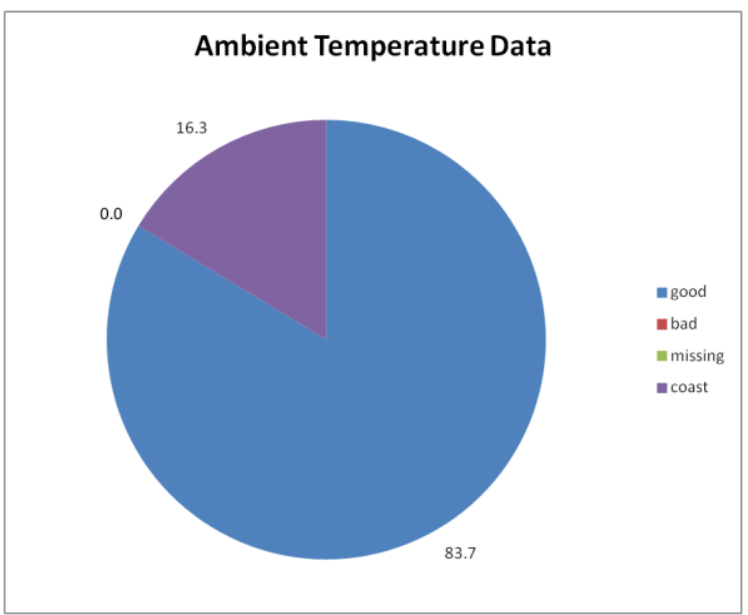

Figure L-21

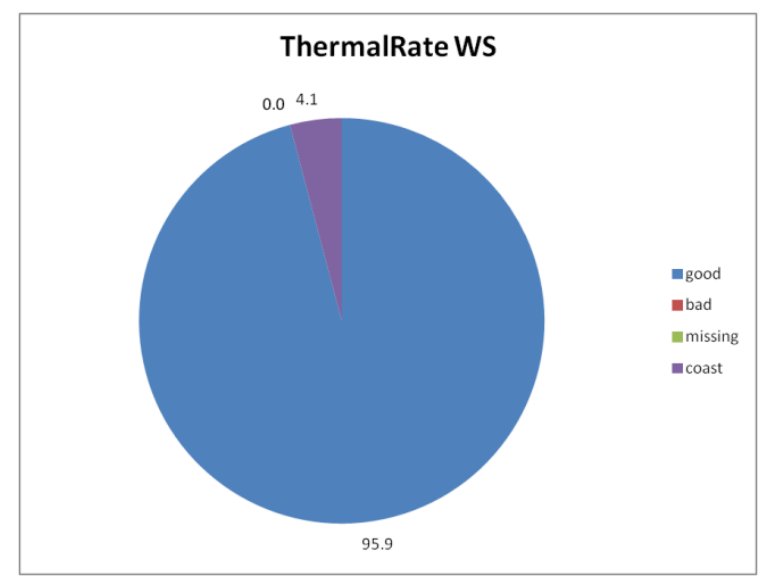

Figure L-23

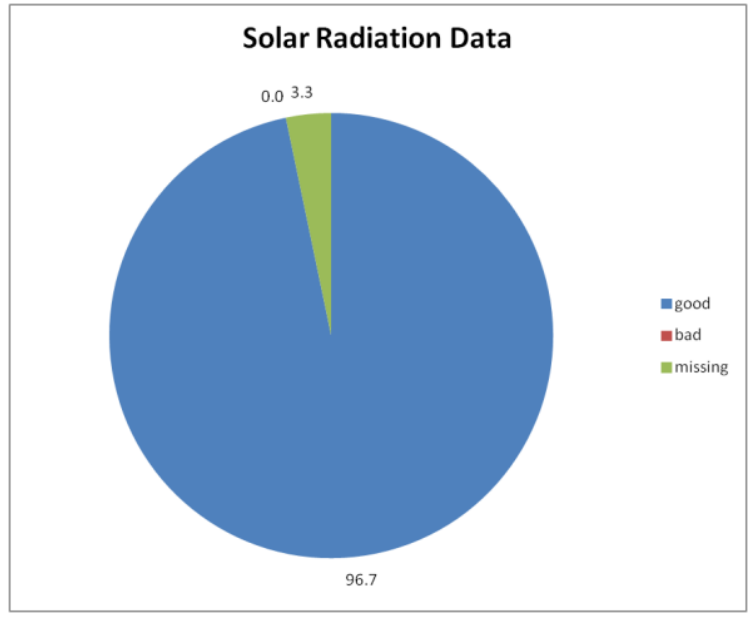

Figure L-22

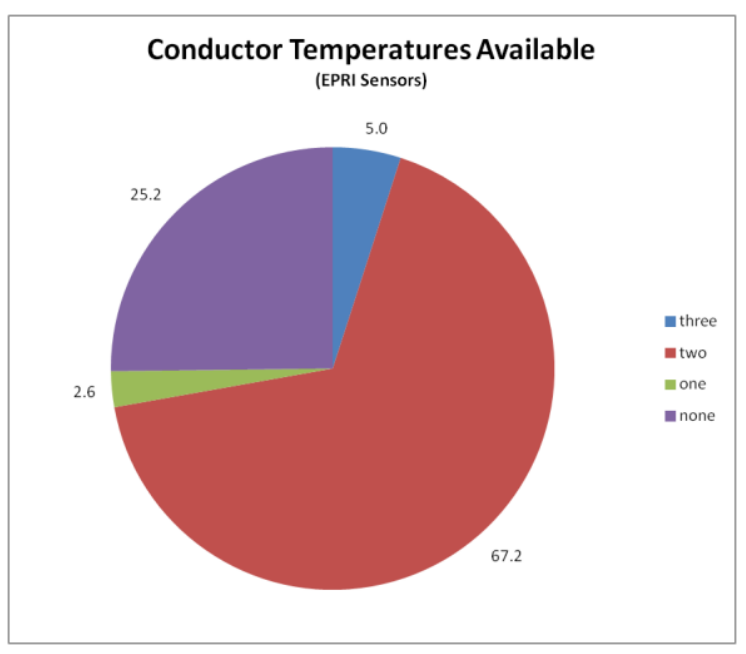

Figure L-24 


\section{April 2011 Site 3}

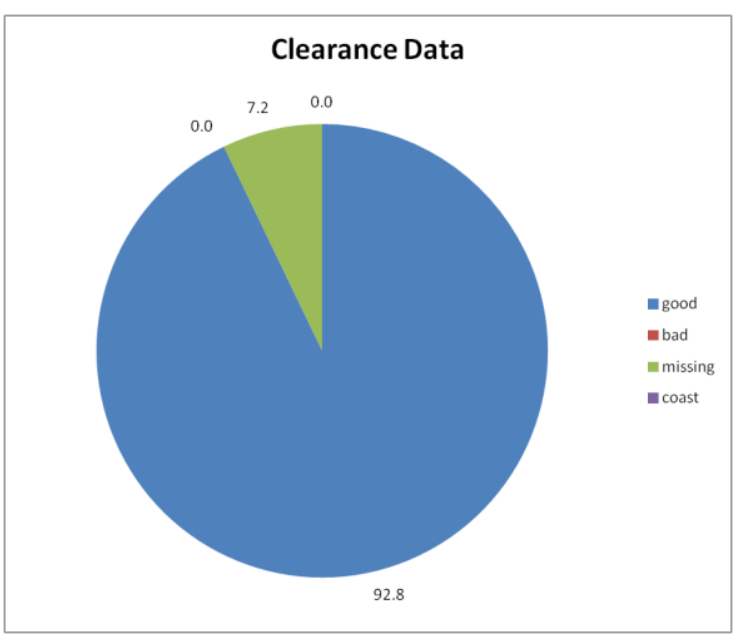

Figure L-25

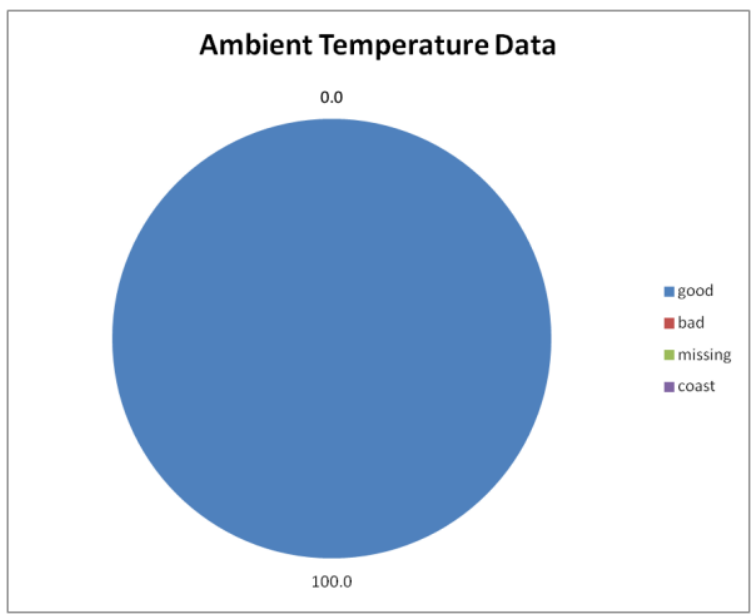

Figure L-27

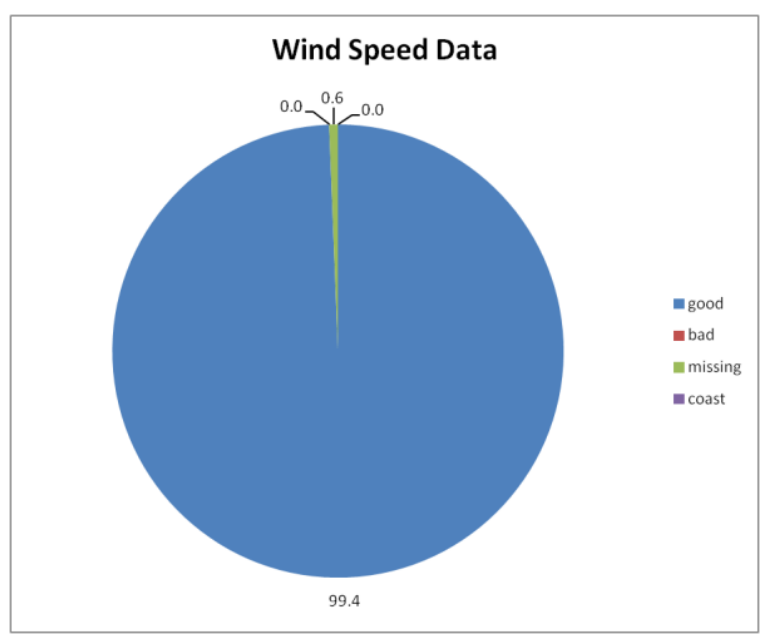

Figure L-26

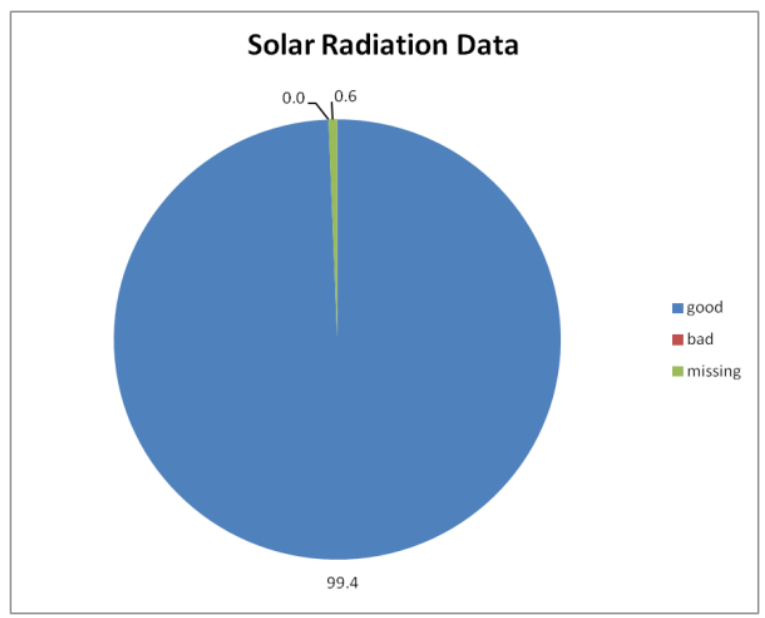

Figure L-28 


\section{April 2011 Site 3}

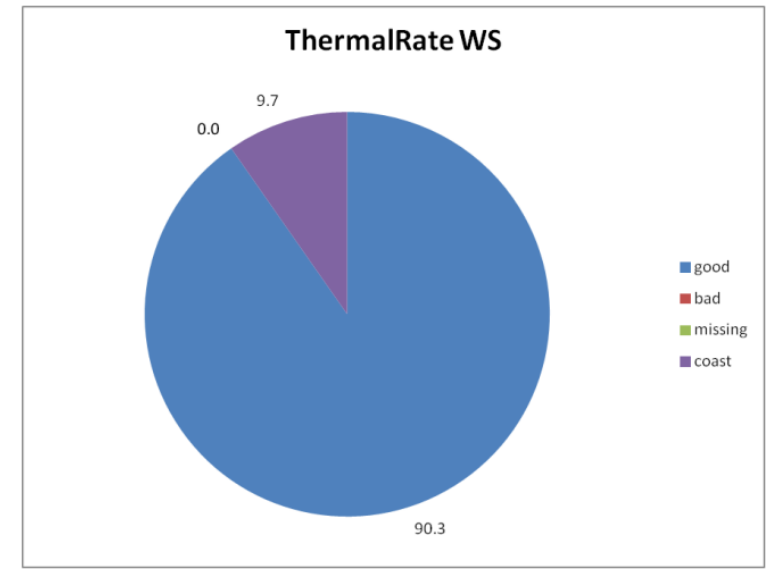

Figure L-29

\section{May 2011 Site 3}

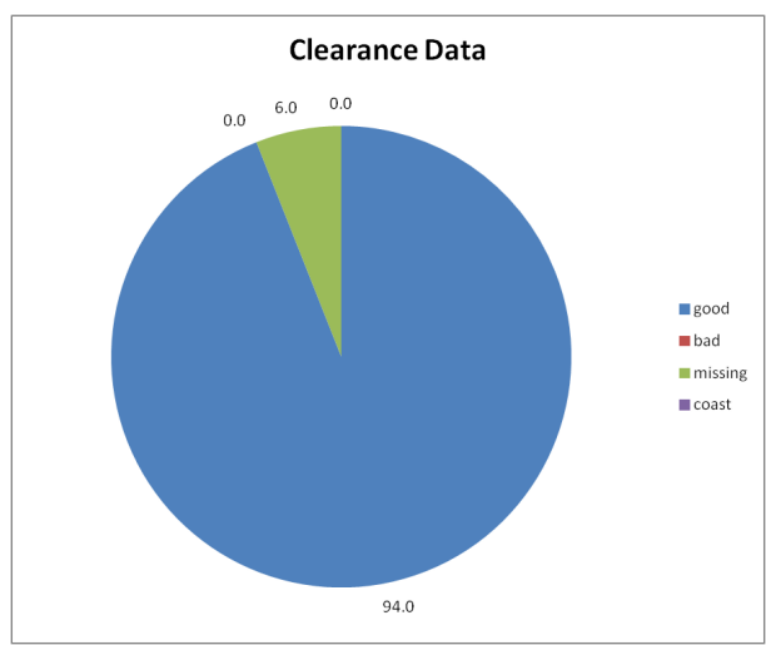

Figure L-31

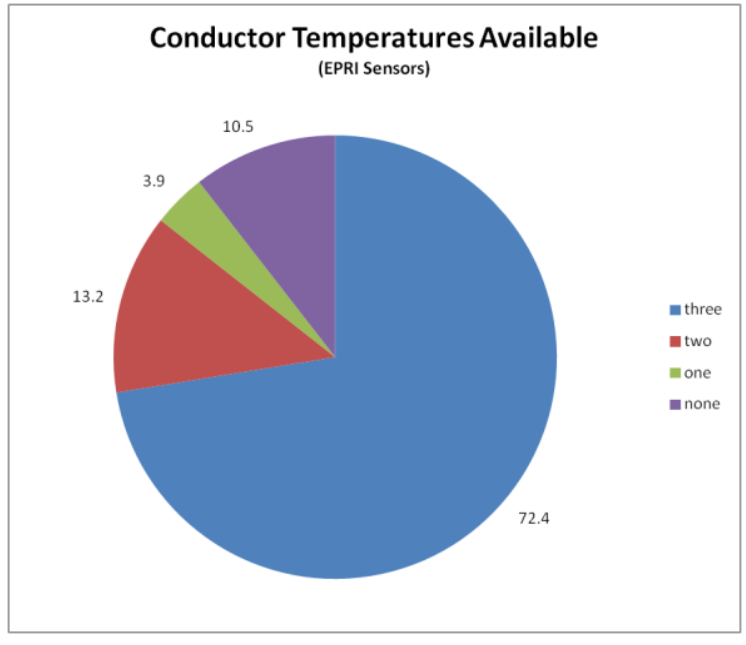

Figure L-30

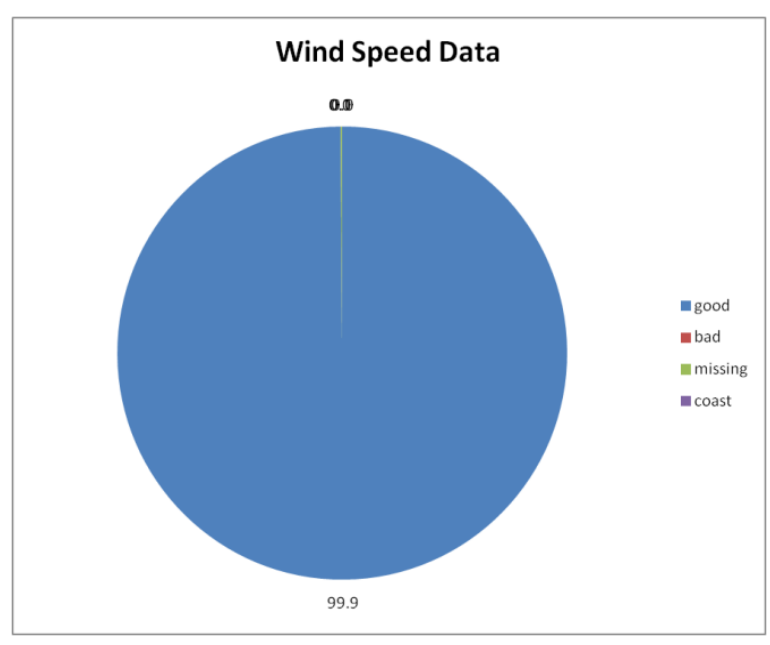

Figure L-32 
May 2011 Site 3

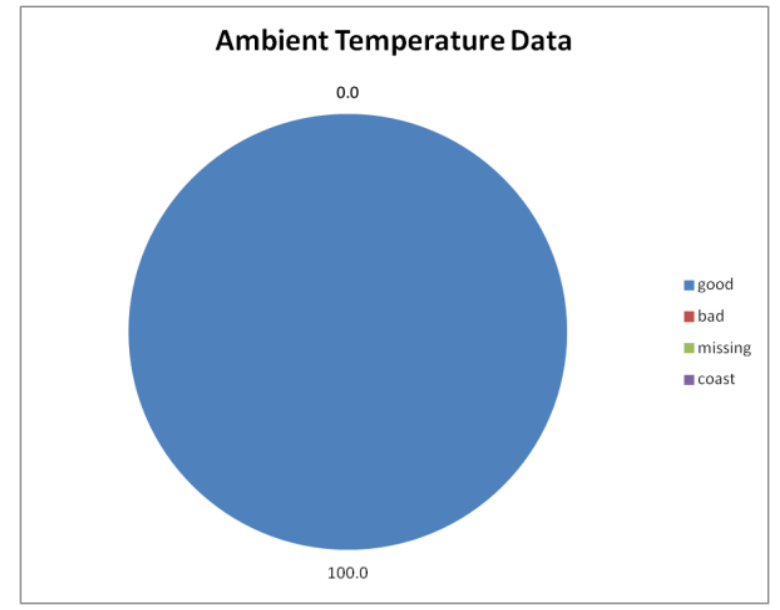

Figure L-33

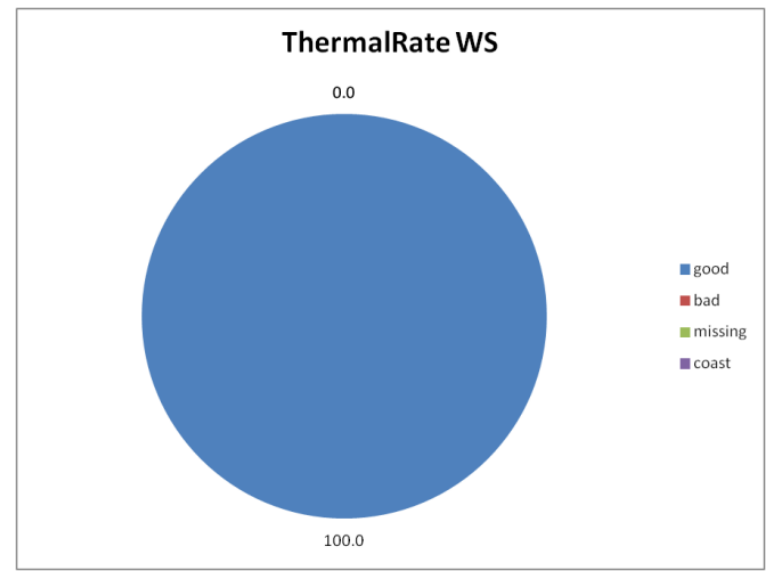

Figure L-35

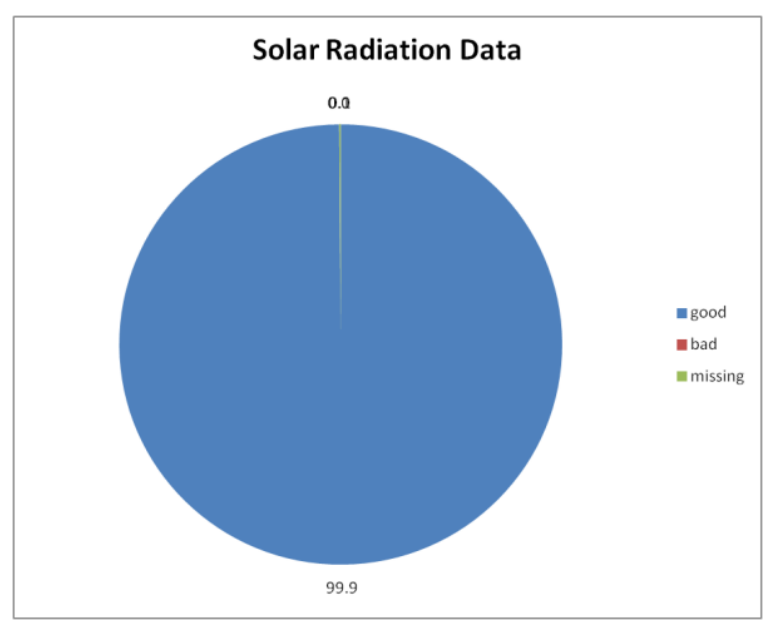

Figure L-34

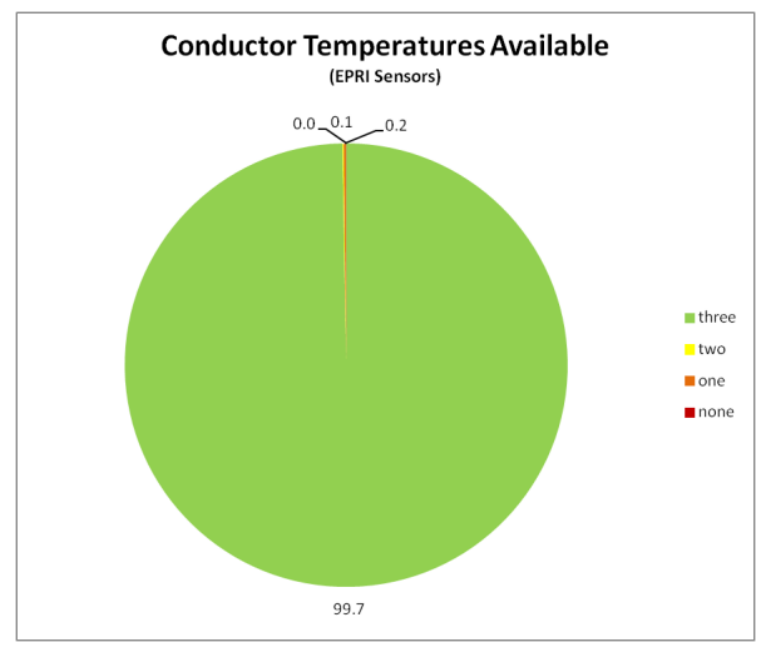

Figure L-36 


\section{June 2011 Site 3}

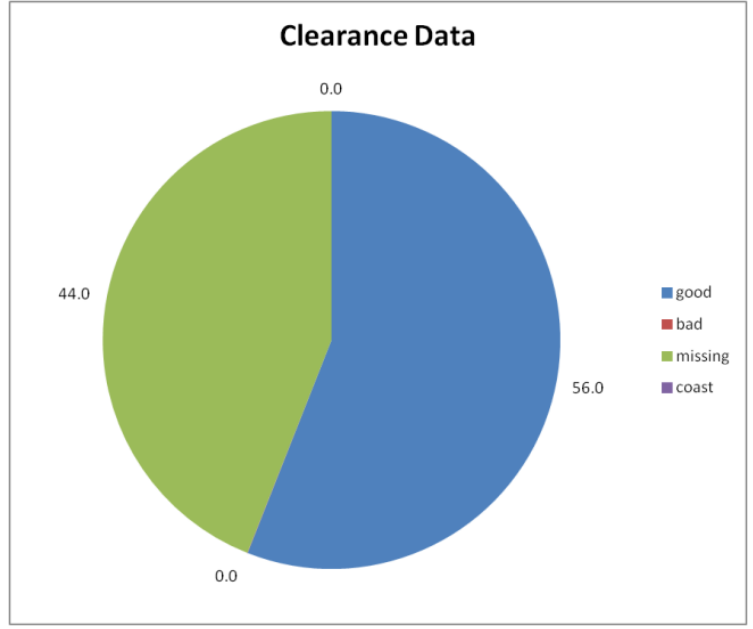

Figure L-37

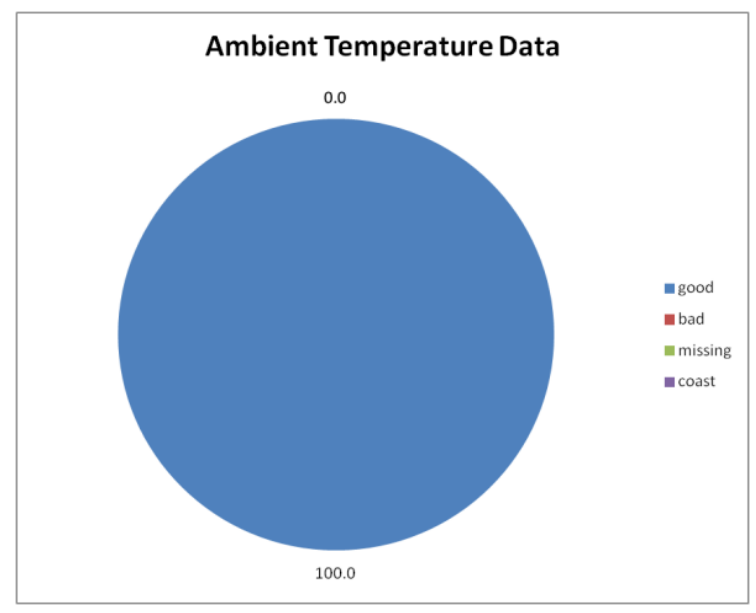

Figure L-39

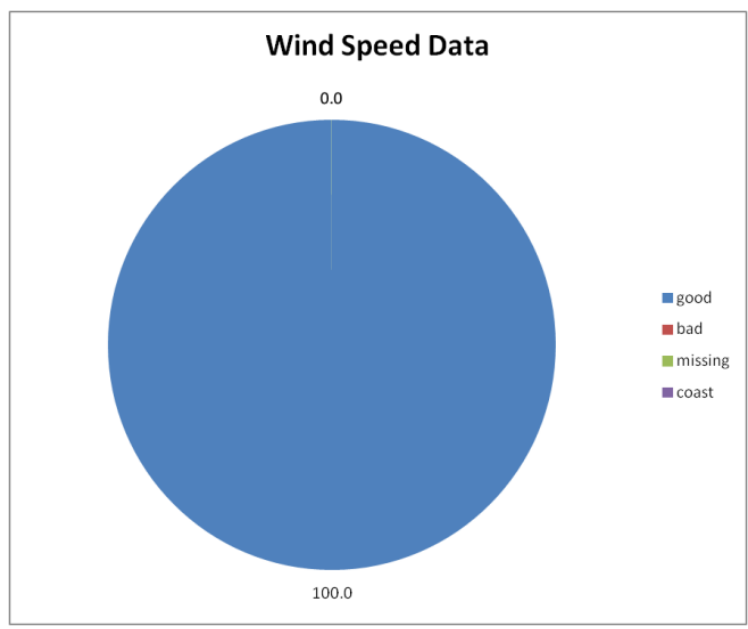

Figure L-38

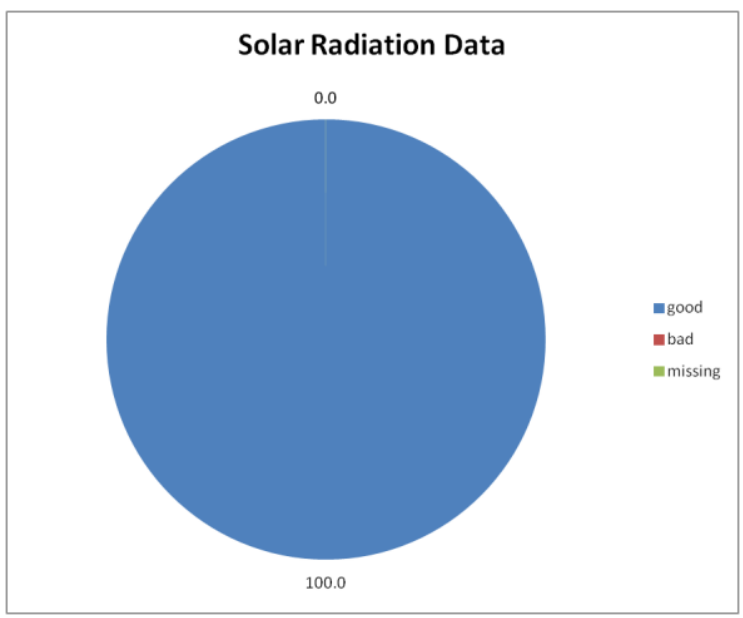

Figure L-40 


\section{June 2011 Site 3}

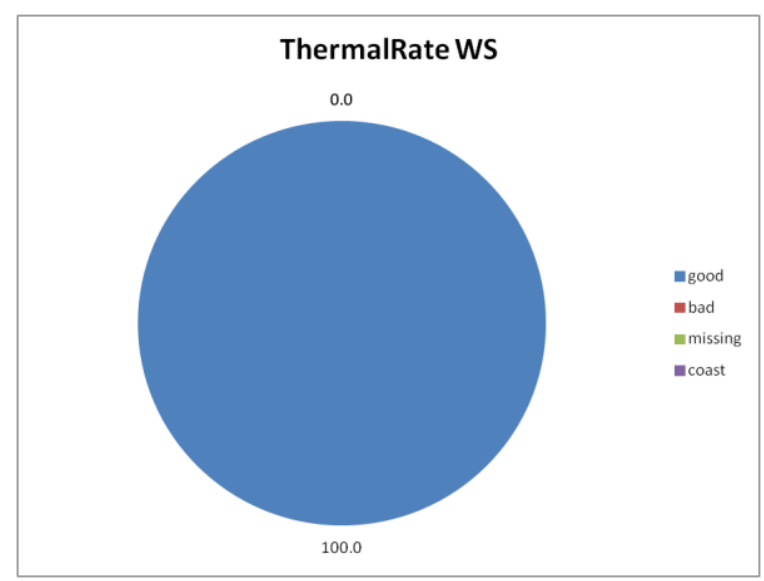

Figure L-41

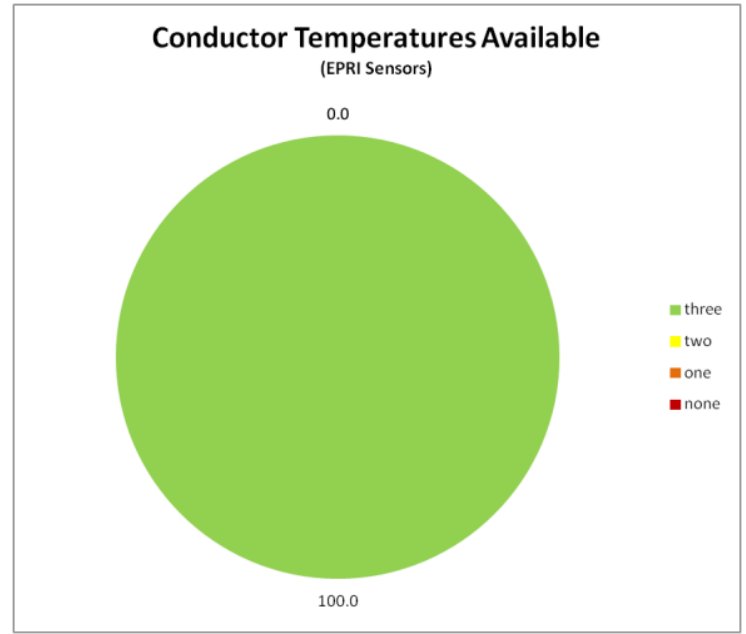

Figure L-42 


\section{July 2011 Site 3}

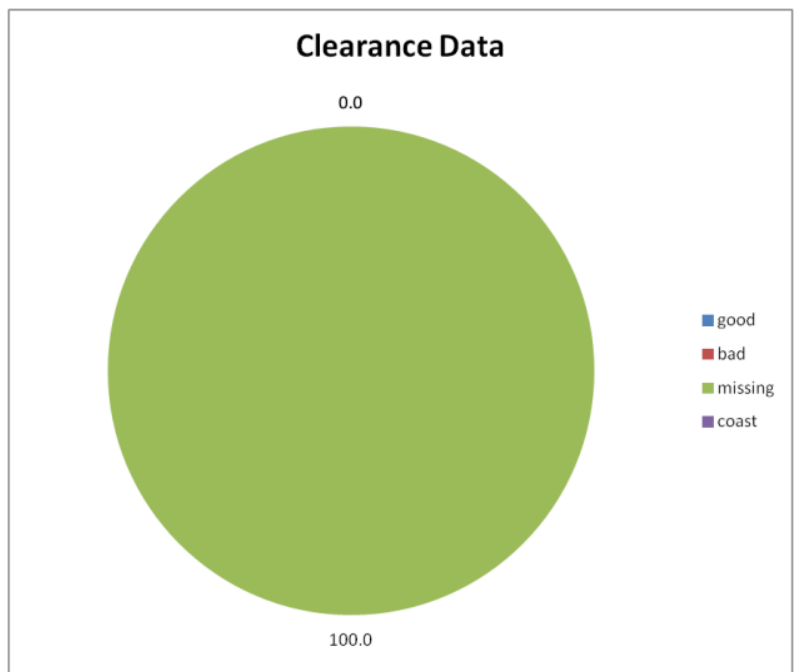

Figure L-43

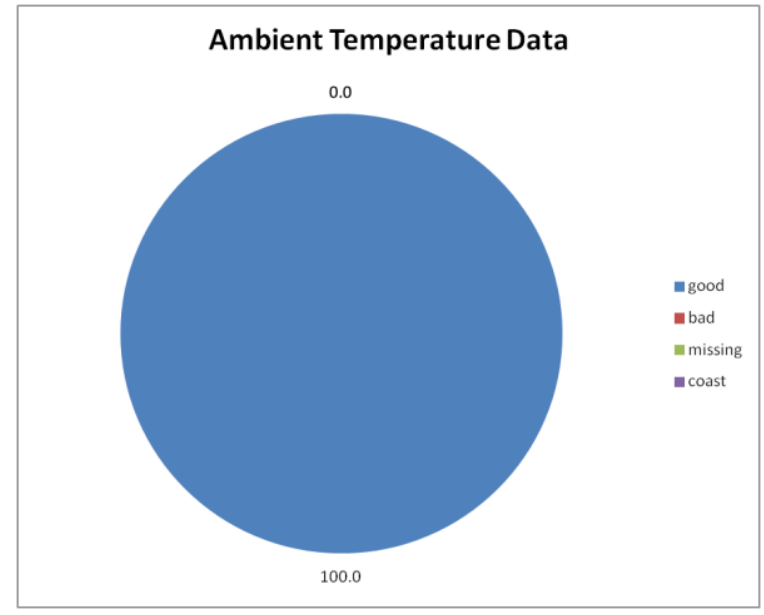

Figure L-45

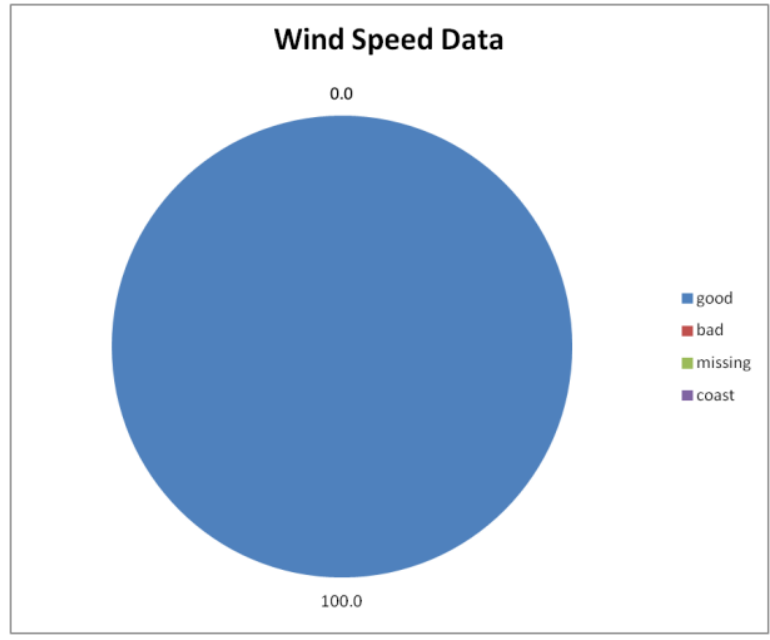

Figure L-44

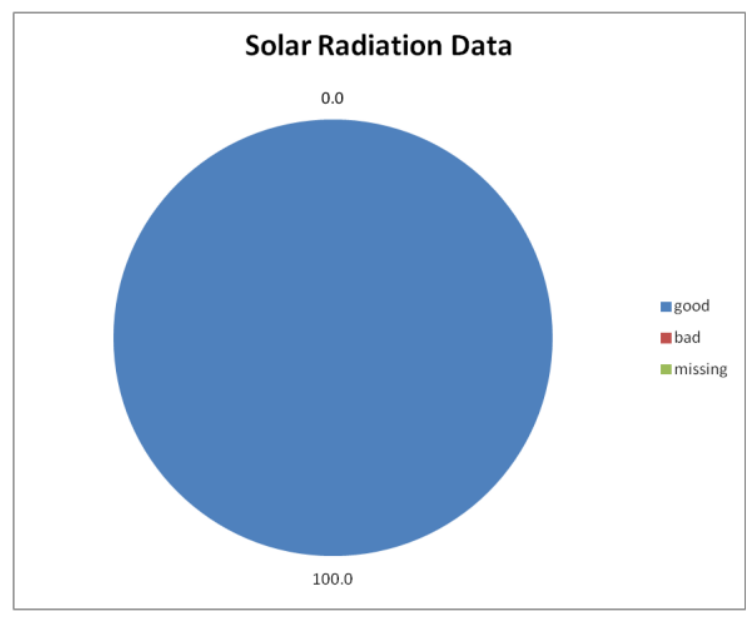

Figure L-46 


\section{July 2011 Site 3}

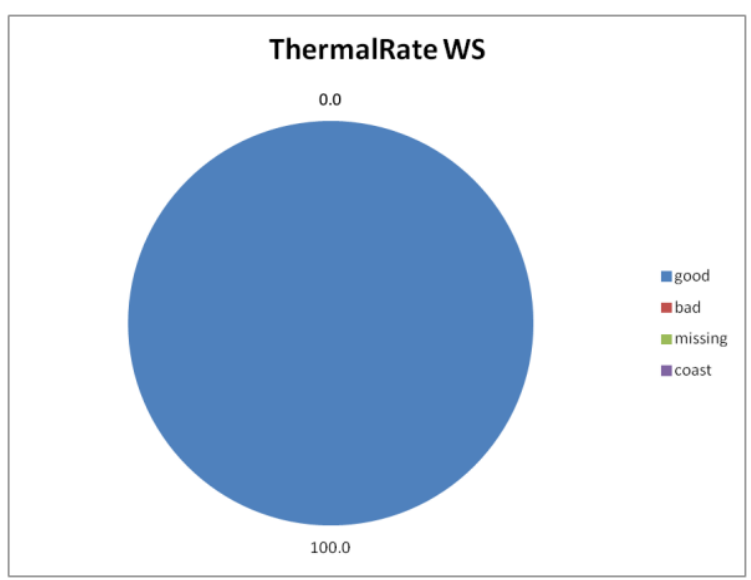

Figure L-47

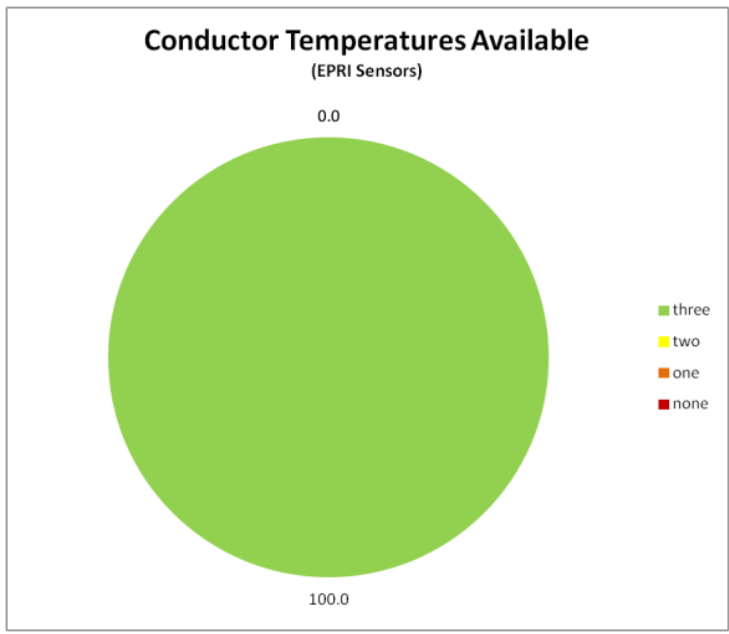

Figure L-48

\section{August 2011 Site 3}
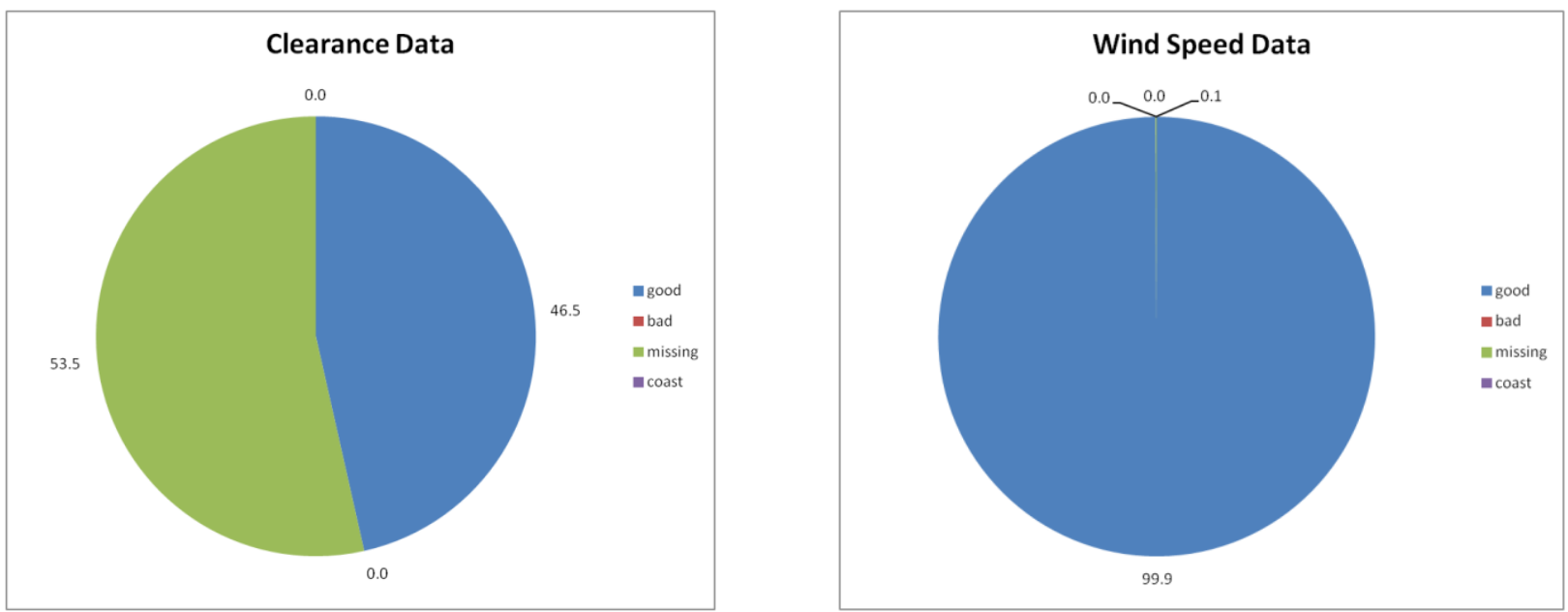

Figure L-49

Figure L-50 


\section{August 2011 Site 3}

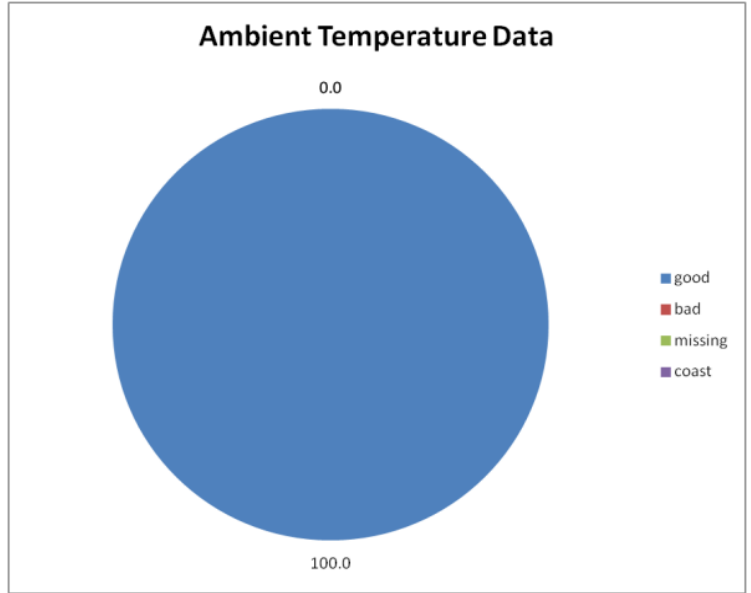

Figure L-51

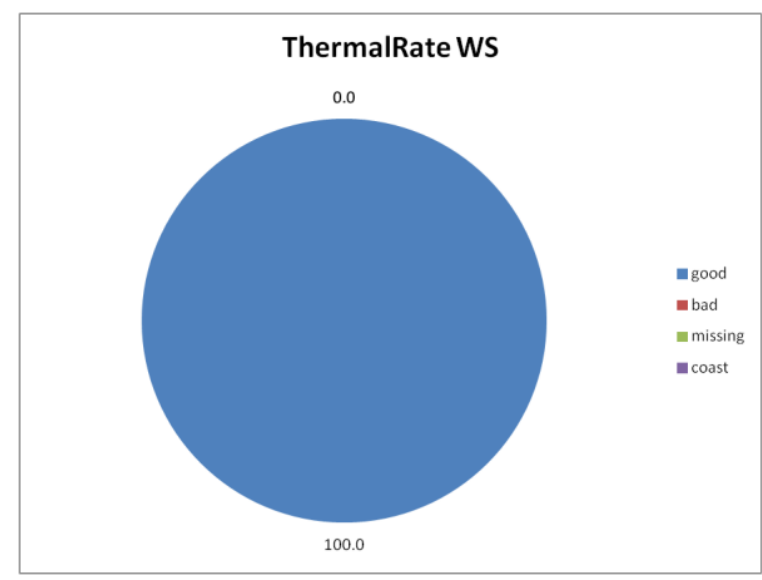

Figure L-53

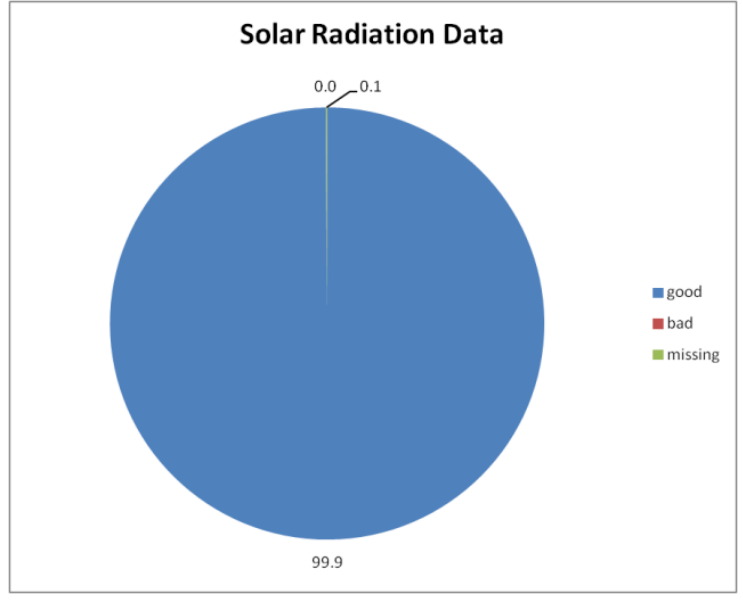

Figure L-52

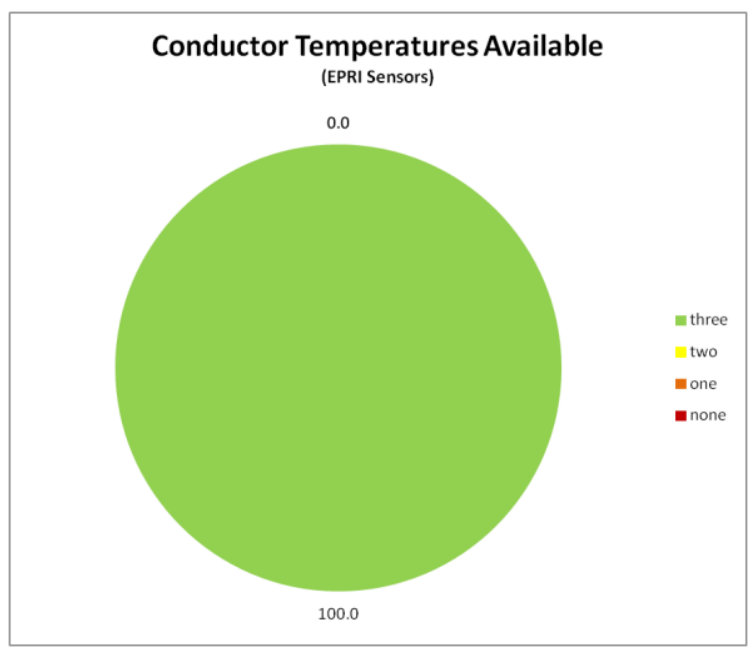

Figure L-54 


\section{September 2011 Site 3}

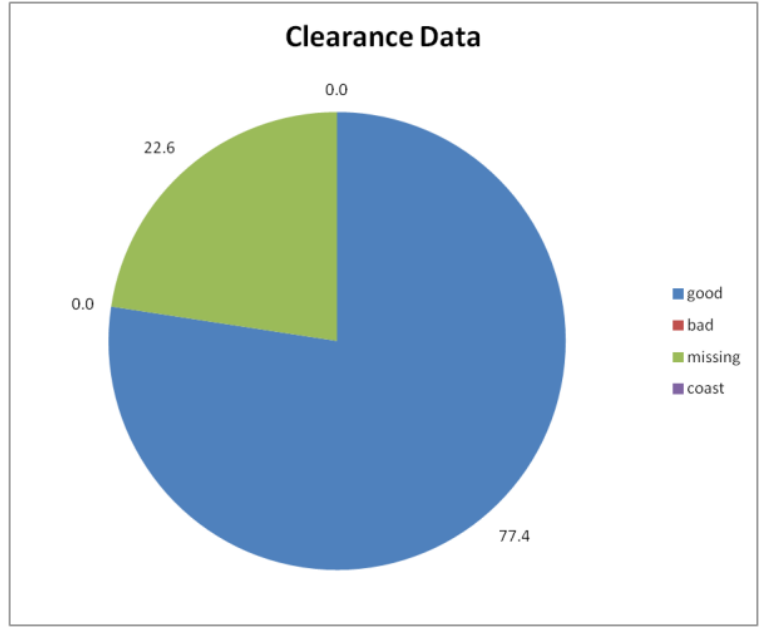

Figure L-55

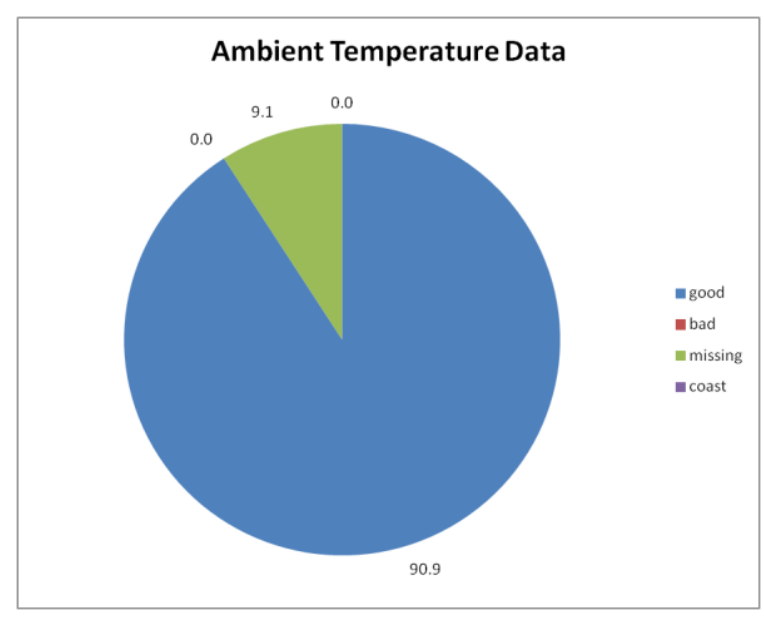

Figure L-57

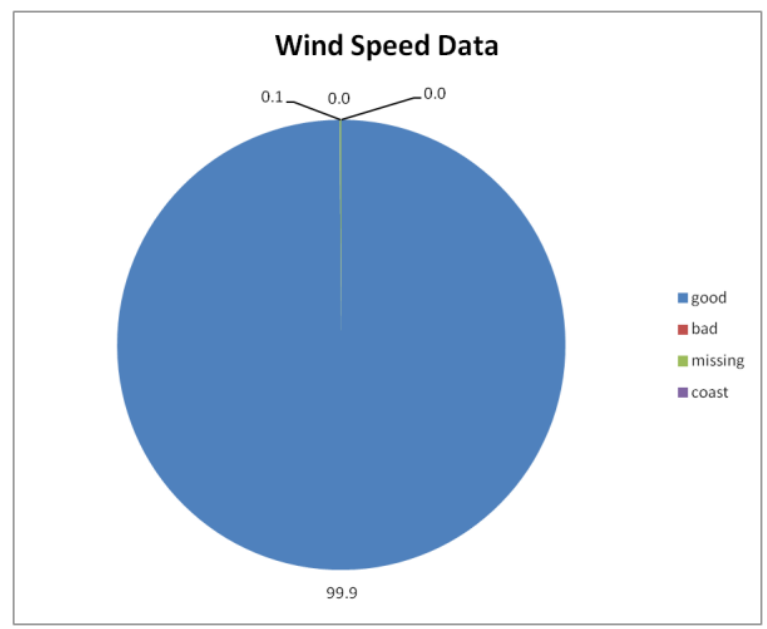

Figure L-56

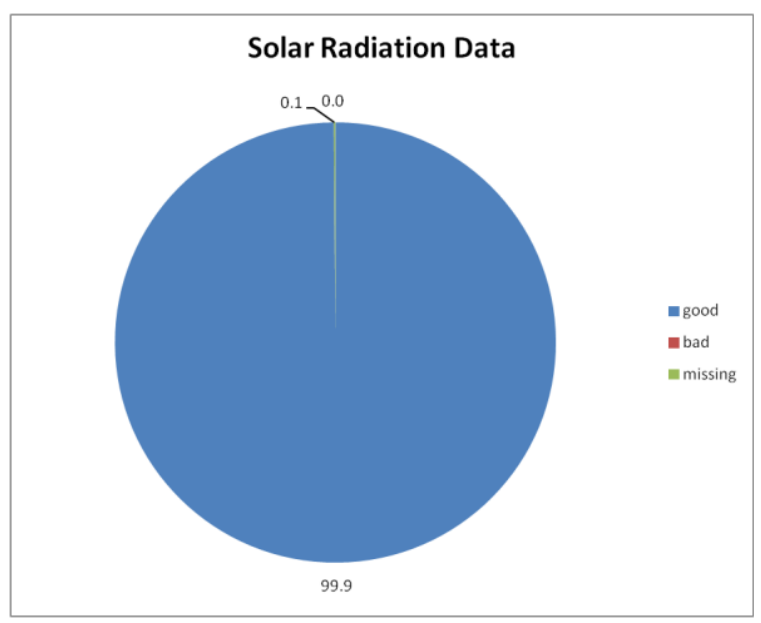

Figure L-58 


\section{September 2011 Site 3}

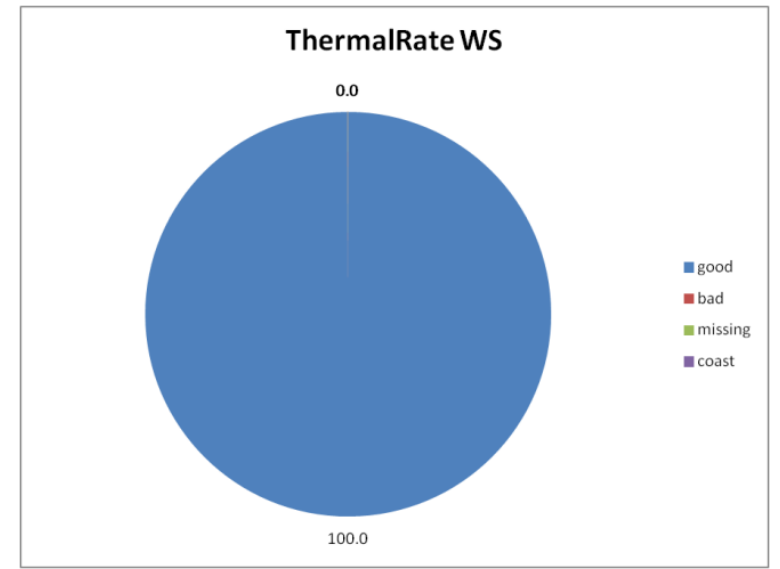

Figure L-59

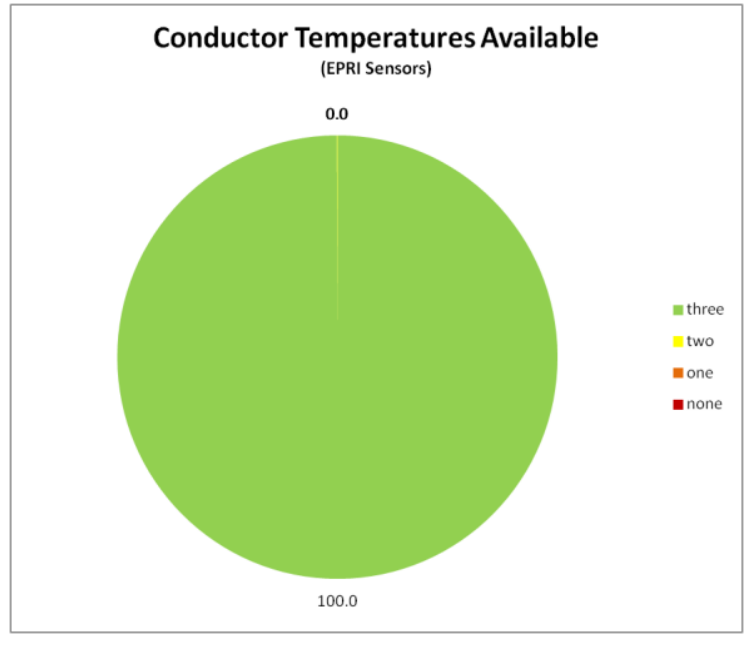

Figure L-60

\section{October 2011 Site 3}

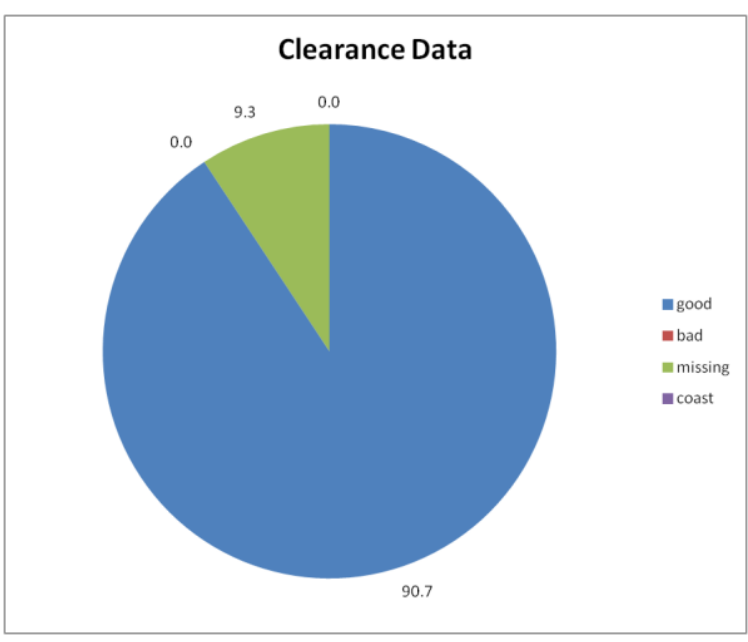

Figure L-61

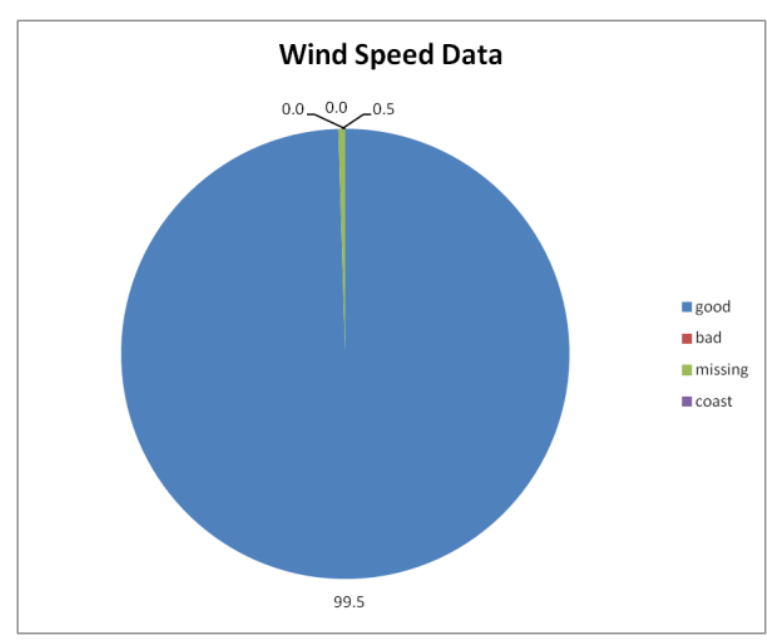

Figure L-62 


\section{October 2011 Site 3}

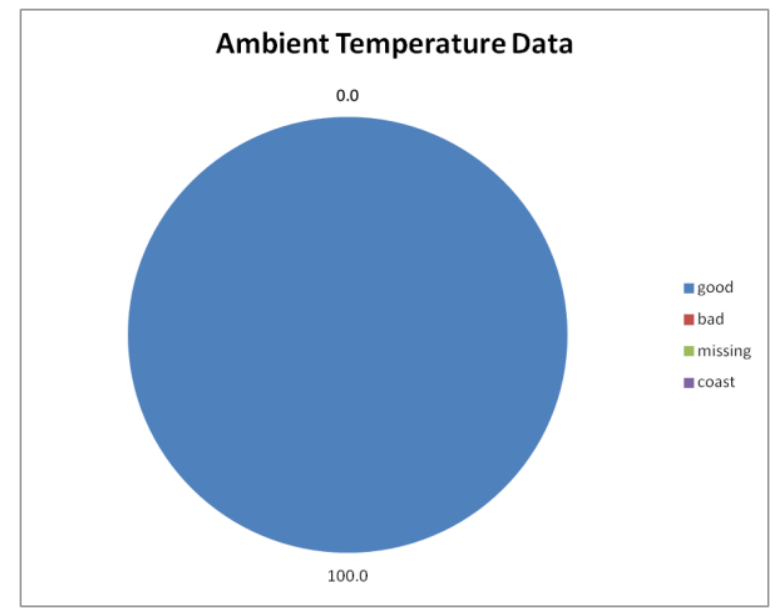

Figure L-63

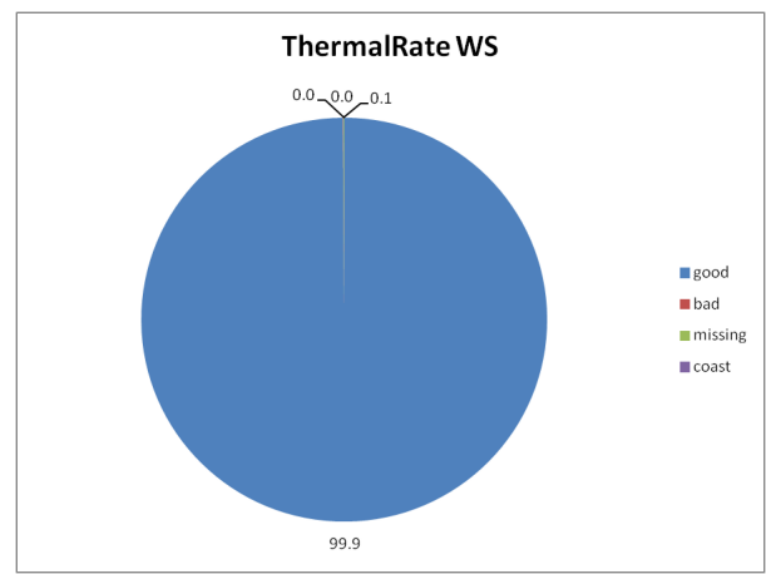

Figure L-65

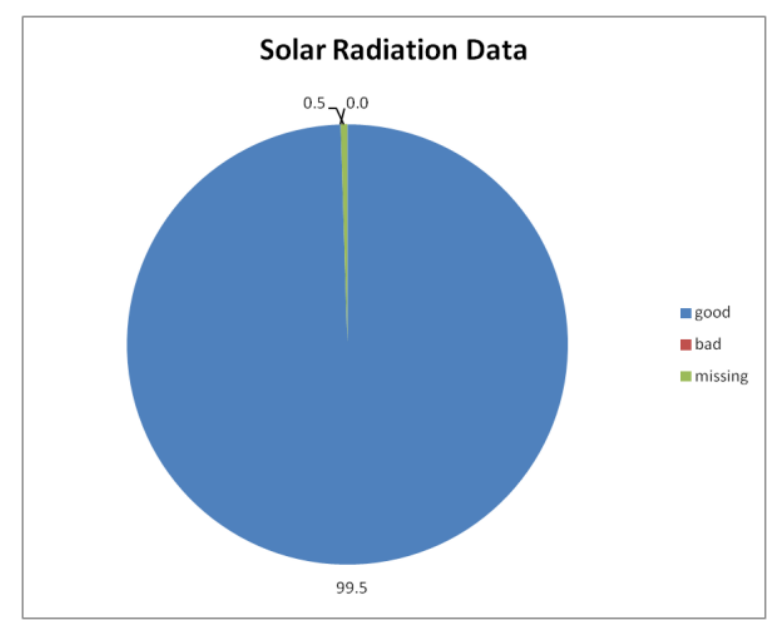

Figure L-64

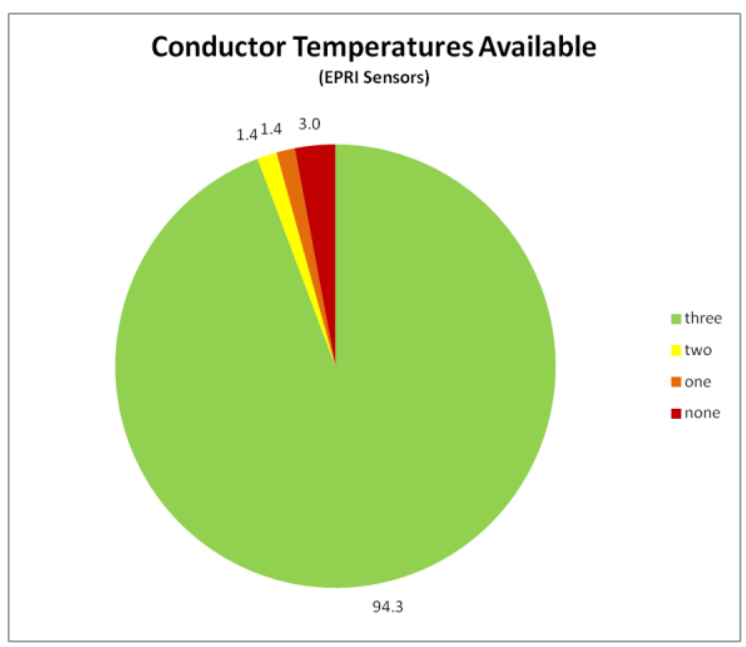

Figure L-66 


\section{November 2011 Site 3}

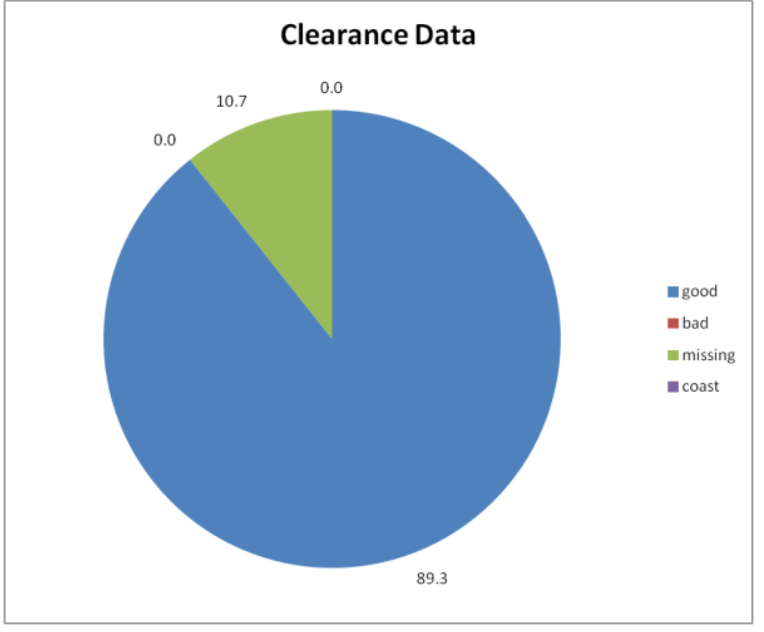

Figure L-67

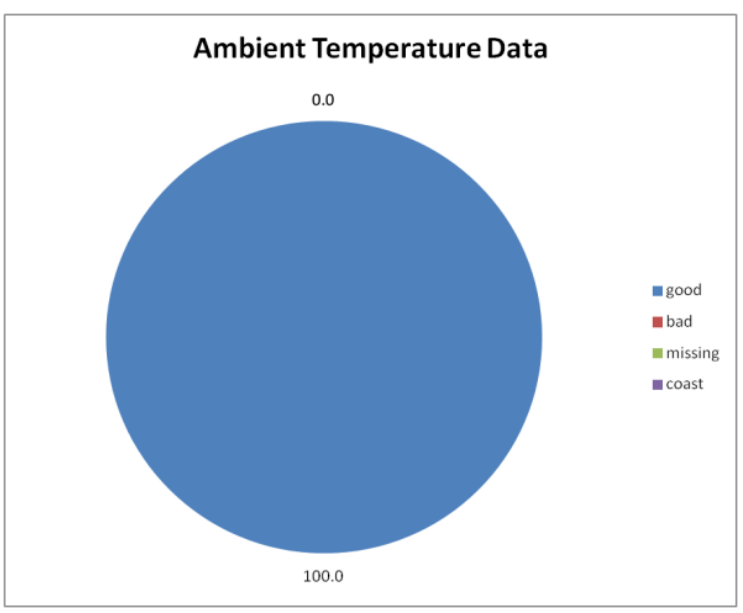

Figure L-69

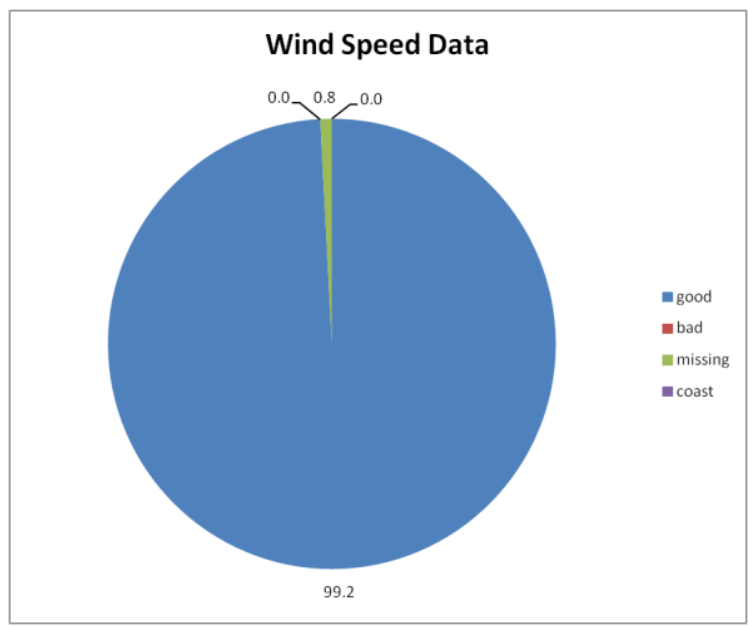

Figure L-68

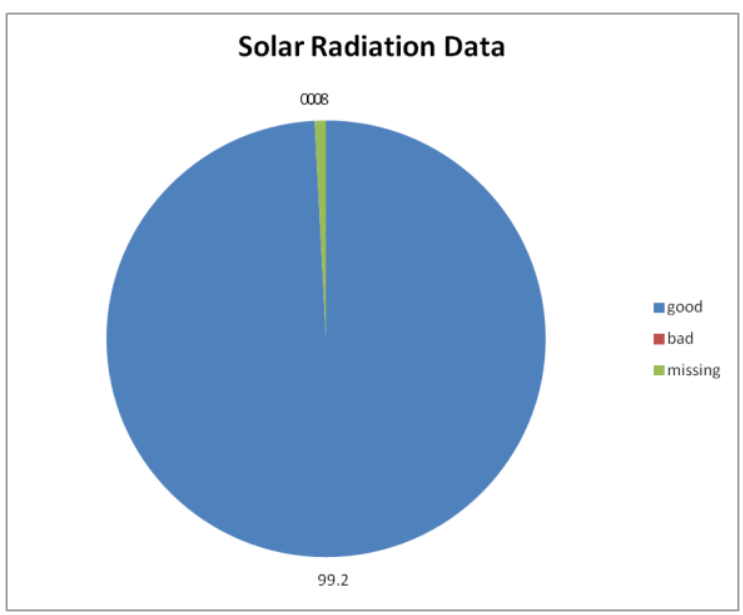

Figure L-70 
November 2011 Site 3

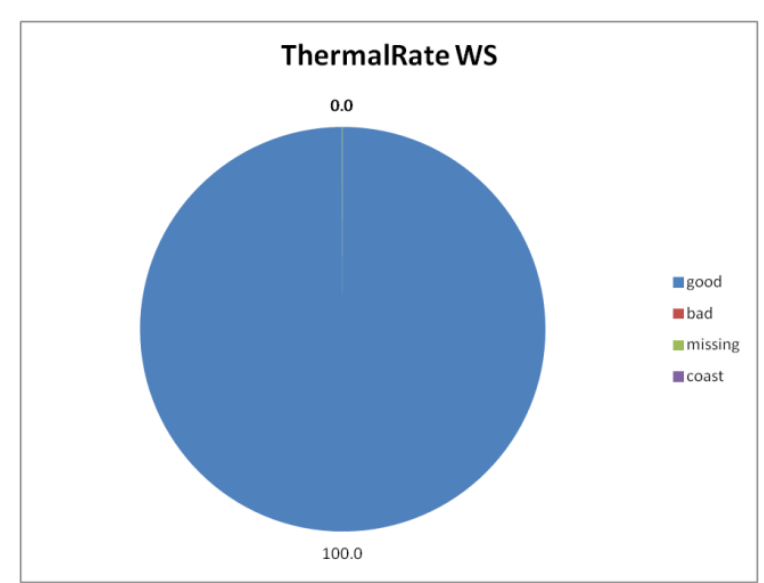

Figure L-71

\section{December 2011 Site 3}

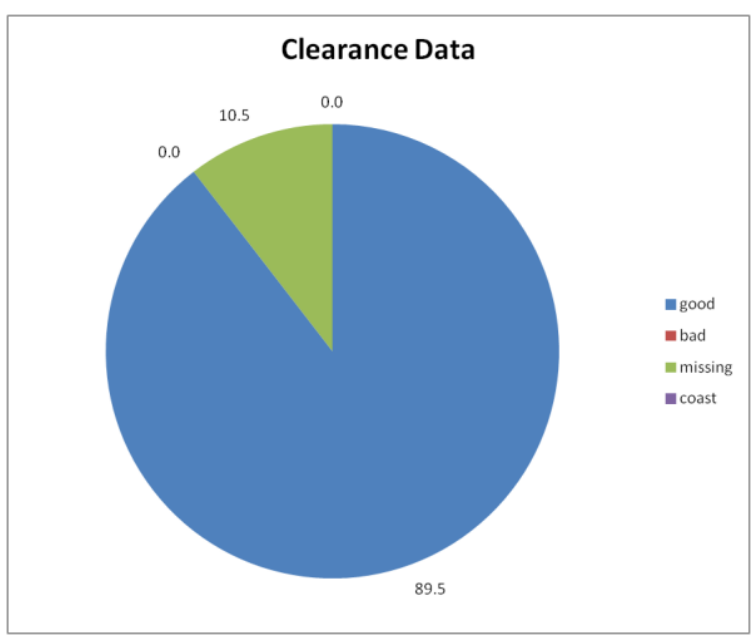

Figure L-73

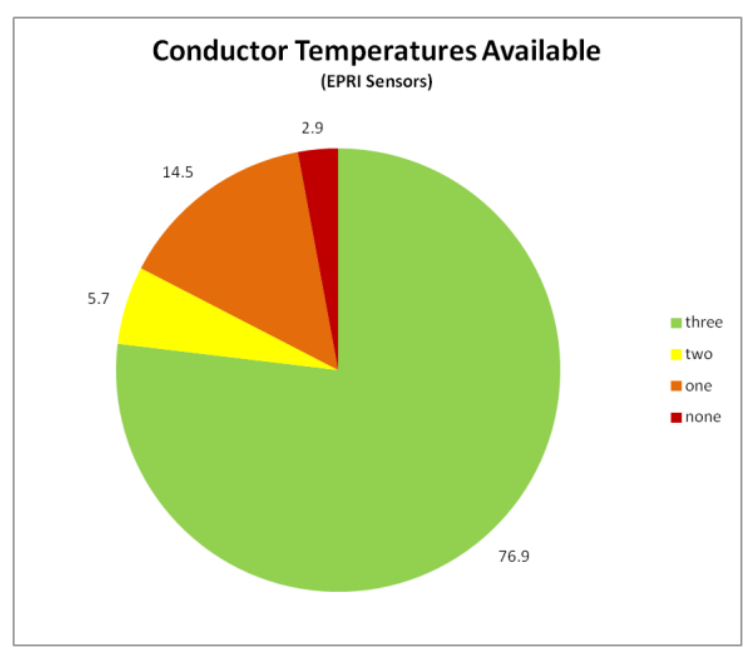

Figure L-72

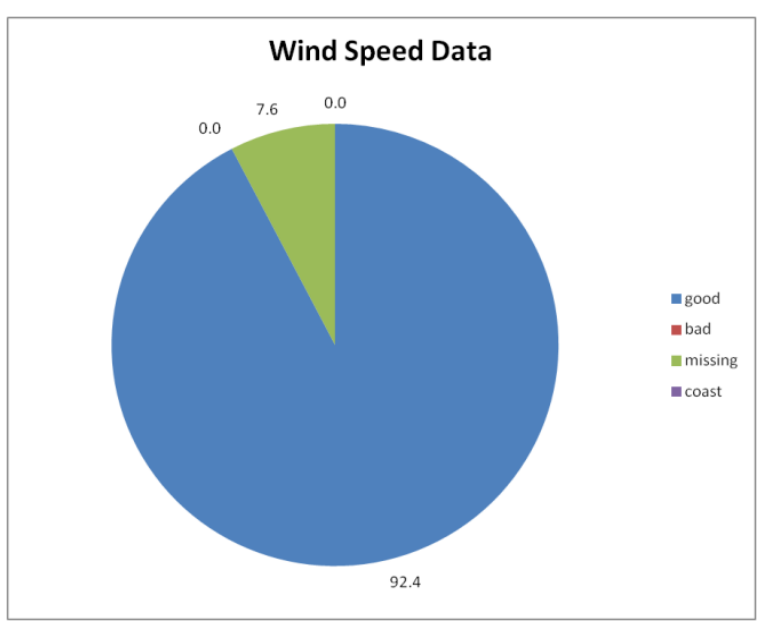

Figure L-74 


\section{December 2011 Site 3}

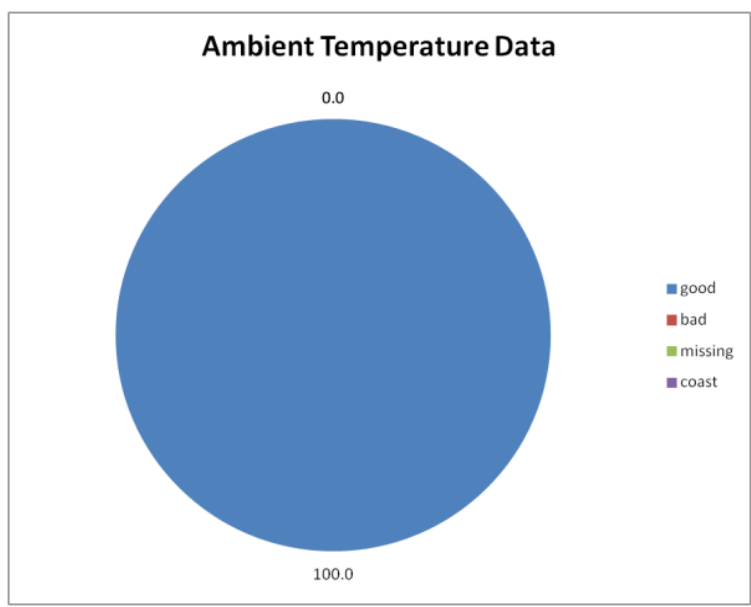

Figure L-75

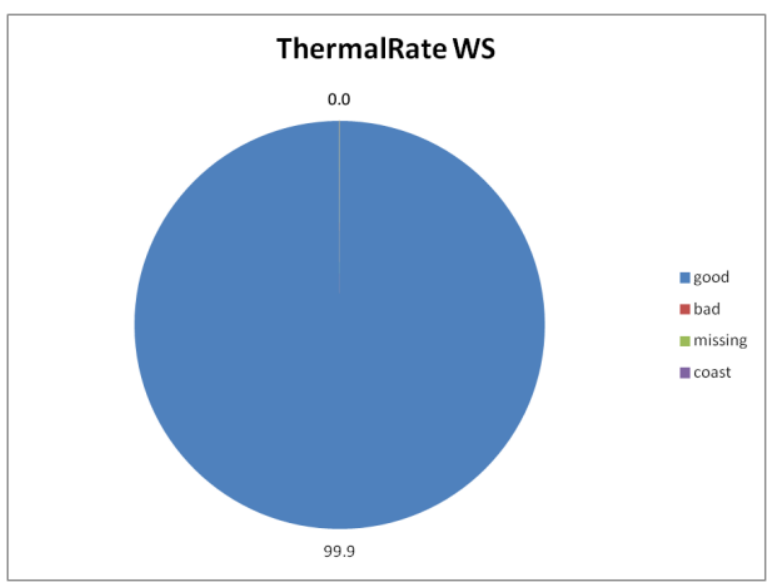

Figure L-77

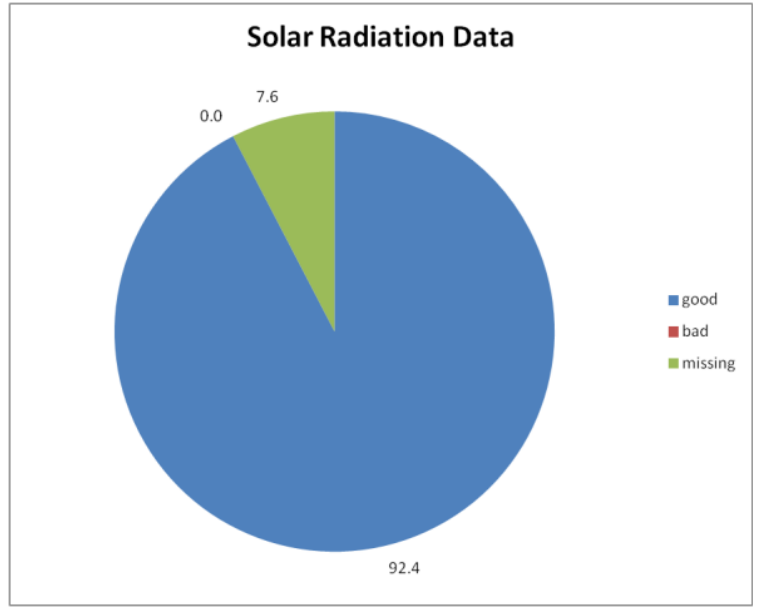

Figure L-76

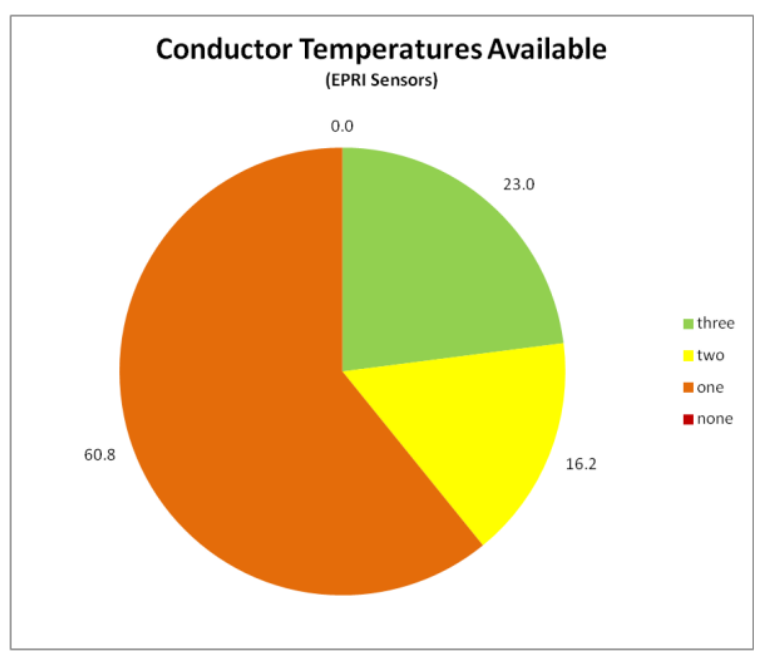

Figure L-78 


\section{January 2012 Site 3}

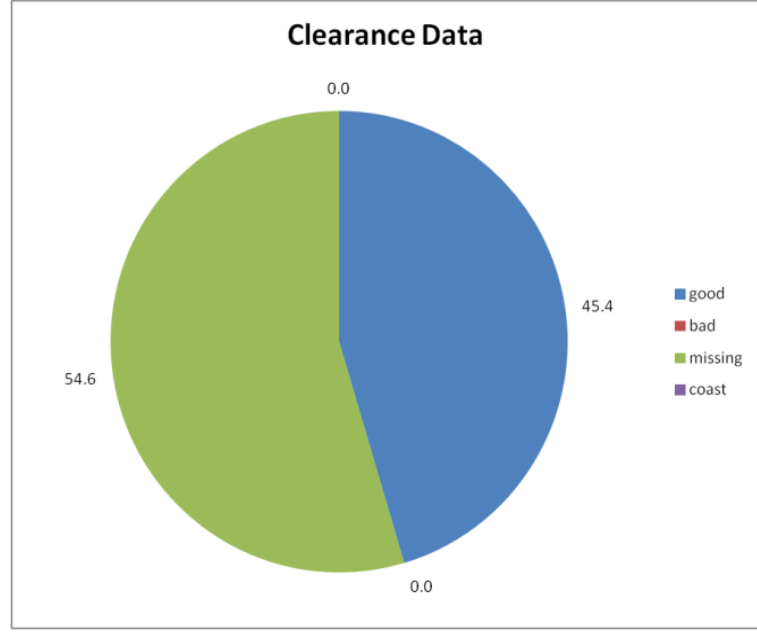

Figure L-79

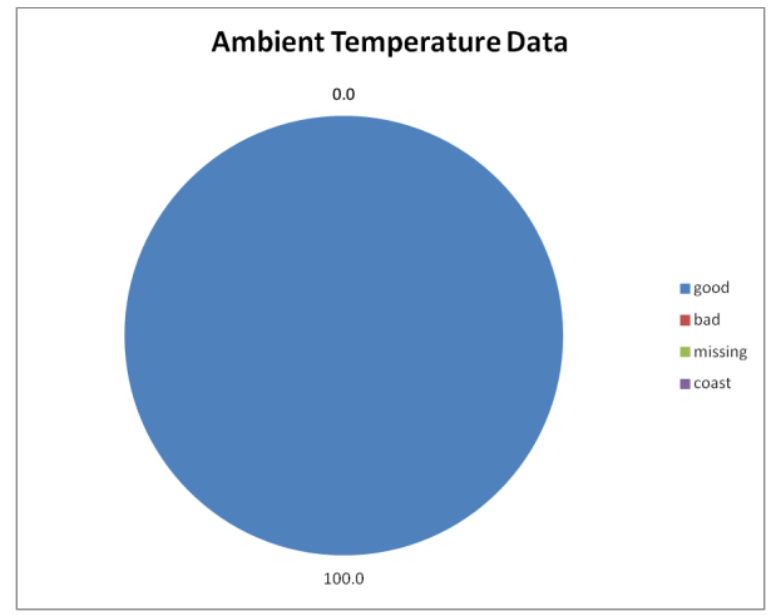

Figure L-81

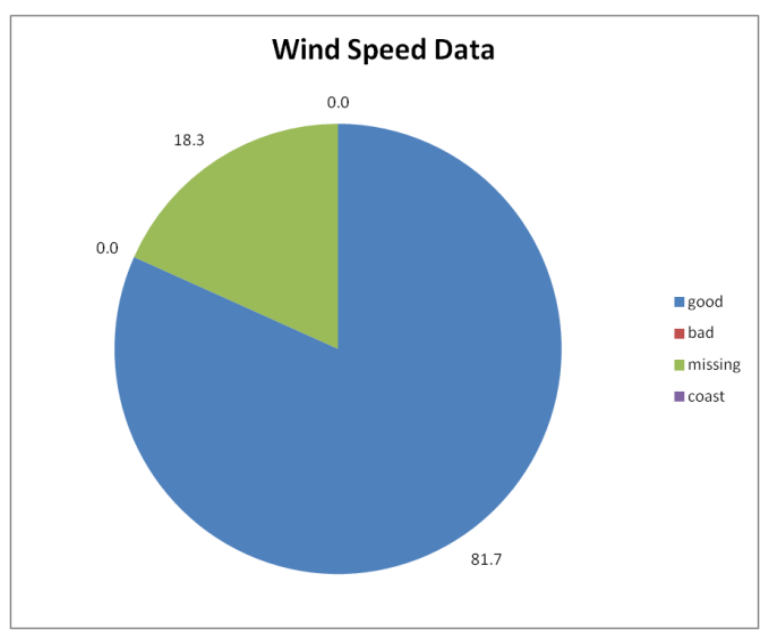

Figure L-80

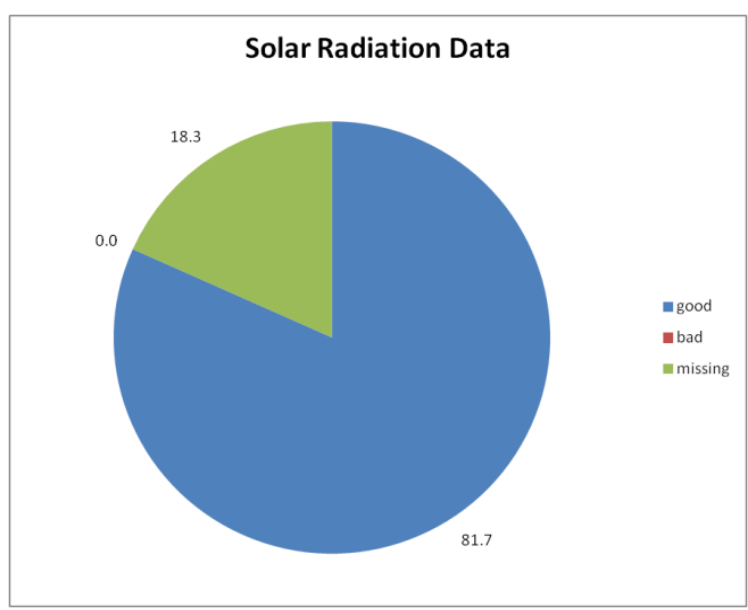

Figure L-82 


\section{January 2012 Site 3}

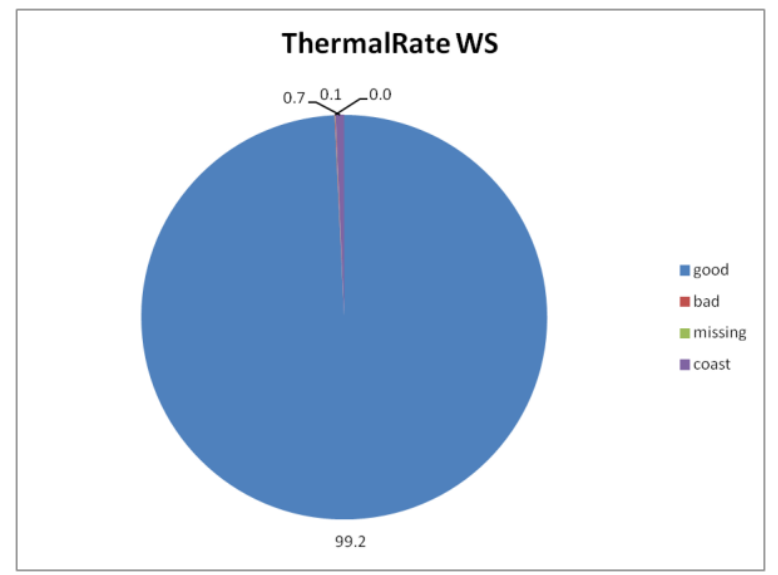

Figure L-83

\section{February 2012 Site 3}

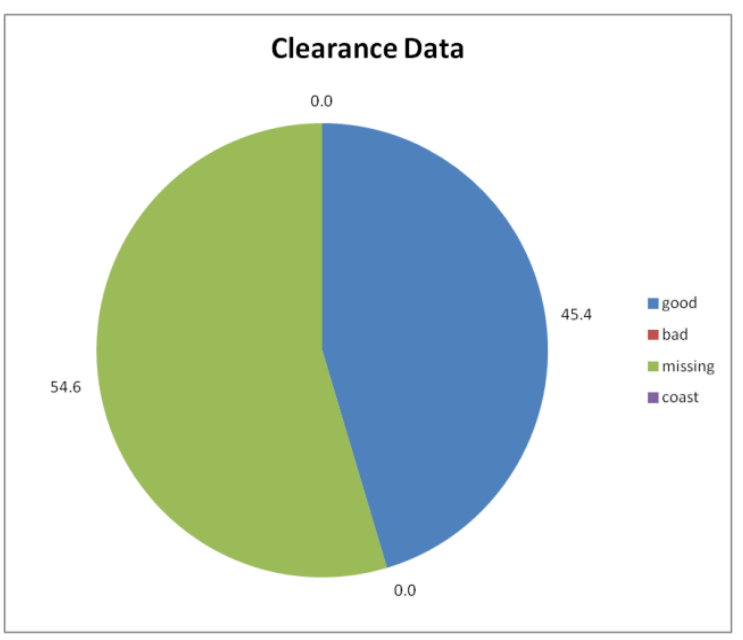

Figure L-85

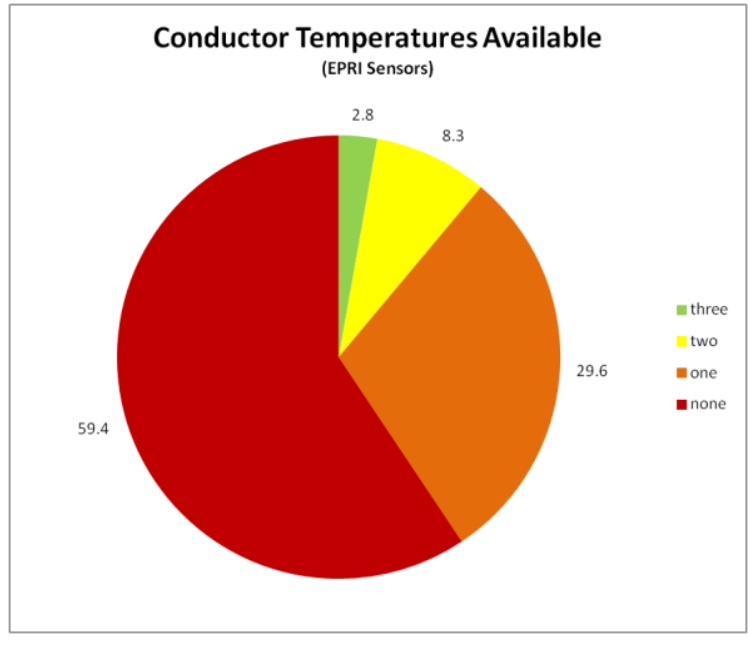

Figure L-84

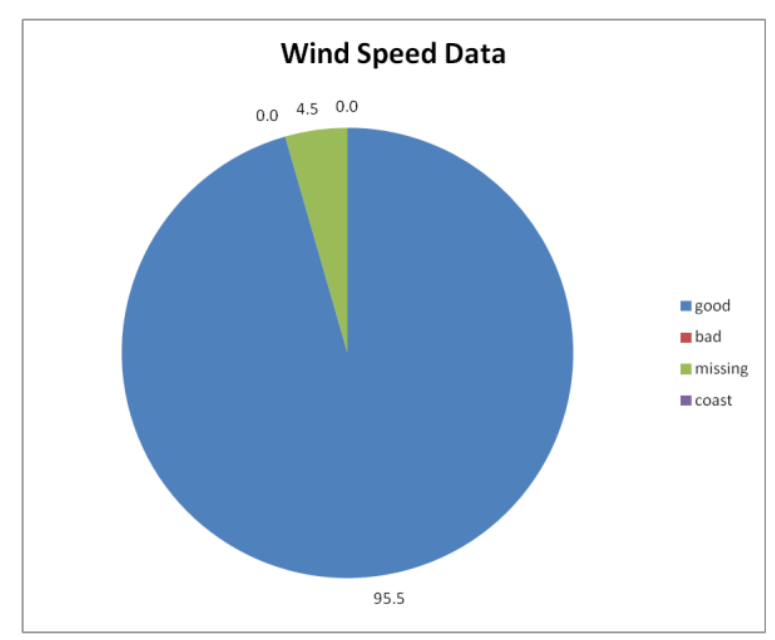

Figure L-86 
February 2012 Site 3

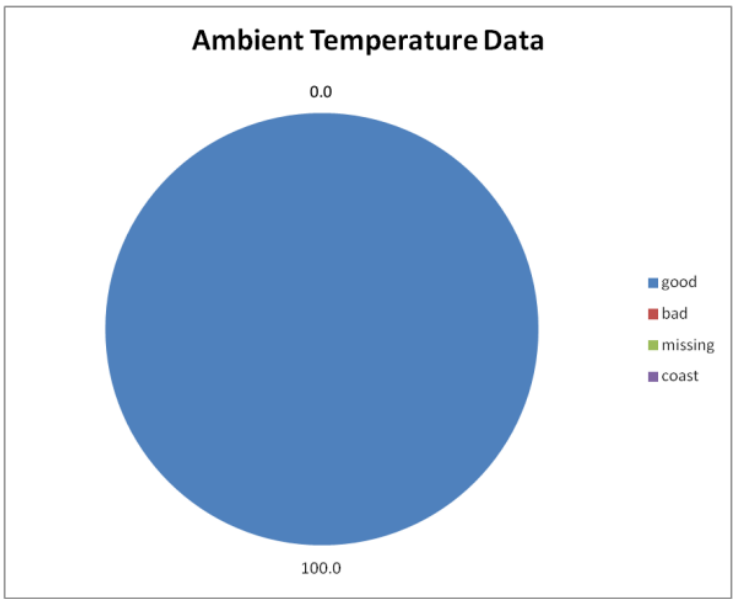

Figure L-87

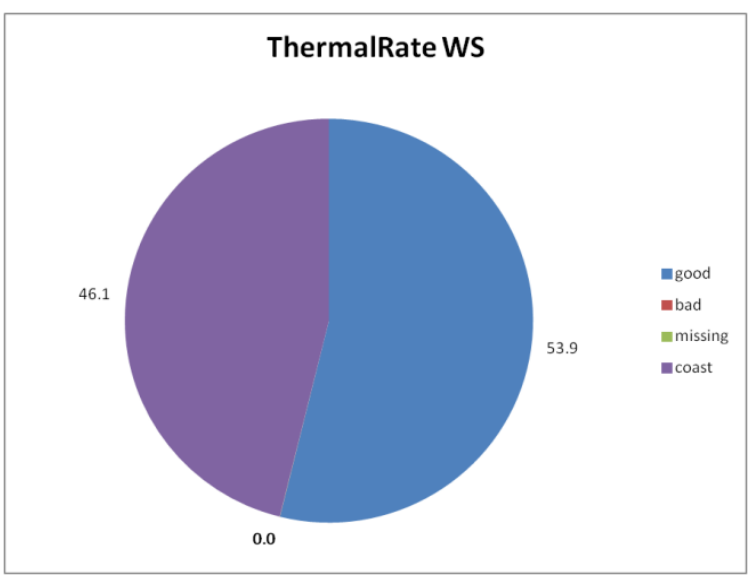

Figure L-89

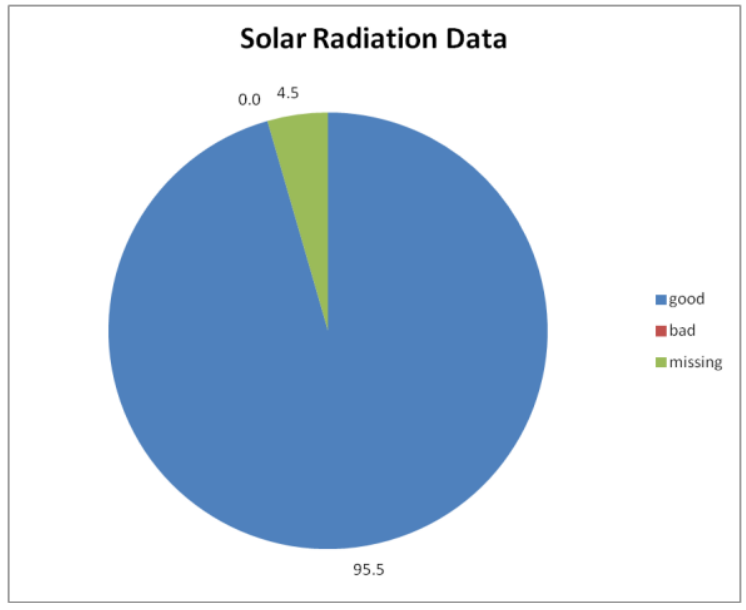

Figure L-88

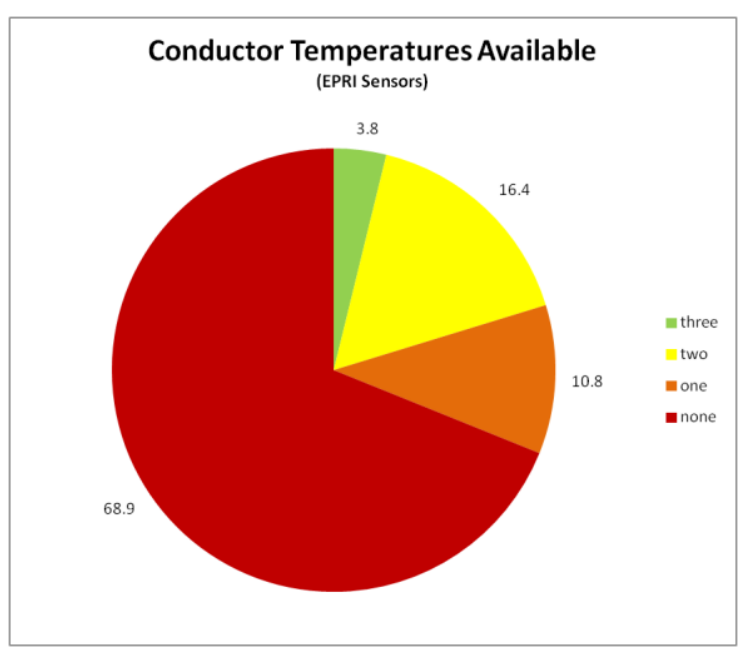

Figure L-90 


\section{March 2012 Site 3}

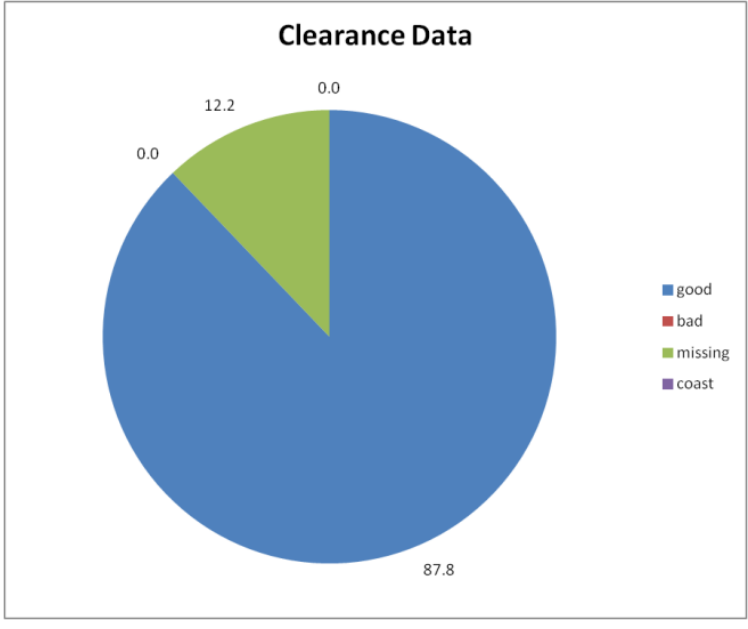

Figure L-91

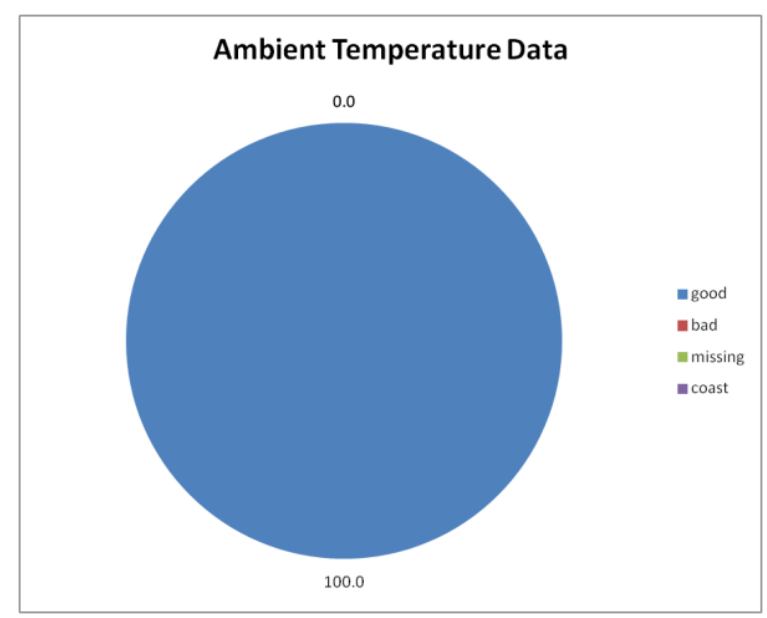

Figure L-93

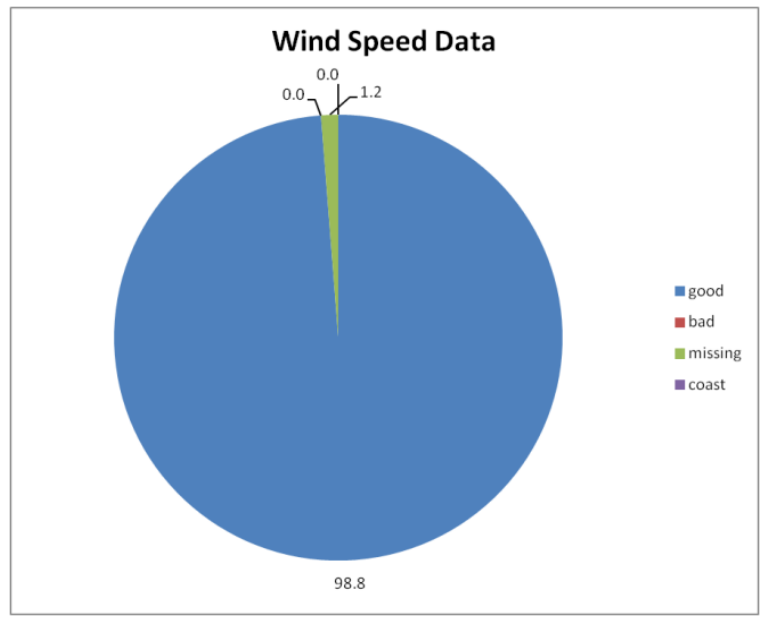

Figure L-92

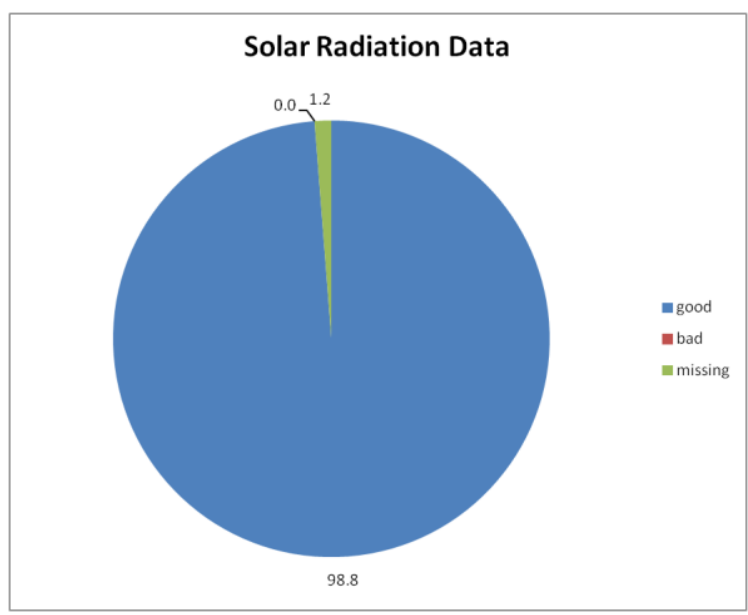

Figure L-94 
March 2012 Site 3

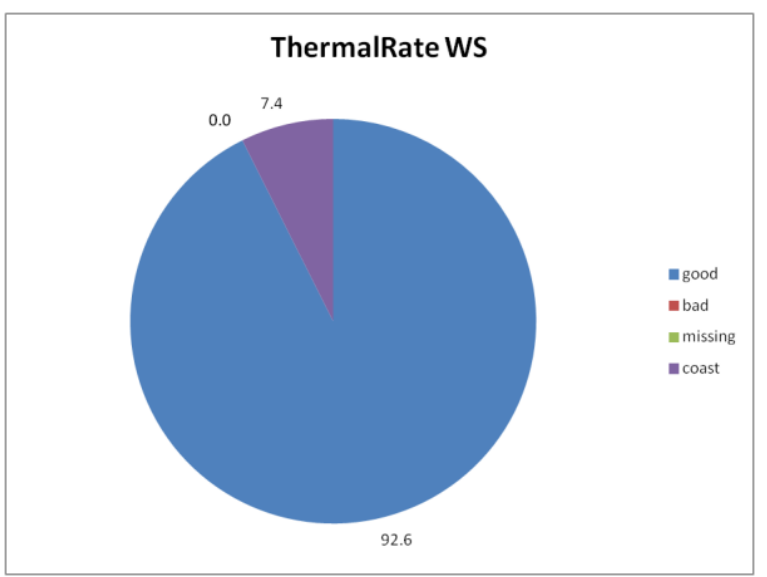

Figure L-95

April 2012 Site 3

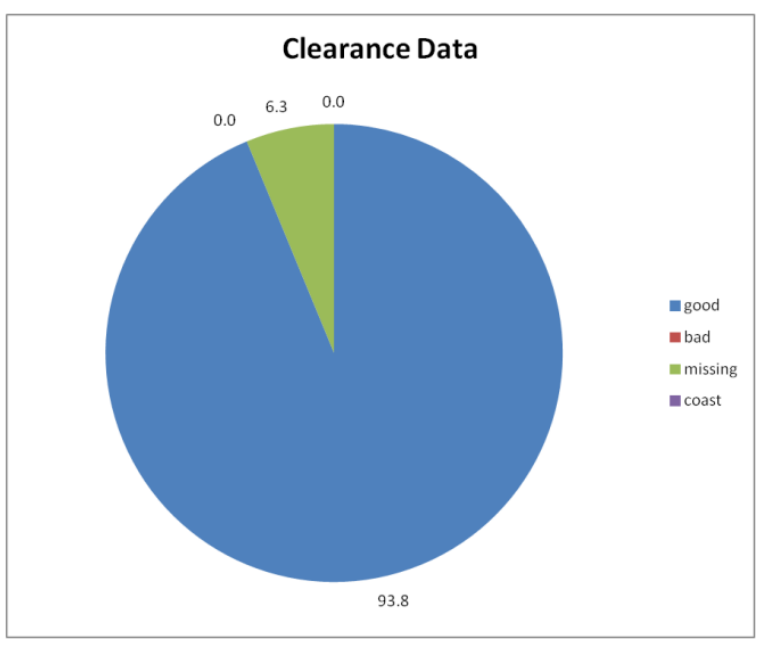

Figure L-97

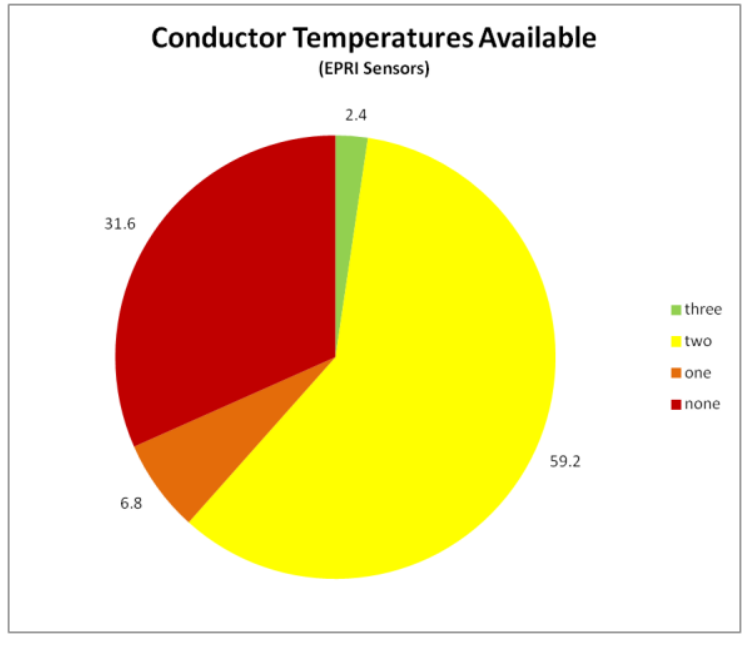

Figure L-96

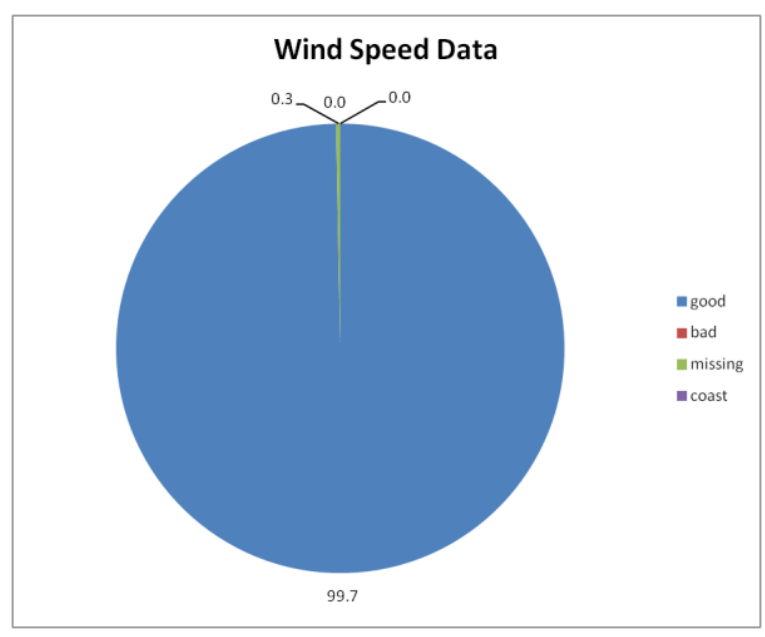

Figure L-98 


\section{April 2012 Site 3}

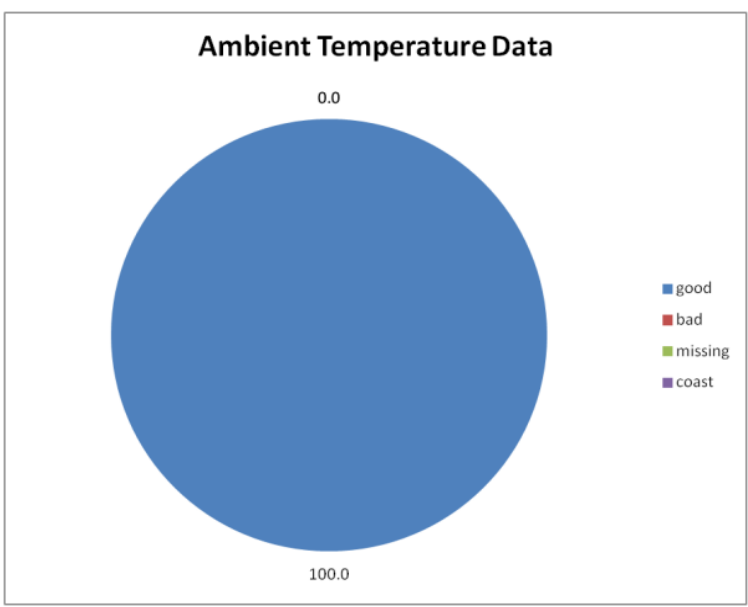

Figure L-99

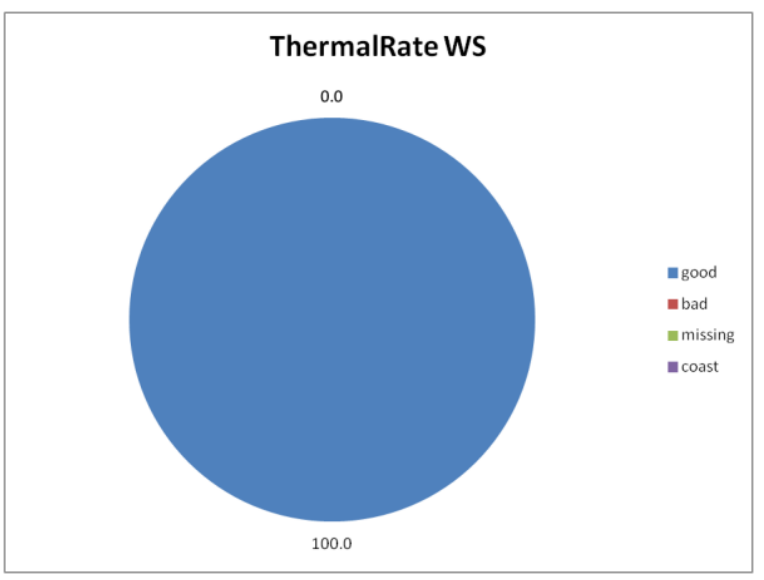

Figure L-101

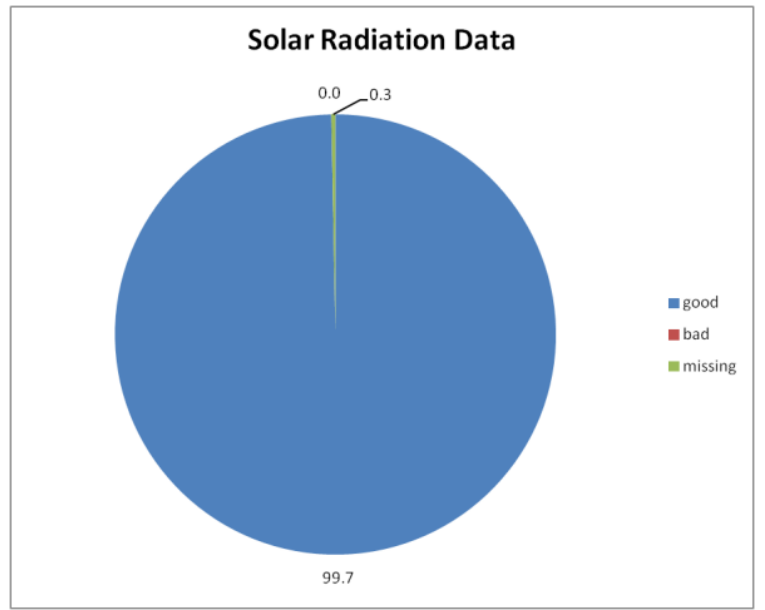

Figure L-100

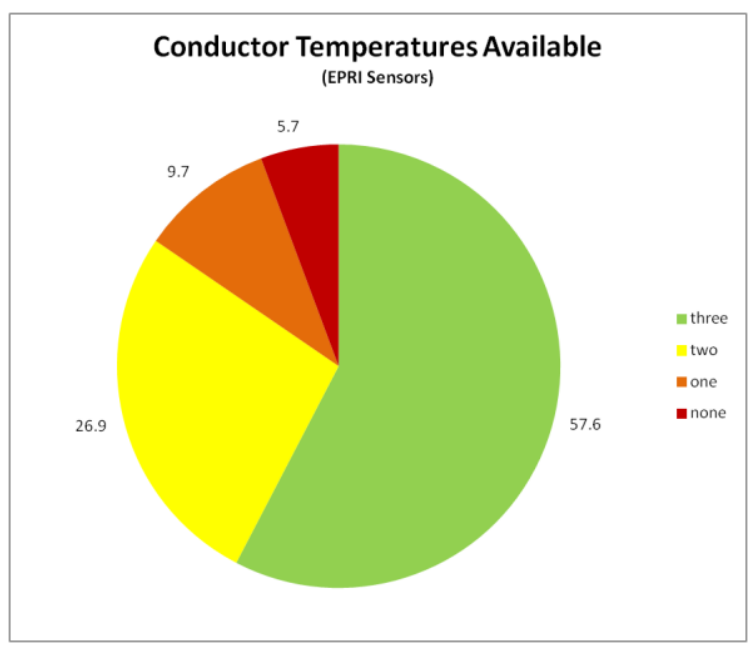

Figure L-102 
May 2012 Site 3

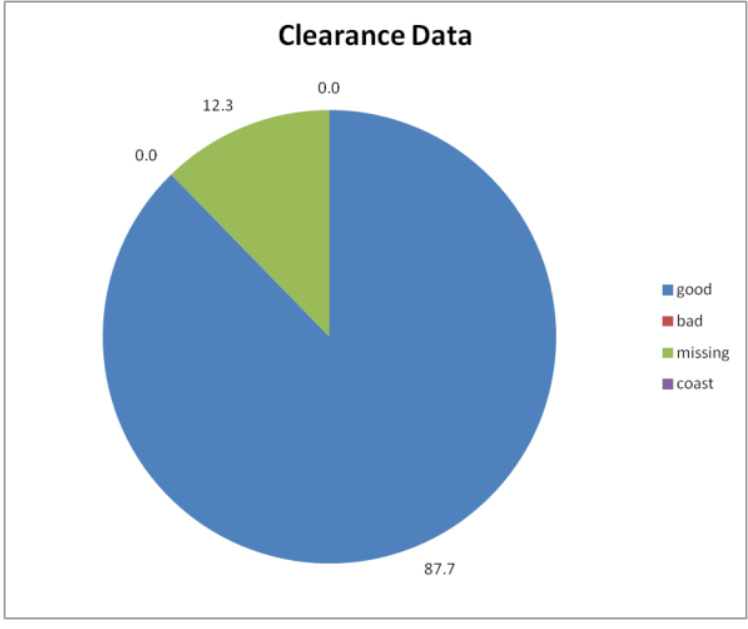

Figure L-103

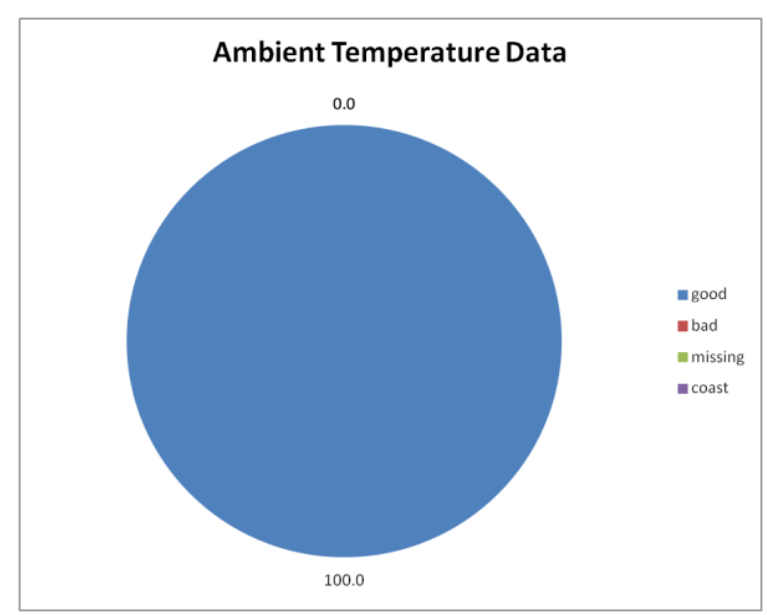

Figure L-105

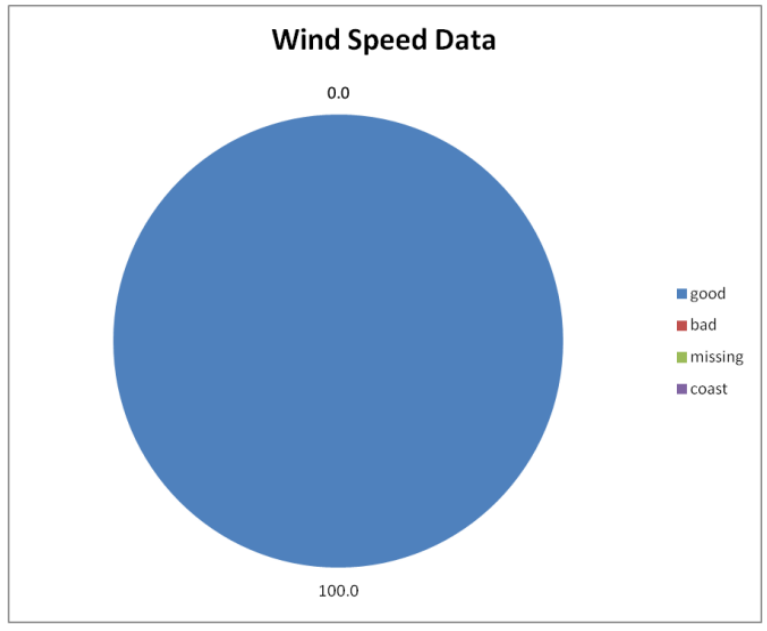

Figure L-104

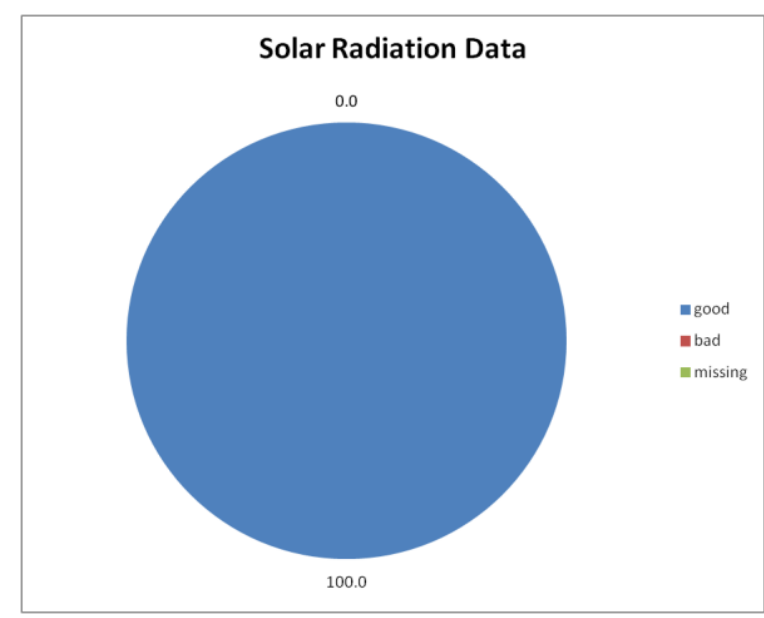

Figure L-106 
May 2012 Site 3

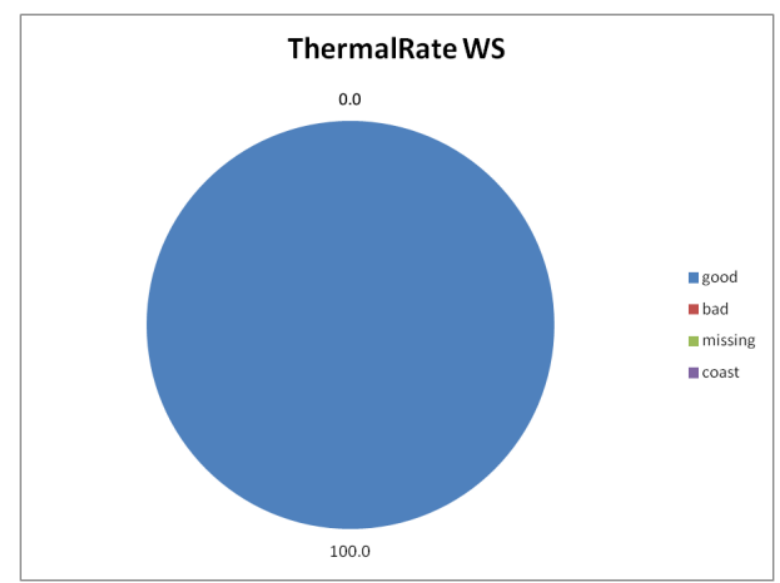

Figure L-107

\section{June 2012 Site 3}

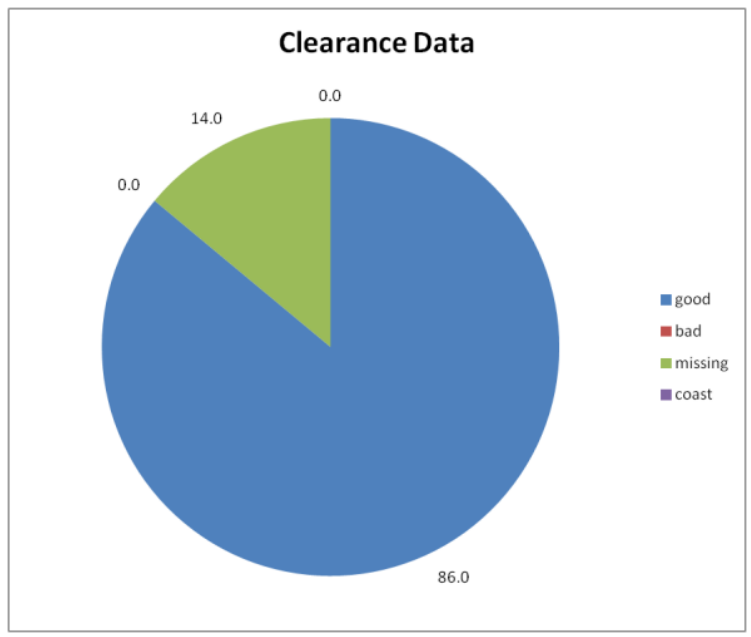

Figure L-109

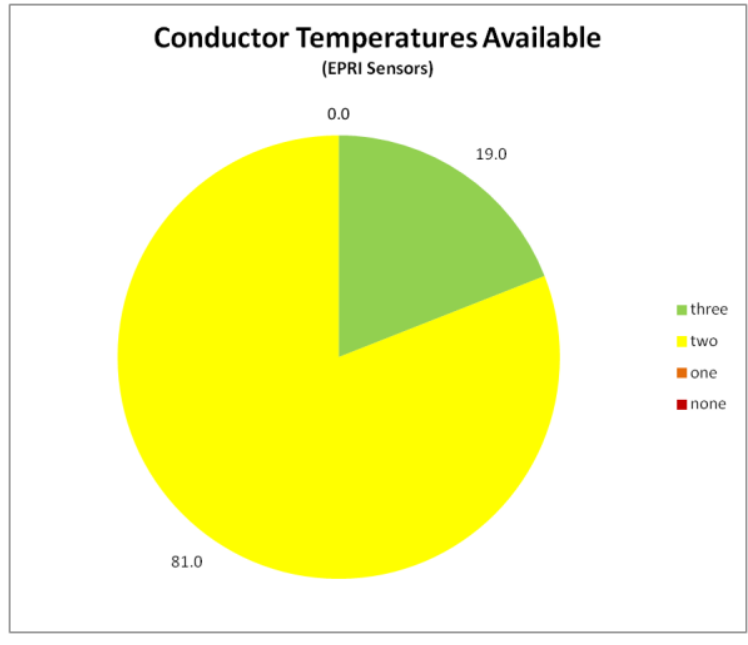

Figure L-108

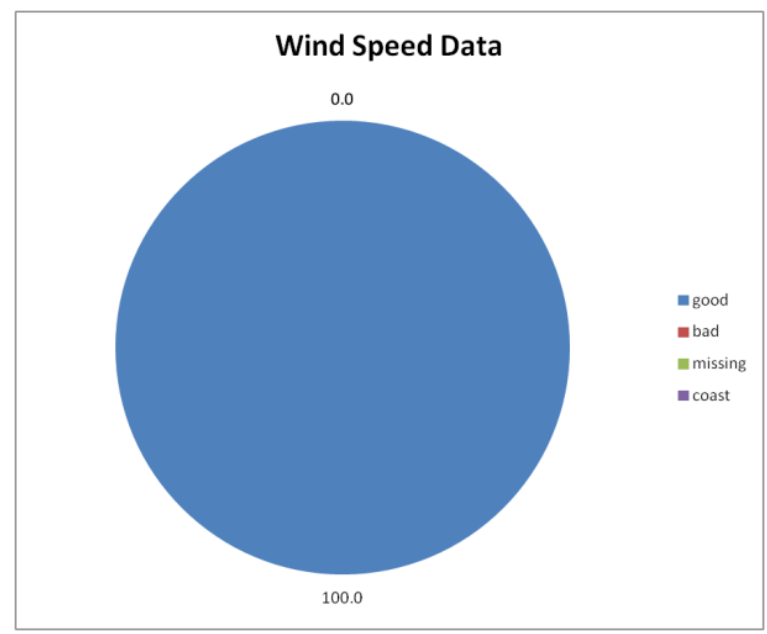

Figure L-110 


\section{June 2012 Site 3}

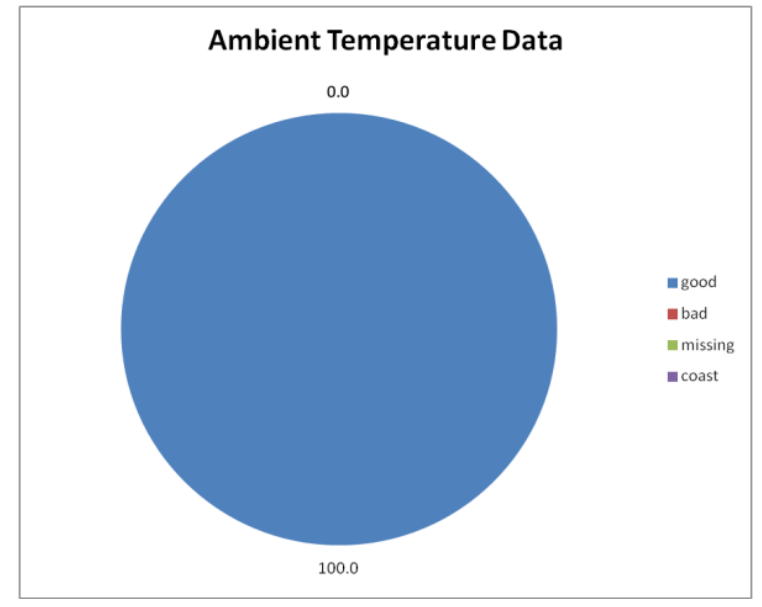

Figure L-111

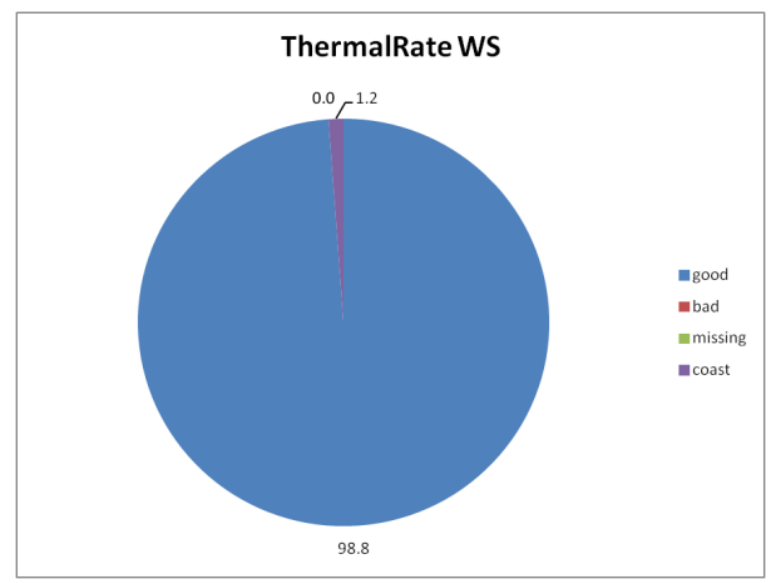

Figure L-113

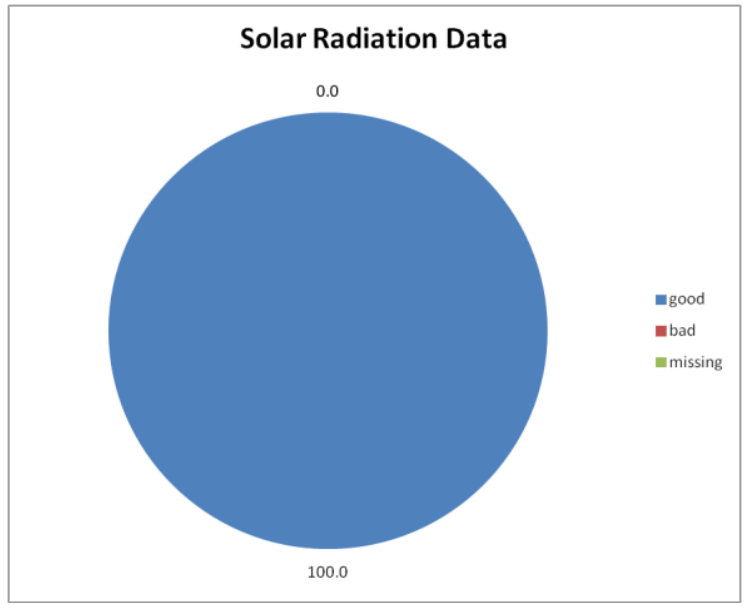

Figure L-112

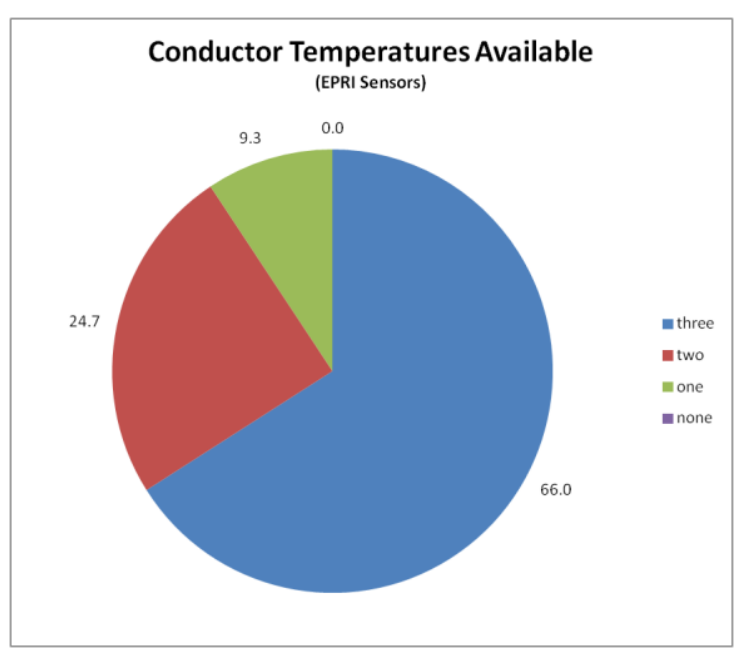

Figure L-114 


\section{July 2012 Site 3}

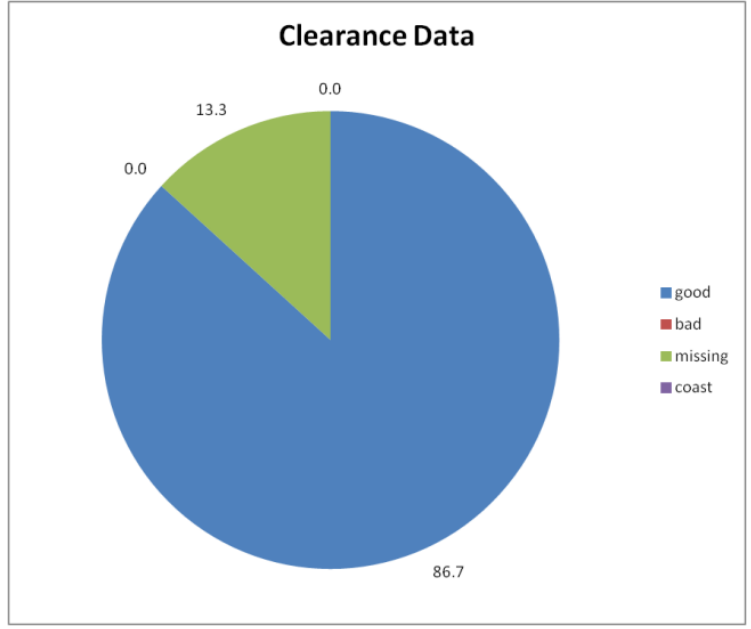

Figure L-115

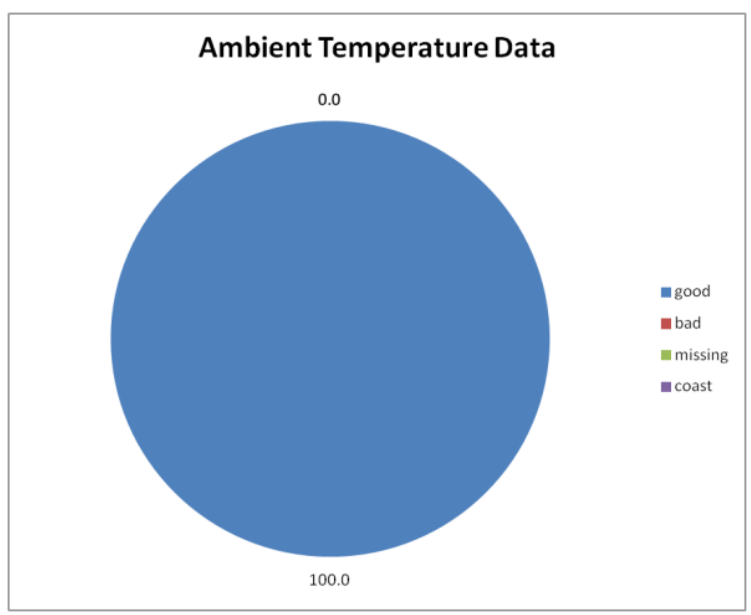

Figure L-117

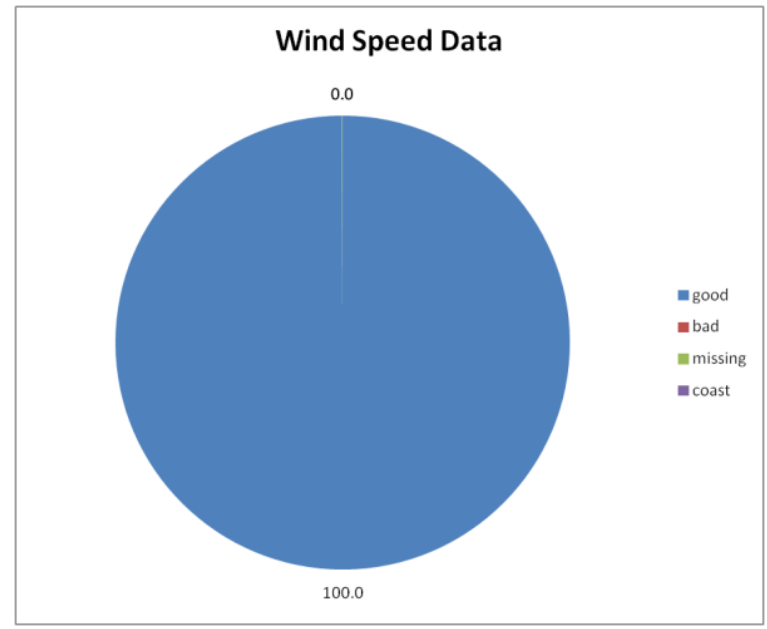

Figure L-116

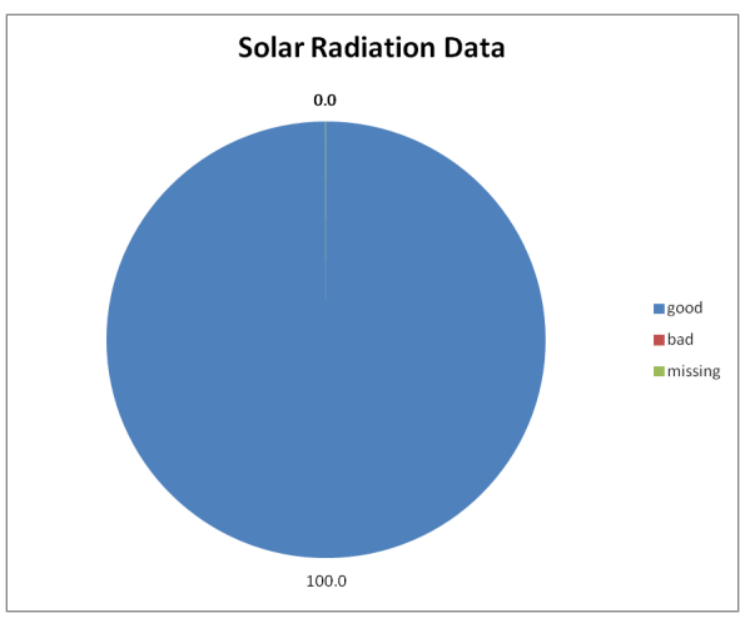

Figure L-118 


\section{July 2012 Site 3}

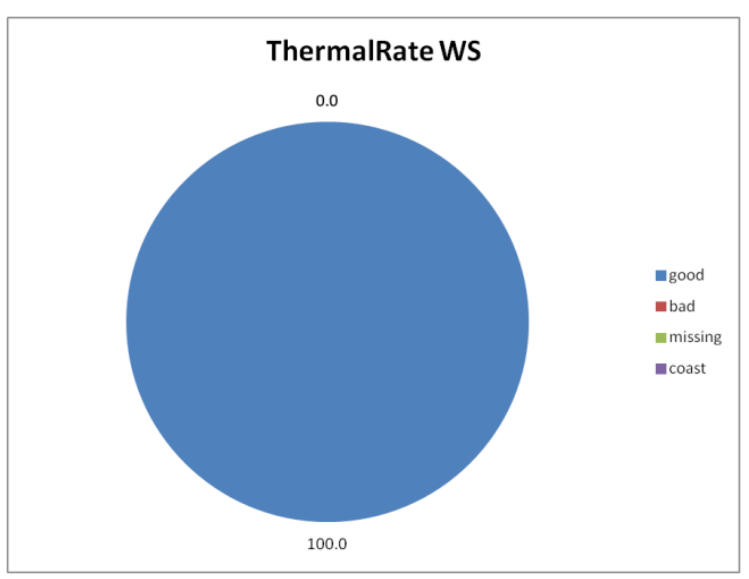

Figure L-119

\section{August 2012 Site 3}

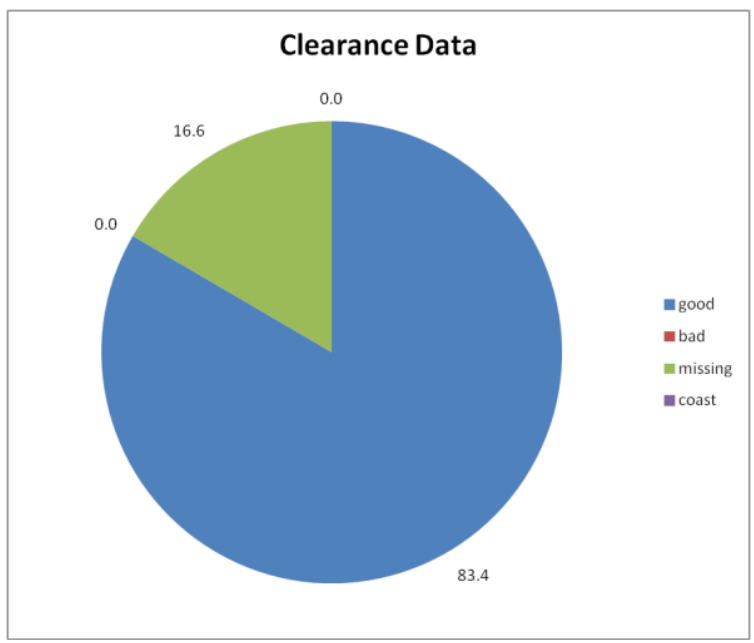

Figure L-121

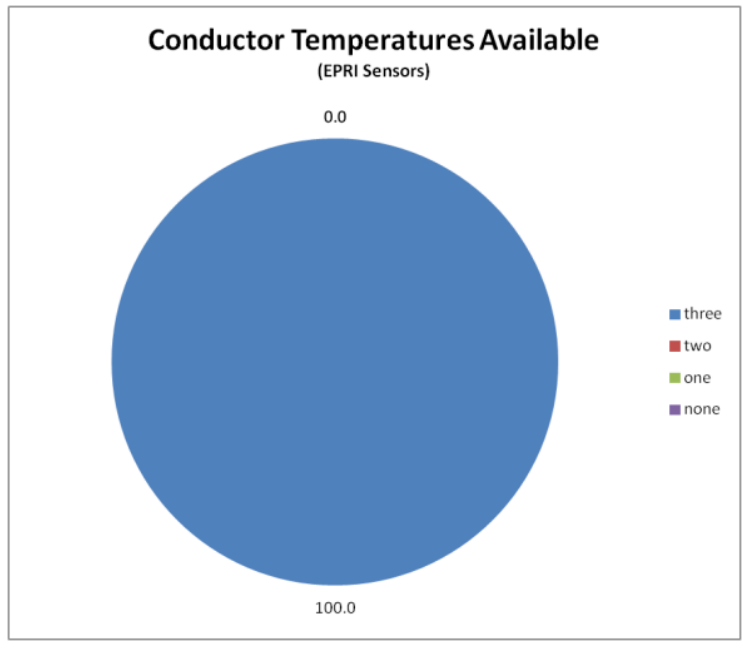

Figure L-120

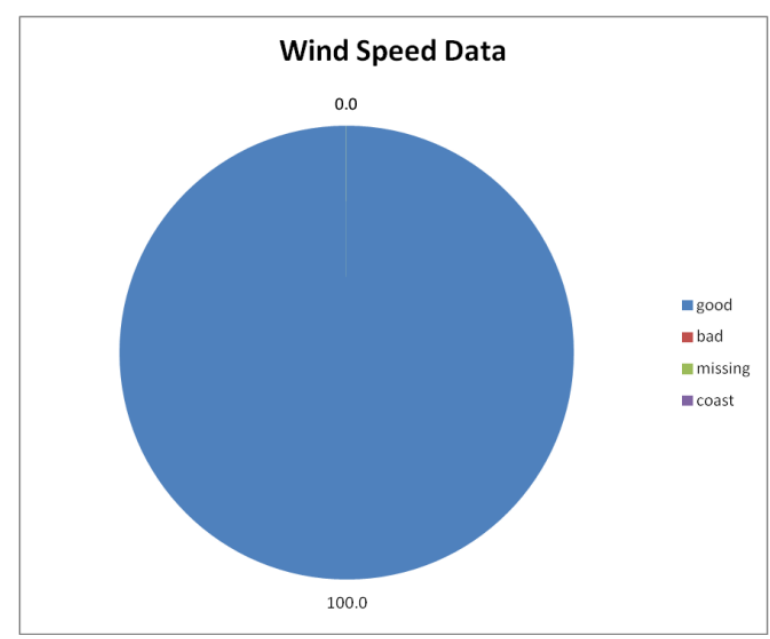

Figure L-122 


\section{August 2012 Site 3}

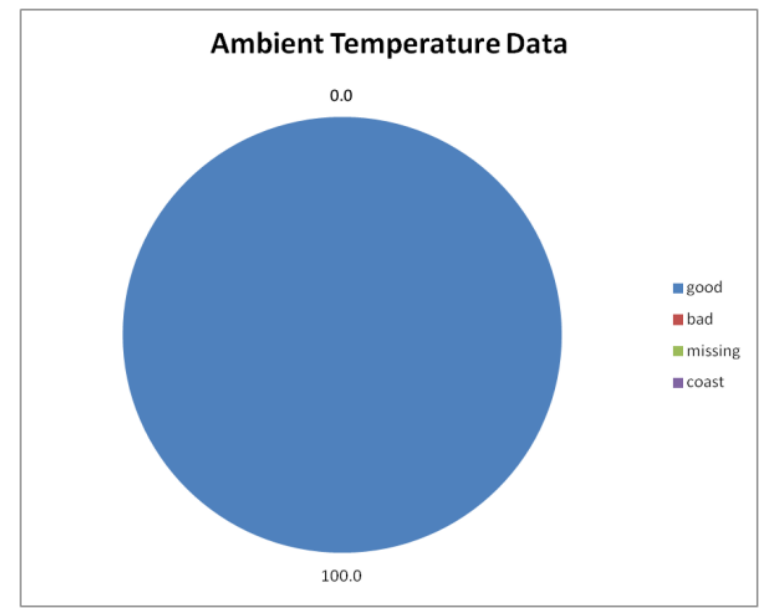

Figure L-123

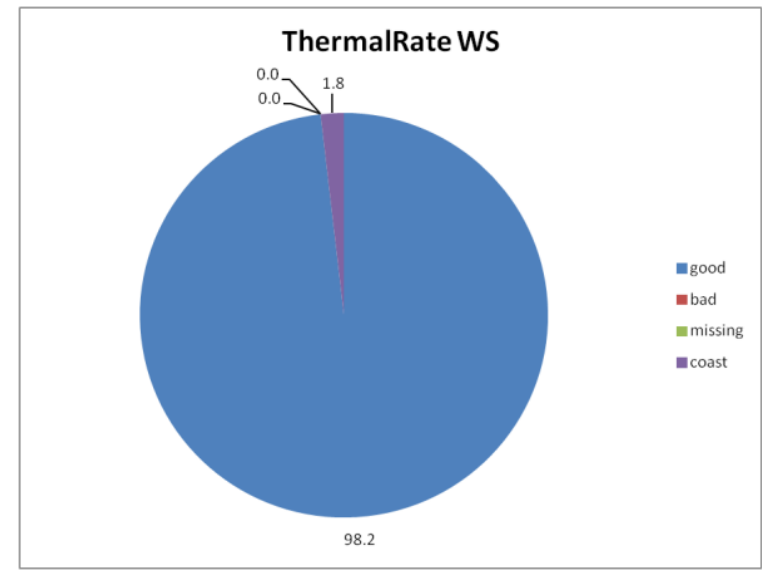

Figure L-125

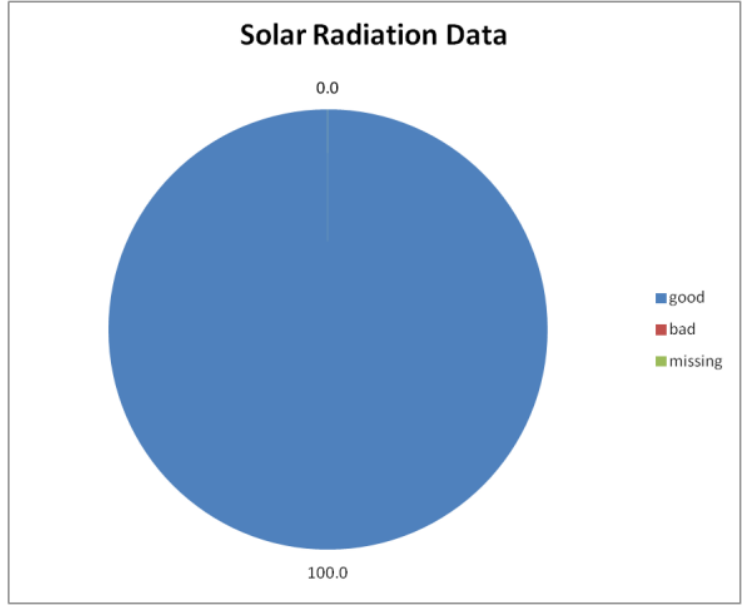

Figure L-124

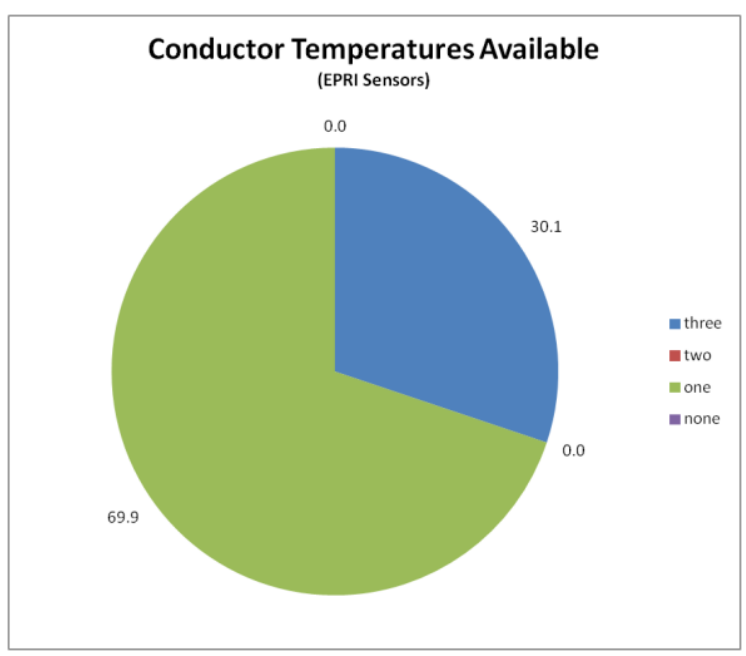

Figure L-126 
September 2012 Site 3

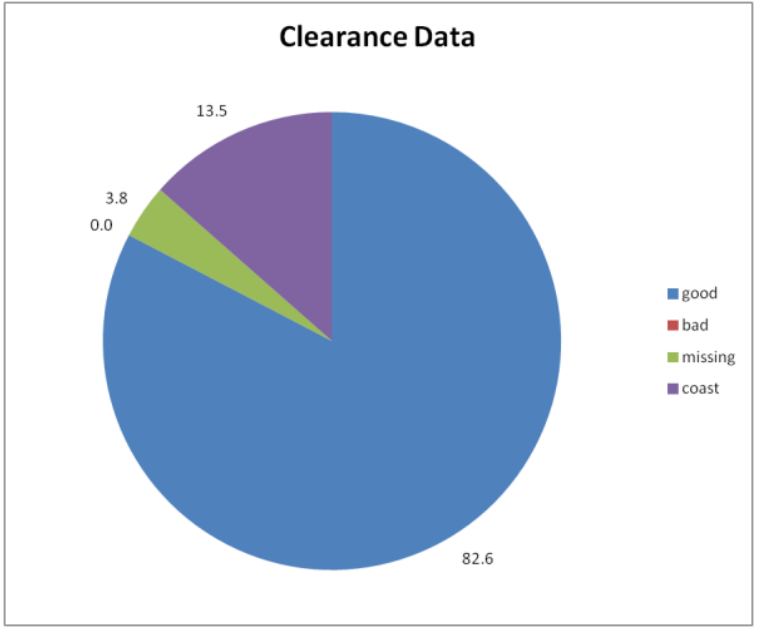

Figure L-127

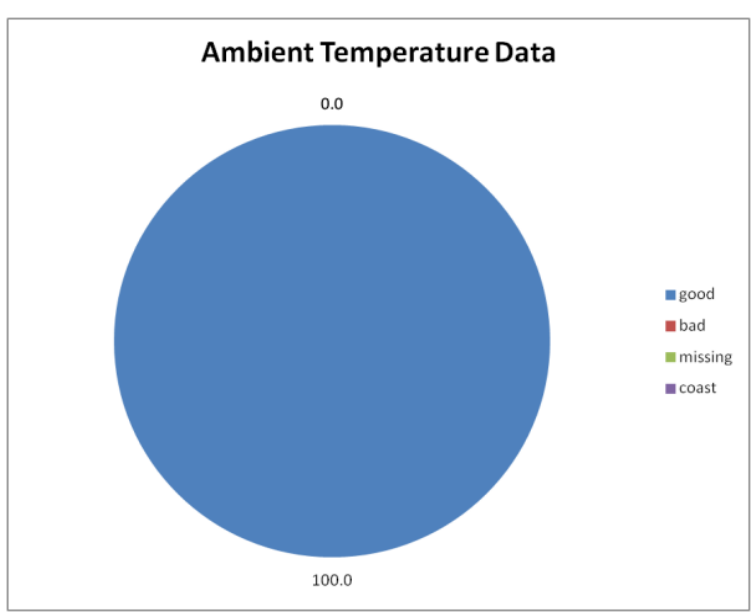

Figure L-129

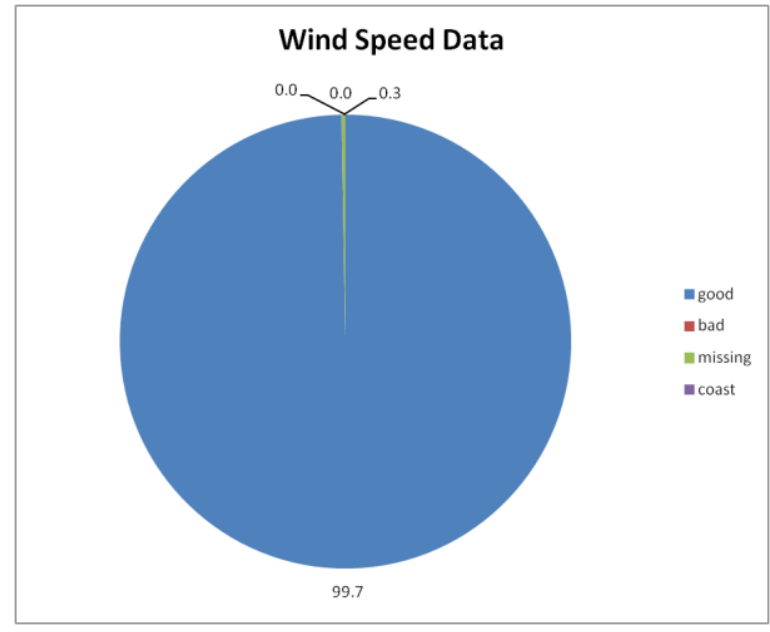

Figure L-128

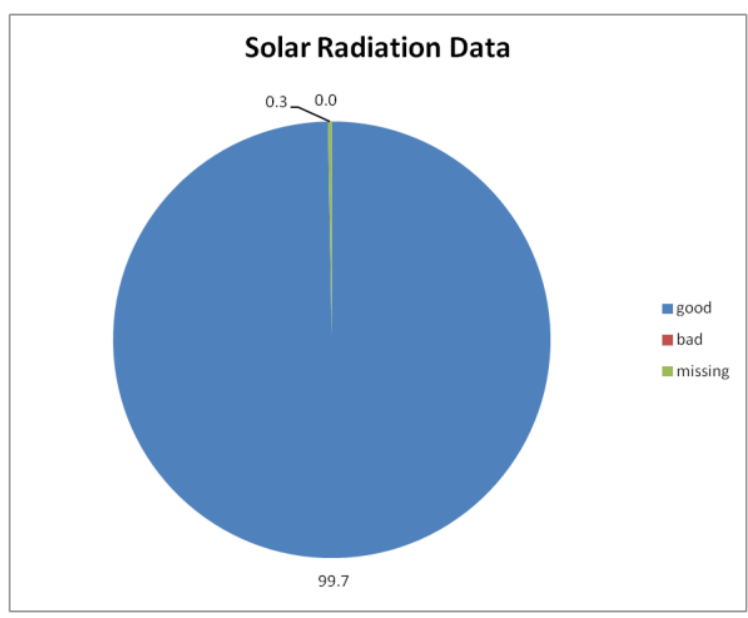

Figure L-130 


\section{September 2012 Site 3}

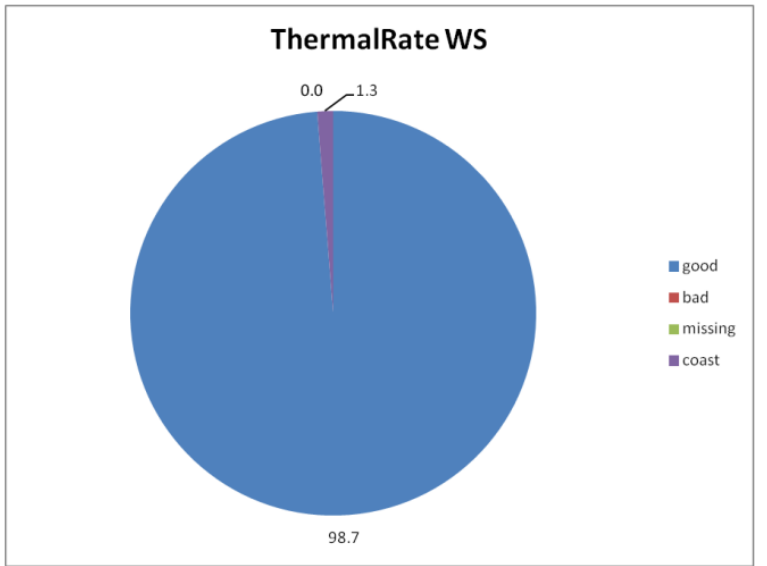

Figure L-131

\section{October 2012 Site 3}

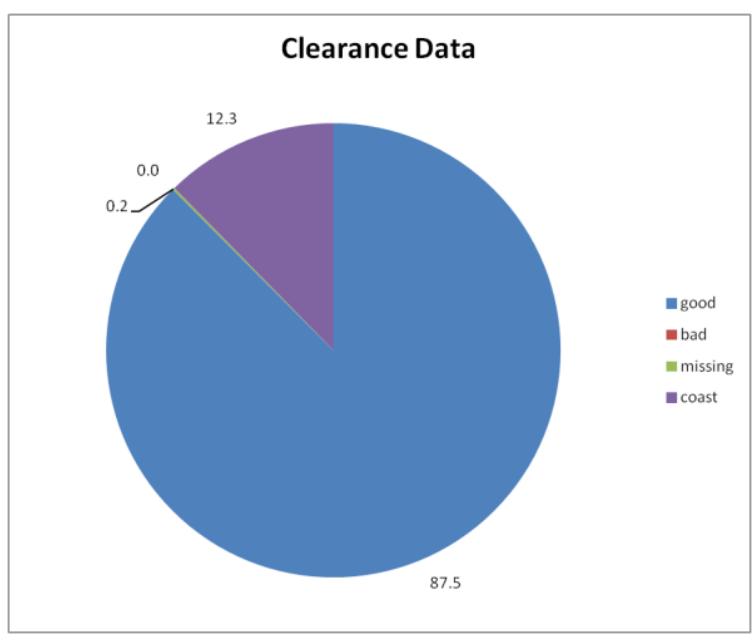

Figure L-133

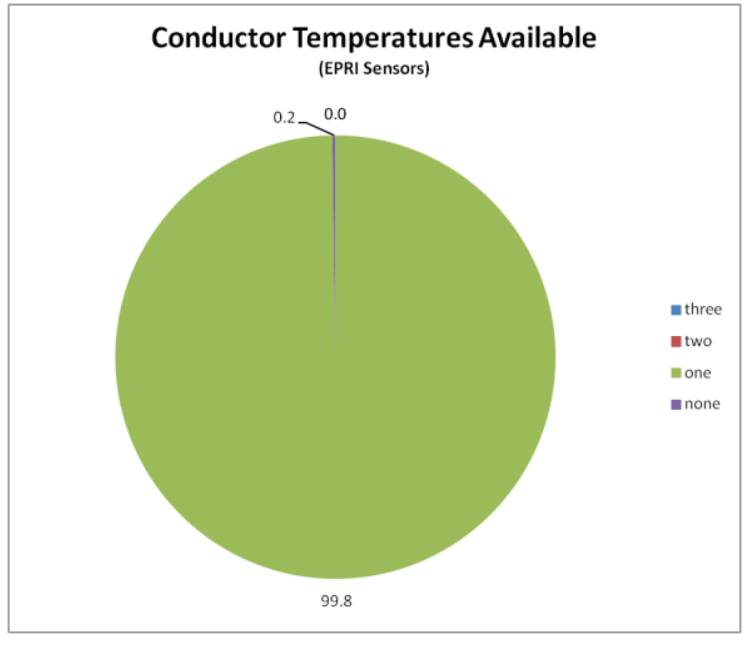

Figure L-132

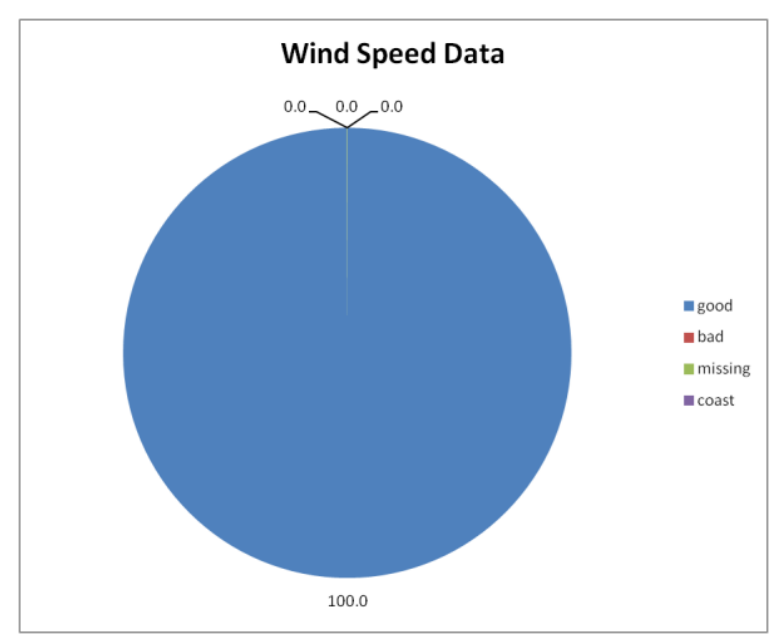

Figure L-134 


\section{October 2012 Site 3}

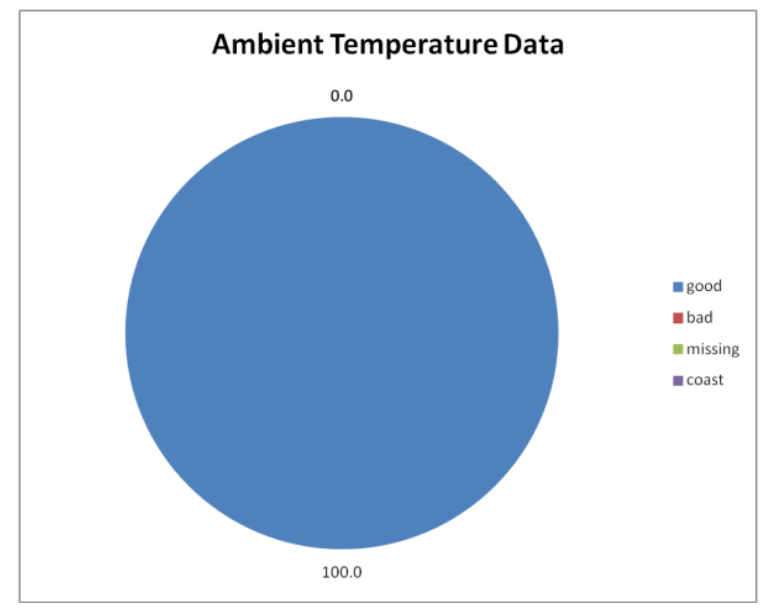

Figure L-135

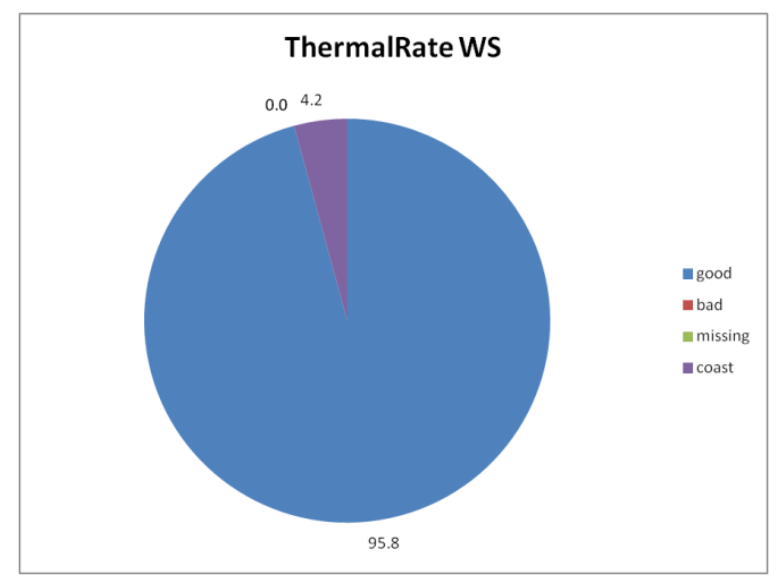

Figure L-137

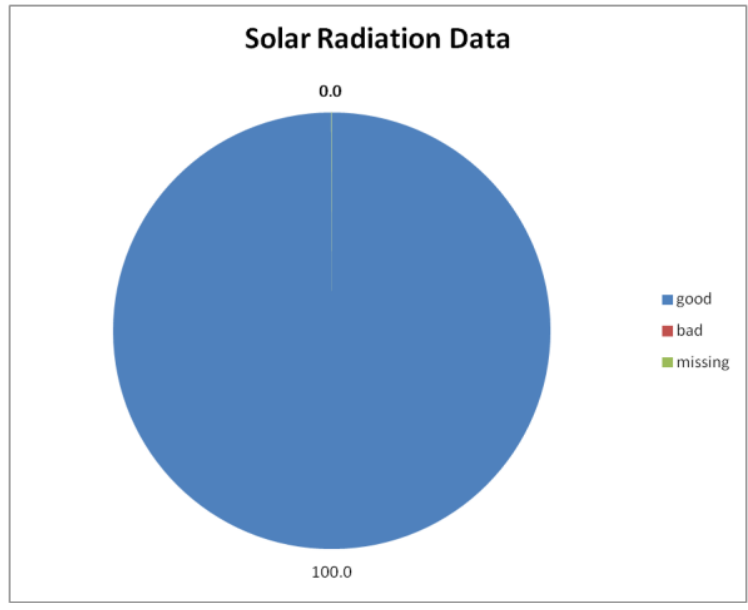

Figure L-136

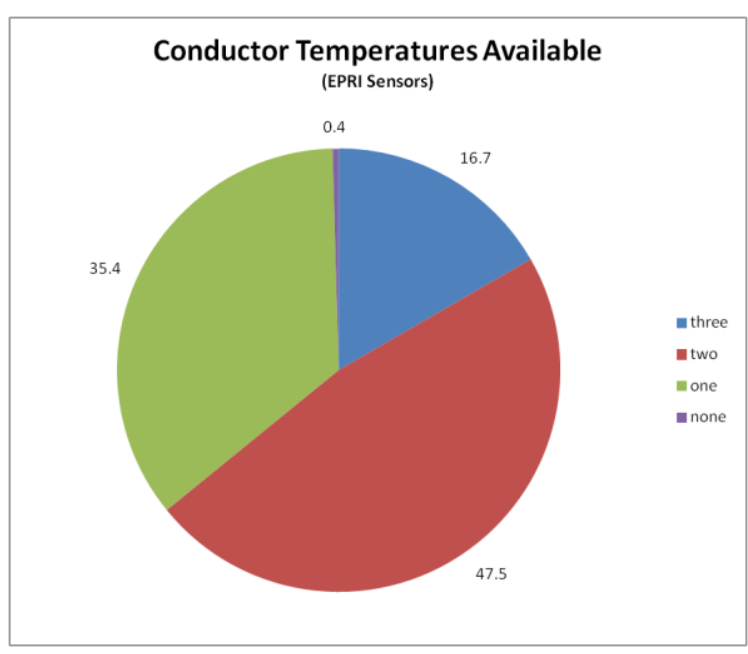

Figure L-138 


\section{December 2010 Site 2}

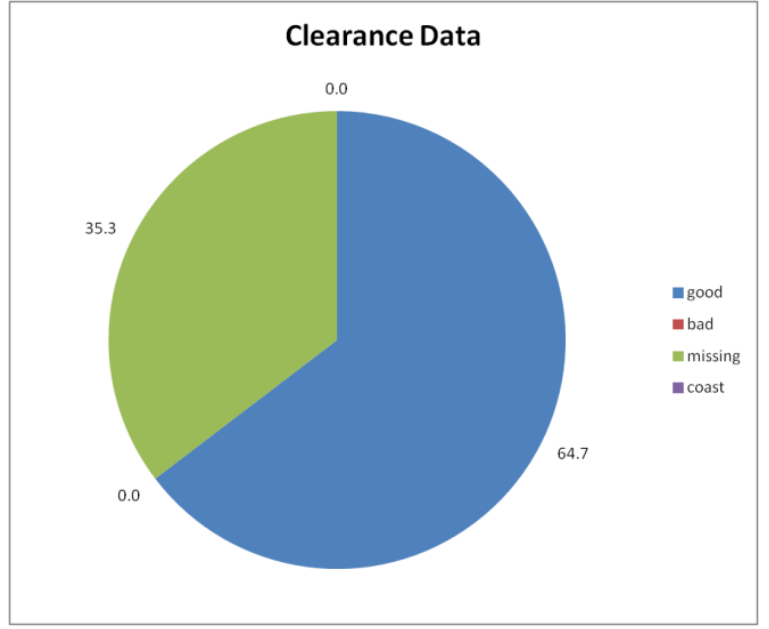

Figure L-139

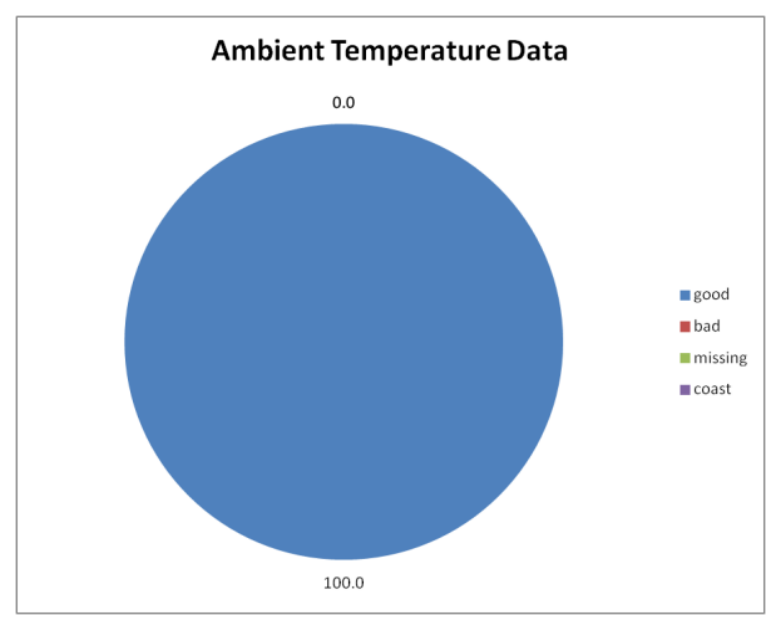

Figure L-141

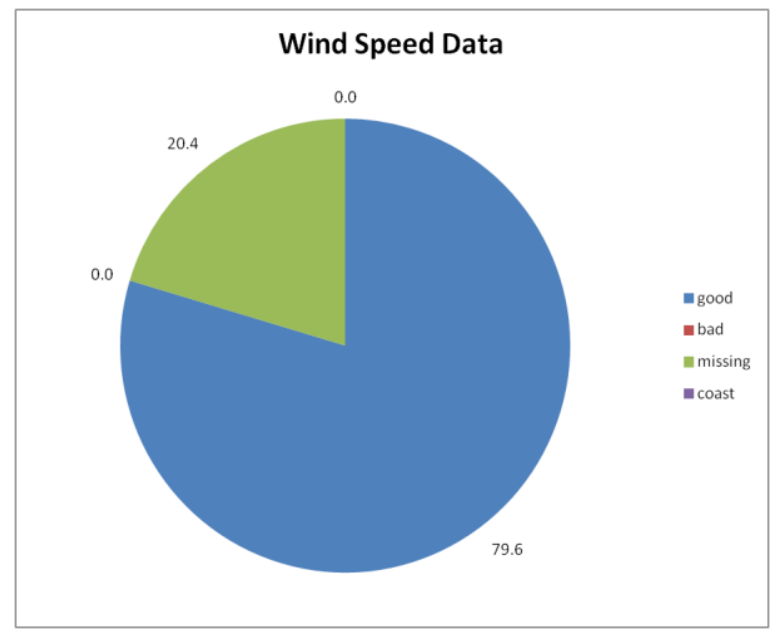

Figure L-140

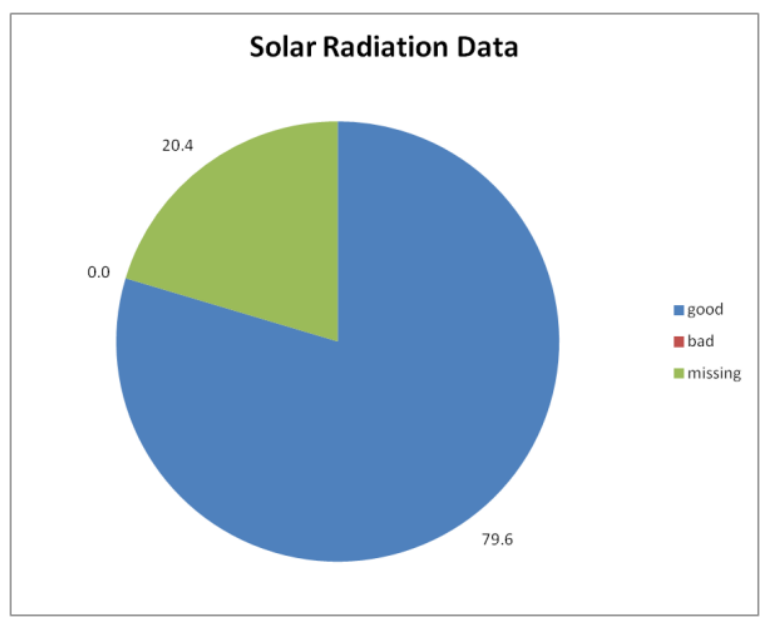

Figure L-142 


\section{December 2010- Site 2}

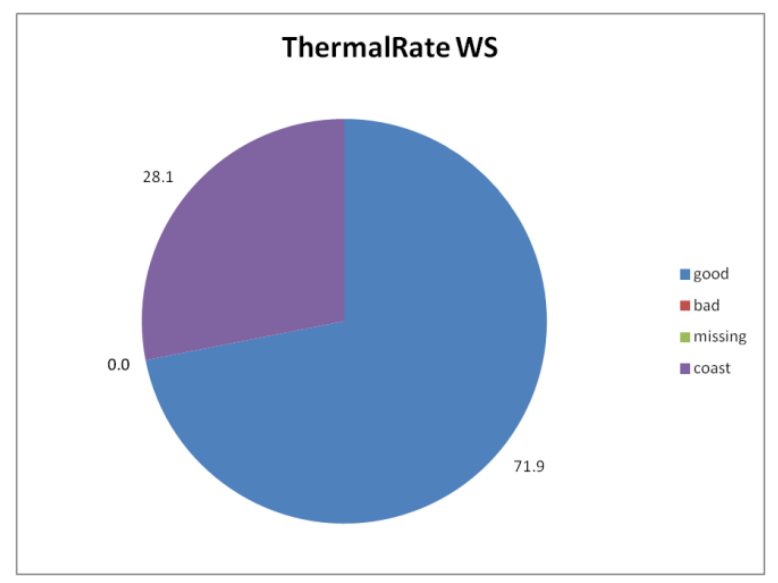

Figure L-143

\section{January 2011 Site 2}

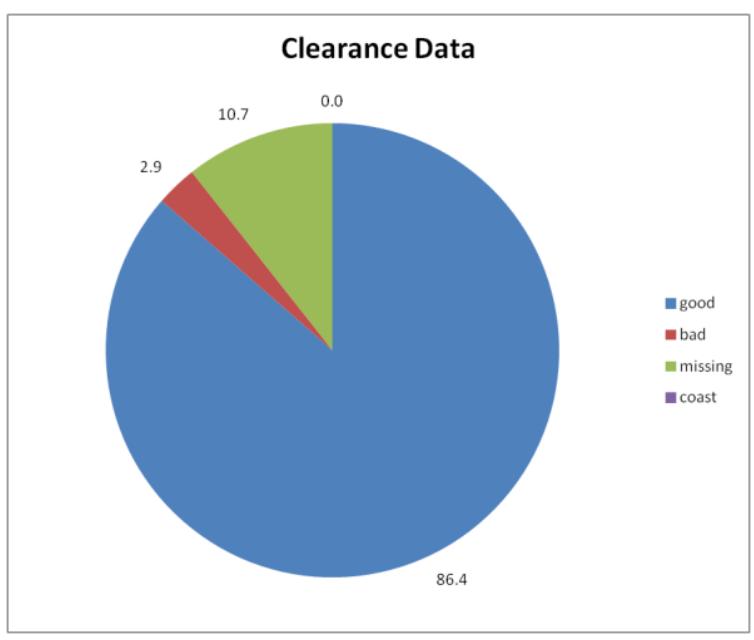

Figure L-145

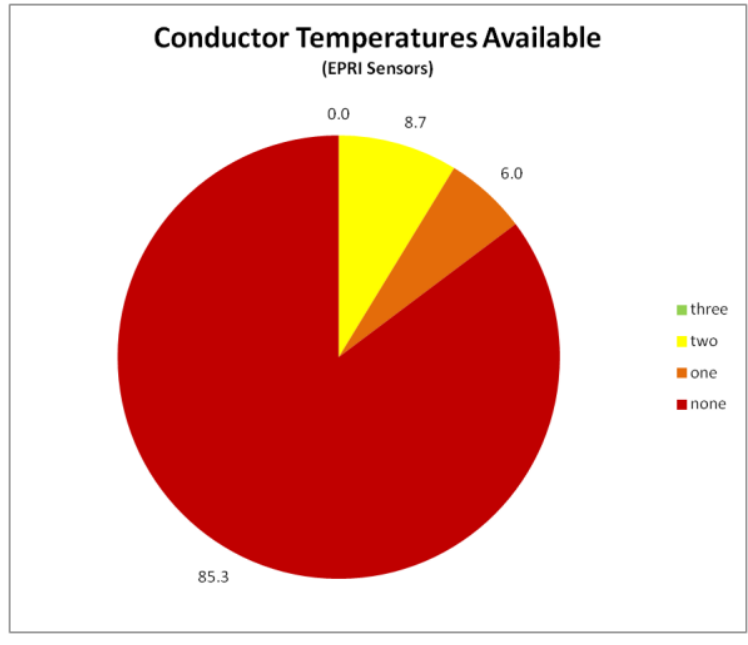

Figure L-144

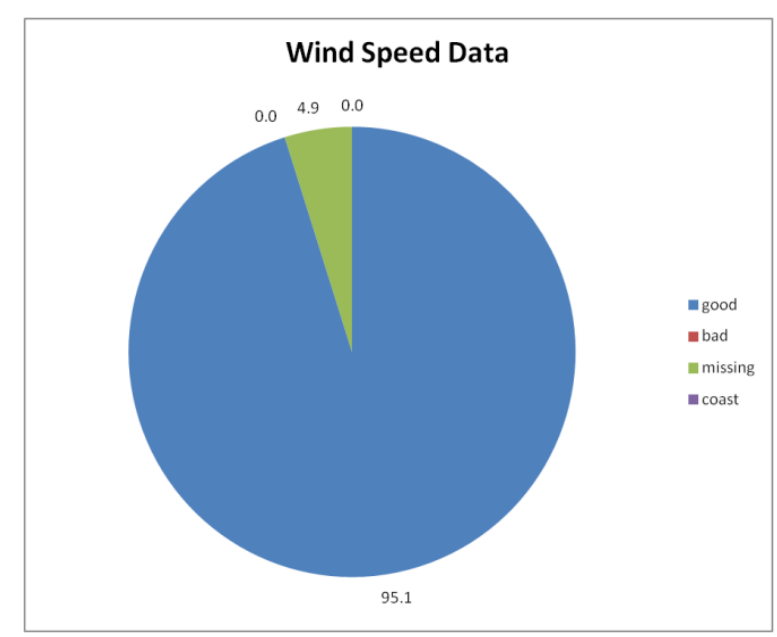

Figure L-146 


\section{January 2011 Site 2}

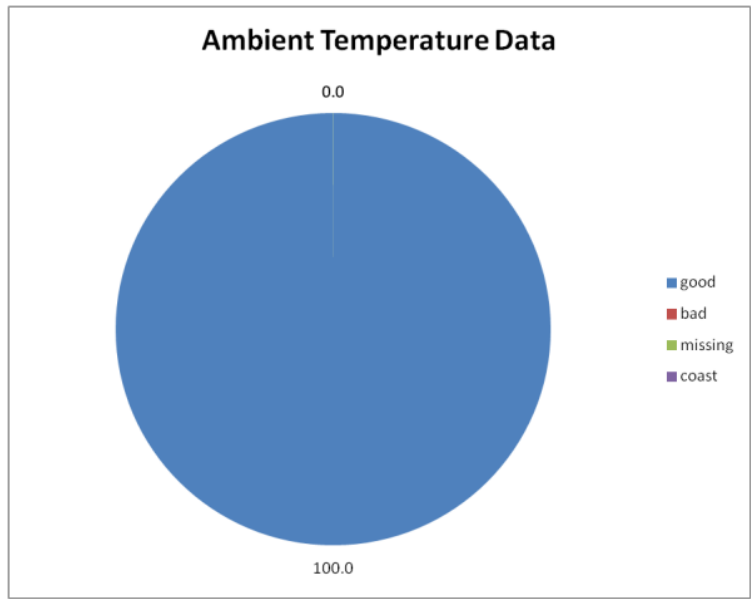

Figure L-147

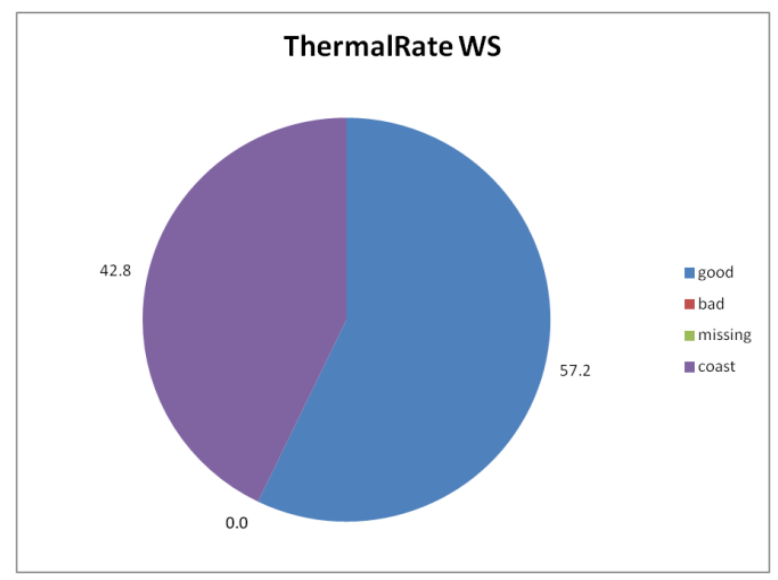

Figure L-149

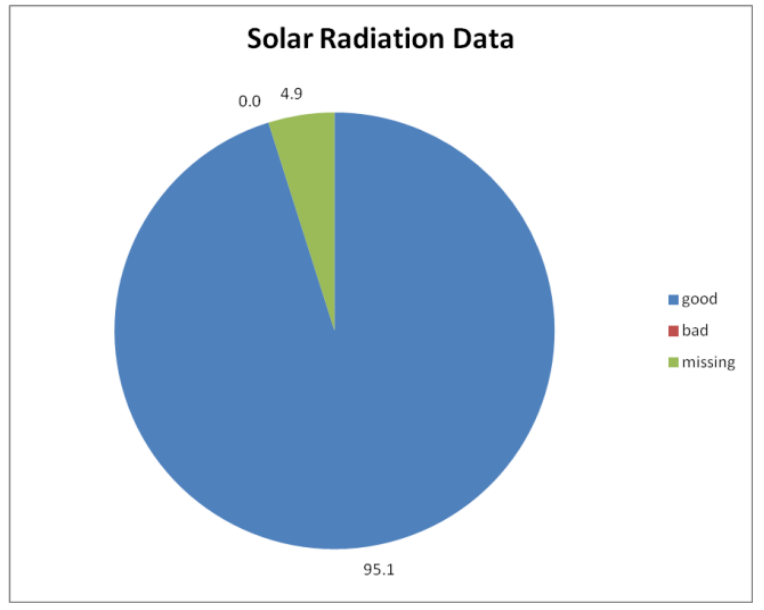

Figure L-148

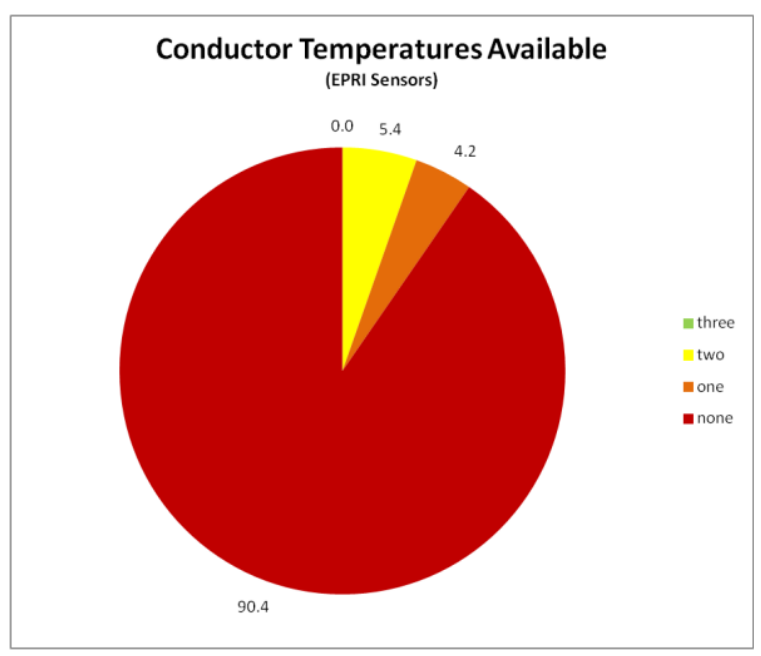

Figure L-150 
February 2011 Site 2

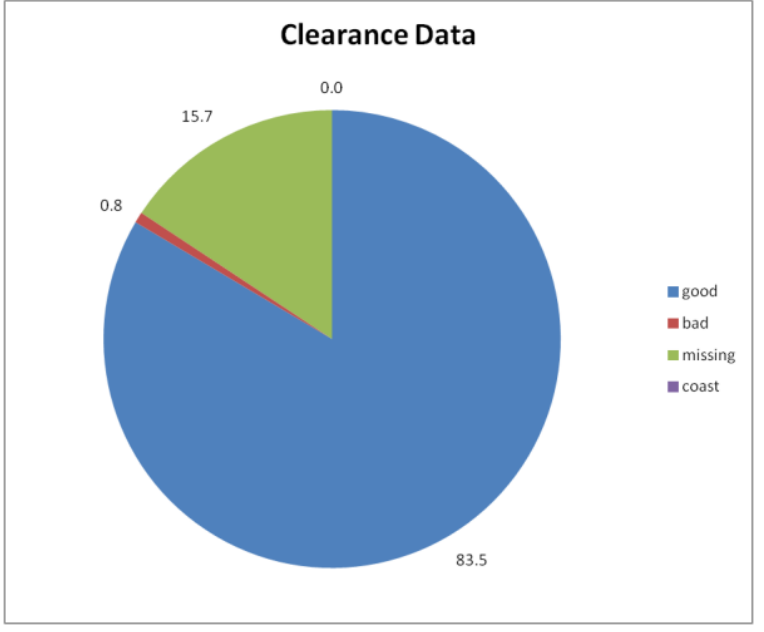

Figure L-151

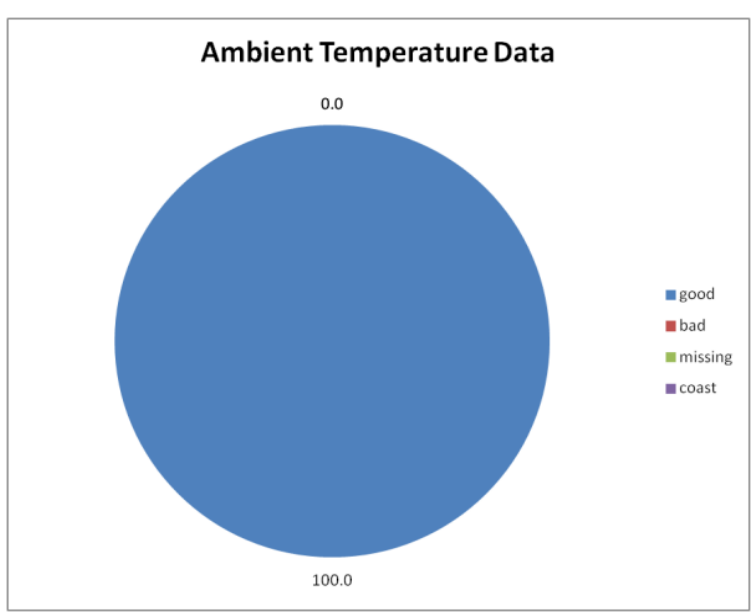

Figure L-153

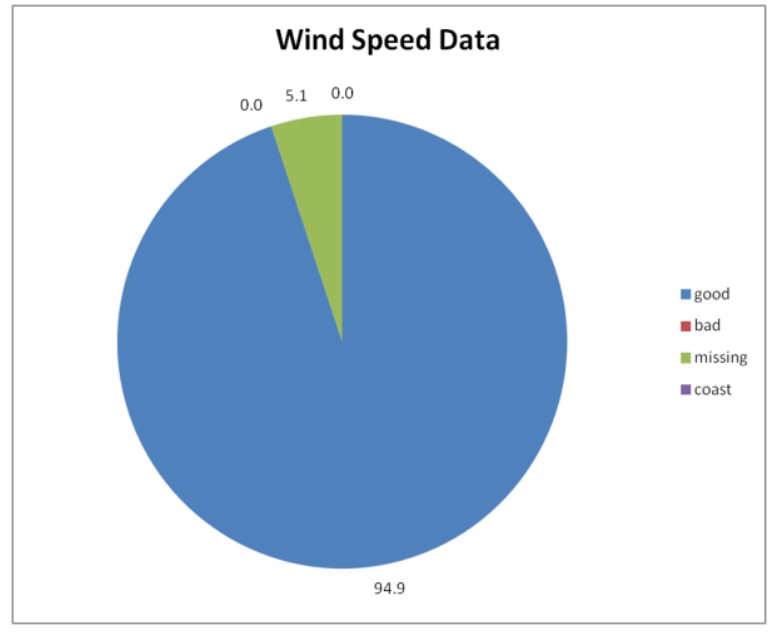

Figure L-152

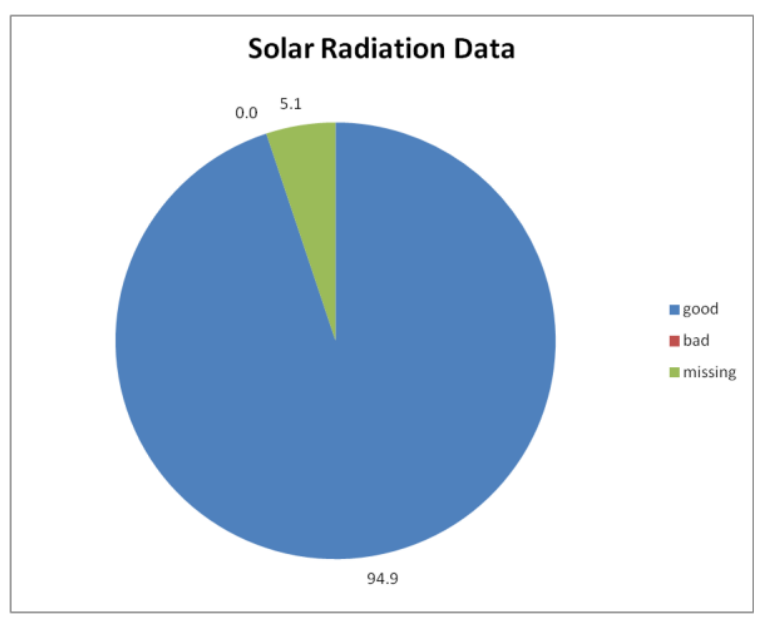

Figure L-154 


\section{February 2011 Site 2}

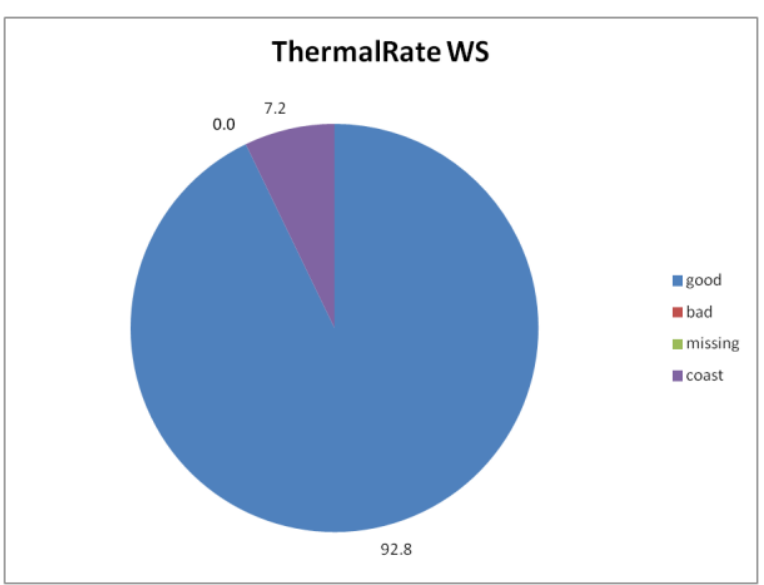

Figure L-155

\section{March 2011 Site 2}

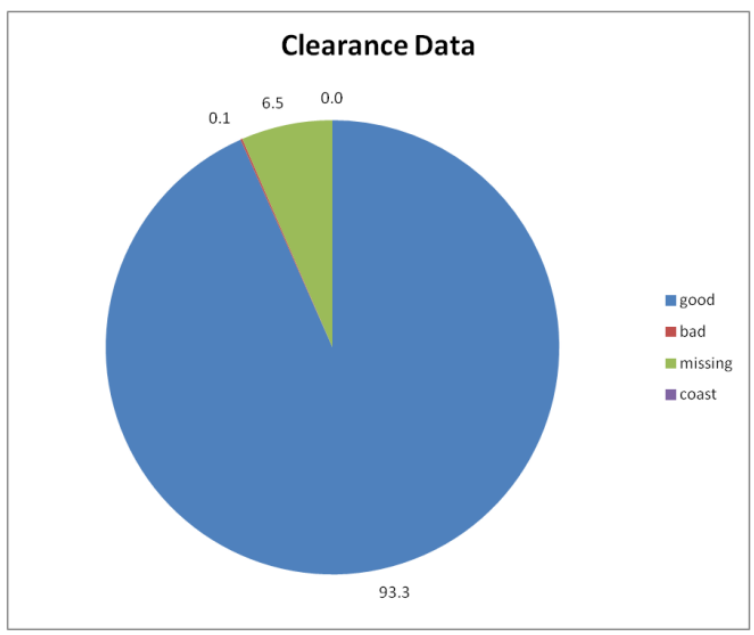

Figure L-157

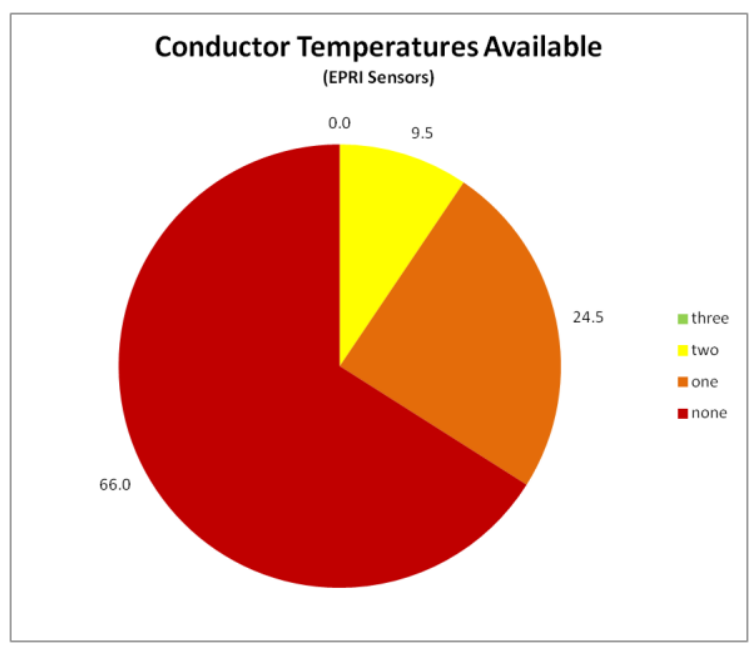

Figure L-156

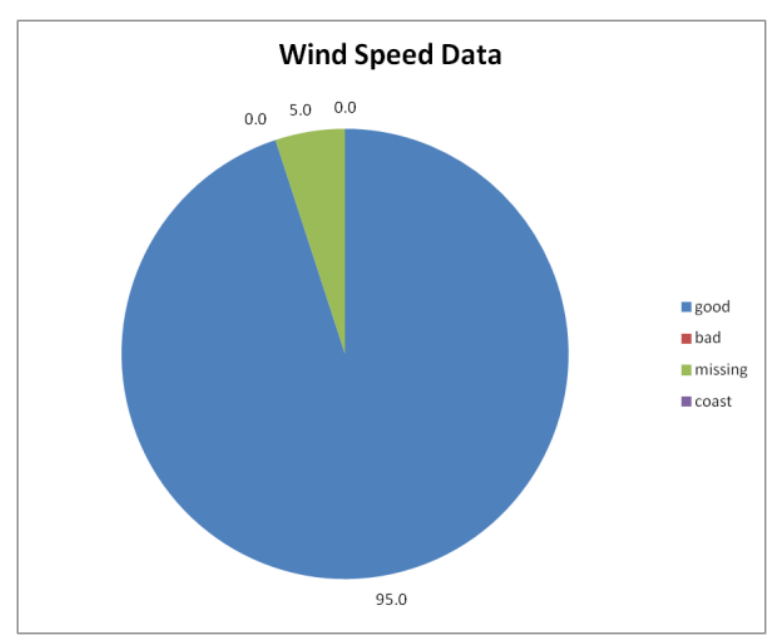

Figure L-158 
March 2011 Site 2

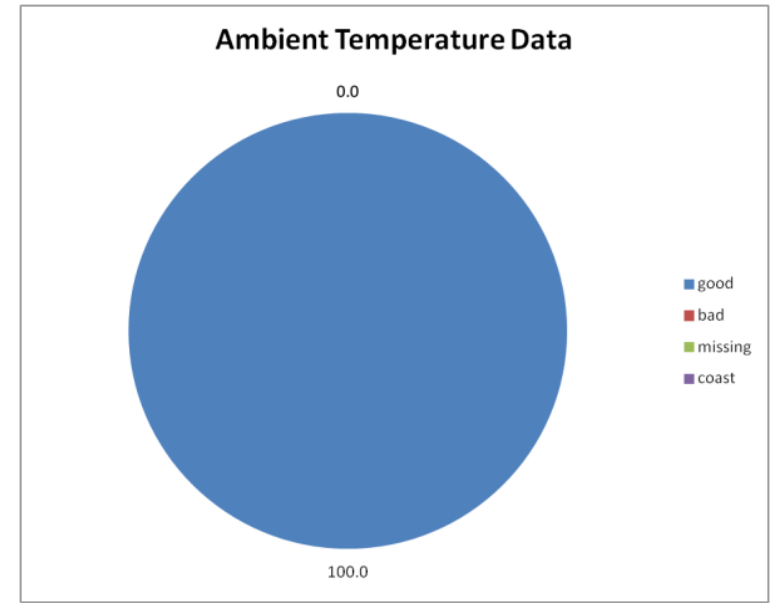

Figure L-159

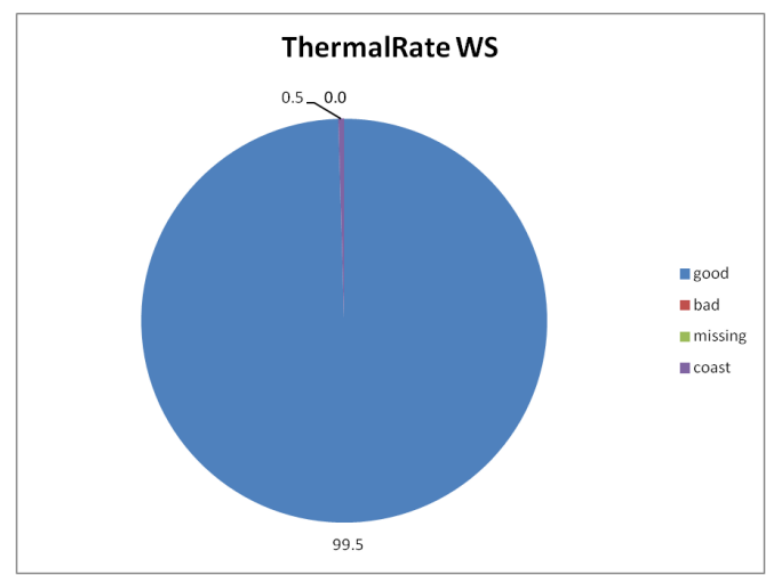

Figure L-161

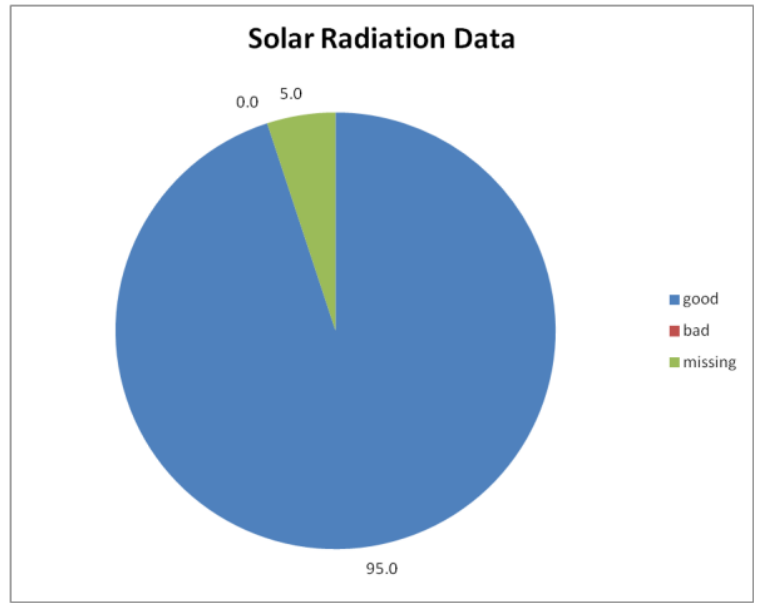

Figure L-160

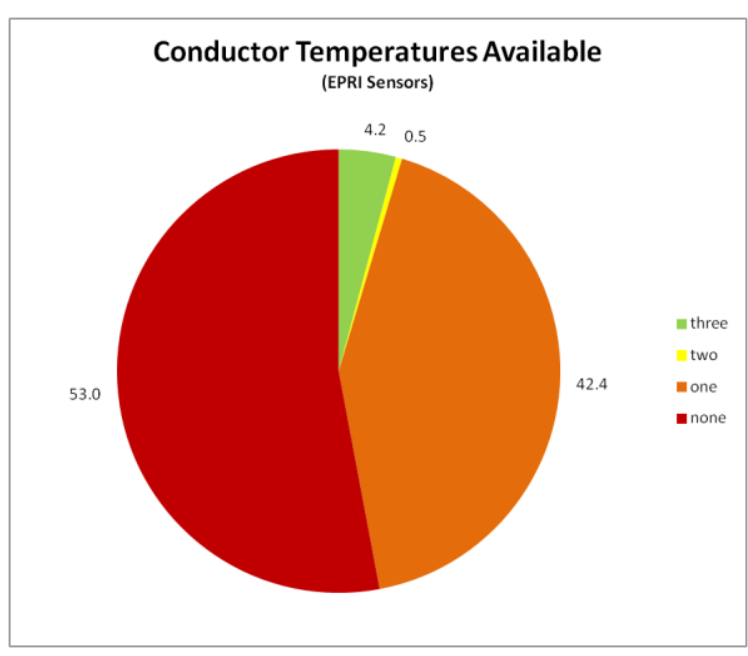

Figure L-162 


\section{April 2011 Site 2}

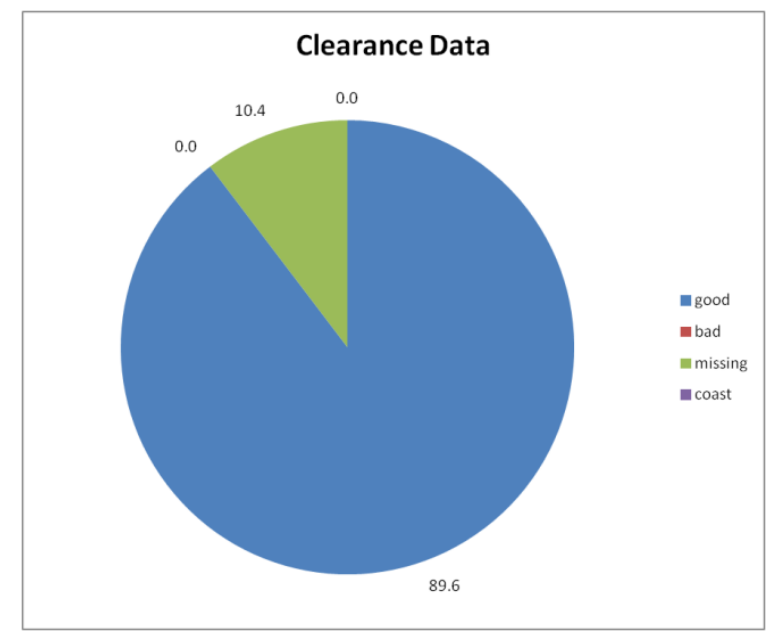

Figure L-163

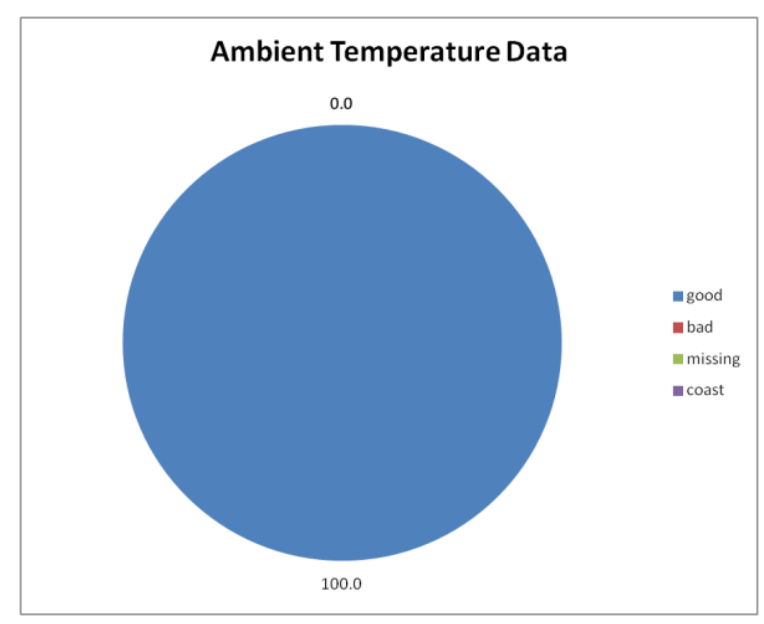

Figure L-165

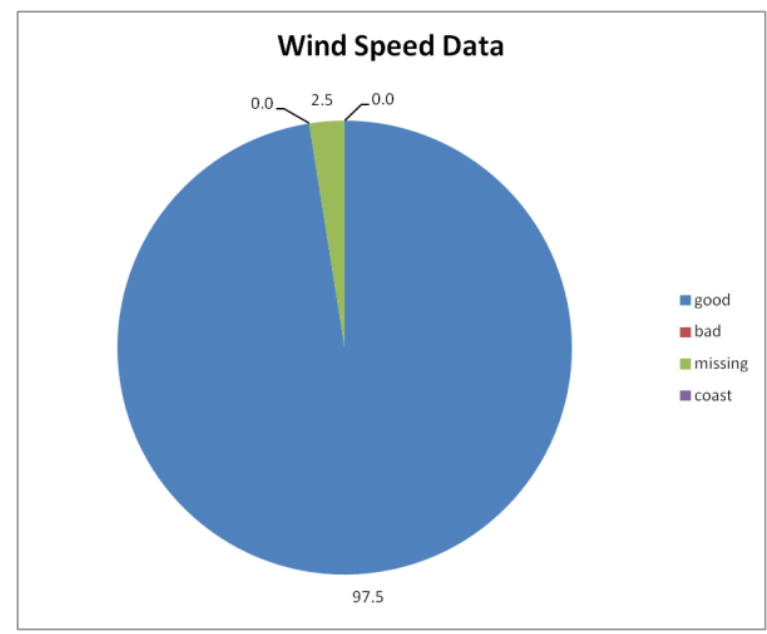

Figure L-164

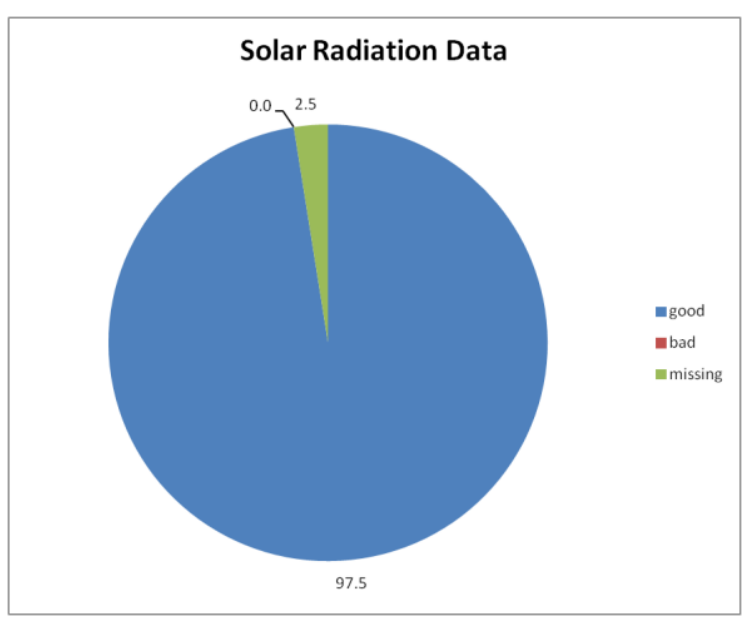

Figure L-166 


\section{April 2011 Site 2}

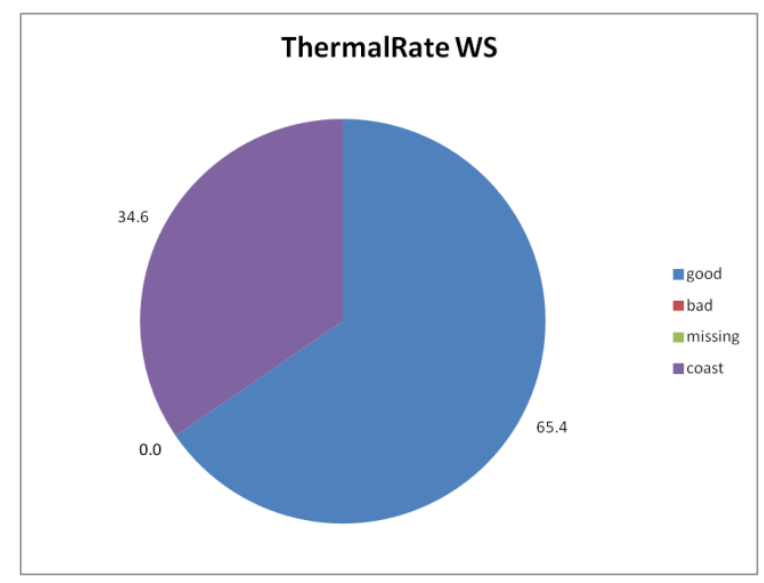

Figure L-167

\section{May 2011 Site 2}

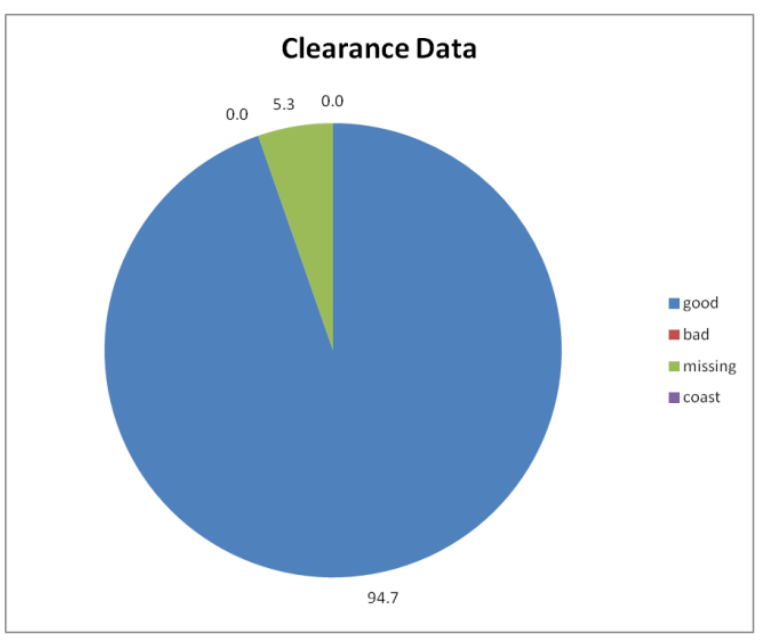

Figure L-169

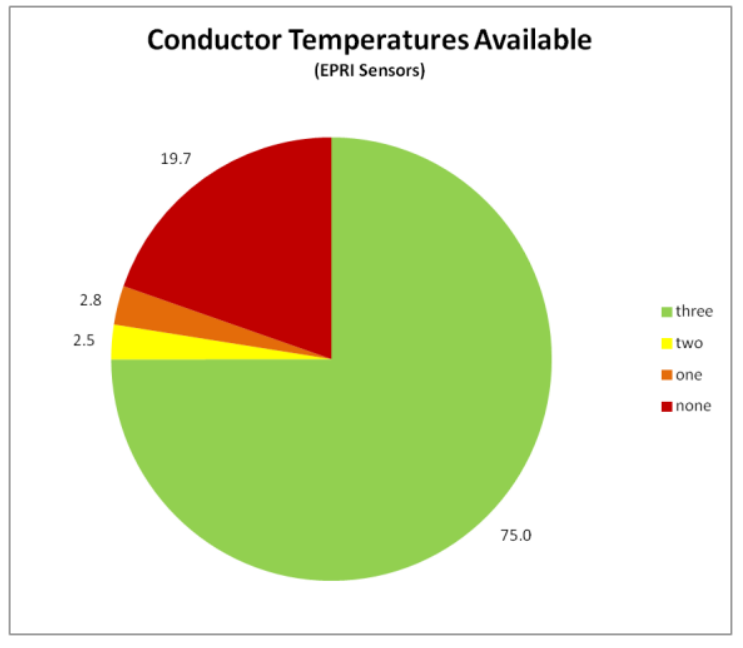

Figure L-168

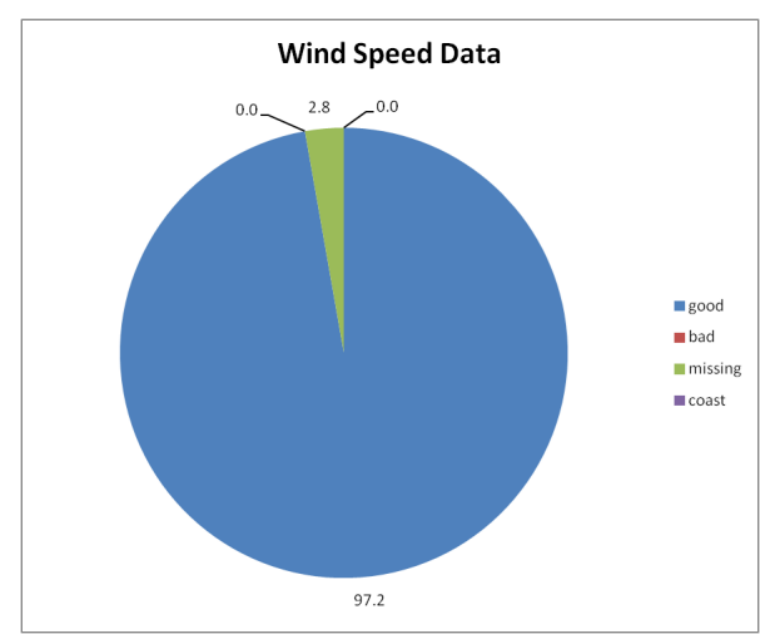

Figure L-170 
May 2011 Site 2

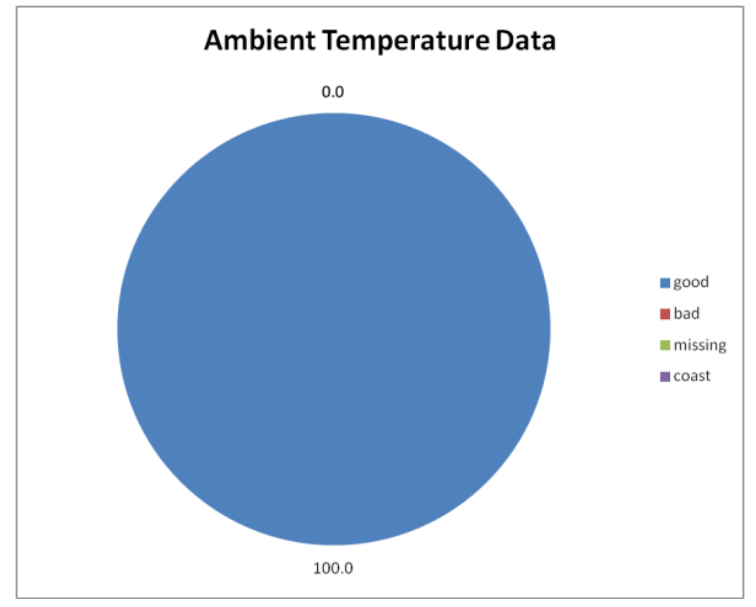

Figure L-171

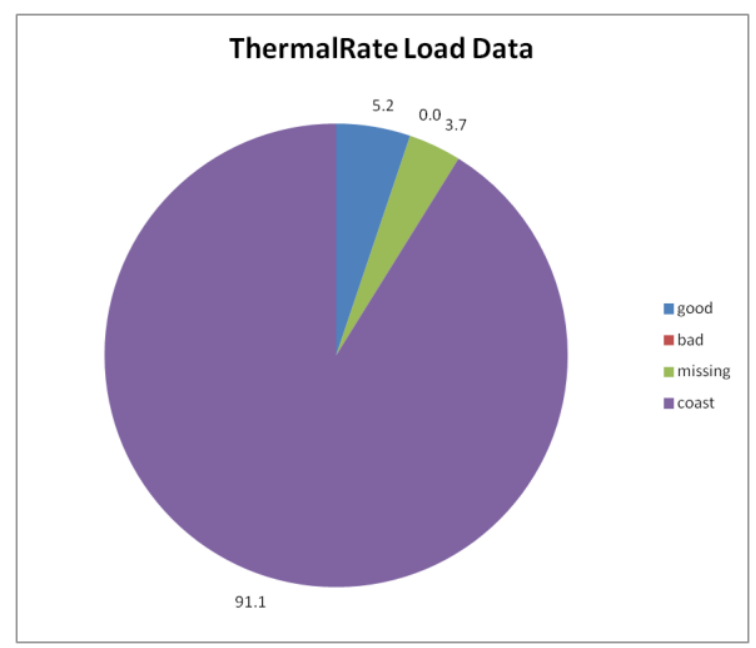

Figure L-173

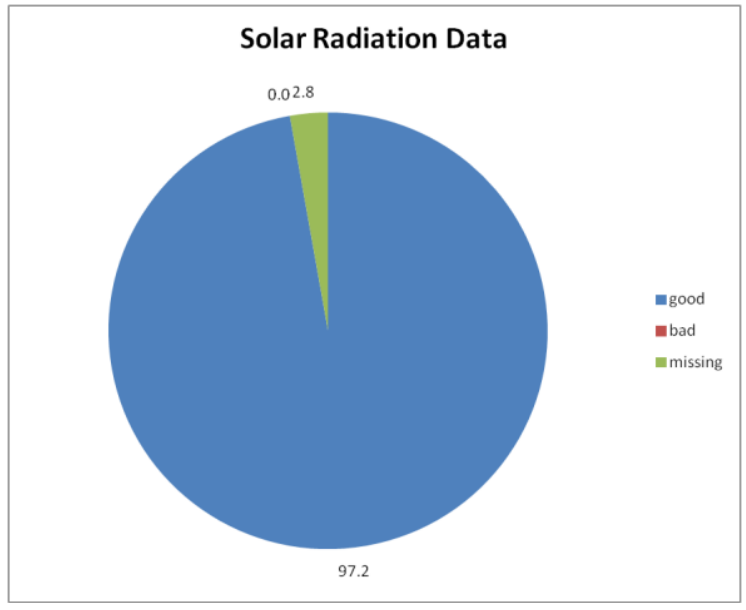

Figure L-172

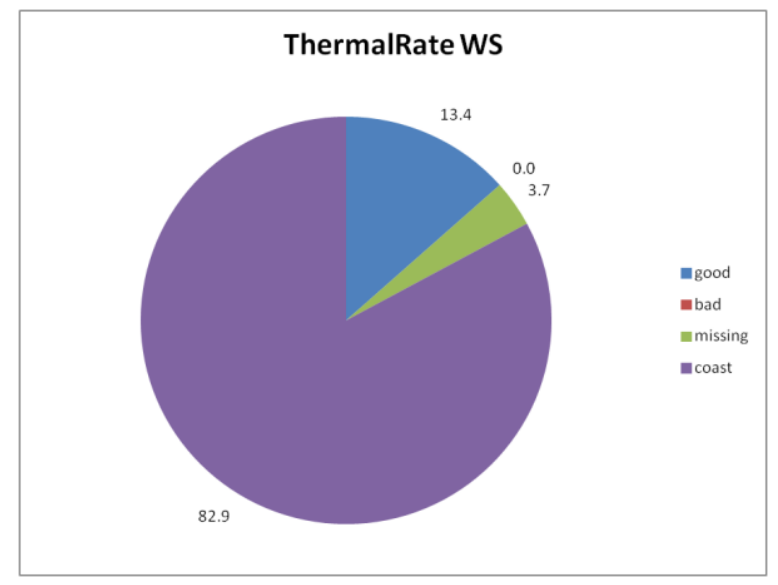

Figure L-174 
May 2011 Site 2

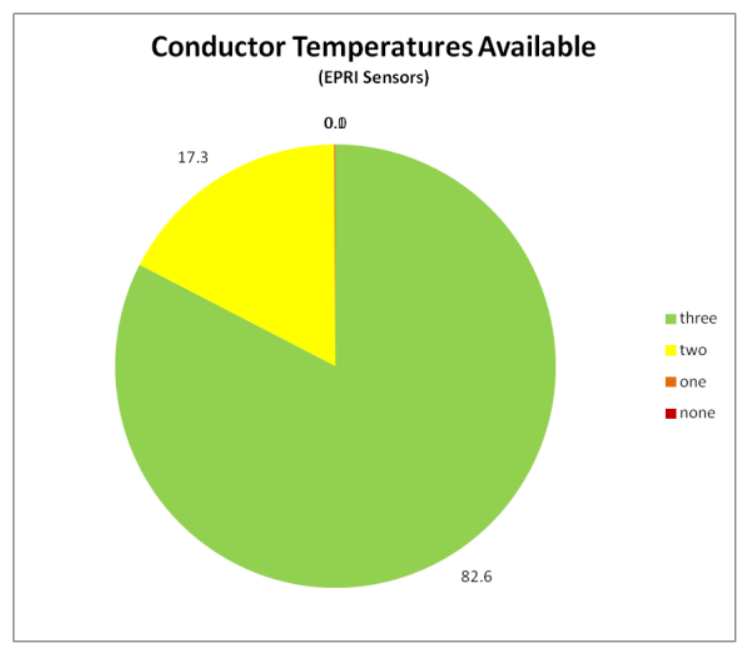

Figure L-175

June 2012 Site 2

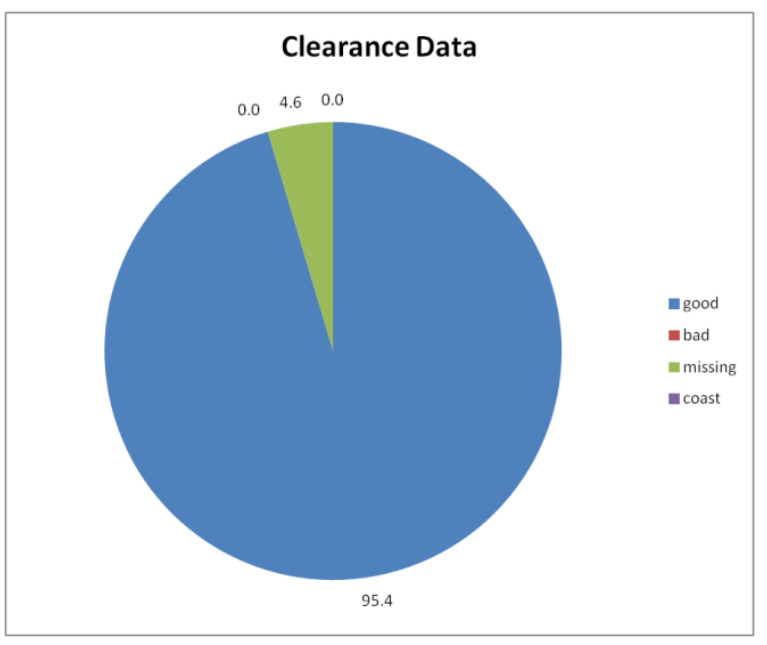

Figure L-176

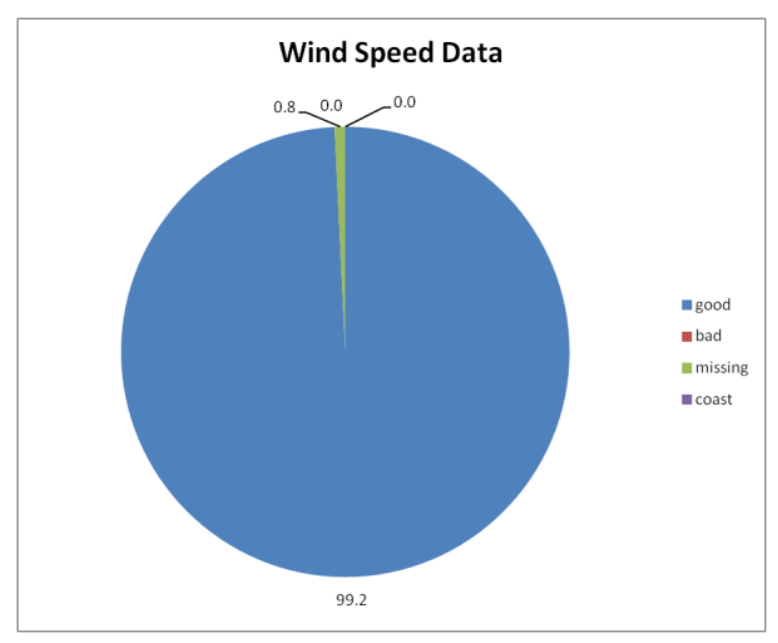

Figure L-177 


\section{June 2012 Site 2}

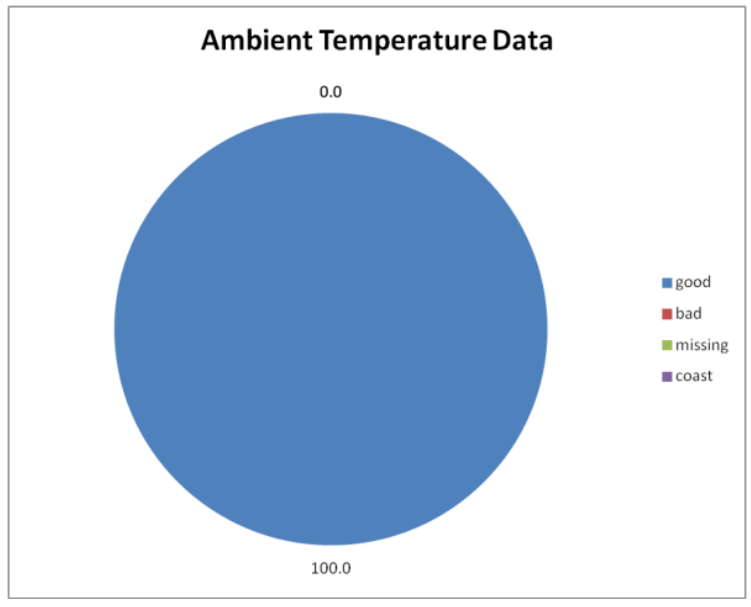

Figure L-178

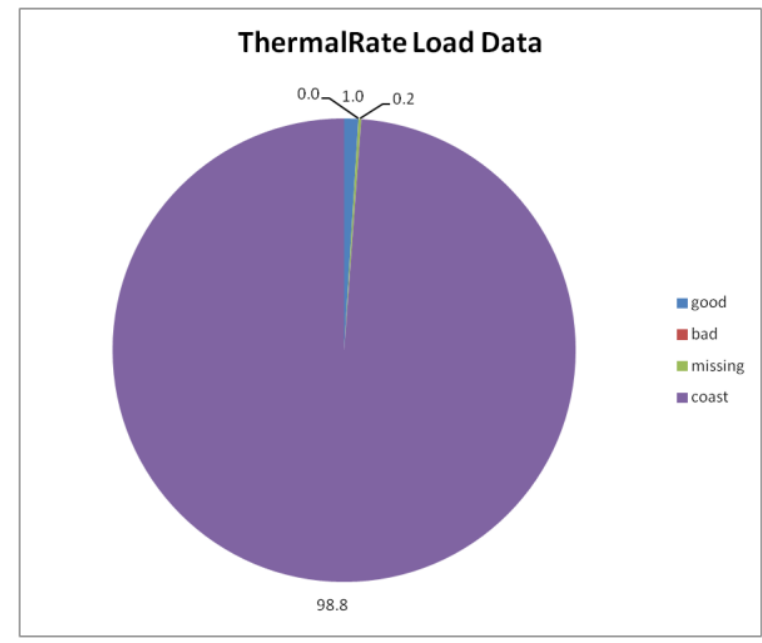

Figure L-180

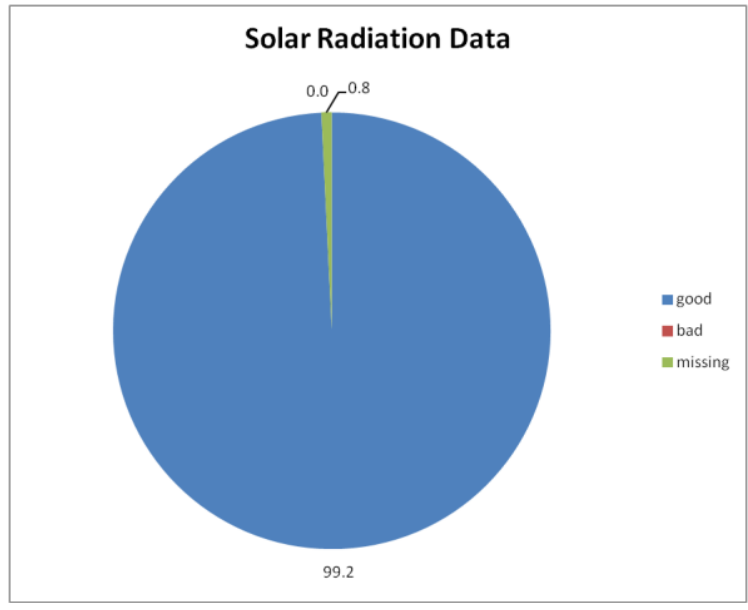

Figure L-179

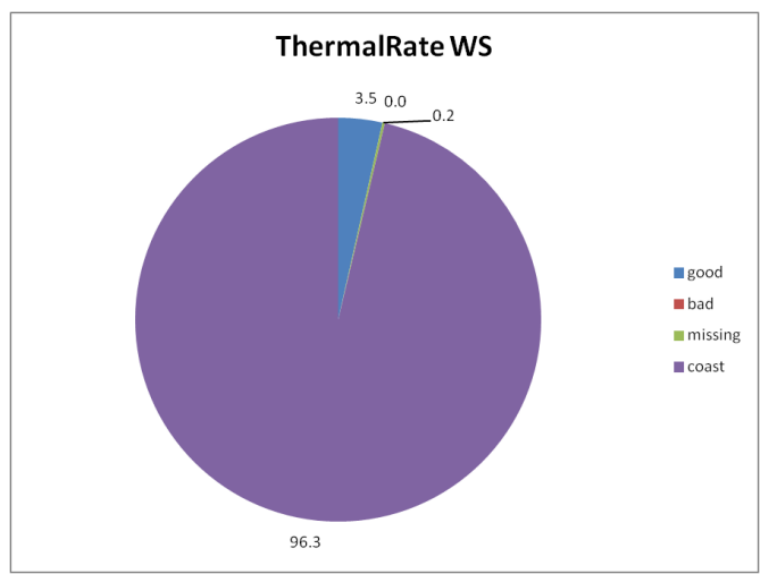

Figure L-181 


\section{June 2012 Site 2}

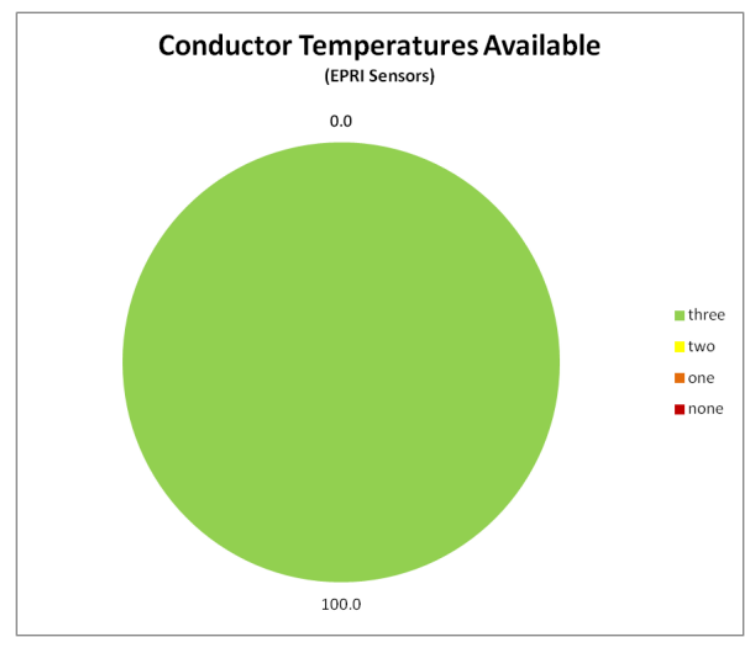

Figure L-182

\section{July 2011 Site 2}

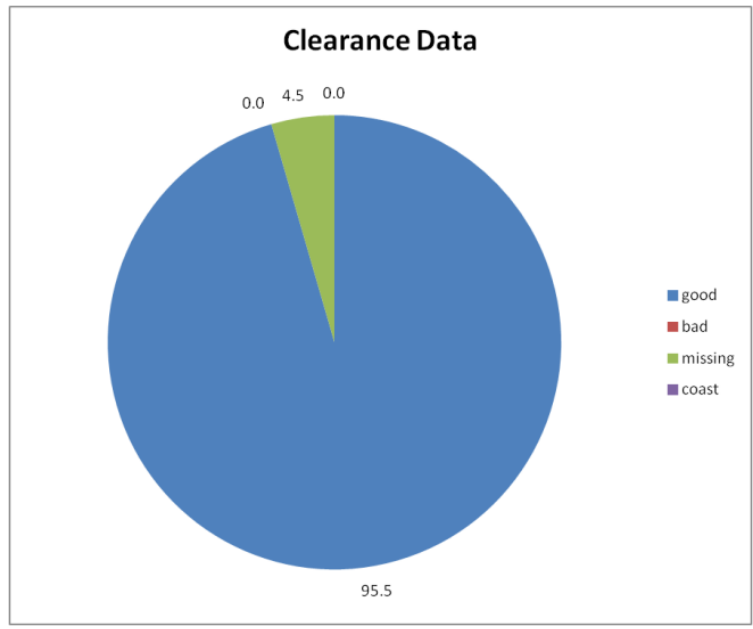

Figure L-183

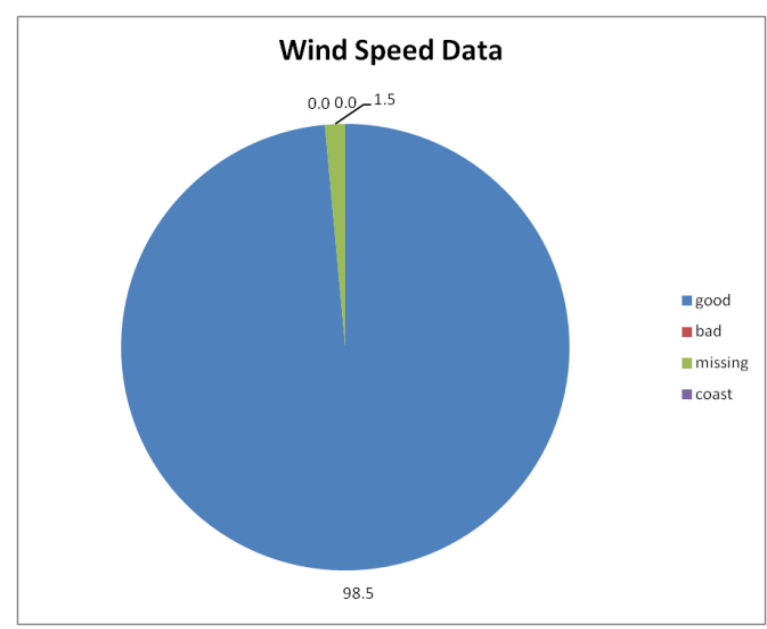

Figure L-184 


\section{July 2011 Site 2}

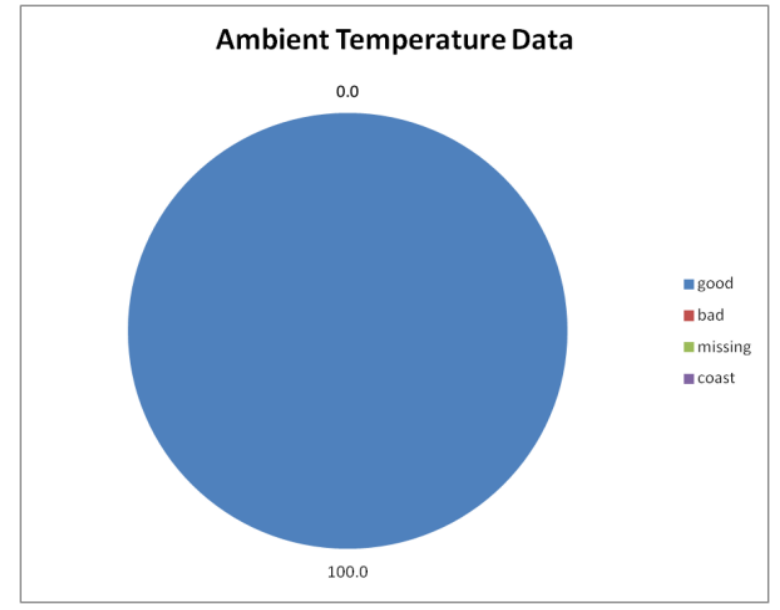

Figure L-185

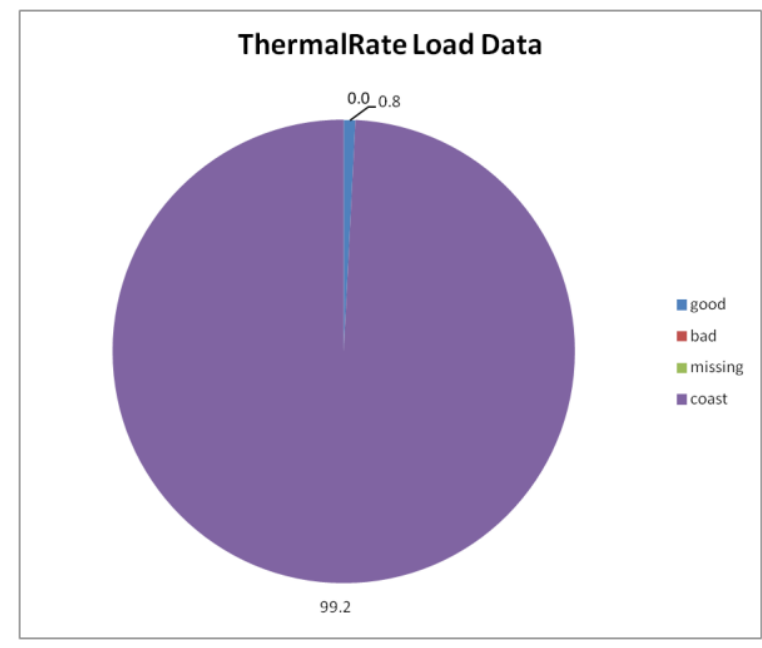

Figure L-187

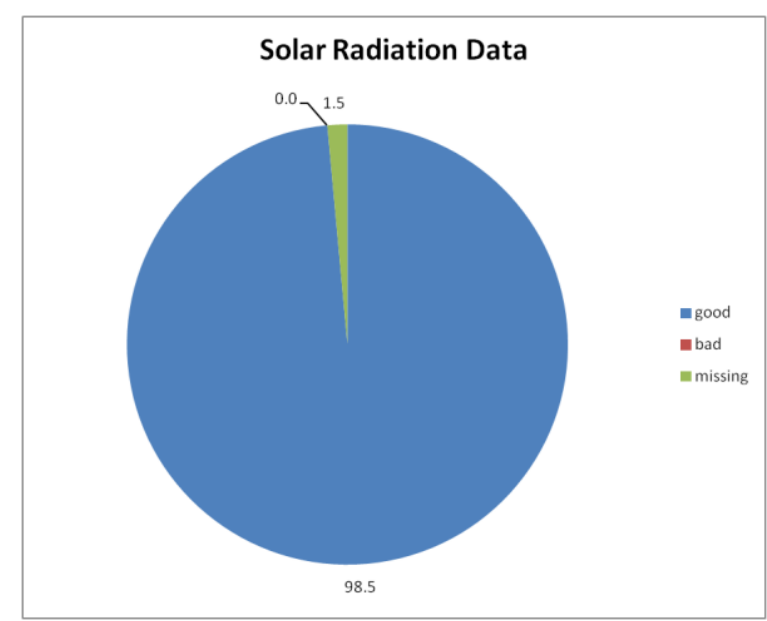

Figure L-186

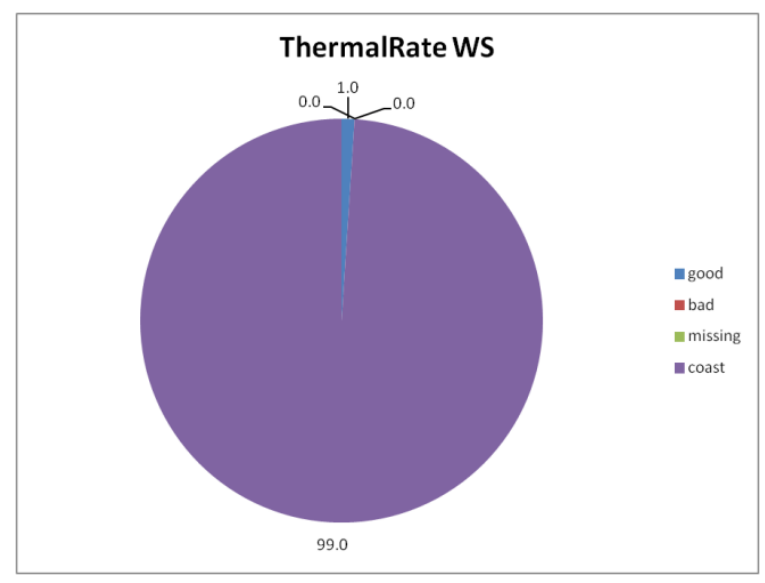

Figure L-188 
July 2011 Site 2

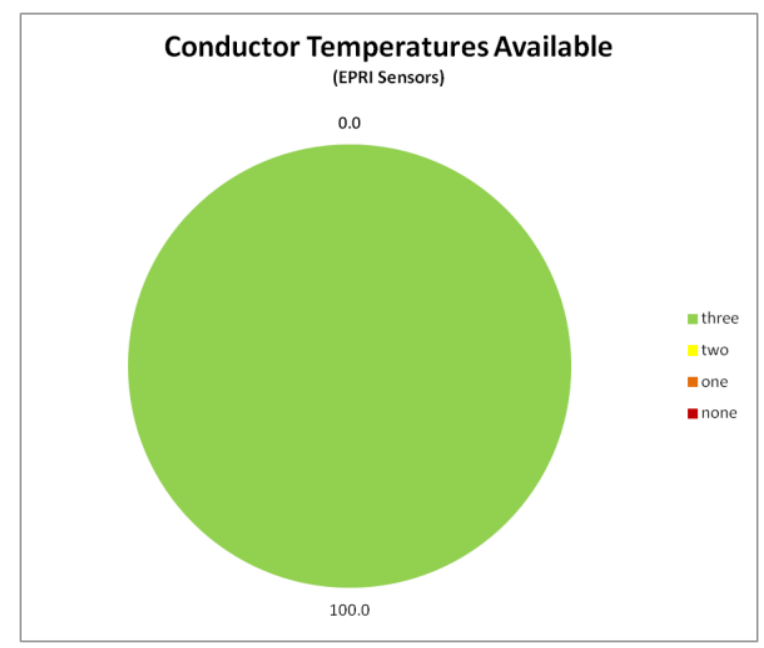

Figure L-189

\section{August 2011 Site 2}

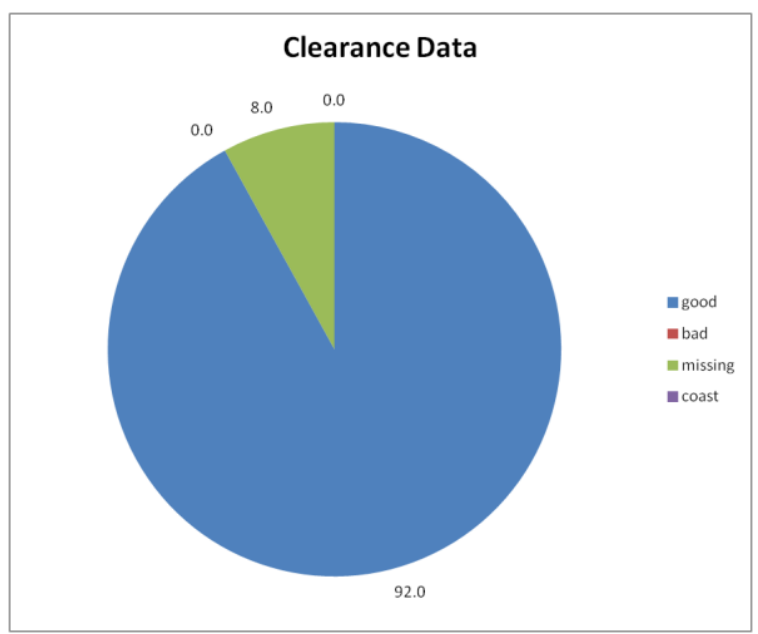

Figure L-190

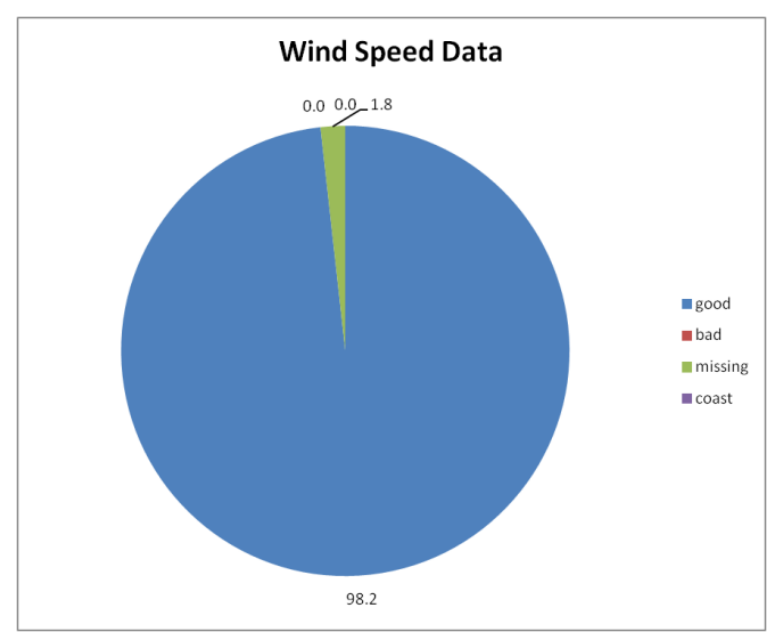

Figure L-191 


\section{August 2011 Site 2}

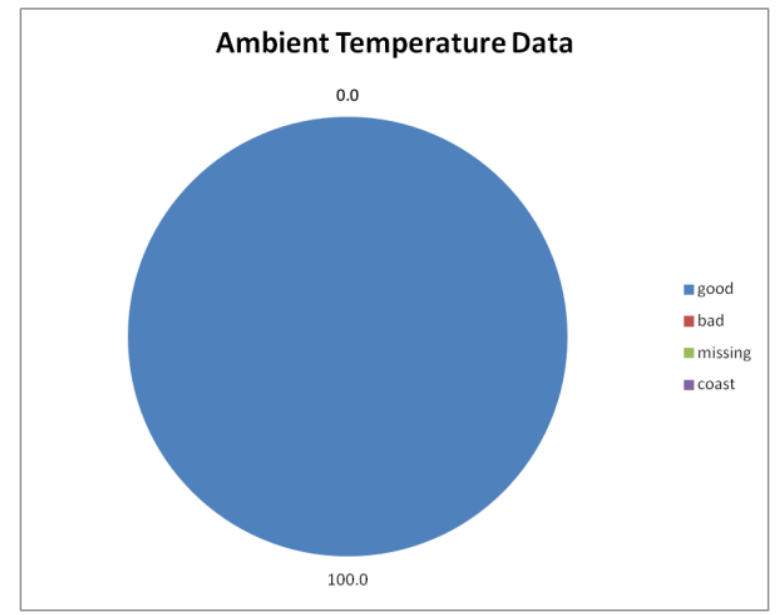

Figure L-192

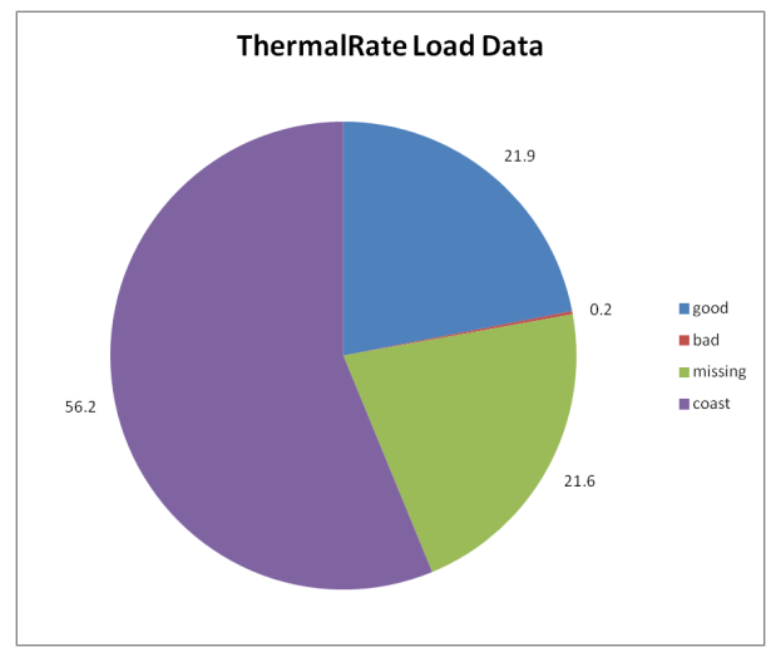

Figure L-194

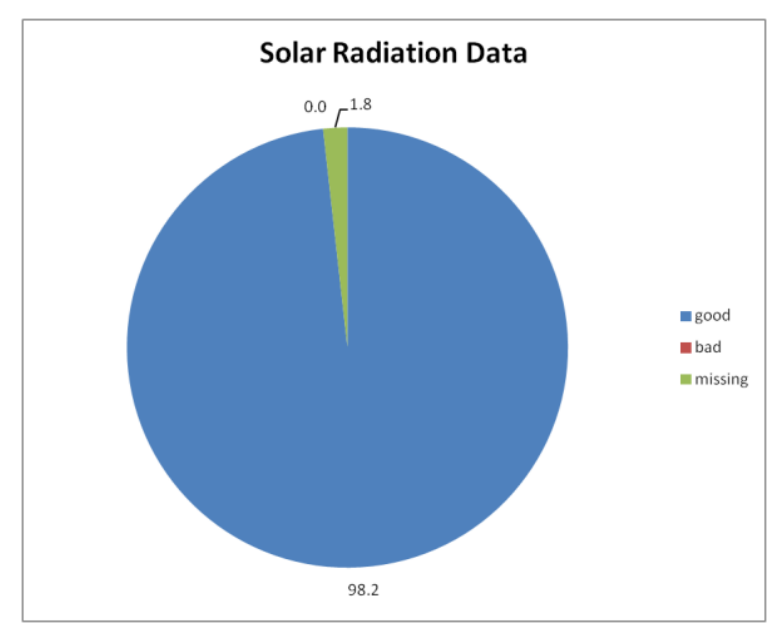

Figure L-193

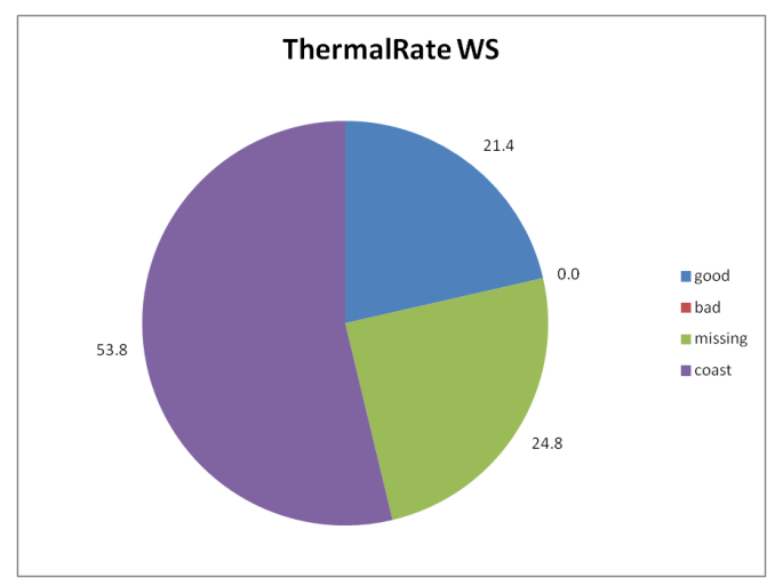

Figure L-195 


\section{August 2011 Site 2}

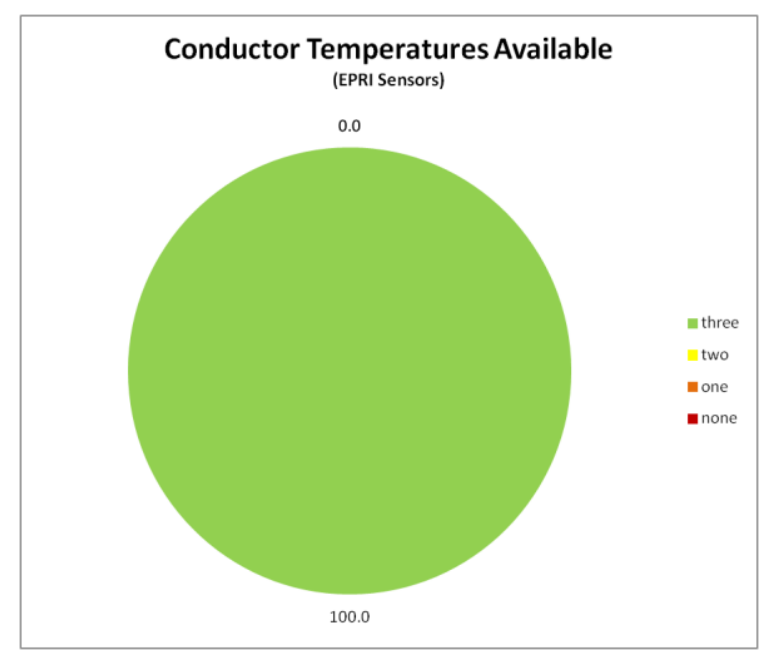

Figure L-196

\section{September 2011 Site 2}

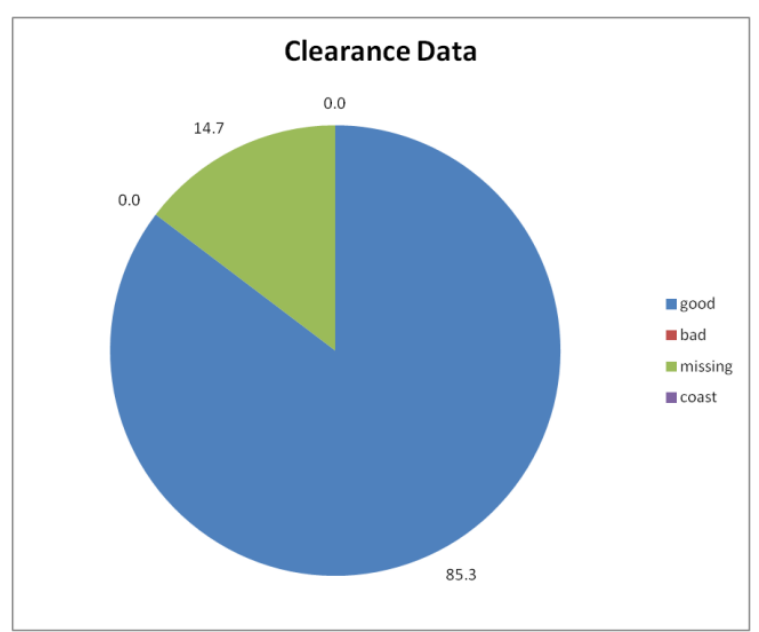

Figure L-197

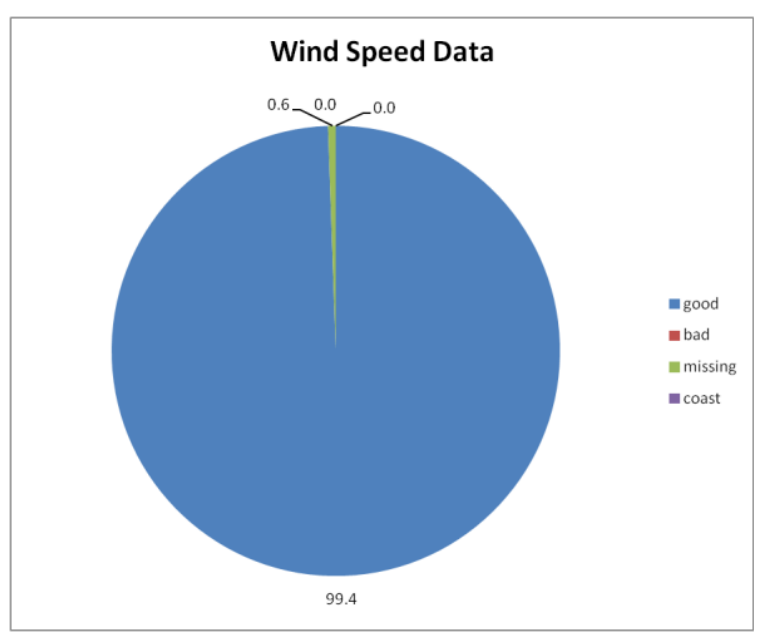

Figure L-198 


\section{September 2011 Site 2}

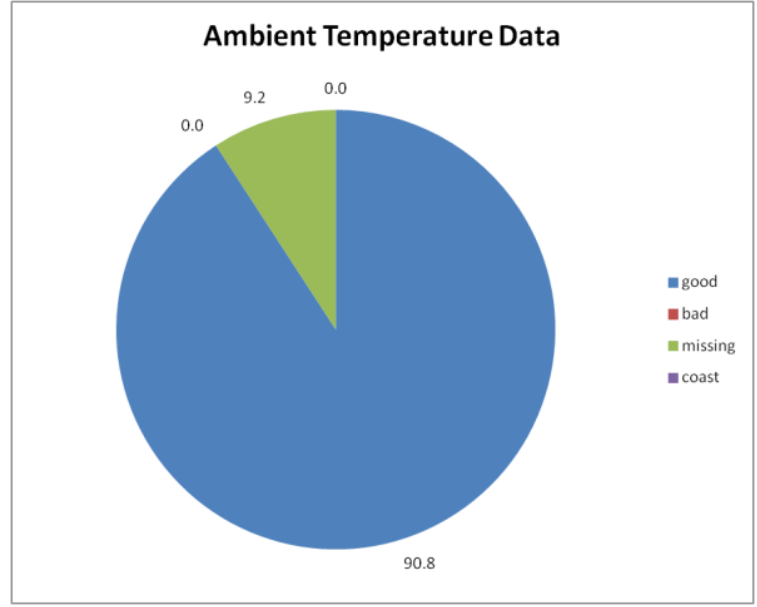

Figure L-199

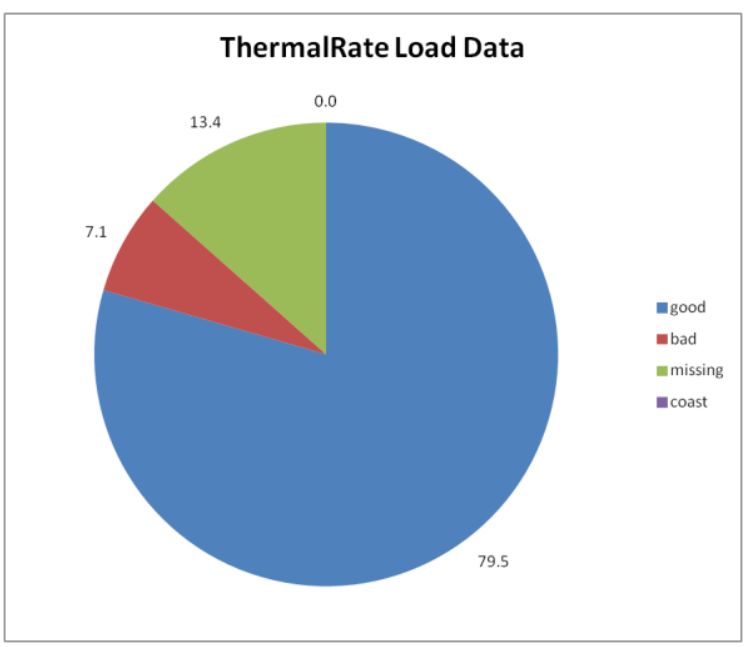

Figure L-201

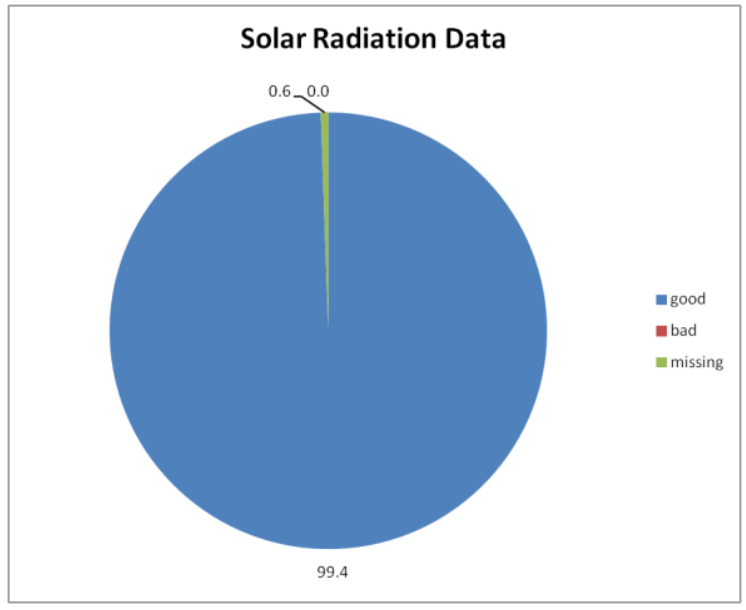

Figure L-200

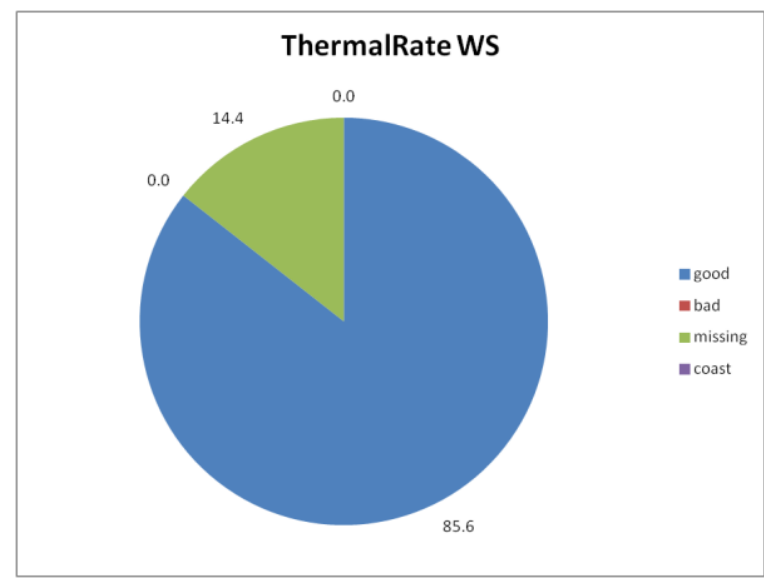

Figure L-202 
September 2011 Site 2

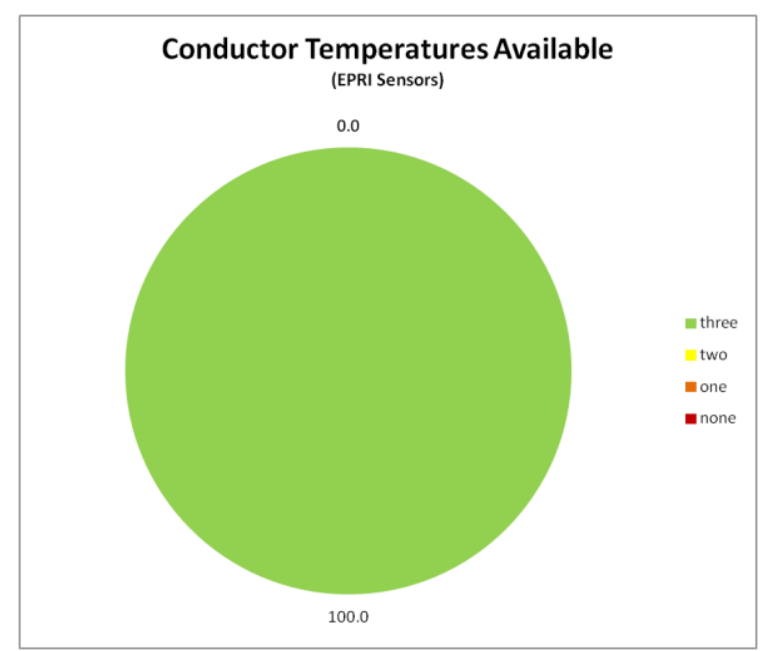

Figure L-203

\section{October 2011 Site 2}

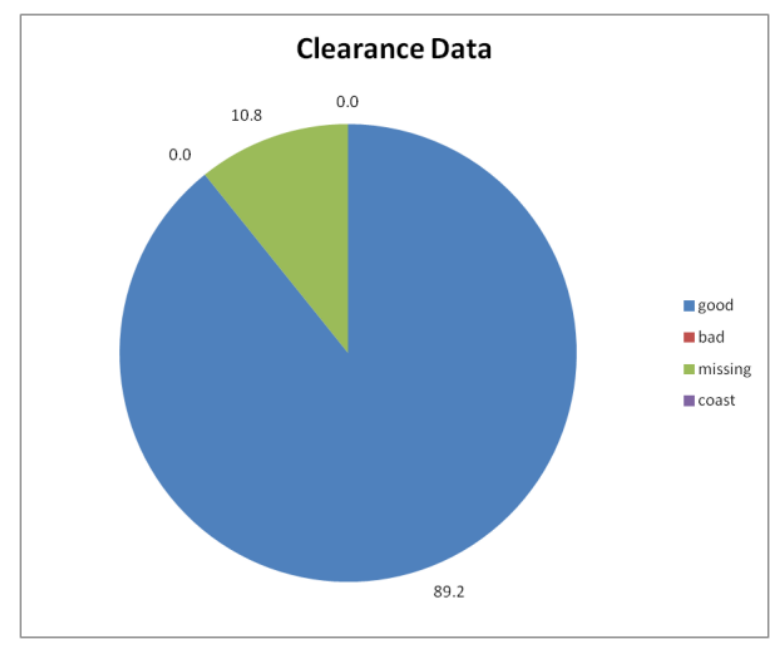

Figure L-204

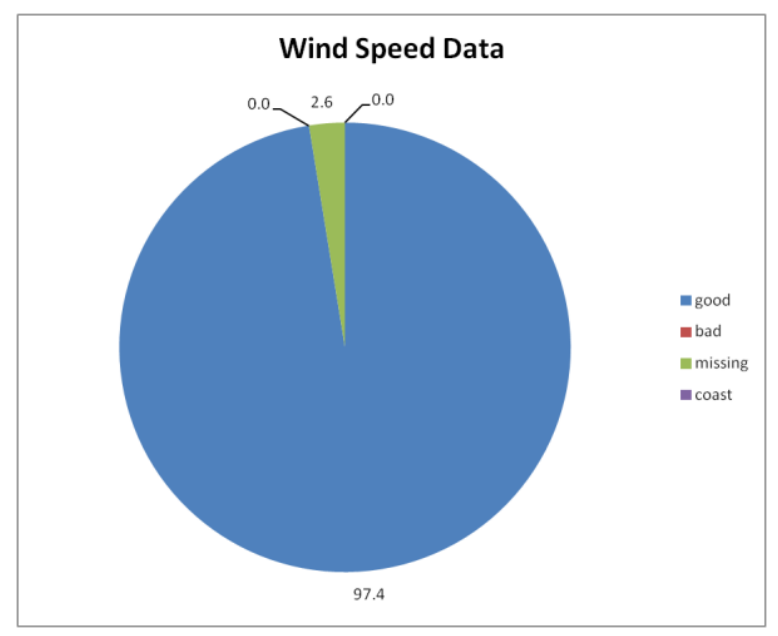

Figure L-205 


\section{October 2011 Site 2}

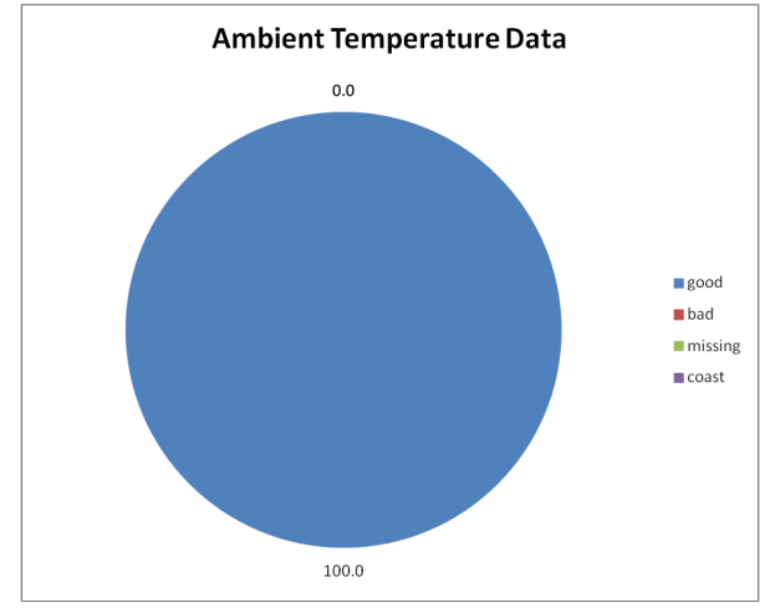

Figure L-206

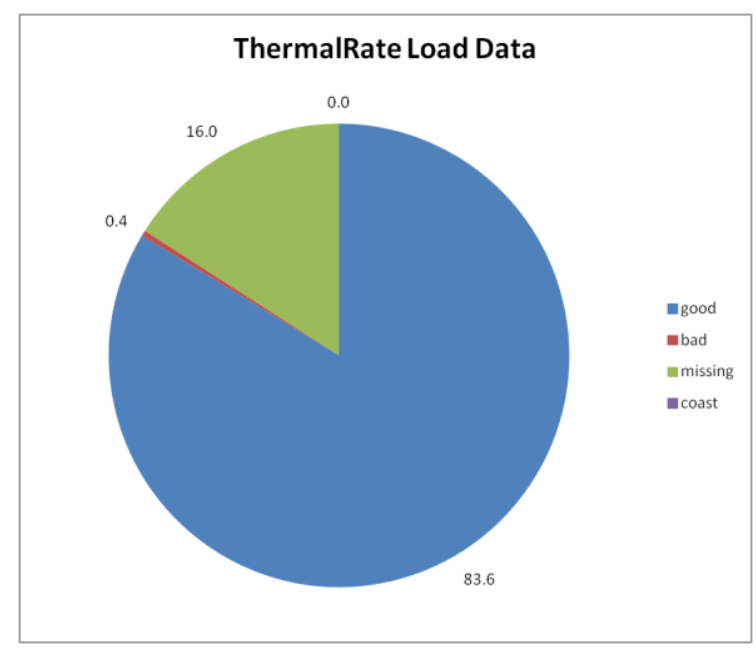

Figure L-208

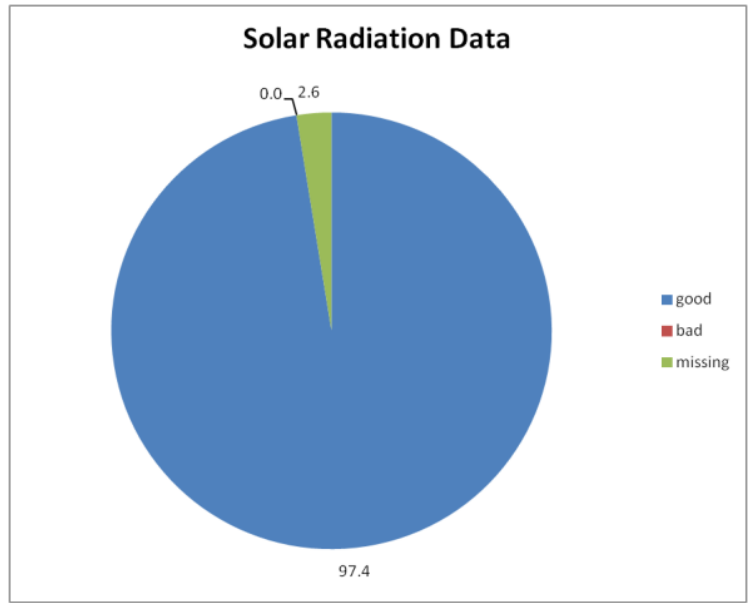

Figure L-207

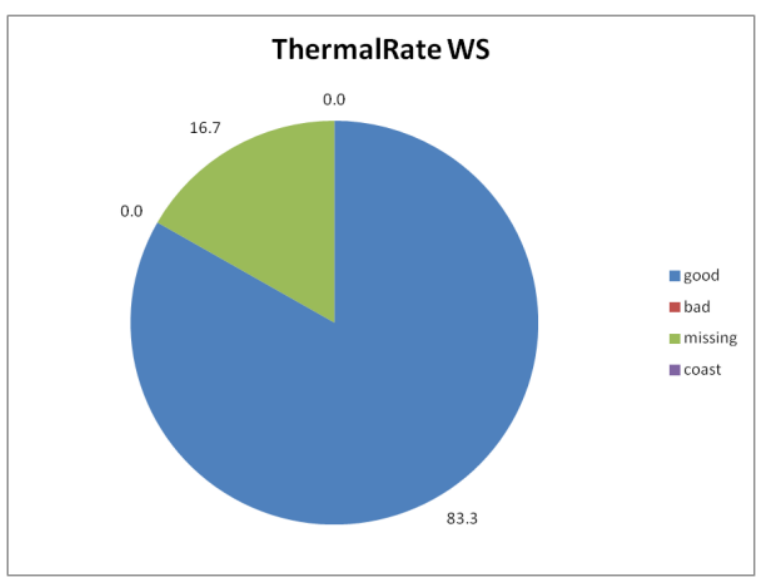

Figure L-209 


\section{October 2011 Site 2}

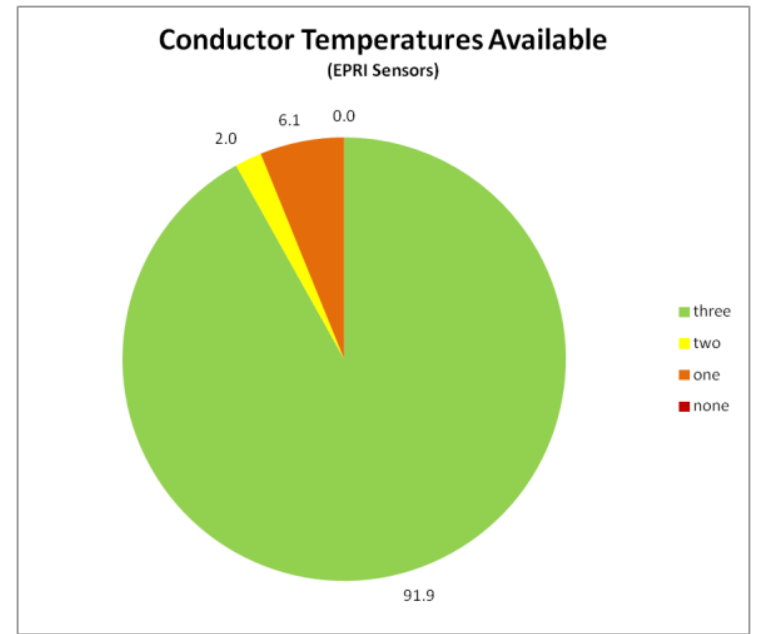

Figure L-210

\section{November 2011 Site 2}

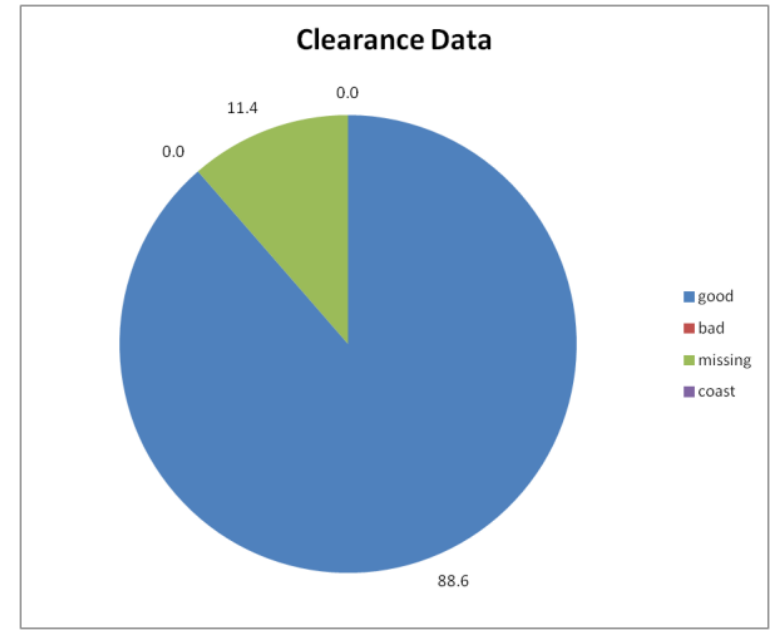

Figure L-211

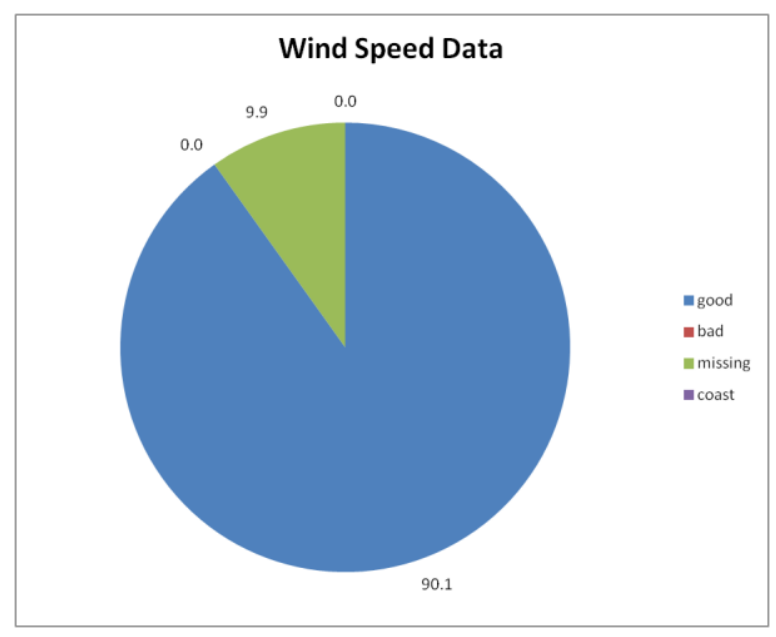

Figure L-212 


\section{November 2011 Site 2}

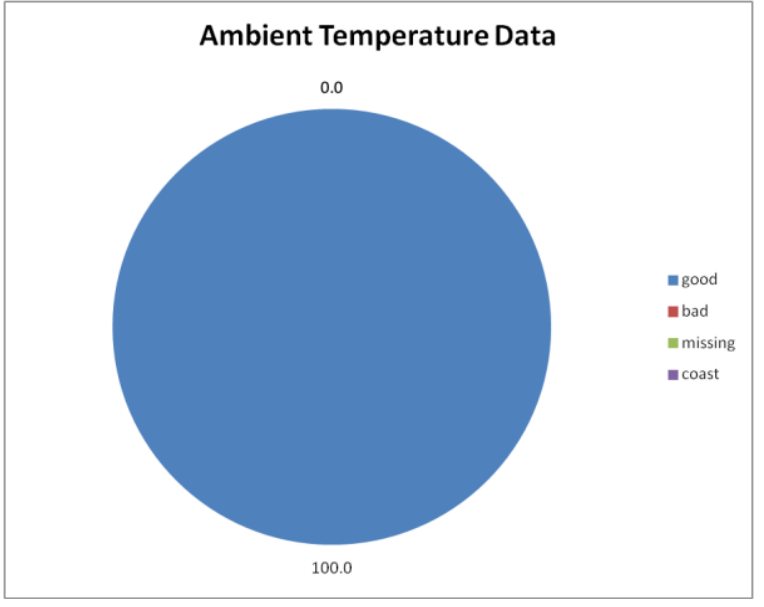

Figure L-213

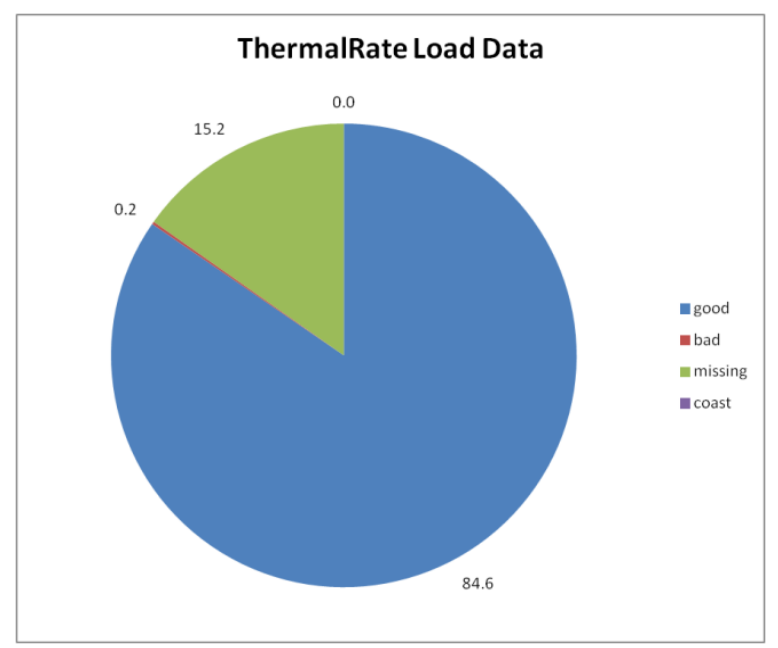

Figure L-215

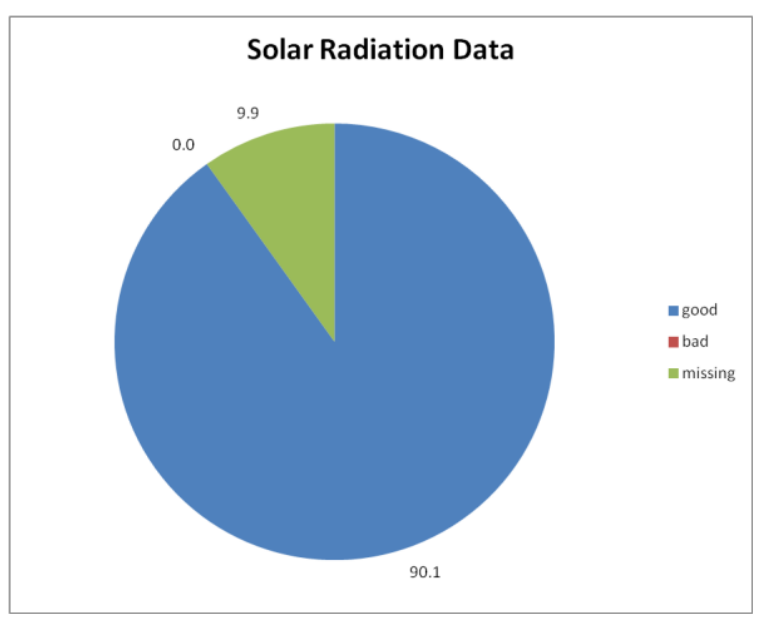

Figure L-214

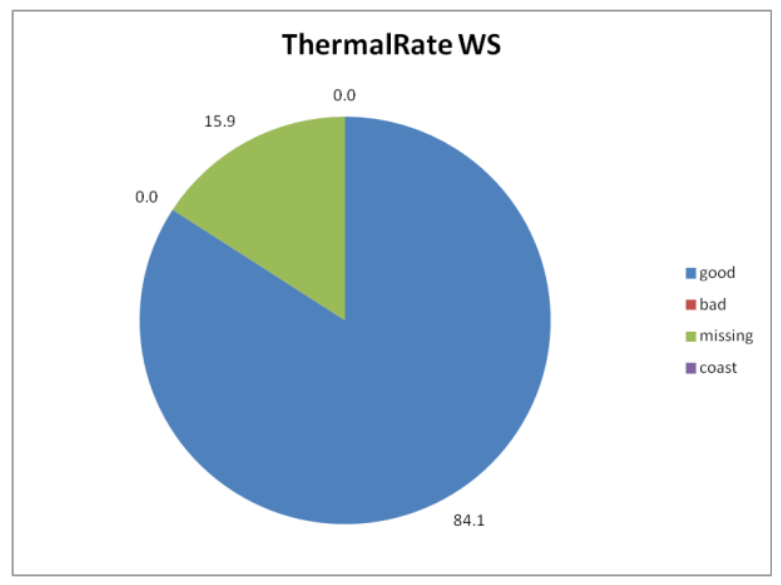

Figure L-216 
November 2011 Site 2

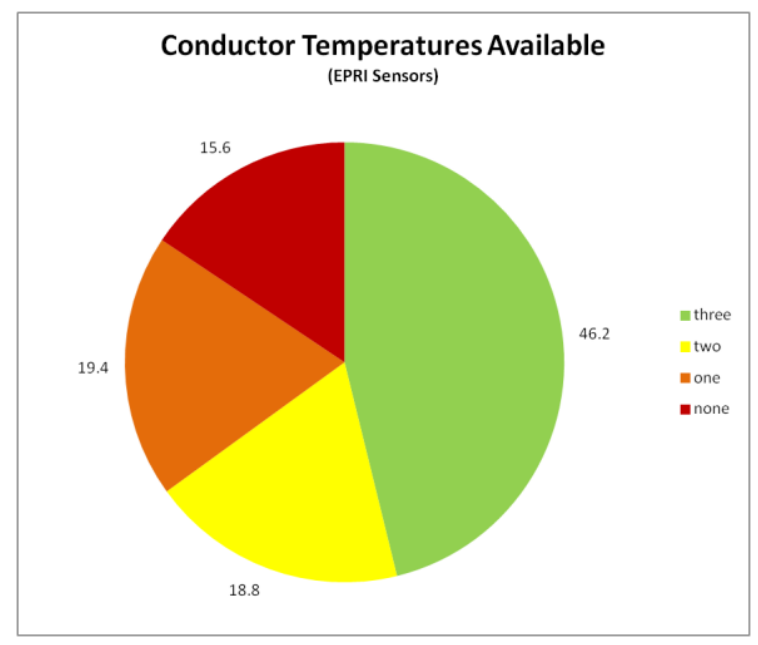

Figure L-217

\section{December 2011 Site 2}

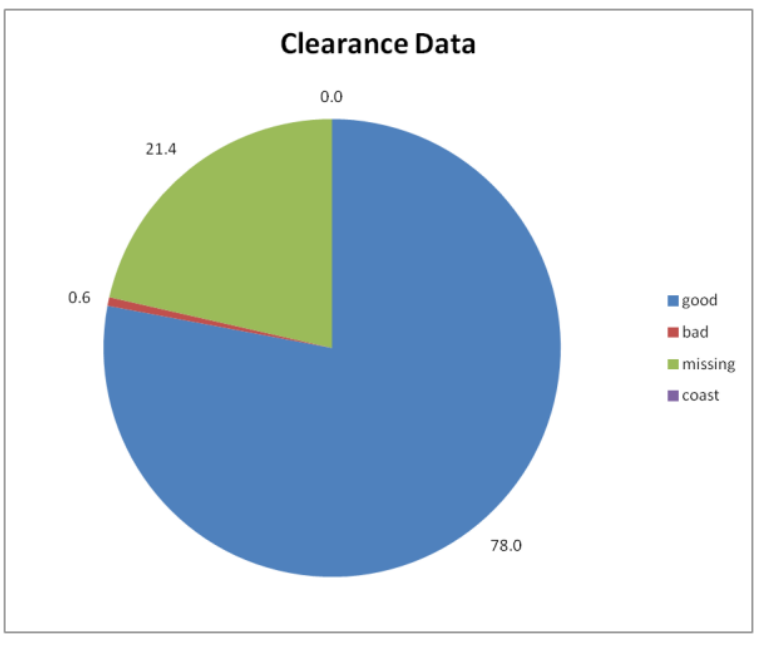

Figure L-218

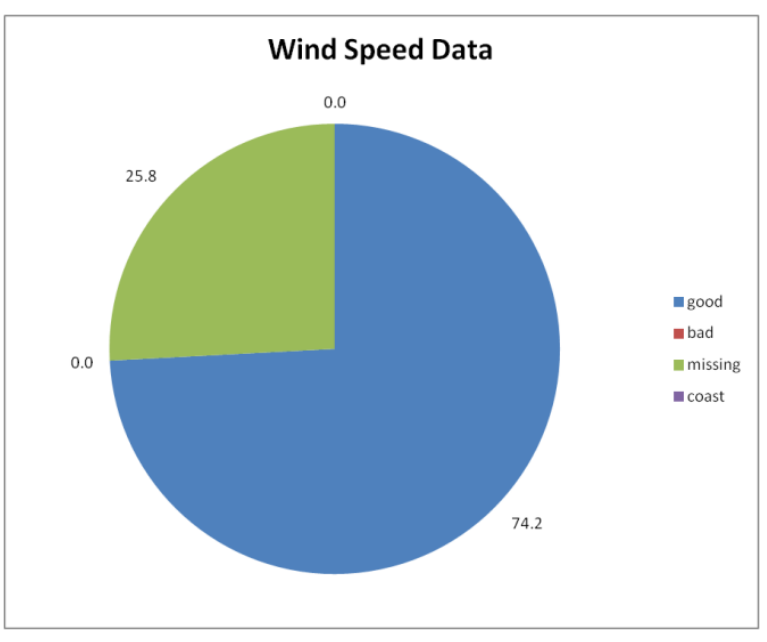

Figure L-219 


\section{December 2011 Site 2}

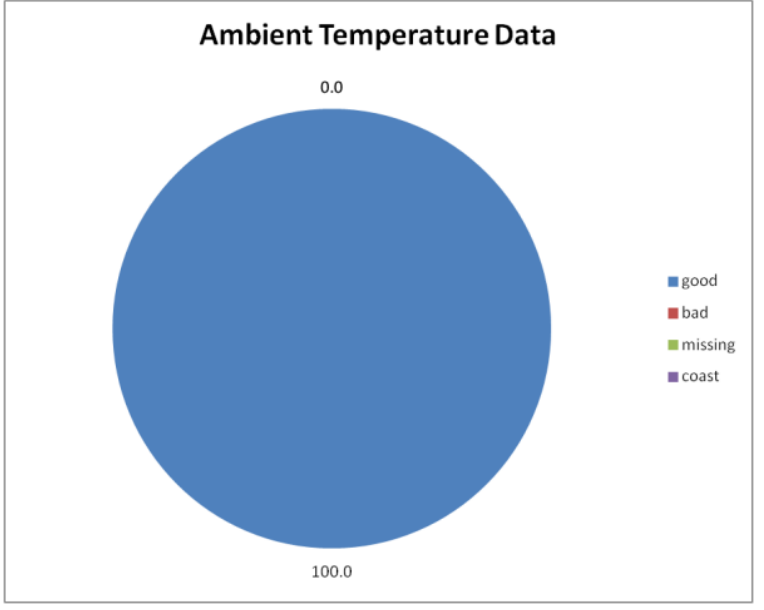

Figure L-220

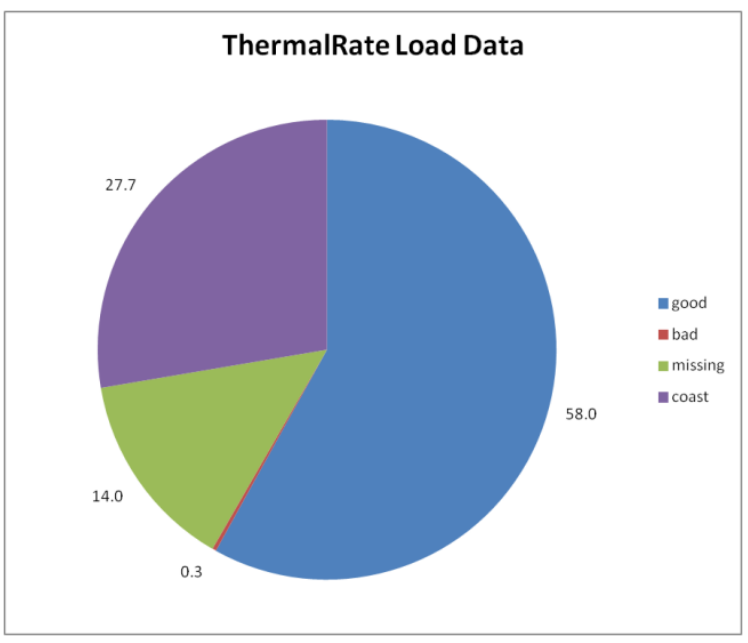

Figure L-222

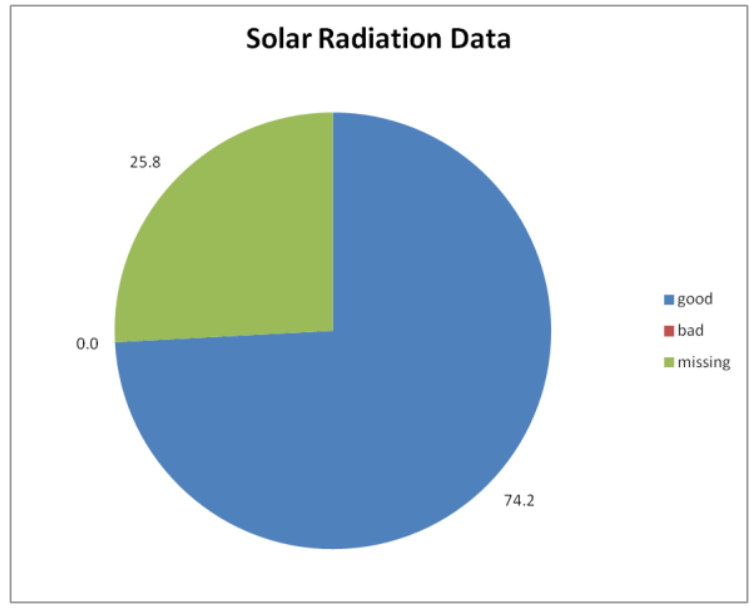

Figure L-221

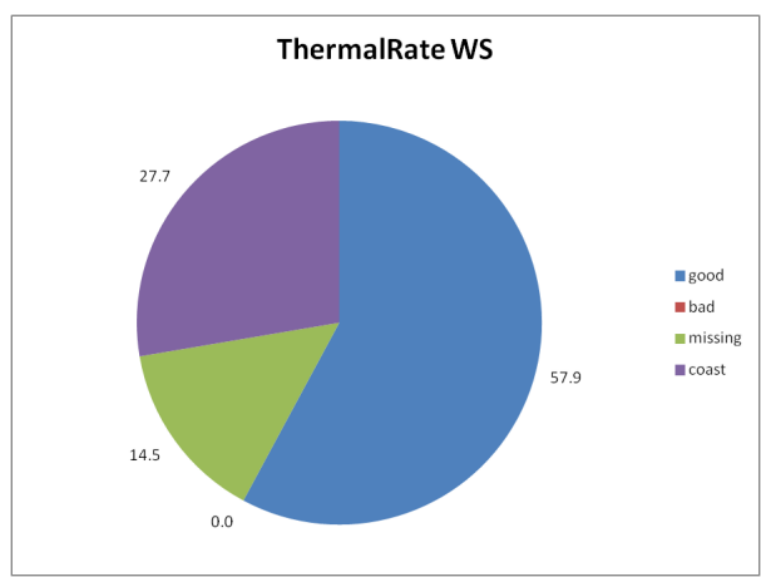

Figure L-223 
December 2011 Site 2

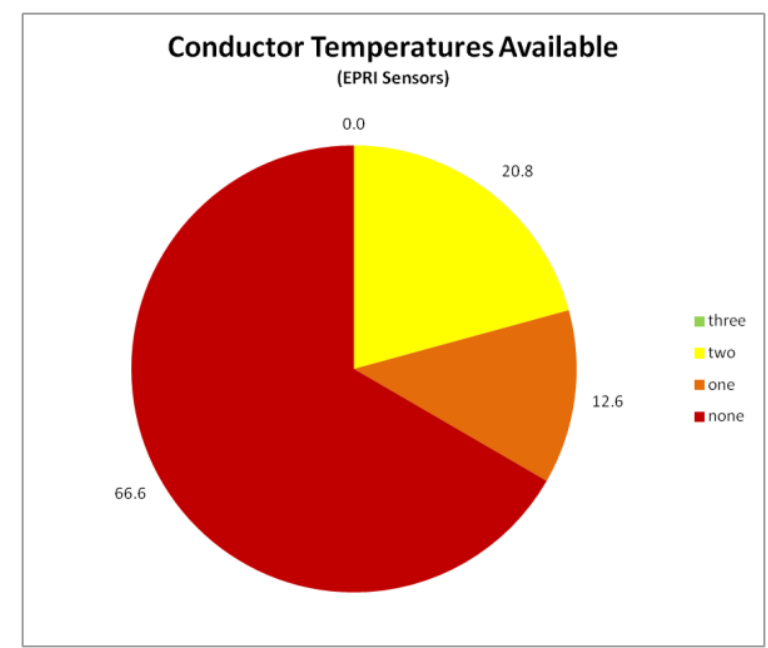

Figure L-224

\section{January 2012 Site 2}

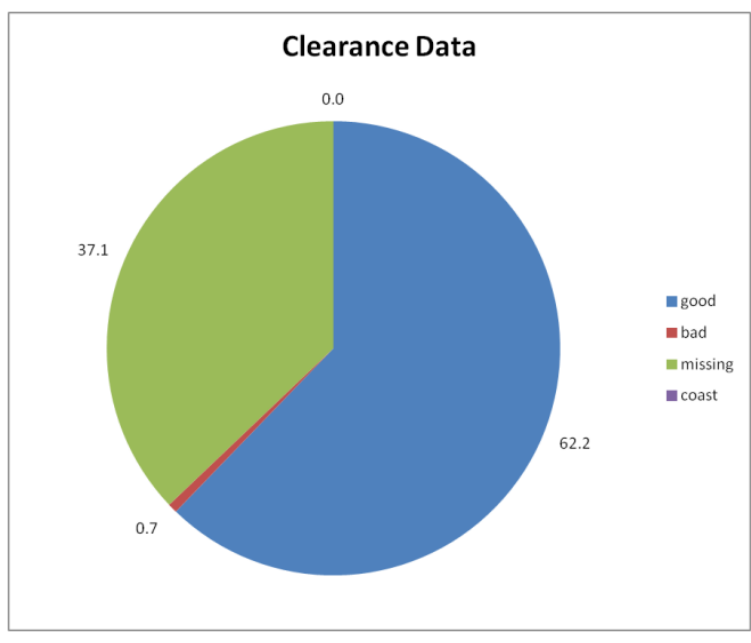

Figure L-225

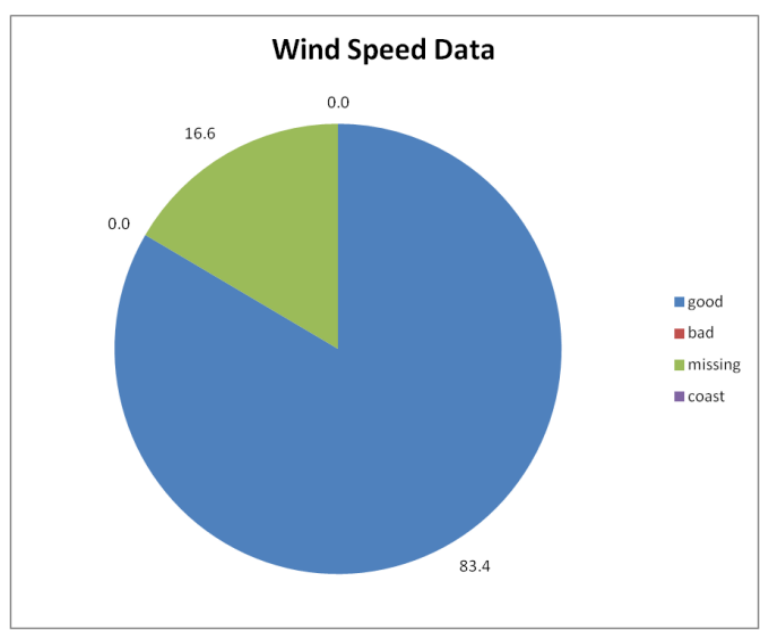

Figure L-226 


\section{January 2012 Site 2}

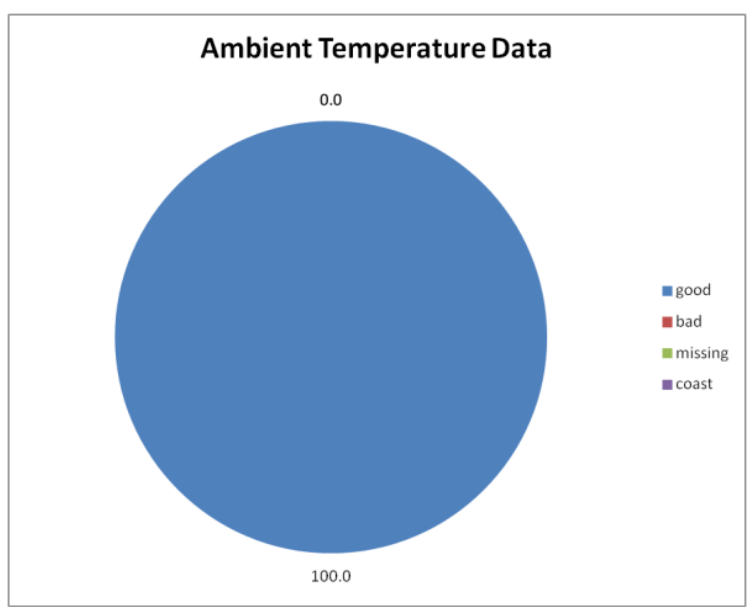

Figure L-227

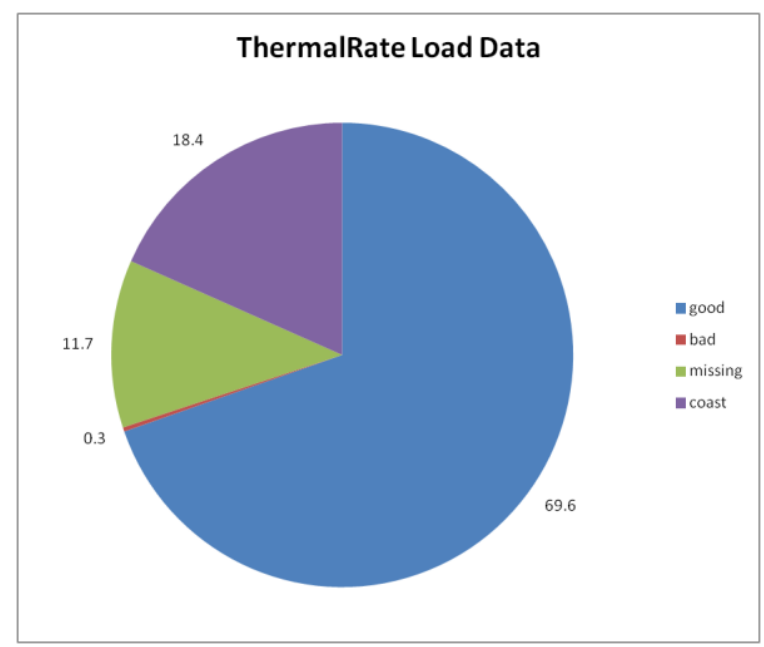

Figure L-229

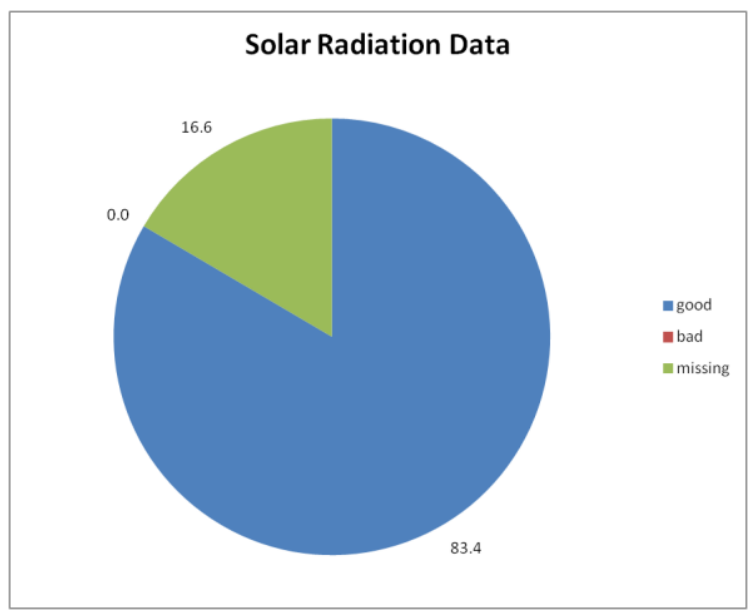

Figure L-228

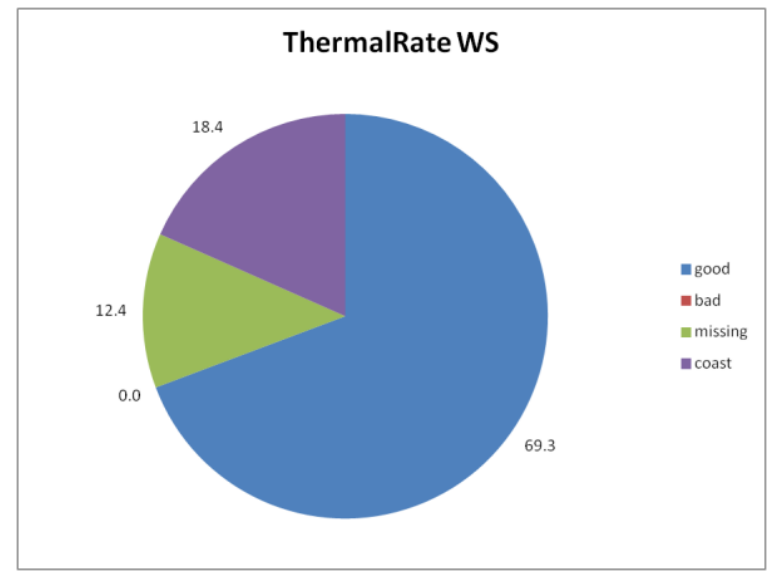

Figure L-230 


\section{January 2012 Site 2}

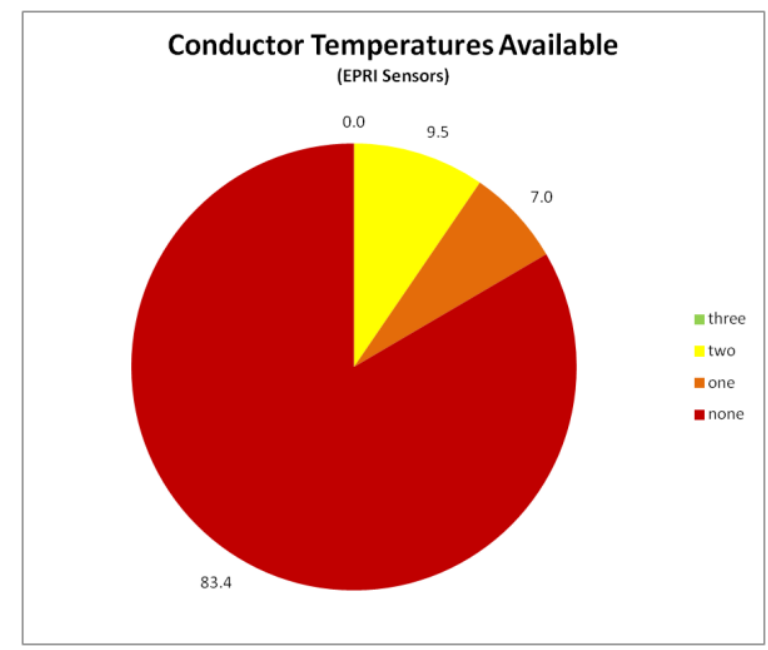

Figure L-231

\section{February 2012 Site 2}

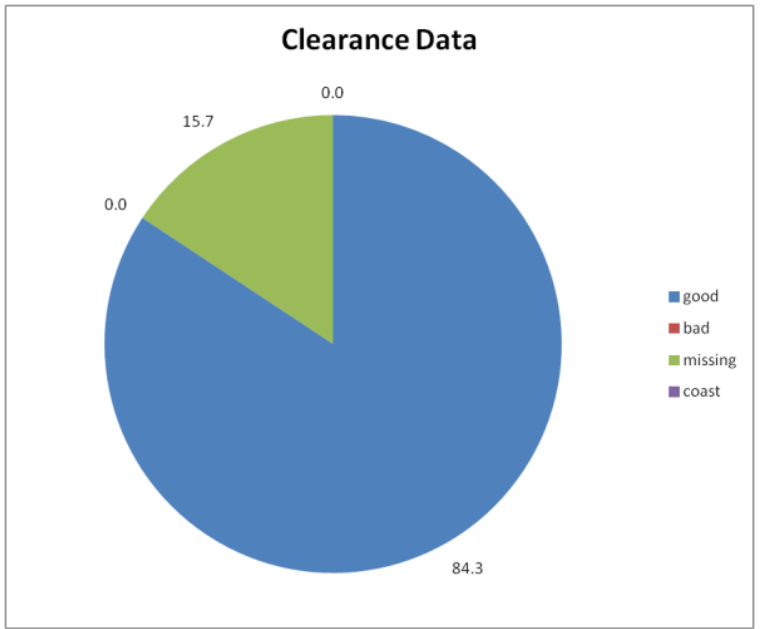

Figure L-232

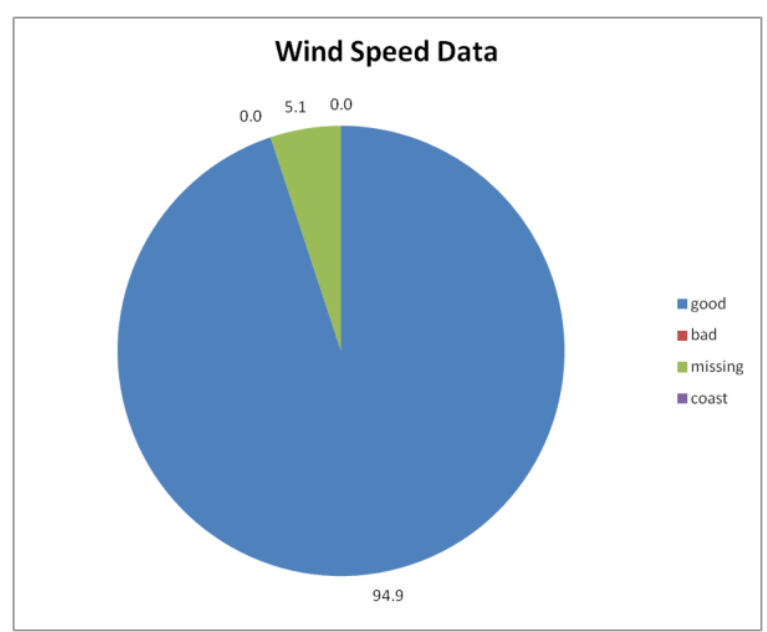

Figure L-233 


\section{February 2012 Site 2}

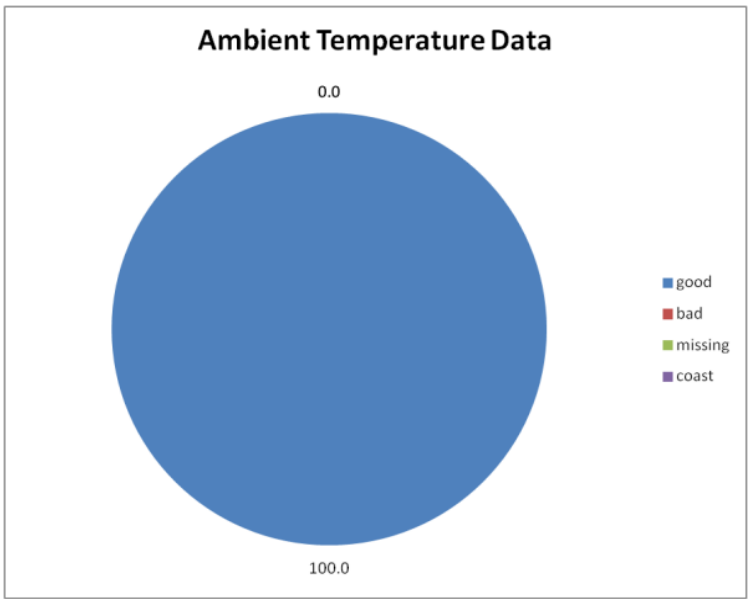

Figure L-234

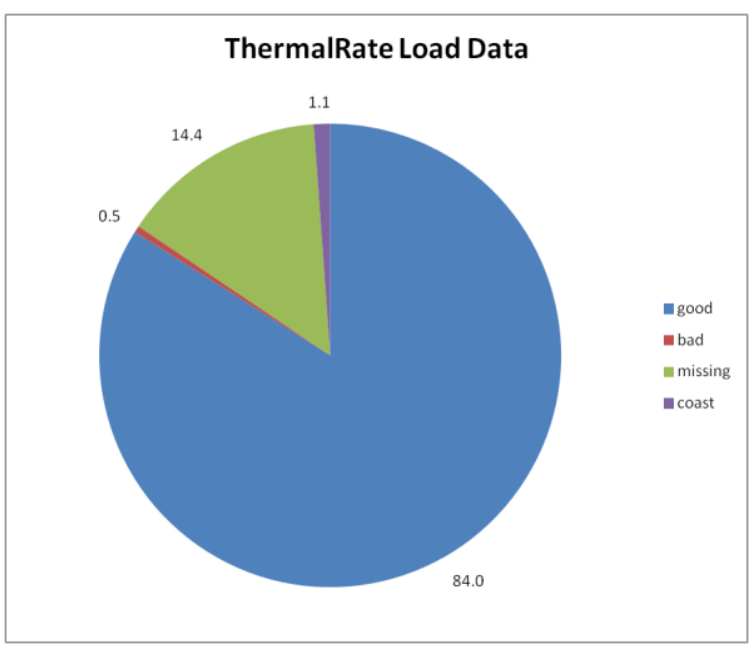

Figure L-236

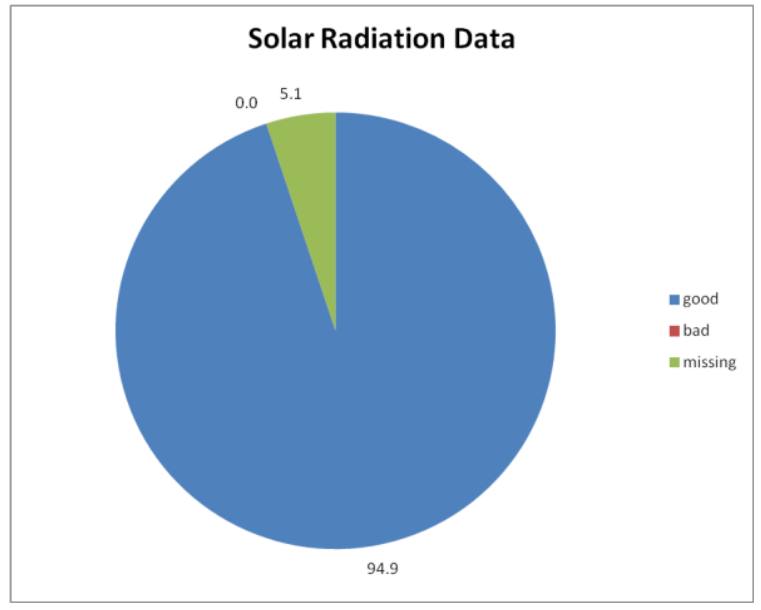

Figure L-235

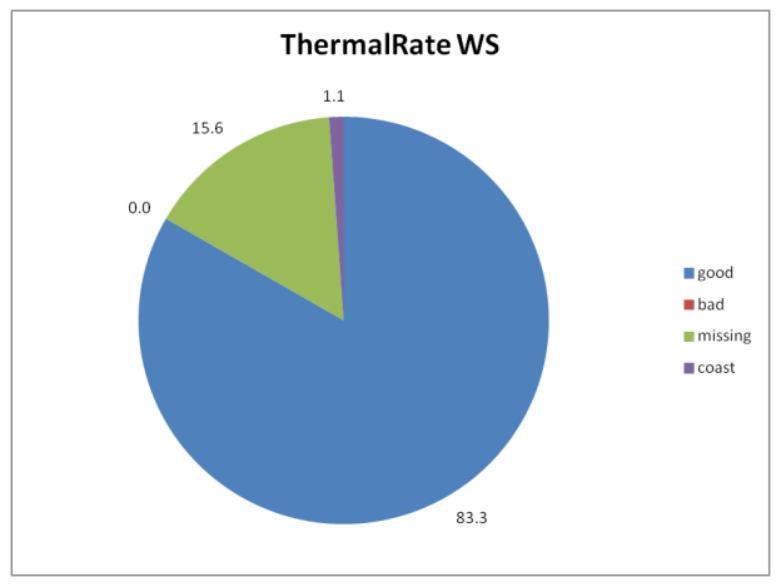

Figure L-237 
February 2012 Site 2

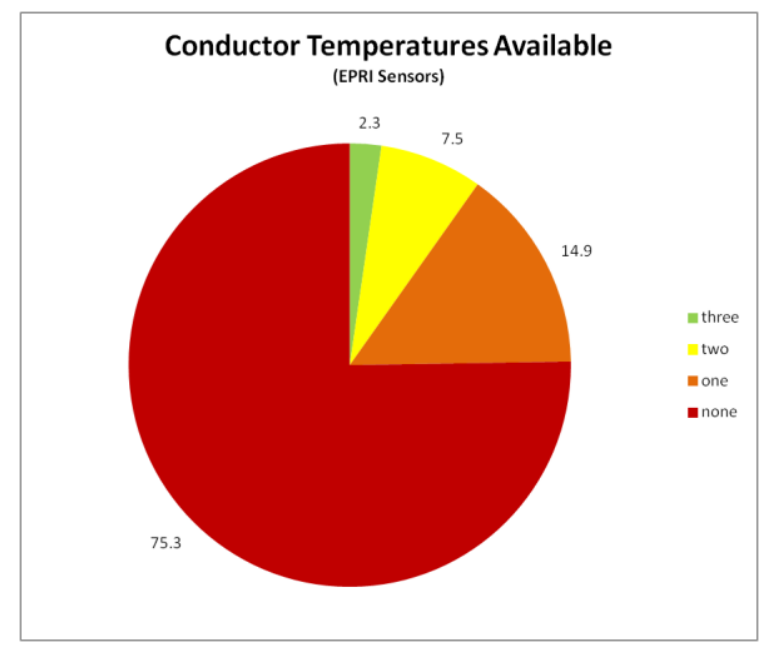

Figure L-238

\section{March 2012 Site 2}

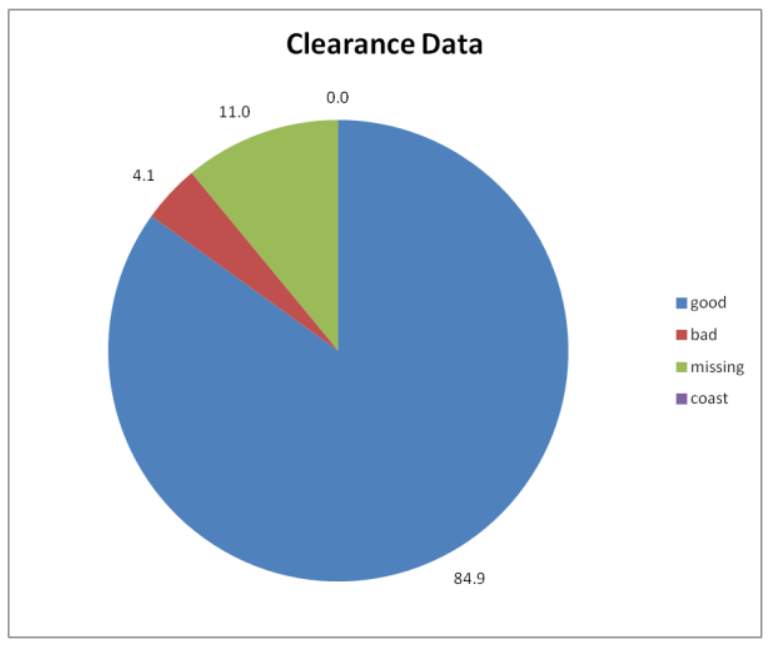

Figure L-239

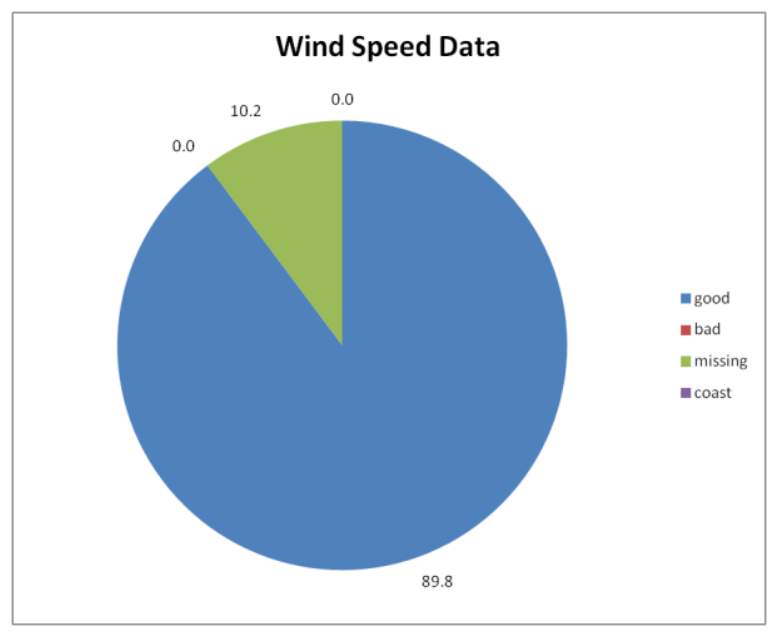

Figure L-240 


\section{March 2012 Site 2}

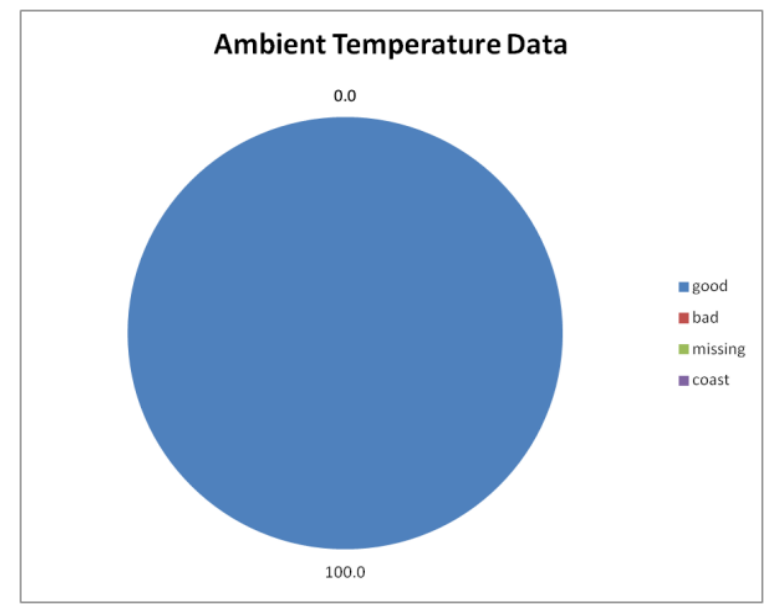

Figure L-241

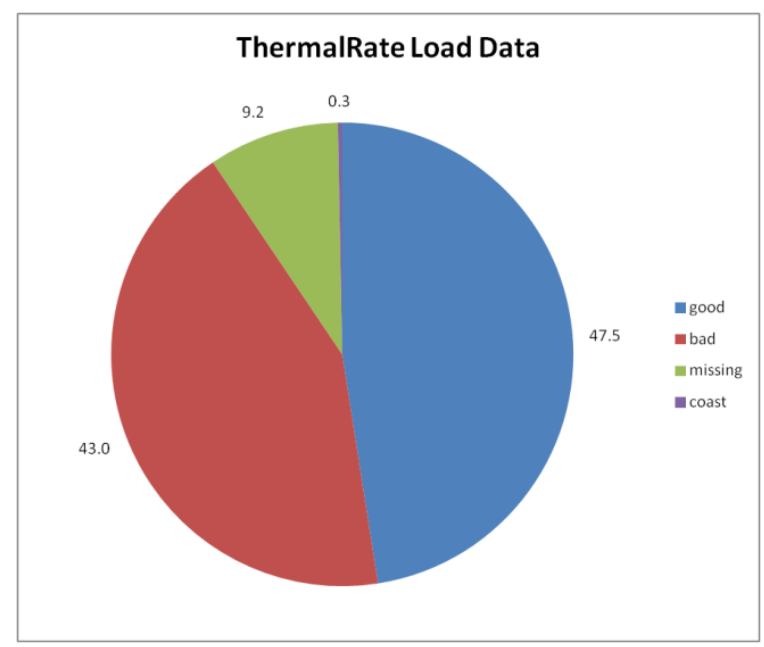

Figure L-243

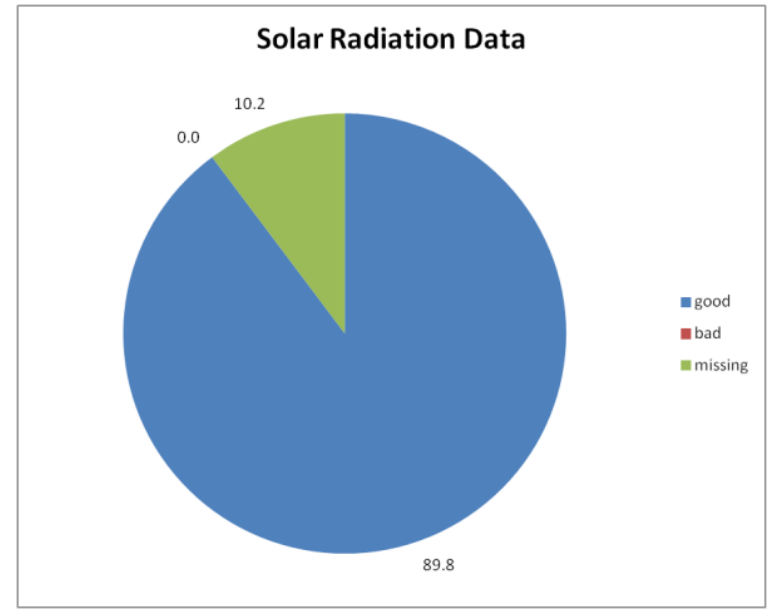

Figure L-242

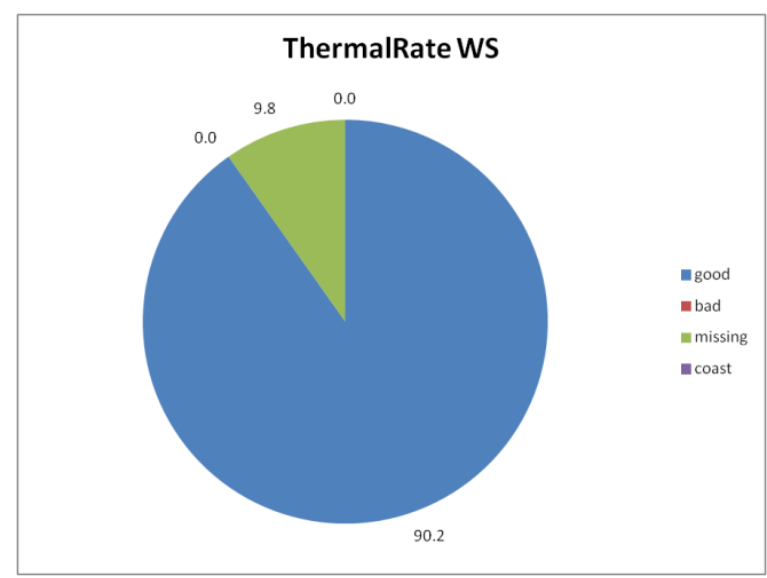

Figure L-244 
March 2012 Site 2

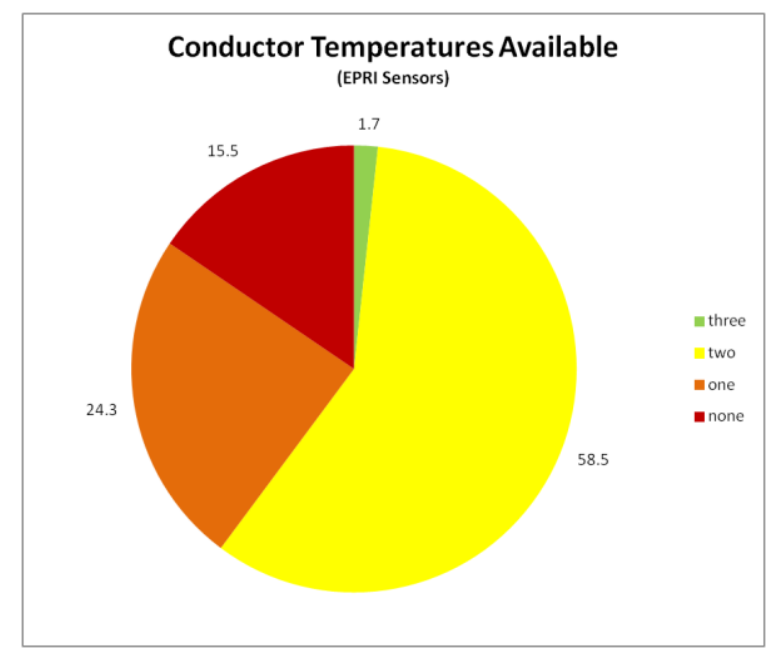

Figure L-245

\section{April 2012 Site 2}

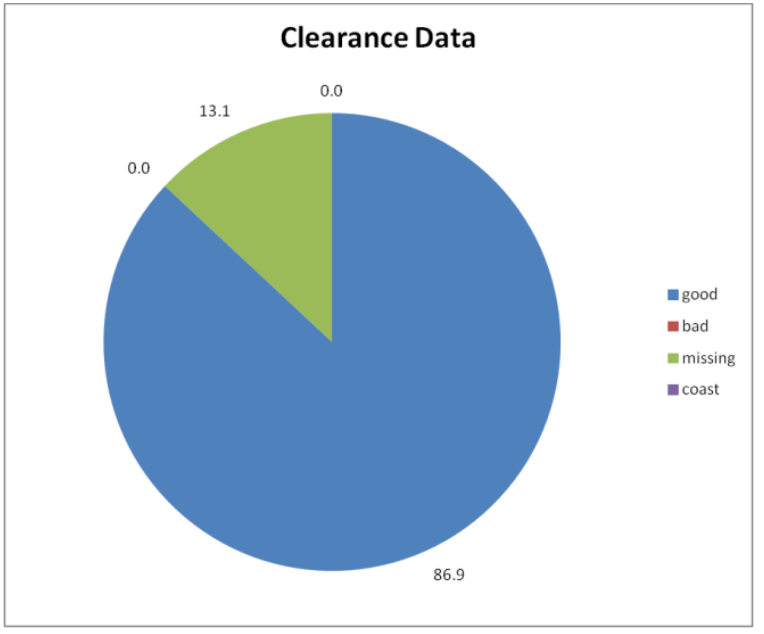

Figure L-246

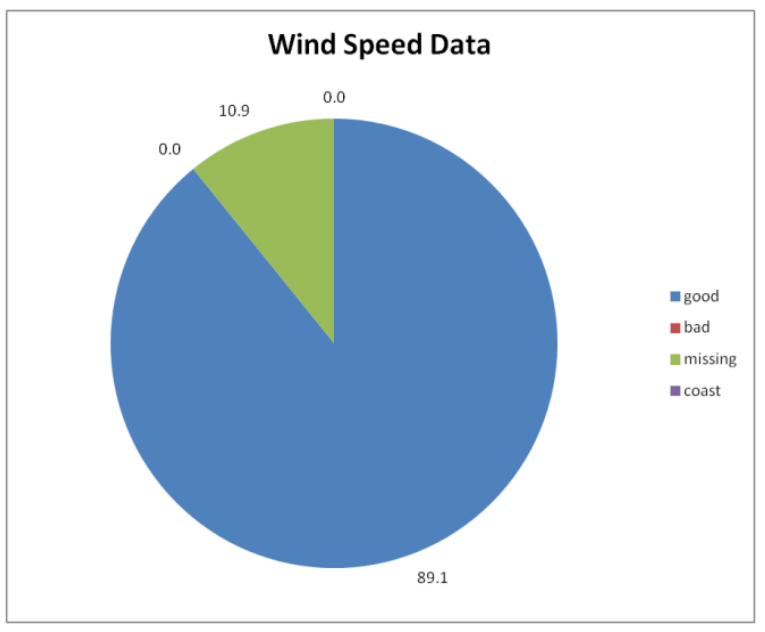

Figure L-247 


\section{April 2012 Site 2}

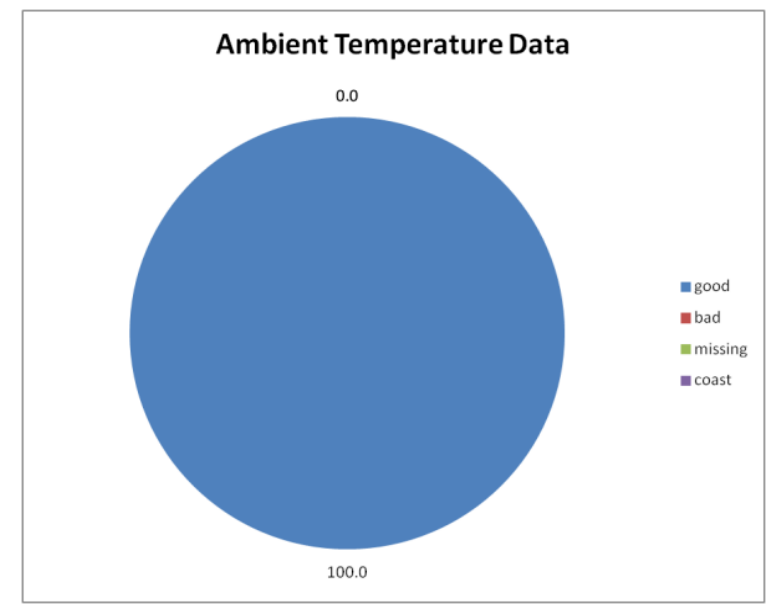

Figure L-248

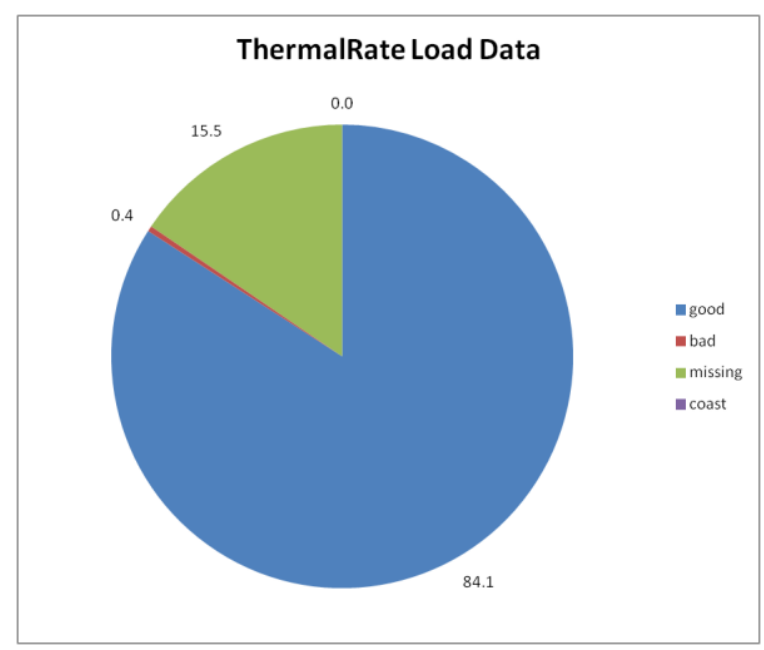

Figure L-250

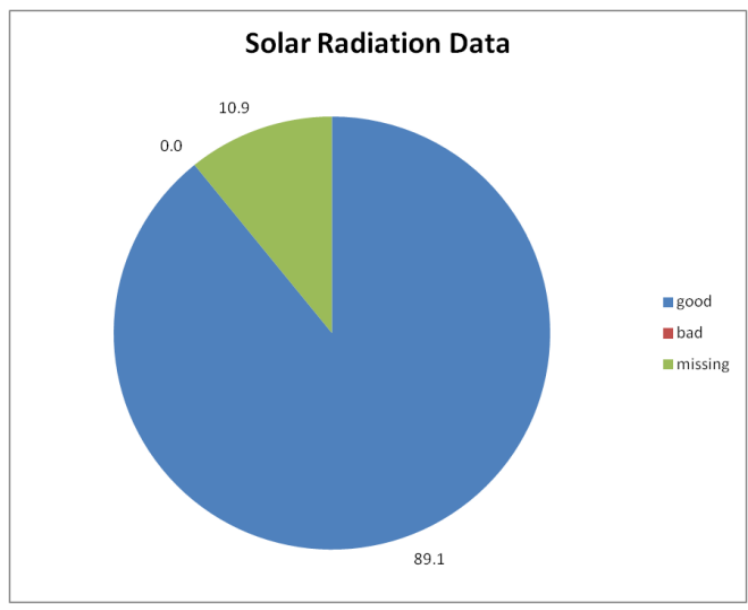

Figure L-249

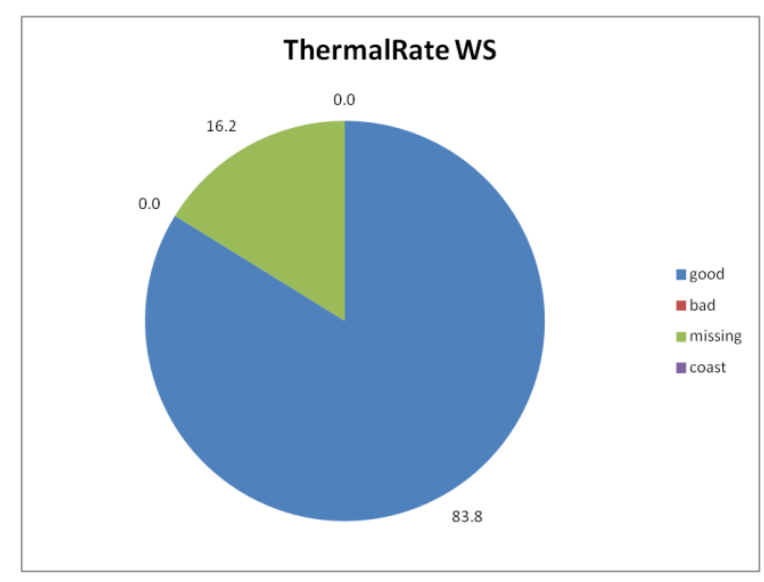

Figure L-251 


\section{April 2012 Site 2}

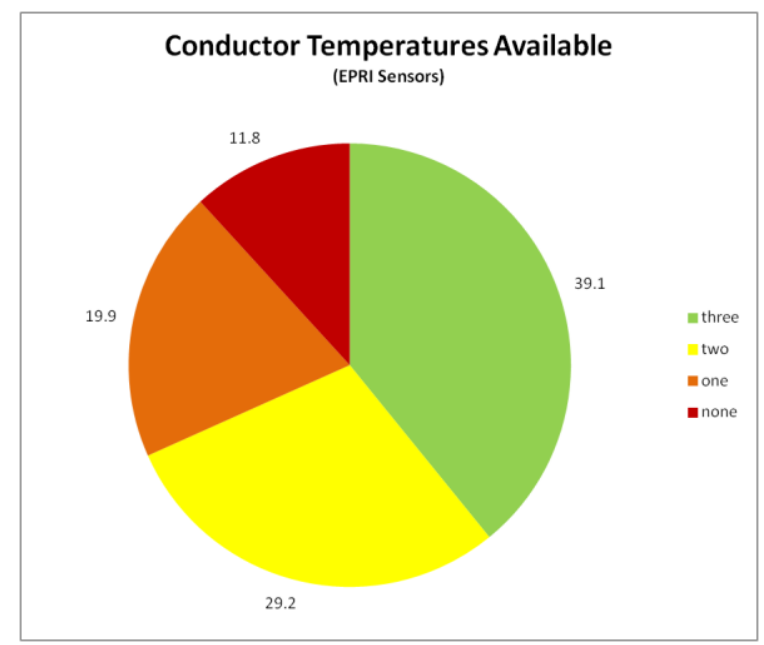

Figure L-252

\section{April 2012 Site 2}

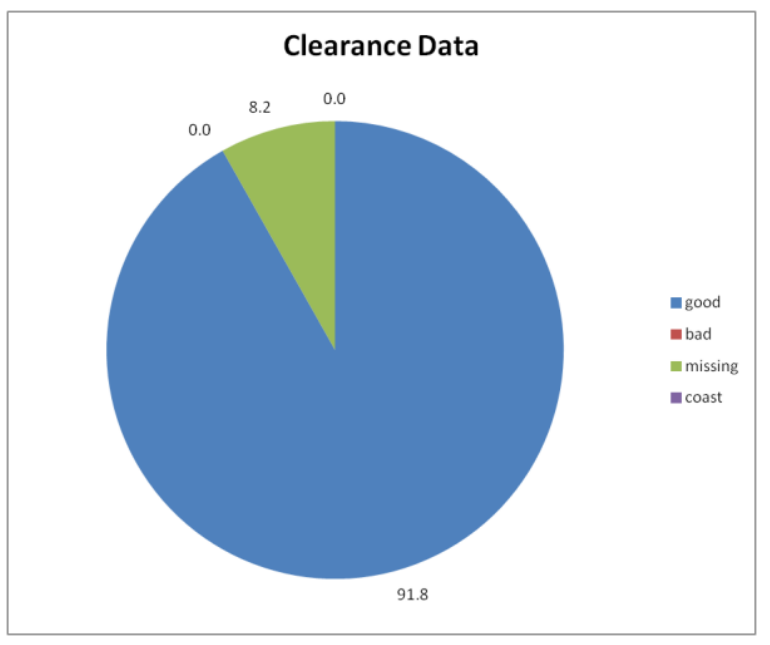

Figure L-253

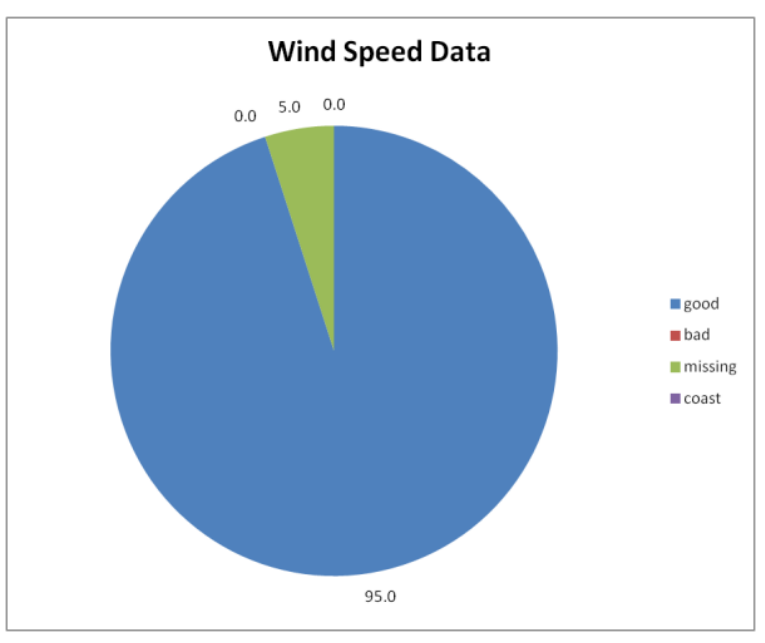

Figure L-254 


\section{April 2012 Site 2}

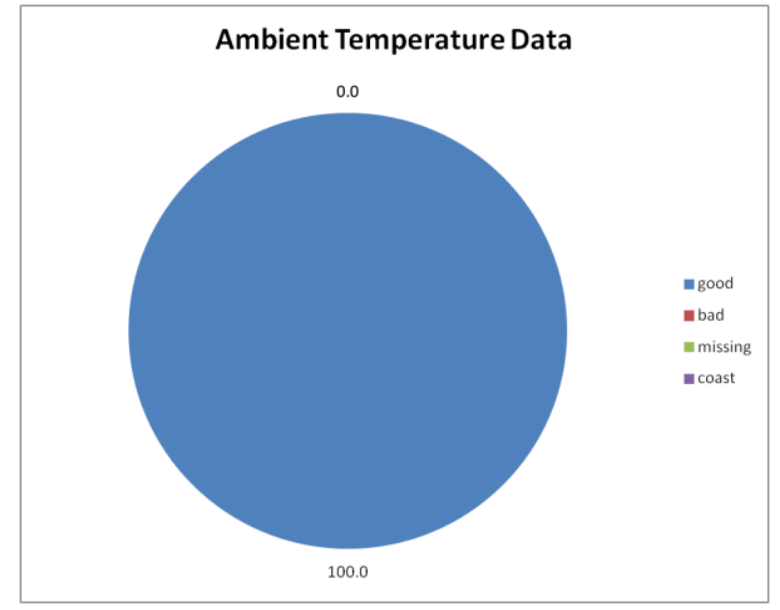

Figure L-255

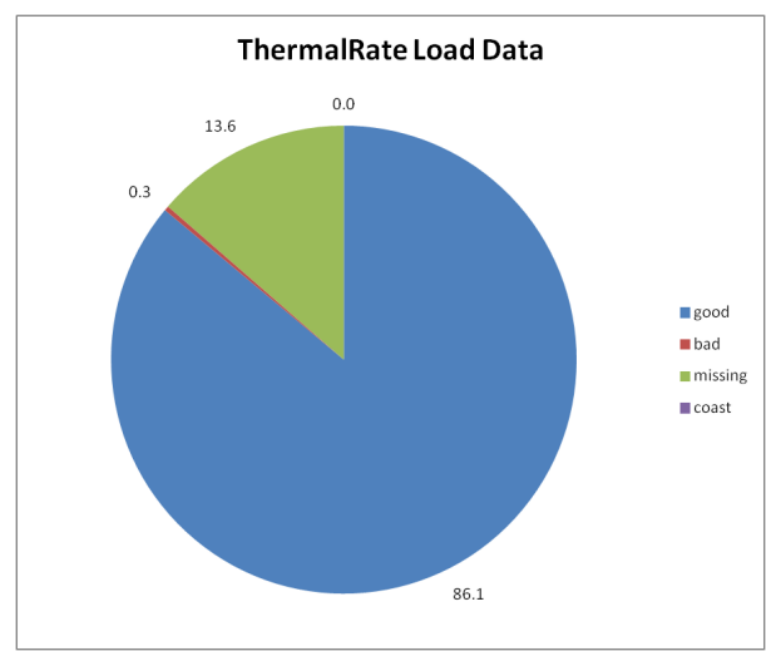

Figure L-257

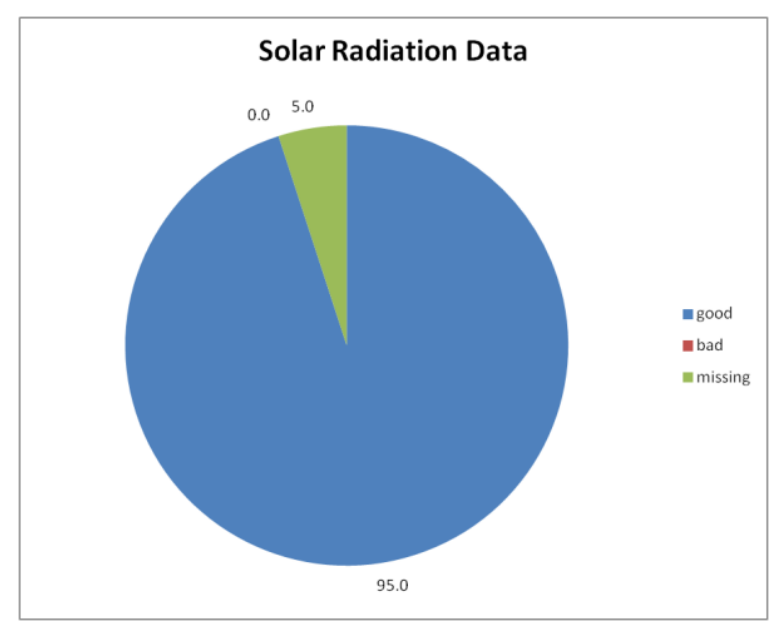

Figure L-256

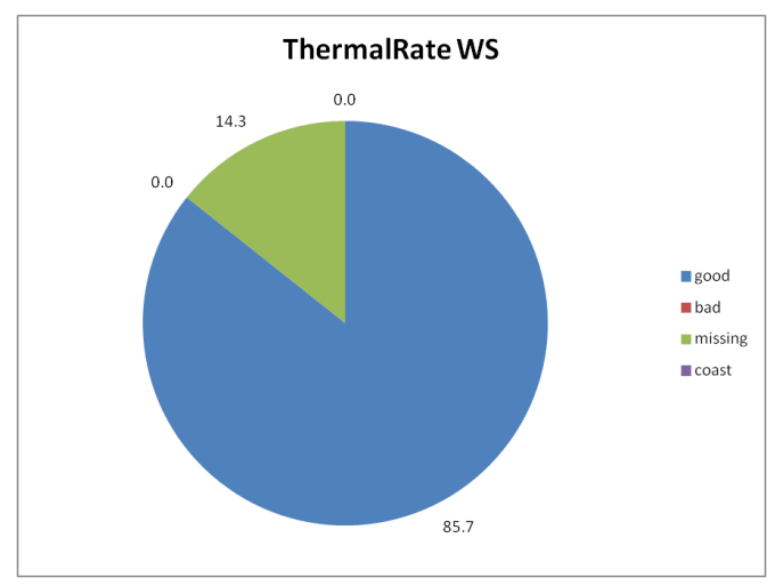

Figure L-258 


\section{April 2012 Site 2}

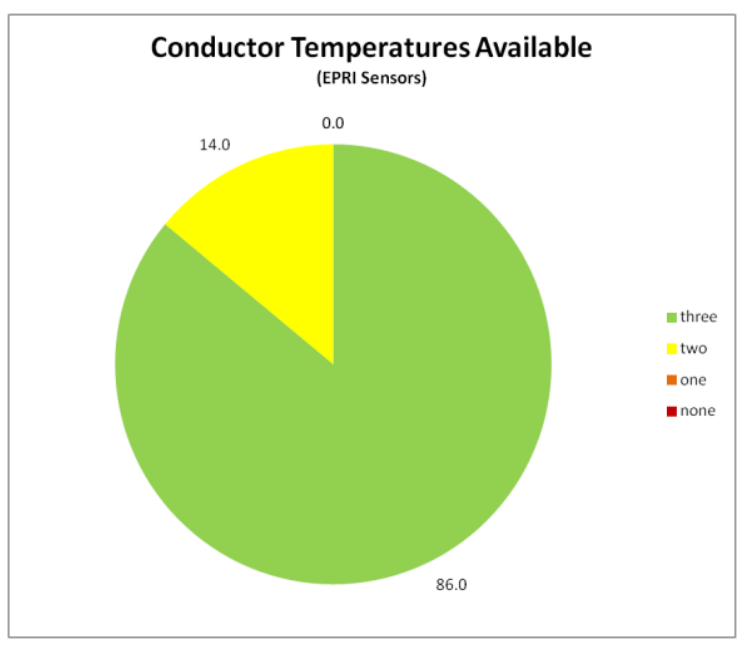

Figure L-259

\section{June 2012 Site 2}

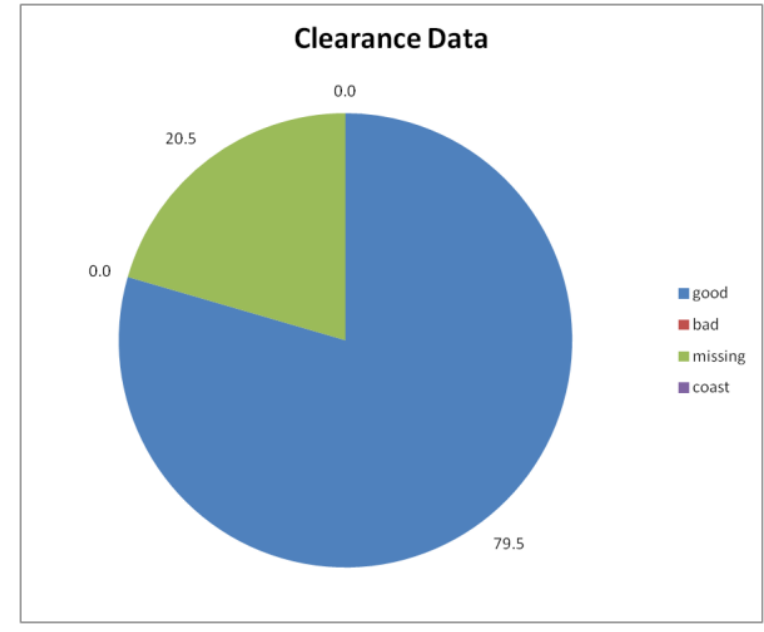

Figure L-260

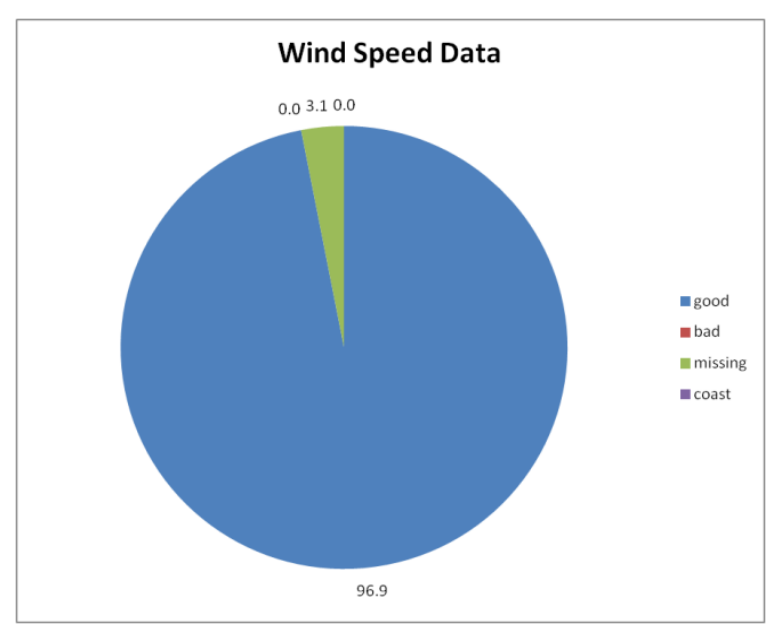

Figure L-261 


\section{June 2012 Site 2}

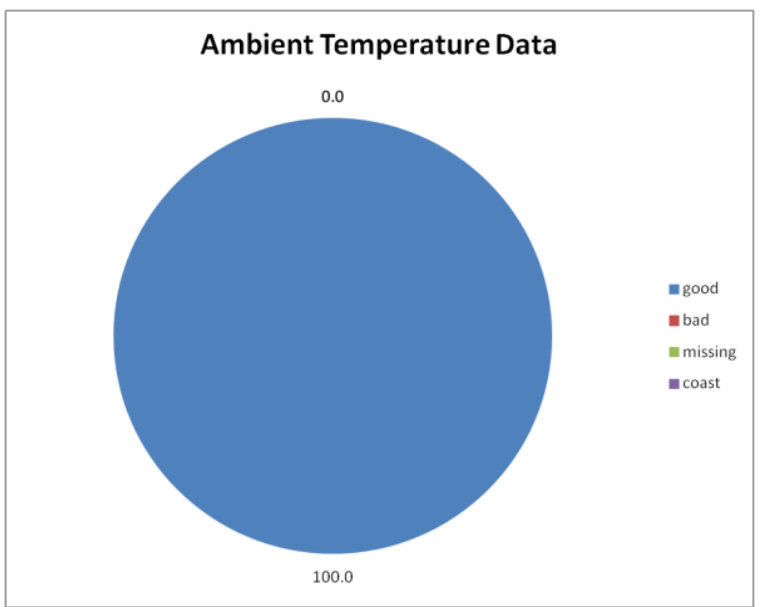

Figure L-262

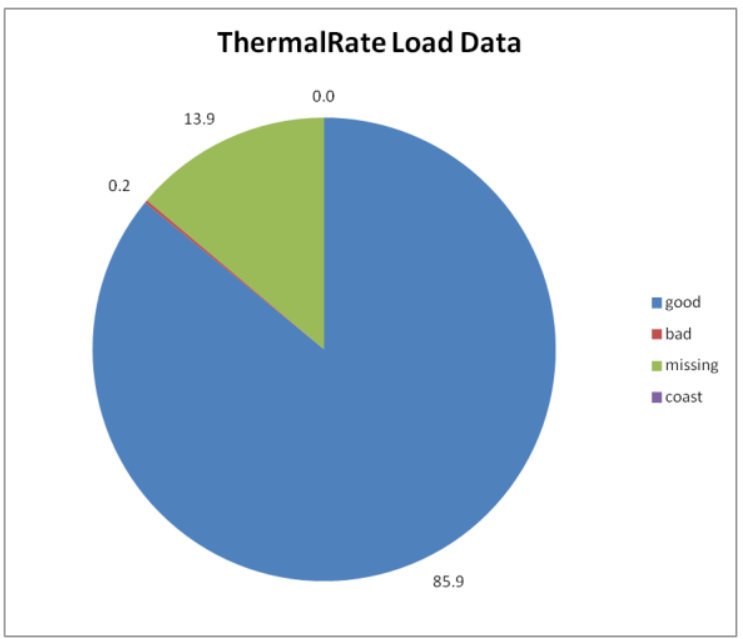

Figure L-264

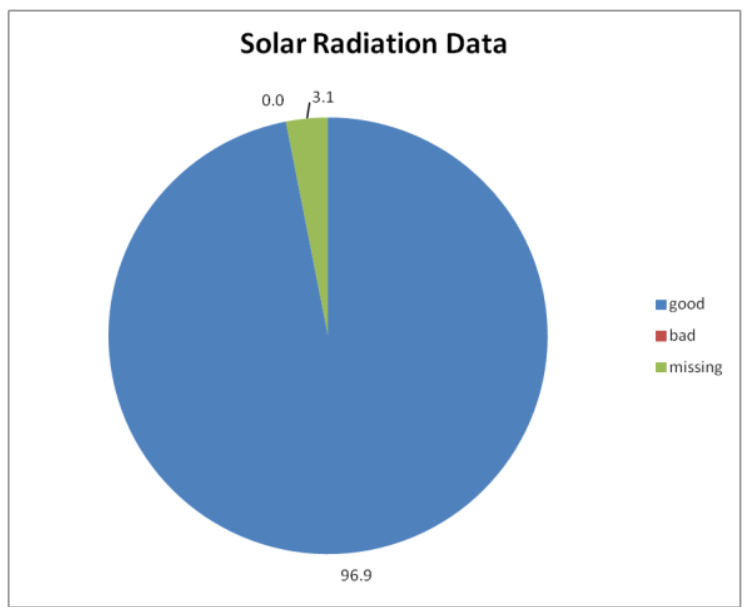

Figure L-263

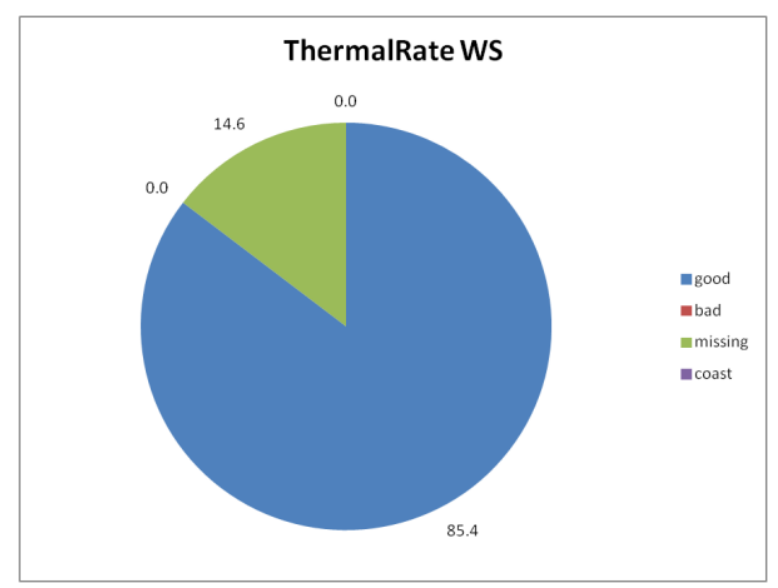

Figure L-265 


\section{June 2012 Site 2}

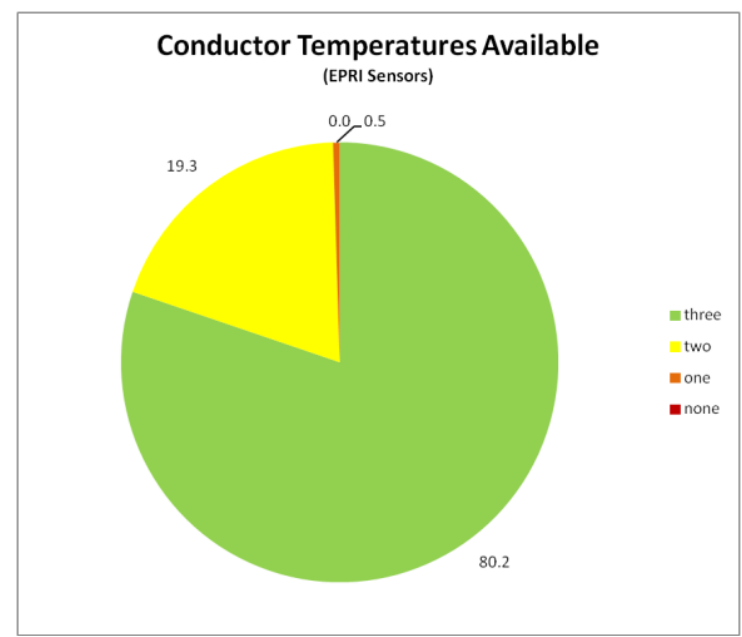

Figure L-266

\section{July 2012 Site 2}

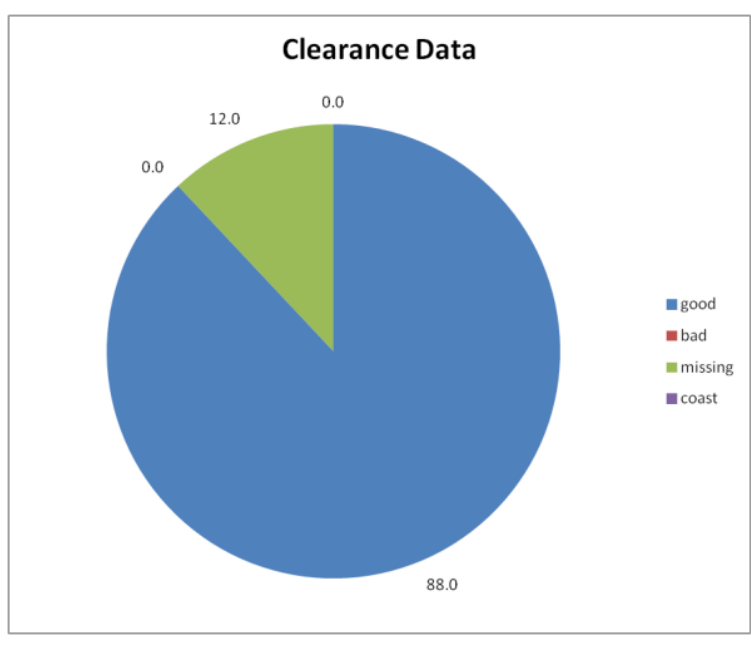

Figure L-267

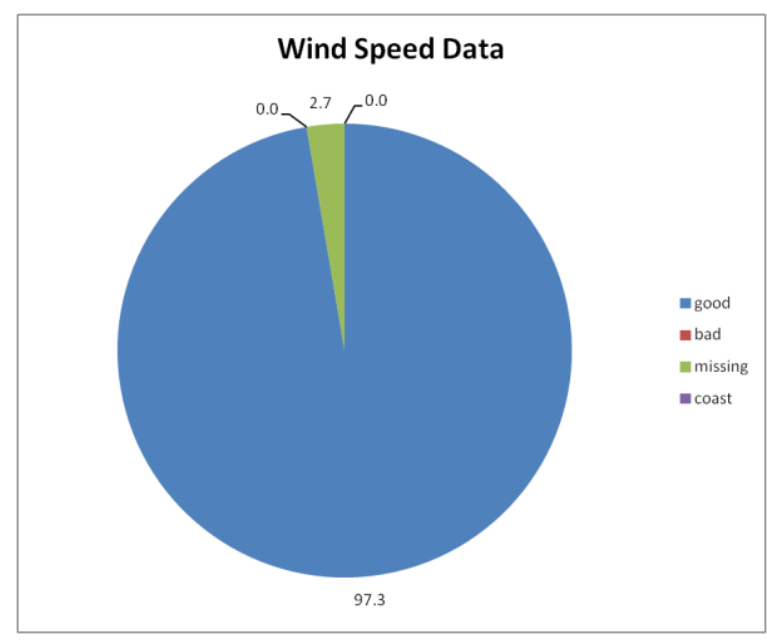

Figure L-268 


\section{July 2012 Site 2}

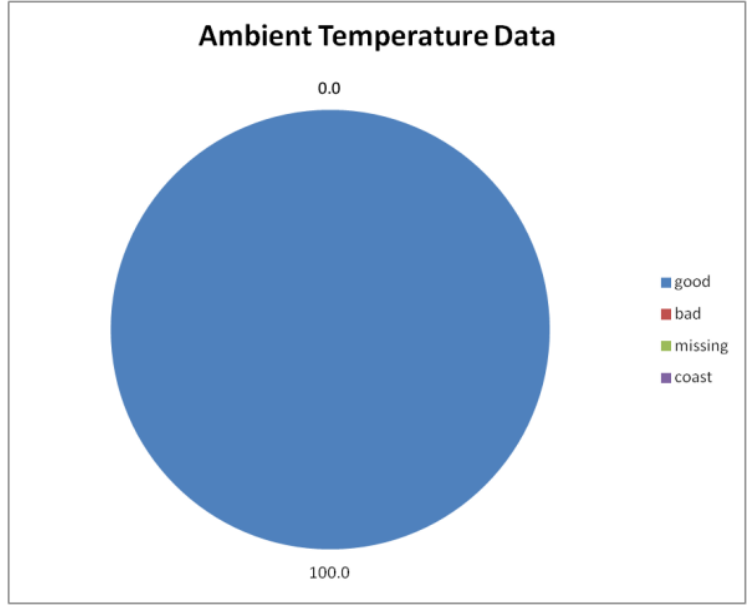

Figure L-269

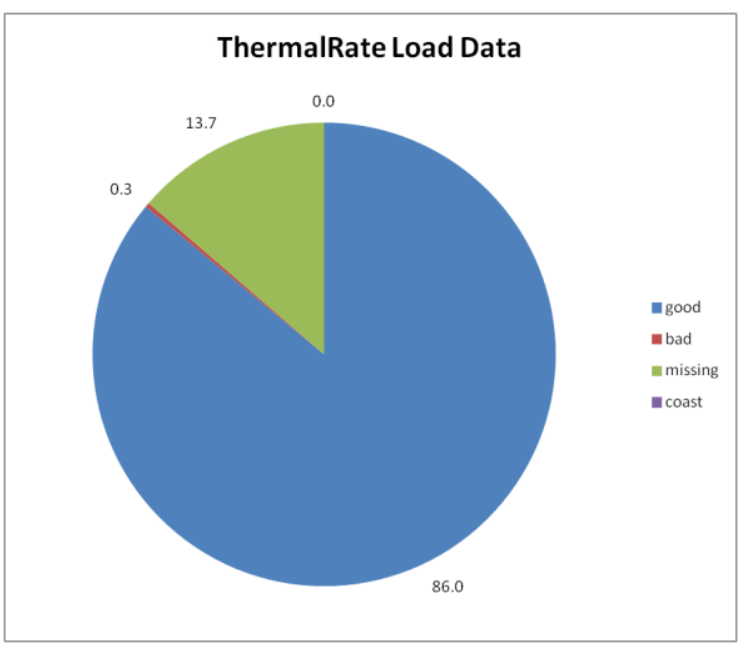

Figure L-271

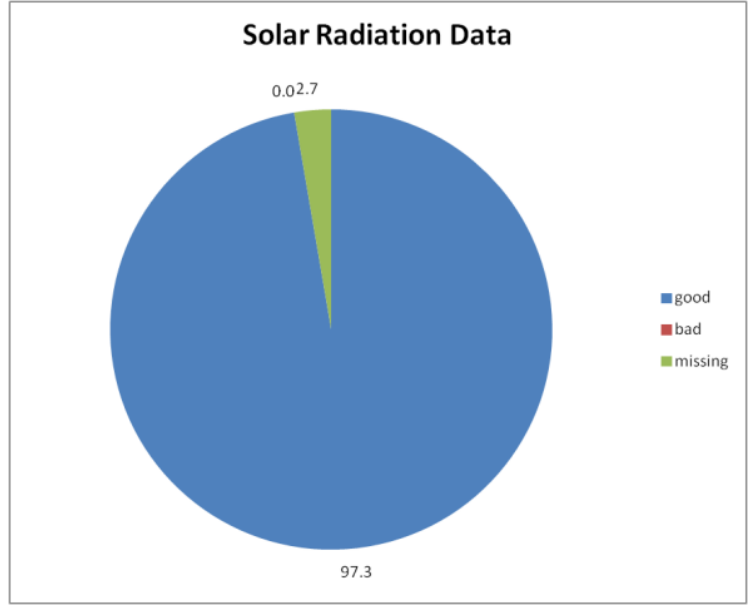

Figure L-270

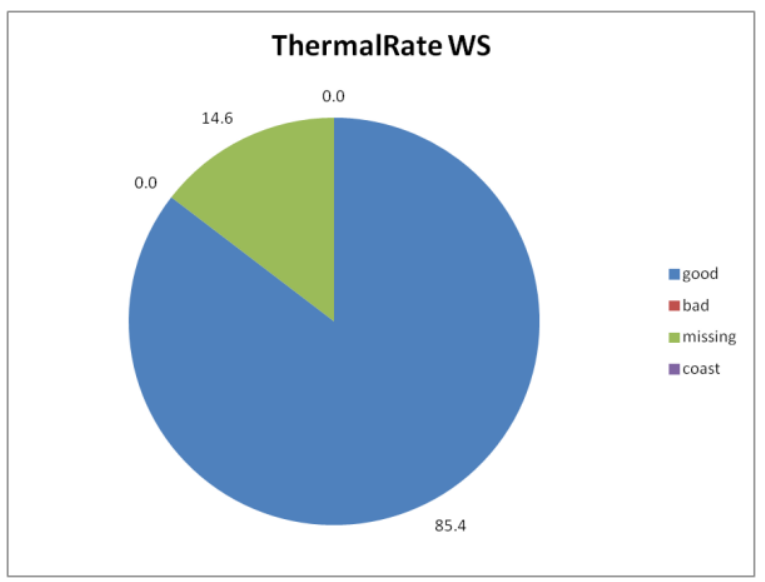

Figure L-272 
July 2012 Site 2

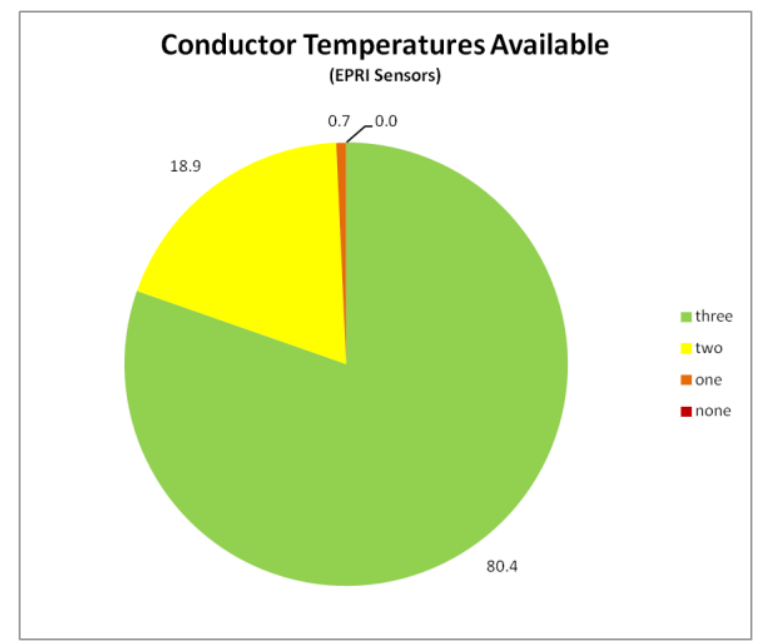

Figure L-273

\section{August 2012 Site 2}

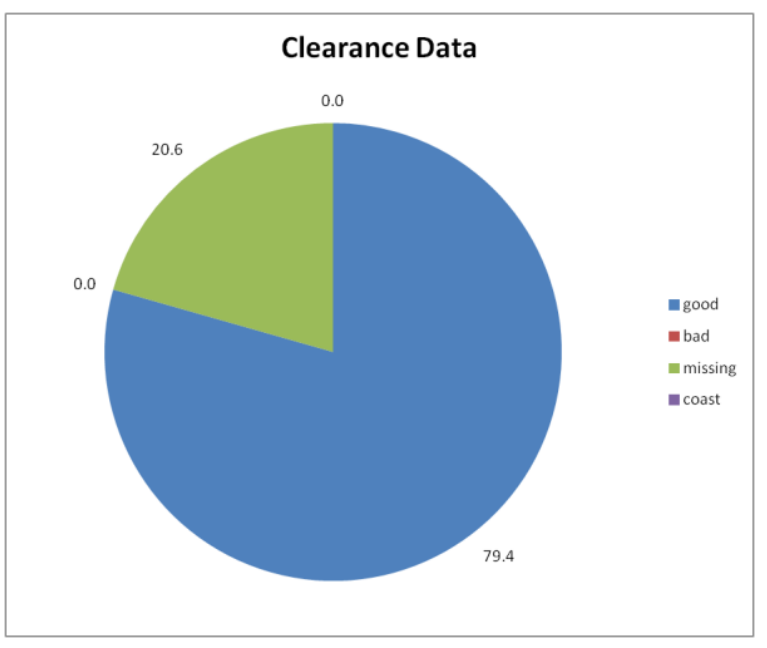

Figure L-274

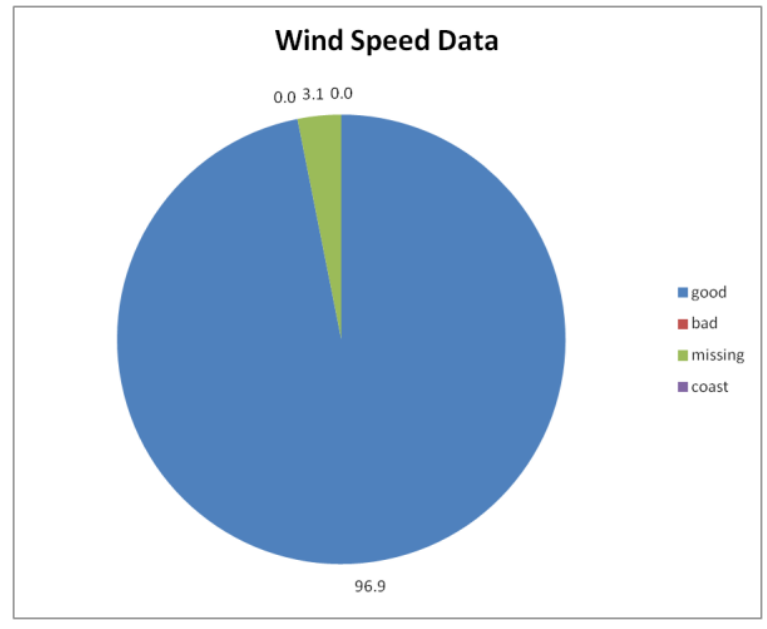

Figure L-275 


\section{August 2012 Site 2}

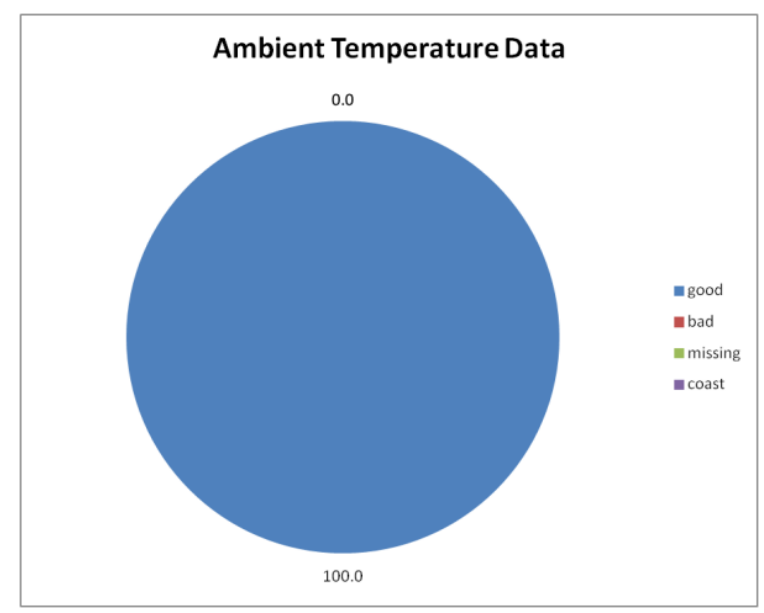

Figure L-276

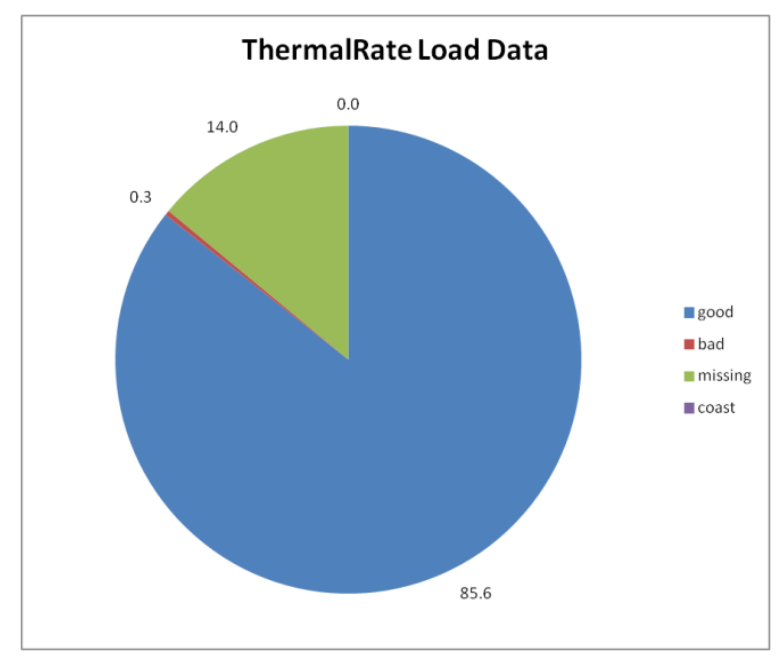

Figure L-278

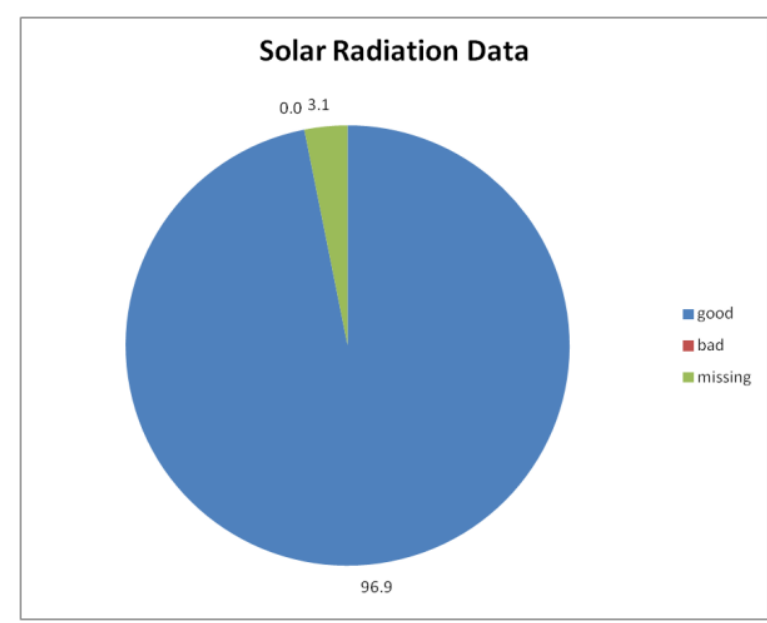

Figure L-277

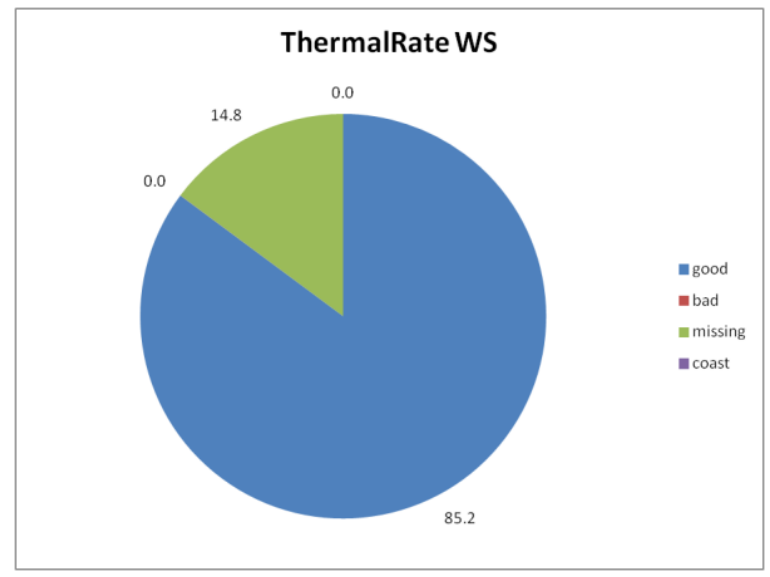

Figure L-279 


\section{August 2012 Site 2}

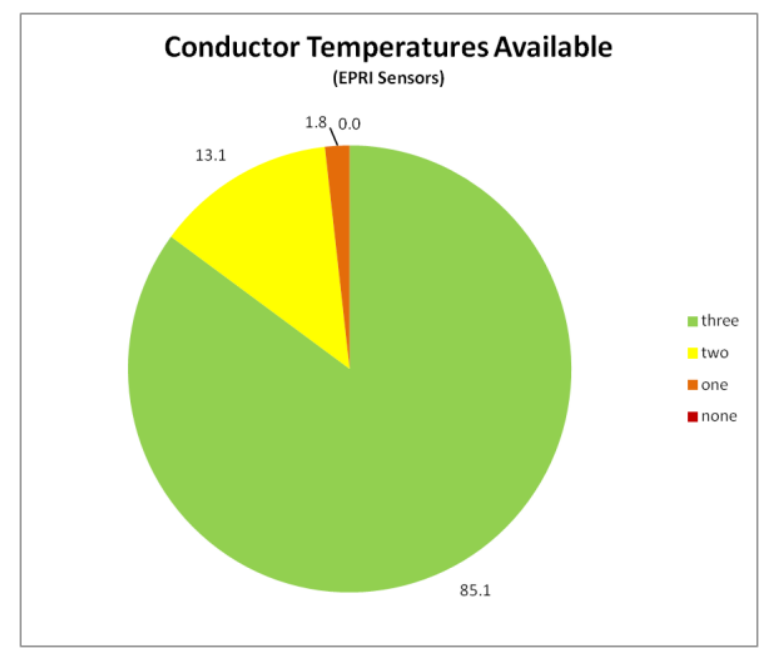

Figure L-280

\section{September 2012 Site 2}

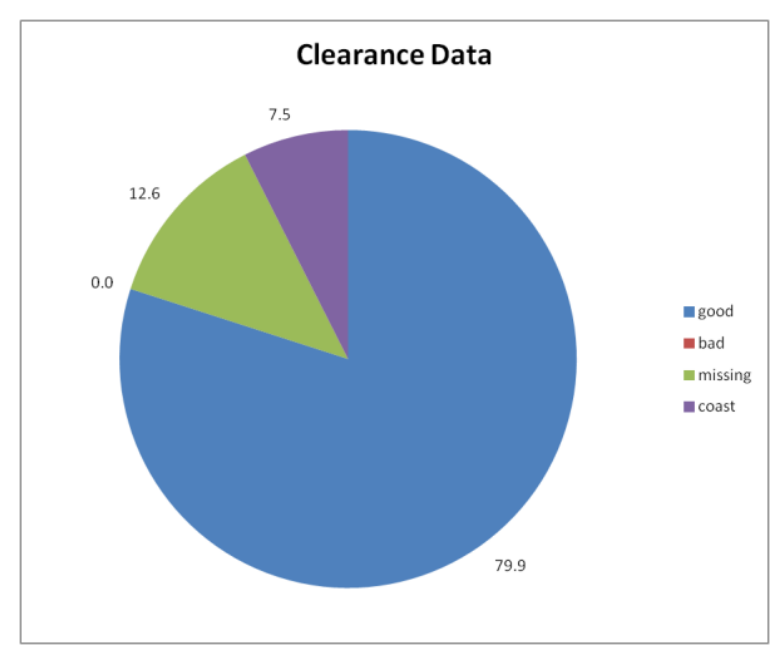

Figure L-281

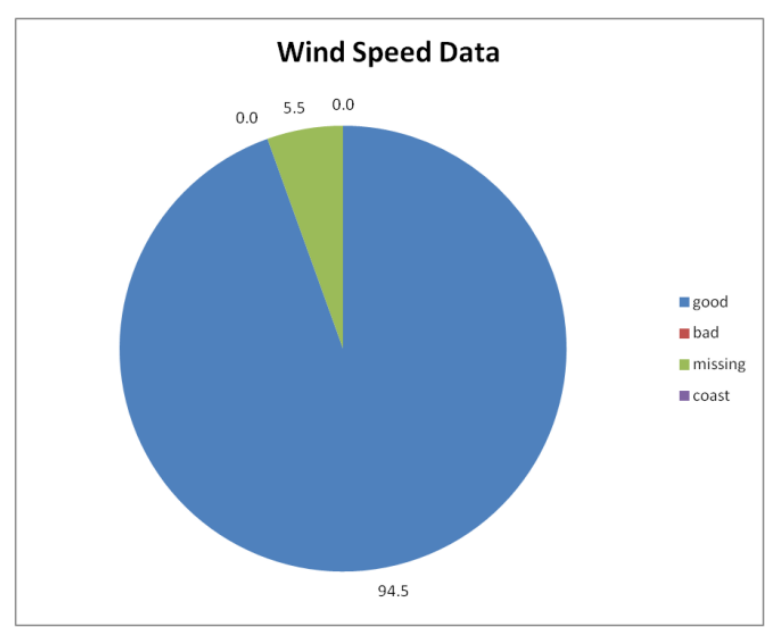

Figure L-282 


\section{September 2012 Site 2}

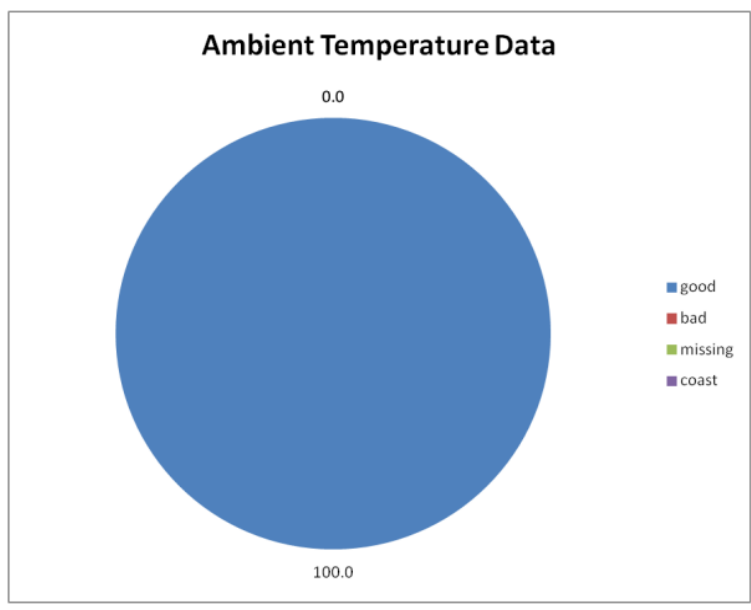

Figure L-283

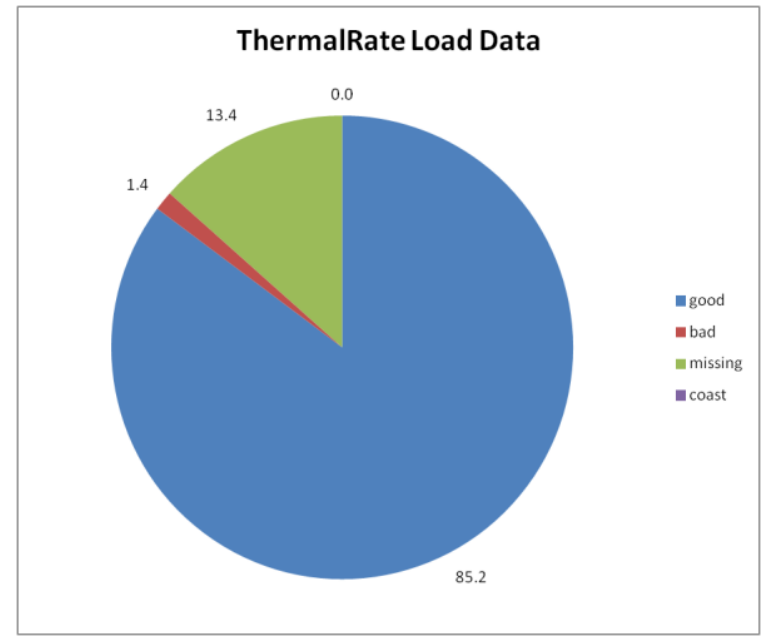

Figure L-285

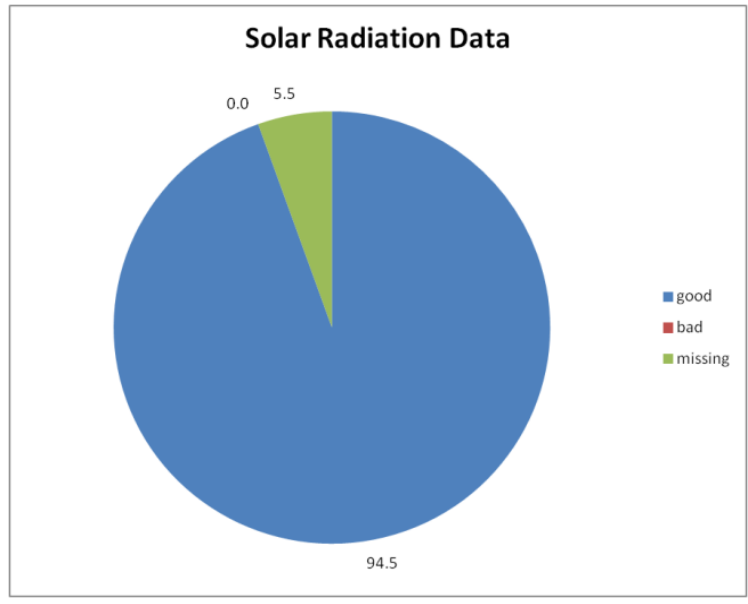

Figure L-284

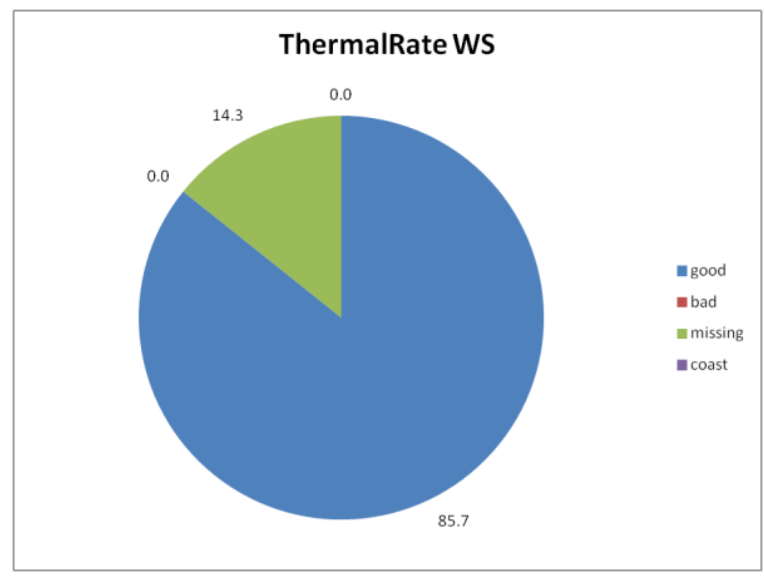

Figure L-286 
September 2012 Site 2

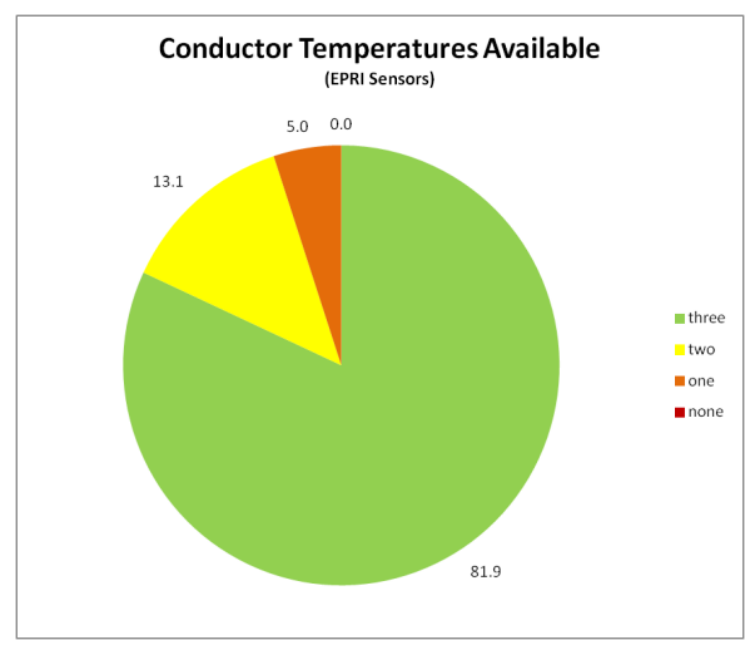

Figure L-287

\section{October 2012 Site 2}

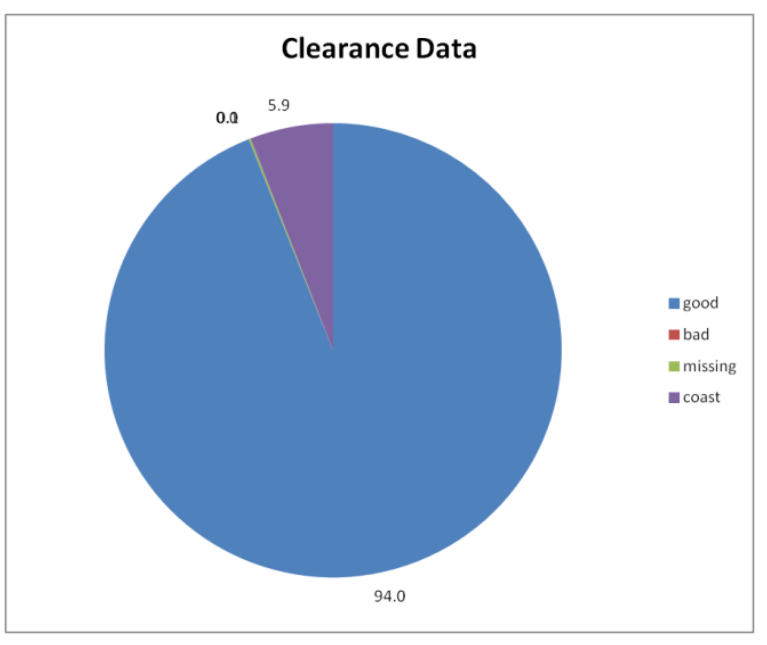

Figure L-288

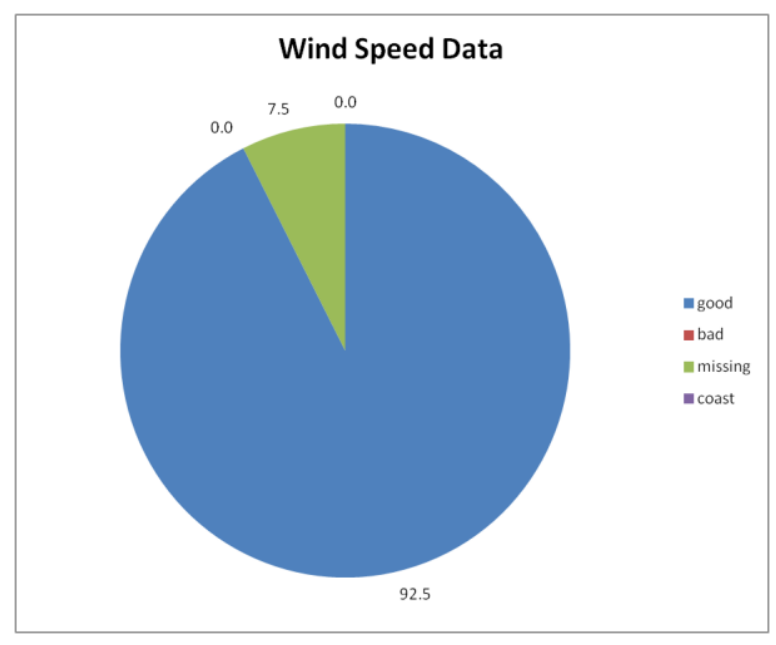

Figure L-289 


\section{October 2012 Site 2}

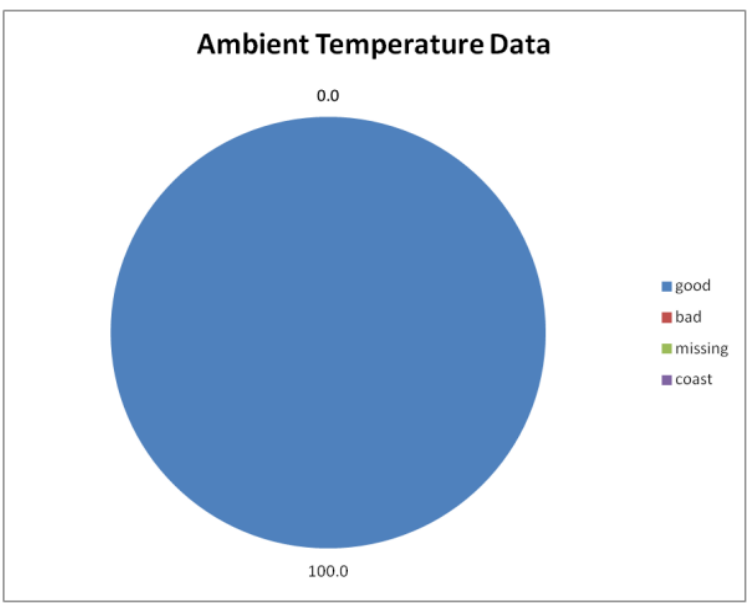

Figure L-290

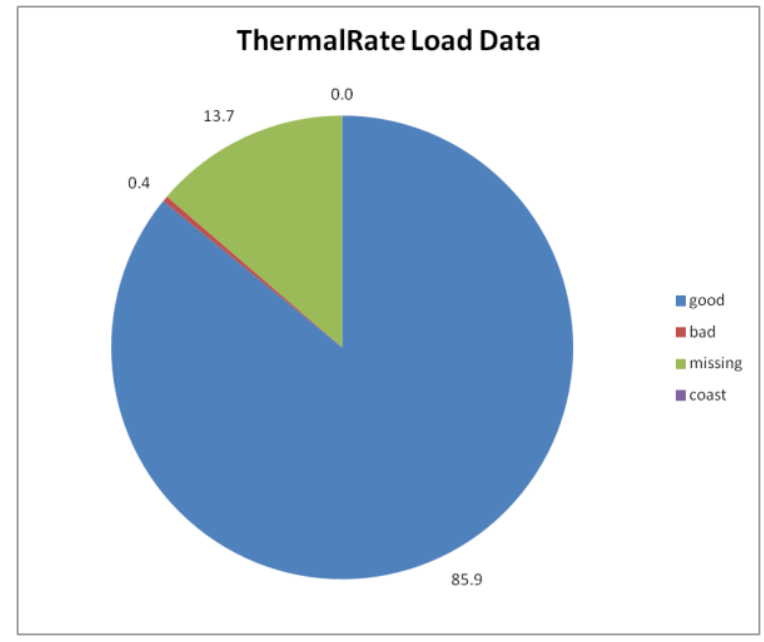

Figure L-292

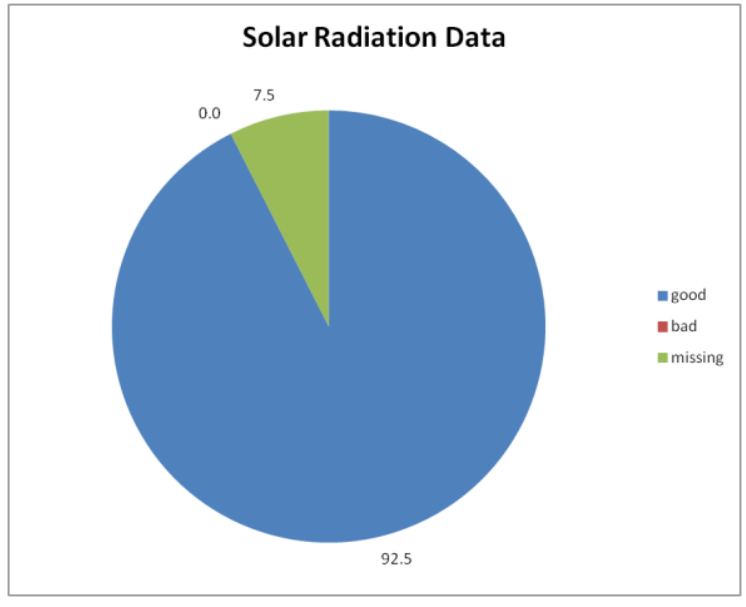

Figure L-291

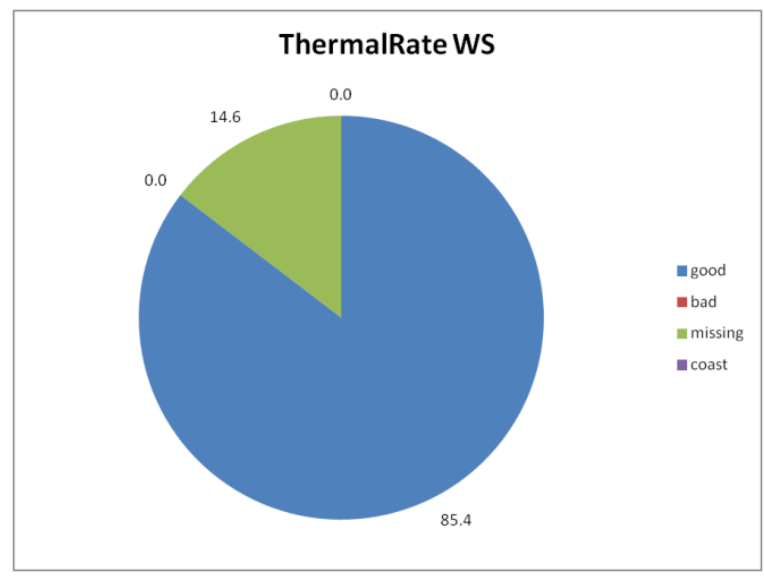

Figure L-293 


\section{October 2012 Site 2}

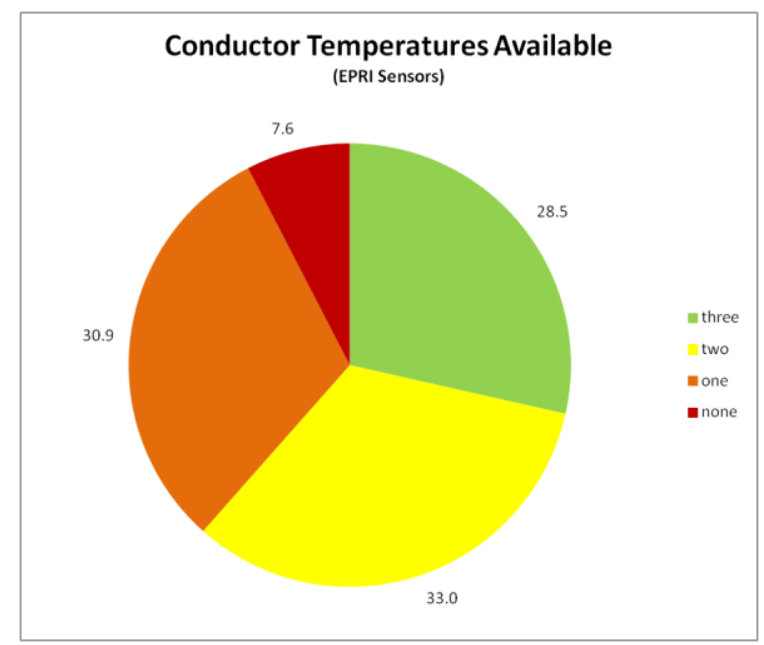

Figure L-294

\section{December 2010 Site 1}

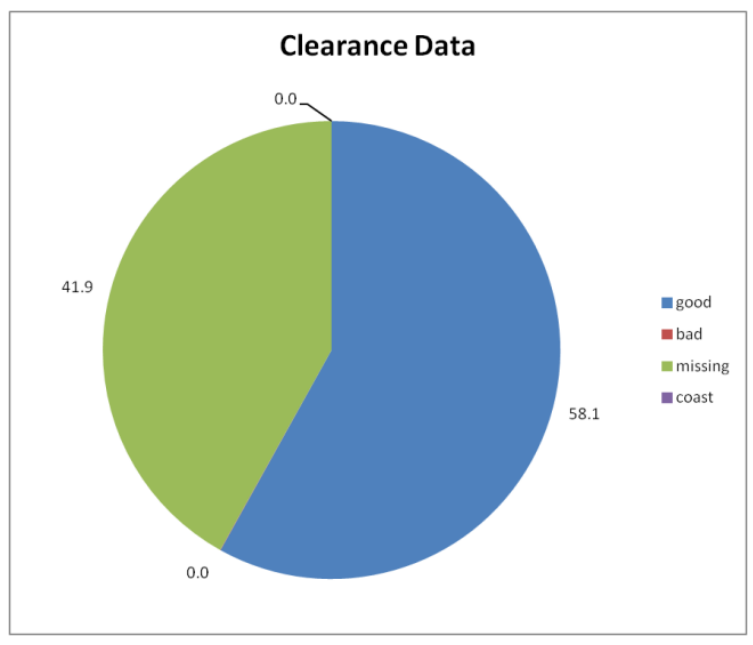

Figure L-295

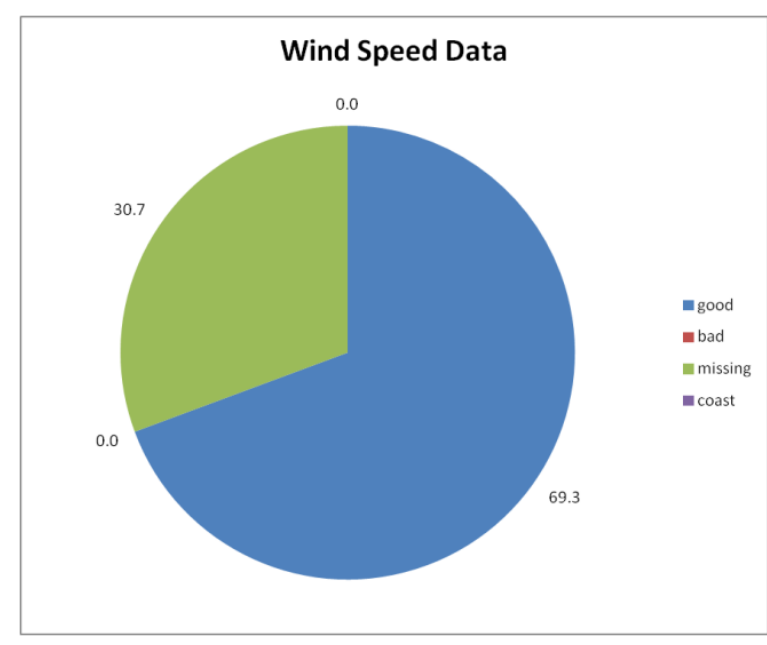

Figure L-296 


\section{December 2010 Site 1}

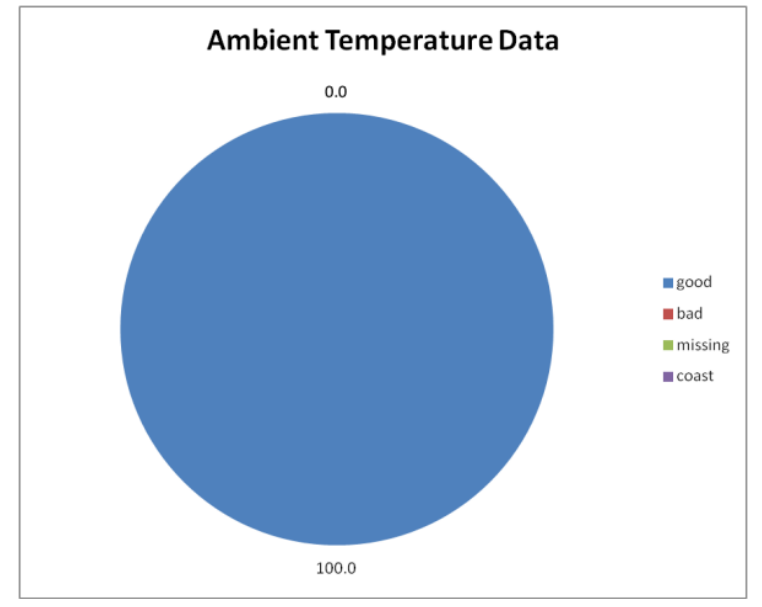

Figure L-297

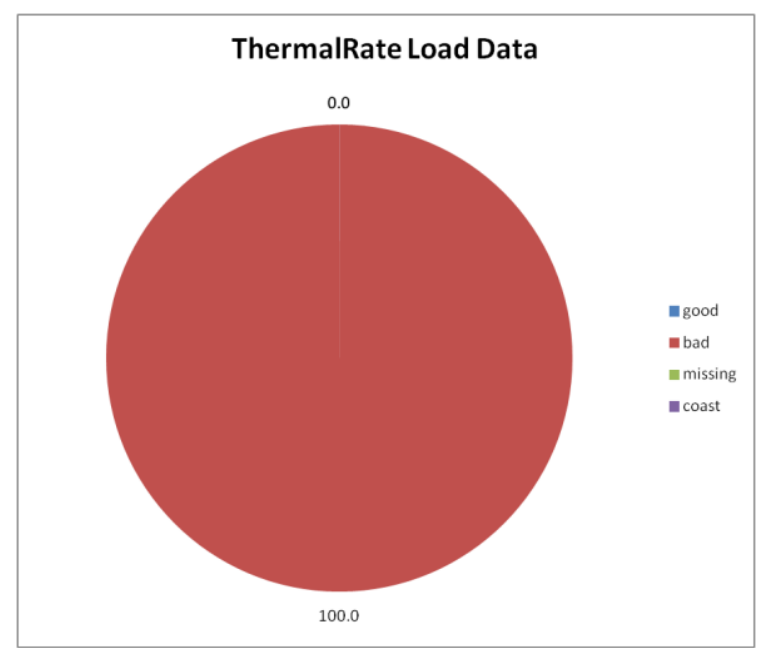

Figure L-299

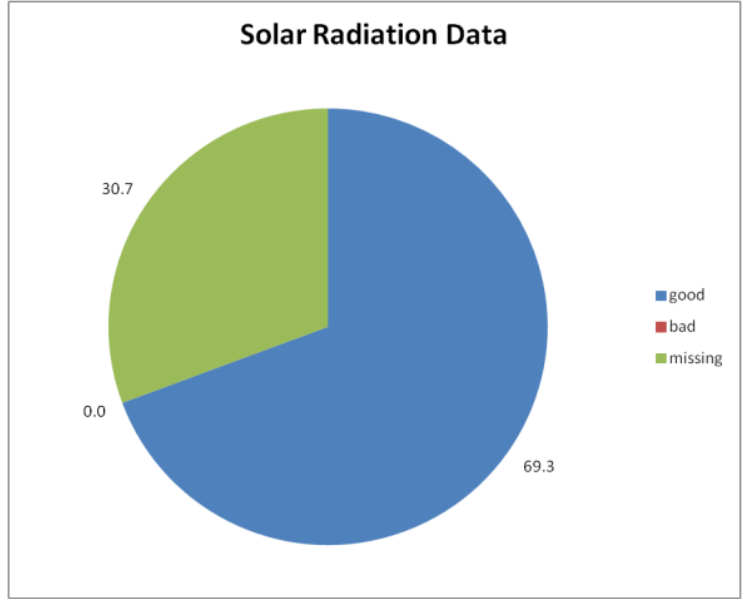

Figure L-298

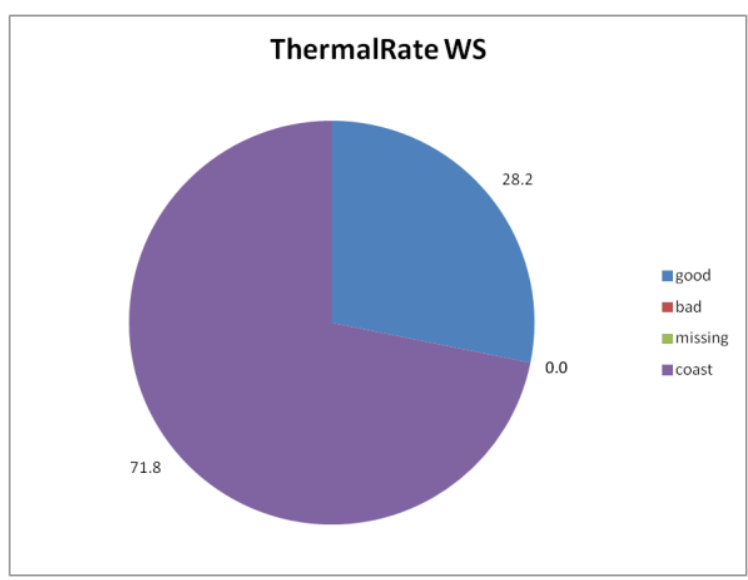

Figure L-300 


\section{December 2010 Site 1}

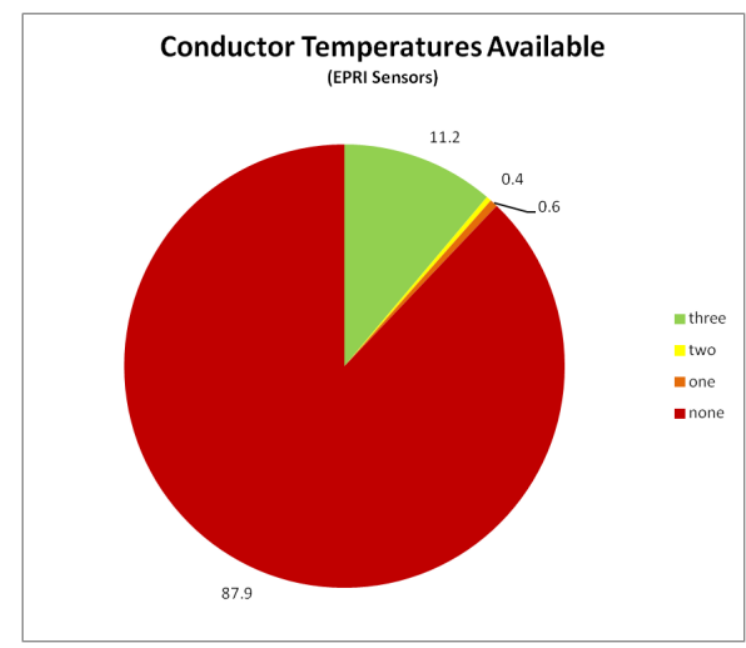

Figure L-301

\section{January 2011 Site 1}

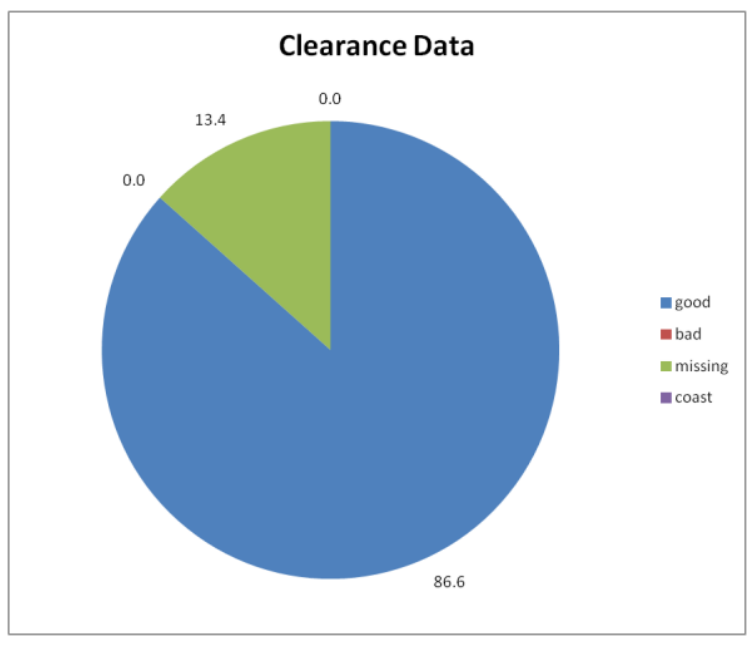

Figure L-302

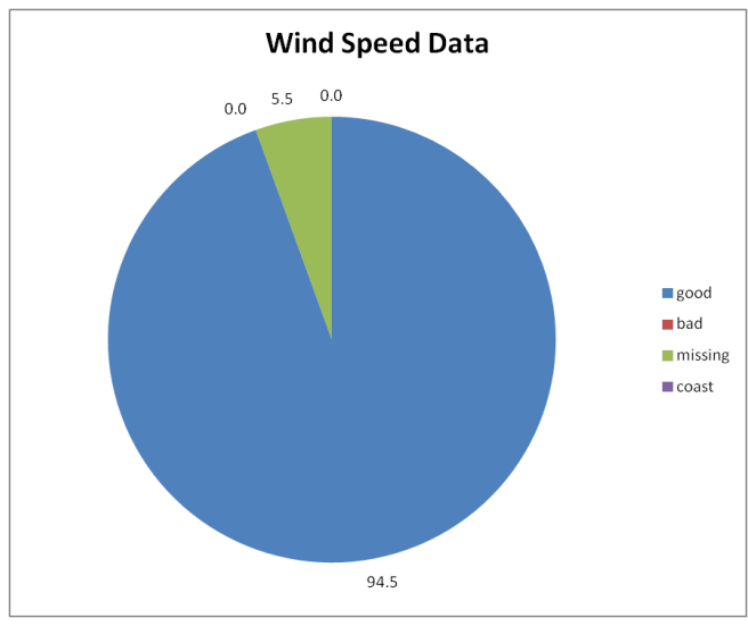

Figure L-303 


\section{January 2011 Site 1}

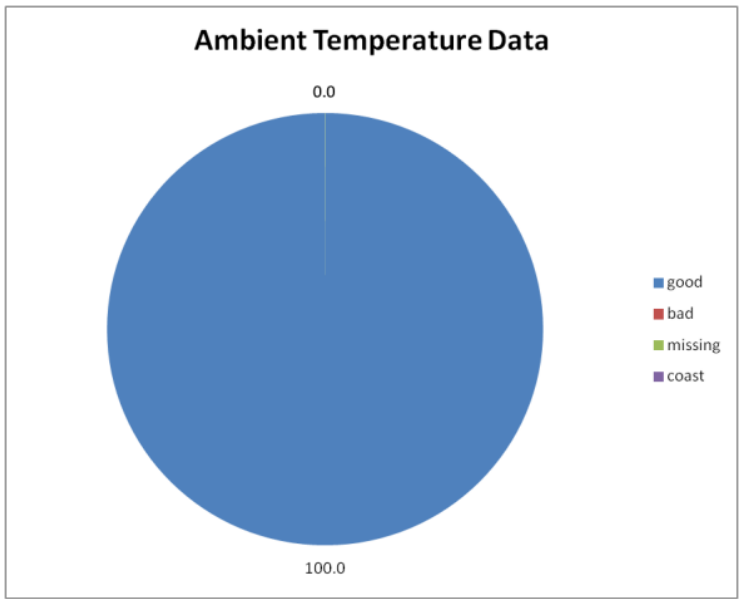

Figure L-304

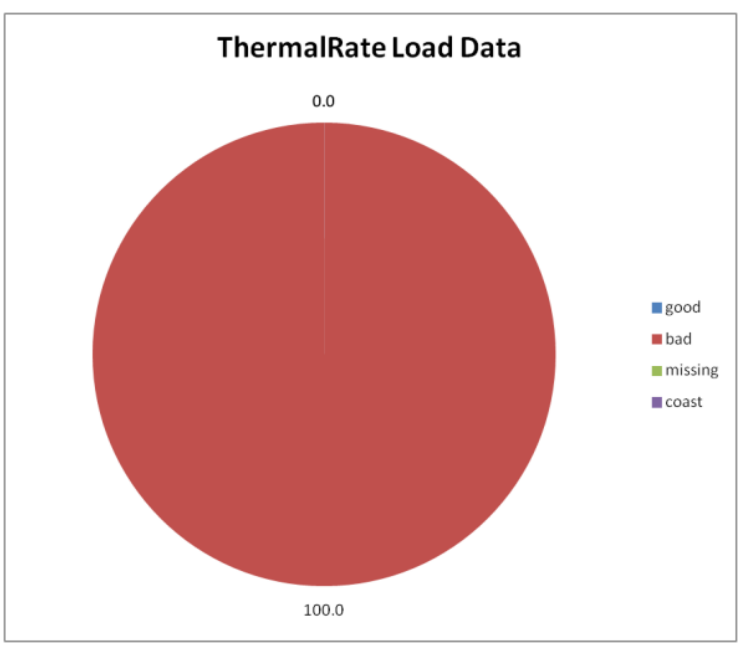

Figure L-306

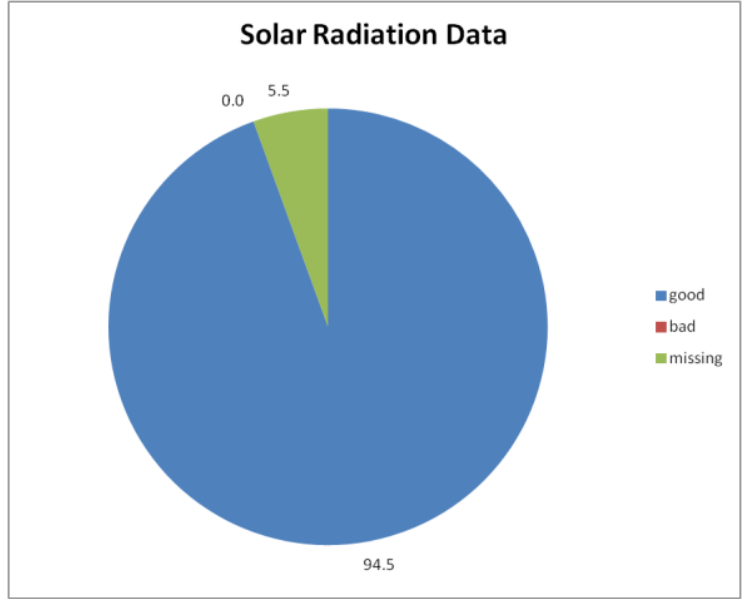

Figure L-305

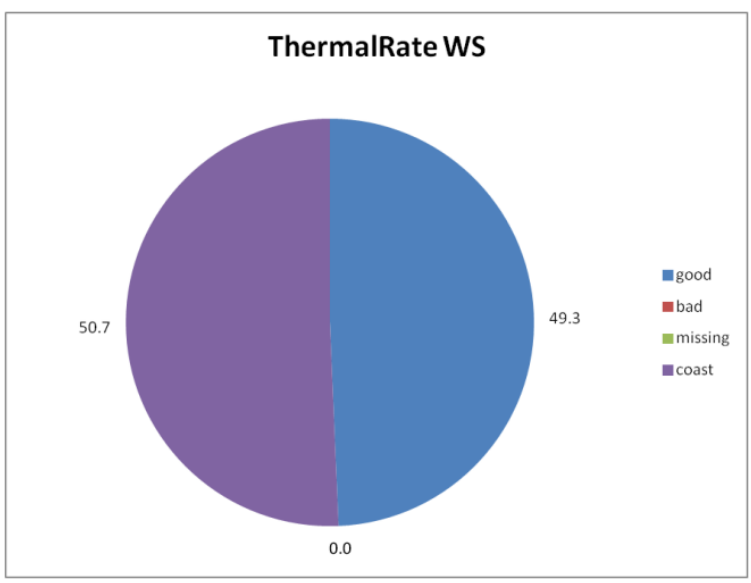

Figure L-307 


\section{January 2011 Site 1}

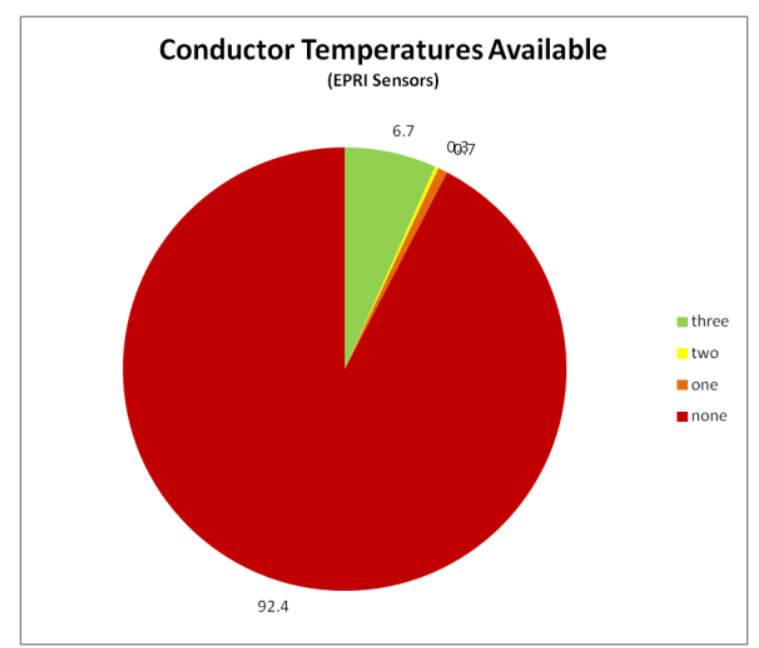

Figure L-308

\section{February 2011 Site 1}

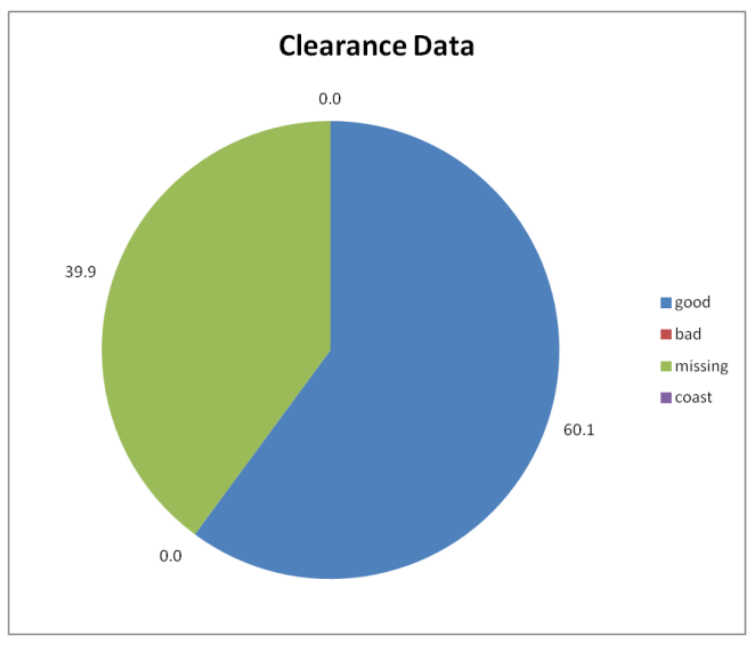

Figure L-309

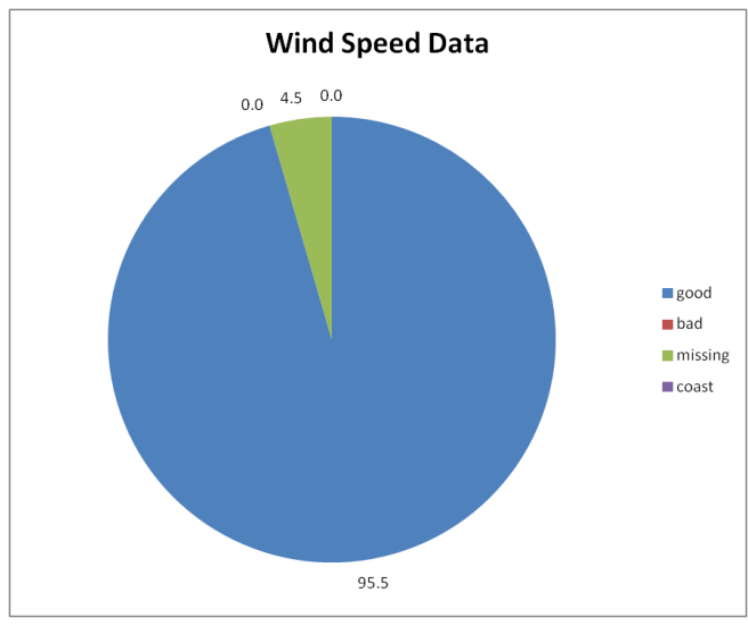

Figure L-310 


\section{February 2011 Site 1}

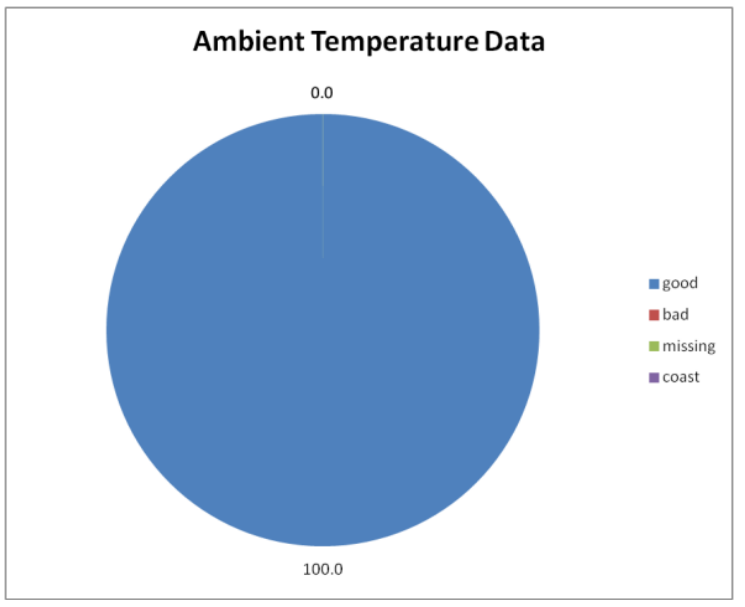

Figure L-311

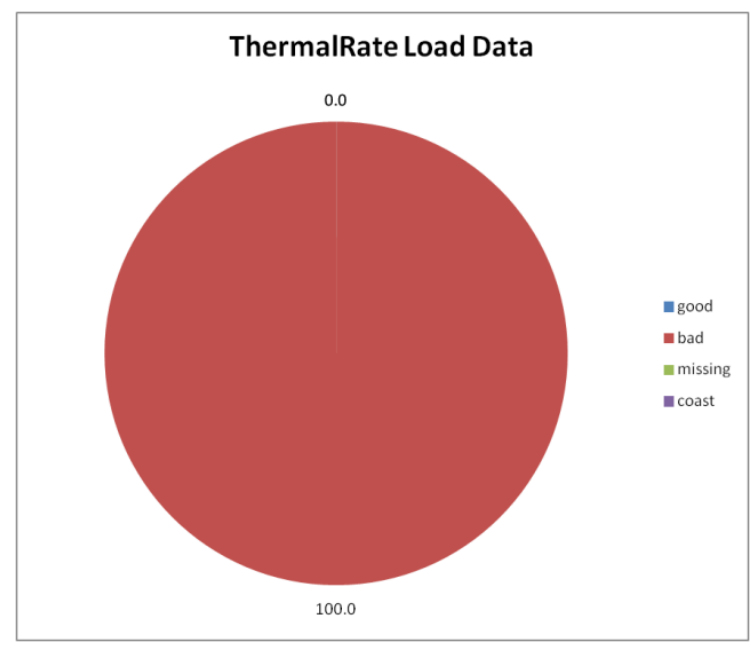

Figure L-313

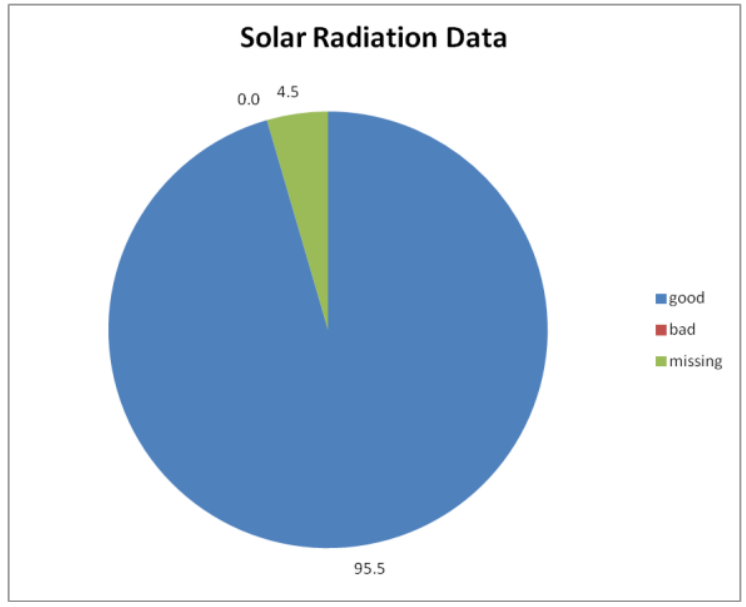

Figure L-312

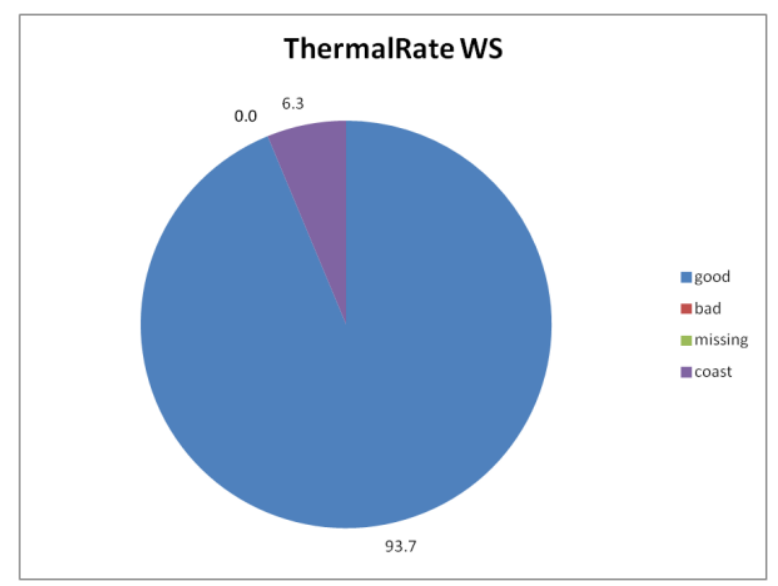

Figure L-314 
February 2011 Site 1

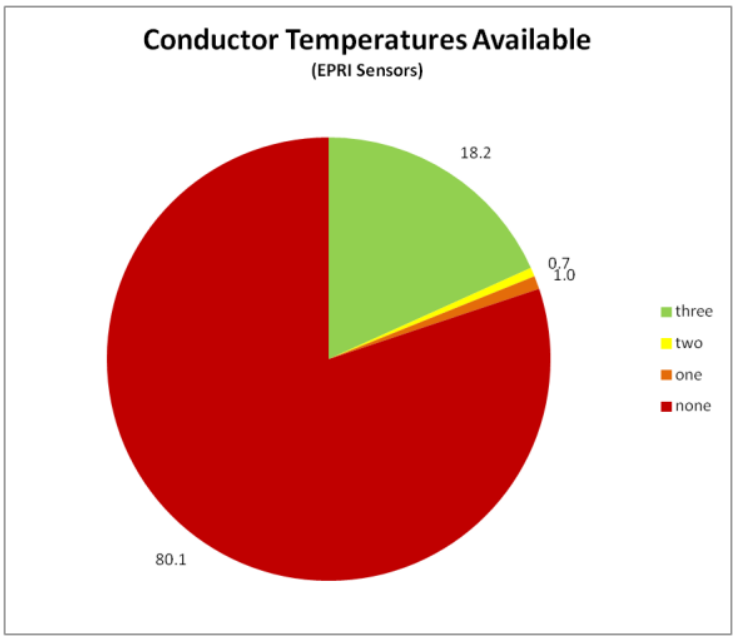

Figure L-315

\section{March 2011 Site 1}

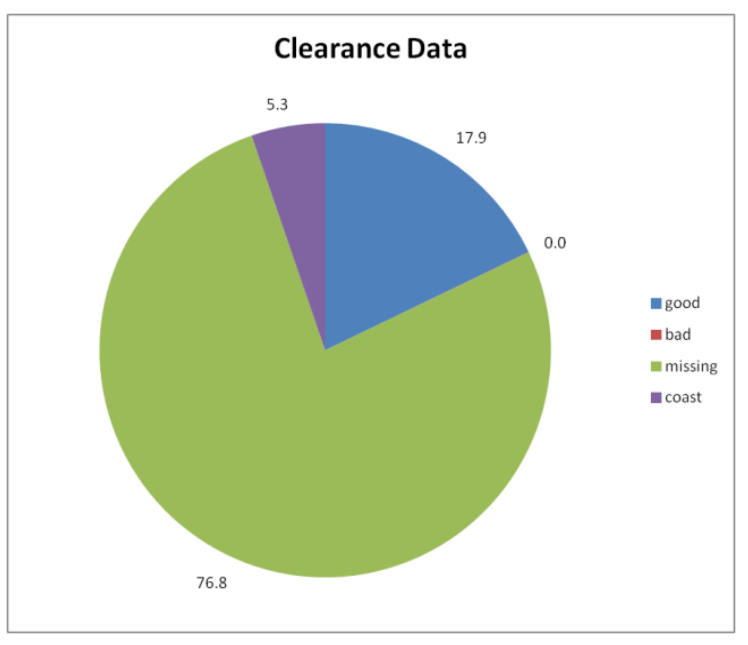

Figure L-316

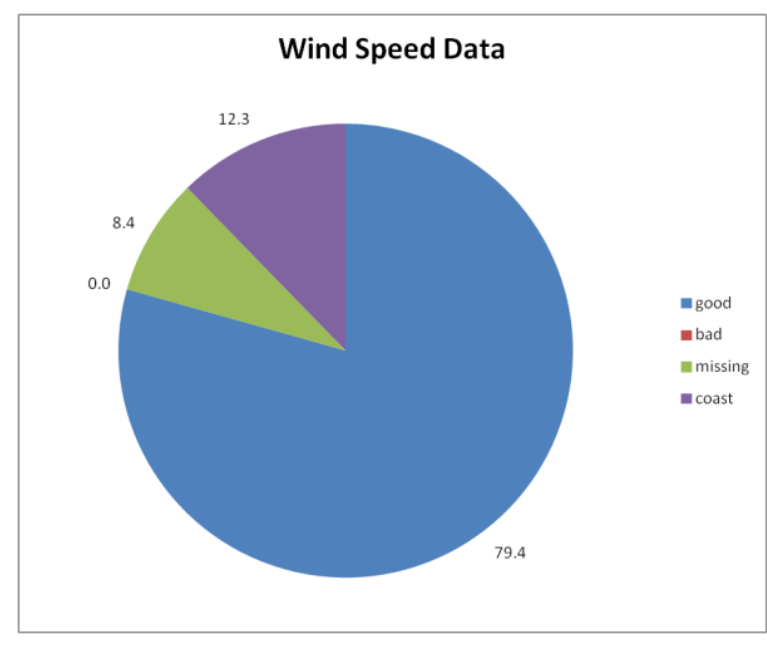

Figure L-317 


\section{March 2011 Site 1}

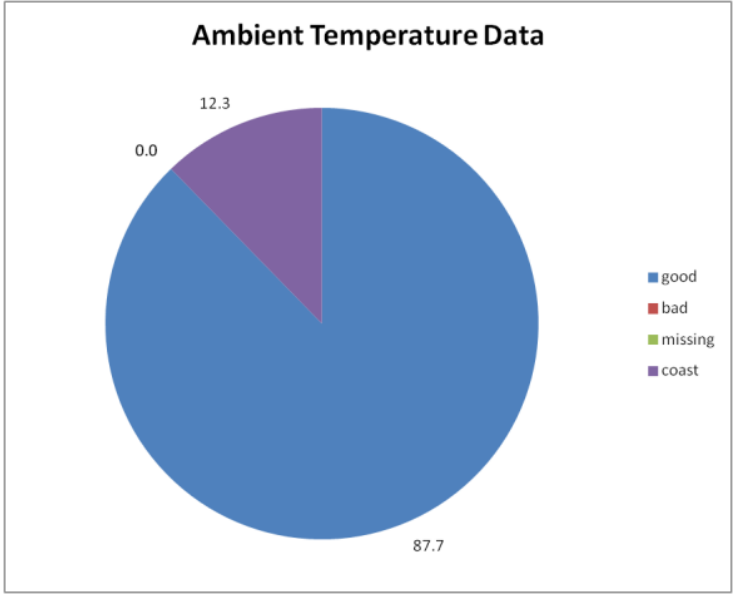

Figure L-318

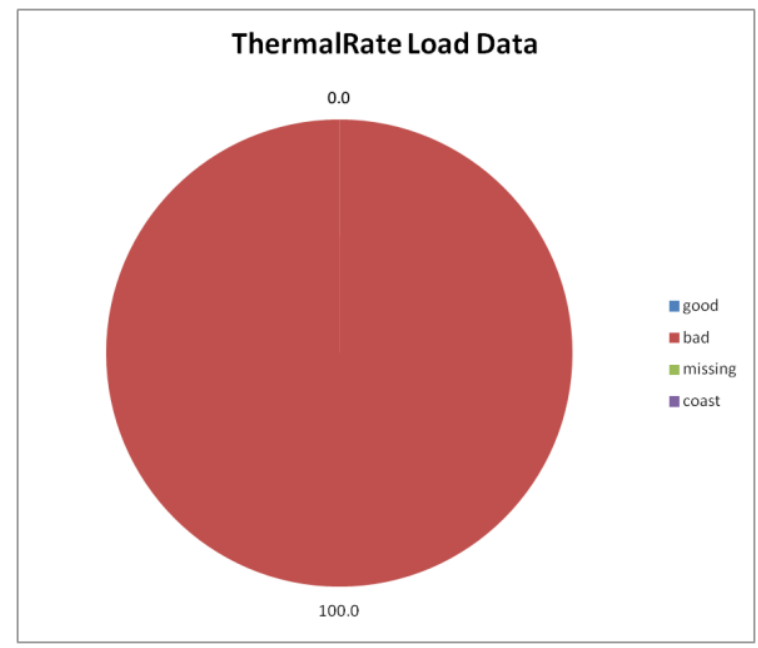

Figure L-320

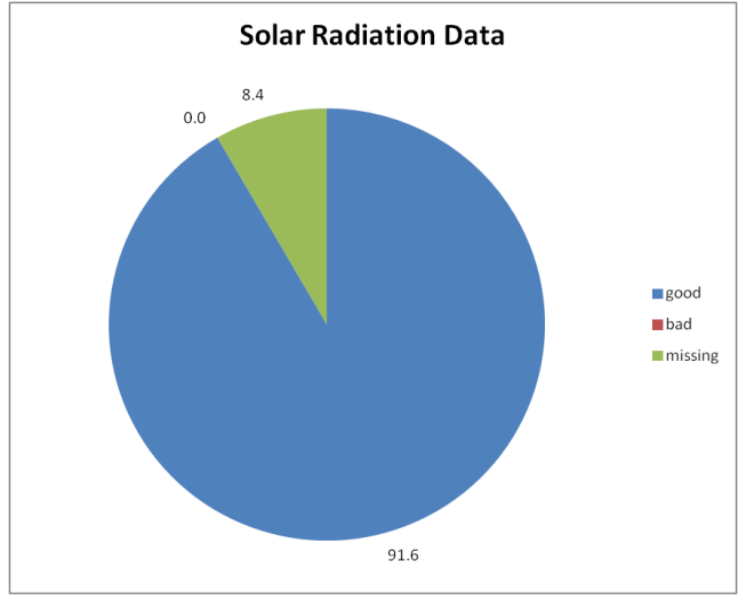

Figure L-319

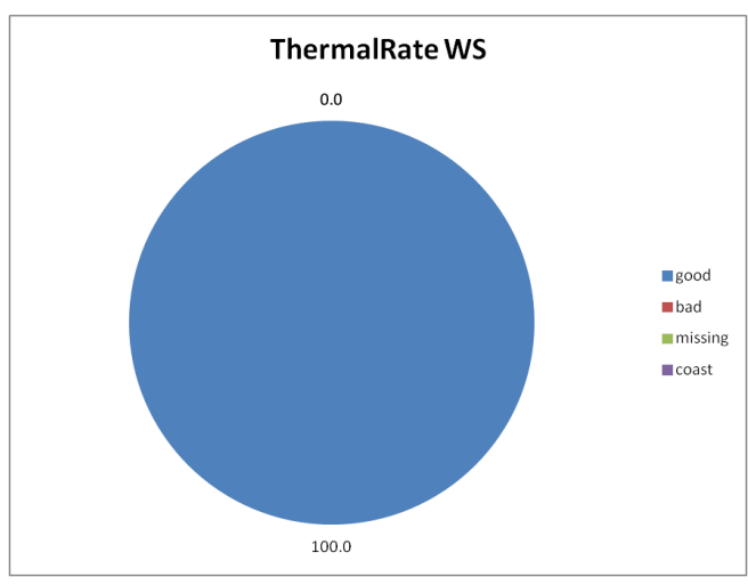

Figure L-321 
March 2011 Site 1

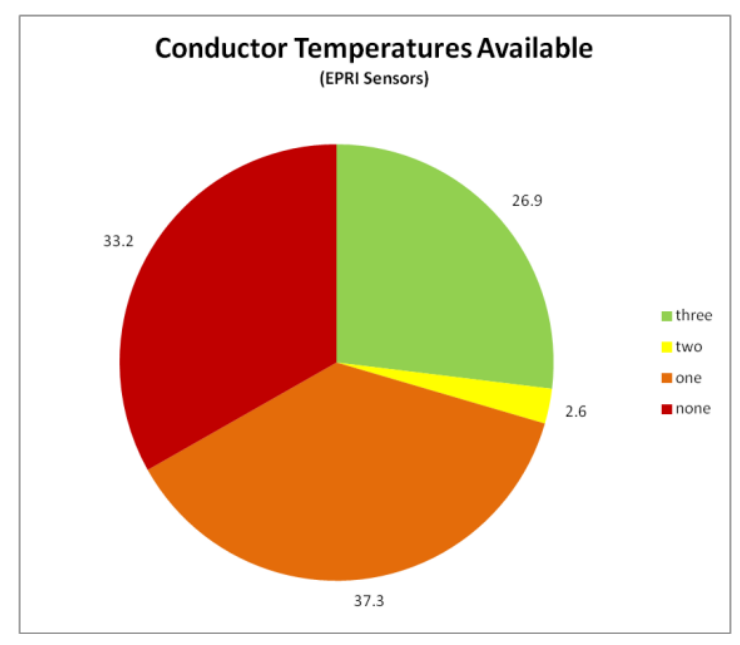

Figure L-322

\section{April 2011Site 1}

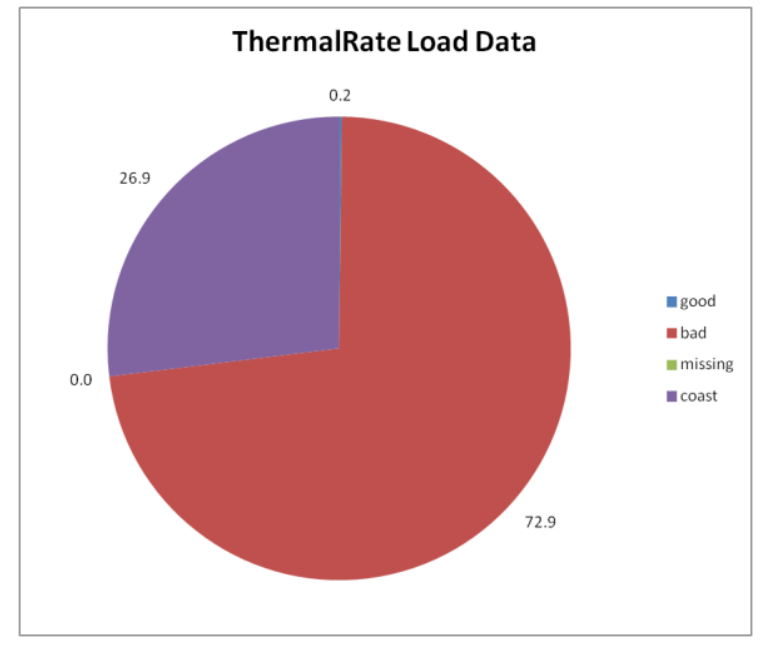

Figure L-323

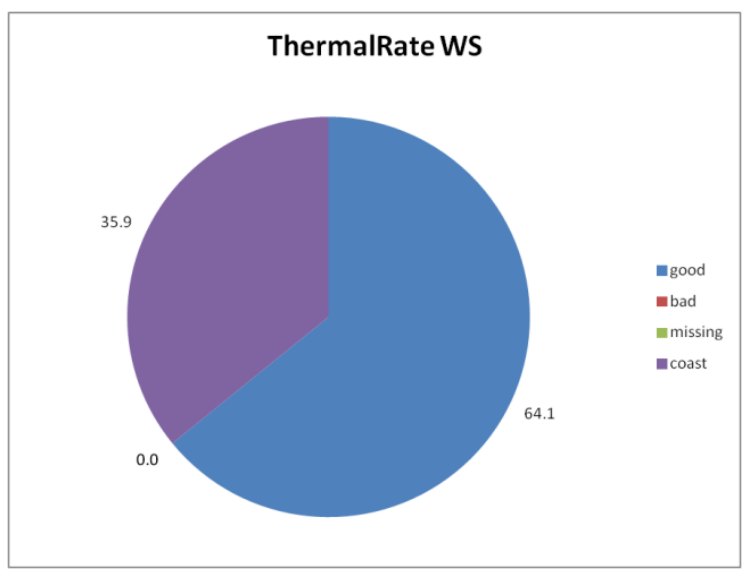

Figure L-324 


\section{April 2011Site 1}

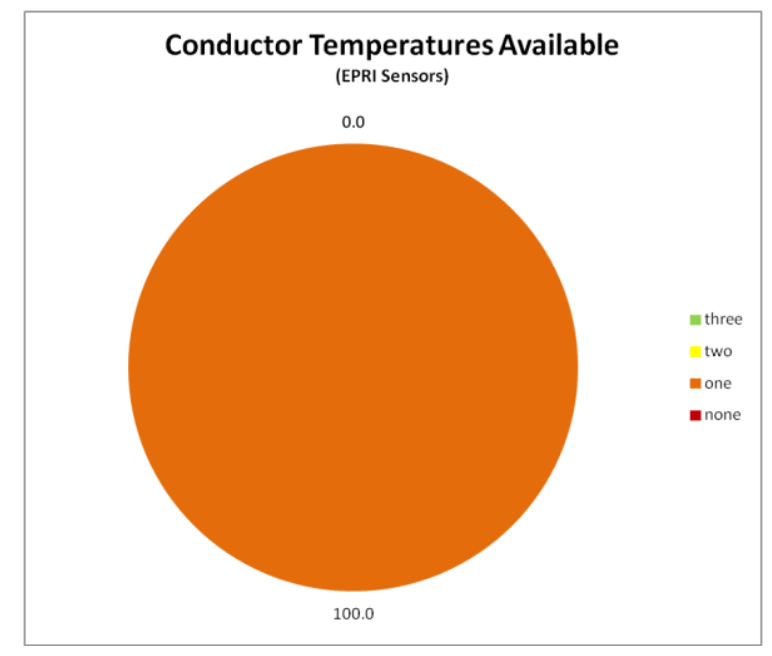

Figure L-325

\section{May 2011 Site 1}

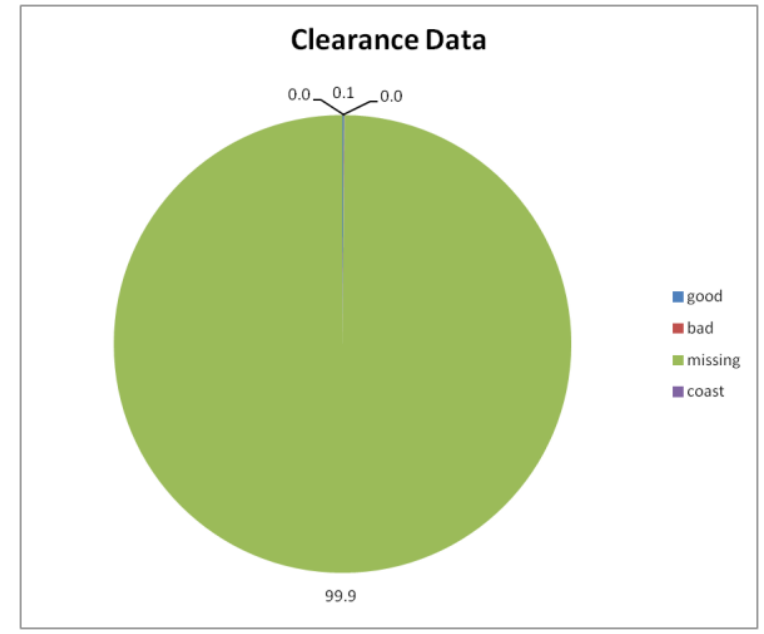

Figure L-326

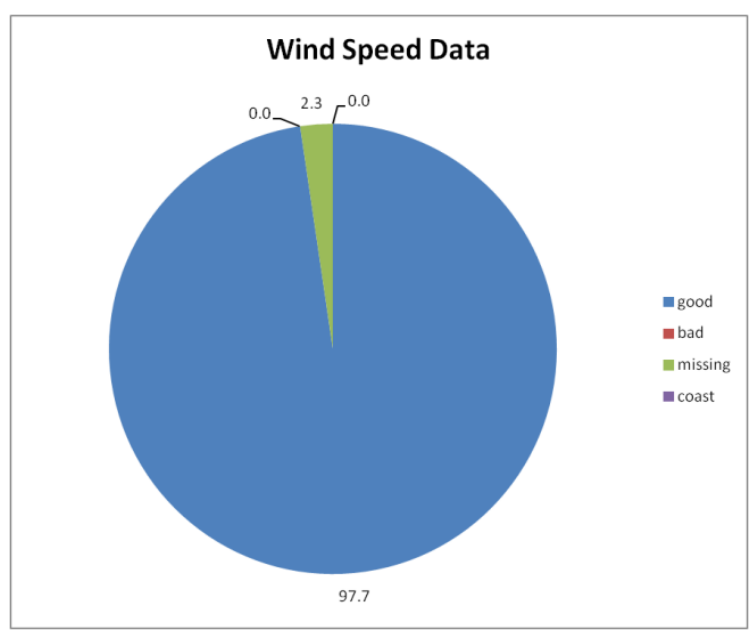

Figure L-327 
May 2011 Site 1

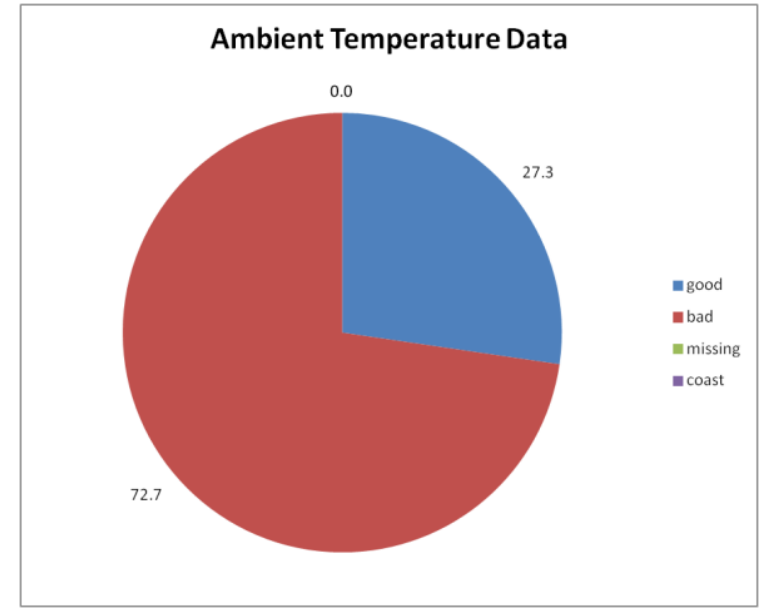

Figure L-328

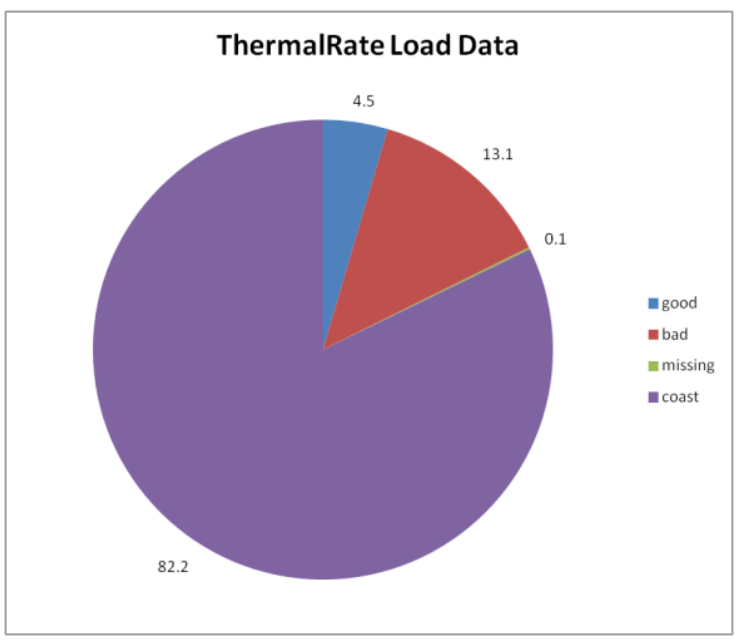

Figure L-330

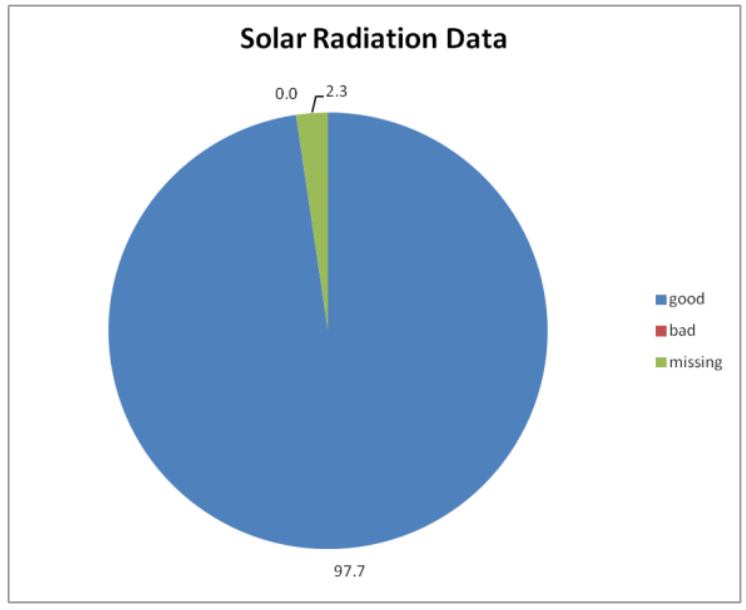

Figure L-329

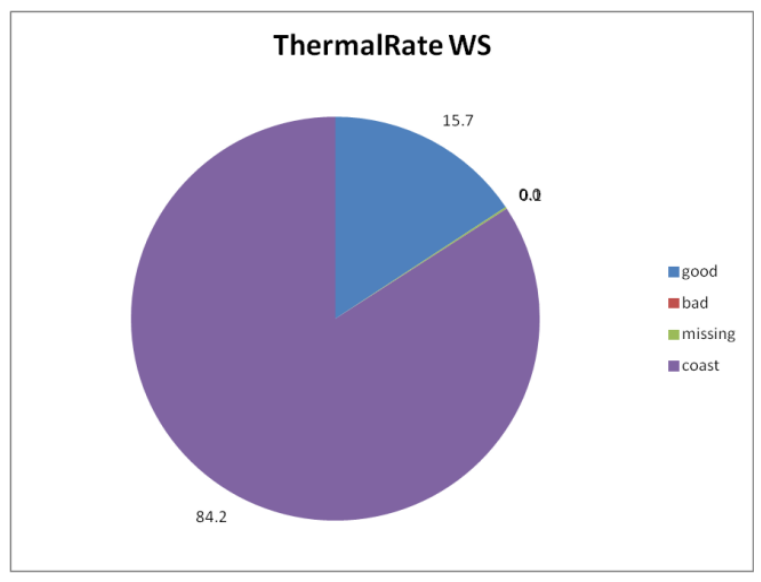

Figure L-331 
May 2011 Site 1

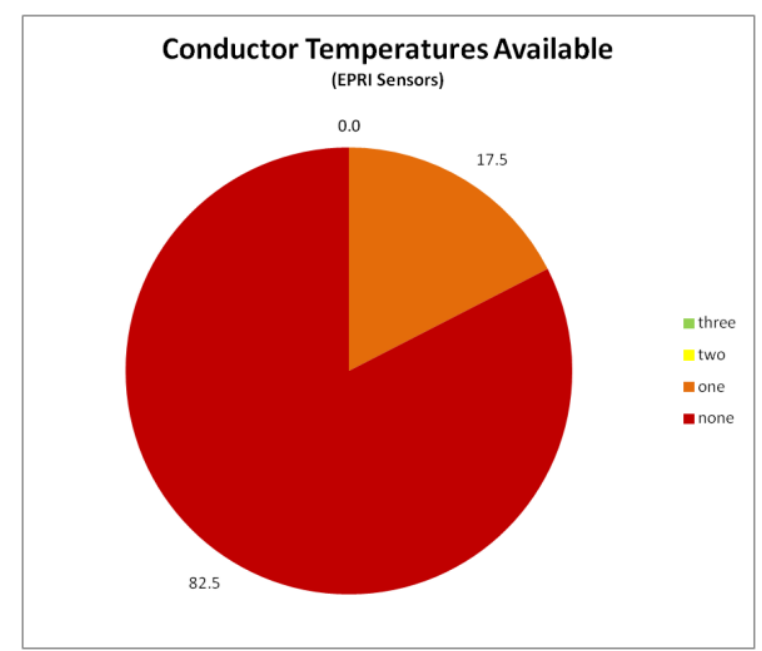

Figure L-332

\section{June 2012 Site 1}

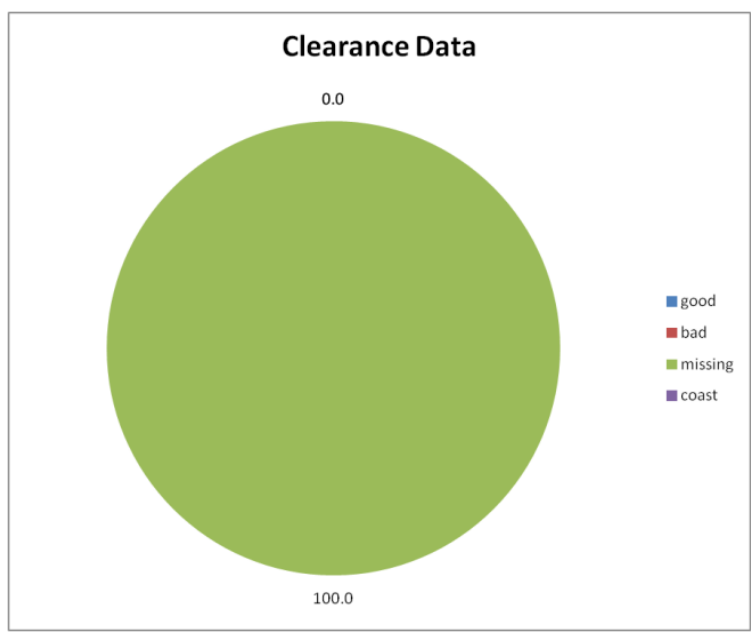

Figure L-333

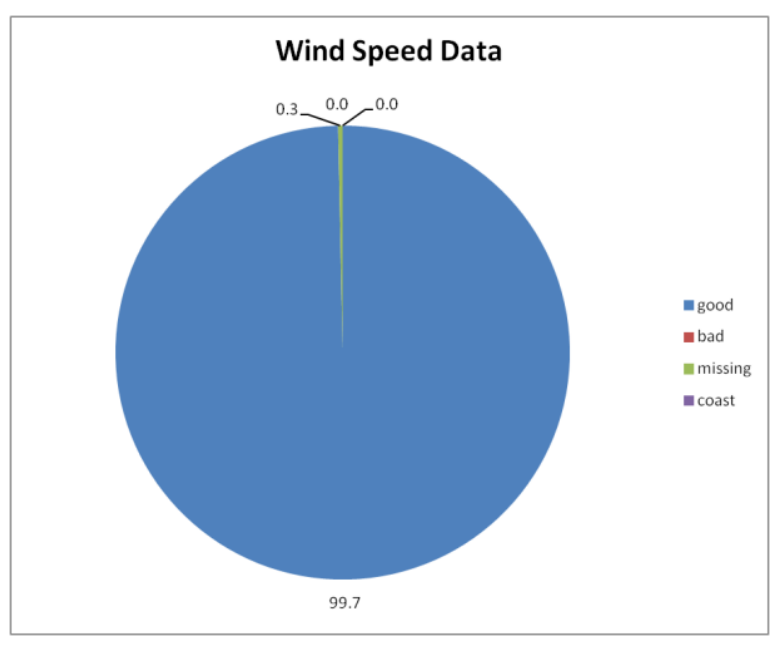

Figure L-334 


\section{June 2012 Site 1}

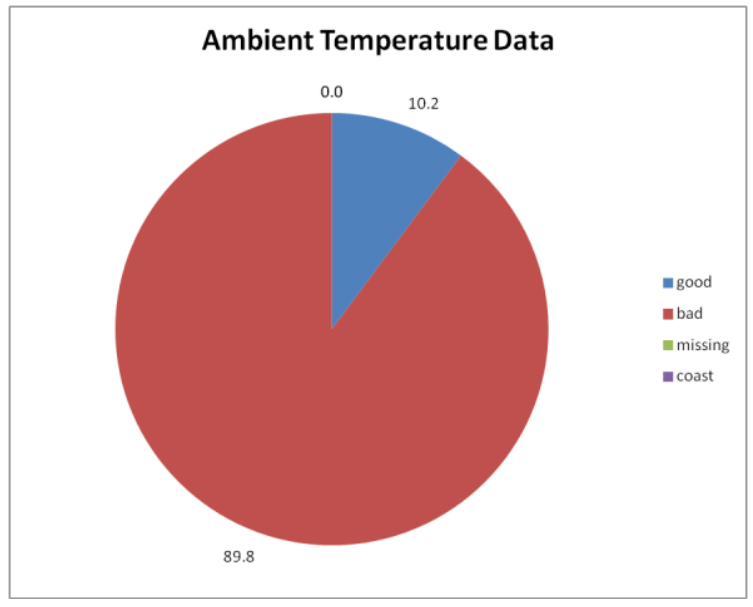

Figure L-335

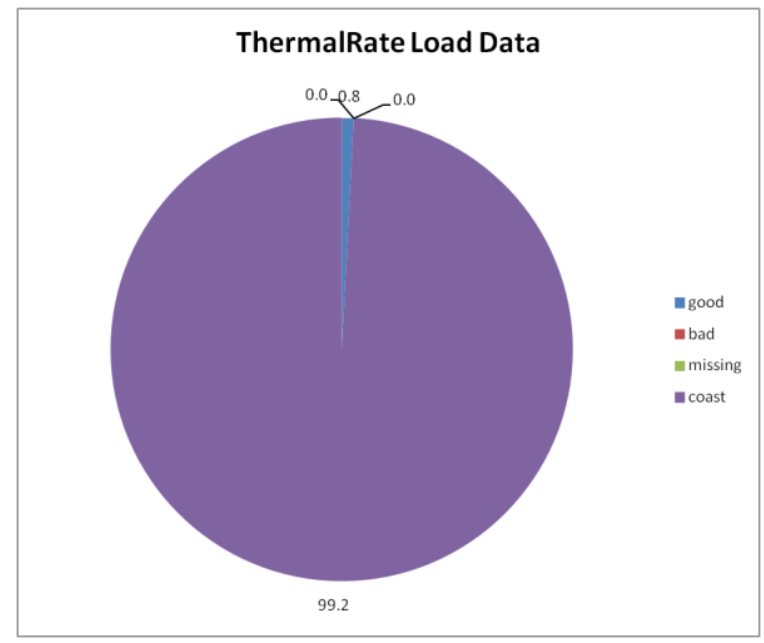

Figure L-337

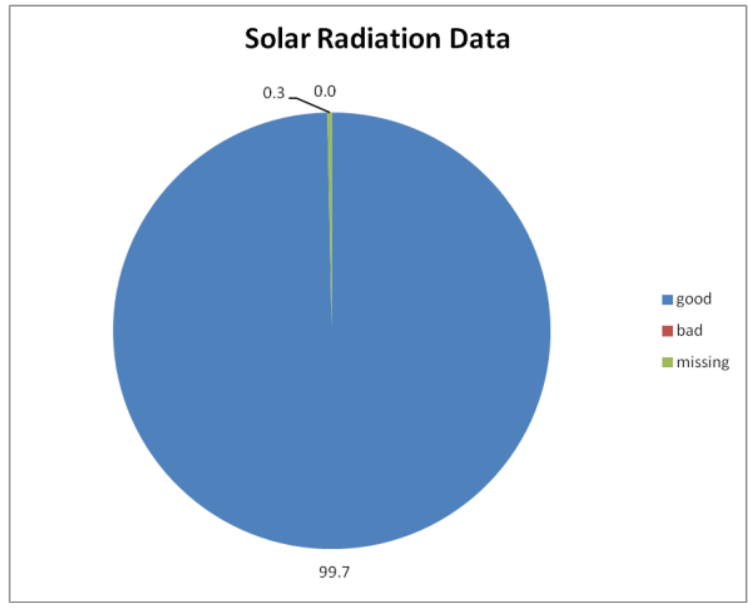

Figure L-336

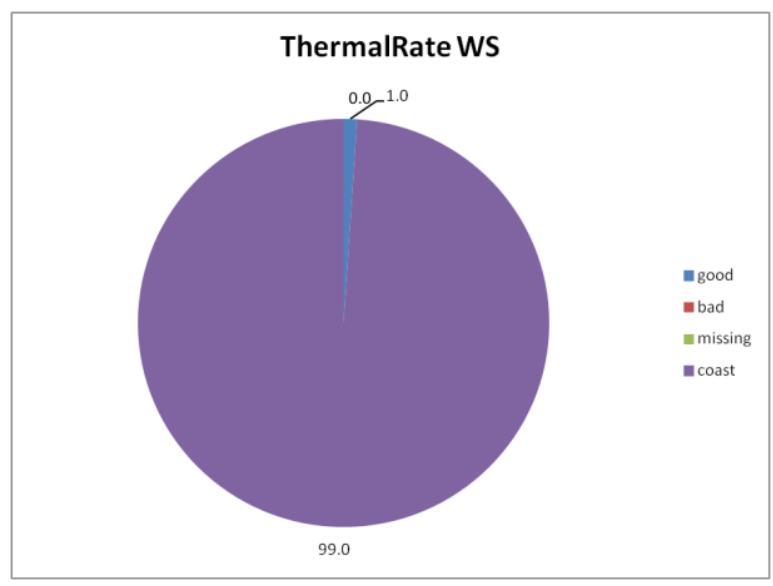

Figure L-338 


\section{June 2012 Site 1}

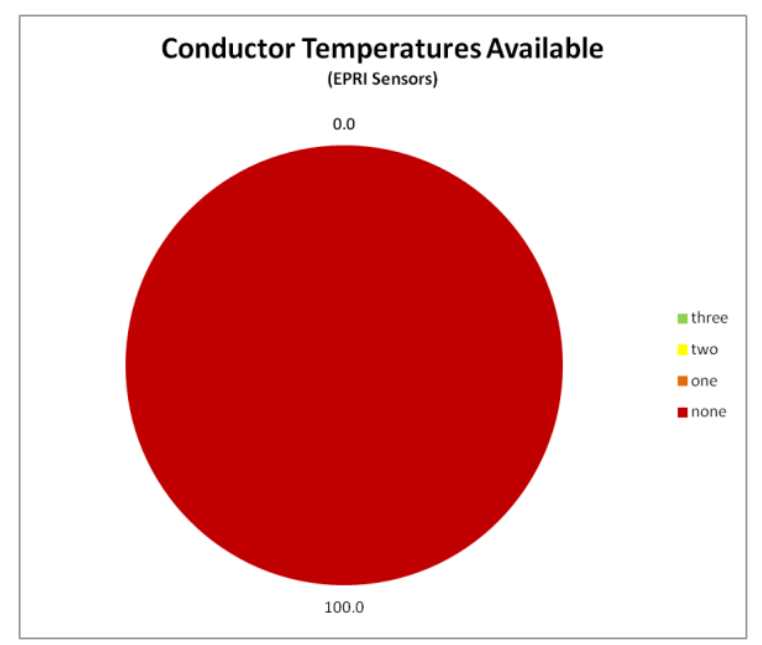

Figure L-339

\section{July 2012 Site 1}

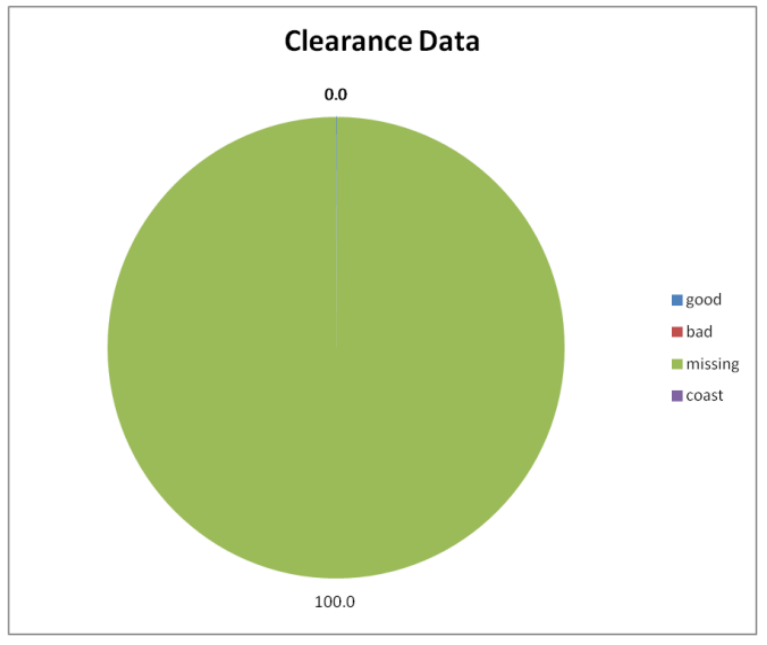

Figure L-340

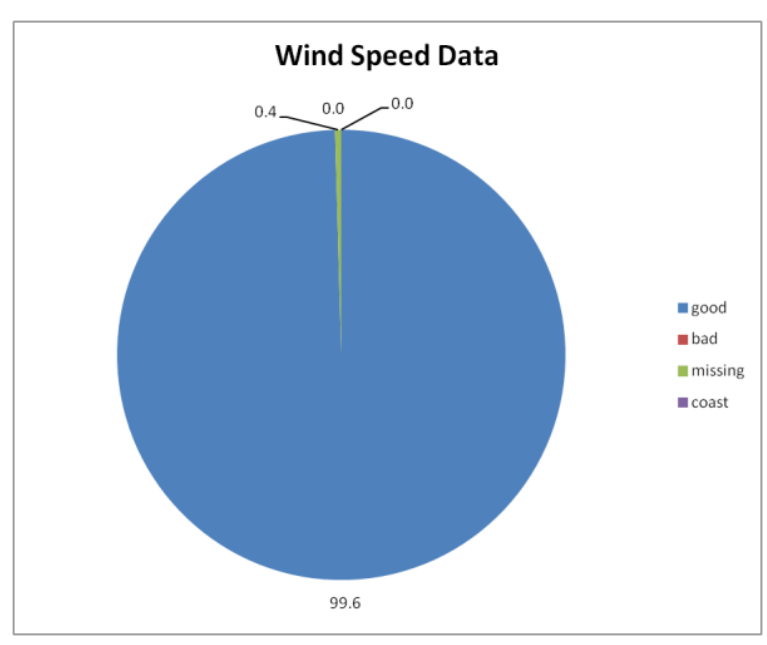

Figure L-341 


\section{July 2012 Site 1}

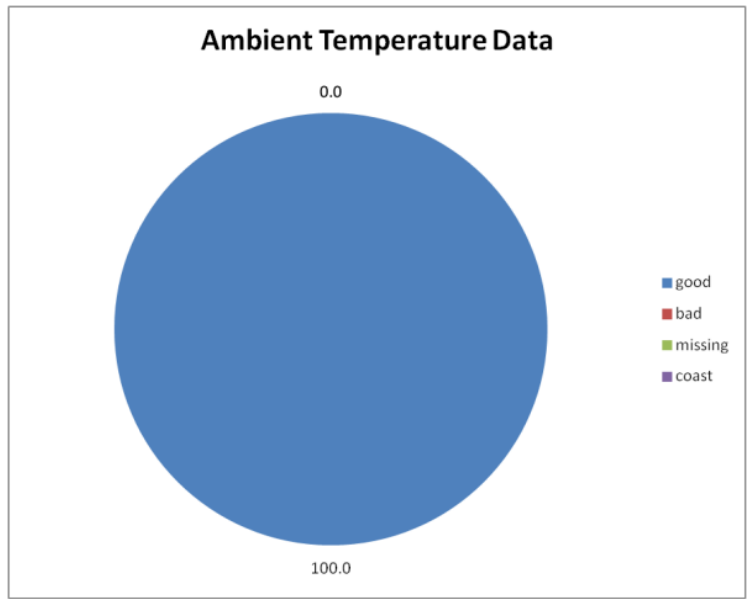

Figure L-342

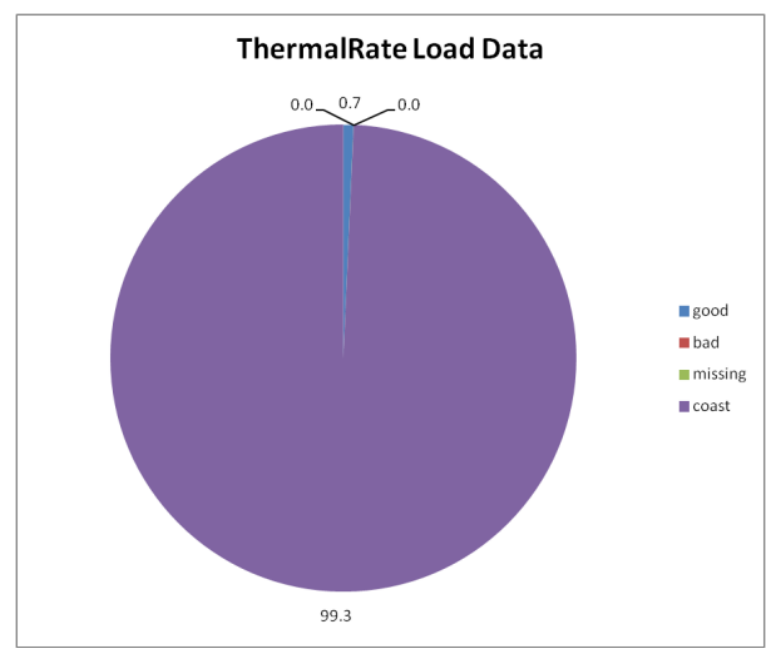

Figure L-344

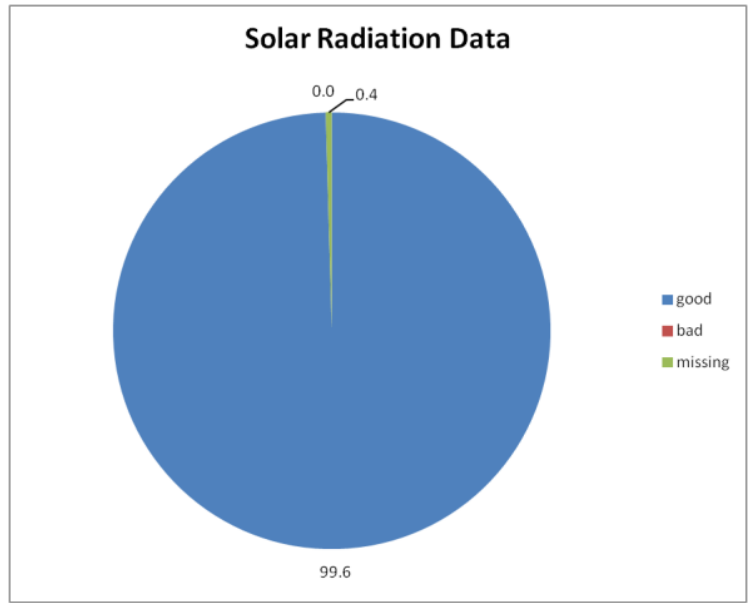

Figure L-343

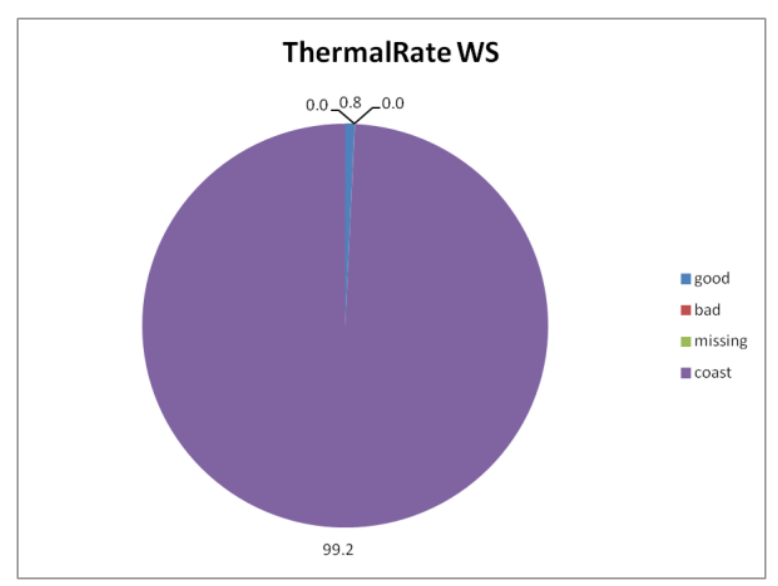

Figure L-345 


\section{July 2012 Site 1}

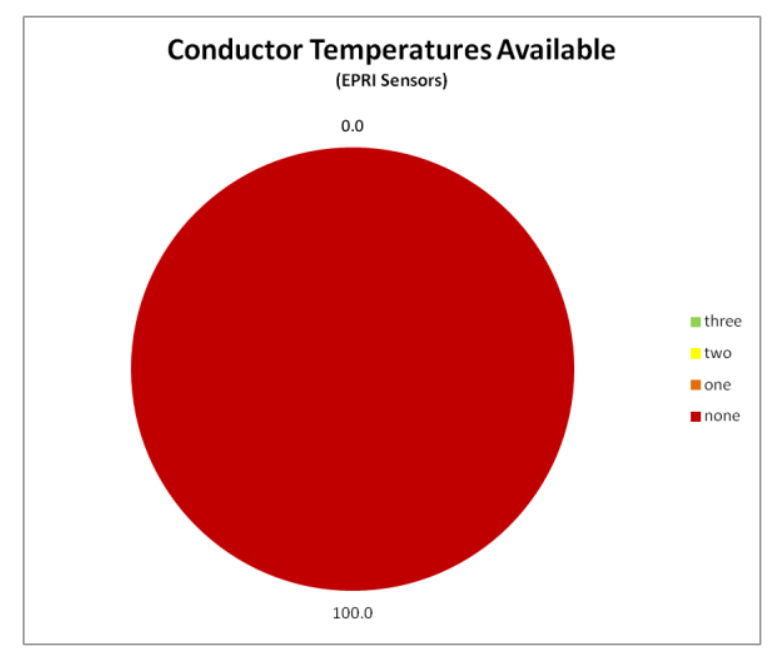

Figure L-346

\section{July 2012 Site 1}

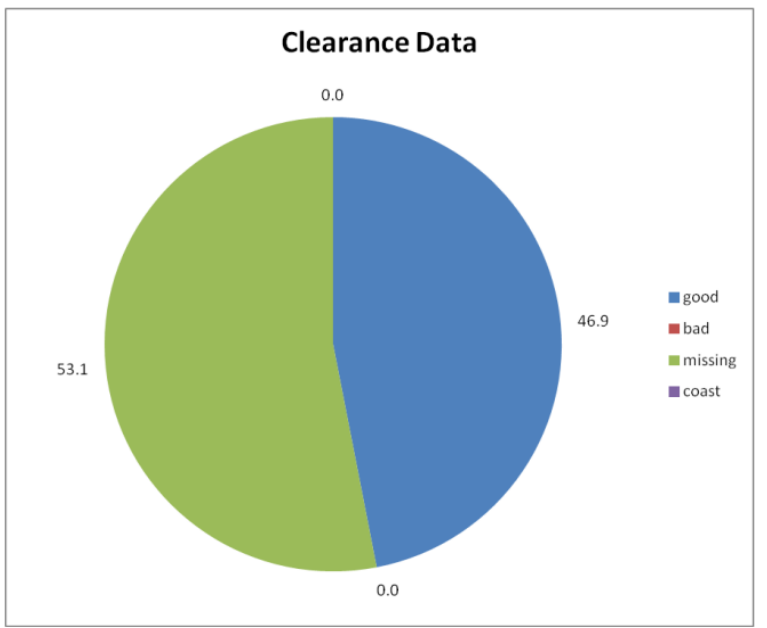

Figure L-347

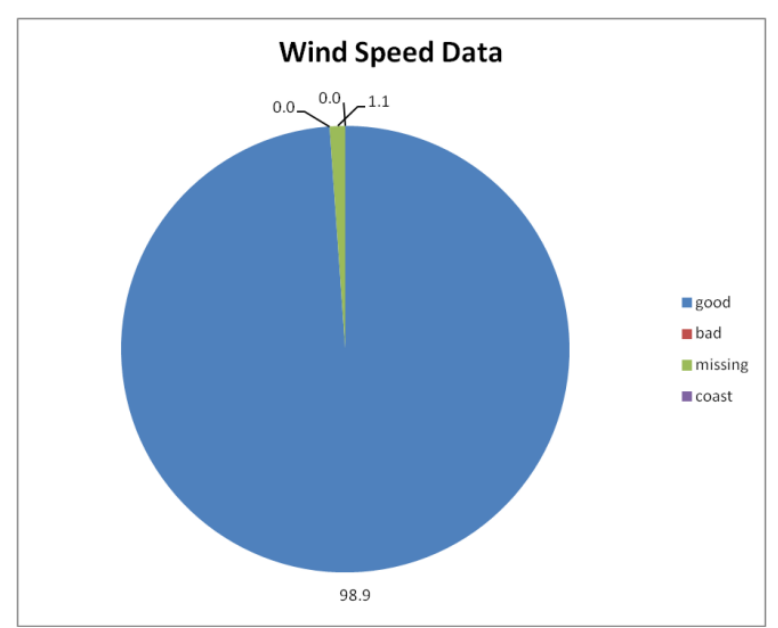

Figure L-348 


\section{July 2012 Site 1}

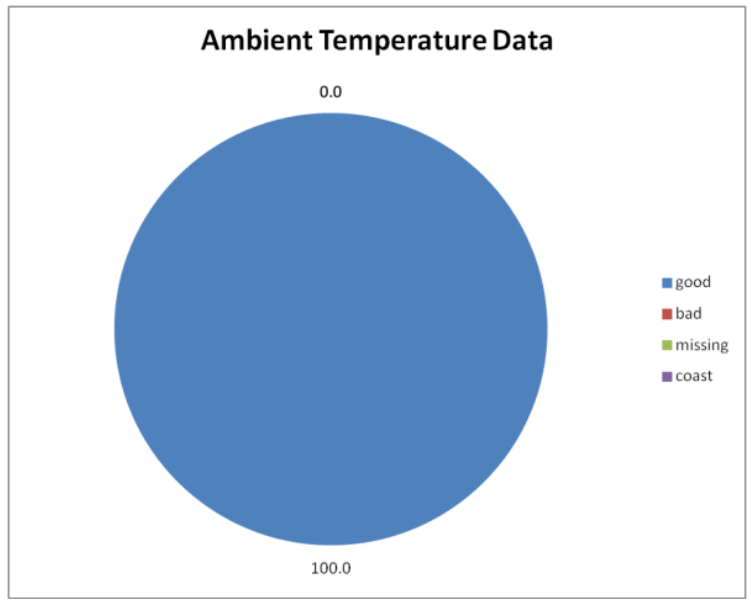

Figure L-349

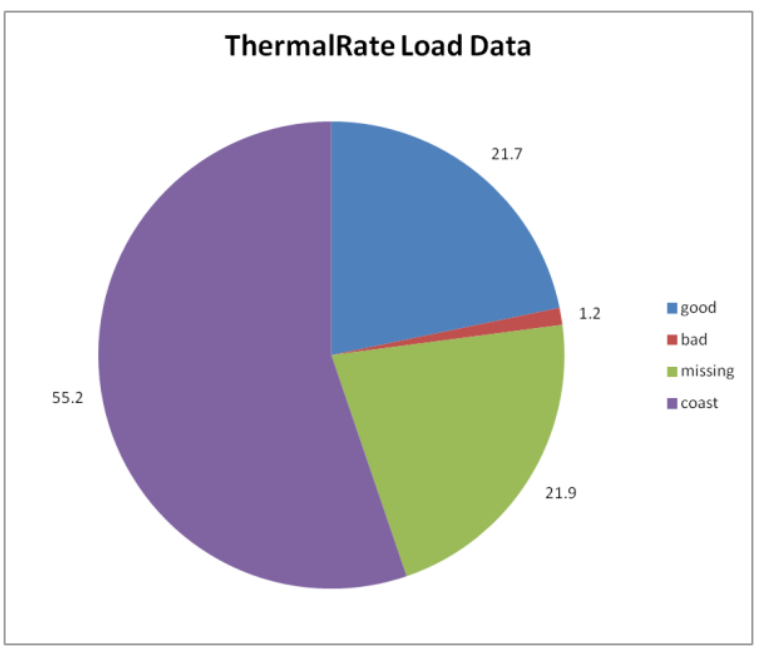

Figure L-351

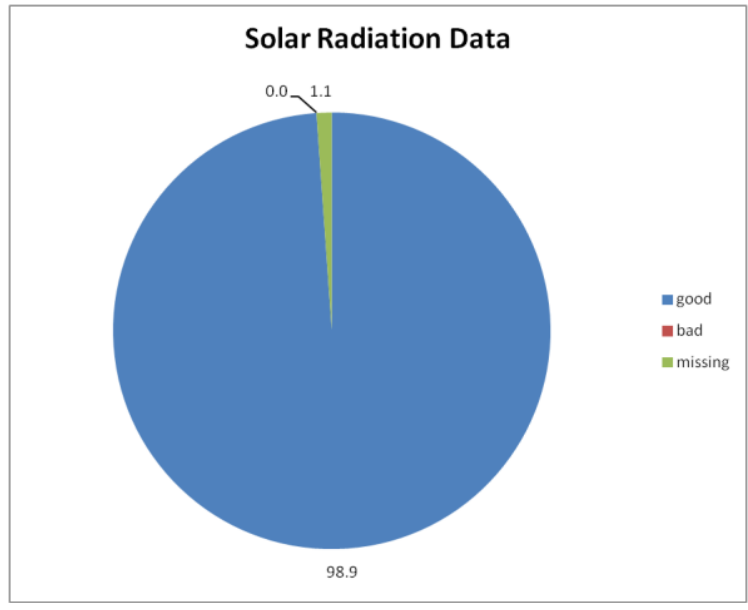

Figure L-350

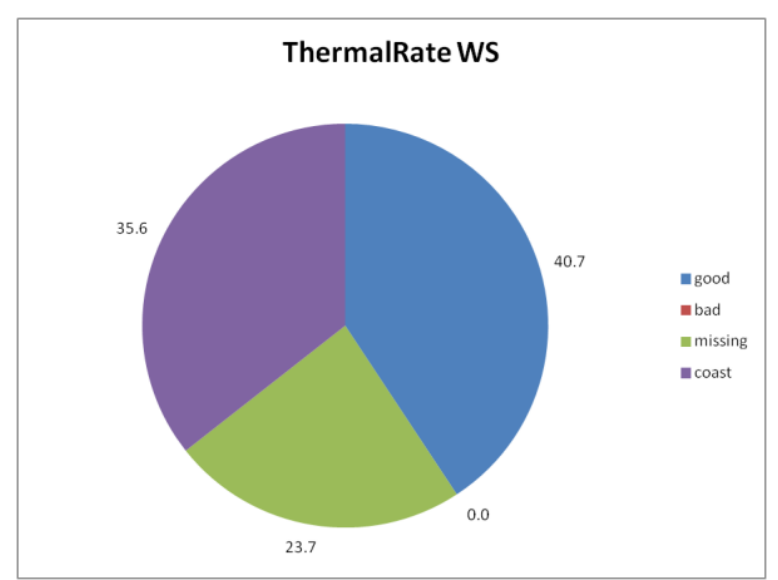

Figure L-352 


\section{July 2012 Site 1}

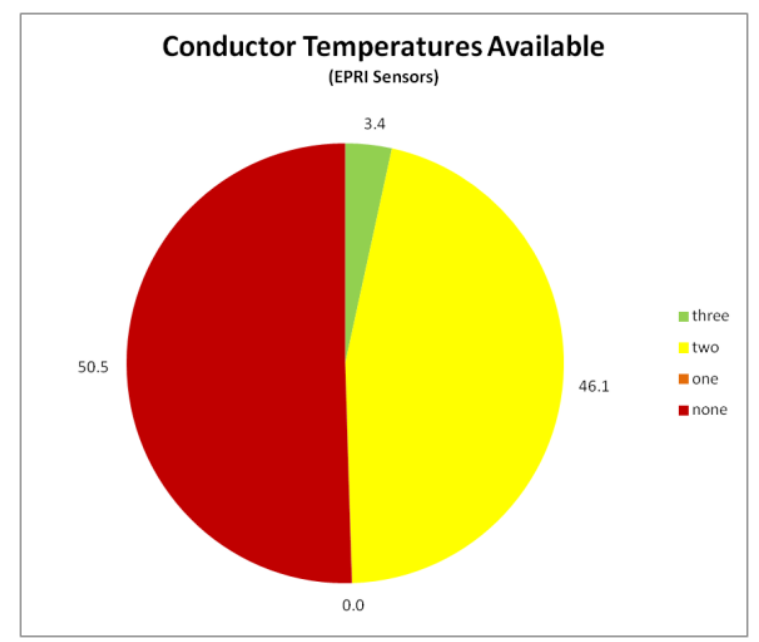

Figure L-353

\section{September 2011 Site 1}

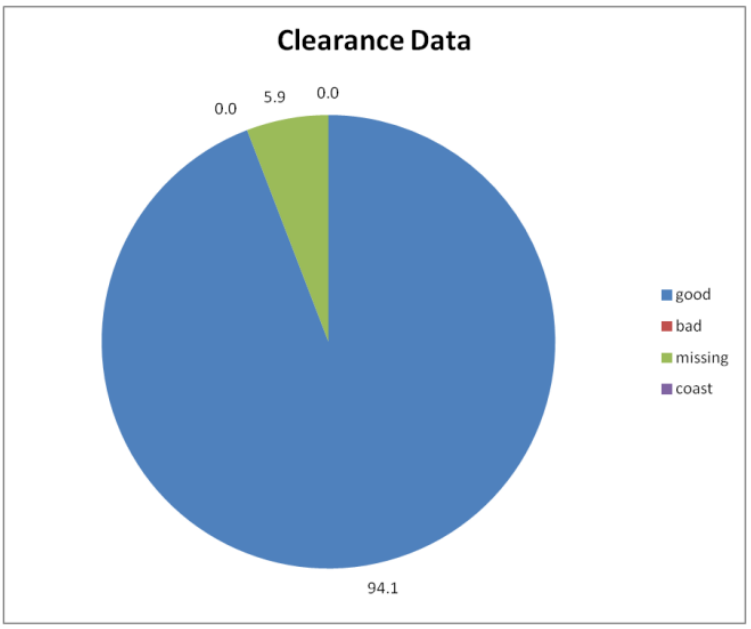

Figure L-354

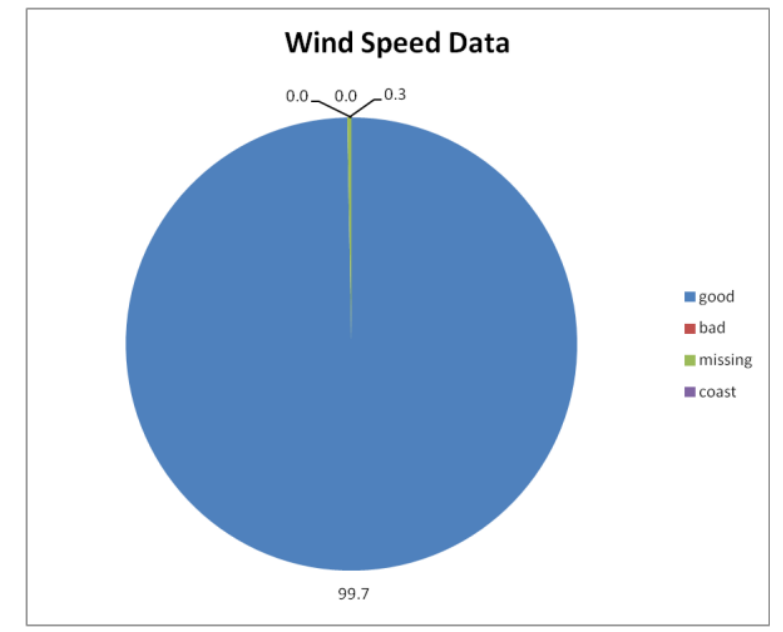

Figure L-355 
September 2011 Site 1

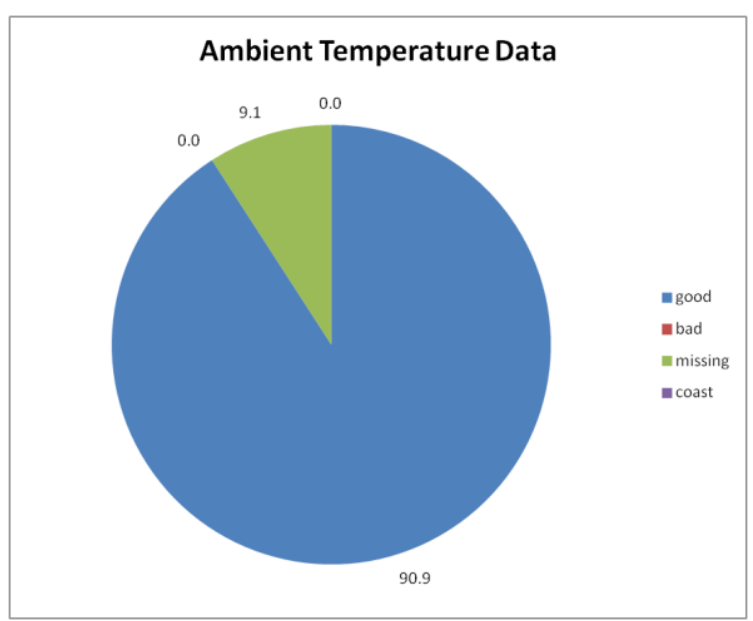

Figure L-356

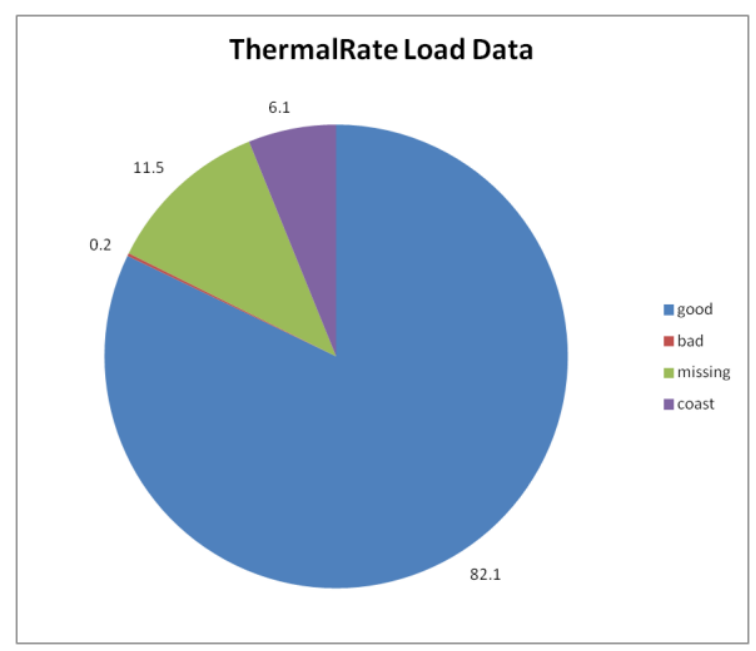

Figure L-358

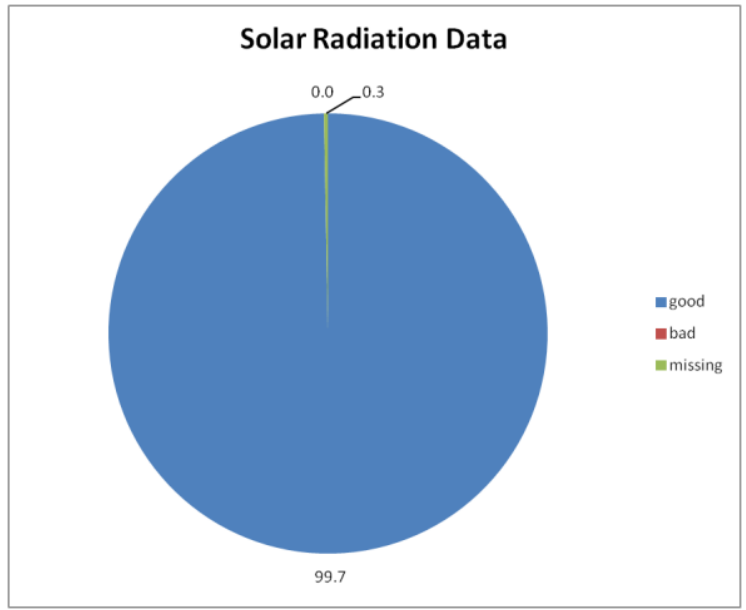

Figure L-357

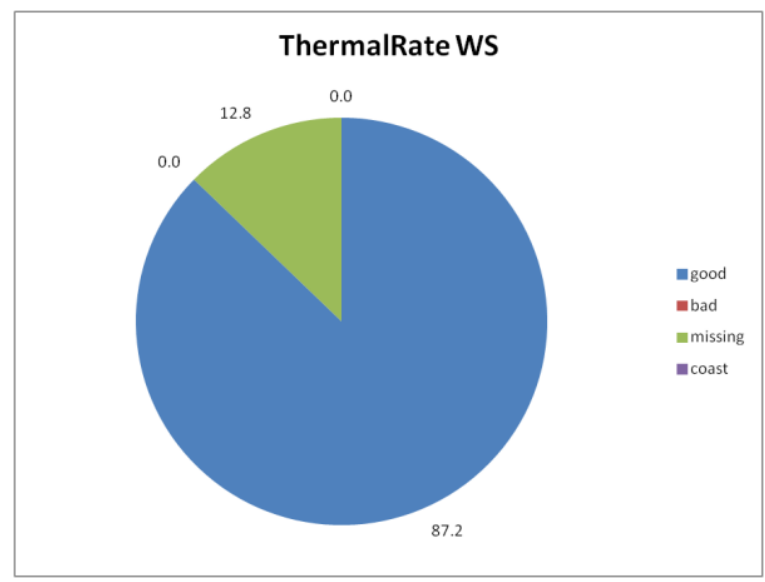

Figure L-359 


\section{September 2011 Site 1}

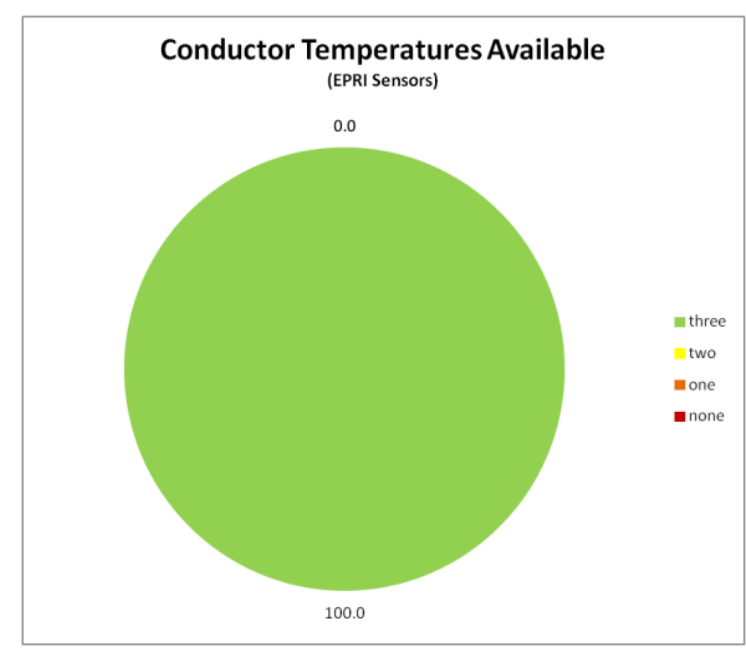

Figure L-360

\section{October 2011 Site 1}

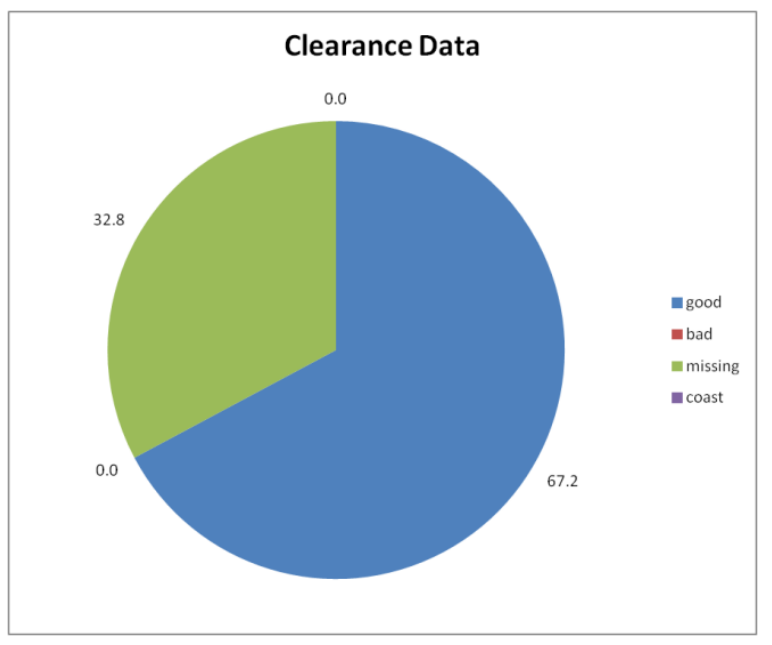

Figure L-361

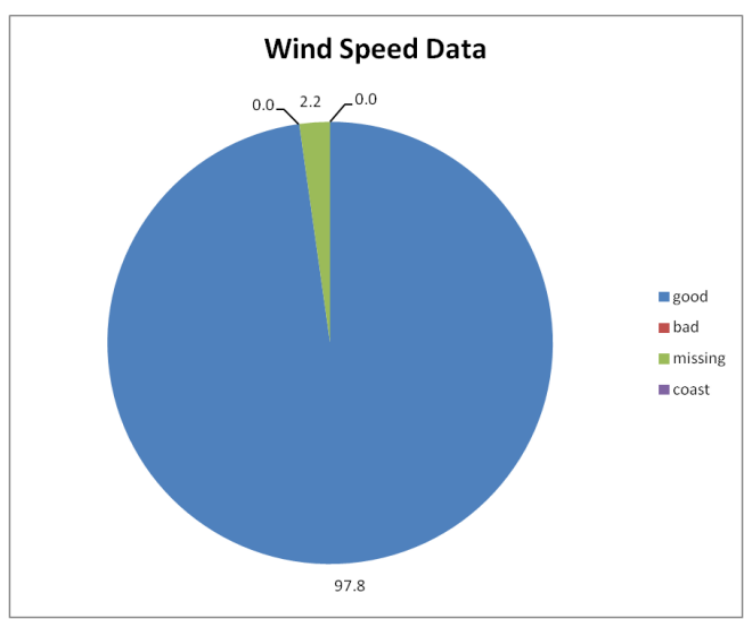

Figure L-362 


\section{October 2011 Site 1}

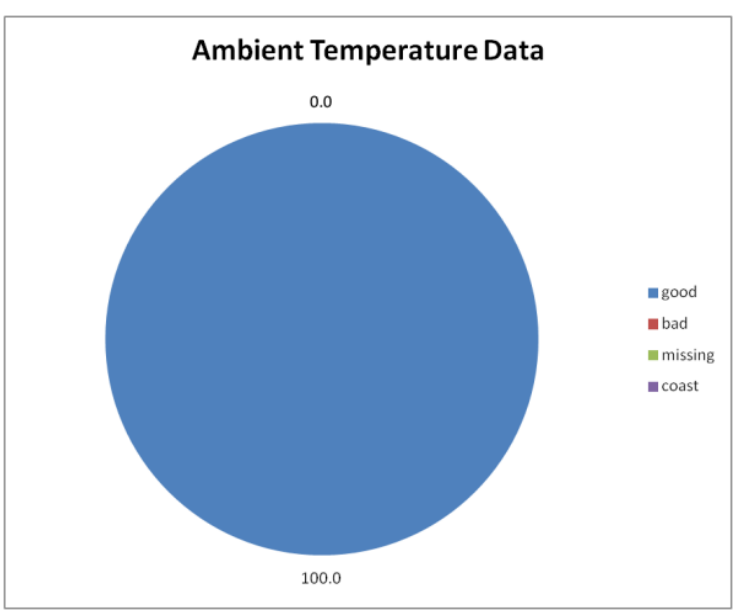

Figure L-363

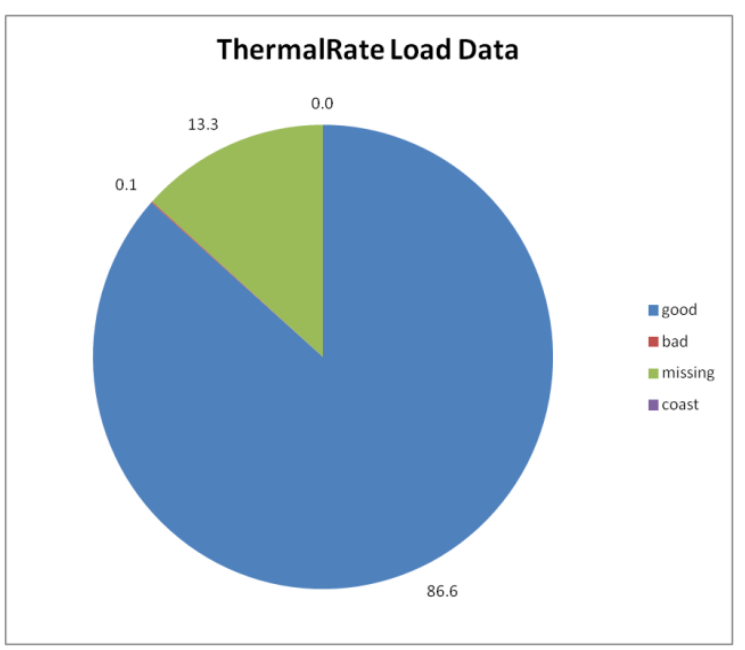

Figure L-365

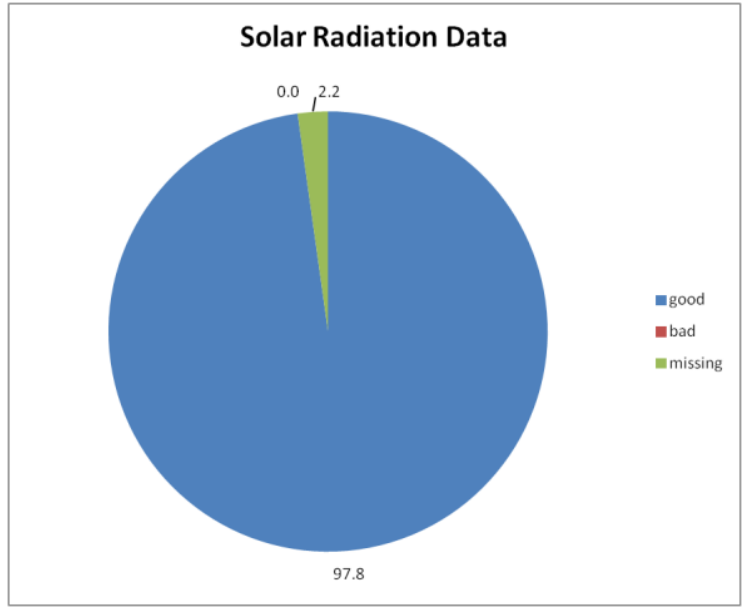

Figure L-364

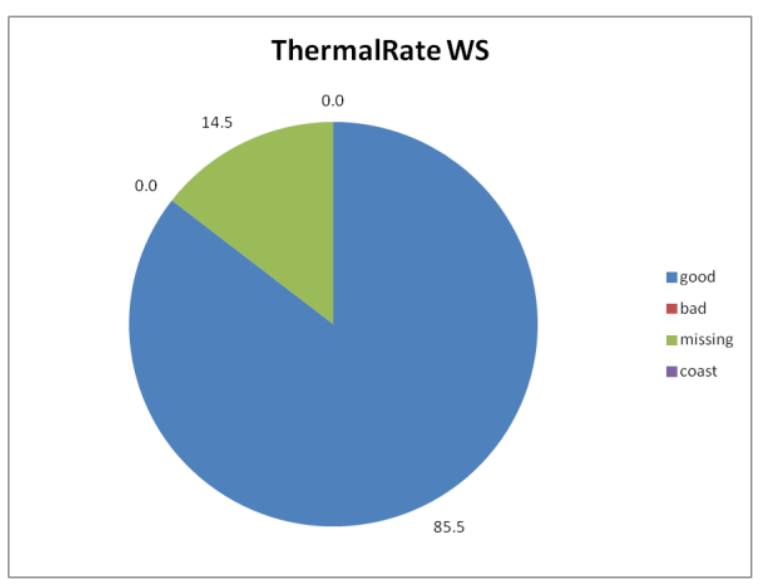

Figure L-366 


\section{October 2011 Site 1}

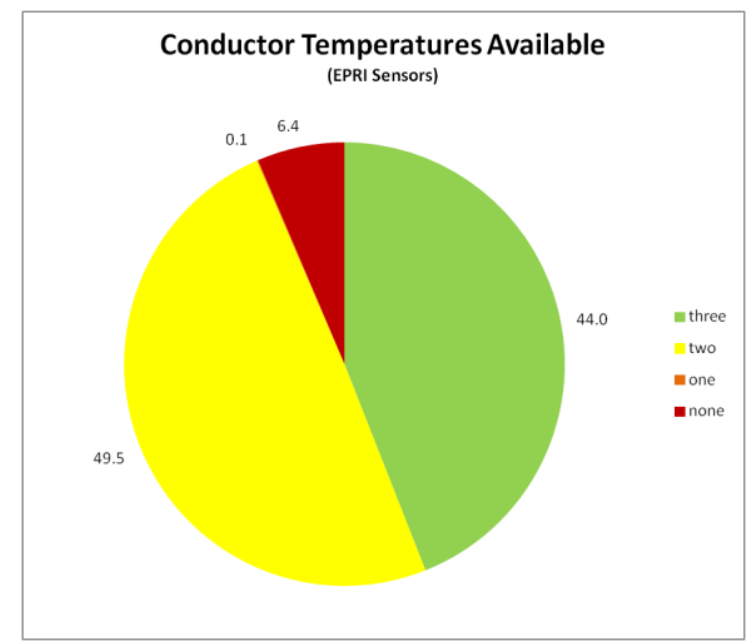

Figure L-367

\section{November 2011 Site 1}

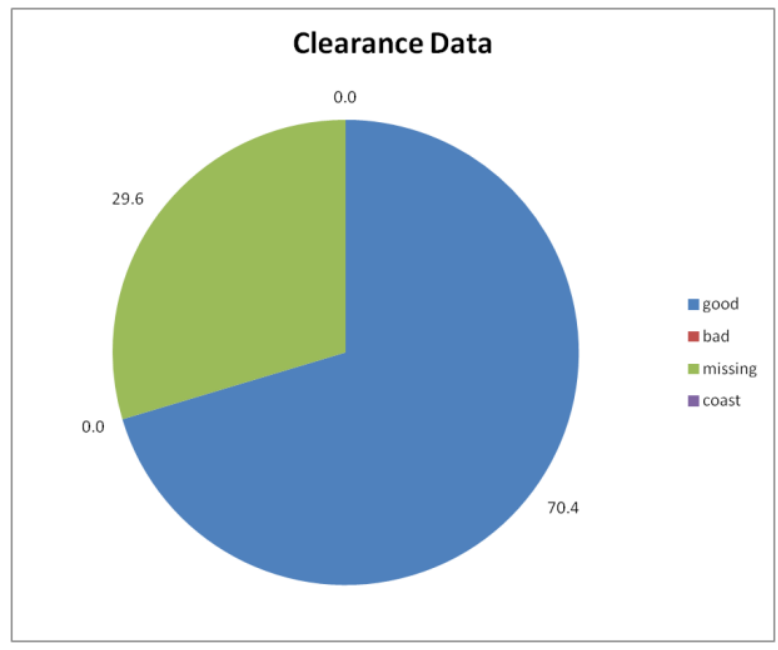

Figure L-368

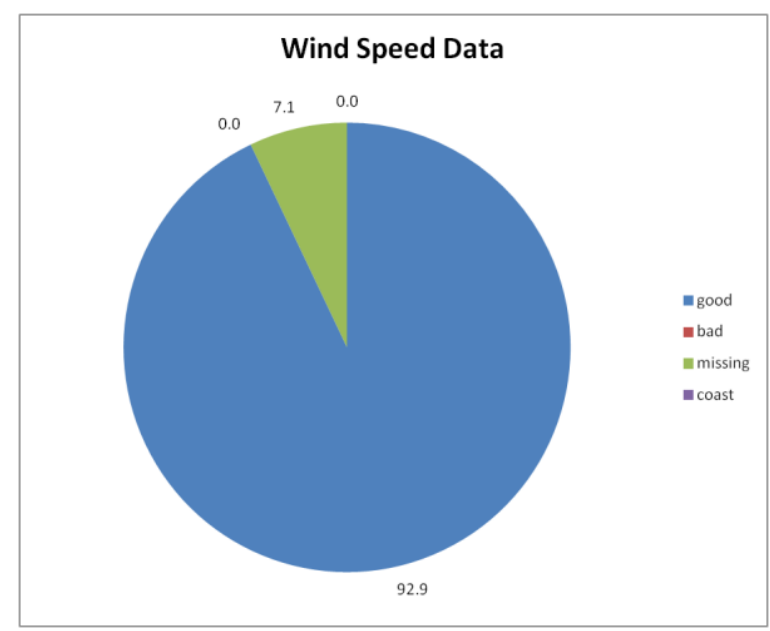

Figure L-369 
November 2011 Site 1

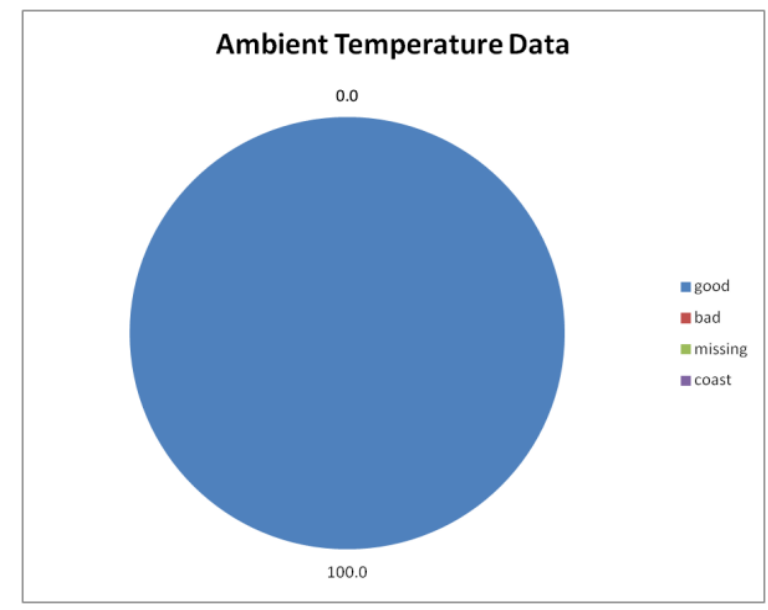

Figure L-370

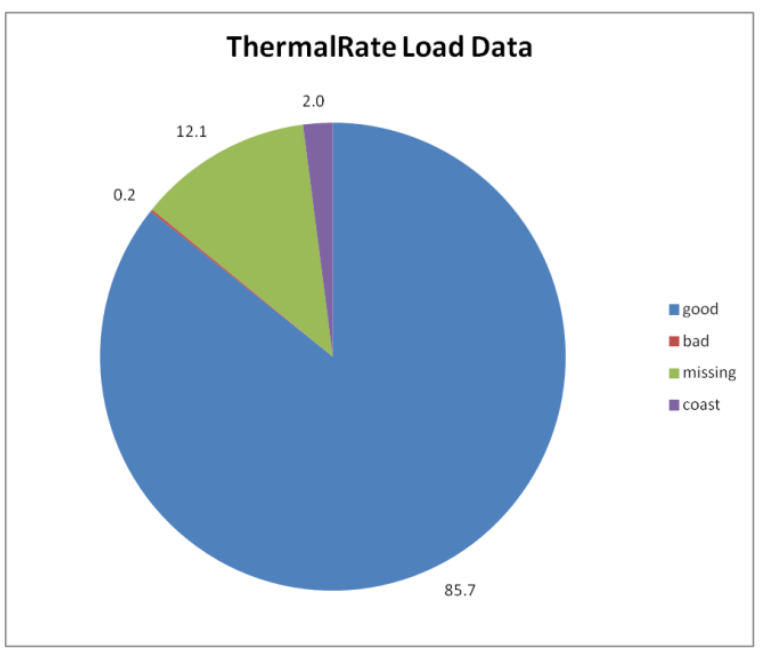

Figure L-372

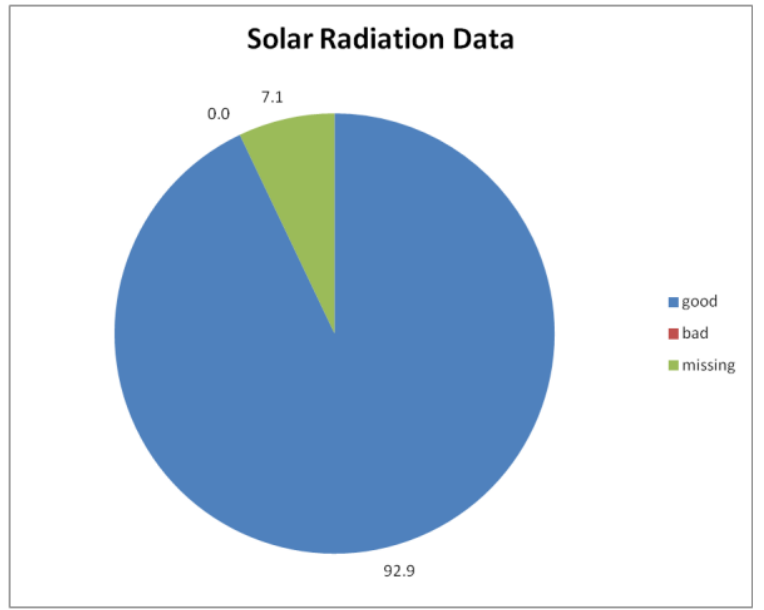

Figure L-371

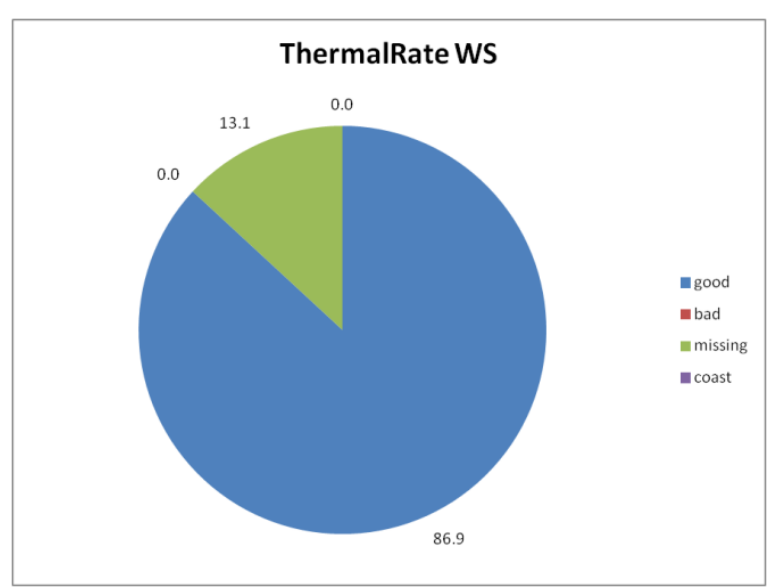

Figure L-373 
November 2011 Site 1

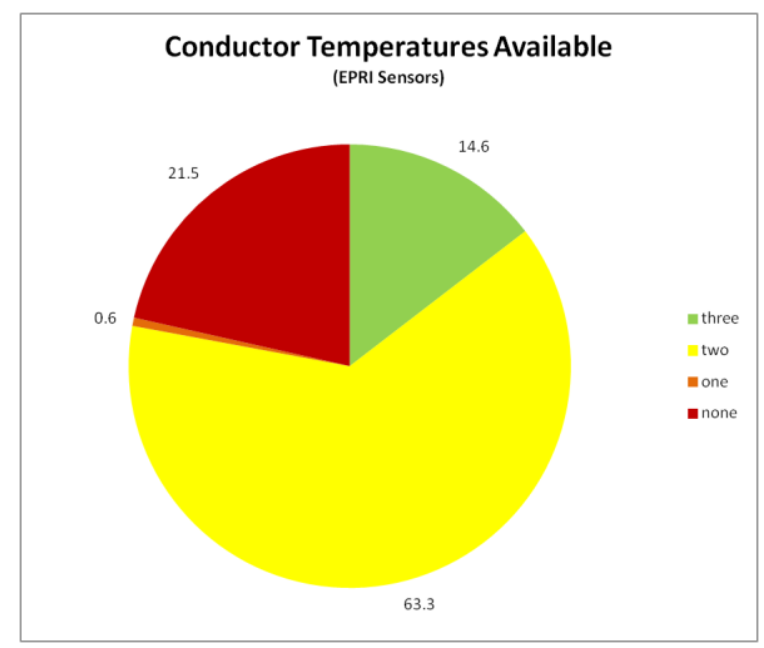

Figure L-374

\section{December 2011 Site 1}

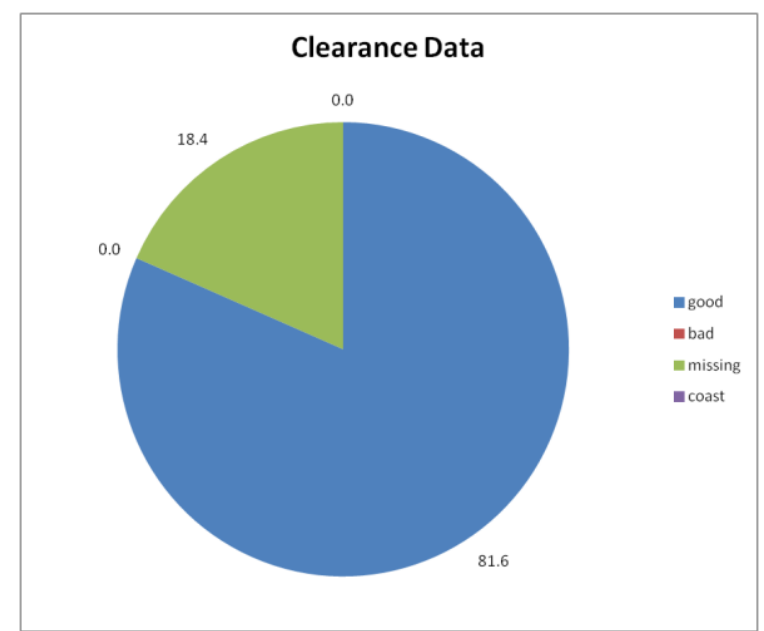

Figure L-375

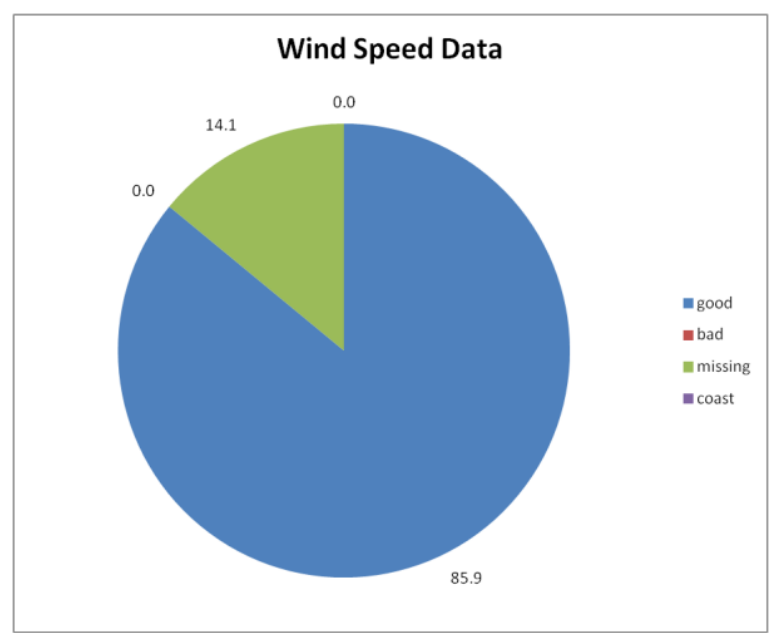

Figure L-376 


\section{December 2011 Site 1}

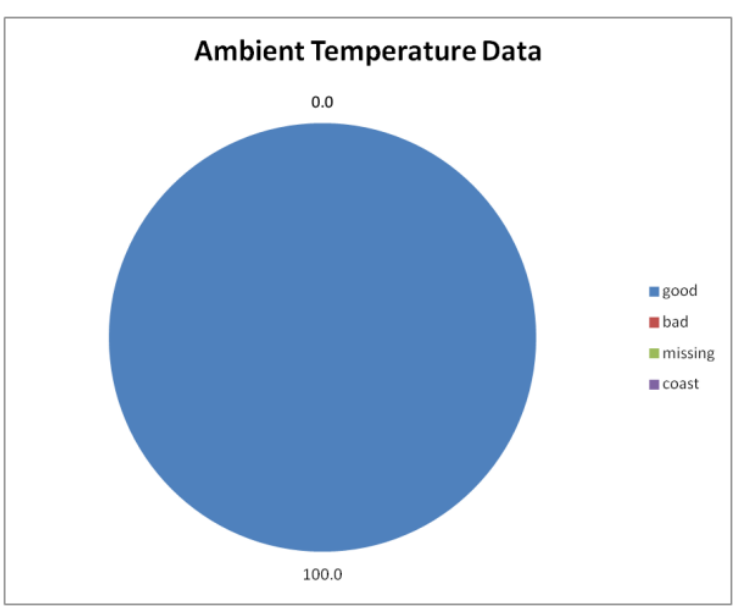

Figure L-377

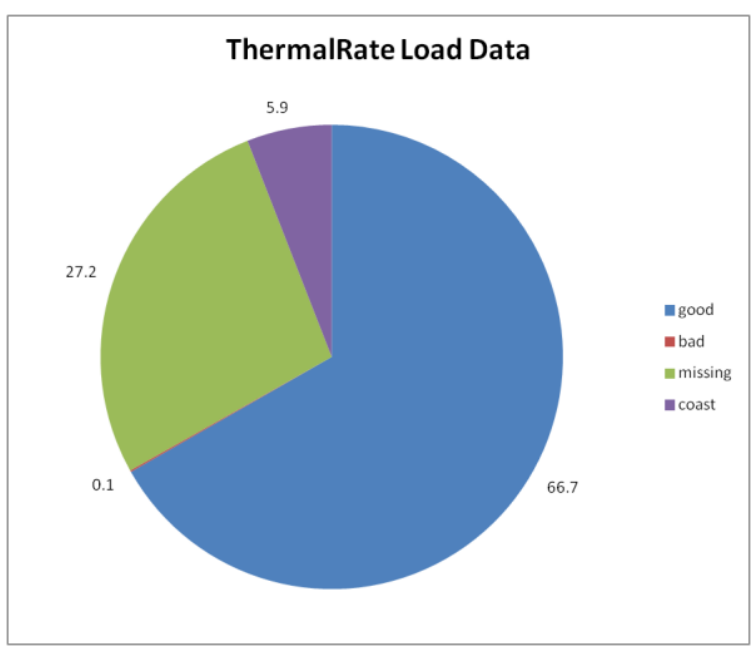

Figure L-379

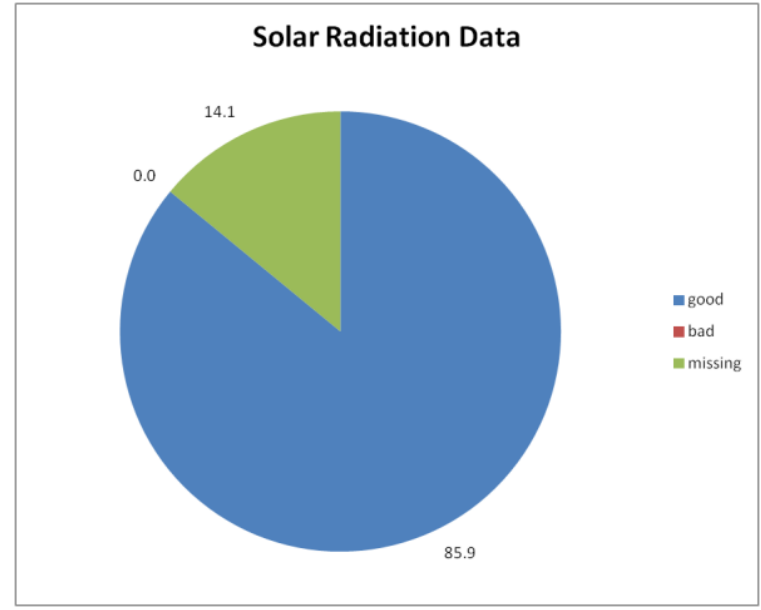

Figure L-378

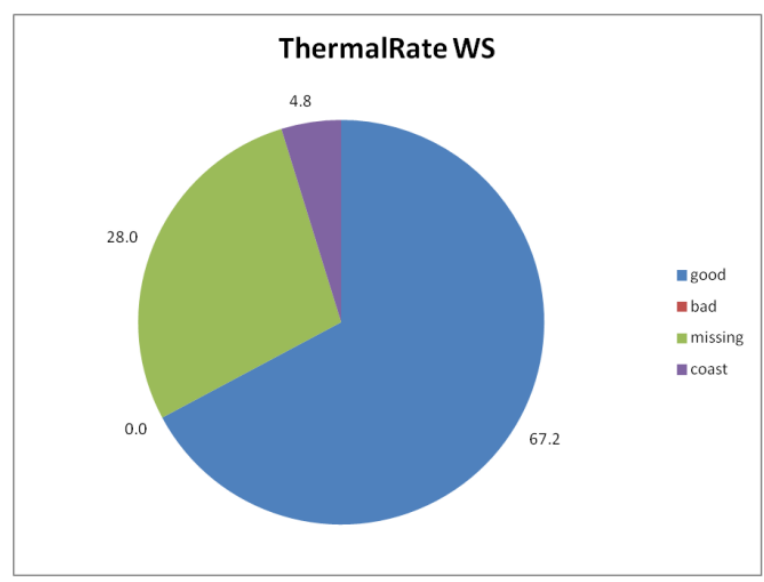

Figure L-380 


\section{December 2011 Site 1}

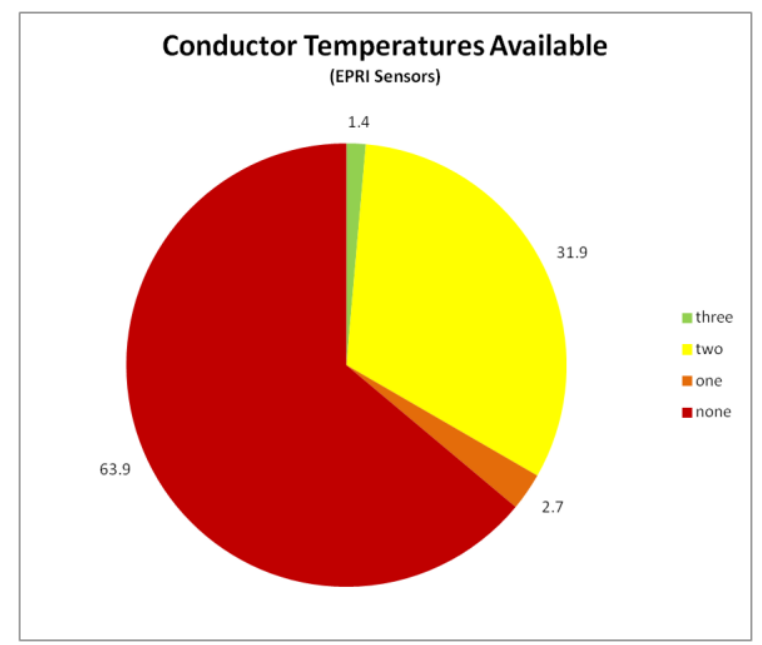

Figure L-381

\section{January 2012 Site 1}

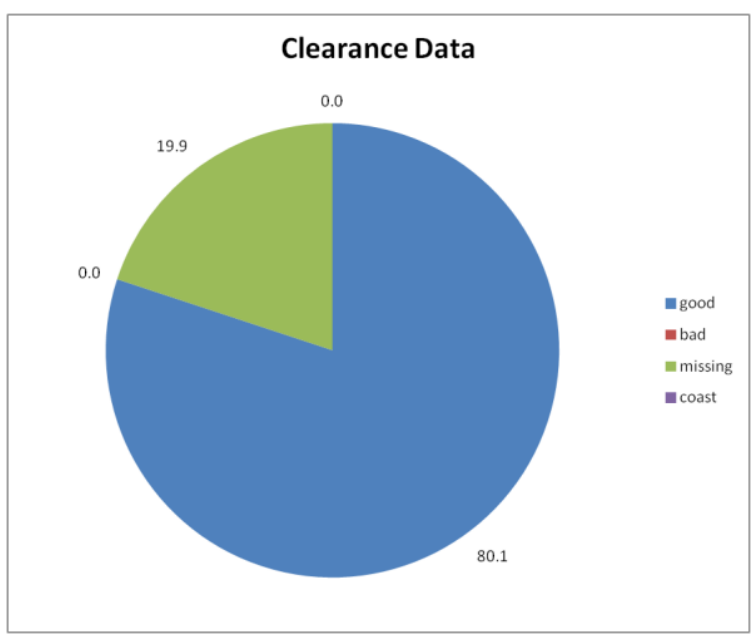

Figure L-382

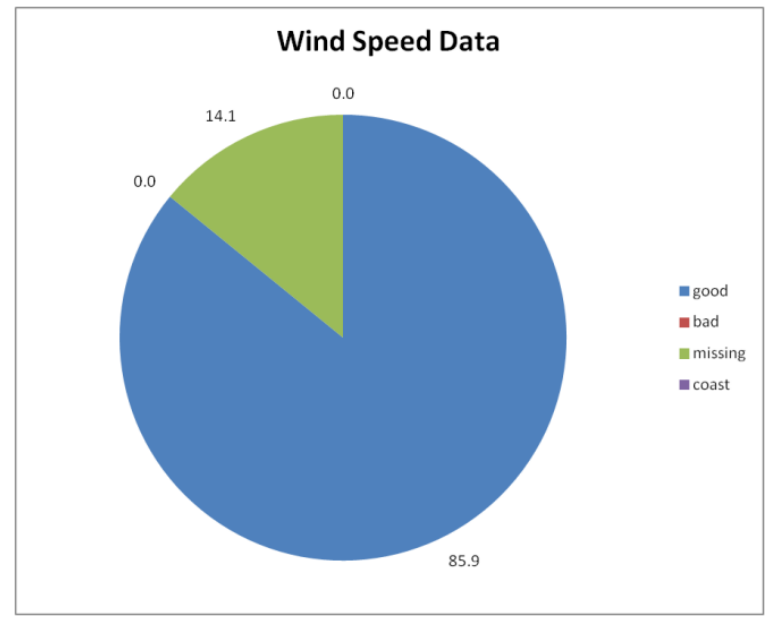

Figure L-383 


\section{January 2012 Site 1}

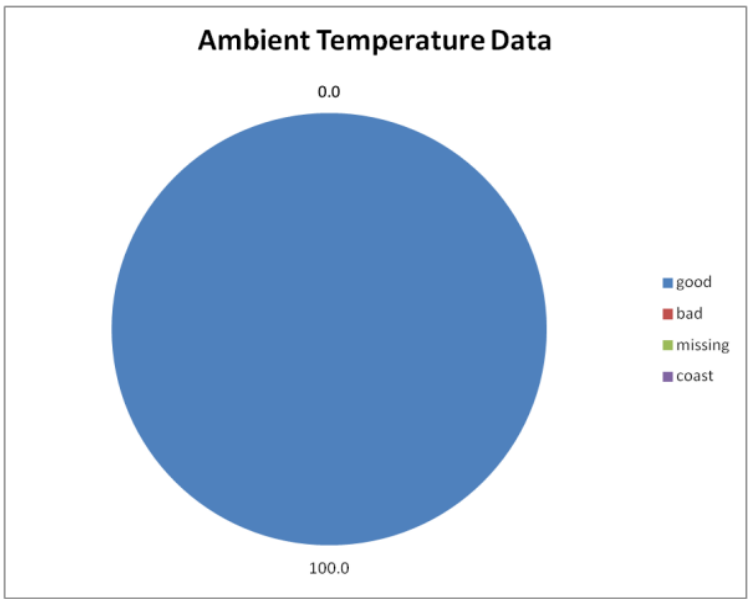

Figure L-384

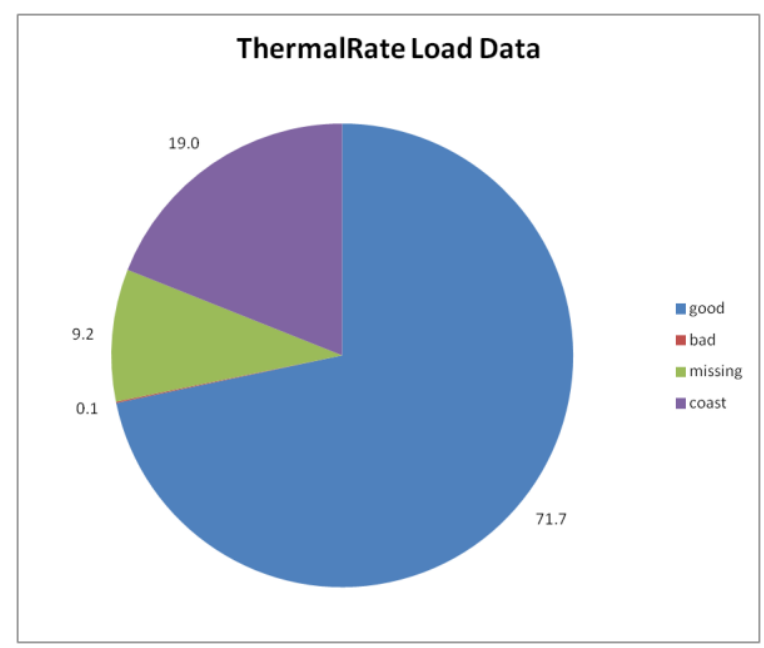

Figure L-386

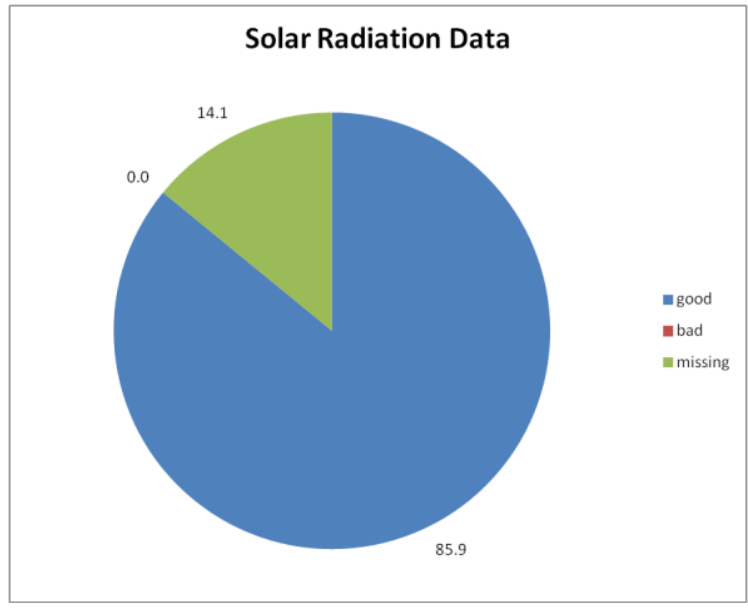

Figure L-385

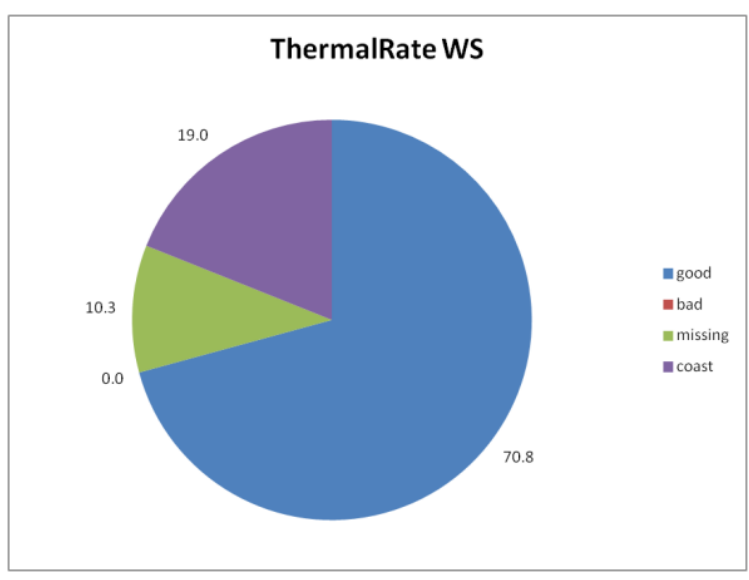

Figure L-387 


\section{January 2012 Site 1}

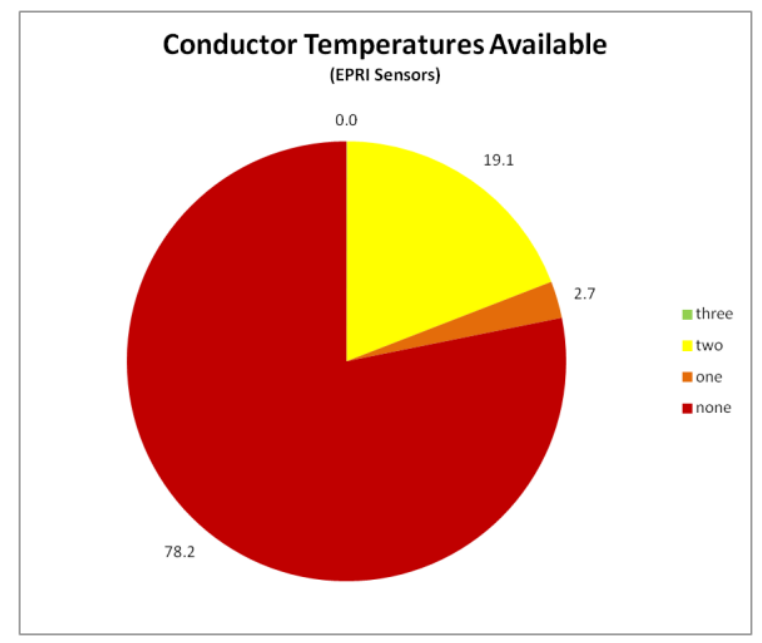

Figure L-388

\section{February 2012 Site 1}

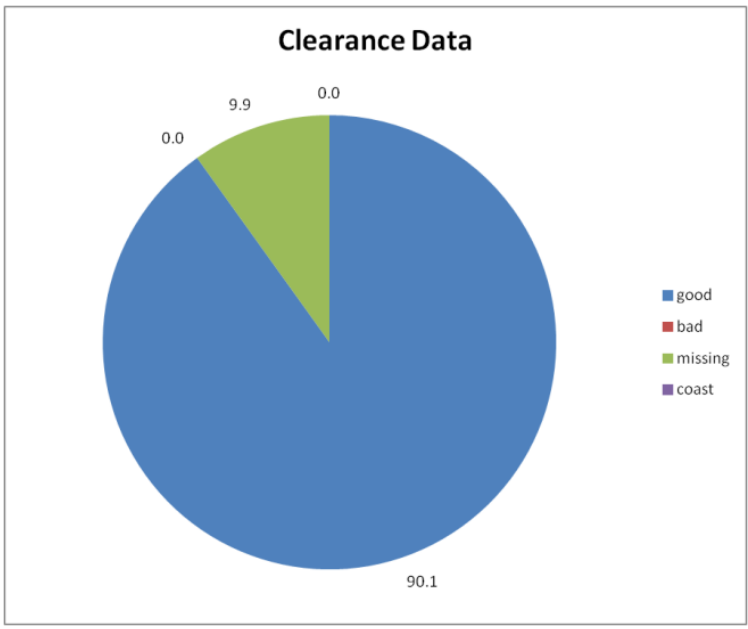

Figure L-389

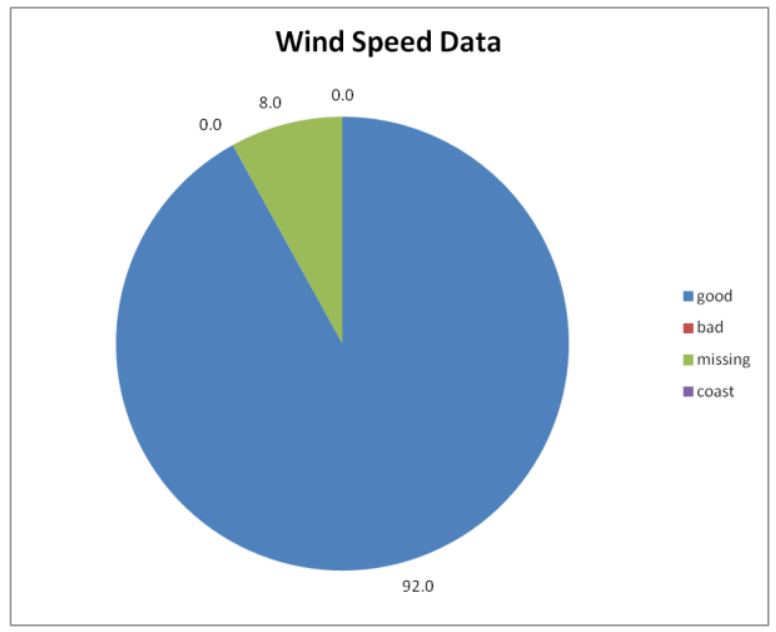

Figure L-390 
February 2012 Site 1

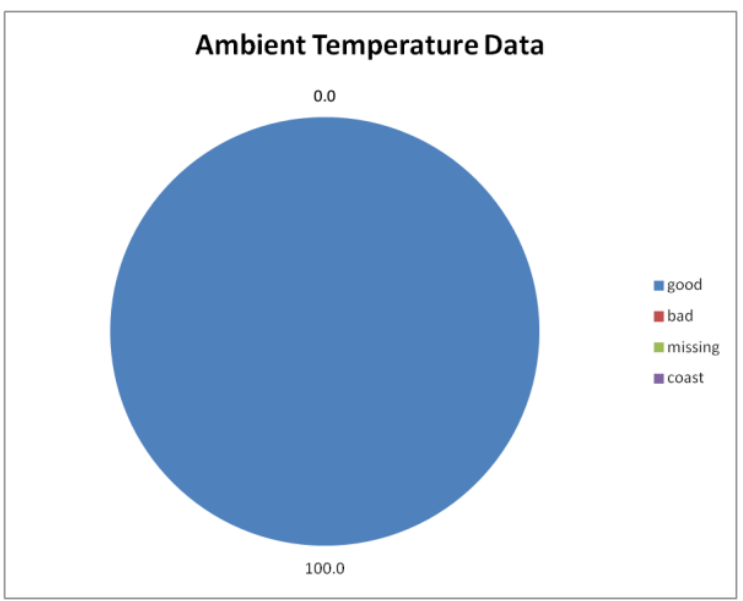

Figure L-391

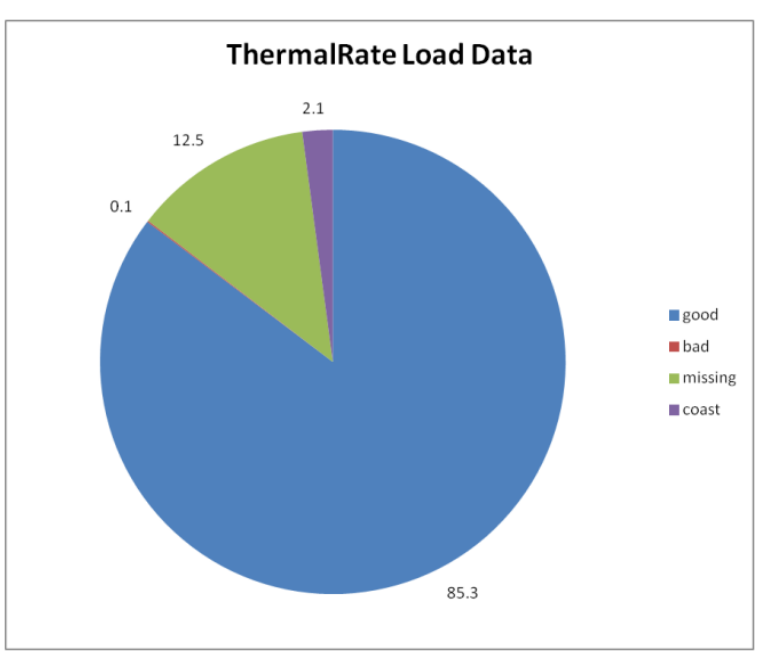

Figure L-393

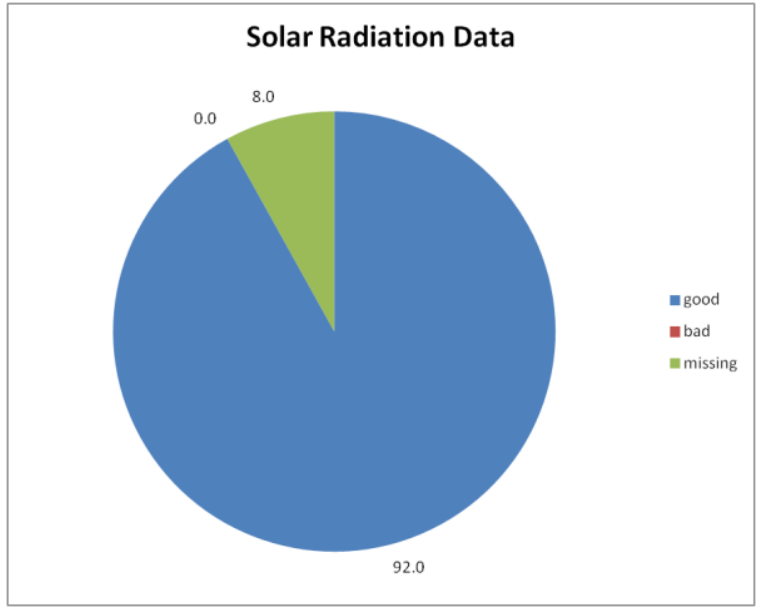

Figure L-392

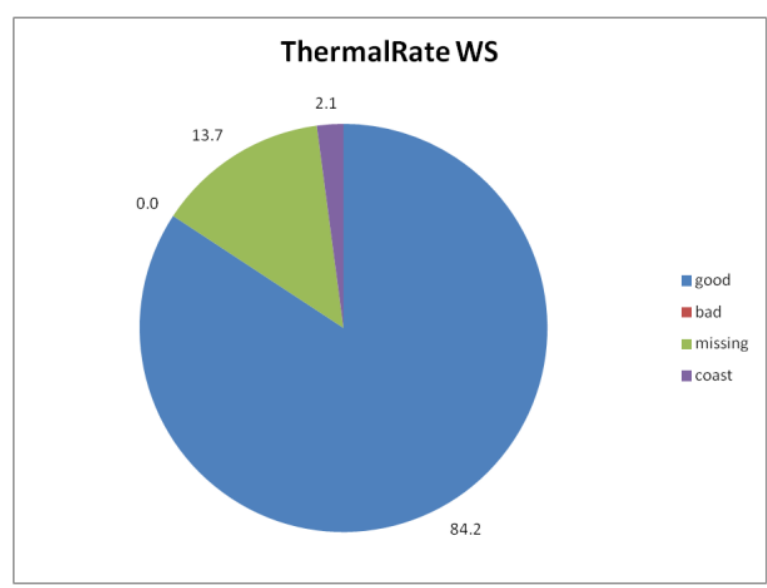

Figure L-394 


\section{February 2012 Site 1}

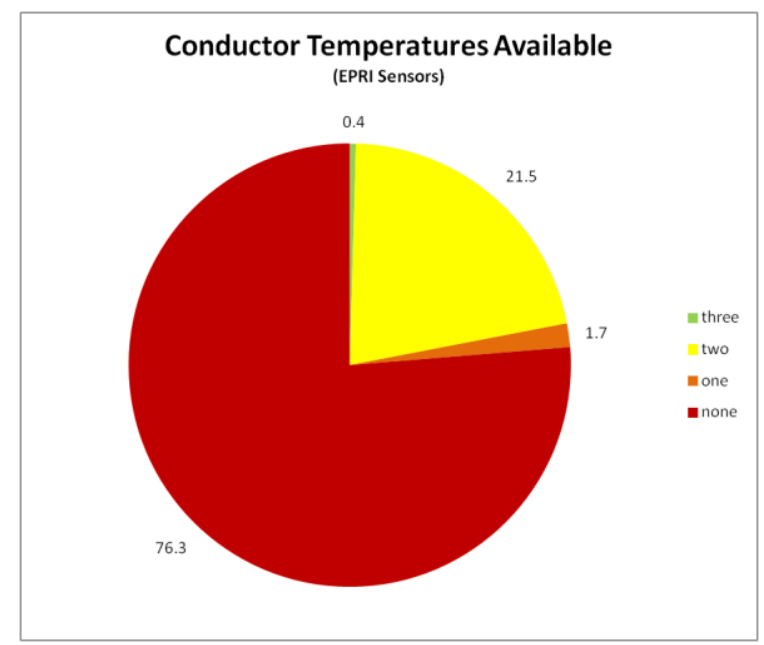

Figure L-395

\section{March 2012 Site 1}

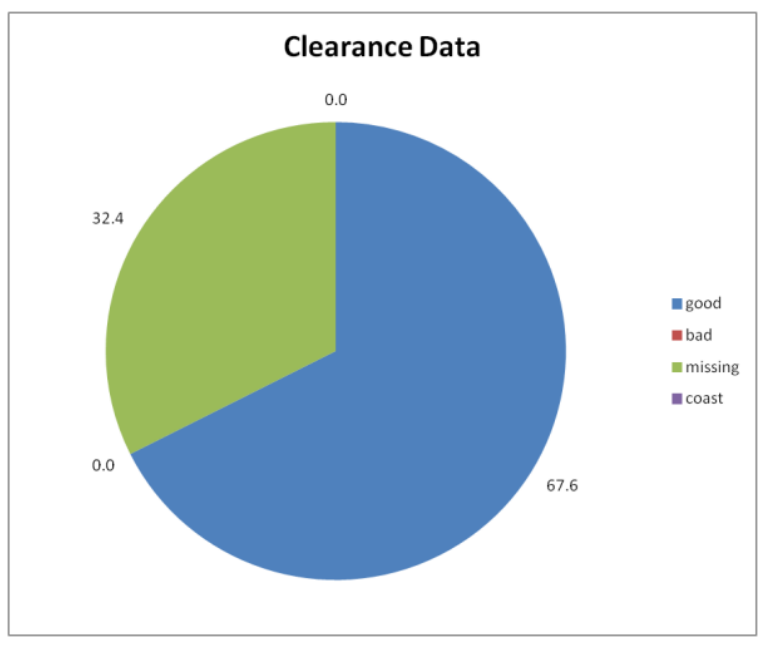

Figure L-396

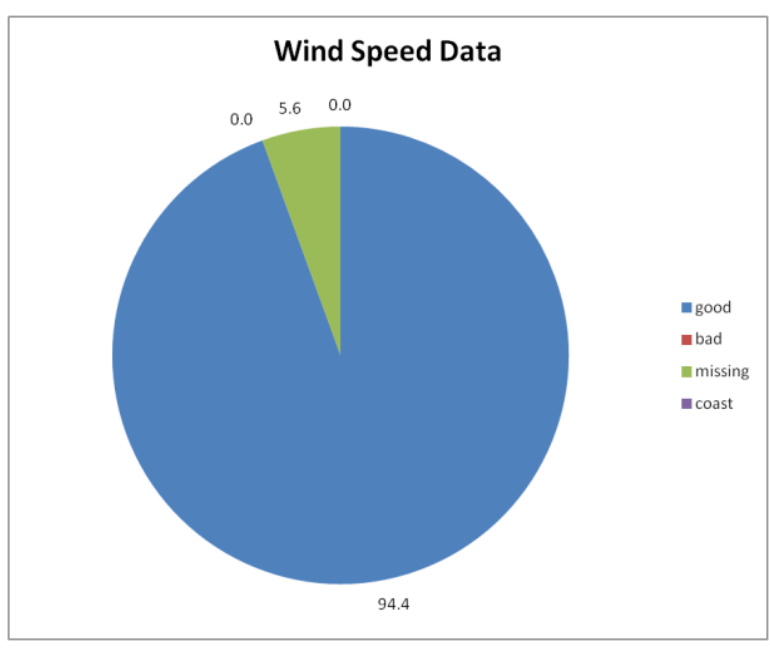

Figure L-397 
March 2012 Site 1

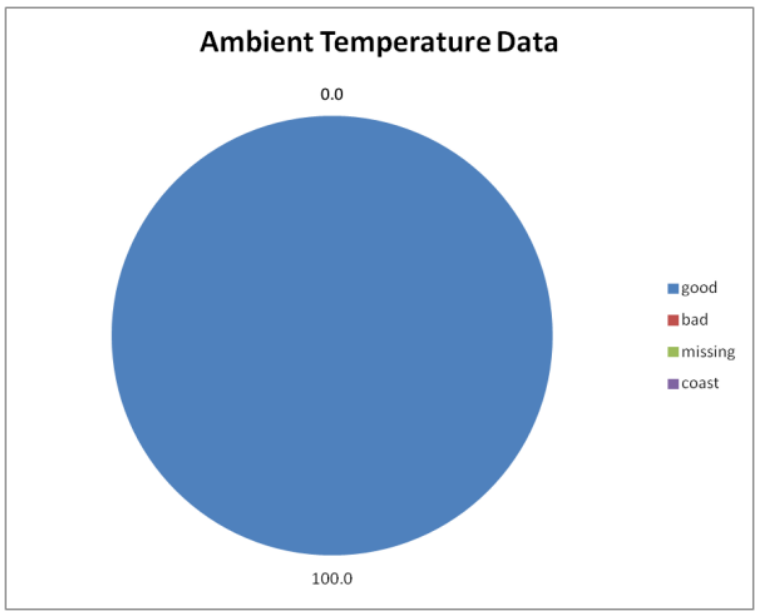

Figure L-398

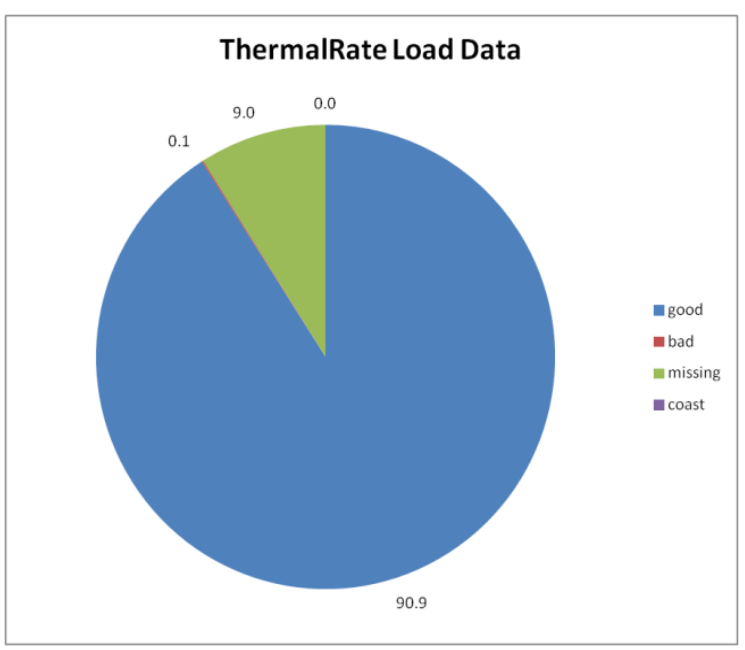

Figure L-400

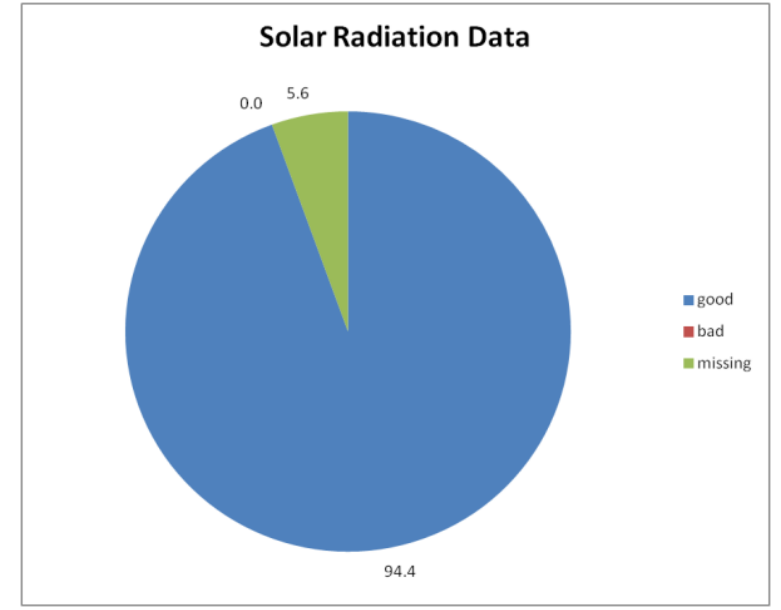

Figure L-399

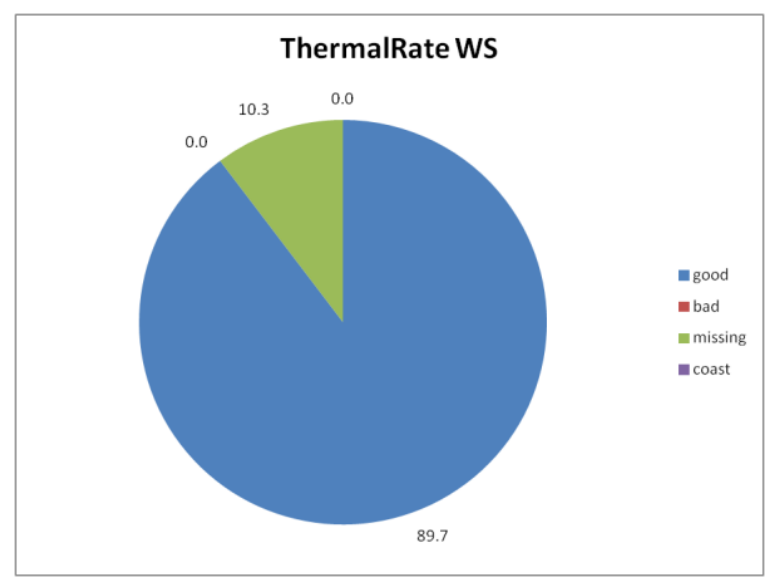

Figure L-401 


\section{March 2012 Site 1}

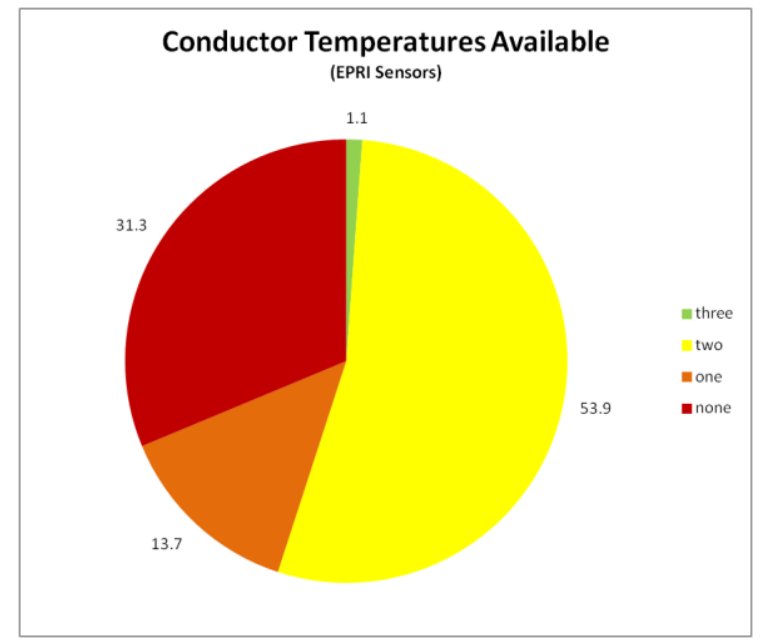

Figure L-402

\section{April 2012 Site 1}

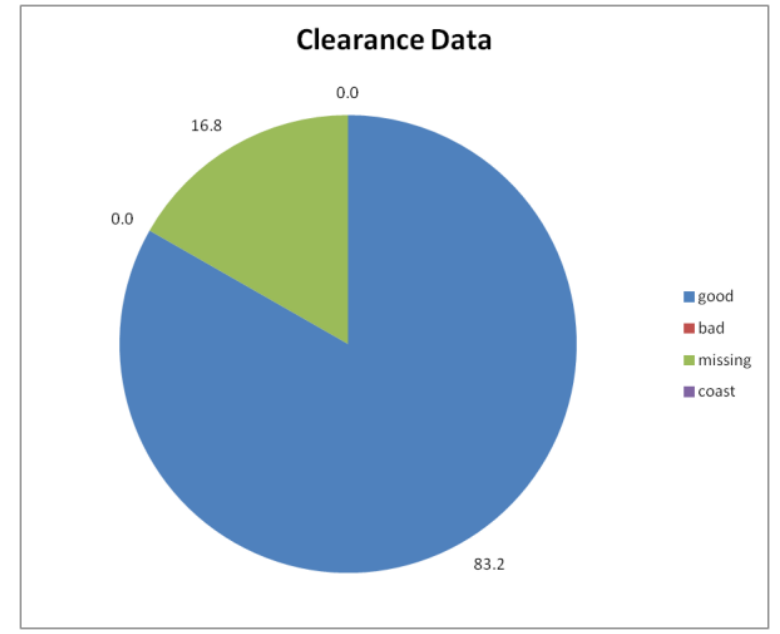

Figure L-403

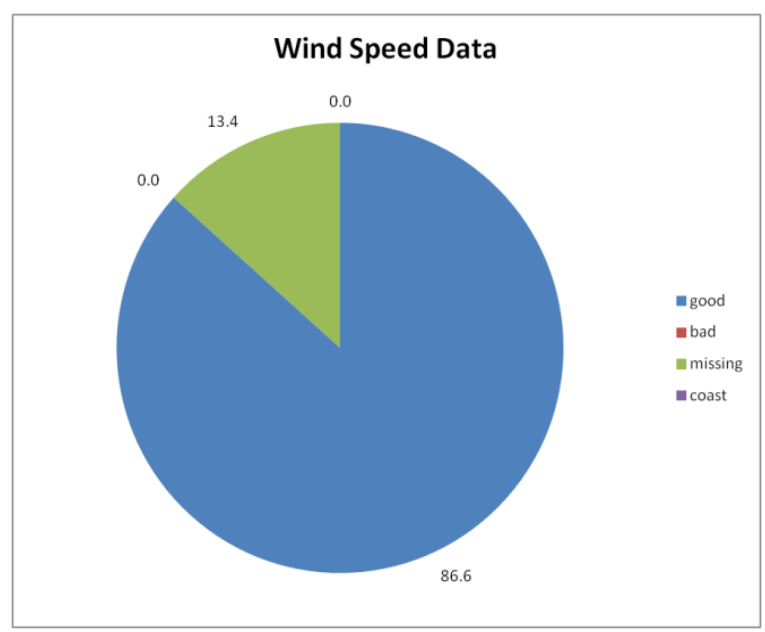

Figure L-404 


\section{April 2012 Site 1}

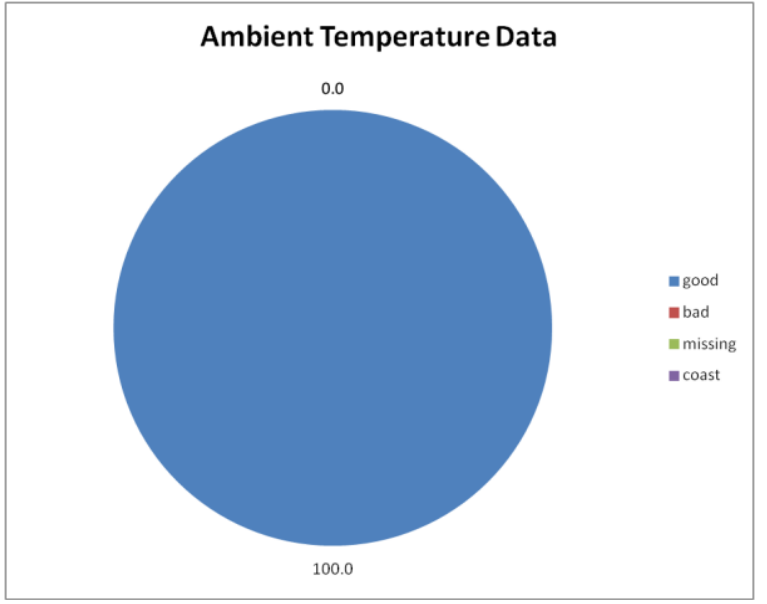

Figure L-405

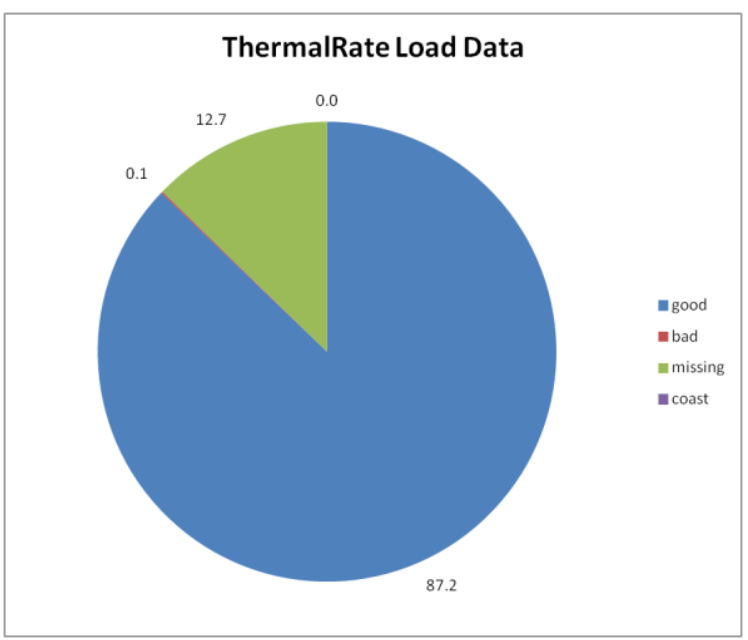

Figure L-407

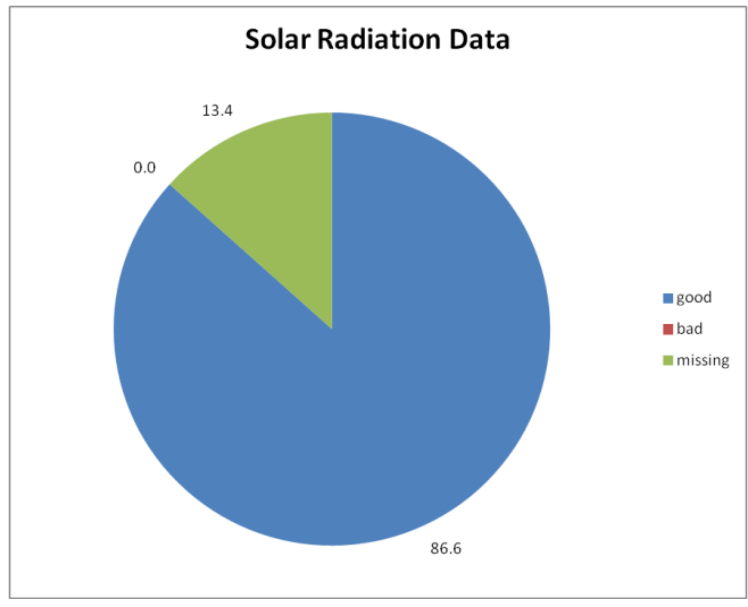

Figure L-406

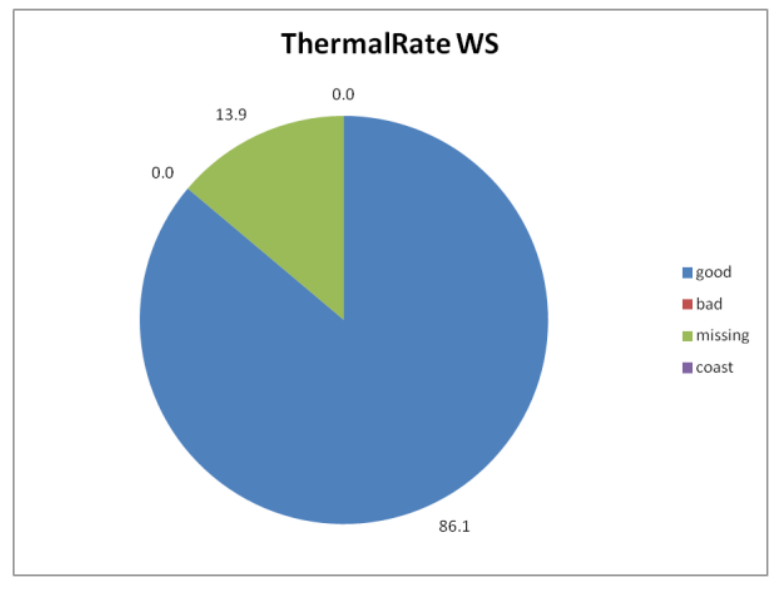

Figure L-408 


\section{April 2012 Site 1}

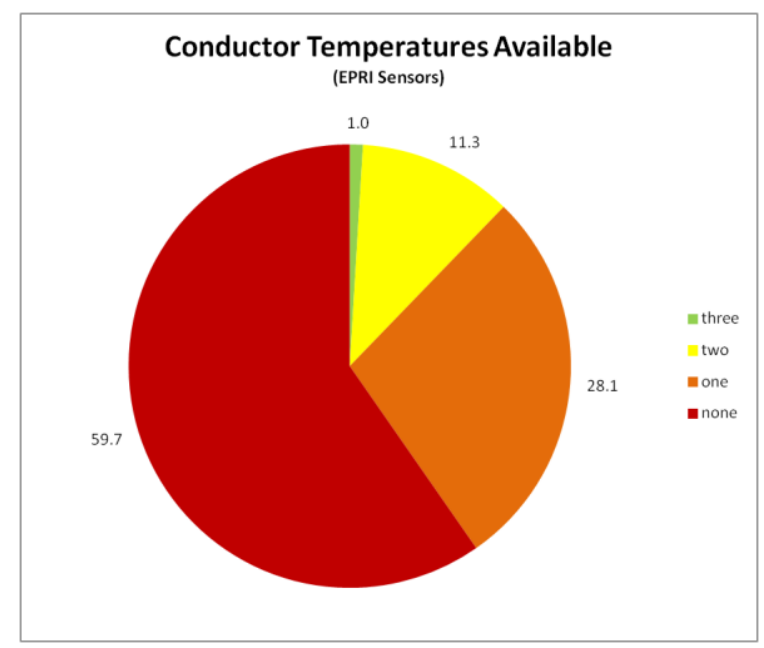

Figure L-409

\section{May 2012 Site 1}

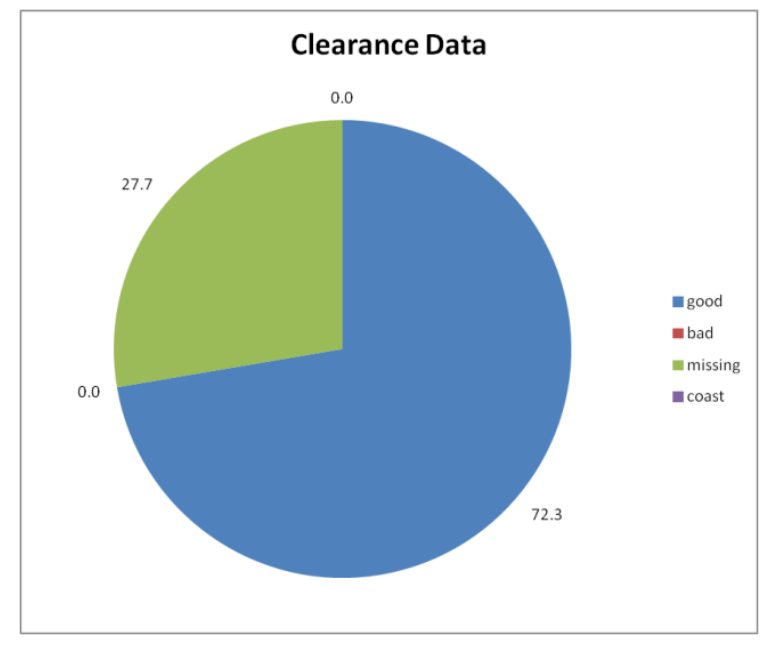

Figure L-410

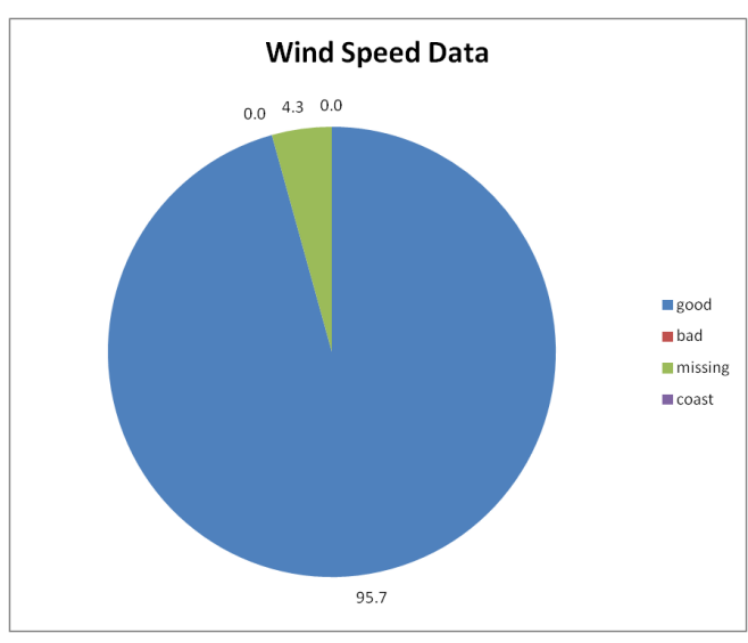

Figure L-411 
May 2012 Site 1

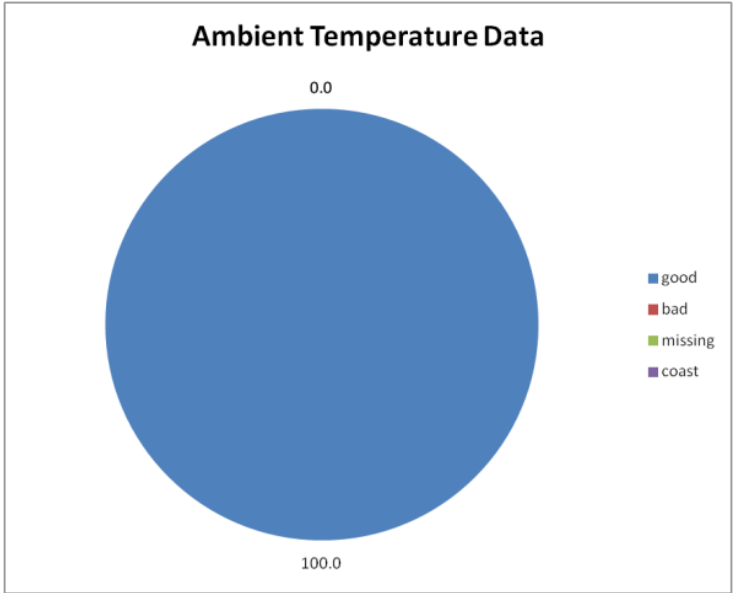

Figure L-412

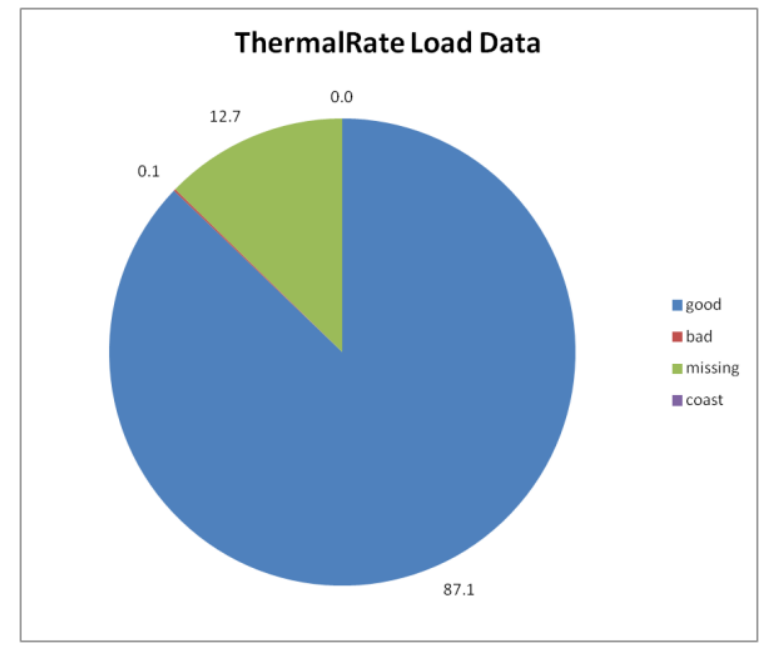

Figure L-414

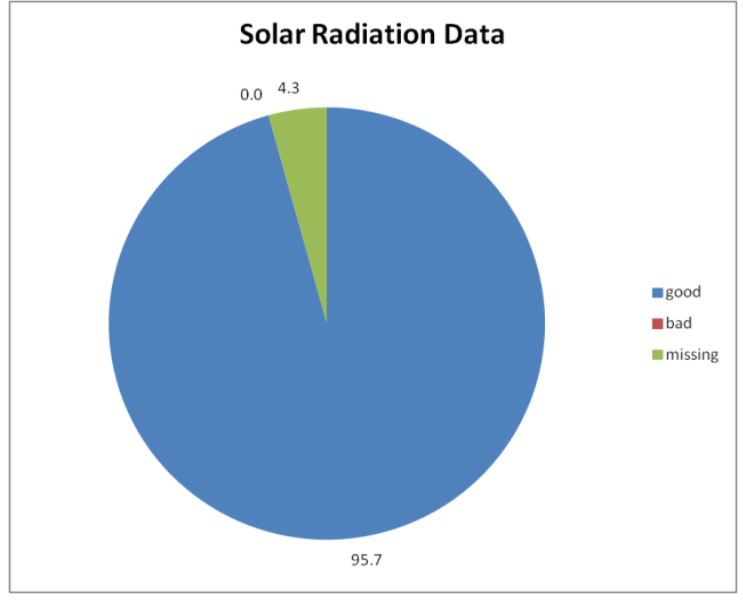

Figure L-413

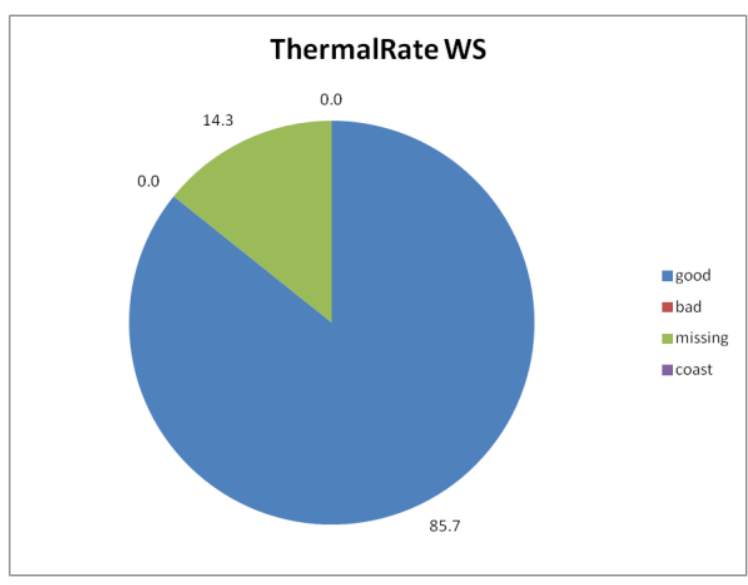

Figure L-415 


\section{May 2012 Site 1}

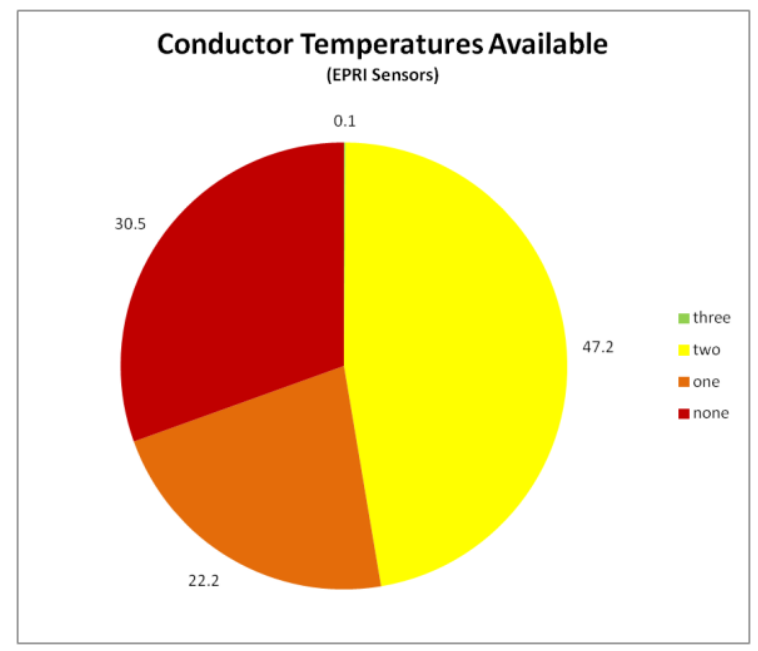

Figure L-416

\section{June 2012 Site 1}

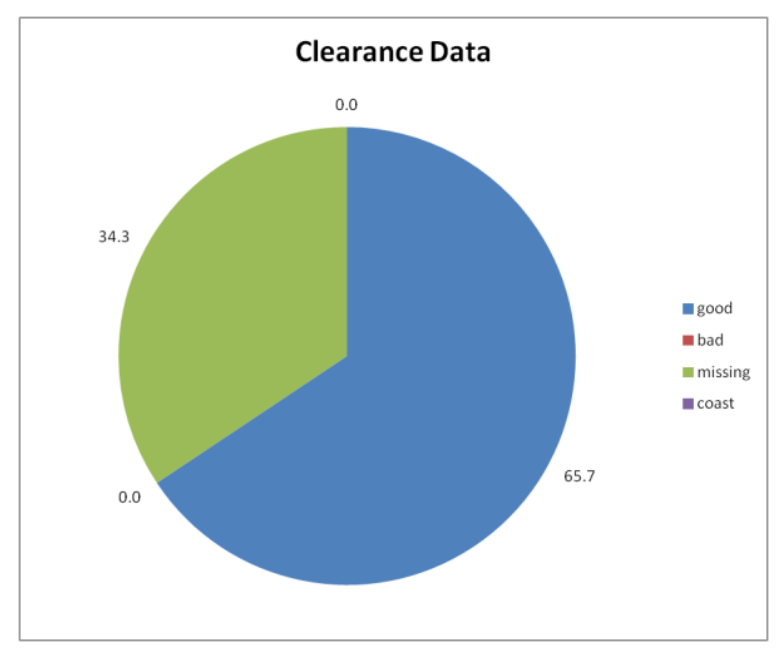

Figure L-417

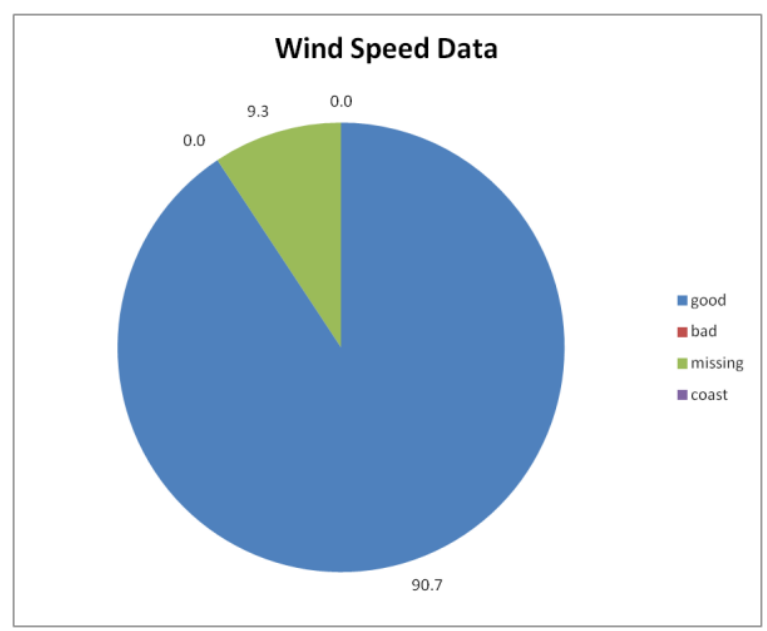

Figure L-418 


\section{June 2012 Site 1}

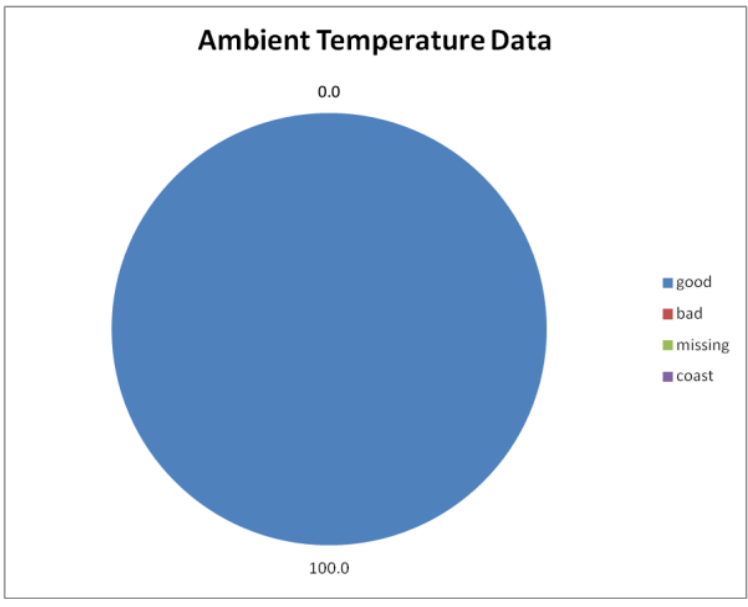

Figure L-419

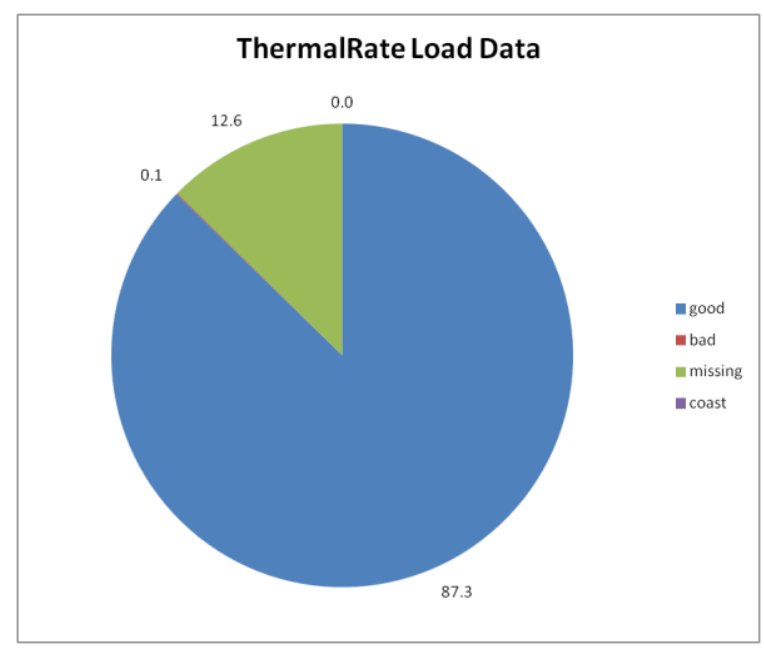

Figure L-421

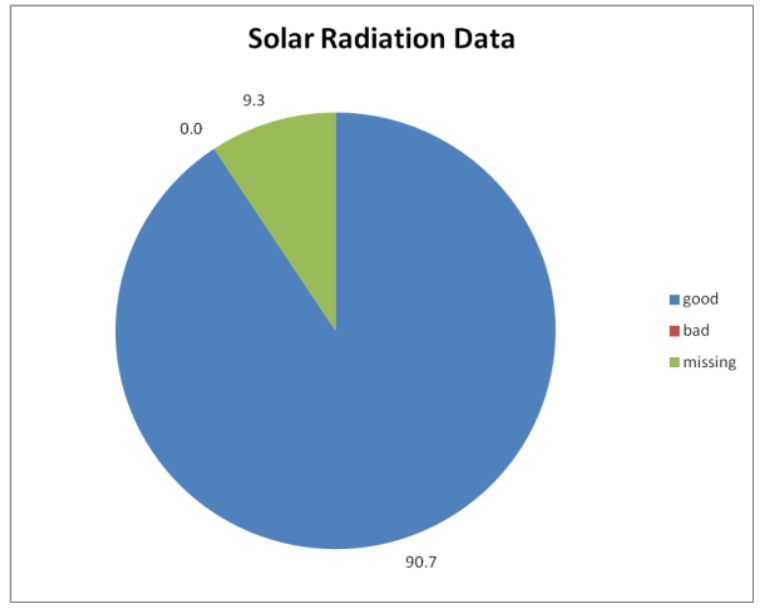

Figure L-420

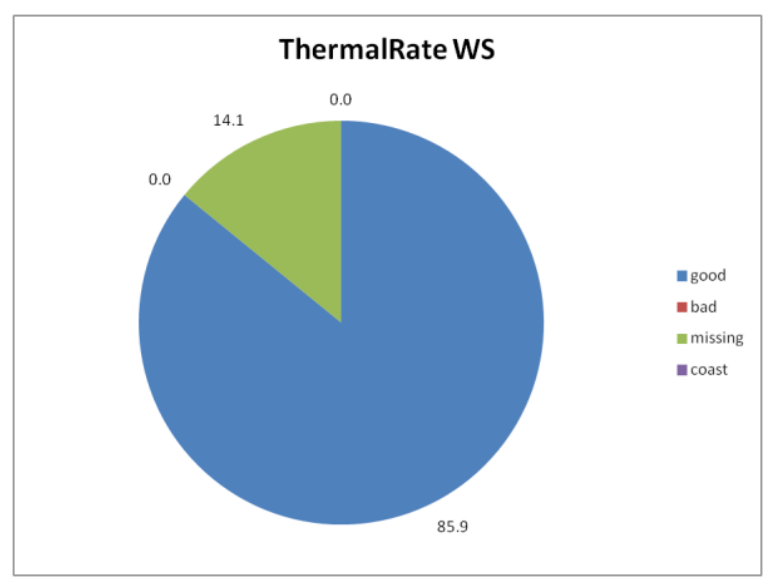

Figure L-422 


\section{June 2012 Site 1}

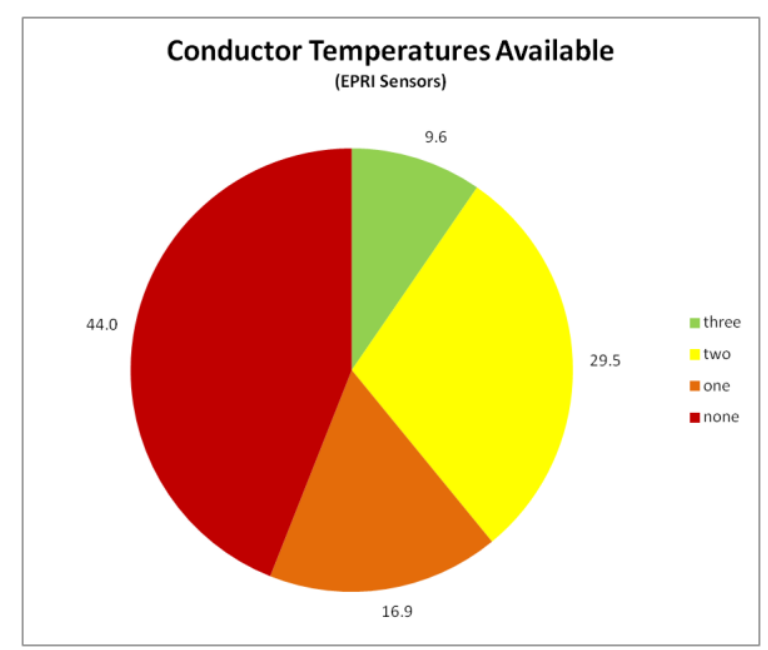

Figure L-423

\section{July 2012 Site 1}

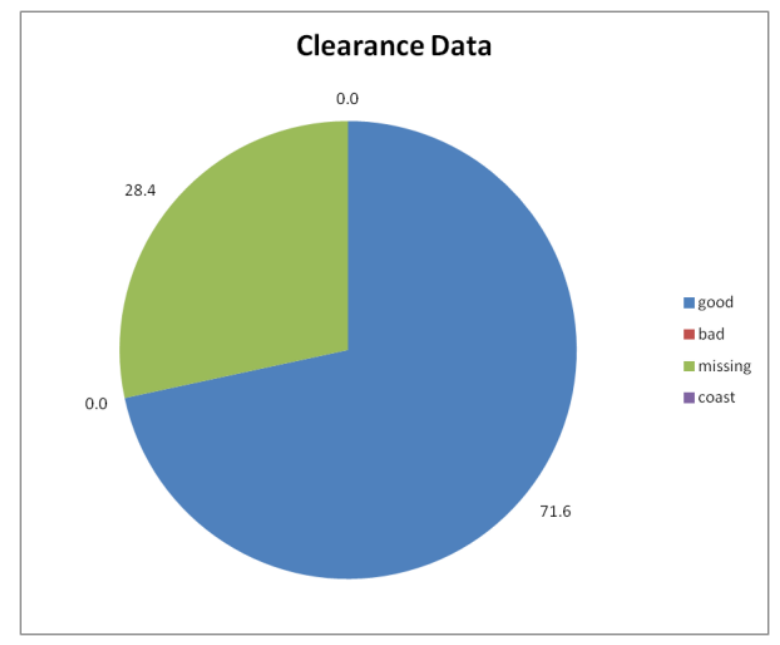

Figure L-424

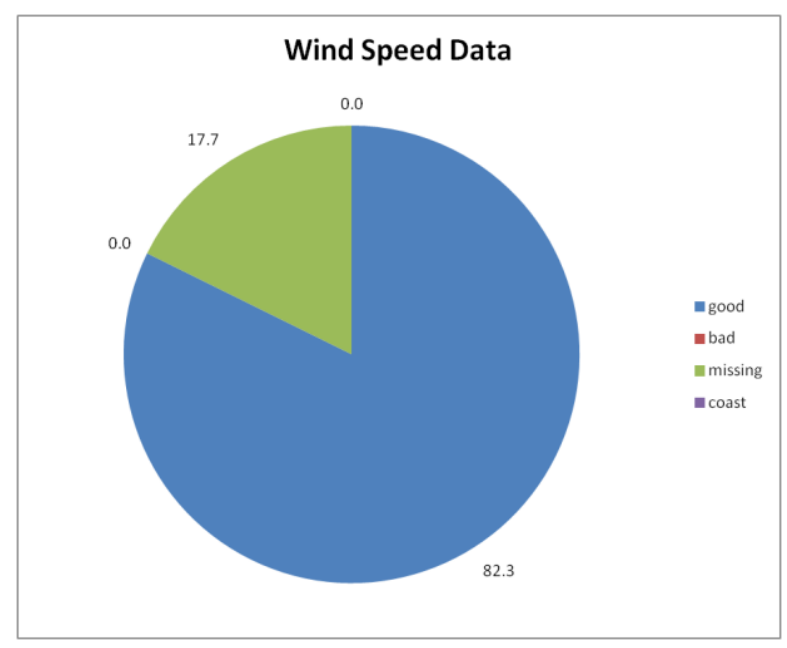

Figure L-425 


\section{July 2012 Site 1}

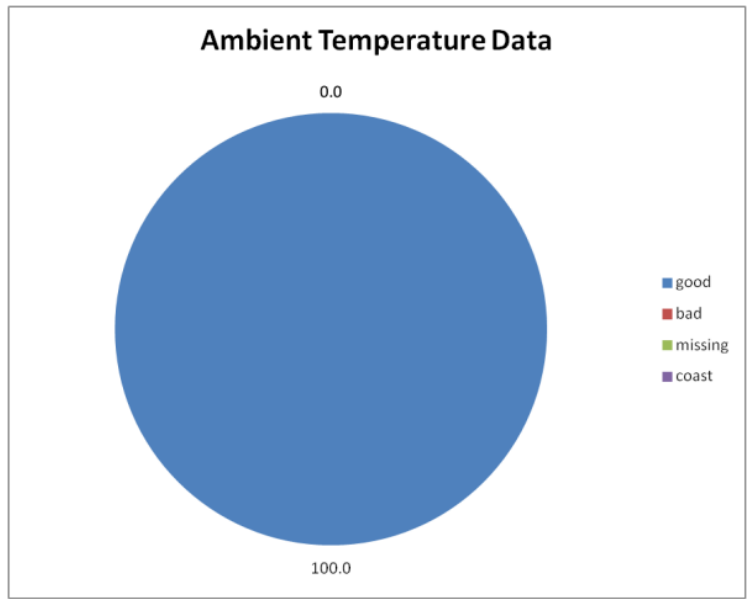

Figure L-426

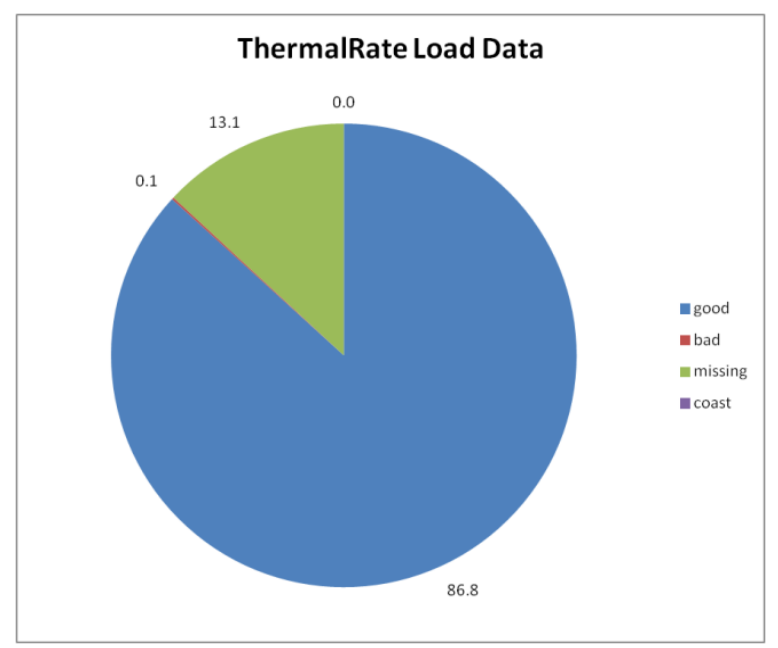

Figure L-428

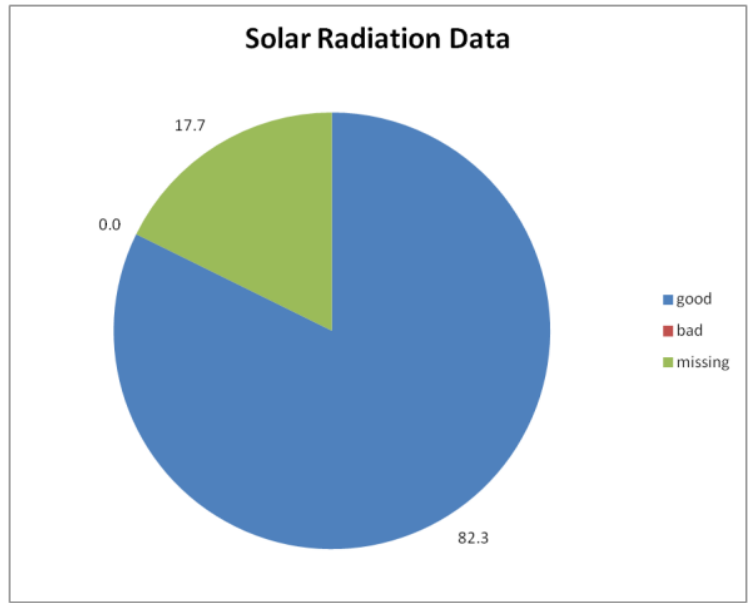

Figure L-427

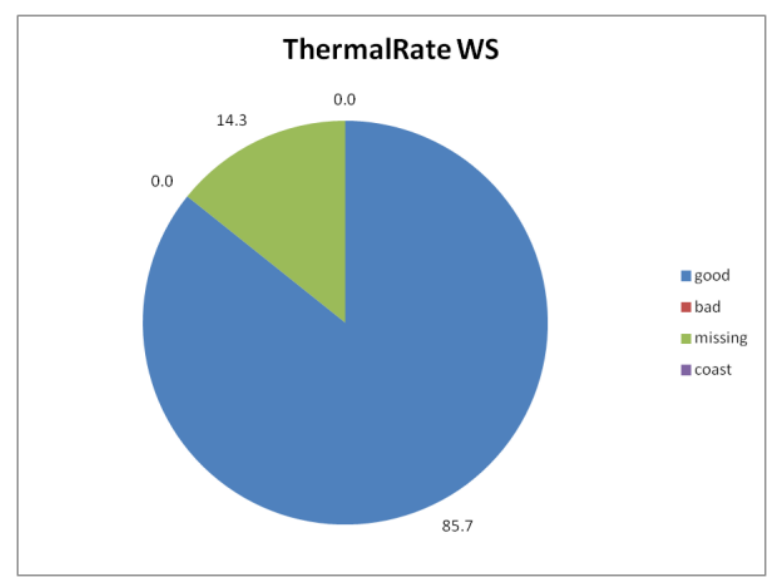

Figure L-429 


\section{July 2012 Site 1}

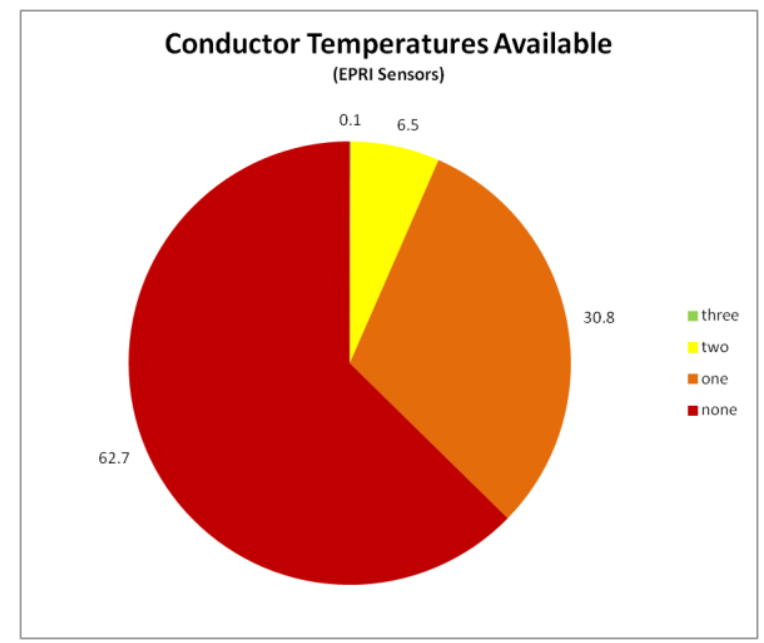

Figure L-430

\section{August 2012 Site 1}

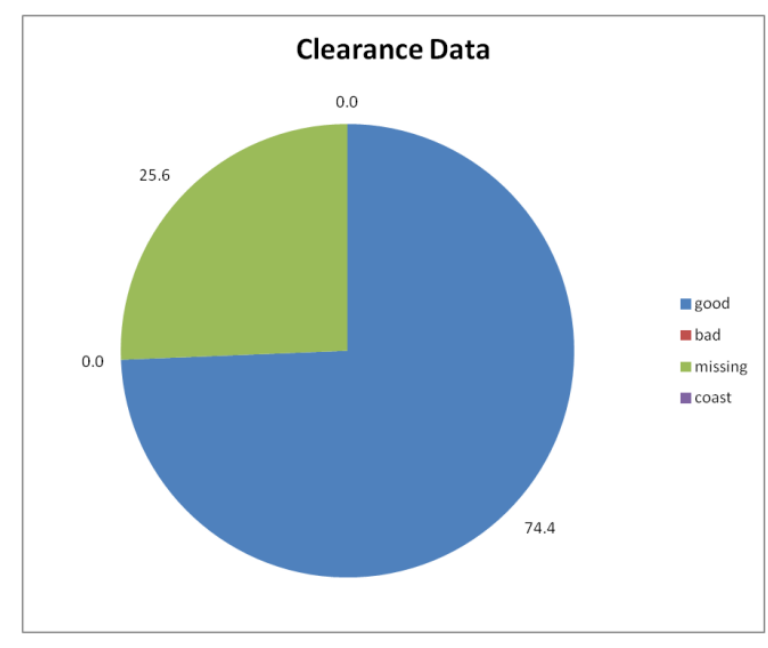

Figure L-431

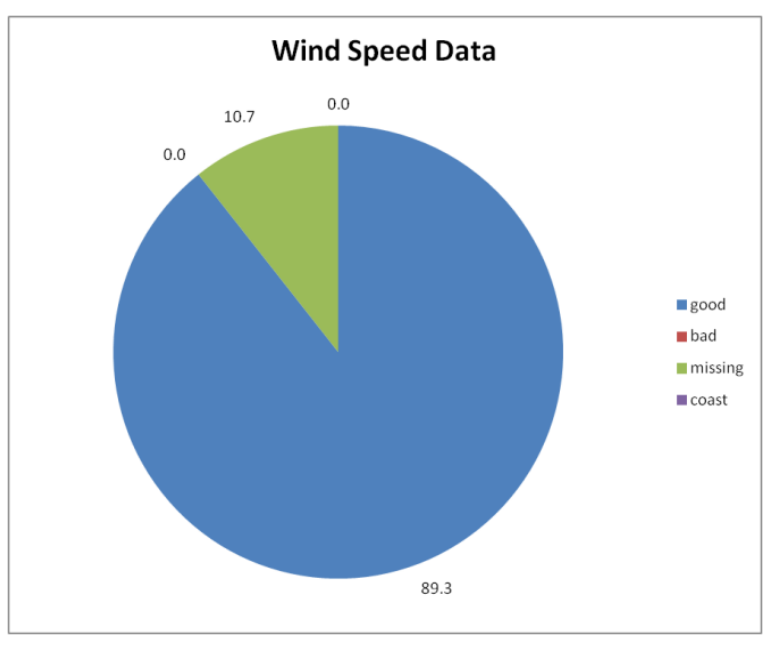

Figure L-432 


\section{August 2012 Site 1}

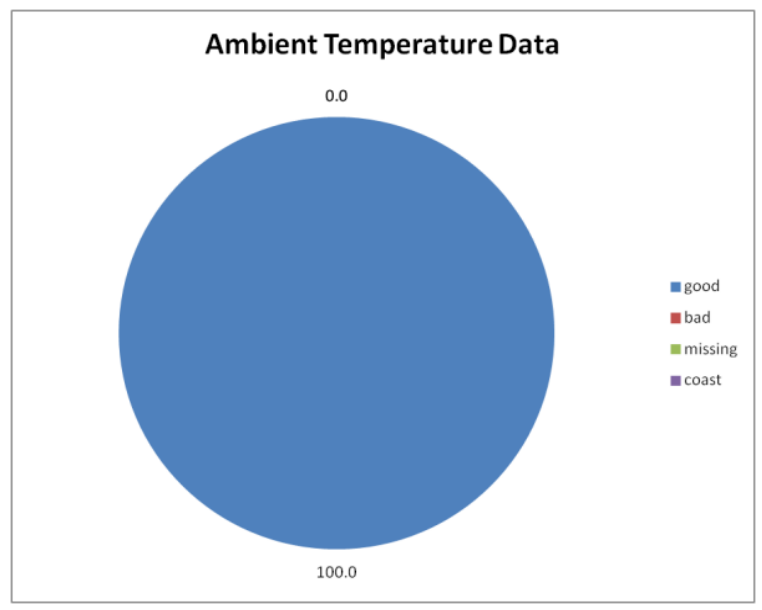

Figure L-433

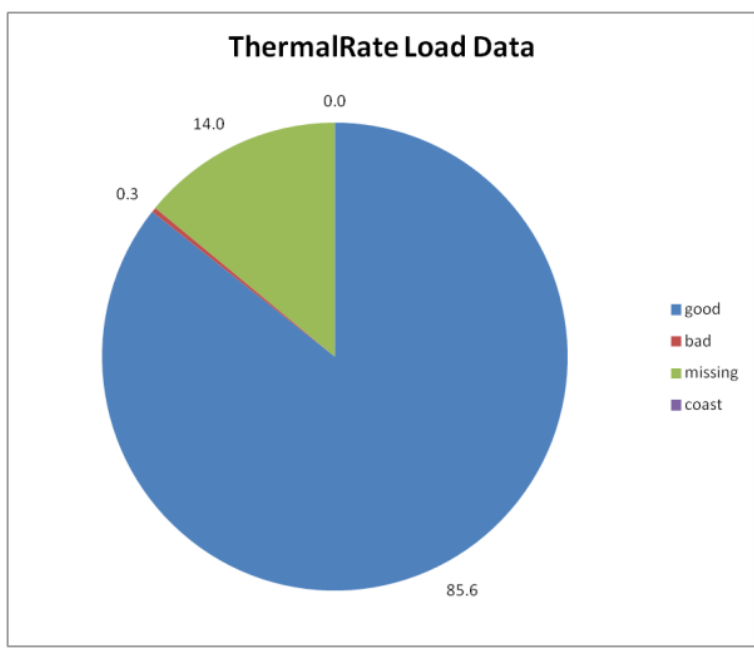

Figure L-435

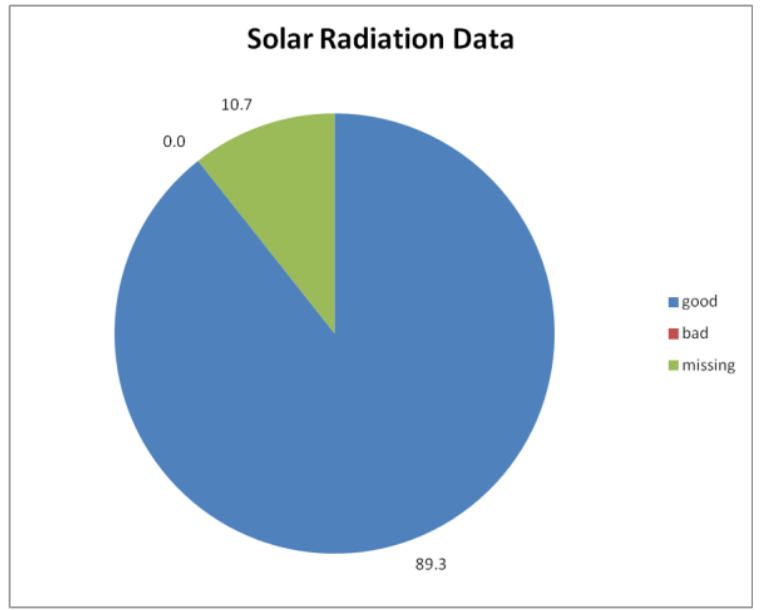

Figure L-434

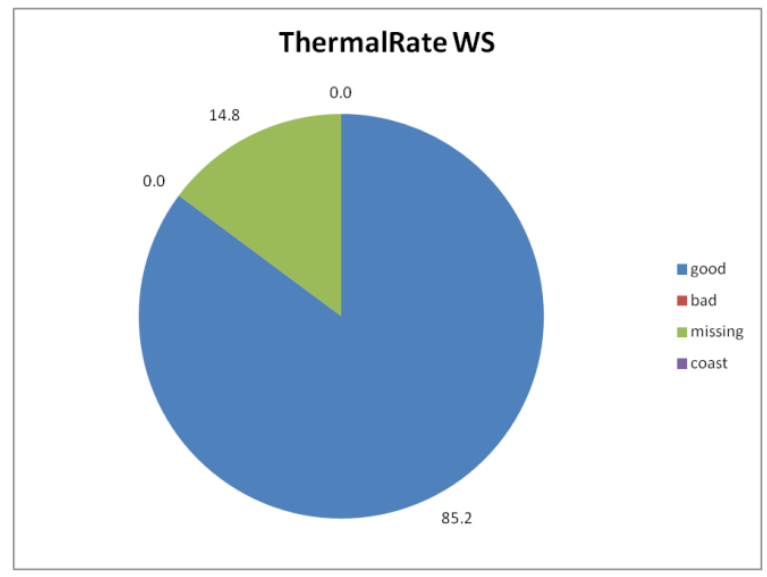

Figure L-436 


\section{August 2012 Site 1}

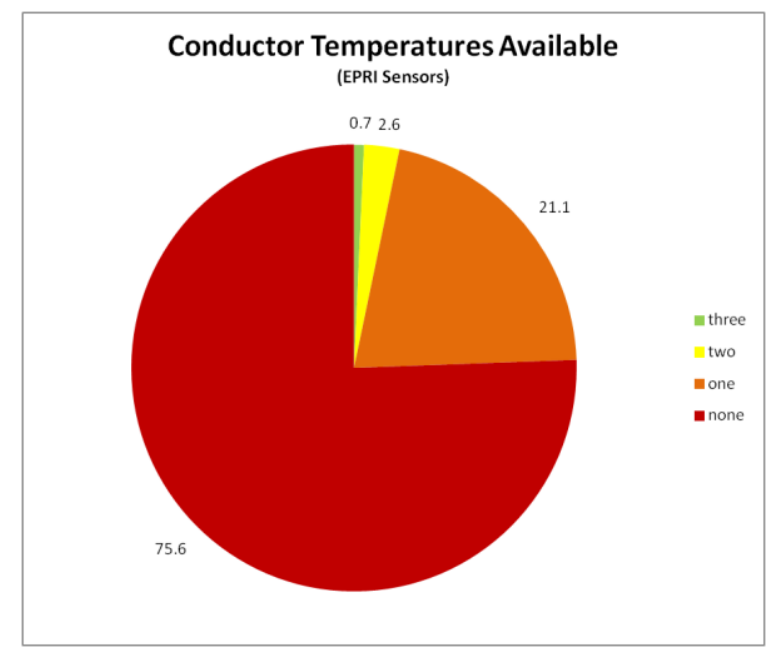

Figure L-437

\section{September 2012 Site 1}

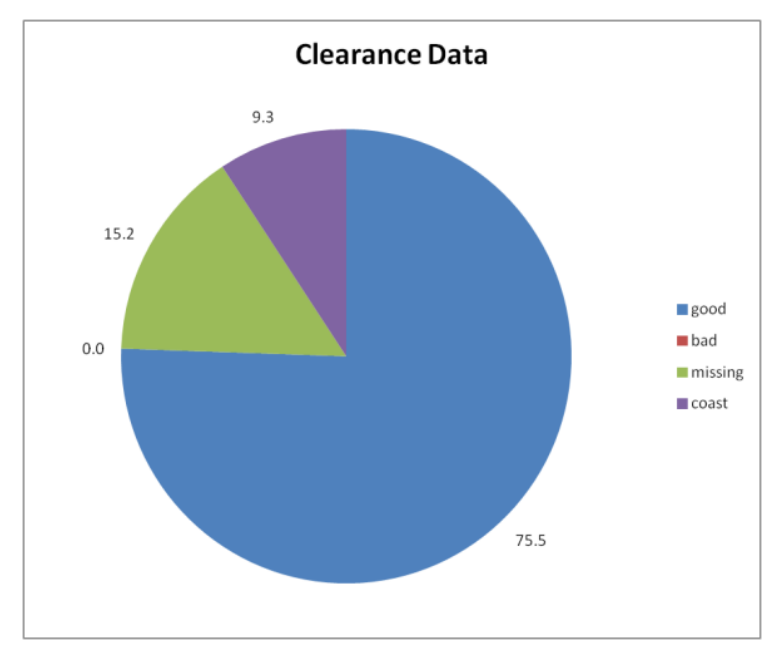

Figure L-438

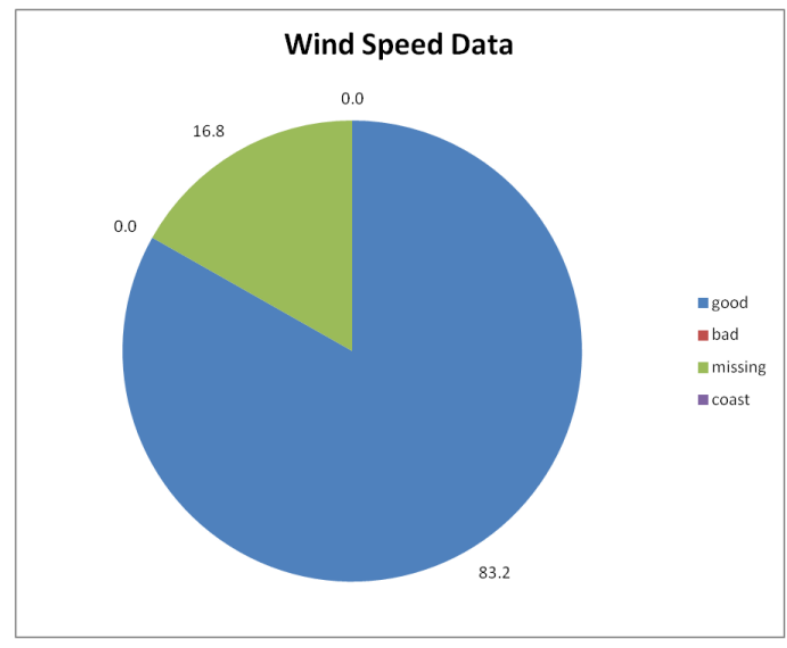

Figure L-439 
September 2012 Site 1

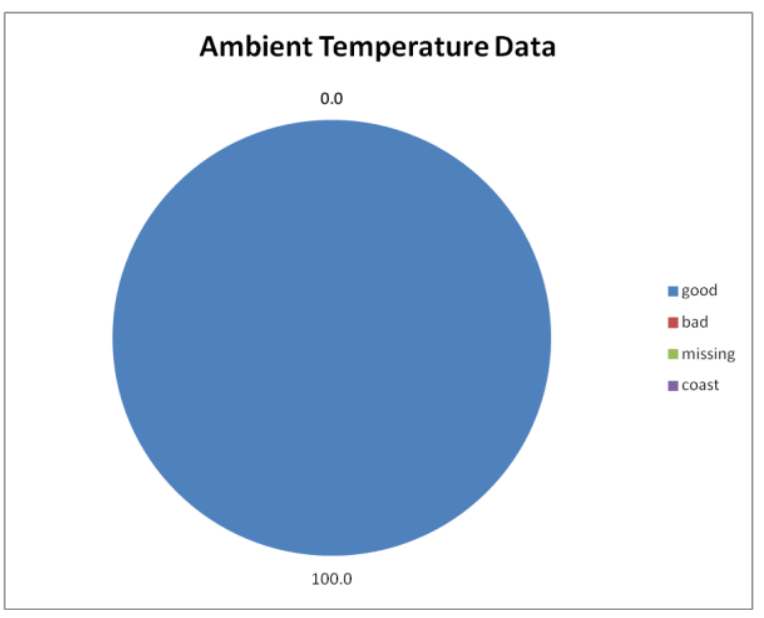

Figure L-440

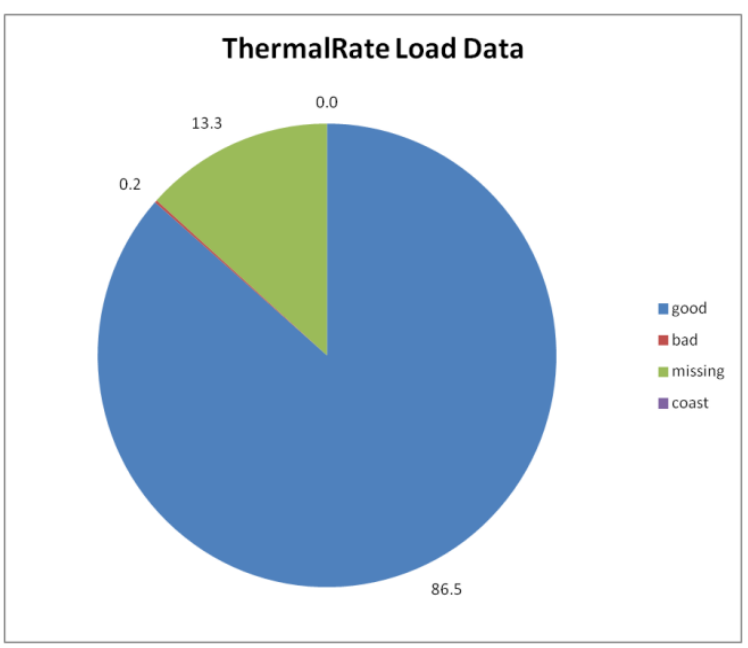

Figure L-442

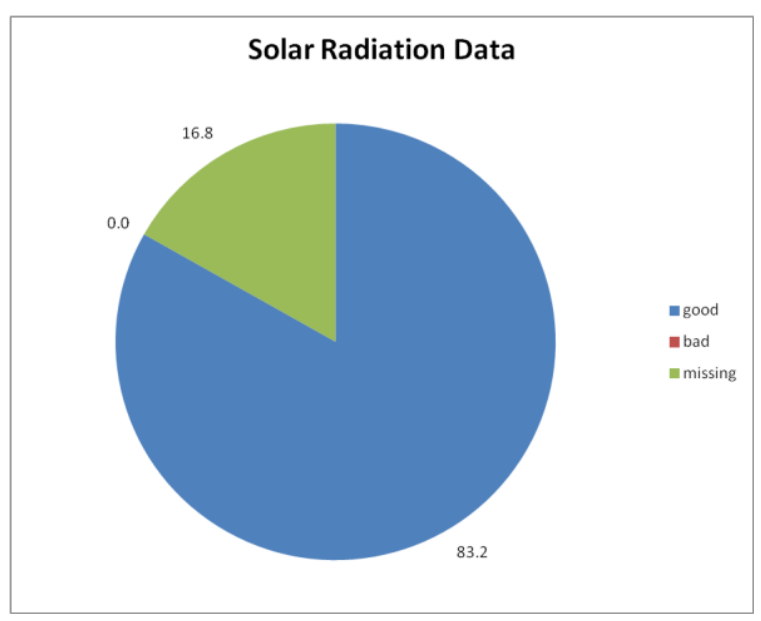

Figure L-441

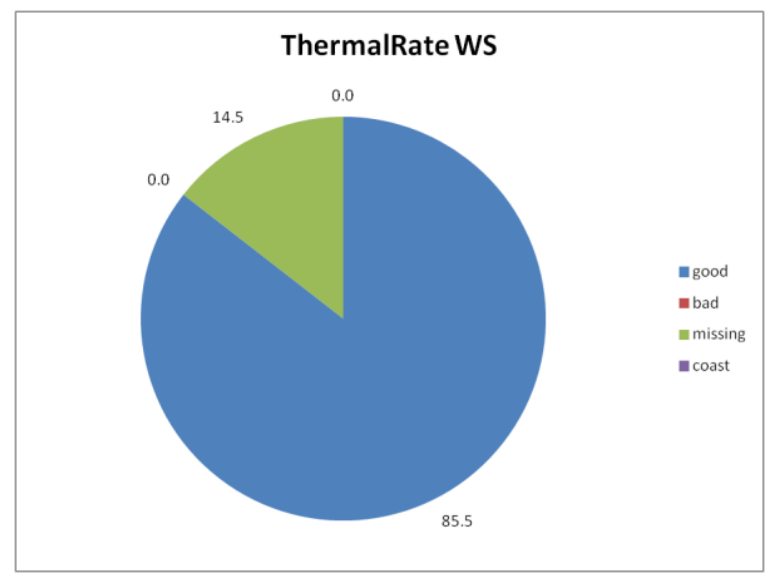

Figure L-443 


\section{September 2012 Site 1}

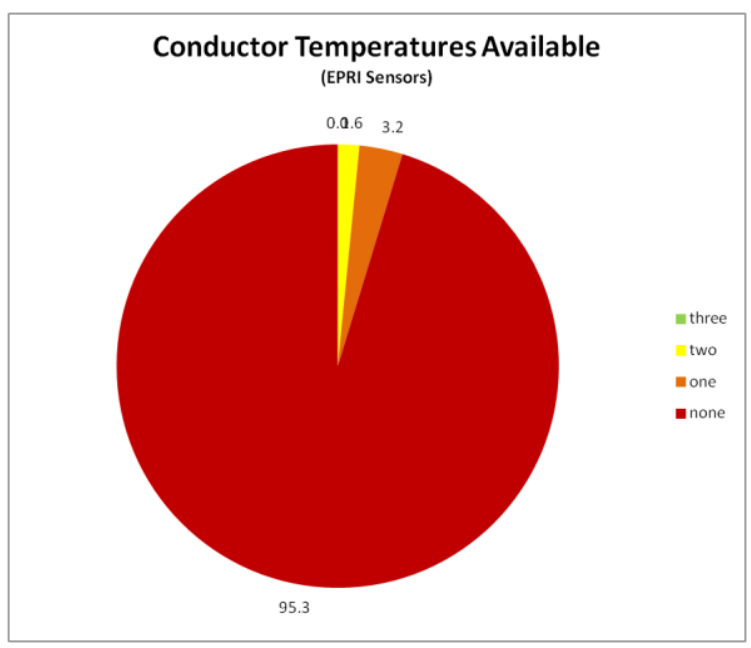

Figure L-444

\section{October 2012 Site 1}

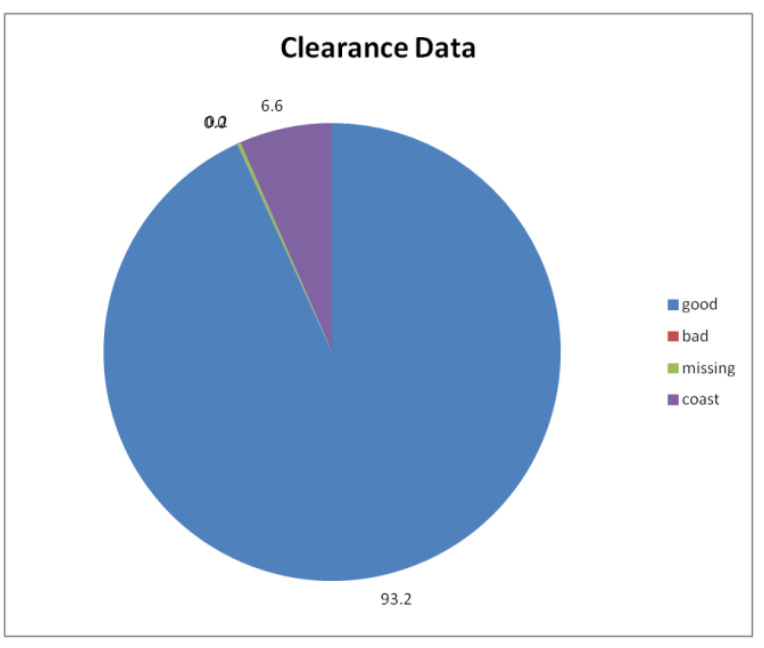

Figure L-445

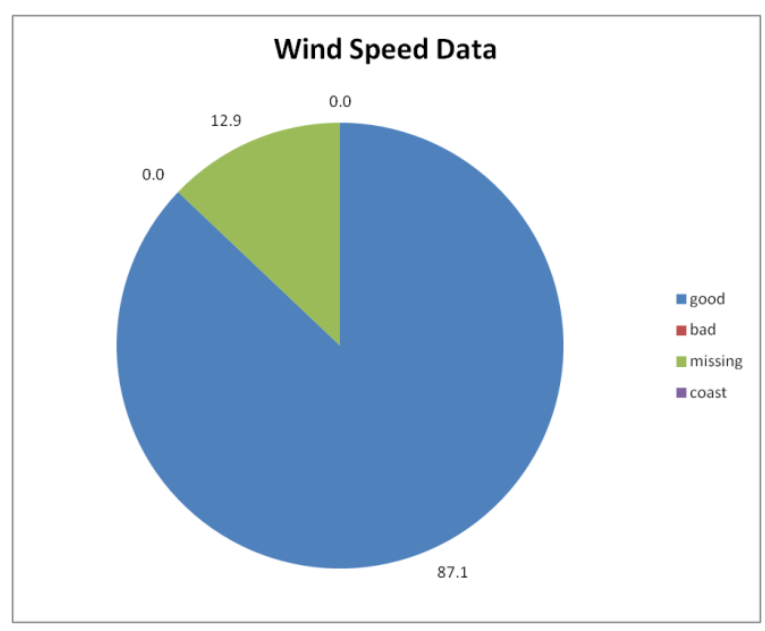

Figure L-446 


\section{October 2012 Site 1}

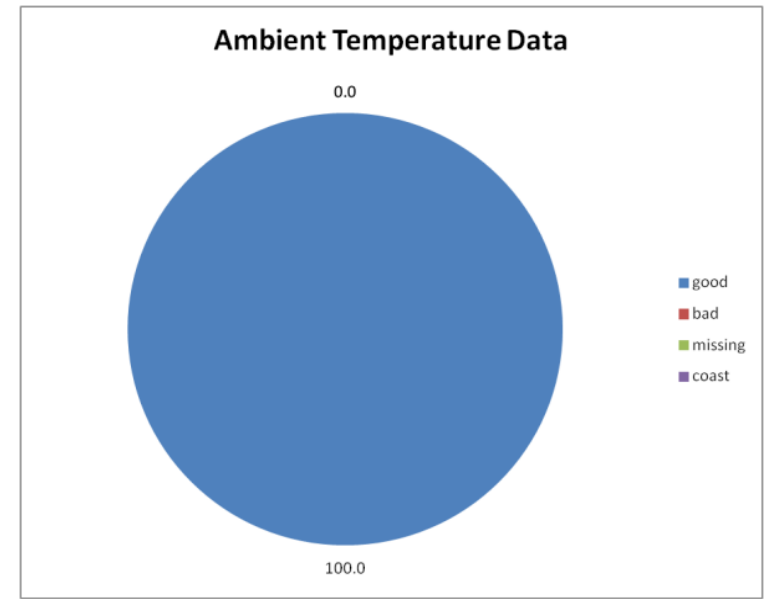

Figure L-447

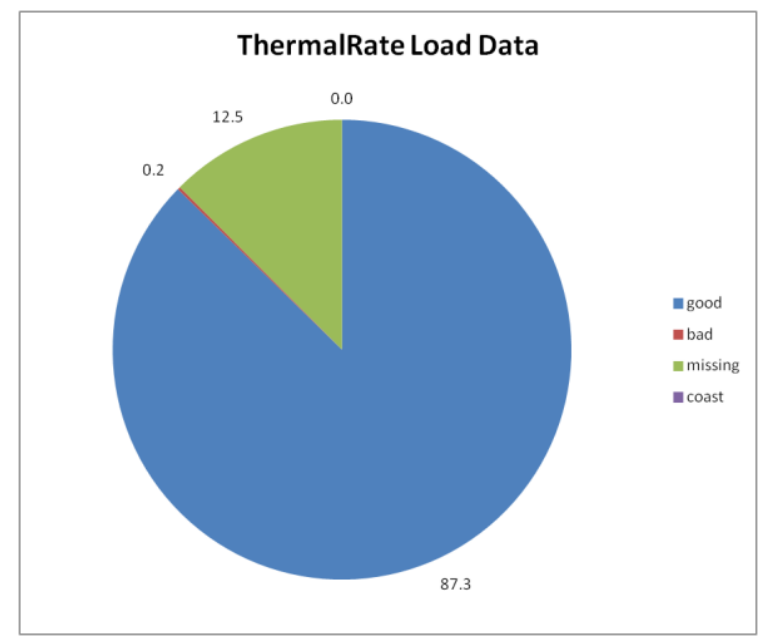

Figure L-449

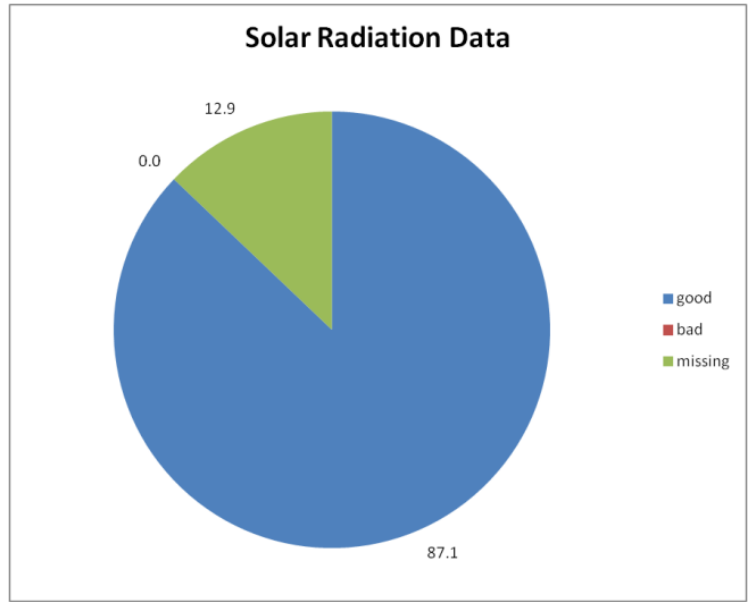

Figure L-448

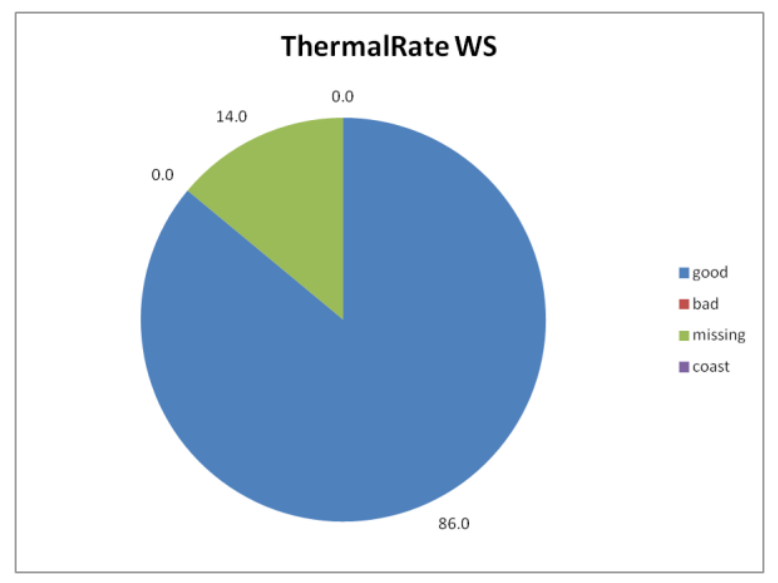

Figure L-450 


\section{October 2012 Site1}

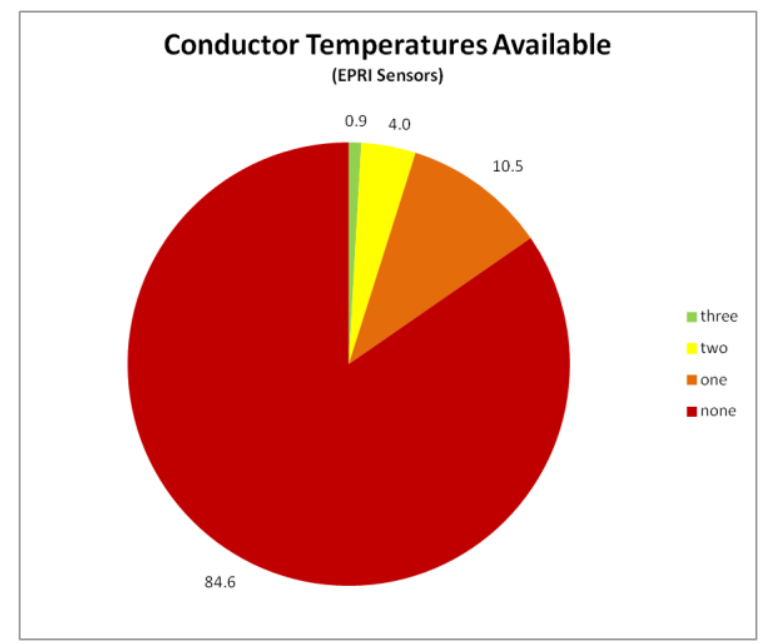

Figure L-451 